\title{
J.A. Bittencourt
}

\section{FUNDAMENTALS OF}
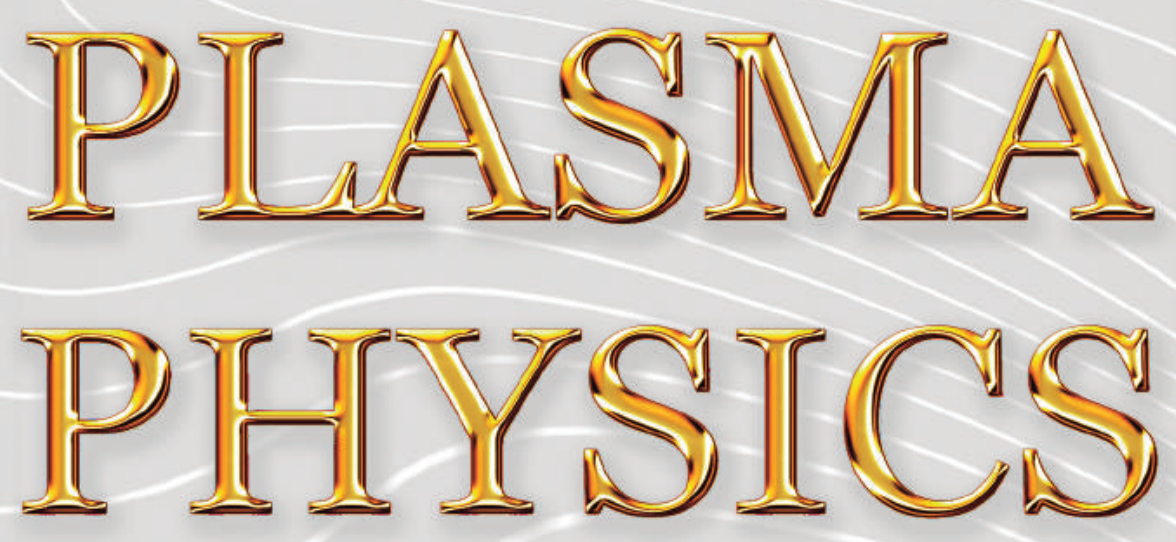

FOURTH EDITION 

Fundamentals of

\section{Plasma Physics}





\section{J. A. Bittencourt}

\section{Fundamentals of}

\section{Plasma Physics}

Fourth Edition 


\section{FUNDAMENTALS OF PLASMA PHYSICS}

J. A. Bittencourt, Ph.D.

Research Scientist and Professor

National Institute for Space Research - INPE

Av. Dos Astronautas, 1758

12227-010 São José dos Campos, SP, Brazil

www.inpe.br

FOURTH EDITION - 2018

Revised edition

(First Edition - 1986; Second Edition - 1995; Third Edition - 2004) 


\section{Preface}

This text is intended as a general introduction to plasma physics and was designed with the main purpose of presenting a comprehensive, logical, and unified treatment of the fundamentals of plasma physics based on statistical kinetic theory. It should be useful primarily for advanced undergraduate and first-year graduate students meeting the subject of plasma physics for the first time and presupposes only a basic elementary knowledge of vector analysis, differential equations, and complex variables, as well as courses on classical mechanics and electromagnetic theory beyond sophomore level. Some effort has been made to make the book self-contained by including in the text developments of fluid mechanics and kinetic theory that are needed.

Throughout the text the emphasis is on clarity, rather than formality. The various derivations are explained in detail and, wherever possible, the physical interpretations are emphasized. The equations are presented in such a way that they connect together, without requiring the reader to do extensive algebra to bridge the gap. The features of clarity and completeness make the book suitable for self-learning and for self-paced courses.

The structure of this book is as follows. Chapter 1 consists of a basic introduction to plasma physics, at a descriptive level, intended to give the reader an overall view of the subject. The motion of charged particles under the influence of specified electric and magnetic fields is treated in detail in Chapters 2, 3, and 4. In the next five chapters the fundamental equations necessary for an elementary description of plasma 
phenomena are developed. Chapter 5 introduces the concepts of phase space and distribution function, and derives the basic differential kinetic equation that governs the evolution of the distribution function in phase space. The definitions of the macroscopic variables in terms of the phase space distribution function are presented in Chapter 6 and their physical interpretations are discussed. The Maxwell-Boltzmann equilibrium distribution function is introduced in Chapter 7, as the equilibrium solution of the Boltzmann equation, and its kinetic properties are analyzed in some detail. In Chapter 8 the macroscopic transport equations for a plasma considered as a mixture of various interpenetrating fluids are derived, whereas the macroscopic transport equations for the whole plasma as a single conducting fluid are developed in Chapter 9.

The remainder of the book is devoted to applications of these basic equations in the description of a variety of important phenomena in plasmas. The problems of electrical conductivity and diffusion in plasmas are analyzed in Chapter 10, and other basic plasma phenomena, such as electron plasma oscillations and Debye shielding, are treated in Chapter 11. Simple applications of the magnetohydrodynamic equations, such as in plasma confinement by magnetic fields and the pinch effect, are presented in Chapters 12 and 13. The subject of wave phenomena in plasmas is organized in the next six chapters. A review of the basic concepts related to electromagnetic wave propagation in free space is given in Chapter 14 . The propagation of very low frequency waves in a highly conducting fluid is analyzed in Chapter 15, under the title of magnetohydrodynamic waves. The various modes of wave propagation in cold and warm plasmas are considered in Chapters 16 and 17, respectively. In Chapters 18 and 19 the various properties of wave propagation in hot nonmagnetized plasmas and in hot magnetized plasmas, respectively, are analyzed. Collisional phenomena in plasmas are treated in Chapter 20, and the derivations of the Boltzmann collision integral and of the Fokker-Planck collision term are presented in Chapter 21. Finally, in Chapter 22 some applications of the Boltzmann equation to the analysis of transport phenomena in plasmas are presented.

Problems are provided at the end of each chapter, which illustrate additional applications of the theory and supplement the textual material. Most of the problems are designed in such a way as to provide a guideline for the student, including intermediate steps and answers in their statements.

The numbering of the equations, within each chapter, starts over again at each section. When reference is made to an equation using three 
numbers, the first number indicates the chapter and the last two numbers indicate the section and the equation, respectively. Within the same chapter the first number is omitted. Vector quantities are represented by boldface type letters (such as $\mathbf{r}$ ) and unit vectors by a circumflex above the corresponding letter ( $\operatorname{such}$ as $\widehat{\mathbf{r}}$ ). Dyadic and triadic quantities are represented by calligraphic type letters (such as $\mathcal{Q}$ ).

The system of units used in this text is the rationalized MKSA. This system is based on four primary quantities: length, mass, time, and current. Its name derives from the units meter $(\mathrm{m})$, kilogram $(\mathrm{kg})$, second (s), and ampere (A).

The book contains more material than what can normally be covered in one semester. This permits some freedom in the selection of topics depending on the level and desired emphasis of the course, and on the interests of the students. The whole text can also be adequately covered within two semesters.

In this, as in any introductory book, the topics included clearly do not cover all areas of plasma physics. No attempt was made to present the experimental aspects of the subject. Moreover, there are some important theoretical topics that are covered only very briefly and some that have been left for more advanced courses on plasma physics, such as plasma instabilities, plasma radiation, nonlinear plasma theory, and plasma turbulence.

I am grateful to the many people who contributed to this book, both directly and indirectly, and especially to the many students to whom I had the opportunity to test my ideas in the various courses I taught over the last forty years. The amount of digitalized information in a book such as this is truly enormous, and some minor errors may be bound to occur. Further feedback from readers will be appreciated. I wish to thank the many professors, students, and researchers who have used the previous editions of this book, all over the world, and contributed to its improvement. 



\section{Contents}

PREFACE V v

1. INTRODUCTION 1

1. General Properties of Plasmas 1

1.1 Definition of a Plasma 1

1.2 Plasma as the Fourth State of Matter 1

1.3 Plasma Production 2

1.4 Particle Interactions and Collective Effects 3

1.5 Some Basic Plasma Phenomena 4

2. Criteria for the Definition of a Plasma 6

2.1 Macroscopic Neutrality 6

$\begin{array}{lll}2.2 & \text { Debye Shielding } & 7\end{array}$

2.3 The Plasma Frequency 9

3. The Occurrence of Plasmas in Nature $\quad 11$

3.1 The Sun and its Atmosphere 13

3.2 The Solar Wind 13

3.3 The Magnetosphere and the Van Allen Radiation Belts 14

$\begin{array}{ll}3.4 & \text { The Ionosphere } \\ 3.5\end{array}$

3.5 Plasmas Beyond the Solar System 17

4. Applications of Plasma Physics $\quad 17$

4.1 Controlled Thermonuclear Fusion 18

4.2 The Magnetohydrodynamic Generator $\quad 22$

4.3 Plasma Propulsion 23

4.4 Other Plasma Devices 23 
5. Theoretical Description of Plasma Phenomena

5.1 General Considerations on a Self-Consistent Formulation 25

5.2 Theoretical Approaches 27

$\begin{array}{ll}\text { Problems } & 28\end{array}$

2. CHARGED PARTICLE MOTION IN CONSTANT AND UNIFORM ELECTROMAGNETIC FIELDS

1. Introduction

2. Energy Conservation $\quad 34$

3. Uniform Electrostatic Field 36

4. Uniform Magnetostatic Field 37

4.1 Formal Solution of the Equation of Motion 37

4.2 Solution in Cartesian Coordinates 40

4.3 Magnetic Moment 44

4.4 Magnetization Current 46

5. Uniform Electrostatic and Magnetostatic Fields 49

5.1 Formal Solution of the Equation of Motion 49

5.2 Solution in Cartesian Coordinates 52

6. Drift Due to an External Force 54

$\begin{array}{ll}\text { Problems } & 56\end{array}$

\section{CHARGED PARTICLE MOTION IN NONUNIFORM MAGNETOSTATIC FIELDS

1. Introduction $\quad 59$

2. Spatial Variation of the Magnetic Field 61

2.1 Divergence Terms 62

2.2 Gradient and Curvature Terms 64

2.3 Shear Terms 65

3. Equation of Motion in the First-Order Approximation 66

4. Average Force Over One Gyration Period 68

4.1 Parallel Force $\quad 70$

$\begin{array}{lll}4.2 & \text { Perpendicular Force } & 72\end{array}$

4.3 Total Average Force $\quad 73$ 
6. Parallel Acceleration of the Guiding Center

6.1 Invariance of the Orbital Magnetic Moment and of the Magnetic Flux

6.2 Magnetic Mirror Effect

6.3 The Longitudinal Adiabatic Invariant

7. Curvature Drift

8. Combined Gradient-Curvature Drift

Problems

\section{CHARGED PARTICLE MOTION IN TIME-VARYING ELECTROMAGNETIC FIELDS}

1. Introduction

2. Slowly Time-Varying Electric Field

2.2 Plasma Dielectric Constant

3. Electric Field with Arbitrary Time Variation 100

3.1 Solution of the Equation of Motion

3.2 Physical Interpretation

3.3 Mobility Dyad

3.4 Plasma Conductivity Dyad

3.5 Cyclotron Resonance

4. Time-Varying Magnetic Field and Space-Varying Electric Field

4.1 Equation of Motion and Adiabatic Invariants

4.2 Magnetic Heating of a Plasma

5. Summary of Guiding Center Drifts and Current Densities

5.1 Guiding Center Drifts

5.2 Current Densities 


\section{ELEMENTS OF PLASMA KINETIC THEORY}

1. Introduction

2. Phase Space

2.1 Single-Particle Phase Space

2.2 Many-Particle Phase Space

2.3 Volume Elements

3. Distribution Function $\quad 126$

4. Number Density and Average Velocity 128

5. The Boltzmann Equation $\quad 129$

5.1 Collisionless Boltzmann Equation 129

5.2 Jacobian of the Transformation in Phase Space 132

5.3 Effects of Particle Interactions 133

6. Relaxation Model for the Collision Term 135

7. The Vlasov Equation 136

$\begin{array}{ll}\text { Problems } & 138\end{array}$

6. AVERAGE VALUES AND MACROSCOPIC VARIABLES 141

1. Average Value of a Physical Quantity 141

2. Average Velocity and Peculiar Velocity 142

3. Flux 143

4. Particle Current Density 146

5. Momentum Flow Dyad or Tensor 147

6. Pressure Dyad or Tensor 148

6.1 Concept of Pressure 148

6.2 Force per Unit Area 149

6.3 Force per Unit Volume 151

6.4 Scalar Pressure and Absolute Temperature 152

7. Heat Flow Vector $\quad 154$

8. Heat Flow Triad 154

9. Total Energy Flux Triad 155 
10. Higher Moments of the Distribution Function

1. The Equilibrium State Distribution Function

1.1 The General Principle of Detailed Balance and Binary Collisions

1.2 Summation Invariants

1.3 Maxwell-Boltzmann Distribution Function

1.4 Determination of the Constant Coefficients

1.5 Local Maxwell-Boltzmann Distribution Function

2. The Most Probable Distribution

3. Mixture of Various Particle Species

4. Properties of the Maxwell-Boltzmann Distribution Function

4.1 Distribution of a Velocity Component 171

4.2 Distribution of Speeds 174

4.3 Mean Values Related to the Molecular Speeds 176

4.4 Distribution of Thermal Kinetic Energy 178

4.5 Random Particle Flux 178

4.6 Kinetic Pressure and Heat Flux 181

5. Equilibrium in the Presence of an External Force 181

6. Degree of Ionization in Equilibrium and the Saha Equation

\section{MACROSCOPIC TRANSPORT EQUATIONS}

1. Moments of the Boltzmann Equation

2. General Transport Equation

3. Conservation of Mass

3.2 Derivation by the Method of Fluid Dynamics

3.3 The Collision Term 
4. Conservation of Momentum 200

4.1 Derivation of the Equation of Motion 200

4.2 The Collision Term 203

5. Conservation of Energy 204

5.1 Derivation of the Energy Transport Equation 204

5.2 Physical Interpretation 207

5.3 Simplifying Approximations 207

6. The Cold Plasma Model 210

7. The Warm Plasma Model 211

$\begin{array}{ll}\text { Problems } & 212\end{array}$

9. MACROSCOPIC EQUATIONS FOR A CONDUCTING FLUID 219

1. Macroscopic Variables for a

Plasma as a Conducting Fluid $\quad 219$

2. Continuity Equation 222

3. Equation of Motion 223

4. Energy Equation $\quad 224$

5. Electrodynamic Equations for a Conducting Fluid $\quad 227$ 5.1 Maxwell Curl Equations 228

5.2 Conservation of Electric Charge 228

5.3 Generalized Ohm's Law 229

6. Simplified Magnetohydrodynamic Equations 234

$\begin{array}{ll}\text { Problems } & 236\end{array}$

\section{PLASMA CONDUCTIVITY AND DIFFUSION 238}

1. Introduction $\quad 238$

2. The Langevin Equation 238

3. Linearization of the Langevin Equation 240

4. DC Conductivity and Electron Mobility 242

4.1 Isotropic Plasma 242

4.2 Anisotropic Magnetoplasma 243 
5. AC Conductivity and Electron Mobility 247

6. Conductivity with Ion Motion 249

7. Plasma as a Dielectric Medium 250

8. Free Electron Diffusion 251

9. Electron Diffusion in a Magnetic Field 254

10. Ambipolar Diffusion $\quad 256$

11. Diffusion in a Fully Ionized Plasma 260

$\begin{array}{ll}\text { Problems } & 262\end{array}$

\section{SOME BASIC PLASMA PHENOMENA}

1. Electron Plasma Oscillations $\quad 269$

2. The Debye Shielding Problem 273

3. Debye Shielding Using the Vlasov Equation 278

4. Plasma Sheath $\quad 279$

4.1 Physical Mechanism 280

4.2 Electric Potential on the Wall 281

4.3 Inner Structure of the Plasma Sheath 282

5. Plasma Probe 288

$\begin{array}{ll}\text { Problems } & 291\end{array}$

12. SIMPLE APPLICATIONS OF MAGNETOHYDRODYNAMICS 299

1. Fundamental Equations of Magnetohydrodynamics 299

1.1 Parker Modified Momentum Equation 300

1.2 The Double Adiabatic Equations of Chew, Goldberger, and Low (CGL) 302

1.3 Special Cases of the Double Adiabatic Equations 305

1.4 Energy Integral 307

2. Magnetic Viscosity and Reynolds Number 309

3. Diffusion of Magnetic Field Lines 311 
4. Freezing of Magnetic Field Lines to the Plasma

5. Magnetic Pressure $\quad 316$

6. Isobaric Surfaces 318

7. Plasma Confinement in a Magnetic Field 319 Problems

\section{THE PINCH EFFECT}

1. Introduction

2. The Equilibrium Pinch

3. The Bennett Pinch

4. Dynamic Model of the Pinch

5. Instabilities in a Pinched Plasma Column

6. The Sausage Instability

7. The Kink Instability

8. Convex Field Configurations

\section{ELECTROMAGNETIC WAVES IN FREE SPACE}

1. The Wave Equation

2. Solution in Plane Waves

3. Harmonic Waves

4. Polarization

5. Energy Flow

6. Wave Packets and Group Velocity 
1. Introduction

375

1.1 Alfvén Waves

376

1.2 Magnetosonic Waves

377

2. MHD Equations for a Compressible

Nonviscous Conducting Fluid

2.1 Basic Equations $\quad 379$

2.2 Development of an Equation for the Fluid Velocity 381

3. Propagation Perpendicular to the Magnetic Field 382

4. Propagation Parallel to the Magnetic Field 383

5. Propagation at Arbitrary Directions $\quad 384$

5.1 Pure Alfvén Wave 386

5.2 Fast and Slow MHD Waves 387

5.3 Phase Velocities 387

5.4 Wave Normal Surfaces 388

6. Effect of Displacement Current 390

6.1 Basic Equations 392

6.2 Equation for the Fluid Velocity 392

6.3 Propagation Across the Magnetostatic Field 393

6.4 Propagation Along the Magnetostatic Field 393

7. Damping of MHD Waves 394

7.1 Alfvén Waves 395

7.2 Sound Waves 396

7.3 Magnetosonic Waves 396

$\begin{array}{ll}\text { Problems } & 397\end{array}$

16. WAVES IN COLD PLASMAS 400

1. Introduction 400

2. Basic Equations of Magnetoionic Theory 401

3. Plane Wave Solutions and Linearization 402

4. Wave Propagation in Isotropic Electron Plasmas 403

4.1 Derivation of the Dispersion Relation 403

4.2 Collisionless Plasma 405 
4.3 Time-Averaged Poynting Vector 407

4.4 The Effect of Collisions 410

5. Wave Propagation in Magnetized Cold Plasmas 413

5.1 Derivation of the Dispersion Relation 414

5.2 The Appleton-Hartree Equation 418

6. Propagation Parallel to $B_{0} \quad 419$

7. Propagation Perpendicular to $B_{0}$

8. Propagation at Arbitrary Directions 430

8.1 Resonances and Reflection Points 430

8.2 Wave Normal Surfaces 432

8.3 The CMA Diagram 434

9. Some Special Wave Phenomena in Cold Plasmas 439

9.1 Atmospheric Whistlers 439

9.2 Helicons 442

9.3 Faraday Rotation 444

$\begin{array}{ll}\text { Problems } & 447\end{array}$

\section{WAVES IN WARM PLASMAS 453}

1. Introduction 453

2. Waves in a Fully Ionized Isotropic Warm Plasma 453

2.1 Derivation of the Equations

for the Electron and Ion Velocities 453

2.2 Longitudinal Waves 456

2.3 Transverse Wave 458

3. Basic Equations for Waves in a Warm Magnetoplasma 460

4. Waves in a Warm Electron Gas in a Magnetic Field 462

4.1 Derivation of the Dispersion Relation 462

4.2 Wave Propagation Along the Magnetic Field 463

4.3 Wave Propagation Normal to the Magnetic Field 466

4.4 Wave Propagation at Arbitrary Directions 469

5. Waves in a Fully Ionized Warm Magnetoplasma 470

5.1 Derivation of the Dispersion Relation 471

5.2 Wave Propagation Along the Magnetic Field 473

5.3 Wave Propagation Normal to the Magnetic Field 477 


\section{WAVES IN HOT ISOTROPIC PLASMAS}

\section{Introduction}

2. Basic Equations

3. General Results for a Plane Wave in a Hot Isotropic Plasma

3.1 Perturbation Charge Density and Current Density

3.2 Solution of the Linearized Vlasov Equation

3.3 Expression for the Current Density

3.4 Separation into the Various Modes

\section{Electrostatic Longitudinal Wave} in a Hot Isotropic Plasma

4.1 Development of the Dispersion Relation

4.2 Limiting Case of a Cold Plasma

4.3 High Phase Velocity Limit

4.4 Dispersion Relation for

Maxwellian Distribution Function

5. Transverse Wave in a Hot Isotropic Plasma

5.1 Development of the Dispersion Relation

5.2 Cold Plasma Result

5.3 Dispersion Relation for Maxwellian Distribution Function

5.4 Landau Damping of the Transverse Wave

6. The Two-Stream Instability

7. Summary

7.2 Transverse Mode 


\section{WAVES IN HOt MAGNETIZED PLASMAS}

1. Introduction

2. Wave Propagation Along the

Magnetostatic Field in a Hot Plasma

516

2.1 Linearized Vlasov Equation $\quad 516$

2.2 Solution of the Linearized Vlasov Equation 517

2.3 Perturbation Current Density 522

2.4 Separation into the Various Modes 524

2.5 Longitudinal Plasma Wave 525

2.6 Transverse Electromagnetic Waves 526

2.7 Temporal Damping of the

Transverse Electromagnetic Waves 529

2.8 Cyclotron Damping of the RCP Transverse Wave 531

2.9 Instabilities in the RCP Transverse Wave 532

3. Wave Propagation Across the

Magnetostatic Field in a Hot Plasma

534

3.1 Solution of the Linearized Vlasov Equation 536

3.2 Current Density and the Conductivity Tensor 538

3.3 Evaluation of the Integrals 540

3.4 Separation into the Various Modes 544

3.5 Dispersion Relations $\quad 545$

3.6 The Quasistatic Mode 546

3.7 The TEM Mode 550

4. Summary 552

4.1 Propagation Along $\mathbf{B}_{0}$ in Hot Magnetoplasmas 552

4.2 Propagation Across $\mathbf{B}_{0}$ in Hot Magnetoplasmas 553

Problems $\quad 554$

\section{PARTICLE INTERACTIONS IN PLASMAS 560}

1. Introduction 560

2. Binary Collisions 561

3. Dynamics of Binary Collisions 566

4. Evaluation of the Scattering Angle $\quad 569$

4.1 Two Perfectly Elastic Hard Spheres 570 
5. Cross Sections

5.1 Differential Scattering Cross Section

5.2 Total Scattering Cross Section

576

5.3 Momentum Transfer Cross Section

6. Cross Sections for the Hard Sphere Model 578

6.1 Differential Scattering Cross Section 578

6.2 Total Scattering Cross Section $\quad 579$

6.3 Momentum Transfer Cross Section 579

7. Cross Sections for the Coulomb Potential 580

7.1 Differential Scattering Cross Section 580

7.2 Total Scattering Cross Section 581

7.3 Momentum Transfer Cross Section 581

8. Screening of the Coulomb Potential 582

$\begin{array}{ll}\text { Problems } & 586\end{array}$

\section{THE BOLTZMANN AND THE FOKKER-PLANCK EQUATIONS 589}

1. Introduction $\quad 589$

2. The Boltzmann Equation $\quad \mathbf{5 9 0}$

2.1 Derivation of the Boltzmann Collision Integral 590

2.2 Jacobian of the Transformation 594

2.3 Assumptions in the Derivation of the Boltzmann Collision Integral 596

2.4 Rate of Change of a Physical Quantity as a Result of Collisions $\quad 597$

3. The Boltzmann's H Function 598

3.1 Boltzmann's H Theorem 599

3.2 Analysis of Boltzmann's H Theorem 601

3.3 Maximum Entropy or Minimum H Approach for Deriving the Equilibrium Distribution Function 604

3.4 Mixture of Various Particle Species 606

4. Boltzmann Collision Term for a Weakly Ionized Plasma 
4.1 Spherical Harmonic Expansion of the Distribution Function

4.2 Approximate Expression for the Boltzmann Collision Term

4.3 Rate of Change of Momentum Due to Collisions

5. The Fokker-Planck Equation

5.1 Derivation of the Fokker-Planck Collision Term

5.2 The Fokker-Planck Coefficients for Coulomb Interactions

5.3 Application to Electron-Ion Collisions

\section{TRANSPORT PROCESSES IN PLASMAS}

1. Introduction

2. Electric Conductivity in a Nonmagnetized Plasma

2.1 Solution of the Boltzmann Equation

2.2 Electric Current Density and Conductivity

2.3 Conductivity for Maxwellian Distribution Function

633

3. Electric Conductivity in a Magnetized Plasma

3.1 Solution of Boltzmann Equation

3.2 Electric Current Density and Conductivity

4.1 Perturbation Distribution Function

4.2 Particle Flux

641

4.3 Free Diffusion Coefficient

5. Diffusion in a Magnetic Field

5.2 Particle Flux and Diffusion Coefficients

6.1 General Expression for the Heat Flow Vector 647

6.2 Thermal Conductivity for a Constant Kinetic Pressure 648

6.3 Thermal Conductivity for the Adiabatic Case 649 
APPENDIX A

Useful Vector Relations

655

APPENDIX B

Useful Relations in Cartesian and in Curvilinear Coordinates

APPENDIX C

Physical Constants (MKSA)

APPENDIX D

Conversion Factors for Physical Units

\section{APPENDIX E}

Some Important Plasma Parameters

APPENDIX F

Approximate Magnitudes in Some Typical Plasmas 



\section{$\mathbb{1}$}

\section{INTRODUCTION}

\section{GENERAL PROPERTIES OF PLASMAS}

\subsection{Definition of a Plasma}

The word plasma is used to describe a wide variety of macroscopically neutral substances containing many interacting free electrons and ionized atoms or molecules, which exhibit collective behavior due to the long-range coulomb forces. Not all media containing charged particles, however, can be classified as plasmas. For a collection of interacting charged and neutral particles to exhibit plasma behavior it must satisfy certain conditions, or criteria, for plasma existence. These criteria will be discussed in some detail in the next section.

The word plasma comes from the Greek and means something molded. It was applied for the first time by Tonks and Langmuir, in 1929, to describe the inner region, remote from the boundaries, of a glowing ionized gas produced by electric discharge in a tube, the ionized gas as a whole remaining electrically neutral.

\subsection{Plasma as the Fourth State of Matter}

From a scientific point of view, matter in the known universe is often classified in terms of four states: solid, liquid, gaseous, and plasma. The basic distinction among solids, liquids, and gases lies in the difference between the strength of the bonds that hold their constituent particles 
together. These binding forces are relatively strong in a solid, weak in a liquid, and essentially almost absent in the gaseous state. Whether a given substance is found in one of these states depends on the random kinetic energy (thermal energy) of its atoms or molecules, i.e., on its temperature. The equilibrium between this particle thermal energy and the interparticle binding forces determines the state.

By heating a solid or liquid substance, the atoms or molecules acquire more thermal kinetic energy until they are able to overcome the binding potential energy. This leads to phase transitions, which occur at a constant temperature for a given pressure. The amount of energy required for the phase transition is called the latent heat.

If sufficient energy is provided, a molecular gas will gradually dissociate into an atomic gas as a result of collisions between those particles whose thermal kinetic energy exceeds the molecular binding energy. At sufficiently elevated temperatures an increasing fraction of the atoms will possess enough kinetic energy to overcome, by collisions, the binding energy of the outermost orbital electrons, and an ionized gas or plasma results. However, this transition from a gas to a plasma is not a phase transition in the thermodynamic sense, since it occurs gradually with increasing temperature.

\subsection{Plasma Production}

A plasma can be produced by raising the temperature of a substance until a reasonably high fractional ionization is obtained. Under thermodynamic equilibrium conditions, the degree of ionization and the electron temperature are closely related. This relation is given by the Saha equation (see Chapter 7). Although plasmas in local thermodynamic equilibrium are found in many places in nature, as is the case for many astrophysical plasmas, they are not very common in the laboratory.

Plasmas can also be generated by ionization processes that raise the degree of ionization much above its thermal equilibrium value. There are many different methods of creating plasmas in the laboratory and, depending on the method, the plasma may have a high or low density, high or low temperature, it may be steady or transient, stable or unstable, and so on. In what follows, a brief description is presented of the most commonly known processes of photoionization and electric discharge in gases.

In the photoionization process, ionization occurs by absorption of incident photons whose energy is equal to, or greater than, the ionization 
potential of the absorbing atom. The excess energy of the photon is transformed into kinetic energy of the electron-ion pair formed. For example, the ionization potential energy for the outermost electron of atomic oxygen is $13.6 \mathrm{eV}$, which can be supplied by radiation of wavelength smaller than about $91 \mathrm{~nm}$, i.e., in the far ultraviolet. Ionization can also be produced by x-rays or gamma rays, which have much smaller wavelengths. The Earth's ionosphere, for example, is a natural photoionized plasma (see section 3).

In a gas discharge, an electric field is applied across the ionized gas, which accelerates the free electrons to energies sufficiently high to ionize other atoms by collisions. One characteristic of this process is that the applied electric field transfers energy much more efficiently to the light electrons than to the relatively heavy ions. The electron temperature in gas discharges is therefore usually higher than the ion temperature, since the transfer of thermal energy from the electrons to the heavier particles is very slow.

When the ionizing source is turned off, the ionization decreases gradually because of recombination until it reaches an equilibrium value consistent with the temperature of the medium. In the laboratory the recombination usually occurs so fast that the plasma completely disappears in a small fraction of a second.

\subsection{Particle Interactions and Collective Effects}

The properties of a plasma are markedly dependent upon the particle interactions. One of the basic features that distinguish the behavior of plasmas from that of ordinary fluids and solids is the existence of collective effects. Due to the long range of electromagnetic forces, each charged particle in the plasma interacts simultaneously with a considerable number of other charged particles, resulting in important collective effects that are responsible for the wealth of physical phenomena that take place in a plasma.

The particle dynamics in a plasma is governed by the internal fields due to the nature and motion of the particles themselves, and by externally applied fields. The basic particle interactions are electromagnetic in character. Quantum effects are negligible, except for some cases of close collisions.

In a plasma we must distinguish between charge-charge and chargeneutral interactions. A charged particle is surrounded by an electric field and interacts with the other charged particles according to the coulomb 
force law, with its dependence on the inverse of the square of the separation distance. Furthermore, a magnetic field is associated with a moving charged particle, which also produces a force on other moving charges. The charged and neutral particles interact through electric polarization fields produced by distortion of the neutral particle's electronic cloud during a close passage of the charged particle. The field associated with neutral particles involves short-range forces, such that their interaction is effective only for interatomic distances sufficiently small to perturb the orbital electrons. It is appreciable when the distance between the centers of the interacting particles is of the order of their diameter, but nearly zero when they are farther apart. Its characteristics can be adequately described only by quantum-mechanical considerations. In many cases this interaction involves permanent or induced electric dipole moments.

A distinction can be made between weakly ionized and strongly ionized plasmas in terms of the nature of the particle interactions. In a weakly ionized plasma the charge-neutral interactions dominate over the multiple coulomb interactions. When the degree of ionization is such that the multiple coulomb interactions become dominant, the plasma is considered strongly ionized. As the degree of ionization increases, the coulomb interactions become increasingly important so that in a fully ionized plasma all particles are subjected to the multiple coulomb interactions.

\subsection{Some Basic Plasma Phenomena}

The fact that some or all of the particles in a plasma are electrically charged and therefore capable of interacting with electromagnetic fields, as well as of creating them, gives rise to many novel phenomena that are not present in ordinary fluids and solids. The presence of the magnetic field used, for example, in the heating and confinement of plasmas in controlled thermonuclear research greatly accentuates the novelty of plasma phenomena. To explore all features of plasma phenomena, the plasma behavior is usually studied in the presence of both electric and magnetic fields.

Because of the high electron mobility, plasmas are generally very good electrical conductors, as well as good thermal conductors. As a consequence of their high electrical conductivity they do not support electrostatic fields except, to a certain extent, in a direction normal to any magnetic field present, which inhibits the flow of charged particles in this direction.

The presence of density gradients in a plasma causes the particles to 
diffuse from dense regions to regions of lower density. Although the diffusion problem in nonmagnetized plasmas is somewhat similar to that which occurs in ordinary fluids, there is nevertheless a fundamental difference. Because of their lower mass, the electrons tend to diffuse faster than the ions, generating a polarization electric field as a result of charge separation. This field enhances the diffusion of the ions and decreases that of the electrons, in such a way as to make ions and electrons diffuse at approximately the same rate. This type of diffusion is called ambipolar diffusion. When there is an externally applied magnetic field, the diffusion of charged particles across the field lines is reduced, which indicates that strong magnetic fields are helpful in plasma confinement. The diffusion of charged particles across magnetic field lines when the diffusion coefficient is proportional to $1 / B^{2}$, where $B$ denotes the magnetic induction magnitude, is called classical diffusion, in contrast to the so-called Bohm diffusion in which the diffusion coefficient is proportional to $1 / B$ (see Chapter 10).

An important characteristic of plasmas is their ability to sustain a great variety of wave phenomena. Examples include longitudinal electrostatic plasma waves and high-frequency transverse electromagnetic waves. In the low-frequency regime important wave modes in a magnetized plasma are the so-called Alfvén waves and magnetosonic waves. Each of the various possible modes of wave propagation can be characterized by a dispersion relation, which is a functional relation between the wave frequency $\omega$ and the wave number $k$, and by its polarization. The study of waves in plasmas provides significant information on plasma properties and is very useful for plasma diagnostics.

Dissipative processes, such as collisions, produce damping of the wave amplitude. This means that energy is transferred from the wave field to the plasma particles. An essentially noncollisional mechanism of wave attenuation also exists in a plasma, which is known as Landau damping. The mechanism responsible for Landau damping is the trapping of some plasma particles (the ones that are moving with velocities close to the wave phase velocity) in the energy potential well of the wave, the net result being the transfer of energy from the wave to the particles.

It is also possible to have modes with growing amplitudes, as a result of instabilities, which transfer energy from the plasma particles to the wave field. Instability phenomena are important in a wide variety of physical situations involving dynamic processes in plasmas. The existence of many different types of instabilities in a plasma greatly complicates the confinement of a hot plasma in the laboratory. The study of these instabilities is of essential importance for controlled thermonuclear fusion 
research.

Another important aspect of plasma behavior is the emission of radiation. The main interest in plasma radiation lies in the fact that it can be used to infer plasma properties. The mechanisms that cause plasmas to emit or absorb radiation can be grouped into two categories: radiation from emitting atoms or molecules, and radiation from accelerated charges. At the same time that ionization is produced in a plasma, the opposite process, recombination of the ions and electrons to form neutral particles, is normally also occurring. As a result of the recombination process, radiation is often emitted as the excited particles formed during recombination decay to the ground state. This radiation constitutes the line spectra of plasmas. On the other hand, any accelerated charged particle emits radiation. The radiation emitted whenever a charged particle is decelerated by making some kind of collisional interaction is called bremsstrahlung. If the charged particle remains unbound, both before and after the encounter, the process is called free-free bremsstrahlung. Radiation of any wavelength can be emitted or absorbed in bremsstrahlung. If the originally unbound charged particle is captured by another particle, as it emits the radiation, the process is called free-bound radiation. Cyclotron radiation, which occurs in magnetized plasmas, is due to the magnetic centripetal acceleration of the charged particles as they spiral about the magnetic field lines. Blackbody radiation emitted from plasmas in thermodynamic equilibrium is important only in astrophysical plasmas, in view of the large size needed for a plasma to radiate as a blackbody.

\section{CRITERIA FOR THE DEFINITION OF A PLASMA}

\subsection{Macroscopic Neutrality}

In the absence of external disturbances a plasma is macroscopically neutral. This means that under equilibrium conditions with no external forces present, in a volume of the plasma sufficiently large to contain a large number of particles and yet sufficiently small compared to the characteristic lengths for variation of macroscopic parameters such as density and temperature, the net resulting electric charge is zero. In the interior of the plasma the microscopic space charge fields cancel each other and no net space charge exists over a macroscopic region.

If this macroscopic neutrality was not maintained, the potential energy associated with the resulting coulomb forces could be enormous compared to the thermal particle kinetic energy. Consider, for example, a 
plasma with a charged particle number density of $10^{20} \mathrm{~m}^{-3}$ and suppose that the electron number density $\left(n_{e}\right)$ in a spherical volume of $10^{-3} \mathrm{~m}$ radius $(r)$ were to differ by $1 \%$ from the positive ion number density $\left(n_{i}\right)$. Denoting the ion charge by $e$ and the electron charge by $-e$, the total net charge $(q)$ inside the sphere would be

$$
q=\frac{4}{3} \pi r^{3}\left(n_{i}-n_{e}\right) e
$$

and the electric potential $(\phi)$ at the surface of the sphere would be

$$
\phi=\frac{1}{4 \pi \epsilon_{o}} \frac{q}{r}=\frac{r^{2} e}{3 \epsilon_{o}}\left(n_{i}-n_{e}\right)
$$

where $\epsilon_{o}$ is the permittivity of free space. Plugging numerical values into (2.2) yields $\phi=6 \times 10^{3}$ volts. Recalling that $1 \mathrm{eV}=1.602 \times 10^{-19}$ joule, we find that $k T=1 \mathrm{eV}$ when $T=11,600 \mathrm{~K}$, where $k$ is Boltzmann's constant $\left(1.380 \times 10^{-23}\right.$ joule/K). Therefore, a plasma temperature of several millions of degrees Kelvin would be required to balance the electric potential energy with the average thermal particle energy.

Departures from macroscopic electrical neutrality can naturally occur only over distances in which a balance is obtained between the thermal particle energy, which tends to disturb the electrical neutrality, and the electrostatic potential energy resulting from any charge separation, which tends to restore the electrical neutrality. This distance is of the order of a characteristic length parameter of the plasma, called the Debye length. In the absence of external forces, the plasma cannot support departures from macroscopic neutrality over larger distances than this, since the charged particles are able to move freely to neutralize any regions of excess space charge in response to the large coulomb forces that appear.

\subsection{Debye Shielding}

The Debye length is an important physical parameter for the description of a plasma. It provides a measure of the distance over which the influence of the electric field of an individual charged particle (or of a surface at some nonzero potential) is felt by the other charged particles inside the plasma. The charged particles arrange themselves in such a way as to effectively shield any electrostatic fields within a distance of the order of the Debye length. This shielding of electrostatic fields is a consequence of the collective effects of the plasma particles. A calculation of the shielding 
distance was first performed by Debye, for an electrolyte. In Chapter 11 it will be shown that the Debye length $\left(\lambda_{D}\right)$ is directly proportional to the square root of the temperature $(T)$ and inversely proportional to the square root of the electron number density $\left(n_{e}\right)$ according to

$$
\lambda_{D}=\left(\frac{\epsilon_{o} k T}{n_{e} e^{2}}\right)^{1 / 2}
$$

As mentioned before, the Debye length can also be regarded as a measure of the distance over which fluctuating electric potentials may appear in a plasma, corresponding to a conversion of the thermal particle kinetic energy into electrostatic potential energy.

When a boundary surface is introduced in a plasma, the perturbation produced extends only up to a distance of the order of $\lambda_{D}$ from the surface. In the neighborhood of any surface inside the plasma there is a layer of width of the order of $\lambda_{D}$, known as the plasma sheath, inside which the condition of macroscopic electrical neutrality may not be satisfied. Beyond the plasma sheath region there is the plasma region, where macroscopic neutrality is maintained.

Generally, $\lambda_{D}$ is very small. For example, in a gas discharge, where typical values for $T$ and $n_{e}$ are around $10^{4} \mathrm{~K}$ and $10^{16} \mathrm{~m}^{-3}$, respectively, we have $\lambda_{D}=10^{-4} \mathrm{~m}$. For the Earth's ionosphere, typical values can be taken as $n_{e}=10^{12} \mathrm{~m}^{-3}$ and $T=10^{3} \mathrm{~K}$, yielding $\lambda_{D}=10^{-3} \mathrm{~m}$. In the interstellar plasma, on the other hand, the Debye length can be several meters long.

It is convenient to define a Debye sphere as a sphere inside the plasma of radius equal to $\lambda_{D}$. Any electrostatic fields originated outside a Debye sphere are effectively screened by the charged particles and do not contribute significantly to the electric field existing at its center. Consequently, each charge in the plasma interacts collectively only with the charges that lie inside its Debye sphere, its effect on the other charges being effectively negligible. The number of electrons $N_{D}$, inside a Debye sphere, is given by

$$
N_{D}=\frac{4}{3} \pi \lambda_{D}^{3} n_{e}=\frac{4}{3} \pi\left(\frac{\epsilon_{o} k T}{n_{e} e^{1 / 3} e^{2}}\right)^{3 / 2}
$$

The Debye shielding effect is a characteristic of all plasmas, although it does not occur in every medium that contains charged particles. A necessary and obvious requirement for the existence of a plasma is that the physical dimensions of the system be large compared to $\lambda_{D}$. Otherwise there is just not sufficient space for the collective shielding effect to 
take place, and the collection of charged particles will not exhibit plasma behavior. If $L$ is a characteristic dimension of the plasma, a first criterion for the definition of a plasma is therefore

$$
L \gg \lambda_{D}
$$

Since the shielding effect is the result of the collective particle behavior inside a Debye sphere, it is also necessary that the number of electrons inside a Debye sphere be very large. A second criterion for the definition of a plasma is therefore

$$
n_{e} \lambda_{D}^{3} \gg 1
$$

This means that the average distance between electrons, which is roughly given by $n_{e}^{-1 / 3}$, must be very small compared to $\lambda_{D}$. The quantity defined by

$$
g=\frac{1}{n_{e} \lambda_{D}^{3}}
$$

is known as the plasma parameter and the condition $g \ll 1$ is called the plasma approximation. This parameter is also a measure of the ratio of the mean interparticle potential energy to the mean plasma kinetic energy.

Note that the requirement (2.5) already implies in macroscopic charge neutrality if it is realized that deviations from neutrality can naturally occur only over distances of the order of $\lambda_{D}$. Nevertheless, macroscopic neutrality is sometimes considered as a third criterion for the existence of a plasma, although it is not an independent one, and can be expressed as

$$
n_{e}=\sum_{i} n_{i}
$$

\subsection{The Plasma Frequency}

An important plasma property is the stability of its macroscopic space charge neutrality. When a plasma is instantaneously disturbed from the equilibrium condition, the resulting internal space charge fields give rise to collective particle motions that tend to restore the original charge neutrality. These collective motions are characterized by a natural frequency of oscillation known as the plasma frequency. Since these collective oscillations are high-frequency oscillations, the ions, because of their heavy mass, are to a certain extent unable to follow the motion of the electrons. The electrons oscillate collectively about the heavy ions, the necessary collective restoring force being provided by the ion-electron coulomb attraction. 
The period of this natural oscillation constitutes a meaningful time scale against which can be compared the dissipative mechanisms tending to destroy the collective electron motions.

Consider a plasma initially uniform and at rest, and suppose that by some external means a small charge separation is produced inside it (see Fig. 1). When the external disturbing force is removed instantaneously, the internal electric field resulting from charge separation collectively accelerates the electrons in an attempt to restore the charge neutrality. However, because of their inertia, the electrons move beyond the equilibrium position, and an electric field is produced in the opposite direction. This sequence of movements repeats itself periodically, with a continuous transformation of kinetic energy into potential energy, and vice versa, resulting in fast collective oscillations of the electrons about the more massive ions. On the average the plasma maintains its macroscopic charge neutrality. It will be shown in Chapter 11 that the angular frequency of this collective electron oscillations, called the (electron) plasma frequency, is given by

$$
\omega_{p e}=\left(\frac{n_{e} e^{2}}{m_{e} \epsilon_{o}}\right)^{1 / 2}
$$

Collisions between electrons and neutral particles tend to damp these collective oscillations and gradually diminish their amplitude. If the oscillations are to be only slightly damped, it is necessary that the electronneutral collision frequency $\left(\nu_{e n}\right)$ be smaller than the electron plasma frequency,

$$
\nu_{p e}>\nu_{e n}
$$

where $\nu_{p e}=\omega_{p e} / 2 \pi$. Otherwise, the electrons will not be able to behave in an independent way, but will be forced by collisions to be in complete equilibrium with the neutrals, and the medium can be treated as a neutral gas. Eq. (2.10) constitutes, therefore, the fourth criterion for the existence of a plasma. This criterion can be alternatively written as

$$
\omega \tau>1
$$

where $\tau=1 / \nu_{\text {en }}$ represents the average time an electron travels between collisions with neutrals, and $\omega$ stands for the angular frequency of typical plasma oscillations. It implies that the average time between electronneutral collisions must be large compared to the characteristic time during which the plasma physical parameters are changing. 


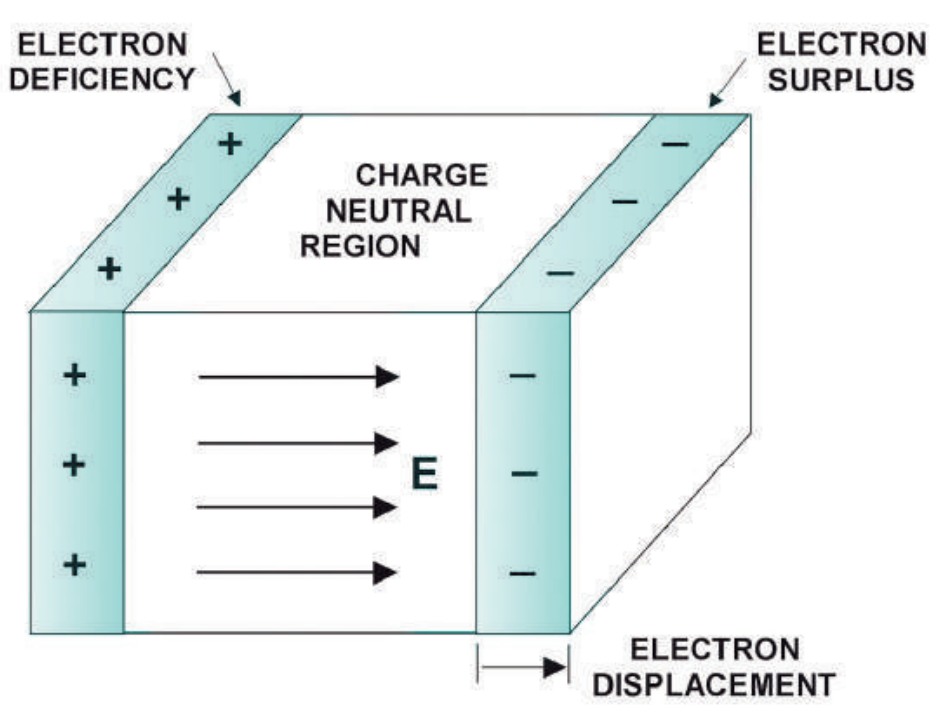

Fig. 1 The electric field resulting from charge separation provides the force that generates the electron plasma oscillations.

Consider, for example, a gas with an electron number density equal to $10^{10} \mathrm{~m}^{-3}$ at a temperature of $10^{3} \mathrm{~K}$, which satisfies both criteria $L \gg \lambda_{D}$ and $n_{e} \lambda_{D}^{3} \gg 1$. If the neutral particle number density $\left(n_{n}\right)$ is relatively small, as in the interstellar gas for example, $\tau$ is relatively large and the electrons will behave independently, so that the medium can then be treated as a plasma. On the other hand, if $n_{n}$ is many orders of magnitude greater than $n_{e}$, then the motion of the electrons will be coupled to that of the neutrals and their effect will be negligible.

The basic characteristics of various laboratory and cosmic plasmas are given in Fig. 2 in terms of their temperature $T$ and electron number density $n_{e}$, as well as of parameters that depend upon $T$ and $n_{e}$, such as the Debye shielding distance $\lambda_{D}$, the electron plasma frequency $\omega_{p e}$, and the number of electrons $N_{D}$ inside a Debye sphere.

\section{THE OCCURRENCE OF PLASMAS IN NATURE}

With the progress made in astrophysics and in theoretical physics during the last century, it was realized that most of the matter in the known universe, with a few exceptions such as the surface of cold planets (the Earth, for example) exists as a plasma. 


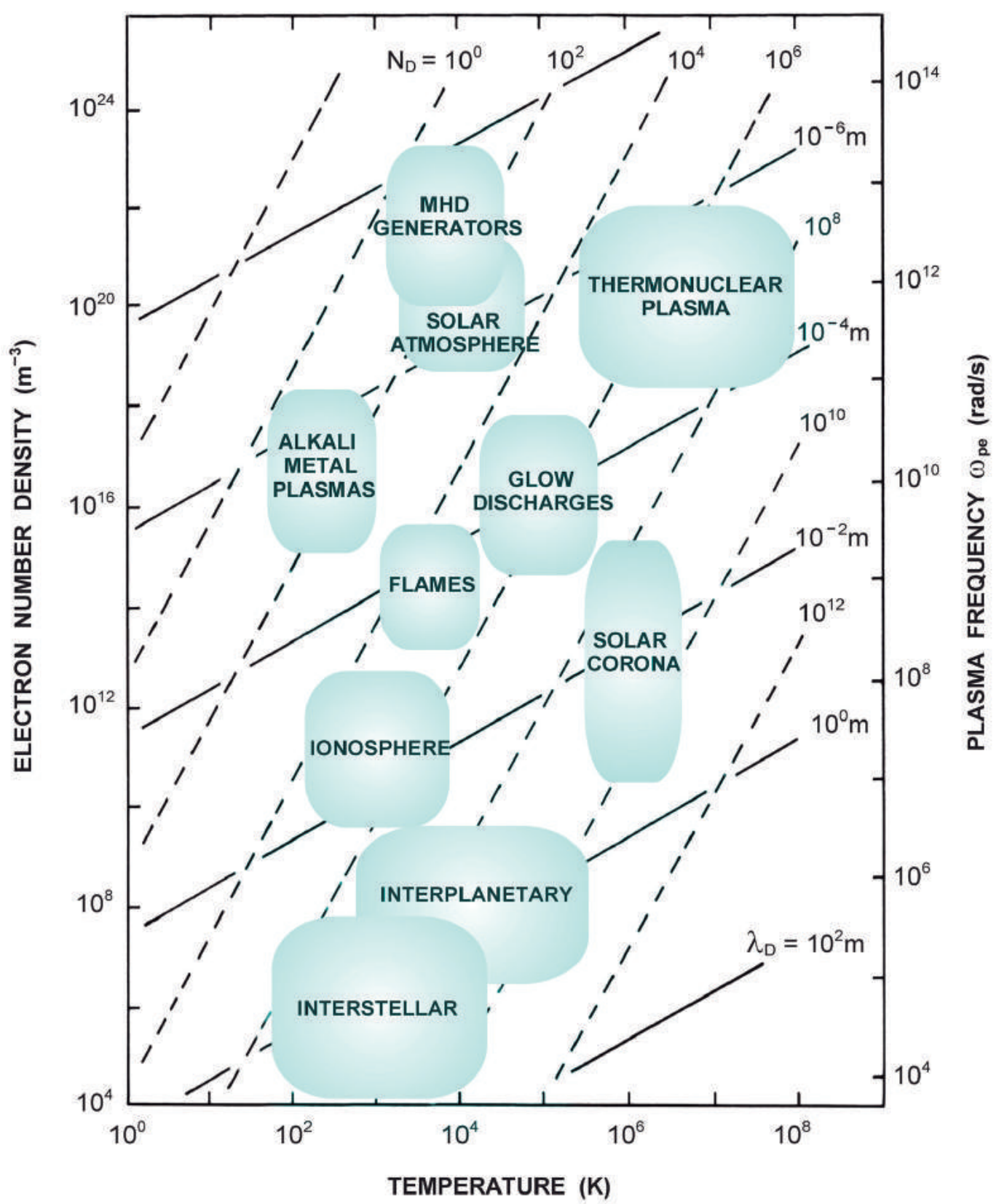

Fig. 2 Ranges of temperature and electron density for several laboratory and cosmic plasmas and their characteristic physical parameters: Debye length $\lambda_{D}$, plasma frequency $\omega_{p e}$, and number of electrons $N_{D}$ in a Debye sphere. MHD, magnetohydrodynamic. 


\subsection{The Sun and Its Atmosphere}

The sun, which is our nearest star and upon which the existence of life on Earth fundamentally depends, is a plasma phenomenon. Its energy output is derived from thermonuclear fusion reactions of protons forming helium ions deep in its interior, where temperatures exceed $1.2 \times 10^{7} \mathrm{~K}$. The high temperature of its interior and the consequent thermonuclear reactions keep the entire sun gaseous. Due to its large mass $\left(2 \times 10^{30} \mathrm{~kg}\right)$, the sun's gravitational force is sufficient to prevent the escape of all but the most energetic particles and, of course, radiation from the hot solar plasma.

There is no sharp boundary surface to the sun. Its visible part is known as the solar atmosphere, which is divided into three general regions or layers. The photosphere, with a temperature of about $6,000 \mathrm{~K}$, comprises the visible disk, the layer in which the gases become opaque, and is a few hundred kilometers thick. Surrounding the photosphere there is a reddish ring called the chromosphere, approximately 10,000 km thick, above which flame-like prominences rise with temperatures of the order of 100,000 K. Surrounding the chromosphere there is a tenuous hot plasma, extending millions of kilometers into space, known as the corona. A steep temperature gradient extends from the chromosphere to the hotter corona, where the temperature exceeds $10^{6} \mathrm{~K}$.

The sun possesses a variable magnetic field, which at its surface is typically of the order of $10^{-4}$ tesla, but in the regions of sunspots (regions of relatively cooler gases) the solar magnetic field rises to about 0.1 tesla.

\subsection{The Solar Wind}

A highly conducting tenuous plasma called the solar wind, composed mainly of protons and electrons, is continuously emitted by the sun at very high speeds into interplanetary space, as a result of the supersonic expansion of the hot solar corona. The solar magnetic field tends to remain frozen in the streaming plasma due to its very high conductivity. Because of solar rotation, the field lines are carried into Archimedean spirals by the radial motion of the solar wind (see Fig. 3). Typical values of the parameters in the solar wind are: electron density $n_{e} \simeq 5 \times 10^{6} \mathrm{~m}^{-3}$, electron and ion temperatures $T_{e} \simeq 5 \times 10^{4} \mathrm{~K}, T_{i} \simeq 10^{4} \mathrm{~K}$, magnetic field $B \simeq 5 \times 10^{-9}$ tesla, and drift velocity $u_{e} \simeq 3 \times 10^{5} \mathrm{~m} / \mathrm{s}$. 


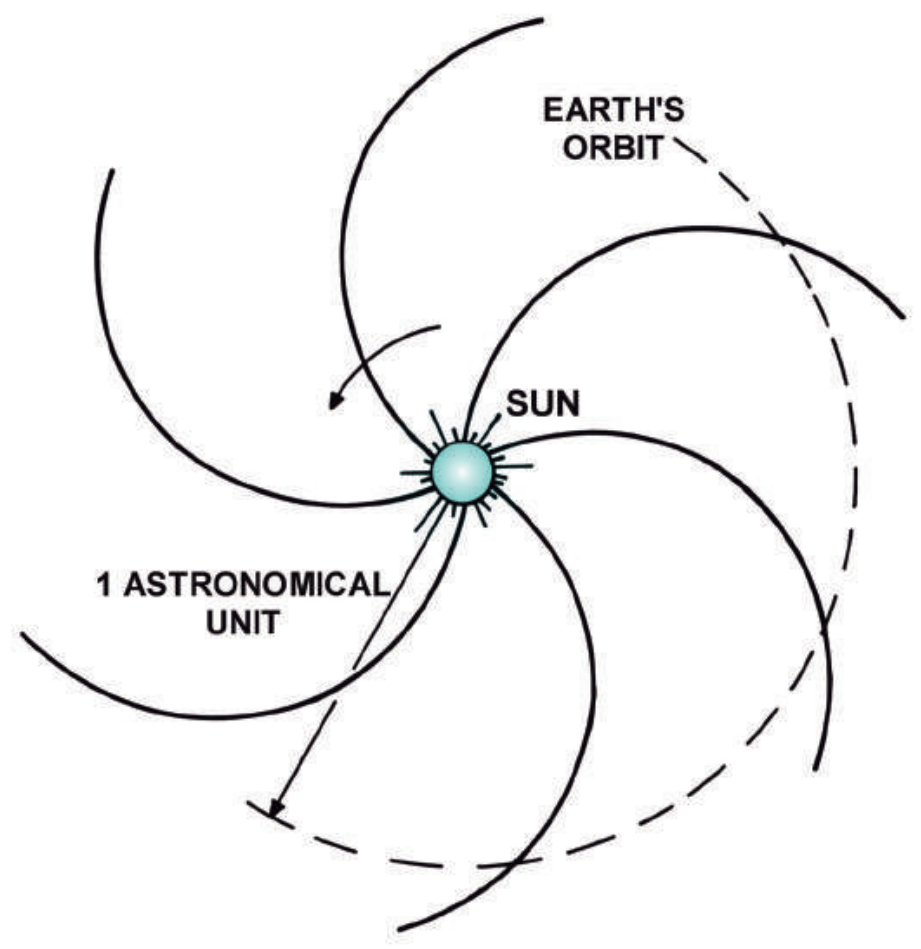

Fig. 3 Schematic representation of the Archimedes spiral structure of the interplanetary magnetic field in the ecliptic plane.

\subsection{The Magnetosphere and the Van Allen Radiation Belts}

As the highly conducting solar wind impinges on the Earth's magnetic field, it compresses the field on the sunward side and flows around it at supersonic speeds. This creates a boundary, called the magnetopause, which is roughly spherical on the sunward side and roughly cylindrical in the anti-sun direction (see Fig. 4). The inner region, from which the solar wind is excluded and which contains the compressed Earth's magnetic field, is called the magnetosphere.

Inside the magnetosphere we find the Van Allen radiation belts, in which energetic charged particles (mainly electrons and protons) are trapped into regions where they execute complicated trajectories that spiral along the geomagnetic field lines and, at the same time, drift slowly around the Earth. The origin of the inner belt is ascribed to cosmic rays, which penetrate into the atmosphere and form proton-electron pairs that are then trapped by the Earth's magnetic field. The outer belt is considered to be due to and maintained by streams of plasma consisting mainly 


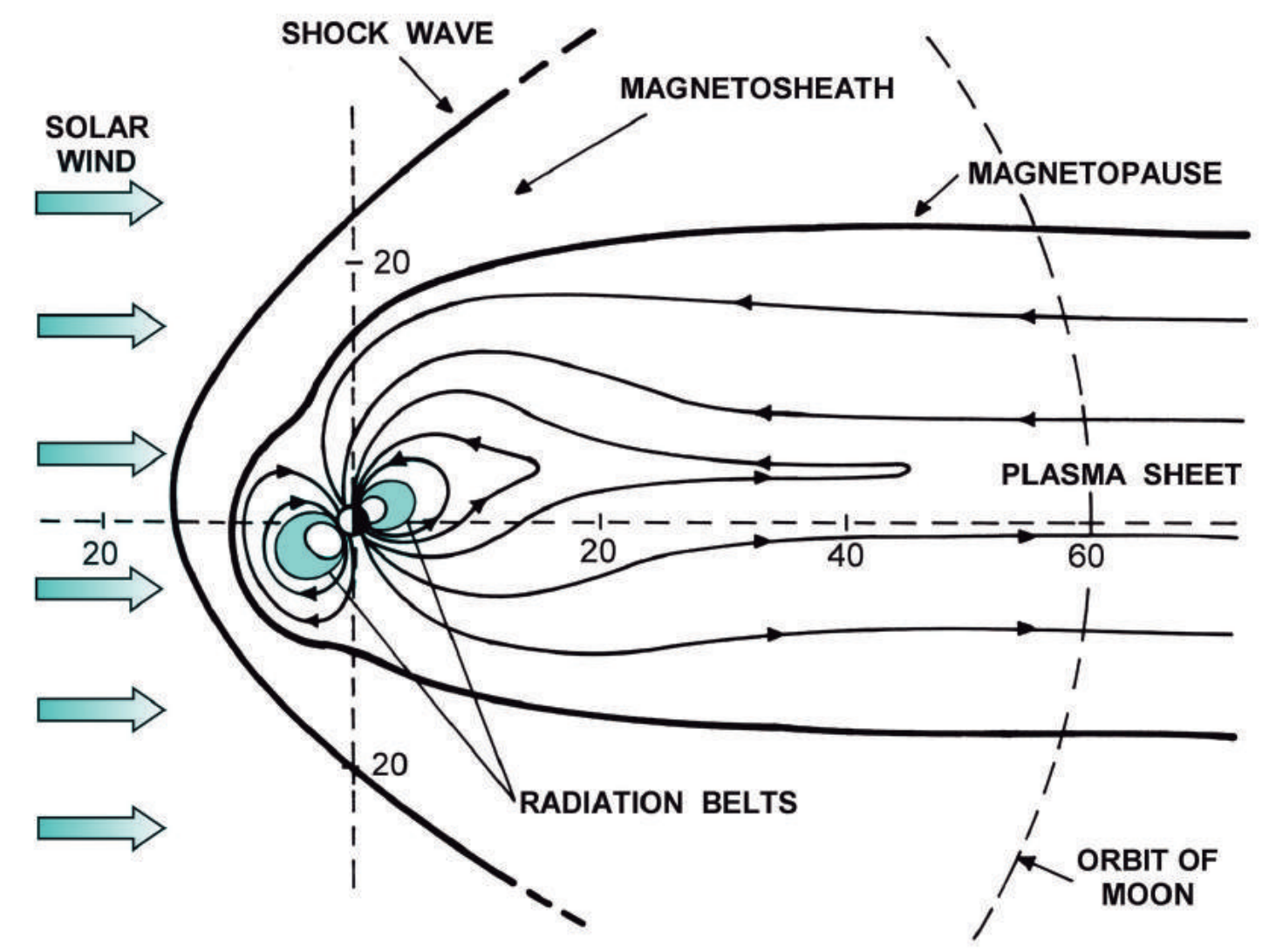

Fig. 4 Schematic configuration of the magnetosphere in the noon-midnight plane. The dark crescents represent the regions of trapped energetic particles (Van Allen radiation belts). The turbulent region between the shock wave (bow shock) and the magnetopause is known as the magnetosheath. Geocentric distances are indicated in units of Earth radii.

of protons and electrons that are ejected from time to time by the sun. Depending on solar activity, particularly violent solar eruptions may occur with the projection of hot streams of plasma material into space. The separation into inner and outer belts reflects only an altitude-dependent energy spectrum, rather than two separate trapping regions. 


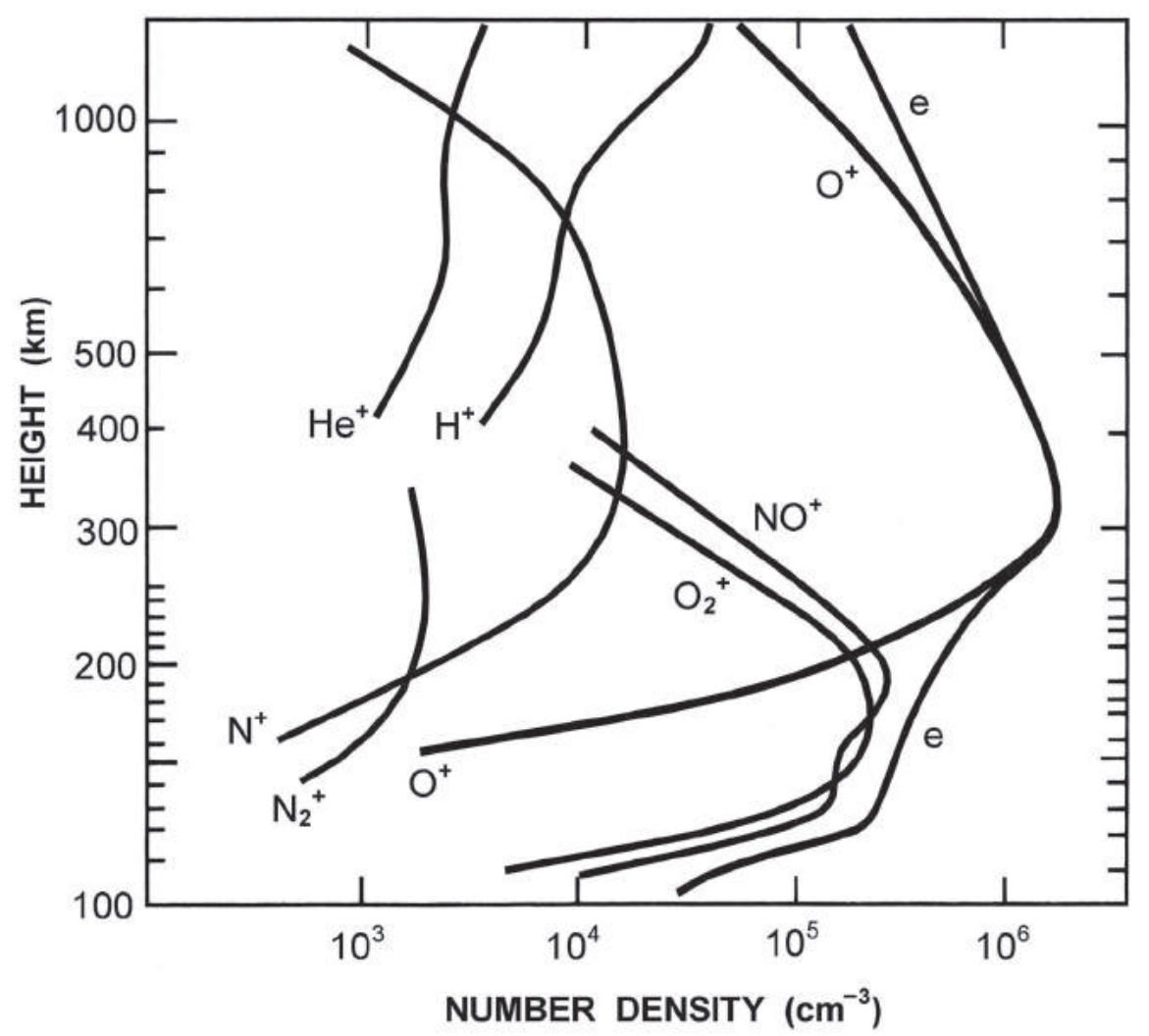

Fig. 5 Height distribution of the electrons and of the principal positive ions, typical of the daytime ionosphere, for average solar conditions.

\subsection{The Ionosphere}

The large natural blanket of plasma in the atmosphere, which envelopes the Earth from an altitude of approximately $60 \mathrm{~km}$ to several thousands of kilometers, is called the ionosphere. The ionized particles in the ionosphere are produced during the daytime through absorption of solar extreme ultraviolet and x-ray radiation by the atmospheric species. As the ionizing radiation from the sun penetrates deeper and deeper into the Earth's atmosphere, it encounters a larger and larger density of gas particles, producing more and more electrons per unit volume. However, since radiation is absorbed in this process, there is a height where the rate of electron production reaches a maximum. Below this height the rate of electron production decreases, in spite of the increase in atmospheric density, since most of the ionizing radiation was already absorbed at the higher altitudes. 
Fig. 5 provides some information on the relative concentration and altitude distribution of the electrons and of the principal positive ions, typical of the daytime ionosphere, for average solar conditions. The Earth's magnetic field exerts a great influence on the dynamic behavior of the ionospheric plasma. An interesting phenomenon that occurs in the ionospheric polar regions is the aurora. It consists of electromagnetic radiation emitted by the atmospheric species and induced by energetic particles of solar and cosmic origin that penetrate into the atmosphere along the geomagnetic field lines near the poles.

\subsection{Plasmas Beyond the Solar System}

Beyond the solar system we find a great variety of natural plasmas in stars, interstellar space, galaxies, intergalactic space, and far beyond to systems quite unknown before the start of astronomy from space vehicles. There we find a variety of phenomena of great cosmological and astrophysical significance, including interstellar shock waves from remote supernova explosions, rapid variations of x-ray fluxes from neutron stars with densities like that of atomic nuclei, pulsating radio stars or pulsars (which are theoretically pictured as rapidly rotating neutron stars with plasmas emitting synchrotron radiation from the surface), and the plasma phenomena around the remarkable black holes (which are considered to be singular regions of space into which matter has collapsed, possessing such a powerful gravitational field that nothing, whether material objects or even light itself, can escape from them).

The behavior of plasmas in the universe involves the interaction between plasmas and magnetic fields. The crab nebula, for example, is a rich source of plasma phenomena because it contains a magnetic field. The widespread existence of magnetic fields in the universe has been demonstrated by independent measurements, and a wide range of field magnitudes has been found, varying from $10^{-9}$ tesla in interstellar space to 1 tesla on the surface of magnetic variable stars.

\section{APPLICATIONS OF PLASMA PHYSICS}

A wide variety of plasma experiments have been perfomed in the laboratory to aid in the understanding of plasmas, as well as to test and help expand plasma theory. The progress in plasma research has led to a wide range of plasma applications. A brief description of some important practical applications of plasma physics is presented in this section. 


\subsection{Controlled Thermonuclear Fusion}

The most important application of man-made plasmas is in the control of thermonuclear fusion reactions, which holds a vast potential for the generation of power. Nuclear fusion is the process whereby two light nuclei combine to form a heavier one, the total final mass being slightly less than the total initial mass. The mass difference $(\Delta m)$ appears as energy $(E)$ according to Einstein's famous law $E=(\Delta m) c^{2}$, where $c$ denotes the speed of light. The nuclear fusion reaction is the source of energy in the stars, including the sun. The confinement of the hot plasma in this case is provided by the self-gravity of the stars.

In the nuclear fusion of hydrogen the principal reactions involve the deuterium $\left({ }^{2} H\right)$ and tritium $\left({ }^{3} H\right)$ isotopes of hydrogen, as follows:

$$
\begin{gathered}
{ }^{2} \mathrm{H}+{ }^{2} \mathrm{H} \longrightarrow{ }^{3} \mathrm{He}+{ }^{1} n+3.27 \mathrm{MeV} \\
{ }^{2} \mathrm{H}+{ }^{2} \mathrm{H} \longrightarrow{ }^{3} \mathrm{H}+{ }^{1} \mathrm{H}+4.03 \mathrm{MeV} \\
{ }^{2} \mathrm{H}+{ }^{3} \mathrm{H} \longrightarrow{ }^{4} \mathrm{He}+{ }^{1} n+17.58 \mathrm{MeV} \\
{ }^{2} \mathrm{H}+{ }^{3} \mathrm{He} \longrightarrow{ }^{4} \mathrm{He}+{ }^{1} \mathrm{H}+18.34 \mathrm{MeV}
\end{gathered}
$$

where ${ }^{1} n$ represents a neutron. The basic problem in achieving controlled fusion is to generate a plasma at very high temperatures (with thermal energies at least in the $10 \mathrm{keV}$ range) and hold its particles together long enough for a substantial number of fusion reactions to take place. The need for high temperatures comes from the fact that, in order to undergo fusion, the positively charged nuclei must come very close together (within a distance of the order of $10^{-14} \mathrm{~m}$ ), which requires sufficient kinetic energy to overcome the electrostatic coulomb repulsion.

Fig. 6 presents the cross sections, as a function of the incident particle energy, for the nuclear fusion reactions of hydrogen given in (4.1). They are appreciable only for incident particles with energies above at least 10 $\mathrm{keV}$. This means that the plasma must have temperatures of the order of $10^{8} \mathrm{~K}$. Other fusion reactions involving nuclei with larger values of the atomic number $\mathrm{Z}$ require even higher energies to overcome the coulomb repulsion.

Many confinement schemes have been suggested and built that use some type of magnetic field configuration. The main experimental efforts for achieving plasma conditions for fusion can be grouped into four approaches: (1) open systems (magnetic mirrors); (2) closed systems (toruses); (3) theta pinch devices; and (4) laser-pellet fusion. 


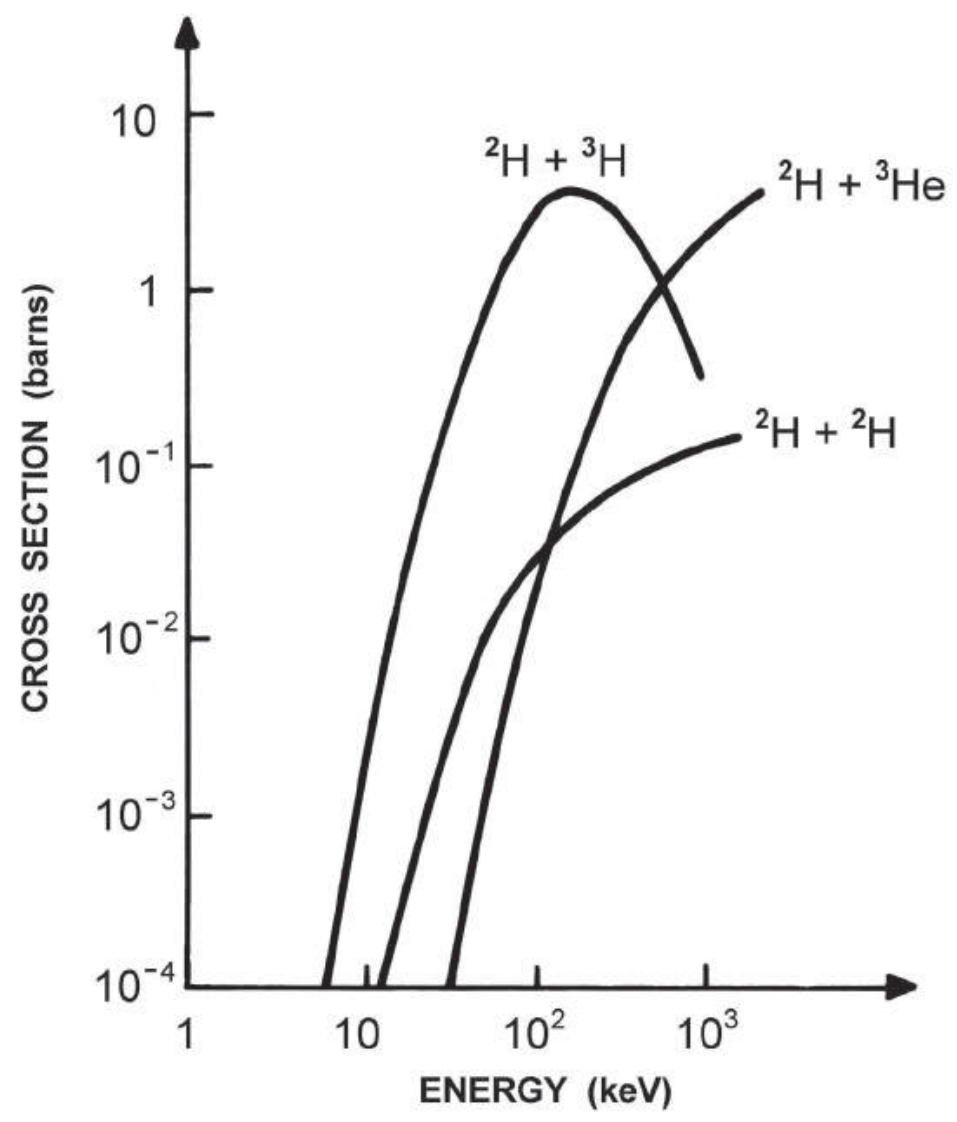

Fig. 6 Fusion cross sections, in barns $\left(1\right.$ barn $\left.=10^{-28} \mathrm{~m}^{2}\right)$, as a function of energy, in $\mathrm{keV}$, for the hydrogen reactions given in (4.1).

The mirror machines are linear devices with an axial magnetic field to keep the particles away from the wall, and with magnetic mirrors (regions of converging magnetic field lines) at the ends to reduce the number of particles escaping at each end (see Fig. 7).

The four principal toroidal systems differ in the way they twist the magnetic field lines. They are the stellarators (in which the twisting of the field lines is produced by external helical conductors), the tokamaks (in which a poloidal field produced by an internal plasma current is superposed on the toroidal field), the multipoles (which have their magnetic field lines primarily in the poloidal direction and produced by internal conductors), and the Astron (in which internal relativistic particle beams modify a mirror field into a form having stable confinement regions with closed lines of force). 


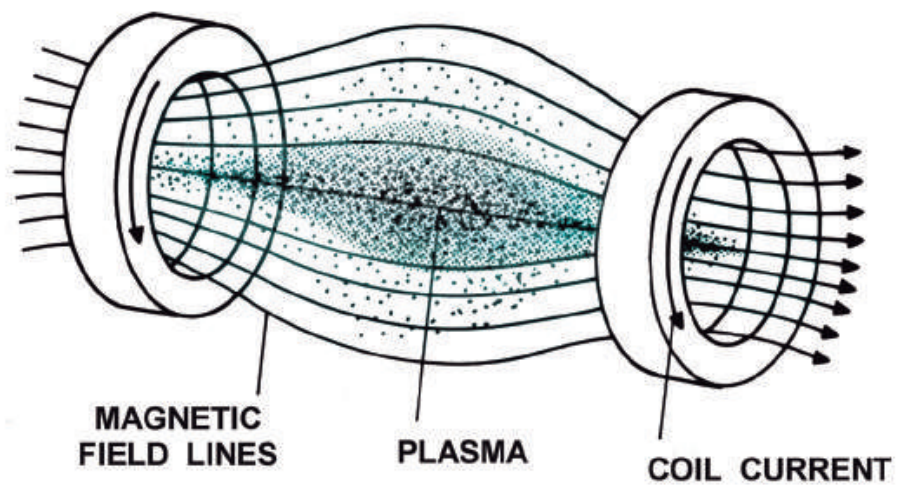

(a)

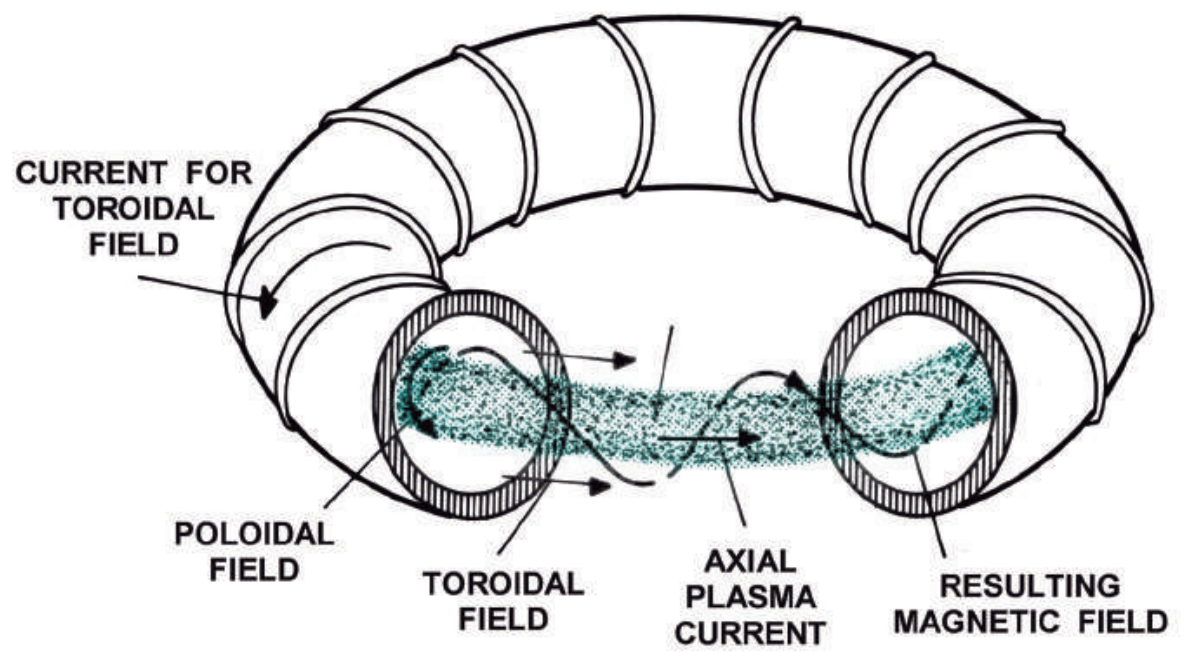

(b)

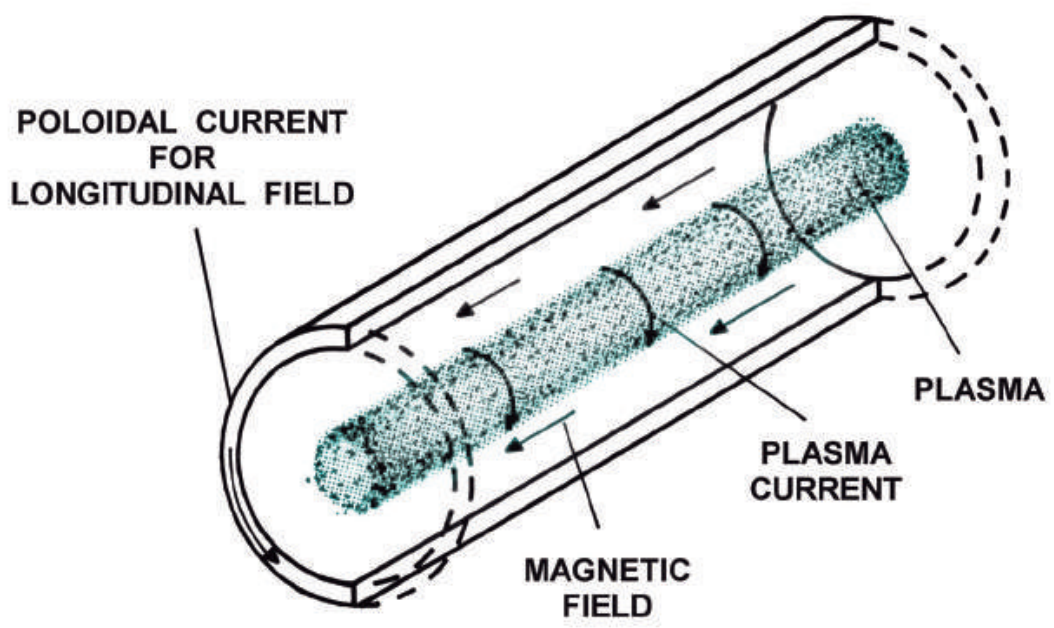

(c)

Fig. 7 Schematic illustration showing the magnetic field configurations of some basic schemes for plasma confinement. (a) Magnetic mirror system. (b) Tokamak. (c) Linear $\theta$ pinch. 


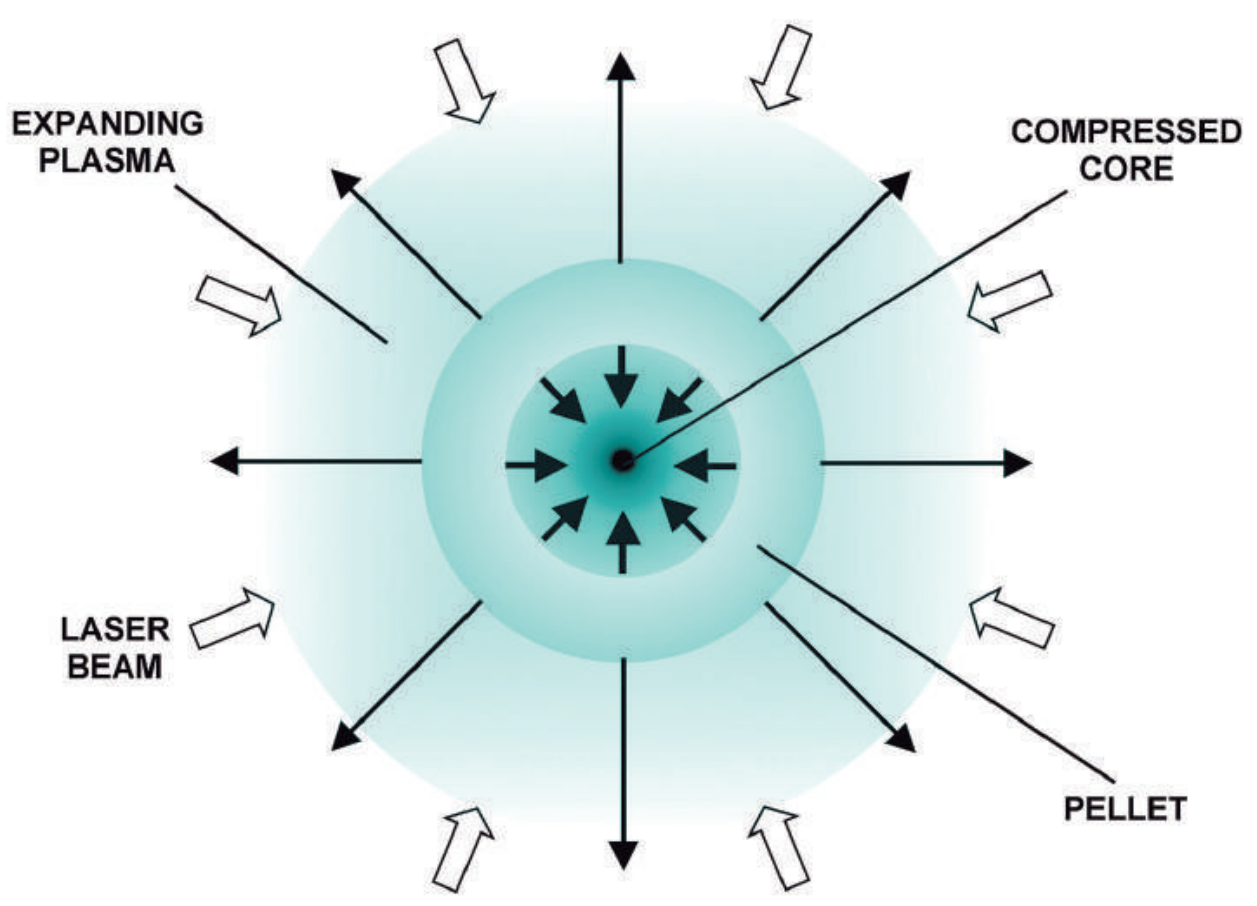

Fig. 8 Illustrating laser-pellet fusion.

In the theta pinch devices, a plasma current in the azimuthal direction and a longitudinal magnetic field produce a force that compresses the cross-sectional area of the plasma.

Finally, the scheme to ignite a fusion reaction using pulsed lasers consists in focusing converging laser beams on a small pellet of solid deuterium-tritium material producing a rapid symmetrical heating of the plasma, followed by an expansion of the heated surrounding shell and compression of the pellet core by the recoil (see Fig. 8).

In addition to the plasma heating and confinement problems, attention must be given to the energy loss by radiation (predominantly electronion bremsstrahlung and electron cyclotron radiation). These radiation losses constitute a serious problem in maintaining a self-sustaining fusion device. To generate more energy by fusion than is required to heat and confine the plasma, and to supply the radiation losses, a condition is imposed on the plasma density $(n)$ and the confinement time $(\tau)$, as well as on the temperature. It turns out that the product $n \tau$ must be higher 


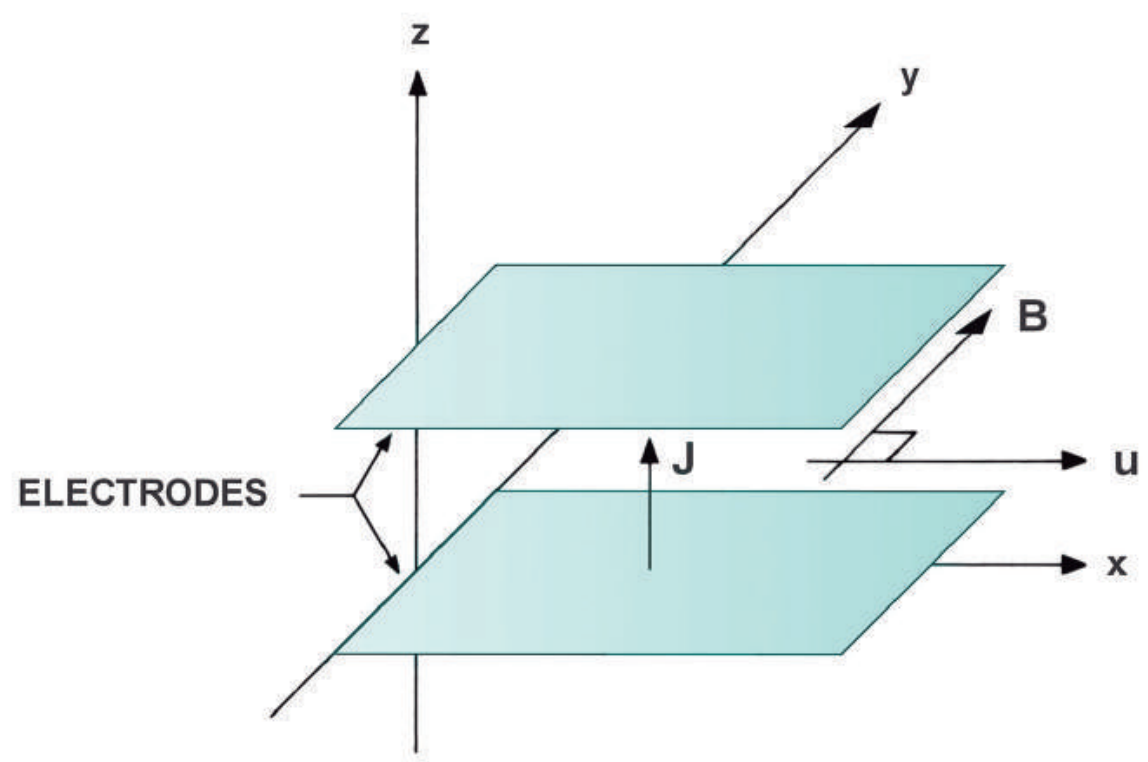

Fig. 9 Schematic diagram illustrating the basic principle of the magnetohydrodynamic energy generator.

than a minimum value, which, for example, is estimated to be about $10^{20} \mathrm{~m}^{-3} \mathrm{~s}$ for deuterium-tritium (with $T>10^{7} \mathrm{~K}$ ) and about $10^{22} \mathrm{~m}^{-3} \mathrm{~s}$ for deuterium-deuterium (with $T>10^{8} \mathrm{~K}$ ). This condition is known as the Lawson criterion. Consequently, controlled fusion can be achieved either by having a large number density of hot plasma particles confined for a short period of time, or by having a smaller number density of particles confined for a longer period of time. For this reason some fusion experiments operate in the regime of high density and short confinement time utilizing a pulsed mode of operation.

Since controlled nuclear fusion can provide an almost limitless source of energy, it is certainly one of the most important scientific challenges man faces today, and its achievement will cause an enormous impact on our civilization.

\subsection{The Magnetohydrodynamic Generator}

The magnetohydrodynamic (MHD) energy generator converts the kinetic energy of a dense plasma flowing across a magnetic field into electrical energy. While a rigorous discussion of this device becomes quite involved, its basic principle is quite simple. 
Suppose that a plasma flows with velocity $\mathbf{u}$ (along the $x$ direction) across an applied magnetic field $\mathbf{B}$ (in the $y$ direction), as shown schematically in Fig. 9. The Lorentz force $q(\mathbf{u} \times \mathbf{B})$ causes the ions to drift upward (in the $z$ direction) and the electrons downward, so that if electrodes are placed in the walls of the channel and connected to an external circuit, then a current density $\mathbf{J}=\sigma \mathbf{E}_{\text {ind }}=\sigma \mathbf{u} \times \mathbf{B}$ (where $\sigma$ denotes the plasma conductivity and $\mathbf{E}_{\text {ind }}$ is the induced electric field) flows across the plasma stream in the $z$ direction. This current density, in turn, produces a force density $\mathbf{J} \times \mathbf{B}$ (in the $x$ direction), which decelerates the flowing plasma. The net result is the conversion of some of the plasma kinetic energy entering the generator into electrical energy that can be applied to an external load. This process has the advantage that it operates without the inefficiency of a heat cycle.

\subsection{Plasma Propulsion}

Plasma propulsion systems for rocket engines are based on a process that converts electrical energy into plasma kinetic energy, that is, the reverse of the MHD generator process.

The plasma rocket engine is accomplished by having both electric and magnetic fields applied perpendicular to each other, across a plasma (see Fig. 10). The resulting current density $\mathbf{J}$ flowing in the direction of the applied $\mathbf{E}$ field gives rise to a $\mathbf{J} \times \mathbf{B}$ force, per unit volume, which accelerates the plasma out of the rocket. The associated reaction force, due to conservation of momentum, accelerates the rocket in the direction opposite to the plasma flow. The ejected plasma must always be neutral, otherwise the rocket will become charged to a large electric potential.

An important characteristic of plasma propulsion systems is that they are capable of generating a certain amount of thrust (although small) over a very long time period, contrarily to chemical propulsion systems. Since the force the plasma rocket engine provides is too modest to overcome the Earth's gravitational field, chemical rockets must still be used as the first stage of any plasma propulsion system in order to produce the extremely high values of thrust required to leave the Earth's gravity. The plasma rocket engine is appropriate for long interplanetary and interstellar space travel.

\subsection{Other Plasma Devices}

A number of other practical applications of plasma physics should be 


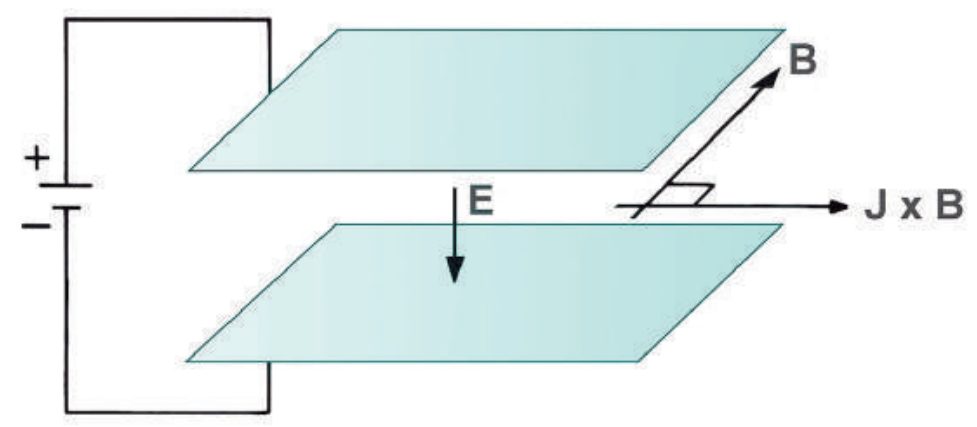

Fig. 10 Schematic diagram illustrating the basic principle of the plasma rocket engine.

mentioned in addition to controlled fusion, MHD energy conversion, and plasma propulsion.

The thermionic energy converter is a device that utilizes a cesium plasma between two electrodes to convert thermal energy into electrical energy. The cathode is heated, so that electrons are emitted from the surface, and the anode is cooled. Due to the presence of the cesium plasma, very large electrical currents can be produced at the expense of a significant fraction of the thermal energy applied to the cathode.

Examples of applications involving gas discharges include the ordinary fluorescent tubes and neon lights used for illumination and for signs, mercury rectifiers, spark gaps, a number of specialized tubes like the hydrogen thyratrons and the ignitrons, which are used for switching, and the arc discharges or plasma jets, which are the source of temperatures two or more times as high as the hottest gas flames and which are used in metallurgy for cutting, melting, and welding metals.

Two major applications in the area of communications are the longdistance radio wave propagation by reflection in the ionospheric plasma and the communication with a space vehicle through the plasma layer that forms around it during the reentry period into the Earth's atmosphere.

Finally, there is the realm of solid state plasmas. If the usual lattice temperature is considered, it can be easily verified that solids do not satisfy the plasma shielding criterion $N_{D} \gg 1$. Nevertheless, quantum mechanical effects, associated with the uncertainty principle, give some solids an effective electron temperature high enough to make $N_{D}$ sufficiently large, 
so that plasma behavior can be observed. It has been demonstrated that the free electrons and holes in appropriate solid materials, particularly semiconductors, exhibit the same sort of oscillations and instabilities as gaseous plasmas. The most likely application of solid state plasmas is in electronic circuitry.

\section{THEORETICAL DESCRIPTION OF PLASMA PHENOMENA}

The dynamic behavior of a plasma is governed by the interaction between the plasma particles and the internal fields produced by the particle themselves, and the externally applied fields. As the charged particles in a plasma move around, they can generate local concentrations of positive or negative charges, which give rise to electric fields. Their motion can also generate electric currents and therefore magnetic fields. The particle dynamics in a plasma is adequately described by the laws of classical (nonquantum) mechanics. Generally, the momentum of the plasma particles is high and the density low enough to keep their De Broglie wavelengths much smaller than the interparticle distance. Quantum effects turn out to be important only at very high densities and very low temperatures.

\subsection{General Considerations on a Self-Consistent Formulation}

The interaction of charged particles with electromagnetic fields is governed by the Lorentz force. For a typical particle of charge $q$ and mass $m$, moving with velocity $\mathbf{v}$, in the presence of electric $(\mathbf{E})$ and magnetic induction (B) fields, the equation of motion is

$$
\frac{d \mathbf{p}}{d t}=q(\mathbf{E}+\mathbf{v} \times \mathbf{B})
$$

where $\mathbf{p}=m \mathbf{v}$ denotes the particle momentum. It is conceivable, at least in principle, to describe the dynamics of a plasma by solving the equations of motion for each particle in the plasma under the combined influence of the externally applied fields and the internal fields generated by all the other plasma particles.

If the total number of particles is $N$, we will have $N$ nonlinear coupled differential equations of motion to solve simultaneously. A self-consistent formulation must be used since the fields and the particle trajectories are intrinsically coupled, that is, the internal fields associated with the presence and motion of the plasma particles influence their motions, 
which, in turn, modify the internal fields. The electromagnetic fields obey Maxwell equations

$$
\begin{gathered}
\nabla \times \mathbf{E}=-\frac{\partial \mathbf{B}}{\partial t} \\
\nabla \times \mathbf{B}=\mu_{o}\left(\mathbf{J}+\epsilon_{o} \frac{\partial \mathbf{E}}{\partial t}\right) \\
\nabla \cdot \mathbf{E}=\frac{\rho}{\epsilon_{o}} \\
\nabla \cdot \mathbf{B}=0
\end{gathered}
$$

where $\rho, \mathbf{J}, \epsilon_{o}$, and $\mu_{o}$ denote, respectively, the total charge density, the total electric current density, the electric permittivity, and the magnetic permeability of free space. The plasma charge and current densities can be expressed, respectively, as

$$
\begin{gathered}
\rho_{p}=\frac{1}{\delta V} \sum_{i} q_{i} \\
\mathbf{J}_{p}=\frac{1}{\delta V} \sum_{i} q_{i} \mathbf{v}_{i}
\end{gathered}
$$

where the summation is over all charged particles contained inside a suitably chosen small volume element $\delta V$. Note that since we are dealing with a discrete distribution of charges and therefore also of current densities, $\rho_{p}$ and $\mathbf{J}_{p}$ should actually be expressed in terms of Dirac delta functions. If point charges are considered, the problem gets even more complicated because the fields become singular at the particle positions. However, if $\delta V$ is chosen big enough to contain a fairly large number of particles, then (5.6) and (5.7) should give smooth functions for $\rho_{p}$ and $\mathbf{J}_{p}$ which are suitable for analytical calculations.

Although this self-consistent approach is conceivable in principle, it cannot be carried out in practice without introducing some averaging scheme, in view of the extremely large number of variables involved. According to the laws of classical mechanics, in order to determine the position and velocity of each particle in the plasma as a function of time under the action of known forces, it is necessary to know the initial position and velocity of each particle. For a system consisting of a very large number of interacting particles these initial conditions are obviously unknown. Furthermore, in order to explain and predict the macroscopic phenomena 
observed in nature and in the laboratory, it is not of interest to know the detailed individual motion of each particle, since the observable macroscopic properties of a plasma are due to the average collective behavior of a large number of particles. We must discard, therefore, the possibility of analytically solving the set of simultaneous equations of motion for a large number of interacting particles.

With the advent of large and fast computers, it is nowadays possible to numerically follow the nonlinear motion of many particles in their own internal self-consistent and externally applied fields and, using some averaging or smoothing scheme for the internal fields, to determine the macroscopic variables from the individual particle motion properties. This method, known as plasma computer simulation via particles, provides a profound view of plasma phenomena in the microscopic and macroscopic levels, and complements the analytical theoretical models and experimental observations.

\subsection{Theoretical Approaches}

For the theoretical description of plasma phenomena, there are basically four principal approaches with several different choices of approximations, each of which applies to different circumstances.

One useful approximation, known as particle orbit theory, consists in studying the motion of each charged particle in the presence of specified electric and magnetic fields. This approach is not really plasma theory, but rather the dynamics of a charged particle in given fields. Nevertheless it is important, since it provides some physical insight for a better understanding of the dynamic processes in plasmas. It has proven to be useful for predicting the behavior of very low density plasmas, which is determined primarily by the interaction of the particles with external fields. This is the case, for example, of the highly rarefied plasmas of the Van Allen radiation belts and the solar corona, as well as of cosmic rays, high energy accelerators, and cathode ray tubes.

Since a plasma consists of a very large number of interacting particles, in order to provide a macroscopic description of plasma phenomena it is appropriate to adopt a statistical approach. This implies a great reduction in the amount of information to be handled. In the kinetic theory statistical description it is necessary to know only the distribution function for the system of particles under consideration. The problem consists in solving the appropriate kinetic equations that govern the evolution of the distribution function in phase space. One example of differential 
kinetic equation is the Vlasov equation, in which the interaction between the charged particles is described by smeared out internal electromagnetic fields consistent with the distributions of electric charge density and current density inside the plasma, and the effects of short-range correlations (close collisions) are neglected.

When collisions between the plasma particles are very frequent, so that each species is able to maintain a local equilibrium distribution function, then each species can be treated as a fluid described by a local density, local macroscopic velocity and local temperature. In this case the plasma is treated as a mixture of two or more interpenetrating fluids. This theory is called two-fluid or many-fluid theory, depending on the number of different species considered. In addition to the usual electrodynamic equations, there is a set of hydrodynamic equations expressing conservation of mass, of momentum, and of energy for each particle species in the plasma.

Another approach consists in treating the whole plasma as a single conducting fluid using lumped macroscopic variables and their corresponding hydrodynamic conservation equations. This theory is usually referred to as the one-fluid theory. An appropriately simplified form of this theory, applicable to the study of very low frequency phenomena in highly conducting fluids immersed in magnetic fields, is usually referred to as the magnetohydrodynamic (MHD) approximation.

\section{PROBLEMS}

1.1 The interatomic or intermolecular forces are usually represented in terms of a potential energy function $V(r)$ such that $F(r)=-d V(r) / d r$. For neutral particles, at large internuclear distances, there is a slight attractive potential between the particles called the van der Waals potential (which is the long-range part of the Lennard-Jones potential). For like atoms or molecules in like states the van der Waals interaction potential can be represented by

$$
V(r)=-C\left(a_{o} / r\right)^{6} R y
$$

where $C$ is a constant (which depends on the type of particle), $a_{o}$ is the Bohr radius $(0.0529 \mathrm{~nm})$, and $R y$ denotes the Rydberg energy unit $(13.605 \mathrm{eV})$. Calculate the van der Waals force of attraction between two hydrogen molecules (for which $C=24.0$ ), and compare with the coulomb 


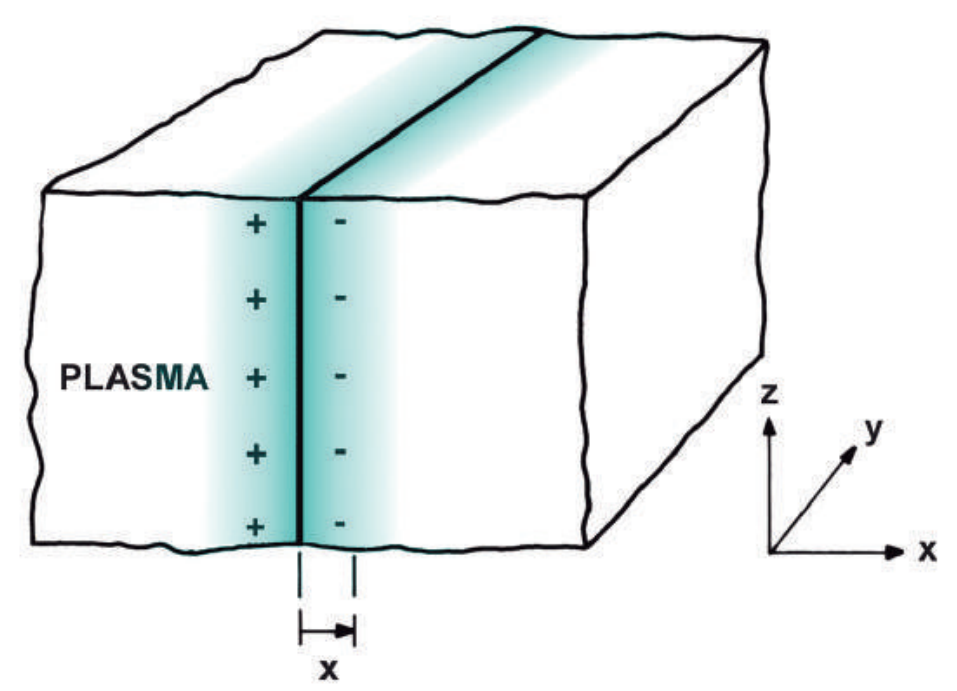

Fig. 11 One-dimensional perturbation in which the electrons, in the $(y, z)$ plane, are displaced by a small amount $\mathrm{x}$.

force between a proton and an electron at a distance $r=N a_{o}$, where $N \gg 1$.

1.2 Consider an initially uniform plasma in which the electron and ion number densities are each equal to $n$. By some external means, let a onedimensional perturbation occur such that the electrons in an infinite plane (the $(y, z)$ plane) are displaced by a small amount $x$, as indicated in Fig. 11.

(a) Using Gauss's law, show that the electric field that appears across the perturbed plane is given by

$$
E=\left(\frac{n e}{\epsilon_{o}}\right) x
$$

(b) Show that the equation of motion (Newton's second law) for each electron, under the action of this electric field, is

$$
\frac{d^{2} x}{d t^{2}}+\left(\frac{n e^{2}}{m \epsilon_{o}}\right) x=0
$$

Verify that this is the equation of a harmonic oscillator of frequency

$$
\omega_{p e}=\left(\frac{n e^{2}}{m \epsilon_{o}}\right)^{1 / 2}
$$


1.3 (a) Calculate the amount of energy released by the fusion of $1 \mathrm{~g}$ of deuterium according to the nuclear reactions indicated in (4.1), considering as end products ${ }^{4} \mathrm{He},{ }^{1} \mathrm{H}$, and ${ }^{1} \mathrm{n}$. Assume that the two possible results shown in (4.1), for the reaction ${ }^{2} H+{ }^{2} H$, occur with equal probabilities. (b) How much energy can be released from the fusion of all the deuterium that exists in one liter of ordinary water? Compare this much energy with the energy obtained from the combustion of one liter of gasoline.

1.4 Calculate the coulomb repulsion force and the associated electric potential energy due to the coulomb interaction of two deuterium nuclei when brought together at a distance of $10^{-14} \mathrm{~m}$. What temperature must have the nuclei in a deuterium plasma, if their average thermal kinetic energy is to be equal to this electric potential energy?

1.5 In a MHD generator a plasma of conductivity $\sigma$ is driven with velocity $\mathbf{u}$ (in the $x$ direction) across a magnetic field $\mathbf{B}$ (in the $y$ direction). Two electrode plates, each of area $A$ and separated by a distance $d$, are placed oriented parallel to the $(x, y)$ plane, as shown in Fig. 9 of Chapter 1.

(a) Show that the open-circuit electric potential difference between the two electrode plates is given by

$$
\phi=B u d
$$

(b) If an external load of resistance $R_{L}$ is connected between the electrodes, show that the current that flows is given by

$$
I=\frac{B u d}{\left(R_{L}+R_{p}\right)}
$$

where $R_{p}$ denotes the internal plasma resistance.

(c) Show that the power delivered to the load is

$$
P_{L}=\frac{B^{2} u^{2} d^{2} R_{L}}{\left(R_{L}+R_{p}\right)^{2}}
$$

Verify that this power has a maximum $\left(d P_{L} / d R_{L}=0\right)$ when $R_{L}=R_{p}$ and show that the maximum power that can be delivered to the load is given by

$$
P_{L, \max }=\frac{1}{4} B^{2} u^{2} d A \sigma
$$

(d) Determine numerical results for items (a), (b), and (c) when $B=1$ tesla, $u=100 \mathrm{~m} / \mathrm{s}, \sigma=100 \mathrm{mho} / \mathrm{m}, d=0.1 \mathrm{~m}$, and $A=1 \mathrm{~m}^{2}$. 
1.6 Consider a rocket once it is beyond the Earth's gravitational field. Let:

$v=$ constant velocity of the exhaust gas relative to the rocket.

$u(t)=$ instantaneous velocity of the rocket.

$M(t)=$ instantaneous total mass of the rocket.

$-d M / d t=$ constant time rate of decrease of $M(t)$, that is, the mass expelled per unit time.

(a) Verify that the equation of motion (Newton's second law) for the rocket is

$$
\frac{d}{d t}[M(t) u(t)]=\frac{d M}{d t}[v-u(t)]
$$

and show that the instantaneous acceleration of the rocket is

$$
\frac{d u}{d t}=-\frac{v}{M(t)} \frac{d M}{d t}
$$

(b) Integrate the equation of motion to show that

$$
u(t)=u\left(t_{o}\right)+v \ln \left[M\left(t_{o}\right) / M(t)\right]
$$

(c) If the rocket burns for a time interval $\delta t=t-t_{o}$ and if $M(t) \ll M\left(t_{o}\right)$, show that the initial acceleration of the rocket is

$$
\left(\frac{d u}{d t}\right)_{t_{o}}=\frac{v}{M\left(t_{o}\right)} \frac{M\left(t_{o}\right)-M(t)}{\delta t} \simeq \frac{v}{\delta t}
$$

(d) Calculate numerically $(d u / d t)_{t_{o}}$ and $u(t)$ for a chemical rocket with $v=10^{3} \mathrm{~m} / \mathrm{s}$ and $\delta t=10 \mathrm{~s}$; and also for a plasma propulsion system with $v=10^{4} \mathrm{~m} / \mathrm{s}$ and $\delta t=100$ days. For the calculation of $u(t)$ consider $u\left(t_{o}\right)=0$ and $M\left(t_{o}\right)=10 M(t)$.

1.7 Using Maxwell equations (5.3) and (5.4) derive the charge conservation equation

$$
\frac{\partial \rho}{\partial t}+\nabla \cdot \mathbf{J}=0
$$

This result shows that conservation of electric charge is already implied by Maxwell equations.

1.8 From Maxwell curl equation (5.2) derive the equation

$$
\nabla \cdot \mathbf{B}=\text { constant }
$$


Therefore, (5.5) can be considered as an initial condition for (5.2) since, if $\nabla \cdot \mathbf{B}=0$ at a given initial time, then (5.2) implies that this condition will remain satisfied for all subsequent times.

1.9 Using Maxwell equations derive the following energy conservation law for electromagnetic fields, known as Poynting's theorem,

$$
\frac{\partial}{\partial t} \int_{V}\left(\frac{1}{2} \epsilon E^{2}+\frac{1}{2} \mu H^{2}\right) d^{3} r+\oint_{S}(\mathbf{E} \times \mathbf{H}) \cdot d \mathbf{S}=-\int_{V}(\mathbf{J} \cdot \mathbf{E}) d^{3} r
$$

for a linear isotropic medium, for which $\mathbf{D}=\epsilon \mathbf{E}$ and $\mathbf{B}=\mu \mathbf{H}$. Give the physical interpretation for each term in this equation. What are the physical dimensions of these terms?

1.10 Consider the following Maxwell equations:

$$
\begin{gathered}
\nabla \times \mathbf{B}=\mu_{o}\left(\mathbf{J}_{t}+\epsilon_{o} \frac{\partial \mathbf{E}}{\partial t}\right) \\
\nabla \times \mathbf{H}=\mathbf{J}+\frac{\partial \mathbf{D}}{\partial t} \\
\nabla \cdot \mathbf{E}=\frac{\rho_{t}}{\epsilon_{o}} \\
\nabla \cdot \mathbf{D}=\rho
\end{gathered}
$$

For a general medium for which

$$
\begin{gathered}
\mathbf{D}=\epsilon_{o} \mathbf{E}+\mathbf{P} \\
\mathbf{B}=\mu_{o}(\mathbf{H}+\mathbf{M})
\end{gathered}
$$

where $\mathbf{P}$ is the polarization vector and $\mathbf{M}$ is the magnetization vector, show that the total electric charge density $\left(\rho_{t}\right)$ and current density $\left(\mathbf{J}_{t}\right)$ are given by

$$
\begin{gathered}
\rho_{t}=\rho-\nabla \cdot \mathbf{P} \\
\mathbf{J}_{t}=\mathbf{J}+\frac{\partial \mathbf{P}}{\partial t}+\nabla \times \mathbf{M}
\end{gathered}
$$

Explain why $\mathbf{E}$ and $\mathbf{B}$ are considered as fundamental fields, whereas $\mathbf{D}$ and $\mathbf{H}$ are partial fields. 


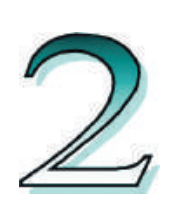

\section{Charged particle motion IN CONSTANT AND UNIFORM ELECTROMAGNETIC FIELDS}

\section{INTRODUCTION}

In this and in the following two chapters we investigate the motion of charged particles in the presence of electric and magnetic fields known as functions of position and time. Thus, the electric and magnetic fields are assumed to be prescribed and are not affected by the charged particles. This chapter, in particular, considers the fields to be constant in time and spatially uniform. This subject is considered in some detail, since many of the more complex situations, considered in Chapters 3 and 4, can be treated as perturbations to this problem.

The study of the motion of charged particles in specified fields is important, since it provides a good physical insight for understanding some of the dynamic processes in plasmas. It also facilitates obtaining information on some macroscopic phenomena that are due to the collective behavior of a large number of particles. Not all of the components of the detailed microscopic particle motion contribute to macroscopic effects, but it is possible to isolate the components of the individual motion that contribute to the collective plasma behavior. Nevertheless, the macroscopic parameters can be obtained much more easily and conveniently from the macroscopic transport equations presented in Chapters 8 and 9.

The equation of motion for a particle of charge $q$, under the action of the Lorentz force $\mathbf{F}$ due to electric (E) and magnetic induction (B) fields, can be written as 


$$
\frac{d \mathbf{p}}{d t}=\mathbf{F}=q(\mathbf{E}+\mathbf{v} \times \mathbf{B})
$$

where $\mathbf{p}$ represents the momentum of the particle and $\mathbf{v}$ its velocity. This equation is relativistically correct if we take

$$
\mathbf{p}=\gamma m \mathbf{v}
$$

where $m$ is the rest mass of the particle and $\gamma$ is the so-called Lorentz factor defined by

$$
\gamma=\left(1-v^{2} / c^{2}\right)^{-1 / 2}
$$

where $c$ is the speed of light in vacuum. In the relativistic case, (1.1) can also be written in the form

$$
\gamma m \frac{d \mathbf{v}}{d t}+q\left(\frac{\mathbf{v}}{c^{2}}\right)(\mathbf{v} \cdot \mathbf{E})=q(\mathbf{E}+\mathbf{v} \times \mathbf{B})
$$

noting that the time rate of change of the total relativistic energy $\left(U=\gamma m c^{2}\right)$ is given by $d U / d t=q(\mathbf{v} \cdot \mathbf{E})$ and that $d \mathbf{p} / d t=d\left(U \mathbf{v} / c^{2}\right) / d t$.

In many situations of practical interest, however, the term $v^{2} / c^{2}$ is negligible compared to unity. For $v^{2} / c^{2} \ll 1$ we have $\gamma \simeq 1$ and $m$ can be considered constant (independent of $v$ ), so that (1.4) reduces to the following nonrelativistic expression

$$
m \frac{d \mathbf{v}}{d t}=q(\mathbf{E}+\mathbf{v} \times \mathbf{B})
$$

If the velocity obtained from (1.5) does not satisfy the condition $v^{2} \ll c^{2}$, then the corresponding result is not valid and the relativistic expression (1.4) must be used instead of (1.5). Relativistic effects become important only for highly energetic particles (a $1 \mathrm{MeV}$ proton, for instance, has a velocity of $1.4 \times 10^{7} \mathrm{~m} / \mathrm{s}$, with $\left.v^{2} / c^{2} \simeq 0.002\right)$. For the situations to be considered here it is assumed that the restriction $v^{2} \ll c^{2}$, implicit in (1.5), is not violated. Also, all radiation effects are neglected.

\section{ENERGY CONSERVATION}

In the absence of an electric field $(\mathbf{E}=0)$, the equation of motion (1.5) reduces to

$$
m \frac{d \mathbf{v}}{d t}=q(\mathbf{v} \times \mathbf{B})
$$

Since the magnetic force is perpendicular to $\mathbf{v}$, it does no work on the particle. Taking the dot product of (2.1) with $\mathbf{v}$ and noting that for any 
vector $\mathbf{v}$, we have $(\mathbf{v} \times \mathbf{B}) \cdot \mathbf{v}=0$, we obtain

$$
m \frac{d \mathbf{v}}{d t} \cdot \mathbf{v}=\frac{d}{d t}\left(\frac{1}{2} m v^{2}\right)=0
$$

which shows that the particle kinetic energy $\left(m v^{2} / 2\right)$ and the magnitude of its velocity (speed $v$ ) are both constants . Therefore, a static magnetic field does not change the particle kinetic energy. This result is valid whatever the spatial dependence of the magnetic flux density $\mathbf{B}$. However, if $\mathbf{B}$ varies with time, then, according to Maxwell equations, an electric field such that $\nabla \times \mathbf{E}=-\partial \mathbf{B} / \partial t$ is also present that does work on the particle changing its kinetic energy.

When both magnetostatic and electrostatic fields are present, we obtain from (1.5)

$$
\frac{d}{d t}\left(\frac{1}{2} m v^{2}\right)=q(\mathbf{E} \cdot \mathbf{v})
$$

Since $\nabla \times \mathbf{E}=0$, we can express the electrostatic field in terms of the electrostatic potential according to $\mathbf{E}=-\nabla \phi$, so that

$$
\frac{d}{d t}\left(\frac{1}{2} m v^{2}\right)=-q(\nabla \phi) \cdot \mathbf{v}=-q(\nabla \phi) \cdot \frac{d \mathbf{r}}{d t}=-q \frac{d \phi}{d t}
$$

This result can be rearranged in the following conservation form:

$$
\frac{d}{d t}\left(\frac{1}{2} m v^{2}+q \phi\right)=0
$$

which shows that the sum of the particle kinetic and electric potential energies remains constant in the presence of static electromagnetic fields. Note that the electric potential $\phi$ can be considered as the potential energy per unit charge.

When the fields are time-dependent we have $\nabla \times \mathbf{E} \neq 0$ and $\mathbf{E}$ is not the gradient of a scalar function. But, since $\nabla \cdot \mathbf{B}=0$, we can define a magnetic vector potential $\mathbf{A}$ by $\mathbf{B}=\nabla \times \mathbf{A}$ and write (1.5.2) (Eq. 5.2 in Chapter 1) as

$$
\nabla \times \mathbf{E}+\frac{\partial \mathbf{B}}{\partial t}=\nabla \times \mathbf{E}+\frac{\partial}{\partial t}(\nabla \times \mathbf{A})=\nabla \times\left(\mathbf{E}+\frac{\partial \mathbf{A}}{\partial t}\right)=0
$$

Hence, we can express the electric field in the form

$$
\mathbf{E}=-\nabla \phi-\frac{\partial \mathbf{A}}{\partial t}
$$

In this case the system is not conservative in the usual sense and there is no energy integral, but the analysis may be performed using a Lagrangian function $L$ for a charged particle in electromagnetic fields, defined by 


$$
L=\frac{1}{2} m v^{2}-U
$$

where $U$ is a velocity-dependent potential energy given by

$$
U=q(\phi-\mathbf{v} \cdot \mathbf{A})
$$

The energy considerations presented in this section assume that the particle energy changes only as a result of the work done by the fields. This assumption is not strictly correct since every charged particle when accelerated irradiates energy in the form of electromagnetic waves. For the situations to be considered here this effect is usually small and can be neglected.

\section{UNIFORM ELECTROSTATIC FIELD}

According to (1.1) the motion of a charged particle in an electric field obeys the following differential equation:

$$
\frac{d \mathbf{p}}{d t}=q \mathbf{E}
$$

For a constant $\mathbf{E}$ field, (3.1) can be integrated directly giving

$$
\mathbf{p}(t)=q \mathbf{E} t+\mathbf{p}_{o}
$$

where $\mathbf{p}_{o}=\mathbf{p}(0)$ denotes the initial particle momentum. Using the nonrelativistic expression

$$
\mathbf{p}=m \mathbf{v}=m \frac{d \mathbf{r}}{d t}
$$

and performing a second integration in (3.2), we obtain the following expression for the particle position as a function of time:

$$
\mathbf{r}(t)=\frac{1}{2}\left(\frac{q \mathbf{E}}{m}\right) t^{2}+\mathbf{v}_{o} t+\mathbf{r}_{o}
$$

where $\mathbf{r}_{o}$ denotes the particle initial position and $\mathbf{v}_{o}$ its initial velocity. Therefore, the particle moves with a constant acceleration, $q \mathbf{E} / m$, in the direction of $\mathbf{E}$ if $q>0$, and in the opposite direction if $q<0$. In a direction perpendicular to the electric field there is no acceleration and the particle state of motion remains unchanged. 


\section{UNIFORM MAGNETOSTATIC FIELD}

\subsection{Formal Solution of the Equation of Motion}

For a particle of charge $q$ and mass $m$, moving with velocity $\mathbf{v}$ in a region of space where there is only a magnetic induction $\mathbf{B}$ (no electric field $\mathbf{E}$ ), the equation of motion is

$$
m \frac{d \mathbf{v}}{d t}=q(\mathbf{v} \times \mathbf{B})
$$

It is convenient to separate $\mathbf{v}$ in components parallel $\left(\mathbf{v}_{\|}\right)$and perpendicular $\left(\mathbf{v}_{\perp}\right)$ to the magnetic field,

$$
\mathbf{v}=\mathbf{v}_{\|}+\mathbf{v}_{\perp}
$$

as indicated in Fig. 1. Substituting (4.2) into (4.1) and noting that $\left(\mathbf{v}_{\|} \times \mathbf{B}=0\right)$ we obtain

$$
\frac{d \mathbf{v}_{\|}}{d t}+\frac{d \mathbf{v}_{\perp}}{d t}=\frac{q}{m}\left(\mathbf{v}_{\perp} \times \mathbf{B}\right)
$$

Since the term $\left(\mathbf{v}_{\perp} \times \mathbf{B}\right)$ is perpendicular to $\mathbf{B}$, the parallel component equation can be written as

$$
\frac{d \mathbf{v}_{\|}}{d t}=0
$$

and the perpendicular component equation as

$$
\frac{d \mathbf{v}_{\perp}}{d t}=\frac{q}{m}\left(\mathbf{v}_{\perp} \times \mathbf{B}\right)
$$

Eq. (4.4) shows that the particle velocity along $\mathbf{B}$ does not change and is equal to the particle initial velocity. For motion in the plane perpendicular to $\mathbf{B}$, we can write (4.5) in the form

$$
\frac{d \mathbf{v}_{\perp}}{d t}=\Omega_{c} \times \mathbf{v}_{\perp}
$$

where $\boldsymbol{\Omega}_{c}$ is a vector defined by

$$
\boldsymbol{\Omega}_{c}=-\frac{q \mathbf{B}}{m}=\frac{|q| B}{m} \widehat{\boldsymbol{\Omega}}_{c}=\Omega_{c} \widehat{\boldsymbol{\Omega}}_{c}
$$




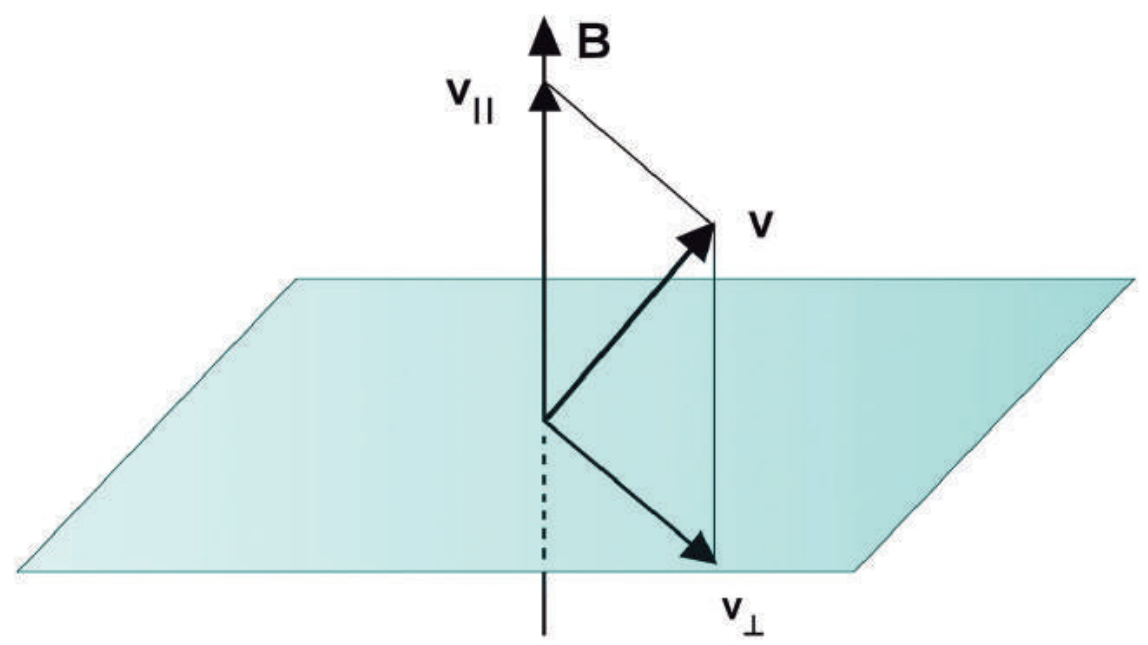

Fig. 1 Decomposition of the velocity vector into components parallel $\left(\mathbf{v}_{\|}\right)$and perpendicular $\left(\mathbf{v}_{\perp}\right)$ to the magnetic field.

Thus, $\widehat{\boldsymbol{\Omega}}_{c}$ points in the direction of $\mathbf{B}$ for a negatively charged particle $(q<0)$ and in the opposite direction for a positively charged particle $(q>0)$. Its magnitude $\Omega_{c}$ is always positive $\left(\Omega_{c}=|q| B / m\right)$. The unit vector $\widehat{\boldsymbol{\Omega}}_{c}$ points along $\boldsymbol{\Omega}_{c}$.

Since $\boldsymbol{\Omega}_{c}$ is constant and, from conservation of kinetic energy, $v_{\perp}$ (the magnitude of $\mathbf{v}_{\perp}$ ) is also constant, (4.6) shows that the particle acceleration is constant in magnitude and its direction is perpendicular to both $\mathbf{v}_{\perp}$ and $\mathbf{B}$. Thus, this acceleration corresponds to a rotation of the velocity vector $\mathbf{v}_{\perp}$ in the plane perpendicular to $\mathbf{B}$ with the constant angular velocity $\boldsymbol{\Omega}_{c}$. We can integrate (4.6) directly, noting that $\boldsymbol{\Omega}_{c}$ is constant and taking $\mathbf{v}_{\perp}=d \mathbf{r}_{c} / d t$, to obtain

$$
\mathbf{v}_{\perp}=\Omega_{c} \times \mathbf{r}_{c}
$$

where the vector $\mathbf{r}_{c}$ is interpreted as the particle position vector with respect to a point $G$ (the center of gyration) in the plane perpendicular to B which contains the particle. Since the particle speed $v_{\perp}$ is constant, the magnitude $r_{c}$ of the position vector is also constant. Therefore, (4.8) shows that the velocity $\mathbf{v}_{\perp}$ corresponds to a rotation of the position vector $\mathbf{r}_{c}$ about the point $\mathrm{G}$ in the plane perpendicular to $\mathbf{B}$ with constant angular velocity $\boldsymbol{\Omega}_{c}$. The component of the motion in the plane perpendicular to $\mathbf{B}$ is therefore a circle of radius $r_{c}$. The instantaneous center of gyration of the particle (the point $\mathrm{G}$ at the distance $r_{c}$ from the particle) is called the 


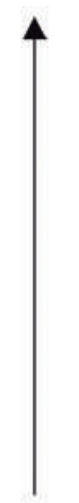

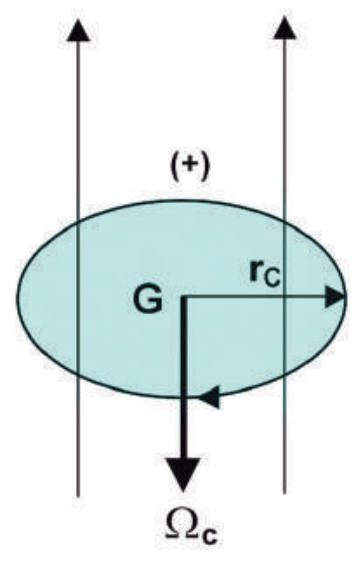

$q>0$

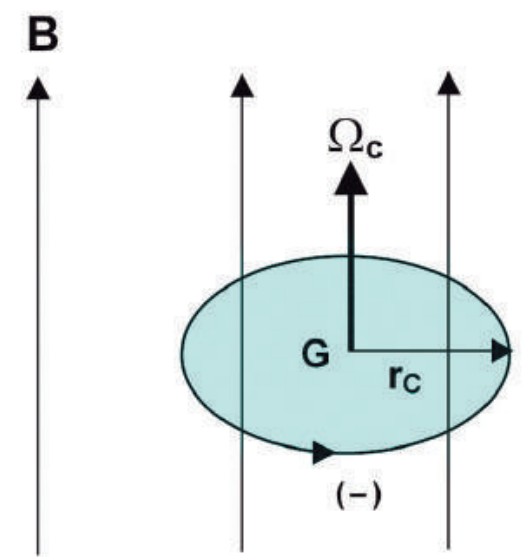

$q<0$

Fig. 2 Circular motion of a charged particle about the guiding center in a uniform magnetostatic field.

guiding center. This circular motion about the guiding center is illustrated in Fig. 2.

Note that according to the definition of $\boldsymbol{\Omega}_{c}$, given in (4.7), $\widehat{\boldsymbol{\Omega}}_{c}$ always points in the same direction as the particle angular momentum vector $\left(\mathbf{r}_{c} \times \mathbf{p}\right)$, irrespective of its charge.

The resulting trajectory of the particle is given by the superposition of a uniform motion along $\mathbf{B}$ (with the constant velocity $\mathbf{v}_{\|}$) and a circular motion in the plane normal to $\mathbf{B}$ (with the constant speed $v_{\perp}$ ). Hence, the particle describes a helix (see Fig. 3). The angle between $\mathbf{B}$ and the direction of motion of the particle is called the pitch angle and is given by

$$
\alpha=\sin ^{-1}\left(\frac{v_{\perp}}{v}\right)=\tan ^{-1}\left(\frac{v_{\perp}}{v_{\|}}\right)
$$

where $v$ is the total speed of the particle $\left(v^{2}=v_{\|}^{2}+v_{\perp}^{2}\right)$. When $v_{\|}=0$ but $v_{\perp} \neq 0$, we have $\alpha=\pi / 2$ and the particle trajectory is a circle in the plane normal to $\mathbf{B}$. On the other hand, when $v_{\perp}=0$ but $v_{\|} \neq 0$, we have $\alpha=0$ and the particle moves along $\mathbf{B}$ with the velocity $\mathbf{v}_{\|}$.

The magnitude of the angular velocity,

$$
\Omega_{c}=\frac{|q| B}{m}
$$


is known as the angular frequency of gyration, and is also called the gyrofrequency, or cyclotron frequency or Larmor frequency. For an electron $|q|=1.602 \times 10^{-19}$ coulomb and $m=9.109 \times 10^{-31} \mathrm{~kg}$, so that

$$
\Omega_{c}(\text { electron })=1.76 \times 10^{11} B \quad(\mathrm{rad} / \mathrm{s})
$$

with $B$ in tesla (or, equivalently, weber $/ \mathrm{m}^{2}$ ). Similarly, for a proton $m=1.673 \times 10^{-27} \mathrm{~kg}$, so that

$$
\Omega_{c}(\text { proton })=9.58 \times 10^{7} B \quad(\mathrm{rad} / \mathrm{s})
$$

The radius of the circular orbit, given by

$$
r_{c}=\frac{v_{\perp}}{\Omega_{c}}=\frac{m v_{\perp}}{|q| B}
$$

is called the radius of gyration, or gyroradius, or cyclotron radius, or Larmor radius. It is important to note that $\Omega_{c}$ is directly proportional to B. Consequently, as B increases, the gyrofrequency increases and the radius decreases. Also, the smaller the particle mass, the larger will be its gyrofrequency and the smaller its gyroradius. Multiplying (4.13) by $B$ gives

$$
B r_{c}=\frac{m v_{\perp}}{|q|}=\frac{p_{\perp}}{|q|}
$$

which shows that the magnitude of $B$ times the particle gyroradius is equal to the particle momentum per unit charge. This quantity is often called the magnetic rigidity.

\subsection{Solution in Cartesian Coordinates}

The treatment presented so far in this section was not related to any particular frame of reference. Consider now a Cartesian coordinate system $(x, y, z)$ such that $\mathbf{B}=B \widehat{\mathbf{z}}$. In this case, the cross product between $\mathbf{v}$ and $\mathbf{B}$ can be written as

$$
\mathbf{v} \times \mathbf{B}=\operatorname{det}\left|\begin{array}{ccc}
\widehat{\mathbf{x}} & \widehat{\mathbf{y}} & \widehat{\mathbf{z}} \\
v_{x} & v_{y} & v_{z} \\
0 & 0 & B
\end{array}\right|=B\left(v_{y} \widehat{\mathbf{x}}-v_{x} \widehat{\mathbf{y}}\right)
$$

and the equation of motion (4.1) becomes

$$
\frac{d \mathbf{v}}{d t}=\frac{q B}{m}\left(v_{y} \widehat{\mathbf{x}}-v_{x} \widehat{\mathbf{y}}\right)= \pm \Omega_{c}\left(v_{y} \widehat{\mathbf{x}}-v_{x} \widehat{\mathbf{y}}\right)
$$




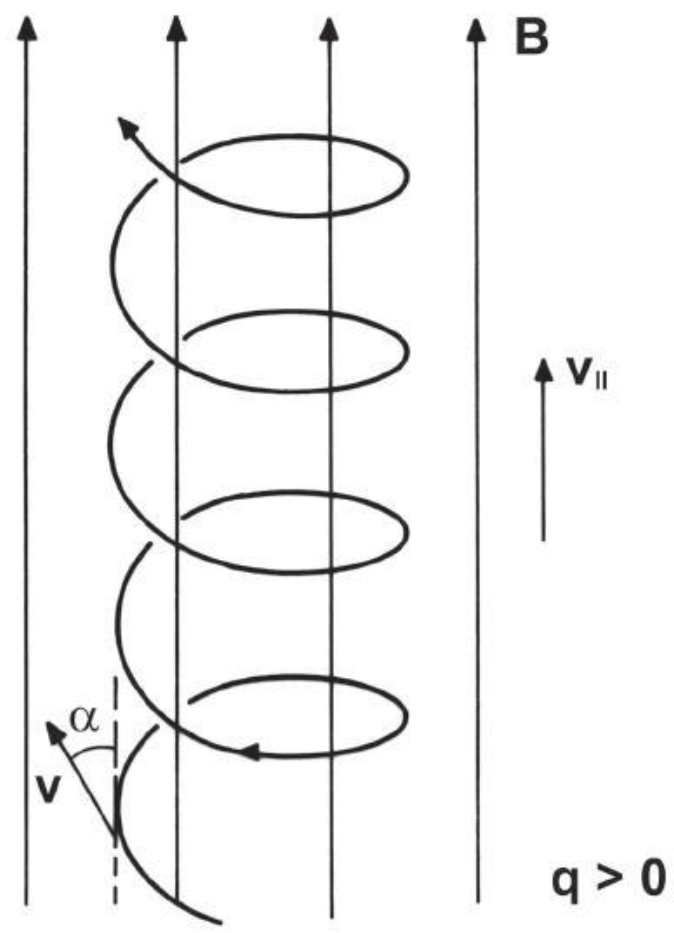

Fig. 3 Helicoidal trajectory of a positively charged particle in a uniform magnetostatic field.

The ( +$)$ sign in front of $\Omega_{c}$ applies to a positively charged particle $(q>0)$ and the $(-)$ sign to a negatively charged particle $(q<0)$, since $\Omega_{c}$ is always positive, according to its definition given in (4.10). In what follows we shall consider a positively charged particle. The results for a negative charge can be obtained by changing the sign of $\Omega_{c}$ in the results for the positive charge.

The Cartesian components of (4.16) are (for $q>0$ )

$$
\begin{gathered}
\frac{d v_{x}}{d t}=\Omega_{c} v_{y} \\
\frac{d v_{y}}{d t}=-\Omega_{c} v_{x} \\
\frac{d v_{z}}{d t}=0
\end{gathered}
$$

The last of these equations gives $v_{z}(t)=v_{z}(0)=v_{\|}$, which is the initial value of the velocity component parallel to $\mathbf{B}$. To obtain the solution of (4.17) and (4.18), we take the derivative of (4.17) with respect to time and 
substitute this result into (4.18), getting

$$
\frac{d^{2} v_{x}}{d t^{2}}+\Omega_{c}^{2} v_{x}=0
$$

This is the homogeneous differential equation for a harmonic oscillator of frequency $\Omega_{c}$, whose solution is

$$
v_{x}(t)=v_{\perp} \sin \left(\Omega_{c} t+\theta_{o}\right)
$$

where $v_{\perp}$ is the constant speed of the particle in the $(x, y)$ plane (normal to $\mathbf{B})$ and $\theta_{o}$ is a constant of integration that depends on the relation between the initial velocities $v_{x}(0)$ and $v_{y}(0)$, according to

$$
\tan \left(\theta_{o}\right)=v_{x}(0) / v_{y}(0)
$$

To determine $v_{y}(t)$ we substitute (4.21) in the left-hand side of (4.17), obtaining

$$
v_{y}(t)=v_{\perp} \cos \left(\Omega_{c} t+\theta_{o}\right)
$$

Note that $v_{x}^{2}+v_{y}^{2}=v_{\perp}^{2}$. The equations for the components of $\mathbf{v}$ can be further integrated with respect to time, yielding

$$
\begin{gathered}
x(t)=-\frac{v_{\perp}}{\Omega_{c}} \cos \left(\Omega_{c} t+\theta_{o}\right)+X_{o} \\
y(t)=\frac{v_{\perp}}{\Omega_{c}} \sin \left(\Omega_{c} t+\theta_{o}\right)+Y_{o} \\
z(t)=v_{\|} t+z_{o}
\end{gathered}
$$

where we have defined

$$
\begin{aligned}
& X_{o}=x_{o}+\frac{v_{\perp}}{\Omega_{c}} \cos \left(\theta_{o}\right) \\
& Y_{o}=y_{o}-\frac{v_{\perp}}{\Omega_{c}} \sin \left(\theta_{o}\right)
\end{aligned}
$$

The vector $\mathbf{r}=x_{o} \widehat{\mathbf{x}}+y_{o} \widehat{\mathbf{y}}+z_{o} \widehat{\mathbf{z}}$ gives the initial particle position. From (4.24) and (4.25) we see that

$$
\left(x-X_{o}\right)^{2}+\left(y-Y_{o}\right)^{2}=\left(v_{\perp} / \Omega_{c}\right)^{2}=r_{c}^{2}
$$




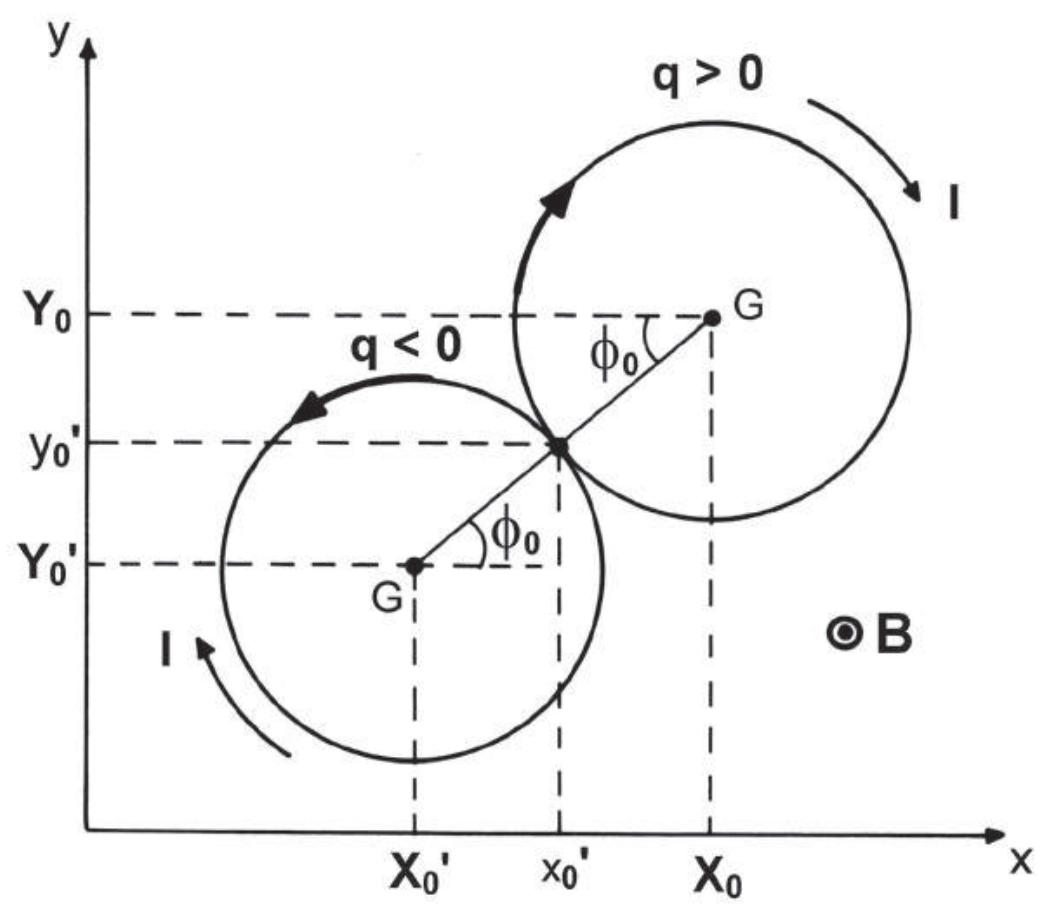

Fig. 4 Circular trajectory of a charged particle in a uniform and constant $\mathbf{B}$ field (directed out of the paper), and the direction of the associated electric current.

The particle trajectory in the plane normal to $\mathbf{B}$ is therefore a circle with center at $\left(X_{o}, Y_{o}\right)$ and radius equal to $\left(v_{\perp} / \Omega_{c}\right)$. The motion of the point $\left[X_{o}, Y_{o}, z(t)\right]$, at the instantaneous center of gyration, corresponds to the trajectory of the guiding center. Thus, the guiding center moves with constant velocity $v_{\|}$along $\mathbf{B}$.

In the $(x, y)$ plane, the argument $\phi(t)$, defined by

$$
\phi(t)=\tan ^{-1} \frac{\left(y-Y_{o}\right)}{\left(x-X_{o}\right)}=-\left(\Omega_{c} t+\theta_{o}\right) ; \quad \phi_{o}=-\theta_{o}
$$

decreases with time for a positively charged particle. For a magnetic field pointing towards the observer, a positive charge describes a circle in the clockwise direction. For a negatively charged particle $\Omega_{c}$ must be replaced by $-\Omega_{c}$ in the results of this subsection. Hence, (4.30) shows that for a negative charge $\phi(t)$ increases with time and the particle moves in a circle in the counterclockwise direction, as shown in Fig. 4. The resulting particle motion is a cylindrical helix of constant pitch angle. Fig. 5 shows the parameters of the helix with reference to a Cartesian coordinate system. 


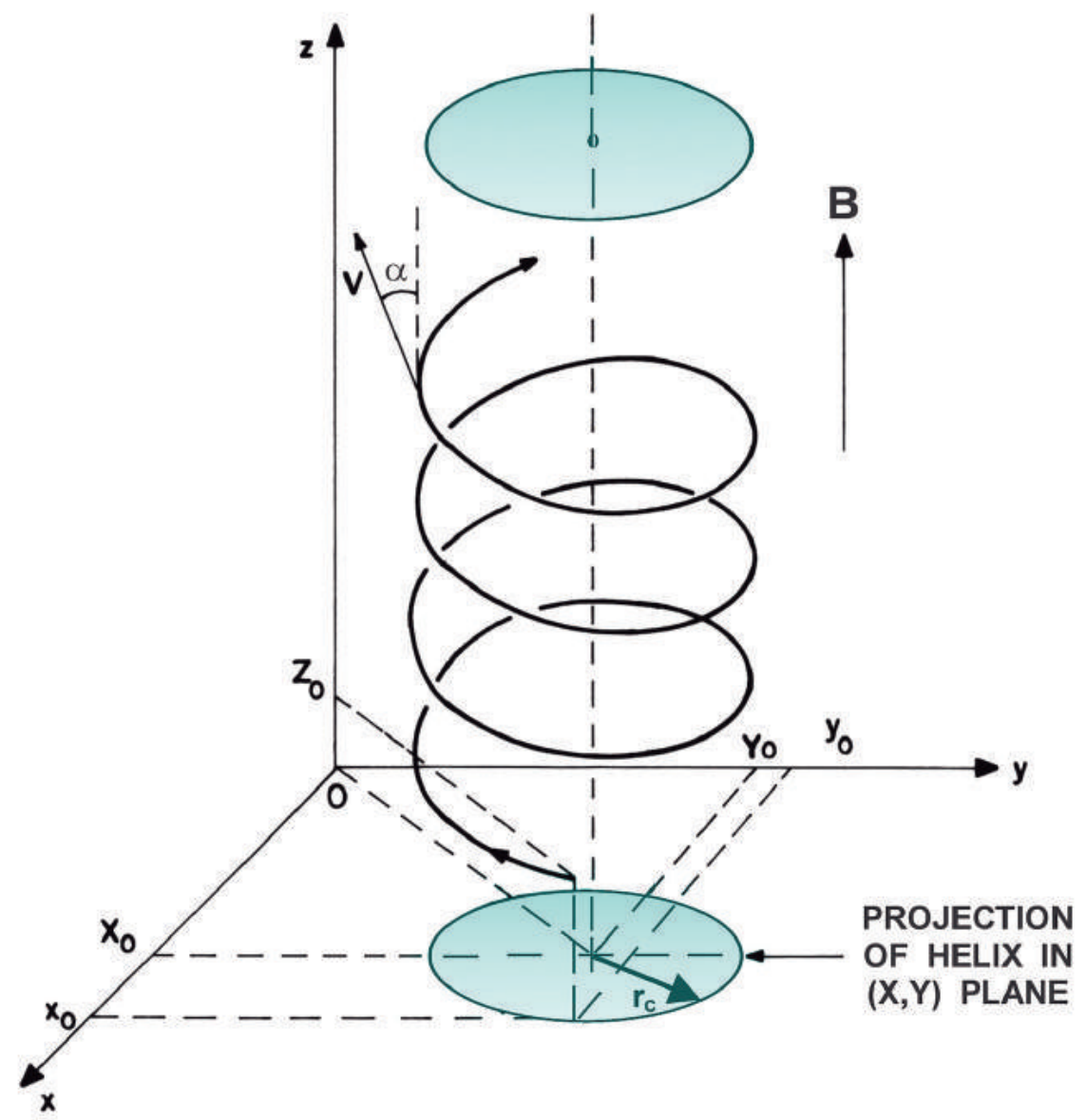

Fig. 5 Parameters of the helicoidal trajectory of a positively charged particle with reference to a Cartesian coordinate system.

\subsection{Magnetic Moment}

To the circular motion of a charged particle in a magnetic field there is associated a circulating electric current $I$. This current flows in the clockwise direction for a $\mathbf{B}$ field pointing towards the observer (Fig. 4). From Ampere's law, the direction of the magnetic field associated with this circulating current is given by the right-hand rule, i.e., with the right thumb pointing in the direction of the current $I$, the right fingers curl in the direction of the associated magnetic field. Therefore, the $\mathbf{B}$ field produced by the circular motion of a charged particle is opposite to the externally applied $\mathbf{B}$ field inside the particle orbit, but in the same direction outside the orbit. The magnetic field generated by the ring current $I$, at distances much larger than $r_{c}$, is similar to that of a dipole (Fig. 6). 


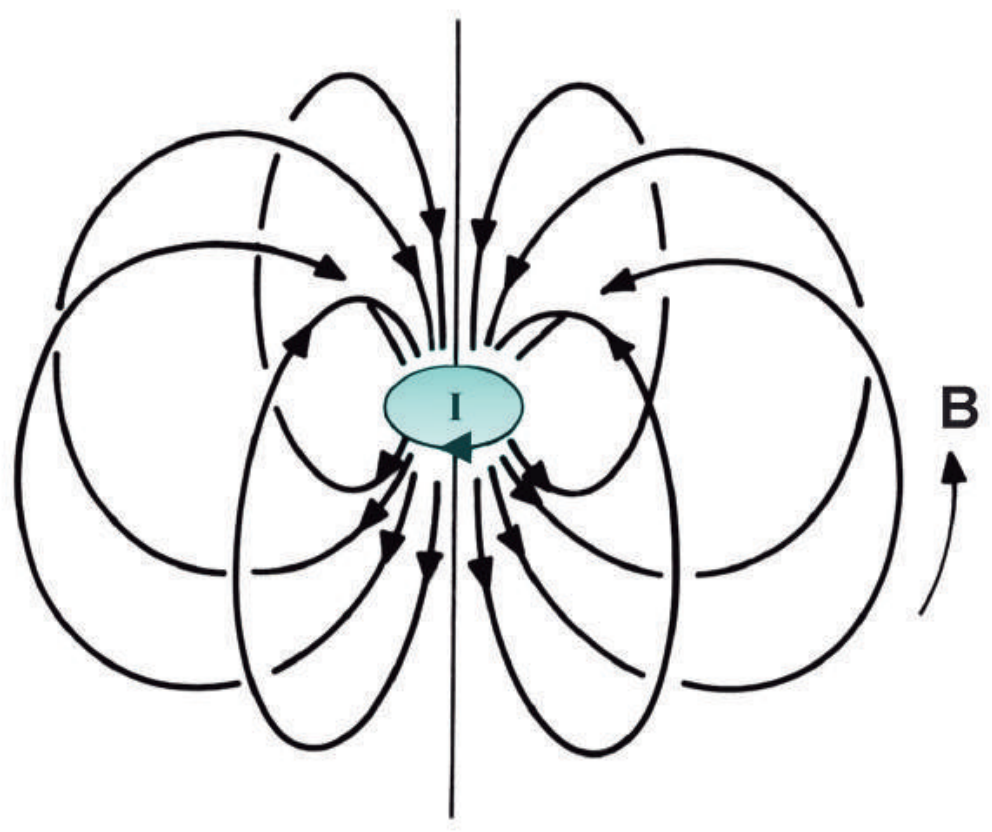

Fig. 6 The magnetic field generated by a small ring current is that of a magnetic dipole.

Since a plasma is a collection of charged particles, it possesses therefore diamagnetic properties.

The magnetic moment $\mathbf{m}$ associated with the circulating current is normal to the area $A$ bounded by the particle orbit and points in the direction opposite to the externally applied $\mathbf{B}$ field, as shown in Fig. 7. Its magnitude is given by

$$
|\mathbf{m}|=(\text { current }) \cdot(\text { orbital area })=I A
$$

This circulating current corresponds to a flow of charge and is given by

$$
I=\frac{|q|}{T_{c}}=\frac{|q| \Omega_{c}}{2 \pi}
$$

where $T_{c}=2 \pi / \Omega_{c}$ is the period of the particle orbit, known as the $c y$ clotron period or Larmor period. The magnitude of $\mathbf{m}$ is therefore

$$
|\mathbf{m}|=\frac{|q| \Omega_{c}}{2 \pi} \pi r_{c}^{2}=\frac{1}{2}|q| \Omega_{c} r_{c}^{2}
$$

Using the relations $\Omega_{c}=|q| B / m$ and $r_{c}=v_{\perp} / \Omega_{c}$, (4.33) becomes

$$
|\mathbf{m}|=\frac{\frac{1}{2} m v_{\perp}^{2}}{B}=\frac{W_{\perp}}{B}
$$




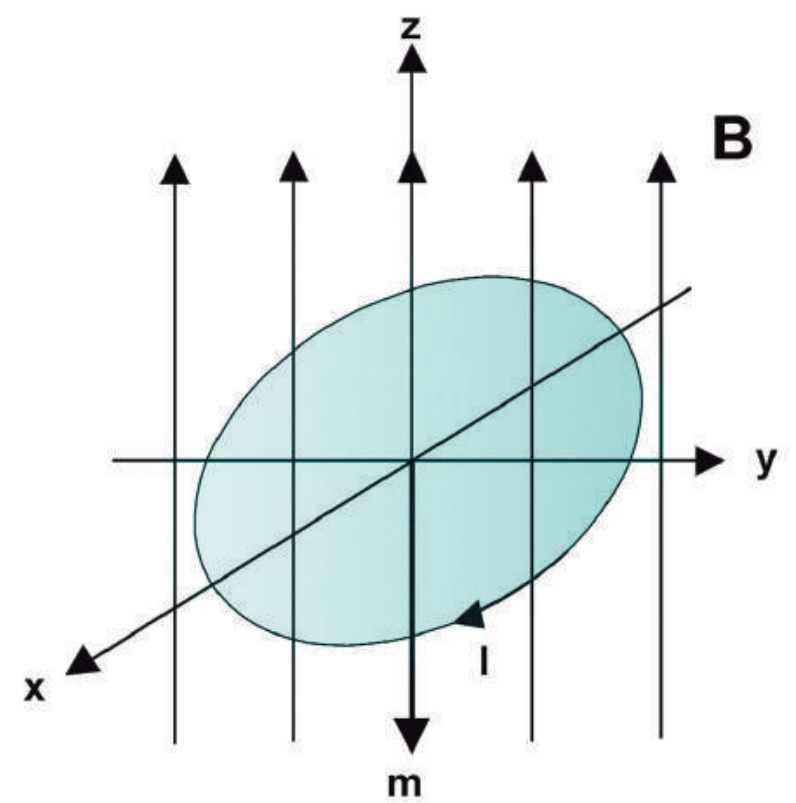

Fig. 7 Magnetic moment $\mathbf{m}$ associated with a circulating current due to the circular motion of a charged particle in an external B field.

where $W_{\perp}$ denotes the part of the particle kinetic energy associated with the transverse velocity $v_{\perp}$. Thus, in vector form,

$$
\mathbf{m}=-\frac{W_{\perp}}{B^{2}} \mathbf{B}
$$

\subsection{Magnetization Current}

Consider now a collection of charged particles, positive and negative in equal numbers (in order to have no internal macroscopic electrostatic fields), instead of just one single particle. For instance, consider the case of a low-density plasma in which the particle collisions can be neglected (collisionless plasma). The condition for this is that the average time between collisions be much greater than the cyclotron period. This condition is fulfilled for many space plasmas, for example. For a collisionless plasma in an external magnetic field, the magnetic moments due to the orbital motion of the charged particles act together, giving rise to a resultant magnetic field that may be strong enough to appreciably change the externally applied $\mathbf{B}$ field. The magnetic field produced by the orbital motion of the charged particles can be determined from the net electric current density associated with their motion. 


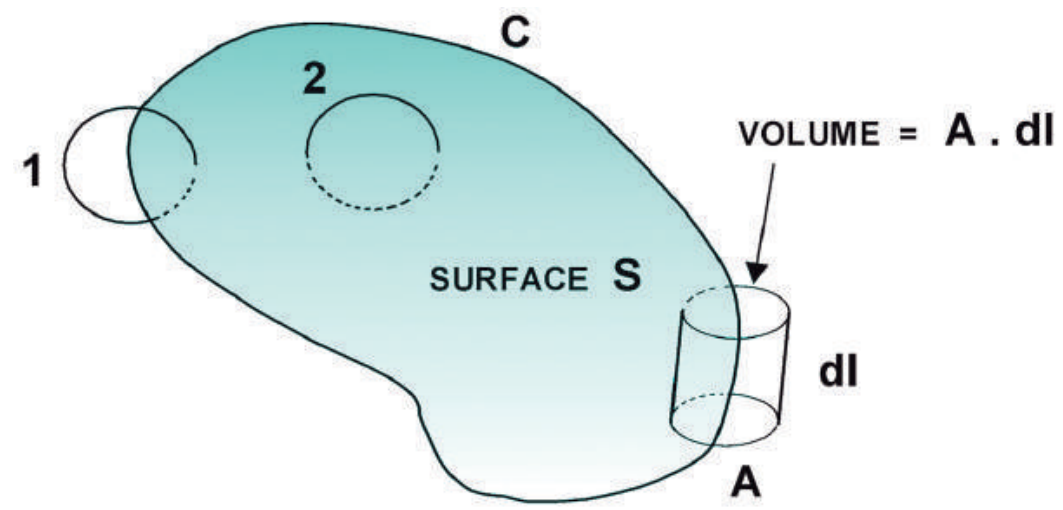

(a)

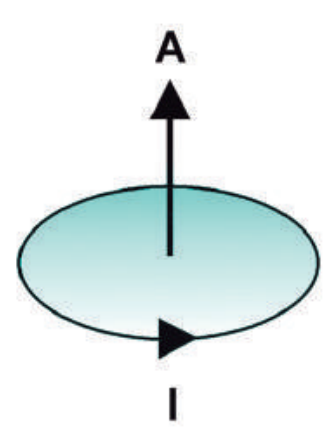

(b)

Fig. 8 (a) Electric current orbits crossing the surface element $S$ bounded by the curve $C$, in a macroscopic volume containing a large number of particles. (b) Positive direction of the vector area $\mathbf{A}$.

To calculate the resultant electric current density, let us consider a macroscopic volume containing a large number of particles. Let $S$ be an element of area in this volume, bounded by the curve $C$, as shown in Fig. 8(a). Orbits such as (1), which encircle the bounded surface only once, contribute to the resultant current, whereas orbits such as (2), which cross the surface twice, do not contribute to the net current. If $d \mathbf{l}$ is an element of arc along the curve $C$, the number of orbits encircling $d \mathbf{l}$ is given by $n \mathbf{A} \cdot d \mathbf{l}$, where $n$ is the number of orbits of current $I$, per unit volume, and $\mathbf{A}$ is the vector area bounded by each orbit. The direction of $\mathbf{A}$ is that of the normal to the orbital area $\mathbf{A}$, the positive sense being related to the sense of circulation in the way the linear motion of a right-hand screw is related to its rotary motion. Thus, A points in the direction of the observer when $I$ flows counterclockwise, as shown in Fig. 8 (b). The net resultant current crossing $S$ is therefore given by the current encircling $d \mathbf{l}$ integrated along the curve $C$,

$$
I_{n}=\oint \operatorname{In} \mathbf{A} \cdot d \mathbf{l}
$$

Since $\mathbf{m}=I \mathbf{A}$, the magnetic moment, per unit volume, $\mathbf{M}$ (also called the magnetization vector), is given by

$$
\mathbf{M}=n \mathbf{m}=n I \mathbf{A}
$$


Hence, (4.36) can be written as

$$
I_{n}=\oint \mathbf{M} \cdot d \mathbf{l}=\int_{S}(\nabla \times \mathbf{M}) \cdot d \mathbf{S}
$$

where we have applied Stokes's theorem. We may define an average magnetization current density, $\mathbf{J}_{M}$, crossing the surface $S$, by

$$
I_{n}=\int_{S} \mathbf{J}_{M} \cdot d \mathbf{S}
$$

Consequently, from (4.38) and (4.39) we obtain the magnetization current density as

$$
\mathbf{J}_{M}=\nabla \times \mathbf{M}
$$

where, from (4.37) and (4.35),

$$
\mathbf{M}=n \mathbf{m}=-\left(\frac{n W_{\perp}}{B^{2}}\right) \mathbf{B}
$$

and $n W_{\perp}$ denotes the kinetic energy, per unit volume, associated with the transverse particle velocity.

The charge density $\rho_{M}$ associated with the magnetization current density $\mathbf{J}_{M}$ can be deduced from the equation of continuity,

$$
\frac{\partial \rho_{M}}{\partial t}+\nabla \cdot \mathbf{J}_{M}=0
$$

Since $\mathbf{J}_{M}=\nabla \times \mathbf{M}$ and since for any vector $\mathbf{a}$, we have $\nabla \cdot(\nabla \times \mathbf{a})=0$, it follows that the charge density $\rho_{M}$ is constant.

In the following Maxwell equation,

$$
\nabla \times \mathbf{B}=\mu_{o}\left(\mathbf{J}+\epsilon_{o} \frac{\partial \mathbf{E}}{\partial t}\right)
$$

we can separate the total current density $\mathbf{J}$ into two parts: a magnetization current density $\mathbf{J}_{M}$ and a current density $\mathbf{J}^{\prime}$ due to other sources,

$$
\mathbf{J}=\mathbf{J}_{M}+\mathbf{J}^{\prime}
$$

Expressing $\mathbf{J}_{M}$ in terms of $\mathbf{M}$, through (4.40), and substituting in (4.43), we obtain

$$
\nabla \times \mathbf{B}=\mu_{o}\left(\nabla \times \mathbf{M}+\mathbf{J}^{\prime}+\epsilon_{o} \frac{\partial \mathbf{E}}{\partial t}\right)
$$


which can be rearranged as

$$
\nabla \times\left(\frac{1}{\mu_{o}} \mathbf{B}-\mathbf{M}\right)=\mathbf{J}^{\prime}+\epsilon_{o} \frac{\partial \mathbf{E}}{\partial t}
$$

Defining an effective magnetic field $\mathbf{H}$ by the relation

$$
\mathbf{B}=\mu_{o}(\mathbf{H}+\mathbf{M})
$$

we can write (4.46) as

$$
\nabla \times \mathbf{H}=\mathbf{J}^{\prime}+\epsilon_{o} \frac{\partial \mathbf{E}}{\partial t}
$$

Thus, the effective magnetic field $\mathbf{H}$ is related to the current due to other sources $\mathbf{J}^{\prime}$, in the way $\mathbf{B}$ is related to the total current $\mathbf{J}$. Eqs. (4.40) and (4.47) constitute the basic relations for the classical treatment of magnetic materials.

A simple linear relation between $\mathbf{B}$ and $\mathbf{H}$ exists when $\mathbf{M}$ is proportional to $\mathbf{B}$ or $\mathbf{H}$,

$$
\mathbf{M}=\chi_{m} \mathbf{H}
$$

where the constant $\chi_{m}$ is called the magnetic susceptibility of the medium. However, for a plasma we have seen that $M \propto 1 / B$ [see (4.41)], so that the relation between $\mathbf{H}$ and $\mathbf{B}$ (or $\mathbf{M}$ ) is not linear. Within this context it is generally not convenient to treat a plasma as a magnetic medium.

\section{UNIFORM ELECTROSTATIC AND MAGNETOSTATIC FIELDS}

\subsection{Formal Solution of the Equation of Motion}

We consider now the motion of a charged particle in the presence of both electric and magnetic fields that are constant in time and spatially uniform. The nonrelativistic equation of motion is

$$
m \frac{d \mathbf{v}}{d t}=q(\mathbf{E}+\mathbf{v} \times \mathbf{B})
$$

Taking components parallel and perpendicular to $\mathbf{B}$,

$$
\begin{gathered}
\mathbf{v}=\mathbf{v}_{\|}+\mathbf{v}_{\perp} \\
\mathbf{E}=\mathbf{E}_{\|}+\mathbf{E}_{\perp}
\end{gathered}
$$


we can resolve (5.1) into two component equations:

$$
\begin{gathered}
m \frac{d \mathbf{v}_{\|}}{d t}=q \mathbf{E}_{\|} \\
m \frac{d \mathbf{v}_{\perp}}{d t}=q\left(\mathbf{E}_{\perp}+\mathbf{v}_{\perp} \times \mathbf{B}\right)
\end{gathered}
$$

Eq. (5.4) is similar to (3.1) and represents a motion with constant acceleration $q \mathbf{E}_{\|} / m$ along the $\mathbf{B}$ field. Hence, according to (3.2) and (3.4),

$$
\begin{gathered}
\mathbf{v}_{\|}(t)=\left(\frac{q \mathbf{E}_{\|}}{m}\right) t+\mathbf{v}_{\|}(0) \\
\mathbf{r}_{\|}(t)=\frac{1}{2}\left(\frac{q \mathbf{E}_{\|}}{m}\right) t^{2}+\mathbf{v}_{\|}(0) t+\mathbf{r}_{\|}(0)
\end{gathered}
$$

To solve (5.5) it is convenient to separate $\mathbf{v}_{\perp}$ into two components,

$$
\mathbf{v}_{\perp}(t)=\mathbf{v}_{\perp}^{\prime}(t)+\mathbf{v}_{E}
$$

where $\mathbf{v}_{E}$ is a constant velocity in the plane normal to $\mathbf{B}$. Hence, $\mathbf{v}_{\perp}^{\prime}$ represents the particle velocity as seen by an observer in a frame of reference moving with the constant velocity $\mathbf{v}_{E}$. Substituting (5.8) into (5.5), and writing the component of the electric field perpendicular to $\mathbf{B}$ in the form (see Fig. 9)

$$
\mathbf{E}_{\perp}=-\left(\frac{\mathbf{E}_{\perp} \times \mathbf{B}}{B^{2}}\right) \times \mathbf{B}
$$

we obtain

$$
m \frac{d \mathbf{v}_{\perp}^{\prime}}{d t}=q\left(\mathbf{v}_{\perp}^{\prime}+\mathbf{v}_{E}-\frac{\mathbf{E}_{\perp} \times \mathbf{B}}{B^{2}}\right) \times \mathbf{B}
$$

This equation shows that in a coordinate system moving with the constant velocity

$$
\mathbf{v}_{E}=\frac{\mathbf{E}_{\perp} \times \mathbf{B}}{B^{2}}
$$

the particle motion in the plane normal to $\mathbf{B}$ is governed entirely by the magnetic field, according to

$$
m \frac{d \mathbf{v}_{\perp}^{\prime}}{d t}=q\left(\mathbf{v}_{\perp}^{\prime} \times \mathbf{B}\right)
$$

Thus, in this frame of reference, the electric field component $\mathbf{E}_{\perp}$ is transformed away, whereas the magnetic field is left unchanged. Eq. (5.12) is 


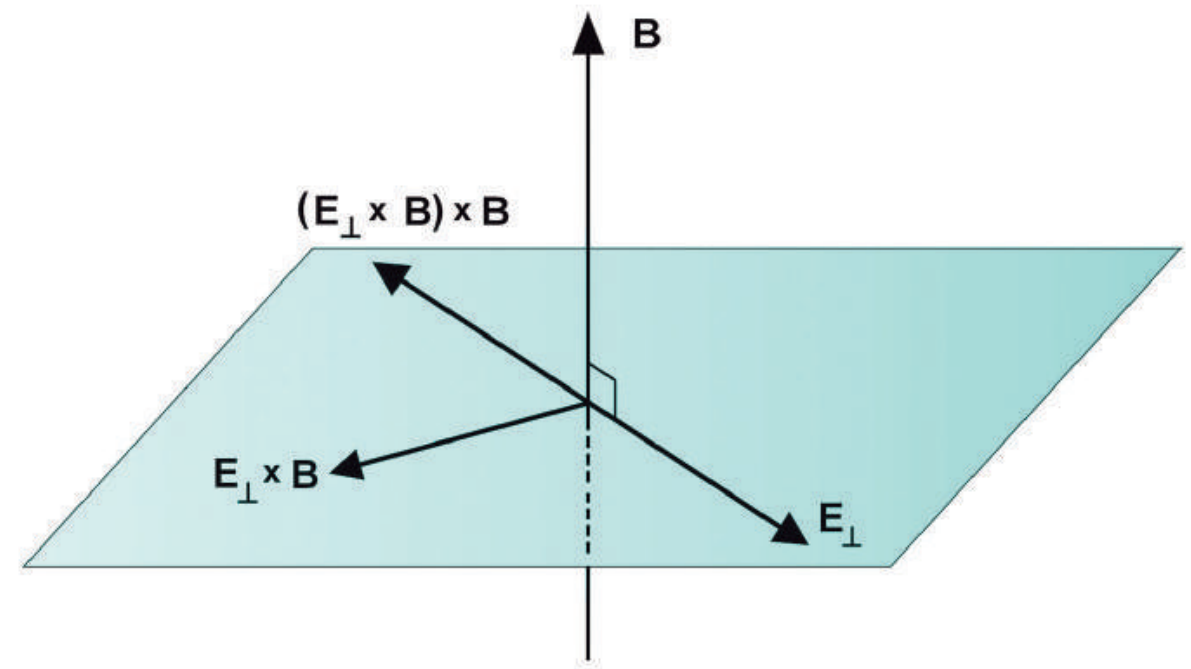

Fig. 9 Vector products appearing in Eq. (5.9) $(\widehat{\mathbf{B}}=\mathbf{B} / B)$.

identical to (4.5) and implies that in the reference system moving with the constant velocity $\mathbf{v}_{E}$, given by (5.11), the particle describes a circular motion at the cyclotron frequency $\Omega_{c}$ with radius $r_{c}$,

$$
\mathbf{v}_{\perp}^{\prime}=\Omega_{c} \times \mathbf{r}_{c}
$$

The results obtained so far indicate that the resulting particle motion is described by a superposition of a circular motion in the plane normal to $\mathbf{B}$, with a uniform motion with the constant velocity $\mathbf{v}_{E}$ perpendicular to both $\mathbf{B}$ and $\mathbf{E}_{\perp}$, plus a uniform acceleration $q \mathbf{E}_{\|} / m$ along $\mathbf{B}$. The particle velocity can be expressed in vector form, independently of a coordinate system, as

$$
\mathbf{v}(t)=\boldsymbol{\Omega}_{c} \times \mathbf{r}_{c}+\frac{\mathbf{E}_{\perp} \times \mathbf{B}}{B^{2}}+\frac{q \mathbf{E}_{\|}}{m} t+\mathbf{v}_{\|}(0)
$$

The first term in the right-hand side of (5.14) represents the cyclotron circular motion, and the following ones represent, respectively, the drift velocity of the guiding center (perpendicular to both $\mathbf{E}_{\perp}$ and $\mathbf{B}$ ), the constant acceleration of the guiding center along $\mathbf{B}$, and the initial velocity parallel to $\mathbf{B}$.

Note that the velocity $\mathbf{v}_{E}$ is independent of the mass and of the sign of the charge and therefore is the same for both positive and negative 
particles. It is usually called the plasma drift velocity or the electromagnetic plasma drift. Since $\mathbf{E}_{\|} \times \mathbf{B}=0$, (5.11) can also be written as

$$
\mathbf{v}_{E}=\frac{\mathbf{E} \times \mathbf{B}}{B^{2}}
$$

The resulting motion of the particle in the plane normal to $\mathbf{B}$ is, in general, a cycloid, as shown in Fig. 10. The physical explanation for this cycloidal motion is a follows. The electric force $q \mathbf{E}_{\perp}$, acting simultaneously with the magnetic force, accelerates the particle so as to increase or decrease its velocity, depending on the relative direction of the particle motion with respect to the direction of $\mathbf{E}_{\perp}$ and on the charge sign. According to (4.13) the radius of gyration increases with velocity, and hence the radius of curvature of the particle path varies under the action of $\mathbf{E}_{\perp}$. This results in a cycloidal trajectory with a net drift in the direction perpendicular to both $\mathbf{E}$ and $\mathbf{B}$. Different trajectories are obtained, depending on the initial conditions and on the magnitude of the applied electric and magnetic fields.

The ions are much more massive than the electrons, and therefore the Larmor radius for ions is correspondingly greater and the Larmor frequency correspondingly smaller than for electrons. Consequently, the arcs of cycloid for ions are greater than for electrons, but there is a larger number of arcs of cycloid per second for electrons, such that the drift velocity is the same for both species.

In a collisionless plasma the drift velocity does not imply an electric current, since both positive and negative particles move together. When collisions between charged and neutral particles are important, this drift gives rise to an electric current, since the ion-neutral collision frequency is greater than the electron-neutral collision frequency, causing the ions to move slower than the electrons. This current is normal to both $\mathbf{E}$ and $\mathbf{B}$, and is in the direction opposite to $\mathbf{v}_{E}$. It is known as the Hall current.

\subsection{Solution in Cartesian Coordinates}

Let us choose a Cartesian coordinate system with the $z$ axis pointing in the direction of $\mathbf{B}$, so that

$$
\begin{gathered}
\mathbf{B}=B \widehat{\mathbf{z}} \\
\mathbf{E}=E_{x} \widehat{\mathbf{x}}+E_{y} \widehat{\mathbf{y}}+E_{z} \widehat{\mathbf{z}}
\end{gathered}
$$




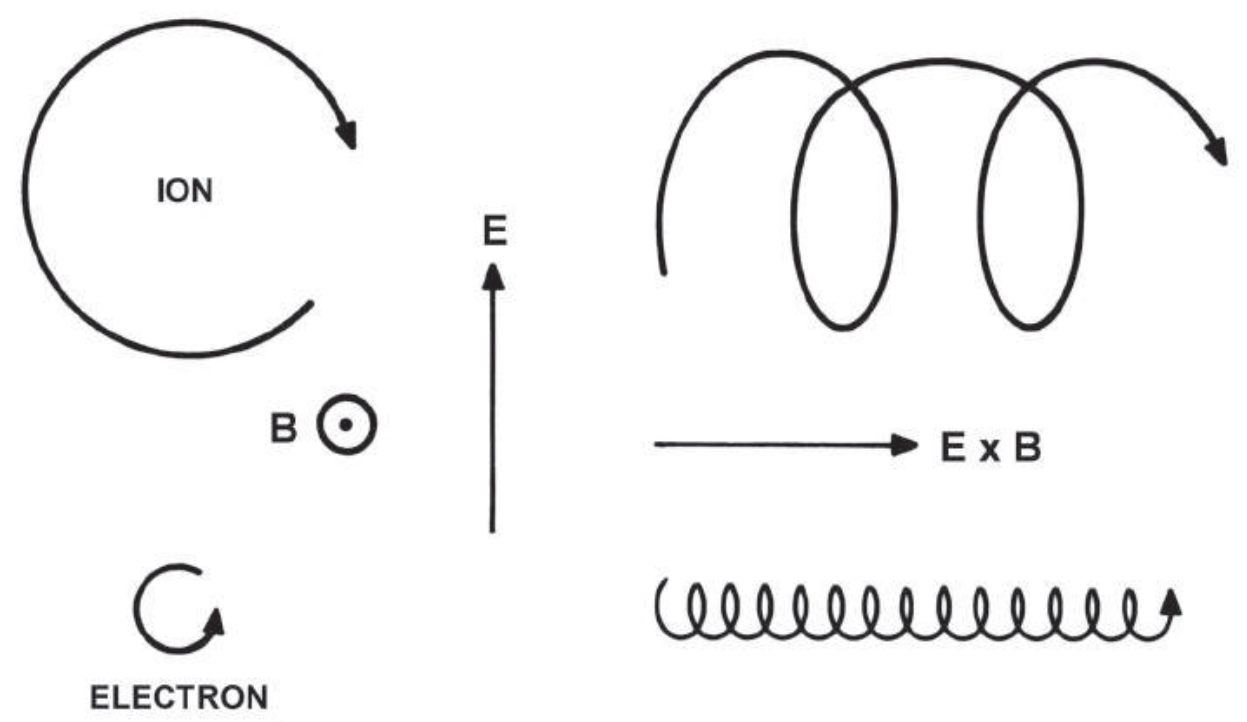

Fig. 10 Cycloidal trajectories described by ions and electrons in crossed electric and magnetic fields. The electric field $\mathbf{E}$ acting together with the magnetic flux density $\mathbf{B}$ gives rise to a drift velocity in the direction given by $\mathbf{E} \times \mathbf{B}$.

Using (4.15), the equation of motion (5.1) can be written as

$$
\frac{d \mathbf{v}}{d t}=\frac{q}{m}\left[\left(E_{x}+v_{y} B\right) \widehat{\mathbf{x}}+\left(E_{y}-v_{x} B\right) \widehat{\mathbf{y}}+E_{z} \widehat{\mathbf{z}}\right]
$$

As before, we consider, in what follows, a positive charge. The results for a negative charge can be obtained by changing the sign of $\Omega_{c}$ in the results for the positive charge.

The $z$ component of (5.18) can be integrated directly and gives the same results expressed in (5.6) and (5.7). For the $x$ and $y$ components, we first take the derivative of $d v_{x} / d t$ with respect to time and substitute the expression for $d v_{y} / d t$, which gives

$$
\frac{d^{2} v_{x}}{d t^{2}}+\Omega_{c}^{2} v_{x}=\Omega_{c}^{2} \frac{E_{y}}{B}
$$

This is the inhomogeneous differential equation for a harmonic oscillator of frequency $\Omega_{c}$. Its solution is given by the sum of the homogeneous equation solution, given in (4.21), with a particular solution (which is clearly given by $\left.E_{y} / B\right)$. Thus,

$$
v_{x}(t)=v_{\perp}^{\prime} \sin \left(\Omega_{c} t+\theta_{o}\right)+\frac{E_{y}}{B}
$$


where $v_{\perp}^{\prime}$ and $\theta_{o}$ are integration constants. The solution for $v_{y}(t)$ can be obtained by substituting (5.20) directly into (5.18). Hence,

$$
v_{y}(t)=\frac{1}{\Omega_{c}} \frac{d v_{x}}{d t}-\frac{E_{x}}{B}=v_{\perp}^{\prime} \cos \left(\Omega_{c} t+\theta_{o}\right)-\frac{E_{x}}{B}
$$

Therefore, the velocity components $v_{x}(t)$ and $v_{y}(t)$, in the plane perpendicular to $\mathbf{B}$, oscillate at the cyclotron frequency $\Omega_{c}$ and with amplitude $v_{\perp}^{\prime}$. This motion is superposed to a constant drift velocity $\mathbf{v}_{E}$ given by

$$
\mathbf{v}_{E}=\frac{E_{y}}{B} \widehat{\mathbf{x}}-\frac{E_{x}}{B} \widehat{\mathbf{y}}
$$

This expression corresponds to (5.11) when $\mathbf{B}=B \widehat{\mathbf{z}}$.

One more integration of (5.20) and (5.21) gives the particle trajectory in the $(x, y)$ plane

$$
\begin{gathered}
x(t)=-\frac{v_{\perp}^{\prime}}{\Omega_{c}} \cos \left(\Omega_{c} t+\theta_{o}\right)+\frac{E_{y}}{B} t+X_{o} \\
y(t)=\frac{v_{\perp}^{\prime}}{\Omega_{c}} \sin \left(\Omega_{c} t+\theta_{o}\right)-\frac{E_{x}}{B} t+Y_{o}
\end{gathered}
$$

where $X_{o}$ and $Y_{o}$ are defined according to (4.27) and (4.28), but with $v_{\perp}$ replaced by $v_{\perp}^{\prime}$.

In summary, the motion of a charged particle in uniform electrostatic and magnetostatic fields consists of three components:

(a) A constant acceleration $q \mathbf{E}_{\|} / m$ along the $\mathbf{B}$ field. If $\mathbf{E}_{\|}=0$, the particle moves along $\mathbf{B}$ with its initial velocity.

(b) A rotation in the plane normal to $\mathbf{B}$ at the cyclotron frequency $\Omega_{c}=|q| B / m$ and radius $r_{c}=v_{\perp}^{\prime} / \Omega_{c}$.

(c) An electromagnetic drift velocity $\mathbf{v}_{E}=(\mathbf{E} \times \mathbf{B}) / B^{2}$, perpendicular to both $\mathbf{B}$ and $\mathbf{E}$.

\section{DRIFT DUE TO AN EXTERNAL FORCE}

If some additional force $\mathbf{F}$ (gravitational force or inertial force, if the motion is considered in a noninertial system, for example) is present, the equation of motion (1.5) must be modified to include this force,

$$
m \frac{d \mathbf{v}}{d t}=q(\mathbf{E}+\mathbf{v} \times \mathbf{B})+\mathbf{F}
$$



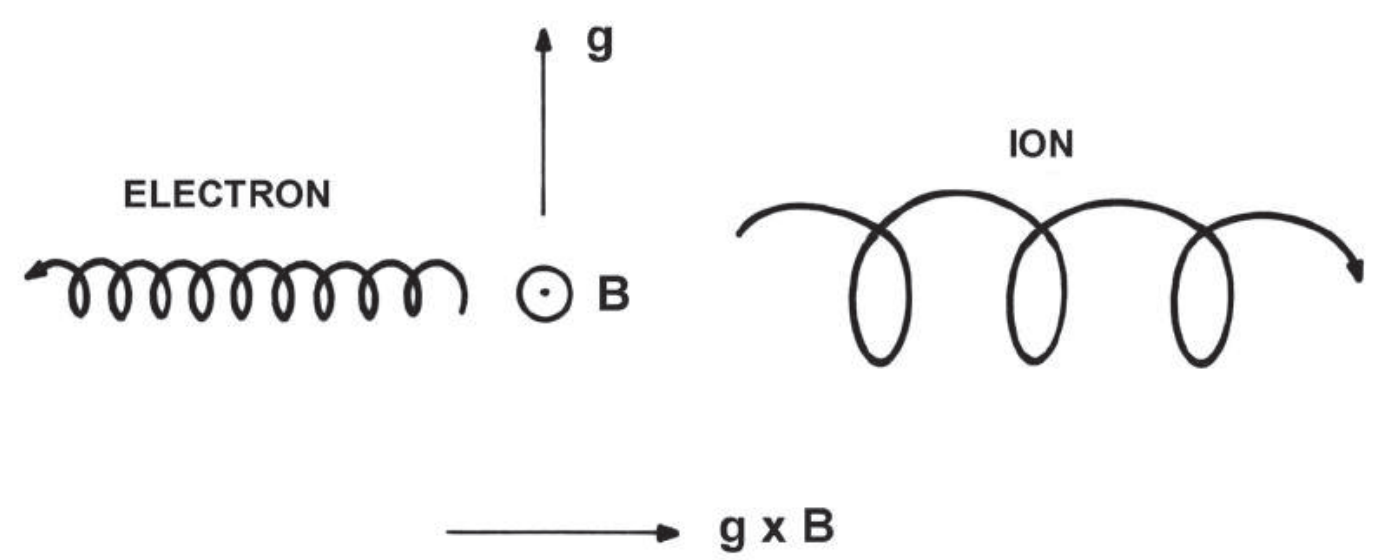

Fig. 11 Drift of a gyrating particle in crossed gravitational and magnetic fields.

The effect of this force is, in a formal sense, analogous to the effect of the electric field. We assume here that $\mathbf{F}$ is uniform and constant. In analogy with the electromagnetic drift velocity $\mathbf{v}_{E}$, given in (5.15), the drift produced by the force $\mathbf{F}$ having a component normal to $\mathbf{B}$ is given by

$$
\mathbf{v}_{F}=\frac{\mathbf{F} \times \mathbf{B}}{q B^{2}}
$$

In the case of a uniform gravitational field, for example, we have $\mathbf{F}=m \mathbf{g}$, where $\mathbf{g}$ is the acceleration due to gravity, and the drift velocity is given by

$$
\mathbf{v}_{g}=\frac{m}{q} \frac{\mathbf{g} \times \mathbf{B}}{B^{2}}
$$

This drift velocity depends on the ratio $m / q$ and therefore it is in opposite directions for particles of opposite charge (Fig. 11). We have seen that in a coordinate system moving with the velocity $\mathbf{v}_{E}$, the electric field component $\mathbf{E}_{\perp}$ is transformed away, leaving the magnetic field unchanged. The gravitational field, however, cannot, in this context, be transformed away.

In a collisionless plasma, associated with the gravitational drift velocity there is an electric current density, $\mathbf{J}_{g}$, in the direction of $\mathbf{g} \times \mathbf{B}$, which can be expressed as

$$
\mathbf{J}_{g}=\frac{1}{\delta V} \sum_{i} q_{i} \mathbf{v}_{g i}
$$


where the summation is over all charged particles contained in a suitably chosen small volume element $\delta V$. Using (6.3) we obtain

$$
\mathbf{J}_{g}=\frac{1}{\delta V}\left(\sum_{i} m_{i}\right) \frac{\mathbf{g} \times \mathbf{B}}{B^{2}}=\rho_{m} \frac{\mathbf{g} \times \mathbf{B}}{B^{2}}
$$

where $\rho_{m}$ denotes the total mass density of the charged particles.

A comment on the validity of (6.2) is appropriate here. Since we have used the nonrelativistic equation of motion, there is a limitation on the magnitude of the force $\mathbf{F}$ in order that (6.2) be applicable. The magnitude of the transverse drift velocity is given by

$$
v_{D}=\frac{F_{\perp}}{q B}
$$

Hence, for the nonrelativistic equation of motion to be applicable we must have

$$
\frac{F_{\perp}}{q B} \ll c
$$

or, if $\mathbf{F}$ is due to an electrostatic field $\mathbf{E}$,

$$
\frac{E_{\perp}}{B} \ll c
$$

For a magnetic field of 1 tesla, for example, (6.2) may be used as long as $E_{\perp}$ is much less than $10^{8}$ volts $/ \mathrm{m}$. If these conditions are not satisfied, the problem must be treated using the relativistic equation of motion. Although the relativistic equation of motion can be integrated exactly for constant $\mathbf{B}, \mathbf{E}$, and $\mathbf{F}$, we shall not analyze this problem here. It is left as an exercise for the reader.

\section{PROBLEMS}

2.1 Calculate the cyclotron frequency and the cyclotron radius for: (a) An electron in the Earth's ionosphere at $300 \mathrm{~km}$ altitude, where the magnetic flux density $B \simeq 0.5 \times 10^{-4}$ tesla, considering that the electron moves at the thermal velocity $(k T / m)$, with $T=1000 \mathrm{~K}$, where $k$ is Boltzmann's constant.

(b) A $50 \mathrm{MeV}$ proton in the Earth's inner Van Allen radiation belt at 
about $1.5 R_{E}$ (where $R_{E}=6370 \mathrm{~km}$ is the Earth's radius) from the center of the Earth in the equatorial plane, considering $B \simeq 10^{-5}$ tesla.

(c) A $1 \mathrm{MeV}$ electron in the Earth's outer Van Allen radiation belt at about $4 R_{E}$ from the center of the Earth in the equatorial plane, where $B \simeq 10^{-7}$ tesla.

(d) A proton in the solar wind with a streaming velocity of $100 \mathrm{~km} / \mathrm{s}$, in a magnetic flux density $B \simeq 10^{-9}$ tesla.

(e) A $1 \mathrm{MeV}$ proton in a sunspot region of the solar photosphere, considering $B \simeq 0.1$ tesla.

2.2 For an electron and an oxygen ion $O^{+}$in the Earth's ionosphere, at $300 \mathrm{~km}$ altitude in the equatorial plane, where $B \simeq 0.5 \times 10^{-4}$ tesla, calculate:

(a) The gravitational drift velocity $\mathbf{v}_{g}$.

(b) the gravitational current density $\mathbf{J}_{g}$, considering $n_{e}=n_{i}=10^{12} \mathrm{~m}^{-3}$.

Assume that $\mathbf{g}$ is perpendicular to $\mathbf{B}$.

2.3 Consider a particle of mass $m$ and charge $q$ moving in the presence of constant and uniform electromagnetic fields given by $\mathbf{E}=E_{o} \widehat{\mathbf{y}}$ and $\mathbf{B}=B_{o} \widehat{\mathbf{z}}$. Assuming that initially $(t=0)$ the particle is at rest at the origin of a Cartesian coordinate system, show that it moves on the cycloid

$$
\begin{gathered}
x(t)=\frac{E_{o}}{B_{o}}\left[t-\frac{1}{\Omega_{c}} \sin \left(\Omega_{c} t\right)\right] \\
y(t)=\frac{E_{o}}{B_{o}} \frac{1}{\Omega_{c}}\left[1-\cos \left(\Omega_{c} t\right)\right]
\end{gathered}
$$

Plot the trajectory of the particle in the $z=0$ plane for $q>0$ and for $q<0$, and consider the cases when $v_{\perp}>v_{E}, v_{\perp}=v_{E}$, and $v_{\perp}<v_{E}$, where $v_{\perp}$ denotes the particle cyclotron motion velocity and $v_{E}$ is the electromagnetic drift velocity.

2.4 In general the trajectory of a charged particle in crossed electric and magnetic fields is a cycloid. Show that, if $\mathbf{v}=v_{o} \widehat{\mathbf{x}}, \mathbf{B}=B_{o} \widehat{\mathbf{z}}$, and $\mathbf{E}=E_{o} \widehat{\mathbf{y}}$, then for $v_{o}=E_{o} / B_{o}$ the path is a straight line. Explain how this situation can be exploited to design a mass spectrometer.

2.5 Derive the relativistic equation of motion in the form (1.4), starting from (1.1) and the relation (1.2). 
2.6 Write down, in vector form, the relativistic equation of motion for a charged particle in the presence of a uniform magnetostatic field $\mathbf{B}=B_{o} \widehat{\mathbf{z}}$, and show that its Cartesian components are given by

$$
\begin{gathered}
\frac{d}{d t}\left(\gamma v_{x}\right)=\left(\frac{q B_{o}}{m}\right) v_{y} \\
\frac{d}{d t}\left(\gamma v_{y}\right)=-\left(\frac{q B_{o}}{m}\right) v_{x} \\
\frac{d}{d t}\left(\gamma v_{z}\right)=0
\end{gathered}
$$

where

$$
\gamma=\frac{1}{\left(1-\beta^{2}\right)^{1 / 2}}
$$

and where $\beta=v / c$. Show that the velocity and trajectory of the charged particle are given by the same formulas as in the nonrelativistic case, but with $\Omega_{c}$ replaced by $|q| B_{o} /(m \gamma)$.

2.7 Analyze the motion of a relativistic charged particle in the presence of crossed electric (E) and magnetic (B) fields that are constant in time and uniform in space. What coordinate transformation must be made in order to transform away the transversal electric field? Derive equations for the velocity and trajectory of the charged particle. 


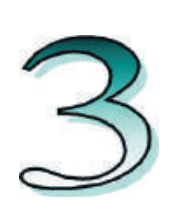

\section{ChARged PARTICLE MOTION IN NONUNIFORM MAGNETOSTATIC FIELDS}

\section{INTRODUCTION}

When the fields are spatially nonuniform, or when they vary with time, the integration of the equation of motion (2.1.1) (Eq. 1.1 in Chapter 2) can be a mathematical problem of great difficulty. In this case, since the equation of motion is nonlinear, the theory may become extremely involved, and rigorous analytic expressions for the charged particle trajectory cannot, in general, be obtained in closed form. Numerical methods of integration must be used in order to obtain all the details of the motion.

There is one particularly important case, however, in which it becomes possible to obtain an approximate, but otherwise general, solution without recourse to numerical integration, if the details of the particle motion are not of interest. This is the case when the magnetic field is strong and slowly varying in both space and time, and when the electric field is weak. In a wide variety of situations of interest the fields are approximately constant and uniform, at least on the distance and time scales seen by the particle during one gyration about the magnetic field. This is the case for many laboratory plasmas, including those of relevance to the problem of controlled thermonuclear reactions, and also for a great number of astrophysical plasmas.

In this chapter we investigate the motion of a charged particle in a static magnetic field slightly inhomogeneous in space. The word slightly here means that the spatial variation of the magnetic field inside the particle orbit is small compared to the magnitude of $\mathbf{B}$. In other words, we shall consider only magnetostatic fields whose spatial change in a distance 


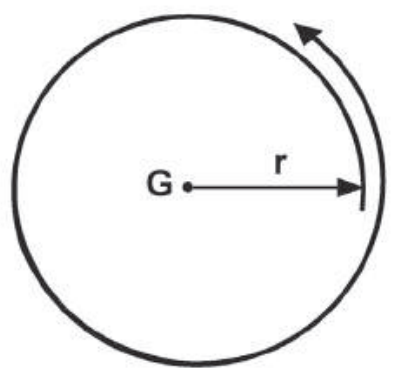

Fig. 1 The motion of a charged particle in a slightly inhomogeneous magnetic field is nearly circular.

of the order of the Larmor radius, $r_{c}$, is much smaller than the magnitude of the field itself.

To specify more quantitatively this assumption, let $\delta B$ represent the spatial change in the magnitude of $\mathbf{B}$ in a distance of the order of $r_{c}$, that is, $\delta B=r_{c}|\nabla B|$, where $\nabla B$ is the gradient of the magnitude of $\mathbf{B}$. It is assumed therefore that $\delta B \ll B$. Consequently, in what follows we limit our discussion to problems where the deviations from uniformity are small and solve for the particle trajectory only in the first-order approximation. The analysis of charged particle motion in stationary fields based on this approximation in often referred to as the first-order orbit theory. This theory was first used systematically by the Swedish scientist Alfvén, and it is also known as the Alfvén approximation or the guiding center approximation.

The concept of guiding center is of great utility in the development of this theory. We have seen that in a uniform magnetic field the particle motion can be regarded as a superposition of a circular motion about the direction of $\mathbf{B}$, with a motion of the guiding center along $\mathbf{B}$. In the case of a nonuniform $\mathbf{B}$ field, satisfying the condition $\delta B \ll B$, the value of $\mathbf{B}$ at the particle position differs only slightly from its value at the guiding center. The component of the particle motion, in a plane normal to the field line that passes through the guiding center instantaneous position, will still be nearly circular (Fig. 1). However, due to the spatial variation of $\mathbf{B}$, we expect in this case a gradual drift of the guiding center across $\mathbf{B}$, as well as a gradual change of its velocity along $\mathbf{B}$.

The rapid gyrations of the charged particle about the direction of $\mathbf{B}$ are not usually of great interest, and it is convenient to eliminate them 
from the equations of motion, and focus attention on the guiding center motion. In the motion of the guiding center, the small oscillations (of amplitude small compared to the cyclotron radius) occurring during one gyration period may be averaged out, since they represent the effect of perturbations due to the spatial variation of the magnetic field. The problem is thus reduced to the calculation of the average values over one gyration period (and not the instantaneous values) of the guiding center transverse drift velocity and parallel acceleration.

\section{SPATIAL VARIATION OF THE MAGNETIC FIELD}

Any of the three components of the magnetic flux density, $\mathbf{B}=B_{x} \widehat{\mathbf{x}}+$ $B_{y} \widehat{\mathbf{y}}+B_{z} \widehat{\mathbf{z}}$, may vary with respect to the three coordinates $x, y$, and $z$. Consequently, nine parameters are needed to completely specify the spatial variation of $\mathbf{B}$. These parameters can be conveniently represented by the dyad (or tensor) $\nabla \mathbf{B}$, which can be written in matrix form as

$$
\nabla \mathbf{B}=\left(\begin{array}{lll}
\widehat{\mathbf{x}} & \widehat{\mathbf{y}} & \widehat{\mathbf{z}}
\end{array}\right)\left(\begin{array}{lll}
\partial B_{x} / \partial x & \partial B_{y} / \partial x & \partial B_{z} / \partial x \\
\partial B_{x} / \partial y & \partial B_{y} / \partial y & \partial B_{z} / \partial y \\
\partial B_{x} / \partial z & \partial B_{y} / \partial z & \partial B_{z} / \partial z
\end{array}\right)\left(\begin{array}{l}
\widehat{\mathbf{x}} \\
\widehat{\mathbf{y}} \\
\widehat{\mathbf{z}}
\end{array}\right)
$$

Of these nine components only eight are independent, since the following Maxwell equation

$$
\nabla \cdot \mathbf{B}=\frac{\partial B_{x}}{\partial x}+\frac{\partial B_{y}}{\partial y}+\frac{\partial B_{z}}{\partial z}=0
$$

shows that only two of the divergence terms are independent.

If the condition $\mathbf{J}=0$ is also satisfied, in the region where the particle is moving, then other restrictions are imposed on the number of independent components of $\mathbf{B}$ since, under these circumstances, the relation $\nabla \times \mathbf{B}=0$ holds. This means that, in regions where there are no electric currents, $\mathbf{B}$ can be written as the gradient of a scalar magnetic potential,

$$
\mathbf{B}=\nabla \phi_{m}
$$

where the magnetic potential $\phi_{m}$ satisfies the Laplace equation

$$
\nabla^{2} \phi_{m}=0
$$

In regions where an electric current density exists, we have $\nabla \times \mathbf{B}=\mu_{0} \mathbf{J}$ and we cannot define a scalar magnetic potential $\phi_{m}$ as indicated. The 
number of independent components of $\nabla \mathbf{B}$ cannot, in this case, be reduced without knowing the electric current density $\mathbf{J}$.

Let us consider a Cartesian coordinate system such that at the origin the magnetic field is in the $z$ direction,

$$
\mathbf{B}(0,0,0)=\mathbf{B}_{0}=B_{0} \widehat{\mathbf{z}}
$$

The nine components of $\nabla \mathbf{B}$ can be conveniently grouped into four categories:

(a) Divergence terms:

$$
\partial B_{x} / \partial x, \quad \partial B_{y} / \partial y, \quad \partial B_{z} / \partial z
$$

(b) Gradient terms:

$$
\partial B_{z} / \partial x, \quad \partial B_{z} / \partial y
$$

(c) Curvature terms:

$$
\partial B_{x} / \partial z, \quad \partial B_{y} / \partial z
$$

(d) Shear terms:

$$
\partial B_{x} / \partial y, \quad \partial B_{y} / \partial x
$$

\subsection{Divergence Terms}

We shall initially discuss the magnetic field line geometry corresponding to the divergence terms of $\nabla \mathbf{B}$. The presence of a small variation in the component $B_{z}$ in the $z$ direction (i.e., $\partial B_{z} / \partial z \neq 0$ ), implies that at least one of the terms $\partial B_{x} / \partial x$ or $\partial B_{y} / \partial y$ is also present, as can be seen from (2.2). It is of great utility to make use here of the concept of magnetic flux lines, which, at any point, are parallel to the $\mathbf{B}$ field at that point and whose density at each point is proportional to the local magnitude of B. To determine the differential equation of a line of force, let

$$
d \mathbf{s}=d x \widehat{\mathbf{x}}+d y \widehat{\mathbf{y}}+d z \widehat{\mathbf{z}}
$$

be an element of arc along the magnetic field line. Then, we must have

$$
d \mathbf{s} \times \mathbf{B}=0
$$

since ds is parallel to $\mathbf{B}$, which gives by expansion of the cross product,

$$
\frac{d x}{B_{x}}=\frac{d y}{B_{y}}=\frac{d z}{B_{z}}
$$




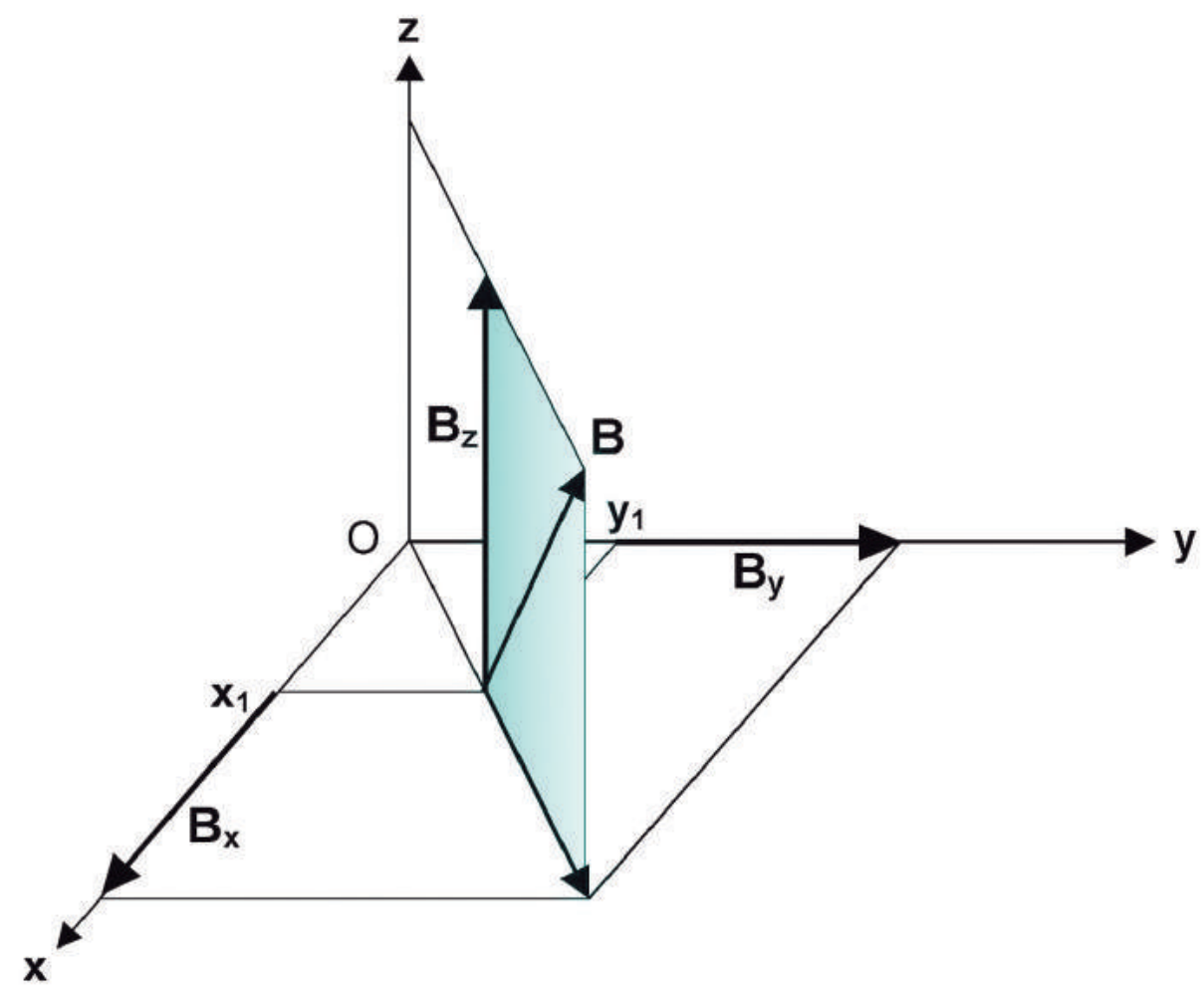

Fig. 2 The magnetic field components $B_{x}$ and $B_{y}$ at the points $\left(x_{1}, 0,0\right)$ and $\left(0, y_{1}, 0\right)$, near the origin.

Since we are focusing attention only on the divergence terms of $\mathbf{B}$, and since in the region of interest the field is considered to be mainly in the $z$ direction, we may expand $B_{x}$ and $B_{y}$ in a Taylor series about the origin (see Fig. 2), as follows,

$$
\begin{gathered}
B_{x}\left(x_{1}, 0,0\right)=B_{x}(0,0,0)+\left(\frac{\partial B_{x}}{\partial x}\right) x_{1}=\left(\frac{\partial B_{x}}{\partial x}\right) x_{1} \\
B_{y}\left(0, y_{1}, 0\right)=B_{y}(0,0,0)+\left(\frac{\partial B_{y}}{\partial y}\right) y_{1}=\left(\frac{\partial B_{y}}{\partial y}\right) y_{1}
\end{gathered}
$$

where the second and higher order terms were neglected. Note that at the origin $B_{x}=B_{y}=0$. Therefore, the magnetic field line crossing the $z=0$ plane at the point $\left(x_{1}, y_{1}, 0\right)$, when projected on the $(x, z)$ plane $(y=0)$ and on the $(y, z)$ plane $(x=0)$, satisfies the following differential equations, respectively,

$$
\frac{d x}{d z}=\frac{B_{x}}{B_{z}}=\frac{1}{B_{z}}\left(\frac{\partial B_{x}}{\partial x}\right) x_{1} \quad(y=0)
$$




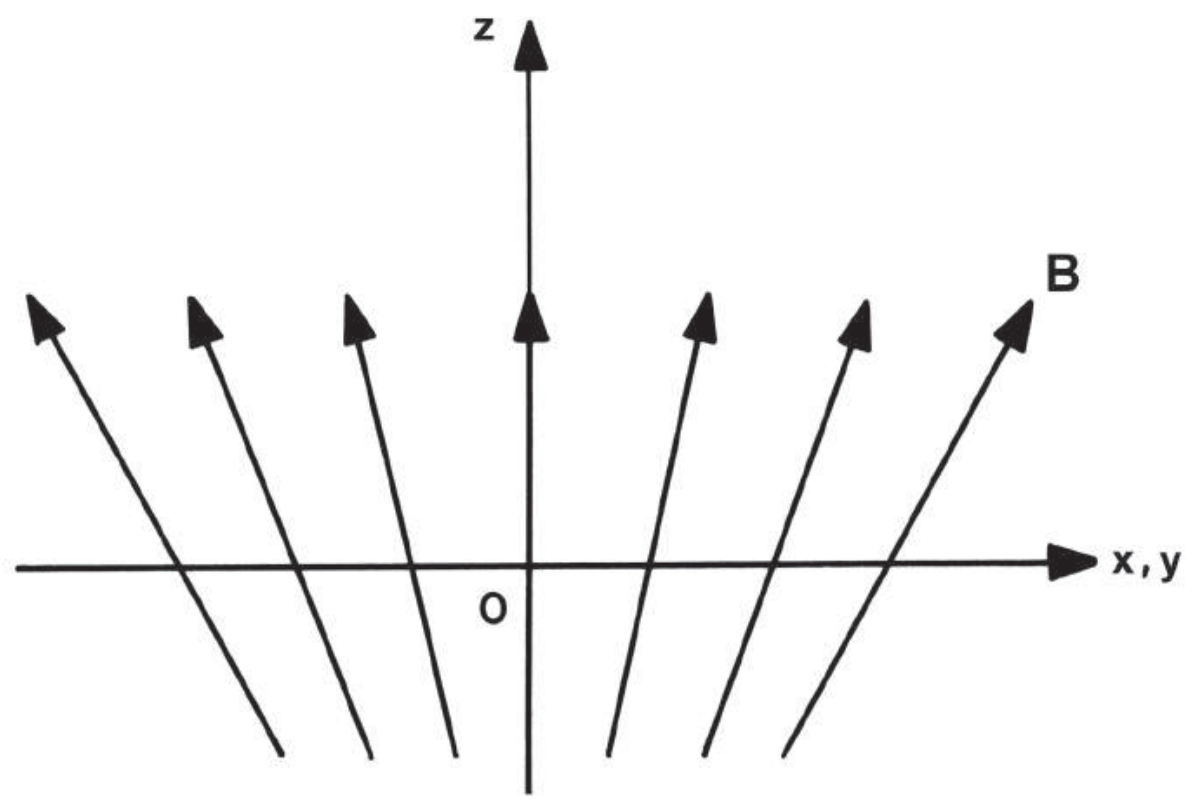

Fig. 3 Geometry of the magnetic field lines corresponding to the divergence terms $\partial B_{x} / \partial x$ or $\partial B_{y} / \partial y$, when they are positive.

$$
\frac{d y}{d z}=\frac{B_{y}}{B_{z}}=\frac{1}{B_{z}}\left(\frac{\partial B_{y}}{\partial y}\right) y_{1} \quad(x=0)
$$

These equations show that the field lines converge or diverge in the $(x, z)$ plane or in the $(y, z)$ plane, depending on the sine of the divergence terms of B. Fig. 3 illustrates the field line geometry when $\partial B_{x} / \partial x$ and $\partial B_{y} / \partial y$ are positive.

\subsection{Gradient and Curvature Terms}

The following vector field has a gradient in the $x$ direction, as shown schematically in Fig. 4:

$$
\mathbf{B}=B_{z} \widehat{\mathbf{z}}=B_{0}(1+\alpha x) \widehat{\mathbf{z}}
$$

We must note, however, that in a region where $\mathbf{J}=0$ this vector field does not satisfy the Maxwell equation $\nabla \times \mathbf{B}=0$, so that we must add to (2.14) a term of curvature, given by $B_{x} \widehat{\mathbf{x}}=B_{0} \alpha z \widehat{\mathbf{x}}$. Therefore, a magnetic field having gradient and curvature terms, and which satisfies $\nabla \times \mathbf{B}=0$, is

$$
\mathbf{B}=B_{0}[\alpha z \widehat{\mathbf{x}}+(1+\alpha x) \widehat{\mathbf{z}}]
$$




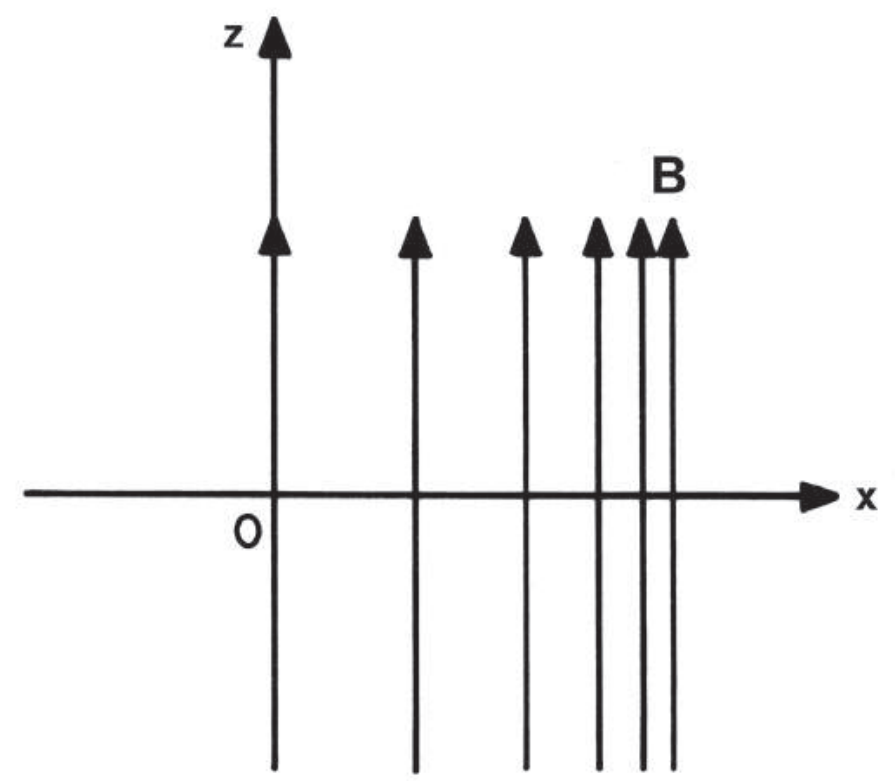

Fig. 4 Geometry of the magnetic field lines when $\mathbf{B}$ has a gradient in the $x$ direction, according to Eq. (2.14). This field geometry does not satisfy $\nabla \times \mathbf{B}=0$.

The geometry of the magnetic field lines corresponding to this expression is schematically indicated in Fig. 5.

Generally, all terms corresponding to divergence, gradient, and curvature are simultaneously present. Fig. 6 illustrates a $\mathbf{B}$ field having divergence, gradient, and curvature. An example is the Earth's magnetic field (refer to Fig. 4 in Chapter 1). Later in this section we will investigate separately the effects of each one of these terms on the charged particle motion. Since in the first-order approximation the equations are linear, the net effect will be the sum of the effects due to each one of them.

\subsection{Shear Terms}

The shear terms of (2.6) enter into the $z$ component of $\nabla \times \mathbf{B}$, that is, into $\mathbf{B} \cdot(\nabla \times \mathbf{B})$, and cause twisting of the magnetic field lines about each other. They do not produce any first-order drifts, although the shape of the orbit can be slightly changed. They do not give rise to any particularly interesting effects on the motion of charged particles and will not be considered any further. 


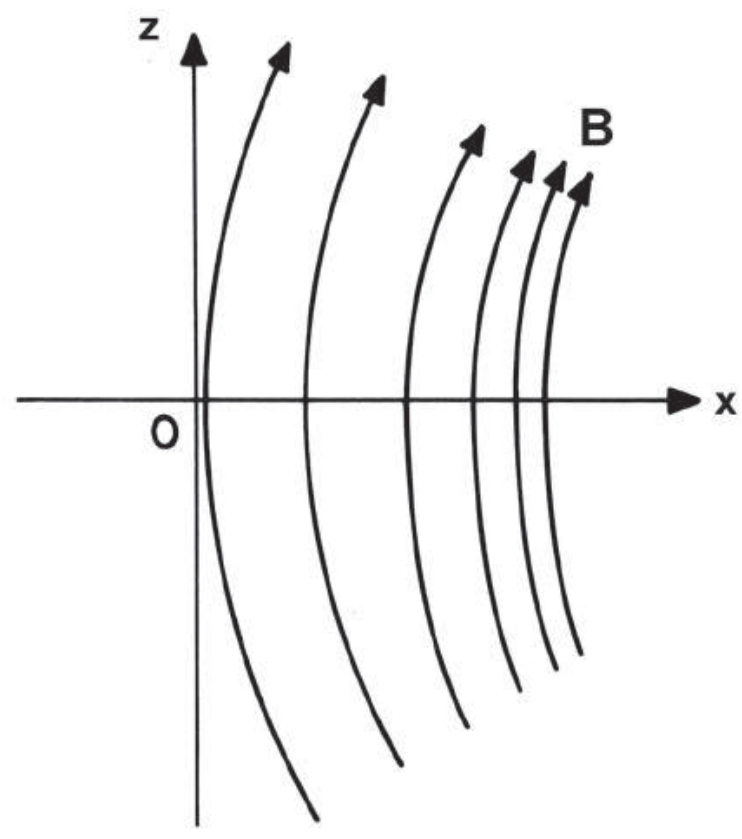

Fig. 5 Geometry of the magnetic field lines corresponding to (2.15), with gradient and curvature terms.

\section{EQUATION OF MOTION IN THE FIRST-ORDER APPROXIMATION}

We consider that the magnetic field $\mathbf{B}_{0}$ that exists at the origin in the guiding center coordinate system is in the $z$ direction,

$$
\mathbf{B}(0,0,0) \equiv \mathbf{B}_{0}=B_{0} \widehat{\mathbf{z}}
$$

The particle motion in the neighborhood of the origin can be described by considering only a linear approximation to the magnetic field near the origin. Let $\mathbf{r}$ be the momentary position vector of the particle in the guiding center coordinate system (see Fig. 1). In the region of interest (near the origin) the magnetic field can be expressed by a Taylor expansion about the origin,

$$
\mathbf{B}(\mathbf{r})=\mathbf{B}_{0}+\mathbf{r} \cdot(\nabla \mathbf{B})+\cdots
$$

where the derivatives of $\mathbf{B}$ are to be calculated at the origin. Note that the instantaneous position of the particle guiding center actually moves slightly during one period of rotation, while the origin is kept fixed during this time. 


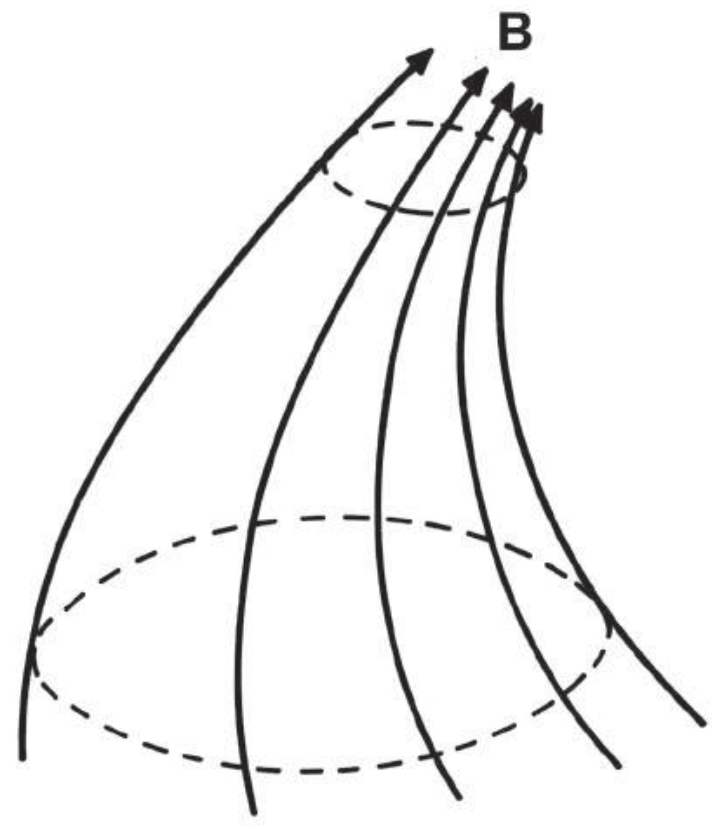

Fig. 6 Schematic representation of a magnetic field having divergence, gradient, and curvature terms.

Since we are assuming that the spatial variation of $\mathbf{B}$ in a distance of the order of the Larmor radius is much smaller than the magnitude of $\mathbf{B}$ itself, the higher order terms of (3.2) can be neglected. The condition

$$
\delta B=|\mathbf{r} \cdot(\nabla \mathbf{B})| \ll\left|\mathbf{B}_{0}\right|
$$

is clearly met (see section 1). Thus, the magnetic field at the particle position differs only slightly from that existing at the guiding center. The first-order term $\mathbf{r} \cdot(\nabla \mathbf{B})$ can be written explicitly as

$$
\begin{gathered}
\mathbf{r} \cdot(\nabla \mathbf{B})=(\mathbf{r} \cdot \nabla) \mathbf{B}=\left(x \frac{\partial}{\partial x}+y \frac{\partial}{\partial y}+z \frac{\partial}{\partial z}\right) \mathbf{B}= \\
\left(x \frac{\partial B_{x}}{\partial x}+y \frac{\partial B_{x}}{\partial y}+z \frac{\partial B_{x}}{\partial z}\right) \widehat{\mathbf{x}}+\left(x \frac{\partial B_{y}}{\partial x}+y \frac{\partial B_{y}}{\partial y}+z \frac{\partial B_{y}}{\partial z}\right) \widehat{\mathbf{y}}+ \\
\left(x \frac{\partial B_{z}}{\partial x}+y \frac{\partial B_{z}}{\partial y}+z \frac{\partial B_{z}}{\partial z}\right) \widehat{\mathbf{z}}
\end{gathered}
$$

where the partial derivatives are to be calculated at the origin. Substituting (3.2) into the equation of motion (2.1.5), with $\mathbf{E}=0$, gives

$$
m \frac{d \mathbf{v}}{d t}=q\left(\mathbf{v} \times \mathbf{B}_{0}\right)+q \mathbf{v} \times[\mathbf{r} \cdot(\nabla \mathbf{B})]
$$


The last term in the right-hand side is of first-order compared to the first one. The particle velocity can be written as a superposition,

$$
\mathbf{v}=\mathbf{v}^{(0)}+\mathbf{v}^{(1)}=\frac{d \mathbf{r}^{(0)}}{d t}+\frac{d \mathbf{r}^{(1)}}{d t}
$$

where $\mathbf{v}^{(1)}$ is a first-order perturbation

$$
\left|\mathbf{v}^{(1)}\right| \ll\left|\mathbf{v}^{(0)}\right|
$$

and $\mathbf{v}^{(0)}$ is the solution of the zero-order equation

$$
m \frac{d \mathbf{v}^{(0)}}{d t}=q\left(\mathbf{v}^{(0)} \times \mathbf{B}_{0}\right)
$$

which has already been discussed in section 4 of Chapter 2. Neglecting second-order terms we can write, therefore,

$$
\mathbf{v} \times[\mathbf{r} \cdot(\nabla \mathbf{B})]=\mathbf{v}^{(0)} \times\left[\mathbf{r}^{(0)} \cdot(\nabla \mathbf{B})\right]
$$

The equation of motion (3.5) becomes, under these approximations,

$$
m \frac{d \mathbf{v}}{d t}=q\left(\mathbf{v} \times \mathbf{B}_{0}\right)+q \mathbf{v}^{(0)} \times\left[\mathbf{r}^{(0)} \cdot(\nabla \mathbf{B})\right]
$$

The second term in the right-hand side constitutes the force term of (2.6.1) (Eq. 6.1 in Chapter 2). This additional force, however, is not constant since it depends on the instantaneous particle position. Thus, small oscillations occur during one period of gyration. Since we are interested in the smoothed motion of the guiding center, we shall eliminate these small oscillations by averaging this force term over one gyration period. Therefore, in what follows we will be involved in calculating the average value over one gyration period of the force term $q \mathbf{v}^{(0)} \times\left[\mathbf{r}^{(0)} \cdot(\nabla \mathbf{B})\right]$, which will allow us to determine the parallel acceleration of the guiding center and its transverse drift velocity using (2.6.2).

\section{AVERAGE FORCE OVER ONE GYRATION PERIOD}

Consider initially the case when the particle initial velocity along $\mathbf{B}$ is zero, so that the particle path differs but little from a circle. In a uniform magnetic field this would be equivalent to observing the particle motion in a coordinate system moving with the guiding center velocity $\mathbf{v}_{\|}$. However, 
when the field lines are bent, a coordinate system gliding along $\mathbf{B}$ is not an inertial system. The curvature of the field lines give rise to inertial forces and therefore to a curvature drift of the particle. This effect will be investigated later in section 7 . For the moment we will assume that the field lines are not curved and that the coordinate system moves with velocity $\mathbf{v}_{\|}$.

Under the conditions indicated above, the zero-order variables, $\mathbf{v}^{(0)}$ and $\mathbf{r}^{(0)}$, are seen to be situated in the $(x, y)$ plane. The force term

$$
\mathbf{F}=q \mathbf{v}^{(0)} \times\left[\mathbf{r}^{(0)} \cdot(\nabla \mathbf{B})\right]
$$

can be separated into a component $\mathbf{F}_{\|}$along $\mathbf{B}_{0}(z$ axis $)$ and a component $\mathbf{F}_{\perp}$ normal to $\mathbf{B}_{0}$, in the $(x, y)$ plane. Using a local cylindrical coordinate system $(r, \theta, z)$ with the $z$ axis pointing along $\mathbf{B}_{0}$ at the origin (refer to Fig. 7), we have

$$
\mathbf{r}^{(0)} \cdot(\nabla \mathbf{B})=r^{(0)} \frac{\partial \mathbf{B}}{\partial r}
$$

Of the three components of $\mathbf{B}=B_{r} \widehat{\mathbf{r}}+B_{\theta} \widehat{\boldsymbol{\Theta}}+B_{z} \widehat{\mathbf{z}}$, the $\theta$-component is parallel to $\mathbf{v}^{(0)}$ and therefore gives no contribution to $\mathbf{F}$, while $B_{r} \widehat{\mathbf{r}}$ contributes to $\mathbf{F}_{\|}$and $B_{z} \widehat{\mathbf{z}}$ contributes to $\mathbf{F}_{\perp}$. Hence, from (4.1) and $(4.2)$,

$$
\begin{gathered}
\mathbf{F}_{\|}=q\left(\mathbf{v}^{(0)} \times \widehat{\mathbf{r}}\right) r^{(0)} \frac{\partial B_{r}}{\partial r}=|q| v^{(0)} r^{(0)} \frac{\partial B_{r}}{\partial r} \widehat{\mathbf{z}} \\
\mathbf{F}_{\perp}=q\left(\mathbf{v}^{(0)} \times \widehat{\mathbf{z}}\right) r^{(0)} \frac{\partial B_{z}}{\partial r}=-|q| v^{(0)} r^{(0)} \frac{\partial B_{z}}{\partial r} \widehat{\mathbf{r}}
\end{gathered}
$$

Note that if $q>0$ we have $\mathbf{v}^{(0)} \times \widehat{\mathbf{r}}=v^{(0)} \widehat{\mathbf{z}}$, whereas if $q<0$ we have $\mathbf{v}^{(0)} \times \widehat{\mathbf{r}}=-v^{(0)} \widehat{\mathbf{z}}$. Now, $r^{(0)}$ is the cyclotron radius corresponding to $\mathbf{B}_{0}$,

$$
r^{(0)}=\frac{v^{(0)}}{\Omega_{c}}=\frac{m v^{(0)}}{|q| B_{0}}
$$

and using the expression for the magnitude of the magnetic moment (2.4.34) (Eq. 4.34 in Chapter 2), we can write (4.3) and (4.4) as

$$
\begin{gathered}
\mathbf{F}_{\|}=2|\mathbf{m}| \frac{\partial B_{r}}{\partial_{r}} \widehat{\mathbf{z}} \\
\mathbf{F}_{\perp}=-2|\mathbf{m}| \frac{\partial B_{z}}{\partial r} \widehat{\mathbf{r}}
\end{gathered}
$$

There results apply to both positively and negatively charged particles. 


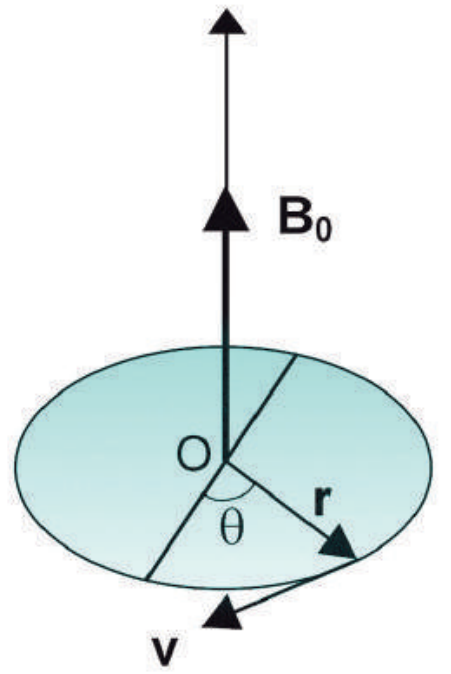

$(q>0)$

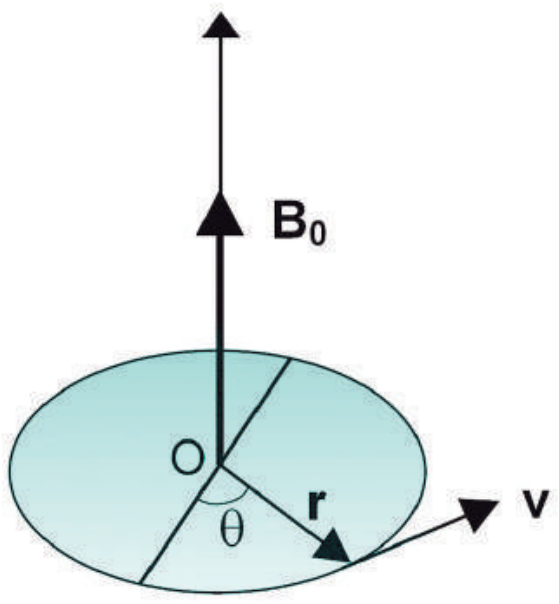

$(q<0)$

Fig. 7 Local cylindrical coordinate system with the $z$ axis pointing in the direction of the field $\mathbf{B}_{0}$ at the origin.

The average values of $\mathbf{F}_{\|}$and $\mathbf{F}_{\perp}$ over one gyration period are given by

$$
\begin{gathered}
<\mathbf{F}_{\|}>=2|\mathbf{m}| \widehat{\mathbf{z}}\left(\frac{1}{2 \pi} \oint \frac{\partial B_{r}}{\partial r} d \theta\right)=2|\mathbf{m}| \widehat{\mathbf{z}}<\left(\frac{\partial B_{r}}{\partial r}\right)> \\
<\mathbf{F}_{\perp}>=-2|\mathbf{m}|\left(\frac{1}{2 \pi} \oint \frac{\partial B_{z}}{\partial r} \widehat{\mathbf{r}} d \theta\right)=-2|\mathbf{m}|<\widehat{\mathbf{r}}\left(\frac{\partial B_{z}}{\partial r}\right)>
\end{gathered}
$$

The average force $\left\langle\mathbf{F}_{\|}\right\rangle$, given in (4.8), produces the guiding center parallel acceleration, while $\left\langle\mathbf{F}_{\perp}\right\rangle$, given in (4.9), is responsible for the guiding center transverse drift velocity. The first one is the result of the divergence terms of $\mathbf{B}$, and the second one of the gradient terms. We proceed now to evaluate each force term separately.

\subsection{Parallel Force}

Note that from $\nabla \cdot \mathbf{B}=0$ we have, in cylindrical coordinates,

$$
\frac{1}{r} \frac{\partial}{\partial r}\left(r B_{r}\right)+\frac{1}{r} \frac{\partial}{\partial \theta}\left(B_{\theta}\right)+\frac{\partial}{\partial z}\left(B_{z}\right)=0
$$


The first term can be expanded as

$$
\frac{1}{r} \frac{\partial}{\partial r}\left(r B_{r}\right)=\frac{\partial B_{r}}{\partial r}+\frac{B_{r}}{r}
$$

Since at $r=0$ we have $B_{r}=0$, and since near the origin $B_{r}$ changes only very slightly with $r$, we can take

$$
\frac{B_{r}}{r}=\frac{\partial B_{r}}{\partial r}
$$

Consequently, from (4.12) and (4.11)

$$
\frac{\partial B_{r}}{\partial r}=-\frac{1}{2}\left(\frac{1}{r} \frac{\partial B_{\theta}}{\partial \theta}+\frac{\partial B_{z}}{\partial z}\right)
$$

Hence, taking the average over one gyration period,

$$
<\left(\frac{\partial B_{r}}{\partial r}\right)>=-\frac{1}{2}<\frac{1}{r}\left(\frac{\partial B_{\theta}}{\partial \theta}\right)>-\frac{1}{2}<\left(\frac{\partial B_{z}}{\partial z}\right)>
$$

Now, since $B$ is single-valued,

$$
<\frac{1}{r}\left(\frac{\partial B_{\theta}}{\partial \theta}\right)>=\frac{1}{2 \pi} \oint \frac{1}{r}\left(\frac{\partial B_{\theta}}{\partial \theta}\right) d \theta=0
$$

Furthermore, since $\partial B_{z} / \partial z$ is a very slowly varying function inside the particle orbit, it can be taken outside the integral sign, so that we have approximately,

$$
<\left(\frac{\partial B_{z}}{\partial z}\right)>=\frac{1}{2 \pi} \oint\left(\frac{\partial B_{z}}{\partial z}\right) d \theta=\frac{\partial B_{z}}{\partial z}=\frac{\partial B}{\partial z}
$$

It is justifiable to replace $B_{z}$ by $B$ in (4.16), since all the spatial variations of the magnetic field in the region of interest are very small. Therefore, we have finally from (4.14), (4.15), and (4.16),

$$
<\left(\frac{\partial B_{r}}{\partial r}\right)>=-\frac{1}{2}\left(\frac{\partial B}{\partial z}\right)
$$

Using this result, the parallel force (4.8) becomes

$$
<\mathbf{F}_{\|}>=-|\mathbf{m}| \frac{\partial B}{\partial z} \widehat{\mathbf{z}}=-|\mathbf{m}|(\nabla B)_{\|}
$$

or, equivalently,

$$
<\mathbf{F}_{\|}>=(\mathbf{m} \cdot \nabla) B \widehat{\mathbf{z}}=-\frac{|\mathbf{m}|}{B}[(\mathbf{B} \cdot \nabla) \mathbf{B}]_{\|}
$$




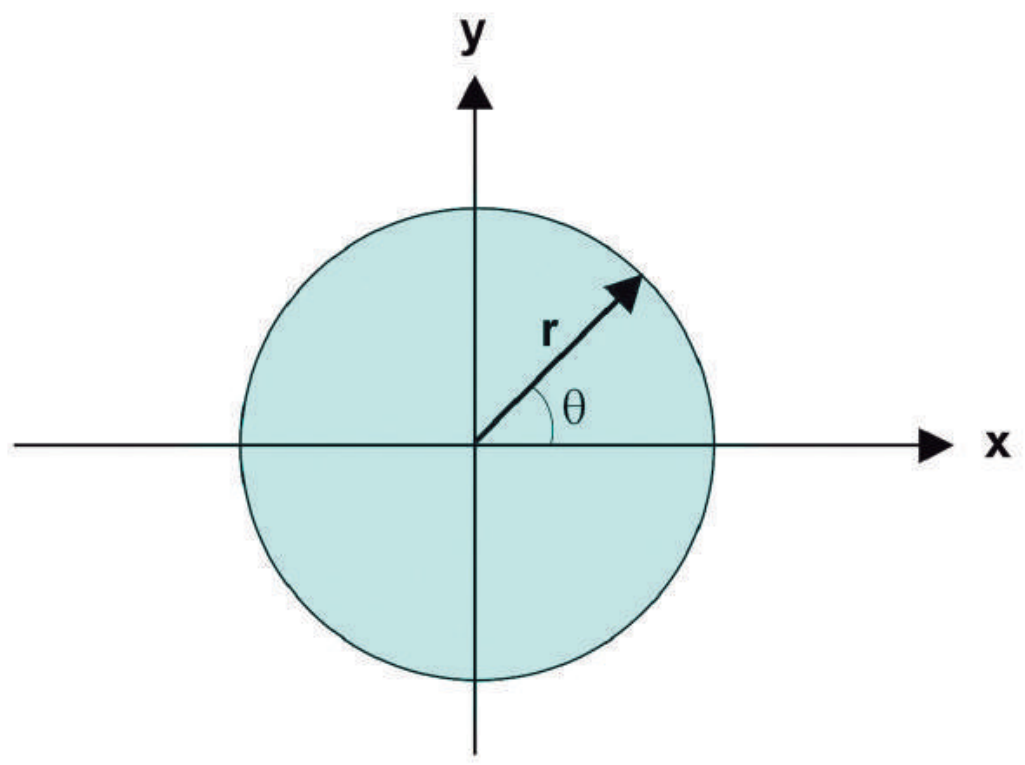

Fig. 8 Two-dimensional coordinate system in the perpendicular plane, used in the evaluation of $\left\langle\mathbf{F}_{\perp}\right\rangle$.

since $\mathbf{m}=-|\mathbf{m}| \widehat{\mathbf{z}}=-|\mathbf{m}| \mathbf{B} / B$, and where the derivatives are evaluated at the origin.

\subsection{Perpendicular Force}

It is convenient to consider a two-dimensional Cartesian coordinate system $(x, y)$ in the perpendicular plane, such that $x=r \cos (\theta)$ and $y=r \sin (\theta)$, as shown in Fig. 8. Hence,

$$
\begin{gathered}
\widehat{\mathbf{r}}=\cos (\theta) \widehat{\mathbf{x}}+\sin (\theta) \widehat{\mathbf{y}} \\
\frac{\partial}{\partial r}=\frac{d x}{d r} \frac{\partial}{\partial x}+\frac{d y}{d r} \frac{\partial}{\partial y}=\cos (\theta) \frac{\partial}{\partial x}+\sin (\theta) \frac{\partial}{\partial y}
\end{gathered}
$$

Therefore, we obtain

$$
\begin{gathered}
<\widehat{\mathbf{r}}\left(\frac{\partial B_{z}}{\partial r}\right)>=<[\cos (\theta) \widehat{\mathbf{x}}+\sin (\theta) \widehat{\mathbf{y}}]\left[\cos (\theta) \frac{\partial B_{z}}{\partial x}+\sin (\theta) \frac{\partial B_{z}}{\partial y}\right]> \\
=<\cos ^{2}(\theta) \frac{\partial B_{z}}{\partial x} \widehat{\mathbf{x}}>+<\sin (\theta) \cos (\theta) \frac{\partial B_{z}}{\partial x} \widehat{\mathbf{y}}>+
\end{gathered}
$$




$$
<\cos (\theta) \sin (\theta) \frac{\partial B_{z}}{\partial y} \widehat{\mathbf{x}}>+<\sin ^{2}(\theta) \frac{\partial B_{z}}{\partial y} \widehat{\mathbf{y}}>
$$

Next we approximate $\left(\partial B_{z} / \partial x\right)$ by $(\partial B / \partial x)$, and $\left(\partial B_{z} / \partial y\right)$ by $(\partial B / \partial y)$, since these terms are slowly varying functions inside the particle orbit, so that they can be taken outside the integral sign contained in the average values of (4.22). Noting that $\langle\sin (\theta) \cos (\theta)\rangle=0$ and $\left\langle\cos ^{2}(\theta)\right\rangle=$ $<\sin ^{2}(\theta)>=1 / 2$, we obtain

$$
<\widehat{\mathbf{r}} \frac{\partial B_{z}}{\partial r}>=\frac{1}{2} \frac{\partial B}{\partial x} \widehat{\mathbf{x}}+\frac{1}{2} \frac{\partial B}{\partial y} \widehat{\mathbf{y}}
$$

Substituting this result into (4.9), yields

$$
<\mathbf{F}_{\perp}>=-|\mathbf{m}|\left(\frac{\partial B}{\partial x} \widehat{\mathbf{x}}+\frac{\partial B}{\partial y} \widehat{\mathbf{y}}\right)=-|\mathbf{m}|(\nabla B)_{\perp}
$$

\subsection{Total Average Force}

We proceed now to write down a general expression for the total average force $\langle\mathbf{F}\rangle=\left\langle\mathbf{F}_{\|}\right\rangle+\left\langle\mathbf{F}_{\perp}\right\rangle$. From (4.18) and (4.24) we have

$$
<\mathbf{F}>=-|\mathbf{m}|(\nabla B)_{\|}-|\mathbf{m}|(\nabla B)_{\perp}=-|\mathbf{m}| \nabla B
$$

Alternatively, we can use the vector identity

$$
(\nabla \times \mathbf{B}) \times \mathbf{B}=(\mathbf{B} \cdot \nabla) \mathbf{B}-\nabla\left(\frac{1}{2} B^{2}\right)
$$

and write (4.25) in the form

$$
<\mathbf{F}>=-\frac{|\mathbf{m}|}{B}[(\mathbf{B} \cdot \nabla) \mathbf{B}-(\nabla \times \mathbf{B}) \times \mathbf{B}]
$$

Since $\mathbf{m}=-|\mathbf{m}| \mathbf{B} / B$, we have

$$
<\mathbf{F}>=(\mathbf{m} \cdot \nabla) \mathbf{B}+\mathbf{m} \times(\nabla \times \mathbf{B})
$$

This is the usual expression for the force acting on a small ring current immersed in a magnetic field with spatial variation. The first term on the right-hand side of (4.28) alone gives the force acting on a magnetic dipole. 


\section{GRADIENT DRIFT}

From (2.6.2) and (4.24) we see that $\left\langle\mathbf{F}_{\perp}\right\rangle$ causes the guiding center to drift with the velocity

$$
\mathbf{v}_{G}=\frac{<\mathbf{F}_{\perp}>\times \mathbf{B}}{q B^{2}}=-\frac{|\mathbf{m}|}{q} \frac{(\nabla B) \times \mathbf{B}}{B^{2}}
$$

This gradient drift is perpendicular to $\mathbf{B}$ and to the field gradient, and its direction depends on the charge sign. Thus, positive and negative charges drift in opposite directions, giving rise to an electric current (see Fig. 9).

The physical reason for this gradient drift can be seen as follows. Since the Larmor radius of the particle orbit decreases as the magnetic field increases, the radius of curvature of the orbit is smaller in the regions of stronger $\mathbf{B}$ field. The positive ions gyrate in the clockwise direction for B pointing towards the observer, while the electrons gyrate in the counterclockwise direction, as shown in Fig. 9, so that the positive ions drift to the left and the electrons to the right.

In the case of a collisionless plasma, associated with this gradient drift across $\mathbf{B}$ there is a magnetization current density $\mathbf{J}_{G}$, given by

$$
\mathbf{J}_{G}=\frac{1}{\delta V} \sum_{i} q_{i} \mathbf{v}_{G i}
$$

where the summation is over all charged particles contained in a suitably chosen element of volume $\delta V$. From (5.1) and (5.2), we have

$$
\mathbf{J}_{G}=-\left(\frac{1}{\delta V} \sum_{i}\left|\mathbf{m}_{i}\right|\right) \frac{(\nabla B) \times \mathbf{B}}{B^{2}}
$$

\section{PARALLEL ACCELERATION OF THE GUIDING CENTER}

The expression (4.18) for $\left\langle\mathbf{F}_{\|}\right\rangle$shows that, when the magnetic field has a longitudinal variation (i.e., convergence or divergence of the field lines along the $z$ direction, as shown in Fig. 3), an axial force along $z$ accelerates the particle in the direction of decreasing magnetic field, irrespective of whether the particle is positively or negatively charged. This is illustrated in Fig. 10. There are several important consequences of this repulsion of gyrating charges from a region of converging magnetic field lines, which we proceed to discuss. 
B OUT OF PAGE

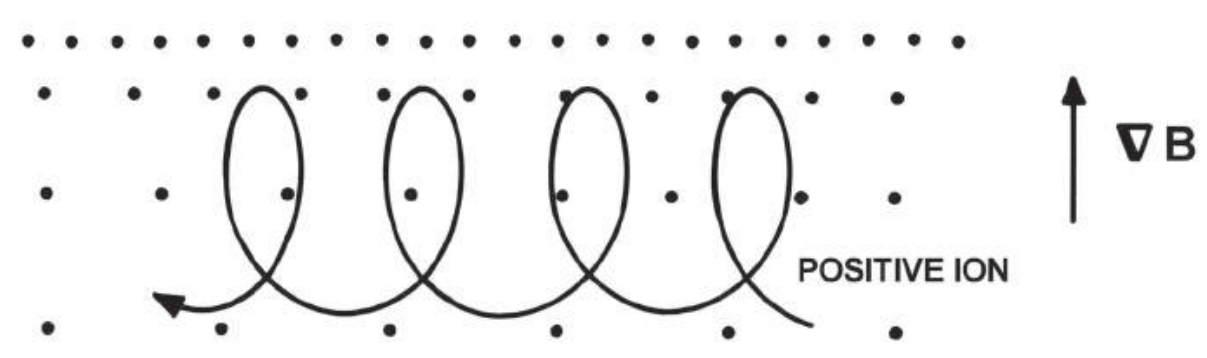

\section{lellllles eLECTRON}

Fig. 9 Charged particle drifts due to a $\mathbf{B}$ field gradient perpendicular to $\mathbf{B}$.

\subsection{Invariance of the Orbital Magnetic Moment and of the Magnetic Flux}

Using (4.18), the component of the equation of motion along $\mathbf{B}$ can be written as

$$
m \frac{d v_{\|}}{d t} \widehat{\mathbf{z}}=<\mathbf{F}_{\|}>=-|\mathbf{m}| \frac{\partial B}{\partial z} \widehat{\mathbf{z}}
$$

If we multiply both sides of this equation by $v_{\|}=d z / d t$, we obtain (replacing $|\mathbf{m}|$ by $\left.W_{\perp} / B\right)$,

$$
m v_{\|} \frac{d v_{\|}}{d t}=\frac{d}{d t}\left(\frac{1}{2} m v_{\|}^{2}\right)=-\frac{W_{\perp}}{B} \frac{\partial B}{\partial z} \frac{d z}{d t}
$$

where $W_{\perp}=m v_{\perp}^{2} / 2$ denotes the part of the particle kinetic energy associated with its transverse velocity. Since the total kinetic energy of a charged particle in a magnetostatic field is constant, that is,

$$
W_{\|}+W_{\perp}=\text { constant }
$$

it follows that

$$
\frac{d}{d t}\left(W_{\perp}\right)=-\frac{d}{d t}\left(W_{\|}\right)=-\frac{d}{d t}\left(\frac{1}{2} m v_{\|}^{2}\right)
$$




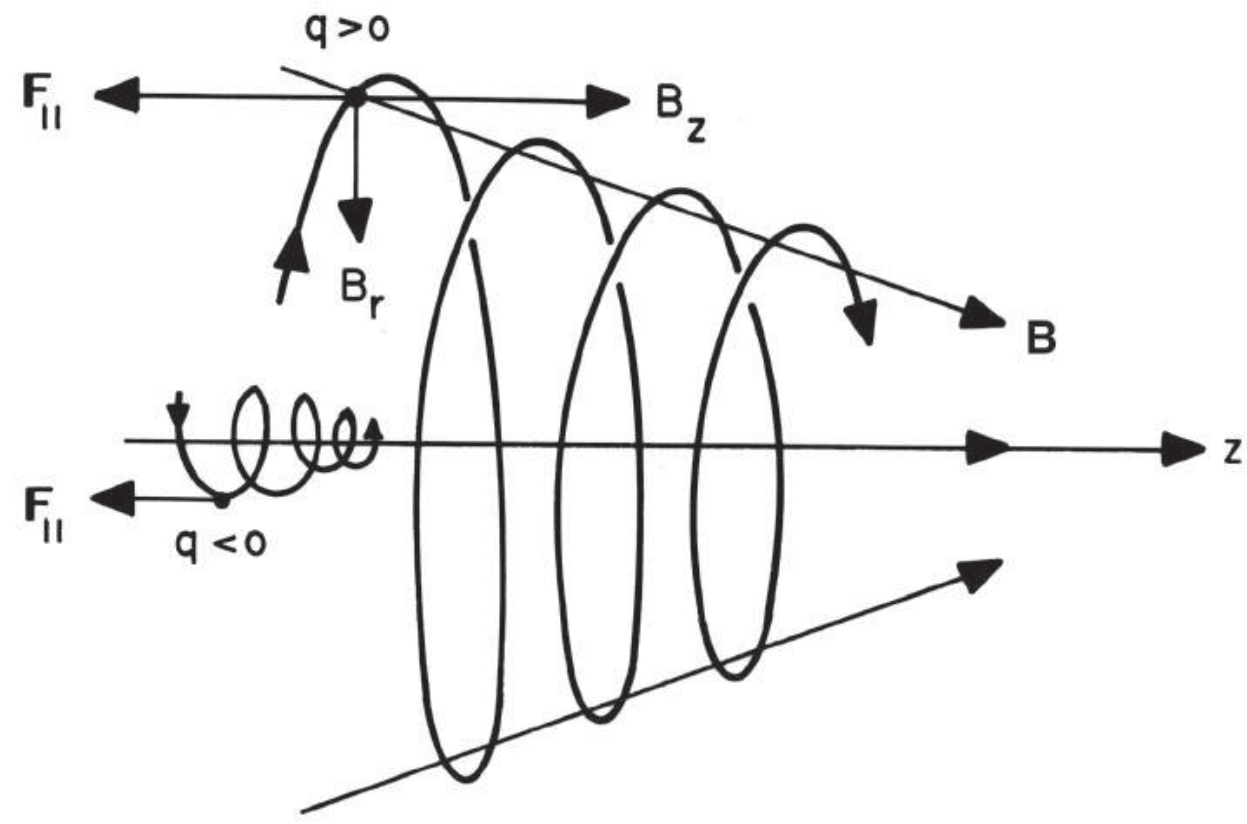

Fig. 10 Repulsion of gyrating charges from a region of converging magnetic field lines.

Therefore, from (6.2) and (6.4),

$$
\frac{d}{d t}\left(W_{\perp}\right)=\frac{W_{\perp}}{B} \frac{\partial B}{\partial z} \frac{d z}{d t}=\frac{W_{\perp}}{B} \frac{d B}{d t}
$$

where $d B / d t$ represents the rate of change of $B$ as seen by the particle as it moves in the spatially varying magnetic field (i.e., in the particle frame of reference). Comparing this result with the following identity,

$$
\frac{d}{d t}\left(W_{\perp}\right)=\frac{d}{d t}\left(\frac{W_{\perp} B}{B}\right)=\frac{W_{\perp}}{B} \frac{d B}{d t}+B \frac{d}{d t}\left(\frac{W_{\perp}}{B}\right)
$$

we conclude that

$$
\frac{d}{d t}\left(\frac{W_{\perp}}{B}\right)=0
$$

or, equivalently,

$$
|\mathbf{m}|=\frac{W_{\perp}}{B}=\text { constant }
$$


Therefore, as the particle moves into regions of converging or diverging $\mathbf{B}$ its cyclotron radius changes, but the magnetic moment remains constant. This constancy of the particle magnetic moment holds only within the approximation used, that is, when the spatial variation of $\mathbf{B}$ inside the particle orbit is small compared to the magnitude of $\mathbf{B}$. Consequently, the orbital magnetic moment is said to be an adiabatic invariant. It is usually referred to as the first adiabatic invariant.

The magnetic flux, $\Phi_{m}$, enclosed by one orbit of the particle is given by

$$
\Phi_{m}=\int_{S} \mathbf{B} \cdot d \mathbf{S}=\pi r_{c}^{2} B=\pi \frac{m^{2} v_{\perp}^{2}}{q^{2} B^{2}} B=\frac{2 \pi m}{q^{2}}\left(\frac{W_{\perp}}{B}\right)
$$

Therefore,

$$
\frac{d}{d t}\left(\Phi_{m}\right)=\frac{2 \pi m}{q^{2}} \frac{d}{d t}|\mathbf{m}|=0
$$

in view of the invariance of $|\mathbf{m}|$. Hence, as the charged particle moves in a region of converging $\mathbf{B}$ field, it will orbit with increasingly smaller radius, so that the magnetic flux enclosed by the orbit remains constant.

\subsection{Magnetic Mirror Effect}

As a consequence of the adiabatic invariance of $|\mathbf{m}|$ and $\Phi_{m}$, as the particle moves into a region of converging magnetic field lines its transverse kinetic energy $W_{\perp}$ increases, while its parallel kinetic energy $W_{\|}$decreases, in order to keep $|\mathbf{m}|$ and the total energy constant. Ultimately, if the B field becomes strong enough, the particle velocity in the direction of increasing field may eventually come to zero and then be reversed. After reversion, the particle is speeded up in the direction of decreasing field, while its transverse velocity diminishes. Thus, the particle is reflected from the region of converging magnetic field lines. This phenomenon is called the magnetic mirror effect and is the basis for one of the primary schemes of plasma confinement.

When two coaxial magnetic mirrors are considered, as illustrated in Fig. 11, the charged particles may be reflected by the magnetic mirrors and may travel back and forth in the space between them, becoming trapped. This trapping region has been called a magnetic bottle and it has been used in laboratory for plasma confinement.

The trapping in a magnetic mirror system is not perfect, however. The effectiveness of a coaxial magnetic mirror system in the trapping of charged particles can be measured by the mirror ratio $B_{m} / B_{0}$, where $B_{m}$ 


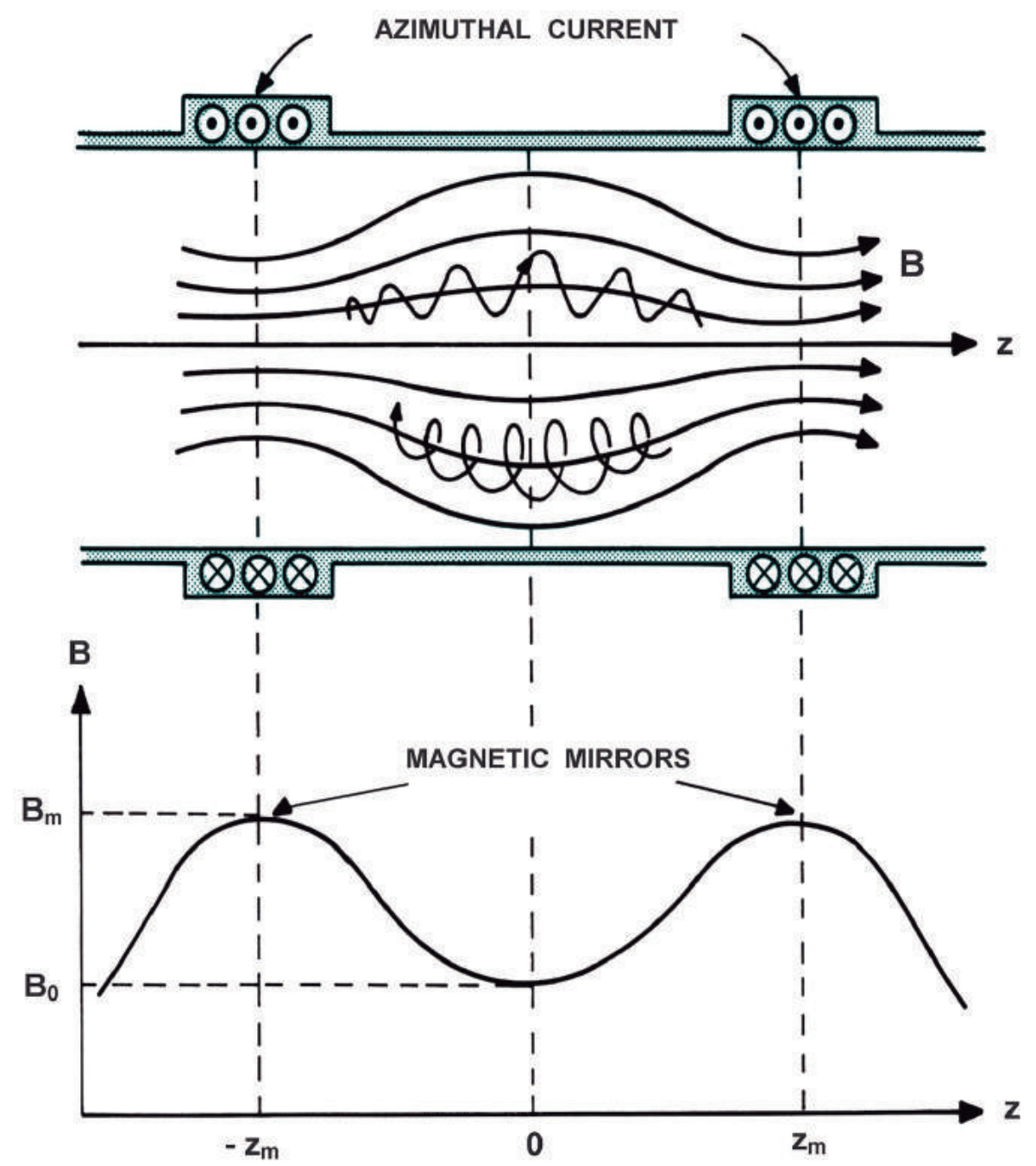

Fig. 11 Schematic diagram showing the arrangement of coils to produce two coaxial magnetic mirrors facing each other, for plasma confinement, and the relative intensity variation of the magnetic field.

is the intensity of the magnetic field at the point of reflection (where the pitch angle of the particle is $\pi / 2$ ) and $B_{0}$ is the intensity of the magnetic field at the center of the magnetic bottle.

Consider a charged particle having a pitch angle $\alpha_{0}$ at the center of the magnetic bottle. If $v$ is the particle speed, which in a static magnetic field 


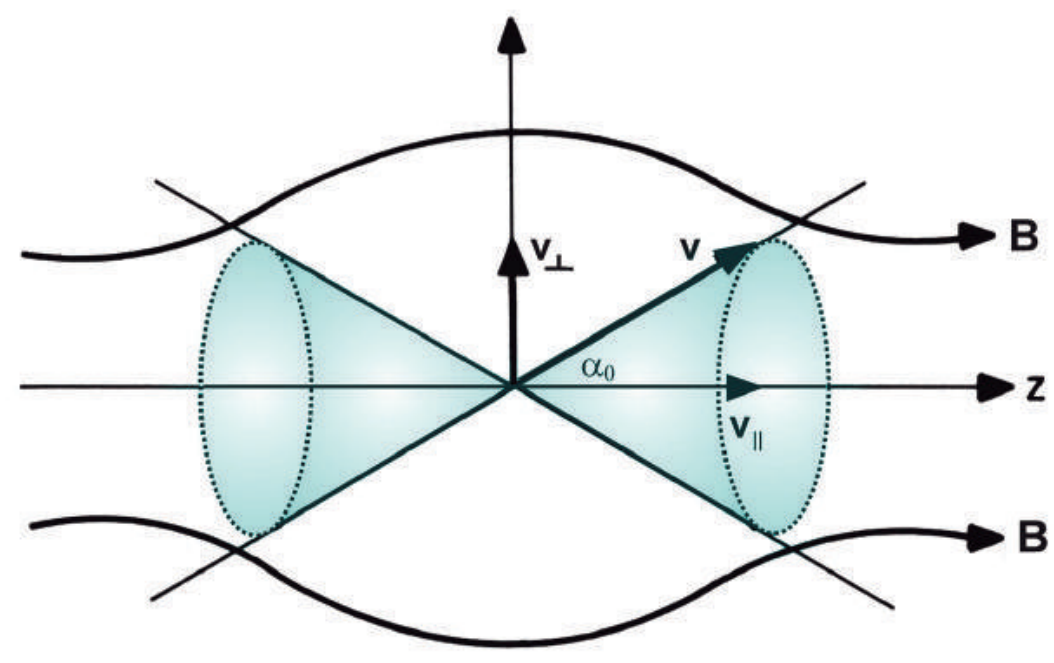

Fig. 12 The loss cone in a coaxial magnetic mirror system.

remains constant, the constancy of the magnetic moment $|\mathbf{m}|=W_{\perp} / B$ leads to

$$
\frac{1}{2} m v^{2}\left(\sin ^{2} \alpha\right) / B=\frac{1}{2} m v^{2}\left(\sin ^{2} \alpha_{0}\right) / B_{0}
$$

where $\alpha$ is the particle pitch angle at a position where the magnetic field intensity is $B$. Thus, at any point inside the magnetic bottle, for this particle,

$$
\frac{\sin ^{2} \alpha(z)}{B(z)}=\frac{\sin ^{2} \alpha_{0}}{B_{0}}
$$

Suppose now that this particle is reflected at the throat of the mirror, that is, $\alpha=\pi / 2$ for $B(z)=B_{m}$. Therefore, from (6.12),

$$
\left(\sin ^{2} \alpha_{0}\right) / B_{0}=1 / B_{m}
$$

This means that a particle having a pitch angle $\alpha_{0}$ given by

$$
\alpha_{0}=\sin ^{-1}\left[\left(B_{0} / B_{m}\right)^{1 / 2}\right]=\sin ^{-1}\left(v_{\perp} / v\right)_{0}
$$

at the center of the bottle, is reflected at a point where the intensity of the field is $B_{m}$. Therefore, for a magnetic bottle with a fixed mirror ratio $B_{m} / B_{0}$, the plasma particles having a pitch angle at the center greater than $\alpha_{0}$, as given by (6.14), will be reflected before the ends of the magnetic bottle. On the other hand, if the pitch angle of the particle at the center is 


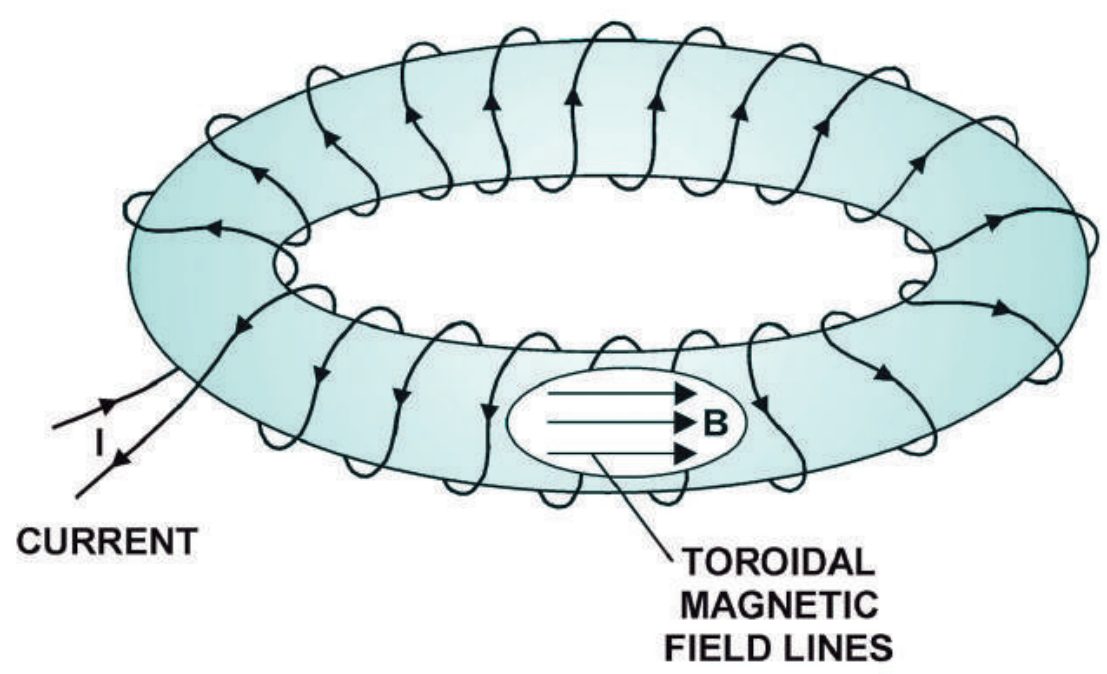

Fig. 13 Magnetic field with toroidal geometry.

less than $\alpha_{0}$, its pitch angle will never reach the value $\pi / 2$, which implies that at the ends of the bottle the particle has a non-vanishing parallel velocity and hence escapes through the ends of the mirror system. There is, therefore, a loss cone, a cone of half-angle $\alpha_{0}$ with its vertex at the center, as shown in Fig. 12, where particles that have velocity vectors with a pitch angle falling inside it are not trapped. The loss cone is determined by the mirror ratio $B_{m} / B_{0}$, according to (6.14).

Devices that have no ends, with geometries such that the magnetic field lines close on themselves, offer many advantages for plasma confinement. Toroidal geometries (Fig. 13), for example, have no ends, but it turns out that confinement of a plasma inside a toroidal magnetic field does not provide a plasma equilibrium situation, because of the radial inhomogeneity of the field. In this case a poloidal magnetic field is normally superposed on the toroidal field, resulting in helical field lines (as in the Tokamak). The major problem in most plasma confinement schemes, however, is that instabilities and small fluctuations from the desired equilibrium configuration are always present, which lead to a rapid escape of the particles from the magnetic bottle. This instability problem is a fundamental one, and it is likely to occur in any conceivable magnetic confinement scheme.

A good example of a natural magnetic bottle is the Earth's magnetic field, which traps charged particles of solar and cosmic origin. These 


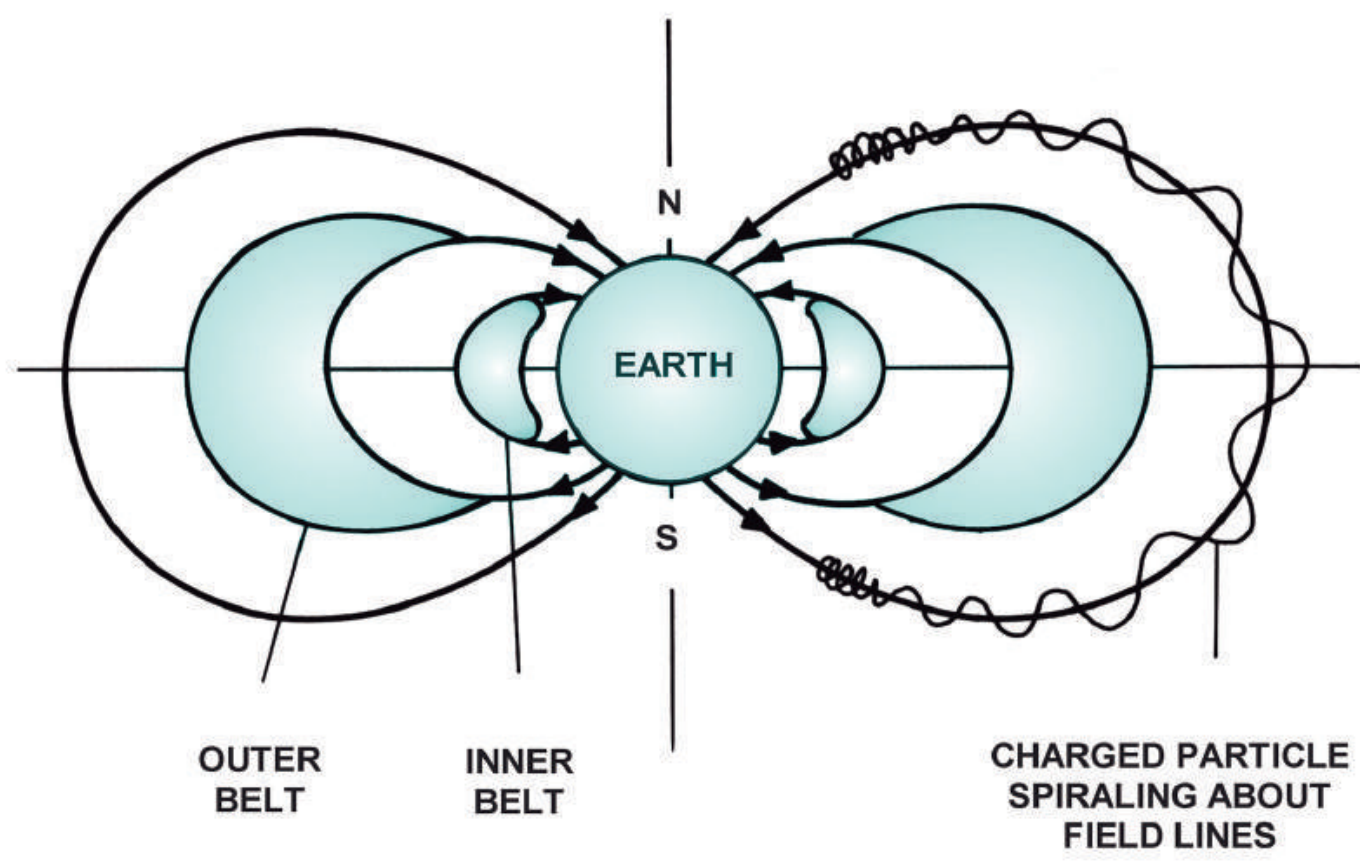

Fig. 14 Dipole approximation of the Earth's magnetic field. The distance of the Van Allen radiation belts from the center of the Earth, at the equator, is about 1.5 Earth radii for the highenergy protons and about 3 to 4 Earth radii for the high-energy electrons.

charged particles trapped in the Earth's magnetic field constitute the socalled Van Allen radiation belts. As shown in Fig. 14, the geomagnetic field near the Earth is approximately that of a dipole, with the field lines converging towards the north and south magnetic poles.

The electrons and protons that are trapped in the Van Allen radiation belts spiral in almost helical paths along the field lines, and towards the magnetic poles, where they are eventually reflected. These particles bounce back and forth between the poles. In addition to this bouncing motion, these trapped charged particles are also subject to a gradient drift and a curvature drift in the east-west direction, to be discussed later in this chapter.

\subsection{The Longitudinal Adiabatic Invariant}

Consider a particle trapped between two magnetic mirrors and bouncing between them. Suppose that the separation distance between the two 


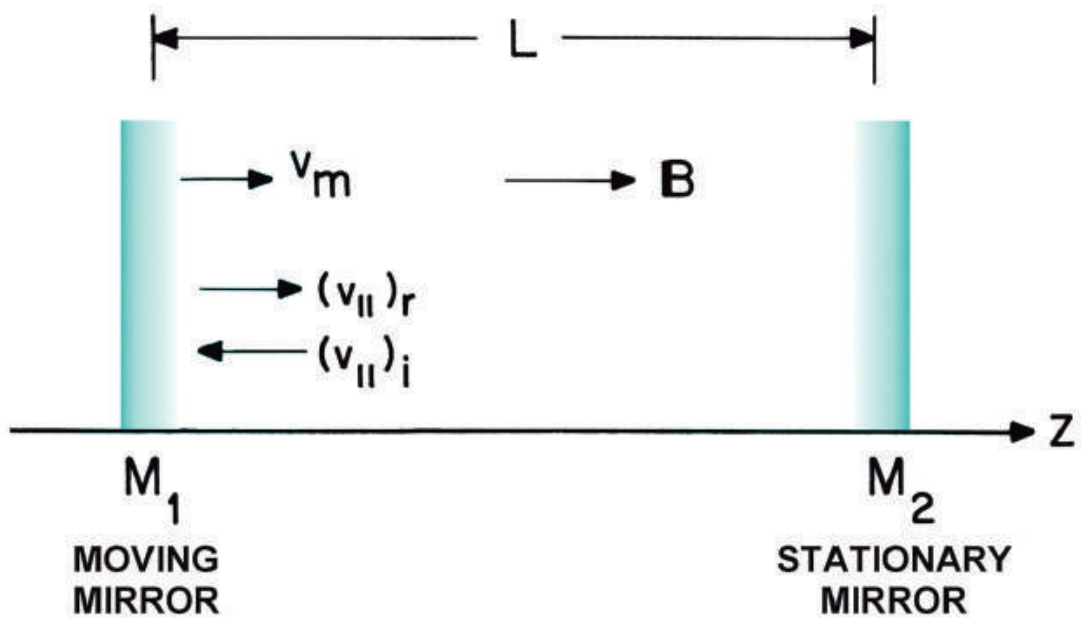

Fig. 15 Schematic representation of a system of two coaxial magnetic mirrors approaching each other.

mirrors changes very slowly in time as compared to the bounce period. With the periodic motion of the particle between the two magnetic mirrors (whose separation varies slowly in time) there is associated an adiabatic invariant called the longitudinal adiabatic invariant, defined by the integral

$$
J=\oint \mathbf{v} \cdot d \mathbf{l}=\oint v_{\|} d l
$$

taken over one period of oscillation of the particle back and forth between the mirror points.

For a simple proof of the adiabatic invariance of $J$, consider the idealized situation illustrated in Fig. 15, where the existing $\mathbf{B}$ field in the $z$ direction is uniform in space, except near the points $M_{1}$ and $M_{2}$, where the field increases to form the two mirrors separated by a distance $L$. Suppose that the mirror $M_{1}$ approaches the other one with velocity

$$
v_{m}=-\frac{d L}{d t}
$$

the negative sign being due to the fact that $L$ decreases with time. It is assumed that this velocity is much smaller than the longitudinal component of the particle velocity, that is, $v_{m} \ll v_{\|}$. Thus, the distance moved by the mirror $M_{1}$ during one period of oscillation of the particle is small compared to the distance $L$ between the mirrors. 
Further, since $\mathbf{B}$ is assumed to be uniform throughout the space between the mirrors (except near the ends), the longitudinal particle speed $v_{\|}$ may be taken to be constant in the space between the mirrors. Neglecting the small end effects at the two mirrors, we can take

$$
J=\int_{0}^{2 L} v_{\|} d l=2 v_{\|} L
$$

The time rate of change of $J$ is

$$
\frac{d J}{d t}=2 v_{\|} \frac{d L}{d t}+2 L \frac{d v_{\|}}{d t}=-2 v_{\|} v_{m}+2 L \frac{d v_{\|}}{d t}
$$

where use was made of (6.16). To calculate $d v_{\|} / d t$, we set

$$
\frac{d v_{\|}}{d t}=\frac{\Delta v_{\|}}{\Delta t}=\frac{\Delta v_{\|}}{\left(2 L / v_{\|}\right)}
$$

where $\Delta v_{\|}$denotes the change in the particle speed $v_{\|}$on reflection from the moving mirror, and $\Delta t=\left(2 L / v_{\|}\right)$is the period of oscillation between the mirrors. In order to find $\Delta v_{\|}$it is convenient to transform to a coordinate system moving with the magnetic mirror $M_{1}$, at the speed $v_{m}$. Let us denote this moving coordinate system by a prime and the incident and reflected particle speeds by subscripts $i$ and $r$, respectively. Thus,

$$
\begin{aligned}
& \left(v_{\|}\right)_{i}^{\prime}=\left(v_{\|}\right)_{i}+v_{m} \\
& \left(v_{\|}\right)_{r}^{\prime}=\left(v_{\|}\right)_{r}-v_{m}
\end{aligned}
$$

which gives for the change in the particle speed, in one reflection,

$$
\Delta v_{\|}=\left(v_{\|}\right)_{r}-\left(v_{\|}\right)_{i}=2 v_{m}
$$

since in the moving coordinate system $\left(v_{\|}\right)_{i}^{\prime}=\left(v_{\|}\right)_{r}^{\prime}$ with only their directions reversed. Therefore, (6.19) becomes

$$
\frac{d v_{\|}}{d t}=\frac{2 v_{m}}{\left(2 L / v_{\|}\right)}=\frac{v_{m} v_{\|}}{L}
$$

On substituting this result into (6.18) we find

$$
\frac{d J}{d t}=\frac{d}{d t}\left(2 v_{\|} L\right)=0
$$


which shows that $J$ is an adiabatic invariant. This quantity is also referred to as the second adiabatic invariant. The parallel kinetic energy of a charged particle trapped between the two mirrors is (taking $J=2 v_{\|} L$ )

$$
W_{\|}=\frac{1}{2} m v_{\|}^{2}=\frac{m J^{2}}{8 L^{2}}
$$

which increases rapidly as $L$ decreases. The Italian physicist Fermi suggested this process as as mechanism for the acceleration of charged particles in order to explain the origin of high-energy cosmic rays. Fermi proposed that two stellar clouds moving towards each other, and having a magnetic field greater than in the space between them, may trap and accelerate the cosmic charged particles. There is a limit, however, in the particle longitudinal speed increase, since the direction of the particle velocity at the center of the mirror system may eventually enter the loss cone and escape through the ends of the system. It should be noted that a magnetic mirror moving towards a stationary one involves in fact time-varying B fields and, consequently, electric fields, which can lead to a change in the particle kinetic energy.

\section{CURVATURE DRIFT}

So far the effects associated with the curvature of the magnetic field lines have not been considered. As stated previously, a $\mathbf{B}$ field with only curvature terms does not satisfy the equation $\nabla \times \mathbf{B}=0$, so that in practice the gradient and the curvature drifts will always be present simultaneously. In first-order orbit theory the effects corresponding to each of the components of $\mathbf{B}$ are additive.

We investigate now the effect of the curvature terms $\partial B_{x} / \partial z$ and $\partial B_{y} / \partial z$, referred to in (2.6c), on the motion of a charged particle. We will assume that these terms are so small that the radius of curvature of the magnetic field lines is very large compared to the particle cyclotron radius. Let us introduce a local coordinate system gliding along the magnetic field line with the particle longitudinal velocity $\mathbf{v}_{\|}$. Since this is not an inertial system because of the curvature of the field lines, a centrifugal force will be present. This local coordinate system can be specified by the orthogonal set of unit vectors $\widehat{\mathbf{B}}, \widehat{\mathbf{n}}_{1}$, and $\widehat{\mathbf{n}}_{2}$, where $\widehat{\mathbf{B}}$ is along the field line, $\widehat{\mathbf{n}}_{1}$ is along the principal normal to the field line, and $\widehat{\mathbf{n}}_{2}$ is along the binormal to the curved magnetic field line, as indicated in Fig. 16. 

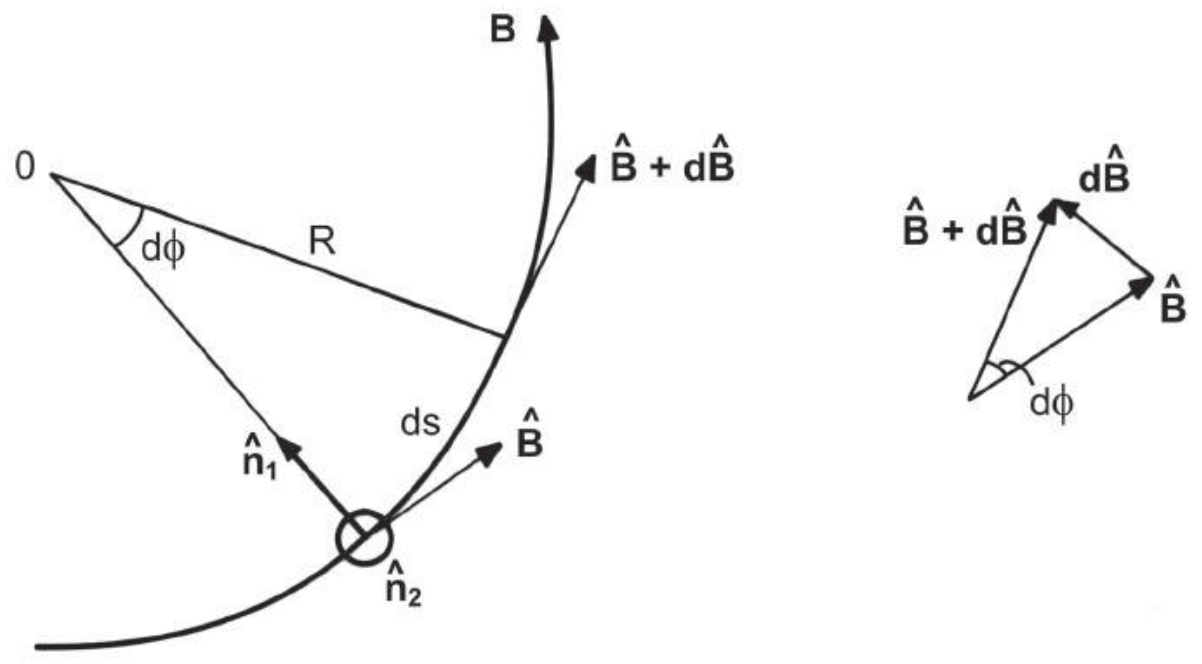

Fig. 16 Curved magnetic field line showing the unit vector $\widehat{\mathbf{B}}$ along the field line, the principal normal $\widehat{\mathbf{n}}_{1}$, and the binormal $\widehat{\mathbf{n}}_{2}$, at an arbitrary point. Note that $\widehat{\mathbf{n}}_{1} \times \widehat{\mathbf{n}}_{2}=\widehat{\mathbf{B}}$. The local radius of curvature is $R$.

The centrifugal force $\mathbf{F}_{c}$ acting on the particle, as seen from this noninertial system, is given by

$$
\mathbf{F}_{c}=-\frac{m v_{\|}^{2}}{R} \widehat{\mathbf{n}}_{1}
$$

where $R$ denotes the local radius of curvature of the magnetic field line and $v_{\|}$is the particle instantaneous longitudinal speed. From (2.6.2) the curvature drift associated with this force is

$$
\mathbf{v}_{C}=\frac{\mathbf{F}_{c} \times \mathbf{B}}{q B^{2}}=-\frac{m v_{\|}^{2}}{R q B^{2}}\left(\widehat{\mathbf{n}}_{1} \times \mathbf{B}\right)
$$

To express the unit vector $\widehat{\mathbf{n}}_{1}$ in terms of the unit vector $\widehat{\mathbf{B}}$ along the magnetic field line, we let $d s$ represent an element of arc along the field line subtending an angle $d \phi$,

$$
d s=R d \phi
$$

If $d \widehat{\mathbf{B}}$ denotes the change in $\mathbf{B}$ due to the displacement $d s$ (see Fig. 16), then $d \widehat{\mathbf{B}}$ is in the direction of $\widehat{\mathbf{n}}_{1}$ and its magnitude is

$$
|d \widehat{\mathbf{B}}|=|\widehat{\mathbf{B}}| d \phi=d \phi
$$


Consequently,

$$
d \widehat{\mathbf{B}}=\widehat{\mathbf{n}}_{1} d \phi
$$

Dividing this equation by (7.3) side by side, gives

$$
\frac{d \widehat{\mathbf{B}}}{d s}=\frac{\widehat{\mathbf{n}}_{1}}{R}
$$

The derivative $d / d s$ along $\mathbf{B}$ may be written as $(\widehat{\mathbf{B}} \cdot \nabla)$, so that $(7.6)$ becomes

$$
\frac{\widehat{\mathbf{n}}_{1}}{R}=(\widehat{\mathbf{B}} \cdot \nabla) \widehat{\mathbf{B}}
$$

Incorporating this result into equation (7.1), we obtain

$$
\mathbf{F}_{c}=-m v_{\|}^{2}(\widehat{\mathbf{B}} \cdot \nabla) \widehat{\mathbf{B}}
$$

This force is obviously perpendicular to the magnetic field $\mathbf{B}$, since it is in the $-\widehat{\mathbf{n}}_{1}$ direction as indicated in (7.1), and gives rise to a curvature drift whose velocity is

$$
\mathbf{v}_{C}=-\frac{m v_{\|}^{2}}{q B^{2}}[(\widehat{\mathbf{B}} \cdot \nabla) \widehat{\mathbf{B}}] \times \mathbf{B}
$$

Since $\mathbf{B}=B \widehat{\mathbf{B}}$ and writing $W_{\|}=m v_{\|}^{2} / 2$ for the particle longitudinal kinetic energy, (7.8) and (7.9) can be written, respectively, as

$$
\begin{gathered}
\mathbf{F}_{c}=-\frac{2 W_{\|}}{B^{2}}[(\mathbf{B} \cdot \nabla) \mathbf{B}]_{\perp} \\
\mathbf{v}_{C}=-\frac{2 W_{\|}}{q B^{4}}[(\mathbf{B} \cdot \nabla) \mathbf{B}] \times \mathbf{B}
\end{gathered}
$$

Thus, at each point, the curvature drift is perpendicular to the osculating plane of the magnetic field line, as shown in Fig. 17.

An electric current is associated with the curvature drift, since it is in opposite directions for particles of opposite sign. From (7.11) and from the definition of the electric current density, we obtain for the curvature drift current density

$$
\mathbf{J}_{C}=\frac{1}{\delta V} \sum_{i}\left(q_{i} \mathbf{v}_{C i}\right)=-2\left(\frac{1}{\delta V} \sum_{i} W_{\|_{i}}\right) \frac{[(\mathbf{B} \cdot \nabla) \mathbf{B}] \times \mathbf{B}}{B^{4}}
$$




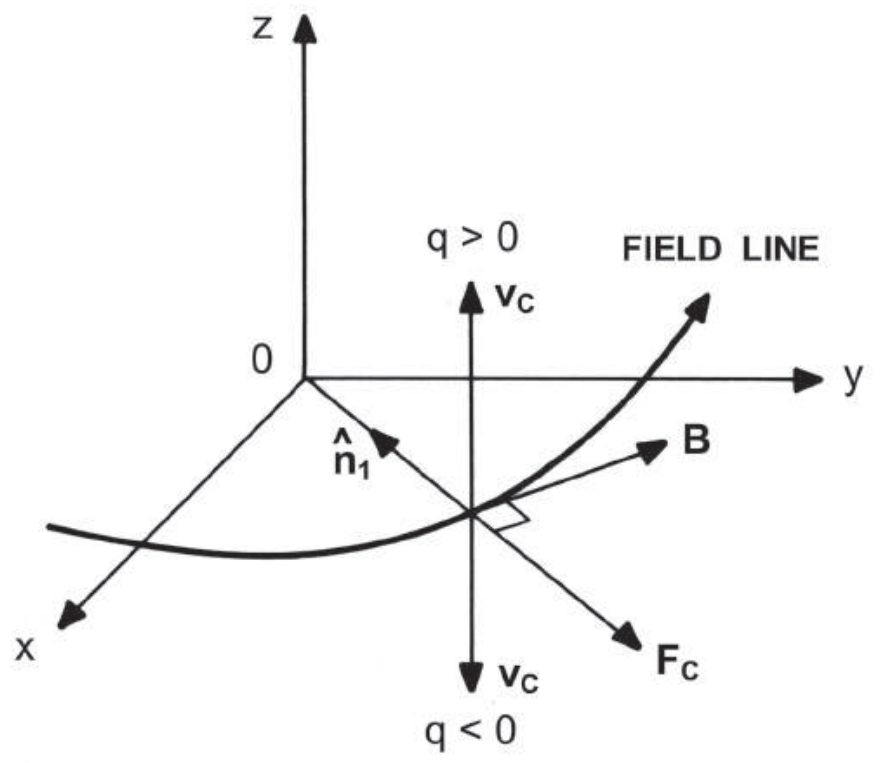

Fig. 17 Relative direction of the particle guiding center drift velocity $\mathbf{v}_{C}$, due to the curvature of the magnetic field line.

where the summation extends over all charged particles contained in the small volume element $\delta V$.

\section{COMBINED GRADIENT-CURVATURE DRIFT}

The curvature drift and the gradient drift always appear together and both point in the same direction, since the term $\nabla B$ points in the direction opposite to $\mathbf{F}_{c}$ (see Fig. 5). These two drifts, therefore, can be added up to form the combined gradient-curvature drift. Thus, from (5.1) and (7.11),

$$
\mathbf{v}_{G C}=\mathbf{v}_{G}+\mathbf{v}_{C}=-\frac{\frac{1}{2} m v_{\perp}^{2}}{q B^{3}}(\nabla B) \times \mathbf{B}-\frac{m v_{\|}^{2}}{q B^{4}}[(\mathbf{B} \cdot \nabla) \mathbf{B}] \times \mathbf{B}
$$

When volume currents are not present (in a vacuum field, for example) so that $\nabla \times \mathbf{B}=0$, the vector identity (4.26) allows the expression (8.1) to be written in the compact form

$$
\mathbf{v}_{G C}=-\frac{m}{q B^{4}}\left(v_{\|}^{2}+\frac{1}{2} v_{\perp}^{2}\right)\left(\nabla \frac{1}{2} B^{2}\right) \times \mathbf{B}
$$

In the Earth's magnetosphere, near the equatorial plane, both the curvature and the gradient drifts ( $\mathrm{B}$ decreases with altitude) cause the 


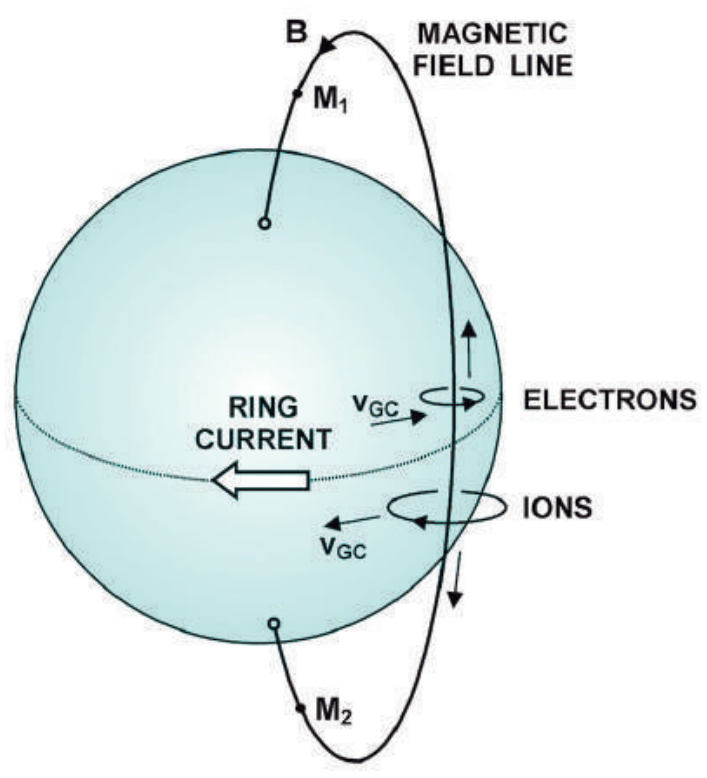

Fig. 18 Sketch (not on scale) illustrating the motion of charged particles in the Earth's magnetic field. The longitudinal drift velocity $\mathbf{v}_{G C}$, due to the gradient and curvature of $\mathbf{B}$, results in an east to west current called the ring current.

positively charged particles to slowly drift westward and the negative ones eastward, resulting in an east to west current, known as the ring current. Fig. 18 illustrates schematically the motion of a charged particle trapped in the Earth's magnetic field. The particle bounces back and forth along the field line between the mirror points $M_{1}$ and $M_{2}$, and drifts in longitude as a result of the gradient and curvature of the field lines. The trajectory described by the particle is therefore contained in a tire-shaped shell encircling the Earth (Fig. 19). This tire-shaped shell encircling the Earth defines a surface on which the particle guiding center drifts slowly around the Earth.

Connected with the periodic motion of the particle on this drift surface there is an adiabatic invariant, called the third adiabatic invariant, which is the total magnetic flux enclosed by the drift surface. Clearly, in a static situation this flux is obviously constant. The significant fact here is that the total magnetic flux, $\Phi_{m}$, enclosed by the drift surface, remains invariant when the field varies slowly in time, that is, when the period of motion of the particle on the drift surface is small compared to the time 


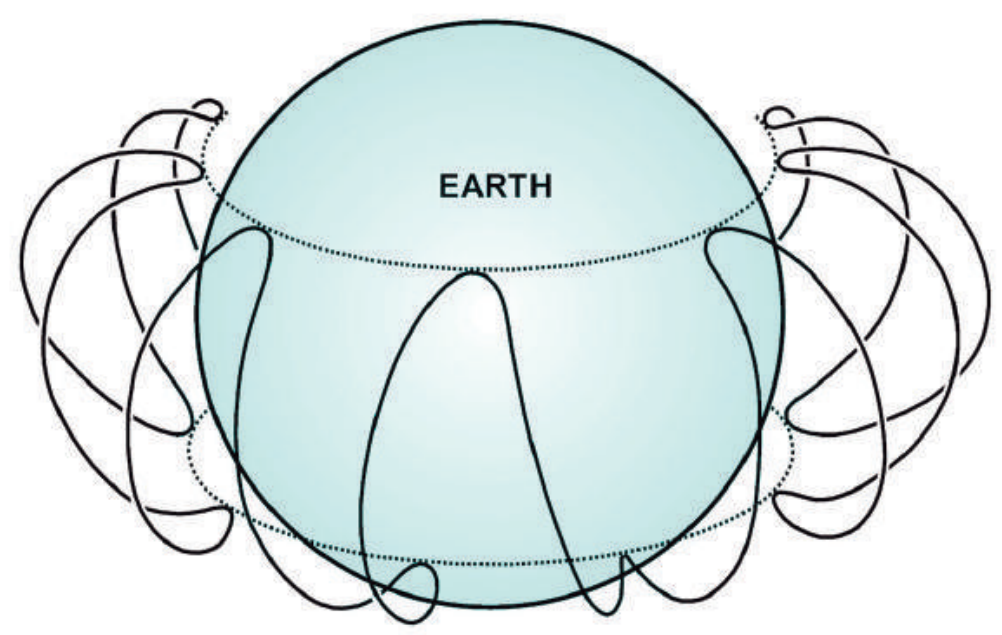

Fig. 19 Schematic representation of the longitudinal drift of charged particles around the Earth.

scale for the magnetic field to change significantly. This invariant has few applications because most fluctuations of $\mathbf{B}$ occur on a time scale that is small compared to the drift period.

\section{PROBLEMS}

3.1 Describe semiquantitatively the motion of an electron under the presence of a constant electric field in the $x$ direction,

$$
\mathbf{E}=E_{0} \widehat{\mathbf{x}}
$$

and a space varying magnetic field given by

$$
\mathbf{B}=B_{0} \alpha(x+z) \widehat{\mathbf{x}}+B_{0}[1+\alpha(x-z)] \widehat{\mathbf{z}}
$$

where $E_{0}, B_{0}$, and $\alpha$ are positive constants, $|\alpha x| \ll 1$ and $|\alpha z| \ll 1$. Assume that initially the electron moves with constant velocity in the $z$ direction, $\mathbf{v}(t=0)=v_{0} \widehat{\mathbf{z}}$. Verify if this magnetic field satisfies the Maxwell equation $\nabla \times \mathbf{B}=0$. 
3.2 Verify if there is any drift velocity for a charged particle in a magnetic field given by

$$
\mathbf{B}=B_{y}(x) \widehat{\mathbf{y}}+B_{0} \widehat{\mathbf{z}}
$$

where $B_{y}(x)$ and $\partial B_{y} / \partial x$ are very small quantities. Does this field satisfy the Maxwell equation $\nabla \times \mathbf{B}=0$ ?

3.3 Consider a system of two coaxial magnetic mirrors whose axis coincides with the $z$ axis, being symmetrical about the plane $z=0$, as shown schematically in Fig. 20. Describe semiquantitatively the motion of a charged particle in this magnetic mirror system considering that at $z=0$ the particle has $v_{\|}=v_{\|}^{0}$ and $v_{\perp}=v_{\perp}^{0}$. What relation must exist between $\mathbf{B}_{0}=B(z=0) \widehat{\mathbf{z}}, \mathbf{B}_{m}=B\left(z= \pm z_{m}\right) \widehat{\mathbf{z}}$ and $\alpha_{0}$ (particle pitch angle at $z=0)$ for the particle to be reflected at $z_{m}$ ?

3.4 For the magnetic mirror system of problem 3.3 suppose that the axial magnetic field changes in time, that is $\mathbf{B}_{\text {axial }}=B(z, t) \widehat{\mathbf{z}}$. Considering that the magnetic moment

$$
|\mathbf{m}|=\frac{\frac{1}{2} m v_{\perp}^{2}(z, t)}{B(z, t)}
$$

is an adiabatic invariant (note that its value is the same at $z=0$ and at $z= \pm z_{m}$, and that $v^{2}=v_{\|}^{2}+v_{\perp}^{2}$ ), show that the longitudinal adiabatic invariant can be written in the form

$$
\int_{-z_{m}}^{z_{m}}\left[B\left(z_{m}, t\right)-B(z, t)\right]^{1 / 2} d z=\text { constant }
$$

3.5 Consider the magnetic mirror system shown in Fig. 20. Suppose that the axial magnetic field is given by

$$
B(z)=B_{0}\left[1+\left(z / a_{0}\right)^{2}\right]
$$

where $B_{0}$ and $a_{0}$ are positive constants, and that the mirroring planes are given by $z=-z_{m}$ and $z=z_{m}$.

(a) For a charged particle trapped in this mirror system, show that the $z$ component of the particle velocity is given by

$$
v_{\|}(z)=\left(\frac{2|\mathbf{m}| B_{0}}{m}\right)^{1 / 2}\left[\left(\frac{z_{m}}{a_{0}}\right)^{2}-\left(\frac{z}{a_{0}}\right)^{2}\right]^{1 / 2}
$$




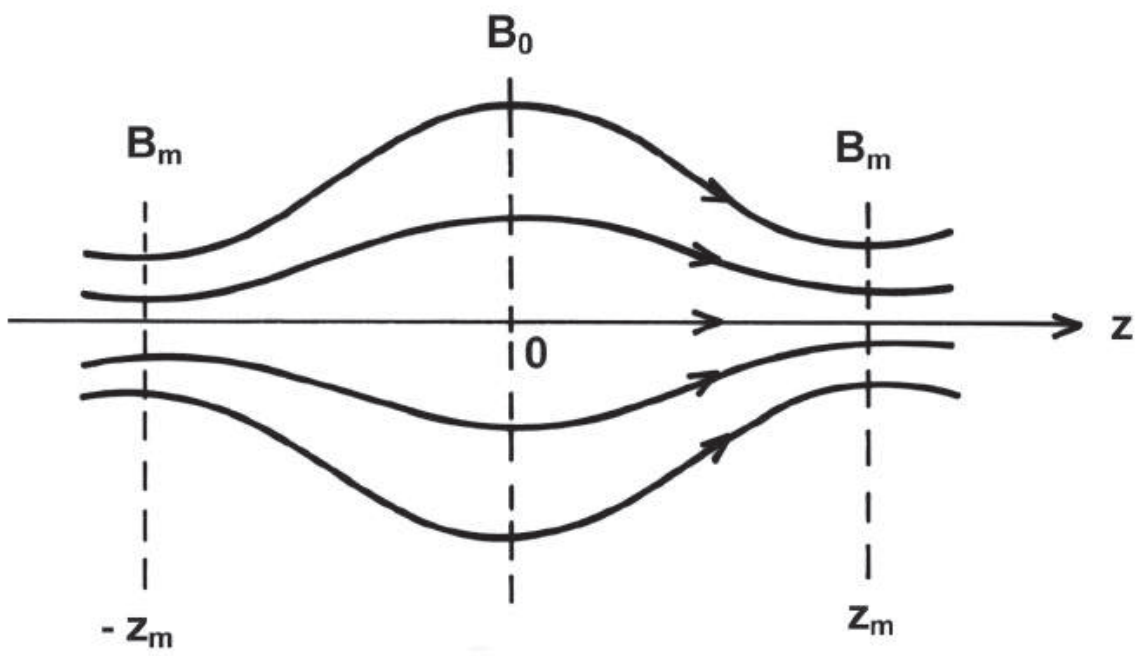

Fig. 20 Magnetic field line geometry for a system of two coaxial magnetic mirrors whose axis coincides with the $z$ axis, being symmetrical about the plane $z=0$.

(b) The average force acting on the particle guiding center, along the $z$ axis, is given by

$$
<\mathbf{F}_{\|}>=-|\mathbf{m}|\left(\frac{\partial B}{\partial z}\right) \widehat{\mathbf{z}}
$$

Show that the particle performs a simple harmonic motion between the mirroring planes, with a period given by

$$
T=2 \pi a_{0}\left(\frac{m}{2|\mathbf{m}| B_{0}}\right)^{1 / 2}
$$

(c) If the motion of the particle is to be limited to the region $|z|<z_{m}$, what restriction must be imposed on the total energy and on the magnetic moment?

3.6 Consider a toroidal magnetic field, as shown in Fig. 21.

(a) Show that the magnetic flux density along the axis of the torus is given by

$$
\mathbf{B}=B_{a}\left(\frac{a}{r}\right) \widehat{\mathbf{\Phi}}
$$

where $B_{a}$ denotes the magnitude of $\mathbf{B}$ at the radial distance $r=a$.

(b) In what direction is the gradient drift associated with the radial variation of $B_{\phi}$ ? Examine qualitatively the type of charge separation that occurs. Neglect the effect of the magnetic field line curvature. 


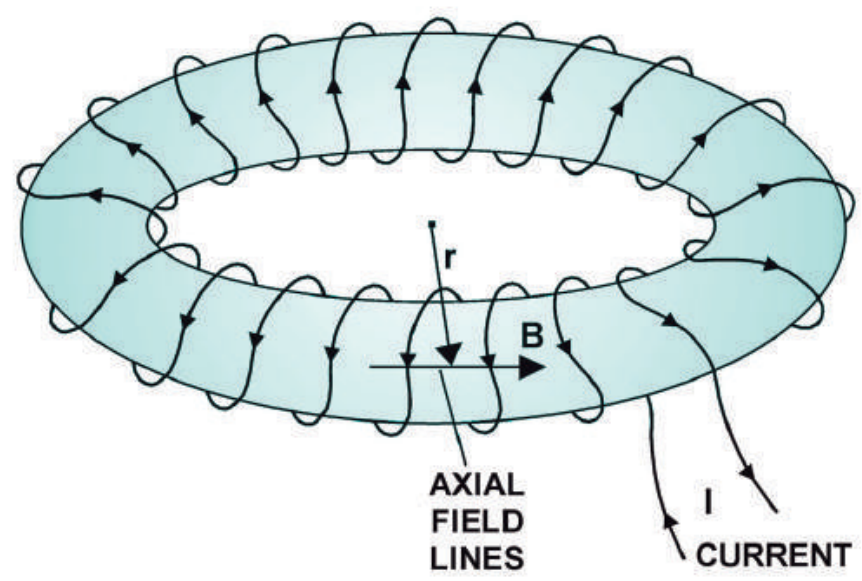

Fig. 21 Magnetic field line with toroidal geometry.

(c) If $\mathbf{E}$ denotes the induced electric field due to charge separation, in what direction is the $\mathbf{E} \times \mathbf{B}$ drift?

(d) Show that it is not possible to confine a plasma in a purely toroidal magnetic field, because of the gradient drift and the $\mathbf{E} \times \mathbf{B}$ drift.

3.7 Consider a spatially nonuniform magnetostatic field expressed in terms of a Cartesian coordinate system by

$$
\mathbf{B}(x, z)=B_{0}[\alpha z \widehat{\mathbf{x}}+(1+\alpha x) \widehat{\mathbf{z}}]
$$

where $B_{0}$ and $\alpha$ are positive constants, $|\alpha x| \ll 1$ and $|\alpha z| \ll 1$.

(a) Show that this magnetic field is consistent with Maxwell equations, so that both gradient and curvature terms are present. Determine the equation of a magnetic flux line.

(b) Write down the Cartesian components of the equation of motion for an electron moving in the region near the origin under the action of this magnetic field.

(c) Consider the following initial conditions for the electron:

$$
\begin{gathered}
\mathbf{r}(0)=\left(x_{0}+v_{\perp 0} / \Omega_{c}\right) \widehat{\mathbf{x}} \\
\mathbf{v}(0)=v_{\perp 0} \widehat{\mathbf{y}}+v_{z 0} \widehat{\mathbf{z}}
\end{gathered}
$$

Solve the equation of motion using a perturbation technique, retaining only terms up to the first order in the small parameter $\alpha$. Show that 
the leading terms in the velocity components, after eliminating the time periodic parts, are given by

$$
\begin{gathered}
\mathbf{v}_{x}=\alpha v_{z 0}^{2} t \widehat{\mathbf{x}} \\
\mathbf{v}_{y}=-\left(\alpha / \Omega_{c}\right)\left(\frac{1}{2} v_{\perp 0}^{2}+v_{z 0}^{2}\right) \widehat{\mathbf{y}} \\
\mathbf{v}_{z}=v_{z 0} \widehat{\mathbf{z}}
\end{gathered}
$$

(d) Show that the average position of the electron in the $(x, z)$ plane follows the magnetic flux line that passes through its initial position.

(e) Show that the gradient and curvature drift velocities are given, respectively, by

$$
\begin{gathered}
\mathbf{v}_{G}=-\left(\alpha / \Omega_{c}\right)\left(\frac{1}{2} v_{\perp}^{2}\right) \widehat{\mathbf{y}} \\
\mathbf{v}_{C}=-\left(\alpha / \Omega_{c}\right) v_{z 0}^{2} \widehat{\mathbf{y}}
\end{gathered}
$$

so that the total drift velocity is precisely the nonperiodic part of $\mathbf{v}_{y}$.

3.8 The Earth's magnetic field can be represented, in a first approximation, by a magnetic dipole placed in the Earth's center, at least up to distances of a few Earth radii $\left(R_{E}\right)$.

(a) Using the fact that, at one of the magnetic poles, the field has a magnitude of approximately 0.5 gauss near the surface, calculate the dipole magnetic moment.

(b) Consider the motion of an electron of energy $E_{0}$ at a radial distance $r_{0}$, where $r_{0}>R_{E}$. Calculate its cyclotron frequency and gyroradius.

(c) Assuming that the electron is confined to move in the equatorial plane, calculate its gradient and curvature drift velocities, and determine the time it takes to drift once around the Earth, at the radial distance $r_{0}$.

(d) Calculate the period of the bounce motion of the electron, as it gets reflected back and forth between the magnetic mirrors near the poles. What is the altitude of the reflection points? Assume that $W_{\|}=W_{\perp}$ at the magnetic equatorial plane.

(e) Obtain numerical values for the results of items (b), (c), and (d), considering $E_{0}=1 \mathrm{MeV}$ and $r_{0}=4 R_{E}$. Examine these results in terms of typical values for charged particles in the outer Van Allen radiation belt. (f) Assuming that there is an isotropic population of $1 \mathrm{MeV}$ protons and $100 \mathrm{keV}$ electrons at about $4 R_{E}$, each having a density $n_{e}=n_{i}=10^{7} \mathrm{~m}^{-3}$ in the equatorial plane, calculate the ring current density in ampere $/ \mathrm{m}^{2}$.

3.9 Imagine an infinite straight wire carrying a current $I$ and uniformly charged to a negative electrostatic potential $\phi$. Analyze the motion of an 
electron in the vicinity of this wire using first-order orbit theory. Sketch the path described by the electron, indicating the relative directions of the electromagnetic, gradient, and curvature drift velocities.

3.10 The field of a magnetic monopole can be represented by

$$
\mathbf{B}(\mathbf{r})=\lambda \frac{\mathbf{r}}{r^{3}}
$$

where $\lambda$ is a constant. Solve the equation of motion to determine the trajectory of a charged particle in this field. (You may refer to the book by B. Rossi and S. Olbert, Introduction to the Physics of Space, Chapter 2, McGraw-Hill, 1970).

3.11 Analyze the motion of a charged particle in the field of a magnetic dipole. Determine the two constants of the motion and analyze their physical meaning. (For this problem, you may refer to the book by S. Stormer, The Polar Aurora, University Oxford Press, 1955, or to B. Rossi and S. Olbert, Introduction to the Physics of Space, Chapter 3, McGraw-Hill, 1970). 


\section{4}

\section{ChARGED PARTICLE MOTION IN TIME-VARYING ELECTROMAGNETIC FIELDS}

\section{INTRODUCTION}

In this chapter we analyze the motion of charged particles in the presence of time-varying fields. Initially, in the two following sections, we consider a time-varying electric field and a constant magnetic field, both fields being spatially uniform. The assumption of a constant and spatially uniform $\mathbf{B}$ field is well justified if the externally applied magnetostatic field is much larger than the magnetic field associated with the time-varying E field. Also, the assumption of an electric field that is spatially uniform is valid if the charged particle cyclotron radius is much smaller than the scale length of the spatial variation of $\mathbf{E}$. Both these requirements are assumed to hold in the analysis presented in sections 2 and 3. In section 4 we consider a time-varying magnetic field and the corresponding spacevarying electric field.

\section{SLOWLY TIME-VARYING ELECTRIC FIELD}

\subsection{Equation of Motion and Polarization Drift}

For the moment we shall assume that the characteristic time scale for variation of the electric field is much larger than the particle cyclotron period. The component of the charged particle motion along the magnetic field lines is given by (2.5.4) (Eq. 5.4 in Chapter 2), from which we can write in general

$$
\mathbf{v}_{\|}(t)-\mathbf{v}_{\|}(0)=\frac{q}{m} \int_{0}^{t} \mathbf{E}_{\|}\left(t^{\prime}\right) d t^{\prime}
$$


This result, however, does not lead to any new interesting information.

Since the $\mathbf{E}$ field is varying slowly in time, the component of the motion across the magnetic field lines is expected to be not very different from that for a constant $\mathbf{E}$ field. Therefore, it is reasonable to seek the solution for $\mathbf{v}_{\perp}$ in a form similar to (2.5.8). Hence, we take

$$
\mathbf{v}_{\perp}=\mathbf{v}_{\perp}^{\prime}+\mathbf{v}_{E}+\mathbf{v}_{p}
$$

where $\mathbf{v}_{E}=\mathbf{E} \times \mathbf{B} / B^{2}$ is the electromagnetic plasma drift velocity (2.5.11). Note that $\mathbf{v}_{E}$ varies slowly in time, since $\mathbf{E}$ is slowly time-varying. The substitution of (2.2) into the perpendicular component equation of motion (2.5.5) yields

$$
m \frac{d}{d t}\left(\mathbf{v}_{\perp}^{\prime}+\mathbf{v}_{E}+\mathbf{v}_{p}\right)=q\left[\mathbf{E}_{\perp}+\left(\mathbf{v}_{\perp}^{\prime}+\mathbf{v}_{E}+\mathbf{v}_{p}\right) \times \mathbf{B}\right]
$$

From (2.5.11) $\mathbf{v}_{E}=\left(\mathbf{E}_{\perp} \times \mathbf{B}\right) / B^{2}$, so that we can write (2.3) as

$$
m \frac{d \mathbf{v}_{\perp}^{\prime}}{d t}+m \frac{d}{d t}\left(\frac{\mathbf{E}_{\perp} \times \mathbf{B}}{B^{2}}\right)+m \frac{d \mathbf{v}_{p}}{d t}=q \mathbf{v}_{\perp}^{\prime} \times \mathbf{B}+q \mathbf{v}_{p} \times \mathbf{B}
$$

Thus, if we set

$$
\mathbf{v}_{p}=\frac{m}{q B^{2}}\left(\frac{\partial \mathbf{E}_{\perp}}{\partial t}\right)
$$

we can write (2.4) in the form

$$
m \frac{d \mathbf{v}_{\perp}^{\prime}}{d t}+m \frac{d \mathbf{v}_{p}}{d t}=q \mathbf{v}_{\perp}^{\prime} \times \mathbf{B}
$$

When the second term on the left-hand side can be neglected, this equation becomes identical to (2.5.12), which describes a circular motion about the magnetic field lines. Comparing the relative magnitudes of the second term on the left, with that on the right-hand side of (2.6), we find

$$
\begin{gathered}
\frac{\left|m d \mathbf{v}_{p} / d t\right|}{\left|q \mathbf{v}_{\perp}^{\prime} \times \mathbf{B}\right|}=\frac{\left|\left(m^{2} / q B^{2}\right)\left(\partial^{2} E_{\perp} / \partial t^{2}\right)\right|}{\left|q v_{\perp}^{\prime} B\right|}= \\
\left|\left(E_{\perp} / B\right) / v_{\perp}^{\prime}\right|\left(\omega^{2} m^{2}\right) /\left(q^{2} B^{2}\right)=\left|v_{E} / v_{\perp}^{\prime}\right|\left(\omega / \Omega_{c}\right)^{2}
\end{gathered}
$$

where we have assumed that $\mathbf{E}_{\perp}$ has a harmonic time dependence with a characteristic angular frequency $\omega$. Considering that this characteristic frequency is much smaller than the cyclotron frequency,

$$
\omega \ll \Omega_{c}
$$


and, further, if $\left|v_{E} / v_{\perp}^{\prime}\right|$ is also small, then the term $m\left(d \mathbf{v}_{p} / d t\right)$ can be neglected in comparison to the other terms of (2.6), and we obtain

$$
m \frac{d \mathbf{v}_{\perp}^{\prime}}{d t}=q \mathbf{v}_{\perp}^{\prime} \times \mathbf{B}
$$

which is identical to (2.5.12). Therefore, $\mathbf{v}_{\perp}^{\prime}$ corresponds to the usual circular motion of the charged particle about the magnetic field, and is independent of the variations of the electric field. Superposed upon this circular motion velocity are the drift velocities

$$
\mathbf{v}_{E}=\frac{\mathbf{E}_{\perp} \times \mathbf{B}}{B^{2}}
$$

and

$$
\mathbf{v}_{p}=\frac{m}{q B^{2}}\left(\frac{\partial \mathbf{E}_{\perp}}{\partial t}\right)
$$

Thus, the effect of a slowly varying electric field is the addition of the drift velocity $\mathbf{v}_{p}$ called the polarization drift velocity.

Since $\mathbf{v}_{p}$ is in opposite directions for charges of opposite sign, the time-dependent electric field produces a net polarization current in a neutral plasma, so that the plasma medium behaves like a dielectric. The polarization current density $\mathbf{J}_{p}$ is the rate of flow of positive and negative charges across unit area, and is given by

$$
\mathbf{J}_{p}=\frac{1}{\delta V} \sum_{i} q_{i} \mathbf{v}_{p i}=\left(\frac{1}{\delta V} \sum_{i} m_{i}\right) \frac{1}{B^{2}}\left(\frac{\partial \mathbf{E}_{\perp}}{\partial t}\right)=\frac{\rho_{m}}{B^{2}}\left(\frac{\partial \mathbf{E}_{\perp}}{\partial t}\right)
$$

where the summation is over all positive and negative charges contained in the small volume element $\delta V$, and $\rho_{m}$ is the mass density of the plasma.

\subsection{Plasma Dielectric Constant}

The polarization effect in a plasma is due to the time variation of the electric field. The application of a steady $\mathbf{E}$ field does not result in a polarization field, since the ions and electrons can move around to preserve quasineutrality. Since the plasma behaves like a dielectric, the polarization current density $\mathbf{J}_{p}$ can be taken into account through the introduction of the dielectric constant of the plasma.

For this purpose, we can separate the total current density $\mathbf{J}$ into the polarization current density $\mathbf{J}_{p}$ and the current density $\mathbf{J}_{0}$ due to other sources,

$$
\mathbf{J}=\mathbf{J}_{p}+\mathbf{J}_{0}
$$


Thus, combining $\mathbf{J}_{p}$ with the term $\epsilon_{0} \partial \mathbf{E}_{\perp} / \partial t$, which appears on the righthand side of Maxwell $\nabla \times \mathbf{B}$ equation, we obtain

$$
\epsilon_{0} \frac{\partial \mathbf{E}_{\perp}}{\partial t}+\frac{\rho_{m}}{B^{2}} \frac{\partial \mathbf{E}_{\perp}}{\partial t}=\epsilon_{0}\left(1+\frac{\rho_{m}}{\epsilon_{0} B^{2}}\right) \frac{\partial \mathbf{E}_{\perp}}{\partial t}=\epsilon \frac{\partial \mathbf{E}_{\perp}}{\partial t}
$$

where

$$
\epsilon=\epsilon_{0} \epsilon_{r}=\epsilon_{0}\left(1+\frac{\rho_{m}}{\epsilon_{0} B^{2}}\right)
$$

is the effective electric permittivity perpendicular to the magnetic field. In some cases the relative permittivity $\epsilon_{r}$ of a plasma can be very high. As an example, if we consider a number density of $10^{20}$ particles $/ \mathrm{m}^{3}$ and $B=1$ tesla, we have $\epsilon_{r}=10^{4}$.

The resulting charge density $\rho_{p}$, which accumulates as a result of the polarization current density $\mathbf{J}_{p}$, satisfies the charge continuity equation

$$
\frac{\partial \rho_{p}}{\partial t}+\nabla \cdot \mathbf{J}_{p}=0
$$

From (2.16) and (2.12) we have

$$
\rho_{p}=-\frac{\rho_{m}}{B^{2}} \nabla \cdot \mathbf{E}_{\perp}
$$

The total charge density $\rho$ can be separated as

$$
\rho=\rho_{0}+\rho_{p}
$$

where $\rho_{0}$ corresponds to $\mathbf{J}_{0}$. Assuming that the parallel component of the electric field vanishes, we see that

$$
\nabla \cdot \mathbf{E}=\frac{1}{\epsilon_{0}}\left(\rho_{0}+\rho_{p}\right)=\frac{\rho_{0}}{\epsilon_{0}}-\frac{\rho_{m}}{\epsilon_{0} B^{2}} \nabla \cdot \mathbf{E}
$$

from which we find, using (2.15),

$$
\nabla \cdot \mathbf{E}=\frac{\rho_{0}}{\epsilon}
$$

Thus, the resulting charge density $\rho_{p}$ can also be correctly taken into account by the introduction of an effective electric permittivity $\epsilon$.

We can further verify the correctness of introducing the effective electric permittivity of the plasma, by calculating the total energy density 
associated with the $\mathbf{E}$ field, which for an ordinary dielectric medium of effective permittivity $\epsilon$ is given by $\epsilon E^{2} / 2$. The energy density in the electric field is given by

$$
W_{E}=\frac{1}{2} \epsilon_{0} E^{2}
$$

To calculate the additional drift kinetic energy acquired by the particle as a result of the polarization drift, we note that for a change $\Delta \mathbf{E}_{\perp}$ in the electric field, in a time interval $\Delta t$, the displacement $\Delta \mathbf{r}$ of the guiding center, due to this change, is

$$
\Delta \mathbf{r}=\mathbf{v}_{p} \Delta t=\frac{m}{q B^{2}}\left(\frac{\partial \mathbf{E}_{\perp}}{\partial t}\right) \Delta t=\frac{m}{q B^{2}} \Delta \mathbf{E}_{\perp}
$$

The corresponding work done by the electric field is, using (2.22),

$$
\Delta W=q \mathbf{E}_{\perp} \cdot(\Delta \mathbf{r})=\frac{m}{B^{2}} \mathbf{E}_{\perp} \cdot\left(\Delta \mathbf{E}_{\perp}\right)=\Delta\left(\frac{1}{2} m E_{\perp}^{2} / B^{2}\right)
$$

Hence, using (2.10), the change in the particle kinetic energy associated with the polarization drift is given by

$$
\Delta W=\Delta\left(\frac{1}{2} m v_{E}^{2}\right)
$$

This result shows that the work done by the electric field, during polarization, is equal to the change in the kinetic energy associated with the particle motion at the electromagnetic drift velocity $\mathbf{v}_{E}$. Note that $\mathbf{v}_{E}$ does not lead to any energy exchange between field and particle, since the displacement associated with $\mathbf{v}_{E}$ is perpendicular to the electric field. Summing (2.24) over all particles in a unit volume gives the change in the total kinetic energy density of the system

$$
\Delta W_{V}=\Delta\left(\frac{1}{2} \rho_{m} v_{E}^{2}\right)=\Delta\left(\frac{1}{2} \rho_{m} E_{\perp}^{2} / B^{2}\right)
$$

The kinetic energy density associated with the circular motion of the particles is not affected by changes in the electric field. Thus, the total energy density $\left(W_{T}=W_{E}+W_{V}\right)$ associated with the electric field is

$$
W_{T}=\frac{1}{2} \epsilon_{0} E^{2}+\frac{1}{2} \rho_{m} v_{E}^{2}=\frac{1}{2} \epsilon_{0} E^{2}\left(1+\frac{\rho_{m}}{\epsilon_{0} B^{2}}\right)=\frac{1}{2} \epsilon E^{2}
$$

assuming that there is no parallel component of the electric field. This result completes our discussion about the legitimacy of the introduction of an effective electric permittivity for the plasma. 


\section{ELECTRIC FIELD WITH ARBITRARY TIME VARIATION}

\subsection{Solution of the Equation of Motion}

We consider now an arbitrary time variation of the electric field, but, again, the field is spatially uniform. The applied magnetic field is static and uniform, as before. Without loss of generality, the time variation of $\mathbf{E}$ can be assumed to be harmonic with angular frequency $\omega$,

$$
\mathbf{E}(t)=\mathbf{E}_{0} e^{-i \omega t}
$$

where the complex amplitude $\mathbf{E}_{0}$ is independent of time. According to the usual convention, only the real part of this expression is to be taken for the physical interpretation of the results. An arbitrary time variation of $\mathbf{E}$ can be written as a superposition of terms similar to (3.1), corresponding to all possible values of $\omega$, since the equation of motion (2.5.5) is linear. Using (3.1), the equation of motion becomes

$$
m \frac{d \mathbf{v}}{d t}=q\left(\mathbf{E}_{0} e^{-i \omega t}+\mathbf{v} \times \mathbf{B}\right)
$$

It is natural to expect the forced oscillations of the charged particle to have the same frequency as that of the forcing electric field. Thus, the particle velocity vector may be conveniently decomposed into two parts,

$$
\mathbf{v}=\mathbf{v}_{m}+\mathbf{v}_{e} e^{-i \omega t}
$$

where $\mathbf{v}_{m}$ is the velocity associated with the magnetic field alone and, thus, contains no time variation at the angular frequency $\omega$, while $\mathbf{v}_{e}$ is due to the oscillating electric field. The substitution of (3.3) into (3.2) gives

$$
m \frac{d \mathbf{v}_{m}}{d t}-i \omega m \mathbf{v}_{e} e^{-i \omega t}=q\left(\mathbf{E}_{0} e^{-i \omega t}+\mathbf{v}_{m} \times \mathbf{B}+\mathbf{v}_{e} \times \mathbf{B} e^{-i \omega t}\right)
$$

The terms containing the periodicity at the angular frequency $\omega$ are decoupled from those that do not, so that (3.4) separates into two equations, one involving only the velocity $\mathbf{v}_{m}$ associated with the magnetic field according to

$$
m \frac{d \mathbf{v}_{m}}{d t}=q \mathbf{v}_{m} \times \mathbf{B}
$$


and the other involving only the velocity $\mathbf{v}_{e}$,

$$
-i \omega m \mathbf{v}_{e}=q\left(\mathbf{E}_{0}+\mathbf{v}_{e} \times \mathbf{B}\right)
$$

Equation (3.5) corresponds to the usual circular motion of the particle about the magnetic field lines at the cyclotron frequency $\boldsymbol{\Omega}_{c}$.

To solve (3.6) for $\mathbf{v}_{e}$ it is appropriate to separate this equation into components parallel and perpendicular to $\mathbf{B}$. The parallel velocity component is obtained immediately as

$$
\mathbf{v}_{e \|}=\frac{i q}{\omega m} \mathbf{E}_{0 \|}
$$

while the perpendicular velocity component satisfies the equation

$$
\left(-i \omega+\frac{q}{m} \mathbf{B} \times\right) \mathbf{v}_{e \perp}=\frac{q}{m} \mathbf{E}_{0 \perp}
$$

Introducing the cyclotron frequency vector, defined by

$$
\boldsymbol{\Omega}_{c}=-\frac{q}{m} \mathbf{B}
$$

we can rewrite (3.8) in the form

$$
\left(i \omega+\boldsymbol{\Omega}_{c} \times\right) \mathbf{v}_{e \perp}=-\frac{q}{m} \mathbf{E}_{0 \perp}
$$

In order to solve this equation for $\mathbf{v}_{e \perp}$, we multiply both sides by the conjugate operator $-\left(i \omega-\boldsymbol{\Omega}_{c} \times\right)$. First, we note that

$$
\begin{gathered}
\left(i \omega-\boldsymbol{\Omega}_{c} \times\right)\left(i \omega+\boldsymbol{\Omega}_{c} \times\right) \mathbf{v}_{e \perp}=(i \omega)^{2} \mathbf{v}_{e \perp}-\boldsymbol{\Omega}_{c} \times\left(\boldsymbol{\Omega}_{c} \times \mathbf{v}_{e \perp}\right)= \\
\left(\Omega_{c}^{2}-\omega^{2}\right) \mathbf{v}_{e \perp}
\end{gathered}
$$

Therefore, (3.10) becomes

$$
\mathbf{v}_{e \perp}=\frac{q}{m} \frac{\left(i \omega-\Omega_{c} \times\right)}{\left(\omega^{2}-\Omega_{c}^{2}\right)} \mathbf{E}_{0 \perp}
$$

Combining the results contained in (3.12), (3.7), and (3.5), we obtain the following expression for the total velocity vector (3.3):

$$
\mathbf{v}=\mathbf{v}_{m}+\frac{q}{m}\left[\frac{i}{\omega} \mathbf{E}_{0 \|}+\frac{\left(i \omega-\Omega_{c} \times\right)}{\left(\omega^{2}-\Omega_{c}^{2}\right)} \mathbf{E}_{0 \perp}\right] e^{-i \omega t}
$$




\subsection{Physical Interpretation}

The result contained in (3.13) shows that along the magnetic field lines the particle oscillates with frequency $\omega$ and amplitude $\mathbf{v}_{e \|}$, as given by (3.7), so that the velocity oscillation lags $90^{\circ}$ behind the oscillation of the applied electric field. This result is easily seen by taking the real part of the vectors $\mathbf{v}_{e \|} e^{-i \omega t}$ and $\mathbf{E}_{0 \|} e^{-i \omega t}$,

$$
\begin{gathered}
\Re\left\{\mathbf{v}_{e \|} e^{-i \omega t}\right\}=\frac{q}{\omega m} \mathbf{E}_{0 \|} \sin (\omega t) \\
\Re\left\{\mathbf{E}_{0 \|} e^{-i \omega t}\right\}=\mathbf{E}_{0 \|} \cos (\omega t)
\end{gathered}
$$

which clearly are $90^{\circ}$ out of phase.

In the plane perpendicular to $\mathbf{B}$ the particle motion is the superposition of the circular motion at the cyclotron frequency $\boldsymbol{\Omega}_{c}$, with an oscillation at the frequency $\omega$ and amplitude given by (3.12).

In order to analyze the physical meaning of the motion in the plane perpendicular to $\mathbf{B}$, it is convenient to decompose the oscillating electric field vector into two circularly polarized components, with opposite directions of rotation. The advantage of using the two circularly polarized components is that (3.12), for the perpendicular velocity component $\mathbf{v}_{e \perp}$, uncouples into two separate equations pertaining to the two circular polarizations rotating in opposite directions. Thus, we take

$$
\mathbf{E}_{\perp}=\mathbf{E}_{R}+\mathbf{E}_{L}=\left(\mathbf{E}_{0 R}+\mathbf{E}_{0 L}\right) e^{-i \omega t}
$$

with

$$
\begin{aligned}
& \mathbf{E}_{0 R}=\frac{1}{2}(1+i \widehat{\mathbf{B}} \times) \mathbf{E}_{0 \perp} \\
& \mathbf{E}_{0 L}=\frac{1}{2}(1-i \widehat{\mathbf{B}} \times) \mathbf{E}_{0 \perp}
\end{aligned}
$$

where $\widehat{\mathbf{B}}=\mathbf{B} / B$ is a unit vector pointing in the direction of the magnetic field. The component $\mathbf{E}_{R}$ represents a circularly polarized field with its electric vector rotating to the right (clockwise direction), and the component $\mathbf{E}_{L}$ represents a circularly polarized field with its electric vector rotating to the left (counterclockwise direction), as seen by an observer looking in the direction of the $\mathbf{B}$ field.

To understand the physical meaning of this decomposition, let us consider a Cartesian coordinate system with the $z$ axis pointing along $\mathbf{B}$ and the $x$ axis pointing along $\mathbf{E}_{\perp}$. Then, we have

$$
\widehat{\mathbf{B}} \times \mathbf{E}_{0 \perp}=\widehat{\mathbf{z}} \times\left(\widehat{\mathbf{x}} E_{0 \perp}\right)=\widehat{\mathbf{y}} E_{0 \perp}
$$




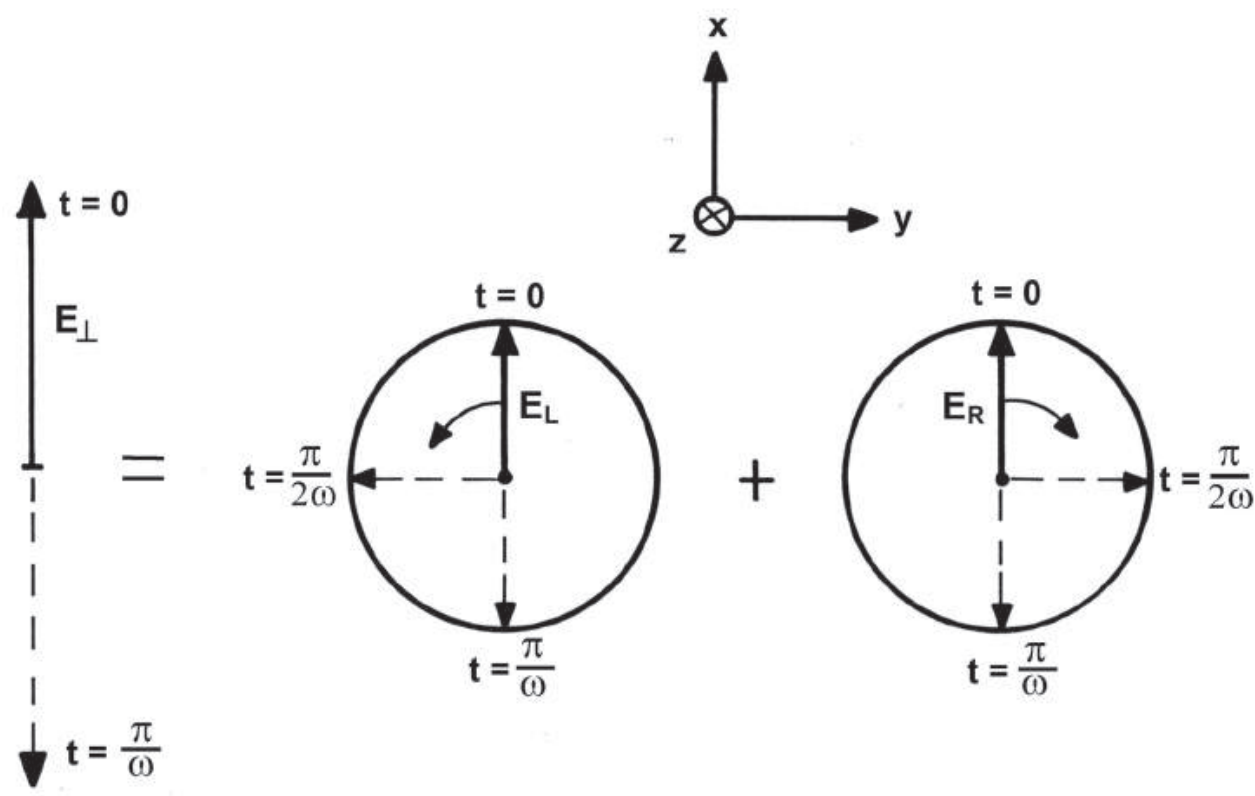

Fig. 1 The electric field of a plane polarized wave, represented by the double-headed arrow $\mathbf{E}_{\perp}$, is equivalent to the vector sum of left and right circularly polarized waves $\mathbf{E}_{L}$ and $\mathbf{E}_{R}$.

and from (3.17) and (3.18),

$$
\begin{aligned}
& \mathbf{E}_{R}=\mathbf{E}_{0 R} e^{-i \omega t}=\frac{1}{2} E_{0 \perp}(\widehat{\mathbf{x}}+i \widehat{\mathbf{y}}) e^{-i \omega t} \\
& \mathbf{E}_{L}=\mathbf{E}_{0 L} e^{-i \omega t}=\frac{1}{2} E_{0 \perp}(\widehat{\mathbf{x}}-i \widehat{\mathbf{y}}) e^{-i \omega t}
\end{aligned}
$$

The components of the actual electric field are obtained by taking the real part of these two equations,

$$
\begin{aligned}
& \Re\left\{\mathbf{E}_{R}\right\}=\frac{1}{2} E_{0 \perp}(\widehat{\mathbf{x}} \cos \omega t+\widehat{\mathbf{y}} \sin \omega t) \\
& \Re\left\{\mathbf{E}_{L}\right\}=\frac{1}{2} E_{0 \perp}(\widehat{\mathbf{x}} \cos \omega t-\widehat{\mathbf{y}} \sin \omega t)
\end{aligned}
$$

Thus, the fields $\mathbf{E}_{R}$ and $\mathbf{E}_{L}$ are constant in magnitude, but sweep around in a circle at the frequency $\omega$. For $\mathbf{E}_{R}$ the rotation is clockwise looking in the direction of $\mathbf{B}$, and it is called a right circularly polarized (RCP) component, while for $\mathbf{E}_{L}$ the rotation is counterclockwise and it is called a left circularly polarized (LCP) component. This behavior is illustrated in Fig. 1. 
Proceeding in the analysis of the particle motion in the perpendicular plane, we must now substitute the decomposed form (3.16) into (3.12). The operator $\left(i \omega-\boldsymbol{\Omega}_{c} \times\right)$ when applied on the right circularly polarized component $\mathbf{E}_{0 R}$ gives

$$
\begin{gathered}
\left(i \omega-\boldsymbol{\Omega}_{c} \times\right) \mathbf{E}_{0 R}=\frac{1}{2}\left(i \omega-\boldsymbol{\Omega}_{c} \times\right)(1+i \widehat{\mathbf{B}} \times) \mathbf{E}_{0 \perp}= \\
\frac{1}{2} i \omega\left(\mathbf{E}_{0 \perp}+i \widehat{\mathbf{B}} \times \mathbf{E}_{0 \perp}\right)+\frac{1}{2}(q B / m) \widehat{\mathbf{B}} \times \mathbf{E}_{0 \perp}-\frac{1}{2} i(q B / m) \mathbf{E}_{0 \perp}= \\
\frac{1}{2} i \omega\left(\mathbf{E}_{0 \perp}+i \widehat{\mathbf{B}} \times \mathbf{E}_{0 \perp}\right)-\frac{1}{2} i(q B / m)\left(\mathbf{E}_{0 \perp}+i \widehat{\mathbf{B}} \times \mathbf{E}_{0 \perp}\right)= \\
i(\omega-q B / m) \mathbf{E}_{0 R}
\end{gathered}
$$

In a similar fashion we find for the left circularly polarized component,

$$
\left(i \omega-\boldsymbol{\Omega}_{c} \times\right) \mathbf{E}_{0 L}=i(\omega+q B / m) \mathbf{E}_{0 L}
$$

Therefore, both $\mathbf{E}_{0 R}$ and $\mathbf{E}_{0 L}$ are eigenvectors of the complex operator appearing in (3.12). Using (3.24) and (3.25), (3.12) becomes

$$
\mathbf{v}_{e \perp}=i\left(\frac{q}{m}\right)\left[\frac{1}{\left(\omega \pm \Omega_{c}\right)} \mathbf{E}_{0 R}+\frac{1}{\left(\omega \mp \Omega_{c}\right)} \mathbf{E}_{0 L}\right]
$$

In this equation the signs are coupled such that the upper signs apply to a positively charged particle and the lower signs to a negatively charged particle, since by definition $\Omega_{c}=|q| B / m$ is always positive. Thus, $\mathbf{v}_{e \perp}$ also separates into two vectors rotating in opposite directions,

$$
\mathbf{v}_{e \perp}=\mathbf{v}_{R}+\mathbf{v}_{L}
$$

where

$$
\begin{aligned}
& \mathbf{v}_{R}=i\left(\frac{q}{m}\right) \frac{1}{\left(\omega \pm \Omega_{c}\right)} \mathbf{E}_{0 R} \\
& \mathbf{v}_{L}=i\left(\frac{q}{m}\right) \frac{1}{\left(\omega \mp \Omega_{c}\right)} \mathbf{E}_{0 L}
\end{aligned}
$$

For a positive ion we see that, as $\omega$ approaches the ion cyclotron frequency $\left(\Omega_{c i}\right)$, there is resonance between the ion and the left circularly polarized component of the electric field. For an electron, as $\omega$ approaches the electron cyclotron frequency $\left(\Omega_{c e}\right)$, resonance occurs with the right circularly polarized component. 


\subsection{Mobility Dyad}

The expression for $\mathbf{v}_{e}=\mathbf{v}_{e \|}+\mathbf{v}_{R}+\mathbf{v}_{L}$ can be written in a compact form through the introduction of the mobility dyad $\mathcal{M}$ defined by

$$
\mathbf{v}_{e}=\mathcal{M} \cdot \mathbf{E}_{0}
$$

Using (3.7), (3.28), and (3.29), we see that the mobility dyad $\mathcal{M}$ is diagonal in the rotating system, so that (3.30) becomes in matrix form,

$$
\left(\begin{array}{c}
v_{R} \\
v_{L} \\
v_{e \|}
\end{array}\right)=\frac{i q}{\omega m}\left(\begin{array}{ccc}
\frac{\omega}{\left(\omega \pm \Omega_{c}\right)} & 0 & 0 \\
0 & \frac{\omega}{\left(\omega \mp \Omega_{c}\right)} & 0 \\
0 & 0 & 1
\end{array}\right)\left(\begin{array}{c}
E_{0 R} \\
E_{0 L} \\
E_{0 \|}
\end{array}\right)
$$

If, instead of the rotating system, we use a stationary Cartesian coordinate system with the $z$ axis pointing along the magnetostatic field, we can write (3.7) and (3.12) in matrix form as

$$
\left(\begin{array}{l}
v_{e x} \\
v_{e y} \\
v_{e z}
\end{array}\right)=\frac{i q}{\omega m}\left(\begin{array}{ccc}
\frac{\omega^{2}}{\left(\omega^{2}-\Omega_{c}^{2}\right)} & \pm \frac{i \omega \Omega_{c}}{\left(\omega^{2}-\Omega_{c}^{2}\right)} & 0 \\
\mp \frac{i \omega \Omega_{c}}{\left(\omega^{2}-\Omega_{c}^{2}\right)} & \frac{\omega^{2}}{\left(\omega^{2}-\Omega_{c}^{2}\right)} & 0 \\
0 & 0 & 1
\end{array}\right)\left(\begin{array}{c}
E_{0 x} \\
E_{0 y} \\
E_{0 z}
\end{array}\right)
$$

\subsection{Plasma Conductivity Dyad}

Denoting by $n_{0}$ the number density of electrons (charge $-e$ ) and ions (charge $+e$ ) in a plasma, the electric current density can be expressed as

$$
\mathbf{J}=-n_{0} e \mathbf{v}_{e}+n_{0} e \mathbf{v}_{i}=n_{0} e\left(\mathcal{M}_{i}-\mathcal{M}_{e}\right) \cdot \mathbf{E}
$$

where $\mathcal{M}_{e}$ and $\mathcal{M}_{i}$ are the mobility dyads for the electrons and ions, respectively. Introducing the conductivity dyad $\mathcal{S}$ by

$$
\mathbf{J}=\mathcal{S} \cdot \mathbf{E}=\left(\mathcal{S}_{e}+\mathcal{S}_{i}\right) \cdot \mathbf{E}
$$

we obtain from (3.33), for the electron and ion conductivities, respectively,

$$
\begin{gathered}
\mathcal{S}_{e}=-n_{0} e \mathcal{M}_{e} \\
\mathcal{S}_{i}=n_{0} e \mathcal{M}_{i}
\end{gathered}
$$

With the help of (3.32), these conductivities can be expressed in matrix form, in a Cartesian coordinate system, as 


$$
\begin{gathered}
\mathcal{S}_{e}=\frac{i n_{0} e^{2}}{\omega m_{e}}\left(\begin{array}{ccc}
\frac{\omega^{2}}{\left(\omega^{2}-\Omega_{c e}^{2}\right)} & -\frac{i \omega \Omega_{c e}}{\left(\omega^{2}-\Omega_{c e}^{2}\right)} & 0 \\
\frac{i \omega \Omega_{c e}}{\left(\omega^{2}-\Omega_{c e}^{2}\right)} & \frac{\omega^{2}}{\left(\omega^{2}-\Omega_{c e}^{2}\right)} & 0 \\
0 & 0 & 1
\end{array}\right) \\
\mathcal{S}_{i}=\frac{i n_{0} e^{2}}{\omega m_{i}}\left(\begin{array}{ccc}
\frac{\omega^{2}}{\left(\omega^{2}-\Omega_{c i}^{2}\right)} & \frac{i \omega \Omega_{c i}}{\left(\omega^{2}-\Omega_{c i}^{2}\right)} & 0 \\
-\frac{i \omega \Omega_{c i}}{\left(\omega^{2}-\Omega_{c i}^{2}\right)} & \frac{\omega^{2}}{\left(\omega^{2}-\Omega_{c i}^{2}\right)} & 0 \\
0 & 0 & 1
\end{array}\right)
\end{gathered}
$$

The fact that the conductivity dyad $\mathcal{S}$ is imaginary implies that $\mathbf{J}$ and $\mathbf{E}$ are 90 degrees out of phase, since the actual physical expressions for $\mathbf{J}$ and $\mathbf{E}$ are obtained by taking the real part of (3.34) and (3.1), respectively.

\subsection{Cyclotron Resonance}

The particle velocities, given in (3.7), (3.28), and (3.29), do not represent correctly the motion of the particle when the frequency $\omega$ of the applied electric field is equal to the particle cyclotron frequency $\Omega_{c}$. For example, when the forcing electric field rotates in the counterclockwise direction, looking along $\mathbf{B}$ (LCP component), a positive particle is able to absorb energy from the electric field, so that its speed increases continuously and indefinitely in time. The same holds for an electron, say, and the RCP component of the forcing electric field when $\omega=\Omega_{c e}$. This phenomenon is called cyclotron resonance.

To investigate the particle motion under resonance conditions, it is necessary to go back to the original equation of motion and solve the problem for the case when $\omega=\Omega_{c}$. For simplicity, let us assume that the component of the $\mathbf{E}$ field along $\mathbf{B}$ vanishes, that is, $\mathbf{E}=\mathbf{E}_{\perp}$. Hence, we take

$$
\mathbf{E}=\mathbf{E}_{0 \perp} e^{-i \Omega_{c} t}
$$

where $\Omega_{c}=|q| B / m$. In view of this assumption, the particle velocity along $\mathbf{B}$ is constant and is equal to the initial parallel velocity. The component of the equation of motion in the plane normal to the magnetic field becomes

$$
\frac{d \mathbf{v}_{\perp}(t)}{d t}=\frac{q}{m}\left[\mathbf{E}_{0 \perp} e^{-i \Omega_{c} t}+\mathbf{v}_{\perp}(t) \times \mathbf{B}\right]
$$


Taking the derivative of this equation with respect to time, yields

$$
\frac{d^{2} \mathbf{v}_{\perp}}{d t^{2}}=\frac{q}{m}\left(-i \Omega_{c} \mathbf{E}_{0 \perp} e^{-i \Omega_{c} t}+\frac{d \mathbf{v}_{\perp}}{d t} \times \mathbf{B}\right)
$$

and using (3.40) to eliminate $\left(d \mathbf{v}_{\perp} / d t\right)$ in (3.41), we obtain, after rearranging the terms,

$$
\frac{d^{2} \mathbf{v}_{\perp}}{d t^{2}}+\Omega_{c}^{2} \mathbf{v}_{\perp}=-i \Omega_{c} \frac{q}{m} \mathbf{E}_{0 \perp} e^{-i \Omega_{c} t}-\frac{q^{2}}{m^{2}}\left(\mathbf{B} \times \mathbf{E}_{0 \perp}\right) e^{-i \Omega_{c} t}
$$

The solution of this inhomogeneous differential equation is given by the sum of the solution of the homogeneous equation plus a particular integral of the inhomogeneous equation. The solution of the homogeneous equation

$$
\frac{d^{2} \mathbf{v}_{\perp}}{d t^{2}}+\Omega_{c}^{2} \mathbf{v}_{\perp}=0
$$

is just the cyclotron motion described previously by the velocity $\mathbf{v}_{m}$. A particular integral of (3.42) is provided by the function

$$
\mathbf{v}_{\perp}=\mathbf{A} t e^{-i \Omega_{c} t}
$$

To determine the constant vector $\mathbf{A}$ we differentiate (3.44) twice with respect to time:

$$
\begin{gathered}
\frac{d \mathbf{v}_{\perp}}{d t}=\mathbf{A}\left(1-i \Omega_{c} t\right) e^{-i \Omega_{c} t} \\
\frac{d^{2} \mathbf{v}_{\perp}}{d t^{2}}=-2 i \Omega_{c} \mathbf{A} e^{-i \Omega_{c} t}-\Omega_{c}^{2} \mathbf{v}_{\perp}
\end{gathered}
$$

Comparing (3.46) with (3.42) we see that (3.44) satisfies (3.42) provided we take

$$
\mathbf{A}=\frac{q}{2 m} \mathbf{E}_{0 \perp}-\frac{i q^{2}}{2 \Omega_{c} m^{2}}\left(\mathbf{B} \times \mathbf{E}_{0 \perp}\right)
$$

Therefore, the complete solution of (3.42) is

$$
\mathbf{v}_{\perp}=\mathbf{v}_{m}+\frac{q}{2 m}\left(\mathbf{E}_{0 \perp} \mp i \widehat{\mathbf{B}} \times \mathbf{E}_{0 \perp}\right) t e^{-i \Omega_{c} t}
$$

where we have replaced $q B / m$ by $\pm \Omega_{c}$, the upper and lower signs corresponding to positive and negative charges, respectively. Using (3.17) and (3.18), which define the right and left circularly polarized components of 
the electric field, respectively, we can write (3.48) for a positively charged particle $(q>0)$ as

$$
\mathbf{v}_{\perp}=\mathbf{v}_{m}+\frac{q}{m} \mathbf{E}_{0 L} t e^{-i \Omega_{c} t}
$$

and for a negatively charged particle $(q<0)$ as

$$
\mathbf{v}_{\perp}=\mathbf{v}_{m}+\frac{q}{m} \mathbf{E}_{0 R} t e^{-i \Omega_{c} t}
$$

Hence, the particle velocity increases indefinitely with time. Note that the expressions $(q / m) \mathbf{E}_{0 L}$ and $(q / m) \mathbf{E}_{0 R}$ represent a constant acceleration. A positive charge resonates with $\mathbf{E}_{L}$ and a negative one with $\mathbf{E}_{R}$. The particle moves in circles of ever-increasing radii, with its velocity increasing continuously during this spiral motion at the expense of the electric field energy. A typical resonant spiral for an electron is shown in Fig. 2.

This phenomenon can be used as a method of increasing the particle speed and hence the kinetic temperature of a plasma through particle collisions. This method is known as radio frequency heating of the plasma by cyclotron resonance.

\section{TIME-VARYING MAGNETIC FIELD AND SPACE-VARYING ELECTRIC FIELD}

From Maxwell equations it is seen that a time-varying magnetic field is also accompanied by a space-varying electric field. The $\mathbf{E}$ field associated with the time-dependent $\mathbf{B}$ field satisfies

$$
\nabla \times \mathbf{E}=-\frac{\partial \mathbf{B}}{\partial t}
$$

We shall assume that the fractional change in the magnetic field, in a time interval of the order of the cyclotron period, is very small.

\subsection{Equation of Motion and Adiabatic Invariants}

Consider the magnetic field in the $z$ direction and suppose that it is spatially uniform and increases with time within the circular orbit of the particle. From Faraday's law, an electric field is induced along the path of the particle orbit (see Fig. 3), which accelerates the particle, with the result that the orbit is no longer a circle. However, since the time variation 


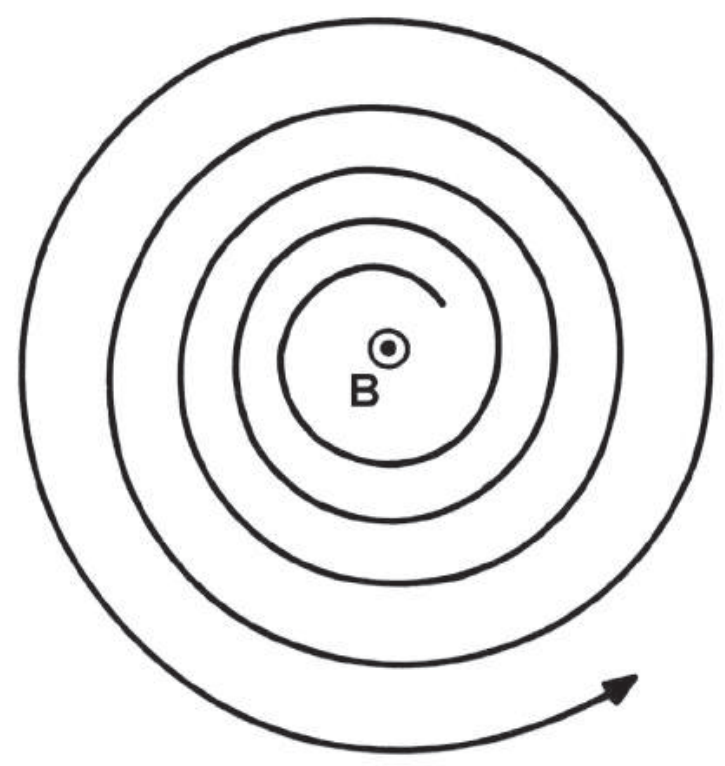

Fig. 2 Outward spiral motion of an electron, in the plane normal to the magnetic field, under cyclotron resonance.

of the $\mathbf{B}$ field is small, the azimuthal component of the electric field $\mathbf{E}$ is also small, and the orbit will be nearly a circle.

Expressing (4.1) in cylindrical coordinates, taking $\mathbf{B}=B \widehat{\mathbf{z}}$, and considering $\mathbf{E}=\widehat{\Theta} E_{\theta}(r)$, we find

$$
\frac{1}{r} \frac{\partial}{\partial r}\left(r E_{\theta}\right)=-\frac{\partial B}{\partial t}
$$

Integrating with respect to $r$, noting that $(\partial B / \partial t)$ can be taken outside the integral sign since B is a slowly varying function, yields

$$
\int_{0}^{r} \frac{\partial}{\partial r^{\prime}}\left(r^{\prime} E_{\theta}\right) d r^{\prime}=-\frac{\partial B}{\partial t} \int_{0}^{r} r^{\prime} d r^{\prime}
$$

from which we obtain the induced electric field as

$$
E_{\theta}=-\frac{1}{2} r \frac{\partial B}{\partial t}
$$

Further, since $\widehat{\mathbf{r}} \times \widehat{\mathbf{z}}=-\widehat{\Theta}$, (4.4) can be written in vector form as

$$
\mathbf{E}_{\theta}=\frac{1}{2} \mathbf{r} \times \frac{\partial \mathbf{B}}{\partial t}
$$




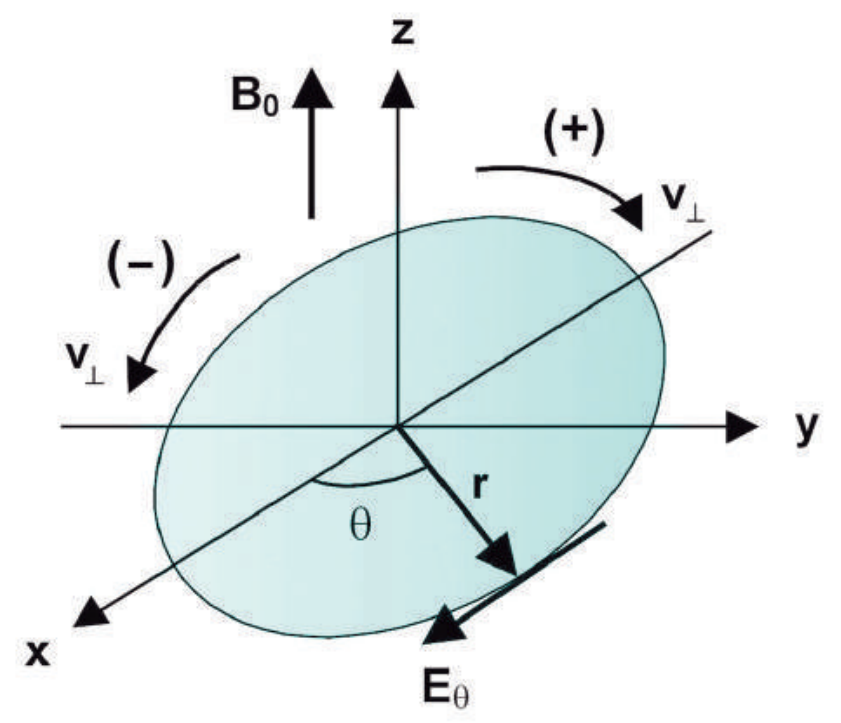

Fig. 3 Azimuthal electric field $\mathbf{E}_{\theta}$ induced by a time-varying magnetic field. The magnetic field inside the orbit is uniform, parallel to the $z$ axis, and increases slowly in time.

Using this result in the Lorentz force equation, we obtain for the equation of motion, after some rearrangement,

$$
\frac{d \mathbf{v}}{d t}=-\frac{1}{2} \mathbf{r} \times \frac{\partial \boldsymbol{\Omega}_{c}}{\partial t}+\boldsymbol{\Omega}_{c} \times \mathbf{v}
$$

Instead of solving (4.6) directly, we shall determine a relation between the radius vector $\mathbf{r}$ and the time rate of change of $\mathbf{B}$, by calculating the change in the particle transverse kinetic energy over one gyration period, which results from the action of the induced electric field. Since the force acting on the particle due to the electric field is given by $q \mathbf{E}_{\theta}$, the increase in the transverse kinetic energy over one gyration period is given by

$$
\delta\left(\frac{1}{2} m v_{\perp}^{2}\right)=q \oint \mathbf{E}_{\theta} \cdot d \mathbf{r}
$$

where $d \mathbf{r}$ denotes an element of path along the particle trajectory, so that $\mathbf{v}_{\perp}=d \mathbf{r} / d t$. Since the field changes very slowly, we can calculate the line integral in (4.7) as if the orbit were closed. Using Stokes's theorem we replace the line integral by a surface integral over the unperturbed orbit,

$$
\delta\left(\frac{1}{2} m v_{\perp}^{2}\right)=q \int_{S}\left(\nabla \times \mathbf{E}_{\theta}\right) \cdot d \mathbf{S}=-q \int_{S} \frac{\partial \mathbf{B}}{\partial t} \cdot d \mathbf{S}
$$


where use was made of (4.1). Here $\mathbf{S}$ denotes the surface enclosed by the cyclotron orbit, its direction being such that $\mathbf{B} \cdot d \mathbf{S}<0$ for ions, and $\mathbf{B} \cdot d \mathbf{S}>0$ for electrons, in view of the diamagnetic character of a plasma. Thus (4.8) becomes

$$
\delta\left(\frac{1}{2} m v_{\perp}^{2}\right)=|q| \frac{\partial B}{\partial t} \pi r_{c}^{2}
$$

Now, the change in the magnetic field over one gyration period $\left(2 \pi / \Omega_{c}\right)$ is

$$
\delta B=\left(\frac{\partial B}{\partial t}\right) \frac{2 \pi}{\Omega_{c}}
$$

and using the relations $r_{c}^{2}=v_{\perp}^{2} / \Omega_{c}^{2}$ and $\Omega_{c}=|q| B / m$, (4.9) can be rewritten as

$$
\delta\left(\frac{1}{2} m v_{\perp}^{2}\right)=\left(\frac{1}{2} m v_{\perp}^{2} / B\right) \delta B=|\mathbf{m}| \delta B
$$

where the quantity $|\mathbf{m}|=\left(\frac{1}{2} m v_{\perp}^{2}\right) / B$ is the orbital magnetic moment of the charged particle. Now, since the left-hand side of $(4.11)$ is $\delta(|\mathbf{m}| B)$, we obtain

$$
\delta|\mathbf{m}|=0
$$

This result shows that the magnetic moment is invariant in slowly varying magnetic fields for which $(\partial B / \partial t)\left(2 \pi / \Omega_{c}\right) \ll B$.

From the constancy of the magnetic moment, we can easily verify that

$$
B \pi r_{c}^{2}=\text { constant }
$$

Therefore, as the magnetic field increases, the radius of gyration decreases, as shown in Fig. 4. Further, since the magnetic flux $\Phi_{m}$ through a Larmor orbit is given by

$$
\Phi_{m}=B S=B \pi r_{c}^{2}
$$

it is clear that the magnetic flux through the particle orbit is also an adiabatic invariant. Hence, as the magnetic field strength increases, the radius of the orbit decreases in such a way that the particle always encircles the same number of magnetic flux lines.

When the time variation of the magnetic field is not spatially uniform within the particle orbit, but if it occurs in an unsymmetrical way, then the induced electric field acting on the particle can considerably modify its orbit from the one shown in Fig. 4. In the most general cases the particle orbit can be extremely complicated. In order to obtain a general idea of the particle orbit, let us consider the simple case of a magnetic field varying in time with cylindrical symmetry over a region of radius $R$, 
which is much larger that the cyclotron radius $r_{c}$, as shown in Fig. 5. The azimuthal component of the induced electric field $\mathbf{E}_{\theta}$, at the point $P$ (see Fig. 5) is given, from (4.5), by

$$
\mathbf{E}_{\theta}=\frac{1}{2} \mathbf{R} \times \frac{\partial \mathbf{B}}{\partial t}
$$

A charged particle located at the point $P$ is now acted upon by crossed electric and magnetic fields, resulting in a drift velocity given by

$$
\mathbf{v}_{E}=\frac{\mathbf{E}_{\theta} \times \mathbf{B}}{B^{2}}=\frac{1}{2}\left(\mathbf{R} \times \frac{\partial \mathbf{B}}{\partial t}\right) \times \frac{\mathbf{B}}{B^{2}}
$$

Since $\mathbf{B}$ is in the $z$ direction (normal to the vector $\mathbf{R}$ ), (4.16) yields

$$
\mathbf{v}_{E}=-\frac{1}{2}\left(\frac{\partial B}{\partial t}\right) \frac{\mathbf{R}}{B}
$$

Therefore, the particle guiding center drifts radially inward with the drift velocity $\mathbf{v}_{E}$ given in (4.17). As the particle drifts radially inward, its radius of gyration decreases in such a way that the flux encircled by the gyrating particle remains constant (see Fig. 5). Since the density of the magnetic flux lines increases as the magnetic field strength increases, this radial particle drift can be pictured as a radially inward motion of the magnetic flux lines at the velocity $\mathbf{v}_{E}$, with the guiding center attached to a given flux line.

\subsection{Magnetic Heating of a Plasma}

The adiabatic invariance of the particle orbital magnetic moment $\left(|\mathbf{m}|=\frac{1}{2} m v_{\perp}^{2} / B=\right.$ constant $)$ implies that when the magnetic field increases, the particle transverse kinetic energy $\left(W_{\perp}=\frac{1}{2} m v_{\perp}^{2}\right)$ increases linearly with B. Further, since the magnetic flux encircled by the gyrating particle is also constant, as the magnetic flux density increases, the magnetic flux tube contracts and the particle guiding center moves radially inward, accompanying the radial displacement of the magnetic field lines, as if it were frozen in the field lines. Consequently, the increase in the magnetic flux density causes the charged particles in a plasma to approach each other, resulting in a magnetic compression. In the present case of two-dimensional compression, since the increase in the number density $(n)$ of the particles is proportional to the cross-sectional area $\pi r_{c}^{2}$, 


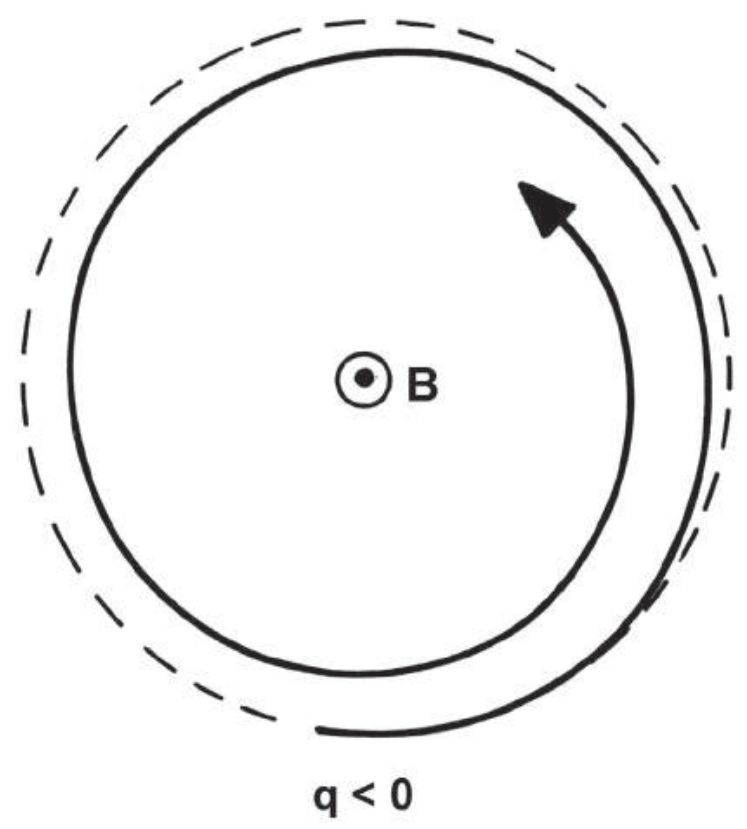

Fig. 4 Motion of an electron in a time-varying magnetic field. The field is spatially uniform and increases in time.

it follows that under magnetic compression in two dimensions $n$ increases linearly with $B$. Similarly, when $B$ decreases, $n$ also decreases, resulting in a magnetic decompression. Thus, for two-dimensional compression we have $W_{\perp} \propto B \propto n$.

This property is used as a method of plasma heating, known as magnetic pumping, which consists in periodic magnetic compressions and decompressions of the plasma. The compression and the decompression must take place in a time interval very large compared to the Larmor period but, at the same time, very small compared to the relaxation time necessary for the achievement of thermal equilibrium. For the present case of two-dimensional compression-decompression, obtained by varying the axial magnetic field, only the velocity and the energy in the two directions normal to the magnetic field are changed.

In order to be able to heat the plasma in a compression-decompression cycle, it is necessary to transfer part of the energy increase in $W_{\perp}$, obtained by compression, to the energy $W_{\|}$, which is unaffected by decompression. This energy transfer is brought about by particle collisions, therefore increasing the particle thermal kinetic energy at the expense of 


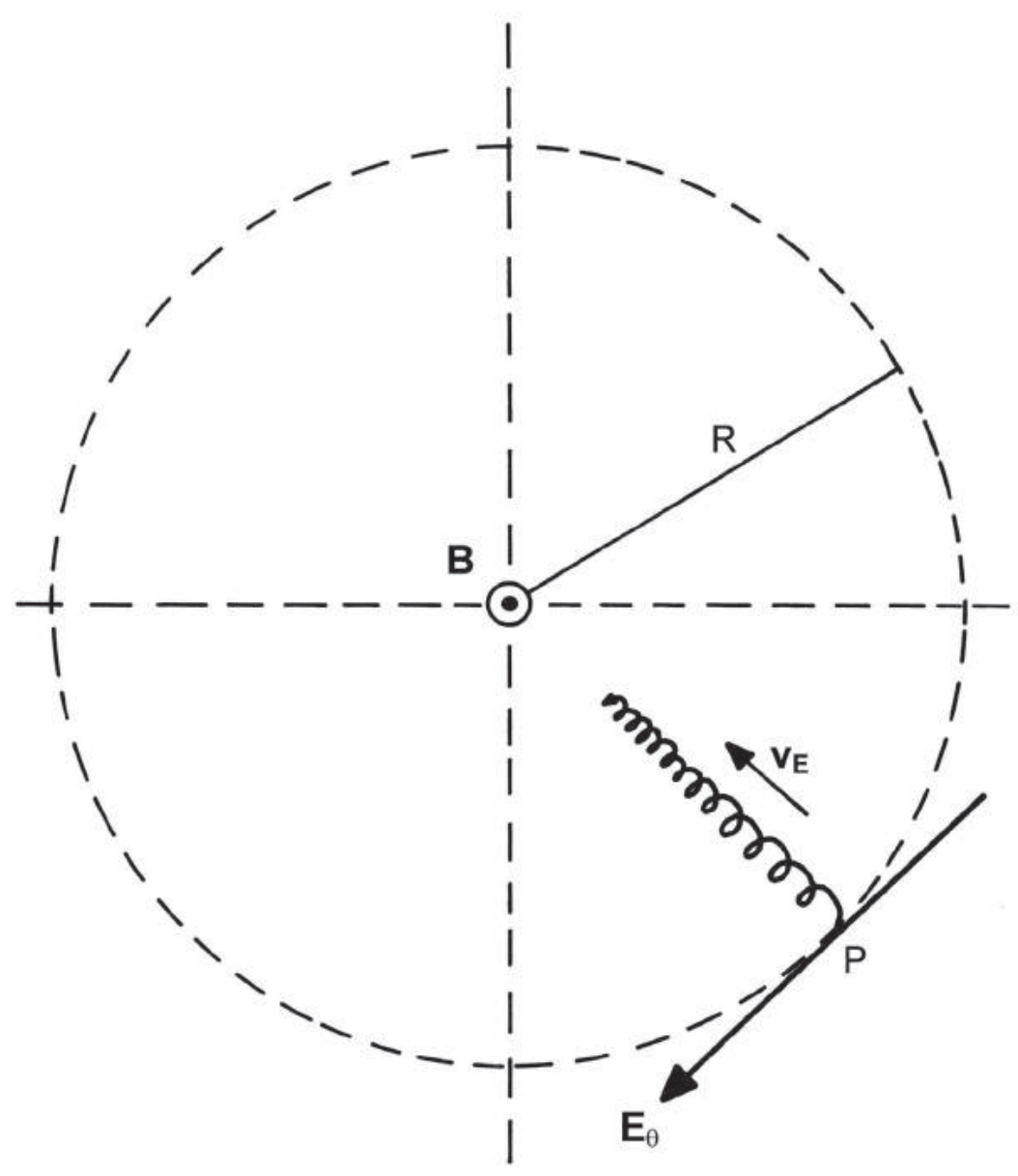

Fig. 5 Motion of a negatively charged particle in an increasing magnetic field (directed out of the page) with cylindrical symmetry over a region of radius $R$ much greater than the Larmor radius $r_{c}$.

the electric field energy. Thus, in a complete cycle consisting of a compression, a relaxation time, and a decompression, part of the energy increase in $W_{\perp}$ achieved during compression is transferred to the degree of freedom parallel to $B$, as a result of collisions, causing an increase in $W_{\|}$, which is not affected during the decompression, whereas $W_{\perp}$ is decreased correspondingly. Therefore, by a periodic repetition of these cycles of adiabatic compression-decompression, the plasma thermal energy and therefore the temperature are increased. 


\section{SUMMARY OF GUIDING CENTER DRIFTS AND CURRENT DENSITIES}

\subsection{Guiding Center Drifts}

Electric field:

$$
\mathbf{v}_{E}=\frac{\mathbf{E} \times \mathbf{B}}{B^{2}}
$$

Gravitational field:

$$
\mathbf{v}_{g}=\frac{m \mathbf{g} \times \mathbf{B}}{q B^{2}}
$$

General force:

$$
\mathbf{v}_{F}=\frac{\mathbf{F} \times \mathbf{B}}{q B^{2}}
$$

Gradient of B:

$$
\mathbf{v}_{G}=-\frac{|\mathbf{m}|(\nabla B) \times \mathbf{B}}{q B^{2}}
$$

Curvature of B:

$$
\mathbf{v}_{C}=-\frac{m v_{\|}^{2}[(\mathbf{B} \cdot \nabla) \mathbf{B}] \times \mathbf{B}}{q B^{4}}
$$

Gradient-curvature of $\mathbf{B}$ (in vacuum field):

$$
\mathbf{v}_{G C}=-\frac{m\left(v_{\|}^{2}+\frac{1}{2} v_{\perp}^{2}\right)\left(\frac{1}{2} \nabla B^{2}\right) \times \mathbf{B}}{q B^{4}}
$$

Polarization:

$$
\mathbf{v}_{p}=\frac{m\left(\partial \mathbf{E}_{\perp} / \partial t\right)}{q B^{2}}
$$

\subsection{Current Densities}

Magnetization:

$$
\mathbf{J}_{M}=\nabla \times \mathbf{M}
$$

Gravitational:

$$
\mathbf{J}_{g}=\frac{\rho_{m} \mathbf{g} \times \mathbf{B}}{B^{2}}
$$

* The first number in these equation numbers is the chapter number. 
Gradient of B:

$$
\mathbf{J}_{G}=-\left(\frac{1}{\delta V} \sum_{i}\left|\mathbf{m}_{i}\right|\right) \frac{(\nabla B) \times \mathbf{B}}{B^{2}}
$$

Curvature of $\mathbf{B}$ :

$$
\mathbf{J}_{C}=-2\left(\frac{1}{\delta V} \sum_{i} W_{i \|}\right) \frac{[(\mathbf{B} \cdot \nabla) \mathbf{B}] \times \mathbf{B}}{B^{4}}
$$

Polarization:

$$
\mathbf{J}_{p}=\frac{\rho_{m}\left(\partial \mathbf{E}_{\perp} / \partial t\right)}{B^{2}}
$$

\section{PROBLEMS}

4.1 With reference to a magnetic field pointing along the $z$ axis $(\mathbf{B}=$ $\left.B_{0} \widehat{\mathbf{z}}\right)$, describe the type of polarization of the following electric field:

$$
\mathbf{E}=E_{0}(\widehat{\mathbf{x}} \cos \omega t-\widehat{\mathbf{y}} \sin \omega t)
$$

Make a drawing that shows the orientation of $\mathbf{E}$ for the instants $t=0$, $t=\pi /(2 \omega)$, and $t=\pi / \omega$. How can you represent this electric field in complex notation?

4.2 Describe, in a semiquantitative way, the motion of an electron in the presence of a constant magnetic field $\mathbf{B}=B_{0} \widehat{\mathbf{z}}$ and a time-varying electric field given by

$$
\mathbf{E}=\frac{1}{2} E_{0}(\widehat{\mathbf{x}}+i \widehat{\mathbf{y}}) e^{-i \Omega_{c} t}
$$

where $E_{0}$ and $B_{0}$ are positive constants and $\Omega_{c}=e B_{0} / m_{e}$. What type of polarization has this electric field?

4.3 Solve the equation of motion to determine the transient response of a charged particle in the presence of a spatially uniform $\mathrm{AC}$ electric field $\mathbf{E}(t)=\widehat{\mathbf{x}} E \sin (\omega t)$, which is switched on at $t=0$. Assume that initially, at $t=0$, the particle is at rest at the origin. Make a plot of the particle trajectory and velocity as a function of time.

4.4 Consider an electron acted upon by a constant and uniform magnetic field $\mathbf{B}=B_{0} \widehat{\mathbf{z}}$, and a uniform but time-varying electric field $\mathbf{E}=$ 
$\widehat{\mathbf{y}} E_{y 0} \sin (\omega t)$. Assume that the initial conditions are such that the motion takes place in the $(x, y)$ plane and that at $t=0$ the electron is at rest $\left(\mathbf{v}_{0}=0\right)$ at the origin.

(a) Show that the orbit of the electron is given by

$$
\begin{gathered}
x(t)=-\frac{e E_{y 0}}{m\left(\omega^{2}-\Omega_{c}^{2}\right)}\left[\frac{\omega}{\Omega_{c}}\left(\cos \Omega_{c} t-1\right)-\frac{\Omega_{c}}{\omega}(\cos \omega t-1)\right] \\
y(t)=-\frac{e E_{y 0}}{m\left(\omega^{2}-\Omega_{c}^{2}\right)}\left(\frac{\omega}{\Omega_{c}} \sin \Omega_{c} t-\sin \omega t\right)
\end{gathered}
$$

(b) In the low-frequency limit, $\omega \ll \Omega_{c}$, show that the electron orbits at the angular frequency $\omega$ around an ellipse that has its major axis perpendicular to the electric field. Determine the ratio of the minor to the major axis of the ellipse.

(c) In the high-frequency limit, $\omega \gg \Omega_{c}$, show that the electron moves in a circle at the cyclotron frequency $\Omega_{c}$.

4.5 Integrate (3.49) and (3.50) to determine the particle trajectory in the plane normal to $\mathbf{B}$ and sketch the path of the particle for $q>0$ and $q<0$.

4.6 Consider the motion of an electron in the presence of a uniform magnetostatic field $\mathbf{B}=B_{0} \widehat{\mathbf{z}}$, and an electric field that oscillates in time at the electron cyclotron frequency $\Omega_{c}$, according to

$$
\mathbf{E}(t)=E_{0}\left(\widehat{\mathbf{x}} \cos \Omega_{c} t+\widehat{\mathbf{y}} \sin \Omega_{c} t\right)
$$

(a) What type of polarization has this electric field?

(b) Obtain the following uncoupled differential equations satisfied by the velocity components $v_{x}(t)$ and $v_{y}(t)$ :

$$
\begin{gathered}
\frac{d^{2} v_{x}}{d t^{2}}+\Omega_{c}^{2} v_{x}=2 \frac{e E_{0}}{m} \Omega_{c} \sin \Omega_{c} t \\
\frac{d^{2} v_{y}}{d t^{2}}+\Omega_{c}^{2} v_{y}=-2 \frac{e E_{0}}{m} \Omega_{c} \cos \Omega_{c} t
\end{gathered}
$$

(c) Assume that, at $t=0$, the electron is located at the origin of the coordinate system, with zero velocity. Neglect the time-varying part of $\mathbf{B}$. Show that the electron velocity is given by

$$
v_{x}(t)=-\frac{e E_{0}}{m} t \cos \Omega_{c} t
$$




$$
v_{y}(t)=-\frac{e E_{0}}{m} t \sin \Omega_{c} t
$$

(d) Show that the electron trajectory is given by

$$
\begin{gathered}
x(t)=-\frac{e E_{0}}{m}\left(\frac{1}{\Omega_{c}^{2}} \cos \Omega_{c} t+\frac{t}{\Omega_{c}} \sin \Omega_{c} t-\frac{1}{\Omega_{c}^{2}}\right) \\
y(t)=-\frac{e E_{0}}{m}\left(\frac{1}{\Omega_{c}^{2}} \sin \Omega_{c} t-\frac{t}{\Omega_{c}} \cos \Omega_{c} t\right)
\end{gathered}
$$

4.7 Solve the equation of motion to determine the velocity and the trajectory of an electron in the presence of a uniform magnetostatic field $\mathbf{B}=B_{0} \widehat{\mathbf{z}}$, and an oscillating electric field given by

$$
\mathbf{E}(t)=\widehat{\mathbf{x}} E_{x} \sin \omega t+\widehat{\mathbf{z}} E_{z} \cos \omega t
$$

Consider the same assumptions and initial conditions as in the previous problem.

4.8 Consider the motion of an electron in a spatially uniform magnetic field $\mathbf{B}=B_{z} \widehat{\mathbf{z}}$, such that $B_{z}$ has a slow time variation given by

$$
B_{z}(t)=B_{0}(1-\alpha t)
$$

where $B_{0}$ and $\alpha$ are positive constants, and $|\alpha t| \ll 1$. Assume the following initial conditions: $\mathbf{r}(0)=\left(r_{c}, 0,0\right)$ and $\mathbf{v}(0)=\left(0, v_{\perp 0}, 0\right)$, where $r_{c}$ is the Larmor radius, $v_{\perp 0}=\Omega_{c} r_{c}$ and $\Omega_{c}=|q| B_{0} / \mathrm{m}$.

(a) Write the equation of motion, considering the Lorentz force, and solve it by a perturbation technique including only terms up to the first order in the small parameter $\alpha$. Show that the particle velocity is given by

$$
\begin{aligned}
& v_{x}(t)=-\Omega_{c} r_{c} \sin \Omega_{c} t+\frac{1}{2} \alpha \Omega_{c} r_{c} t\left(\sin \Omega_{c} t+\Omega_{c} t \cos \Omega_{c} t\right) \\
& v_{y}(t)=\Omega_{c} r_{c} \cos \Omega_{c} t+\frac{1}{2} \alpha \Omega_{c} r_{c} t\left(-\cos \Omega_{c} t+\Omega_{c} t \sin \Omega_{c} t\right)
\end{aligned}
$$

(b) Show that the particle orbit is given by

$$
\begin{gathered}
x(t)=r_{c}\left(1+\frac{1}{2} \alpha t\right) \cos \Omega_{c} t+\frac{r_{c} \alpha}{2 \Omega_{c}}\left(\Omega_{c}^{2} t^{2}-1\right) \sin \Omega_{c} t \\
y(t)=r_{c}\left(1+\frac{1}{2} \alpha t\right) \sin \Omega_{c} t-\frac{r_{c} \alpha}{2 \Omega_{c}}\left(\Omega_{c}^{2} t^{2}-1\right) \cos \Omega_{c} t-\frac{r_{c} \alpha}{2 \Omega_{c}} \\
z(t)=v_{z 0} t
\end{gathered}
$$


(c) Determine the orbital magnetic moment and verify its adiabatic invariance, retaining only terms up to the first order in $\alpha$.

4.9 Consider the motion of a charged particle in a spatially uniform magnetic field that varies slowly in time as compared to the particle cyclotron period.

(a) Show that the equation of motion can be written in vector form as

$$
\frac{d \mathbf{v}(t)}{d t}=\boldsymbol{\Omega}_{c}(t) \times \mathbf{v}(t)-\frac{1}{2} \mathbf{r}(t) \times \frac{\partial \boldsymbol{\Omega}_{c}(t)}{\partial t}
$$

where $\boldsymbol{\Omega}_{c}(t)=-q \mathbf{B}(t) / m$.

(b) Considering that $\mathbf{B}(t)=\widehat{\mathbf{z}} B_{0} f(t)$, where $B_{0}$ is constant, obtain the following equations for the motion of the particle in the plane normal to B:

$$
\begin{aligned}
& \frac{d^{2} x(t)}{d t^{2}}-\Omega_{c}\left[f(t) \frac{d y(t)}{d t}+\frac{1}{2} y(t) \frac{d f(t)}{d t}\right]=0 \\
& \frac{d^{2} y(t)}{d t^{2}}+\Omega_{c}\left[f(t) \frac{d x(t)}{d t}+\frac{1}{2} x(t) \frac{d f(t)}{d t}\right]=0
\end{aligned}
$$

where $\Omega_{c}=|q| B / m$.

(c) Define a complex variable $u(t)=x(t)+i y(t)$ and a function $\xi(t)$ by

$$
\xi(t)=u(t) \exp \left[\frac{1}{2} i \Omega_{c} \int_{0}^{t} f\left(t^{\prime}\right) d t^{\prime}\right]
$$

and show that the equation satisfied by $\xi(t)$ is

$$
\frac{d^{2} \xi(t)}{d t^{2}}+\frac{\Omega_{c}^{2}}{4} f^{2}(t) \xi(t)=0
$$

(d) If $\xi_{1}(t)$ and $\xi_{2}(t)$ are two linearly independent solutions of this equation, subject to the initial conditions

$$
\begin{array}{ll}
\xi_{1}(0)=0 & \left(d \xi_{1}(t) / d t\right)_{t=0}=1 \\
\xi_{2}(0)=1 & \left(d \xi_{2}(t) / d t\right)_{t=0}=0
\end{array}
$$

show that the solution for $u(t)$ can be written as

$$
u(t)=\left\{u_{0} \xi_{2}(t)+\xi_{1}(t)\left[d u_{0} / d t+\frac{1}{2} i \Omega_{c} f(0) u_{0}\right]\right\} \exp \left[-\frac{1}{2} i \Omega_{c} \int_{0}^{t} f\left(t^{\prime}\right) d t^{\prime}\right]
$$


where $u_{0}$ and $d u_{0} / d t$ represent the initial position and velocity, respectively.

(e) Considering now that the particle is initially $(t=0)$ at the origin and moving with velocity $v_{0}$ along the negative $y$ axis, that is, $u_{0}=0$ and $d u_{0} / d t=-i v_{0}$, show that

$$
u(t)=-i v_{0} \xi_{1}(t) \exp \left[-\frac{1}{2} i \Omega_{c} \int_{0}^{t} f\left(t^{\prime}\right) d t^{\prime}\right]
$$

and, consequently,

$$
\begin{gathered}
x(t)=v_{0} \xi_{1}(t) \sin \left[\frac{1}{2} \Omega_{c} \int_{0}^{t} f\left(t^{\prime}\right) d t^{\prime}\right] \\
y(t)=-v_{0} \xi_{1}(t) \cos \left[\frac{1}{2} \Omega_{c} \int_{0}^{t} f\left(t^{\prime}\right) d t^{\prime}\right]
\end{gathered}
$$

4.10 (a) Assume that $f(t)$, in problem 4.9, is given by exp $(-\alpha t)$. Show that, in this case, $\xi(t)$ satisfies the Bessel equation of zero order,

$$
\frac{d^{2} \xi(\tau)}{d \tau^{2}}+\frac{1}{\tau} \frac{d \xi(\tau)}{d \tau}+\xi(\tau)=0
$$

where $\tau=\left(\Omega_{c} / 2 \alpha\right)$ exp $(-\alpha t)$. Determine the two solutions of this equation which satisfy the initial conditions stated in problem 4.9 and interpret them physically.

(b) Considering now that $f(t)=(1-\alpha t)$, solve the equation for $\xi(t)$ in problem 4.9 in a power series in $\alpha$, and determine the particle trajectory to order $\alpha$. Show that the ratio $\left(v_{x}^{2}+v_{y}^{2}\right) / B(t)$ has no terms of order $\alpha$, thus verifying the adiabatic invariance of the magnetic moment. Compare these results with those of problem 4.8.

4.11 For an electron with initial velocity $v_{0} \widehat{\mathbf{x}}$ and initial position $x_{0} \widehat{\mathbf{x}}$, acted upon by an electric field $\mathbf{E}=\widehat{\mathbf{x}} E \cos (k x-\omega t)$, show that its velocity is given by

$$
v(t)=v_{0}-\frac{e E}{m_{e}} \int_{0}^{t} \cos \left(k x-\omega t^{\prime}\right) d t^{\prime}
$$

Using a perturbation approach, in which to lowest order $\mathbf{E}=0$, show that

$$
v(t)=v_{0}-\frac{e E}{m_{e}\left(k v_{0}-\omega\right)}\left\{\sin \left[k x_{0}+\left(k v_{0}-\omega\right) t\right]-\sin \left(k x_{0}\right)\right\}
$$


Notice that the velocity perturbation will be large only when $v_{0}$ is close to the phase velocity $\omega / k$.

4.12 Using the Maxwell equation (1.5.3) (Eq. 5.3 in Chapter 1) and the equation (3.34) which defines the plasma conductivity dyad $\mathcal{S}$, and considering the time variation indicated in (3.1), show that

$$
\nabla \times \mathbf{B}=-i \omega \mu_{0} \mathcal{E} \cdot \mathbf{E}
$$

where $\mathcal{E}$ is the plasma electric permittivity dyad given by

$$
\mathcal{E}=\epsilon_{0}\left(\mathbf{1}+\frac{i}{\omega \epsilon_{0}} \mathcal{S}\right)
$$

where 1 denotes the unit dyad, which in Cartesian coordinates can be written as

$$
\mathbf{1}=\widehat{\mathbf{x}} \widehat{\mathbf{x}}+\widehat{\mathbf{y}} \widehat{\mathbf{y}}+\widehat{\mathbf{z}} \widehat{\mathbf{z}}
$$




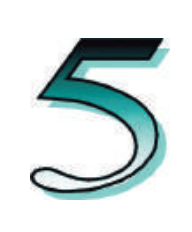

\section{ELEMENTS OF}

\section{PLASMA KINETIC THEORY}

\section{INTRODUCTION}

A plasma is a system containing a very large number of interacting charged particles, so that for its analysis it is appropriate and convenient to use a statistical approach. In this chapter we present the basic elements of kinetic theory, introducing the concepts of phase space and distribution function, which are necessary for a statistical description.

All physically interesting information about the system is contained in the distribution function. From knowledge of the distribution function the macroscopic variables of physical interest, necessary for a macroscopic description of the plasma behavior, can be systematically deduced. These macroscopic variables are functions of position and time and are directly related to the average values of the various particle physical quantities of interest, which can be considered as functions of the particle velocities, with the distribution function used as a weighting function in phase space.

The differential kinetic equation satisfied by the distribution function, generally known as the Boltzmann equation, is deduced in section 5. At this point, the effects due to particle collisions are incorporated into this kinetic equation only through a general, unspecified collision term. In Chapter 21 we shall deduce explicit expressions for the collision term, in particular for the Boltzmann collision integral and for the Fokker-Planck collision term. Only a simple approximate expression for the collision term is presented at this point, the so-called relaxation model or Krook collision term. The Vlasov equation for a plasma is introduced in the last section. 


\section{PHASE SPACE}

At any instant of time each particle in the plasma can be localized by a position vector $\mathbf{r}$ drawn from the origin of a coordinate system to the center of mass of the particle. In a Cartesian frame of reference, as illustrated in Fig. 1, we have

$$
\mathbf{r}=x \widehat{\mathbf{x}}+y \widehat{\mathbf{y}}+z \widehat{\mathbf{z}}
$$

where $\widehat{\mathbf{x}}, \widehat{\mathbf{y}}$, and $\widehat{\mathbf{z}}$ denote unit vectors along the axes $x, y$, and $z$, respectively. The linear velocity of the center of mass of the particle can be represented by the vector

$$
\mathbf{v}=v_{x} \widehat{\mathbf{x}}+v_{y} \widehat{\mathbf{y}}+v_{z} \widehat{\mathbf{z}}
$$

with $v_{x}=d x / d t, v_{y}=d y / d t$, and $v_{z}=d z / d t$.

In analogy with the configuration space defined by the position coordinates $(x, y, z)$, it is convenient to introduce the velocity space defined by the velocity coordinates $\left(v_{x}, v_{y}, v_{z}\right)$. In this space the velocity vector $\mathbf{v}$ can be viewed as a position vector drawn from the origin of the coordinate system $\left(v_{x}, v_{y}, v_{z}\right)$ to the center of mass of the particle, as indicated schematically in Fig. 1.

\subsection{Single-Particle Phase Space}

From the point of view of classical mechanics the instantaneous $d y$ namic state of each particle can be specified by its position and velocity vectors. It is convenient, therefore, to consider the phase space defined by the six coordinates $\left(x, y, z, v_{x}, v_{y}, v_{z}\right)$.

In this six-dimensional space the dynamic state of each particle is appropriately represented by a single point. The coordinates $(\mathbf{r}, \mathbf{v})$ of the representative point give the position and velocity of the particle. When the particle moves, its representative point describes a trajectory in phase space. At each instant of time the dynamic state of a system of $N$ particles is represented by $N$ points in phase space.

\subsection{Many-Particle Phase Space}

The phase space just defined, often called $\mu$-space, is the phase space for a single particle, in contrast with the many-particle phase space or 


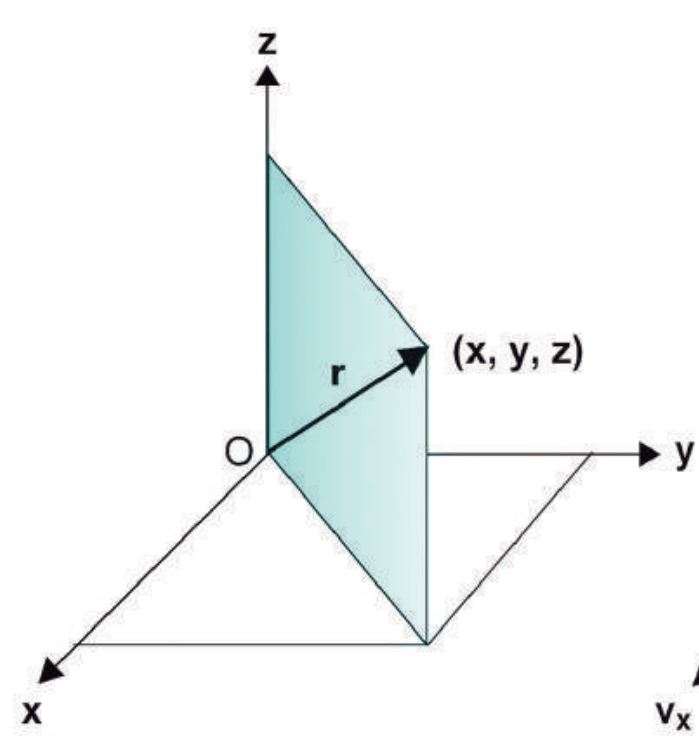

(a)

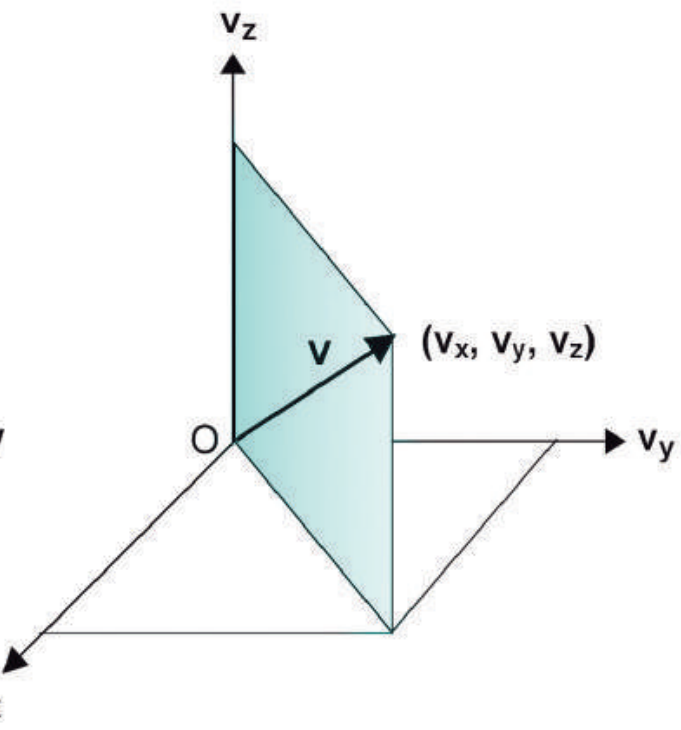

(b)

Fig. 1 Position vectors (a) in configuration space and (b) in velocity space.

$\Gamma$-space for the whole system of particles. In the latter, a system consisting of $N$ particles, with no internal degrees of freedom, is represented by a single point in a $6 \mathrm{~N}$-dimensional space defined by the $3 \mathrm{~N}$ position coordinates $\left(\mathbf{r}_{1}, \mathbf{r}_{2}, \ldots, \mathbf{r}_{N}\right)$ and the $3 N$ velocity coordinates $\left(\mathbf{v}_{1}, \mathbf{v}_{2}, \ldots, \mathbf{v}_{N}\right)$. Thus, a point in $\Gamma$-space corresponds to a single microscopic state for the whole system of particles. This many-particle phase space is often used in statistical mechanics and advanced kinetic theory. The single-particle phase space is the one normally used in elementary kinetic theory and basic plasma physics, and is the space that will be considered in what follows.

\subsection{Volume Elements}

A small element of volume in configuration space is represented by $d^{3} r=d x d y d z$. This differential element of volume should not be taken literally as a mathematically infinitesimal quantity but as a finite element of volume, sufficiently large to contain a very large number of particles, yet sufficiently small in comparison with the characteristic lengths associated with the spatial variation of physical parameters of interest such as, for example, density and temperature. In a gas containing $10^{18}$ molecules $/ \mathrm{m}^{3}$, 


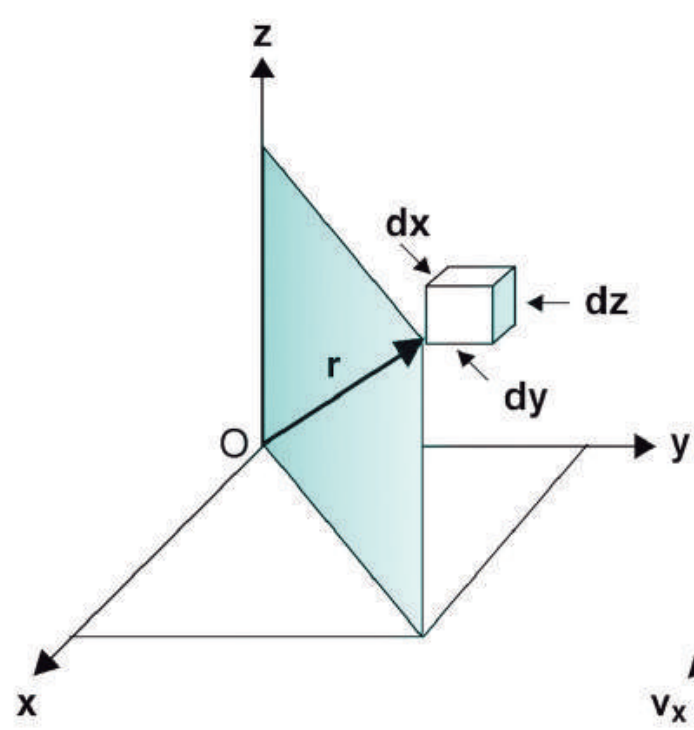

(a)

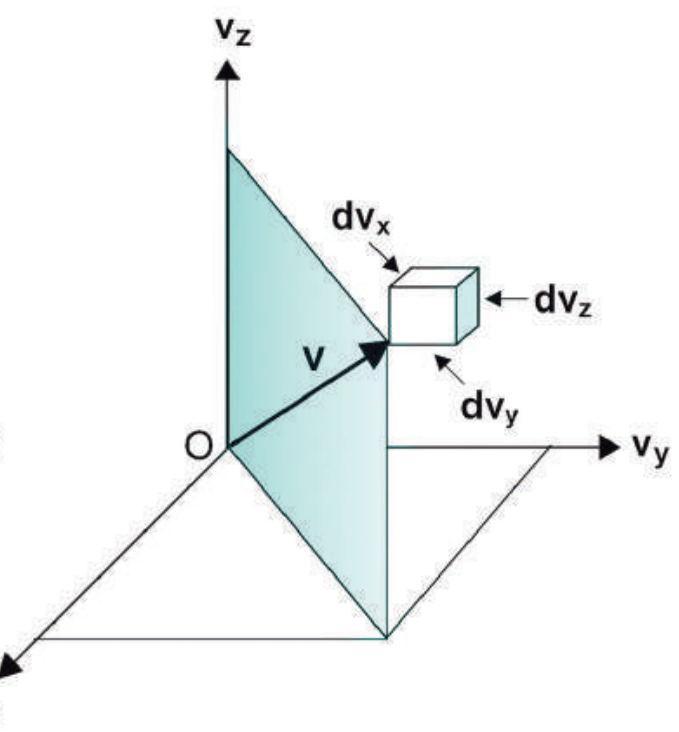

(b)

Fig. 2 (a) The element of volume $d^{3} r=d x d y d z$ around the terminal point of $\mathbf{r}$, in configuration space, and (b) the element of volume $d^{3} v=d v_{x} d v_{y} d v_{z}$, in velocity space, around the terminal point of $\mathbf{v}$.

for example, if we take $d^{3} r=10^{-12} \mathrm{~m}^{3}$, which in a macroscopic scale can be considered as a point, there are still $10^{6}$ molecules inside $d^{3} r$. Plasmas that do not allow a choice of differential volume elements as indicated, cannot be analyzed statistically.

When we refer to a particle as being situated inside $d^{3} r$, at $\mathbf{r}$, it is meant that the $x$ coordinate of the particle lies between $x$ and $x+d x$, the $y$ coordinate lies between $y$ and $y+d y$, and the $z$ coordinate lies between $z$ and $z+d z$, that is, inside the volume element $d x d y d z$ situated around the terminal point of the position vector $\mathbf{r}=x \widehat{\mathbf{x}}+y \widehat{\mathbf{y}}+z \widehat{\mathbf{z}}$. It is important to note that the particles localized inside $d^{3} r$, at $\mathbf{r}$, may have completely arbitrary velocities that would be represented by scattered points in velocity space.

A small element of volume in velocity space is represented by $d^{3} v=$ $d v_{x} d v_{y} d v_{z}$. For a particle to be included in $d^{3} v$, around the terminal point of the velocity vector $\mathbf{v}$, its $v_{x}$ velocity component must lie between $v_{x}$ and $v_{x}+d v_{x}$, the $v_{y}$ component between $v_{y}$ and $v_{y}+d v_{y}$, and the $v_{z}$ component between $v_{z}$ and $v_{z}+d v_{z}$. The differential elements of volume $d^{3} r$ and $d^{3} v$ are schematically represented in Fig. 2. 


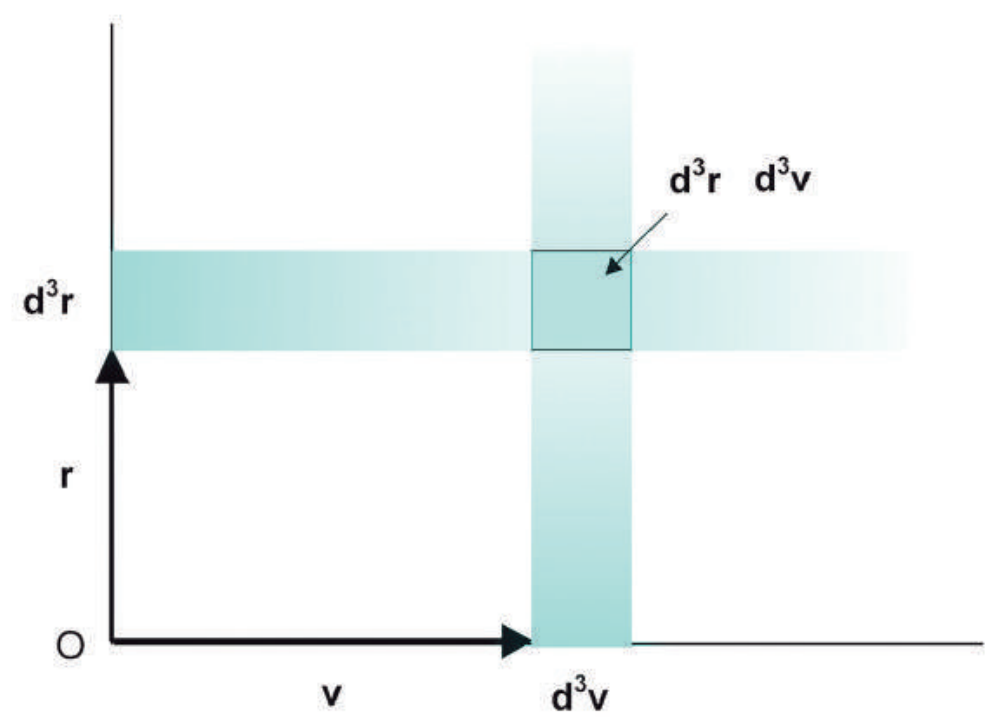

Fig. 3 Schematic representation of the element of volume $d^{3} r d^{3} v$ in the six-dimensional phase space, around the representative point $(\mathbf{r}, \mathbf{v})$.

In phase space ( $\mu$-space) a differential element of volume may be imagined as a six-dimensional cube, represented by

$$
d^{3} r d^{3} v=d x d y d z d v_{x} d v_{y} d v_{z}
$$

as shown schematically in Fig. 3. Note that inside $d^{3} r d^{3} v$, at the position $(\mathbf{r}, \mathbf{v})$ in phase space, there are only the particles inside $d^{3} r$ around $\mathbf{r}$ whose velocities lie inside $d^{3} v$ about $\mathbf{v}$. The number of representative points inside the volume element $d^{3} r d^{3} v$ is, in general, a function of time and of the position of this element in phase space. It is important to note that the coordinates $\mathbf{r}$ and $\mathbf{v}$ of phase space are considered to be independent variables, since they represent the position of individual volume elements (containing many particles) in phase space.

\section{DISTRIBUTION FUNCTION}

Let $d^{6} \mathcal{N}_{\alpha}(\mathbf{r}, \mathbf{v}, t)$ denote the number of particles of type $\alpha$ inside the volume element $d^{3} r d^{3} v$ around the phase space coordinates $(\mathbf{r}, \mathbf{v})$, at the instant $t$. The distribution function in phase space, $f_{\alpha}(\mathbf{r}, \mathbf{v}, t)$, is defined 
as the density of representative points of the type $\alpha$ particles in phase space, that is,

$$
f_{\alpha}(\mathbf{r}, \mathbf{v}, t)=\frac{d^{6} \mathcal{N}_{\alpha}(\mathbf{r}, \mathbf{v}, t)}{d^{3} r d^{3} v}
$$

It is assumed that the density of representative points in phase space does not vary rapidly from one element of volume to the neighboring element, so that $f_{\alpha}(\mathbf{r}, \mathbf{v}, t)$ can be considered as a continuous function of its arguments. According to its definition $f_{\alpha}(\mathbf{r}, \mathbf{v}, t)$ is also a positive and finite function at any instant of time. In a volume element $d^{3} r d^{3} v$, whose velocity coordinates $\left(v_{x}, v_{y}, v_{z}\right)$ are very large, the number of representative points is relatively small since, in any macroscopic system, there must be relatively few particles with very large velocities. Physical considerations require therefore that $f_{\alpha}(\mathbf{r}, \mathbf{v}, t)$ must tend to zero as the velocity becomes infinitely large.

The distribution function is, in general, a function of the position vector $\mathbf{r}$. When this is the case the corresponding plasma is said to be inhomogeneous. In the absence of external forces, however, a plasma initially inhomogeneous reaches, in the course of time, an equilibrium state as a result of the mutual particle interactions. In this homogeneous state the distribution function does not depend on $\mathbf{r}$.

In velocity space the distribution function can be anisotropic, when it depends on the orientation of the velocity vector $\mathbf{v}$, or isotropic, when it does not depend on the orientation of $\mathbf{v}$ but only on its magnitude, i.e., on the particle speed $v=|\mathbf{v}|$.

The statistical description of different types of plasmas requires the use of inhomogeneous or homogeneous, as well as anisotropic or isotropic distribution functions. A plasma in thermal equilibrium, for example, is characterized by a homogeneous, isotropic, and time-independent distribution function.

In a statistical sense the distribution function provides a complete description of the system under consideration. Knowing $f_{\alpha}(\mathbf{r}, \mathbf{v}, t)$ we can deduce all the macroscopic variables of physical interest for the type $\alpha$ species. One of the primary problems of kinetic theory consists in determining the distribution function for a given system. The differential equation that governs the temporal and spatial variation of the distribution function under given conditions, known generally as the Boltzmann equation, will be derived in section 5 . 


\section{NUMBER DENSITY AND AVERAGE VELOCITY}

The number density, $n_{\alpha}(\mathbf{r}, t)$, is a macroscopic variable defined in configuration space as the number of particles of type $\alpha$, per unit volume, irrespective of velocity. It can be obtained by integrating $d^{6} \mathcal{N}_{\alpha}(\mathbf{r}, \mathbf{v}, t)$ over all of velocity space and dividing the result by the volume element $d^{3} r$ of configuration space,

$$
n_{\alpha}(\mathbf{r}, t)=\frac{1}{d^{3} r} \int_{v} d^{6} \mathcal{N}_{\alpha}(\mathbf{r}, \mathbf{v}, t)
$$

or, using the definition (3.1),

$$
n_{\alpha}(\mathbf{r}, t)=\int_{v} f_{\alpha}(\mathbf{r}, \mathbf{v}, t) d^{3} v
$$

The single integral sign indicated here represents in fact a triple integral extending over all velocity space, that is, over each one of the variables $v_{x}, v_{y}$, and $v_{z}$ from $-\infty$ to $+\infty$. For convenience and simplification of notation only a single integral sign will be indicated, being implicit the fact that the integral extends over all velocity space.

The average velocity $\mathbf{u}_{\alpha}(\mathbf{r}, t)$ is defined as the macroscopic flow velocity of the particles of type $\alpha$ in the neighborhood of the position vector $\mathbf{r}$ at the instant $t$. In order to relate $\mathbf{u}_{\alpha}(\mathbf{r}, t)$ to the distribution function, consider the particles of type $\alpha$ contained in the volume element $d^{3} r d^{3} v$ about $(\mathbf{r}, \mathbf{v})$ at the instant $t$, which we have denoted by $d^{6} \mathcal{N}_{\alpha}(\mathbf{r}, \mathbf{v}, t)$. The average velocity of the particles of type $\alpha$ can be obtained as follows. First we multiply $d^{6} \mathcal{N}_{\alpha}(\mathbf{r}, \mathbf{v}, t)$ by the particle velocity $\mathbf{v}$, next we integrate over all possible velocities, and finally we divide the result by the total number of type $\alpha$ particles contained in $d^{3} r$, irrespective of velocity. Therefore,

$$
\mathbf{u}_{\alpha}(\mathbf{r}, t)=\frac{1}{n_{\alpha}(\mathbf{r}, t) d^{3} r} \int_{v} \mathbf{v} d^{6} \mathcal{N}_{\alpha}(\mathbf{r}, \mathbf{v}, t)
$$

The procedure just described is the usual statistical definition of average values. Using the definition of $f_{\alpha}(\mathbf{r}, \mathbf{v}, t)$, given in (3.1), we obtain

$$
\mathbf{u}_{\alpha}(\mathbf{r}, t)=\frac{1}{n_{\alpha}(\mathbf{r}, t)} \int_{v} \mathbf{v} f_{\alpha}(\mathbf{r}, \mathbf{v}, t) d^{3} v
$$

Note that both $n_{\alpha}(\mathbf{r}, t)$ and $\mathbf{u}_{\alpha}(\mathbf{r}, t)$ are macroscopic variables that depend only upon the coordinates $\mathbf{r}$ and $t$. 
A systematic method for deducing the macroscopic variables (such as momentum flux, pressure, temperature, heat flux, and so on), in terms of the distribution function, is formally presented in Chapter 6 .

\section{THE BOLTZMANN EQUATION}

In order to calculate the average values of the particle physical properties (the macroscopic variables of interest), it is necessary to know the distribution function for the system under consideration. The dependence of the distribution function on the independent variables $\mathbf{r}, \mathbf{v}$, and $t$ is governed by an equation known as the Boltzmann equation. We present in this section a derivation of the collisionless Boltzmann equation and the general form it takes when the effects of the particle interactions are taken into account, without explicity deriving any particular expression for the collision term.

\subsection{Collisionless Boltzmann Equation}

Recall that

$$
d^{6} \mathcal{N}_{\alpha}(\mathbf{r}, \mathbf{v}, t)=f_{\alpha}(\mathbf{r}, \mathbf{v}, t) d^{3} r d^{3} v
$$

represents the number of particles of type $\alpha$ that, at the instant $t$, are situated within the volume element $d^{3} r d^{3} v$ of phase space, about the coordinates $(\mathbf{r}, \mathbf{v})$. Suppose that each particle is subjected to an external force $\mathbf{F}$. In the absence of particle interactions, a particle of type $\alpha$ with coordinates about $(\mathbf{r}, \mathbf{v})$ in phase space, at the instant $t$, will be found after a time interval $d t$ about the new coordinates $\left(\mathbf{r}^{\prime}, \mathbf{v}^{\prime}\right)$ such that

$$
\begin{aligned}
\mathbf{r}^{\prime}(t+d t) & =\mathbf{r}(t)+\mathbf{v} d t \\
\mathbf{v}^{\prime}(t+d t) & =\mathbf{v}(t)+\mathbf{a} d t
\end{aligned}
$$

where $\mathbf{a}=\mathbf{F} / m_{\alpha}$ is the particle acceleration and $m_{\alpha}$ its mass. Thus, all particles of type $\alpha$ inside the volume element $d^{3} r d^{3} v$ of phase space, about $(\mathbf{r}, \mathbf{v})$ at the instant $t$, will occupy a new volume element $d^{3} r^{\prime} d^{3} v^{\prime}$, about $\left(\mathbf{r}^{\prime}, \mathbf{v}^{\prime}\right)$ after the interval $d t$ (see Fig. 4). Since we are considering the same particles at $t$ and at $t+d t$, we must have, in the absence of collisions,

$$
f_{\alpha}\left(\mathbf{r}^{\prime}, \mathbf{v}^{\prime}, t+d t\right) d^{3} r^{\prime} d^{3} v^{\prime}=f_{\alpha}(\mathbf{r}, \mathbf{v}, t) d^{3} r d^{3} v
$$




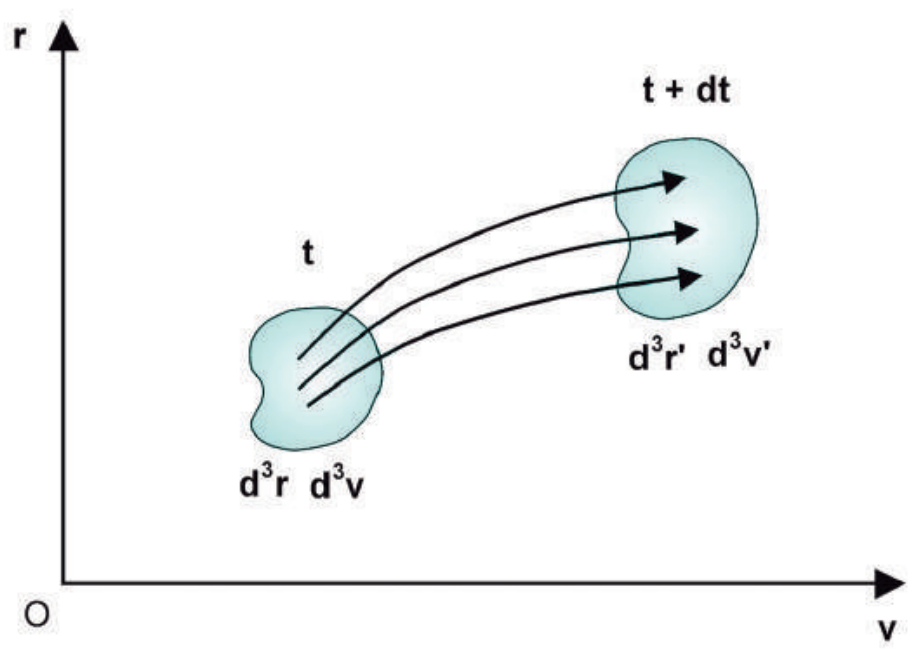

Fig. 4 In the absence of collisions the particles within the volume element $d^{3} r d^{3} v$ about $(\mathbf{r}, \mathbf{v})$, at an instant $t$, will occupy after a time interval $d t$ a new volume element $d^{3} r^{\prime} d^{3} v^{\prime}$, about $\left(\mathbf{r}^{\prime}, \mathbf{v}^{\prime}\right)$.

The element of volume $d^{3} r d^{3} v$ may become distorted in shape as a result of the particle motion. The relation between the new element of volume and the initial one is given by

$$
d^{3} r^{\prime} d^{3} v^{\prime}=|J| d^{3} r d^{3} v
$$

where $J$ stands for the Jacobian of the transformation from the initial coordinates $(\mathbf{r}, \mathbf{v})$ to the final ones $\left(\mathbf{r}^{\prime}, \mathbf{v}^{\prime}\right)$. It will be shown in the next subsection that for the transformation defined by (5.2) and (5.3) we have $|J|=1$, so that

$$
d^{3} r^{\prime} d^{3} v^{\prime}=d^{3} r d^{3} v
$$

and (5.4) becomes

$$
\left[f_{\alpha}\left(\mathbf{r}^{\prime}, \mathbf{v}^{\prime}, t+d t\right)-f_{\alpha}(\mathbf{r}, \mathbf{v}, t)\right] d^{3} r d^{3} v=0
$$

The first term on the left-hand side of (5.7) can be expanded in a Taylor series about $f_{\alpha}(\mathbf{r}, \mathbf{v}, t)$ as follows:

$$
f_{\alpha}(\mathbf{r}+\mathbf{v} d t, \mathbf{v}+\mathbf{a} d t, t+d t)=f_{\alpha}(\mathbf{r}, \mathbf{v}, t)+\left[\frac{\partial f_{\alpha}}{\partial t}+\right.
$$




$$
\left.\left(v_{x} \frac{\partial f_{\alpha}}{\partial x}+v_{y} \frac{\partial f_{\alpha}}{\partial y}+v_{z} \frac{\partial f_{\alpha}}{\partial z}\right)+\left(a_{x} \frac{\partial f_{\alpha}}{\partial v_{x}}+a_{y} \frac{\partial f_{\alpha}}{\partial v_{y}}+a_{z} \frac{\partial f_{\alpha}}{\partial v_{z}}\right)\right] d t
$$

neglecting terms of order $(d t)^{2}$ and higher. Using the del operator notation

$$
\nabla=\widehat{\mathbf{x}} \frac{\partial}{\partial x}+\widehat{\mathbf{y}} \frac{\partial}{\partial y}+\widehat{\mathbf{z}} \frac{\partial}{\partial z}
$$

and, in a similar way, defining a del operator in velocity space by

$$
\nabla_{v}=\widehat{\mathbf{x}} \frac{\partial}{\partial v_{x}}+\widehat{\mathbf{y}} \frac{\partial}{\partial v_{y}}+\widehat{\mathbf{z}} \frac{\partial}{\partial v_{z}}
$$

we obtain from (5.8)

$$
\begin{gathered}
f_{\alpha}(\mathbf{r}+\mathbf{v} d t, \mathbf{v}+\mathbf{a} d t, t+d t)=f_{\alpha}(\mathbf{r}, \mathbf{v}, t)+ \\
{\left[\frac{\partial f_{\alpha}(\mathbf{r}, \mathbf{v}, t)}{\partial t}+\mathbf{v} \cdot \nabla f_{\alpha}(\mathbf{r}, \mathbf{v}, t)+\mathbf{a} \cdot \nabla_{v} f_{\alpha}(\mathbf{r}, \mathbf{v}, t)\right] d t}
\end{gathered}
$$

Substituting this result into (5.7) gives

$$
\frac{\partial f_{\alpha}(\mathbf{r}, \mathbf{v}, t)}{\partial t}+\mathbf{v} \cdot \nabla f_{\alpha}(\mathbf{r}, \mathbf{v}, t)+\mathbf{a} \cdot \nabla_{v} f_{\alpha}(\mathbf{r}, \mathbf{v}, t)=0
$$

which is the Boltzmann equation in the absence of collisions.

This equation can be rewritten as

$$
\frac{\mathcal{D} f_{\alpha}(\mathbf{r}, \mathbf{v}, t)}{\mathcal{D} t}=0
$$

where the operator

$$
\frac{\mathcal{D}}{\mathcal{D} t}=\frac{\partial}{\partial t}+\mathbf{v} \cdot \nabla+\mathbf{a} \cdot \nabla_{v}
$$

represents the total derivative with respect to time, in phase space. Eq. (5.13) is a statement of the conservation of the density of representative points in phase space. If we move along with a representative point in phase space and observe the density of representative points $f_{\alpha}(\mathbf{r}, \mathbf{v}, t)$ in its neighborhood, we find that this density remains constant in time. This result is known as Liouville's theorem. Note that this result applies only 
to the special case in which collisions, as well as radiation losses, processes of production, and loss of particles, are unimportant.

\subsection{Jacobian of the Transformation in Phase Space}

To determine the Jacobian of the transformation defined by (5.2) and (5.3) recall that, from its definition, we have

$$
J=\frac{\partial\left(\mathbf{r}^{\prime}, \mathbf{v}^{\prime}\right)}{\partial(\mathbf{r}, \mathbf{v})}=\frac{\partial\left(x^{\prime}, y^{\prime}, z^{\prime}, v_{x}^{\prime}, v_{y}^{\prime}, v_{z}^{\prime}\right)}{\partial\left(x, y, z, v_{x}, v_{y}, v_{z}\right)}
$$

which corresponds to the determinant of the $6 \times 6$ matrix

$$
J=\left(\begin{array}{cccc}
\partial x^{\prime} / \partial x & \partial y^{\prime} / \partial x & \cdots & \partial v_{z}^{\prime} / \partial x \\
\partial x^{\prime} / \partial y & \partial y^{\prime} / \partial y & \cdots & \partial v_{z}^{\prime} / \partial y \\
\cdots & \cdots & \cdots & \cdots \\
\partial x^{\prime} / \partial v_{z} & \partial y^{\prime} / \partial v_{z} & \cdots & \partial v_{z}^{\prime} / \partial v_{z}
\end{array}\right)
$$

We can separate the external force $\mathbf{F}$ into two parts,

$$
\mathbf{F}=\mathbf{F}^{\prime}+q_{\alpha}(\mathbf{v} \times \mathbf{B})
$$

where $\mathbf{F}^{\prime}$ represents a velocity-independent force and the second term is the velocity-dependent force due to an externally applied magnetic field $\mathbf{B}$, the only velocity-dependent force that may concern us in this treatment. The partial derivatives appearing in the matrix $J$ are

$$
\begin{gathered}
\frac{\partial x_{i}^{\prime}}{\partial x_{j}}=\delta_{i j}, \quad \frac{\partial v_{i}^{\prime}}{\partial x_{j}}=\frac{1}{m_{\alpha}} \frac{\partial F_{i}^{\prime}}{\partial x_{j}} d t \\
\frac{\partial x_{i}^{\prime}}{\partial v_{j}}=\delta_{i j} d t \quad, \quad \frac{\partial v_{i}^{\prime}}{\partial v_{j}}=\delta_{i j}+\frac{q_{\alpha}}{m_{\alpha}} \frac{\partial(\mathbf{v} \times \mathbf{B})_{i}}{\partial v_{j}} d t
\end{gathered}
$$

where (5.2), (5.3), and (5.17) have been used, and where $x_{i, j}=x, y, z$ and $v_{i, j}=v_{x}, v_{y}, v_{z}$. The symbol $\delta_{i j}$ is the Kronecker delta. The matrix (5.16) can be written in the form

$$
J=\left(\begin{array}{ll}
(J)_{1} & (J)_{2} \\
(J)_{3} & (J)_{4}
\end{array}\right)
$$

where the $(J)_{i}^{\prime} s$, with $i=1,2,3,4$, represent the following $3 \times 3$ submatrices: 


$$
\begin{aligned}
& (J)_{1}=\left(\begin{array}{ccc}
1 & 0 & 0 \\
0 & 1 & 0 \\
0 & 0 & 1
\end{array}\right) \\
& (J)_{2}=\frac{d t}{m_{\alpha}}\left(\begin{array}{lll}
\partial F_{x}^{\prime} / \partial x & \partial F_{y}^{\prime} / \partial x & \partial F_{z}^{\prime} / \partial x \\
\partial F_{x}^{\prime} / \partial y & \partial F_{y}^{\prime} / \partial y & \partial F_{z}^{\prime} / \partial y \\
\partial F_{x}^{\prime} / \partial z & \partial F_{y}^{\prime} / \partial z & \partial F_{z}^{\prime} / \partial z
\end{array}\right) \\
& (J)_{3}=\left(\begin{array}{ccc}
d t & 0 & 0 \\
0 & d t & 0 \\
0 & 0 & d t
\end{array}\right) \\
& (J)_{4}=\left(\begin{array}{ccc}
1 & -a B_{z} & a B_{y} \\
a B_{z} & 1 & -a B_{x} \\
-a B_{y} & a B_{x} & 1
\end{array}\right)
\end{aligned}
$$

where the constant $a$ stands for $\left(q_{\alpha} / m_{\alpha}\right) d t$. Neglecting terms of order $(d t)^{2}$, it can be easily verified that $|J|=1$. Thus, up to and including the terms of first order in the infinitesimal $d t$, we have

$$
d^{3} r^{\prime} d^{3} v^{\prime}=d^{3} r d^{3} v
$$

which is the result (5.6) used in the previous subsection.

\subsection{Effects of Particle Interactions}

When the effects due to the particle interactions are taken into account, (5.12) needs to be modified. As a result of collisions during the time interval $d t$, some of the particles of type $\alpha$ that were initially within the volume element $d^{3} r d^{3} v$ of phase space may be removed from it, and particles of type $\alpha$ initially outside this volume element may end up inside it. This is indicated schematically in Fig. 5. Generally, the number of particles of type $\alpha$ inside $d^{3} r d^{3} v$ about the coordinates $(\mathbf{r}, \mathbf{v})$, at an instant $t$, will be different from the number of particles of type $\alpha$ inside this same volume element about the coordinates $\left(\mathbf{r}^{\prime}, \mathbf{v}^{\prime}\right)$ at the instant $t+d t$. We shall denote this net gain or loss of particles of type $\alpha$, as a result of collisions during the interval $d t$, in the volume element $d^{3} r d^{3} v$, by

$$
\left[\frac{\delta f_{\alpha}(\mathbf{r}, \mathbf{v}, t)}{\delta t}\right]_{\text {coll }} d^{3} r d^{3} v d t
$$




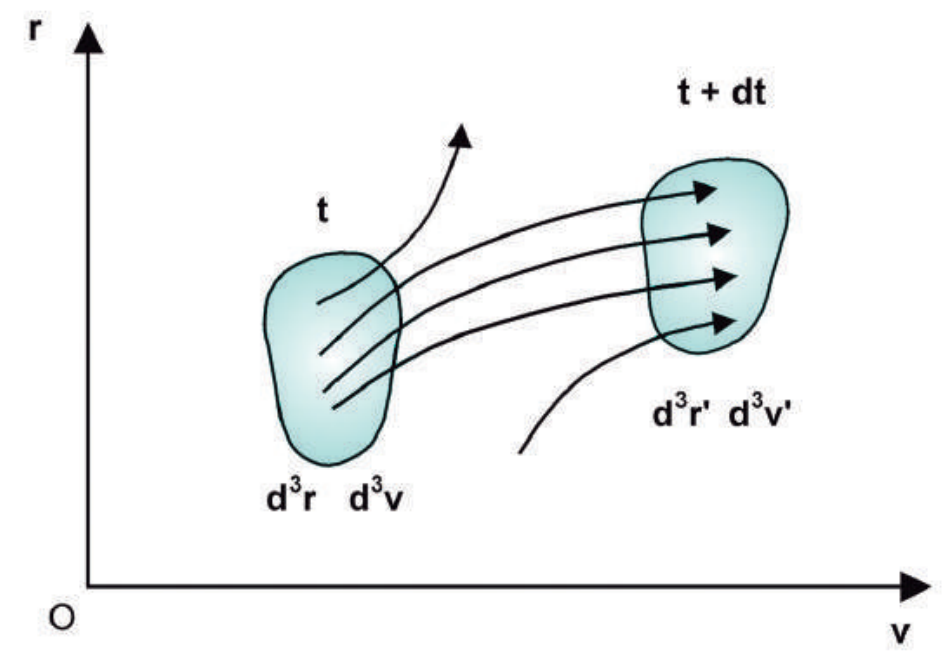

Fig. 5 Schematic representation of the motion of the volume element $d^{3} r d^{3} v$ in phase space, showing particles entering and leaving this volume element, as a result of collisions during the time interval $d t$.

where $\left(\delta f_{\alpha} / \delta t\right)_{\text {coll }}$ represents the rate of change of $f_{\alpha}(\mathbf{r}, \mathbf{v}, t)$ due to collisions. Thus, when collisions are considered, (5.7) becomes

$$
\left[f_{\alpha}\left(\mathbf{r}^{\prime}, \mathbf{v}^{\prime}, t+d t\right)-f_{\alpha}(\mathbf{r}, \mathbf{v}, t)\right] d^{3} r d^{3} v=\left(\frac{\delta f_{\alpha}}{\delta t}\right)_{\text {coll }} d^{3} r d^{3} v d t
$$

and the following modified form of Eq. (5.12) results

$$
\frac{\partial f_{\alpha}}{\partial t}+\mathbf{v} \cdot \nabla f_{\alpha}+\mathbf{a} \cdot \nabla_{v} f_{\alpha}=\left(\frac{\delta f_{\alpha}}{\delta t}\right)_{\text {coll }}
$$

Using the total time derivative operator, defined in (5.14), we can rewrite this equation in compact form as

$$
\frac{\mathcal{D} f_{\alpha}}{\mathcal{D} t}=\left(\frac{\delta f_{\alpha}}{\delta t}\right)_{\text {coll }}
$$

This equation is obviously incomplete, since the precise form of the collision term is not known. In the following section we will consider a very simple expression for the collision term, known as the Krook model or relaxation model. More elaborate expressions, such as the Boltzmann 
collision integral and the Fokker-Planck collision term, will be considered in Chapter 21.

\section{RELAXATION MODEL FOR THE COLLISION TERM}

A very simple method for taking into account collision effects is provided by the relaxation model. In this model it is assumed that the effect of collisions is to restore a situation of local equilibrium, characterized by a local equilibrium distribution function $f_{\alpha 0}(\mathbf{r}, \mathbf{v})$. In the absence of external forces, it assumes that a situation initially not in equilibrium, described by a distribution function $f_{\alpha}(\mathbf{r}, \mathbf{v}, t)$ different from $f_{\alpha 0}(\mathbf{r}, \mathbf{v})$, reaches a local equilibrium condition exponentially with time, as a result of collisions, with a relaxation time $\tau$. This relaxation time is of the order of the time between collisions and may also be written as $\nu^{-1}$ where $\nu$ represents a relaxation collision frequency. This model was originally developed by Krook and can be expressed mathematically as

$$
\left(\frac{\delta f_{\alpha}}{\delta t}\right)_{\text {coll }}=-\frac{\left(f_{\alpha}-f_{\alpha 0}\right)}{\tau}
$$

According to this expression for the collision term, when $f_{\alpha}=f_{\alpha 0}$ we have $\left(\delta f_{\alpha} / \delta t\right)_{\text {coll }}=0$, so that in a state of local equilibrium the distribution function is not altered as a result of collisions.

In order to bring out the physical meaning of the relaxation model, let us consider the Boltzmann equation with this collision term, in the absence of external forces and spatial gradients, and when $f_{\alpha 0}$ and $\tau$ are time-independent,

$$
\frac{\partial f_{\alpha}}{\partial t}=-\frac{\left(f_{\alpha}-f_{\alpha 0}\right)}{\tau}
$$

which can be rewritten as

$$
\frac{\partial f_{\alpha}}{\partial t}+\frac{f_{\alpha}}{\tau}=\frac{f_{\alpha 0}}{\tau}
$$

This simple inhomogeneous differential equation has $C e^{-t / \tau}$ as the homogeneous solution, where $C$ is a constant, and $f_{\alpha 0}$ as a particular integral. Therefore, the complete solution is

$$
f_{\alpha}(\mathbf{v}, t)=f_{\alpha 0}+\left[f_{\alpha}(\mathbf{v}, 0)-f_{\alpha 0}\right] e^{-t / \tau}
$$

Thus, the difference between $f_{\alpha}$ and $f_{\alpha 0}$ decreases exponentially in time at a rate governed by the relaxation collision frequency $\nu=1 / \tau$. 
This collisional model has proved to be useful and, in some cases, leads to results almost identical to the ones obtained using the Boltzmann collision integral (deduced in Chapter 21). It is particularly applicable to a weakly ionized plasma in which only charge-neutral collisions are important. However, it oversimplifies the entire relaxation phenomena and does not predict correctly the different relaxation collision frequencies for the various physical quantities of interest, such as the macroscopic velocity, momentum, and energy. According to the relaxation model, these macroscopic physical variables approach equilibrium at the same rate $\nu$. A detailed analysis of the collision process, however, shows that this is not the case, and the relaxation times for the various macroscopic variables differ to some extent. For nonrelativistic velocities, while the relaxation times for the average velocity and the momentum are found to be the same, approximately $\tau$, that of the average thermal energy is approximately $\left(m_{\beta} / 2 m_{\alpha}\right) \tau$. Hence, for collisions between electrons and neutral particles, the relaxation time for the kinetic energy of the electrons is longer than that for the average velocity by a factor that is of the order of the ratio of the neutral particle mass to the electron mass. The relaxation model is therefore strictly applicable only to the cases of collisions between particles of the same mass. In spite of this limitation, the relaxation model is still useful partly because of its simplicity and partly because it usually gives a first approximation to the problem under consideration.

\section{THE VLASOV EQUATION}

A very useful approximate way to describe the dynamics of a plasma is to consider that the plasma particle motions are governed by the applied external fields plus the macroscopic average internal fields, smoothed in space and time, due to the presence and motion of all plasma particles. The problem of obtaining the macroscopic (smoothed) internal electromagnetic fields, however, is still a complex one and requires that a selfconsistent solution be obtained.

The Vlasov equation is a partial differential equation that describes the time evolution of the distribution function in phase space and that directly incorporates the smoothed macroscopic internal electromagnetic fields. It may be obtained from the Boltzmann equation (5.27) with the collision term $\left(\delta f_{\alpha} / \delta t\right)_{\text {coll }}$ equal to zero, but including the internal smoothed fields in the force term,

$$
\frac{\partial f_{\alpha}}{\partial t}+\mathbf{v} \cdot \nabla f_{\alpha}+\frac{1}{m_{\alpha}}\left[\mathbf{F}_{e x t}+q_{\alpha}\left(\mathbf{E}_{i}+\mathbf{v} \times \mathbf{B}_{i}\right)\right] \cdot \nabla_{v} f_{\alpha}=0
$$


Here $\mathbf{F}_{\text {ext }}$ represents the external force, including the Lorentz force associated with any externally applied electric and magnetic fields, and $\mathbf{E}_{i}$ and $\mathbf{B}_{i}$ are internal smoothed electric and magnetic fields due to the presence and motion of all charged particles inside the plasma. In order that the internal macroscopic electromagnetic fields $\mathbf{E}_{i}$ and $\mathbf{B}_{i}$ be consistent with the macroscopic charge and current densities existing in the plasma itself, they must satisfy Maxwell equations

$$
\begin{gathered}
\nabla \cdot \mathbf{E}_{i}=\frac{\rho}{\epsilon_{0}} \\
\nabla \cdot \mathbf{B}_{i}=0 \\
\nabla \times \mathbf{E}_{i}=-\frac{\partial \mathbf{B}_{i}}{\partial t} \\
\nabla \times \mathbf{B}_{i}=\mu_{0}\left(\mathbf{J}+\epsilon_{0} \frac{\partial \mathbf{E}_{i}}{\partial t}\right)
\end{gathered}
$$

with the plasma charge density $\rho$ and the plasma current density $\mathbf{J}$ given by the expressions

$$
\begin{gathered}
\rho(\mathbf{r}, t)=\sum_{\alpha} q_{\alpha} n_{\alpha}(\mathbf{r}, t)=\sum_{\alpha} q_{\alpha} \int_{v} f_{\alpha}(\mathbf{r}, \mathbf{v}, t) d^{3} v \\
\mathbf{J}(\mathbf{r}, t)=\sum_{\alpha} q_{\alpha} n_{\alpha}(\mathbf{r}, t) \mathbf{u}_{\alpha}(\mathbf{r}, t)=\sum_{\alpha} q_{\alpha} \int_{v} \mathbf{v} f_{\alpha}(\mathbf{r}, \mathbf{v}, t) d^{3} v
\end{gathered}
$$

the summations being over the different charged particle species in the plasma. Here $\mathbf{u}_{\alpha}(\mathbf{r}, t)$ denotes the macroscopic average velocity for the particles of type $\alpha$, given in (4.4).

Eqs. (7.1) to (7.7) constitute a complete set of self-consistent equations to be solved simultaneously. For example, in an iterative procedure assuming starting approximate values for $\mathbf{E}_{i}(\mathbf{r}, t)$ and $\mathbf{B}_{i}(\mathbf{r}, t)$, Eq. (7.1) can be solved to yield $f_{\alpha}(\mathbf{r}, \mathbf{v}, t)$ for the various different species. Using the calculated $f_{\alpha}^{\prime} s$ in (7.6) and (7.7) leads to values for the charge and current densities ( $\rho$ and $\mathbf{J}$ ) in the plasma, which can be substituted into Maxwell equations and solved for $\mathbf{E}_{i}(\mathbf{r}, t)$ and $\mathbf{B}_{i}(\mathbf{r}, t)$. These values are then plugged back into the Vlasov equation, and so on, in order to obtain a self-consistent solution for the single particle distribution function.

Although the Vlasov equation does not explicitly include a collision term in its right-hand side and, hence, does not take into account shortrange collisions, it is not so restrictive as it may appear, since a significant 
part of the effects of the particle interactions has already been included in the Lorentz force, through the internal self-consistent smoothed electromagnetic fields.

\section{PROBLEMS}

5.1 Consider a system of particles uniformly distributed in space, with a constant particle number density $n_{0}$, and characterized by a velocity distribution function $f(v)$ such that

$$
\begin{aligned}
& f(v)=K_{0} \quad \text { for } \quad\left|v_{i}\right| \leq v_{0} \quad(i=x, y, z) \\
& f(v)=0 \quad \text { otherwise, }
\end{aligned}
$$

where $K_{0}$ is a nonzero positive constant. Determine the value of $K_{0}$ in terms of $n_{0}$ and $v_{0}$.

5.2 Consider the following two-dimensional Maxwellian distribution function:

$$
f\left(v_{x}, v_{y}\right)=n_{0}\left(\frac{m}{2 \pi k T}\right) \exp \left[-\frac{m\left(v_{x}^{2}+v_{y}^{2}\right)}{2 k T}\right]
$$

(a) Verify that $n_{0}$ represents correctly the particle number density, that is, the number of particles per unit area.

(b) Sketch, in a three-dimensionsal perspective view, the surface for this distribution function, plotting $f\left(v_{x}, v_{y}\right)$ in terms of $v_{x}$ and $v_{y}$. Draw, on this surface, curves of constant $v_{x}$, curves of constant $v_{y}$, and curves of constant $f$.

5.3 The electrons inside a system of two coaxial magnetic mirrors can be described by the so-called loss-cone distribution function

$$
f(\mathbf{v})=\frac{n_{0}}{\pi^{3 / 2} \alpha_{\perp}^{2} \alpha_{\|}}\left(\frac{v_{\perp}}{\alpha_{\perp}}\right)^{2} \exp \left[-\left(\frac{v_{\perp}}{\alpha_{\perp}}\right)^{2}-\left(\frac{v_{\|}}{\alpha_{\|}}\right)^{2}\right]
$$

where $v_{\|}$and $v_{\perp}$ denote the magnitudes of the electron velocities in the directions parallel and perpendicular to the magnetic bottle axis, respectively, and where $\alpha_{\|}^{2}=2 k T_{\|} / m$ and $\alpha_{\perp}^{2}=2 k T_{\perp} / m$.

(a) Verify that the number density of the electrons in the magnetic bottle is given by $n_{0}$. 
(b) Justify the applicability of the loss-cone distribution function to a magnetic mirror bottle by analyzing its dependence on $v_{\|}$and $v_{\perp}$. Sketch, in a three-dimensionsal perspective view, the surface for $f(\mathbf{v})$ as a function of $v_{\|}$and $v_{\perp}$.

5.4 Consider the motion of charged particles, in one dimension only, in the presence of an electric potential $V(x)$. Show, by direct substitution, that a function of the form

$$
f=f\left(\frac{1}{2} m v^{2}+q V\right)
$$

is a solution of the Boltzmann equation under steady-state conditions.

5.5 (a) Show that the Boltzmann equation, in cylindrical coordinates, can be written as

$$
\begin{gathered}
\frac{\partial f}{\partial t}+\dot{r} \frac{\partial f}{\partial r}+\dot{\phi} \frac{\partial f}{\partial \phi}-r \dot{\phi}^{2} \frac{\partial f}{\partial \dot{r}}+\frac{2 \dot{r} \dot{\phi}}{r} \frac{\partial f}{\partial \dot{\phi}}+\dot{z} \frac{\partial f}{\partial z}+ \\
\frac{1}{m}\left(F_{r} \frac{\partial f}{\partial \dot{r}}+\frac{F_{\phi}}{r} \frac{\partial f}{\partial \dot{\phi}}+F_{z} \frac{\partial f}{\partial \dot{z}}\right)=\left(\frac{\delta f}{\delta t}\right)_{\text {coll }}
\end{gathered}
$$

where the dot over the symbols stands for the time derivative operator $d / d t$ and where $F_{r}=m\left(d^{2} r / d t^{2}\right), F_{\phi}=m r\left(d^{2} \phi / d t^{2}\right)$, and $F_{z}=m\left(d^{2} z / d t^{2}\right)$.

(b) Show, by direct substitution, that in the presence of an azimuthally symmetric magnetic field (in the $z$ direction) a function of the form

$$
f=f\left(\frac{1}{2} m v^{2}, m r^{2} \dot{\phi}+q r A_{\phi}\right)
$$

is a solution of the Boltzmann equation under steady conditions, where the constant canonical momentum is given by $p_{\phi}=m r^{2} \dot{\phi}+q r A_{\phi}$, and where $A_{\phi}$ denotes the $\phi$ component of the magnetic potential $\mathbf{A}$, defined such that $\mathbf{B}=\nabla \times \mathbf{A}$.

5.6 Show that the Vlasov equation for a homogeneous plasma under the influence of a uniform external magnetostatic field $\mathbf{B}_{0}$, in the equilibrium state, is satisfied by any homogeneous distribution function, $f\left(v_{\|}, v_{\perp}\right)$, which is cylindrically symmetric with respect to the magnetostatic field.

5.7 The entropy of a system can be expressed, in terms of the distribution function, as

$$
S=-k \int_{r} \int_{v} f \ln (f) d^{3} r d^{3} v
$$


Show that, for a system that obeys the collisionless Boltzmann equation, the total time derivative of the entropy vanishes.

5.8 Consider a one-dimensional harmonic oscillator whose total energy can be expressed by

$$
E=\frac{1}{2}\left(m v^{2}+c x^{2}\right)
$$

where $c$ is a constant and $x$ its displacement coordinate. Show that the trajectory described by the representative point of the oscillator, in phase space, is an ellipse. 


\section{6}

\section{Average VALUES AND MACROSCOPIC VARIABLES}

\section{AVERAGE VALUE OF A PHYSICAL QUANTITY}

A systematic method for obtaining the average values of functions of particle velocities is presented in this chapter. The macroscopic variables, such as number density, flow velocity, kinetic pressure, thermal energy flux, and so on, can be considered as average values of physical quantities, involving the collective behavior of a large number of particles. These macroscopic variables are related to the the various moments of the distribution function. A formal definition of the moments of the distribution function is presented in section 10 .

To each particle in the plasma we can associate some molecular property, $\chi(\mathbf{r}, \mathbf{v}, t)$, which in general may be a function of the position $\mathbf{r}$ of the particle, of its velocity $\mathbf{v}$, and of the time $t$. This property may be, for example, the mass, the velocity, the momentum, or the energy of the particle.

In order to calculate the average value of the property $\chi(\mathbf{r}, \mathbf{v}, t)$, recall that $d^{6} \mathcal{N}_{\alpha}(\mathbf{r}, \mathbf{v}, t)$ represents the number of particles of type $\alpha$ inside the phase space volume element $d^{3} r d^{3} v$ about $(\mathbf{r}, \mathbf{v})$, at the instant $t$. Thus, the total value of $\chi(\mathbf{r}, \mathbf{v}, t)$ for all the particles of type $\alpha$ inside $d^{3} r d^{3} v$ is given by

$$
\chi(\mathbf{r}, \mathbf{v}, t) d^{6} \mathcal{N}_{\alpha}(\mathbf{r}, \mathbf{v}, t)=\chi(\mathbf{r}, \mathbf{v}, t) f_{\alpha}(\mathbf{r}, \mathbf{v}, t) d^{3} r d^{3} v
$$

The total value of $\chi(\mathbf{r}, \mathbf{v}, t)$ for all the particles of type $\alpha$ inside the volume element $d^{3} r$ of configuration space, irrespective of velocity, is obtained by 
integrating (1.1) over all possible velocities,

$$
d^{3} r \int_{v} \chi(\mathbf{r}, \mathbf{v}, t) f_{\alpha}(\mathbf{r}, \mathbf{v}, t) d^{3} v
$$

The average value of $\chi(\mathbf{r}, \mathbf{v}, t)$ can now be obtained by dividing (1.2) by the number of particles of type $\alpha$ inside $d^{3} r$ about $\mathbf{r}$, at the instant $t$, i.e., by $n_{\alpha}(\mathbf{r}, t) d^{3} r$. We define, therefore, the average value of the property $\chi(\mathbf{r}, \mathbf{v}, t)$ for the particles of type $\alpha$ by

$$
<\chi(\mathbf{r}, \mathbf{v}, t)>_{\alpha}=\frac{1}{n_{\alpha}(\mathbf{r}, t)} \int_{v} \chi(\mathbf{r}, \mathbf{v}, t) f_{\alpha}(\mathbf{r}, \mathbf{v}, t) d^{3} v
$$

The symbol $<>_{\alpha}$ denotes the average value with respect to velocity space for the particles of type $\alpha$. Note that the average value is always independent of $\mathbf{v}$, being a function of only $\mathbf{r}$ and $t$.

If we take $\chi(\mathbf{r}, \mathbf{v}, t)=1$ in (1.3), the expression for the number density $n_{\alpha}(\mathbf{r}, t)$, given in (5.4.2), is obtained.

\section{AVERAGE VELOCITY AND PECULIAR VELOCITY}

Consider now $\chi(\mathbf{r}, \mathbf{v}, t)$ as being the velocity $\mathbf{v}$ of the type $\alpha$ particles in the vicinity of the position $\mathbf{r}$, at the instant $t$. Replacing $\chi(\mathbf{r}, \mathbf{v}, t)$ in (1.3) by $\mathbf{v}$ gives the macroscopic average velocity or flow velocity $\mathbf{u}_{\alpha}(\mathbf{r}, t)$ for the particles of type $\alpha$,

$$
\mathbf{u}_{\alpha}(\mathbf{r}, t)=<\mathbf{v}>_{\alpha}=\frac{1}{n_{\alpha}(\mathbf{r}, t)} \int_{v} \mathbf{v} f_{\alpha}(\mathbf{r}, \mathbf{v}, t) d^{3} v
$$

which is the same expression given in (5.4.4) (Eq. 4.4 in Chapter 5).

Note that $\mathbf{r}, \mathbf{v}$, and $t$ are taken as independent variables, whereas the average velocity $\mathbf{u}_{\alpha}(\mathbf{r}, t)$ depends on $\mathbf{r}$ and $t$. For the cases in which $\chi(\mathbf{r}, \mathbf{v}, t)$ is independent of the particle velocity, we have

$$
<\chi(\mathbf{r}, t)>_{\alpha}=\chi_{\alpha}(\mathbf{r}, t)
$$

so that, for example, $\left\langle\mathbf{u}_{\alpha}\right\rangle=\mathbf{u}_{\alpha}$. In what follows, the index $\alpha$ after the average value symbol will be omitted whenever it is redundant, that is, $<\mathbf{u}_{\alpha}>_{\alpha} \equiv \mathbf{u}_{\alpha}$.

The peculiar velocity or random velocity $\mathbf{c}_{\alpha}$ is defined as the velocity of a type $\alpha$ particle relative to the average velocity $\mathbf{u}_{\alpha}(\mathbf{r}, t)$,

$$
\mathbf{c}_{\alpha}=\mathbf{v}-\mathbf{u}_{\alpha}
$$


Consequently, we always have $\left\langle\mathbf{c}_{\alpha}\right\rangle=0$, since $\left\langle\mathbf{v}_{\alpha}\right\rangle=\mathbf{u}_{\alpha}(\mathbf{r}, t)$. The peculiar velocity $\mathbf{c}_{\alpha}$ is the one associated with the random or thermal motions of the particles. When $\mathbf{u}_{\alpha}(\mathbf{r}, t)$ vanishes, we have $\mathbf{c}_{\alpha} \equiv \mathbf{v}$.

\section{FLUX}

From the concept of distribution function many other macroscopic variables can be defined in terms of average values. Macroscopic variables such as the particle current density (or particle flux), the pressure dyad or tensor, and the heat flow vector (or thermal energy flux), involve the flux of some molecular property $\chi(\mathbf{r}, \mathbf{v}, t)$. The flux of $\chi(\mathbf{r}, \mathbf{v}, t)$ is defined as the amount of the quantity $\chi(\mathbf{r}, \mathbf{v}, t)$ transported across some given surface, per unit area and per unit time.

Consider a surface element $d \mathbf{S}$ inside the plasma. If the distribution of velocities is isotropic, the flux will be independent of the relative orientation in space of the surface element $d \mathbf{S}$. However, more generally, when the velocity distribution is anisotropic the flux will depend on the relative spatial orientation of $d \mathbf{S}$. Suppose, therefore, that the surface element of magnitude $d S$ is oriented along some direction specified by the unit vector $\widehat{\mathbf{n}}$,

$$
d \mathbf{S}=d S \widehat{\mathbf{n}}
$$

$\widehat{\mathbf{n}}$ being normal to the surface element. In the case of an open surface there are two possible directions for the normal $\widehat{\mathbf{n}}$, one opposite to the other. The direction that is taken as positive is related to the positive sense of traversing the perimeter (bounding curve) of the open surface, according to the following convention: if the positive sense of traversal of the perimeter of a horizontal open surface is taken as counterclockwise, then the positive normal to the open surface is up; if the positive sense of traversal of the perimeter is clockwise, then the positive normal to the open surface is down, as shown in Fig. 1. For a closed surface the normal unit vector is conventionally chosen to point outward.

The particles inside the plasma, due to their velocities, will move across the surface element $d \mathbf{S}$ carrying the property $\chi(\mathbf{r}, \mathbf{v}, t)$ with them. We want to calculate the number of particles of type $\alpha$ that move across $d \mathbf{S}$ during the time interval $d t$.

The particles with velocity between $\mathbf{v}$ and $\mathbf{v}+d \mathbf{v}$ that will cross $d \mathbf{S}$ in the interval between $t$ and $t+d t$ must lie initially in the volume of the prism of base $d S$ and side $v d t$, as indicated in Fig. 2. The volume of this prism is

$$
d^{3} r=d \mathbf{S} \cdot \mathbf{v} d t=\widehat{\mathbf{n}} \cdot \mathbf{v} d S d t
$$



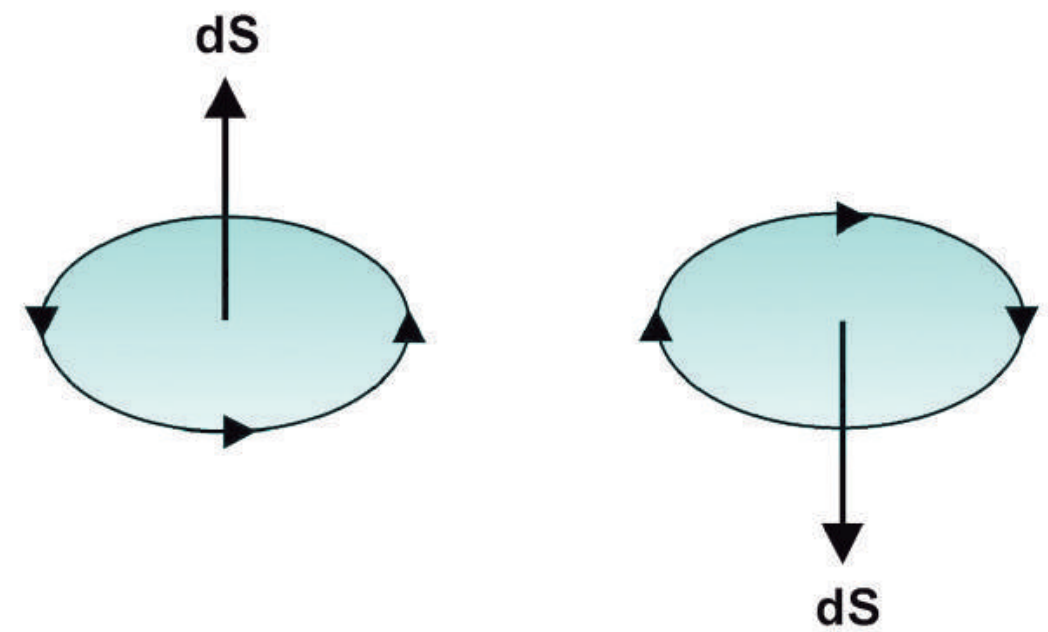

Fig. 1 Direction of the positive normal to the surface element $d \mathbf{S}$ as related to the sense of traversing the perimeter of $d \mathbf{S}$.

From the definition of $f_{\alpha}(\mathbf{r}, \mathbf{v}, t)$, the number of particles of type $\alpha$ in the volume of this prism that have velocities between $\mathbf{v}$ and $\mathbf{v}+d \mathbf{v}$ is

$$
f_{\alpha}(\mathbf{r}, \mathbf{v}, t) d^{3} r d^{3} v=f_{\alpha}(\mathbf{r}, \mathbf{v}, t) \widehat{\mathbf{n}} \cdot \mathbf{v} d S d t d^{3} v
$$

so that the total amount of $\chi(\mathbf{r}, \mathbf{v}, t)$ transported across $d \mathbf{S}$, in the interval $d t$, is obtained by multiplying this number of particles by $\chi(\mathbf{r}, \mathbf{v}, t)$ and integrating the result over all possible velocities,

$$
\int_{v} \chi(\mathbf{r}, \mathbf{v}, t) f_{\alpha}(\mathbf{r}, \mathbf{v}, t) \widehat{\mathbf{n}} \cdot \mathbf{v} d^{3} v d S d t
$$

Note that the contributions corresponding to a rotation of the segment $v d t$ over all possible directions about $d \mathbf{S}$ are taken into account in the integration over velocity space. Particles that cross $d \mathbf{S}$ in a direction such that $\widehat{\mathbf{n}} \cdot \mathbf{v}$ is positive give a positive contribution to the flux in the direction of $\widehat{\mathbf{n}}$, while particles that cross $d \mathbf{S}$ in a direction such that $\widehat{\mathbf{n}} \cdot \mathbf{v}$ is negative give a negative contribution to the flux in the direction of $\widehat{\mathbf{n}}$, as illustrated in Fig. 3.

The net amount of the quantity $\chi(\mathbf{r}, \mathbf{v}, t)$ transported by the particles of type $\alpha$, per unit area and per unit time, is obtained by dividing expression (3.4) by $d S d t$. The flux in the direction $\widehat{\mathbf{n}}, \Phi_{\alpha n}(\chi)$, is therefore given by

$$
\Phi_{\alpha n}(\chi)=\int_{v} \chi(\mathbf{r}, \mathbf{v}, t) f_{\alpha}(\mathbf{r}, \mathbf{v}, t) \widehat{\mathbf{n}} \cdot \mathbf{v} d^{3} v
$$




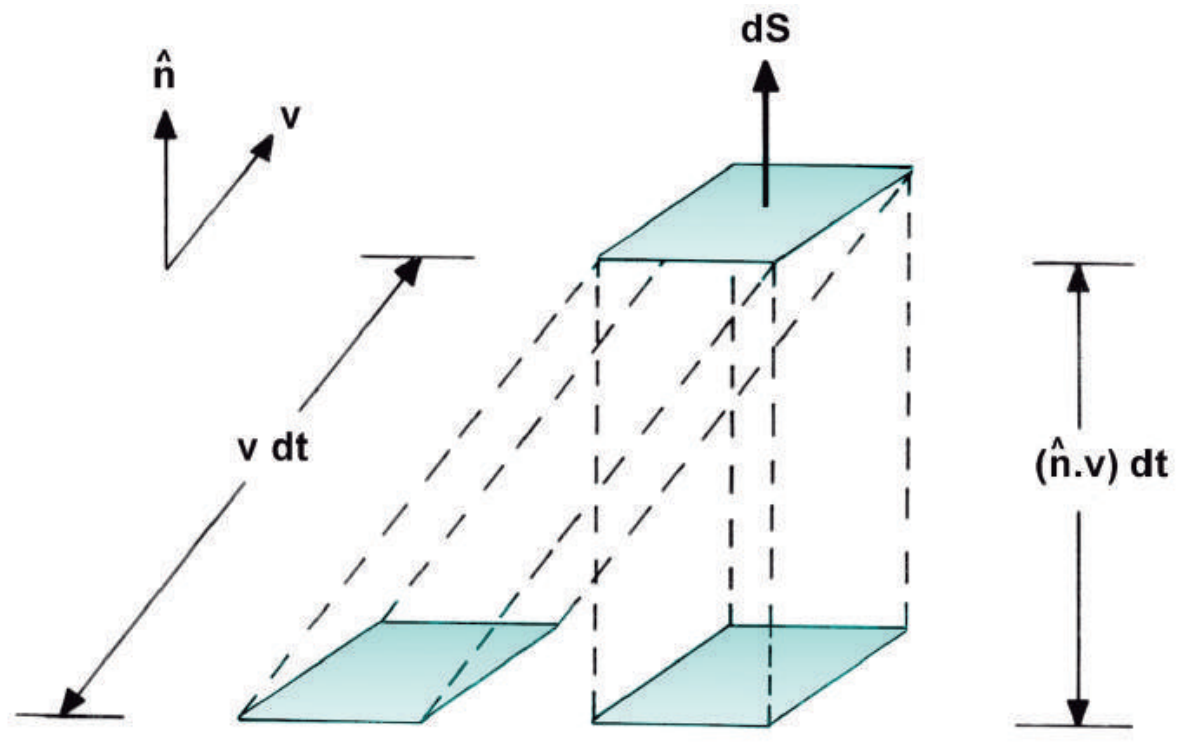

Fig. 2 Prism of volume $d^{3} r=d \mathbf{S} \cdot \mathbf{v} d t=\widehat{\mathbf{n}} \cdot \mathbf{v} d S d t$ containing the particles of type $\alpha$ with velocities between $\mathbf{v}$ and $\mathbf{v}+d \mathbf{v}$, and which will cross $d \mathbf{S}$ in the time interval $d t$.

or, using the average value symbol,

$$
\Phi_{\alpha n}(\chi)=n_{\alpha}(\mathbf{r}, t)<\chi(\mathbf{r}, \mathbf{v}, t) \widehat{\mathbf{n}} \cdot \mathbf{v}>_{\alpha}=n_{\alpha}<\chi v_{n}>_{\alpha}
$$

where $v_{n}=\widehat{\mathbf{n}} \cdot \mathbf{v}$ denotes the component of $\mathbf{v}$ along the direction specified by the unit vector $\widehat{\mathbf{n}}$.

When $\chi(\mathbf{r}, \mathbf{v}, t)$ is a scalar quantity, $\Phi_{\alpha n}(\chi)$ can be considered as the component, along $\widehat{\mathbf{n}}$, of a vector flux $\boldsymbol{\Phi}_{\alpha}(\chi)$, that is,

$$
\Phi_{\alpha n}(\chi)=\widehat{\mathbf{n}} \cdot \Phi_{\alpha}(\chi)
$$

with

$$
\boldsymbol{\Phi}_{\alpha}(\chi)=n_{\alpha}<\chi \mathbf{v}>_{\alpha}
$$

If $\chi(\mathbf{r}, \mathbf{v}, t)$ represents a vector quantity, which in this case we shall denote by $\mathbf{X}(\mathbf{r}, \mathbf{v}, t)$, then we will have a flux dyad (or tensor),

$$
\Phi_{\alpha}(\chi)=n_{\alpha}<\mathbf{X} \mathbf{v}>_{\alpha}
$$

and if it represents a dyad quantity we will have a flux triad, and so on. 


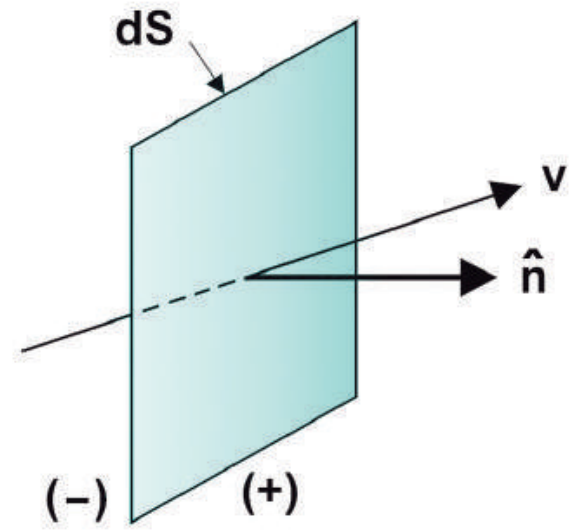

(a)

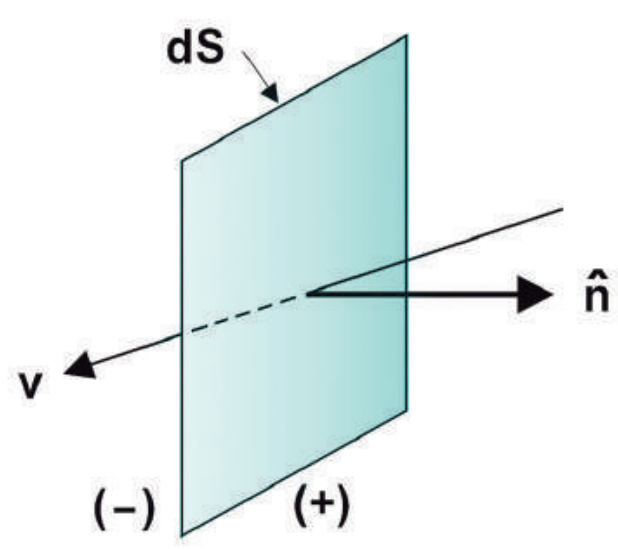

(b)

Fig. 3 (a) Particles that cross $d \mathbf{S}$ from the (-) region to the $(+)$ region contribute positively to the flux in the direction $\widehat{\mathbf{n}}$, while (b) particles that cross $d \mathbf{S}$ from the $(+)$ region to the (-) region contribute negatively to the flux in the direction $\widehat{\mathbf{n}}$.

In many situations of practical interest it is important to separately consider the contribution to the flux due to the average velocity $\mathbf{u}_{\alpha}(\mathbf{r}, t)$, and that due to the random velocity $\mathbf{c}_{\alpha}$ of the particles of type $\alpha$. Substituting $\mathbf{v}=\mathbf{c}_{\alpha}+\mathbf{u}_{\alpha}$ in (3.6), gives

$$
\Phi_{\alpha n}(\chi)=n_{\alpha}<\chi c_{\alpha n}>+n_{\alpha}<\chi u_{\alpha n}>
$$

where $c_{\alpha n}=\widehat{\mathbf{n}} \cdot \mathbf{c}_{\alpha}$ and $u_{\alpha n}=\widehat{\mathbf{n}} \cdot \mathbf{u}_{\alpha}$.

For the cases in which the flow velocity $\mathbf{u}_{\alpha}$ is zero or, equivalently, if we take $d \mathbf{S}$ to be in a frame of reference moving with the average velocity $\mathbf{u}_{\alpha},(3.10)$ becomes

$$
\Phi_{\alpha n}(\chi)=n_{\alpha}<\chi c_{\alpha n}>
$$

which is the flux of $\chi(\mathbf{r}, \mathbf{v}, t)$ along $\widehat{\mathbf{n}}$ due to the random motions of the particles of type $\alpha$.

\section{PARTICLE CURRENT DENSITY}

The particle current density (or particle flux) is defined as the number of particles passing through a given surface, per unit area and per unit 
time. Taking $\chi(\mathbf{r}, \mathbf{v}, t)=1$ in (3.6), we obtain the flux of particles of type $\alpha$ in the direction $\widehat{\mathbf{n}}$,

$$
\Gamma_{\alpha n}(\mathbf{r}, t)=n_{\alpha}<v_{n}>_{\alpha}=n_{\alpha} u_{\alpha n}
$$

since $\left\langle c_{\alpha n}\right\rangle=0$. When $\mathbf{u}_{\alpha}$ vanishes, it is of interest to consider only the flux in the positive direction instead of the resulting net flux. The number of particles of type $\alpha$ that cross a given surface along the direction $\widehat{\mathbf{n}}$ from the same side, per unit area and time, due to their random motions, is given by

$$
\Gamma_{\alpha n}^{(+)}(\mathbf{r}, t)=\int_{v(+)} \widehat{\mathbf{n}} \cdot \mathbf{c}_{\alpha} f_{\alpha}(\mathbf{r}, \mathbf{v}, t) d^{3} v
$$

where the integral in velocity space is over only the velocities for which $\widehat{\mathbf{n}} \cdot \mathbf{c}_{\alpha}>0$.

The random mass flux in the positive direction of $\widehat{\mathbf{n}}$ is consequently given by $m_{\alpha} \Gamma_{\alpha n}^{(+)}(\mathbf{r}, t)$, where $m_{\alpha}$ is the mass of the type $\alpha$ particle.

\section{MOMENTUM FLOW DYAD OR TENSOR}

This quantity is defined as the net momentum transported per unit area and time through some surface element $\widehat{\mathbf{n}} d S$. If we take, in (3.6), $\chi(\mathbf{r}, \mathbf{v}, t)$ as the component of momentum of the type $\alpha$ particles along some direction specified by the unit vector $\widehat{\mathbf{j}}$, that is,

$$
\chi_{j}=m_{\alpha} \mathbf{v} \cdot \widehat{\mathbf{j}}=m_{\alpha} v_{j}
$$

we obtain the element $\Pi_{\alpha j n}(\mathbf{r}, t)$ of the momentum flow tensor

$$
\Pi_{\alpha j n}(\mathbf{r}, t)=n_{\alpha}<m_{\alpha}(\widehat{\mathbf{j}} \cdot \mathbf{v})(\mathbf{v} \cdot \widehat{\mathbf{n}})>_{\alpha}=\rho_{m \alpha}<v_{j} v_{n}>_{\alpha}
$$

where $\rho_{m \alpha}=n_{\alpha} m_{\alpha}$ denotes the mass density of the type $\alpha$ particles. Thus, the momentum flow element $\Pi_{\alpha j n}(\mathbf{r}, t)$ represents the flux of the $j^{\text {th }}$ component of the momentum of the type $\alpha$ particles through a surface element whose normal is oriented along $\widehat{\mathbf{n}}$. Since $\mathbf{v}=\mathbf{c}_{\alpha}+\mathbf{u}_{\alpha}$, we obtain

$$
\Pi_{\alpha j n}(\mathbf{r}, t)=\rho_{m \alpha}<c_{\alpha j} c_{\alpha n}>+\rho_{m \alpha} u_{\alpha j} u_{\alpha n}
$$

or, in dyadic form,

$$
\Pi_{\alpha}(\mathbf{r}, t)=\rho_{m \alpha}<\mathbf{c}_{\alpha} \mathbf{c}_{\alpha}>+\rho_{m \alpha} \mathbf{u}_{\alpha} \mathbf{u}_{\alpha}
$$


where we have used the result $\left\langle\mathbf{u}_{\alpha} \mathbf{c}_{\alpha}\right\rangle=\mathbf{u}_{\alpha}\left\langle\mathbf{c}_{\alpha}\right\rangle=0$.

In a Cartesian coordinate system $(x, y, z)$ the momentum flow dyad can be written in the following form, in terms of its components,

$$
\begin{aligned}
\Pi_{\alpha} & =\widehat{\mathbf{x}} \widehat{\mathbf{x}} \Pi_{\alpha x x}+\widehat{\mathbf{x}} \widehat{\mathbf{y}} \Pi_{\alpha x y}+\widehat{\mathbf{x}} \widehat{\mathbf{z}} \Pi_{\alpha x z} \\
& +\widehat{\mathbf{y}} \widehat{\mathbf{x}} \Pi_{\alpha y x}+\widehat{\mathbf{y}} \widehat{\mathbf{y}} \Pi_{\alpha y y}+\widehat{\mathbf{y}} \widehat{\mathbf{z}} \Pi_{\alpha y z} \\
& +\widehat{\mathbf{z}} \widehat{\mathbf{x}} \Pi_{\alpha z x}+\widehat{\mathbf{z}} \widehat{\mathbf{y}} \Pi_{\alpha z y}+\widehat{\mathbf{z}} \widehat{\mathbf{z}} \Pi_{\alpha z z}
\end{aligned}
$$

From the rules of matrix multiplication $\Pi_{\alpha}$ can be expressed as

$$
\Pi_{\alpha}=\left(\begin{array}{lll}
\widehat{\mathbf{x}} & \widehat{\mathbf{y}} & \widehat{\mathbf{z}}
\end{array}\right)\left(\begin{array}{lll}
\Pi_{\alpha x x} & \Pi_{\alpha x y} & \Pi_{\alpha x z} \\
\Pi_{\alpha y x} & \Pi_{\alpha y y} & \Pi_{\alpha y z} \\
\Pi_{\alpha z x} & \Pi_{\alpha z y} & \Pi_{\alpha z z}
\end{array}\right)\left(\begin{array}{c}
\widehat{\mathbf{x}} \\
\widehat{\mathbf{y}} \\
\widehat{\mathbf{z}}
\end{array}\right)
$$

It is usual, however, to omit the pre- and post-multiplicative dyadic signs, such as $\widehat{\mathbf{x}} \widehat{\mathbf{x}}$, and so on, and denote the dyad only by the $3 \times 3$ matrix containing the elements $\Pi_{\alpha i j}$. Thus, $\Pi_{\alpha i j}$ corresponds to the element of the $i^{\text {th }}$ row and the $j^{\text {th }}$ column. From (5.3) it is clear that $\Pi_{\alpha i j}=\Pi_{\alpha j i}$ and, consequently, the $3 \times 3$ matrix in (5.6) is symmetric. Therefore, only six of the components of the momentum flow dyad are independent.

\section{PRESSURE DYAD OR TENSOR}

\subsection{Concept of Pressure}

The pressure of a gas is usually defined as the force per unit area exerted by the gas molecules through collisions with the walls of the containing vessel. This force is equal to the rate of transfer of molecular momentum to the walls of the container. This definition applies also to any surface immersed in the gas as, for example, the surface of a material body.

We may generalize this definition of pressure so that it can be applied to any point inside the gas. To this end, we will define pressure in terms of an imaginary surface element $d \mathbf{S}=\widehat{\mathbf{n}} d S$, inside the gas, moving with its average flow velocity. The pressure on $d \mathbf{S}$ is then defined as the rate of transport of molecular momentum per unit area, that is, the flux of momentum across $d \mathbf{S}$ due to the random particle motions.

When different species of particles are present, as in a plasma, it is useful to define a (partial) pressure due to the particles of type $\alpha$, as the 
flux of momentum transported by the type $\alpha$ particles as they move back and forth across the surface element $\widehat{\mathbf{n}} d S$, which is moving at the average velocity $\mathbf{u}_{\alpha}(\mathbf{r}, t)$. In the reference frame of $d \mathbf{S}$ (3.11) applies, and taking $\chi(\mathbf{r}, \mathbf{v}, t)$ as the $j^{\text {th }}$ component of momentum of the type $\alpha$ particles, $m_{\alpha} c_{\alpha j}$, we obtain the element $P_{\alpha j n}$ of the pressure tensor,

$$
P_{\alpha j n}=\rho_{m \alpha}<c_{\alpha j} c_{\alpha n}>
$$

The pressure dyad is therefore given by

$$
\mathcal{P}_{\alpha}=\rho_{m \alpha}<\mathbf{c}_{\alpha} \mathbf{c}_{\alpha}>
$$

From (5.4) we find the following relation between the pressure dyad $\mathcal{P}_{\alpha}$ and the momentum flow dyad $\Pi_{\alpha}$,

$$
\mathcal{P}_{\alpha}=\Pi_{\alpha}-\rho_{m \alpha} \mathbf{u}_{\alpha} \mathbf{u}_{\alpha}
$$

They are equal only when the flow velocity $\mathbf{u}_{\alpha}(\mathbf{r}, t)$ vanishes.

\subsection{Force per Unit Area}

Consider now a small element of volume inside the plasma, bounded by the closed surface $S$, and let $d \mathbf{S}=\widehat{\mathbf{n}} d S$ be an element of area belonging to $S$, with the unit vector $\widehat{\mathbf{n}}$ normal to the surface element and pointing outward (see Fig. 4). The force per unit area, $\mathbf{f}_{\alpha}$, acting on the area element $\widehat{\mathbf{n}} d S$, as the result of the random particle motions, is given by

$$
\mathbf{f}_{\alpha}=-\mathcal{P}_{\alpha} \cdot \widehat{\mathbf{n}}=-\rho_{m \alpha}<\mathbf{c}_{\alpha}\left(\mathbf{c}_{\alpha} \cdot \widehat{\mathbf{n}}\right)>
$$

The reason for the minus sign can be seen as follows. Suppose, for the moment, that all type $\alpha$ particles have the same velocity $\mathbf{c}_{\alpha}$. If $\mathbf{c}_{\alpha}$ forms an angle of less than $90^{\circ}$ with $\widehat{\mathbf{n}}$, then the quantity $n_{\alpha}\left(\mathbf{c}_{\alpha} \cdot \widehat{\mathbf{n}}\right) d S$ is the number of type $\alpha$ particles leaving, per unit time, the volume enclosed by the closed surface $S$, through $d \mathbf{S}$. The corresponding change (decrease) in the momentum of the plasma enclosed by the surface $S$ is given by the expression $-n_{\alpha} m_{\alpha} \mathbf{c}_{\alpha}\left(\mathbf{c}_{\alpha} \cdot \widehat{\mathbf{n}}\right) d S$, since $\left(\mathbf{c}_{\alpha} \cdot \widehat{\mathbf{n}}\right)$ is positive. On the other hand, if $\mathbf{c}_{\alpha}$ forms an angle greater than $90^{\circ}$ with $\widehat{\mathbf{n}}$, then $-n_{\alpha}\left(\mathbf{c}_{\alpha} \cdot \widehat{\mathbf{n}}\right) d S$ represents the number of particles entering, per unit time, the bounded volume through $d \mathbf{S}$, and the corresponding change (increase) in the momentum of the plasma within the closed surface $S$ is again $-n_{\alpha} m_{\alpha} \mathbf{c}_{\alpha}\left(\mathbf{c}_{\alpha} \cdot \widehat{\mathbf{n}}\right) d S$, since now $\left(\mathbf{c}_{\alpha} \cdot \widehat{\mathbf{n}}\right)$ is negative. 


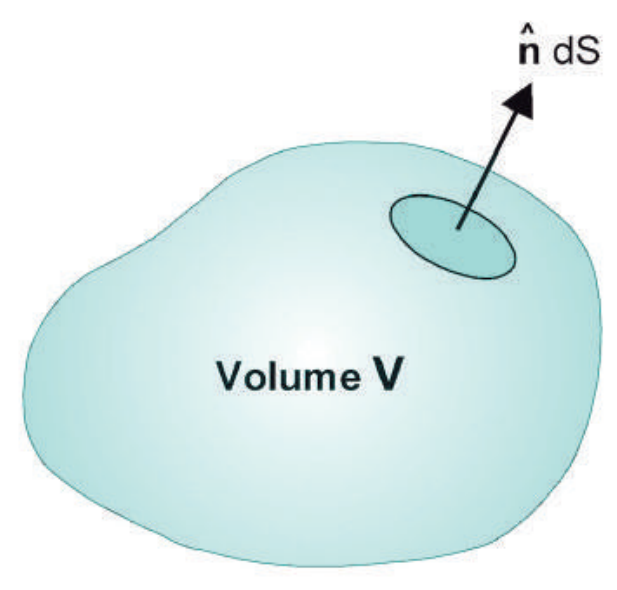

Fig. 4 Element of volume $V$ bounded by a closed surface $S$, with the surface element $\widehat{\mathbf{n}} d S$ pointing outward.

We conclude, by generalizing this result, that for any arbitrary distribution of individual velocities, the vector quantity

$$
-n_{\alpha} m_{\alpha}<\mathbf{c}_{\alpha}\left(\mathbf{c}_{\alpha} \cdot \widehat{\mathbf{n}}\right)>d S=-\mathcal{P}_{\alpha} \cdot \widehat{\mathbf{n}} d S
$$

represents the rate of change of the plasma momentum within the closed surface $S$, due to the exchange of type $\alpha$ particles through the surface element $\widehat{\mathbf{n}} d S$. Therefore, the force per unit area exerted on an element of area oriented along the unit vector $\widehat{\mathbf{n}}$ is $-\mathcal{P}_{\alpha} \cdot \widehat{\mathbf{n}}$. If we take, for example, an element of area oriented along the $x$ direction, that is, $\widehat{\mathbf{n}}=\widehat{\mathbf{x}}$, we have

$$
-\mathcal{P}_{\alpha} \cdot \widehat{\mathbf{n}}=-\widehat{\mathbf{x}} P_{\alpha x x}-\widehat{\mathbf{y}} P_{\alpha y x}-\widehat{\mathbf{z}} P_{\alpha z x}
$$

where $P_{\alpha x x}$ is normal to the surface and towards it, just like a hydrostatic pressure, whereas the components $P_{\alpha y x}$ and $P_{\alpha z x}$ are pressures due to shear forces that are tangential to the surface, as indicated in Fig. 5. All other components of $\mathcal{P}_{\alpha}$ are interpreted in an analogous way. Generally, the force per unit area $P_{\alpha j n}$ acts along the negative direction of the axis denoted by the first subscript $(j)$ on a surface whose outward normal is parallel to the axis indicated by the second subscript $(n)$. Alternatively, if the outward normal to the surface is in the negative direction of the axis indicated by the second subscript $(n)$, then the force acts in the same direction as the axis denoted by the first subscript $(j)$. 


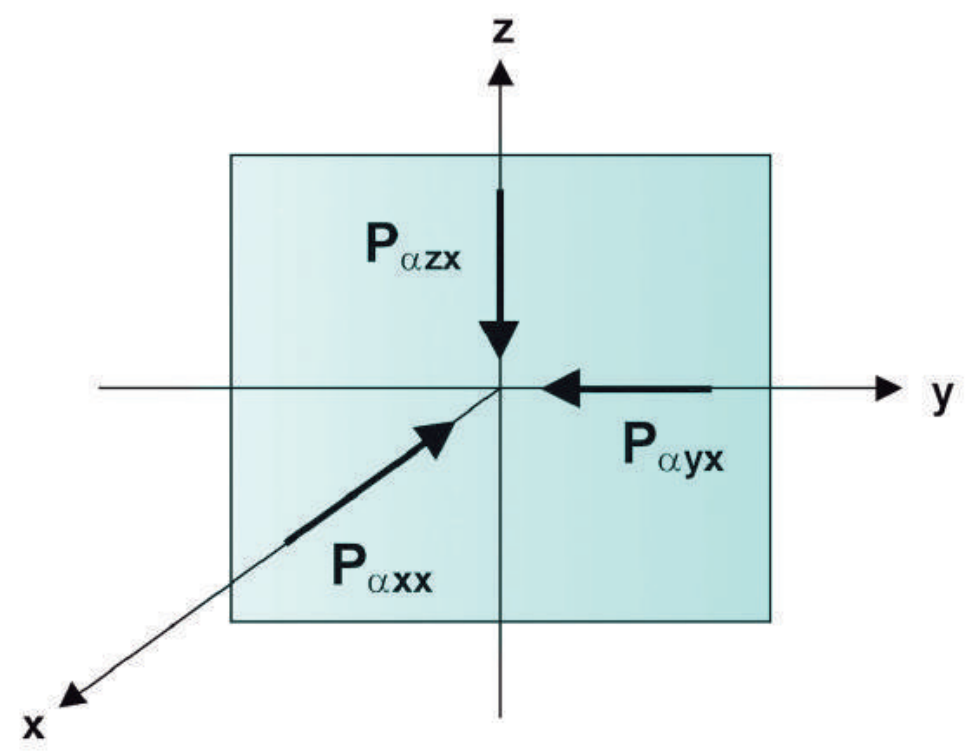

Fig. 5 Components of the pressure tensor $\mathcal{P}$ corresponding to the tangential shear stresses $P_{\alpha y x}$ and $P_{\alpha z x}$, and to the normal stress $P_{\alpha x x}$, acting on a surface element whose normal is oriented along the unit vector $\widehat{\mathbf{x}}$.

\subsection{Force per Unit Volume}

The force per unit volume inside the plasma, due to the random particle motions, can be obtained by integrating (6.5) over the closed surface $S$ bounding the volume element $V$, dividing the result by $V$, and then taking the limit as $V$ tends to zero. This procedure is just the definition of the divergence,

$$
-\nabla \cdot \mathcal{P}_{\alpha}=-\lim _{V \rightarrow 0}\left[\frac{1}{V} \oint_{S} \mathcal{P}_{\alpha} \cdot \widehat{\mathbf{n}} d S\right]
$$

and, from the Gauss's divergence theorem,

$$
-\oint_{S} \mathcal{P}_{\alpha} \cdot \widehat{\mathbf{n}} d S=-\int_{V} \nabla \cdot \mathcal{P}_{\alpha} d^{3} r
$$

We conclude, therefore, that the negative divergence of the kinetic pressure dyad $\left(-\nabla \cdot \mathcal{P}_{\alpha}\right)$ is the force exerted on a unit volume of the 
plasma due to the random particle motions, and $\mathcal{P}_{\alpha} \cdot \widehat{\mathbf{n}}$ is the force acting on a unit area of a surface normal to the unit vector $\widehat{\mathbf{n}}$.

\subsection{Scalar Pressure and Absolute Temperature}

An important macroscopic variable is the scalar pressure, or mean hydrostatic pressure, $p_{\alpha}$. It is defined as one-third the trace of the pressure tensor,

$$
p_{\alpha}=\frac{1}{3} \sum_{i, j} P_{\alpha i j} \delta_{i j}=\frac{1}{3} \sum_{i} P_{\alpha i i}=\frac{1}{3}\left(P_{\alpha x x}+P_{\alpha y y}+P_{\alpha z z}\right)
$$

where $\delta_{i j}$ is the Kronecker delta, defined such that $\delta_{i j}=1$ for $i=j$ and $\delta_{i j}=0$ for $i \neq j$. The pressure tensor elements $P_{\alpha i i}$, with $i=x, y, z$, are just the hydrostatic pressures normal to the surfaces described by $i=$ constant. Using (6.1),

$$
p_{\alpha}=\frac{1}{3} \rho_{m \alpha}<c_{\alpha x}^{2}+c_{\alpha y}^{2}+c_{\alpha z}^{2}>
$$

Since $c_{\alpha}^{2}=c_{\alpha x}^{2}+c_{\alpha y}^{2}+c_{\alpha z}^{2}$, we have

$$
p_{\alpha}=\frac{1}{3} \rho_{m \alpha}<c_{\alpha}^{2}>
$$

Another important parameter for a macroscopic description of a plasma is its temperature. The absolute temperature $T_{\alpha}$, for the type $\alpha$ particles, is a measure of the mean kinetic energy of the random particle motions. According to the thermodynamic definition of absolute temperature, there is a mean thermal energy of $k T_{\alpha i} / 2$ associated with each translational degree of freedom $(i=x, y, z)$, so that

$$
\frac{1}{2} k T_{\alpha i}=\frac{1}{2} m_{\alpha}<c_{\alpha i}^{2}>
$$

where $k$ is Boltzmann's constant.

When the distribution of random velocities is isotropic, as is the case of the Maxwell-Boltzmann distribution function (to be considered in the next chapter), which characterizes the state of thermal equilibrium of a gas, we have $c_{\alpha x}^{2}=c_{\alpha y}^{2}=c_{\alpha z}^{2}=c_{\alpha}^{2} / 3$, and therefore,

$$
p_{\alpha}=P_{\alpha x x}=P_{\alpha y y}=P_{\alpha z z}=\rho_{m \alpha}<c_{\alpha i}^{2}>
$$


Combining (6.13) and (6.12), gives

$$
p_{\alpha}=n_{\alpha} k T_{\alpha}
$$

which is the equation of state of an ideal gas. For the Maxwell-Boltzmann distribution function the nondiagonal elements of the kinetic pressure dyad are all zero and it reduces to

$$
\mathcal{P}_{\alpha}=(\widehat{\mathbf{x}} \widehat{\mathbf{x}}+\widehat{\mathbf{y}} \widehat{\mathbf{y}}+\widehat{\mathbf{z}} \widehat{\mathbf{z}}) p_{\alpha}=\mathbf{1} p_{\alpha}
$$

where 1 stands for the unit dyad, which in matrix form can be written as

$$
\mathbf{1}=\left(\begin{array}{lll}
1 & 0 & 0 \\
0 & 1 & 0 \\
0 & 0 & 1
\end{array}\right)
$$

In this case the negative divergence of the pressure dyad becomes

$$
-\nabla \cdot \mathcal{P}_{\alpha}=-\left(\widehat{\mathbf{x}} \frac{\partial}{\partial x} p_{\alpha}+\widehat{\mathbf{y}} \frac{\partial}{\partial y} p_{\alpha}+\widehat{\mathbf{z}} \frac{\partial}{\partial z} p_{\alpha}\right)=-\nabla p_{\alpha}
$$

Thus, for an isotropic velocity distribution, the force per unit volume due to the random variations of the peculiar velocities is given by the negative gradient of the scalar pressure.

In some problems a simplification of practical interest for the general form of the kinetic pressure dyad consists in taking

$$
\mathcal{P}_{\alpha}=\widehat{\mathbf{x}} \widehat{\mathbf{x}} P_{\alpha x x}+\widehat{\mathbf{y}} \widehat{\mathbf{y}} P_{\alpha y y}+\widehat{\mathbf{z}} \mathbf{z} P_{\alpha z z}
$$

or, in matrix form,

$$
\mathcal{P}_{\alpha}=\left(\begin{array}{ccc}
P_{\alpha x x} & 0 & 0 \\
0 & P_{\alpha y y} & 0 \\
0 & 0 & P_{\alpha z z}
\end{array}\right)
$$

where the diagonal elements are different from one another but all nondiagonal elements vanish. This expression corresponds to an anisotropy of the peculiar velocities and the absence of shear forces and viscous drag. The effects of viscosity and shear stresses are incorporated in the nondiagonal elements of the pressure dyad. Usually the effects of viscosity are relatively unimportant for most plasmas and the nondiagonal elements of $\mathcal{P}_{\alpha}$ can, in many cases, be neglected. 
In this anisotropic case, different absolute temperatures $T_{\alpha i}$ can be defined for each direction in space, according to (6.12).

\section{HEAT FLOW VECTOR}

The component of the heat flow vector, $q_{\alpha n}$, is defined as the flux of random or thermal energy across a surface whose normal points in the direction of the unit vector $\widehat{\mathbf{n}}$. Taking $\chi(\mathbf{r}, \mathbf{v}, t)$, in (3.11), as the kinetic energy of random motion of the particles of type $\alpha$, that is, $\chi=m_{\alpha} c_{\alpha}^{2} / 2$, we obtain for the component of the heat flow vector along $\widehat{\mathbf{n}}$,

$$
q_{\alpha n}=\mathbf{q}_{\alpha} \cdot \widehat{\mathbf{n}}=\frac{1}{2} \rho_{m \alpha}<c_{\alpha}^{2} \mathbf{c}_{\alpha} \cdot \widehat{\mathbf{n}}>
$$

The heat flow vector is therefore given by

$$
\mathbf{q}_{\alpha}=\frac{1}{2} \rho_{m \alpha}<c_{\alpha}^{2} \mathbf{c}_{\alpha}>
$$

\section{HEAT FLOW TRIAD}

It is convenient, at this point, to introduce a triad of thermal energy flux defined by

$$
\mathcal{Q}_{\alpha}=\rho_{m \alpha}<\mathbf{c}_{\alpha} \mathbf{c}_{\alpha} \mathbf{c}_{\alpha}>
$$

Its components are, explicity,

$$
Q_{\alpha i j k}=\rho_{m \alpha}<c_{\alpha i} c_{\alpha j} c_{\alpha k}>
$$

Using Cartesian coordinates, the thermal energy flux triad can be written in the form

$$
\mathcal{Q}_{\alpha}=\mathcal{Q}_{\alpha x} \widehat{\mathbf{x}}+\mathcal{Q}_{\alpha y} \widehat{\mathbf{y}}+\mathcal{Q}_{\alpha z} \widehat{\mathbf{z}}
$$

where each of the dyads $\mathcal{Q}_{\alpha n}$, with $n=x, y, z$, can be expressed in matrix form as

$$
\mathcal{Q}_{\alpha n}=\left(\begin{array}{lll}
Q_{\alpha x x n} & Q_{\alpha x y n} & Q_{\alpha x z n} \\
Q_{\alpha y x n} & Q_{\alpha y y n} & Q_{\alpha y z n} \\
Q_{\alpha z x n} & Q_{\alpha z y n} & Q_{\alpha z z n}
\end{array}\right)
$$

To obtain a relation between the heat flow vector $\mathbf{q}_{\alpha}$ and the thermal energy flux triad $\mathcal{Q}_{\alpha}$, note that (7.1) can be written as

$$
q_{\alpha n}=\frac{1}{2} \rho_{m \alpha}\left(<c_{\alpha x}^{2} c_{\alpha n}>+<c_{\alpha y}^{2} c_{\alpha n}>+<c_{\alpha z}^{2} c_{\alpha n}>\right)
$$


and comparing this equation with (8.2), we see that $q_{\alpha n}$ can be written as

$$
q_{\alpha n}=\frac{1}{2}\left(Q_{\alpha x x n}+Q_{\alpha y y n}+Q_{\alpha z z n}\right)
$$

\section{TOTAL ENERGY FLUX TRIAD}

In analogy with the definition of the heat flow triad $\mathcal{Q}_{\alpha}$ (with components denoted by $Q_{\alpha i j k}$ ), consider now the quantity

$$
E_{\alpha i j k}(\mathbf{r}, t)=\rho_{m \alpha}<v_{i} v_{j} v_{k}>_{\alpha}
$$

which represents one of the nine components of the total energy flux triad $\mathcal{E}_{\alpha}(\mathbf{r}, t)$. This quantity can be considered as the sum of three parts. Substituting $v_{i}=u_{\alpha i}+c_{\alpha i}$ in (9.1) and expanding,

$$
\begin{aligned}
<v_{i} v_{j} v_{k}>_{\alpha}= & <c_{\alpha i} c_{\alpha j} c_{\alpha k}+u_{\alpha i} c_{\alpha j} c_{\alpha k}+u_{\alpha j} c_{\alpha k} c_{\alpha i} \\
& +u_{\alpha k} c_{\alpha i} c_{\alpha j}+u_{\alpha i} u_{\alpha j} c_{\alpha k}+u_{\alpha j} u_{\alpha k} c_{\alpha i} \\
& +u_{\alpha k} u_{\alpha i} c_{\alpha j}+u_{\alpha i} u_{\alpha j} u_{\alpha k}>
\end{aligned}
$$

Noting that $\left\langle u_{\alpha i}\right\rangle=u_{\alpha i}$ and $\left\langle c_{\alpha i}\right\rangle=0$, and using (8.2) and (6.1), we obtain

$$
\rho_{m \alpha}<v_{i} v_{j} v_{k}>_{\alpha}=\rho_{m \alpha} u_{\alpha i} u_{\alpha j} u_{\alpha k}+\left(\mathbf{u}_{\alpha}, \mathcal{P}_{\alpha}\right)_{i j k}+Q_{\alpha i j k}
$$

where the following notation was used

$$
\left(\mathbf{u}_{\alpha}, \mathcal{P}_{\alpha}\right)_{i j k}=u_{\alpha i} P_{\alpha j k}+u_{\alpha j} P_{\alpha k i}+u_{\alpha k} P_{\alpha i j}
$$

Therefore, we can write (9.1) in triadic form as

$$
\mathcal{E}_{\alpha}(\mathbf{r}, t)=\rho_{m \alpha}<\mathbf{v} \mathbf{v} \mathbf{v}>_{\alpha}=\rho_{m \alpha} \mathbf{u}_{\alpha} \mathbf{u}_{\alpha} \mathbf{u}_{\alpha}+\left(\mathbf{u}_{\alpha}, \mathcal{P}_{\alpha}\right)+\mathcal{Q}_{\alpha}
$$

The total energy flux triad, therefore, can be considered as the sum of the energy flux transported by the convective particle motions, represented by the first two terms in the right-hand side of (9.5), and the thermal energy flux $\mathcal{Q}_{\alpha}$ due to the random thermal motions of the particles of type $\alpha$.

The physical interpretation of the heat flow triad $\mathcal{Q}_{\alpha}$ is, in some sense, analogous to the physical interpretation of the heat flow vector $\mathbf{q}_{\alpha}$. For this purpose, consider the quantity

$$
\frac{1}{2} \rho_{m \alpha}<v^{2} \mathbf{v}>_{\alpha}
$$


which represents the average energy flux transported by the particles of type $\alpha$. This quantity can be written as the sum of three terms. Substituting $\mathbf{v}=\mathbf{c}_{\alpha}+\mathbf{u}_{\alpha}$ in expression (9.6) and expanding,

$$
\begin{aligned}
\frac{1}{2} \rho_{m \alpha} & <v^{2} \mathbf{v}>_{\alpha}=\frac{1}{2} \rho_{m \alpha}<u_{\alpha}^{2} \mathbf{u}_{\alpha}+2\left(\mathbf{u}_{\alpha} \cdot \mathbf{c}_{\alpha}\right) \mathbf{u}_{\alpha} \\
& +c_{\alpha}^{2} \mathbf{u}_{\alpha}+u_{\alpha}^{2} \mathbf{c}_{\alpha}+2\left(\mathbf{u}_{\alpha} \cdot \mathbf{c}_{\alpha}\right) \mathbf{c}_{\alpha}+c_{\alpha}^{2} \mathbf{c}_{\alpha}>
\end{aligned}
$$

and since $\left\langle\mathbf{c}_{\alpha}\right\rangle=0$ and $\left\langle\mathbf{u}_{\alpha}\right\rangle=\mathbf{u}_{\alpha}$, we obtain

$$
\begin{gathered}
\frac{1}{2} \rho_{m \alpha}<v^{2} \mathbf{v}>_{\alpha}=\frac{1}{2} \rho_{m \alpha}\left(u_{\alpha}^{2}+<c_{\alpha}^{2}>\right) \mathbf{u}_{\alpha} \\
+\rho_{m \alpha} \mathbf{u}_{\alpha} \cdot<\mathbf{c}_{\alpha} \mathbf{c}_{\alpha}>+\frac{1}{2} \rho_{m \alpha}<c_{\alpha}^{2} \mathbf{c}_{\alpha}>
\end{gathered}
$$

If we now use (6.2) and (7.2), which define $\mathcal{P}_{\alpha}$ and $\mathbf{q}_{\alpha}$, respectively, we obtain the identity

$$
\frac{1}{2} \rho_{m \alpha}<v^{2} \mathbf{v}>_{\alpha}=W_{\alpha} \mathbf{u}_{\alpha}+\mathbf{u}_{\alpha} \cdot \mathcal{P}_{\alpha}+\mathbf{q}_{\alpha}
$$

where $W_{\alpha}$ is the mean kinetic energy density of the type $\alpha$ particles,

$$
W_{\alpha}=\frac{1}{2} \rho_{m \alpha} u_{\alpha}^{2}+\frac{1}{2} \rho_{m \alpha}<c_{\alpha}^{2}>
$$

Eq. (9.9) is written in a form analogous to (9.5). It shows that the flux (rate of transport per unit area) of the average energy of the type $\alpha$ particles can be separated into three parts: the first term in the right-hand side of (9.9) represents the flux of the mean kinetic energy transported convectively, the second term is the rate of work per unit area done by the kinetic pressure dyad, and the third term is the random thermal energy flux transported by the particles due to their random thermal motions. It is instructive to note that in a frame of reference moving with the average velocity $\mathbf{u}_{\alpha}(\mathbf{r}, t)$, the particle velocities become identical to their random velocities, that is, $\mathbf{v}=\mathbf{c}_{\alpha}$, so that (9.9) reduces to (7.2), which defines the thermal energy flux vector $\mathbf{q}_{\alpha}$.

When the thermal velocities $\mathbf{c}_{\alpha}$ are distributed uniformly in all directions, that is isotropically, it turns out that $\mathbf{q}_{\alpha}=0$ (since the integrand is an odd function of $\mathbf{c}_{\alpha}$ ). Consequently, $\mathbf{q}_{\alpha}$ can be considered as a partial measure of the anisotropies in the distribution of the thermal velocities. The thermal energy flux triad $\mathcal{Q}_{\alpha}$ considerably extends the concept of the heat flux vector and in this sense can be considered as a complete measure of the anisotropies in the distribution of the thermal velocities of the particles. 


\section{HIGHER MOMENTS OF THE DISTRIBUTION FUNCTION}

The first four moments of the distribution function $f_{\alpha}(\mathbf{r}, \mathbf{v}, t)$ are related, respectively, to the number density $n_{\alpha}(\mathbf{r}, t)$, to the average velocity $\mathbf{u}_{\alpha}(\mathbf{r}, t)$, to the momentum flow dyad $\Pi_{\alpha}(\mathbf{r}, t)$, and to the total energy flow triad $\mathcal{E}_{\alpha}(\mathbf{r}, t)$. For easy reference and convenience we gather here their mathematical expressions,

$$
\begin{gathered}
n_{\alpha}(\mathbf{r}, t)=\int_{v} f_{\alpha}(\mathbf{r}, \mathbf{v}, t) d^{3} v \\
u_{\alpha i}(\mathbf{r}, t)=<v_{i}>_{\alpha}=\frac{1}{n_{\alpha}(\mathbf{r}, t)} \int_{v} v_{i} f_{\alpha}(\mathbf{r}, \mathbf{v}, t) d^{3} v \\
\Pi_{\alpha i j}(\mathbf{r}, t)=\rho_{m \alpha}<v_{i} v_{j}>_{\alpha}=m_{\alpha} \int_{v} v_{i} v_{j} f_{\alpha}(\mathbf{r}, \mathbf{v}, t) d^{3} v \\
E_{\alpha i j k}(\mathbf{r}, t)=\rho_{m \alpha}<v_{i} v_{j} v_{k}>_{\alpha}=m_{\alpha} \int_{v} v_{i} v_{j} v_{k} f_{\alpha}(\mathbf{r}, \mathbf{v}, t) d^{3} v
\end{gathered}
$$

When the average velocity $\mathbf{u}_{\alpha}(\mathbf{r}, t)$ vanishes, we have $\mathbf{v}=\mathbf{c}_{\alpha}$, the momentum flow dyad $\Pi_{\alpha}$ becomes the same as the pressure dyad $\mathcal{P}_{\alpha}$, and the total energy flux triad $\mathcal{E}_{\alpha}$ becomes the same as the thermal energy flux $\operatorname{triad} \mathcal{Q}_{\alpha}$.

As a formal extension of these definitions we may, whenever necessary, consider higher moments of the distribution function. The moment of order $N$ can be defined by the expression

$$
M_{\alpha i j \ldots k}^{(N)}(\mathbf{r}, t)=\int_{v} v_{i} v_{j} \ldots v_{k} f_{\alpha}(\mathbf{r}, \mathbf{v}, t) d^{3} v
$$

where the velocity components $v_{i}$ appear $N$ times in the integrand of expression (10.5).

\section{PROBLEMS}

6.1 Consider a system of particles characterized by the distribution function given in problem 5.1 (in Chapter 5).

(a) Show that the absolute temperature of the system is given by

$$
T=\frac{m v_{0}^{2}}{3 k}
$$


where $m$ is the mass of each particle and $k$ is Boltzmann's constant.

(b) Obtain the following expression for the pressure dyad

$$
\mathcal{P}=\frac{1}{3} \rho_{m} v_{0}^{2} \mathbf{1}
$$

where $\rho_{m}=n m$ and $\mathbf{1}$ is the unit dyad.

(c) Verify that the heat flow vector $\mathbf{q}=0$.

6.2 Suppose that the peculiar (random) velocities of the electrons in a given plasma satisfy the following modified Maxwell-Boltzmann distribution function (considering $\mathbf{u}=0$ ),

$$
f(\mathbf{c})=n_{0}\left(\frac{m}{2 \pi k T_{\perp}}\right)\left(\frac{m}{2 \pi k T_{\|}}\right)^{1 / 2} \exp \left[-\frac{m}{2 k}\left(\frac{c_{x}^{2}+c_{y}^{2}}{T_{\perp}}+\frac{c_{z}^{2}}{T_{\|}}\right)\right]
$$

(a) Verify that the electron number density is given by $n_{0}$.

(b) Considering a Cartesian coordinate system with the $z$ axis coincident with the parallel direction, show that the kinetic pressure dyad is given by

$$
\mathcal{P}=n_{0} k\left[T_{\perp}(\widehat{\mathbf{x}} \widehat{\mathbf{x}}+\widehat{\mathbf{y}} \widehat{\mathbf{y}})+T_{\|} \widehat{\mathbf{z}} \widehat{\mathbf{z}}\right]
$$

which indicates the presence of an anisotropy in the $z$ direction.

(c) Calculate the heat flow vector $\mathbf{q}$.

(d) Show that

$$
\begin{aligned}
& \frac{1}{2} m<v_{\|}^{2}>=\frac{1}{2} k T_{\|} \\
& \frac{1}{2} m<v_{\perp}^{2}>=k T_{\perp}
\end{aligned}
$$

6.3 For the loss-cone distribution function of problem 5.3 (in Chapter 5), show that

$$
\begin{aligned}
& \frac{1}{2} m<v_{\|}^{2}>=\frac{1}{4} m \alpha_{\|}^{2} \\
& \frac{1}{2} m<v_{\perp}^{2}>=m \alpha_{\perp}^{2}
\end{aligned}
$$

Compare these results with those of problem 6.2(d) and provide physical arguments to justify the difference in the perpendicular part of the average thermal energy.

6.4 Verify, by symmetry arguments, that there are only ten independent elements in the thermal energy flux triad $\mathcal{Q}$. Note that, according to its definition, $Q_{i j k}=n m<c_{i} c_{j} c_{k}>$ is symmetric under the interchange of any two of its three indices. 
6.5 A plasma is made up of a mixture of various particle species, the type $\alpha$ species having mass $m_{\alpha}$, number density $n_{\alpha}$, average macroscopic velocity $\mathbf{u}_{\alpha}$, random velocity $\mathbf{c}_{\alpha}=\mathbf{v}-\mathbf{u}_{\alpha}$, scalar pressure $p_{\alpha}=n_{\alpha} k T_{\alpha}$, temperature $\left.T_{\alpha}=\left(m_{\alpha} / 3 k\right)<c_{\alpha}^{2}\right\rangle$, pressure dyad $\mathcal{P}_{\alpha}=n_{\alpha} m_{\alpha}\left\langle\mathbf{c}_{\alpha} \mathbf{c}_{\alpha}\right\rangle$, and heat flow vector $q_{\alpha}=\left(n_{\alpha} m_{\alpha} / 2\right)<c_{\alpha}^{2} \mathbf{c}_{\alpha}>$. Similar quantities can be defined for the plasma as a whole, for example, we can define the total number density by

$$
n_{0}=\sum_{\alpha} n_{\alpha}
$$

the average mass by

$$
m_{0}=\frac{1}{n_{0}} \sum_{\alpha} n_{\alpha} m_{\alpha}
$$

and the average flow velocity by

$$
\mathbf{u}_{0}=\frac{1}{n_{0} m_{0}} \sum_{\alpha} n_{\alpha} m_{\alpha} \mathbf{u}_{\alpha}
$$

We can also define an alternative random velocity for the type $\alpha$ species, with reference to $\mathbf{u}_{0}$, as $\mathbf{c}_{\alpha 0}=\mathbf{v}-\mathbf{u}_{0}$, as well as an alternative absolute temperature by

$$
T_{\alpha 0}=\frac{m_{\alpha}<c_{\alpha 0}^{2}>}{3 k}
$$

a corresponding pressure dyad by

$$
\mathcal{P}_{\alpha 0}=n_{\alpha} m_{\alpha}<\mathbf{c}_{\alpha 0} \mathbf{c}_{\alpha 0}>
$$

and heat flow vector by

$$
\mathbf{q}_{\alpha 0}=\frac{1}{2} n_{\alpha} m_{\alpha}<c_{\alpha 0}^{2} \mathbf{c}_{\alpha 0}>
$$

(a) Show that, for the plasma as a whole, the total pressure dyad is given by

$$
\mathcal{P}_{0}=\sum_{\alpha}\left(\mathcal{P}_{\alpha}+n_{\alpha} m_{\alpha} \mathbf{w}_{\alpha} \mathbf{w}_{\alpha}\right)
$$

and the total scalar pressure by

$$
p_{0}=\sum_{\alpha}\left(p_{\alpha}+\frac{1}{3} n_{\alpha} m_{\alpha} w_{\alpha}^{2}\right)
$$


where $\mathbf{w}_{\alpha}=\mathbf{u}_{\alpha}-\mathbf{u}_{0}$ is the macroscopic diffusion velocity.

(b) If $\mathbf{c}_{\alpha}$ is isotropic, that is, $\left\langle c_{\alpha i}^{2}\right\rangle=(1 / 3)<c_{\alpha}^{2}>$, for $i=x, y, z$, show that the total heat flow vector is given by

$$
\mathbf{q}_{0}=\sum_{\alpha}\left(\mathbf{q}_{\alpha}+\frac{5}{2} p_{\alpha} \mathbf{w}_{\alpha}+\frac{1}{2} n_{\alpha} m_{\alpha} w_{\alpha}^{2} \mathbf{w}_{\alpha}\right)
$$

(c) If an average temperature $T_{0}$, for the plasma as a whole, is defined by requiring that $p_{0}=n_{0} k T_{0}$, show that

$$
T_{0}=\frac{1}{n_{0}} \sum_{\alpha} n_{\alpha}\left(T_{\alpha}+\frac{m_{\alpha} w_{\alpha}^{2}}{3 k}\right)
$$

(d) Verify that

$$
\frac{1}{2} \sum_{\alpha} n_{\alpha} m_{\alpha}<c_{\alpha 0}^{2}>=\frac{3}{2} n_{0} k T_{0}
$$

so that there is an average thermal energy of $k T_{0} / 2$ per degree of freedom.

6.6 (a) Show that the time rate of increase of momentum in an infinitesimal volume element $d^{3} r=d x d y d z$ inside a gas of number density $n$, as a result of particles of mass $m$ entering $d^{3} r$ with average velocity $\mathbf{u}$, is given by $-\nabla \cdot(n m \mathbf{u u}) d^{3} r$.

(b) If the infinitesimal volume element $d^{3} r$ moves with the average particle velocity $\mathbf{u}$, show that, because of the work done by the kinetic pressure dyad $\mathcal{P}$, the particle energy inside $d^{3} r$ increases at a time rate given by $-\nabla \cdot(\mathbf{u} \cdot \mathcal{P}) d^{3} r$.

(c) Verify, by expansion, that $(\mathcal{P} \cdot \widehat{\mathbf{n}}) \cdot \mathbf{u}=(\mathbf{u} \cdot \mathcal{P}) \cdot \widehat{\mathbf{n}}$, where $\widehat{\mathbf{n}}$ denotes an outward unit vector, normal to the surface bounding the volume element.

6.7 Consider (5.6.4), which is the solution of the Boltzmann equation with the relaxation model for the collision term, in the absence of external forces and spatial gradients, and when $f_{\alpha_{0}}$ and the relaxation time $\tau$ are time-independent. Show that, according to this simplified equation, we have

$$
G_{\alpha}(t)=G_{\alpha 0}+\left[G_{\alpha}(0)-G_{\alpha 0}\right] \exp (-t / \tau)
$$

where

$$
\begin{aligned}
& G_{\alpha}(t)=\int_{v} f_{\alpha} \chi d^{3} v=n_{\alpha}<\chi>_{\alpha} \\
& G_{\alpha 0}=\int_{v} f_{\alpha 0} \chi d^{3} v=n_{\alpha}<\chi>_{\alpha 0}
\end{aligned}
$$

Thus, according to the relaxation model for the collision term, every average value $\langle\chi\rangle_{\alpha}$ approaches equilibrium with the same relaxation time. 


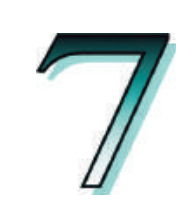

\section{THE EQUILIBRIUM STATE}

\section{THE EQUILIBRIUM STATE DISTRIBUTION FUNCTION}

The equilibrium distribution function is the time-independent solution of the Boltzmann equation in the absence of external forces. In the equilibrium state the particle interactions do not cause any change in the distribution function with time and there are no spatial gradients in the particle number density. We deduce in this section an expression for the equilibrium distribution function, known as the Maxwell-Boltzmann or Maxwellian velocity distribution function.

For simplicity we will consider a gas consisting of only one particle species. The extension to a mixture will be indicated in a subsequent section of this chapter. We assume that there are no external forces acting on the system $\left(\mathbf{F}_{\text {ext }}=0\right)$ and that the particles are uniformly distributed in space. Under these conditions the distribution function is homogeneous $(\nabla f=0)$ and, since we are looking for a steady-state solution of the Boltzmann equation, it is also time-independent $(\partial f / \partial t=0)$. Therefore, it can be denoted by $f(\mathbf{v})$. According to the Boltzmann equation (5.5.27) (Eq. 5.27 in Chapter 5), the equilibrium distribution function satisfies the following condition:

$$
\left(\frac{\delta f}{\delta t}\right)_{\text {coll }}=0
$$

Hence, under equilibrium conditions, there are no changes in the distribution function as a result of collisions between the particles. In Chapter 21 we shall derive the expression for the equilibrium distribution function using the Boltzmann collision integral. For the moment, however, in order 
to simplify matters, it is appropriate to consider a simple derivation based on the general principle of detailed balance of statistical mechanics.

\subsection{The General Principle of Detailed Balance and Binary Collisions}

In general, this principle asserts that, under equilibrium conditions, the probability of occurrence of any physical process is equal to the probability of occurrence of the inverse process. Hence, for the case of a system of interacting particles in the state of equilibrium, the principle of detailed balance asserts that the effect of each type of collision is exactly compensated by the effect of the corresponding inverse collision.

Consider an elastic collision between two particles having velocities $\mathbf{v}$ and $\mathbf{v}_{1}$ before collision, and $\mathbf{v}^{\prime}$ and $\mathbf{v}_{1}^{\prime}$ after collision. The corresponding inverse collision refers to an elastic collision in which a particle with initial velocity $\mathbf{v}^{\prime}$ collides with another particle with velocity $\mathbf{v}_{1}^{\prime}$, the velocities after collision being $\mathbf{v}$ and $\mathbf{v}_{1}$, respectively. Such events are illustrated schematically in Fig. 1, in a reference frame in which one of the particles is at rest.

Assuming that the velocities of the particles before collision are uncorrelated, the number of binary collisions occurring per unit time in a given volume $d^{3} r$, about the position $\mathbf{r}$ in configuration space, between particles having velocities within the velocity space element $d^{3} v$, about $\mathbf{v}$, and particles with velocities within $d^{3} v_{1}$, about $\mathbf{v}_{1}$, in the same configuration space element $d^{3} r$ (see Fig. 2), is proportional to the product of the respective number of particles, that is, to $\left(f d^{3} r d^{3} v\right)\left(f_{1} d^{3} r d^{3} v_{1}\right)$, where $f_{1}$ represents $f\left(\mathbf{v}_{1}\right)$.

In a similar way, assuming the particle velocities to be uncorrelated, the number of corresponding inverse binary collisions occurring per unit time in the same volume element $d^{3} r$, about $\mathbf{r}$, in configuration space, between particles having velocities within $d^{3} v^{\prime}$, about $\mathbf{v}^{\prime}$, and particles with velocities within $d^{3} v_{1}^{\prime}$, about $\mathbf{v}_{1}^{\prime}$, is proportional to the product $\left(f^{\prime} d^{3} r d^{3} v^{\prime}\right)\left(f_{1}^{\prime} d^{3} r d^{3} v_{1}^{\prime}\right)$, where $f^{\prime}=f\left(\mathbf{v}^{\prime}\right)$ and $f_{1}^{\prime}=f\left(\mathbf{v}_{1}^{\prime}\right)$.

According to the principle of detailed balance, in the equilibrium state the effect of each direct collision is compensated by the effect of the corresponding inverse collision, so that

$$
f f_{1} d^{3} v d^{3} v_{1}=f^{\prime} f_{1}^{\prime} d^{3} v^{\prime} d^{3} v_{1}^{\prime}
$$



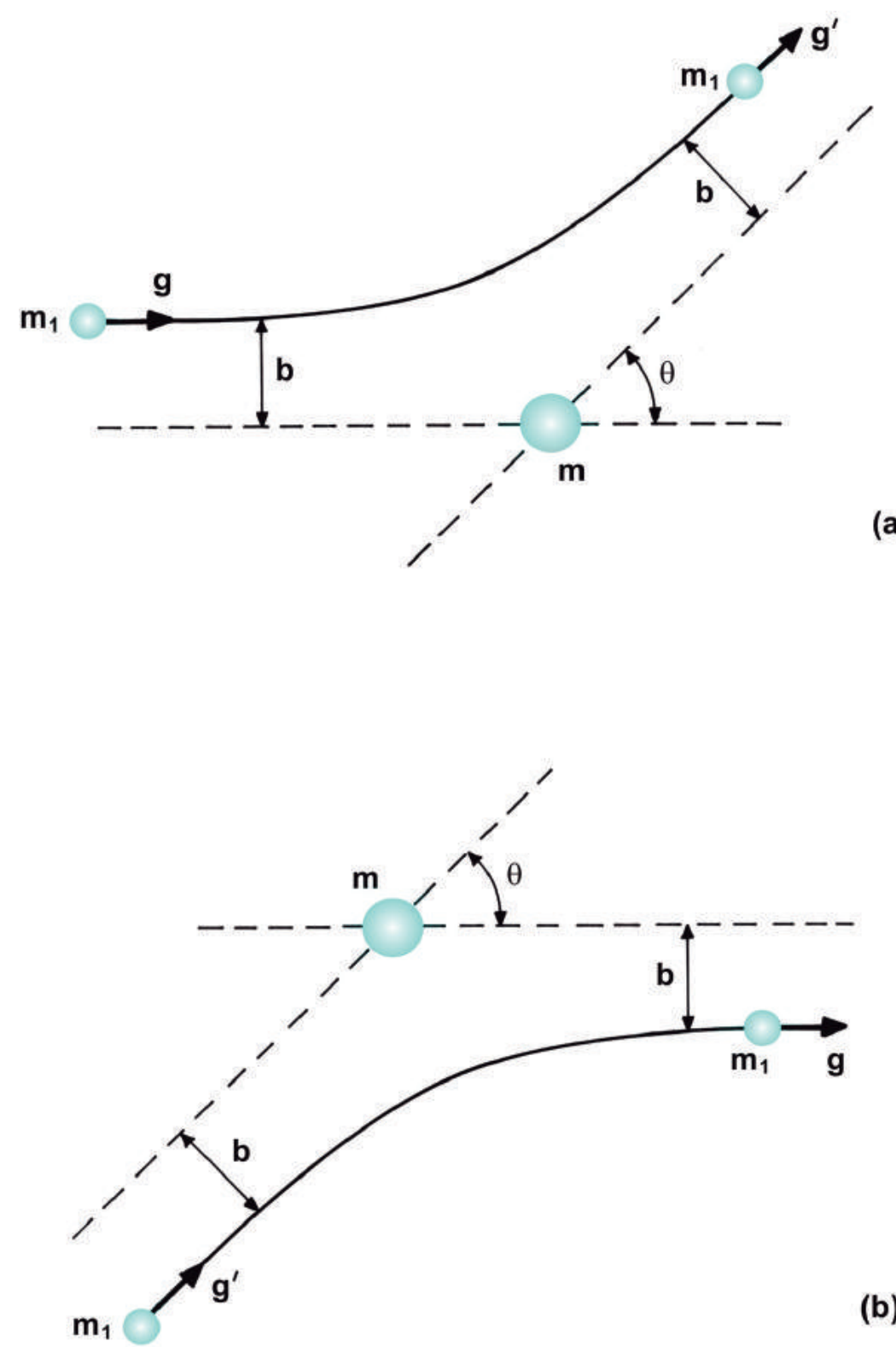

(a)

(b)

Fig. 1 (a) Direct binary collision and (b) the corresponding inverse binary collision. Here $\mathbf{g}=\mathbf{v}_{1}-\mathbf{v}$ and $\mathbf{g}^{\prime}=\mathbf{v}_{1}^{\prime}-\mathbf{v}^{\prime}$.

Since it can be shown that we have $d^{3} v d^{3} v_{1}=d^{3} v^{\prime} d^{3} v_{1}^{\prime}$ (see section 2 in Chapter 21), Eq. (1.2) yields

$$
f(\mathbf{v}) f\left(\mathbf{v}_{1}\right)=f\left(\mathbf{v}^{\prime}\right) f\left(\mathbf{v}_{1}^{\prime}\right)
$$




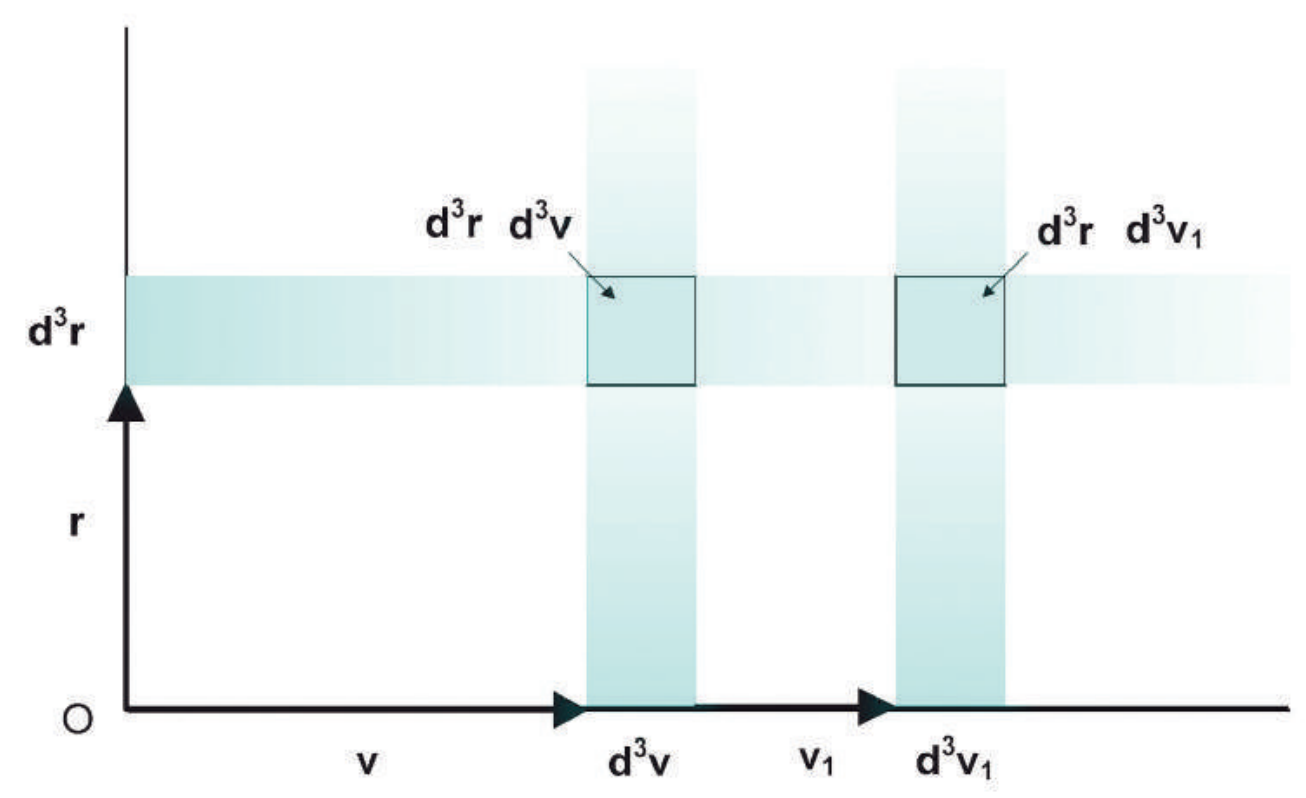

Fig. 2 Schematic representation of the volume elements $d^{3} r d^{3} v$ and $d^{3} r d^{3} v_{1}$ in phase space.

The assumption that the particle velocities are uncorrelated is known as the molecular chaos assumption. It is well justified when the density of the gas is sufficiently small so that the mean free path is larger than the characteristic range of the interparticle forces. Although this is certainly not a general situation for a plasma, the validity of the Maxwell-Boltzmann distribution function is very well justified experimentally.

\subsection{Summation Invariants}

It is convenient to introduce at this moment the concept of summation invariants. Consider a collision between two particles and let $\chi(\mathbf{v})$ be a physical quantity (scalar or vector) associated with each particle, which in general may be a function of the particle velocity. If the sum of the quantity $\chi(\mathbf{v})$ for the two particles is conserved during the collision process, then $\chi(\mathbf{v})$ is called a summation invariant. For a collision between two particles having initial velocities $\mathbf{v}$ and $\mathbf{v}_{1}$, and velocities after collision $\mathbf{v}^{\prime}$ and $\mathbf{v}_{1}^{\prime}$, respectively, we have for a summation invariant quantity

$$
\chi(\mathbf{v})+\chi\left(\mathbf{v}_{1}\right)=\chi\left(\mathbf{v}^{\prime}\right)+\chi\left(\mathbf{v}_{1}^{\prime}\right)
$$


From the laws of conservation of mass, of momentum, and of energy, it is clear that these physical quantities are summation invariants in binary elastic collisions. Denoting the masses of the two colliding particles by $m$ and $m_{1}$, we can express the laws of conservation of mass, of momentum, and of energy as

$$
\begin{gathered}
m+m_{1}=m+m_{1} \\
m \mathbf{v}+m_{1} \mathbf{v}_{1}=m \mathbf{v}^{\prime}+m_{1} \mathbf{v}_{1}^{\prime} \\
\frac{1}{2} m v^{2}+\frac{1}{2} m_{1} v_{1}^{2}=\frac{1}{2} m\left(\mathbf{v}^{\prime}\right)^{2}+\frac{1}{2} m_{1}\left(\mathbf{v}_{1}^{\prime}\right)^{2}
\end{gathered}
$$

Eq. (1.5) is a trivial one and does not lead to any new information. It only indicates that a numerical constant is a summation invariant. Eq. (1.6) represents three equations, one for each component of the momentum. The four equations in (1.6) and (1.7), together with the equations involving the impact parameter $b$ and the angle of the collision plane $\epsilon$ (for more details refer to the analysis of the dynamics of binary collisions, presented in Chapter 20), constitute six equations to be solved for the determination of the six unknown quantities, which are the components of the after-collision velocities $\mathbf{v}^{\prime}$ and $\mathbf{v}_{1}^{\prime}$, in terms of the initial velocities $\mathbf{v}$ and $\mathbf{v}_{1}$. The binary collision problem, therefore, is uniquely determined by these summation invariants. Any other summation invariant in the collision process gives no additional information and cannot be an independent one and may be expressed as a linear combination of the summation invariants defined by (1.5), (1.6), and (1.7).

\subsection{Maxwell-Boltzmann Distribution Function}

We proceed now to derive the equilibrium velocity distribution function starting from (1.3) and the concept of summation invariants. Taking the natural logarithm of both sides of (1.3) gives

$$
\ln f+\ln f_{1}=\ln f^{\prime}+\ln f_{1}^{\prime}
$$

which shows that $\ln f$ is a summation invariant in the collision process. Therefore, it can be written as a linear combination of the summation invariants $m, m \mathbf{v}$, and $m v^{2} / 2$,

$$
\ln f=m\left(a_{0}+\mathbf{a}_{1} \cdot \mathbf{v}-\frac{1}{2} a_{2} v^{2}\right)
$$


where $a_{0}, \mathbf{a}_{1}=a_{1 x} \widehat{\mathbf{x}}+a_{1 y} \widehat{\mathbf{y}}+a_{1 z} \widehat{\mathbf{z}}$, and $a_{2}$ are constants. The negative sign in front of $a_{2}$ is chosen for convenience in the equations that follow. Completing the squares in the right-hand side of (1.9), using Cartesian coordinates, gives

$$
\begin{gathered}
\ln f=m\left[a_{0}+\left(a_{1 x}^{2}+a_{1 y}^{2}+a_{1 z}^{2}\right) /\left(2 a_{2}\right)\right] \\
-\frac{1}{2} m a_{2}\left[\left(v_{x}-a_{1 x} / a_{2}\right)^{2}+\left(v_{y}-a_{1 y} / a_{2}\right)^{2}\right. \\
\left.\quad+\left(v_{z}-a_{1 z} / a_{2}\right)^{2}\right] \\
=m\left[a_{0}+a_{1}^{2} /\left(2 a_{2}\right)\right]-\frac{1}{2} m a_{2}\left(\mathbf{v}-\mathbf{a}_{1} / a_{2}\right)^{2}
\end{gathered}
$$

Defining new constants by

$$
\begin{gathered}
\ln C=m\left[a_{0}+a_{1}^{2} /\left(2 a_{2}\right)\right] \\
\mathbf{v}_{0}=\mathbf{a}_{1} / a_{2}
\end{gathered}
$$

we can write (1.10) in the form

$$
f=C \exp \left[-\frac{1}{2} m a_{2}\left(\mathbf{v}-\mathbf{v}_{0}\right)^{2}\right]
$$

This expression is known as the Maxwell-Boltzmann, or Maxwellian equilibrium distribution function.

\subsection{Determination of the Constant Coefficients}

The Maxwellian distribution function (1.13) contains five constant coefficients to be determined, namely $C, a_{2}, v_{0 x}, v_{0 y}$, and $v_{0 z}$. Note that this is exactly the same number of coefficients in the original equation (1.9). These constants can be expressed in terms of observable physical properties of the system, such as the number density $n$, the average velocity $\mathbf{u}$, and the kinetic temperature $T$ (or the scalar pressure $p$, since from the equation of state we have $p=n k T)$. To relate the observables $n, \mathbf{u}$, and $T$ with the constant coefficients $C, a_{2}$, and $\mathbf{v}_{0}$, we proceed as follows.

From the definition of the number density we must have

$$
n=\int_{v} f d^{3} v
$$

Substituting the Maxwellian distribution function (1.13) into (1.14), results in

$$
n=C \int_{v} \exp \left[-\frac{1}{2} m a_{2}\left(\mathbf{v}-\mathbf{v}_{0}\right)^{2}\right] d^{3} v
$$


If we use the notation $A=m a_{2} / 2$ and $\xi_{i}=\left(v_{i}-v_{0 i}\right)$, with $i=x, y, z$, (1.15) becomes

$$
n=C \iiint_{-\infty}^{+\infty} \exp \left[-A\left(\xi_{x}^{2}+\xi_{y}^{2}+\xi_{z}^{2}\right)\right] d \xi_{x} d \xi_{y} d \xi_{z}
$$

Performing the integrals over all possible values of $\xi_{x}, \xi_{y}$, and $\xi_{z}$, yields

$$
n=C\left(\frac{\pi}{A}\right)^{3 / 2}=C\left(\frac{2 \pi}{m a_{2}}\right)^{3 / 2}
$$

From the definition of the average velocity we have

$$
\mathbf{u}=<\mathbf{v}>=\frac{1}{n} \int_{v} f \mathbf{v} d^{3} v
$$

and substituting the Maxwellian distribution function (1.13),

$$
\mathbf{u}=\frac{C}{n} \int_{v} \mathbf{v} \exp \left[-\frac{1}{2} m a_{2}\left(\mathbf{v}-\mathbf{v}_{0}\right)^{2}\right] d^{3} v
$$

Using the same notation as in (1.16), we can write

$$
\begin{aligned}
\mathbf{u}=\frac{C}{n} \iiint_{-\infty}^{+\infty}\left(\xi_{x} \widehat{\mathbf{x}}+\xi_{y} \widehat{\mathbf{y}}+\xi_{z} \widehat{\mathbf{z}}\right) \exp \left[-A\left(\xi_{x}^{2}+\xi_{y}^{2}+\xi_{z}^{2}\right)\right] d \xi_{x} d \xi_{y} d \xi_{z} \\
+\frac{C}{n} \mathbf{v}_{0} \iiint_{-\infty}^{+\infty} \exp \left[-A\left(\xi_{x}^{2}+\xi_{y}^{2}+\xi_{z}^{2}\right)\right] d \xi_{x} d \xi_{y} d \xi_{z}
\end{aligned}
$$

The first triple integral in the right-hand side of (1.20), over all possible values of $\xi_{x}, \xi_{y}$, and $\xi_{z}$, vanishes, since the integrand is an odd function of $\xi_{i}$. According to (1.16) the second triple integral is equal to $n / C$. Thus, we obtain

$$
\mathbf{u}=\mathbf{v}_{0}
$$

which shows that the constant $\mathbf{v}_{0}$ represents the average (flow) velocity of the particles. Recall that the particle velocity $\mathbf{v}$ can be written as the sum of the peculiar (random) velocity $\mathbf{c}$ and the average velocity $\mathbf{u}$, that is, $\mathbf{v}=\mathbf{c}+\mathbf{u}$. If the system has no translational motion as a whole, then $\mathbf{v}_{0}=\mathbf{u}=0$.

Consider now the thermodynamic definition of the kinetic temperature $T$,

$$
\frac{3}{2} n k T=\frac{1}{2} n m<c^{2}>=\frac{1}{2} m \int_{v} f c^{2} d^{3} v
$$


where $k$ is Boltzmann's constant. Substituting the Maxwellian distribution function (1.13), noting that $\mathbf{c}=\mathbf{v}-\mathbf{u}$ and $d^{3} v=d^{3} c$, we obtain

$$
\frac{3}{2} n k T=\frac{1}{2} m C \int_{c} c^{2} \exp \left(-A c^{2}\right) d^{3} c
$$

Performing the triple integral over all possible values of $c_{x}, c_{y}$, and $c_{z}$, gives

$$
k T=\left(\frac{C}{n a_{2}}\right)\left(\frac{2 \pi}{m a_{2}}\right)^{3 / 2}
$$

We can now solve (1.17) and (1.24) for $C$ and $a_{2}$, to obtain

$$
\begin{gathered}
C=n\left(\frac{m}{2 \pi k T}\right)^{3 / 2} \\
a_{2}=\frac{1}{k T}
\end{gathered}
$$

Substituting the results just obtained into (1.13), the Maxwellian distribution of random velocities becomes

$$
f(c)=n\left(\frac{m}{2 \pi k T}\right)^{3 / 2} \exp \left(-\frac{m c^{2}}{2 k T}\right)
$$

This is the equilibrium distribution function for a system of particles uniformly distributed in space and free from the action of external forces. Note that the number density $n$ and the temperature $T$ are constants, independent of $\mathbf{r}$ and $t$. This distribution function represents the only permanent mode for the distribution of the particle velocities in the gas, for specified values of $n$ and $T$. Whatever may be the velocity distribution function of a gas, initially not in equilibrium, it tends to the distribution function (1.27) in the course of time, if the gas is maintained isolated from the action of external forces.

When the system has no translational motion as a whole (if it is maintained inside a container, for example), the average (flow) velocity $\mathbf{u}$ is zero and consequently we have $\mathbf{c}=\mathbf{v}$ in (1.27). The equilibrium distribution function depends only on the magnitude of the random velocity $\mathbf{c}$ so that, when a perfectly reflecting surface is immersed in the gas, $f(c)$ remains unchanged since the magnitude of the random velocity does not change when the particles are reflected at the surface.

For a plasma under equilibrium conditions, in which the various particle species such as electrons, ions, and neutrals have the same temperature, the random velocities of each species are separately described by a 
Maxwell-Boltzmann distribution function with the corresponding number density.

\subsection{Local Maxwell-Boltzmann Distribution Function}

In many situations of interest we are dealing with a gas that, although not in equilibrium, is not very far from it. It is then a good approximation to consider that, in the neighborhood of any point in the gas, there is an equilibrium situation described by a local Maxwell-Boltzmann distribution function of the form

$$
f(\mathbf{r}, \mathbf{v}, t)=n(\mathbf{r}, t)\left[\frac{m}{2 \pi k T(\mathbf{r}, t)}\right]^{3 / 2} \exp \left\{-\frac{m[\mathbf{v}-\mathbf{u}(\mathbf{r}, t)]^{2}}{2 k T(\mathbf{r}, t)}\right\}
$$

where the number density $n$, the temperature $T$, and the average velocity $\mathbf{u}$ are slowly varying functions of $\mathbf{r}$ and $t$.

\section{THE MOST PROBABLE DISTRIBUTION}

We have seen that the Maxwell-Boltzmann distribution function is the solution of the Boltzmann equation representing the equilibrium state of a gas, in the absence of external forces. One of the important conclusions obtained from the derivation of this distribution function is that it is independent of the cross section for the particle collisions. This means that the Maxwell-Boltzmann function is, in a certain way, universal in the description of the equilibrium state, and it should be possible to derive it without explicitly considering the particle interactions. A derivation in these terms is in fact presented in statistical mechanics, where it is shown that the Maxwell-Boltzmann distribution function represents the most probable distribution satisfying the macroscopic conditions (or constraints) imposed on the system.

In statistical mechanics, to a given macroscopic system there corresponds a very large number of possible microscopic states that lead to the same macroscopic parameters specifying the system, such as number density $n$, average velocity $\mathbf{u}$, and absolute temperature $T$. Each microscopic state is considered to be equiprobable. If we choose, at random, any particular microscopic state for the system, among all the possible microscopic states consistent with the specified macroscopic parameters (such as $n, \mathbf{u}, T$ ), the probability of choosing a Maxwellian distribution is overwhelmingly larger than that of any other distribution. It is also 
shown that the entropy is proportional to the probability of having a given distribution. Consequently, the state having maximum entropy is the most probable state consistent with the macroscopic constraints imposed on the system.

The meaning of the Maxwell-Boltzmann distribution function can be further illustrated by the following example. If a dilute gas is prepared in an arbitrary nonequilibrium initial state, and if there are interactions between the particles so as to allow the gas to pass from the initial state to other states, as time passes the gas will certainly reach the Maxwellian state, since essentially almost all possible microscopic states, consistent with the macroscopic constraints, have a Maxwellian distribution.

The statistical mechanics derivation of the most probable distribution function provides information only on the equilibrium state, and cannot possibly tell, for example, how long (which depends on the collision cross section) a given distribution function, initially not the equilibrium one, takes to become Maxwellian. The Boltzmann equation, on the other hand, is much more general and provides information also for nonequilibrium situations.

\section{MIXTURE OF VARIOUS PARTICLE SPECIES}

For the case of a mixture containing different species of particles, each species having its own number density $n_{\alpha}$, average velocity $\mathbf{u}_{\alpha}$, and temperature $T_{\alpha}$, we can still perform a calculation to determine the most probable distribution subjected to these macroscopic constraints. This requires only that we set $f_{\alpha} f_{\alpha 1}=f_{\alpha}^{\prime} f_{\alpha 1}^{\prime}$ for each particle species, but not necessarily $f_{\alpha} f_{\beta 1}=f_{\alpha}^{\prime} f_{\beta 1}^{\prime}$ for $\alpha \neq \beta$. This condition, therefore, does not describe an equilibrium situation, unless the temperatures and mean velocities of all species are the same. To determine the most probable distribution function for this nonequilibrium gas mixture (each species having its own number density, mean velocity, and temperature), we independently apply (1.3) to each species, in order to maximize the entropy for each species. This also maximizes the entropy for the gas mixture under the specified macroscopic constraints. The problem is completely analogous to the one just solved for a one-component gas and leads, in identical fashion, to

$$
f_{\alpha}(v)=n_{\alpha}\left(\frac{m_{\alpha}}{2 \pi k T_{\alpha}}\right)^{3 / 2} \exp \left[-\frac{m_{\alpha}\left(\mathbf{v}-\mathbf{u}_{\alpha}\right)^{2}}{2 k T_{\alpha}}\right]
$$

Thus, each species has a Maxwellian distribution of velocities, but with its own density, average velocity, and temperature. Although this is not 
an equilibrium distribution for the system, since the equilibrium condition $f_{\alpha} f_{\beta 1}=f_{\alpha}^{\prime} f_{\beta 1}^{\prime}$ for all $\alpha$ and $\beta$ is not satisfied, it is, nevertheless, the most probable distribution under the specified constraints. Only if the temperatures and average velocities of all species are equal will this be an equilibrium situation. Indeed, if two systems with different species and at different temperatures are brought together, then, as time passes, there will be a transfer of energy through collisions between the different species, until equilibrium is reached with the various species at the same temperature and mean velocity.

\section{PROPERTIES OF THE MAXWELL-BOLTZMANN DISTRIBUTION FUNCTION}

Due to the importance of the equilibrium distribution function, we present in this section some of its basic properties. We consider a gas in thermal equilibrium having no average (flow) velocity, $\mathbf{u}=0$. If, however, this average velocity is not zero, we suppose that the observer is moving with the average velocity of the gas. Thus, in either case, $\mathbf{v}=\mathbf{c}$. According to the definition of the distribution function, the number of particles per unit volume having velocities between $\mathbf{v}$ and $\mathbf{v}+d \mathbf{v}$ is given by

$$
f(v) d^{3} v=n\left(\frac{m}{2 \pi k T}\right)^{3 / 2} \exp \left(-\frac{m v^{2}}{2 k T}\right) d^{3} v
$$

\subsection{Distribution of a Velocity Component}

The distribution function for one component of the velocity, $g\left(v_{i}\right)$, is defined such that $g\left(v_{i}\right) d v_{i}$ represents the number of particles per unit volume that have the $i$ component of the velocity between $v_{i}$ and $v_{i}+d v_{i}$, irrespective of the values of the other two velocity components.

For the $x$ component, for example, $g\left(v_{x}\right) d v_{x}$ is obtained by integrating $f(v)$ over all possible values of the velocity components $v_{y}$ and $v_{z}$,

$$
g\left(v_{x}\right) d v_{x}=\int_{v_{y}} \int_{v_{z}} f(v) d v_{x} d v_{y} d v_{z}
$$

Substituting the Maxwell-Boltzmann distribution function, yields

$$
g\left(v_{x}\right) d v_{x}=n\left(\frac{m}{2 \pi k T}\right)^{3 / 2} \exp \left(-\frac{m v_{x}^{2}}{2 k T}\right) d v_{x} \int_{-\infty}^{+\infty} \exp \left(-\frac{m v_{y}^{2}}{2 k T}\right) d v_{y}
$$




$$
\int_{-\infty}^{+\infty} \exp \left(-\frac{m v_{z}^{2}}{2 k T}\right) d v_{z}
$$

Each integral in $(4.3)$ is equal to $(2 \pi k T / m)^{1 / 2}$. Therefore,

$$
g\left(v_{x}\right) d v_{x}=n\left(\frac{m}{2 \pi k T}\right)^{1 / 2} \exp \left(-\frac{m v_{x}^{2}}{2 k T}\right) d v_{x}
$$

Obviously this expression applies to any of the velocity components. It shows that each of the velocity components has a Gaussian distribution, which is symmetric about the average value $\left\langle v_{i}\right\rangle=0$, for $i=x, y, z$. The distribution function given in (4.4) is plotted in Fig. 3. Note that it is properly normalized so that

$$
\int_{-\infty}^{+\infty} g\left(v_{x}\right) d v_{x}=n
$$

The fact that the average value $\left\langle v_{i}\right\rangle$ vanishes is physically evident by symmetry, since each component of the velocity can be equally positive or negative. Mathematically, we have

$$
\begin{gathered}
<v_{i}>=\frac{1}{n} \int_{-\infty}^{+\infty} g\left(v_{i}\right) v_{i} d v_{i} \\
=\left(\frac{m}{2 \pi k T}\right)^{1 / 2} \int_{-\infty}^{+\infty} \exp \left(-\frac{m v_{i}^{2}}{2 k T}\right) v_{i} d v_{i}=0
\end{gathered}
$$

since the integrand is an odd function of $v_{i}$. Consequently, if $l$ represents any odd integer number,

$$
<v_{i}^{l}>=0 \quad l=1,3,5, \ldots
$$

On the other hand, $\left\langle v_{i}^{2}\right\rangle$ is intrinsically positive and represents the dispersion or variance of $v_{i}$,

$$
<v_{i}^{2}>=\frac{1}{n} \int_{-\infty}^{+\infty} g\left(v_{i}\right) v_{i}^{2} d v_{i}=\frac{k T}{m}
$$

This result is consistent with the theorem of equipartition of energy, according to which

$$
\frac{1}{2} m<v_{i}^{2}>=\frac{1}{2} k T
$$




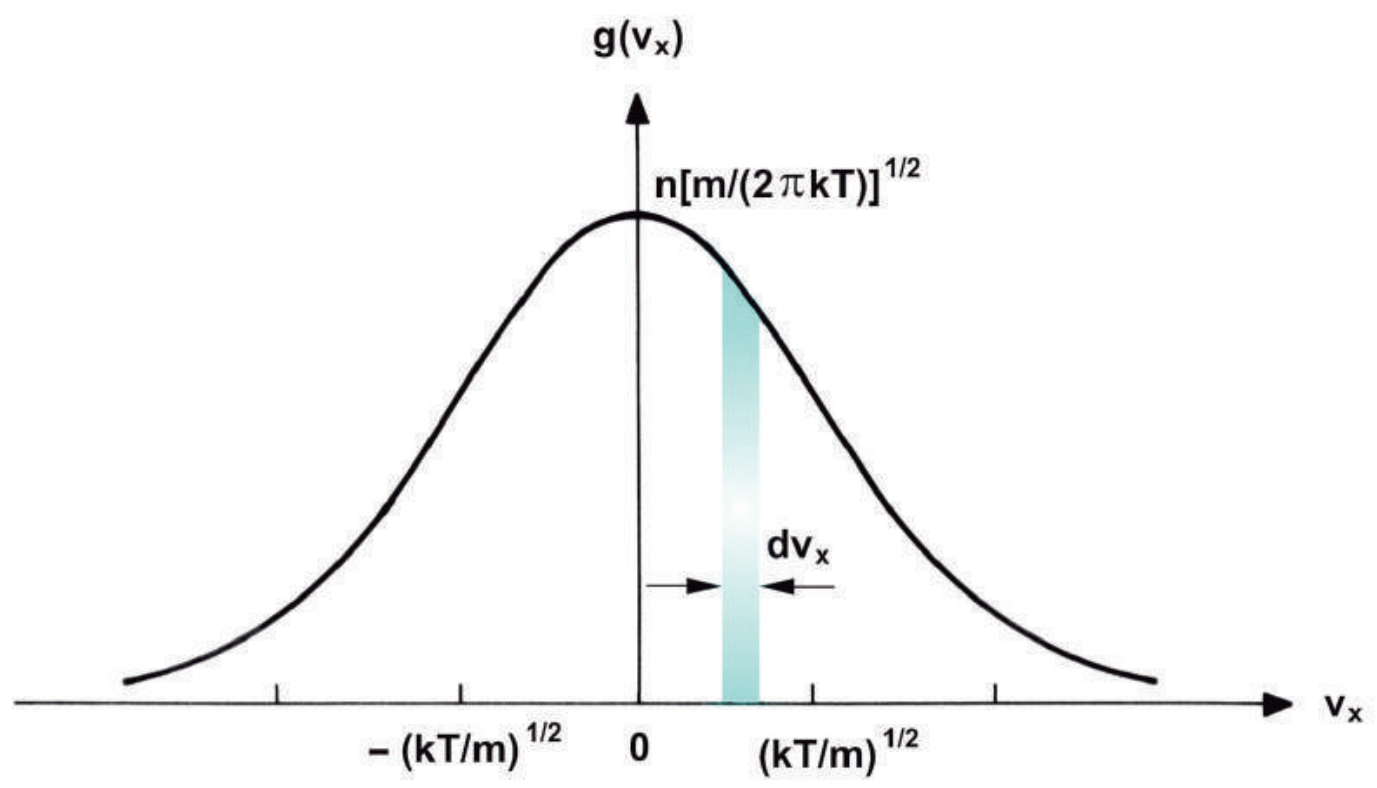

Fig. 3 The equilibrium Maxwellian distribution function for each velocity component is a Gaussian distribution having zero expectation $\left(\left\langle v_{x}\right\rangle=0\right)$ and root-mean-square width given by $<v_{x}^{2}>^{1 / 2}=(k T / m)^{1 / 2}$.

for $i=x, y, z$. The root-mean-square width of the Gaussian distribution $g\left(v_{i}\right)$ is therefore given by

$$
<v_{i}^{2}>^{1 / 2}=\left(\frac{k T}{m}\right)^{1 / 2}
$$

which shows that the higher the temperature, the larger will be the width of the distribution function $g\left(v_{i}\right)$.

The velocity components behave, individually, like statistically independent quantities. Since $v^{2}=v_{x}^{2}+v_{y}^{2}+v_{z}^{2}$, the probability that the particle velocity lies between $\mathbf{v}$ and $\mathbf{v}+d \mathbf{v}$ is equal to the product of the probabilities that the velocity components lie between $v_{i}$ and $v_{i}+d v_{i}$, for $i=x, y, z$, that is

$$
\frac{f(v) d^{3} v}{n}=\frac{g\left(v_{x}\right) d v_{x}}{n} \cdot \frac{g\left(v_{y}\right) d v_{y}}{n} \cdot \frac{g\left(v_{z}\right) d v_{z}}{n}
$$




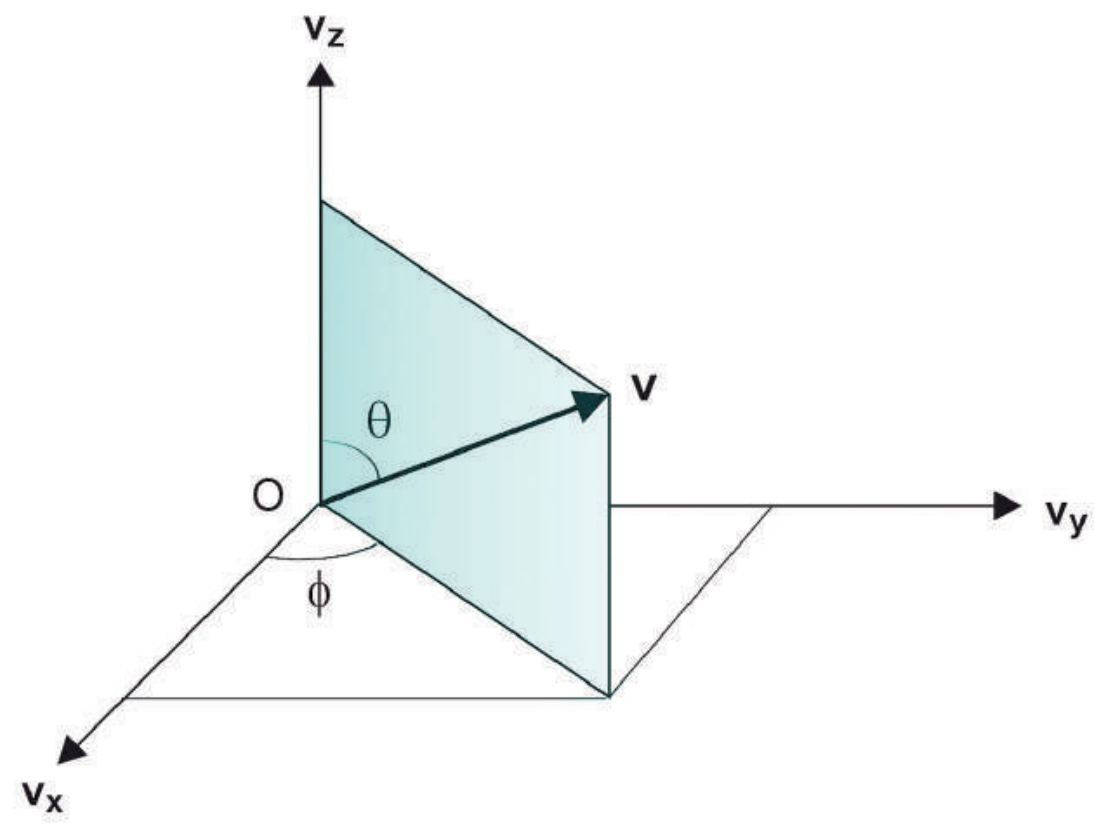

Fig. 4 Spherical coordinate system $(v, \theta, \phi)$ in velocity space.

\subsection{Distribution of Speeds}

Since the Maxwell-Boltzmann velocity distribution function is isotropic, it is of interest to define a distribution function of speeds $v=|\mathbf{v}|$. For this purpose, consider a spherical polar coordinate system in velocity space $(v, \theta, \phi)$, as shown in Fig. 4 . The element of volume $d^{3} v$, in velocity space, between the coordinates $(v, \theta, \phi)$ and $(v+d v, \theta+d \theta, \phi+d \phi)$, is given by

$$
d^{3} v=v^{2} \sin \theta d \theta d \phi
$$

The distribution function of speeds $F(v)$ is defined such that $F(v) d v$ is the number of particles per unit volume having speed between $v$ and $v+d v$, irrespective of the direction in space of the velocity vector $\mathbf{v}$. Hence, to determine $F(v)$ we integrate $f(v)$ over all velocities whose magnitude lies between $v$ and $v+d v$, irrespective of the values of $\theta$ and $\phi$, that is, whose velocity vector ends in a spherical shell in velocity space of internal radius $v$ and external radius $v+d v$, as shown in Fig. 5. Therefore,

$$
F(v) d v=\int_{\theta} \int_{\phi} f(v) v^{2} \sin \theta d \theta d \phi d v
$$




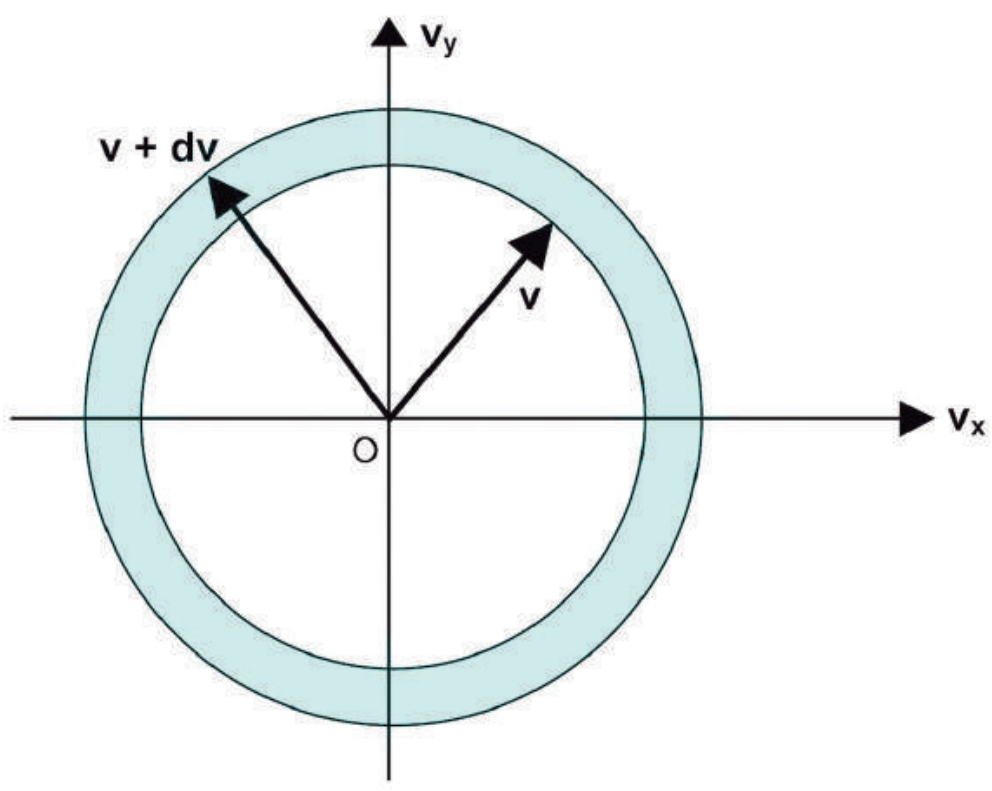

Fig. 5 Schematic representation, in two dimensions, of a spherical shell in velocity space containing all particles with velocity having magnitude between $v$ and $v+d v$.

Since $f(v)$ depends only on the magnitude of $\mathbf{v}$, but not on its direction,

$$
F(v) d v=f(v) v^{2} d v \int_{0}^{\pi} \sin \theta d \theta \int_{0}^{2 \pi} d \phi=4 \pi v^{2} f(v) d v
$$

Note that $4 \pi v^{2} d v$ is the volume of the spherical shell in velocity space shown in Fig. 5. Substituting the Maxwell-Boltzmann distribution function for $f(v)$, we obtain the distribution of speeds,

$$
F(v)=4 \pi n\left(\frac{m}{2 \pi k T}\right)^{3 / 2} v^{2} \exp \left(-\frac{m v^{2}}{k T}\right)
$$

This expression is properly normalized, so that

$$
\int_{0}^{\infty} F(v) d v=n
$$

From the expression for $F(v)$ we see that, as $v$ increases, the exponential factor decreases faster than $v^{2}$ increases, resulting in a maximum in $F(v)$ 


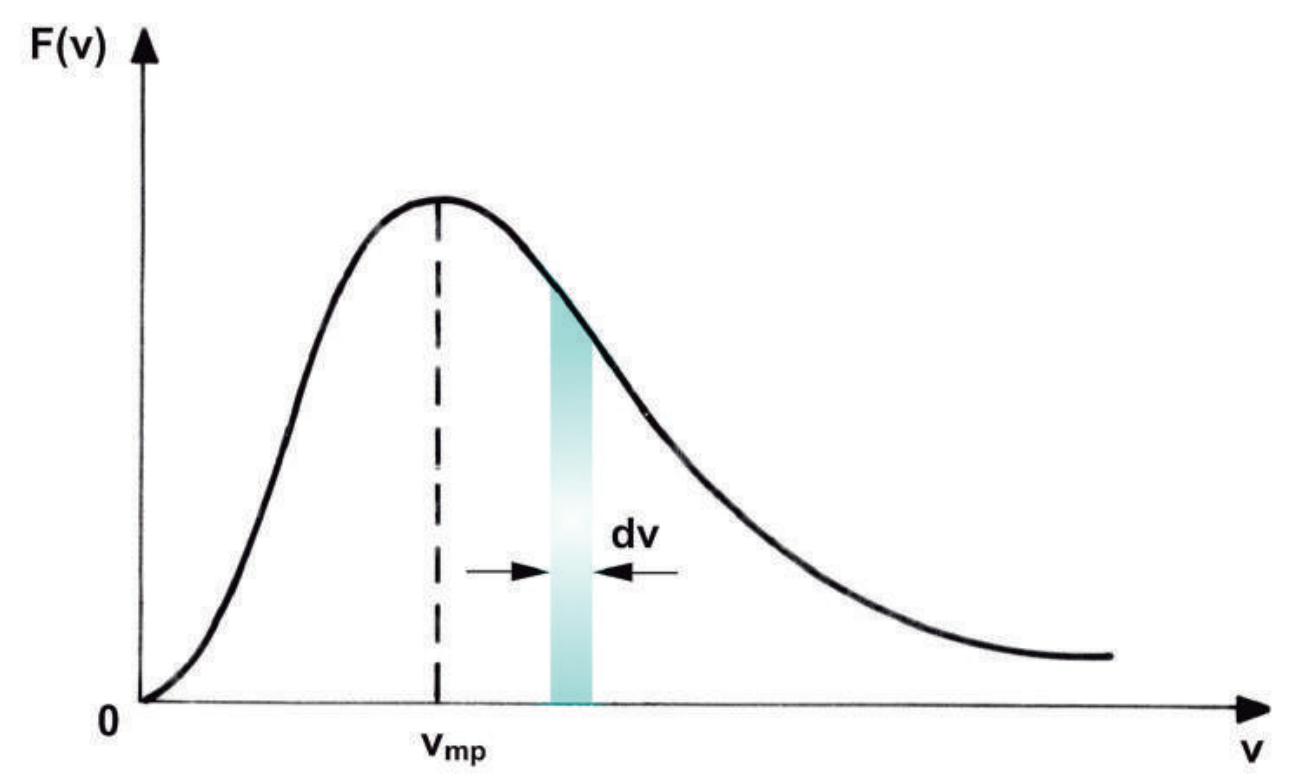

Fig. 6 Maxwellian distribution of speeds, showing the most probable speed $v_{m p}$.

for a given value of $v$ which is called the most probable speed. The curve for $F(v)$ is shown in Fig. 6.

\subsection{Mean Values Related to the Molecular Speeds}

The average value of the speed is given by

$$
<v>=\frac{1}{n} \int_{v} f v d^{3} v=\frac{1}{n} \iiint_{-\infty}^{+\infty} f v d v_{x} d v_{y} d v_{z}
$$

or, equivalently, by

$$
<v>=\frac{1}{n} \int_{0}^{\infty} F(v) v d v
$$

It is intrinsically a positive quantity, since $v=|\mathbf{v}|$ is always positive. Using expression (4.15) for $F(v)$, we get

$$
\begin{gathered}
<v>=4 \pi\left(\frac{m}{2 \pi k T}\right)^{3 / 2} \int_{0}^{\infty} v^{3} \exp \left(-\frac{m v^{2}}{2 k T}\right) d v \\
=4 \pi\left(\frac{m}{2 \pi k T}\right)^{3 / 2} \frac{1}{2}\left(\frac{2 k T}{m}\right)^{2}
\end{gathered}
$$


Therefore,

$$
<v>=(8 / \pi)^{1 / 2}(k T / m)^{1 / 2}
$$

Integrals of the type

$$
I(j)=\int_{0}^{\infty} x^{j} \exp \left(-\alpha x^{2}\right) d x
$$

where $j$ represents a positive integer number, occur frequently in the computation of average values using the Maxwellian distribution of speeds. For future reference we present here the results for some integrals of the type (4.21):

$$
\begin{array}{rlrl}
I(0) & =\frac{1}{2} \pi^{1 / 2} \alpha^{-1 / 2} & I(1) & =\frac{1}{2} \alpha^{-1} \\
I(2) & =\frac{1}{4} \pi^{1 / 2} \alpha^{-3 / 2} & I(3) & =\frac{1}{2} \alpha^{-2} \\
I(4) & =\frac{3}{8} \pi^{1 / 2} \alpha^{-5 / 2} & I(5) & =\alpha^{-3}
\end{array}
$$

The average of the square of the speed is given by

$$
<v^{2}>=\frac{1}{n} \iiint_{-\infty}^{+\infty} f v^{2} d v_{x} d v_{y} d v_{z}=\frac{4 \pi}{n} \int_{0}^{\infty} v^{4} f(v) d v
$$

Substituting the Maxwellian distribution function for $f(v)$,

$$
<v^{2}>=4 \pi\left(\frac{m}{2 \pi k T}\right)^{3 / 2} \int_{0}^{\infty} v^{4} \exp \left(-\frac{m v^{2}}{2 k T}\right) d v
$$

which gives

$$
<v^{2}>=3 k T / m
$$

This result can also be obtained from (4.8), noting that $v^{2}=v_{x}^{2}+v_{y}^{2}+v_{z}^{2}$ and that $\left\langle v_{x}^{2}\right\rangle=\left\langle v_{y}^{2}\right\rangle=\left\langle v_{z}^{2}\right\rangle$. The root-mean-square speed is given by

$$
v_{r m s}=\left\langle v^{2}>^{1 / 2}=(3 k T / m)^{1 / 2}\right.
$$

The most probable speed $v_{m p}$ corresponds to the speed for which $F(v)$ is maximum, and can be obtained by the condition

$$
\left(\frac{d F(v)}{d v}\right)_{v=v_{m p}}=0
$$

Differentiating (4.15) with respect to $v$, yields

$$
\frac{d F(v)}{d v}=2 v \exp \left(-\frac{m v^{2}}{2 k T}\right)+v^{2}\left(-\frac{m v}{k T}\right) \exp \left(-\frac{m v^{2}}{2 k T}\right)
$$


which, for the condition of maximum expressed in (4.27), gives

$$
v_{m p}=(2 k T / m)^{1 / 2}
$$

Note that the mean speeds $\langle v\rangle, v_{r m s}$, and $v_{m p}$ are all proportional to $(k T / m)^{1 / 2}$ and are such that $v_{m p}<(<v>)<v_{r m s}$. Therefore, they increase with the temperature and, for a given temperature, particles having a larger mass will move with a smaller speed. We have also seen that the average kinetic energy of the random particle motions satisfies the relation

$$
\frac{1}{2} m<v^{2}>=\frac{3}{2} k T
$$

\subsection{Distribution of Thermal Kinetic Energy}

The distribution of thermal kinetic energy $G(E)$, where $E=m v^{2} / 2$, is defined such that $G(E) d E$ is the number of particles per unit volume having random kinetic energy between $E$ and $E+d E$. It can be obtained from (4.15) substituting $v$ by $(2 E / m)^{1 / 2}$ and $d v$ by $d E /(2 m E)^{1 / 2}$. Hence,

$$
G(E) d E=4 \pi n\left(\frac{m}{2 \pi k T}\right)^{3 / 2}\left(\frac{2 E}{m}\right) \exp \left(-\frac{E}{k T}\right) \frac{d E}{(2 m E)^{1 / 2}}
$$

Simplifying this expression,

$$
G(E) d E=\frac{2 n E^{1 / 2}}{\pi^{1 / 2}(k T)^{3 / 2}} \exp \left(-\frac{E}{k T}\right) d E
$$

The function $G(E)$ is displayed in Fig. 7 .

\subsection{Random Particle Flux}

We have seen in Chapter 6 that the particle flux, in a given direction specified by the unit vector $\widehat{\mathbf{n}}$, is given by

$$
\Gamma_{n}=n<v_{n}>=\int_{v} f \mathbf{v} \cdot \widehat{\mathbf{n}} d^{3} v
$$

Let us consider a surface element inside the gas. We are interested in determining the number of particles that reach this surface element, per 


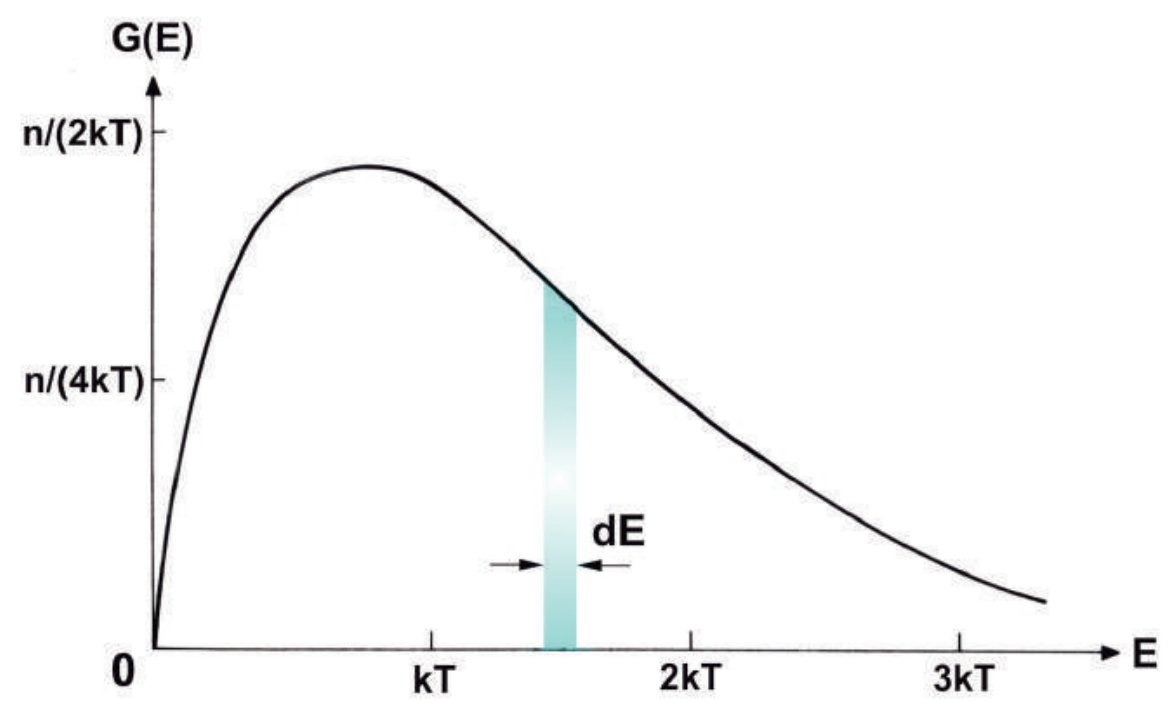

Fig. 7 Maxwellian distribution of thermal kinetic energies. The shadowed area represents the number of particles that have random kinetic energy between $E$ and $E+d E$.

unit area and time, due to the random particle motions. Eq. (4.33) takes into account particles that reach the surface element, oriented along the direction of $\widehat{\mathbf{n}}$, coming from all possible directions. Since we are assuming that the average velocity $\mathbf{u}$ is zero, the flux given by (4.33) is obviously zero, since $\langle\mathbf{c}\rangle=0$. In this case, it is of interest to consider only the flux of particles that cross the surface element from the same side (such that $\mathbf{v} \cdot \widehat{\mathbf{n}}$ is positive, say), due to their random motions.

Let $d \mathbf{S}$ be a surface element situated at the origin of a Cartesian coordinate system $(x, y, z)$ and oriented along the $z$ axis, that is, $d \mathbf{S}=$ $\widehat{\mathbf{z}} d S$, as shown in Fig. 8. Consider the particles that cross $\widehat{\mathbf{z}} d S$ coming from the region $z<0$, having velocities between $\mathbf{v}$ and $\mathbf{v}+d \mathbf{v}$, making an angle $\theta$ with the $z$ axis, so that $\mathbf{v} \cdot \widehat{\mathbf{z}}=v \cos (\theta)$. Expressing $d^{3} v$ in terms of spherical coordinates $(v, \theta, \phi)$,

$$
d^{3} v=v^{2} \sin \theta d \theta d \phi d v
$$

the random particle current density, crossing $\widehat{\mathbf{z}} d S$ from the region $z<0$, is given by

$$
\Gamma_{z}=\int_{0}^{\infty} f v^{3} d v \int_{0}^{\pi / 2} \sin \theta \cos \theta d \theta \int_{0}^{2 \pi} d \phi=\pi \int_{0}^{\infty} f v^{3} d v
$$




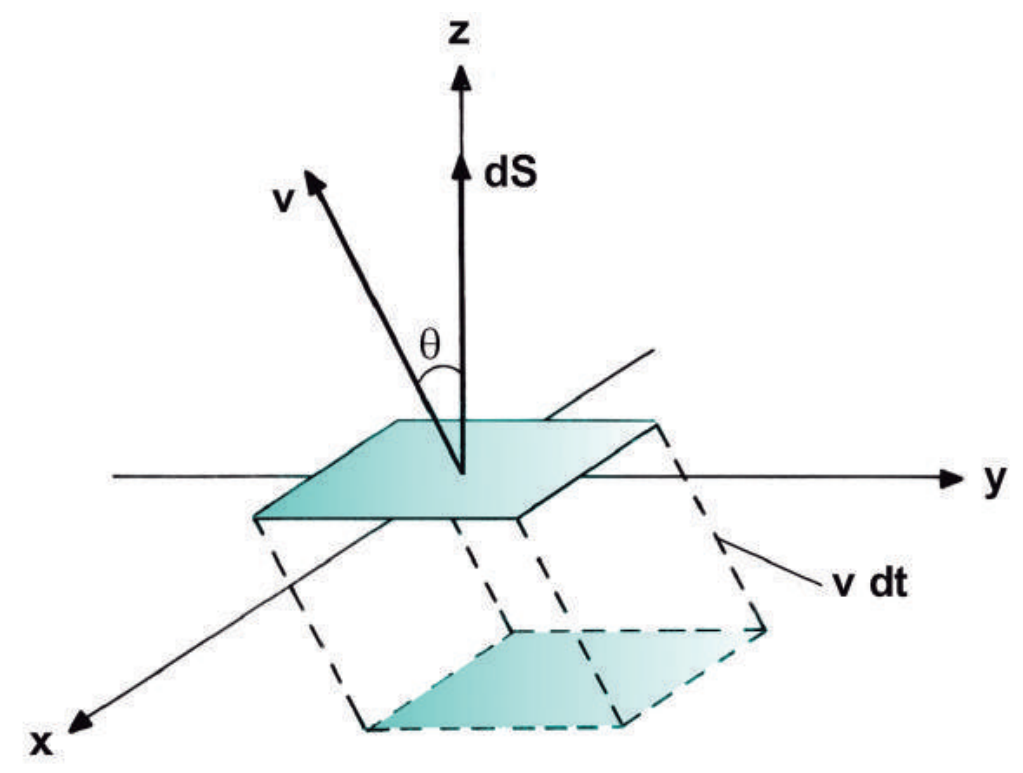

Fig. 8 Prism of base $d \mathbf{S}=\widehat{\mathbf{z}} d S$ containing the particles that have velocity between $\mathbf{v}$ and $\mathbf{v}+d \mathbf{v}$ that will cross $d \mathbf{S}$ during the interval $d t$.

Substituting the Maxwellian distribution for $f(v)$, we find

$$
\Gamma_{z}=\pi n\left(\frac{m}{2 \pi k T}\right)^{3 / 2} \int_{0}^{\infty} \exp \left(-\frac{m v^{2}}{2 k T}\right) v^{3} d v
$$

and solving the integral, we obtain

$$
\Gamma=n\left(\frac{k T}{2 \pi m}\right)^{1 / 2}=\frac{1}{4} n<v>
$$

In this result we have eliminated the index $z$ from $\Gamma$, since the Maxwellian distribution function is isotropic, so that (4.37) applies to any direction inside the gas.

It is important to note that the random particle flux is inversely proportional to the square root of the particle mass. In a plasma the particle current density for the electrons is therefore much larger than that for the ions (the ratio of the electron mass to the proton mass, for example, is 1/1836). This difference in the thermal particle flux between electrons and ions plays a very important role in the interaction of a plasma with a material body immersed in it (see Chapter 11). 


\subsection{Kinetic Pressure and Heat Flux}

From the definitions of the kinetic pressure dyad

$$
\mathcal{P}=\rho_{m}<\mathbf{c} \mathbf{c}>=m \int_{v} \mathbf{c} \mathbf{c} f d^{3} v
$$

and of the heat flux vector

$$
\mathbf{q}=\frac{1}{2} \rho_{m}<c^{2} \mathbf{c}>=\frac{1}{2} m \int_{v} c^{2} \mathbf{c} f d^{3} v
$$

we obtain, using the Maxwellian distribution function,

$$
\begin{gathered}
\mathcal{P}=\rho_{m}\left(<c_{x}^{2}>\widehat{\mathbf{x}} \widehat{\mathbf{x}}+<c_{y}^{2}>\widehat{\mathbf{y}} \widehat{\mathbf{y}}+<c_{z}^{2}>\widehat{\mathbf{z}} \widehat{\mathbf{Z}}\right) \\
=n k T(\widehat{\mathbf{x}} \widehat{\mathbf{x}}+\widehat{\mathbf{y}} \widehat{\mathbf{y}}+\widehat{\mathbf{z}} \mathbf{\mathbf { Z }})
\end{gathered}
$$

and

$$
\mathbf{q}=0
$$

since the integrals having an odd integrand vanish. The scalar pressure, therefore, is

$$
p=n k T
$$

\section{EQUILIBRIUM IN THE PRESENCE OF AN EXTERNAL FORCE}

A gas under steady-state conditions and immersed in a conservative force field is characterized by a distribution function that differs from the Maxwell-Boltzmann distribution by an exponential factor, known as the Boltzmann factor. The conservative force field can be specified in terms of a potential energy $U(\mathbf{r})$, such that

$$
\mathbf{F}(\mathbf{r})=-\nabla U(\mathbf{r})
$$

Since the conservative force field is a function only of the position vector $\mathbf{r}$, we expect the steady-state solution of the Boltzmann equation for this case to be of the form

$$
f(\mathbf{r}, v)=f_{0}(v) \psi(\mathbf{r})
$$


where $f_{0}(v)$ represents the Maxwell-Boltzmann equilibrium distribution function and $\psi(\mathbf{r})$ is a scalar function of $\mathbf{r}$ only, still to be determined. The function $\psi(\mathbf{r})$ can be determined by requiring (5.2) to satisfy the Boltzmann equation under equilibrium conditions in the presence of the conservative field,

$$
\mathbf{v} \cdot \nabla\left[f_{0}(v) \psi(\mathbf{r})\right]-\frac{1}{m}[\nabla U(\mathbf{r})] \cdot \nabla_{v}\left[f_{0}(v) \psi(\mathbf{r})\right]=0
$$

From the expression for $f_{0}(v)$ it can be easily verified that

$$
\nabla_{v} f_{0}(v)=-\frac{m \mathbf{v}}{k T} f_{0}(v)
$$

Therefore, (5.3) simplifies to

$$
f_{0}(v) \mathbf{v} \cdot\left[\nabla \psi(\mathbf{r})+\frac{1}{k T} \psi(\mathbf{r}) \nabla U(\mathbf{r})\right]=0
$$

from which we can write

$$
\frac{\nabla \psi(\mathbf{r})}{\psi(\mathbf{r})}=-\frac{1}{k T} \nabla U(\mathbf{r})
$$

Since $d \psi=\nabla \psi \cdot d \mathbf{r},(5.6)$ may also be written as

$$
\frac{d \psi(\mathbf{r})}{\psi(\mathbf{r})}=-\frac{1}{k T} d U(\mathbf{r})
$$

The solution of this differential equation is

$$
\psi(\mathbf{r})=A_{0} \exp \left[-\frac{U(\mathbf{r})}{k T}\right]
$$

where $A_{0}$ is a constant that can be determined by requiring that

$$
\int_{v} f(\mathbf{r}, v) d^{3} v=n(\mathbf{r})
$$

from which we get

$$
n(\mathbf{r})=A_{0} \exp \left[-\frac{U(\mathbf{r})}{k T}\right] \int_{v} f_{0}(v) d^{3} v
$$


Denoting by $n_{0}$ the number density in a region where $U(\mathbf{r})=0$, under equilibrium conditions, that is,

$$
n_{0}=\int_{v} f_{0}(v) d^{3} v
$$

we must choose $A_{0}=1$. Therefore, the equilibrium distribution function (with $\mathbf{u}=0$ ), under the presence of a conservative force field, is

$$
\begin{gathered}
f(\mathbf{r}, v)=f_{0}(v) \exp \left[-\frac{U(\mathbf{r})}{k T}\right] \\
=n_{0}\left(\frac{m}{2 \pi k T}\right)^{3 / 2} \exp \left[-\frac{\left(\frac{1}{2} m v^{2}+U\right)}{k T}\right]
\end{gathered}
$$

The number density, for a system described by this velocity distribution function, is therefore given by

$$
n(\mathbf{r})=n_{0} \exp \left[-\frac{U(\mathbf{r})}{k T}\right]
$$

The factor $\exp [-U(\mathbf{r}) / k T]$, responsible for the inhomogeneity of $f(\mathbf{r}, v)$ in (5.12), is known as the Boltzmann factor.

An important example is provided by a plasma in the presence of a conservative force due to an electrostatic field

$$
\mathbf{E}=-\nabla \phi(\mathbf{r})
$$

where $\phi(\mathbf{r})$ is the electrostatic scalar potential. The potential energy, in this case, is

$$
U(\mathbf{r})=q \phi(\mathbf{r})
$$

The number density for particles of charge $q$ in equilibrium under the action of an electrostatic field is therefore

$$
n(\mathbf{r})=n_{0} \exp \left[-\frac{q \phi(\mathbf{r})}{k T}\right]
$$

This expression is very useful for the analysis of electrostatic shielding in a plasma (see Chapter 11). 


\section{DEGREE OF IONIZATION IN EQUILIBRIUM AND THE SAHA EQUATION}

From the methods of statistical mechanics we can determine the degree of ionization in a gas in thermal equilibrium at some temperature $T$, without considering the details of the ionization process. In order to ionize an atom or molecule, it is necessary to provide a certain amount of energy. This ionization energy is conveniently expressed in electron volts, and is normally called the ionization potential. Values for the first ionization potential of some atoms are given in Table 1 . Note that to provide a mean thermal energy $k T$ of $1 \mathrm{eV}$ requires a temperature of 11,600 K. Hence, it is apparent that only at very high temperatures does the mean thermal kinetic energy $3 k T / 2$ of a particle exceed the ionization energy. However, we will show that a considerable degree of ionization can be achieved even when the mean thermal energy of the particles is far below the ionization energy, since some of the particles, the ones with the largest velocities (in the tail of the Maxwellian distribution function), have enough energy to produce ionization by collisions. The degree of ionization, under thermal equilibrium conditions, is then determined by a balance between the rate of ionization by collisions and the rate of recombination.

To calculate the relative numbers of ionized and neutral atoms in a plasma, at a given temperature, it is appropriate to use a particle distribution function similar to that given in (5.13). However, the physical situation is somewhat different because of the necessary quantum mechanical aspects of the problem. Denoting by $n_{a}$ and $n_{b}$ the number density of the particles having energies $U_{a}$ and $U_{b}$, respectively, the ratio $n_{a} / n_{b}$ is given, from statistical mechanics, by

$$
\frac{n_{a}}{n_{b}}=\frac{g_{a}}{g_{b}} \exp \left[-\frac{\left(U_{a}-U_{b}\right)}{k T}\right]
$$

where $g_{a}$ and $g_{b}$ are the statistical weights associated with the energies $U_{a}$ and $U_{b}$, that is, the degeneracy factors giving the number of states having the energies $U_{a}$ and $U_{b}$, respectively. For the particular case of a system having only two energy levels, $U_{a}$ and $U_{b}$, the fraction $\alpha$ of all the particles that are in the higher energy state $U_{a}$ is given by

$$
\alpha=\frac{n_{a}}{\left(n_{a}+n_{b}\right)}=\frac{n_{a}}{n_{b}}\left(\frac{n_{a}}{n_{b}}+1\right)^{-1}
$$

or, using (6.1) with $U=U_{a}-U_{b}$,

$$
\alpha=\frac{\left(g_{a} / g_{b}\right) \exp (-U / k T)}{\left(g_{a} / g_{b}\right) \exp (-U / k T)+1}
$$


TABLE 1

IONIZATION POTENTIAL ENERGY U OF SOME ATOMS FOR THE FIRST ELECTRON

\begin{tabular}{|c|r|}
\hline Element & $\mathbf{U}(\mathbf{e V})$ \\
\hline Helium (He) & 24.59 \\
Argon (A) & 15.76 \\
Nitrogen (N) & 14.53 \\
Oxygen (O) & 13.62 \\
Hydrogen (H) & 13.60 \\
Mercury (Hg) & 10.44 \\
Iron (Fe) & 7.87 \\
Sodium (Na) & 5.14 \\
Potassium (K) & 4.34 \\
Cesium (Cs) & 3.89 \\
\hline
\end{tabular}

For the ionization problem, state $a$ is taken as that of the ion-electron pair, state $b$ is that of the neutral atom, and $U=U_{a}-U_{b}$ is the ionization energy. The temperature $T$ for which $\alpha=0.5$, that is, for which $50 \%$ of all the atoms are in the ionized state $\left(n_{a}=n_{b}\right)$, can be determined by taking

$$
\frac{g_{a}}{g_{b}} \exp \left(-\frac{U}{k T_{1 / 2}}\right)=1
$$

which gives

$$
T_{1 / 2}=\frac{U}{k \ln \left(g_{a} / g_{b}\right)}
$$

Fig. 9 shows the plot of $\alpha$ as a function of $T$, according to (6.3).

The fraction of particles in the ionized state changes from nearly zero to nearly one over a small temperature range. An estimate of this temperature range can be obtained from the temperature difference $\Delta T$ that would exist between $\alpha=0$ and $\alpha=1$, if the curve of $\alpha(T)$ were a straight line with the slope of the true $\alpha(T)$ curve at $T_{1 / 2}$. Hence, we take

$$
\left(\frac{d \alpha(T)}{d T}\right)_{T_{1 / 2}}=\frac{1}{\Delta T}
$$

From (6.3) we obtain, assuming $d\left(g_{a} / g_{b}\right) / d T=0$,

$$
\left(\frac{d \alpha(T)}{d T}\right)_{T_{1 / 2}}=\left[\frac{U \alpha^{2}}{T^{2}\left(g_{a} / g_{b}\right) \exp (-U / k T)}\right]_{T_{1 / 2}}=\frac{U}{4 T_{1 / 2}^{2}}
$$




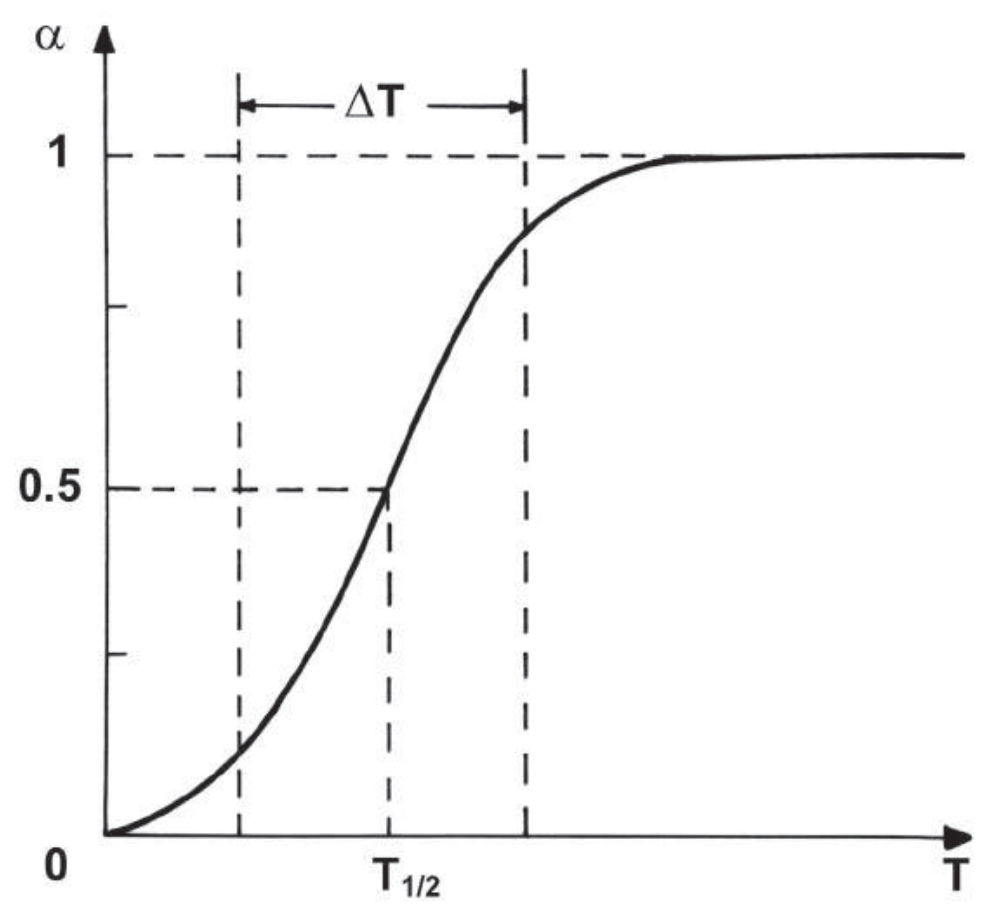

Fig. 9 The function $\alpha(T)$, which gives the fraction of particles in the ionized state as a function of temperature $T$.

so that

$$
\Delta T=\frac{4 T_{1 / 2}}{k \ln \left(g_{a} / g_{b}\right)}=\frac{4 U}{\left[k \ln \left(g_{a} / g_{b}\right)\right]^{2}}
$$

From this result we can see that, the larger is $g_{a} / g_{b}$, the smaller is $\Delta T$.

Since the ionized state is much more degenerate than the neutral state $\left(g_{a} \gg g_{b}\right)$, the curve of $\alpha(T)$ presents a very steep inclination near $T_{1 / 2}$, with most of the transitions from the neutral state to the ionized state occurring near $T_{1 / 2}$, given by (6.5). Thus, for $g_{a} \gg g_{b}$, the curve of $\alpha(T)$ will look approximately like a step function with the ionization occurring near $T_{1 / 2}$.

The degeneracy factors $g_{a}$ and $g_{b}$ can be obtained from a quantum mechanical calculation. If we neglect the small interaction potential between the ion and the free electron, and also the internal degrees of freedom of all the particles, it turns out that

$$
\frac{g_{a}}{g_{b}}=\left(\frac{2 \pi m_{e} k T}{h^{2}}\right)^{3 / 2} \frac{1}{n_{i}}
$$


where $h$ is Planck's constant and $n_{i}$ is the ion number density. For $T$ expressed in degrees Kelvin and $n_{i}$ in $m^{-3}$,

$$
\frac{g_{a}}{g_{b}}=2.405 \times 10^{21} T^{3 / 2} \frac{1}{n_{i}}
$$

Using this result in (6.1), we obtain the following equation

$$
\frac{n_{i}}{n_{n}}=2.405 \times 10^{21} T^{3 / 2} \frac{1}{n_{i}} \exp \left(-\frac{U}{k T}\right)
$$

which is known as the Saha equation. Since $1 \mathrm{eV}=k T$ for $T=11,600 \mathrm{~K}$, we can also write the Saha equation as

$$
\frac{n_{i}}{n_{n}}=3.00 \times 10^{27} T^{3 / 2} \frac{1}{n_{i}} \exp \left(-\frac{U}{T}\right)
$$

with $T$ in $\mathrm{eV}$ and $n_{i}$ in $\mathrm{m}^{-3}$. Thus, when the total number density $n_{t}=n_{i}+n_{n}$ is sufficiently low, a considerable degree of ionization can be achieved for temperatures that are well below the ionization energy. This point is illustrated in Fig. 10, which shows the degree of ionization of hydrogen as a function of temperature, for values of the total number density of $10^{16}, 10^{19}, 10^{22}$, and $10^{25} \mathrm{~m}^{-3}$. It is clear that, as the number density decreases, the values of $\Delta T$ and $T_{1 / 2}$ decrease significantly, and a significant degree of ionization can be obtained at temperatures far below the ionization energy of atomic hydrogen $(13.60 \mathrm{eV})$. In a gas like cesium, whose ionization energy is only $3.89 \mathrm{eV}$, a high degree of ionization can be obtained even at relatively low temperatures of the order of $1000 \mathrm{~K}$.

\section{PROBLEMS}

7.1 A two-dimensional gas, consisting of only one species and whose particles are restricted to move in a plane (the $z=0$ plane), is characterized by a homogeneous, isotropic, two-dimensional Maxwell-Boltzmann distribution function (with $\mathbf{u}=0$ ),

$$
f(v)=n_{0}\left(\frac{m}{2 \pi k T}\right) \exp \left[-\frac{m\left(v_{x}^{2}+v_{y}^{2}\right)}{2 k T}\right]
$$

where $n_{0}$ represents the number of particles per unit area.

(a) Show that the most probable speed of the particles is

$$
v_{m p}=(k T / m)^{1 / 2}
$$




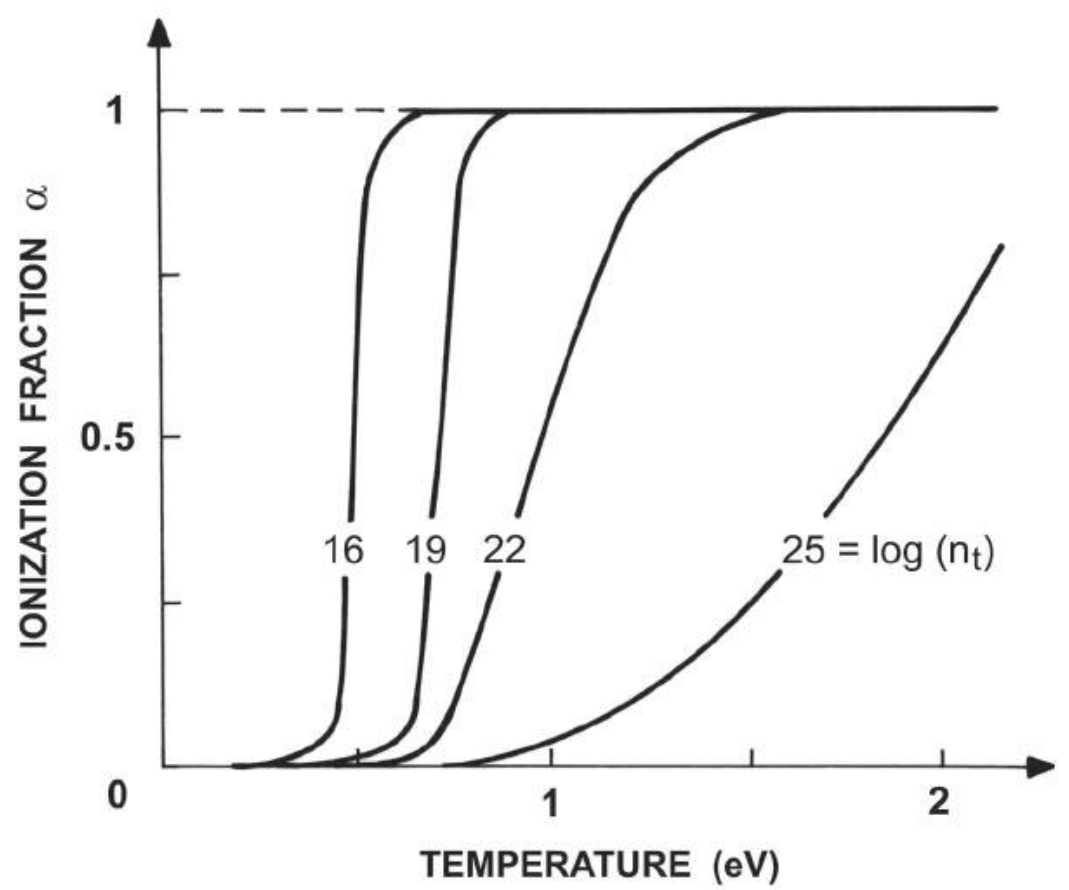

Fig. 10 Degree of ionization $\alpha=n_{i} /\left(n_{i}+n_{n}\right)$ as a function of temperature for atomic hydrogen $(U=13.60 \mathrm{eV})$. The various curves refer to different number densities $n_{t}=n_{i}+n_{n}$ in $m^{-3}$.

(b) Show that the fraction of the number of particles per unit area, which have speeds greater than the most probable speed, is given by $(1 / e)^{1 / 2}$, where $e$ is the base of natural logarithms.

(c) Show that the number of particles crossing a unit length per unit time (flux), from one side only, is given by

$$
\Gamma=\frac{n_{0}}{\pi}<v>=n_{0}\left(\frac{k T}{2 \pi m}\right)^{1 / 2}
$$

(d) Show that the kinetic pressure dyad is given by

$$
\mathcal{P}=n_{0} k T(\widehat{\mathbf{x}} \widehat{\mathbf{x}}+\widehat{\mathbf{y}} \widehat{\mathbf{y}})
$$

7.2 Consider a gas of particles consisting of only one species and characterized by the Maxwell-Boltzmann equilibrium distribution function (with $\mathbf{u}=0$ )

$$
f(v)=n_{0}\left(\frac{m}{2 \pi k T}\right)^{3 / 2} \exp \left(-\frac{m v^{2}}{2 k T}\right)
$$


(a) Show that the total number of particles crossing a unit area per unit time, lying within an element $d \Omega$ of solid angle, is given by

$$
\frac{n_{0}}{\pi}\left(\frac{k T}{2 \pi m}\right)^{1 / 2} \cos \theta d \Omega
$$

where $\theta$ denotes the angle between the solid angle orientation and the direction of the normal to the area considered.

(b) Show that the fraction of particles that cross a unit area perpendicular to the $x$ axis per unit time, from the same side, having the velocity components in the range $d^{3} v=d v_{x} d v_{y} d v_{z}$, about $\mathbf{v}$, is given by

$$
\frac{1}{2 \pi}\left(\frac{m}{k T}\right)^{2} v_{x} \exp \left(-\frac{m v^{2}}{2 k T}\right) d^{3} v
$$

(c) Calculate the thermal energy flux triad for the Maxwellian gas.

7.3 The distribution of thermal kinetic energies $E$, for a gas in the Maxwellian state, is given by

$$
G(E)=\frac{2 n E^{1 / 2}}{\pi^{1 / 2}(k T)^{3 / 2}} \exp \left(-\frac{E}{k T}\right)
$$

Calculate the most probable energy and show that the velocity of the particles, which have this energy, is equal to $(k T / m)^{1 / 2}$.

7.4 The entropy of a system can be expressed in terms of the distribution function as

$$
S=-k \int_{r} \int_{v} f \ln f d^{3} v d^{3} r
$$

Prove that, for a Maxwellian distribution function, the entropy satisfies the following thermodynamic relations:

$$
\begin{aligned}
& \left(\frac{\partial S}{\partial E}\right)_{V, N}=\frac{1}{T} \\
& \left(\frac{\partial S}{\partial V}\right)_{E, N}=\frac{p}{T}
\end{aligned}
$$

where $N$ is the total number of particles in the system, $V$ is the total volume, and $E=3 N k T / 2$ is the total energy. 
7.5 Derive an expression for the Doppler intensity profile (thermal broadening) of a spectral line emitted near the central frequency $\nu_{0}$, assuming that the emitting atoms have a Maxwellian velocity distribution. Ignore all other factors that contribute to the shape of the line.

Hints: (1) The change in frequency due to the Doppler effect associated with the relative (nonrelativistic) motion of the emitting atoms, with respect to the direction of observation (e.g., $x$ direction), is given by

$$
\nu-\nu_{0}=-\nu_{0} \frac{v_{x}}{c}
$$

where in this expression $c$ denotes the speed of light in vacuum. (2) The observed intensity in the frequency range between $\nu$ and $\nu+d \nu$, that is, $I(\nu) d \nu$, is proportional to the number of emitting atoms per unit volume, which have velocities along the direction of observation ( $x$ direction) between $v_{x}$ and $v_{x}+d v_{x}$.

7.6 Consider a gas mixture containing $n_{e}$ electrons and $n_{i}$ oxygen ions per unit volume, all in thermal equilibrium at a temperature $T$ and having no drift velocity.

(a) Resolve the motion of the particle species into the motion in space of the center of mass plus the relative motion of one species with respect to the other, but using the reduced mass. Calculate the Jacobian $J$ of this velocity transformation and show that $|J|=1$.

(b) Show that the center of mass velocities have a Maxwellian distribution and that the relative velocities also have a Maxwellian distribution, but with the reduced mass.

(c) What must be the magnitude of $T$ such that $1 / 5$ of the electrons have a relative kinetic energy greater than $2 \mathrm{eV}$ ? The following integral will be useful:

$$
\int_{x_{0}}^{\infty} x^{2} \exp \left(-\alpha x^{2}\right) d x=\frac{x_{0} \exp \left(-\alpha x_{0}^{2}\right)}{2 \alpha}+\frac{\pi^{1 / 2}}{4 \alpha^{3 / 2}} \operatorname{erfc}\left(\alpha^{1 / 2} x_{0}\right)
$$

where erfc $\left(\alpha^{1 / 2} x_{0}\right)$ denotes the complementary error function.

7.7 A gas of $O_{2}$ molecules is in the equilibrium state with number density $n$ and absolute temperature $T$. Calculate the average value of the reciprocal of the particle velocity, that is, $\langle 1 / v\rangle$. 
7.8 A plasma is in equilibrium under the action of an external electrostatic field $\mathbf{E}$ and a gravitational field $\mathbf{g}$. Consider that the plasma as a whole is moving with constant velocity $\mathbf{u}$, with respect to the observer's frame of reference. Write down the distribution function for the species of type $\alpha$ for this plasma.

7.9 Consider the particles in the Earth's atmosphere under equilibrium conditions in the presence of the Earth's gravitationl field. Assume a horizontally stratified ( $x, y$ plane) atmosphere with constant temperature $T$ and consider a constant value $\mathbf{g}=-g \widehat{\mathbf{z}}$ for the acceleration due to gravity. Derive an expression for the number density $n_{\alpha}(z)$ as a function of height $z$, for the type $\alpha$ species, in terms of the number density $n_{\alpha}\left(z_{0}\right)$ at a base level $z_{0}$ and of the scale height $H_{\alpha}=k T / m_{\alpha} g$. How is the expression for $n_{\alpha}(z)$ modified, when $T$ and $g$ vary with height?

7.10 The temperature of a plasma, in thermal equilibrium with a neutral gas, can be determined experimentally by measuring the electron density $n_{e}$ with a microwave transmission experiment, for example, and the neutrals number density in a particular excited state through the rate of transitions to a lower state. Determine the temperature of a plasma that has only one type of ions, with the electron number density equal to $10^{20} \mathrm{~m}^{-3}$, and that is in equilibrium with a state of ionization potential equal to $2 \mathrm{eV}$ whose population is $10^{15} \mathrm{~m}^{-3}$.

7.11 Consider two large chambers that communicate with each other only through a small aperture of area $A$ in a very thin wall, as indicated in Fig. 11. The chambers contain an ideal gas at a very low pressure, such that the particle mean free path is much larger than the dimensions of $A$. The temperatures of the chambers are $T_{1}$ and $T_{2}$. Determine the ratio $p_{1} / p_{2}$ of pressure in the two chambers assuming that, under equilibrium conditions, the flux of particles through the aperture $A$ from one chamber must equal that from the other. What would be the result in the case of normal conditions of pressure? Give a physical explanation for the two different results.

7.12 Use the laws of conservation of momentum and of energy in a collision to show that the Maxwell-Boltzmann distribution function 


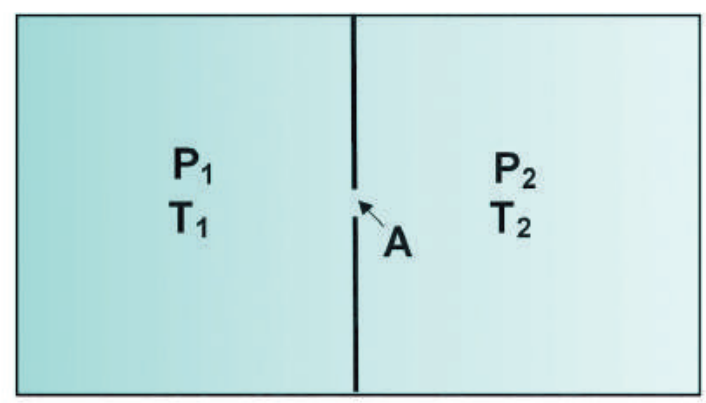

Fig. 11 Two chambers connected through a small aperture of area $A$, at very low pressure, as illustration for problem 7.11.

$$
f(\mathbf{v})=n\left(\frac{m}{2 \pi k T}\right)^{3 / 2} \exp \left[-\frac{m(\mathbf{v}-\mathbf{u})^{2}}{2 k T}\right]
$$

satisfies the following equation of detailed balance

$$
f^{\prime} f_{1}^{\prime}=f f_{1}
$$

7.13 Show that the average thermal energy per particle, for a gas in thermodynamic equilibrium, is equal to $1.292 \times 10^{-4} \mathrm{eV} / \mathrm{K}$. 


\section{8}

\section{MACROSCOPIC TRANSPORT EQUATIONS}

\section{MOMENTS OF THE BOLTZMANN EQUATION}

In the previous chapters we have seen that the macroscopic variables of physical interest for a plasma, such as number density $n_{\alpha}$, mean velocity $\mathbf{u}_{\alpha}$, temperature $T_{\alpha}$, and so on, can be calculated if we know the distribution function for the system under consideration. For the case of a system in thermal equilibrium we have calculated, in Chapter 7, several of these macroscopic parameters using the Maxwell-Boltzmann distribution function. In principle, the distribution function for a system not in equilibrium can be obtained by solving the Boltzmann equation. However, the solution of the Boltzmann equation is generally a matter of great difficulty. We will see, in this chapter, that it is not necessary to solve the Boltzmann equation for the distribution function in order to determine the macroscopic variables of physical interest. The differential equations governing the temporal and spatial variations of these macroscopic variables can be derived directly from the Boltzmann equation without solving it. These differential equations are known as the macroscopic transport equations, and their solutions, under certain assumptions, give us directly the macroscopic variables.

The macroscopic variables are related to the moments of the distribution function and the transport equations satisfied by these variables can be obtained by taking the various moments of the Boltzmann equation. The first three moments of the Boltzmann equation, obtained by multiplying it by $m_{\alpha}, m_{\alpha} \mathbf{v}$, and $m_{\alpha} v^{2} / 2$, respectively, and integrating over all of velocity space, give us the equation of conservation of mass, 
the equation of conservation of momentum, and the equation of conservation of energy. However, at each step of the hierarchy of moments of the Boltzmann equation, the resulting set of transport equations is not complete in the sense that the number of equations is not sufficient to determine all the macroscopic variables that appear in them. Each time a higher moment of the Boltzmann equation is calculated in an attempt to obtain a complete set of transport equations, a new macroscopic variable appears. It is necessary, therefore, to truncate the system of transport equations at some point of the hierarchy and to introduce a simplifying assumption concerning the highest moment of the distribution function that appears in the system. Thus, with such simplifying approximation, we can obtain a complete set of transport equations sufficient to determine all the macroscopic variables appearing in the system. Since a plasma is composed of more than one particle species (electrons, ions, and neutral particles), there is, consequently, a system of transport equations for each species.

There are several different complete sets of transport equations (or hydrodynamic equations) that can be formed, depending on the assumptions considered. Among the possible complete systems of macroscopic equations, there are two that are widely used and that characterize the so-called cold and warm plasma models. The equations that describe these two simple models and the corresponding approximations are discussed in sections 6 and 7 of this chapter.

\section{GENERAL TRANSPORT EQUATION}

We derive now a general partial differential equation that describes the temporal and spatial variation of the physically relevant macroscopic parameters. Let $\chi(\mathbf{v})$ represent some physical property of the particles in the plasma, which may be, in general, a function of the particle velocity. Since the average value of $\chi(\mathbf{v})$ is obtained by multiplying the distribution function by the property $\chi(\mathbf{v})$, integrating the product over all of velocity space and dividing the result by the particle number density, the differential equation governing the temporal and spatial variation of the average value of $\chi(\mathbf{v})$ can be obtained in a similar way by multiplying the Boltzmann equation by the function $\chi(\mathbf{v})$ and integrating the resulting equation over all of velocity space.

Consider the Boltzmann equation for the type $\alpha$ particles in the general form 


$$
\frac{\partial f_{\alpha}}{\partial t}+\mathbf{v} \cdot \nabla f_{\alpha}+\mathbf{a} \cdot \nabla_{v} f_{\alpha}=\left(\frac{\delta f_{\alpha}}{\delta t}\right)_{c o l l}
$$

As indicated, we now multiply each term by $\chi(\mathbf{v})$ and integrate the resulting equation over all of velocity space to obtain

$$
\begin{gathered}
\int_{v} \chi \frac{\partial f_{\alpha}}{\partial t} d^{3} v+\int_{v} \chi \mathbf{v} \cdot \nabla f_{\alpha} d^{3} v+\int_{v} \chi \mathbf{a} \cdot \nabla_{v} f_{\alpha} d^{3} v \\
=\int_{v} \chi\left(\frac{\delta f_{\alpha}}{\delta t}\right)_{\text {coll }} d^{3} v
\end{gathered}
$$

We proceed next to evaluate separately each of the terms in (2.2).

The first term of (2.2) may be rewritten as

$$
\int_{v} \chi \frac{\partial f_{\alpha}}{\partial t} d^{3} v=\frac{\partial}{\partial t}\left(\int_{v} \chi f_{\alpha} d^{3} v\right)-\int_{v} f_{\alpha} \frac{\partial \chi}{\partial t} d^{3} v
$$

since the limits of integration do not depend upon the space and time variables, and therefore the partial time derivative can be taken inside or outside the integral sign. The last integral in (2.3) vanishes since $\chi(\mathbf{v})$ does not depend upon $t$. Using the definition of average values, as presented in Chapter 6, we obtain

$$
\int_{v} \chi \frac{\partial f_{\alpha}}{\partial t} d^{3} v=\frac{\partial}{\partial t}\left(n_{\alpha}<\chi>_{\alpha}\right)
$$

Similarly, for the second term of (2.2) we can write

$$
\begin{aligned}
\int_{v} \chi \mathbf{v} \cdot \nabla f_{\alpha} d^{3} v= & \nabla \cdot\left(\int_{v} \mathbf{v} \chi f_{\alpha} d^{3} v\right)-\int_{v} f_{\alpha} \mathbf{v} \cdot \nabla \chi d^{3} v \\
& -\int_{v} f_{\alpha} \chi \nabla \cdot \mathbf{v} d^{3} v
\end{aligned}
$$

The term involving $\nabla \cdot \mathbf{v}$ is zero, since $\mathbf{r}, \mathbf{v}$, and $t$ are independent variables, as well as the term involving $\nabla \chi$, since $\chi(\mathbf{v})$ does not depend upon the space variables. Thus, the second term in (2.2) becomes

$$
\int_{v} \chi \mathbf{v} \cdot \nabla f_{\alpha} d^{3} v=\nabla \cdot\left(n_{\alpha}<\chi \mathbf{v}>_{\alpha}\right)
$$


For the third term of (2.2) we have, in a similar way,

$$
\begin{gathered}
\int_{v} \chi \mathbf{a} \cdot \nabla_{v} f_{\alpha} d^{3} v=\int_{v} \nabla_{v} \cdot\left(\mathbf{a} \chi f_{\alpha}\right) d^{3} v-\int_{v} f_{\alpha} \mathbf{a} \cdot \nabla_{v} \chi d^{3} v- \\
\int_{v} f_{\alpha} \chi \nabla_{v} \cdot \mathbf{a} d^{3} v
\end{gathered}
$$

The last integral in (2.7) vanishes if we assume that

$$
\nabla_{v} \cdot \mathbf{a}=\frac{1}{m_{\alpha}} \nabla_{v} \cdot \mathbf{F}=0
$$

that is, if the force component $F_{i}$ is independent of the corresponding velocity component $v_{i}$, for $i=x, y, z$. Note that this restriction does not exclude the force due to a magnetic field, $\mathbf{F}=q_{\alpha} \mathbf{v} \times \mathbf{B}$, since in this case $F_{i}$ is still independent of $v_{i}$. For the $x$ component, for example, we have

$$
F_{x}=q_{\alpha}\left(v_{y} B_{z}-v_{z} B_{y}\right)
$$

which is independent of $v_{x}$, and the same holds true for the other two components. The first integral in the right-hand side of (2.7) consists of a sum of three triple integrals,

$$
\int_{v} \nabla_{v} \cdot\left(\mathbf{a} \chi f_{\alpha}\right) d^{3} v=\sum_{i} \iiint_{-\infty}^{+\infty} \frac{\partial}{\partial v_{i}}\left(a_{i} \chi f_{\alpha}\right) d v_{x} d v_{y} d v_{z}
$$

For each one of these triple integrals $(i=x, y, z)$ we have the result

$$
\begin{gathered}
\iiint_{-\infty}^{+\infty} \frac{\partial}{\partial v_{x}}\left(a_{x} \chi f_{\alpha}\right) d v_{x} d v_{y} d v_{z}= \\
\iint_{-\infty}^{+\infty} d v_{y} d v_{z}\left(\left.a_{x} \chi f_{\alpha}\right|_{-\infty} ^{+\infty}\right)=0
\end{gathered}
$$

since $f_{\alpha}(\mathbf{r}, \mathbf{v}, t)$ must be zero when $v_{i}$ becomes infinitely large, as there are no particles with infinite velocity. Consequently, the first integral in the right-hand side of (2.7) vanishes. Therefore,

$$
\int_{v} \chi \mathbf{a} \cdot \nabla_{v} f_{\alpha} d^{3} v=-n_{\alpha}<\mathbf{a} \cdot \nabla_{v} \chi>_{\alpha}
$$


Combining the results contained in Eqs. (2.4), (2.6), and (2.12), we obtain the general transport equation,

$$
\begin{aligned}
\frac{\partial}{\partial t}\left(n_{\alpha}<\chi>_{\alpha}\right)+ & \nabla \cdot\left(n_{\alpha}<\chi \mathbf{v}>_{\alpha}\right)-n_{\alpha}<\mathbf{a} \cdot \nabla_{v} \chi>_{\alpha}= \\
& {\left[\frac{\delta}{\delta t}\left(n_{\alpha}<\chi>_{\alpha}\right)\right]_{\text {coll }} }
\end{aligned}
$$

where the term in the right denotes the time rate of change of the quantity $\chi$ per unit volume, for the particles of type $\alpha$, due to collisions,

$$
\left[\frac{\delta}{\delta t}\left(n_{\alpha}<\chi>_{\alpha}\right)\right]_{\text {coll }}=\int_{v} \chi\left(\frac{\delta f_{\alpha}}{\delta t}\right)_{\text {coll }} d^{3} v
$$

The equations to be derived in the subsequent sections of this chapter are very general and are not specifically dependent on any particular form of the collision term. A derivation of the general transport equation for the case when the property $\chi$ is a function of $\mathbf{r}, \mathbf{v}$, and $t$ is included in problem 8.6.

\section{CONSERVATION OF MASS}

\subsection{Derivation of the Continuity Equation}

The transport equation (2.13) is a general expression and it applies to any arbitrary function $\chi(\mathbf{v})$. The equation of continuity, or of conservation of mass, can be obtained by taking $\chi=m_{\alpha}$ in (2.13). Hence, consider

$$
\begin{gathered}
<\chi>_{\alpha}=m_{\alpha} \\
<\chi \mathbf{v}>_{\alpha}=m_{\alpha}<\mathbf{v}>_{\alpha}=m_{\alpha} \mathbf{u}_{\alpha} \\
\nabla_{v} \chi=\nabla_{v} m_{\alpha}=0
\end{gathered}
$$

The substitution of these results into the general transport equation gives the continuity equation,

$$
\frac{\partial \rho_{m \alpha}}{\partial t}+\nabla \cdot\left(\rho_{m \alpha} \mathbf{u}_{\alpha}\right)=S_{\alpha}
$$

where $\rho_{m \alpha}=n_{\alpha} m_{\alpha}$ represents the mass density and where the collision term $S_{\alpha}$, defined by

$$
S_{\alpha}=m_{\alpha} \int_{v}\left(\frac{\delta f_{\alpha}}{\delta t}\right)_{\text {coll }} d^{3} v=\left(\frac{\delta \rho_{m \alpha}}{\delta t}\right)_{\text {coll }}
$$

represents the rate per unit volume at which particles of type $\alpha$ (with mass $m_{\alpha}$ ) are produced or lost as a result of collisions. Contributions to 
this term are due to processes of type $\alpha$ particle production or destruction such as ionization, recombination, attachment, charge transfer, and so on. In the absence of interactions leading to production or loss of particles of type $\alpha$, the collision term (3.3) is equal to zero, since in this case the mass is conserved in the collision process. When $S_{\alpha}=0$ the continuity equation reduces to

$$
\frac{\partial \rho_{m \alpha}}{\partial t}+\nabla \cdot\left(\rho_{m \alpha} \mathbf{u}_{\alpha}\right)=0
$$

Dividing each term in (3.4) by $m_{\alpha}$, the continuity equation can be written in terms of the number density $n_{\alpha}$, as

$$
\frac{\partial n_{\alpha}}{\partial t}+\nabla \cdot\left(n_{\alpha} \mathbf{u}_{\alpha}\right)=0
$$

The equation of conservation of electric charge follows from (3.5) by multiplying it by the particle charge $q_{\alpha}$,

$$
\frac{\partial \rho_{\alpha}}{\partial t}+\nabla \cdot \mathbf{J}_{\alpha}=0
$$

where $\rho_{\alpha}=n_{\alpha} q_{\alpha}$ is the charge density and $\mathbf{J}_{\alpha}=\rho_{\alpha} \mathbf{u}_{\alpha}$ is the charge current density.

\subsection{Derivation by the Method of Fluid Dynamics}

The continuity equation can also be derived using the method of fluid dynamics, since $n_{\alpha}(\mathbf{r}, t)$ and $\mathbf{u}_{\alpha}(\mathbf{r}, t)$ are macroscopic variables. Consider a volume $V$ in the fluid, limited by the closed surface $S$, and let $d \mathbf{S}=\widehat{\mathbf{n}} d S$ be an element of area on this surface, such that the unit normal vector $\widehat{\mathbf{n}}$ points outward, as shown in Fig. 1. The average number of particles of type $\alpha$ that leave the volume $V$ through the element of area $d \mathbf{S}$ per unit time is given by

$$
n_{\alpha} \mathbf{u}_{\alpha} \cdot d \mathbf{S}
$$

Therefore, the number of particles of type $\alpha$ that leave the volume $V$ through the whole closed surface $S$ per unit time is obtained by integrating expression (3.7) over the whole surface,

$$
\oint_{S} n_{\alpha} \mathbf{u}_{\alpha} \cdot d \mathbf{S}
$$




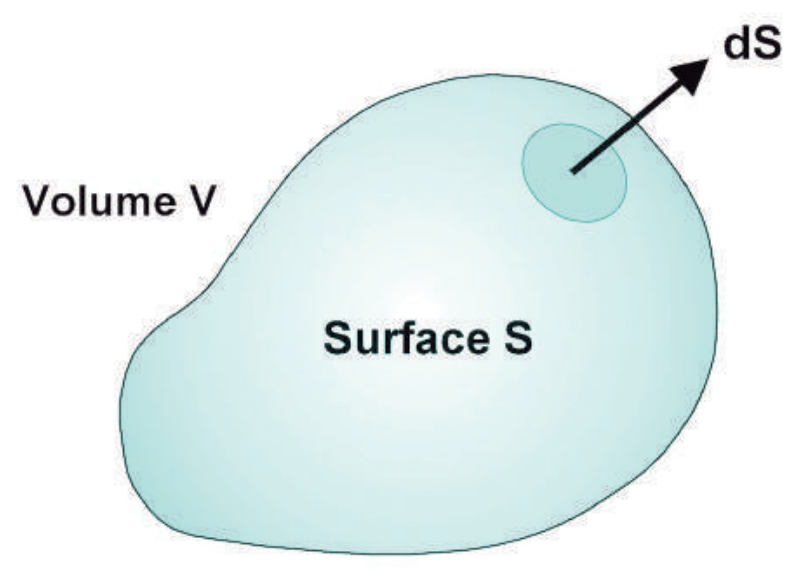

Fig. 1 Closed surface $S$ surrounding the arbitrary volume $V$ inside the fluid, and the element of area $d \mathbf{S}=\widehat{\mathbf{n}} d S$ pointing outwards.

On the other hand, the total number of particles of type $\alpha$ contained in $V$, at any time, is given by

$$
\int_{V} n_{\alpha} d^{3} r
$$

If we consider that there are no production or loss of particles inside the volume $V$, then the number of type $\alpha$ particles leaving $V$ must equal the time rate of decrease of the number of type $\alpha$ particles inside $V$. We must have, therefore,

$$
\oint_{S} n_{\alpha} \mathbf{u}_{\alpha} \cdot d \mathbf{S}=-\frac{\partial}{\partial t} \int_{V} n_{\alpha} d^{3} r
$$

Using Gauss's divergence theorem we can write

$$
\oint_{S} n_{\alpha} \mathbf{u}_{\alpha} \cdot d \mathbf{S}=\int_{V} \nabla \cdot\left(n_{\alpha} \mathbf{u}_{\alpha}\right) d^{3} r
$$

and (3.10) becomes

$$
\int_{V}\left[\frac{\partial n_{\alpha}}{\partial t}+\nabla \cdot\left(n_{\alpha} \mathbf{u}_{\alpha}\right)\right] d^{3} r=0
$$

This result must be valid for any arbitrary volume $V$, which implies that the integrand of (3.12) vanishes identically. Hence, we obtain the expression (3.5) for the continuity equation. 


\subsection{The Collision Term}

Let us consider now the form of the collision term $S_{\alpha}$ for some mechanisms of production and loss of particles in plasmas. The processes leading to production and loss of particles are usually related to inelastic collisions, such as those involving ionization, recombination, or electron attachment, for example.

The effect of ionization can be included in the continuity equation through a rate coefficient for ionization, $k_{i}$, defined such that the number of electrons produced per unit time is given by $k_{i} n_{e}$.

An important process leading to the loss of electrons and ions in a plasma is ion-electron recombination. Let $k_{r}$ denote the recombination coefficient, which can be determined experimentally. The rate of electron recombination is proportional to the product of the electron and ion number densities. Assuming that there is only one ion species present, we have $n_{i}=n_{e}$, and the electron loss term, due to recombination, can be written as $k_{r} n_{e}^{2}$.

Another important mechanism for electron loss is the process of electron attachment. In this case, the electron loss rate is proportional to the product of the electron number density and the neutral particle number density. In a weakly ionized plasma the neutral particle number density can be considered to be approximately constant, and the loss term for the electrons due to attachment can be written as $k_{a} n_{e}$, where $k_{a}$ is the attachment collision frequency, which can be determined experimentally.

For these inelastic collision mechanisms just described, the collision term $S_{\alpha}$ for the electrons can be expressed as

$$
S_{e}=m_{e}\left(k_{i} n_{e}-k_{r} n_{e}^{2}-k_{a} n_{e}\right)
$$

\section{CONSERVATION OF MOMENTUM}

\subsection{Derivation of the Equation of Motion}

In order to derive the momentum transport equation, we replace $\chi(\mathbf{v})$ by $m_{\alpha} \mathbf{v}$ in the general transport equation (2.13). Taking $\mathbf{v}=\mathbf{c}_{\alpha}+\mathbf{u}_{\alpha}$ and noting that $\left\langle\mathbf{c}_{\alpha}\right\rangle=0$, the terms of the general transport equation (2.13) become

$$
\frac{\partial}{\partial t}\left(\rho_{m \alpha}<\mathbf{v}>_{\alpha}\right)=\rho_{m \alpha} \frac{\partial \mathbf{u}_{\alpha}}{\partial t}+\mathbf{u}_{\alpha} \frac{\partial \rho_{m \alpha}}{\partial t}
$$




$$
\begin{gathered}
\nabla \cdot\left(\rho_{m \alpha}<\mathbf{v} \mathbf{v}>_{\alpha}\right)=\nabla \cdot\left[\rho _ { m \alpha } \left(\mathbf{u}_{\alpha} \mathbf{u}_{\alpha}+\mathbf{u}_{\alpha}<\mathbf{c}_{\alpha}>+<\mathbf{c}_{\alpha}>\mathbf{u}_{\alpha}+\right.\right. \\
\left.\left\langle\mathbf{c}_{\alpha} \mathbf{c}_{\alpha}>\right)\right]=\nabla \cdot\left(\rho_{m \alpha} \mathbf{u}_{\alpha} \mathbf{u}_{\alpha}+\rho_{m \alpha}<\mathbf{c}_{\alpha} \mathbf{c}_{\alpha}>\right) \\
-n_{\alpha}<\mathbf{F} \cdot \nabla_{v} \mathbf{v}>_{\alpha}=-n_{\alpha}<\left(F_{x} \frac{\partial}{\partial v_{x}}+F_{y} \frac{\partial}{\partial v_{y}}+F_{z} \frac{\partial}{\partial v_{z}}\right) \mathbf{v}>_{\alpha} \\
=-n_{\alpha}<F_{x} \widehat{\mathbf{x}}+F_{y} \widehat{\mathbf{y}}+F_{z} \widehat{\mathbf{z}}>_{\alpha}=-n_{\alpha}<\mathbf{F}>_{\alpha}
\end{gathered}
$$

Substituting these expressions into (2.13), results in the momentum conservation equation

$$
\begin{gathered}
\rho_{m \alpha} \frac{\partial \mathbf{u}_{\alpha}}{\partial t}+\mathbf{u}_{\alpha} \frac{\partial \rho_{m \alpha}}{\partial t}+\nabla \cdot\left(\rho_{m \alpha} \mathbf{u}_{\alpha} \mathbf{u}_{\alpha}\right)+\nabla \cdot\left(\rho_{m \alpha}<\mathbf{c}_{\alpha} \mathbf{c}_{\alpha}>\right)- \\
n_{\alpha}<\mathbf{F}>_{\alpha}=\mathbf{A}_{\alpha}
\end{gathered}
$$

where $\mathbf{A}_{\alpha}$ denotes the collision term

$$
\mathbf{A}_{\alpha}=m_{\alpha} \int_{v} \mathbf{v}\left(\frac{\delta f_{\alpha}}{\delta t}\right)_{c o l l} d^{3} v=\left[\frac{\delta\left(\rho_{m \alpha} \mathbf{u}_{\alpha}\right)}{\delta t}\right]_{c o l l}
$$

The expression $\rho_{m \alpha}\left\langle\mathbf{c}_{\alpha} \mathbf{c}_{\alpha}>\right.$ is the kinetic pressure dyad $\mathcal{P}_{\alpha}$ defined in (6.6.2) (Eq. 6.2 in Chapter 6). Therefore,

$$
\nabla \cdot\left(\rho_{m \alpha}<\mathbf{c}_{\alpha} \mathbf{c}_{\alpha}>\right)=\nabla \cdot \mathcal{P}_{\alpha}
$$

The third term in the left-hand side of (4.2) can be expanded as follows:

$$
\begin{aligned}
& \nabla \cdot\left(\rho_{m \alpha} \mathbf{u}_{\alpha} \mathbf{u}_{\alpha}\right)=\frac{\partial}{\partial x}\left(\rho_{m \alpha} u_{\alpha x} \mathbf{u}_{\alpha}\right)+\frac{\partial}{\partial y}\left(\rho_{m \alpha} u_{\alpha y} \mathbf{u}_{\alpha}\right)+\frac{\partial}{\partial z}\left(\rho_{m \alpha} u_{\alpha z} \mathbf{u}_{\alpha}\right) \\
&= \rho_{m \alpha}\left(u_{\alpha x} \frac{\partial \mathbf{u}_{\alpha}}{\partial x}+u_{\alpha y} \frac{\partial \mathbf{u}_{\alpha}}{\partial y}+u_{\alpha z} \frac{\partial \mathbf{u}_{\alpha}}{\partial z}\right)+ \\
& \mathbf{u}_{\alpha}\left[\frac{\partial\left(\rho_{m \alpha} u_{\alpha x}\right)}{\partial x}+\frac{\partial\left(\rho_{m \alpha} u_{\alpha y}\right)}{\partial y}+\frac{\partial\left(\rho_{m \alpha} u_{\alpha z}\right)}{\partial z}\right] \\
&=\rho_{m \alpha}\left(\mathbf{u}_{\alpha} \cdot \nabla\right) \mathbf{u}_{\alpha}+\mathbf{u}_{\alpha}\left[\nabla \cdot\left(\rho_{m \alpha} \mathbf{u}_{\alpha}\right)\right]
\end{aligned}
$$

Substituting (4.4) and (4.5) into (4.2), and using the continuity equation (3.2), we obtain

$$
\rho_{m \alpha}\left[\frac{\partial \mathbf{u}_{\alpha}}{\partial t}+\left(\mathbf{u}_{\alpha} \cdot \nabla\right) \mathbf{u}_{\alpha}\right]+\nabla \cdot \mathcal{P}_{\alpha}-n_{\alpha}<\mathbf{F}>_{\alpha}=\mathbf{A}_{\alpha}-\mathbf{u}_{\alpha} S_{\alpha}
$$


For the terms within brackets in this last equation we can use the total (or substantial) time derivative operator

$$
\frac{D}{D t}=\frac{\partial}{\partial t}+\mathbf{u}_{\alpha} \cdot \nabla
$$

which corresponds to the time variation observed in a reference frame moving with the mean velocity $\mathbf{u}_{\alpha}$. If the electromagnetic Lorentz force and the gravitational force are considered, the last term in the left-hand side of (4.6) becomes

$$
-n_{\alpha}<\mathbf{F}>_{\alpha}=-n_{\alpha} q_{\alpha}\left(\mathbf{E}+\mathbf{u}_{\alpha} \times \mathbf{B}\right)-n_{\alpha} m_{\alpha} \mathbf{g}
$$

where the fields $\mathbf{E}$ and $\mathbf{B}$, in this equation, represent smoothed macroscopic fields. The equation of motion, therefore, can be written as

$$
\rho_{m \alpha} \frac{D \mathbf{u}_{\alpha}}{D t}=n_{\alpha} q_{\alpha}\left(\mathbf{E}+\mathbf{u}_{\alpha} \times \mathbf{B}\right)+\rho_{m \alpha} \mathbf{g}-\nabla \cdot \mathcal{P}_{\alpha}+\mathbf{A}_{\alpha}-\mathbf{u}_{\alpha} S_{\alpha}
$$

Physically, this equation states that the time rate of change of the mean momentum, in each fluid element, is due to the external forces applied in the fluid, plus the shear (viscosity) and pressure forces of the fluid itself, plus the internal forces associated with the collisional interactions. Thus, the equation of motion establishes the condition necessary to guarantee conservation of momentum, just as the continuity equation establishes the condition necessary to guarantee conservation of mass (or number of particles).

In Chapter 6 we have seen that the term $-\nabla \cdot \mathcal{P}_{\alpha}$ represents the force exerted in a unit volume of the plasma, due to the random variations in the particle peculiar velocities. This force per unit volume includes forces associated with the scalar pressure and tangential shear forces (viscous forces). In many cases, the effect of viscosity is relatively unimportant in plasmas, and the nondiagonal terms of $\mathcal{P}_{\alpha}$ can be neglected. Furthermore, in the special case when the distribution of peculiar velocities is isotropic, the diagonal terms of $\mathcal{P}_{\alpha}$ are all equal and correspond to a scalar kinetic pressure $p_{\alpha}$. Thus, neglecting viscosity effects and considering an isotropic velocity distribution, we have $\mathcal{P}_{\alpha}=p_{\alpha} \mathbf{1}$, and the force per unit volume becomes $-\nabla \cdot \mathcal{P}_{\alpha}=-\nabla p_{\alpha}$, according to (6.6.18) (Eq. 6.18 in Chapter 6).

With these simplifying approximations, and neglecting collisions leading to production or loss of particles $\left(S_{\alpha}=0\right)$, the momentum equation becomes

$$
\rho_{m \alpha} \frac{D \mathbf{u}_{\alpha}}{D t}=n_{\alpha} q_{\alpha}\left(\mathbf{E}+\mathbf{u}_{\alpha} \times \mathbf{B}\right)+\rho_{m \alpha} \mathbf{g}-\nabla p_{\alpha}+\mathbf{A}_{\alpha}
$$


The momentum conservation equation can also be derived using a fluid-dynamics approach, in a way similar to the derivation of the mass conservation equation presented in section 3.2 , which we shall not discuss here.

\subsection{The Collision Term}

The symbol $\mathbf{A}_{\alpha}$ denotes the rate of change of the mean momentum per unit volume, due to collisions. As a consequence of conservation of the total momentum in an elastic collision, the change in the momentum of one of the particles must be equal and opposite to the change in momentum of the other particle participating in the collision event. This means that, for collisions involving particles of the same species, there is no variation in the total momentum per unit volume and therefore, in this case, $\mathbf{A}_{\alpha}=0$. However, for a fluid composed of particles of different species, as in a plasma, the collision term $\mathbf{A}_{\alpha}$ is not zero in general. For collisions between electrons and neutral particles there is a net momentum transfer from the electron gas to the neutral gas. Collisions between electrons and ions also modify the total momentum of the electron gas. Therefore, for the case of collisions between particles of different species, a collision term must be included in the equation of momentum conservation.

An expression often used for the term of momentum transfer by collisions is

$$
\mathbf{A}_{\alpha}=-\rho_{m \alpha} \sum_{\beta} \nu_{\alpha \beta}\left(\mathbf{u}_{\alpha}-\mathbf{u}_{\beta}\right)
$$

which assumes that the force per unit volume exerted on the particles of type $\alpha$ due to collisions with particles of some other type $(\beta)$ is proportional to the difference between the mean velocities of these particles. The proportionality constant $\nu_{\alpha \beta}$ (which has dimensions of $\sec ^{-1}$ ) is called the collision frequency for momentum transfer between the particles of type $\alpha$ and those of type $\beta$. Since the total momentum must be conserved during a collision, we must have

$$
\rho_{m \alpha} \nu_{\alpha \beta}\left(\mathbf{u}_{\alpha}-\mathbf{u}_{\beta}\right)+\rho_{m \beta} \nu_{\beta \alpha}\left(\mathbf{u}_{\beta}-\mathbf{u}_{\alpha}\right)=0
$$

The collision frequencies $\nu_{\alpha \beta}$ and $\nu_{\beta \alpha}$ satisfy, therefore, the following important relation:

$$
\rho_{m \alpha} \nu_{\alpha \beta}=\rho_{m \beta} \nu_{\beta \alpha}
$$

The collision term $\mathbf{A}_{\alpha}$, defined in (4.3), will be considered in more detail in Chapter 21. We will see, then, that the expression (4.11) is not 
generally valid, although this result is obtained when the difference between the mean velocities of the various particle species in the plasma is relatively small and when each particle species has a Maxwellian velocity distribution.

\section{CONSERVATION OF ENERGY}

\subsection{Derivation of the Energy Transport Equation}

To derive the energy transport equation, we substitute $\chi(\mathbf{v})$ by the particle kinetic energy $m_{\alpha} v^{2} / 2$ in the general transport equation (2.13). In this case, we have

$$
\begin{gathered}
n_{\alpha}<\chi>_{\alpha}=\frac{1}{2} \rho_{m \alpha}<c_{\alpha}^{2}>+\frac{1}{2} \rho_{m \alpha} u_{\alpha}^{2}= \\
\frac{1}{2}\left(3 p_{\alpha}+\rho_{m \alpha} u_{\alpha}^{2}\right) \\
\nabla_{v} \chi=\frac{1}{2} m_{\alpha} \nabla_{v}(\mathbf{v} \cdot \mathbf{v})=m_{\alpha}\left(\mathbf{v} \cdot \nabla_{v}\right) \mathbf{v}=m_{\alpha} \mathbf{v}
\end{gathered}
$$

Therefore, the terms in the left-hand side of the general transport equation (2.13) become

$$
\begin{gathered}
\frac{\partial}{\partial t}\left(n_{\alpha}<\chi>_{\alpha}\right)=\frac{3}{2} \frac{\partial p_{\alpha}}{\partial t}+\frac{\partial}{\partial t}\left(\frac{1}{2} \rho_{m \alpha} u_{\alpha}^{2}\right) \\
\nabla \cdot\left(n_{\alpha}<\chi \mathbf{v}>_{\alpha}\right)=\nabla \cdot\left[\frac{1}{2} \rho_{m \alpha}<(\mathbf{v} \cdot \mathbf{v}) \mathbf{v}>_{\alpha}\right] \\
-n_{\alpha}<\left(\mathbf{F} / m_{\alpha}\right) \cdot \nabla_{v} \chi>_{\alpha}=-n_{\alpha}<\mathbf{F} \cdot \mathbf{v}>_{\alpha}
\end{gathered}
$$

Adding these terms, results in the following energy conservation equation:

$$
\begin{gathered}
\frac{3}{2} \frac{\partial p_{\alpha}}{\partial t}+\frac{\partial}{\partial t}\left(\frac{1}{2} \rho_{m \alpha} u_{\alpha}^{2}\right)+\nabla \cdot\left[\frac{1}{2} \rho_{m \alpha}<(\mathbf{v} \cdot \mathbf{v}) \mathbf{v}>_{\alpha}\right]- \\
n_{\alpha}<\mathbf{F} \cdot \mathbf{v}>_{\alpha}=M_{\alpha}
\end{gathered}
$$

where $M_{\alpha}$ represents the rate of energy density change due to collisions,

$$
M_{\alpha}=\frac{1}{2} m_{\alpha} \int_{v} v^{2}\left(\frac{\delta f_{\alpha}}{\delta t}\right)_{c o l l} d^{3} v=\left[\frac{\delta\left(\frac{1}{2} \rho_{m \alpha}<v^{2}>_{\alpha}\right)}{\delta t}\right]_{c o l l}
$$

The energy conservation equation (5.4) can be written in an alternative form as follows. Consider, initially, the third term in the left-hand 
side of (5.4). Taking $\mathbf{v}=\mathbf{c}_{\alpha}+\mathbf{u}_{\alpha}$, the quantity $\langle(\mathbf{v} \cdot \mathbf{v}) \mathbf{v}\rangle_{\alpha}$ can be expanded as

$$
\begin{gathered}
<\left[\left(\mathbf{u}_{\alpha}+\mathbf{c}_{\alpha}\right) \cdot\left(\mathbf{u}_{\alpha}+\mathbf{c}_{\alpha}\right)\right]\left(\mathbf{u}_{\alpha}+\mathbf{c}_{\alpha}\right)>=<\left(u_{\alpha}^{2}+2 \mathbf{u}_{\alpha} \cdot \mathbf{c}_{\alpha}+c_{\alpha}^{2}\right)\left(\mathbf{u}_{\alpha}+\mathbf{c}_{\alpha}\right)> \\
=u_{\alpha}^{2} \mathbf{u}_{\alpha}+<c_{\alpha}^{2}>\mathbf{u}_{\alpha}+2<\mathbf{c}_{\alpha} \mathbf{c}_{\alpha}>\cdot \mathbf{u}_{\alpha}+<c_{\alpha}^{2} \mathbf{c}_{\alpha}>
\end{gathered}
$$

The term $\rho_{m \alpha}\left\langle\mathbf{c}_{\alpha} \mathbf{c}_{\alpha}>\right.$ represents the kinetic pressure dyad $\mathcal{P}_{\alpha}$ and $\frac{1}{2} \rho_{m \alpha}<c_{\alpha}^{2} \mathbf{c}_{\alpha}>$ is the heat flux vector $\mathbf{q}_{\alpha}$, defined in Chapter 6 . We have also seen that $\frac{1}{2} \rho_{m \alpha}<c_{\alpha}^{2}>=3 p_{\alpha} / 2$. Therefore,

$$
\begin{gathered}
\nabla \cdot\left[\frac{1}{2} \rho_{m \alpha}<(\mathbf{v} \cdot \mathbf{v}) \mathbf{v}>_{\alpha}\right]=\nabla \cdot\left[\frac{1}{2} \rho_{m \alpha} u_{\alpha}^{2} \mathbf{u}_{\alpha}+\frac{1}{2}\left(3 p_{\alpha}\right) \mathbf{u}_{\alpha}+\mathcal{P}_{\alpha} \cdot \mathbf{u}_{\alpha}+\mathbf{q}_{\alpha}\right]= \\
\nabla \cdot\left(\frac{1}{2} \rho_{m \alpha} u_{\alpha}^{2} \mathbf{u}_{\alpha}\right)+\frac{1}{2}\left(3 p_{\alpha}\right)\left(\nabla \cdot \mathbf{u}_{\alpha}\right)+ \\
\frac{1}{2}\left(\mathbf{u}_{\alpha} \cdot \nabla\right)\left(3 p_{\alpha}\right)+\nabla \cdot\left(\mathcal{P}_{\alpha} \cdot \mathbf{u}_{\alpha}\right)+\nabla \cdot \mathbf{q}_{\alpha}
\end{gathered}
$$

Substituting this result into (5.4) and using the notation $D / D t$ for the total time derivative (4.7), we obtain

$$
\begin{gathered}
\frac{D}{D t}\left(\frac{3 p_{\alpha}}{2}\right)+\left(\frac{3 p_{\alpha}}{2}\right) \nabla \cdot \mathbf{u}_{\alpha}+\frac{\partial}{\partial t}\left(\frac{1}{2} \rho_{m \alpha} u_{\alpha}^{2}\right)+\nabla \cdot\left(\frac{1}{2} \rho_{m \alpha} u_{\alpha}^{2} \mathbf{u}_{\alpha}\right)+ \\
\nabla \cdot\left(\mathcal{P}_{\alpha} \cdot \mathbf{u}_{\alpha}\right)+\nabla \cdot \mathbf{q}_{\alpha}-n_{\alpha}<\mathbf{F} \cdot \mathbf{v}>_{\alpha}=M_{\alpha}
\end{gathered}
$$

The third and fourth terms in the left-hand side can be written as

$$
\begin{gathered}
\frac{\partial}{\partial t}\left(\frac{1}{2} \rho_{m \alpha} \mathbf{u}_{\alpha} \cdot \mathbf{u}_{\alpha}\right)+\nabla \cdot\left[\frac{1}{2} \rho_{m \alpha}\left(\mathbf{u}_{\alpha} \cdot \mathbf{u}_{\alpha}\right) \mathbf{u}_{\alpha}\right]= \\
\frac{1}{2} u_{\alpha}^{2} \frac{\partial \rho_{m \alpha}}{\partial t}+\rho_{m \alpha} \mathbf{u}_{\alpha} \cdot \frac{\partial \mathbf{u}_{\alpha}}{\partial t}+ \\
\frac{1}{2} u_{\alpha}^{2} \nabla \cdot\left(\rho_{m \alpha} \mathbf{u}_{\alpha}\right)+\rho_{m \alpha} \mathbf{u}_{\alpha} \cdot\left[\left(\mathbf{u}_{\alpha} \cdot \nabla\right) \mathbf{u}_{\alpha}\right]= \\
\frac{1}{2} u_{\alpha}^{2}\left[\frac{\partial \rho_{m \alpha}}{\partial t}+\nabla \cdot\left(\rho_{m \alpha} \mathbf{u}_{\alpha}\right)\right]+\rho_{m \alpha} \mathbf{u}_{\alpha} \cdot \frac{D \mathbf{u}_{\alpha}}{D t}
\end{gathered}
$$

Using the continuity equation (3.2) and the equation of motion (4.6), this last equation becomes

$$
\frac{1}{2} u_{\alpha}^{2} S_{\alpha}+n_{\alpha} \mathbf{u}_{\alpha} \cdot<\mathbf{F}>_{\alpha}-\mathbf{u}_{\alpha} \cdot\left(\nabla \cdot \mathcal{P}_{\alpha}\right)+\mathbf{u}_{\alpha} \cdot \mathbf{A}_{\alpha}-u_{\alpha}^{2} S_{\alpha}
$$

Taking this result back into (5.8) we obtain

$$
\frac{D}{D t}\left(\frac{3 p_{\alpha}}{2}\right)+\frac{3 p_{\alpha}}{2} \nabla \cdot \mathbf{u}_{\alpha}+\nabla \cdot\left(\mathcal{P}_{\alpha} \cdot \mathbf{u}_{\alpha}\right)-\mathbf{u}_{\alpha} \cdot\left(\nabla \cdot \mathcal{P}_{\alpha}\right)-
$$




$$
\begin{gathered}
n_{\alpha}<\mathbf{F} \cdot \mathbf{v}>_{\alpha}+n_{\alpha} \mathbf{u}_{\alpha} \cdot<\mathbf{F}>_{\alpha}+\nabla \cdot \mathbf{q}_{\alpha}= \\
M_{\alpha}-\mathbf{u}_{\alpha} \cdot \mathbf{A}_{\alpha}+\frac{1}{2} u_{\alpha}^{2} S_{\alpha}
\end{gathered}
$$

The third and fourth terms in the left-hand side of this equation can be combined into one single term,

$$
\nabla \cdot\left(\mathcal{P}_{\alpha} \cdot \mathbf{u}_{\alpha}\right)-\mathbf{u}_{\alpha} \cdot\left(\nabla \cdot \mathcal{P}_{\alpha}\right)=\left(\mathcal{P}_{\alpha} \cdot \nabla\right) \cdot \mathbf{u}_{\alpha}
$$

as well as the fifth and the sixth terms, which give

$$
-n_{\alpha}<\mathbf{F} \cdot \mathbf{v}>_{\alpha}+n_{\alpha} \mathbf{u}_{\alpha} \cdot\left\langle\mathbf{F}>_{\alpha}=-n_{\alpha}<\mathbf{F} \cdot \mathbf{c}_{\alpha}>\right.
$$

since

$$
\left\langle\mathbf{F} \cdot \mathbf{v}>_{\alpha}=\left\langle\mathbf{F} \cdot\left(\mathbf{u}_{\alpha}+\mathbf{c}_{\alpha}\right)>=\left\langle\mathbf{F}>_{\alpha} \cdot \mathbf{u}_{\alpha}+\left\langle\mathbf{F} \cdot \mathbf{c}_{\alpha}\right\rangle\right.\right.\right.
$$

For a velocity-independent force (5.13) vanishes, since, in this case,

$$
<\mathbf{F} \cdot \mathbf{c}_{\alpha}>=\mathbf{F} \cdot\left\langle\mathbf{c}_{\alpha}>=0\right.
$$

For the force due to a magnetic field $\mathbf{B}$, the only velocity-dependent force that we are interested here, (5.13) also vanishes,

$$
\begin{gathered}
<\mathbf{F} \cdot \mathbf{c}_{\alpha}>=q_{\alpha}<(\mathbf{v} \times \mathbf{B}) \cdot \mathbf{c}_{\alpha}>= \\
q_{\alpha}\left(\mathbf{u}_{\alpha} \times \mathbf{B}\right) \cdot<\mathbf{c}_{\alpha}>+q_{\alpha}<\left(\mathbf{c}_{\alpha} \times \mathbf{B}\right) \cdot \mathbf{c}_{\alpha}>=0
\end{gathered}
$$

where both terms vanish since $\left\langle\mathbf{c}_{\alpha}\right\rangle=0$ and $\left(\mathbf{c}_{\alpha} \times \mathbf{B}\right)$ is normal to $\mathbf{c}_{\alpha}$. We obtain, finally, the following alternative form for the equation of conservation of energy:

$$
\begin{gathered}
\frac{D}{D t}\left(\frac{3 p_{\alpha}}{2}\right)+\frac{3 p_{\alpha}}{2} \nabla \cdot \mathbf{u}_{\alpha}+\left(\mathcal{P}_{\alpha} \cdot \nabla\right) \cdot \mathbf{u}_{\alpha}+\nabla \cdot \mathbf{q}_{\alpha}= \\
M_{\alpha}-\mathbf{u}_{\alpha} \cdot \mathbf{A}_{\alpha}+\frac{1}{2} u_{\alpha}^{2} S_{\alpha}
\end{gathered}
$$




\subsection{Physical Interpretation}

The physical interpretation of this equation is as follows. The first term in the left-hand side represents the total rate of change of the particle thermal energy density in a volume element moving with the mean fluid velocity $\mathbf{u}_{\alpha}$. Note that the thermal energy density is given by $3 p_{\alpha} / 2=$ $\rho_{m \alpha}\left\langle c_{\alpha}^{2}>/ 2\right.$. The other terms of (5.17) contribute to some extent to this total rate of change of the thermal energy density. The second term in the left-hand side of (5.17) can be interpreted as the change in the thermal energy density due to particles entering the volume element with the mean velocity $\mathbf{u}_{\alpha}$. The third term is related to the work done on the unit volume by the kinetic pressure dyad acting on its surface, whereas the fourth term represents the change in the thermal energy density due to the heat flux. Finally, the terms in the right-hand side of (5.17) represent the rate of change in the thermal energy density as a consequence of collisions. In the case of a fluid containing only one type of particles, the collision terms vanish, as indicated previously.

The first two terms in the energy equation may also be combined, making use of the continuity equation (3.2). Expanding $\nabla \cdot\left(\rho_{m \alpha} \mathbf{u}_{\alpha}\right)$, (3.2) becomes

$$
\left(\frac{\partial}{\partial t}+\mathbf{u}_{\alpha} \cdot \nabla\right) \rho_{m \alpha}+\rho_{m \alpha} \nabla \cdot \mathbf{u}_{\alpha}=S_{\alpha}
$$

which gives

$$
\nabla \cdot \mathbf{u}_{\alpha}=-\frac{1}{\rho_{m \alpha}}\left(\frac{D \rho_{m \alpha}}{D t}-S_{\alpha}\right)
$$

Substituting this result into (5.17), taking $\rho_{m \alpha}=n_{\alpha} m_{\alpha}$ and $p_{\alpha}=n_{\alpha} k T_{\alpha}$, yields the following alternative form for the energy equation in terms of the temperature $T_{\alpha}$ :

$$
\frac{3}{2} n_{\alpha} k \frac{D T_{\alpha}}{D t}+\left(\mathcal{P}_{\alpha} \cdot \nabla\right) \cdot \mathbf{u}_{\alpha}+\nabla \cdot \mathbf{q}_{\alpha}=M_{\alpha}-\mathbf{u}_{\alpha} \cdot \mathbf{A}_{\alpha}+\left(\frac{1}{2} u_{\alpha}^{2}-\frac{3}{2} \frac{k T_{\alpha}}{m_{\alpha}}\right) S_{\alpha}
$$

\subsection{Simplifying Approximations}

Several simplifying approximations can be considered for the energy equation, depending on the situation of interest.

(a) When the collision terms vanish, or are negligible, and when the mean 
fluid velocity $\mathbf{u}_{\alpha}$ is equal to zero, (5.20) reduces to a diffusion equation for $T_{\alpha}$, if we take the heat flux vector as

$$
\mathbf{q}_{\alpha}=-K \nabla T_{\alpha}
$$

where $K$ denotes the thermal conductivity. Thus, in this case, (5.20) reduces to

$$
\frac{3}{2} n_{\alpha} k \frac{D T_{\alpha}}{D t}=\nabla \cdot\left(K \nabla T_{\alpha}\right)
$$

The thermal conductivity coefficient $K$ is related to the fluid viscosity coefficient.

(b) Consider now a nonviscous fluid, in which the pressure dyad reduces to a scalar pressure without thermal conductivity $\left(\mathbf{q}_{\alpha}=0\right)$. If we consider also that the collision terms vanish, the energy conservation equation (5.17) becomes

$$
\frac{D}{D t}\left(\frac{3 p_{\alpha}}{2}\right)+\frac{3 p_{\alpha}}{2}\left(\nabla \cdot \mathbf{u}_{\alpha}\right)+p_{\alpha}\left(\nabla \cdot \mathbf{u}_{\alpha}\right)=0
$$

Substituting (5.19) for $\left(\nabla \cdot \mathbf{u}_{\alpha}\right)$, with $S_{\alpha}=0$, yields

$$
\frac{D}{D t}\left(\frac{3 p_{\alpha}}{2}\right)-\frac{5 p_{\alpha}}{2 \rho_{m \alpha}} \frac{D \rho_{m \alpha}}{D t}=0
$$

from which results

$$
\frac{D p_{\alpha}}{p_{\alpha}}=\frac{5}{3} \frac{D \rho_{m \alpha}}{\rho_{m \alpha}}
$$

Integrating this equation gives

$$
\frac{p_{\alpha}}{p_{0}}=\left(\frac{\rho_{m \alpha}}{\rho_{m 0}}\right)^{5 / 3}
$$

where $p_{0}$ and $\rho_{m 0}$ are constants, that is,

$$
p_{\alpha} \rho_{m \alpha}^{-5 / 3}=\text { constant }
$$

This is the adiabatic energy equation for a gas in which the ratio of the specific heats at constant pressure and at constant volume, $\gamma$, is equal to $5 / 3$. We emphasize here that the energy equation reduces to this adiabatic equation only when the effects of viscosity, thermal conductivity, and energy transfer due to collisions are neglected. 
The parameter $\gamma$ is related to the number of degrees of freedom, $N$, of a gas by the condition

$$
\gamma=(2+N) / N
$$

For particles that have no internal degrees of freedom, as for example in a monoatomic gas, where the only degrees of freedom are those associated with the three possible directions of translational motion, we have $N=3$ and therefore $\gamma=5 / 3$. Other degrees of freedom exist in the case of diatomic or poliatomic molecules. The adiabatic energy equation often used in thermodynamics is

$$
p \rho_{m}^{-\gamma}=\text { constant }
$$

Differentiating this equation, yields

$$
\rho_{m}^{-\gamma} d p-\gamma p \rho_{m}^{-(\gamma+1)} d \rho_{m}=0
$$

or, equivalently,

$$
d p=\left(\frac{\gamma p}{\rho_{m}}\right) d \rho_{m}=V_{s}^{2} d \rho_{m}
$$

where we have defined

$$
V_{s}=\left(\gamma p / \rho_{m}\right)^{1 / 2}=(\gamma k T / m)^{1 / 2}
$$

which is the adiabatic speed of sound for the fluid.

(c) An equation that is also used in thermodynamics when the temperature is constant inside the fluid, is the isothermal energy equation. It can be easily obtained from the equation of state for an ideal gas, $p=n k T$. For an isothermal process ( $T=$ constant), we have

$$
d p=k T d n=\left(p / \rho_{m}\right) d \rho_{m}=V_{T}^{2} d \rho_{m}
$$

where the isothermal speed of sound is

$$
V_{T}=\left(p / \rho_{m}\right)^{1 / 2}=(k T / m)^{1 / 2}
$$




\section{THE COLD PLASMA MODEL}

In the previous sections we have seen that the differential equations governing the temporal and spatial variation of the macroscopic variables can be obtained by taking the various moments of the Boltzmann equation. The macroscopic parameters are all related to the moments of the distribution function $f_{\alpha}(\mathbf{r}, \mathbf{v}, t)$. The first four moments of the distribution function give us the number density $n_{\alpha}$, the mean velocity $\mathbf{u}_{\alpha}$, the momentum flow dyad $\Pi_{\alpha}$, and the energy flow triad $\mathcal{E}_{\alpha}$.

The first moment of the Boltzmann equation gives us the continuity equation, which relates the number density $n_{\alpha}$ (or the mass density $\rho_{m \alpha}$ ) with the mean velocity $\mathbf{u}_{\alpha}$ for the particles of type $\alpha$. In order to determine these two macroscopic variables we need two independent macroscopic transport equations. Thus, we need to consider the second moment of the Boltzmann equation, which gives us the equation of motion (or momentum conservation equation), and which relates the mean velocity $\mathbf{u}_{\alpha}$ with the number density $n_{\alpha}$ and the kinetic pressure dyad $\mathcal{P}_{\alpha}$. This gives us now two transport equations involving three independent variables. We find, therefore, that the set of transport equations derived from the moments of the Boltzmann equation always includes more variables than independent equations. This situation is clearly evident in the three transport equations derived in this chapter. The energy equation, besides the variables $n_{\alpha}$, $\mathbf{u}_{\alpha}$, and $\mathcal{P}_{\alpha}$, also includes the heat flow vector $\mathbf{q}_{\alpha}$. A more general energy conservation equation would include the energy flow triad $\mathcal{E}_{\alpha}$.

Any finite set of transport equations, therefore, is insufficient to form a closed system of equations. Consequently, it is necessary to introduce a scheme of approximation to eliminate some of the independent variables, or to express some of these variables in terms of the others. It is common, therefore, to arbitrarily truncate the system of transport equations at some point in the hierarchy of moments of the Boltzmann equation, and consider some simplifying approximation for the highest moment of the distribution function appearing in the system.

The simplest closed system of macroscopic transport equations that can be formed is known as the cold plasma model. This simple model encompasses only the equations of conservation of mass and of momentum. The highest moment of the distribution function, appearing in the momentum equation, is the kinetic pressure dyad, which, in this model, is taken equal to zero. This means that the effects due to the thermal motion of the particles and the force due to the divergence of the kinetic pressure dyad are neglected. For convenience, we collect here the two transport 
equations pertinent to the cold plasma model,

$$
\begin{gathered}
\frac{\partial \rho_{m \alpha}}{\partial t}+\nabla \cdot\left(\rho_{m \alpha} \mathbf{u}_{\alpha}\right)=S_{\alpha} \\
\rho_{m \alpha} \frac{D \mathbf{u}_{\alpha}}{D t}=n_{\alpha} q_{\alpha}\left(\mathbf{E}+\mathbf{u}_{\alpha} \times \mathbf{B}\right)+\rho_{m \alpha} \mathbf{g}+\mathbf{A}_{\alpha}-\mathbf{u}_{\alpha} S_{\alpha}
\end{gathered}
$$

In the absence of processes leading to production and loss of particles of type $\alpha$ (such as ionization and recombination), we have $S_{\alpha}=0$. The expression normally used for the collision term for momentum transfer $\mathbf{A}_{\alpha}$ is the one indicated in (4.11). The cold plasma model assumes, in fact, a zero plasma temperature, so that the corresponding distribution function is a Dirac delta function centered at the macroscopic flow velocity, $f_{\alpha}(\mathbf{r}, \mathbf{v}, t)=\delta[\mathbf{v}-\mathbf{u}(\mathbf{r}, t)]$.

This model has been successfully applied, e.g., in the investigation of the properties of small-amplitude electromagnetic waves propagating in plasmas, with phase velocities much larger than the thermal velocity of the particles. The theory of high-frequency waves propagating in cold magnetized plasmas is commonly known as the magnetoionic theory.

\section{THE WARM PLASMA MODEL}

In this closed system of transport equations, the simplifying approximation is introduced in the equation of conservation of energy, in which we neglect the term involving the heat flux vector. Thus, the approximation consists in taking $\nabla \cdot \mathbf{q}_{\alpha}=0$, which means that the processes occurring in the plasma are such that there is no thermal energy flux. This approximation is also called the adiabatic approximation. Since the thermal conductivity is zero in this case, it follows that the plasma is nonviscous and, consequently, the nondiagonal terms of the kinetic pressure dyad are all equal to zero. Further, the diagonal terms of $\mathcal{P}_{\alpha}$ are assumed to be equal and the kinetic pressure dyad is replaced by a scalar pressure $p_{\alpha}$. Thus, the term $\nabla \cdot \mathcal{P}_{\alpha}$ in the momentum equation degenerates to $\nabla p_{\alpha}$.

The three macroscopic variables appearing in this case are the number density $n_{\alpha}$, the mean velocity $\mathbf{u}_{\alpha}$, and the scalar pressure $p_{\alpha}$. The three transport equations pertaining to the warm plasma model are, therefore,

$$
\begin{gathered}
\frac{\partial \rho_{m \alpha}}{\partial t}+\nabla \cdot\left(\rho_{m \alpha} \mathbf{u}_{\alpha}\right)=S_{\alpha} \\
\rho_{m \alpha} \frac{D \mathbf{u}_{\alpha}}{D t}=n_{\alpha} q_{\alpha}\left(\mathbf{E}+\mathbf{u}_{\alpha} \times \mathbf{B}\right)+\rho_{m \alpha} \mathbf{g}-\nabla p_{\alpha}+\mathbf{A}_{\alpha}-\mathbf{u}_{\alpha} S_{\alpha}
\end{gathered}
$$




$$
\frac{D}{D t}\left(\frac{3 p_{\alpha}}{2}\right)+\frac{5 p_{\alpha}}{2}\left(\nabla \cdot \mathbf{u}_{\alpha}\right)=M_{\alpha}-\mathbf{u}_{\alpha} \cdot \mathbf{A}_{\alpha}+\frac{1}{2} u_{\alpha}^{2} S_{\alpha}
$$

Considering the additional approximation that the change in energy, as a result of collisions, is negligible, the energy equation (7.3) reduces to the following adiabatic equation (as shown in section 5),

$$
p_{\alpha} \rho_{m \alpha}^{-\gamma}=\text { constant }
$$

Generally, the warm plasma model gives a more precise description of the behavior of plasma phenomena as compared to the cold plasma model.

In the most general cases, in which the plasma is not in a state of local equilibrium, and when heat flow and viscosity need to be taken into account, it is more convenient and simple to work directly with the phase space distribution function. In this case, the plasma is usually said to be hot. After determining the distribution function $f_{\alpha}$ by solving the differential kinetic equation that governs the evolution of $f_{\alpha}$ in phase space, for the specific problem under consideration, the macroscopic variables can be obtained from $f_{\alpha}$ according to the systematic method presented in Chapter 6.

\section{PROBLEMS}

8.1 Consider the following simplified steady-state equation of motion, for each species in a fluid plasma,

$$
n q(\mathbf{E}+\mathbf{u} \times \mathbf{B})-\nabla p=0
$$

where the electric $(\mathbf{E})$ and magnetic $(\mathbf{B})$ fields are uniform, but the number density $(n)$ and the kinetic pressure $(p)$ have a spatial gradient. Taking the cross-product of this equation with $\mathbf{B}$ show that, besides the $\mathbf{E} \times \mathbf{B}$ drift, there is also a diamagnetic drift given by

$$
\mathbf{v}_{D}=-\frac{1}{n q B^{2}}(\nabla p) \times \mathbf{B}
$$

Provide physical arguments to justify the physical reason for this fluid drift. Explain if there is any motion of the particle guiding centers associated with this fluid drift, and why it does not appear in single particle orbit theory. 
8.2 (a) From Maxwell equations,

$$
\begin{gathered}
\nabla \cdot \mathbf{E}=\frac{\rho}{\epsilon_{0}} \\
\nabla \cdot \mathbf{H}=0 \\
\nabla \times \mathbf{E}=-\mu_{0} \frac{\partial \mathbf{H}}{\partial t} \\
\nabla \times \mathbf{H}=\mathbf{J}+\epsilon_{0} \frac{\partial \mathbf{E}}{\partial t}
\end{gathered}
$$

where $\mathbf{E}$ and $\mathbf{H}$ denote the electric and magnetic fields in a plasma, $\rho$ denotes the electric charge density $n q$, and $\mathbf{J}$ the electric current density $n q \mathbf{u}$, show that

$$
\epsilon_{0} \mu_{0} \frac{\partial}{\partial t}(\mathbf{E} \times \mathbf{H})=\nabla \cdot \mathcal{T}-n q(\mathbf{E}+\mathbf{u} \times \mathbf{B})
$$

where $\epsilon_{0} \mu_{0}(\mathbf{E} \times \mathbf{H})$ is the electromagnetic momentum density, and $\mathcal{T}$ is the electromagnetic stress dyad whose components are given by

$$
T_{i j}=\epsilon_{0} E_{i} E_{j}+\mu_{0} H_{i} H_{j}-\frac{1}{2}\left(\epsilon_{0} E^{2}+\mu_{0} H^{2}\right) \delta_{i j}
$$

where $\delta_{i j}$ is the Kronecker delta.

(b) From this equation, which expresses conservation of the electromagnetic momentum density, and the continuity equation

$$
\frac{\partial n}{\partial t}+\nabla \cdot(n \mathbf{u})=0
$$

show that the momentum transport equation

$$
n m \frac{D \mathbf{u}}{D t}=n q(\mathbf{E}+\mathbf{u} \times \mathbf{B})-\nabla \cdot \mathcal{P}
$$

can be written in the form

$$
\frac{\partial \mathbf{G}}{\partial t}+\nabla \cdot \mathbf{\Pi}=0
$$

where $\mathbf{G}$ denotes the total momentum density

$$
\mathbf{G}=n m \mathbf{u}+\epsilon_{0} \mu_{0}(\mathbf{E} \times \mathbf{H})
$$


and $\boldsymbol{\Pi}$ is the total momentum flux dyad (rate of transport of momentum through unit area)

$$
\Pi=n m \mathbf{u} \mathbf{u}+\mathcal{P}-\mathcal{T}
$$

(c) Using Maxwell curl equations, show that the energy transport equation

$$
\begin{gathered}
\frac{\partial}{\partial t}\left(\frac{1}{2} n m<c^{2}>\right)+\frac{\partial}{\partial t}\left(\frac{1}{2} n m u^{2}\right)=-\nabla \cdot\left(\frac{1}{2} n m<c^{2}>\mathbf{u}\right)- \\
\nabla \cdot\left(\frac{1}{2} n m u^{2} \mathbf{u}\right)-\nabla \cdot \mathbf{q}-\nabla \cdot(\mathcal{P} \cdot \mathbf{u})+n q \mathbf{u} \cdot \mathbf{E}
\end{gathered}
$$

can be written in the form $[$ note that $\mathbf{u} \cdot(\mathbf{u} \times \mathbf{B})=0$ ]

$$
\frac{\partial W}{\partial t}+\nabla \cdot \mathbf{S}=0
$$

where $W$ denotes the total energy density

$$
W=\frac{1}{2}\left(\epsilon_{0} E^{2}+\mu_{0} H^{2}+n m u^{2}+n m<c^{2}>\right)
$$

and $\mathbf{S}$ is the total energy flux (power per unit area)

$$
\mathbf{S}=\mathbf{E} \times \mathbf{H}+\mathcal{P} \cdot \mathbf{u}+\frac{1}{2} n m \mathbf{u}\left(u^{2}+<c^{2}>\right)+\mathbf{q}
$$

8.3 In order to investigate the effect of the collision term (4.11) in the macroscopic fluid motion, consider a uniform mixture of different fluids (all spatial derivatives vanish), with no external forces, so that the equation of motion for the $\alpha$ species reduces to

$$
\frac{d \mathbf{u}_{\alpha}}{d t}=-\sum_{\beta} \nu_{\alpha \beta}\left(\mathbf{u}_{\alpha}-\mathbf{u}_{\beta}\right)
$$

Solve this equation to determine $\mathbf{u}(t)$, for a two-fluid mixture and for a three-fluid mixture (in the case of the three-fluid mixture it is convenient to use Laplace transforms). Notice that, at equilibrium (when $d \mathbf{u}_{\alpha} / d t=0$ ), the velocities of all species must be the same.

8.4 Consider a uniform mixture of different fluids (all spatial derivatives vanish), with no external forces, such that the equation of motion for the $\alpha$ species becomes

$$
\frac{d \mathbf{u}_{\alpha}}{d t}=-\sum_{\beta} \nu_{\alpha \beta}\left(\mathbf{u}_{\alpha}-\mathbf{u}_{\beta}\right)
$$


(a) Show that the time rate of change of the total fluid kinetic energy density, $W_{k}$, is given by

$$
\frac{d W_{k}}{d t}=-\sum_{\alpha, \beta} \frac{1}{2} \rho_{m \alpha} \nu_{\alpha \beta}\left(\mathbf{u}_{\alpha}-\mathbf{u}_{\beta}\right)^{2}
$$

where

$$
W_{k}=\sum_{\alpha} \frac{1}{2} \rho_{m \alpha} u_{\alpha}^{2}
$$

(b) Consider now the total fluid thermal energy density,

$$
W_{T}=\sum_{\alpha} \frac{3}{2} n_{\alpha} k T_{\alpha}
$$

If the energy equation for a homogeneous fluid mixture, with no external forces, is

$$
\frac{d T_{\alpha}}{d t}=-\sum_{\beta} \frac{2 m_{\alpha} \nu_{\alpha \beta}}{\left(m_{\alpha}+m_{\beta}\right)}\left[\left(T_{\alpha}-T_{\beta}\right)-\frac{m_{\beta}}{3 k}\left(\mathbf{u}_{\alpha}-\mathbf{u}_{\beta}\right)^{2}\right]
$$

then show that the time rate of change of $W_{T}$ is given by

$$
\frac{d W_{T}}{d t}=\sum_{\alpha, \beta} \frac{1}{2} \rho_{m \alpha} \nu_{\alpha \beta}\left(\mathbf{u}_{\alpha}-\mathbf{u}_{\beta}\right)^{2}
$$

Thus, the total thermal energy density $W_{T}$ increases at exactly the same rate as the total kinetic energy density $W_{k}$ decreases. As a hint for this problem, notice that for any function that is summed over two indices, the result is unchanged if we interchange the indices, so that

$$
\sum_{\alpha, \beta} f_{\alpha \beta}=\sum_{\alpha, \beta} f_{\beta \alpha}
$$

or

$$
\sum_{\alpha, \beta} f_{\alpha \beta}=\sum_{\alpha, \beta} \frac{1}{2}\left(f_{\alpha \beta}+f_{\beta \alpha}\right)
$$


8.5 Explain the reason why there is no term containing the magnetic flux density $\mathbf{B}$ in the energy equation (5.17).

8.6 Derive the following general transport equation, similar to (2.13), for the case when the quantity $\chi$ depends on $\mathbf{r}, \mathbf{v}$, and $t$,

$$
\begin{gathered}
\frac{\partial}{\partial t}\left(n_{\alpha}<\chi>_{\alpha}\right)-n_{\alpha}<\frac{\partial \chi}{\partial t}>_{\alpha}+\nabla \cdot\left(n_{\alpha}<\chi \mathbf{v}>_{\alpha}\right)- \\
n_{\alpha}<(\mathbf{v} \cdot \nabla) \chi>_{\alpha}-n_{\alpha}<\left(\mathbf{a} \cdot \nabla_{v}\right) \chi>_{\alpha}=\left[\frac{\delta\left(n_{\alpha}<\chi>_{\alpha}\right)}{\delta t}\right]_{\text {coll }}
\end{gathered}
$$

8.7 Consider the general transport equation of the previous problem and let the property $\chi(\mathbf{r}, \mathbf{v}, t)$ be the random flux of thermal kinetic energy, that is, $\frac{1}{2} m_{\alpha} c_{\alpha}^{2} \mathbf{c}_{\alpha}$, where $\mathbf{c}_{\alpha}=\mathbf{v}-\mathbf{u}_{\alpha}(\mathbf{r}, t)$. Show that (considering the Lorentz force for $\mathbf{F}$ )

$$
\begin{gathered}
\frac{\partial}{\partial t}\left(n_{\alpha}<\chi>_{\alpha}\right)=\frac{\partial \mathbf{q}_{\alpha}}{\partial t} \\
n_{\alpha}<\frac{\partial \chi}{\partial t}>_{\alpha}=-\left(\frac{\partial \mathbf{u}_{\alpha}}{\partial t}\right) \cdot\left(\mathcal{P}_{\alpha}+\frac{3}{2} p_{\alpha} \mathbf{1}\right) \\
\nabla \cdot\left(n_{\alpha}<\chi \mathbf{v}>_{\alpha}\right)=\nabla \cdot\left(\frac{1}{2} \rho_{m \alpha}<c_{\alpha}^{2} \mathbf{c}_{\alpha} \mathbf{c}_{\alpha}>+\mathbf{u}_{\alpha} \mathbf{q}_{\alpha}\right) \\
n_{\alpha}<(\mathbf{v} \cdot \nabla) \chi>_{\alpha}=-\left(\mathcal{Q}_{\alpha} \cdot \nabla\right) \cdot \mathbf{u}_{\alpha}-\left(\mathbf{q}_{\alpha} \cdot \nabla\right) \mathbf{u}_{\alpha}- \\
{\left[\left(\mathbf{u}_{\alpha} \cdot \nabla\right) \mathbf{u}_{\alpha}\right] \cdot\left(\mathcal{P}_{\alpha}+\frac{3}{2} p_{\alpha} \mathbf{1}\right)} \\
n_{\alpha}<\left(\mathbf{a} \cdot \nabla_{v}\right) \chi>_{\alpha}=\rho_{m \alpha}<\mathbf{a} \cdot\left(\mathbf{c}_{\alpha} \mathbf{c}_{\alpha}+\frac{1}{2} c_{\alpha}^{2} \mathbf{1}\right)>= \\
\frac{q_{\alpha}}{m_{\alpha}}\left[\left(\mathbf{E}+\mathbf{u}_{\alpha} \times \mathbf{B}\right) \cdot\left(\mathcal{P}_{\alpha}+\frac{3}{2} p_{\alpha} \mathbf{1}\right)+\mathbf{q}_{\alpha} \times \mathbf{B}\right]
\end{gathered}
$$

Using these results in the general transport equation, derive the following equation, known as the heat flow equation,

$$
\begin{gathered}
\frac{\partial \mathbf{q}_{\alpha}}{\partial t}-\frac{1}{\rho_{m \alpha}}\left(\nabla \cdot \mathcal{P}_{\alpha}\right) \cdot\left(\mathcal{P}_{\alpha}+\frac{3}{2} p_{\alpha} \mathbf{1}\right)+ \\
\nabla \cdot\left(\frac{1}{2} \rho_{m \alpha}<c_{\alpha}^{2} \mathbf{c}_{\alpha} \mathbf{c}_{\alpha}>+\mathbf{u}_{\alpha} \mathbf{q}_{\alpha}\right)+\left(\mathcal{Q}_{\alpha} \cdot \nabla\right) \cdot \mathbf{u}_{\alpha}+ \\
\left(\mathbf{q}_{\alpha} \cdot \nabla\right) \mathbf{u}_{\alpha}-\frac{q_{\alpha}}{m_{\alpha}}\left(\mathbf{q}_{\alpha} \times \mathbf{B}\right)=\left(\frac{\delta \mathbf{q}_{\alpha}}{\delta t}\right)_{\text {coll }}
\end{gathered}
$$


8.8 In the general transport equation of problem 8.6, consider that the property $\chi(\mathbf{r}, \mathbf{v}, t)$ is the random momentum flux, that is, $m_{\alpha} c_{\alpha j} c_{\alpha k}$. Show that, in this case,

$$
\begin{gathered}
\frac{\partial}{\partial t}\left(n_{\alpha}<\chi>_{\alpha}\right)=\frac{\partial P_{\alpha j k}}{\partial t} \\
n_{\alpha}<\frac{\partial \chi}{\partial t}>_{\alpha}=0 \\
\frac{\partial}{\partial x_{i}}\left(n_{\alpha}<\chi v_{i}>_{\alpha}\right)=\frac{\partial}{\partial x_{i}}\left(Q_{\alpha i j k}+u_{\alpha i} P_{\alpha j k}\right) \\
n_{\alpha}<v_{i} \frac{\partial \chi}{\partial x_{i}}>_{\alpha}=-P_{\alpha i j} \frac{\partial u_{\alpha k}}{\partial x_{i}}-P_{\alpha i k} \frac{\partial u_{\alpha j}}{\partial x_{i}} \\
n_{\alpha}<a_{i} \frac{\partial \chi}{\partial v_{i}}>_{\alpha}=\rho_{m \alpha}<a_{j} c_{\alpha k}+a_{k} c_{\alpha j}>
\end{gathered}
$$

where the summation convention on repeated indices is being used. Plug these results in the general transport equation to derive the following equation, known as the viscous stress equation:

$$
\begin{gathered}
\frac{\partial P_{\alpha j k}}{\partial t}+\frac{\partial}{\partial x_{i}}\left(Q_{\alpha i j k}+u_{\alpha i} P_{\alpha j k}\right)+P_{\alpha i j} \frac{\partial u_{\alpha k}}{\partial x_{i}}+P_{\alpha i k} \frac{\partial u_{\alpha j}}{\partial x_{i}}- \\
\rho_{m \alpha}<a_{j} c_{\alpha k}+a_{k} c_{\alpha j}>=\left(\frac{\delta P_{\alpha j k}}{\delta t}\right)_{\text {coll }}
\end{gathered}
$$

8.9 Verify that the energy conservation equation, for the random kinetic energy $\frac{1}{2} m_{\alpha} c_{\alpha}^{2}$, can be obtained from the viscous stress equation (see problem 8.8) by letting $j=k$, and summing over $k$.

8.10 From the heat flow equation, derived in problem 8.7, obtain the following simplified equation for heat flow in a stationary $(\mathbf{u}=0)$ electron gas:

$$
\frac{5 p_{e}}{2} \nabla\left(\frac{p_{e}}{\rho_{m e}}\right)+\Omega_{c e}\left(\mathbf{q}_{e} \times \mathbf{B}\right)=\left(\frac{\delta \mathbf{q}_{e}}{\delta t}\right)_{\text {coll }}
$$

State all the assumptions necessary to obtain this result. 
8.11 Using the relaxation model (or Krook collision model) for the collision term,

$$
\left(\frac{\delta f_{\alpha}}{\delta t}\right)_{\text {coll }}=-\nu\left(f_{\alpha}-f_{\alpha 0}\right)
$$

and the ideal gas law $p_{e}=n_{e} k T_{e}$, show that the heat flow equation of problem 8.10 becomes

$$
\mathbf{q}_{e}+\frac{\Omega_{c e}}{\nu}\left(\mathbf{q}_{e} \times \mathbf{B}\right)=-K_{0} \nabla T_{e}
$$

where

$$
K_{0}=\frac{5 k p_{e}}{2 m_{e} \nu}
$$

is the thermal conductivity. 


\section{9}

\section{MACROSCOPIC EQUATIONS FOR A CONDUCTING FLUID}

\section{MACROSCOPIC VARIABLES FOR A PLASMA AS A CONDUCTING FLUID}

A plasma can also be considered as a conducting fluid, without specifying its various individual species. The macroscopic transport equations, derived in the previous chapter, describe the macroscopic behavior of each individual plasma species (electrons, ions, and neutral particles). We will determine now the set of transport equations that describe the macroscopic behavior of the plasma as a whole, without considering the individual species present. Each macroscopic variable is combined, by adding the contributions of the various particle species in the plasma. This procedure yields the total macroscopic parameters of interest, such as the total mass and charge densities, the total mass and charge current densities (or flux), the total kinetic pressure dyad, and the total heat flux vector.

The mass density is the mass per unit volume of fluid and can be expressed as

$$
\rho_{m}=\sum_{\alpha} \rho_{m \alpha}=\sum_{\alpha} n_{\alpha} m_{\alpha}
$$

The electric charge density is the electric charge per unit volume of fluid,

$$
\rho=\sum_{\alpha} n_{\alpha} q_{\alpha}
$$

The mean fluid velocity, $\mathbf{u}$, is defined such that the total momentum density is the same as the sum of the momentum density of each species, 
according to

$$
\rho_{m} \mathbf{u}=\sum_{\alpha} \rho_{m \alpha} \mathbf{u}_{\alpha}
$$

The mean velocity of the plasma, $\mathbf{u}$, therefore, is a weighted mean value, where the velocity of each species is weighted proportionally to its mass density.

The mean velocity of each particle species, when considered in a reference frame moving with the global mean velocity $\mathbf{u}$ of the plasma, is called the diffusion velocity $\mathbf{w}_{\alpha}$,

$$
\mathbf{w}_{\alpha}=\mathbf{u}_{\alpha}-\mathbf{u}=\mathbf{u}_{\alpha}-\frac{1}{\rho_{m}} \sum_{\alpha} \rho_{m \alpha} \mathbf{u}_{\alpha}
$$

The mass current density or mass flux is given by

$$
\mathbf{J}_{m}=\sum_{\alpha} n_{\alpha} m_{\alpha} \mathbf{u}_{\alpha}=\rho_{m} \mathbf{u}
$$

and the electric current density or charge flux is expressed as

$$
\mathbf{J}=\sum_{\alpha} n_{\alpha} q_{\alpha} \mathbf{u}_{\alpha}=\rho \mathbf{u}+\sum_{\alpha} n_{\alpha} q_{\alpha} \mathbf{w}_{\alpha}
$$

Note that in (1.5) we have $\sum_{\alpha} \rho_{m \alpha} \mathbf{w}_{\alpha}=0$, in virtue of (1.4), which defines the diffusion velocity $\mathbf{w}_{\alpha}$.

The kinetic pressure dyad for each particle species in the plasma is defined in (6.6.2) as

$$
\mathcal{P}_{\alpha}=\rho_{m \alpha}<\mathbf{c}_{\alpha} \mathbf{c}_{\alpha}>
$$

where $\mathbf{c}_{\alpha}=\mathbf{v}-\mathbf{u}_{\alpha}$ is the peculiar or random velocity of the type $\alpha$ particles. Note that the pressure is defined as the time rate in which momentum is transported by the particles through a surface element moving with the particle mean velocity. For the plasma as a whole it is necessary to define an alternative peculiar velocity $\mathbf{c}_{\alpha 0}$, for the particles of type $\alpha$, relative to the global plasma mean velocity $\mathbf{u}$,

$$
\mathbf{c}_{\alpha 0}=\mathbf{v}-\mathbf{u}
$$

Thus, the total pressure is defined as the rate of momentum transfer due to all particles in the plasma, through a surface element moving with the 
global mean velocity $\mathbf{u}$. The total kinetic pressure dyad $\mathcal{P}$ is therefore given by

$$
\mathcal{P}=\sum_{\alpha} \rho_{m \alpha}<\mathbf{c}_{\alpha 0} \mathbf{c}_{\alpha 0}>
$$

To relate $\mathcal{P}$, given in (1.9), with $\mathcal{P}_{\alpha}$, given in (1.7), we substitute $\mathbf{u}$ by $\mathbf{u}_{\alpha}-\mathbf{w}_{\alpha}$ and $\mathbf{v}$ by $\mathbf{c}_{\alpha}+\mathbf{u}_{\alpha}$ in (1.8), to obtain

$$
\mathbf{c}_{\alpha 0}=\mathbf{c}_{\alpha}+\mathbf{w}_{\alpha}
$$

Consequently,

$$
\mathcal{P}=\sum_{\alpha} \rho_{m \alpha}<\left(\mathbf{c}_{\alpha}+\mathbf{w}_{\alpha}\right)\left(\mathbf{c}_{\alpha}+\mathbf{w}_{\alpha}\right)>
$$

and expanding this expression,

$$
\mathcal{P}=\sum_{\alpha} \rho_{m \alpha}\left(<\mathbf{c}_{\alpha} \mathbf{c}_{\alpha}>+<\mathbf{c}_{\alpha} \mathbf{w}_{\alpha}>+<\mathbf{w}_{\alpha} \mathbf{c}_{\alpha}>+<\mathbf{w}_{\alpha} \mathbf{w}_{\alpha}>\right)
$$

From the definition of $\mathbf{w}_{\alpha}$ we see that $\left\langle\mathbf{w}_{\alpha}\right\rangle=\mathbf{w}_{\alpha}$, since it is a macroscopic variable and therefore $\left\langle\mathbf{c}_{\alpha} \mathbf{w}_{\alpha}\right\rangle=\left\langle\mathbf{c}_{\alpha}\right\rangle \mathbf{w}_{\alpha}=0$. Thus, (1.12) becomes

$$
\mathcal{P}=\sum_{\alpha} \mathcal{P}_{\alpha}+\sum_{\alpha} \rho_{m \alpha} \mathbf{w}_{\alpha} \mathbf{w}_{\alpha}
$$

Note that $\mathcal{P}_{\alpha}$ is a pressure relative to $\mathbf{u}_{\alpha}$, whereas $\mathcal{P}$ is relative to the global mean velocity $\mathbf{u}$.

The total scalar pressure $p$ is defined as one-third the trace of $\mathcal{P}$,

$$
p=\frac{1}{3} \sum_{i} P_{i i}=\frac{1}{3} \sum_{i} \sum_{\alpha} \rho_{m \alpha}<c_{\alpha 0 i} c_{\alpha 0 i}>=\frac{1}{3} \sum_{\alpha} \rho_{m \alpha}<c_{\alpha 0}^{2}>
$$

Using (1.13) we can write

$$
p=\sum_{\alpha} p_{\alpha}+\frac{1}{3} \sum_{\alpha} \rho_{m \alpha} w_{\alpha}^{2}
$$

Finally, we define the total heat flux vector $\mathbf{q}$ as

$$
\mathbf{q}=\frac{1}{2} \sum_{\alpha} \rho_{m \alpha}<c_{\alpha 0}^{2} \mathbf{c}_{\alpha 0}>
$$


and the thermal energy density of the plasma as a whole as

$$
\frac{3 p}{2}=\frac{1}{2} \sum_{\alpha} \rho_{m \alpha}<c_{\alpha 0}^{2}>
$$

It is useful to relate $\mathbf{q}$, defined in (1.16), with the heat flux vector $\mathbf{q}_{\alpha}$ for the particles of type $\alpha$,

$$
\mathbf{q}_{\alpha}=\frac{1}{2} \rho_{m \alpha}<c_{\alpha}^{2} \mathbf{c}_{\alpha}>
$$

For this purpose, we substitute $\mathbf{c}_{\alpha 0}$ by $\mathbf{c}_{\alpha}+\mathbf{w}_{\alpha}$ in (1.16) and expand the resulting expression, obtaining

$$
\begin{gathered}
\mathbf{q}=\frac{1}{2} \sum_{\alpha} \rho_{m \alpha}\left[<c_{\alpha}^{2} \mathbf{c}_{\alpha}>+w_{\alpha}^{2}<\mathbf{c}_{\alpha}>+2<\left(\mathbf{w}_{\alpha} \cdot \mathbf{c}_{\alpha}\right) \mathbf{c}_{\alpha}>+\right. \\
\left.<c_{\alpha}^{2}>\mathbf{w}_{\alpha}+w_{\alpha}^{2} \mathbf{w}_{\alpha}+2\left(<\mathbf{c}_{\alpha}>\cdot \mathbf{w}_{\alpha}\right) \mathbf{w}_{\alpha}\right]
\end{gathered}
$$

The second and sixth terms in the right-hand side of this equation are equal to zero, since $\left\langle\mathbf{c}_{\alpha}\right\rangle=0$. Therefore,

$$
\mathbf{q}=\frac{1}{2} \sum_{\alpha} \rho_{m \alpha}\left[<c_{\alpha}^{2} \mathbf{c}_{\alpha}>+2 \mathbf{w}_{\alpha} \cdot<\mathbf{c}_{\alpha} \mathbf{c}_{\alpha}>+<c_{\alpha}^{2}>\mathbf{w}_{\alpha}+w_{\alpha}^{2} \mathbf{w}_{\alpha}\right]
$$

Using (1.18), (1.7), and the relation $p_{\alpha}=\rho_{m \alpha}<c_{\alpha}^{2}>/ 3$, we can write $(1.20)$ as

$$
\mathbf{q}=\sum_{\alpha}\left(\mathbf{q}_{\alpha}+\mathbf{w}_{\alpha} \cdot \mathcal{P}_{\alpha}+\frac{3}{2} p_{\alpha} \mathbf{w}_{\alpha}+\frac{1}{2} \rho_{m \alpha} w_{\alpha}^{2} \mathbf{w}_{\alpha}\right)
$$

In particular, for the isotropic case in which $\mathcal{P}_{\alpha}=p_{\alpha} \mathbf{1}$, we have $\mathbf{w}_{\alpha} \cdot \mathcal{P}_{\alpha}=\mathbf{w}_{\alpha} p_{\alpha}$, so that (1.21) becomes

$$
\mathbf{q}=\sum_{\alpha}\left(\mathbf{q}_{\alpha}+\frac{5}{2} p_{\alpha} \mathbf{w}_{\alpha}+\frac{1}{2} \rho_{m \alpha} w_{\alpha}^{2} \mathbf{w}_{\alpha}\right)
$$

\section{CONTINUITY EQUATION}

To obtain the continuity equation for the plasma as a whole, we add (8.3.2) (Eq. 3.2 in Chapter 8) over all particle species in the plasma,

$$
\sum_{\alpha} \frac{\partial \rho_{m \alpha}}{\partial t}+\sum_{\alpha} \nabla \cdot\left(\rho_{m \alpha} \mathbf{u}_{\alpha}\right)=\sum_{\alpha} S_{\alpha}
$$


which gives

$$
\frac{\partial \rho_{m}}{\partial t}+\nabla \cdot\left(\rho_{m} \mathbf{u}\right)=0
$$

with $\rho_{m}$ and $\mathbf{u}$ given by (1.1) and (1.3), respectively. The collision term $S_{\alpha}$, when summed over all particle species, must certainly vanish, as a consequence of conservation of the total mass of the system. It is of interest to note that, using the total time derivative operator $D / D t=\partial / \partial t+\mathbf{u} \cdot \nabla$, (2.2) can also be written in the form

$$
\frac{D \rho_{m}}{D t}+\rho_{m} \nabla \cdot \mathbf{u}=0
$$

\section{EQUATION OF MOTION}

Similarly, adding the equation of conservation of momentum (8.4.9) over all particle species in the plasma, yields

$$
\begin{gathered}
\sum_{\alpha} \rho_{m \alpha}\left[\frac{\partial \mathbf{u}_{\alpha}}{\partial t}+\left(\mathbf{u}_{\alpha} \cdot \nabla\right) \mathbf{u}_{\alpha}\right]=\sum_{\alpha} n_{\alpha} q_{\alpha} \mathbf{E}+\sum_{\alpha} n_{\alpha} q_{\alpha}\left(\mathbf{u}_{\alpha} \times \mathbf{B}\right)+ \\
\sum_{\alpha} \rho_{m \alpha} \mathbf{g}-\sum_{\alpha} \nabla \cdot \mathcal{P}_{\alpha}+\sum_{\alpha} \mathbf{A}_{\alpha}-\sum_{\alpha} \mathbf{u}_{\alpha} S_{\alpha}
\end{gathered}
$$

Since the total momentum of the particles in the plasma is conserved, the collision term for momentum transfer vanishes when summed over all species. Using the definitions (1.1), (1.2), and (1.6), and the relation (1.13), we can write (3.1) as

$$
\begin{gathered}
\sum_{\alpha} \rho_{m \alpha}\left[\frac{\partial \mathbf{u}_{\alpha}}{\partial t}+\left(\mathbf{u}_{\alpha} \cdot \nabla\right) \mathbf{u}_{\alpha}\right]=\rho \mathbf{E}+\mathbf{J} \times \mathbf{B}+\rho_{m} \mathbf{g}-\nabla \cdot \mathcal{P}+ \\
\sum_{\alpha} \nabla \cdot\left(\rho_{m \alpha} \mathbf{w}_{\alpha} \mathbf{w}_{\alpha}\right)-\sum_{\alpha} \mathbf{u}_{\alpha} S_{\alpha}
\end{gathered}
$$

The term involving $S_{\alpha}$ can be eliminated using the equation of continuity,

$$
\sum_{\alpha} \mathbf{u}_{\alpha} S_{\alpha}=\sum_{\alpha} \mathbf{u}_{\alpha}\left[\frac{\partial \rho_{m \alpha}}{\partial t}+\nabla \cdot\left(\rho_{m \alpha} \mathbf{u}_{\alpha}\right)\right]
$$

Combining this expression with the terms in the left-hand side of (3.2), results in the expression 


$$
\sum_{\alpha}\left[\frac{\partial\left(\rho_{m \alpha} \mathbf{u}_{\alpha}\right)}{\partial t}+\nabla \cdot\left(\rho_{m \alpha} \mathbf{u}_{\alpha} \mathbf{u}_{\alpha}\right)\right]
$$

We can now substitute the mean velocity $\mathbf{u}_{\alpha}$ by $\mathbf{w}_{\alpha}+\mathbf{u}$ and expand the result. Noting that

$$
\sum_{\alpha} \rho_{m \alpha} \mathbf{w}_{\alpha}=\sum_{\alpha} \rho_{m \alpha}\left(\mathbf{u}_{\alpha}-\mathbf{u}\right)=\rho_{m} \mathbf{u}-\rho_{m} \mathbf{u}=0
$$

we can express (3.4) as

$$
\begin{gathered}
\sum_{\alpha}\left[\frac{\partial\left(\rho_{m \alpha} \mathbf{u}_{\alpha}\right)}{\partial t}+\nabla \cdot\left(\rho_{m \alpha} \mathbf{u}_{\alpha} \mathbf{u}_{\alpha}\right)\right]=\frac{\partial\left(\rho_{m} \mathbf{u}\right)}{\partial t}+\nabla \cdot\left(\rho_{m} \mathbf{u u}\right)+ \\
\sum_{\alpha} \nabla \cdot\left(\rho_{m \alpha} \mathbf{w}_{\alpha} \mathbf{w}_{\alpha}\right)=\rho_{m}\left[\frac{\partial \mathbf{u}}{\partial t}+(\mathbf{u} \cdot \nabla) \mathbf{u}\right]+\mathbf{u}\left[\frac{\partial \rho_{m}}{\partial t}+\nabla \cdot\left(\rho_{m} \mathbf{u}\right)\right]+ \\
\sum_{\alpha} \nabla \cdot\left(\rho_{m \alpha} \mathbf{w}_{\alpha} \mathbf{w}_{\alpha}\right)=\rho_{m} \frac{D \mathbf{u}}{D t}+\sum_{\alpha} \nabla \cdot\left(\rho_{m \alpha} \mathbf{w}_{\alpha} \mathbf{w}_{\alpha}\right)
\end{gathered}
$$

where we have used the continuity equation (2.2) and the total time derivative operator $D / D t$. Taking this result back into the equation of motion (3.2), we obtain the following momentum equation for the plasma as a whole,

$$
\rho_{m} \frac{D \mathbf{u}}{D t}=\rho \mathbf{E}+\mathbf{J} \times \mathbf{B}+\rho_{m} \mathbf{g}-\nabla \cdot \mathcal{P}
$$

This equation is an expression of Newton's second law of motion.

\section{ENERGY EQUATION}

To obtain the equation of conservation of energy for the plasma as a conducting fluid, we start from the energy equation (8.5.4) for the particles of type $\alpha$, and add this equation over all plasma species,

$$
\begin{gathered}
\sum_{\alpha} \frac{\partial}{\partial t}\left(\frac{1}{2} \rho_{m \alpha}<v^{2}>_{\alpha}\right)+\sum_{\alpha} \nabla \cdot\left(\frac{1}{2} \rho_{m \alpha}<v^{2} \mathbf{v}>_{\alpha}\right)- \\
\sum_{\alpha} n_{\alpha}<\mathbf{F} \cdot \mathbf{v}>_{\alpha}=0
\end{gathered}
$$

where the collision term $M_{\alpha}$ vanishes when summed over all species of particles. We substitute now $\mathbf{v}$ by $\mathbf{c}_{\alpha 0}+\mathbf{u}$ and expand each term of (4.1). 
For the first term we have

$$
\begin{gathered}
\frac{\partial}{\partial t}\left(\sum_{\alpha} \frac{1}{2} \rho_{m \alpha}<\mathbf{v} \cdot \mathbf{v}>_{\alpha}\right)=\frac{\partial}{\partial t}\left[\sum_{\alpha} \frac{1}{2} \rho_{m \alpha}\left(<c_{\alpha 0}^{2}>+u^{2}+2 \mathbf{w}_{\alpha} \cdot \mathbf{u}\right)\right] \\
=\frac{\partial}{\partial t}\left(\sum_{\alpha} \frac{1}{2} \rho_{m \alpha}<c_{\alpha 0}^{2}>\right)+\frac{\partial}{\partial t}\left(\frac{1}{2} \rho_{m} u^{2}\right) \\
=\frac{\partial}{\partial t}\left(\frac{3 p}{2}\right)+\frac{\partial}{\partial t}\left(\frac{1}{2} \rho_{m} u^{2}\right)
\end{gathered}
$$

where we have used the definition (1.17) and the fact that $\sum_{\alpha} \rho_{m \alpha} \mathbf{w}_{\alpha}=0$. For the second term we note initially that

$$
\begin{gathered}
<v^{2} \mathbf{v}>_{\alpha}=<\left(c_{\alpha 0}^{2}+u^{2}+2 \mathbf{c}_{\alpha 0} \cdot \mathbf{u}\right)\left(\mathbf{c}_{\alpha 0}+\mathbf{u}\right)> \\
=<c_{\alpha 0}^{2} \mathbf{c}_{\alpha 0}>+u^{2} \mathbf{w}_{\alpha}+2<\mathbf{c}_{\alpha 0} \mathbf{c}_{\alpha 0}>\cdot \mathbf{u}+ \\
<c_{\alpha 0}^{2}>\mathbf{u}+u^{2} \mathbf{u}+2\left(\mathbf{w}_{\alpha} \cdot \mathbf{u}\right) \mathbf{u}
\end{gathered}
$$

since $\mathbf{c}_{\alpha 0}=\mathbf{c}_{\alpha}+\mathbf{w}_{\alpha}$ and $\left\langle\mathbf{c}_{\alpha}\right\rangle=0$. Therefore,

$$
\begin{gathered}
\nabla \cdot\left(\sum_{\alpha} \frac{1}{2} \rho_{m \alpha}<v^{2} \mathbf{v}>_{\alpha}\right)=\nabla \cdot\left(\sum_{\alpha} \frac{1}{2} \rho_{m \alpha}<c_{\alpha 0}^{2} \mathbf{c}_{\alpha 0}>\right)+ \\
\nabla \cdot\left(\sum_{\alpha} \rho_{m \alpha}<\mathbf{c}_{\alpha 0} \mathbf{c}_{\alpha 0}>\cdot \mathbf{u}\right)+\nabla \cdot\left(\sum_{\alpha} \frac{1}{2} \rho_{m \alpha}<c_{\alpha 0}^{2}>\mathbf{u}\right)+ \\
\nabla \cdot\left(\sum_{\alpha} \frac{1}{2} \rho_{m \alpha} u^{2} \mathbf{u}\right)
\end{gathered}
$$

Using the definitions of the total heat flux vector $\mathbf{q}$ and of the total kinetic pressure dyad $\mathcal{P}$, we can write (4.4) as

$$
\begin{gathered}
\nabla \cdot\left(\sum_{\alpha} \frac{1}{2} \rho_{m \alpha}<v^{2} \mathbf{v}>_{\alpha}\right)=\nabla \cdot \mathbf{q}+\nabla \cdot(\mathcal{P} \cdot \mathbf{u})+ \\
\nabla \cdot\left(\frac{3 p}{2} \mathbf{u}\right)+\nabla \cdot\left(\frac{1}{2} \rho_{m} u^{2} \mathbf{u}\right)
\end{gathered}
$$

For the third term of (4.1) we have

$$
\sum_{\alpha} n_{\alpha}<\mathbf{F} \cdot \mathbf{v}>_{\alpha}=\sum_{\alpha} n_{\alpha}\left[q_{\alpha}<\mathbf{E} \cdot \mathbf{v}>_{\alpha}+q_{\alpha}<(\mathbf{v} \times \mathbf{B}) \cdot \mathbf{v}>_{\alpha}+\right.
$$




$$
\left.m_{\alpha}<\mathbf{g} \cdot \mathbf{v}>_{\alpha}\right]
$$

where we have considered external forces due to electromagnetic and gravitational fields. Since $\langle\mathbf{v}\rangle_{\alpha}=\mathbf{u}_{\alpha}$ and since, for any vector $\mathbf{v}$, we have $(\mathbf{v} \times \mathbf{B}) \cdot \mathbf{v}=0$, we obtain

$$
\sum_{\alpha} n_{\alpha}<\mathbf{F} \cdot \mathbf{v}>_{\alpha}=\mathbf{J} \cdot \mathbf{E}+\mathbf{J}_{m} \cdot \mathbf{g}
$$

where we have used the definitions (1.5) and (1.6), and where $\mathbf{E}$ and $\mathbf{g}$ are smoothed macroscopic fields.

Combining the results contained in (4.2), (4.5), and (4.7), the energy equation becomes

$$
\begin{gathered}
\frac{\partial}{\partial t}\left(\frac{3 p}{2}\right)+\nabla \cdot\left(\frac{3 p}{2} \mathbf{u}\right)+\frac{\partial}{\partial t}\left(\frac{1}{2} \rho_{m} u^{2}\right)+\nabla \cdot\left(\frac{1}{2} \rho_{m} u^{2} \mathbf{u}\right)+ \\
\nabla \cdot \mathbf{q}+\nabla \cdot(\mathcal{P} \cdot \mathbf{u})-\mathbf{J} \cdot \mathbf{E}-\mathbf{J}_{m} \cdot \mathbf{g}=0
\end{gathered}
$$

This equation can be further simplified as follows. The third and fourth terms of (4.8) can be combined as

$$
\begin{gathered}
\frac{\partial}{\partial t}\left(\frac{1}{2} \rho_{m} u^{2}\right)+\nabla \cdot\left(\frac{1}{2} \rho_{m} u^{2} \mathbf{u}\right)=\frac{1}{2} u^{2}\left[\frac{\partial \rho_{m}}{\partial t}+\nabla \cdot\left(\rho_{m} \mathbf{u}\right)\right]+ \\
\mathbf{u} \cdot\left(\rho_{m} \frac{D \mathbf{u}}{D t}\right)
\end{gathered}
$$

and using the continuity equation (2.2) and the equation of motion (3.7), we can express (4.9) as

$$
\rho \mathbf{u} \cdot \mathbf{E}+\mathbf{u} \cdot(\mathbf{J} \times \mathbf{B})+\mathbf{J}_{m} \cdot \mathbf{g}-\mathbf{u} \cdot(\nabla \cdot \mathcal{P})
$$

Taking this result back into the energy equation (4.8), yields

$$
\begin{gathered}
\frac{D}{D t}\left(\frac{3 p}{2}\right)+\frac{3 p}{2} \nabla \cdot \mathbf{u}+\nabla \cdot \mathbf{q}+(\mathcal{P} \cdot \nabla) \cdot \mathbf{u}= \\
\mathbf{J} \cdot \mathbf{E}-\mathbf{u} \cdot(\mathbf{J} \times \mathbf{B})-\rho \mathbf{u} \cdot \mathbf{E}
\end{gathered}
$$

The first term in the left-hand side of (4.11) represents the time rate of change of the total thermal energy density of the plasma $(3 p / 2)$ in a frame of reference moving with the global mean velocity $\mathbf{u}$. The second term contributes to this rate of change through the thermal energy transferred to this volume element, as a consequence of the particle motions. The 
third term represents the heat flux and the fourth one the work done on the volume element by the pressure forces (normal and tangential). The terms in the right-hand side of (4.11) represent the work done on the volume element by the electric field existing in the frame of reference moving with the global mean velocity $\mathbf{u}$. These last terms can be combined as follows. We note, initially, that the charge current density consists of two parts

$$
\mathbf{J}=\sum_{\alpha} n_{\alpha} q_{\alpha} \mathbf{u}_{\alpha}=\sum_{\alpha} n_{\alpha} q_{\alpha} \mathbf{w}_{\alpha}+\sum_{\alpha} n_{\alpha} q_{\alpha} \mathbf{u}=\mathbf{J}^{\prime}+\rho \mathbf{u}
$$

where $\rho \mathbf{u}$ is the convection charge current density, which represents the flux of the space charge with velocity $\mathbf{u}$, and $\mathbf{J}^{\prime}$ is the conduction charge current density, which represents the charge current density in the frame of reference moving with the global mean velocity $\mathbf{u}$. On the other hand, we can write

$$
\mathbf{u} \cdot(\mathbf{J} \times \mathbf{B})=-\mathbf{J} \cdot(\mathbf{u} \times \mathbf{B})=-\mathbf{J}^{\prime} \cdot(\mathbf{u} \times \mathbf{B})
$$

Substituting (4.13) and (4.12) into the energy equation (4.11), we obtain

$$
\frac{D}{D t}\left(\frac{3 p}{2}\right)+\frac{3 p}{2} \nabla \cdot \mathbf{u}+\nabla \cdot \mathbf{q}+(\mathcal{P} \cdot \nabla) \cdot \mathbf{u}=\mathbf{J}^{\prime} \cdot \mathbf{E}^{\prime}
$$

where $\mathbf{E}^{\prime}=\mathbf{E}+\mathbf{u} \times \mathbf{B}$ is the electric field existing in the reference frame moving with the global mean velocity $\mathbf{u}$. The term $\mathbf{J}^{\prime} \cdot \mathbf{E}^{\prime}$ represents, therefore, the rate of change in the energy density due to joule heating.

\section{ELECTRODYNAMIC EQUATIONS FOR A CONDUCTING FLUID}

In the previous sections we have derived the macroscopic transport equations for conservation of mass, of momentum, and of energy in a conducting fluid. As mentioned before, this set of equations does not constitute a complete system, and it is necessary to truncate the hierarchy of macroscopic equations at some stage and make some simplifying assumptions. The continuity equation relates the mass density $\rho_{m}$ with the global mean velocity $\mathbf{u}$. The equation of motion, which specifies the variation of $\mathbf{u}$, involves also the total kinetic pressure dyad $\mathcal{P}$. The energy equation, specifying the rate of change of the total thermal energy density $(3 p / 2)$, 
includes also the heat flux vector $\mathbf{q}$. A more general energy equation would give us the variation of the total kinetic pressure dyad $\mathcal{P}$, which would include also the total heat flow triad $\mathcal{Q}$. We can continue taking higher order moments of the Boltzmann equation and obtain, for example, the transport equation governing the variation of the heat flow triad $\mathcal{Q}$. To obtain a complete system it is essential, therefore, to truncate the hierarchy of transport equations at some point. However, even after this truncation, the remaining equations include also the following electrodynamic variables: electric field $\mathbf{E}$, magnetic induction $\mathbf{B}$, charge current density $\mathbf{J}$, and charge density $\rho$. Besides the hydrodynamic transport equations, we need, therefore, ten electrodynamic equations that must relate the variations of $\mathbf{E}, \mathbf{B}, \mathbf{J}$, and $\rho$. These equations are considered next.

\subsection{Maxwell Curl Equations}

The following Maxwell equations

$$
\begin{gathered}
\nabla \times \mathbf{E}=-\frac{\partial \mathbf{B}}{\partial t} \\
\nabla \times \mathbf{B}=\mu_{0}\left(\mathbf{J}+\epsilon_{0} \frac{\partial \mathbf{E}}{\partial t}\right)
\end{gathered}
$$

provide six component equations, which can be considered as the equations governing the variations of the electromagnetic fields $\mathbf{E}$ and $\mathbf{B}$.

\subsection{Conservation of Electric Charge}

The electric charge conservation equation can be obtained by multiplying the mass conservation equation (8.3.2) (Eq. 3.2 in Chapter 8) by $q_{\alpha} / m_{\alpha}$, and adding over all species,

$$
\frac{\partial}{\partial t}\left(\sum_{\alpha} n_{\alpha} q_{\alpha}\right)+\nabla \cdot\left(\sum_{\alpha} n_{\alpha} q_{\alpha} \mathbf{u}_{\alpha}\right)=\sum_{\alpha}\left(\frac{q_{\alpha}}{m_{\alpha}}\right) S_{\alpha}
$$

Using the definitions of $\rho$ and $\mathbf{J}$, and noting that the total electric charge does not change as a result of collisions, we obtain

$$
\frac{\partial \rho}{\partial t}+\nabla \cdot \mathbf{J}=0
$$


It is worth noting here that (5.4) can also be derived, in an independent way, from the Maxwell curl equation (5.2) and the Maxwell divergence equation

$$
\nabla \cdot \mathbf{E}=\frac{\rho}{\epsilon_{0}}
$$

Taking the divergence of (5.2), yields

$$
\nabla \cdot \mathbf{J}+\epsilon_{0} \frac{\partial}{\partial t}(\nabla \cdot \mathbf{E})=0
$$

since the divergence of the curl of a vector field vanishes identically. This last equation, combined with (5.5), yields the charge conservation equation (5.4). The equations (5.4) and (5.5), therefore, cannot be considered as independent. As we have just shown, the Maxwell equations (5.2) and (5.5) already imply conservation of electric charge.

Another important aspect of Maxwell equations can be seen by taking the divergence of (5.1), which gives

$$
\frac{\partial}{\partial t}(\nabla \cdot \mathbf{B})=0
$$

or

$$
\nabla \cdot \mathbf{B}=\text { constant }
$$

Therefore, the Maxwell equation

$$
\nabla \cdot \mathbf{B}=0
$$

can be considered as an initial condition for (5.1), since if initially we take $\nabla \cdot \mathbf{B}=0,(5.1)$ implies that this condition will remain satisfied for all subsequent times.

\subsection{Generalized Ohm's Law}

To obtain a differential equation governing the variation of the charge current density, $\mathbf{J}$, we proceed in a way analogous to the derivation of (5.4). Thus, we multiply the momentum conservation equation (8.4.9) by $q_{\alpha} / m_{\alpha}$ and add over all particle species. This procedure leads to

$$
\sum_{\alpha} n_{\alpha} q_{\alpha}\left(\frac{\partial \mathbf{u}_{\alpha}}{\partial t}\right)+\sum_{\alpha} n_{\alpha} q_{\alpha}\left(\mathbf{u}_{\alpha} \cdot \nabla\right) \mathbf{u}_{\alpha}=\sum_{\alpha} n_{\alpha}\left(\frac{q_{\alpha}}{m_{\alpha}}\right)<\mathbf{F}>_{\alpha}-
$$




$$
\nabla \cdot\left[\sum_{\alpha}\left(\frac{q_{\alpha}}{m_{\alpha}}\right) \mathcal{P}_{\alpha}\right]+\sum_{\alpha}\left(\frac{q_{\alpha}}{m_{\alpha}}\right) \mathbf{A}_{\alpha}-\sum_{\alpha}\left(\frac{q_{\alpha}}{m_{\alpha}}\right) \mathbf{u}_{\alpha} S_{\alpha}
$$

We define now the electrokinetic pressure dyad $\mathcal{P}_{\alpha}^{E}$ for the particles of type $\alpha$, by

$$
\mathcal{P}_{\alpha}^{E}=\left(\frac{q_{\alpha}}{m_{\alpha}}\right) \mathcal{P}_{\alpha}=n_{\alpha} q_{\alpha}<\mathbf{c}_{\alpha} \mathbf{c}_{\alpha}>
$$

Consequently, for the plasma as a conducting fluid, we have the following relation analogous to $(1.13)$ :

$$
\mathcal{P}^{E}=\sum_{\alpha} \mathcal{P}_{\alpha}^{E}+\sum_{\alpha} n_{\alpha} q_{\alpha} \mathbf{w}_{\alpha} \mathbf{w}_{\alpha}
$$

The second term in the right-hand side of (5.10), therefore, becomes

$$
-\nabla \cdot\left[\sum_{\alpha}\left(\frac{q_{\alpha}}{m_{\alpha}}\right) \mathcal{P}_{\alpha}\right]=-\nabla \cdot \mathcal{P}^{E}+\nabla \cdot\left(\sum_{\alpha} n_{\alpha} q_{\alpha} \mathbf{w}_{\alpha} \mathbf{w}_{\alpha}\right)
$$

Using the continuity equation (8.3.2) and substituting $\mathbf{u}_{\alpha}$ by $\mathbf{w}_{\alpha}+\mathbf{u}$, the last term in the right-hand side of (5.10) can be written as

$$
\begin{gathered}
-\sum_{\alpha}\left(\frac{q_{\alpha}}{m_{\alpha}}\right) \mathbf{u}_{\alpha} S_{\alpha}=-\sum_{\alpha} \mathbf{w}_{\alpha} \frac{\partial}{\partial t}\left(n_{\alpha} q_{\alpha}\right)-\sum_{\alpha} \mathbf{w}_{\alpha}\left[\nabla \cdot\left(n_{\alpha} q_{\alpha} \mathbf{w}_{\alpha}\right)\right]- \\
\sum_{\alpha} \mathbf{w}_{\alpha}\left[\nabla \cdot\left(n_{\alpha} q_{\alpha} \mathbf{u}\right)\right]-\mathbf{u} \frac{\partial \rho}{\partial t}-\mathbf{u}(\nabla \cdot \mathbf{J})
\end{gathered}
$$

Similarly, the first and second terms in the left-hand side of (5.10) can be combined in the form

$$
\begin{gathered}
\sum_{\alpha} n_{\alpha} q_{\alpha} \frac{\partial \mathbf{w}_{\alpha}}{\partial t}+\sum_{\alpha}\left(n_{\alpha} q_{\alpha} \mathbf{w}_{\alpha} \cdot \nabla\right) \mathbf{w}_{\alpha}+\sum_{\alpha}\left(n_{\alpha} q_{\alpha} \mathbf{u} \cdot \nabla\right) \mathbf{w}_{\alpha}+ \\
\rho \frac{\partial \mathbf{u}}{\partial t}+(\mathbf{J} \cdot \nabla) \mathbf{u}
\end{gathered}
$$

We can now substitute expressions (5.13), (5.14), and (5.15) into (5.10) and simplify the result. Making use of the following identity for two vectors $\mathbf{a}$ and $\mathbf{b}$,

$$
\nabla \cdot(\mathbf{a b})=\mathbf{b}(\nabla \cdot \mathbf{a})+(\mathbf{a} \cdot \nabla) \mathbf{b}
$$

and the relation (4.12), we obtain

$$
\frac{\partial \mathbf{J}}{\partial t}+\nabla \cdot\left(\mathbf{u} \mathbf{J}^{\prime}+\mathbf{J u}\right)+\nabla \cdot \mathcal{P}^{E}=
$$




$$
\sum_{\alpha} n_{\alpha}\left(\frac{q_{\alpha}}{m_{\alpha}}\right)<\mathbf{F}>_{\alpha}+\sum_{\alpha}\left(\frac{q_{\alpha}}{m_{\alpha}}\right) \mathbf{A}_{\alpha}
$$

Equations (5.1), (5.2), (5.4), and (5.17) constitute ten component equations that complement the equations of conservation of mass, of momentum, and of energy for a conducting fluid. Equation (5.17), however, is still in a very general form of little practical value. A very useful and simple expression can be obtained for the case of a completely ionized plasma consisting of electrons and only one type of ions. In what follows, we simplify (5.17) for this case.

The electric charge current density $\mathbf{J}$ and the electric charge density $\rho$ for a completely ionized plasma containing only electrons and one type of ions of charge $e$ are given, respectively, by

$$
\begin{gathered}
\mathbf{J}=\sum_{\alpha} n_{\alpha} q_{\alpha} \mathbf{u}_{\alpha}=e\left(n_{i} \mathbf{u}_{i}-n_{e} \mathbf{u}_{e}\right) \\
\rho=\sum_{\alpha} n_{\alpha} q_{\alpha}=e\left(n_{i}-n_{e}\right)
\end{gathered}
$$

The global mean velocity $\mathbf{u}$, defined in (1.3), becomes

$$
\mathbf{u}=\frac{1}{\rho_{m}}\left(\rho_{m e} \mathbf{u}_{e}+\rho_{m i} \mathbf{u}_{i}\right)
$$

where $\rho_{m}=\rho_{m e}+\rho_{m i}$. Combining this last equation with (5.18) gives

$$
\begin{aligned}
& \mathbf{u}_{i}=\frac{\mu}{\rho_{m i}}\left(\frac{\rho_{m} \mathbf{u}}{m_{e}}+\frac{\mathbf{J}}{e}\right) \\
& \mathbf{u}_{e}=\frac{\mu}{\rho_{m e}}\left(\frac{\rho_{m} \mathbf{u}}{m_{i}}-\frac{\mathbf{J}}{e}\right)
\end{aligned}
$$

where $\mu=m_{e} m_{i} /\left(m_{e}+m_{i}\right)$ denotes the reduced mass.

We assume now that the mean velocity of the electrons and ions, relative to the global mean velocity $\mathbf{u}$, (that is, the diffusion velocities $\mathbf{w}_{e}$ and $\mathbf{w}_{i}$ ) are small compared to the thermal velocities. This condition being satisfied, (5.12) becomes

$$
\mathcal{P}^{E}=\mathcal{P}_{i}^{E}+\mathcal{P}_{e}^{E}=e\left(\frac{\mathcal{P}_{i}}{m_{i}}-\frac{\mathcal{P}_{e}}{m_{e}}\right)
$$

Considering that the conducting fluid is immersed in an electromagnetic field, the term containing the external force in (5.17) becomes

$$
\begin{gathered}
\sum_{\alpha} n_{\alpha}\left(\frac{q_{\alpha}}{m_{\alpha}}\right)<\mathbf{F}>_{\alpha}=\sum_{\alpha} n_{\alpha}\left(\frac{q_{\alpha}}{m_{\alpha}}\right)\left[q_{\alpha}\left(\mathbf{E}+\mathbf{u}_{\alpha} \times \mathbf{B}\right)\right] \\
=e^{2}\left(\frac{n_{i}}{m_{i}}+\frac{n_{e}}{m_{e}}\right) \mathbf{E}+e^{2}\left(\frac{n_{i}}{m_{i}} \mathbf{u}_{i}+\frac{n_{e}}{m_{e}} \mathbf{u}_{e}\right) \times \mathbf{B}
\end{gathered}
$$


Substituting the relations (5.21) and (5.22) in this last equation and simplifying, yields

$$
\begin{aligned}
& \sum_{\alpha} n_{\alpha}\left(\frac{q_{\alpha}}{m_{\alpha}}\right)<\mathbf{F}>_{\alpha}=e^{2}\left(\frac{n_{i}}{m_{i}}+\frac{n_{e}}{m_{e}}\right) \mathbf{E}+ \\
& e^{2}\left(\frac{n_{i}}{m_{e}}+\frac{n_{e}}{m_{i}}\right) \mathbf{u} \times \mathbf{B}+e\left(\frac{1}{m_{i}}-\frac{1}{m_{e}}\right) \mathbf{J} \times \mathbf{B}
\end{aligned}
$$

It is convenient at this moment to simplify this equation by making one additional approximation. Since the ion mass $m_{i}$ is much larger than the electron mass $m_{e}$ (for protons and electrons, for example, $m_{i} / m_{e} \simeq 1836$ ) and assuming macroscopic charge neutrality, $n_{e}=n_{i}=n$, we can take

$$
\begin{aligned}
& \frac{1}{m_{i}}-\frac{1}{m_{e}} \simeq-\frac{1}{m_{e}} \\
& \frac{n_{i}}{m_{i}}+\frac{n_{e}}{m_{e}} \simeq \frac{n}{m_{e}} \\
& \frac{n_{i}}{m_{e}}+\frac{n_{e}}{m_{i}} \simeq \frac{n}{m_{e}}
\end{aligned}
$$

Consequently, from (5.23) we have $\mathcal{P}^{E}=-\left(e / m_{e}\right) \mathcal{P}_{e}$, and from (5.25)

$$
\sum_{\alpha} n_{\alpha}\left(\frac{q_{\alpha}}{m_{\alpha}}\right)<\mathbf{F}>_{\alpha}=\frac{n e^{2}}{m_{e}}(\mathbf{E}+\mathbf{u} \times \mathbf{B})-\frac{e}{m_{e}} \mathbf{J} \times \mathbf{B}
$$

For the collision term in (5.17) we make use of expression (8.4.11), that is,

$$
\begin{aligned}
& \mathbf{A}_{e}=-\rho_{m e} \nu_{e i}\left(\mathbf{u}_{e}-\mathbf{u}_{i}\right) \\
& \mathbf{A}_{i}=-\rho_{m i} \nu_{i e}\left(\mathbf{u}_{i}-\mathbf{u}_{e}\right)
\end{aligned}
$$

From (8.4.13) we have $\rho_{m i} \nu_{i e}=\rho_{m e} \nu_{e i}$, so that

$$
\sum_{\alpha}\left(\frac{q_{\alpha}}{m_{\alpha}}\right) \mathbf{A}_{\alpha}=e \rho_{m e} \nu_{e i}\left(\mathbf{u}_{e}-\mathbf{u}_{i}\right)\left(\frac{1}{m_{i}}+\frac{1}{m_{e}}\right)=-\nu_{e i} \mathbf{J}
$$

where we have used (5.18) for $\mathbf{J}$, and the approximations $m_{i} \gg m_{e}$ and $n_{e}=n_{i}=n$.

We can now substitute the results contained in (5.23), (5.29), and (5.32) into (5.17), to obtain

$$
\frac{\partial \mathbf{J}}{\partial t}+\nabla \cdot\left(\mathbf{u} \mathbf{J}^{\prime}+\mathbf{J u}\right)-\frac{e}{m_{e}} \nabla \cdot \mathcal{P}_{e}=
$$




$$
\frac{n e^{2}}{m_{e}}(\mathbf{E}+\mathbf{u} \times \mathbf{B})-\frac{e}{m_{e}} \mathbf{J} \times \mathbf{B}-\nu_{e i} \mathbf{J}
$$

Note that, since we assumed $n_{e}=n_{i}$, we must have $\rho=0$ and $\mathbf{J}^{\prime}=\mathbf{J}$. In some situations in which $\mathbf{J}$ and $\mathbf{u}$ can be considered as small perturbations, the nonlinear terms involving their product may be neglected compared to the other terms. With this simplifying approximation and using the notation

$$
\sigma_{0}=\frac{n e^{2}}{m_{e} \nu_{e i}}
$$

which represents the longitudinal electrical conductivity, we obtain for $(5.33)$

$$
\frac{m_{e}}{n e^{2}} \frac{\partial \mathbf{J}}{\partial t}-\frac{1}{n e} \nabla \cdot \mathcal{P}_{e}=\mathbf{E}+\mathbf{u} \times \mathbf{B}-\frac{1}{n e} \mathbf{J} \times \mathbf{B}-\frac{1}{\sigma_{0}} \mathbf{J}
$$

This equation is known as the generalized Ohm's law. The terms in the right-hand side are the ones normally retained in magnetohydrodynamics, while all the others are neglected. However, the omission of the terms in the left-hand side of (5.35) is not always justifiable.

For cases in which $\mathbf{J}$ does not vary with time, that is, under steadystate conditions, we have $\partial \mathbf{J} / \partial t=0$. If we consider also that the pressure term in (5.35) is negligible, that is, $\nabla \cdot \mathcal{P}_{e}=0$, then (5.35) simplifies to

$$
\mathbf{J}=\sigma_{0}(\mathbf{E}+\mathbf{u} \times \mathbf{B})-\frac{\sigma_{0}}{n e} \mathbf{J} \times \mathbf{B}
$$

The last term in this equation is related to a phenomenon called the Hall effect in magnetohydrodynamic flow problems and, for this reason, it is normally called the Hall effect term. This term is small when $\sigma_{0}|\mathbf{B}|$ is much less than $n e$, that is, when $\Omega_{c e} \ll \nu_{e i}$. Thus, when the collision frequency is much larger than the magnetic gyrofrequency, the Hall effect term can be neglected and (5.36) reduces to

$$
\mathbf{J}=\sigma_{0}(\mathbf{E}+\mathbf{u} \times \mathbf{B})
$$

In the absence of an external magnetic field, (5.37) reduces further to

$$
\mathbf{J}=\sigma_{0} \mathbf{E}
$$

which is the expression commonly known as Ohm's law. 


\section{SIMPLIFIED MAGNETOHYDRODYNAMIC EQUATIONS}

In the last two sections we have shown that the set of macroscopic transport equations for each individual species in a plasma can be substituted by transport equations for the whole plasma as a conducting fluid, complemented by the electrodynamic equations. These total macroscopic equations for a conducting fluid are usually known as the magnetohydrodynamic (MHD) equations. In their most general form they are essentially equivalent to the set of equations for each individual particle species, except for the loss of information regarding the variables for each individual species.

In practice, however, the MHD equations are seldom used in their general form. Several simplifying approximations are normally considered, based on physical arguments that allow the elimination of some of the terms in the equations. For steady-state situations, or slowly varying problems, the MHD equations are very convenient and, in many cases, lead to important results that would not be easily obtained from the individual equations for each particle species.

One of the approximations normally used in MHD consists in neglecting the term $\epsilon_{0} \partial \mathbf{E} / \partial t$ in the Maxwell equation (5.2). In order to investigate the validity of this approximation, it is convenient to use dimensional analysis, as follows. We can express, in general, the charge current density as $\mathbf{J}=\mathcal{S} \cdot \mathbf{E}$, where $\mathcal{S}$ denotes the conductivity dyad, so that, dimensionally, we have

$$
\begin{gathered}
J \sim \sigma E \\
\epsilon_{0}|\partial \mathbf{E} / \partial t| \sim \epsilon_{0} E / \tau
\end{gathered}
$$

where $\tau$ represents a characteristic time for changes in the electric field and $\sigma$ represents a characteristic conductivity. The ratio of the two terms in the right-hand side of (5.2) becomes, therefore,

$$
\epsilon_{0} \frac{|\partial \mathbf{E} / \partial t|}{J} \sim \frac{\epsilon_{0}}{\sigma \tau}
$$

For most of the fluids normally considered in MHD problems, $\sigma$ is very large, typically greater than $1 \mathrm{mho} / \mathrm{m}$, whereas $\epsilon_{0}$ is of the order of $10^{-11}$ Farad/m. Consequently,

$$
\epsilon_{0} \frac{|\partial \mathbf{E} / \partial t|}{J} \sim \frac{10^{-11}}{\tau}
$$


with $\tau$ in seconds, which shows that this approximation is not valid only when we are considering extremely small characteristic times.

It is also usually assumed that macroscopic electric neutrality is maintained with a high degree of accuracy and therefore the electric density $\rho$ is set equal to zero.

A questionable approximation in the set of MHD equations is the generalized Ohm's law, in the form given in (5.36). In this form, the terms containing the time derivatives and pressure gradient (or divergence of the pressure dyad) are omitted, even though these terms are retained in other equations of the set. This approximation, therefore, is not justifiable in a direct manner. It is common to simply assume that all time derivatives are negligibly small and that the plasma is almost a cold plasma, so that the generalized Ohm's law reduces to the form given in (5.36).

For convenience, we collect here the following set of simplified magnetohydrodynamic equations:

$$
\begin{gathered}
\frac{\partial \rho_{m}}{\partial t}+\nabla \cdot\left(\rho_{m} \mathbf{u}\right)=0 \\
\rho_{m} \frac{D \mathbf{u}}{D t}=\mathbf{J} \times \mathbf{B}-\nabla p \\
\nabla p=V_{s}^{2} \nabla \rho_{m} \\
\nabla \times \mathbf{E}=-\frac{\partial \mathbf{B}}{\partial t} \\
\nabla \times \mathbf{B}=\mu_{0} \mathbf{J} \\
\mathbf{J}=\sigma_{0}(\mathbf{E}+\mathbf{u} \times \mathbf{B})-\frac{\sigma_{0}}{n e} \mathbf{J} \times \mathbf{B}
\end{gathered}
$$

In this set of equations, viscosity and thermal conductivity are neglected, so that the pressure dyad reduces to a scalar pressure. Note that (6.9) implies in

$$
\nabla \cdot \mathbf{J}=0
$$

which is the equation of conservation of electric charge in the absence of changes in the total macroscopic charge density $\rho$. It is for this reason that the equation of conservation of electric charge is not explicity considered in this set of MHD equations. Except in some special circumstances, it is also common to neglect the Hall effect term $\left(\sigma_{0} / e n\right) \mathbf{J} \times \mathbf{B}$ in (6.10).

In some situations where the fluid electrical conductivity is extremely large, it is convenient to consider the idealized approximation of a perfectly 
conducting fluid, in which the conductivity approaches infinity. In this case Ohm's law reduces to

$$
\mathbf{E}=-\mathbf{u} \times \mathbf{B}
$$

and the corresponding set of equations is commonly referred to as the ideal MHD equations.

\section{PROBLEMS}

9.1 Show that the total kinetic energy density of all species in a fluid can be written as the sum of the thermal energy density of the whole fluid plus the kinetic energy of the mass motion, that is

$$
\sum_{\alpha} \frac{1}{2} \rho_{m \alpha}<v^{2}>_{\alpha}=\frac{3 p}{2}+\sum_{\alpha} \frac{1}{2} \rho_{m \alpha} u_{\alpha}^{2}
$$

where

$$
\frac{3 p}{2}=\sum_{\alpha} \frac{1}{2} \rho_{m \alpha}<c_{\alpha 0}^{2}>=\sum_{\alpha} \frac{1}{2} \rho_{m \alpha}<c_{\alpha}^{2}>+\sum_{\alpha} \frac{1}{2} \rho_{m \alpha} w_{\alpha}^{2}
$$

9.2 Show that when there is no heat flow $(\mathbf{q}=0)$, no joule heating $\left(\mathbf{J}^{\prime} \cdot \mathbf{E}^{\prime}=0\right)$, and when the pressure tensor is isotropic given by $\mathcal{P}=p \mathbf{1}$, the energy equation (4.14) reduces to the following adiabatic equation:

$$
p \rho_{m}^{-5 / 3}=\text { constant }
$$

9.3 From the momentum conservation equation with the MHD approximation [see (6.6)], and the generalized Ohm's law in the simplified form (6.10), but without considering the Hall effect term, derive the following equation:

$$
\rho_{m} \frac{D \mathbf{u}}{D t}=\sigma_{0}(\mathbf{E} \times \mathbf{B})+\sigma_{0}(\mathbf{u} \times \mathbf{B}) \times \mathbf{B}-\nabla p
$$

Solve this equation, considering that $\mathbf{E}=0$ and $p=$ constant, to show that the fluid velocity perpendicular to $\mathbf{B}$ is given by

$$
u_{\perp}(t)=u_{\perp}(0) \exp (-t / \tau)
$$


where $\tau$ is a characteristic time for diffusion of the fluid across the magnetic field lines, given by

$$
\tau=\frac{\rho_{m}}{\sigma_{0} B^{2}}
$$

9.4 In equations (1.5) and (1.6), explain the reason why the mass flux $\mathbf{J}_{m}$ is given by $\rho_{m} \mathbf{u}$, whereas the electric charge flux $\mathbf{J}$ is not given by $\rho \mathbf{u}$.

9.5 Obtain an expression for the heat flux triad $\mathcal{Q}$ for the plasma as a whole, defined as

$$
\mathcal{Q}=\sum_{\alpha} \rho_{m \alpha}<\mathbf{c}_{\alpha 0} \mathbf{c}_{\alpha 0} \mathbf{c}_{\alpha 0}>
$$

where $\mathbf{c}_{\alpha 0}=\mathbf{c}_{\alpha}+\mathbf{w}_{\alpha}$, in terms of a summation over the heat flux triad for each species $\mathcal{Q}_{\alpha}$ and of terms involving the diffusion velocity $\mathbf{w}_{\alpha}$. Then, simplify this expression for the isotropic case.

9.6 Derive an energy equation, of higher order than (4.14), involving the total time rate of change of the total pressure dyad, that is, $D \mathcal{P} / D t$.

9.7 For a perfectly conducting fluid characterized by a scalar pressure, under steady-state conditions, use the equation of motion (6.6) and the generalized Ohm's law (6.10) to derive the following equation for the fluid velocity component perpendicular to $\mathbf{B}$ :

$$
\mathbf{u}_{\perp}=\frac{1}{B^{2}}\left(\mathbf{E}-\frac{1}{n e} \nabla p\right) \times \mathbf{B}
$$




\section{0}

\section{Plasma conductivity AND DIFFUSION}

\section{INTRODUCTION}

In the previous chapters we have introduced the fundamental elements of kinetic theory and the macroscopic transport equations necessary for the study of a variety of important phenomena in plasmas. Many plasma phenomena can be analyzed using the macroscopic transport equations, either by considering the plasma as a multiconstituent fluid or by treating the whole plasma as a single conducting fluid. In some cases, however, a satisfactory description can be obtained only through the use of kinetic theory.

In this and in the following chapters we investigate some basic plasma phenomena that illustrate the use of the cold and warm plasma models, and of the phase space distribution function. Phenomena that can be analyzed treating the whole plasma as a single conducting fluid are usually studied under the general title of magnetohydrodynamics (MHD), and will be considered in Chapters 12, 13, and 15 .

\section{THE LANGEVIN EQUATION}

Before we consider the phenomena of plasma conductivity and diffusion, it is convenient to introduce a very simple form of the equation of motion for a weakly ionized cold plasma, known as the Langevin equation. In a weakly ionized plasma the number density of the charged particles is much smaller than that of the neutral particles. In this case the chargeneutral interactions are dominant. The macroscopic equation of motion 
for the electrons under the action of the Lorentz force and the collisional forces, can be written as

$$
m_{e} \frac{D \mathbf{u}_{e}}{D t}=-e\left(\mathbf{E}+\mathbf{u}_{e} \times \mathbf{B}\right)+\left(\mathbf{F}_{\text {coll }}\right)_{e}
$$

where $\mathbf{u}_{e}(\mathbf{r}, t)$ is the average electron velocity and $\left(\mathbf{F}_{\text {coll }}\right)_{e}$ denotes symbolically the rate of change of the average electron momentum due to collisions with neutral particles.

The macroscopic collision term $\left(\mathbf{F}_{\text {coll }}\right)_{e}$ can be expressed in a phenomenological way as the product of the average electron momentum with an effective constant collision frequency $\nu_{c}$ for momentum transfer between the electrons and the heavy (neutral) particles,

$$
\left(\mathbf{F}_{\text {coll }}\right)_{e}=-\nu_{c} m_{e} \mathbf{u}_{e}
$$

In this expression we are neglecting the average motion of the neutral particles, as they are much more massive than the electrons. Note that this does not mean that the velocities of the individual neutral particles are zero, but only that they are completely random so that their average velocity is zero. Using this expression for the collision term, we obtain the following equation, known as the Langevin equation:

$$
m_{e} \frac{D \mathbf{u}_{e}}{D t}=-e\left(\mathbf{E}+\mathbf{u}_{e} \times \mathbf{B}\right)-\nu_{c} m_{e} \mathbf{u}_{e}
$$

The physical meaning of this collision term can be seen as follows. In the absence of electric and magnetic fields, (2.3) reduces to

$$
\frac{D \mathbf{u}_{e}}{D t}=-\nu_{c} \mathbf{u}_{e}
$$

whose solution is

$$
\mathbf{u}_{e}(t)=\mathbf{u}_{e}(0) \exp \left(-\nu_{c} t\right)
$$

Thus, the electron-neutral collisions decrease the average electron velocity exponentially, at a rate governed by the collision frequency.

An equation analogous to (2.3) can be written for the ions,

$$
m_{i} \frac{D \mathbf{u}_{i}}{D t}=Z e\left(\mathbf{E}+\mathbf{u}_{i} \times \mathbf{B}\right)+\left(\mathbf{F}_{\text {coll }}\right)_{i}
$$

where $\mathbf{u}_{i}$ denotes the average ion velocity and $Z e$ the ion charge. In many cases of interest, as in high-frequency phenomena, we can neglect the ion 
motion and assume $\mathbf{u}_{i}=0$, since the ion mass is typically about $10^{3}$ or $10^{4}$ times greater than the electron mass. The type of plasma in which only the electron motion is important is usually called a Lorentz gas. When dealing with very low frequencies, however, the motion of the ions must be considered.

Despite the approximations implicit in the Langevin equation, it has been successfully used to describe a variety of phenomena in plasmas, including the propagation of electromagnetic waves in cold magnetoplasmas. Particularly, the analysis of the characteristics of electromagnetic wave propagation in the Earth's ionosphere has been quite successful. A great advantage of this equation is its simplicity.

\section{LINEARIZATION OF THE LANGEVIN EQUATION}

In the form presented in (2.3) the Langevin equation contains nonlinear terms that involve the product of two variables. In many situations of interest the difficulty inherent in the nonlinear terms can be eliminated through a linearization approximation, which is valid for small-amplitude variations.

The total time derivative contains the nonlinear term $\left(\mathbf{u}_{e} \cdot \nabla\right) \mathbf{u}_{e}$, which is called the inertial term in fluid dynamics. The omission of this inertial term is justified when the average velocity and its space derivatives are small, or when $\mathbf{u}_{e}$ is normal to its gradient (such as in the case of transverse waves).

For the nonlinear term $\mathbf{u}_{e} \times \mathbf{B}$, we can separate the magnetic flux density $\mathbf{B}(\mathbf{r}, t)$ into two parts,

$$
\mathbf{B}(\mathbf{r}, t)=\mathbf{B}_{0}+\mathbf{B}^{\prime}(\mathbf{r}, t)
$$

where $\mathbf{B}_{0}$ is constant and $\mathbf{B}^{\prime}(\mathbf{r}, t)$ is the variable component, so that

$$
q\left(\mathbf{E}+\mathbf{u}_{e} \times \mathbf{B}\right)=q\left(\mathbf{E}+\mathbf{u}_{e} \times \mathbf{B}_{0}+\mathbf{u}_{e} \times \mathbf{B}^{\prime}\right)
$$

For situations in which

$$
\left|\mathbf{u}_{e} \times \mathbf{B}^{\prime}\right| \ll|\mathbf{E}|
$$

the nonlinear term $\mathbf{u}_{e} \times \mathbf{B}^{\prime}$ in (3.2) can be neglected. With these linearization approximations the Langevin equation becomes

$$
m_{e} \frac{\partial \mathbf{u}_{e}}{\partial t}=-e\left(\mathbf{E}+\mathbf{u}_{e} \times \mathbf{B}_{0}\right)-m_{e} \nu_{c} \mathbf{u}_{e}
$$


A case of great practical interest is the one in which the variables $\mathbf{E}$, $\mathbf{B}^{\prime}$, and $\mathbf{u}_{e}$ vary harmonically in space and time. The treatment in terms of plane waves has the advantage of great mathematical simplicity, besides the fact that any complex and physically realizable wave motion can be synthesized in terms of a superposition of plane waves. Let us consider, therefore, plane wave solutions for $\mathbf{E}, \mathbf{B}^{\prime}$, and $\mathbf{u}_{e}$ in the form

$$
\mathbf{E}, \mathbf{B}^{\prime}, \mathbf{u}_{e} \propto \exp [i(\mathbf{k} \cdot \mathbf{r}-\omega t)]
$$

where $\omega$ denotes the wave angular frequency, $\mathbf{k}$ is the wave propagation vector (normal to the wave front), and $\mathbf{r}$ is a position vector drawn from the origin of a coordinate system to the point considered on the wave front (refer to Fig. 1).

For the space and time dependence given in (3.5), the differential operators $\nabla$ and $\partial / \partial t$ are transformed into simple algebraic operators, according to $\nabla \rightarrow i \mathbf{k}$ and $\partial / \partial t \rightarrow-i \omega$. Substituting (3.1) into Maxwell equation $\nabla \times \mathbf{E}=-\partial \mathbf{B} / \partial t$, we obtain

$$
i \mathbf{k} \times \mathbf{E}=i \omega \mathbf{B}^{\prime}
$$

where $\partial \mathbf{B}_{0} / \partial t=0$, since $\mathbf{B}_{0}$ is constant. Therefore,

$$
\mathbf{B}^{\prime}=\frac{\mathbf{k} \times \mathbf{E}}{\omega}
$$

and plugging this result back into (3.3) yields the condition

$$
\left|\mathbf{u}_{e} \times(\mathbf{k} \times \mathbf{E}) / \omega\right| \ll|\mathbf{E}|
$$

The magnitude of the nonlinear term $\mathbf{u}_{e} \times \mathbf{B}^{\prime}$ may be equal to or smaller than $\left|\left(u_{e} k E\right) / \omega\right|$. Hence, the nonlinear term can be neglected if

$$
\left|u_{e}(k / \omega)\right| \ll 1
$$

or, equivalently, if

$$
\left|u_{e}\right| \ll|\omega / k|
$$

The term $(\omega / k)$ represents the phase velocity of the plane wave. Since this term is usually of the order of the speed of light $c$, whereas the magnitude of the mean velocity of the electrons $u_{e}$ is much less than $c$, the nonlinear term can generally be neglected. However, in cases of resonance $\omega / k$ is very 


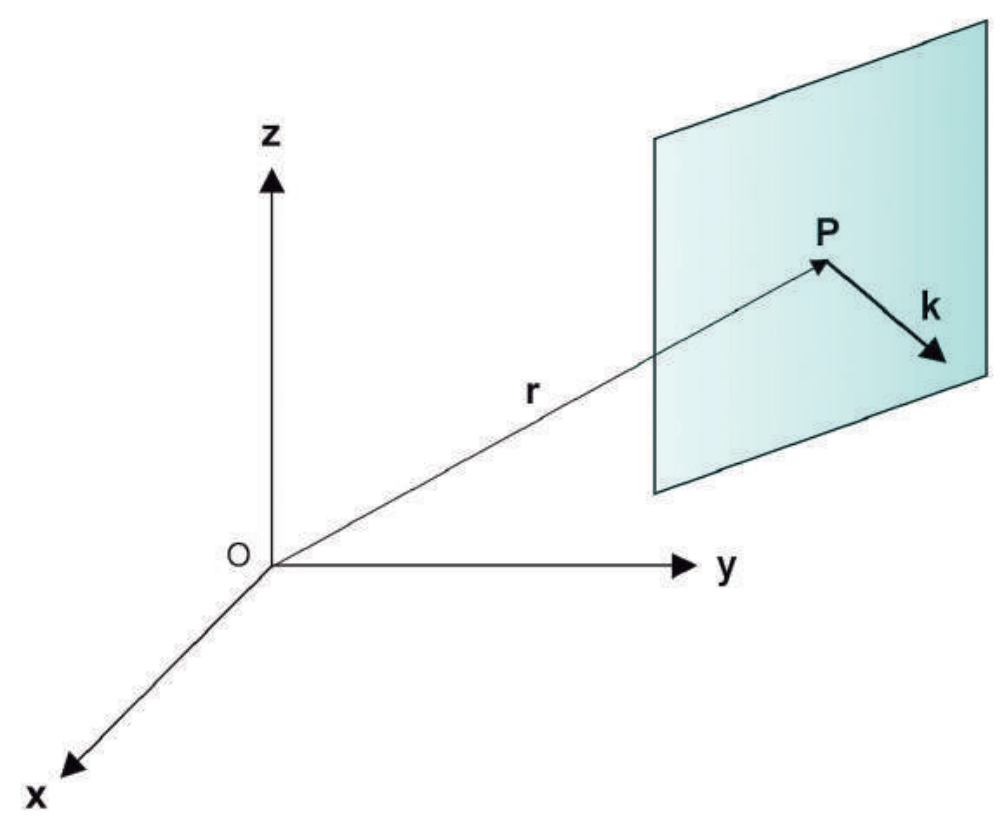

Fig. 1 Position vector $\mathbf{r}$ drawn from the origin of a coordinate system $(x, y, z)$ to a point $P$ on the wave front, whose normal is given by the wave propagation vector $\mathbf{k}$.

small, whereas $u_{e}$ becomes large. Under these conditions the nonlinear terms are important and a nonlinear analysis must be used.

\section{DC CONDUCTIVITY AND ELECTRON MOBILITY}

In this section we apply the steady-state Langevin equation to derive an expression for the DC (direct current) conductivity of a weakly ionized homogeneous plasma, for which the Lorentz model (electron gas) is applicable. The applied electric field is assumed to be constant and uniform.

\subsection{Isotropic Plasma}

In the absence of a magnetic field the steady-state Langevin equation for the electrons becomes

$$
-e \mathbf{E}-m_{e} \nu_{c} \mathbf{u}_{e}=0
$$

In this case the action of the applied electric field is balanced dynamically by the electron-neutral collisions. The electric current density associated 
with the electron motion is

$$
\mathbf{J}=-e n_{e} \mathbf{u}_{e}
$$

Combining (4.1) and (4.2), gives

$$
\mathbf{J}=\frac{n_{e} e^{2}}{m_{e} \nu_{c}} \mathbf{E}
$$

From Ohm's law, $\mathbf{J}=\sigma_{0} \mathbf{E}$, we identify the following expression for the $D C$ conductivity of an isotropic electron gas:

$$
\sigma_{0}=\frac{n_{e} e^{2}}{m_{e} \nu_{c}}
$$

The electron mobility $\mathcal{M}_{e}$ is defined as the ratio of the mean velocity of the electrons to the applied electric field,

$$
\mathcal{M}_{e}=\frac{u_{e}}{E}
$$

Therefore, from (4.1) we obtain

$$
\mathcal{M}_{e}=-\frac{e}{m_{e} \nu_{c}}=-\frac{\sigma_{0}}{n_{e} e}
$$

\subsection{Anisotropic Magnetoplasma}

In the presence of a magnetic field the plasma becomes spatially anisotropic. The steady-state Langevin equation can be written as

$$
-e\left(\mathbf{E}+\mathbf{u}_{e} \times \mathbf{B}_{0}\right)-m_{e} \nu_{c} \mathbf{u}_{e}=0
$$

where $\mathbf{B}_{0}$ is a constant and uniform magnetic field. Using (4.2),

$$
\frac{m_{e} \nu_{c}}{n_{e} e} \mathbf{J}=e\left(\mathbf{E}+\mathbf{u}_{e} \times \mathbf{B}_{0}\right)
$$

which may be written in the form

$$
\mathbf{J}=\sigma_{0}\left(\mathbf{E}+\mathbf{u}_{e} \times \mathbf{B}_{0}\right)
$$


where $\sigma_{0}$ is given in (4.4). This last equation is a simplified form of the generalized Ohm's law (see Chapter 9).

At this point it is worth considering a useful result that arises when the collisional effects are negligible. When $\nu_{c} \rightarrow 0$ the DC conductivity becomes very large $\left(\sigma_{0} \rightarrow \infty\right)$ so that we must have, from (4.9),

$$
\mathbf{E}+\mathbf{u}_{e} \times \mathbf{B}_{0}=0
$$

This expression represents, therefore, the simplified form of the generalized Ohm's law for a plasma with a very large conductivity. In this case, taking the cross-product of (4.10) with $\mathbf{B}_{0}$ and noting that

$$
\left(\mathbf{u}_{e} \times \mathbf{B}_{0}\right) \times \mathbf{B}_{0}=-\mathbf{u}_{e \perp} B_{0}^{2}
$$

we obtain

$$
\mathbf{u}_{e \perp}=\frac{\mathbf{E} \times \mathbf{B}_{0}}{B_{0}^{2}}
$$

This result shows that, in the absence of collisions, the electrons have a drift velocity $\mathbf{u}_{e \perp}$ perpendicular to both the electric and the magnetic fields. Since this result is independent of the particle mass and charge, the same result will be obtained for the ions if their motion is taken into account. This can be easily shown considering the Langevin equation for the ions. Thus, in the absence of collisions, both electrons and ions move together with the drift velocity (4.12), and there is no electric current $(\mathbf{J}=0)$ associated with their motion. When the collisional effects are not negligible, the motion of the ions suffers a larger retardation than that of the electrons as a result of collisions. In this case, there is an electric current given by (assuming $n_{e}=n_{i}$ )

$$
\mathbf{J}_{\perp}=e n_{e}\left(\mathbf{u}_{i \perp}-\mathbf{u}_{e \perp}\right)
$$

which is perpendicular to both $\mathbf{E}$ and $\mathbf{B}_{0}$, known as the Hall current. Note that, since $\mathbf{u}_{e \perp}>\mathbf{u}_{i \perp}$, this current is in the direction of $-\left(\mathbf{E} \times \mathbf{B}_{0}\right)$, that is, opposite to the drift velocity of both types of particles.

Returning now to the generalized Ohm's law in the simplified form (4.9), let us rewrite it in a way that relates the current density directly to the applied electric field. We define, therefore, a $D C$ conductivity dyad (or tensor) $\mathcal{S}$ by the equation

$$
\mathbf{J}=\mathcal{S} \cdot \mathbf{E}
$$


In order to obtain an expression for $\mathcal{S}$, consider a Cartesian coordinate system with the $z$ axis parallel to the magnetic field, $\mathbf{B}_{0}=B_{0} \widehat{\mathbf{z}}$. Replacing $\mathbf{u}_{e}$ in (4.9) by $-\mathbf{J} /\left(e n_{e}\right)$, we get

$$
\mathbf{J}=\sigma_{0} \mathbf{E}-\frac{\sigma_{0} B_{0}}{e n_{e}}(\mathbf{J} \times \widehat{\mathbf{z}})
$$

Noting that

$$
\mathbf{J} \times \widehat{\mathbf{z}}=J_{y} \widehat{\mathbf{x}}-J_{x} \widehat{\mathbf{y}}
$$

we obtain the following set of equations for the $x, y$, and $z$ components of $(4.15)$

$$
\begin{array}{ll}
\widehat{\mathbf{x}}: & J_{x}=\sigma_{0} E_{x}-\frac{\Omega_{c e}}{\nu_{c}} J_{y} \\
\widehat{\mathbf{y}}: & J_{y}=\sigma_{0} E_{y}+\frac{\Omega_{c e}}{\nu_{c}} J_{x} \\
\widehat{\mathbf{z}}: & J_{z}=\sigma_{0} E_{z}
\end{array}
$$

where $\Omega_{c e}$ denotes the electron cyclotron frequency. We can combine (4.17) and (4.18) to eliminate $J_{y}$ from the first one and $J_{x}$ from the second one, obtaining

$$
\begin{aligned}
& J_{x}=\frac{\nu_{c}^{2}}{\left(\nu_{c}^{2}+\Omega_{c e}^{2}\right)} \sigma_{0} E_{x}-\frac{\nu_{c} \Omega_{c e}}{\left(\nu_{c}^{2}+\Omega_{c e}^{2}\right)} \sigma_{0} E_{y} \\
& J_{y}=\frac{\nu_{c} \Omega_{c e}}{\left(\nu_{c}^{2}+\Omega_{c e}^{2}\right)} \sigma_{0} E_{x}+\frac{\nu_{c}^{2}}{\left(\nu_{c}^{2}+\Omega_{c e}^{2}\right)} \sigma_{0} E_{y}
\end{aligned}
$$

In matrix form we can write, therefore,

$$
\left(\begin{array}{c}
J_{x} \\
J_{y} \\
J_{z}
\end{array}\right)=\sigma_{0}\left(\begin{array}{ccc}
\frac{\nu_{c}^{2}}{\left(\nu_{c}^{2}+\Omega_{c e}^{2}\right)} & -\frac{\nu_{c} \Omega_{c e}}{\left(\nu_{c}^{2}+\Omega_{c e}^{2}\right)} & 0 \\
\frac{\nu_{c} \Omega_{c e}}{\left(\nu_{c}^{2}+\Omega_{c e}^{2}\right)} & \frac{\nu_{c}^{2}}{\left(\nu_{c}^{2}+\Omega_{c e}^{2}\right)} & 0 \\
0 & 0 & 1
\end{array}\right)\left(\begin{array}{c}
E_{x} \\
E_{y} \\
E_{z}
\end{array}\right)
$$

which is now in the form given in (4.14). The DC conductivity dyad, therefore, is given by

$$
\mathcal{S}=\left(\begin{array}{ccc}
\sigma_{\perp} & -\sigma_{H} & 0 \\
\sigma_{H} & \sigma_{\perp} & 0 \\
0 & 0 & \sigma_{\|}
\end{array}\right)
$$


where we have used the notation

$$
\begin{gathered}
\sigma_{\perp}=\frac{\nu_{c}^{2}}{\left(\nu_{c}^{2}+\Omega_{c e}^{2}\right)} \sigma_{0} \\
\sigma_{H}=\frac{\nu_{c} \Omega_{c e}}{\left(\nu_{c}^{2}+\Omega_{c e}^{2}\right)} \sigma_{0} \\
\sigma_{\|} \equiv \sigma_{0}=\frac{n_{e} e^{2}}{m_{e} \nu_{c}}
\end{gathered}
$$

To illustrate the physical meaning of the components of $\mathcal{S}$ it is convenient to separate the applied electric field in a component parallel to $\mathbf{B}_{0}, \mathbf{E}_{\|}$, and a component in the plane normal to $\mathbf{B}_{0}, \mathbf{E}_{\perp}$, as shown in Fig. 2. The element $\sigma_{\perp}$ is called the perpendicular or transverse conductivity (also known as Pedersen conductivity), since it governs the flow of electric current in the direction of the electric field component normal to the magnetic field ( $\| \mathbf{E}_{\perp}, \perp \mathbf{B}_{0}$ ), while $\sigma_{H}$ (known as the Hall conductivity) governs the flow of electric current in the direction perpendicular to both the electric and magnetic fields $\left(\perp \mathbf{E}, \perp \mathbf{B}_{0}\right)$. The element $\sigma_{0}$ is the longitudinal conductivity, since it governs the electric current flow in the direction of the electric field component along the magnetic field ( $\| \mathbf{E}_{\|}$, $\left.\| \mathbf{B}_{0}\right)$. Note that the electric current along $\mathbf{B}_{0}$ is governed by the same conductivity $\left(\sigma_{0}\right)$ as in the isotropic plasma.

The dependence of $\sigma_{\perp}$ and $\sigma_{H}$ on the ratio of the cyclotron frequency to the collision frequency is shown in Fig. 3. As the ratio $\left(\Omega_{c e} / \nu_{c}\right)$ increases, $\sigma_{\perp}$ and $\sigma_{H}$ decrease rapidly, the effect being more pronounced for $\sigma_{\perp}$. Thus, when $\left(\Omega_{c e} / \nu_{c}\right)$ is relatively large, very little current is produced across the magnetic field lines, as compared to the current produced along the field lines, for the same applied electric field. Note that $\sigma_{0}$ increases as $\nu_{c}$ decreases and it is independent of the magnitude of $\mathbf{B}$ and therefore of $\Omega_{c e}$. Thus, in a rarefied plasma immersed in a relatively strong magnetic field, the electric current flows essentially along the magnetic field lines.

Note that in the absence of a magnetic field $\left(\Omega_{c e}=0\right),(4.24),(4.25)$, and (4.26) give $\sigma_{\perp}=\sigma_{0}$ and $\sigma_{H}=0$, so that the plasma becomes isotropic.

We deduce next an expression for the electron mobility. Due to the anisotropy introduced by the magnetic field we have in this case a mobility dyad $\mathcal{M}_{e}$. We define the electron mobility dyad by the equation

$$
\mathbf{u}_{e}=\mathcal{M}_{e} \cdot \mathbf{E}
$$

Since $\mathbf{J}=-e n_{e} \mathbf{u}_{e}=\mathcal{S} \cdot \mathbf{E}$, we find that

$$
\mathcal{M}_{e}=-\frac{1}{n_{e} e} \mathcal{S}
$$




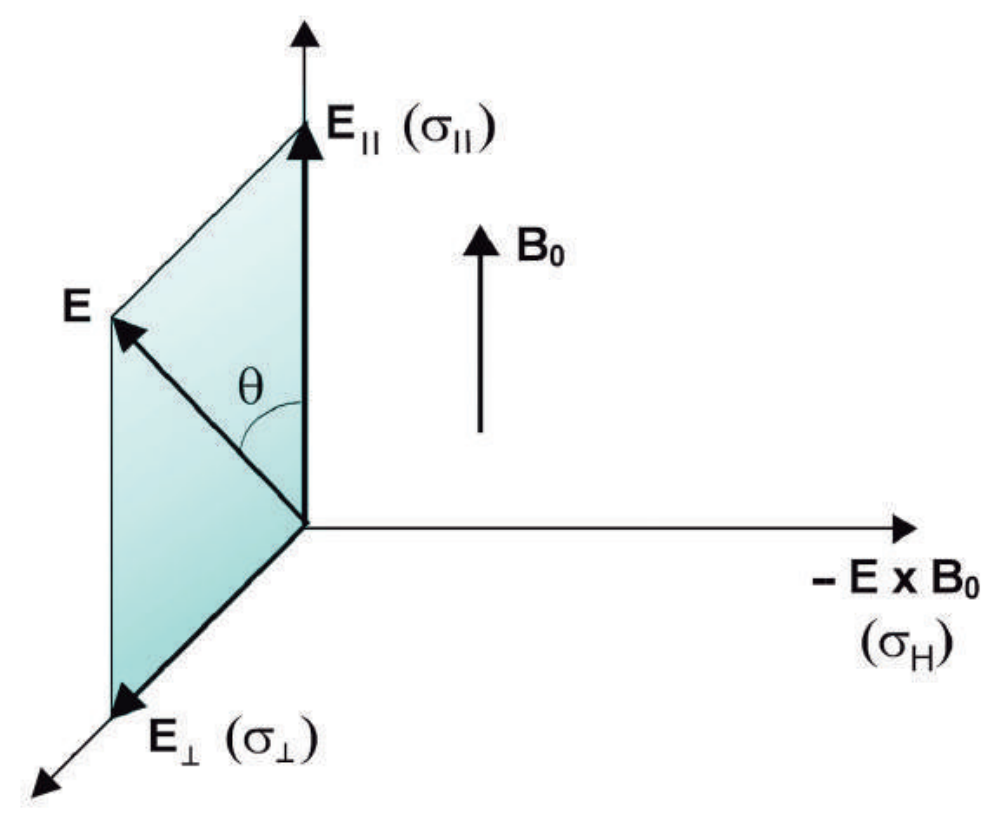

Fig. 2 Relative orientation of the vector fields $\mathbf{E}_{\|}, \mathbf{E}_{\perp}$, and $-\mathbf{E} \times \mathbf{B}_{0}$. The conductivities $\sigma_{\|}, \sigma_{\perp}$, and $\sigma_{H}$ govern the magnitude of the electric currents flowing along these directions, respectively.

Explicit expressions for the components of $\mathcal{M}_{e}$ can be easily written down considering (4.23), (4.24), (4.25), and (4.26).

\section{AC CONDUCTIVITY AND ELECTRON MOBILITY}

Consider now the case when the electric field $\mathbf{E}(\mathbf{r}, t)$ and the mean electron velocity $\mathbf{u}_{e}(\mathbf{r}, t)$ vary harmonically in time, as $\exp (-i \omega t)$. We have seen that for time harmonic disturbances $\partial / \partial t$ is replaced by $-i \omega$. Therefore, the linearized Langevin equation (3.4) becomes

$$
-i \omega m_{e} \mathbf{u}_{e}=-e\left(\mathbf{E}+\mathbf{u}_{e} \times \mathbf{B}_{0}\right)-m_{e} \nu_{c} \mathbf{u}_{e}
$$

which can be written as

$$
-e\left(\mathbf{E}+\mathbf{u}_{e} \times \mathbf{B}_{0}\right)-m_{e}\left(\nu_{c}-i \omega\right) \mathbf{u}_{e}=0
$$

This equation is identical to (4.7), except for the change in the collision frequency $\nu_{c}$ to $\left(\nu_{c}-i \omega\right)$. We obtain, therefore, solutions similar to the ones 


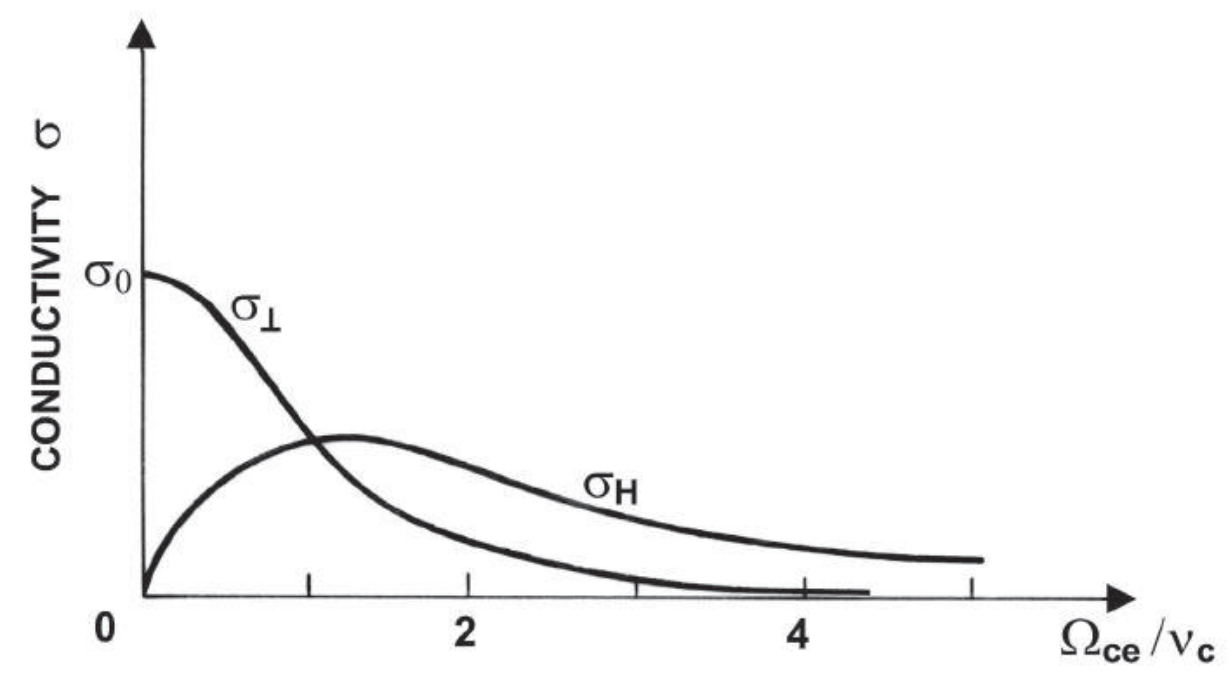

Fig. 3 Dependence of the Hall conductivity $\sigma_{H}$ and of the perpendicular conductivity $\sigma_{\perp}$ on the ratio of the cyclotron frequency $\Omega_{c e}$ to the collision frequency $\nu_{c}$.

obtained for the DC conductivity dyad in the previous section, except that now we must replace $\nu_{c}$ by $\left(\nu_{c}-i \omega\right)$ in each element of the dyad. Therefore, the expressions for the frequency-dependent perpendicular conductivity, Hall conductivity, and longitudinal conductivity are, respectively,

$$
\begin{gathered}
\sigma_{\perp}=\frac{\left(\nu_{c}-i \omega\right)^{2}}{\left(\nu_{c}-i \omega\right)^{2}+\Omega_{c e}^{2}} \sigma_{0} \\
\sigma_{H}=\frac{\left(\nu_{c}-i \omega\right) \Omega_{c e}}{\left(\nu_{c}-i \omega\right)^{2}+\Omega_{c e}^{2}} \sigma_{0} \\
\sigma_{0}=\frac{n_{e} e^{2}}{m_{e}\left(\nu_{c}-i \omega\right)}=\frac{n_{e} e^{2}\left(\nu_{c}+i \omega\right)}{m_{e}\left(\nu_{c}^{2}+\omega^{2}\right)}
\end{gathered}
$$

When the electron-neutral collisions can be neglected $\left(\nu_{c}=0\right)$, the expressions for the components of the $\mathrm{AC}$ (alternating current) conductivity dyad become

$$
\begin{aligned}
\sigma_{\perp} & =\frac{\omega^{2}}{\left(\omega^{2}-\Omega_{c e}^{2}\right)} \sigma_{0} \\
\sigma_{H} & =\frac{i \omega \Omega_{c e}}{\left(\omega^{2}-\Omega_{c e}^{2}\right)} \sigma_{0}
\end{aligned}
$$




$$
\sigma_{0}=i \frac{n_{e} e^{2}}{m_{e} \omega}
$$

A complex conductivity means that there is a phase difference between the current density and the applied electric field.

The electron mobility, in any of the cases considered in this section, can be easily written down considering the relation (4.28).

\section{CONDUCTIVITY WITH ION MOTION}

The evaluation of the conductivity dyad, when the contribution due to the motion of the ions is included, can be performed in a straightforward way. For this purpose, consider the linearized Langevin equation for the type $\alpha$ species,

$$
m_{\alpha} \frac{\partial \mathbf{u}_{\alpha}}{\partial t}=q_{\alpha}\left(\mathbf{E}+\mathbf{u}_{\alpha} \times \mathbf{B}_{0}\right)-m_{\alpha} \nu_{c \alpha} \mathbf{u}_{\alpha}
$$

where $\nu_{c \alpha}$ is an effective collision frequency or damping term for the type $\alpha$ species resulting from collisions with neutral particles. Note that the Langevin equations, for each charged particle species, are uncoupled. Therefore, the net current density is given by

$$
\mathbf{J}=\sum_{\alpha} n_{\alpha} q_{\alpha} \mathbf{u}_{\alpha}=\sum_{\alpha} \mathbf{J}_{\alpha}=\left(\sum_{\alpha} \mathcal{S}_{\alpha}\right) \cdot \mathbf{E}
$$

and the total conductivity dyad is simply

$$
\mathcal{S}=\sum_{\alpha} \mathcal{S}_{\alpha}
$$

For a plasma with electrons and several types of ions (index $j$ ) we obtain, using (5.3), (5.4), and (5.5), in terms of the plasma frequency $\omega_{p \alpha}$ and $\epsilon_{0}$,

$$
\begin{gathered}
\sigma_{\perp}=\epsilon_{0}\left[\frac{\omega_{p e}^{2}\left(\nu_{c e}-i \omega\right)}{\left(\nu_{c e}-i \omega\right)^{2}+\Omega_{c e}^{2}}+\sum_{j} \frac{\omega_{p j}^{2}\left(\nu_{c j}-i \omega\right)}{\left(\nu_{c j}-i \omega\right)^{2}+\Omega_{c j}^{2}}\right] \\
\sigma_{H}=\epsilon_{0}\left[\frac{\omega_{p e}^{2} \Omega_{c e}}{\left(\nu_{c e}-i \omega\right)^{2}+\Omega_{c e}^{2}}-\sum_{j} \frac{\omega_{p j}^{2} \Omega_{c j}}{\left(\nu_{c j}-i \omega\right)^{2}+\Omega_{c j}^{2}}\right]
\end{gathered}
$$




$$
\sigma_{\|}=\epsilon_{0}\left[\frac{\omega_{p e}^{2}}{\left(\nu_{c e}-i \omega\right)}+\sum_{j} \frac{\omega_{p j}^{2}}{\left(\nu_{c j}-i \omega\right)}\right]
$$

\section{PLASMA AS A DIELECTRIC MEDIUM}

The plasma can also be treated as a dielectric medium characterized by a dielectric dyad, in which the internal particle behavior is not considered. So far, we have treated the plasma as a collection of charged and neutral particles moving about in their own internal fields. Thus, as far as the constitutive relations are concerned, we have taken

$$
\begin{aligned}
\mathbf{D} & =\epsilon_{0} \mathbf{E} \\
\mathbf{B} & =\mu_{0} \mathbf{H}
\end{aligned}
$$

which are applicable for free space, and the plasma effects show up through the motion and interaction of the charged particles inside the plasma.

A different approach is provided by the use of a dielectric dyad, in which we are concerned only with the gross macroscopic properties of the plasma and not with the elementary particle motions. Thus, instead of the Langevin equation, let us consider the following Maxwell equation,

$$
\nabla \times \mathbf{B}=\mu_{0}\left(\mathbf{J}+\epsilon_{0} \frac{\partial \mathbf{E}}{\partial t}\right)
$$

and incorporate the effects of the plasma in the conductivity dyad $\mathcal{S}$, defined by the equation

$$
\mathbf{J}=\mathcal{S} \cdot \mathbf{E}
$$

Substituting (7.4) into (7.3), and assuming time-harmonic variations of the form $\exp (-i \omega t)$, we obtain

$$
\nabla \times \mathbf{B}=\mu_{0} \mathcal{S} \cdot \mathbf{E}-i \omega \mu_{0} \epsilon_{0} \mathbf{E}
$$

If we let 1 denote the unit dyad, we can write

$$
\nabla \times \mathbf{B}=-i \omega \mu_{0} \epsilon_{0}\left(\mathbf{1}+\frac{i \mathcal{S}}{\omega \epsilon_{0}}\right) \cdot \mathbf{E}
$$

or, equivalently, as

$$
\nabla \times \mathbf{B}=-i \omega \mu_{0} \mathcal{E} \cdot \mathbf{E}
$$


where

$$
\mathcal{E}=\epsilon_{0}\left(\mathbf{1}+\frac{i \mathcal{S}}{\omega \epsilon_{0}}\right)
$$

is called the dielectric dyad for the plasma. The use of the dielectric dyad represents, therefore, a different approach for the treatment of a plasma, as compared to the one we have used so far. Adopting this approach, (7.1) must be replaced by

$$
\mathbf{D}=\mathcal{E} \cdot \mathbf{E}
$$

and the plasma is considered as a dielectric medium, without bringing into the picture its internal particle behavior. Note that $\mathcal{E}$ depends on the frequency $\omega$.

The dielectric dyad can be written in matrix form as

$$
\mathcal{E}=\epsilon_{0}\left(\begin{array}{ccc}
\epsilon_{1} & -\epsilon_{2} & 0 \\
\epsilon_{2} & \epsilon_{1} & 0 \\
0 & 0 & \epsilon_{3}
\end{array}\right)
$$

where the following notation was introduced:

$$
\begin{gathered}
\epsilon_{1}=1+\frac{i}{\omega \epsilon_{0}} \sigma_{\perp} \\
\epsilon_{2}=\frac{i}{\omega \epsilon_{0}} \sigma_{H} \\
\epsilon_{3}=1+\frac{i}{\omega \epsilon_{0}} \sigma_{0}
\end{gathered}
$$

For the case of a multispecies plasma the total conductivity must be used in (7.8), so that the expressions to be substituted for $\sigma_{\perp}, \sigma_{H}$, and $\sigma_{0}$ are those given in (6.4), (6.5), and (6.6).

\section{FREE ELECTRON DIFFUSION}

The presence of a pressure gradient term in the momentum transport equation provides a force that tends to smooth out any inhomogeneities in the plasma density. The diffusion of particles in a plasma results from this pressure gradient force. To deduce the expression for the electron diffusion coefficient for a warm weakly ionized plasma we will use the momentum transport equation for the electrons, with a constant electron-neutral collision frequency. We assume that the deviations from the equilibrium state 
caused by the inhomogeneities in the density are very small, so that they may be considered as first-order quantities. This means that the mean velocity of the electrons $\mathbf{u}_{e}$ is also a first-order quantity, and considering that the velocity distribution is approximately isotropic, we can replace the pressure dyad $\mathcal{P}_{e}$ by a scalar pressure $p_{e}$.

Consider the case in which $\mathbf{E}$ and $\mathbf{B}$ are zero and the electron temperature $T_{e}$ is constant. For a slightly nonuniform electron number density, we can write

$$
\begin{gathered}
n_{e}(\mathbf{r}, t)=n_{0}+n_{e}^{\prime}(\mathbf{r}, t) \\
p_{e}(\mathbf{r}, t)=n_{e}(\mathbf{r}, t) k T_{e}=\left(n_{0}+n_{e}^{\prime}\right) k T_{e}
\end{gathered}
$$

where $\left|n_{e}^{\prime}\right| \ll n_{0}$ is a first-order quantity and $n_{0}$ is constant. Since $\mathbf{u}_{e}$ is also a first-order quantity, the continuity equation for the electron gas becomes

$$
\frac{\partial n_{e}^{\prime}}{\partial t}+n_{0} \nabla \cdot \mathbf{u}_{e}=0
$$

where the second-order term $n_{e}^{\prime} \mathbf{u}_{e}$ has been neglected. Similarly, for the momentum transport equation,

$$
n_{e} m_{e}\left[\frac{\partial \mathbf{u}_{e}}{\partial t}+\left(\mathbf{u}_{e} \cdot \nabla\right) \mathbf{u}_{e}\right]=-\nabla p_{e}-n_{e} m_{e} \nu_{c} \mathbf{u}_{e}
$$

we obtain, after linearization,

$$
n_{0} \frac{\partial \mathbf{u}_{e}}{\partial t}=-\frac{k T_{e}}{m_{e}} \nabla n_{e}^{\prime}-n_{0} \nu_{c} \mathbf{u}_{e}
$$

Taking the divergence of this equation, we obtain

$$
n_{0} \frac{\partial\left(\nabla \cdot \mathbf{u}_{e}\right)}{\partial t}=-\frac{k T_{e}}{m_{e}} \nabla^{2} n_{e}^{\prime}-n_{0} \nu_{c} \nabla \cdot \mathbf{u}_{e}
$$

Using (8.3) to substitute for $n_{0} \nabla \cdot \mathbf{u}_{e}$, yields

$$
\frac{\partial^{2} n_{e}^{\prime}}{\partial t^{2}}=\frac{k T_{e}}{m_{e}} \nabla^{2} n_{e}^{\prime}-\nu_{c} \frac{\partial n_{e}^{\prime}}{\partial t}
$$

This equation may also be written in the form

$$
\frac{\partial n_{e}^{\prime}}{\partial t}=D_{e} \nabla^{2} n_{e}^{\prime}-\frac{1}{\nu_{c}} \frac{\partial^{2} n_{e}^{\prime}}{\partial t^{2}}
$$


where we have defined

$$
D_{e}=\frac{k T_{e}}{m_{e} \nu_{c}}
$$

which is called the electron free-diffusion coefficient.

To obtain a rough estimate of the order of magnitude of the various terms in (8.8), let $\tau$ and $L$ represent, respectively, a characteristic time and a characteristic length over which $n_{e}^{\prime}$ varies significantly. Thus, any spatial derivative is of the order of $L^{-1}$ and any time derivative is of the order $\tau^{-1}$, so that the order of magnitude of the terms in (8.8) are

$$
\begin{gathered}
\frac{\partial n_{e}^{\prime}}{\partial t} \sim \frac{n_{e}^{\prime}}{\tau} \\
D_{e} \nabla^{2} n_{e}^{\prime} \sim D_{e} \frac{n_{e}^{\prime}}{L^{2}} \\
\frac{1}{\nu_{c}} \frac{\partial^{2} n_{e}^{\prime}}{\partial t^{2}} \sim \frac{n_{e}^{\prime}}{\nu_{c} \tau^{2}}
\end{gathered}
$$

Comparing (8.10) and (8.12) we see that if $\nu_{c} \tau \gg 1$, that is, if the average number of electron-neutral collisions is large during the time interval $\tau$, then the last term in (8.8) can be neglected and it reduces to the following diffusion equation:

$$
\frac{\partial n_{e}^{\prime}}{\partial t}=D_{e} \nabla^{2} n_{e}^{\prime}
$$

Therefore, when the rate of change in the number density is slow compared to the collision frequency, the number density of the electrons is governed by a diffusion equation with a free-diffusion coefficient as given by (8.9).

The condition $\nu_{c} \tau \gg 1$ implies in the omission of the acceleration term in the momentum transport equation, that is, $\partial \mathbf{u}_{e} / \partial t$ is neglected. From the linearized equation (8.5), when there are no time variations in $\mathbf{u}_{e}$, we obtain

$$
n_{0} \nu_{c} \mathbf{u}_{e}=-\frac{k T_{e}}{m_{e}} \nabla n_{e}^{\prime}
$$

which can be written as

$$
\boldsymbol{\Gamma}_{e}=-D_{e} \nabla n_{e}^{\prime}
$$

where $\boldsymbol{\Gamma}_{e}=n_{0} \mathbf{u}_{e}$ denotes the linearized electron flux.

Expression (8.15) is analogous to the simple Ohm's law $\mathbf{J}=\sigma_{0} \mathbf{E}$, replacing $\mathbf{J}$ by $\boldsymbol{\Gamma}_{e}, \sigma_{0}$ by $D_{e}$, and $\mathbf{E}$ by $-\nabla n_{e}^{\prime}$. Thus, we see that the electron flux $\boldsymbol{\Gamma}_{e}$ is caused by a density gradient, in a way analogous to the 
electric current caused by an electric field, under steady-state conditions for $\mathbf{u}_{e}$.

\section{ELECTRON DIFFUSION IN A MAGNETIC FIELD}

Consider now the problem of electron diffusion in the presence of a constant and uniform magnetic field $B_{0}$. We shall make the same assumptions as in the previous section and neglect the acceleration term $\partial \mathbf{u}_{e} / \partial t$ in the equation of motion. In the linearized momentum transport equation (8.5), with the time derivative set equal to zero, we include now a magnetic force term, which results in

$$
\boldsymbol{\Gamma}_{e}=-D_{e} \nabla n_{e}^{\prime}-\frac{e}{m_{e} \nu_{c}}\left(\boldsymbol{\Gamma}_{e} \times \mathbf{B}_{0}\right)
$$

Choosing a Cartesian coordinate system with the $z$ axis pointing in the direction of the constant $\mathbf{B}_{0}$ field, that is, $\mathbf{B}_{0}=B_{0} \widehat{\mathbf{z}}$, we have

$$
\boldsymbol{\Gamma}_{e}=-D_{e} \nabla n_{e}^{\prime}-\frac{\Omega_{c e}}{\nu_{c}}\left(\boldsymbol{\Gamma}_{e} \times \widehat{\mathbf{z}}\right)
$$

This equation is analogous to (4.15), with $\boldsymbol{\Gamma}_{e}$ replacing $\mathbf{J}, D_{e}$ replacing $\sigma_{0}$, and $-\nabla n_{e}^{\prime}$ replacing $\mathbf{E}$. Note that $\Omega_{c e} / \nu_{c}=\sigma_{0} B_{0} /\left(e n_{e}\right)$. Therefore, in analogy with the expression $\mathbf{J}=\mathcal{S} \cdot \mathbf{E}$, we can write

$$
\Gamma_{e}=-\mathcal{D} \cdot \nabla n_{e}^{\prime}
$$

where $\mathcal{D}$ is the dyad coefficient for free-diffusion given in matrix form by

$$
\mathcal{D}=\left(\begin{array}{ccc}
D_{\perp} & D_{H} & 0 \\
-D_{H} & D_{\perp} & 0 \\
0 & 0 & D_{\|}
\end{array}\right)
$$

where the following notation is used:

$$
\begin{gathered}
D_{\perp}=\frac{\nu_{c}^{2}}{\nu_{c}^{2}+\Omega_{c e}^{2}} D_{e} \\
D_{H}=\frac{\nu_{c} \Omega_{c e}}{\nu_{c}^{2}+\Omega_{c e}^{2}} D_{e} \\
D_{\|} \equiv D_{e}=\frac{k T_{e}}{m_{e} \nu_{c}}
\end{gathered}
$$


A diffusion equation for $n_{e}^{\prime}$, when there is a constant and uniform magnetic field present, can also be derived in the same way as in the previous section. First, we write the continuity equation (8.3) in the form

$$
\frac{\partial n_{e}^{\prime}}{\partial t}+\nabla \cdot \Gamma_{e}=0
$$

Substituting (9.3) for $\boldsymbol{\Gamma}_{e}$, yields

$$
\frac{\partial n_{e}^{\prime}}{\partial t}=\nabla \cdot\left(\mathcal{D} \cdot \nabla n_{e}^{\prime}\right)
$$

Using (9.4) we find, by direct calculation in Cartesian coordinates,

$$
\begin{aligned}
& \mathcal{D} \cdot \nabla n_{e}^{\prime}=\widehat{\mathbf{x}}\left(D_{\perp} \frac{\partial n_{e}^{\prime}}{\partial x}+D_{H} \frac{\partial n_{e}^{\prime}}{\partial y}\right)+ \\
& \widehat{\mathbf{y}}\left(-D_{H} \frac{\partial n_{e}^{\prime}}{\partial x}+D_{\perp} \frac{\partial n_{e}^{\prime}}{\partial y}\right)+\widehat{\mathbf{z}} D_{e} \frac{\partial n_{e}^{\prime}}{\partial z}
\end{aligned}
$$

Substituting this result into (9.9), yields

$$
\frac{\partial n_{e}^{\prime}}{\partial t}=D_{\perp}\left(\frac{\partial^{2} n_{e}^{\prime}}{\partial x^{2}}+\frac{\partial^{2} n_{e}^{\prime}}{\partial y^{2}}\right)+D_{e} \frac{\partial^{2} n_{e}^{\prime}}{\partial z^{2}}
$$

Since $D_{\perp}<D_{e}$ and since $D_{\perp}$ decreases with increasing values of $\Omega_{c e} / \nu_{c}$ (similarly to $\sigma_{\perp}$, as shown in Fig. 3) the diffusion of particles in a direction perpendicular to $\mathbf{B}$ is always less than that in the direction parallel to $\mathbf{B}$. For values of $\Omega_{c e}$ much larger than $\nu_{c}$, the diffusion of particles across the magnetic field lines is greatly reduced. Note that, from (9.5) and (9.6), it can be seen that for $\Omega_{c e} \gg \nu_{c}$ we have, approximately, $D_{\perp} \propto 1 / B^{2}$ and $D_{H} \propto 1 / B$.

As a final point in this section we note that the momentum transport equation for an electron gas, neglecting the acceleration term but including the electromagnetic force, and when the temperature is constant, can be written in the general form

$$
\boldsymbol{\Gamma}_{e}=\mathcal{M}_{e}\left(n_{e} \mathbf{E}+\boldsymbol{\Gamma}_{e} \times \mathbf{B}\right)-D_{e} \nabla n_{e}
$$

From this equation we can see that the electron flux is produced by either electromagnetic fields or density gradients, or both. The ratio of the scalar mobility $\mathcal{M}_{e}$ to the diffusion coefficient is known as the Einstein relation and is given by

$$
\frac{\mathcal{M}_{e}}{D_{e}}=-\frac{e}{k T_{e}}
$$




\section{AMBIPOLAR DIFFUSION}

We have seen in section 8 that the steady-state momentum equation, in the absence of electromagnetic forces and when the temperature is constant, gives the following diffusion equation for the electrons:

$$
\Gamma_{e}=-D_{e} \nabla n_{e}^{\prime}
$$

where the electron free-diffusion coefficient is given by

$$
D_{e}=\frac{k T_{e}}{m_{e} \nu_{c e}}
$$

The subscript $e$ has been added here to $\nu_{c}$ to indicate that the effective collision frequency $\nu_{c e}$ refers to electron-neutral collisions.

If we consider similar equations for the ions in a weakly ionized plasma, under the same assumptions, we obtain the following ion diffusion equation:

$$
\boldsymbol{\Gamma}_{i}=-D_{i} \nabla n_{i}^{\prime}
$$

where

$$
D_{i}=\frac{k T_{i}}{m_{i} \nu_{c i}}
$$

denotes the ion free-diffusion coefficient and $\nu_{c i}$ is the effective ion-neutral collision frequency.

In deriving the results given by (10.1) and (10.3), the interaction between the electrons and the ions were not taken into account. Since the diffusion coefficient is inversely proportional to the particle mass, the electrons tend to diffuse faster than the ions, leaving an excess of positive charge behind them. This gives rise to a space charge electric field, which points in the same direction as the particle diffusion, and which accelerates the diffusion of the ions and slows down that of the electrons. The diffusion in which the effect of this space charge electric field is not included is known as free-diffusion.

For most problems of plasma diffusion, however, the space charge electric field cannot be neglected. According to Maxwell equation

$$
\nabla \cdot \mathbf{E}=\frac{\rho}{\epsilon_{0}}=\frac{e\left(n_{i}-n_{e}\right)}{\epsilon_{0}}
$$

it is clear that an electric field is present whenever the electron density differs from the ion density. To estimate the importance of the space 
charge electric field in diffusion problems, let us use dimensional analysis and let $L$ represent a characteristic length over which the number density changes significantly. Thus, from (10.5) we may write

$$
E \sim \frac{e n L}{\epsilon_{0}}
$$

so that the electric force per unit mass $f_{E}$ is of the order

$$
f_{E}=\frac{e E}{m} \sim \frac{e^{2} n L}{m \epsilon_{0}}
$$

The diffusion force per unit mass $f_{D}$, obtained from (10.1), is of the order

$$
f_{D}=\frac{k T}{m n_{0}}|\nabla n| \sim \frac{k T n}{m n_{0} L}
$$

Therefore, the space charge electric field can be neglected only if $f_{E} \ll f_{D}$, or equivalently, if

$$
L^{2} \ll \frac{\epsilon_{0} k T}{n_{0} e^{2}}=\lambda_{D}^{2}
$$

where $\lambda_{D}$ is the Debye length. Since the Debye length is generally very small (see Fig. 2, Chapter 1), the condition $L \ll \lambda_{D}$ is rarely satisfied and for most plasma diffusion problems we cannot neglect the space charge electric field. In what follows we will reexamine, therefore, the problem of plasma diffusion, taking into account the motion of both ions and electrons and including the space charge electric field $\mathbf{E}$. The combined diffusion of the electrons and the ions, forced by the space charge $\mathbf{E}$ field, is known as ambipolar diffusion. Since the electric field is such that the electrons are decelerated and the ions are accelerated, the two kinds of charged particles tend to reach a diffusion rate that is intermediate in value to their free diffusion rates.

To investigate the characteristics of ambipolar diffusion, we assume that the variations for both electrons and ions are small first-order quantities, so that (for $\alpha=e, i$ )

$$
n_{\alpha}(\mathbf{r}, t)=n_{0}+n_{\alpha}^{\prime}(\mathbf{r}, t)
$$

with $\left|n_{\alpha}^{\prime}\right| \ll n_{0}$, and that the mean velocities $\mathbf{u}_{\alpha}$ are of very small amplitude. We obtain, under these assumptions, the following linearized mass conservation equations (for $\alpha=e, i$ ),

$$
\frac{\partial n_{\alpha}^{\prime}}{\partial t}+n_{0} \nabla \cdot \mathbf{u}_{\alpha}=0
$$


The linearized momentum equations, assuming that the temperatures are constant, and without a magnetic field, become (with $\alpha=e, i$ )

$$
\frac{\partial \mathbf{u}_{\alpha}}{\partial t}=\frac{q_{\alpha}}{m_{\alpha}} \mathbf{E}-\frac{k T_{\alpha}}{m_{\alpha} n_{0}} \nabla n_{\alpha}^{\prime}-\nu_{c \alpha} \mathbf{u}_{\alpha}
$$

where the space charge $\mathbf{E}$ field satisfies Maxwell equation (10.5). We are assuming that the neutral mean velocity is zero and we are neglecting electron-ion collisions, since the plasma is weakly ionized. Taking the divergence of (10.12) and using (10.11), we obtain

$$
\frac{\partial^{2} n_{\alpha}^{\prime}}{\partial t^{2}}=-\frac{q_{\alpha} n_{0}}{m_{\alpha}}(\nabla \cdot \mathbf{E})+\frac{k T_{\alpha}}{m_{\alpha}} \nabla^{2} n_{\alpha}^{\prime}-\nu_{c \alpha} \frac{\partial n_{\alpha}^{\prime}}{\partial t}
$$

If we replace $\nabla \cdot \mathbf{E}$ from (10.5), we obtain the following set of coupled equations for the two variables $n_{e}^{\prime}$ and $n_{i}^{\prime}$,

$$
\begin{aligned}
& \frac{\partial^{2} n_{e}^{\prime}}{\partial t^{2}}=\omega_{p e}^{2}\left(n_{i}^{\prime}-n_{e}^{\prime}\right)+\frac{k T_{e}}{m_{e}} \nabla^{2} n_{e}^{\prime}-\nu_{c e} \frac{\partial n_{e}^{\prime}}{\partial t} \\
& \frac{\partial^{2} n_{i}^{\prime}}{\partial t^{2}}=-\omega_{p i}^{2}\left(n_{i}^{\prime}-n_{e}^{\prime}\right)+\frac{k T_{i}}{m_{i}} \nabla^{2} n_{i}^{\prime}-\nu_{c i} \frac{\partial n_{i}^{\prime}}{\partial t}
\end{aligned}
$$

These equations, however, are still too complicated for a detailed analytical treatment, and to go further we will make some additional simplifying assumptions.

Recall that, if $\nu_{c} \tau \gg 1$, that is, if the average electron or ion has many collisions with neutral particles during the characteristic time for diffusion $\tau$, the term $\partial^{2} n_{\alpha}^{\prime} / \partial t^{2}$ (originated from the acceleration term in the momentum equation) can be neglected. With this assumption we neglect the term in the left-hand side of (10.14) and (10.15). Combining these equations, we obtain

$$
k T_{e} \nabla^{2} n_{e}^{\prime}+k T_{i} \nabla^{2} n_{i}^{\prime}-m_{e} \nu_{c e} \frac{\partial n_{e}^{\prime}}{\partial t}-m_{i} \nu_{c i} \frac{\partial n_{i}^{\prime}}{\partial t}=0
$$

As a second approximation we will set $n_{e}^{\prime}=n_{i}^{\prime}=n^{\prime}$ in (10.16) to obtain the following diffusion equation,

$$
k\left(T_{e}+T_{i}\right) \nabla^{2} n^{\prime}-\left(m_{e} \nu_{c e}+m_{i} \nu_{c i}\right) \frac{\partial n^{\prime}}{\partial t}=0
$$


which can be written in the form

$$
\frac{\partial n^{\prime}}{\partial t}=D_{a} \nabla^{2} n^{\prime}
$$

where

$$
D_{a}=\frac{k\left(T_{e}+T_{i}\right)}{m_{e} \nu_{c e}+m_{i} \nu_{c i}}
$$

is the ambipolar diffusion coefficient. Note that the coupling of the two equations (10.14) and (10.15) is a consequence of the electric field term, and that the simplifying approximation $n_{i}^{\prime}=n_{e}^{\prime}$ was introduced only after the two equations were combined into (10.16). This approximation implies that the space charge electric field becomes a negligible perturbation with the result that both ions and electrons diffuse together. This situation is known as perfect ambipolar diffusion, since the coupling between the two types of charged particles is complete.

Instead of taking $n_{i}^{\prime}=n_{e}^{\prime}$, a less restrictive simplifying approximation would be to assume

$$
n_{i}^{\prime}=C n_{e}^{\prime}
$$

where $C$ is a constant. Using this approximation in (10.16) we obtain

$$
k\left(T_{e}+C T_{i}\right) \nabla^{2} n_{e}^{\prime}=\left(m_{e} \nu_{c e}+C m_{i} \nu_{c i}\right) \frac{\partial n_{e}^{\prime}}{\partial t}=0
$$

or

$$
\frac{\partial n_{e}^{\prime}}{\partial t}=D_{a} \nabla^{2} n_{e}^{\prime}
$$

where the ambipolar diffusion coefficient is now given by

$$
D_{a}=\frac{k\left(T_{e}+C T_{i}\right)}{m_{e} \nu_{c e}+C m_{i} \nu_{c i}}
$$

The space charge density is now

$$
\rho=e\left(n_{i}^{\prime}-n_{e}^{\prime}\right)=e n_{e}^{\prime}(C-1)
$$

and the electric field can be obtained from Maxwell equation

$$
\nabla \cdot \mathbf{E}=\frac{e n_{e}^{\prime}(C-1)}{\epsilon_{0}}
$$

The effect of this electric field is to accelerate the diffusion of the ions and to retard the diffusion of the electrons, as compared to their individual 
free-diffusion rates, so that to a good approximation both species diffuse together.

Whenever there is a significant deviation from charge neutrality, the electric field force becomes very strong, as can be seen from the following dimensional argument. A comparison of the magnitude of the electric force per unit mass, $\mathbf{f}_{E}=q_{\alpha} \mathbf{E} / m_{\alpha}$, and the diffusion force per unit mass, $\mathbf{f}_{D}=-\left(k T_{\alpha} / m_{\alpha} n_{0}\right) \nabla n_{\alpha}^{\prime}$, which are of the order

$$
\begin{gathered}
f_{E} \sim \frac{L e^{2} n_{e}^{\prime}(C-1)}{m \epsilon_{0}} \\
f_{D} \sim \frac{k T n_{e}^{\prime}}{m n_{0} L}
\end{gathered}
$$

shows that

$$
\frac{f_{E}}{f_{D}} \sim \frac{L^{2}(C-1)}{\lambda_{D}^{2}}
$$

Since in most cases $L^{2}$ is much larger than $\lambda_{D}^{2}$, we see that if $n_{i}^{\prime}$ differs significantly from $n_{e}^{\prime}$, then the electric field force (which tends to equalize $n_{i}^{\prime}$ and $n_{e}^{\prime}$ ) becomes very strong.

\section{DIFFUSION IN A FULLY IONIZED PLASMA}

Consider now the problem of diffusion in a fully ionized plasma. For simplicity, we shall describe the plasma as a single conducting fluid for which the equation of motion, under steady-state conditions, in the presence of magnetic and pressure gradient forces, is

$$
\mathbf{J} \times \mathbf{B}=\nabla p
$$

where $\mathbf{J}$ denotes the total electric current density, B is the magnetic induction, and $p$ represents the total scalar pressure of the conducting fluid. Note that the electric force is zero, since the plasma, as a whole, is macroscopically neutral $(\rho=0)$. This equation is complemented by the generalized Ohm's law in the following simplified form,

$$
\mathbf{J}=\sigma_{0}(\mathbf{E}+\mathbf{u} \times \mathbf{B})
$$

where $\sigma_{0}$ is the longitudinal electric conductivity and $\mathbf{u}$ is the total macroscopic fluid velocity.

Taking the cross-product of (11.2) with $\mathbf{B}$, yields

$$
\mathbf{J} \times \mathbf{B}=\sigma_{0}\left(\mathbf{E} \times \mathbf{B}-B^{2} \mathbf{u}_{\perp}\right)
$$


where $\mathbf{u}_{\perp}$ is the component of $\mathbf{u}$ in a direction normal to the external field B. Using (11.1) and rearranging, (11.3) gives

$$
\mathbf{u}_{\perp}=\frac{\mathbf{E} \times \mathbf{B}}{B^{2}}-\frac{\nabla p}{\sigma_{0} B^{2}}
$$

This result shows that the total fluid velocity across the magnetic field is given by the $\mathbf{E} \times \mathbf{B}$ drift of the whole plasma plus a diffusion velocity in the direction of $-\nabla p$.

The flux associated with the diffusion velocity only, is given by

$$
\boldsymbol{\Gamma}_{\perp}=n \mathbf{u}_{\perp}=-\frac{n \nabla p}{\sigma_{0} B^{2}}
$$

where $n$ denotes the electron (or total ion) number density. Considering a two-fluid plasma (electrons and one type of ions), we have

$$
p=p_{e}+p_{i}=n k\left(T_{e}+T_{i}\right)
$$

so that (11.5) becomes, assuming the temperatures to be constant,

$$
\Gamma_{\perp}=-\frac{n k\left(T_{e}+T_{i}\right)}{\sigma_{0} B^{2}} \nabla n=-D_{\perp} \nabla n
$$

The quantity

$$
D_{\perp}=\frac{n k\left(T_{e}+T_{i}\right)}{\sigma_{0} B^{2}}
$$

is known as the classical diffusion coefficient for a fully ionized plasma.

This diffusion coefficient is proportional to $1 / B^{2}$, just as in the case of a weakly ionized plasma. Nevertheless, there are some fundamental differences between $D_{\perp}$, as given by (11.8), and the corresponding coefficient for a partially ionized plasma. Initially note that in a fully ionized plasma $D_{\perp}$ is not constant, but depends on the number density $n$. Further, since it can be shown that $\sigma_{0}$ is proportional to $T^{3 / 2}$ for a Maxwellian distribution of velocities, $D_{\perp}$ decreases with increasing temperature in a fully ionized plasma, while the opposite situation holds for a weakly ionized plasma. Finally, the diffusion coefficient $D_{\perp}$ in (11.8) was derived for the whole plasma as a conducting fluid and, since both ions and electrons diffuse together, there is no ambipolar electric field.

In some experiments a dependence of $D_{\perp}$ on the magnetic field as $B^{-1}$, rather than $B^{-2}$, has been observed, and the decay of the plasma was 
found to be exponential, rather than reciprocal, with time. Furthermore, the absolute value of $D_{\perp}$ was found to be much larger than that given in (11.8). This anomalously poor magnetic confinement was first noted in laboratory by Bohm, in 1946, who arrived at the following semiempirical formula:

$$
D_{\perp} \equiv D_{B}=\frac{k T_{e}}{16 e B}
$$

Since this diffusion coefficient does not depend on the density, the decay of the plasma density is exponential with time. This type of diffusion in plasmas is known as Bohm diffusion.

\section{PROBLEMS}

10.1 Consider a solid-state plasma with the same number of electrons $(e)$ and holes $(h)$. Using the linearized Langevin equation (with $\alpha=e, h$ )

$$
m_{\alpha} \frac{\partial \mathbf{u}_{\alpha}}{\partial t}=q_{\alpha}\left(\mathbf{E}+\mathbf{u}_{\alpha} \times \mathbf{B}_{0}\right)-\nu_{c \alpha} m_{\alpha} \mathbf{u}_{\alpha}
$$

taking $m_{e}=m_{h}, \nu_{c e}=\nu_{c h}$, assuming a time dependence for both $\mathbf{E}$ and $\mathbf{u}_{\alpha}$ of the form $\exp (-i \omega t)$, and choosing a Cartesian coordinate system with the $z$ axis pointing along the constant and uniform magnetic field $\mathbf{B}_{0}$, show that the conductivity dyad is given by

$$
\mathcal{S}=2\left(\begin{array}{ccc}
\sigma_{\perp} & 0 & 0 \\
0 & \sigma_{\perp} & 0 \\
0 & 0 & \sigma_{0}
\end{array}\right)
$$

with $\sigma_{\perp}$ and $\sigma_{0}$ given by (5.3) and (5.5), respectively. Explain, in physical terms, why we have $\sigma_{H}=0$ in this case.

10.2 Assume that the average velocities of the electrons and ions in a completely ionized plasma, in the presence of constant and uniform electric (E) and magnetic $\left(\mathbf{B}_{0}\right)$ fields, satisfy, respectively, the following equations of motion:

$$
\begin{aligned}
m_{e} \frac{\partial \mathbf{u}_{e}}{\partial t} & =-e\left(\mathbf{E}+\mathbf{u}_{e} \times \mathbf{B}_{0}\right)-m_{e} \nu\left(\mathbf{u}_{e}-\mathbf{u}_{i}\right) \\
m_{i} \frac{\partial \mathbf{u}_{i}}{\partial t} & =e\left(\mathbf{E}+\mathbf{u}_{i} \times \mathbf{B}_{0}\right)-m_{e} \nu\left(\mathbf{u}_{i}-\mathbf{u}_{e}\right)
\end{aligned}
$$


(a) Determine expressions for the steady-state DC conductivities $\sigma_{H}, \sigma_{\perp}$, and $\sigma_{0}$.

(b) For $\mathbf{u}_{e}, \mathbf{u}_{i}$, and $\mathbf{E}$ all proportional to $\exp (-i \omega t)$ and $\mathbf{B}_{0}$ constant, calculate the AC conductivity dyad for the plasma.

10.3 Consider the equation $\mathbf{J}=\mathcal{S} \cdot \mathbf{E}$, with $\mathcal{S}$ as given in (4.23). If we choose a Cartesian coordinate system such that $E_{x}=E_{\perp}, E_{y}=0$, $E_{z}=E_{\|}$, and $\mathbf{B}_{0}=B_{0} \widehat{\mathbf{z}}$ (refer to Fig. 2), verify that in this coordinate system we have

$$
\begin{gathered}
J_{x}=\sigma_{\perp} E_{\perp} \\
J_{y}=\sigma_{H} E_{\perp} \\
J_{z}=\sigma_{\|} E_{\|}
\end{gathered}
$$

Interpret physically this result with reference to Fig. 2.

10.4 What is the physical meaning of a complex conductivity, as given in (5.7) and (5.8)? Consider, for example, that $\mathbf{E}(\mathbf{r}, t)=\mathbf{E}(\mathbf{r}) \exp (-i \omega t)$, and calculate the real parts of $\mathbf{E}(\mathbf{r}, t)$ and of $\mathbf{J}(\mathbf{r}, t)=\mathcal{S} \cdot \mathbf{E}(\mathbf{r}, t)$. Interpret physically the results considering the phase differences between $\mathbf{J}$ and $\mathbf{E}$.

10.5 Write expressions for the components of the dielectric dyad $\mathcal{E}$ of a multiconstituent magnetized plasma.

10.6 Consider the electrons in a plasma acted upon by a small, constant, and uniform external electric field E. Under steady-state conditions with no spatial gradients, obtain an expression for the nonequilibrium distribution function $f$ for the electrons, by applying a perturbation technique to the Boltzmann equation (take $f=f_{0}+f_{1}$ with $\left|f_{1}\right| \ll f_{0}$ and neglect all second-order terms), using the relaxation model for the collision term

$$
\left(\frac{\delta f}{\delta t}\right)_{\text {coll }}=-\nu\left(f-f_{0}\right)
$$

where $\nu$ is a relaxation collision frequency and $f_{0}$ is the equilibrium Maxwellian distribution function. Assuming that $\nu$ is independent of velocity, obtain an expression for the electric conductivity $\sigma_{0}$ of the plasma, by taking $\mathbf{J}=\sigma_{0} \mathbf{E}$.

10.7 Same as problem 10.6, but including also a constant and uniform magnetic field $\mathbf{B}_{0}$. 
10.8 Imagine a horizontally stratified ionosphere in the absence of a magnetic field, constituted only of electrons (density $n$, temperature $T$, charge $-e$, mass $m_{e}$ ) and one type of ions (density $n$, temperature $T$, charge $+e$, mass $\left.m_{i}\right)$, subjected to the gravitational field $(\mathbf{g})$, vertical pressure gradient $(\nabla p)$, and the internal electric field $(\mathbf{E})$ due to the charge separation associated with ambipolar diffusion. Neglect the gravitational force for the electrons and consider the system in equilibrium. Using the collisionless equations of motion for the electrons and the ions, show that the internal electric field acts downward on the electrons with a force $m_{i} g / 2$, and upward on the ions with the same force. Consequently, the net effect is the same as if both ions and electrons had mass $m_{i} / 2$.

10.9 (a) In order to solve the diffusion equation

$$
\frac{\partial n(\mathbf{r}, t)}{\partial t}=D \nabla^{2} n(\mathbf{r}, t)
$$

by the method of separation of variables, let

$$
n(\mathbf{r}, t)=S(\mathbf{r}) T(t)
$$

and show that

$$
\begin{gathered}
T_{k}(t)=T_{0} \exp \left(-D k^{2} t\right) \\
\left(\nabla^{2}+k^{2}\right) S(\mathbf{r})=0
\end{gathered}
$$

where $k^{2}$ is the separation constant and $T_{0}$ is a constant.

(b) Assuming that $S$ depends only on the $x$ coordinate, show that

$$
S(x)=c(k) \exp (i k x)
$$

where $k$ can be either positive or negative, and that

$$
\begin{gathered}
n(x, t)=\int_{-\infty}^{+\infty} c(k) \exp \left(i k x-D k^{2} t\right) d k \\
n_{0}(x)=\int_{-\infty}^{+\infty} c(k) \exp (i k x) d k
\end{gathered}
$$

where $n_{0}(x)=n(x, 0)$ is the known initial density distribution.

(c) Using Fourier transform theory, show that

$$
c(k)=\frac{1}{2 \pi} \int_{-\infty}^{+\infty} n_{0}(x) \exp (-i k x) d x
$$


and, consequently, that

$$
n(x, t)=\frac{1}{2(\pi D t)^{1 / 2}} \int_{-\infty}^{+\infty} n_{0}\left(x^{\prime}\right) \exp \left[-\frac{\left(x-x^{\prime}\right)^{2}}{4 D t}\right] d x^{\prime}
$$

(d) Taking as initial condition

$$
n_{0}(x)=\exp \left(-x^{2} / x_{0}^{2}\right)
$$

show that

$$
n(x, t)=\left(\frac{\tau_{D}}{\tau_{D}+4 t}\right)^{1 / 2} \exp \left[-\frac{x^{2}}{x_{0}^{2}}\left(\frac{\tau_{D}}{\tau_{D}+4 t}\right)\right]
$$

where $\tau_{D}=x_{0}^{2} / D$ is a characteristic time for diffusion to smooth out the density $n$.

(e) Generalize the problem for the three-dimensional case in Cartesian coordinates, when $\mathrm{S}=\mathrm{S}(\mathbf{r})$.

10.10 Consider the solution of the diffusion equation by separation of variables in the linear geometry of the plasma slab indicated in Fig. 4. Show that the solutions of the equation

$$
\frac{d^{2} S(x)}{d x^{2}}+k^{2} S(x)=0
$$

that satisfy the boundary condition $S=0$ at $x= \pm L$, are

$$
S(x)=\sum_{m} a_{m} \cos [(m+1 / 2) \pi x / L]
$$

and

$$
S(x)=\sum_{m} b_{m} \sin (m \pi x / L)
$$

Explain why the solution as a sine series is not a physically acceptable solution for this case. Consequently, from $n(x, t)=S(x) T(t)$, show that the number density can be expressed as

$$
n(x, t)=\sum_{m} a_{m} \exp \left[-D \pi^{2}(m+1 / 2)^{2} t / L^{2}\right] \cos [\pi(m+1 / 2) x / L]
$$




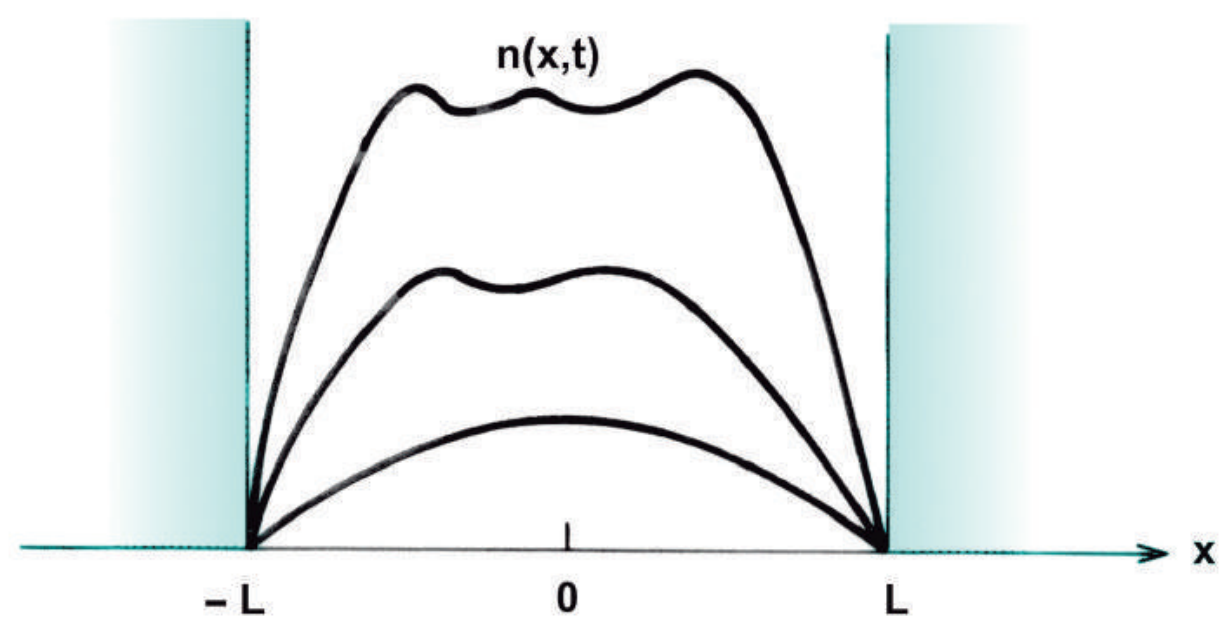

Fig. 4 Geometry of the plasma slab for the solution of the diffusion equation considered in problem 10.10.

Therefore, the decay time constant for the $m^{\text {th }}$ mode is

$$
\tau_{m}=\left[\frac{L}{\pi(m+1 / 2)}\right]^{2} \frac{1}{D}
$$

This result shows that the higher modes decay faster than the lower ones. How are the coefficients $a_{m}$ determined in terms of $n_{0}(x)$ ?

10.11 Show that the solution of the diffusion equation in the case of cylindrical geometry (see Fig. 5),

$$
\frac{d^{2} S(r)}{d r^{2}}+\frac{1}{r} \frac{d S(r)}{d r}+k^{2} S(r)=0
$$

can be written in terms of Bessel's functions $J_{m}(k r)$. Explain how $k$ must be determined so that $n(\mathbf{r}, t)$ satisfies the boundary condition $n=0$ at $r=R_{0}$.

10.12 Verify that plane wave solutions to the diffusion equation

$$
\frac{\partial n(\mathbf{r}, t)}{\partial t}=D \nabla^{2} n(\mathbf{r}, t)
$$




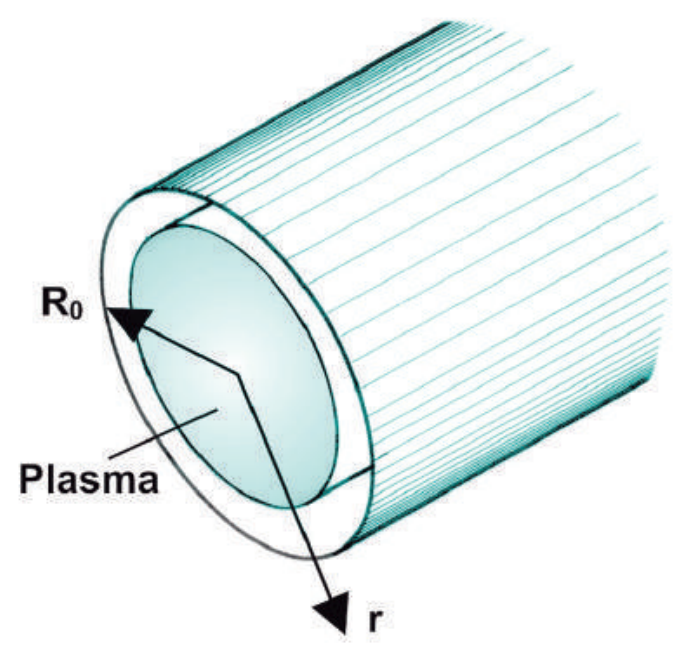

Fig. 5 Cylindrical geometry of the plasma column for the solution of the diffusion equation considered in problem 10.11 .

yields the following dispersion relation between $k$ and $\omega$,

$$
k^{2} D=i \omega
$$

Then show that for free electron diffusion we obtain

$$
k^{2} V_{s e}^{2}=i \omega \nu_{c e}
$$

where $V_{s e}=\left(k_{B} T_{e} / m_{e}\right)^{1 / 2}=\left(p_{e} / \rho_{m e}\right)^{1 / 2}$ is the isothermal speed of sound in the electron gas and $k_{B}$ is Boltzmann's constant. Next show that for ambipolar diffusion we obtain

$$
k^{2} V_{s p}^{2}=i \omega \nu_{c i}
$$

where

$$
V_{s p}=\left[\frac{k_{B}\left(T_{e}+T_{i}\right)}{m_{i}}\right]^{1 / 2}=\left(\frac{p_{e}+p_{i}}{\rho_{m i}}\right)^{1 / 2}
$$

is the isothermal plasma sound speed. Calculate the phase velocity and the damping factor for these waves and verify if they are longitudinal or transverse waves.

10.13 Consider a weakly ionized plasma immersed in a uniform magnetostatic field $\mathbf{B}_{0}$ oriented along the $z$ axis of a Cartesian coordinate system. 
(a) Show that the diffusion equation for the electrons (with $D \mathbf{u}_{e} / D t=0$ ) in the presence of the space charge electric field is given by

$$
\boldsymbol{\Gamma}_{e}=-\nabla \cdot\left(\mathcal{D}_{e} n_{e}\right)+n_{e} \mathcal{M}_{e} \cdot \mathbf{E}
$$

where

$$
\mathcal{D}_{e}=\left(\begin{array}{ccc}
D_{e \perp} & D_{e H} & 0 \\
-D_{e H} & D_{e \perp} & 0 \\
0 & 0 & D_{e \|}
\end{array}\right)
$$

with $D_{e \perp}, D_{e H}$, and $D_{e \|}$ given by (9.5), (9.6), and (9.7), respectively, and where

$$
\mathcal{M}_{e}=-\frac{e}{k T_{e}} \mathcal{D}_{e}
$$

(b) Deduce the corresponding equation for the ions in the presence of the space charge electric field $\mathbf{E}$. Combine the equations for the electrons and for the ions in order to eliminate the space charge electric field. Then, assuming that the electron and ion fluxes are equal, $\boldsymbol{\Gamma}_{e}=\boldsymbol{\Gamma}_{i}$, and that their number densities are also equal, $n_{e}=n_{i}$, determine the ambipolar diffusion coefficient. Verify that it is not affected by the presence of the magnetostatic field.

10.14 Consider the following heat flow equation, derived in problem 8.11 (in Chapter 8), for a stationary electron gas immersed in a magnetic field,

$$
\mathbf{q}_{e}+\frac{\Omega_{c e}}{\nu}\left(\mathbf{q}_{e} \times \mathbf{B}\right)=-K_{0} \nabla T_{e}
$$

Show that this equation can be written in the form

$$
\mathbf{q}_{e}=-\mathcal{K} \cdot \nabla T_{e}
$$

where $\mathcal{K}$ denotes the dyadic thermal conductivity coefficient, given by

$$
\mathcal{K}=\left(\begin{array}{ccc}
K_{\perp} & -K_{H} & 0 \\
k_{H} & K_{\perp} & 0 \\
0 & 0 & K_{0}
\end{array}\right)
$$

where

$$
\begin{gathered}
K_{\perp}=\frac{\nu^{2}}{\left(\nu^{2}+\Omega_{c e}^{2}\right)} K_{0} \\
K_{H}=\frac{\nu \Omega_{c e}}{\left(\nu^{2}+\Omega_{c e}^{2}\right)} K_{0} \\
K_{0}=\frac{5 k p_{e}}{2 m_{e} \nu}
\end{gathered}
$$




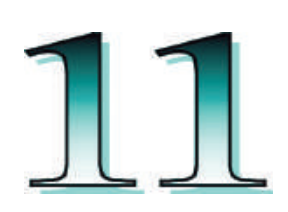

\section{SOME BASIC \\ PLASMA PHENOMENA}

\section{ELECTRON PLASMA OSCILLATIONS}

One of the fundamental properties of a plasma is its tendency to maintain electric charge neutrality on a macroscopic scale under equilibrium conditions. When this macroscopic charge neutrality is disturbed, such as to temporarily produce a significant imbalance of charge, large coulomb forces come into play, which tend to restore the macroscopic charge neutrality. Since these coulomb forces cannot be naturally sustained in the plasma, it breaks into high-frequency electron plasma oscillations, which enable the plasma to maintain on the average its electrical neutrality.

As a simple example, consider a small spherical region inside a plasma and suppose that a perturbation in the form of an excess of negative charge is introduced in this small region. Because of spherical symmetry, the corresponding electric field is radial and points towards the center (see Fig. 1), forcing the electrons to move radially outward. After a small time interval, since the electrons gain kinetic energy in the course of their motion, more electrons leave the spherical plasma region (due to their inertia) than is necessary to resume the state of electrical neutrality. An excess of positive charge results, therefore, inside this region and the reversed (outward, now) electric field causes the electrons to move inward. This sequence of outward and inward electron movement in the spherical plasma region continues periodically, resulting in electron plasma oscillations. In this way the plasma maintains its macroscopic neutrality on the average, since the total charge inside the spherical region, averaged over one period of these oscillations, is zero. The frequency of these oscillations is usually 


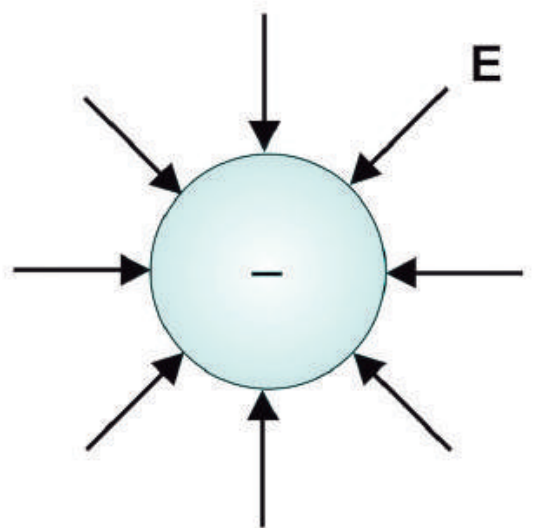

(a)

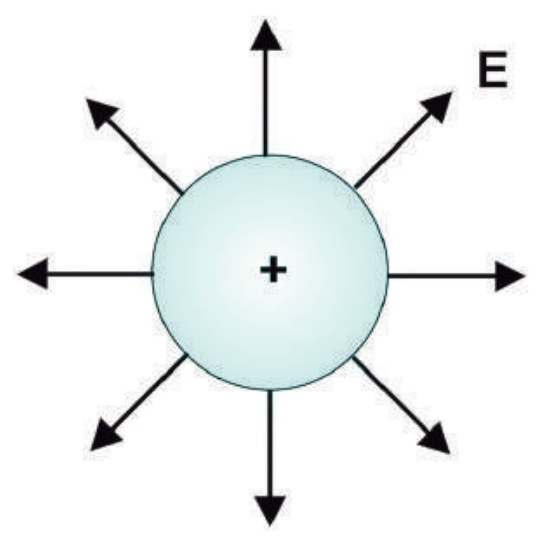

(b)

Fig. 1 The radial electric field $\mathbf{E}$ produced by a spherical distribution of negative charge (a) forces the electrons to move radially outwards, whereas the field produced by a spherical distribution of positive charge (b) forces the electrons to move radially inwards.

very high, and since the ions (in view of their much higher mass) are unable to follow the rapidity of the electron oscillations, their motion is often neglected.

To study the characteristics of the electron plasma oscillations we can use the cold plasma model, in which the particle thermal motion and the pressure gradient force are not taken into account. We shall neglect ion motion and assume a very small electron density perturbation such that

$$
n_{e}(\mathbf{r}, t)=n_{0}+n_{e}^{\prime}(\mathbf{r}, t)
$$

where $n_{0}$ is a constant number density and $\left|n_{e}^{\prime}\right| \ll n_{0}$. Similarly, we assume that the electric field produced, $\mathbf{E}(\mathbf{r}, t)$, and the average electron velocity, $\mathbf{u}_{e}(\mathbf{r}, t)$, are first-order perturbations, so that the linearized equations can be used. The linearized continuity and momentum equations become, respectively,

$$
\begin{gathered}
\frac{\partial n_{e}^{\prime}(\mathbf{r}, t)}{\partial t}+n_{0} \nabla \cdot \mathbf{u}_{e}(\mathbf{r}, t)=0 \\
\frac{\partial \mathbf{u}_{e}(\mathbf{r}, t)}{\partial t}=-\frac{e}{m_{e}} \mathbf{E}(\mathbf{r}, t)
\end{gathered}
$$


In the momentum equation we have assumed that the rate of momentum loss from the electron gas due to collisions is negligible. Considering singly charged ions, the charge density is given by

$$
\rho(\mathbf{r}, t)=-e\left[n_{0}+n_{e}^{\prime}(\mathbf{r}, t)\right]+e n_{0}=-e n_{e}^{\prime}(\mathbf{r}, t)
$$

where the ion density was considered to be constant and uniform, and equal to $n_{0}$ (neglecting ion motion). Therefore,

$$
\nabla \cdot \mathbf{E}(\mathbf{r}, t)=\frac{\rho(\mathbf{r}, t)}{\epsilon_{0}}=-\frac{e}{\epsilon_{0}} n_{e}^{\prime}(\mathbf{r}, t)
$$

Eqs. (1.2), (1.3), and (1.5) constitute a complete set of equations to be solved for the variables $n_{e}^{\prime}(\mathbf{r}, t), \mathbf{u}_{e}(\mathbf{r}, t)$, and $\mathbf{E}(\mathbf{r}, t)$. Taking the divergence of (1.3) and using (1.2) to substitute for $\nabla \cdot \mathbf{u}_{e}$, we obtain

$$
\frac{\partial^{2} n_{e}^{\prime}(\mathbf{r}, t)}{\partial t^{2}}-\frac{e n_{0}}{m_{e}} \nabla \cdot \mathbf{E}(\mathbf{r}, t)=0
$$

Combining (1.5) and (1.6) to eliminate $\nabla \cdot \mathbf{E}$, yields

$$
\frac{\partial^{2} n_{e}^{\prime}(\mathbf{r}, t)}{\partial t^{2}}+\omega_{p e}^{2} n_{e}^{\prime}(\mathbf{r}, t)=0
$$

where

$$
\omega_{p e}=\left(\frac{\left(n_{0} e^{2}\right.}{m_{e} \epsilon_{0}}\right)^{1 / 2}
$$

is called the electron plasma frequency. Equation (1.7) shows that $n_{e}^{\prime}(\mathbf{r}, t)$ varies harmonically in time at the electron plasma frequency,

$$
n_{e}^{\prime}(\mathbf{r}, t)=n_{e}^{\prime}(\mathbf{r}) \exp \left(-i \omega_{p e} t\right)
$$

In fact, all first-order perturbations have a harmonic time variation at the plasma frequency $\omega_{p e}$. To justify this statement it is convenient to start with the assumption that all first-order quantities vary harmonically

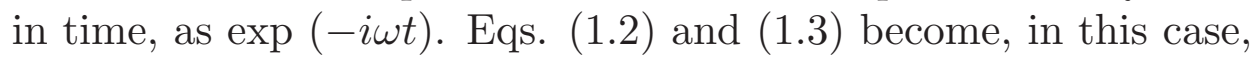

$$
\begin{gathered}
n_{e}^{\prime}=-\frac{i}{\omega} n_{0} \nabla \cdot \mathbf{u}_{e} \\
\mathbf{u}_{e}=-\frac{i e}{\omega m_{e}} \mathbf{E}
\end{gathered}
$$


which can be combined into

$$
n_{e}^{\prime}=-\frac{n_{0} e}{\omega^{2} m_{e}} \nabla \cdot \mathbf{E}
$$

Substituting this expression for $n_{e}^{\prime}$ into (1.5), yields

$$
\left(1-\frac{\omega_{p e}^{2}}{\omega^{2}}\right) \nabla \cdot \mathbf{E}=0
$$

which shows that a nontrivial solution requires $\omega=\omega_{p e}$. Therefore, all the perturbations vary harmonically in time at the electron plasma frequency. Further, for all variables there is no change in phase from point to point, implying the absence of wave propagation. The oscillations are therefore stationary. Also, (1.11) shows that the electron velocity is in the same direction as the electric field, so that these oscillations are longitudinal.

The electron plasma oscillations are also electrostatic in character. In order to show this aspect of the oscillations, consider Maxwell curl equations with a harmonic time variation,

$$
\begin{gathered}
\nabla \times \mathbf{E}=i \omega \mathbf{B} \\
\nabla \times \mathbf{B}=\mu_{0}\left(\mathbf{J}-i \omega \epsilon_{0} \mathbf{E}\right)
\end{gathered}
$$

The electric current density is given by

$$
\mathbf{J}=-e n_{0} \mathbf{u}_{e}=\frac{i n_{0} e^{2}}{\omega m_{e}} \mathbf{E}
$$

where we have used (1.11) for $\mathbf{u}_{e}$. Therefore,

$$
\nabla \times \mathbf{B}=-i \omega \mu_{0} \epsilon_{0} \epsilon_{r} \mathbf{E}
$$

where we have defined a relative permittivity by

$$
\epsilon_{r}=1-\frac{\omega_{p e}^{2}}{\omega^{2}}
$$

For the electron plasma oscillations we have $\omega=\omega_{p e}$, so that $\epsilon_{r}=0$, and (1.17) reduces to

$$
\nabla \times \mathbf{B}=0
$$

Since the curl of the gradient of any scalar function vanishes identically, 
we may write

$$
\mathbf{B}=\nabla \psi
$$

where $\psi$ is a magnetic scalar potential. Substituting (1.20) into (1.14) and taking the divergence of both sides, we obtain the Laplace equation

$$
\nabla \cdot(\nabla \psi)=\nabla^{2} \psi=0
$$

since the divergence of the curl of any vector function vanishes identically. The only solution of this equation, which is not singular and finite at infinity, is $\psi=$ constant, so that $\mathbf{B}=0$. Hence, there is no magnetic field associated with these space charge oscillations.

In summary, the electron plasma oscillations are stationary, longitudinal, and electrostatic. They are also referred to as Langmuir oscillations. When the effect of the pressure gradient force is included in the equation of motion (1.3), complemented by an adiabatic energy equation, these oscillations become propagating disturbances, commonly known as space charge waves or Langmuir waves. Characteristic values of the electron plasma frequency for various laboratory and cosmic plasmas are given in Fig. 2 of Chapter 1.

\section{THE DEBYE SHIELDING PROBLEM}

To examine the mechanism by which the plasma strives to shield its interior from a disturbing electric field, consider a plasma whose equilibrium state is perturbed by an electric field due to an external charged particle. For that matter, this electric field may also be considered to be due to one of the charged particles inside the plasma, isolated for observation. For definiteness, we assume this test particle to have a positive charge $+Q$, and choose a spherical coordinate system whose origin coincides with the position of the test particle. We are interested in determining the electrostatic potential $\phi(\mathbf{r})$ that is established near the test charge $Q$, due to the combined effects of the test charge and the distribution of charged particles surrounding it. Since the positive test charge $Q$ attracts the negatively charged particles and repels the positively charged ones, the number densities of the electrons $n_{e}(\mathbf{r})$ and of the ions $n_{i}(\mathbf{r})$ will be slightly different near the origin (test particle), whereas at large distances from the origin the electrostatic potential vanishes, so that $n_{e}(\infty)=n_{i}(\infty)=n_{0}$. Since this is a steady-state problem under the action of a conservative electric field, we have

$$
\mathbf{E}(\mathbf{r})=-\nabla \phi(\mathbf{r})
$$


and from (7.5.16) it follows that

$$
\begin{gathered}
n_{e}(\mathbf{r})=n_{0} \exp \left[\frac{e \phi(\mathbf{r})}{k T}\right] \\
n_{i}(\mathbf{r})=n_{0} \exp \left[-\frac{e \phi(\mathbf{r})}{k T}\right]
\end{gathered}
$$

where we have assumed that the electrons and ions (of charge $e$ ) have the same temperature $T$.

The total electric charge density $\rho(\mathbf{r})$, including the test charge $Q$, can be expressed as

$$
\rho(\mathbf{r})=-e\left[n_{e}(\mathbf{r})-n_{i}(\mathbf{r})\right]+Q \delta(\mathbf{r})
$$

where $\delta(\mathbf{r})$ denotes the Dirac delta function. Using (2.2) and (2.3),

$$
\rho(\mathbf{r})=-e n_{0}\left\{\exp \left[\frac{e \phi(\mathbf{r})}{k T}\right]-\exp \left[-\frac{e \phi(\mathbf{r})}{k T}\right]\right\}+Q \delta(\mathbf{r})
$$

Substituting (2.1) and (2.5) into the following Maxwell equation,

$$
\nabla \cdot \mathbf{E}(\mathbf{r})=\frac{\rho(\mathbf{r})}{\epsilon_{0}}
$$

gives the differential equation

$$
\nabla^{2} \phi(\mathbf{r})-\frac{e n_{0}}{\epsilon_{0}}\left\{\exp \left[\frac{e \phi(\mathbf{r})}{k T}\right]-\exp \left[-\frac{e \phi(\mathbf{r})}{k T}\right]\right\}=-\frac{Q}{\epsilon_{0}} \delta(\mathbf{r})
$$

which allows the evaluation of the electrostatic potential $\phi(\mathbf{r})$.

In order to proceed analytically, we assume now that the perturbing electrostatic potential is weak so that the electrostatic potential energy is much less than the mean thermal energy, that is,

$$
e \phi(\mathbf{r}) \ll k T
$$

Under this condition we can use the approximation (making a series expansion)

$$
\exp \left[ \pm \frac{e \phi(\mathbf{r})}{k T}\right] \simeq 1 \pm \frac{e \phi(\mathbf{r})}{k T}
$$

Therefore, (2.7) simplifies to

$$
\nabla^{2} \phi(\mathbf{r})-\frac{2}{\lambda_{D}^{2}} \phi(\mathbf{r})=-\frac{Q}{\epsilon_{0}} \delta(\mathbf{r})
$$


where $\lambda_{D}$ denotes the Debye length

$$
\lambda_{D}=\left(\frac{\epsilon_{0} k T}{n_{0} e^{2}}\right)^{1 / 2}=\frac{1}{\omega_{p e}}\left(\frac{k T_{e}}{m_{e}}\right)^{1 / 2}
$$

Since the problem has spherical symmetry, the electrostatic potential depends only on the radial distance $r$ measured from the position of the test particle, being independent of the spatial orientation of $\mathbf{r}$. Thus, using spherical coordinates, (2.10) can be written (for $r \neq 0$ ) as

$$
\frac{1}{r^{2}} \frac{d}{d r}\left[r^{2} \frac{d}{d r} \phi(\mathbf{r})\right]-\frac{2}{\lambda_{D}^{2}} \phi(\mathbf{r})=0 \quad(r \neq 0)
$$

In order to solve this equation we note initially that for an isolated particle of charge $+Q$, in free space, the electric field is directed radially outward and is given by

$$
\mathbf{E}(\mathbf{r})=\frac{1}{4 \pi \epsilon_{0}} \frac{Q}{r^{2}} \widehat{\mathbf{r}}
$$

so that the electrostatic coulomb potential $\phi_{c}(r)$ due to this isolated charged particle in free space is

$$
\phi_{c}(r)=\frac{1}{4 \pi \epsilon_{0}} \frac{Q}{r}
$$

In the very close proximity of the test particle the electrostatic potential should be the same as that for an isolated particle in free space. Hence, it is appropriate to seek the solution of (2.12) in the form

$$
\phi(r)=\phi_{c}(r) F(r)=\frac{Q}{4 \pi \epsilon_{0}} \frac{F(r)}{r}
$$

where the function $F(r)$ must be such that $F(r) \rightarrow 1$ when $r \rightarrow 0$. Furthermore, the electrostatic potential $\phi(r)$ is required to vanish at infinity, that is, $\phi \rightarrow 0$ when $r \rightarrow \infty$. Substituting (2.15) into (2.12) yields the following differential equation for $F(r)$ :

$$
\frac{d^{2} F(r)}{d r^{2}}=\frac{2}{\lambda_{D}^{2}} F(r)
$$

This simple differential equation for $F(r)$ has the solution

$$
F(r)=A \exp \left(\frac{\sqrt{2} r}{\lambda_{D}}\right)+B \exp \left(-\frac{\sqrt{2} r}{\lambda_{D}}\right)
$$


The condition that $\phi(r)$ vanishes for large values of $r$ requires $A=0$. Also, the condition that $F(r)$ tends to one when $r$ tends to zero requires $B=1$. Therefore, the solution of (2.12) is

$$
\phi(r)=\phi_{c}(r) \exp \left(-\frac{\sqrt{2} r}{\lambda_{D}}\right)=\frac{1}{4 \pi \epsilon_{0}} \frac{Q}{r} \exp \left(-\frac{\sqrt{2} r}{\lambda_{D}}\right)
$$

This result is commonly known as the Debye potential, since this nonrigorous derivation was first presented by Debye and Huckel in their theory of electrolytes. It shows that $\phi(r)$ becomes much less than the ordinary coulomb potential once $r$ exceeds the distance $\lambda_{D}$, called the Debye length (see Fig. 2). Hence, we can say in a crude way that a charged particle in a plasma interacts effectively only with particles situated at distances less than one Debye length away, and it has a negligible influence on particles lying at distances greater than one Debye length.

The charge $Q$ of the test particle is neutralized by the charge distribution surrounding the test particle. From (2.5) and (2.9) we obtain for the charge density

$$
\rho(\mathbf{r})=-2 \frac{n_{0} e^{2} \phi(\mathbf{r})}{k T}+Q \delta(\mathbf{r})
$$

Substituting $\phi(\mathbf{r})$ by the Debye potential (2.18), we obtain

$$
\rho(\mathbf{r})=-\frac{Q}{2 \pi r \lambda_{D}^{2}} \exp \left(-\frac{\sqrt{2} r}{\lambda_{D}}\right)+Q \delta(\mathbf{r})
$$

To obtain the total charge $q_{t}$ we integrate (2.20) over all space,

$$
\begin{gathered}
q_{t}=\iiint \rho(\mathbf{r}) d^{3} r= \\
Q \int \frac{Q}{2 \pi \lambda_{D}^{2}} \int_{0}^{\infty} \frac{1}{r} \exp \left(-\frac{\sqrt{2} r}{\lambda_{D}}\right) 4 \pi r^{2} d r+ \\
Q \iint \delta(\mathbf{r}) d^{3} r
\end{gathered}
$$

Since the first integral gives $-Q$, whereas the second one is equal to $+Q$, we find $q_{t}=0$. The principal contribution to the first integral in (2.21) comes from the plasma particles lying in the very close neighborhood of the test particle, since the integrand falls off exponentially with increasing values of $r$. Thus, the neutralization of the test particle takes place effectively on account of the charged particles inside the Debye sphere. From (2.2) and (2.3) we see that in the neighborhood of the test particle the electron number density is larger than the ion number density, on account of the fact that the positive test particle attracts the electrons and repels the ions. Therefore, in the close proximity of the test particle there is an imbalance of charge and, consequently, an electric field. We have seen 


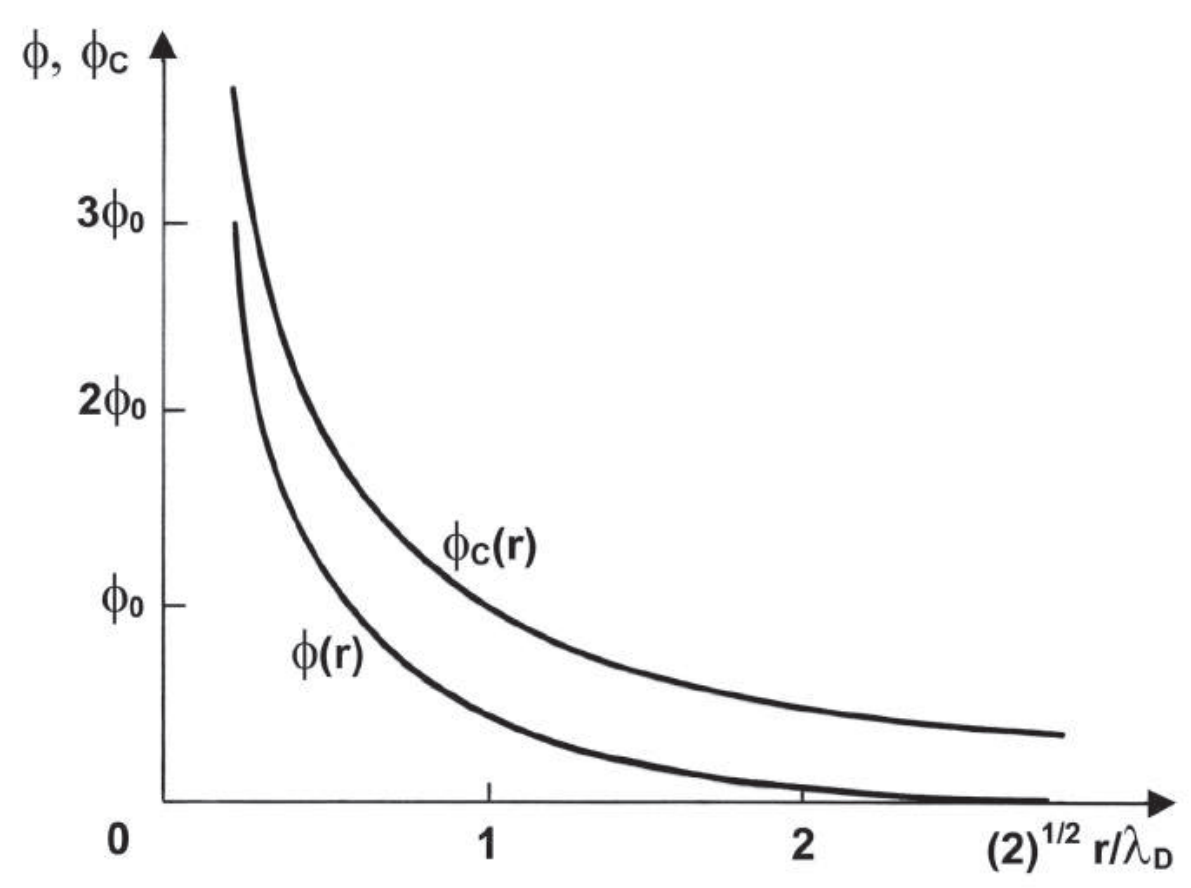

Fig. 2 Electrostatic coulomb potential $\phi_{c}(r)$ and Debye potential $\phi(r)$ as a function of distance $r$ from the test charge $Q$. Here we have $\phi_{0}=\sqrt{2} Q /\left(4 \pi \epsilon_{0} \lambda_{D}\right)$.

that the shielding of this electric field is effectively completed over a distance of the order of $\lambda_{D}$. Thus, for macroscopic neutrality, it is necessary that the plasma typical characteristic dimension $L$ be much greater than $\lambda_{D}$. This quantitative criterion for the definition of a plasma has been previously discussed in Chapter 1 .

An important point to be noted in the result (2.18) is that, for $r \rightarrow 0$, the Debye potential becomes very large and the assumption $e \phi(r) \ll k T$ is unlikely to be fulfilled. To verify the validity of this approximation, and consequently of (2.18), note that using (2.18) with $Q=e$ we have

$$
\frac{e \phi}{k T}=\frac{e^{2} \exp \left(-\sqrt{2} r / \lambda_{D}\right)}{4 \pi \epsilon_{0} r k T}=\frac{\lambda_{D}}{3 N_{D}} \frac{\exp \left(-\sqrt{2} r / \lambda_{D}\right)}{r}
$$

where $N_{D}$ is the number of electrons inside a Debye sphere. Since $N_{D}$ is very large for virtually all plasmas, it is evident that the ratio given in (2.22) is much less than one, except when $r$ is less than $\lambda_{D} / N_{D}$. Therefore, 
the Debye potential (2.18) is consistent with the approximation $e \phi \ll k T$ used to derive it, if we restrict attention to distances from the test particle greater than $\lambda_{D} / N_{D}$.

As a final point, we note that in the derivation of the Debye potential that appears extensively in the literature, it is usual to ignore ion motion and to assume a constant ion number density equal to the unperturbed electron number density. In this case the factor of 2 disappears from (2.10), and the expression for the Debye potential becomes

$$
\phi(r)=\frac{1}{4 \pi \epsilon_{0}} \frac{Q}{r} \exp \left(-r / \lambda_{D}\right)
$$

\section{DEBYE SHIELDING USING THE VLASOV EQUATION}

In this section we analyze the Debye potential problem from the point of view of kinetic theory. As before, we suppose that a test charge $+Q$ is placed at the origin of a spherical coordinate system inside the plasma. In order to determine the steady-state electron and ion distribution functions, $f_{e}$ and $f_{i}$, and the electrostatic potential $\phi(\mathbf{r})$ near the test charge, let us consider the steady-state Vlasov equations for the electrons and the ions, with only the electric field $\mathbf{E}(\mathbf{r})=-\nabla \phi(\mathbf{r})$ in the Lorentz force term,

$$
\begin{aligned}
& \mathbf{v} \cdot \nabla f_{e}+\frac{e}{m_{e}}(\nabla \phi) \cdot \nabla_{v} f_{e}=0 \\
& \mathbf{v} \cdot \nabla f_{i}-\frac{e}{m_{i}}(\nabla \phi) \cdot \nabla_{v} f_{i}=0
\end{aligned}
$$

Since

$$
n_{\alpha}(\mathbf{r})=\int_{v} f_{\alpha}(\mathbf{r}, v) d^{3} v
$$

the total charge density (including the test particle) can be expressed as

$$
\rho(\mathbf{r})=-e \int_{v}\left(f_{e}-f_{i}\right) d^{3} v+Q \delta(\mathbf{r})
$$

and the Poisson equation for this case becomes

$$
\nabla^{2} \phi-\frac{e}{\epsilon_{0}} \int_{v}\left(f_{e}-f_{i}\right) d^{3} v=-\frac{Q}{\epsilon_{0}} \delta(\mathbf{r})
$$


Equations (3.1), (3.2), and (3.5) constitute three equations to be solved simultaneously to determine $f_{e}, f_{i}$, and $\phi$.

The solution of the Vlasov equations (3.1) and (3.2) can be expressed in terms of the Maxwellian distribution function and the Boltzmann factor (see section 5, Chapter 7), as

$$
f_{\alpha}(\mathbf{r}, v)=f_{0 \alpha}(v) \exp \left[-\frac{q_{\alpha} \phi(\mathbf{r})}{k T}\right]
$$

When the electrostatic potential vanishes, this distribution function goes into the Maxwellian distribution $f_{0 \alpha}(v)$, with zero drift velocity. Substituting (3.6), with $\alpha=e, i$, into (3.5), yields

$$
\begin{aligned}
& \nabla^{2} \phi-\frac{e}{\epsilon_{0}}\left[\exp \left(\frac{e \phi}{k T}\right) \int_{v} f_{0 e} d^{3} v-\right. \\
& \left.\exp \left(-\frac{e \phi}{k T}\right) \int_{v} f_{0 i} d^{3} v\right]=-\frac{Q}{\epsilon_{0}} \delta(\mathbf{r})
\end{aligned}
$$

Denoting the electron and ion number densities under equilibrium conditions (when $\phi$ vanishes) by $n_{0}$,

$$
n_{0}=\int_{v} f_{0 \alpha}(v) d^{3} v \quad \alpha=e, i
$$

equation (3.7) becomes

$$
\nabla^{2} \phi-\frac{e n_{0}}{\epsilon_{0}}\left[\exp \left(\frac{e \phi}{k T}\right)-\exp \left(-\frac{e \phi}{k T}\right)\right]=-\frac{Q}{\epsilon_{0}} \delta(\mathbf{r})
$$

This equation is identical to (2.7), yielding the same result as before for the Debye potential.

\section{PLASMA SHEATH}

When a material body is immersed in a plasma, the body acquires a net negative charge and therefore a negative potential with respect to the plasma potential. In the region near the wall of the body there is a boundary layer, known as the plasma sheath, in which the electron and the ion number densities are different. Inside the plasma sheath the potential increases monotonically from a negative value on the wall to the value corresponding to the unperturbed plasma. The thickness of the plasma 
sheath, where departures from macroscopic electric neutrality occur, is found to be of the order of a Debye length.

A satisfactory mathematical treatment of this phenomenon is quite involved and cannot be presented here. However, the basic physical factors responsible for the formation of the plasma sheath are fairly simple to understand. In this section we will set up the problem mathematically and obtain some approximate results. The problem is strongly dependent on the particular geometry under consideration. For simplicity, we shall assume that the wall bounding the plasma is an infinite plane surface at $x=0$, with the plasma in the region $x>0$, and that there are no variations with respect to the coordinates $y$ and $z$.

\subsection{Physical Mechanism}

We begin with a descriptive account of the physical mechanism responsible for the formation of the plasma sheath. The charged particles in the plasma that strike the wall in virtue of their random thermal motions are for the most part lost to the plasma. The ions generally recombine at the wall and return to the plasma as neutral particles, whereas the electrons may either recombine there or enter the conduction band if the surface is a metal. We have seen in section 4 of Chapter 7 that the random particle flux, that is, the number of particles that hit the surface per unit area and per unit time, from one side only, for the case of an isotropic velocity distribution function, is given by [see Eqs. (7.4.35) and (7.4.18)]

$$
\Gamma_{\alpha}=n_{\alpha}<v>_{\alpha} / 4
$$

where $\langle v\rangle_{\alpha}$ is the average particle speed for the $\alpha$ species. For the Maxwell-Boltzmann velocity distribution function we have found that [see Eq. (7.4.20)]

$$
<v>_{\alpha}=\left(\frac{8}{\pi}\right)^{1 / 2}\left(\frac{k T_{\alpha}}{m_{\alpha}}\right)^{1 / 2}
$$

so that a typical value for the random particle flux in this case is

$$
\Gamma_{\alpha}=n_{\alpha}\left(\frac{k T_{\alpha}}{2 \pi m_{\alpha}}\right)^{1 / 2}
$$

It is evident from this result that if initially the electron and the ion number densities are equal, then the random particle flux for the electrons $\left(\Gamma_{e}\right)$ greatly exceeds that for the ions $\left(\Gamma_{i}\right)$, since in general $\left(T_{e} / m_{e}\right)^{1 / 2}$ is much larger than $\left(T_{i} / m_{i}\right)^{1 / 2}$. For the least heavy ion, hydrogen, for 
example, $m_{i} / m_{e}=1836$. Therefore, the wall in contact with the plasma rapidly accumulates a negative charge, since initially more electrons reach the wall than positive ions. This negative potential repels the electrons and attracts the ions so that the electron flux diminishes and the ion flux increases. Eventually, the negative potential at the wall becomes large enough in magnitude to equalize the rate at which electrons and ions hit the surface. At this floating negative potential the wall and the plasma reach a dynamical equilibrium such that the net current at the wall is zero.

\subsection{Electric Potential on the Wall}

To estimate the value of the potential on the wall after the plasma sheath has been established, consider a steady-state situation and let the electric potential $\phi(x)$ at the wall $(x=0)$ be given by

$$
\phi(0)=\phi_{w}
$$

Let us choose the reference potential inside the plasma, at a very large distance from the wall, equal to zero,

$$
\phi(\infty)=0
$$

The electrons and the ions are assumed to be in thermodynamic equilibrium at the same temperature $T$, under the action of the conservative electric field associated with the negative potential on the wall. At $x \rightarrow \infty$ the plasma is unperturbed and the electron and ion number densities are each equal to $n_{0}$. According to the results of section 5, Chapter 7, the electron and ion number densities can be expressed as

$$
\begin{aligned}
& n_{e}(\mathbf{r})=n_{0} \exp \left[\frac{e \phi(\mathbf{r})}{k T}\right] \\
& n_{i}(\mathbf{r})=n_{0} \exp \left[-\frac{e \phi(\mathbf{r})}{k T}\right]
\end{aligned}
$$

It is important to note at this point that (4.6) and (4.7) do not take into account the particle drift velocity towards the wall. Since the electrons and the ions impinging on the wall surface are for the most part lost to the plasma, there must be a steady flux of both species towards the wall to replenish this charged particle loss. Despite this inadequacy, (4.6) 
and (4.7) will still be used to obtain an approximate expression for the potential on the wall. Afterwards, in order to study the inner structure of the plasma sheath, we will take into account the particle drift in an approximate manner using the hydrodynamic equations.

One of the boundary conditions of the problem is that under equilibrium conditions there must be no charge buildup at the wall $(x=0)$, so that

$$
J_{e}(0)=J_{i}(0)
$$

Using (4.3), (4.6), and (4.7), considering singly charged ions,

$$
\left(\frac{1}{m_{e}}\right)^{1 / 2} \exp \left(\frac{e \phi_{w}}{k T}\right)=\left(\frac{1}{m_{i}}\right)^{1 / 2} \exp \left(-\frac{e \phi_{w}}{k T}\right)
$$

which may be written as

$$
\exp \left(-\frac{2 e \phi_{w}}{k T}\right)=\left(\frac{m_{i}}{m_{e}}\right)^{1 / 2}
$$

Taking the natural logarithm of both sides, and solving for the wall potential, we obtain

$$
\phi_{w}=-\left(\frac{k T}{4 e}\right) \ln \left(\frac{m_{i}}{m_{e}}\right)
$$

Other more accurate methods of calculating the wall potential yield results which, for $T_{e}=T_{i}$, agree qualitatively with the one given in (4.11), despite the inadequacy of (4.6) and (4.7), which neglect the particle drift velocity towards the wall.

Note from (4.11) that the magnitude of the potential energy near the wall $\left|e \phi_{w}\right|$ is of the same order as the average thermal energy $k T$ of the particles in the plasma, since

$$
\frac{\left|e \phi_{w}\right|}{k T}=\frac{1}{4} \ln \left(\frac{m_{i}}{m_{e}}\right)
$$

For a hydrogen ion, for example, $\left|e \phi_{w}\right| /(k T)$ is approximately equal to 2 , whereas for heavier ions it may be close to 3 .

\subsection{Inner Structure of the Plasma Sheath}

To investigate the inner structure of the plasma sheath, consider the equations of conservation of particles and momentum for the electrons 
and ions, under steady-state conditions, with spatial dependence only on the $x$ direction. The equation of conservation of particles becomes (with $\alpha=e, i)$

$$
\frac{d\left(n_{\alpha} u_{\alpha}\right)}{d x}=n_{\alpha} \frac{d u_{\alpha}}{d x}+u_{\alpha} \frac{d n_{\alpha}}{d x}=0
$$

In the momentum conservation equation we neglect viscosity effects and approximate the kinetic pressure dyad by a scalar pressure. The ideal gas equation of state, $p_{\alpha}=n_{\alpha} k T_{\alpha}$, can be used to introduce the temperature, which is assumed to be constant. Collisions are neglected, since the thickness of the plasma sheath is much less than the mean free path for the plasma particles. With these assumptions and in the absence of a magnetic field, the equation of motion becomes [taking $\mathbf{E}(\mathbf{r})=-\nabla \phi(\mathbf{r})$, and $\left.D / D t=\partial / \partial t+\mathbf{u}_{\alpha} \cdot \nabla=u_{\alpha} d / d x\right]$

$$
m_{\alpha} u_{\alpha} \frac{d u_{\alpha}}{d x}=-\frac{k T_{\alpha}}{n_{\alpha}} \frac{d n_{\alpha}}{d x}-q_{\alpha} \frac{d \phi}{d x}
$$

In order to simplify the analysis we shall make two approximations. From (4.13), we can write

$$
\frac{d n_{\alpha}}{d x}=-\frac{n_{\alpha}}{u_{\alpha}} \frac{d u_{\alpha}}{d x}
$$

and the ratio of the magnitude of the term in the left-hand side of (4.14) to the first term in the right-hand side can be expressed as

$$
\frac{\left|m_{\alpha} u_{\alpha}\left(d u_{\alpha} / d x\right)\right|}{\left|\left(k T_{\alpha} / n_{\alpha}\right)\left(d n_{\alpha} / d x\right)\right|}=\frac{m_{\alpha} u_{\alpha}^{2}}{k T_{\alpha}}
$$

The two approximations consist in neglecting the left-hand side term of (4.14) for the electrons, whereas for the ions we neglect the first term in the right-hand side of (4.14). Explicitly, we take for the electrons (neglecting electron inertia)

$$
\frac{k T_{e}}{n_{e}} \frac{d n_{e}}{d x}-e \frac{d \phi}{d x}=0
$$

and for the ions (assuming cold ions)

$$
m_{i} u_{i} \frac{d u_{i}}{d x}+e \frac{d \phi}{d x}=0
$$

These two approximations are justified only if the thermal energy of the electrons is much larger than their kinetic energy, and if the thermal energy 
of the ions is much smaller than their kinetic energy. Thus, we require from (4.15) that

$$
m_{e} u_{e}^{2} \ll k T \ll m_{i} u_{i}^{2}
$$

in order to justify the approximations in (4.17) and (4.18). We shall assume that the condition (4.19) is satisfied in the plasma sheath but it remains to be justified later in this section.

If we integrate (4.17), we obtain

$$
e \phi(x)=k T \ln n_{e}(x)+(\text { constant })
$$

and using the condition that $n_{e}=n_{0}$ when $\phi=0$, we find

$$
n_{e}(x)=n_{0} \exp \left[\frac{e \phi(x)}{k T}\right]
$$

This result is identical to (4.6), which is not surprising, since the condition $m_{e} u_{e}^{2} \ll k T$ implies neglecting the electron inertia $\left(m_{e}=0\right)$ and consequently their kinetic energy.

For the ions, we first integrate (4.13) to find

$$
n_{i}(x) u_{i}(x)=C_{1}
$$

and then integrate (4.18) to obtain

$$
e \phi(x)+\frac{1}{2} m_{i} u_{i}^{2}(x)=C_{2}
$$

where $C_{1}$ and $C_{2}$ are constants. The boundary conditions require that at $x \rightarrow \infty$ we must have $\phi(\infty)=0, n_{i}(\infty)=n_{0}$, and $u_{i}(\infty)=u_{0 i}$. Thus,

$$
C_{i}=n_{0} u_{0 i} ; \quad C_{2}=\frac{1}{2} m_{i} u_{0 i}^{2}
$$

and using these results in (4.22) and (4.23), we find

$$
\begin{gathered}
n_{i}(x) u_{i}(x)=n_{0} u_{0 i} \\
e \phi(x)+\frac{1}{2} m_{i} u_{i}^{2}(x)=\frac{1}{2} m_{i} u_{0 i}^{2}
\end{gathered}
$$

These two equations can be combined to eliminate $u_{i}(x)$ and solved for $n_{i}(x)$, giving

$$
n_{i}(x)=n_{0}\left[1-\frac{2 e \phi(x)}{m_{i} u_{0 i}^{2}}\right]^{-1 / 2}
$$


This expression for $n_{i}(x)$ is substantially different from the one given in (4.7), and this difference is due to the importance of the ion drift velocity. We now find that, since $\phi(x)<0$ in the sheath, $n_{i}(x)$ decreases slowly towards the wall rather than increasing as predicted by (4.7). Physically, this behavior is due to the fact that the negative potential on the wall causes $u_{i}(x)$ to increase as the ions approach the wall and since the ion flux $n_{i}(x) u_{i}(x)$ must stay constant, in virtue of (4.25), it turns out that $n_{i}(x)$ must decrease according to (4.27). This behavior is illustrated schematically in Fig. 3.

To obtain the differential equation satisfied by the electrostatic potential $\phi(x)$, we substitute (4.21) and (4.27) into the Poisson equation

$$
\nabla^{2} \phi=\frac{e}{\epsilon_{0}}\left(n_{e}-n_{i}\right)
$$

to obtain

$$
\frac{d^{2} \phi}{d x^{2}}=\frac{n_{0} e}{\epsilon_{0}}\left[\exp \left(\frac{e \phi}{k T}\right)-\left(1-\frac{2 e \phi}{m_{i} u_{0 i}^{2}}\right)^{-1 / 2}\right]
$$

In this equation, the drift velocity $u_{0 i}$, far away from the wall, still needs to be determined. This equation is nonlinear, and in order to facilitate its analytical solution we need to make one more approximation. Since we have seen that $|e \phi|$ ranges from zero in the plasma to a value of the order of $k T$ on the wall and since we have also assumed that $m_{i} u_{0 i}^{2}$ is larger than $k T$, we will restrict our attention to the region near the plasma edge of the sheath and assume further that $|e \phi|$ is small compared to both $k T$ and $m_{i} u_{0 i}^{2}$. Thus, in the region near the edge of the sheath adjacent to the plasma, we can expand the terms in the right-hand side of (4.29), for $e \phi /(k T) \ll 1$ and $e \phi /\left(m_{i} u_{0 i}^{2}\right) \ll 1$, as

$$
\begin{gathered}
\exp \left(\frac{e \phi}{k T}\right) \simeq 1+\frac{e \phi}{k T} \\
\left(1-\frac{2 e \phi}{m_{i} u_{0 i}^{2}}\right)^{-1 / 2} \simeq 1+\frac{e \phi}{m_{i} u_{0 i}^{2}}
\end{gathered}
$$

with the result that (4.29) reduces to

$$
\frac{d^{2} \phi}{d x^{2}}=\frac{\phi}{X^{2}}
$$

where

$$
X^{2}=\lambda_{D}^{2}\left(1-\frac{k T}{m_{i} u_{0 i}^{2}}\right)^{-1}
$$




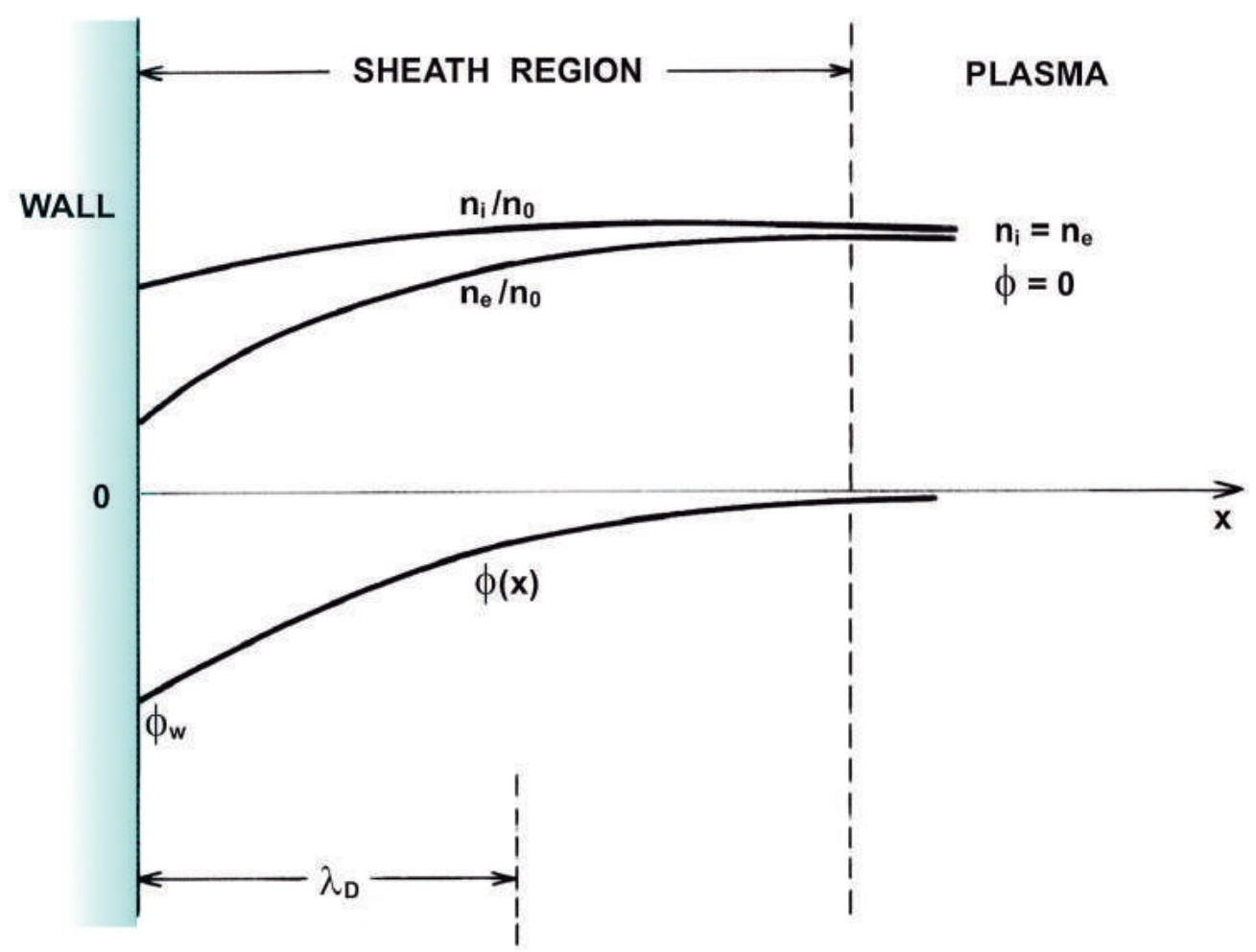

Fig. 3 Diagram showing the variation of the electrostatic potential $\phi(x)$ and the number densities $n_{e}(x)$ and $n_{i}(x)$ inside the plasma sheath near an infinite plane wall.

The solution of (4.32), with the boundary condition $\phi(\infty)=0$, is

$$
\phi(x)=A \exp (-x / X)
$$

where $A$ is a constant. Since we have assumed that $k T \ll m_{i} u_{0 i}^{2}$, it follows from (4.33) that $X$ is real and approximately equal to $\lambda_{D}$.

Therefore, we find that the absolute value of $\phi(x)$ decreases exponentially (note that $A$ must be negative, so that $\phi(x)$ actually increases) as we move from inside the sheath into the plasma and goes asymptotically to zero at very large distances from the wall. Since $X \simeq \lambda_{D}$, this variation effectively takes place within a distance of the order of a Debye length. This solution for $\phi(x)$ is strictly valid only near the plasma edge of the sheath, but if it is continued to apply throughout the plasma sheath, we can impose the boundary condition on the wall, that is, $\phi(0)=\phi_{w}$, which requires that $A=\phi_{w}$. 
If $k T$ were greater than $m_{i} u_{0 i}^{2}$, then $X$ would be imaginary and the electric potential would be an oscillating function of distance near the wall. The condition

$$
k T<m_{i} u_{0 i}^{2}
$$

therefore must be satisfied for the formation of a plasma sheath. It is known as the Bohm criterion.

It is not a trivial matter to determine the potential on the wall using the hydrodynamic equations. All the approximate methods that have been suggested give results that, for $T_{e}=T_{i}$, agree reasonably well with the approximate value for $\phi_{w}$ given in (4.11). Furthermore, there is no consistent way to determine the ion drift velocity $u_{0 i}$ at $x=\infty$, but an approximate estimate can be obtained as follows. Since the ion flux must be constant, from (4.25) we can equate the ion flux $n_{0} u_{0 i}$ at $x=\infty$ to its value $\Gamma_{i}(0)$ at the wall. Using (4.3) with $n_{i}$ as given by (4.7) evaluated at the wall, we find

$$
u_{0 i}=\left(\frac{k T}{2 \pi m_{i}}\right)^{1 / 2} \exp \left(-\frac{e \phi_{w}}{k T}\right)
$$

Similarly, for the electrons we can equate the electron flux $n_{0} u_{0 e}$ at $x=\infty$ to its value $\Gamma_{e}(0)$ at the wall. From (4.3), with $n_{e}$ given by (4.6) evaluated at the wall, we find

$$
u_{0 e}=\left(\frac{k T}{2 \pi m_{e}}\right)^{1 / 2} \exp \left(\frac{e \phi_{w}}{k T}\right)
$$

and using (4.9) we see that

$$
u_{0 e}=u_{0 i}
$$

To verify the validity of the approximations indicated in (4.19), we note first that from the mass conservation equation the particle flux, given by $n_{\alpha}(x) u_{\alpha}(x)$, must be constant for all $x$ and equal to the value $n_{0} u_{0 \alpha}$. From (4.21) we see that the minimum value of $n_{e}(x)$ is $n_{0} \exp \left(e \phi_{w} /(k T)\right.$ since $\phi_{w}$ is negative. Therefore,

$$
u_{e}=\left(\frac{n_{0}}{n_{e}}\right) u_{0 e} \leq u_{0 e} \exp \left(-\frac{e \phi_{w}}{k T}\right)
$$

and using (4.37), we get

$$
u_{e} \leq\left(\frac{k T}{2 \pi m_{e}}\right)^{1 / 2}
$$


or

$$
\frac{k T}{m_{e} u_{e}^{2}} \geq 2 \pi
$$

in agreement with the assumption (4.19). Similarly, from (4.27) the maximum value of $n_{i}(x)$ is $n_{0}$, so that

$$
u_{i}=\left(\frac{n_{0}}{n_{i}}\right) u_{0 i} \geq u_{0 i}
$$

and using (4.36), we obtain

$$
u_{i} \geq\left(\frac{k T}{2 \pi m_{i}}\right)^{1 / 2} \exp \left(-\frac{e \phi_{w}}{k T}\right)
$$

or

$$
\frac{k T}{m_{i} u_{i}^{2}} \leq 2 \pi \exp \left(\frac{2 e \phi_{w}}{k T}\right) \simeq 0.1
$$

where the result on the right has been obtained substituting $\phi_{w}$ by the value given in (4.11). Therefore, in view of (4.41) and (4.44), the thermal energy of the electrons is seen to be greater than their kinetic energy, whereas for the ions the opposite situation is verified. From (4.44) we can also see that the Bohm criterion, for the formation of the plasma sheath, is satisfied.

Although the quantitative aspects of the discussion presented here are very approximate, it provides, however, a satisfactory qualitative picture of the plasma sheath.

\section{PLASMA PROBE}

The plasma probe is a device that has been widely used to measure the temperature and density of a plasma, both in the laboratory and in space. The electrostatic probe was originally developed by Langmuir and Mott-Smith, and the physical mechanism of its operation can be well explained using the theory of plasma sheaths presented in the previous section.

A conducting probe, or electrode, is immersed in a plasma and the current that flows through it is measured for various potentials applied to the probe. The temperature and number density of the electrons can be obtained from the characteristics of the resulting current-potential curve. When the surface of the probe is a plane, the current-potential curve has a shape like that illustrated in Fig. 4. 


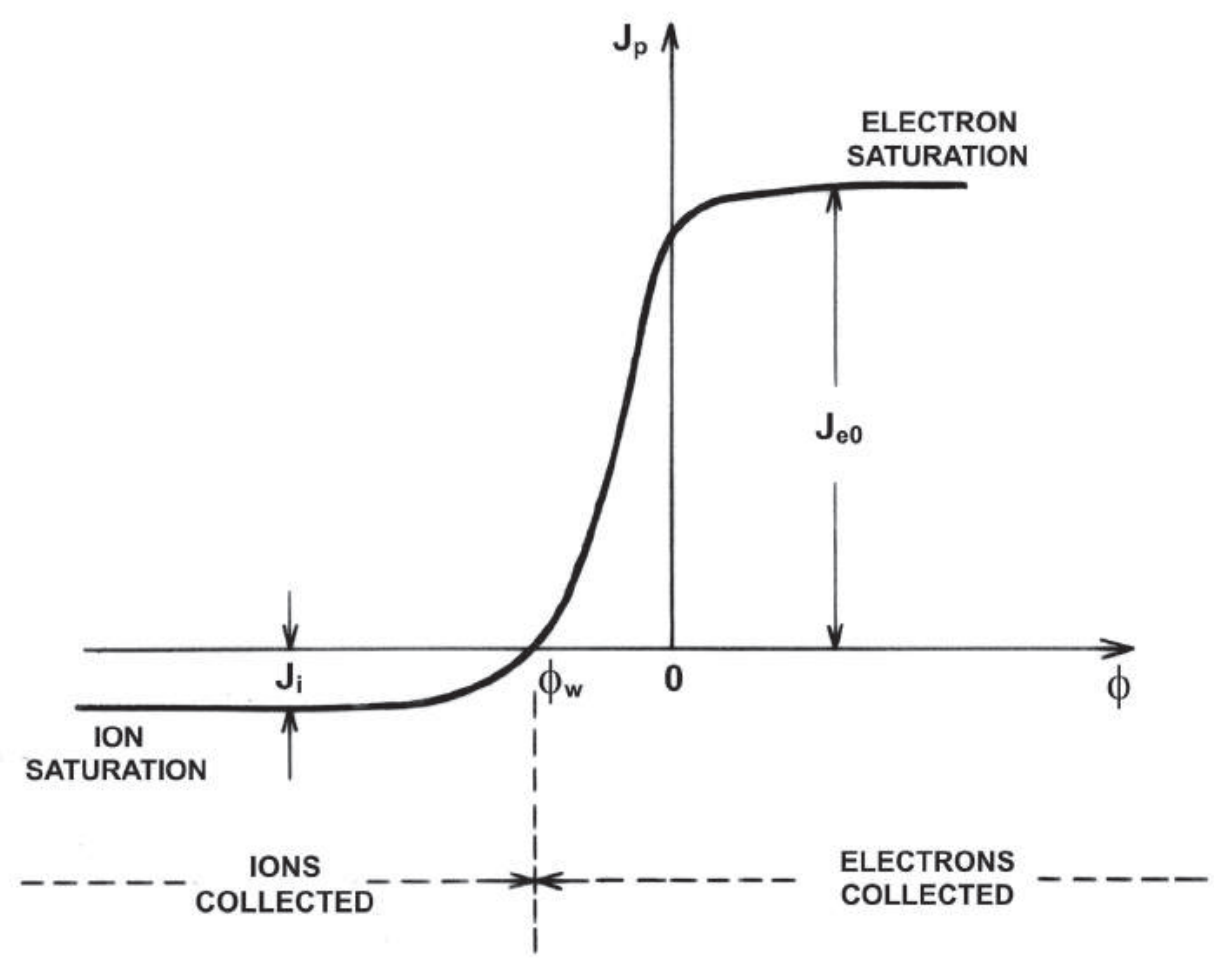

Fig. 4 Characteristics of the current-potential curve of an electrostatic plane probe immersed in a plasma. The probe floating potential, with reference to the plasma potential, is denoted by $\phi_{w}$.

The probe, when inserted in the plasma, is surrounded by a plasma sheath that shields the major portion of the plasma from the disturbing probe field. The thickness of the sheath is of the order of a Debye length. When no current flows through the electrode, it stays at the negative floating potential $\phi_{w}$, which is the wall potential discussed in the previous section. Under these equilibrium conditions, the number of electrons reaching the probe per unit time is equal to the number of positive ions reaching the probe per unit time. We assume the current to be positive when it flows in the direction away from the probe. The current associated with the electron flow is directed away from the probe and therefore it is considered positive. Consequently, the electric current associated with the flow of ions is negative. Under equilibrium conditions there is no net current flowing through the probe and its potential is the floating potential $\phi_{w}$. When the probe potential is made more negative than $\phi_{w}$, the 
electron current is reduced due to the increased repulsive force imposed by the probe electric field on the electrons. As the potential is made more negative, the contribution to the electric current arising from the electrons will eventually become negligible and the total electric current asymptotically approaches a constant negative value, corresponding to the electric current density $J_{i}$ associated with the flow of ions only. The ions that reach the edge of the plasma sheath fall into the potential well and their current is practically unaffected if the potential is made even more negative. On the other hand, when the probe potential is increased from the negative value $\phi_{w}$, more electrons reach the probe than ions per unit time due to the decrease in the repulsion force on the electrons, and the net electric current becomes positive. When the electric potential is zero, that is, when the probe is at the same potential as the plasma, there is no electric field near the electrode and, since the average thermal velocity of the electrons is much greater than that of the ions, the electron current density $J_{e 0}$ (for $\phi=0$ ) is much greater than the ion current density. If the potential is made sufficiently positive, a situation arises in which the current associated with the ions becomes negligible, but all the electrons that reach the edge of the sheath are collected by the probe. The electron current density reaches a fairly constant value for sufficiently high positive values of $\phi$. This plateau region in the probe current-potential curve is called the region of saturation of the electron current. For higher positive values of $\phi$ there are complications in the current-potential characteristic of the electrode due to the occurrence of another phenomenon.

An approximate expression for the magnitude of the electron current density, away from the region of saturation, can be obtained from (4.6) as

$$
J_{e}=J_{e 0} \exp \left(\frac{e \phi}{k T_{e}}\right)
$$

where $J_{e 0}$ is the electron current density when the electric potential is zero. Since for $\phi=0$ we have $\Gamma_{e}=n_{e}\langle v\rangle_{e} / 4$, and using (4.2) for the average electron speed, we obtain

$$
J_{e 0}=e n_{e}\left(\frac{k T_{e}}{2 \pi m_{e}}\right)^{1 / 2}
$$

where $n_{e}$ is the electron number density in the unperturbed plasma region. Note that when $\phi$ is negative the ions reaching the edge of the sheath continue to fall into the negative potential of the probe and hence the ion current density is a constant $\left(J_{i}\right)$ in the negative potential region. Thus, 
we can express the probe current density, in the region where $\phi<0$, as

$$
J_{p}=J_{e 0} \exp \left(\frac{e \phi}{k T_{e}}\right)-J_{i} \quad(\text { for } \phi<0)
$$

From this equation we can deduce the result

$$
T_{e}=\frac{e}{k}\left\{\frac{d}{d \phi}\left[\ln \left(J_{p}+J_{i}\right)\right]\right\}^{-1}
$$

This expression can be used to determine the electron temperature as follows. First, the electrode is made sufficiently negative with reference to the plasma potential, so that the current that flows through the probe is due to the ions only. The measurement of this current gives directly the value $J_{i}$. Then, the current-potential characteristic curve of the probe is measured and a plot of $\ln \left(J_{p}+J_{i}\right)$ as a function of $\phi$ is made. This curve has a straight-line section corresponding to the probe potential less than the plasma potential, and the slope of this straight line gives the value of $(d / d \phi)\left[\ln \left(J_{p}+J_{i}\right)\right]$ which, when substituted in (5.4), gives the electron temperature in the plasma.

After the electron temperature $T_{e}$ has been determined, we can evaluate the electron number density from (5.2), which can be written as

$$
n_{e}=\frac{J_{e 0}}{e}\left(\frac{2 \pi m_{e}}{k T_{e}}\right)^{1 / 2}
$$

The value of $J_{e 0}$ is determined by measuring the probe current corresponding to the plateau (electron saturation) region of the current-potential characteristic of the probe.

\section{PROBLEMS}

11.1 Consider a stationary plasma (electrons and one type of ions) under steady-state conditions at a uniform temperature $T_{0}$, when perturbed by a point charge $+Q$ placed at the origin of a coordinate system. Using the collisionless hydrodynamic equation for the electrons and ions $(\alpha=e, i)$

$$
\rho_{m \alpha} \frac{D \mathbf{u}_{\alpha}}{D t}=n_{\alpha} q_{\alpha} \mathbf{E}-\nabla p_{\alpha}
$$


with the ideal gas law $p_{\alpha}=n_{\alpha} k T_{0}$, and the Poisson equation

$$
\nabla^{2} \phi(\mathbf{r})=-\frac{\rho(\mathbf{r})}{\epsilon_{0}}
$$

obtain the following differential equation

$$
\nabla^{2} \phi(\mathbf{r})-\frac{2}{\lambda_{D}^{2}} \phi(\mathbf{r})=-\frac{Q}{\epsilon_{0}} \delta(\mathbf{r})
$$

for the Debye potential $\phi(\mathbf{r})$. Assume that the number density of each species can be expressed as $n_{\alpha}=n_{0}+n_{\alpha}^{\prime}$, where $n_{0}$ is constant and $\left|n_{\alpha}^{\prime}\right| \ll n_{0}$. What are the approximations necessary to obtain this result?

11.2 Analyze the Debye potential problem considering only the motion of the electrons (ions stay immobile) and show that in this case the differential equation for the electric potential $\phi(\mathbf{r})$ is

$$
\nabla^{2} \phi(\mathbf{r})-\frac{1}{\lambda_{D}^{2}} \phi(\mathbf{r})=-\frac{Q}{\epsilon_{0}} \delta(\mathbf{r})
$$

11.3 When the macroscopic neutrality of a plasma is instantaneously perturbed by external means, the electrons react in a such a way as to give rise to oscillations at the electron plasma frequency $\omega_{p e}=\left[n_{0} e^{2} /\left(m_{e} \epsilon_{0}\right)\right]^{1 / 2}$. Consider these oscillations, but including the motion of the ions. Show that in this case the natural frequency of oscillation of the net charge density is given by

$$
\omega=\left(\omega_{p e}^{2}+\omega_{p i}^{2}\right)^{1 / 2}
$$

where $\omega_{p i}=\left[n_{0} e^{2} /\left(m_{i} \epsilon_{0}\right)\right]^{1 / 2}$. Use the linearized equations of continuity and of momentum for each species, and Poisson equation, considering only the electric force due to the internal charge separation.

11.4 Evaluate the negative electrostatic potential $\phi_{w}$ that appears on an infinite plane wall immersed in a plasma consisting of electrons of charge $-e$ and ions of charge $Z e$, under steady-state conditions. Denote the electron and ion temperatures by $T_{e}$ and $T_{i}$, respectively.

11.5 Deduce an expression for the Debye potential for a test particle of charge $+Q$ immersed in a plasma consisting of electrons (charge $-e$ ) and ions of charge $Z e$, the temperature of the electrons and the ions being 
$T_{e}$ and $T_{i}$, respectively. Show that, if $T_{e} \gg T_{i}$, then the Debye length is governed by the ion temperature $T_{i}$.

11.6 Using the following expressions for the electron and ion number densities

$$
\begin{aligned}
& n_{e}(\mathbf{r})=n_{0} \exp \left[\frac{e \phi(\mathbf{r})}{k T}\right] \\
& n_{i}(\mathbf{r})=n_{0} \exp \left[-\frac{e \phi(\mathbf{r})}{k T}\right]
\end{aligned}
$$

in the plasma sheath region formed between an infinite plane and a semiinfinite plasma, deduce the differential equation satisfied by the electric potential $\phi(x)$ in the plasma sheath. Show that this differential equation can be written in the form

$$
\frac{d^{2} F}{d \xi^{2}}=\sinh (F)
$$

where $F=e \phi / k T$ and $\xi=\sqrt{2} x / \lambda_{D}$. Assuming that at $x=\infty$ we have $n_{e}=n_{i}=n_{0}, F=0$, and $d F / d \xi=0$, show that

$$
F(\xi)=4 \tanh ^{-1}\left\{\exp \left[-\left(\xi-\xi_{0}\right)\right]\right\}
$$

where $\xi_{0}$ is a constant. Denoting the potential at the wall by $\phi_{w}$ and assuming that $e \phi / k T \ll 1$, show that

$$
\phi(x)=\phi_{w} \exp \left(\frac{\sqrt{2} x}{\lambda_{D}}\right)
$$

with

$$
\phi_{w}=\frac{4 k T}{e} \exp \left(\xi_{0}\right)
$$

11.7 For the plasma sheath region formed in the vicinity of a plane wall immersed in a plasma, assume that the ions at the plasma edge of the sheath can be described by a shifted Maxwellian distribution function

$$
f_{i}(v)=n_{0}\left(\frac{m_{i}}{2 \pi k T}\right)^{3 / 2} \exp \left[-\frac{m_{i}\left(\mathbf{v}-\mathbf{u}_{0}\right)^{2}}{2 k T}\right]
$$

with drift velocity $\mathbf{u}_{0}=u_{0} \widehat{\mathbf{x}}$. Prove that the ion flux $\Gamma_{i x}$, at the edge of the sheath, is given by

$$
\Gamma_{i x}=n_{0}\left(\frac{k T}{2 \pi m_{i}}\right)^{1 / 2}\left\{\exp \left(-y^{2}\right)+y \sqrt{\pi}[1+\operatorname{erf}(y)]\right\}
$$


where $y=u_{0}\left[m_{i} /(2 k T)\right]^{1 / 2}$ and erf $(y)$ is the error function, defined by

$$
\operatorname{erf}(y)=\frac{2}{\sqrt{\pi}} \int_{0}^{y} \exp \left(-s^{2}\right) d s
$$

Calculate $d \Gamma_{i x} / d y$. Note that the error function vanishes for $y=0$, increases monotonically as $y$ increases, and tends asymptotically to unity as $y \rightarrow \infty$. Also note that

$$
\frac{d}{d y}[\operatorname{erf}(y)]=\frac{2}{\sqrt{\pi}} \exp \left(-y^{2}\right)
$$

11.8 From an experimental current-potential curve of a Langmuir probe of area $A$ immersed in a plasma, such as shown in Fig. 5, where the electric potentials are measured with respect to a fixed reference potential, explain how you can determine $J_{i}, J_{e o}$, the space potential $\phi_{s}$, the floating potential $\phi_{f}$ with respect to $\phi_{s}$ (note that $\left.\phi_{f}-\phi_{s}=\phi_{w}\right), T_{e}$, and $n_{e}$.

11.9 The Langmuir plasma probe has been widely used in satellites to measure space plasma properties. In one valuable technique, circuits are arranged that measure directly $d I_{p} / d \phi$ and $d^{2} I_{p} / d \phi^{2}$, where $I_{p}=J_{p} A$ and $A$ is the probe's area. Use (5.3) to show that

$$
\frac{\left(d I_{p} / d \phi\right)}{\left(d^{2} I_{p} / d \phi^{2}\right)}=\frac{k T_{e}}{e}
$$

which gives directly the electron temperature $T_{e}$. Next show that $J_{e 0}$ can be calculated from $T_{e}$ and $d I_{p} / d \phi$ at a known value of $\phi$, according to

$$
J_{e 0}=\frac{k T_{e}}{A e} \exp \left(-\frac{e \phi}{k T_{e}}\right) \frac{d I_{p}}{d \phi}
$$

The electron density $n_{e}$ can then be calculated from (5.5).

11.10 An electron gas (Lorentz gas), in a background of stationary ions, is acted upon by a weak, externally applied electric field $\mathbf{E}$, under steadystate conditions. Using the Boltzmann equation for the electrons, with the relaxation model for the collision term (considering a constant collision frequency $\nu$ ),

$$
\left(\frac{\delta f}{\delta t}\right)_{c o l l}=-\nu\left(f-f_{0}\right)
$$




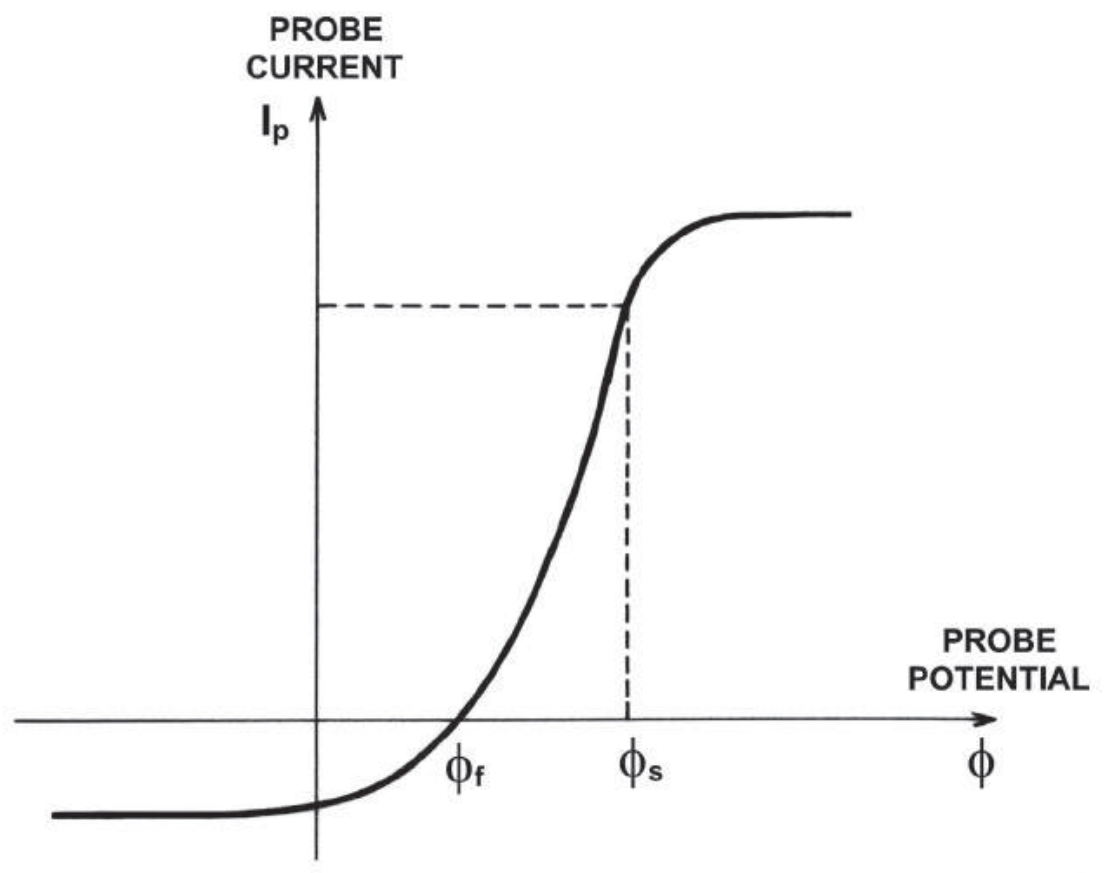

Fig. 5 Typical current-potential curve of a plane Langmuir probe immersed in a plasma.

and considering the adiabatic case for which

$$
n(\mathbf{r})[T(\mathbf{r})]^{-3 / 2}=\text { constant }
$$

show that the electron distribution function is given by

$$
f=f_{0}\left\{1-\frac{1}{\nu}\left[\frac{m v^{2}}{2 k T}\left(\mathbf{v} \cdot \frac{\nabla T}{T}\right)+\frac{e}{k T}(\mathbf{v} \cdot \mathbf{E})\right]\right\}
$$

Assume that $f=f_{0}+f_{1}$, where $\left|f_{1}\right| \ll f_{0}$ and where $f_{0}$ is the following modified Maxwellian distribution

$$
f_{0}(\mathbf{r}, v)=n(\mathbf{r})\left[\frac{m}{2 \pi k T(\mathbf{r})}\right]^{3 / 2} \exp \left[-\frac{m v^{2}}{2 k T(\mathbf{r})}\right]
$$

and neglect all second-order terms in the Boltzmann equation. Consider the term involving $\nabla f_{1}$ as a second-order quantity. 
11.11 Using the distribution function of the previous problem, evaluate the electric current density $\mathbf{J}$ to show that the presence of a temperature gradient gives rise to an electric current associated with thermoelectric effects.

11.12 Consider problem 11.10, but taking $\mathbf{E}=0$ and, instead of the adiabatic case, consider a constant kinetic pressure

$$
p=n(\mathbf{r}) k T(\mathbf{r})=\text { constant }
$$

(a) Show that the electron distribution function is given by

$$
f=f_{0}\left[1-\frac{1}{\nu}\left(-\frac{5}{2}+\frac{m v^{2}}{2 k T}\right) \mathbf{v} \cdot \frac{\nabla T}{T}\right]
$$

(b) Evaluate the heat flux vector $\mathbf{q}$ and show that it can be written as

$$
\mathbf{q}=-K \nabla T(\mathbf{r})
$$

where the thermal conductivity $K$ is equal to $5 k p /(2 m \nu)$.

(c) What is the value of the electric current density $\mathbf{J}$ in this case?

11.13 In the previous problem, consider that $n=$ constant and that $f_{0}$ is the following modified Maxwell-Boltzmann distribution function:

$$
f_{0}(\mathbf{r}, v)=n\left[\frac{m}{2 \pi k T(\mathbf{r})}\right]^{3 / 2} \exp \left[-\frac{m v^{2}}{2 k T(\mathbf{r})}\right]
$$

Calculate the electron distribution function $f(\mathbf{r}, \mathbf{v})$ and show that the heat flux vector is given by

$$
\mathbf{q}=-K \nabla T(\mathbf{r})
$$

Determine the expression for the thermal conductivity $K$.

11.14 In problem 11.12, include the presence of an external magnetostatic field $\mathbf{B}$ in the $z$ direction and deduce the following expression for the nonequilibrium distribution function:

$$
\begin{gathered}
f=f_{0}\left\{1-\frac{1}{\nu}\left(-\frac{5}{2}+\frac{m v^{2}}{2 k T}\right)\left[\frac{\nu \Omega_{c e}}{\left(\nu^{2}+\Omega_{c e}^{2}\right)} v_{x}\left(\frac{\nu}{\Omega_{c e}} \widehat{\mathbf{x}}-\widehat{\mathbf{y}}\right)+\right.\right. \\
\left.\left.\frac{\nu \Omega_{c e}}{\left(\nu^{2}+\Omega_{c e}^{2}\right)} v_{y}\left(\widehat{\mathbf{x}}+\frac{\nu}{\Omega_{c e}} \widehat{\mathbf{y}}\right)+v_{z} \widehat{\mathbf{z}}\right] \cdot \frac{\nabla T}{T}\right\}
\end{gathered}
$$


Show that the heat flux vector $\mathbf{q}$ can be expressed as

$$
\mathbf{q}=-\mathcal{K} \cdot \nabla T(\mathbf{r})
$$

where $\mathcal{K}$ is the dyadic thermal conductivity, which in matrix form can be written as

$$
\mathcal{K}=\left(\begin{array}{ccc}
K_{\perp} & -K_{H} & 0 \\
K_{H} & K_{\perp} & 0 \\
0 & 0 & K_{\|}
\end{array}\right)
$$

with

$$
\begin{aligned}
K_{\perp} & =\frac{\nu^{2}}{\left(\nu^{2}+\Omega_{c e}^{2}\right)} K_{0} \\
K_{H} & =\frac{\nu \Omega_{c e}}{\left(\nu^{2}+\Omega_{c e}^{2}\right)} K_{0} \\
K_{\|} & =\frac{5 k p}{2 m \nu} \equiv K_{0}
\end{aligned}
$$

Note: The solution of the differential equation

$$
\frac{d f_{1}}{d \phi}+\frac{\nu}{\Omega_{c e}} f_{1}=-\frac{1}{\Omega_{c e}} \mathbf{v} \cdot \nabla f_{0}
$$

is given by

$$
f_{1}=-\exp \left(-\frac{\nu}{\Omega_{c e}} \phi\right) \frac{1}{\Omega_{c e}} \int_{-\infty}^{\phi} d \phi^{\prime} \exp \left(\frac{\nu}{\Omega_{c e}} \phi^{\prime}\right) \mathbf{v} \cdot \nabla f_{0}
$$

11.15 The coefficient of viscosity $\eta$ is defined as the shear stress produced by unit velocity gradient. For the $p_{x z}$ component of the kinetic pressure dyad, for example, we have

$$
p_{x z}=-\eta \frac{d}{d z} u_{x}(z)
$$

Assume the following form for the equilibrium velocity distribution function of the electrons

$$
f_{0}(\mathbf{r}, \mathbf{v})=n\left(\frac{m}{2 \pi k T}\right)^{3 / 2} \exp \left[-\frac{m}{2 k T}\left\{\left[v_{x}-u_{x}(z)\right]^{2}+v_{y}^{2}+v_{z}^{2}\right\}\right]
$$

which indicates the presence of an average velocity $u_{x}(z)$ in the $x$ direction having a gradient in the $z$ direction. In the absence of external forces and 
using the relaxation model for the collision term with a constant collision frequency $\nu$, let $f=f_{0}+f_{1}$ with $\left|f_{1}\right| \ll f_{0}$ in the Boltzmann equation, under steady-state conditions, to show that

$$
f_{1}(\mathbf{r}, \mathbf{v})=-\frac{m}{k T \nu} f_{0}(\mathbf{r}, \mathbf{v}) v_{z}\left[v_{x}-u_{x}(z)\right] \frac{\partial}{\partial z} u_{x}(z)
$$

Next, calculate $p_{x z}$ and show that the coefficient of viscosity is given by

$$
\eta=\frac{n k T}{\nu}
$$




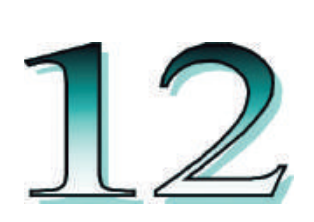

\section{SIMPLE APPLICATIONS OF MAGNETOHYDRODYNAMICS}

\section{FUNDAMENTAL EQUATIONS OF MAGNETOHYDRODYNAMICS}

The basic equations governing the behavior of a conducting fluid have been presented and discussed in Chapter 9. For convenience, we reproduce here the simplified form of the magnetohydrodynamic (MHD) equations. They include the equation of continuity for the whole conducting fluid

$$
\frac{\partial \rho_{m}}{\partial t}+\nabla \cdot\left(\rho_{m} \mathbf{u}\right)=0
$$

the equation of motion in the form

$$
\rho_{m} \frac{D \mathbf{u}}{D t}=\mathbf{J} \times \mathbf{B}-\nabla p
$$

and the adiabatic equation of conservation of energy

$$
\nabla p=V_{s}^{2} \nabla \rho_{m}
$$

where $\rho_{m}$ denotes the total mass density, $\mathbf{u}$ is the average fluid velocity, $\mathbf{J}$ is the electric current density, $\mathbf{B}$ is the magnetic flux density, $p$ is the total scalar pressure, and $V_{s}$ is the adiabatic sound speed, which is equal to $\left(\gamma p / \rho_{m}\right)^{1 / 2}$, where $\gamma$ is the ratio of the specific heats at constant pressure and at constant volume. To these equations we must add Maxwell curl equations, in the following reduced form,

$$
\nabla \times \mathbf{B}=\mu_{0} \mathbf{J}
$$




$$
\nabla \times \mathbf{E}=-\frac{\partial \mathbf{B}}{\partial t}
$$

and the generalized Ohm's law, in the simplified form,

$$
\mathbf{J}=\sigma_{0}(\mathbf{E}+\mathbf{u} \times \mathbf{B})
$$

where $\sigma_{0}$ denotes the electric conductivity of the fluid, and $\mathbf{E}$ is the electric field.

In this closed set of simplified MHD equations it has been assumed that macroscopic electrical neutrality is maintained to a high degree of approximation, so that the electric charge density $\rho$ vanishes. As discussed in section 6 of Chapter 9, the term $\partial \mathbf{E} / \partial t$, in Maxwell equation (1.4), can be neglected for the case of very low frequency phenomena and highly conducting fluids. As far as the generalized Ohm's law (1.6) is concerned, it is assumed that the time derivatives and pressure gradients are negligible, even though these terms are retained in some of the other MHD equations. Also, viscosity and thermal conductivity are neglected and the pressure dyad reduces to a scalar pressure.

The advantage of this approximate set of equations is that they reduce substantially the mathematical complexity of the more general equations for a conducting fluid and therefore facilitate the understanding of the physical processes that take place in highly conducting fluids at very low frequencies.

\subsection{Parker Modified Momentum Equation}

In the presence of a strong magnetic field the pressure tensor of an inviscid conducting fluid is anisotropic. When the cyclotron frequency is much larger than the collision frequency, a charged particle gyrates many times around a magnetic force line during the time between collisions, so that there is equipartition between the particle kinetic energies in the two independent directions normal to $\mathbf{B}$ but not, in general, in the direction along $\mathbf{B}$. If we denote by $p_{\perp}$ and $p_{\|}$the scalar pressures in the plane normal to $\mathbf{B}$ and along $\mathbf{B}$, respectively, and consider a local coordinate system in which the third axis is in the direction of $\mathbf{B}$, we can write the pressure tensor of an inviscid fluid as

$$
\mathcal{P}=\left(\begin{array}{ccc}
p_{\perp} & 0 & 0 \\
0 & p_{\perp} & 0 \\
0 & 0 & p_{\|}
\end{array}\right)
$$


Note that the parallel and perpendicular indexes used here do not refer to vector components but only indicate the part of the scalar pressures associated with the kinetic energy densities of the particle motions along $\mathbf{B}$ and in the plane perpendicular to $\mathbf{B}$, respectively.

When the magnetic field is not constant, the orientation of the axes of the local coordinate system changes from point to point and this change in direction must be taken into account when evaluating the divergence of the pressure tensor. We can express $\mathcal{P}$, in (1.7), as the sum of a hydrostatic scalar pressure $p_{\perp}$ and another tensor referred to the local coordinate system, as

$$
\mathcal{P}=p_{\perp} \mathbf{1}+\left(p_{\|}-p_{\perp}\right) \widehat{\mathbf{B}} \widehat{\mathbf{B}}
$$

where $\mathbf{1}$ is the unit dyad

$$
\mathbf{1}=\left(\begin{array}{lll}
1 & 0 & 0 \\
0 & 1 & 0 \\
0 & 0 & 1
\end{array}\right)
$$

and $\widehat{\mathbf{B}} \widehat{\mathbf{B}}=\mathbf{B B} / B^{2}$ is the dyad formed from the unit vector $\widehat{\mathbf{B}}$,

$$
\widehat{\mathbf{B}} \widehat{\mathbf{B}}=\left(\begin{array}{lll}
0 & 0 & 0 \\
0 & 0 & 0 \\
0 & 0 & 1
\end{array}\right)
$$

The momentum equation, in the form (1.2), must be modified to include the anisotropy of the pressure dyad. Thus, we write

$$
\rho_{m} \frac{D \mathbf{u}}{D t}=\mathbf{J} \times \mathbf{B}-\nabla \cdot \mathcal{P}
$$

To evaluate $\nabla \cdot \mathcal{P}$, with $\mathcal{P}$ as given by (1.8), we note that

$$
\nabla \cdot\left(p_{\perp} \mathbf{1}\right)=\nabla p_{\perp}
$$

and using the following identity,

$$
\begin{gathered}
\nabla \cdot\left[\left(p_{\|}-p_{\perp}\right) \widehat{\mathbf{B}} \widehat{\mathbf{B}}\right]=(\mathbf{B} \cdot \nabla)\left[\left(p_{\|}-p_{\perp}\right) \frac{\mathbf{B}}{B^{2}}\right]+ \\
{\left[\left(p_{\|}-p_{\perp}\right) \frac{\mathbf{B}}{B^{2}}\right](\nabla \cdot \mathbf{B})}
\end{gathered}
$$


where the second term in the right-hand side vanishes in virtue of $\nabla \cdot \mathbf{B}=0$, we obtain

$$
\nabla \cdot \mathcal{P}=\nabla p_{\perp}+(\mathbf{B} \cdot \nabla)\left[\left(p_{\|}-p_{\perp}\right) \frac{\mathbf{B}}{B^{2}}\right]
$$

Furthermore, using Maxwell equation (1.4) we can write the magnetic force per unit volume as

$$
\mathbf{J} \times \mathbf{B}=\frac{1}{\mu_{0}}(\nabla \times \mathbf{B}) \times \mathbf{B}
$$

The term in the right-hand side can be expanded, using a vector identity, with the result

$$
\mathbf{J} \times \mathbf{B}=\frac{1}{\mu_{0}}\left[(\mathbf{B} \cdot \nabla) \mathbf{B}-\nabla\left(\frac{1}{2} B^{2}\right)\right]
$$

Substituting expressions (1.14) and (1.16) into the momentum equation (1.11), we obtain, finally,

$$
\rho_{m} \frac{D \mathbf{u}}{D t}=-\nabla\left(p_{\perp}+\frac{B^{2}}{2 \mu_{0}}\right)+(\mathbf{B} \cdot \nabla)\left[\frac{\mathbf{B}}{\mu_{0}}-\frac{\left(p_{\|}-p_{\perp}\right) \mathbf{B}}{B^{2}}\right]
$$

This equation differs from the usual momentum equation (1.2), for a highly conducting inviscid fluid, only through the term $\left(p_{\|}-p_{\perp}\right) / B^{2}$. It was derived, although in a quite different way, by E.N. Parker in 1957 and, for this reason, it is usually referred to as the Parker modified momentum equation.

\subsection{The Double Adiabatic Equations of Chew, Goldberger, and Low (CGL)}

To complement the momentum equation (1.17), we need equations for the time rate of change of $p_{\|}$and $p_{\perp}$. These equations will take the place of the familiar adiabatic energy equation (1.3), which applies for the isotropic case. From the general energy equation (9.4.14) (Eq. 4.14 in Chapter 9) for a conducting fluid, if we do not take into account heat conduction and joule heating, we have

$$
\frac{D}{D t}\left(\frac{3 p}{2}\right)+\left(\frac{3 p}{2}\right)(\nabla \cdot \mathbf{u})+(\mathcal{P} \cdot \nabla) \cdot \mathbf{u}=0
$$

with the pressure dyad $\mathcal{P}$ as given by (1.8) and where the scalar pressure $p$ is one-third the trace of $\mathcal{P}$, that is,

$$
p=\frac{1}{3}\left(2 p_{\perp}+p_{\|}\right)
$$


Note that $3 p / 2$ represents the total thermal energy density. By direct expansion, using (1.8) for $\mathcal{P}$, we find that

$$
(\mathcal{P} \cdot \nabla) \cdot \mathbf{u}=\left[p_{\perp} \nabla+\left(p_{\|}-p_{\perp}\right)(\widehat{\mathbf{B}} \widehat{\mathbf{B}} \cdot \nabla)\right] \cdot \mathbf{u}
$$

and taking this expression, together with (1.19), into (1.18), we obtain

$$
\frac{D}{D t}\left(2 p_{\perp}+p_{\|}\right)+\left(p_{\|}+4 p_{\perp}\right) \nabla \cdot \mathbf{u}+2\left(p_{\|}-p_{\perp}\right)(\widehat{\mathbf{B}} \widehat{\mathbf{B}} \cdot \nabla) \cdot \mathbf{u}=0
$$

A strong magnetic field constrains the charged particle motion only in the direction transverse to $\mathbf{B}$, but the particles are still free to move large distances along $\mathbf{B}$. Thus, it is reasonable to suppose that the contribution to the total thermal energy, arising from the particle motion parallel to $\mathbf{B}$, also satisfies an energy conservation equation similar to (1.18). This leads to the following equation for the part of the total thermal energy due to the random particle motions along $\mathbf{B}$ :

$$
\frac{D p_{\|}}{D t}+p_{\|} \nabla \cdot \mathbf{u}+2 p_{\|}(\widehat{\mathbf{B}} \widehat{\mathbf{B}} \cdot \nabla) \cdot \mathbf{u}=0
$$

Therefore, decoupling the parallel and perpendicular motions, the equation for $p_{\perp}$ becomes

$$
\frac{D p_{\perp}}{D t}+2 p_{\perp} \nabla \cdot \mathbf{u}-p_{\perp}(\widehat{\mathbf{B}} \widehat{\mathbf{B}} \cdot \nabla) \cdot \mathbf{u}=0
$$

Equations (1.21) and (1.22) can also be obtained from an energy equation of higher order than (9.4.14), involving the total time rate of change of the pressure dyad $\mathcal{P}$. When this equation, involving $D \mathcal{P} / D t$, is contracted with the unit dyad $\mathbf{1}$ we obtain (1.21), and when contracted with the dyad $\widehat{\mathbf{B}} \widehat{\mathbf{B}}$ results in (1.22).

Equations (1.22) and (1.23) enable $p_{\|}$and $p_{\perp}$ to be calculated. They can be written in a more succinct form, as follows. First we note that using Maxwell curl equation

$$
\nabla \times \mathbf{E}=-\frac{\partial \mathbf{B}}{\partial t}
$$

and considering a perfectly conducting fluid for which

$$
\mathbf{E}+\mathbf{u} \times \mathbf{B}=0
$$

we have

$$
\frac{\partial \mathbf{B}}{\partial t}=\nabla \times(\mathbf{u} \times \mathbf{B})
$$


Expanding the right-hand side using the vector identity $\nabla \times(\mathbf{u} \times \mathbf{B})=$ $(\mathbf{B} \cdot \nabla) \mathbf{u}-\mathbf{B}(\nabla \cdot \mathbf{u})-(\mathbf{u} \cdot \nabla) \mathbf{B}+\mathbf{u}(\nabla \cdot \mathbf{B})$, and noting that $\nabla \cdot \mathbf{B}=0$, we obtain

$$
\frac{D \mathbf{B}}{D t}=(\mathbf{B} \cdot \nabla) \mathbf{u}-\mathbf{B}(\nabla \cdot \mathbf{u})
$$

If we now take the scalar product of (1.27) with $\mathbf{B} / B^{2}$, we obtain

$$
\frac{1}{2 B^{2}} \frac{D\left(B^{2}\right)}{D t}=\widehat{\mathbf{B}} \cdot(\widehat{\mathbf{B}} \cdot \nabla) \mathbf{u}-\nabla \cdot \mathbf{u}
$$

which may be written as

$$
\frac{1}{B} \frac{D B}{D t}=(\widehat{\mathbf{B}} \widehat{\mathbf{B}} \cdot \nabla) \cdot \mathbf{u}-\nabla \cdot \mathbf{u}
$$

Furthermore, from the equation of continuity (1.1), we get

$$
\nabla \cdot \mathbf{u}=-\frac{1}{\rho_{m}} \frac{D \rho_{m}}{D t}
$$

and using (1.29) and (1.30), to eliminate $(\widehat{\mathbf{B}} \widehat{\mathbf{B}} \cdot \nabla) \cdot \mathbf{u}$ and $\nabla \cdot \mathbf{u}$ in $(1.22)$ and (1.23), we obtain

$$
\begin{aligned}
& \frac{1}{p_{\|}} \frac{D p_{\|}}{D t}-\frac{3}{\rho_{m}} \frac{D \rho_{m}}{D t}+\frac{2}{B} \frac{D B}{D t}=0 \\
& \frac{1}{p_{\perp}} \frac{D p_{\perp}}{D t}-\frac{1}{\rho_{m}} \frac{D \rho_{m}}{D t}-\frac{1}{B} \frac{D B}{D t}=0
\end{aligned}
$$

These two equations can be written in compact form as

$$
\begin{aligned}
& \frac{D}{D t}\left(\frac{p_{\|} B^{2}}{\rho_{m}^{3}}\right)=0 \\
& \frac{D}{D t}\left(\frac{p_{\perp}}{\rho_{m} B}\right)=0
\end{aligned}
$$

They are known as the double adiabatic equations for a conducting fluid in a strong magnetic field and are due to G.F. Chew, M.L. Goldberger, and F.E. Low (1956). They are also known as the CGL equations. They take the place of the adiabatic energy equation for isotropic plasmas:

$$
\frac{D}{D t}\left(p \rho_{m}^{-\gamma}\right)=0
$$




\subsection{Special Cases of the Double Adiabatic Equations}

As a simple application of the double adiabatic equations, consider initially the case in which the only variations are parallel to the magnetic field as, for example, in sound waves traveling along the field lines. This situation is usually referred to as linear compression parallel to the magnetic field or one-dimensional compression. The magnetic field is assumed to be straight and uniform, and directed along the $z$ axis. Thus, $B_{x}=B_{y}=0$ and $\mathbf{B}=B_{z} \widehat{\mathbf{z}}$, as well as $\partial / \partial x=\partial / \partial y=0$. In this case, we find

$$
(\widehat{\mathbf{B}} \widehat{\mathbf{B}} \cdot \nabla) \cdot \mathbf{u}=\frac{\partial u_{z}}{\partial z}=\nabla \cdot \mathbf{u}
$$

and from (1.29), we see that $B$ stays constant. Equations (1.31) and (1.32), with $D B / D t=0$, then yield

$$
\begin{aligned}
& \frac{D}{D t}\left(\frac{p_{\|}}{\rho_{m}^{3}}\right)=0 \\
& \frac{D}{D t}\left(\frac{p_{\perp}}{\rho_{m}}\right)=0
\end{aligned}
$$

If we compare these results with (1.35), we find that $\gamma$ may be assigned the value 3 along the field lines (one-dimensional compression), and the value 1 across the field lines.

It is useful to introduce a parallel and a perpendicular temperature through the relations

$$
\begin{gathered}
p_{\|}=n k T_{\|} \\
p_{\perp}=n k T_{\perp}
\end{gathered}
$$

Therefore, for the case of one-dimensional compression parallel to $\mathbf{B}$, we have

$$
\begin{gathered}
T_{\|} \propto n^{2} \\
T_{\perp}=\text { constant }
\end{gathered}
$$

which shows that this type of compression is isothermal with respect to the perpendicular temperature $T_{\perp}$. The changes in $p_{\perp}$, therefore, are entirely due to the changes in the number density $n$, whereas those of $p_{\|}$are due to changes in both $n$ and $T_{\|}$.

Another special case of interest is the two-dimensional compression perpendicular to the magnetic field, in which all motion is transverse to the field lines. This situation can be pictured as the motion of magnetic flux 
tubes, identified by the particles contained in them. Assuming straight field lines along the $z$ axis $\left(B_{x}=B_{y}=0, \mathbf{B}=B_{z} \widehat{\mathbf{z}}\right)$ and variations only in the transverse direction $(\partial / \partial z=0)$, we find

$$
(\widehat{\mathbf{B}} \widehat{\mathbf{B}} \cdot \nabla) \cdot \mathbf{u}=\left(\widehat{\mathbf{z}} \frac{\partial}{\partial z}\right) \cdot \mathbf{u}=0
$$

and (1.22) and (1.23) yield

$$
\begin{aligned}
& \frac{D p_{\|}}{D t}-\frac{p_{\|}}{\rho_{m}} \frac{D \rho_{m}}{D t}=0 \\
& \frac{D p_{\perp}}{D t}-\frac{2 p_{\perp}}{\rho_{m}} \frac{D \rho_{m}}{D t}=0
\end{aligned}
$$

Therefore, in the case of cylindrical compression perpendicular to $\mathbf{B}$ the adiabatic equations reduce to

$$
\begin{aligned}
& \frac{D}{D t}\left(\frac{p_{\|}}{\rho_{m}}\right)=0 \\
& \frac{D}{D t}\left(\frac{p_{\perp}}{\rho_{m}^{2}}\right)=0
\end{aligned}
$$

Comparing with (1.35), we see that $\gamma$ takes the value 1 parallel to the magnetic field and 2 transverse to it. From (1.39) and (1.40) we find that for a two-dimensional (cylindrically symmetric) compression perpendicular to B,

$$
\begin{gathered}
T_{\|}=\text {constant } \\
T_{\perp} \propto n
\end{gathered}
$$

so that this type of compression is isothermal with respect to the parallel temperature. The changes in $p_{\|}$are due entirely to variations in the number density $n$, whereas those of $p_{\perp}$ result from variations in $n$ as well as in $T_{\perp}$.

In the case of three-dimensional spherically symmetric compression, we have

$$
p_{\perp}=p_{\|}=p
$$

and (1.21) reduces to

$$
3 \frac{D p}{D t}-\frac{5 p}{\rho_{m}} \frac{D \rho_{m}}{D t}=0
$$


Thus, we obtain

$$
\frac{D}{D t}\left(\frac{p}{\rho_{m}^{5 / 3}}\right)=0
$$

which is the familiar adiabatic equation (1.35) of gas dynamics, with $\gamma=5 / 3$. In any of the cases of adiabatic compression, the fluid has to be subjected to a certain system of forces in order to achieve the desired type of adiabatic compression. The required system of forces has to be determined from the momentum equation in conjunction with the conditions appropriate to the particular problem under analysis.

\subsection{Energy Integral}

As a final consideration in this section, we will show that the system of hydromagnetic equations (1.1) to (1.6) possesses an energy integral. Using Maxwell equation (1.4), to substitute $\mathbf{J}$ in the equation of motion (1.2), yields

$$
\rho_{m} \frac{D \mathbf{u}}{D t}=\frac{1}{\mu_{0}}(\nabla \times \mathbf{B}) \times \mathbf{B}-\nabla p
$$

Now we take the dot product of this equation with $\mathbf{u}$,

$$
\rho_{m} \mathbf{u} \cdot \frac{D \mathbf{u}}{D t}=\frac{1}{\mu_{0}} \mathbf{u} \cdot(\nabla \times \mathbf{B}) \times \mathbf{B}-\mathbf{u} \cdot \nabla p
$$

The term on the left-hand side can be expanded as

$$
\begin{aligned}
\rho_{m} \mathbf{u} \cdot \frac{D \mathbf{u}}{D t}= & \rho_{m} \mathbf{u} \cdot\left[\frac{\partial \mathbf{u}}{\partial t}+(\mathbf{u} \cdot \nabla) \mathbf{u}\right]=\frac{1}{2} \rho_{m}\left[\frac{\partial u^{2}}{\partial t}+(\mathbf{u} \cdot \nabla) u^{2}\right]= \\
& \frac{\partial}{\partial t}\left(\frac{1}{2} \rho_{m} u^{2}\right)-\frac{1}{2} u^{2} \frac{\partial \rho_{m}}{\partial t}+\frac{1}{2} \rho_{m}(\mathbf{u} \cdot \nabla) u^{2}
\end{aligned}
$$

Using the continuity equation (1.1) to eliminate $\partial \rho_{m} / \partial t$, yields

$$
\begin{gathered}
\rho_{m} \mathbf{u} \cdot \frac{D \mathbf{u}}{D t}=\frac{\partial}{\partial t}\left(\frac{1}{2} \rho_{m} u^{2}\right)+\frac{1}{2} u^{2} \nabla \cdot\left(\rho_{m} \mathbf{u}\right)+\frac{1}{2} \rho_{m}(\mathbf{u} \cdot \nabla) u^{2}= \\
\frac{\partial}{\partial t}\left(\frac{1}{2} \rho_{m} u^{2}\right)+\nabla \cdot\left(\frac{1}{2} \rho_{m} u^{2} \mathbf{u}\right)
\end{gathered}
$$

In order to transform the term $(\mathbf{u} \cdot \nabla) p$, let us write the adiabatic energy equation (1.35) as

$$
\rho_{m}^{-\gamma} \frac{D p}{D t}-\gamma p \rho_{m}^{-(\gamma+1)} \frac{D \rho_{m}}{D t}=0
$$


and use the continuity equation in the form

$$
\frac{D \rho_{m}}{D t}=-\rho_{m}(\nabla \cdot \mathbf{u})
$$

Combining these two equations, we obtain

$$
\frac{\partial p}{\partial t}+(\mathbf{u} \cdot \nabla) p+\gamma p(\nabla \cdot \mathbf{u})=0
$$

which may also be written as

$$
\frac{\partial p}{\partial t}+(1-\gamma)(\mathbf{u} \cdot \nabla) p+\gamma \nabla \cdot(p \mathbf{u})=0
$$

from which we get

$$
(\mathbf{u} \cdot \nabla) p=\frac{1}{(\gamma-1)} \frac{\partial p}{\partial t}+\frac{\gamma}{(\gamma-1)} \nabla \cdot(p \mathbf{u})
$$

Finally, for the $\mathbf{u} \cdot(\nabla \times \mathbf{B}) \times \mathbf{B}$ term in (1.54), considering a perfectly conducting fluid for which $\mathbf{E}=-\mathbf{u} \times \mathbf{B}$, and using a vector identity, we can write

$$
\begin{gathered}
\mathbf{u} \cdot(\nabla \times \mathbf{B}) \times \mathbf{B}=-(\mathbf{u} \times \mathbf{B}) \cdot(\nabla \times \mathbf{B})=\mathbf{E} \cdot(\nabla \times \mathbf{B})= \\
\mathbf{B} \cdot(\nabla \times \mathbf{E})-\nabla \cdot(\mathbf{E} \times \mathbf{B})
\end{gathered}
$$

Using Maxwell equation (1.5) we arrive at

$$
\frac{1}{\mu_{0}} \mathbf{u} \cdot(\nabla \times \mathbf{B}) \times \mathbf{B}=-\frac{\partial}{\partial t}\left(\frac{B^{2}}{2 \mu_{0}}\right)-\frac{1}{\mu_{0}} \nabla \cdot(\mathbf{E} \times \mathbf{B})
$$

Substituting (1.56), (1.61), and (1.63), into (1.54) yields the following energy conservation equation:

$$
\begin{gathered}
\frac{\partial}{\partial t}\left(\frac{1}{2} \rho_{m} u^{2}+\frac{p}{\gamma-1}+\frac{B^{2}}{2 \mu_{0}}\right)+ \\
\nabla \cdot\left(\frac{1}{2} \rho_{m} u^{2} \mathbf{u}+\frac{\gamma}{\gamma-1} p \mathbf{u}+\mathbf{E} \times \mathbf{H}\right)=0
\end{gathered}
$$

The first three terms in this equation represent the kinetic energy density associated with the macroscopic motion of the fluid, the thermal 
energy density, and the energy density stored in the magnetic field, respectively, whereas the last three terms denote the flux of macroscopic kinetic energy, the flux of thermal energy transported at the macroscopic mean velocity $\mathbf{u}$, and the flux of electromagnetic energy (given by the Poynting vector $\mathbf{E} \times \mathbf{H}$ ), respectively.

If we integrate (1.64) over the entire fluid-plus-vacuum volume and use Gauss's divergence theorem to transform the divergence term into a surface integral, we find that the first two terms in the surface integral vanish, since $\rho_{m}, p$, and $\mathbf{u}$ are zero outside the fluid. The remaining surface term is the surface integral of the Poynting vector, which, for an isolated system, also vanishes. Therefore, we obtain the following energy conservation integral:

$$
\int_{V}\left(\frac{1}{2} \rho_{m} u^{2}+\frac{p}{\gamma-1}+\frac{B^{2}}{2 \mu_{0}}\right) d^{3} r=\text { constant }
$$

The first integral represents the macroscopic kinetic energy of the fluid, the second term is the thermal free energy, and the last term represents the total energy of the magnetic field. It is usually useful to separate (1.65) into a kinetic energy part

$$
K=\int_{V} \frac{1}{2} \rho_{m} u^{2} d^{3} r
$$

and a potential energy part

$$
U=\int_{V}\left(\frac{p}{\gamma-1}+\frac{B^{2}}{2 \mu_{0}}\right) d^{3} r
$$

with the energy conservation law $K+U=$ constant. In these equations the integration extends over the entire fluid-plus-vacuum volume.

\section{MAGNETIC VISCOSITY AND REYNOLDS NUMBER}

The behavior of the magnetic field is of great importance in many MHD problems. To obtain a simple equation for the variations of $\mathbf{B}$, let us start by taking the curl of the generalized Ohm's law (1.6),

$$
\nabla \times \mathbf{J}=\sigma_{0}[\nabla \times \mathbf{E}+\nabla \times(\mathbf{u} \times \mathbf{B})]
$$

Replacing $\mathbf{J}$ and $\nabla \times \mathbf{E}$, using Maxwell curl equations (1.4) and (1.5),

$$
\nabla \times(\nabla \times \mathbf{B})=\mu_{0} \sigma_{0}\left[-\frac{\partial \mathbf{B}}{\partial t}+\nabla \times(\mathbf{u} \times \mathbf{B})\right]
$$


Making use of the following identity (with $\nabla \cdot \mathbf{B}=0$ ),

$$
\nabla \times(\nabla \times \mathbf{B})=-\nabla^{2} \mathbf{B}
$$

equation (2.2) reduces to

$$
\frac{\partial \mathbf{B}}{\partial t}=\nabla \times(\mathbf{u} \times \mathbf{B})+\eta_{m} \nabla^{2} \mathbf{B}
$$

where $\eta_{m}$ is called the magnetic viscosity,

$$
\eta_{m}=\frac{1}{\mu_{0} \sigma_{0}}
$$

The first term in the right-hand side of (2.4) is called the flow term, while the second term is called the diffusion term. To compare the relative magnitude of these two terms, we can use dimensional analysis and take, approximately,

$$
\begin{gathered}
|\nabla \times(\mathbf{u} \times \mathbf{B})| \simeq \frac{u B}{L} \\
\eta_{m}\left|\nabla^{2} \mathbf{B}\right| \simeq \eta_{m} \frac{B}{L^{2}}
\end{gathered}
$$

where $L$ denotes some characteristic length for variation of the parameters. The ratio of the flow term to the diffusion term is called the magnetic Reynolds number and is given by

$$
R_{m}=\frac{u L}{\eta_{m}}
$$

In most MHD problems one or the other of these two terms is of predominant importance and $R_{m}$ is either very large or very small compared to unity.

It is instructive to compare the magnetic viscosity $\left(\eta_{m}\right)$ and the magnetic Reynolds number $\left(R_{m}\right)$ with the ordinary hydrodynamic viscosity $\left(\eta_{k}\right)$ and hydrodynamic Reynolds number $(R)$. For this purpose, consider the Navier-Stokes equation of hydrodynamics,

$$
\frac{D \mathbf{u}}{D t}=\mathbf{f}-\frac{1}{\rho_{m}} \nabla p+\eta_{k}\left[\nabla^{2} \mathbf{u}+\frac{1}{3} \nabla(\nabla \cdot \mathbf{u})\right]
$$

where $\mathbf{f}$ denotes the average force per unit mass of the fluid, and $\eta_{k}$ is the kinematic viscosity (viscosity divided by density). Comparing this 
equation with (2.4) we see that the role played by $\eta_{m}$, in the rate of change of $\mathbf{B}$, is completely analogous to the role played by $\eta_{k}$, in the rate of change of the mean fluid velocity $\mathbf{u}$. The ordinary Reynolds number is defined as the ratio of the inertia term $(\mathbf{u} \cdot \nabla) \mathbf{u}$ to the viscosity term $\eta_{k} \nabla^{2} \mathbf{u}$. Using dimensional analysis, we have

$$
\begin{aligned}
& |(\mathbf{u} \cdot \nabla) \mathbf{u}| \simeq \frac{u^{2}}{L} \\
& \eta_{k}\left|\nabla^{2} \mathbf{u}\right| \simeq \eta_{k} \frac{u}{L^{2}}
\end{aligned}
$$

which gives the following expression (completely analogous to $R_{m}$ ) for the ordinary Reynolds number:

$$
R=\frac{u L}{\eta_{k}}
$$

\section{DIFFUSION OF MAGNETIC FIELD LINES}

When $R_{m} \ll 1$, that is when the diffusion term dominates, (2.4) becomes approximately,

$$
\frac{\partial \mathbf{B}}{\partial t}=\eta_{m} \nabla^{2} \mathbf{B} \quad\left(R_{m} \ll 1\right)
$$

This is the equation of diffusion of a magnetic field in a stationary conductor, resulting in the decay of the magnetic field. It is analogous to the particle diffusion equation studied in Chapter 10. The characteristic decay time of the magnetic field can be obtained by dimensional analysis, taking

$$
\begin{aligned}
|\partial \mathbf{B} / \partial t| & \simeq \frac{B}{\tau_{D}} \\
\left|\eta_{m} \nabla^{2} \mathbf{B}\right| & \simeq \eta_{m} \frac{B}{L^{2}}
\end{aligned}
$$

where $\tau_{D}$ represents a characteristic time for variation of the plasma parameters. Thus, according to (3.1), the magnetic field diffuses away with a characteristic decay time of the order of

$$
\tau_{D}=\frac{L^{2}}{\eta_{m}}=L^{2} \mu_{0} \sigma_{0}
$$

For ordinary conductors the time of decay is very small. If we consider, for example, a copper sphere of radius $1 \mathrm{~m}$, we find that $\tau_{D}$ is less than 10 seconds. For a celestial body, however, because of the large dimensions, the time of decay can be very large. For the Earth's core, considering it to be molten iron, the time of free decay is approximately 
$10^{4}$ years, while for the general magnetic field of the sun it is found to be of the order of $10^{10}$ years.

\section{FREEZING OF MAGNETIC FIELD LINES TO THE PLASMA}

A completely different type of behavior appears when $R_{m} \gg 1$. In this case, the flow term dominates over the diffusion term and (2.4) reduces to

$$
\frac{\partial \mathbf{B}}{\partial t}=\nabla \times(\mathbf{u} \times \mathbf{B}) \quad\left(R_{m} \gg 1\right)
$$

This equation implies that in a highly conducting fluid the magnetic field lines move along exactly with the fluid, rather than simply diffusing out. Alfvén has expressed this type of behavior by saying that the magnetic field lines are frozen in the conducting fluid. In effect, the fluid can flow freely along the magnetic field lines, but any motion of the conducting fluid, perpendicular to the field lines, carries them with the fluid.

In order to show this implication of (4.1), it is convenient to consider initially the concept of magnetic tubes of force that are used to visually describe the direction and magnitude of $\mathbf{B}$ at various points in space. One can think of the space pervaded by a magnetic field as divided into a large number of elementary magnetic tubes of force, all of them enclosing the same magnetic flux $\Delta \Phi_{B}$. If $\Delta S$ is the local cross-sectional area of an elementary magnetic tube of force (see Fig. 1), then the magnitude of $\mathbf{B}$, at the local point $P$, is equal to $\Delta \Phi_{B} / \Delta S$. According to this definition, the magnitude of $\mathbf{B}$ is everywhere inversely proportional to the cross-sectional area of the elementary tube of force.

Let us now consider a closed line whose points move with velocity $\mathbf{u}$ in a space pervaded by a magnetic field. Assume, for the moment, that $\mathbf{u}$ is an arbitrary function of position and time (not necessarily equal to the fluid velocity), with the result that the closed curve may change in shape, as well as undergo translational and rotational motion. Let $C_{1}$ denote this closed line at time $t$, bounding the open surface $\mathbf{S}(t)=\mathbf{S}_{1}$. At a time $\Delta t$ later, let $C_{2}$ and $\mathbf{S}(t+\Delta t)=\mathbf{S}_{2}$ denote the corresponding closed line and open surface (refer to Fig. 2). The flux of the magnetic field through an open surface $\mathbf{S}$, at time $t$, is given by

$$
\Phi_{B}(t)=\int_{S} \mathbf{B}(\mathbf{r}, t) \cdot d \mathbf{S}
$$




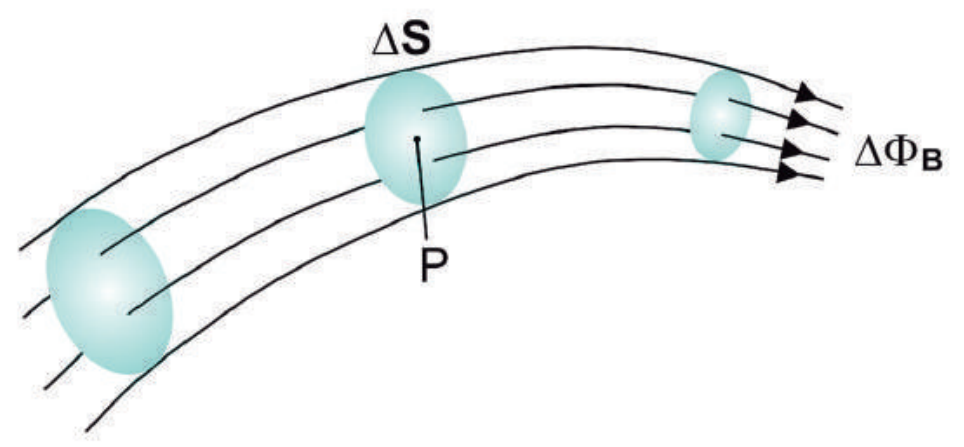

Fig. 1 Elementary magnetic tube of force. The magnitude of B at the point $P$ is equal to $\Delta \Phi_{B} / \Delta S$.

The rate of change of the magnetic flux through an open surface $\mathbf{S}$ can be written as

$$
\begin{gathered}
\frac{d}{d t}\left[\int_{S} \mathbf{B}(\mathbf{r}, t) \cdot d \mathbf{S}\right]= \\
\lim _{\Delta t \rightarrow 0} \frac{1}{\Delta t}\left[\int_{S_{2}} \mathbf{B}(\mathbf{r}, t+\Delta t) \cdot d \mathbf{S}-\int_{S_{1}} \mathbf{B}(\mathbf{r}, t) \cdot d \mathbf{S}\right]
\end{gathered}
$$

If we expand $\mathbf{B}(\mathbf{r}, t+\Delta t)$ in a Taylor series about $\mathbf{B}(\mathbf{r}, t)$, we obtain

$$
\mathbf{B}(\mathbf{r}, t+\Delta t)=\mathbf{B}(\mathbf{r}, t)+\frac{\partial \mathbf{B}(\mathbf{r}, t)}{\partial t} \Delta t+\cdots
$$

so that, in the limit as $\Delta t \rightarrow 0,(4.3)$ reduces to

$$
\begin{gathered}
\frac{d}{d t}\left[\int_{S} \mathbf{B}(\mathbf{r}, t) \cdot d \mathbf{S}\right]=\lim _{\Delta t \rightarrow 0}\left\{\int_{S_{2}} \frac{\partial \mathbf{B}(\mathbf{r}, t)}{\partial t} \cdot d \mathbf{S}+\right. \\
\left.\frac{1}{\Delta t}\left[\int_{S_{2}} \mathbf{B}(\mathbf{r}, t) \cdot d \mathbf{S}-\int_{S_{1}} \mathbf{B}(\mathbf{r}, t) \cdot d \mathbf{S}\right]\right\}
\end{gathered}
$$

To evaluate the term within brackets in the right-hand side of this equation, we can use the fact that for any closed surface at time $t$ we have, from Gauss's divergence theorem,

$$
\oint_{S} \mathbf{B} \cdot d \mathbf{S}=\int_{V} \nabla \cdot \mathbf{B} d^{3} r=0
$$




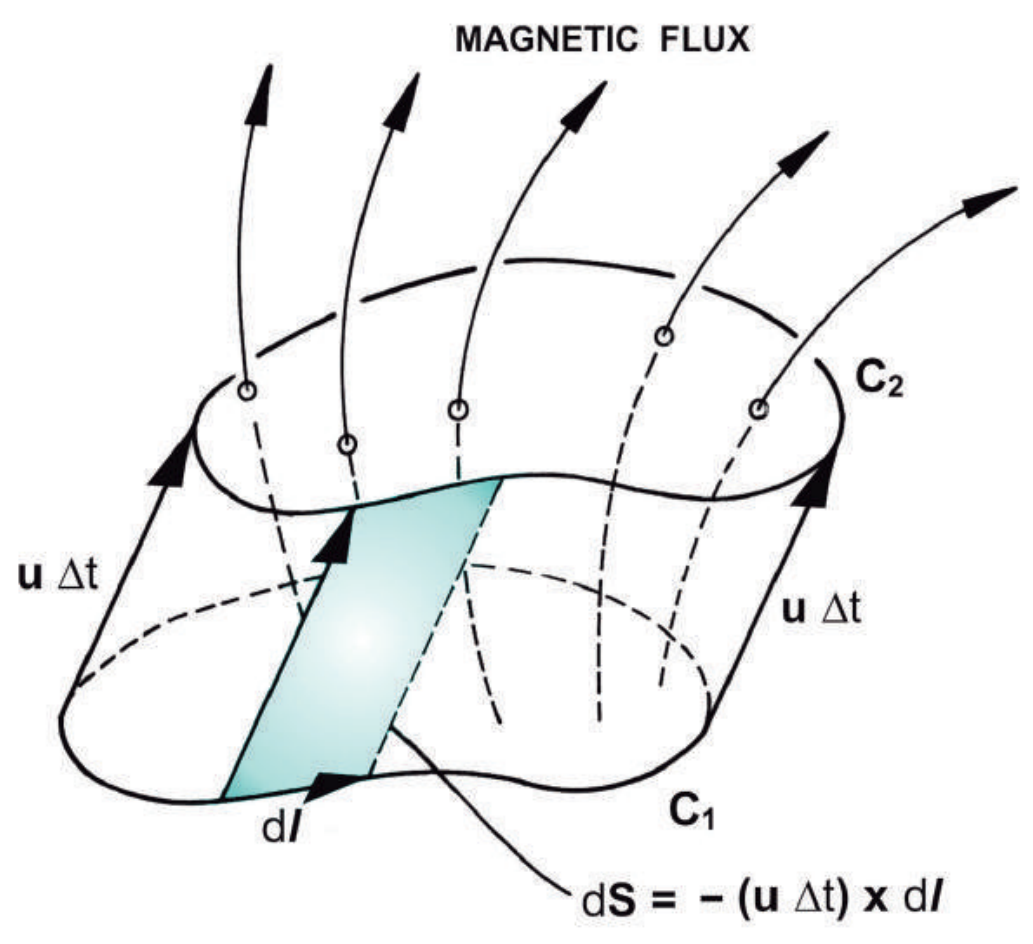

Fig. 2 A closed line bounding an open surface moving in a magnetic field with velocity $\mathbf{u}(\mathbf{r}, t)$, viewed at the instants of time $t$ and $t+\Delta t$. The shaded area is the part of the cylindrical surface described by an element $d \ell$ of the contour curve.

since $\nabla \cdot \mathbf{B}=0$. Thus, if we apply this result to the closed surface consisting of $\mathbf{S}_{1}, \mathbf{S}_{2}$, and the sides of the cylindrical surface of length $\mathbf{u} \Delta t$ shown in Fig. 2, we obtain

$$
-\int_{S_{1}} \mathbf{B}(\mathbf{r}, t) \cdot d \mathbf{S}+\int_{S_{2}} \mathbf{B}(\mathbf{r}, t) \cdot d \mathbf{S}-\oint_{C_{1}} \mathbf{B}(\mathbf{r}, t) \cdot[(\mathbf{u} \Delta t) \times d \mathbf{l}]=0
$$

where the minus sign in the first term on the left-hand side is due to the fact that the outwardly drawn unit normal to the surface $S_{1}$ is in a direction opposite to that of the surface $S_{2}$, and $-(\mathbf{u} \Delta t) \times d \mathbf{l}$ is the element of area (pointing outwards) covered by the vector element $d \mathbf{l}$ of the closed line in the time interval $\Delta t$. If (4.7) is substituted into (4.5) and the limit $\Delta t \rightarrow 0$ is evaluated, noting that in this limit $S_{2}=S_{1}=S(t)$, we obtain

$$
\frac{d}{d t}\left[\int_{S} \mathbf{B}(\mathbf{r}, t) \cdot d \mathbf{S}\right]=\int_{S} \frac{\partial \mathbf{B}(\mathbf{r}, t)}{\partial t} \cdot d \mathbf{S}+\oint_{C} \mathbf{B}(\mathbf{r}, t) \cdot(\mathbf{u} \times d \mathbf{l})
$$


The last term in the right-hand side of this equation can be transformed using the vector identity

$$
\mathbf{B}(\mathbf{r}, t) \cdot(\mathbf{u} \times d \mathbf{l})=-[\mathbf{u} \times \mathbf{B}(\mathbf{r}, t)] \cdot d \mathbf{l}
$$

and from Stokes's theorem we can write

$$
\oint_{C}[\mathbf{u} \times \mathbf{B}(\mathbf{r}, t)] \cdot d \mathbf{l}=\int_{S} \nabla \times[\mathbf{u} \times \mathbf{B}(\mathbf{r}, t)] \cdot d \mathbf{S}
$$

Thus, using this expression in (4.8), we obtain the general result

$$
\frac{d}{d t}\left[\int_{S} \mathbf{B}(\mathbf{r}, t) \cdot d \mathbf{S}\right]=\int_{S}\left\{\frac{\partial \mathbf{B}(\mathbf{r}, t)}{\partial t}-\nabla \times[\mathbf{u} \times \mathbf{B}(\mathbf{r}, t)]\right\} \cdot d \mathbf{S}
$$

Suppose now that the space is filled with a highly conducting fluid so that (4.1), which is valid for $R_{m} \gg 1$, applies. If the velocity $\mathbf{u}$ in (4.11) is taken as the fluid velocity, we conclude, from (4.1) and (4.11), that

$$
\frac{d}{d t}\left[\int_{S} \mathbf{B}(\mathbf{r}, t) \cdot d \mathbf{S}\right]=0
$$

which is a mathematical statement of the fact that the magnetic flux linked by a closed line (bounding the open surface $S$ ) moving with the fluid velocity $\mathbf{u}$ is constant. Note that this conclusion requires that only the velocity component of the closed line perpendicular to $\mathbf{B}$ be the same as the fluid velocity component perpendicular to $\mathbf{B}$, since the velocity component parallel to $\mathbf{B}$ gives no contribution to the term $\mathbf{u} \times \mathbf{B}$. Thus, (4.1) implies that the lines of magnetic flux are frozen into the highly conducting fluid and are carried by any motion of the fluid perpendicular to the field lines. There is no restriction, however, on the motion along the field lines so that the conducting fluid can flow freely in the direction parallel to $\mathbf{B}$.

This result is expected on physical grounds since, as the conducting fluid moves across the magnetic field, it induces an electric field that is proportional to the fluid velocity component perpendicular to B. However, if the fluid conductivity is infinite, this perpendicular velocity component must be infinitesimally small if the flow of electric current is to remain finite.

In a fluid of finite conductivity the result (4.12) is no longer true. Using (2.4) in the general result (4.11), yields

$$
\frac{d \Phi_{B}}{d t}=\frac{1}{\mu_{0} \sigma_{0}} \int_{S} \nabla^{2} \mathbf{B} \cdot d \mathbf{S}
$$

where the right-hand side of this equation gives rise to a slipping of magnetic flux through a closed material line. 


\section{MAGNETIC PRESSURE}

The concept of magnetic pressure is very useful in the study of hightemperature plasma confinement. Under steady-state conditions the MHD equations reduce to the following closed set of magnetohydrostatic equations:

$$
\begin{gathered}
\nabla p=\mathbf{J} \times \mathbf{B} \\
\nabla \times \mathbf{B}=\mu_{0} \mathbf{J} \\
\nabla \cdot \mathbf{B}=0
\end{gathered}
$$

If we eliminate $\mathbf{J}$ from these equations, we obtain the following equivalent set of magnetohydrostatic equations involving only $p$ and $\mathbf{B}$ :

$$
\begin{gathered}
\nabla p=\frac{1}{\mu_{0}}(\nabla \times \mathbf{B}) \times \mathbf{B} \\
\nabla \cdot \mathbf{B}=0
\end{gathered}
$$

The term in the right-hand side of (5.4) can be written as the divergence of the magnetic part of the electromagnetic stress dyad. Using the vector identity

$$
(\nabla \times \mathbf{B}) \times \mathbf{B}=(\mathbf{B} \cdot \nabla) \mathbf{B}-\frac{1}{2} \nabla\left(B^{2}\right)=\nabla \cdot(\mathbf{B B})-\nabla \cdot\left(\frac{1}{2} \mathbf{1} B^{2}\right)
$$

where $\mathbf{1}$ is the unit dyad, and using the following definition of the magnetic stress dyad,

$$
\mathcal{T}^{(m)}=\frac{1}{\mu_{0}}\left(\mathbf{B B}-\frac{1}{2} \mathbf{1} B^{2}\right)
$$

which written out in matrix form (in a Cartesian coordinate system) is

$$
\mathcal{T}^{(m)}=\frac{1}{\mu_{0}}\left(\begin{array}{ccc}
\left(B_{x}^{2}-B^{2} / 2\right) & B_{x} B_{y} & B_{x} B_{z} \\
B_{y} B_{x} & \left(B_{y}^{2}-B^{2} / 2\right) & B_{y} B_{z} \\
B_{z} B_{x} & B_{z} B_{y} & \left(B_{z}^{2}-B^{2} / 2\right)
\end{array}\right)
$$

we can write (5.4) as

$$
\nabla p=\nabla \cdot \mathcal{T}^{(m)}
$$

or, equivalently,

$$
\nabla \cdot\left(\mathbf{1} p-\mathcal{T}^{(m)}\right)=0
$$




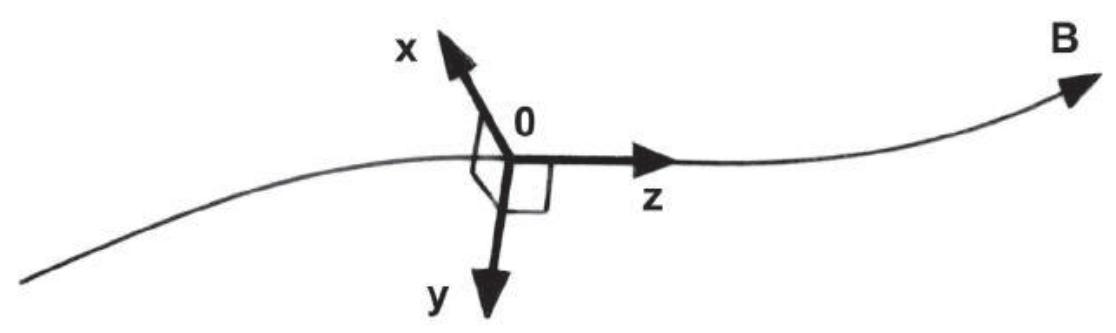

Fig. 3 Local magnetic coordinate system with the $z$ axis pointing along the local direction of $\mathbf{B}$.

The stress is considered to be positive if it is tensile, and negative if it is compressive. Thus, we see that $-\mathcal{T}^{(m)}$ may be defined as the magnetic pressure dyad, playing the same role as the fluid pressure dyad. Note that (5.10) is equivalent to equations (5.1) and (5.2).

It is instructive to consider a local magnetic coordinate system in which the third axis points along the local direction of $\mathbf{B}$, as shown in Fig. 3. For this local coordinate system, the off-diagonal elements of the magnetic stress dyad vanish, since $\mathbf{B}=B \widehat{\mathbf{z}}$, so that

$$
\mathcal{T}^{(m)}=\left(\begin{array}{ccc}
-B^{2} / 2 \mu_{0} & 0 & 0 \\
0 & -B^{2} / 2 \mu_{0} & 0 \\
0 & 0 & B^{2} / 2 \mu_{0}
\end{array}\right)
$$

Therefore, the principal stresses are equivalent to a tension $B^{2} / 2 \mu_{0}$ along the magnetic field lines, and a pressure $B^{2} / 2 \mu_{0}$ perpendicular to the magnetic field lines, similar to a mutual repulsion of the field lines. Alternatively, we can express (5.11) in the form

$$
\mathcal{T}^{(m)}=\left(\begin{array}{ccc}
0 & 0 & 0 \\
0 & 0 & 0 \\
0 & 0 & B^{2} / \mu_{0}
\end{array}\right)+\left(\begin{array}{ccc}
-B^{2} / 2 \mu_{0} & 0 & 0 \\
0 & -B^{2} / 2 \mu_{0} & 0 \\
0 & 0 & -B^{2} / 2 \mu_{0}
\end{array}\right)
$$

so that the stress caused by the magnetic flux can also be thought of as an isotropic magnetic pressure $B^{2} / 2 \mu_{0}$ and a tension $B^{2} / \mu_{0}$ along the magnetic flux lines as if they were elastic cords (see Fig. 4). This latter representation is very useful, since the isotropic pressure $B^{2} / 2 \mu_{0}$ can 


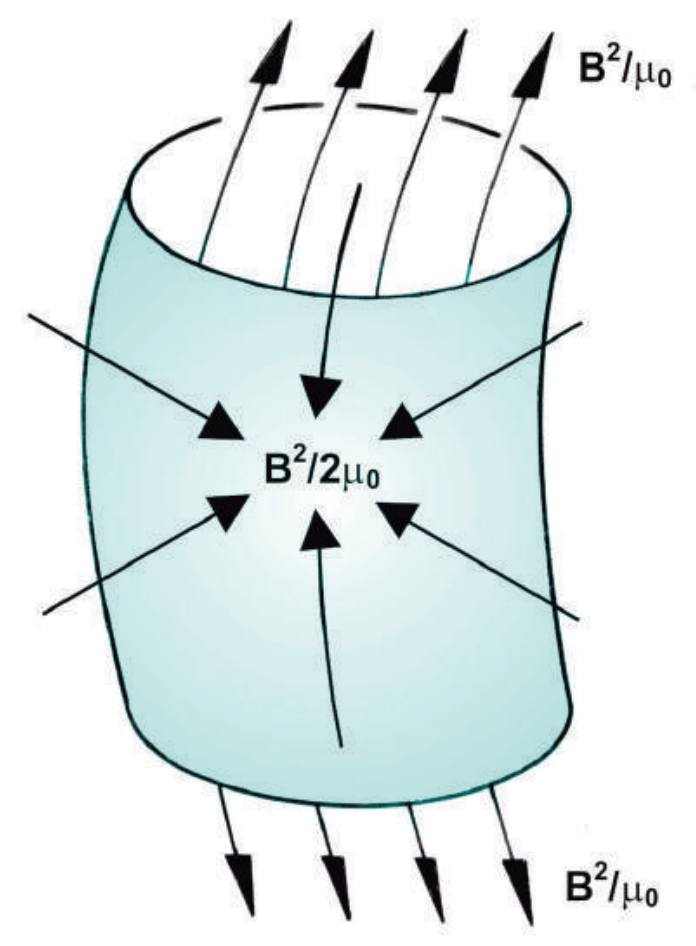

Fig. 4 The stress caused by the magnetic flux can be decomposed into an isotropic magnetic pressure $B^{2} / 2 \mu_{0}$ and a magnetic tension $B^{2} / \mu_{0}$ along the field lines.

always be superposed on the fluid pressure, resulting in a decrease in the pressure exerted by the fluid.

\section{ISOBARIC SURFACES}

It is convenient to consider in the plasma hypothetical surfaces over which the kinetic pressure is constant, called isobaric surfaces. At any point, the vector $\nabla p$ is normal to the isobaric surface passing through the point considered. From (5.1) we see that $\nabla p$ is normal to the plane containing $\mathbf{J}$ and $\mathbf{B}$, that is

$$
\begin{aligned}
& \mathbf{J} \cdot \nabla p=0 \\
& \mathbf{B} \cdot \nabla p=0
\end{aligned}
$$

Therefore, both $\mathbf{J}$ and $\mathbf{B}$ lie on isobaric surfaces. To illustrate this point, consider the particular case in which the isobaric surfaces are closed concentric cylindrical surfaces, with the kinetic pressure increasing in the 
direction towards the central axis of the concentric cylindrical surfaces. Thus, $\nabla p$ is along a radial line directed towards the axis. From (6.1) and (6.2) we see that neither $\mathbf{B}$ nor $\mathbf{J}$ passes through the isobaric surfaces and therefore it follows that the cylindrical isobaric surfaces are formed by a network of magnetic field lines and electric currents. Further, in view of (5.1), the magnetic field lines and electric currents, lying on the isobaric surfaces, must cross each other in such a way that $\mathbf{J} \times \mathbf{B}$ is equal to $\nabla p$. This situation is shown in Fig. 5. The maximum kinetic pressure occurs along the central axis, which also coincides with a magnetic field line. For this reason, this axis is usually called the magnetic axis of the magnetoplasma configuration.

\section{PLASMA CONFINEMENT IN A MAGNETIC FIELD}

The subject of plasma confinement by magnetic fields is of considerable interest in the theory of controlled thermonuclear fusion. Consider, for simplicity, the special case in which the magnetic field is along the $z$ axis, that is $\mathbf{B}=B \widehat{\mathbf{z}}$, so that (5.10) simplifies to

$$
\nabla \cdot\left(\begin{array}{ccc}
\left(p+B^{2} / 2 \mu_{0}\right) & 0 & 0 \\
0 & \left(p+B^{2} / 2 \mu_{0}\right) & 0 \\
0 & 0 & \left(p-B^{2} / 2 \mu_{0}\right)
\end{array}\right)=0
$$

from which we obtain

$$
\begin{aligned}
\frac{\partial}{\partial x}\left(p+\frac{B^{2}}{2 \mu_{0}}\right) & =0 \\
\frac{\partial}{\partial y}\left(p+\frac{B^{2}}{2 \mu_{0}}\right) & =0 \\
\frac{\partial}{\partial z}\left(p-\frac{B^{2}}{2 \mu_{0}}\right) & =0
\end{aligned}
$$

Also, from $\nabla \cdot \mathbf{B}=0$, we have

$$
\frac{\partial B}{\partial z}=0
$$

since, in the local coordinate system, $\mathbf{B}$ is along the $z$ axis. This last equation, together with (7.4), implies that both $p$ and $\mathbf{B}$ do not vary in 


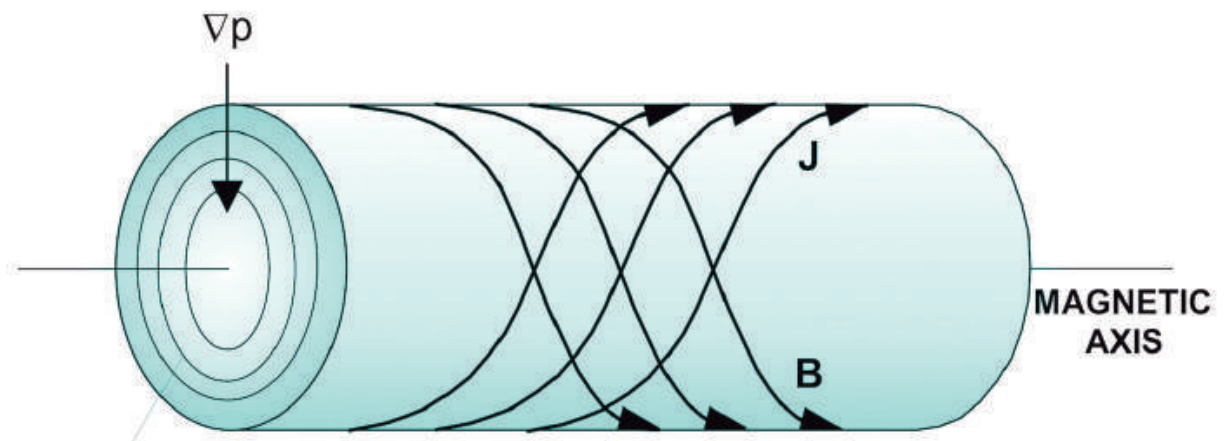

ISOBARIC

SURFACES

Fig. 5 Isobaric concentric cylindrical surfaces, with $\nabla p$ along a radial directed towards the magnetic axis. The lines of $\mathbf{B}$ and $\mathbf{J}$ lie on the isobaric surfaces and cross each other in a such a manner that $\mathbf{J} \times \mathbf{B}$ is equal to $\nabla p$.

the $z$ direction. The solutions of (7.2) and (7.3), combined with this result, give

$$
\left(p+\frac{B^{2}}{2 \mu_{0}}\right)=\text { constant }
$$

Therefore, in the presence of an externally applied magnetic field, if the plasma is bounded, the plasma kinetic pressure decreases from the axis radially outwards, whereas the magnetic pressure increases in the same direction in such a manner that their sum remains constant at each point, according to (7.6). The plasma kinetic pressure can be forced to vanish on an outer surface if the applied magnetic field is sufficiently strong, with the result that the plasma is confined within this outer surface by the magnetic field.

Let $\mathbf{B}_{0}$ be the value of the magnetic induction at the plasma boundary. Since the kinetic pressure at the plasma boundary is zero (ideally), we can evaluate the constant in (7.6) from the pressure equilibrium condition at the plasma boundary. Therefore,

$$
p+\frac{B^{2}}{2 \mu_{0}}=\frac{B_{0}^{2}}{2 \mu_{0}}
$$


The maximum fluid pressure that can be confined for a given applied field $\mathbf{B}_{0}$ is, consequently,

$$
p_{\max }=\frac{B_{0}^{2}}{2 \mu_{0}}
$$

A device that can be used to confine a magnetoplasma by straight parallel field lines is shown in Fig. 6, called a theta $(\theta)$ pinch, since the effect responsible for the confinement is due to electric currents flowing in the plasma in the azimuthal $(\theta)$ direction. The plasma is initially confined inside a hollow cylindrical metal tube, whose side is split in the longitudinal direction in such a way as to form a capacitor. When a high voltage is discharged through the capacitor, the large azimuthal current produced in the metal tube generates a magnetic field in the longitudinal direction inside the plasma. The electric current induced in the plasma is also in the azimuthal direction, but in a sense opposite to that on the metal tube. The resulting $\mathbf{J} \times \mathbf{B}$ force acting on the plasma pushes it inwards, towards the axis, until a balance is reached between the kinetic pressure due to the random particle thermal motions and the magnetic pressure that acts to constrict or pinch the plasma.

A parameter $\beta$, defined as the ratio of the kinetic pressure at a point inside the plasma, to the confining magnetic pressure at the plasma boundary, is usually introduced as a measure of the relative magnitudes of the kinetic and magnetic pressures. It is given by

$$
\beta=\frac{p}{B_{0}^{2} / 2 \mu_{0}}
$$

Note that $\beta$ ranges between 0 and 1 , since the field inside the plasma is less than $B_{0}$. From (7.7) we can also express the parameter $\beta$ as

$$
\beta=1-\left(\frac{B}{B_{0}}\right)^{2}
$$

Two special cases of plasma confinement schemes are the so-called low- $\beta$ and high- $\beta$ devices. In the low- $\beta$ devices, the kinetic pressure is small in comparison to the magnetic pressure at the plasma boundary, whereas in the high- $\beta$ devices they are of an equal order of magnitude $(\beta \simeq 1)$.

An important property of a plasma is its diamagnetic character. Equation (7.7) implies that the magnetic field inside the plasma is less than its value at the plasma boundary. As the kinetic pressure increases inside the plasma, the magnetic field decreases. Under the action of the externally applied $\mathbf{B}$ field, the particle motions give rise to internal electric 


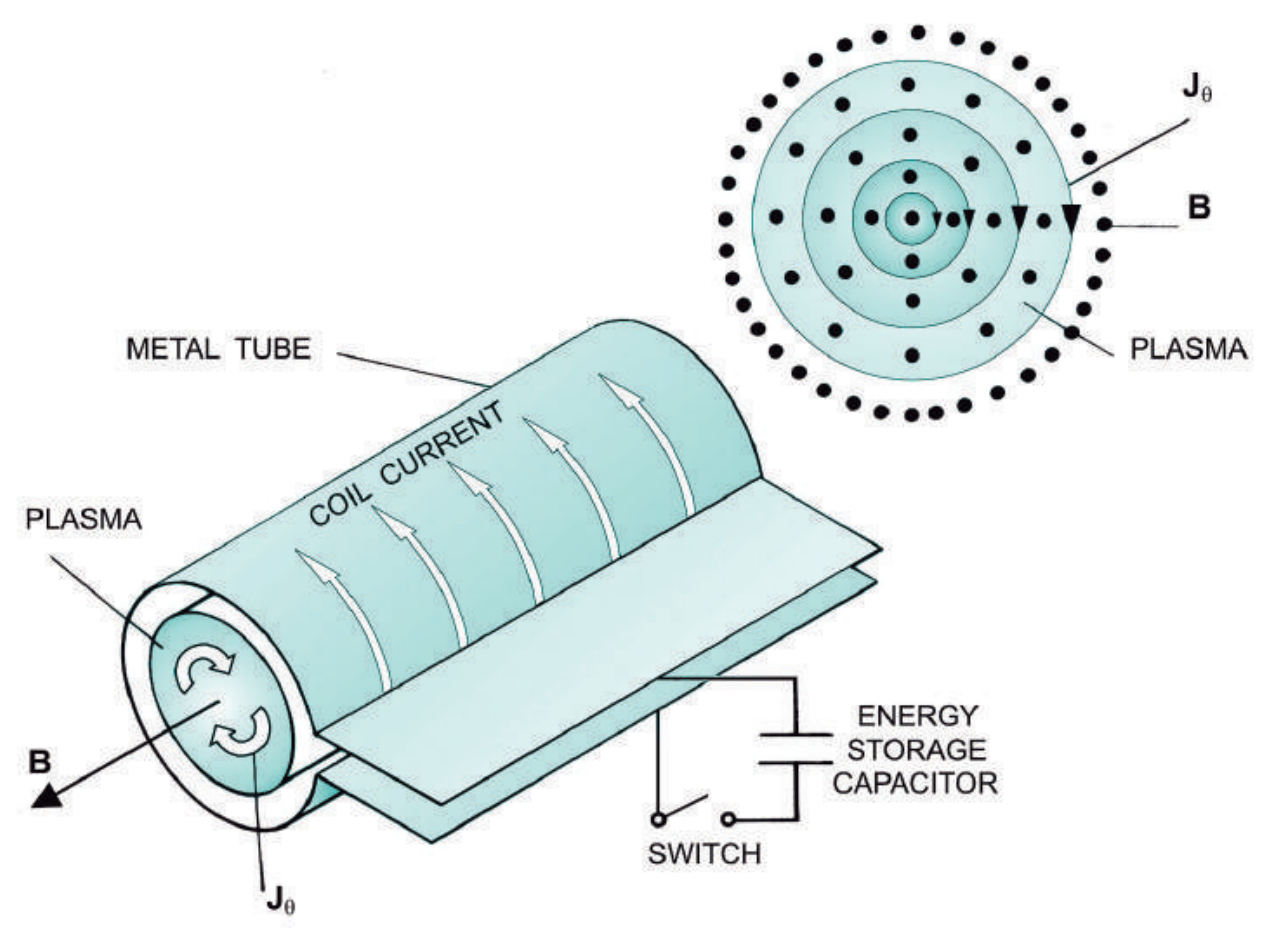

Fig. 6 Magnetoplasma confined by straight parallel field lines in a theta-pinch device.

currents that induce a magnetic field opposite to the externally applied field. Consequently, the resultant magnetic field inside the plasma is reduced to a value less than that at the plasma boundary. The electric current, induced in the plasma, depends on the number density of the charged particles and on their velocity. Therefore, as the plasma kinetic pressure increases, the induced electric current and the induced magnetic field also increase, thus enhancing the diamagnetic effect.

\section{PROBLEMS}

12.1 Consider the energy equation involving the time rate of change of the total pressure dyad $\mathcal{P}$, derived in problem 9.6 in Chapter 9. Show that when this equation is contracted with the unit dyad 1 results in (1.21), whereas when contracted with the dyad BB yields (1.22). 
12.2 Derive an energy conservation equation, similar to (1.64), but considering the Parker modified momentum equation and the CGL energy equations, instead of (1.2) and (1.3).

12.3 Calculate the minimum intensity of the magnetic induction $\left(\mathbf{B}_{0}\right)$ necessary to confine a plasma at

(a) an internal pressure of $100 \mathrm{~atm}$;

(b) a temperature of $10 \mathrm{keV}$ and density of $8 \times 10^{21} \mathrm{~m}^{-3}$.

12.4 A plasma is confined by a unidirectional magnetic induction $\mathbf{B}$ of magnitude 5 weber $/ \mathrm{m}^{2}$. Considering that the plasma temperature is 10 $\mathrm{keV}$ and $\beta=0.4$, calculate the particle number density. If the temperature increases to $50 \mathrm{keV}$, what is the value of the $\mathbf{B}$ field necessary to confine the plasma, assuming that $\beta$ stays the same?

12.5 Calculate the diffusion time $\left(\tau_{D}\right)$ and the magnetic Reynolds number $\left(R_{m}\right)$ for a typical MHD generator, with $L=0.1 \mathrm{~m}, u=10^{3} \mathrm{~m} / \mathrm{s}$, and $\sigma_{0}=100 \mathrm{mho} / \mathrm{m}$. Verify that in this case $\tau_{D}$ is very short, so that inhomogeneities in the magnetic field are smoothed out rapidly.

12.6 Consider a plasma in the form of a straight circular cylinder with a helical magnetic field given by

$$
\mathbf{B}=B_{\theta}(r) \widehat{\boldsymbol{\Theta}}+B_{z}(r) \widehat{\mathbf{z}}
$$

Show that the force per unit volume, associated with the inward magnetic pressure for this configuration, is

$$
-\nabla_{\perp}\left(\frac{B^{2}}{2 \mu_{0}}\right)=-\widehat{\mathbf{r}} \frac{\partial}{\partial r}\left[\frac{B^{2}(r)}{2 \mu_{0}}\right]
$$

and the force per unit volume, associated with the magnetic tension due to the curvature of the magnetic field lines, is

$$
\left(\frac{B^{2}}{\mu_{0}}\right)(\mathbf{B} \cdot \nabla) \mathbf{B}=-\widehat{\mathbf{r}} \frac{B_{\theta}^{2}(r)}{\mu_{0} r}
$$

12.7 Use (4.1), for a perfectly conducting fluid, and the nonlinear equation of continuity (1.1), to show that the change of $\mathbf{B}$ with time in a fluid element is related to changes of density according to

$$
\frac{D}{D t}\left(\frac{\mathbf{B}}{\rho_{m}}\right)=\frac{1}{\rho_{m}}(\mathbf{B} \cdot \nabla) \mathbf{u}
$$


Use this relation to establish that, in a perfectly conducting fluid, the fluid elements that lie initially on a magnetic flux line continue to lie on a flux line.

12.8 The boundary of the Earth's magnetosphere, in the direction of the Earth-sun line, occurs at a distance where the kinetic pressure of the solar wind particles is equal to the (modified) Earth's magnetic field pressure. Show that the distance of the magnetopause from the center of the Earth, along the Earth-sun line, is given approximately by

$$
R_{M}=\left(\frac{2 B_{0}^{2}}{\mu_{0} \rho_{m} u_{s}^{2}}\right)^{1 / 6} R_{E}
$$

where $R_{E}$ is the Earth's radius, $\rho_{m}$ is the mass density of the solar wind, $u_{s}$ is its undisturbed speed, and $B_{0}$ is the surface value of the undisturbed Earth's magnetic field.

12.9 Consider a cylindrically symmetric plasma column $(\partial / \partial z=0$, $\partial / \partial \theta=0)$ under equilibrium conditions, confined by a magnetic field. Verify that in cylindrical coordinates the radial component of (5.1) becomes

$$
\frac{d p(r)}{d r}=J_{\theta}(r) B_{z}(r)-J_{z}(r) B_{\theta}(r)
$$

Using Maxwell equation (5.2), show that

$$
\begin{gathered}
J_{\theta}=-\frac{1}{\mu_{0}} \frac{d B_{z}}{d r} \\
J_{z}=\frac{1}{\mu_{0} r} \frac{d\left(r B_{\theta}\right)}{d r}
\end{gathered}
$$

From these results, obtain the following basic equation for the equilibrium of a plasma column with cylindrical symmetry

$$
\frac{d}{d r}\left(p+\frac{B_{z}^{2}}{2 \mu_{0}}+\frac{B_{\theta}^{2}}{2 \mu_{0}}\right)=-\frac{1}{\mu_{0}} \frac{B_{\theta}^{2}}{r}
$$

Give a physical interpretion for the various terms in this equation. 


\section{3}

\section{THE PINCH EFFECT}

\section{INTRODUCTION}

In view of the importance of plasma confinement by a magnetic field in controlled thermonuclear research, as well as in other applications, we present in this chapter a detailed treatment of plasma confinement for the special case in which the confinement is produced by an azimuthal $(\theta)$ self-magnetic field, due to an axial current in the plasma generated by an appropriately applied electric field.

Consider an infinite cylindrical column of conducting fluid with an axial current density $\mathbf{J}=J_{z}(r) \widehat{\mathbf{z}}$ and a resulting azimuthal magnetic induction $\mathbf{B}=B_{\theta}(r) \widehat{\boldsymbol{\Theta}}$, as depicted in Fig. 1. The $\mathbf{J} \times \mathbf{B}$ force, acting on the plasma, forces the column to contract radially. This radial constriction of the plasma column is known as the pinch effect. In this case the isobaric surfaces, for which $p=$ constant, are concentric cylinders.

As the plasma is compressed radially, the plasma number density and the temperature increase. The plasma kinetic pressure counteracts to hinder the constriction of the plasma column, whereas the magnetic force acts to confine the plasma. When these counteracting forces are balanced, a steady-state condition results in which the plasma is mainly confined within a certain radius $R$, which remains constant in time. This situation is commonly referred to as the equilibrium pinch. When the selfmagnetic pressure exceeds the plasma kinetic pressure, the column radius changes with time, resulting in a situation known as the dynamic pinch. In what follows we investigate first the equilibrium pinch and afterwards the dynamic pinch. 


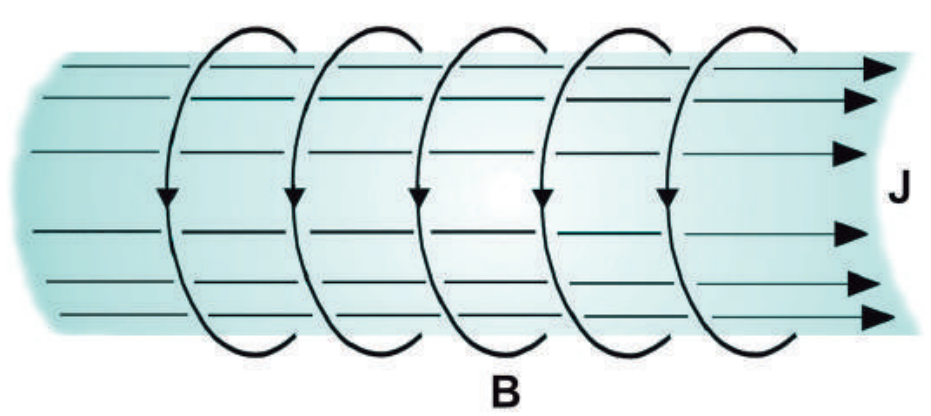

Fig. 1 Pinch configuration in which a magnetoplasma is confined by azimuthal magnetic fields generated by axial currents flowing along the plasma column.

\section{THE EQUILIBRIUM PINCH}

For simplicity, the current density, the magnetic field, and the plasma kinetic pressure are assumed to depend only on the distance from the cylinder axis. For steady-state conditions, none of the variables changes with time. The various parameters of the equilibrium pinch are schematically shown in Fig. 2. Since the system is cylindrically symmetric, only the radial component of (12.5.1) (Eq. 5.1 in Chapter 12) must be considered and we have

$$
\frac{d p(r)}{d r}=-J_{z}(r) B_{\theta}(r)
$$

Inside a cylinder of general radius $r$, the total enclosed current $I_{z}(r)$ is

$$
I_{z}(r)=\int_{0}^{r} J_{z}(r) 2 \pi r d r
$$

Note that the variable $r$ inside the integrand is a dummy variable. From (2.2) we obtain

$$
\frac{d I_{z}(r)}{d r}=2 \pi r J_{z}(r)
$$

Ampère's law in integral form relates $B_{\theta}(r)$ to the total enclosed current giving for the magnetic induction

$$
B_{\theta}(r)=\frac{\mu_{0}}{2 \pi r} I_{z}(r)=\frac{\mu_{0}}{r} \int_{0}^{r} J_{z}(r) r d r
$$



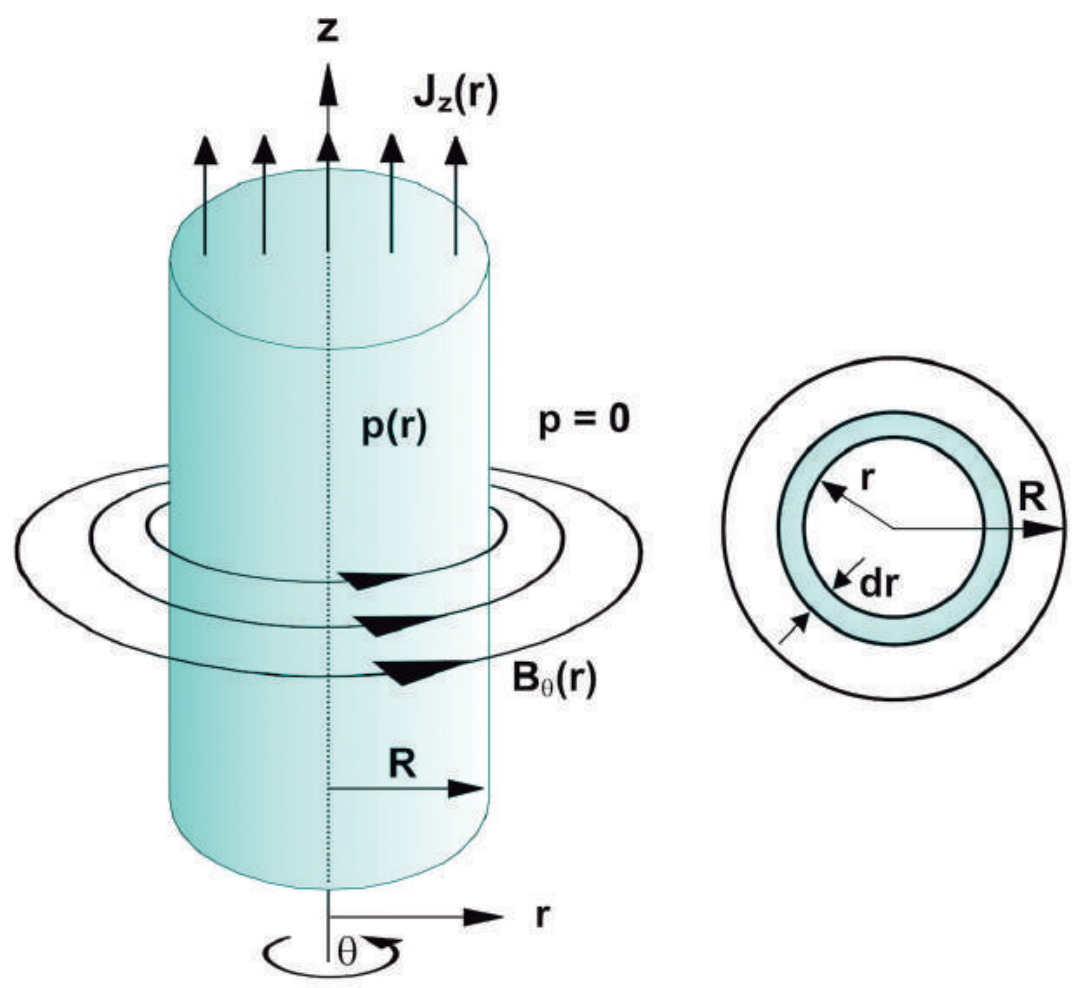

Fig. 2 Schematic diagram illustrating the various parameters relevant to the study of the equilibrium longitudinal pinch configuration.

A number of results can be obtained even without specifying the precise form of $J_{z}(r)$. If the conducting fluid lies almost entirely inside $r=R$, then the magnetic induction $B_{\theta}(r)$ outside the plasma is

$$
B_{\theta}(r)=\frac{\mu_{0} I_{0}}{2 \pi r} \quad(r \geq R)
$$

where

$$
I_{0}=\int_{0}^{R} J_{z}(r) 2 \pi r d r=I_{z}(R)
$$

which is the total current flowing inside the cylindrical plasma column. The substitution of $B_{\theta}(r)$ and $J_{z}(r)$, from (2.4) and (2.3), respectively, into (2.1), gives

$$
\frac{d p(r)}{d r}=-\frac{\mu_{0}}{4 \pi^{2} r^{2}} I_{z}(r) \frac{d I_{z}(r)}{d r}
$$


which can be written as

$$
4 \pi^{2} r^{2} \frac{d p(r)}{d r}=-\frac{d}{d r}\left[\frac{1}{2} \mu_{0} I_{z}^{2}(r)\right]
$$

If we now integrate this equation from $r=0$ to $r=R$, and simplify the left-hand side by an integration by parts, we obtain

$$
\left(\left.4 \pi^{2} r^{2} p(r)\right|_{0} ^{R}\right)-4 \pi \int_{0}^{R} 2 \pi r p(r) d r=-\frac{1}{2} \mu_{0} I_{0}^{2}
$$

where $I_{0}=I_{z}(R)$ is the total current flowing through the entire cross section of the plasma column and, obviously, $I_{z}(0)=0$. Considering the plasma column to be confined to the range $0 \leq r<R$, it follows that $p(r)$ is zero for $r \geq R$ and finite for $0 \leq r<R$, so that the first term in the left-hand side of (2.9) vanishes. Therefore, we find that

$$
I_{0}^{2}=\frac{8 \pi}{\mu_{0}} \int_{0}^{R} 2 \pi r p(r) d r
$$

If the partial pressures of the electrons and ions are governed by the ideal gas law,

$$
\begin{aligned}
& p_{e}(r)=n(r) k T_{e} \\
& p_{i}(r)=n(r) k T_{i}
\end{aligned}
$$

assuming that the electron and ion temperatures, $T_{e}$ and $T_{i}$, respectively, are constants throughout the plasma column, we have

$$
p(r)=p_{e}(r)+p_{i}(r)=n(r) k\left(T_{e}+T_{i}\right)
$$

Therefore, (2.10) becomes

$$
I_{0}^{2}=\frac{8 \pi}{\mu_{0}} k\left(T_{e}+T_{i}\right) \int_{0}^{R} 2 \pi r n(r) d r
$$

which can be rewritten as

$$
I_{0}^{2}=\frac{8 \pi}{\mu_{0}} k\left(T_{e}+T_{i}\right) N_{\ell}
$$

where

$$
N_{\ell}=\int_{0}^{R} 2 \pi r n(r) d r
$$


is the number of particles per unit length of the plasma column.

Equation (2.15) is known as the Bennett relation. It gives the total current that must be discharged through the plasma column in order to confine a plasma at a specified temperature and a given number of particles $\left(N_{\ell}\right)$ per unit length. The current required for the confinement of hot plasmas is usually very large. As an example, suppose that $N_{\ell}=10^{19} \mathrm{~m}^{-1}$ and that the plasma temperature is such that $\left(T_{e}+T_{i}\right)=10^{8} \mathrm{~K}$. Since $\mu_{0}=4 \pi \times 10^{-7} \mathrm{H} / \mathrm{m}$ and $k=1.38 \times 10^{-23} \mathrm{~J} / \mathrm{K}$, it follows that the required current $I_{0}$ is of the order of one million amperes.

To obtain the radial distribution of $p(r)$ in terms of $B_{\theta}(r)$, it is convenient to start from (2.1) and proceed in a different way. First, we note that from Maxwell equation $\nabla \times \mathbf{B}=\mu_{0} \mathbf{J}$ we have, in cylindrical coordinates, with only radial dependence,

$$
\frac{1}{r} \frac{d}{d r}\left[r B_{\theta}(r)\right]=\mu_{0} J_{z}(r)
$$

from which we get

$$
J_{z}(r)=\frac{1}{\mu_{0}} \frac{d B_{\theta}(r)}{d r}+\frac{1}{\mu_{0}} \frac{B_{\theta}(r)}{r}
$$

Substitution of this result for $J_{z}(r)$ into (2.1), yields

$$
\frac{d p(r)}{d r}=-\frac{1}{2 \mu_{0} r^{2}} \frac{d}{d r}\left[r^{2} B_{\theta}^{2}(r)\right]
$$

We now integrate this equation from $r=0$ to a general radius $r$,

$$
p(r)=p(0)-\frac{1}{2 \mu_{0}} \int_{0}^{r} \frac{1}{r^{2}} \frac{d}{d r}\left[r^{2} B_{\theta}^{2}(r)\right] d r
$$

In particular, since for $r=R$ we have $p(R)=0$,

$$
p(0)=\frac{1}{2 \mu_{0}} \int_{0}^{R} \frac{1}{r^{2}} \frac{d}{d r}\left[r^{2} B_{\theta}^{2}(r)\right] d r
$$

and substituting this result into (2.20),

$$
p(r)=\frac{1}{2 \mu_{0}} \int_{r}^{R} \frac{1}{r^{2}} \frac{d}{d r}\left[r^{2} B_{\theta}^{2}(r)\right] d r
$$


The average pressure $\bar{p}$ inside the cylinder can be related to the total current $I_{0}$ and the column radius $R$ without knowing the detailed radial dependence. The average of the kinetic pressure inside the column is defined by

$$
\bar{p}=\frac{1}{\pi R^{2}} \int_{0}^{R} 2 \pi r p(r) d r
$$

Simplifying this expression by an integration by parts, yields

$$
\bar{p}=-\frac{1}{R^{2}} \int_{0}^{R} r^{2} \frac{d p(r)}{d r} d r
$$

since the integrated term is zero, because $p(R)=0$. Replacing $d p(r) / d r$, using (2.19), we get

$$
\bar{p}=\frac{B_{\theta}^{2}(R)}{2 \mu_{0}}=\frac{\mu_{0} I_{0}^{2}}{8 \pi^{2} R^{2}}
$$

This result shows that the average kinetic pressure in the equilibrium plasma column is balanced by the magnetic pressure at the boundary.

From (2.2), (2.4), and (2.22) we can deduce the radial distribution for $I_{z}(r), B_{\theta}(r)$, and $p(r)$ if we know the radial dependence of $J_{z}(r)$. So far, the radial dependence of $J_{z}(r)$ has not been discussed. In what follows, we will consider two simple possibilities, in order to illustrate the use of the equations just derived.

As a simple example consider the case in which the current density $J_{z}(r)$ is constant for $r<R$. Taking $J_{z}=I_{0} / \pi R^{2}$ in (2.4), we obtain for $r<R$,

$$
B_{\theta}(r)=\frac{\mu_{0} I_{0}}{\pi R^{2} r} \int_{0}^{r} r d r=\frac{\mu_{0} I_{0}}{2 \pi R^{2}} r \quad(r<R)
$$

Substituting this result into (2.22) we obtain a parabolic dependence for the pressure versus radius,

$$
p(r)=\frac{1}{2 \mu_{0}} \int_{r}^{R} \frac{1}{r^{2}} \frac{d}{d r}\left(\frac{\mu_{0}^{2} I_{0}^{2} r^{4}}{4 \pi^{2} R^{4}}\right) d r=\frac{\mu_{0} I_{0}^{2}}{4 \pi^{2} R^{2}}\left(1-\frac{r^{2}}{R^{2}}\right)
$$

Note that, in this case, the axial pressure $p(0)$ is twice the average pressure $\bar{p}$ given in (2.25). The radial dependence of the various quantities for this example is shown in Fig. 3.

Another radial distribution of $J_{z}(r)$ that is also of interest in the investigation of the equilibrium pinch is the one in which the current density is confined to a very thin layer on the surface of the column. This 


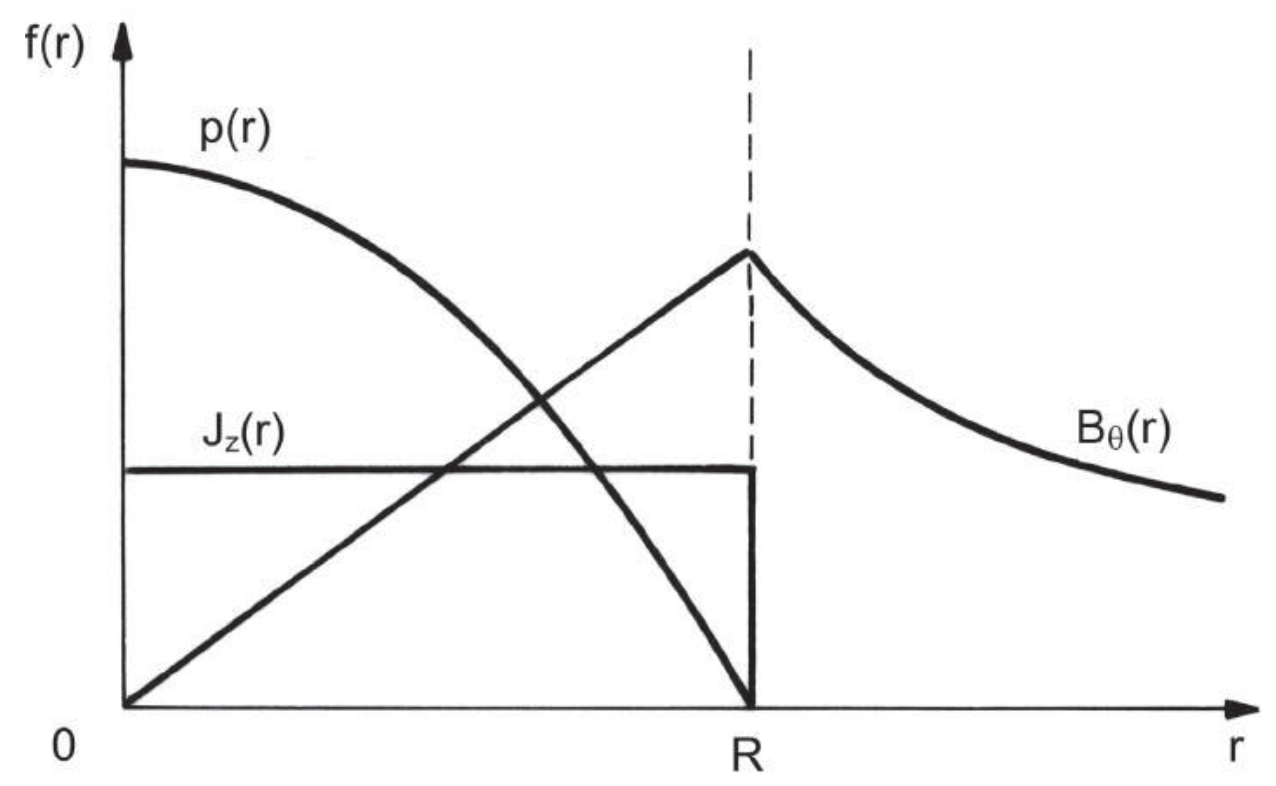

Fig. 3 Radial dependence of the azimuthal magnetic induction $B_{\theta}(r)$ and plasma pressure $p(r)$ in a cylindrical plasma column with a constant current density $J_{z}(r)$. The radius of the column is $R$.

model is appropriate for a highly conducting fluid. In a perfectly conducting plasma, the current cannot penetrate the plasma and exists only on the column surface. This surface current density can be conveniently represented by a Dirac delta function at $r=R$. In this case there is no magnetic field inside the plasma and $B_{\theta}(r)$ exists only for $r>R$. From (2.5) the magnetic induction is given by

$$
B_{\theta}(r)=\frac{\mu_{0} I_{0}}{2 \pi r} \quad(r>R)
$$

where $I_{0}$ is the total axial current. Therefore, from (2.20) we have

$$
p(r)=p(0) \quad(0<r<R)
$$

so that the plasma kinetic pressure is constant inside the cylindrical column and equal to the average value given in (2.25). The radial dependence of the various quantities for this model is sketched in Fig. 4. Thus, for a perfectly conducting plasma column, the magnetic induction vanishes inside the column and falls off as $1 / r$ outside the column. The plasma kinetic pressure is constant inside the column and vanishes outside it. The 


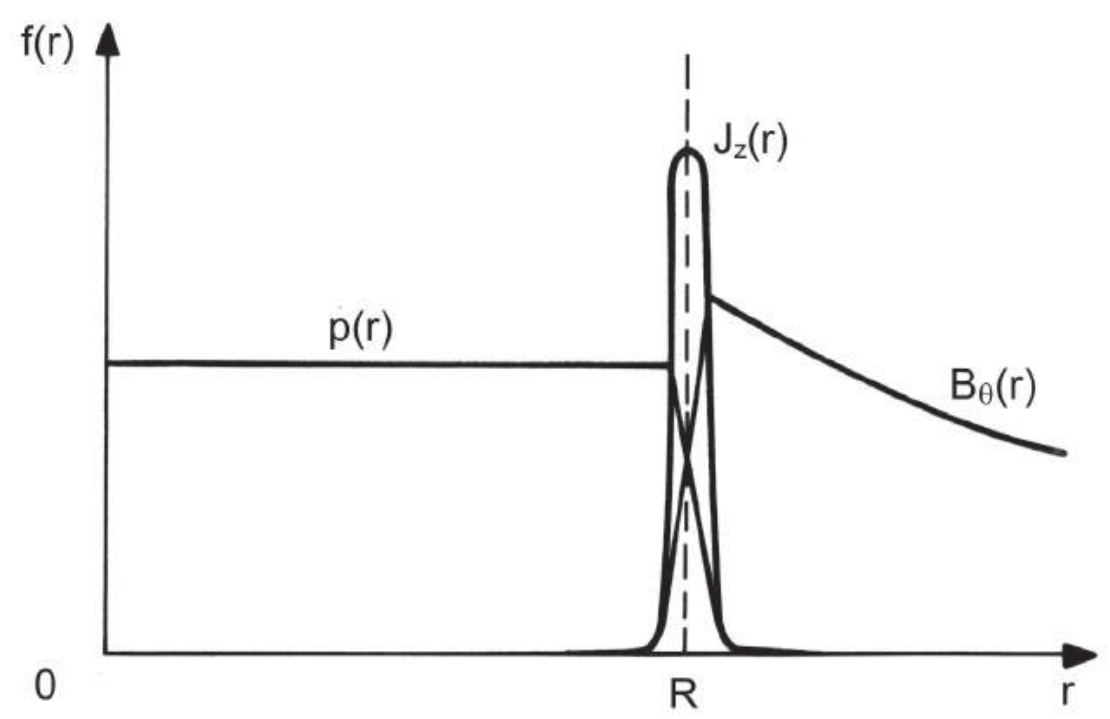

Fig. 4 Radial dependence of the azimuthal magnetic induction $B_{\theta}(r)$ and plasma pressure $p(r)$ in a cylindrical plasma column with a surface current density $J_{z}(r)$. The radius of the column is $R$.

pinch effect, in this special case, can be thought of as due to an abrupt buildup of the magnetic pressure $B_{\theta}^{2} / 2 \mu_{0}$ in the region external to the plasma column.

\section{THE BENNETT PINCH}

W. H. Bennett, the discoverer of the pinch effect, investigated a special model of the equilibrium longitudinal pinch in which the radial distribution of the various quantities are such that the drift velocity of the plasma particles is constant throughout the column cross section. As an instructive application of the previous equations for the equilibrium pinch configuration, we investigate this particular model in what follows. In view of the fact that the ion mass is much larger than the electron mass, the drift velocity of the ions is much smaller than that of the electrons and therefore can be neglected on a first approximation. Thus, we consider the current density to be given by

$$
\mathbf{J}(r)=-e n(r) \mathbf{u}_{e}
$$


Since the applied electric field is in the $z$ direction, we have $\mathbf{J}(r)=J_{z}(r) \widehat{\mathbf{z}}$ and $\mathbf{u}_{e}=-u_{e z} \widehat{\mathbf{z}}$, where $u_{e z}$ is positive and constant, independent of $r$. Therefore,

$$
J_{z}(r)=e n(r) u_{e z}
$$

Substitution of this equation for $J_{z}(r)$, and (2.13) for $p(r)$, into the hydrostatic equation of motion (2.1), yields

$$
k\left(T_{e}+T_{i}\right) \frac{d n(r)}{d r}=-e n(r) u_{e z} B_{\theta}(r)
$$

If we multiply this equation by $r /\left[n(r) k\left(T_{e}+T_{i}\right)\right]$ and differentiate it with respect to $r$, we obtain

$$
\frac{d}{d r}\left[\frac{r}{n(r)} \frac{d n(r)}{d r}\right]=-\frac{e u_{e z}}{k\left(T_{e}+T_{i}\right)} \frac{d}{d r}\left[r B_{\theta}(r)\right]
$$

From (2.17) and (3.2), we have

$$
\frac{d}{d r}\left[r B_{\theta}(r)\right]=\mu_{0} \text { e } u_{e z} r n(r)
$$

and using this result in (3.4),

$$
\frac{d}{d r}\left[\frac{r}{n(r)} \frac{d n(r)}{d r}\right]+\left[\frac{\mu_{0} e^{2} u_{e z}^{2}}{k\left(T_{e}+T_{i}\right)}\right] r n(r)=0
$$

The solution of this nonlinear differential equation gives the radial dependence of the number density $n(r)$. Bennett obtained the solution of this nonlinear equation subjected to the boundary condition that $n(r)$ is symmetric about the $z$ axis, where $r=0$, and is a smoothly varying function of $r$, so that

$$
\left[\frac{d n(r)}{d r}\right]_{r=0}=0
$$

The solution of (3.6), subjected to the boundary condition (3.7), is known as the Bennett distribution and is given by

$$
n(r)=\frac{n_{0}}{\left(1+n_{0} b r^{2}\right)^{2}}
$$

where $n_{0}=n(0)$, which is the number density on the axis, and

$$
b=\frac{\mu_{0} e^{2} u_{e z}^{2}}{8 k\left(T_{e}+T_{i}\right)}
$$




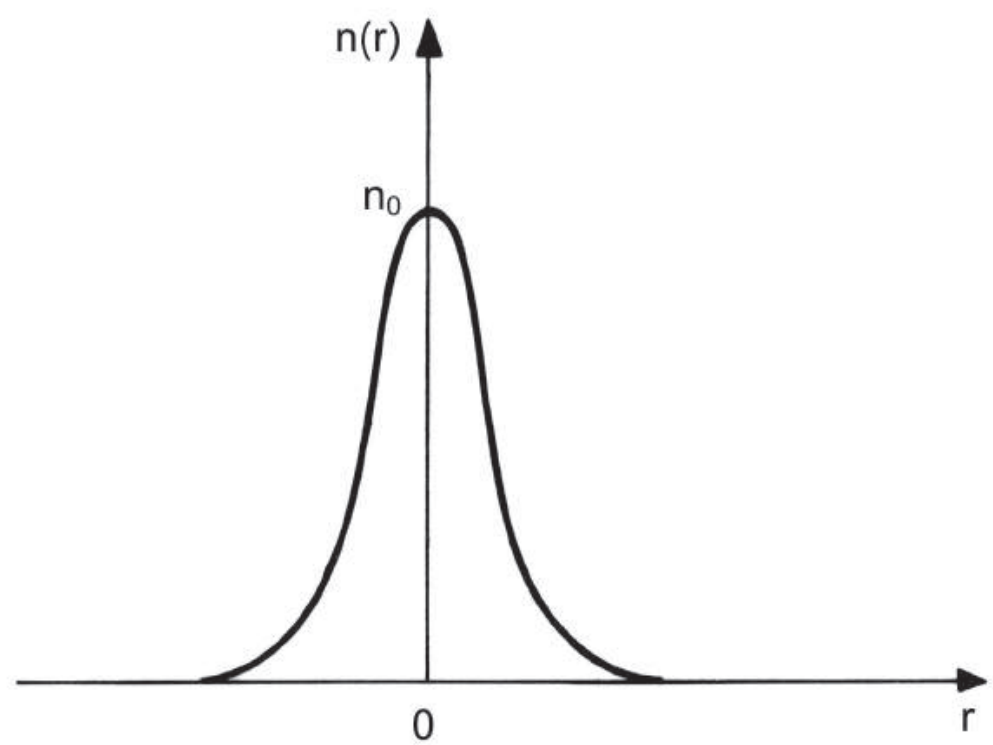

Fig. 5 The Bennett distribution for the particle number density $n(r)$ in an equilibrium pinched plasma column.

which has dimensions of length. This radial dependence of the number density is sketched in Fig. 5. From (3.2) and (2.13) we see that the radial dependence of $J_{z}(r)$ and $p(r)$ is the same as that of $n(r)$. It can be used to determine $B_{\theta}(r)$ according to $(2.4)$.

The Bennett distribution (3.8) shows that particles are present up to infinity but, since $n(r)$ falls off very rapidly with increasing values of $r$, we can consider, for all practical purposes, that the plasma is essentially confined symmetrically in a small cylindrical region about the $z$ axis. Using (3.8) we obtain the number of particles $N_{\ell}(R)$ per unit length contained in a cylindrical column of radius $R$,

$$
N_{\ell}(R)=\int_{0}^{R} n(r) 2 \pi r d r=2 \pi n_{0} \int_{0}^{R} \frac{r}{\left(1+n_{0} b r^{2}\right)^{2}} d r
$$

Evaluating the integral yields

$$
N_{\ell}(R)=\frac{n_{0} \pi R^{2}}{\left(1+n_{0} b R^{2}\right)}
$$

Since particles are present up to infinity, the total number of particles per unit length can be obtained from (3.11) by taking the limit as $R \rightarrow \infty$, 
which gives

$$
N_{\ell}(\infty)=\frac{\pi}{b}
$$

If we let $\alpha$ denote the fraction of the number of particles per unit length that is contained in a cylinder of radius $R$, that is,

$$
\alpha=\frac{N_{\ell}(R)}{N_{\ell}(\infty)}=\frac{b}{\pi} N_{\ell}(R)
$$

and use (3.11), we obtain, after some rearrangement,

$$
\left(n_{0} b\right)^{1 / 2} R=\left(\frac{\alpha}{1-\alpha}\right)^{1 / 2}
$$

Therefore, if $90 \%$ of the plasma particles are confined within the cylindrical plasma column of radius $R$, that is $\alpha=0.9$, we must have

$$
\left(n_{0} b\right)^{1 / 2} R=3
$$

Thus, even though the particles extend up to infinity, the major portion of them lies in a small neighborhood around the $z$ axis. Note that, since $\left(n_{0} b\right)^{1 / 2}$ has dimensions of an inverse length, we can think of $\left(n_{0} b\right)^{1 / 2} R$ as a normalized radius of the cylindrical plasma column. If we assume arbitrarily that a plasma is confined within a cylindrical surface of radius $R$ if $90 \%$ of the particles are within this cylindrical column, then this radius must satisfy $(3.15)$.

\section{DYNAMIC MODEL OF THE PINCH}

The simple theory of the equilibrium pinch, considered previously, is valid when the plasma column radius is constant in time or when it is varying very slowly compared to the time required for the plasma to attain a constant temperature. In actual practice, however, static or quasi-static situations do not arise and it is necessary to consider the dynamic behavior of the pinch effect. Initially, when the current starts flowing down the plasma column, the kinetic pressure is generally too small to resist the force due to the external magnetic pressure, so that the radius of the plasma cylinder is forced inwards and the plasma column is pinched.

The essential dynamic features of the time-varying pinch are illustrated by the following simple model. Suppose that a fully ionized plasma fills the interior region $\left(0<r<R_{0}\right)$ of a hollow dielectric cylinder of 
radius $R_{0}$ and length $L$. A voltage difference $V$ is applied between the ends of the cylinder, so that a current $I$ flows in the plasma. This current produces an azimuthal magnetic induction $B_{\theta}(r)$ that causes the plasma to pinch inwards. The plasma is assumed to be perfectly conducting, so that all the current flows on the surface and there is no magnetic flux inside the plasma. Also, the plasma kinetic pressure is neglected. Let $R(t)$ be the plasma column radius at time $t$ (see Fig. 6). The magnitude of the azimuthal magnetic induction just adjacent to the current sheath at radius $R(t)$ is given by

$$
B_{\theta}(R)=\frac{\mu_{0} I(t)}{2 \pi R}
$$

where $I(t)$ is the total axial current at the instant $t$. In particular for $t=0$ we have $R=R_{0}$ and this equation gives the initial value $B_{\theta}\left(R_{0}\right)$ of the magnetic induction. The magnetic pressure $p_{m}(R)$ produced by this magnetic induction, acting on the current sheath radially inwards, is given by

$$
p_{m}(R)=\frac{B_{\theta}^{2}(R)}{2 \mu_{0}}=\frac{\mu_{0} I^{2}(t)}{8 \pi^{2} R^{2}}
$$

The force per unit length of the current sheath, acting radially inwards, in obtained from (4.2) as

$$
\mathbf{F}(R)=\widehat{\mathbf{r}} F(R)=-2 \pi R p_{m}(R) \widehat{\mathbf{r}}=-\frac{\mu_{0} I^{2}(t)}{4 \pi R} \widehat{\mathbf{r}}
$$

To set up the equation of motion, relating $I(t)$ to the instantaneous radius $R(t)$ of the pinch discharge, we must make some assumption about the plasma. We shall consider the so-called snowplow model, in which the current sheath is imagined to carry along with it all the material that it hits as it moves inward. If $\rho_{m}$ is the original mass density of the plasma, then the mass per unit length carried by the interface as it moves in, at time $t$, when the radius of the current sheath is $R$, is given by

$$
M(R)=\pi\left(R_{0}^{2}-R^{2}\right) \rho_{m}
$$

Fig. 7 illustrates the cross-sectional area swept by the current sheath as it moves inward. From Newton's second law, the magnetic pressure force and the rate of change of momentum are related by

$$
\frac{d}{d t}\left[M(R) \frac{d R}{d t}\right]=F(R)
$$




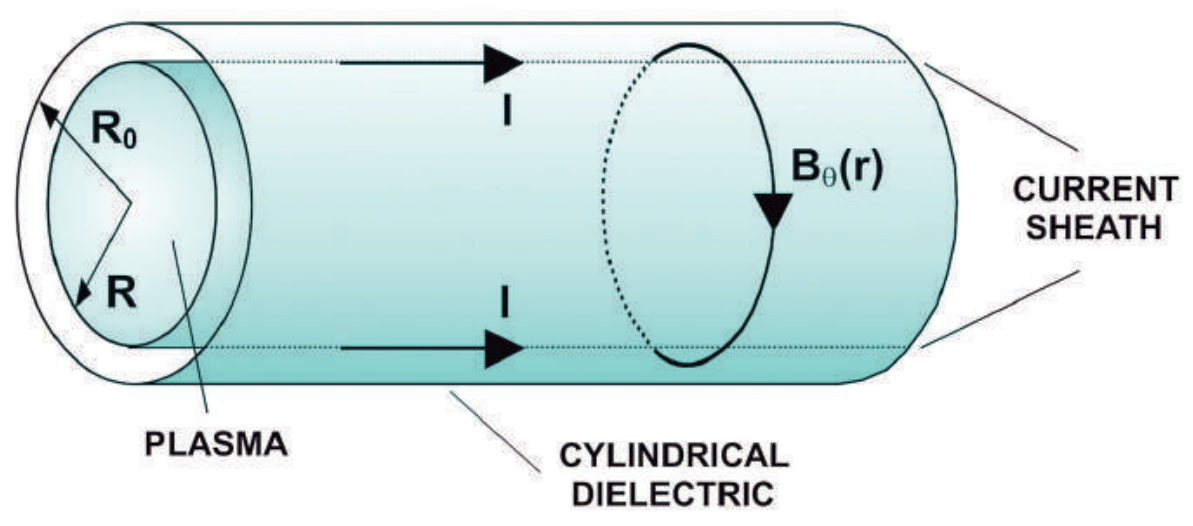

Fig. 6 Plasma column of infinite conductivity, inside a hollow cylindrical dielectric, with a current sheath on its surface.

or, using (4.3) and (4.4),

$$
\frac{d}{d t}\left[\pi \rho_{m}\left(R_{0}^{2}-R^{2}\right) \frac{d R}{d t}\right]=-\frac{\mu_{0} I^{2}(t)}{4 \pi R}
$$

If the functional dependence of the pinch current $I(t)$ is known, (4.6) permits the evaluation of the pinch discharge radius as a function of time.

A standard inductive relation between the applied voltage, the current, and the dimensions (inductance) of the plasma column can be obtained using Faraday's law of induction. For this purpose consider the closed loop shown in Fig. 8, in which the inner arm lies on the interface and moves inward with it. Applying Faraday's law to this dotted loop,

$$
\oint \mathbf{E} \cdot d \mathbf{l}=-\frac{d}{d t}\left(\int_{S} \mathbf{B} \cdot d \mathbf{S}\right)
$$

and noting that the only contribution to the line integral of $\mathbf{E}$ comes from the side of the loop lying in the conducting wall, we obtain

$$
-\frac{V}{L}=-\frac{d}{d t} \int_{R(t)}^{R_{0}} B_{\theta}(r) d r
$$

Using (4.1), and performing the integral, yields

$$
\frac{V}{L}=\frac{\mu_{0}}{2 \pi} \frac{d}{d t}\left\{I(t) \ln \left[\frac{R_{0}}{R(t)}\right]\right\}
$$




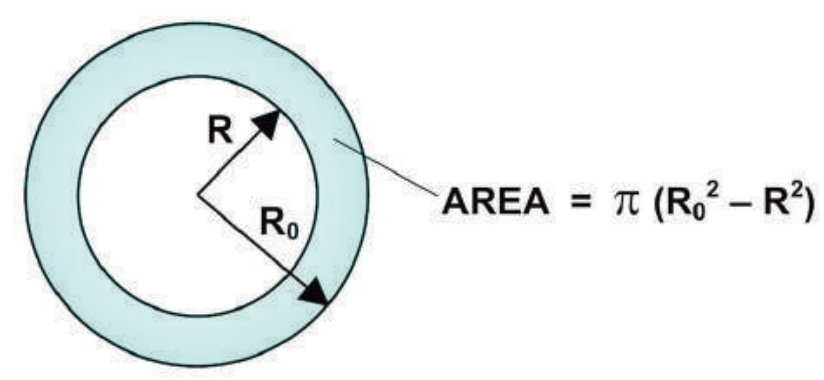

Fig. 7 Area swept by the current sheath as it moves inward from the radius $R_{0}$ to $R(t)$.

If we denote the applied electric field $V / L$ by $E_{0} f(t)$, where the function $f(t)$ is assumed known and is normalized so that the peak value of the applied electric field is $E_{0}$, (4.9) becomes

$$
I(t) \ln \left[\frac{R_{0}}{R(t)}\right]=\frac{2 \pi E_{0}}{\mu_{0}} \int_{0}^{t} f\left(t^{\prime}\right) d t^{\prime}
$$

This equation can be used to eliminate $I(t)$ from the equation of motion (4.6), resulting in the following equation for the rate of change of $R(t)$ :

$$
\frac{d}{d t}\left[\left(R_{0}^{2}-R^{2}\right) \frac{d R}{d t}\right]=-\frac{E_{0}^{2}\left[\int_{0}^{t} f\left(t^{\prime}\right) d t^{\prime}\right]^{2}}{\mu_{0} \rho_{m} R\left[\ln \left(R_{0} / R\right)\right]^{2}}
$$

It is convenient to introduce the following dimensionless variables

$$
\begin{gathered}
x=\frac{R}{R_{0}} \\
\tau=\left(\frac{E_{0}^{2}}{\mu_{0} \rho_{m} R_{0}^{4}}\right)^{1 / 4} t
\end{gathered}
$$

and recast (4.11) in normalized form as

$$
\frac{d}{d \tau}\left[\left(1-x^{2}\right) \frac{d x}{d \tau}\right]=-\frac{1}{x(\ln x)^{2}}\left[\int_{0}^{\tau} f\left(\tau^{\prime}\right) d \tau^{\prime}\right]^{2}
$$

This equation cannot be solved without knowing the function $f(t)$. However, some idea of the results can be obtained, without solving this 


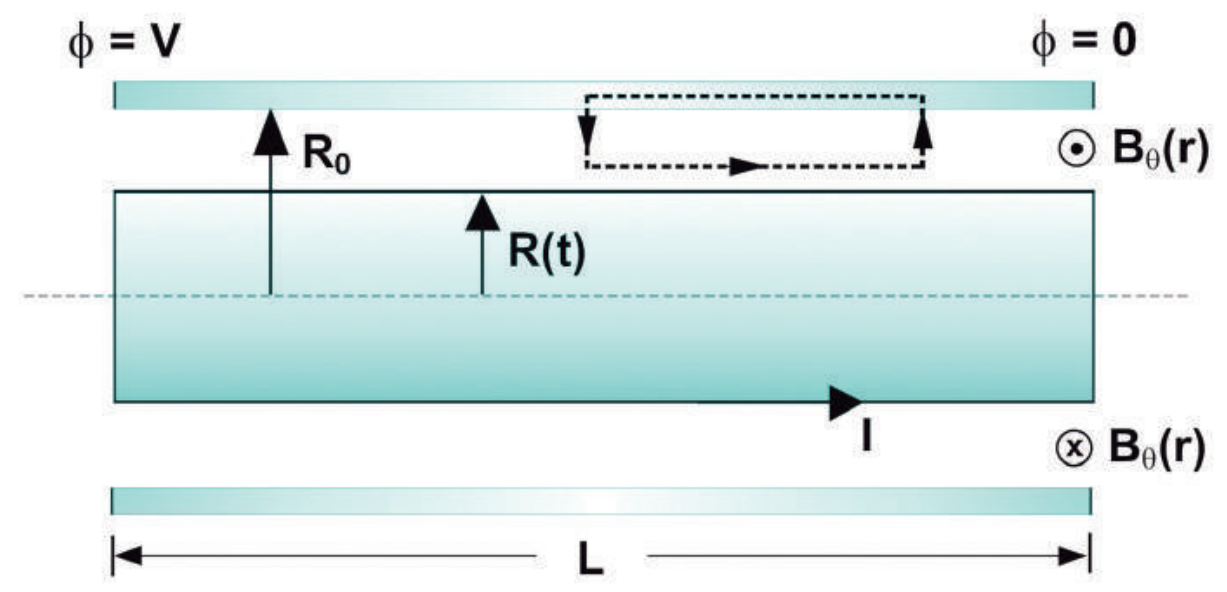

Fig. 8 Schematic representation of a closed loop for application of Faraday's law, with the inner side lying on the interface and moving inwards with it.

equation, by noting that $x$ changes significantly for time periods such that $\tau=1$. Thus, from (4.13), the scaling law for the radial velocity of the pinch is, approximately,

$$
|d R / d t| \simeq v_{0}=\left(\frac{E_{0}^{2}}{\mu_{0} \rho_{m}}\right)^{1 / 4}
$$

The typical experimental conditions involved in a small-scale pinch column of hydrogen or deuterium plasma are initial mass densities of the order of $10^{-8} \mathrm{~g} / \mathrm{cm}^{3}$ and applied electric fields of the order of $10^{3}$ volts $/ \mathrm{cm}$, which give a velocity $v_{0}$ of the order of $10^{7} \mathrm{~cm} / \mathrm{s}$. For these conditions, in a tube of $10 \mathrm{~cm}$ radius, the current measured is of the order of $10^{5}$ or $10^{6}$ amperes.

It is instructive to consider a particular case in which the pinch current varies in time according to

$$
I(t)=I_{0} \sin (\omega t) \simeq I_{0} \omega t
$$

Then, from (4.6) we obtain directly

$$
\frac{d}{d \tau}\left[\left(1-x^{2}\right) \frac{d x}{d \tau}\right]=-\frac{\tau^{2}}{x}
$$




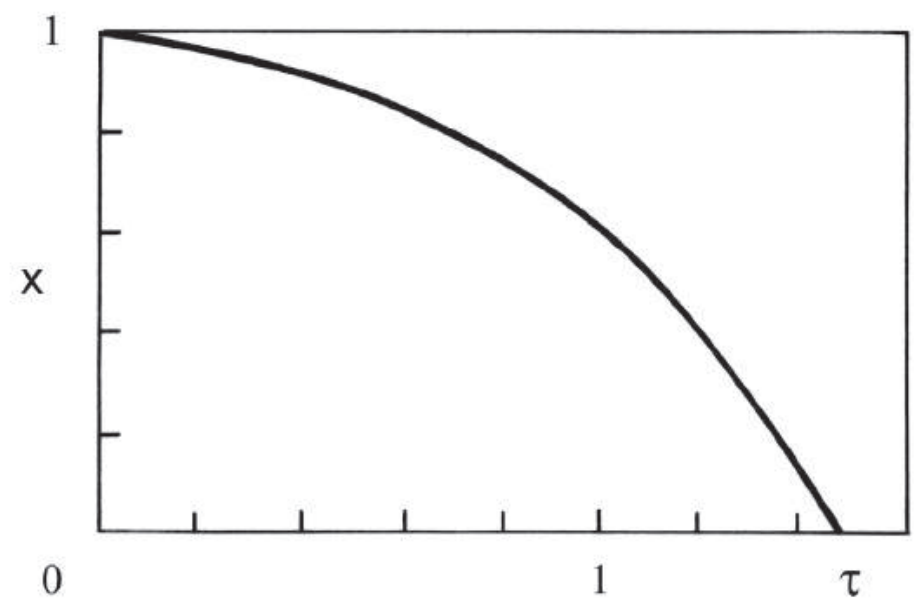

Fig. 9 Normalized radius $x=R / R_{0}$ of the dynamic pinch column as a function of the normalized time $\tau$, according to (4.17).

with $x$ as given by (4.12), and

$$
\tau=\left(\frac{\mu_{0} I_{0}^{2} \omega^{2}}{4 \pi^{2} \rho_{m} R_{0}^{4}}\right)^{1 / 4} t
$$

Equation (4.17) has to be solved numerically to determine $x(\tau)$. The resulting relation between the normalized radius of the dynamic pinch and the normalized time is sketched in Fig. 9. This simplified model indicates that the plasma column radius goes to zero in a time slightly greater than $\tau$. This is a consequence of neglecting the kinetic pressure of the plasma. The above discussion, therefore, is valid only for very short time periods after the onset of the current flow.

An important phenomenon that usually occurs in the dynamic pinch has not been considered in this analysis. As the current sheath moves radially inwards, compressing the plasma, the behavior just discussed is modified. A radial wave motion is usually set up by the pinch, and this wave travels faster than the current sheath. These waves, traveling inwards, get reflected off the axis and move outwards, striking the interface and retarding the inward motion of the current sheath or even reversing it. This phenomenon is known as bouncing. This sequence of events takes place periodically and the amplitude of each succeeding bounce becomes smaller. The plasma column radius presumably reaches an equilibrium state at some radius less than $R_{0}$. Fig. 10 illustrates the general behavior expected for the column radius $R$ as a function of time. 


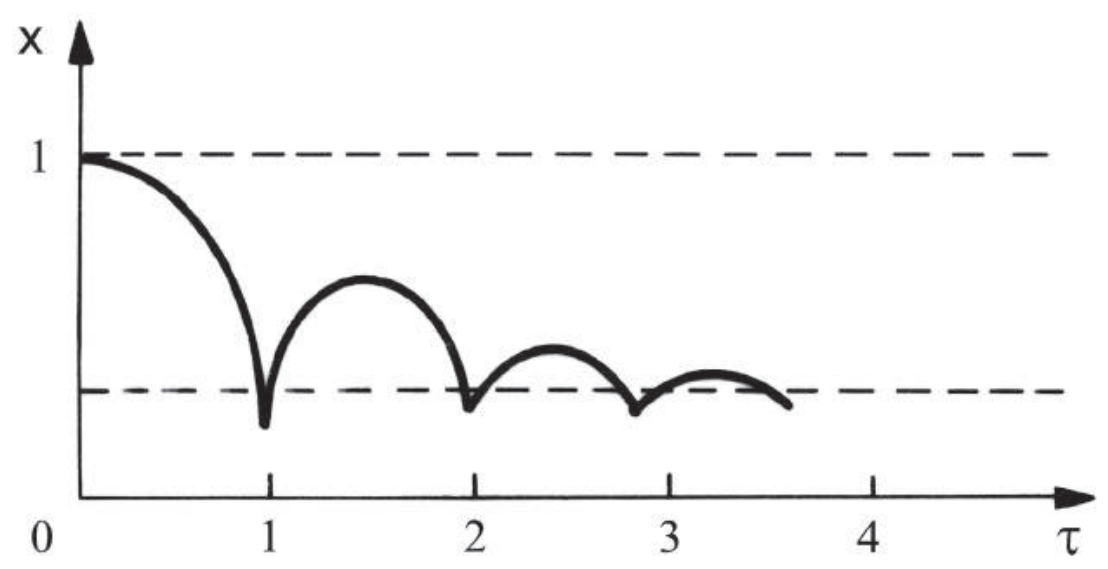

Fig. 10 Normalized radius of the plasma column as a function of the normalized time, illustrating the phenomenon of bouncing.

\section{INSTABILITIES IN A PINCHED PLASMA COLUMN}

Although it is possible to achieve an equilibrium state for plasma confinement with the pinch effect, this equilibrium state is not stable. A small departure from the cylindrical geometry of the equilibrium state results in the growth of the original perturbations with time and in the disintegration of the plasma column. The growth of instabilities is the reason why it is difficult to sustain reasonably long-lived pinched plasmas in the laboratory.

A detailed mathematical treatment of these instabilities is beyond the scope of this text. For simplicity, in the following discussion of instabilities we shall consider a perfectly diamagnetic plasma column confined by a static magnetic field. Since the plasma is perfectly diamagnetic, there is no magnetic field, and consequently no magnetic pressure, inside the plasma column. The plasma kinetic pressure is assumed to be uniform inside the plama and vanishes outside it. In the equilibrium state, the magnetic pressure at the plasma surface $p_{m 0}$ must be equal to the kinetic pressure $p$ of the plasma,

$$
p=p_{m 0}=\frac{B_{0}^{2}}{2 \mu_{0}}
$$

where $B_{0}$ is the magnitude of the magnetic flux density at the plasma surface. This situation of a sharp plasma boundary is an idealized one and is difficult to create in the laboratory, since the plasma particles diffuse 


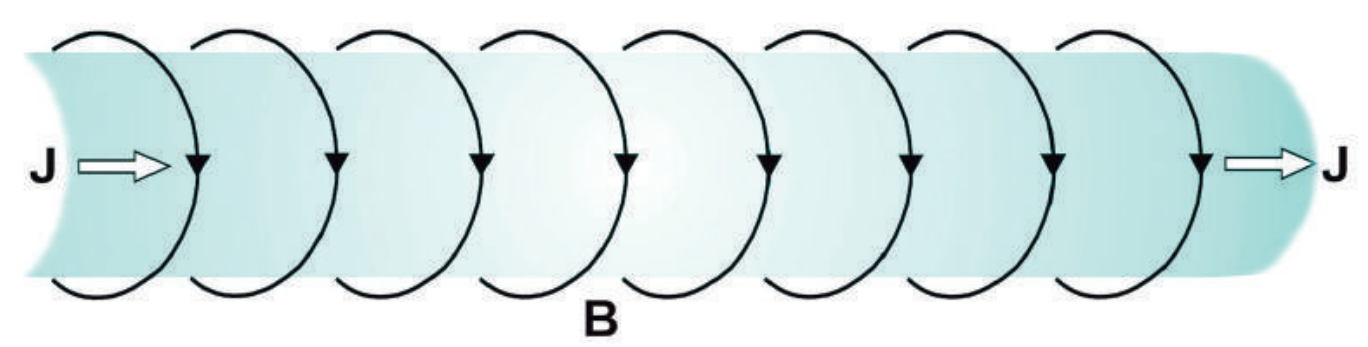

Fig. 11 Unstable equilibrium configuration of a cylindrical plasma column. The azimuthal $\mathbf{B}$ field decreases radially outwards.

through the magnetic field lines in a diffusion time of the order of $\mu_{0} \sigma_{0} L^{2}$, in view of the finite plasma conductivity $\sigma_{0}$, as discussed in section 3 of Chapter 12.

In the cylindrical pinch column, the confining magnetic field lines have a curvature such that they are concave towards the plasma and the field strength decreases with increasing distance from the center of curvature of the field lines (see Fig. 11). According to Ampère's law, this azimuthal magnetic field is inversely proportional to the radial distance $r$ from the column axis.

\section{THE SAUSAGE INSTABILITY}

Suppose that the equilibrium state of the pinched plasma column, shown in Fig. 11, is disturbed by a wave-like perturbation, with the crests and troughs on the surface of the plasma column and cylindrically symmetric about the column axis, as indicated schematically in Fig. 12. We shall consider that the plasma is constricted in some locations and expanded at others, in such a way that its volume does not change. Consequently, the uniform kinetic pressure of the plasma is left unchanged. However, in view of the $1 / r$ radial dependence of the azimuthal magnetic field, the magnitude of this field at the surface of the disturbed plasma column will vary from place to place on the surface. At the locations where the radius has decreased, in relation to the equilibrium value, the magnetic pressure at the constricted plasma surface will be larger than the plasma kinetic pressure, and will force the plasma surface radially inwards, thus enhancing the constriction. At the locations where the radius 


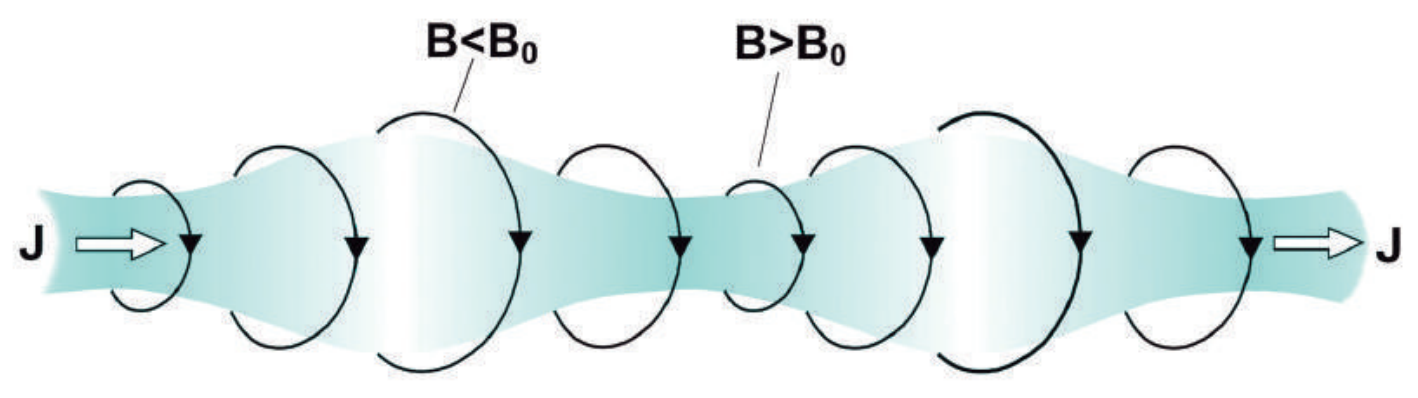

Fig. 12 The sausage instability.

has become larger than the equilibrium value, the plasma kinetic pressure will be larger than the magnetic pressure at the expanded plasma surface and will force the surface radially outwards, increasing the local expansion of the plasma. Therefore, the troughs will become deeper and the crests higher. The initial perturbation gives rise to forces that tend to further increase the initial disturbance, so that the initial equilibrium state is unstable. When the constrictions reach the axis, the column appears like a string of sausages and, for this reason, this type of instability has become known as the sausage instability.

The sausage instability can be inhibited by a longitudinal magnetic field applied inside the plasma column. This longitudinal magnetic field can be produced by passing a current through a solenoidal coil wound around the column. Because of the plasma high electric conductivity, the longitudinal field lines are frozen in the plasma. When the sausage distortion starts to grow, the longitudinal magnetic field lines are compressed at the constrictions, causing an increase in the total pressure inside the plasma that opposes the increased magnetic pressure of the azimuthal field at the constricted surface, and forces the constriction to expand. At the locations where the column radius has increased, the longitudinal field lines move apart with the plasma expansion, thus decreasing the total internal pressure, with the result that the net pressure forces the plasma surface radially inwards. This situation is illustrated schematically in Fig. 13.

We shall next determine what must be the magnitude $B_{z}$ of the longitudinal magnetic flux density, as compared to the magnitude of the azimuthal $B_{\theta}$ field, in order that the longitudinal field be able to stabilize 


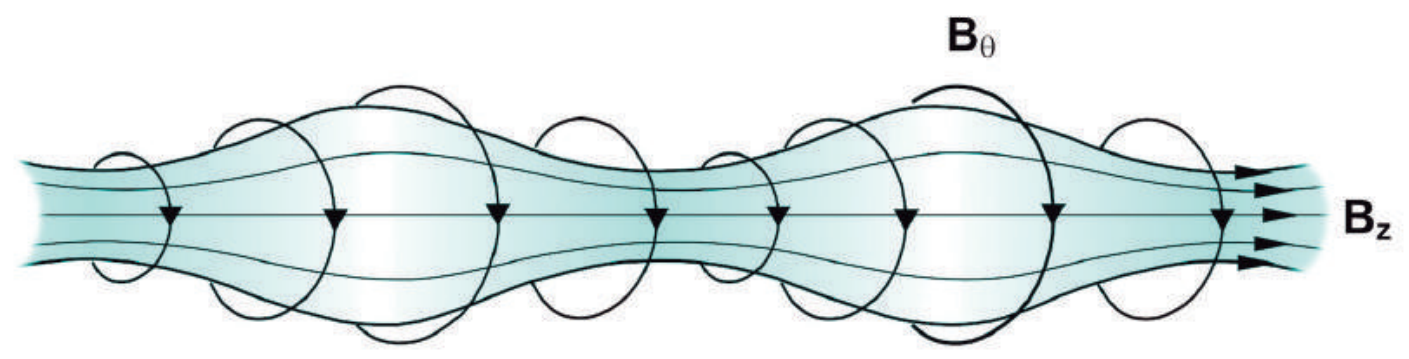

Fig. 13 A longitudinal magnetic flux density $B_{z}$ can be used to inhibit the sausage instability.

the plasma column against the setting of the sausage instability. If the radius $r$ of the column, at the constriction, is decreased by an amount $d r$, and considering that the magnetic flux $\left(\Phi_{m}=B_{z} \pi r^{2}\right)$ through the cross-sectional area of the column remains constant during compression, then we have

$$
d \Phi_{m}=\pi r^{2} d B_{z}+B_{z} 2 \pi r d r=0
$$

Hence, the longitudinal magnetic flux density is increased by the amount

$$
d B_{z}=-2 B_{z} \frac{d r}{r}
$$

Consequently, the corresponding internal magnetic pressure increases by

$$
d p_{z}=\frac{\left(B_{z}+d B_{z}\right)^{2}}{2 \mu_{0}}-\frac{B_{z}^{2}}{2 \mu_{0}}=\frac{1}{\mu_{0}} B_{z} d B_{z}
$$

or, using (6.2),

$$
d p_{z}=-\frac{2 B_{z}^{2}}{\mu_{0}} \frac{d r}{r}
$$

Considering now the azimuthal magnetic flux density $B_{\theta}$, it is easily seen from Ampère's law that external to the column we have

$$
r B_{\theta}(r)=\text { constant }
$$

so that the azimuthal magnetic flux density, at the constricted surface, 


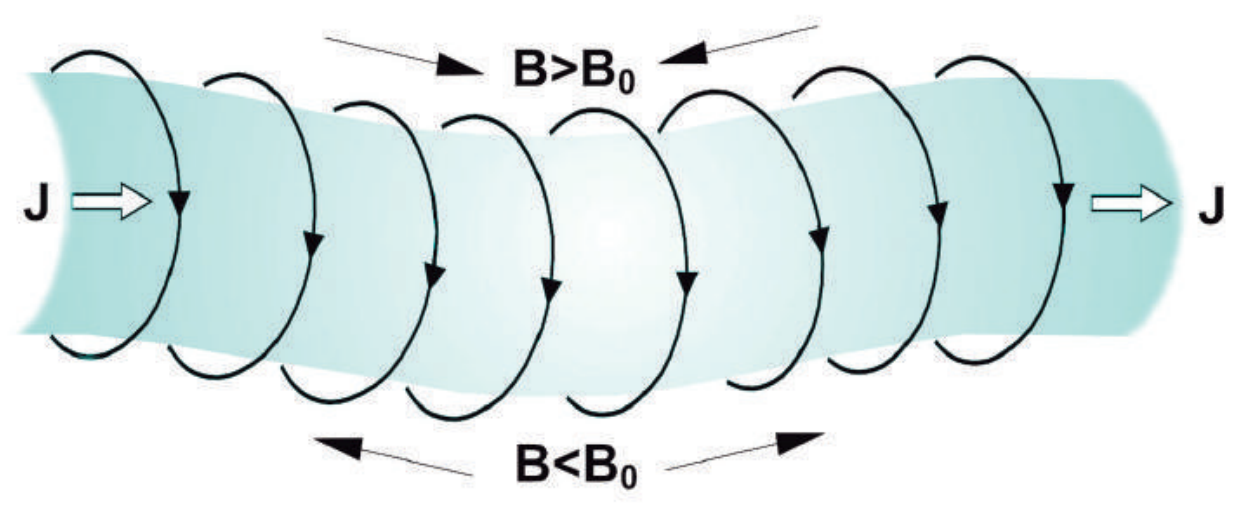

Fig. 14 The kink instability.

increases by the amount

$$
d B_{\theta}=-B_{\theta} \frac{d r}{r}
$$

Hence, the corresponding increase in the external magnetic pressure is

$$
d p_{\theta}=\frac{B_{\theta}}{\mu_{0}} d B_{\theta}=-\frac{B_{\theta}^{2}}{\mu_{0}} \frac{d r}{r}
$$

Therefore, in order that the plasma column be stable against the sausage distortion, we must have $d p_{z}>d p_{\theta}$, or, using (6.4) and (6.7),

$$
B_{z}^{2}>\frac{1}{2} B_{\theta}^{2}
$$

\section{THE KINK INSTABILITY}

Another type of instability of the pinched plasma column is the socalled kink instability. The kink distortion consists of a perturbation in the form of a bend or kink in the column, but with the disturbed column maintaining its uniform circular cross section, as shown in Fig. 14. Usually there may be several kinks along the column length. In the neighborhood 


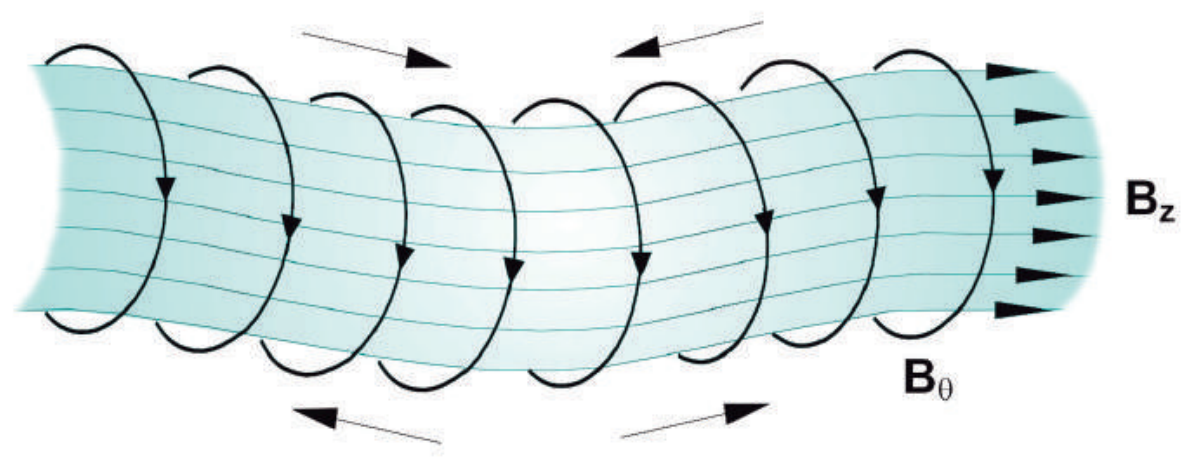

Fig. 15 The increased tension of a longitudinal magnetic field, applied inside the column, inhibits the kink instability.

of the column, where the kink has developed, the magnetic field lines are brought closer together on the concave side, and separated on the convex side, so that the external magnetic pressure is increased on the concave side and decreased on the convex side. Therefore, the changes in the external magnetic pressure are in such a way as to accentuate the distortion still further. This type of distortion is therefore unstable.

The kink instability can be hindered by the application of a longitudinal magnetic field within the plasma column, as in the case of the sausage instability. In the kink distortion, the longitudinal magnetic field lines frozen inside the plasma column are stretched and the increased tension acting along the longitudinal magnetic field lines opposes the external forces. The net result is the stabilization of the column (see Fig. 15).

In actual practice, however, the plasma is not perfectly diamagnetic and other fields may also be present. The calculation of the stability of the pinched plasma column is not, in general, a simple task.

\section{CONVEX FIELD CONFIGURATIONS}

In the linear pinch configuration, the azimuthal magnetic field confining the plasma column is produced by a longitudinal current flowing along the column. The configuration of this field is such that the magnetic flux lines are concave towards the plasma. Configurations of this type are unstable, as we have seen with the sausage and the kink instabilities. 


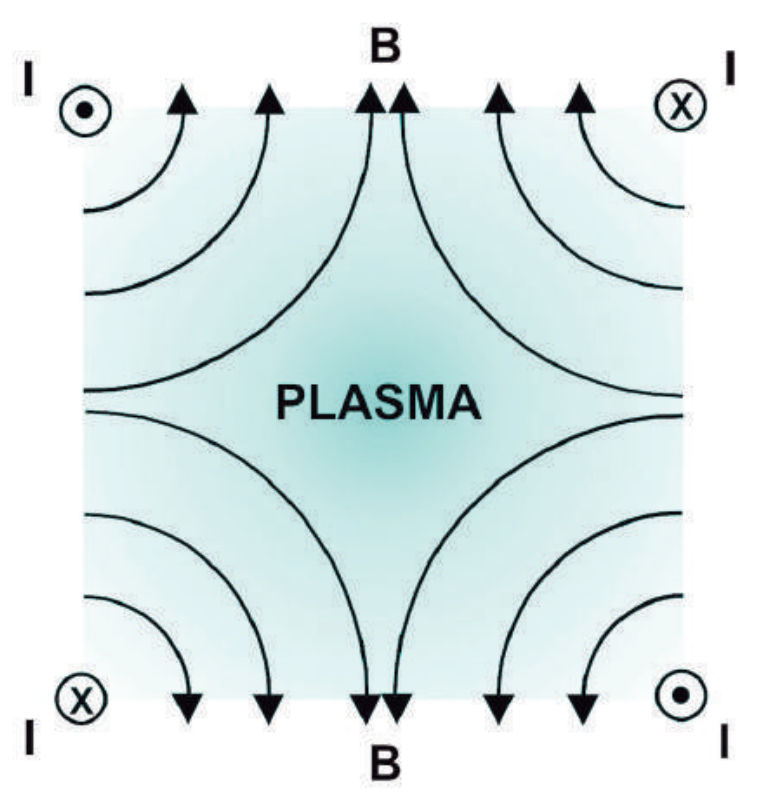

Fig. 16 Plasma confinement by a cusped magnetic field, produced by four current-carrying wires.

Configurations for which the field lines are convex towards the plasma lead to a stable equilibrium, since the magnetic field strength increases in a direction away from the plasma. If the plasma surface is perturbed by a wave-like disturbance, the magnetic pressure at the crests will be larger than the internal kinetic pressure and the plasma is forced to return to its equilibrium configuration (assuming that the kinetic pressure is not affected by the perturbations). At the troughs, the internal kinetic pressure will be larger than the magnetic pressure acting on the plasma surface and will force the plasma to expand. Therefore, for plasma confinement, it is desirable to use a magnetic field configuration in which the magnetic flux lines are everywhere convex towards the plasma. An example of this type of configuration is the cusp field, which can be produced by an array of four current-carrying wires, as shown in Fig. 16. The presence of sharp edges and cusps at the plasma boundary, however, can lead to escape of the plasma particles. Although edges and cusps are characteristics of these configurations, modifications of the cusp field geometry are commonly employed for confinement of high-temperature plasmas.

Higher order cusp fields can be produced by lining up several pairs of current-carrying wires as, for example, in the picket-fence field geometry illustrated in Fig. 17. 


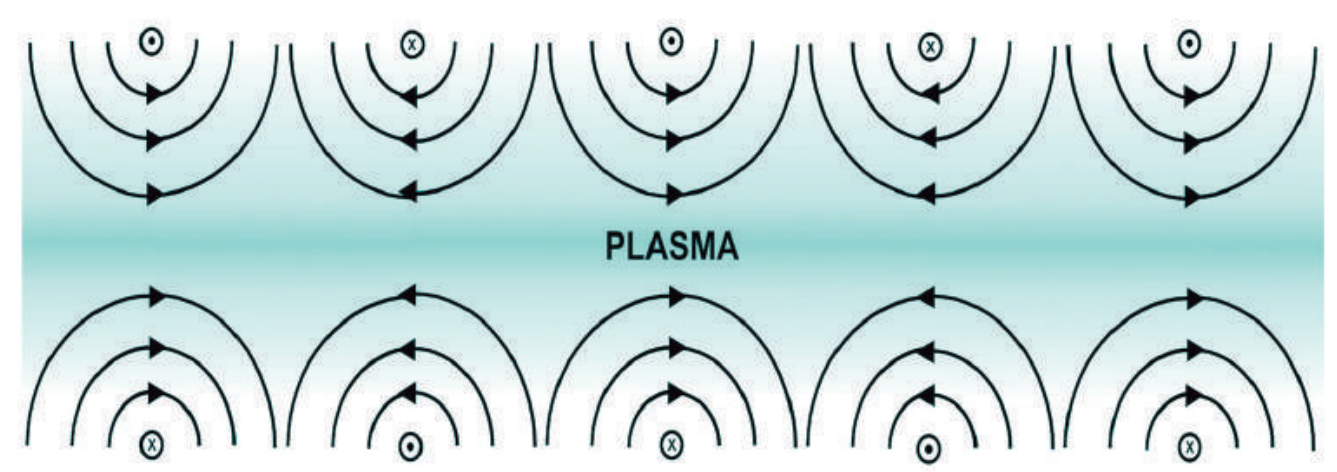

Fig. 17 Picket-fence field configuration for magnetic confinement of a plasma.

\section{PROBLEMS}

13.1 The minimum intensity of the magnetic induction $\left(\mathbf{B}_{0}\right)$ necessary to confine a plasma at an internal pressure of $100 \mathrm{~atm}$ is 5 weber $/ \mathrm{m}^{2}$ (refer to problem 12.3 in Chapter 12). Assuming that this field is produced by an axial current flowing in a cylindrical plasma column (as in the longitudinal pinch effect) of radius $10 \mathrm{~cm}$, show (applying Ampère's law) that the total current necessary to produce this magnetic field at the column surface is $2.5 \times 10^{6}$ ampere $\left(1 \mathrm{~atm}=10^{5}\right.$ newton $/ \mathrm{m}^{2}$ and $\mu_{0}=4 \times 10^{-7}$ henry $\left./ \mathrm{m}\right)$.

13.2 For the equilibrium Bennett pinch with cylindrical geometry, calculate $B_{\theta}(r)$ using (2.4) and the expression for $n(r)$ given in (3.8). Make a plot showing the radial distributions of $p(r), J_{z}(r)$, and $B_{\theta}(r)$.

13.3 For the equilibrium theta pinch produced by an azimuthal current in the theta direction $\left(\mathbf{J}_{\theta}\right)$, as illustrated in Fig. 6 of Chapter 12, determine expressions for the radial distributions of $J_{\theta}(r)$ and $p(r)$ in terms of $B_{z}(r)$. Draw a diagram illustrating these radial distributions for the special case when $B_{z}$ is constant.

13.4 Use the equation for the fluid velocity component $\left(\mathbf{u}_{\perp}\right)$ normal to $\mathbf{B}$, derived in problem 9.7 in Chapter 9, to determine the relative orientations of $\mathbf{u}, \mathbf{B}, \mathbf{E}, \mathbf{J}$, and $\nabla p$ in a theta-pinch device.

13.5 In the longitudinal equilibrium pinch shown schematically in Fig. 1 , assume that the radial dependence of the current density $J_{z}(r)$ is such 
that

$$
\begin{aligned}
& J_{z}=0 \quad \text { for } \quad 0<r<a \\
& J_{z}=J_{0}=\text { constant, for } \quad a<r<b \\
& J_{z}=0 \quad \text { for } r>b
\end{aligned}
$$

Calculate $p(r)$ and $B_{\theta}(r)$ and make a plot showing their radial dependence. Show that, as $a \rightarrow b$, the magnetic pressure $B_{\theta}^{2} / 2 \mu_{0}$ at $r=b$ becomes equal to $p$ at $r=0$, while as $a \rightarrow 0, \quad B_{\theta}^{2} / \mu_{0}$ at $r=b$ becomes equal to $p$ at $r=0$.

13.6 (a) Show that a force-free magnetic field satisfies the relation

$$
(\nabla \times \mathbf{B}) \times \mathbf{B}=0
$$

(b) Let $\nabla \times \mathbf{B}=\alpha(\mathbf{r}) \mathbf{B}$ and show that

$$
\text { B } \cdot \nabla \alpha=0
$$

(c) Verify that the surfaces $\alpha=$ constant are made up of magnetic field lines.

(d) Show that $\alpha(\mathbf{r})$, as defined in part (b), for the force-free field, can be expressed as

$$
\alpha=\widehat{\mathbf{B}} \cdot(\nabla \times \widehat{\mathbf{B}})
$$

(e) Prove that for the force-free field $\nabla B$ lies on the osculating plane, that is, the plane containing $\mathbf{B}$ and the principal normal to the field line.

13.7 Consider the following basic equation for the equilibrium of a plasma column with cylindrical geometry (see problem 12.9 in Chapter 12)

$$
\frac{d}{d r}\left(p+\frac{B_{z}^{2}}{2 \mu_{0}}+\frac{B_{\theta}^{2}}{2 \mu_{0}}\right)=-\frac{1}{\mu_{0}} \frac{B_{\theta}^{2}}{r}
$$

(a) Verify that, for the theta pinch, this equation reduces to

$$
p+\frac{B_{z}^{2}}{2 \mu_{0}}=\text { constant }
$$

whereas, for the longitudinal pinch, it becomes

$$
\frac{d}{d r}\left(p+\frac{B_{\theta}^{2}}{2 \mu_{0}}\right)=-\frac{B_{\theta}^{2}}{\mu_{0} r}
$$


(b) For the cylindrical screw pinch, in which both $B_{\theta}$ and $B_{z}$ are nonzero, assume that the longitudinal current density and the kinetic pressure are given, respectively, by

$$
\begin{array}{ll}
J_{z}(r)=J_{0}\left(1-\frac{r^{2}}{a^{2}}\right) & (r \leq a) \\
p(r)=p_{0}=\text { constant } & (r<a)
\end{array}
$$

Verify that

$$
\begin{array}{ll}
B_{\theta}(r)=\frac{1}{2} \mu_{0} J_{0}\left(1-\frac{r^{2}}{2 a^{2}}\right) r & (r \leq a) \\
B_{\theta}(r)=\frac{\mu_{0} J_{0} a^{2}}{4 r} & (r \geq a)
\end{array}
$$

Show that $B_{z}(r)$ satisfies the equation

$$
\frac{B_{z}^{2}}{2 \mu_{0}}=-\frac{B_{\theta}^{2}}{2 \mu_{0}}-\frac{1}{\mu_{0}} \int_{r} \frac{B_{\theta}^{2}(r)}{r} d r
$$

From this equation determine $B_{z}(r)$ and make a plot showing $p(r), J_{z}(r)$, $B_{\theta}(r)$, and $B_{z}(r)$ as a function of $r$. 


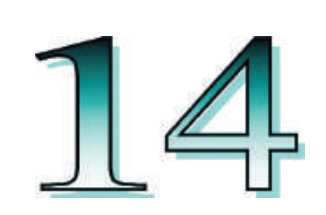

\section{ELECTROMAGNETIC WAVES \\ IN FREE SPACE}

\section{THE WAVE EQUATION}

Plasmas are able to sustain a great variety of wave phenomena. Before we initiate the study of wave phenomena in plasmas, we present in this chapter a review of some of the basic features of electromagnetic waves propagating in free space. The starting point for deriving the partial differential equation for electromagnetic waves in free space is Maxwell equations, which for free space $(\rho=0$ and $\mathbf{J}=0)$ may be written as

$$
\begin{gathered}
\nabla \cdot \mathbf{E}=0 \\
\nabla \cdot \mathbf{B}=0 \\
\nabla \times \mathbf{E}=-\frac{\partial \mathbf{B}}{\partial t} \\
\nabla \times \mathbf{B}=\frac{1}{c^{2}} \frac{\partial \mathbf{E}}{\partial t}
\end{gathered}
$$

Taking the time derivative of both sides of (1.4), yields

$$
\nabla \times \frac{\partial \mathbf{B}}{\partial t}=\frac{1}{c^{2}} \frac{\partial^{2} \mathbf{E}}{\partial t^{2}}
$$

Substituting $(\partial \mathbf{B} / \partial t)$ from $(1.3)$, gives

$$
\nabla \times(\nabla \times \mathbf{E})=-\frac{1}{c^{2}} \frac{\partial^{2} \mathbf{E}}{\partial t^{2}}
$$


Using the vector operator identity

$$
\nabla \times(\nabla \times \mathbf{E})=\nabla(\nabla \cdot \mathbf{E})-\nabla^{2} \mathbf{E}
$$

and noting that $\nabla \cdot \mathbf{E}=0$ in free space, we obtain

$$
\nabla^{2} \mathbf{E}-\frac{1}{c^{2}} \frac{\partial^{2} \mathbf{E}}{\partial t^{2}}=0
$$

In a similar way we can perform the same operations on (1.3) to obtain

$$
\nabla^{2} \mathbf{B}-\frac{1}{c^{2}} \frac{\partial^{2} \mathbf{B}}{\partial t^{2}}=0
$$

Equations (1.8) and (1.9) are the vector wave equations satisfied by the electromagnetic field vectors $\mathbf{E}$ and $\mathbf{B}$ in free space. The velocity of propagation of such waves is $c=1 / \sqrt{\mu_{0} \epsilon_{0}}$. Since these equations are satisfied by each component of the field vectors $\mathbf{E}$ and $\mathbf{B}$, we may as well write a scalar wave equation

$$
\nabla^{2} \psi-\frac{1}{c^{2}} \frac{\partial^{2} \psi}{\partial t^{2}}=0
$$

where $\psi(\mathbf{r}, t)$ is used to denote any one of the components of $\mathbf{E}$ and $\mathbf{B}$.

\section{SOLUTION IN PLANE WAVES}

We are interested in transverse plane wave solutions of the partial differential wave equation (1.10), since these are the simplest and most fundamental electromagnetic waves. For transverse plane waves, the field vectors $\mathbf{E}$ and $\mathbf{B}$ lie on a plane perpendicular to the propagation direction and are functions only of the perpendicular distance from the origin to this plane and, of course, of time also. This plane, normal to the propagation direction, is called the wave front. Let $\zeta$ denote the perpendicular distance from the origin to the wave front plane and let $\widehat{\mathbf{k}}$ be a unit vector normal to this plane (refer to Fig. 1). Any point $P$ on the wave front can be represented by a position vector $\mathbf{r}$ drawn from the origin of the coordinate system. Thus, for any point on a given wave front we have $\widehat{\mathbf{k}} \cdot \mathbf{r}=\zeta=$ constant, which is the equation specifying this wave front plane. The direction cosines of the unit vector $\widehat{\mathbf{k}}$, in a Cartesian coordinate system, are given by the scalar products $\widehat{\mathbf{k}} \cdot \widehat{\mathbf{x}}, \widehat{\mathbf{k}} \cdot \widehat{\mathbf{y}}$, and $\widehat{\mathbf{k}} \cdot \widehat{\mathbf{z}}$. 


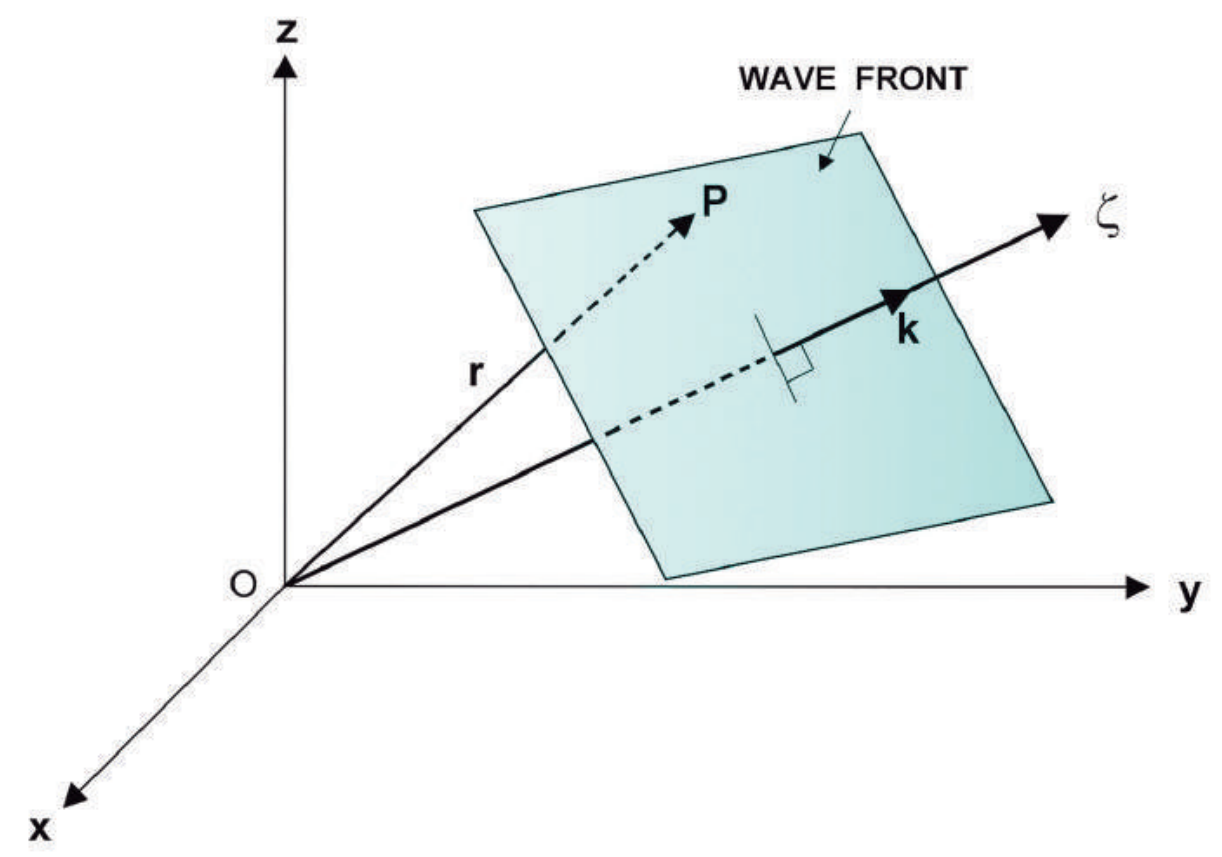

Fig. 1 In a transverse plane wave the field vectors do not vary along a given wave front plane, normal to the direction of propagation $\zeta$. They vary only along the $\zeta$ direction and with time.

Since the field vectors $\mathbf{E}$ and $\mathbf{B}$ are spatially constant along the wave front (normal to $\widehat{\mathbf{k}}$ ), but vary only in the $\zeta$ direction (and with time), the del operator can be written as

$$
\nabla=\widehat{\mathbf{x}} \frac{\partial}{\partial x}+\widehat{\mathbf{y}} \frac{\partial}{\partial y}+\widehat{\mathbf{z}} \frac{\partial}{\partial z}=\widehat{\mathbf{k}} \frac{\partial}{\partial \zeta}
$$

Hence, the wave equation is actually one-dimensional in form for the space variable $\zeta$,

$$
\frac{\partial^{2} \psi}{\partial \zeta^{2}}-\frac{1}{c^{2}} \frac{\partial^{2} \psi}{\partial t^{2}}=0
$$

The general solution of this one-dimensional wave equation is a linear combination of arbitrary functions of the variables $(\zeta-c t)$ and $(\zeta+c t)$,

$$
\psi(\zeta, t)=f(\zeta-c t)+g(\zeta+c t)
$$


The function $f(\zeta-c t)$ represents a plane waveform propagating in the positive $\zeta$ direction, whereas $g(\zeta+c t)$ corresponds to a waveform propagating in the negative $\zeta$ direction, the velocity of propagation being $c=1 / \sqrt{\mu_{0} \epsilon_{0}}$.

\section{HARMONIC WAVES}

A particularly important type of plane waveform is the harmonic wave, which can be written in the form (for propagation in the positive direction)

$$
\psi(\zeta, t)=A \cos [k(\zeta-c t)]=A \cos (k \zeta-\omega t)
$$

where $\omega=k c$ is the angular frequency of the oscillation and $k$ is the wave number or propagation constant. Fig. 2 shows the space and time dependence of the harmonic wave $\psi(\zeta, t)$ given by (3.1). The wavelength $\lambda$ and the period $T$ of the wave motion are given, respectively, by

$$
\lambda=\frac{2 \pi}{k} \quad ; \quad T=\frac{2 \pi}{\omega}=\frac{1}{\nu}
$$

where $\nu$ is the frequency in cycles per second.

If we define a propagation vector $\mathbf{k}$, whose direction is that of the normal to the wave front $(\widehat{\mathbf{k}})$ and whose magnitude is the wave number $(|\mathbf{k}|=k)$, then, since $\zeta$ is the perpendicular distance from the origin to the wave front, we have

$$
\widehat{\mathbf{k}} \cdot \mathbf{r}=(\mathbf{k} / k) \cdot \mathbf{r}=\zeta
$$

Hence, for a harmonic plane wave traveling at some arbitrary direction specified by the unit vector $\widehat{\mathbf{k}}$,

$$
\psi(\mathbf{r}, t)=A \cos (\mathbf{k} \cdot \mathbf{r}-\omega t)
$$

In view of the argument of the cosine function (3.4), the planes of constant phase are defined by the condition

$$
\mathbf{k} \cdot \mathbf{r}-\omega t=k \zeta-\omega t=\mathrm{constant}
$$

The phase velocity is defined as the velocity of propagation of these planes of constant phase $(d \zeta / d t)$ and is found by differentiating (3.5) with respect to time,

$$
v_{p h}=\frac{\omega}{k}
$$



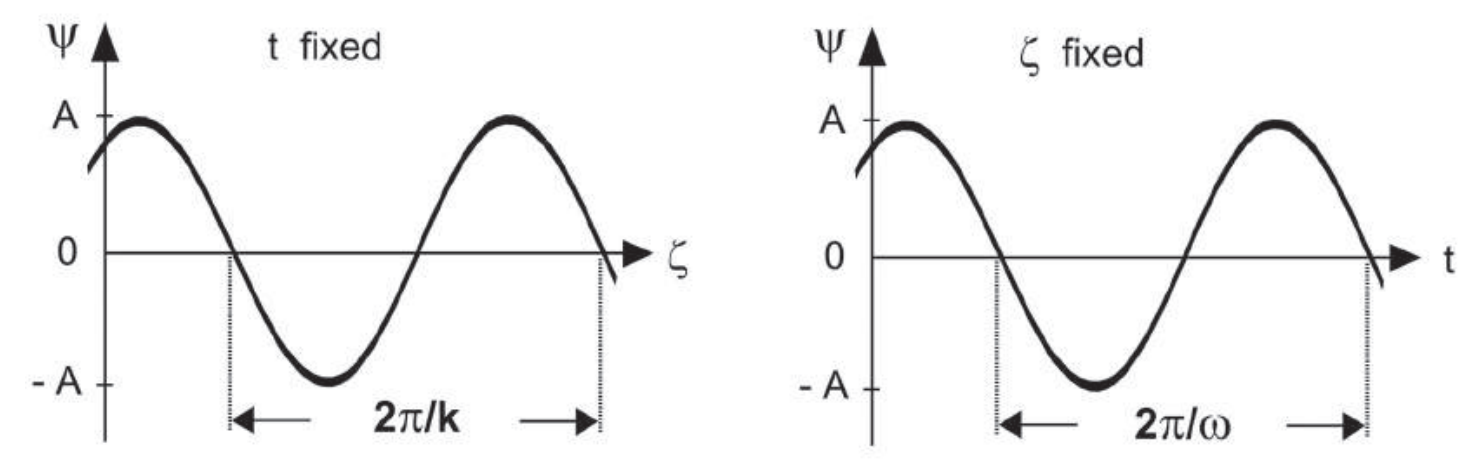

Fig. 2 Amplitude of a harmonic plane wave as a function of space and time.

The phase velocity is positive for a wave moving in the positive $\zeta$ direction, that is, $\zeta$ increases as $t$ increases in order to keep $(k \zeta-\omega t)$ constant. If we had taken

$$
\psi(\mathbf{r}, t)=A \cos (\mathbf{k} \cdot \mathbf{r}+\omega t)
$$

which represents a harmonic plane wave moving in the negative $\zeta$ direction, then the phase velocity would be negative.

It is generally convenient and extremely useful, as we shall see, to write (3.4) in complex form

$$
\psi(\mathbf{r}, t)=A \exp [i(\mathbf{k} \cdot \mathbf{r}-\omega t)]
$$

where implicit in this notation is the understanding that the field quantities are obtained by taking the real part of the complex expressions. The true physical quantity involved (the one we would measure in an experiment) is represented by the real part of the complex form. The use of complex expressions, however, greatly simplifies the mathematical calculations for the case of linear differential equations, as they are transformed into simple algebraic equations.

Using the complex notation (3.8) for the harmonic plane wave solutions for the field vectors $\mathbf{E}$ and $\mathbf{B}$, the operators $\nabla$ and $\partial / \partial t$ become

$$
\nabla=i \mathbf{k} \quad ; \quad \frac{\partial}{\partial t}=-i \omega
$$




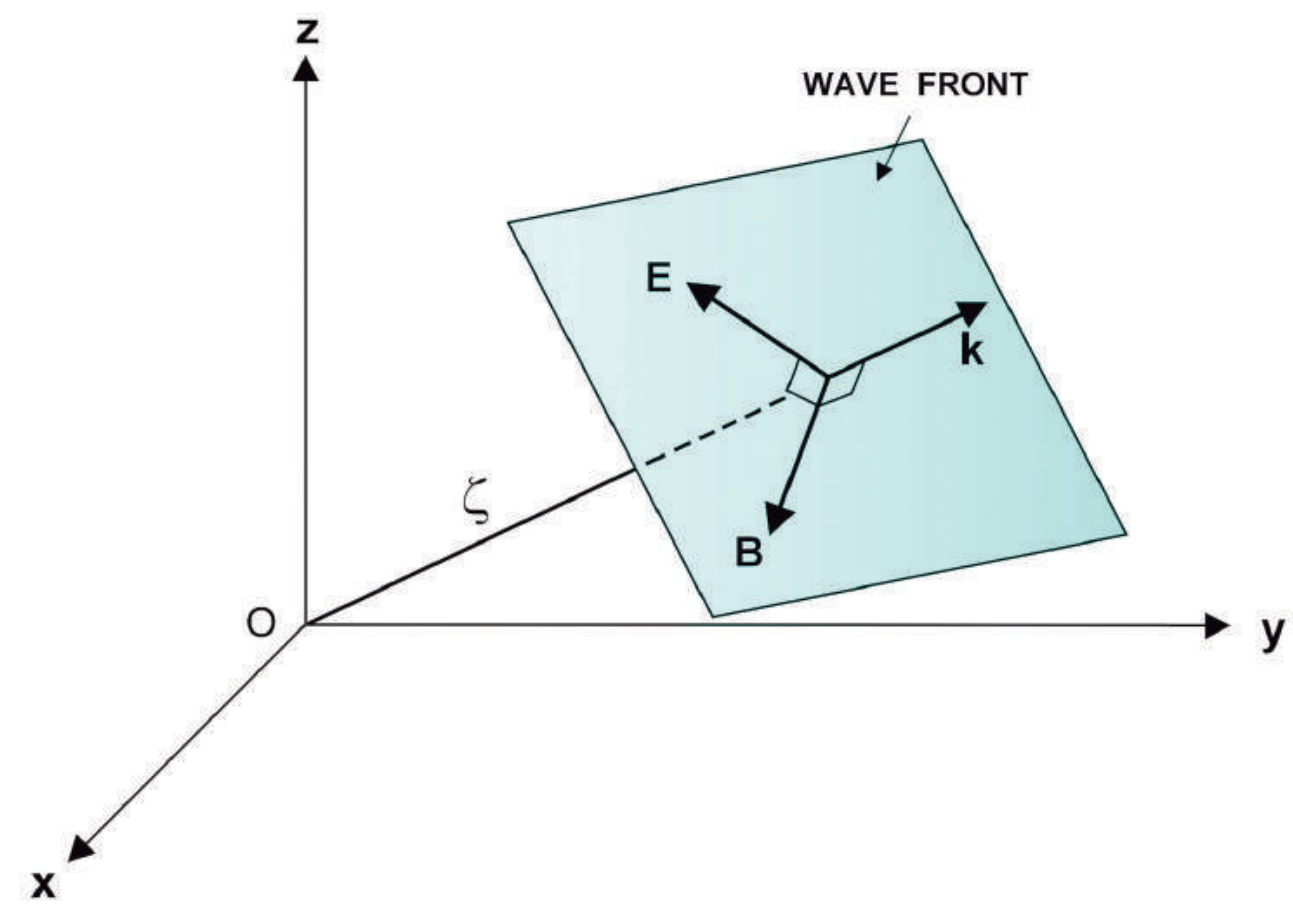

Fig. 3 The propagation vector $\mathbf{k}$ and the two wave field vectors $\mathbf{E}$ and $\mathbf{B}$ are orthogonal to each other.

so that Maxwell equations (1.1) to (1.4) in free space reduce to the following simple (linear, homogeneous) algebraic set of equations:

$$
\begin{gathered}
\mathbf{k} \cdot \mathbf{E}=0 \\
\mathbf{k} \cdot \mathbf{B}=0 \\
\mathbf{k} \times \mathbf{E}=\omega \mathbf{B} \\
\mathbf{k} \times \mathbf{B}=-\left(\frac{\omega}{c^{2}}\right) \mathbf{E}
\end{gathered}
$$

Thus, from (3.10) and (3.11) we see that $\mathbf{E}$ and $\mathbf{B}$ are both perpendicular to $\mathbf{k}$ and, for this reason, these waves are called transverse waves. In addition, from (3.12) or (3.13) we see that $\mathbf{E}$ and $\mathbf{B}$ are also perpendicular to each other. This situation is illustrated in Fig. 3. The set of vectors $(\mathbf{E}, \mathbf{B}, \mathbf{k})$, taken in this order, constitute a right-handed orthogonal set. Fig. 4 illustrates the relation between the $\mathbf{E}$ and $\mathbf{B}$ vectors of a plane electromagnetic wave propagating in free space. 

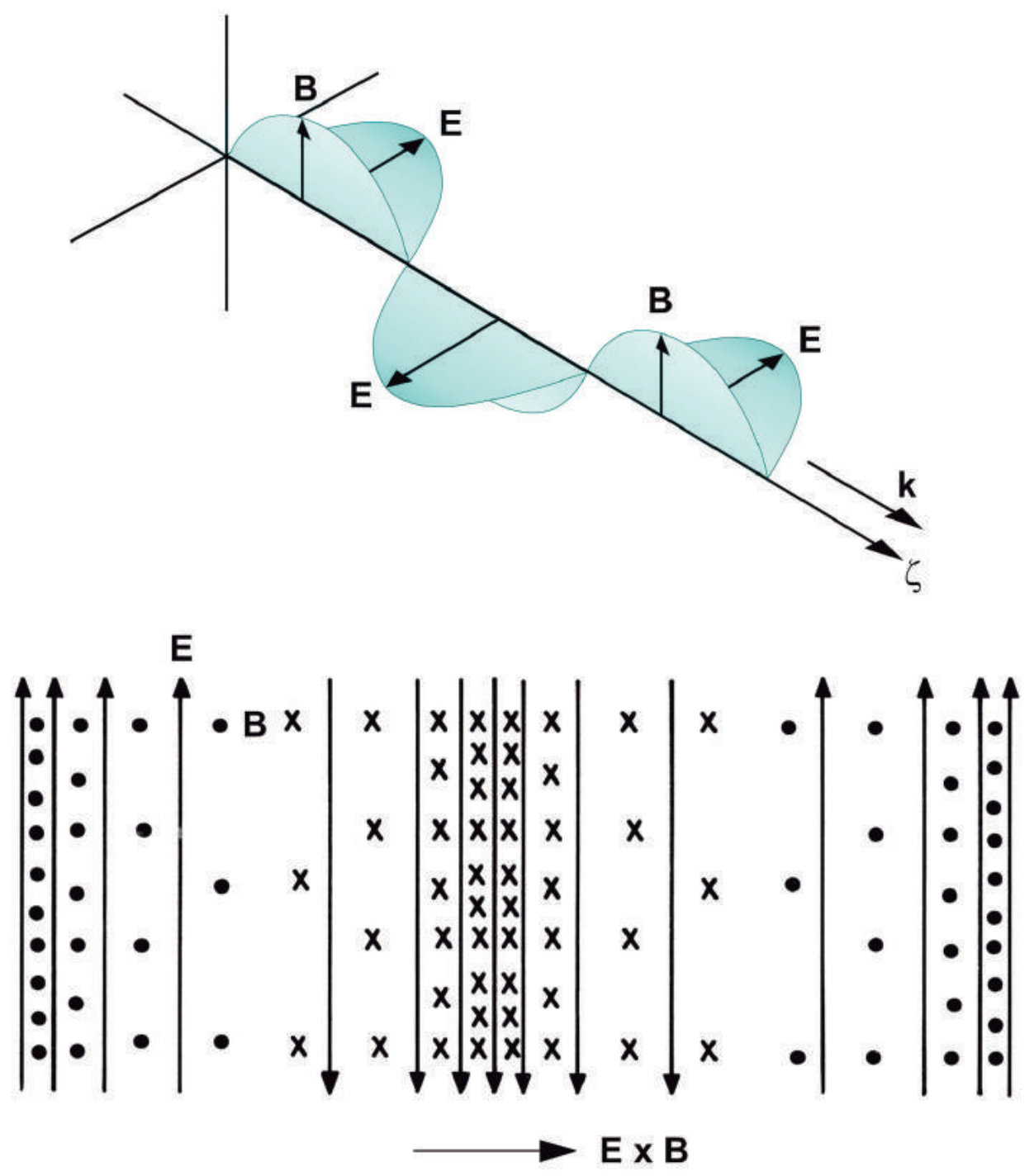

Fig. 4 Illustrating the field vectors $\mathbf{E}$ and $\mathbf{B}$ of a plane electromagnetic wave in free space. The dots and crosses represent magnetic lines of force coming out of the paper and into the paper, respectively, and the vertical lines represent the electric field. The direction of propagation is given by $\mathbf{k}$, which, in free space, is along the direction of $\mathbf{E} \times \mathbf{B}$. 


\section{POLARIZATION}

From (3.8) it is seen that the field vectors $\mathbf{E}(\mathbf{r}, t)$ and $\mathbf{B}(\mathbf{r}, t)$, which are solutions of the wave equation in the form of harmonic plane waves propagating in the positive $\zeta$ direction, can be written as

$$
\begin{aligned}
& \mathbf{E}(\mathbf{r}, t)=\mathbf{E}_{0} \exp [i(\mathbf{k} \cdot \mathbf{r}-\omega t)]=\mathbf{E}_{0} \exp [i(k \zeta-\omega t)] \\
& \mathbf{B}(\mathbf{r}, t)=\mathbf{B}_{0} \exp [i(\mathbf{k} \cdot \mathbf{r}-\omega t)]=\mathbf{B}_{0} \exp [i(k \zeta-\omega t)]
\end{aligned}
$$

where $\mathbf{E}_{0}$ and $\mathbf{B}_{0}$ are constant vector amplitudes, which may also be complex quantities. The phenomenon of polarization can be discussed entirely from the point of view of the electric field vector, since the magnetic induction vector may always be obtained from $\mathbf{E}$ using (3.12) for the case of plane waves.

According to (4.1), the direction of the electric field vector $\mathbf{E}$ is always the same, and the wave is said to be linearly polarized. This is the simplest type of polarization state.

In general, in order to describe an arbitrary state of polarization, we must consider the electric field vector in a given plane as the superposition of two linearly independent, linearly polarized waves. Two such linearly independent waves can be represented by

$$
\begin{aligned}
& \mathbf{E}_{1}(\mathbf{r}, t)=\widehat{\mathbf{e}}_{1} E_{1} \exp [i(\mathbf{k} \cdot \mathbf{r}-\omega t)] \\
& \mathbf{E}_{2}(\mathbf{r}, t)=\widehat{\mathbf{e}}_{2} E_{2} \exp [i(\mathbf{k} \cdot \mathbf{r}-\omega t)]
\end{aligned}
$$

with the associated magnetic induction vectors given, respectively, by

$$
\begin{aligned}
& \mathbf{B}_{1}(\mathbf{r}, t)=\frac{1}{\omega} \mathbf{k} \times \mathbf{E}_{1}(\mathbf{r}, t) \\
& \mathbf{B}_{2}(\mathbf{r}, t)=\frac{1}{\omega} \mathbf{k} \times \mathbf{E}_{2}(\mathbf{r}, t)
\end{aligned}
$$

The unit vectors $\widehat{\mathbf{e}}_{1}$ and $\widehat{\mathbf{e}}_{2}$, called the polarization vectors, are perpendicular to each other and lie in the plane normal to the propagation vector $\mathbf{k}$, and are such that $\left(\widehat{\mathbf{e}}_{1}, \widehat{\mathbf{e}}_{2}, \widehat{\mathbf{k}}\right)$, taken in this order, form a right-handed orthogonal set of unit vectors (see Fig. 5). The amplitudes $\mathbf{E}_{1}$ and $\mathbf{E}_{2}$ may be complex numbers, in order to allow for the possibility of any phase difference between the two waves $\mathbf{E}_{1}(\mathbf{r}, t)$ and $\mathbf{E}_{2}(\mathbf{r}, t)$. Therefore, a general solution for a harmonic plane wave propagating in the direction $\widehat{\mathbf{k}}$ can be 


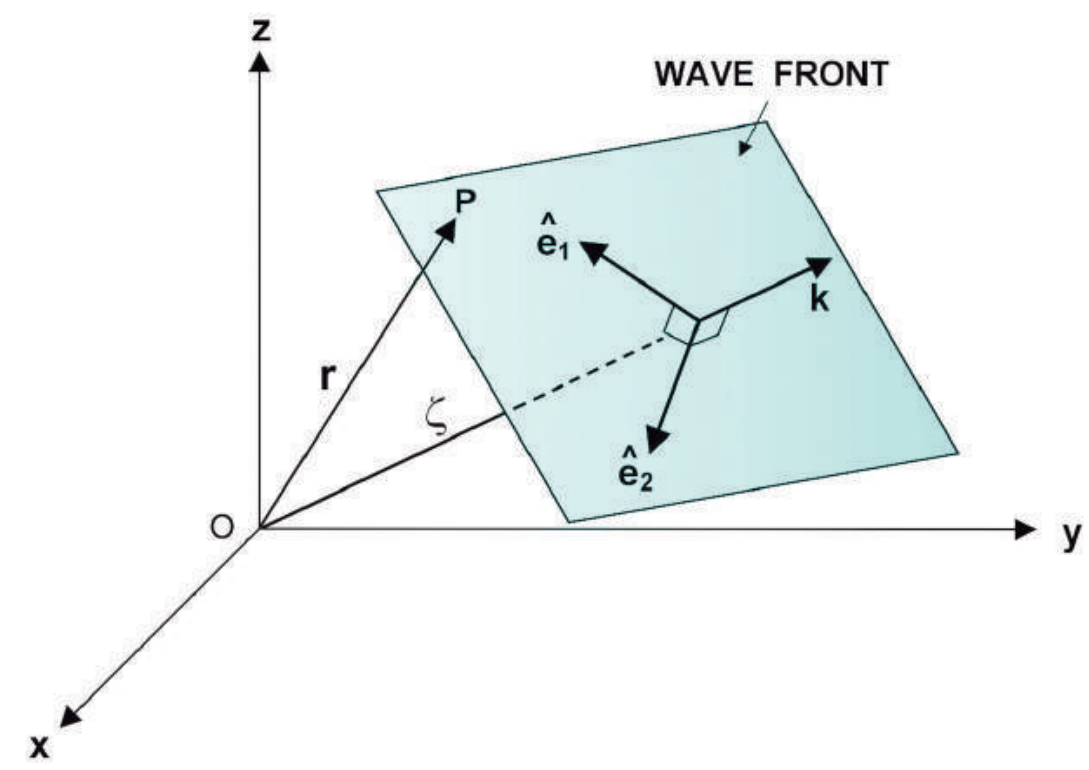

Fig. 5 The orthogonal polarization vectors $\widehat{\mathbf{e}}_{1}$ and $\widehat{\mathbf{e}}_{2}$, and the propagation vector $\mathbf{k}$.

written as a linear combination of the two linearly independent solutions $\mathbf{E}_{1}$ and $\mathbf{E}_{2}$,

$$
\mathbf{E}(\mathbf{r}, t)=\left(\widehat{\mathbf{e}}_{1} E_{1}+\widehat{\mathbf{e}}_{2} E_{2}\right) \exp [i(\mathbf{k} \cdot \mathbf{r}-\omega t)]
$$

Since any complex quantity can be expressed as the product of a real quantity and a complex phase factor, we way write

$$
\begin{aligned}
& E_{1}=E_{1}^{0} \exp \left(i \alpha_{1}\right) \\
& E_{2}=E_{2}^{0} \exp \left(i \alpha_{2}\right)
\end{aligned}
$$

where the amplitudes $E_{1}^{0}$ and $E_{2}^{0}$ are real numbers, and $\alpha_{1}$ and $\alpha_{2}$ represent the phase of the complex amplitudes $E_{1}$ and $E_{2}$, respectively. Note that it is only the phase difference that is physically significant and not the absolute magnitudes of $\alpha_{1}$ and $\alpha_{2}$. Hence, (4.7) becomes

$$
\mathbf{E}(\mathbf{r}, t)=\left[\widehat{\mathbf{e}}_{1} E_{1}^{0} \exp \left(i \alpha_{1}\right)+\widehat{\mathbf{e}}_{2} E_{2}^{0} \exp \left(i \alpha_{2}\right)\right] \exp [i(\mathbf{k} \cdot \mathbf{r}-\omega t)]
$$

If $E_{1}$ and $E_{2}$ have the same phase $\left(\alpha_{1}=\alpha_{2}\right)$, then (4.10) represents a linearly polarized wave. The magnitude of $\mathbf{E}$ is

$$
E=\left(E_{1}^{2}+E_{2}^{2}\right)^{1 / 2}
$$




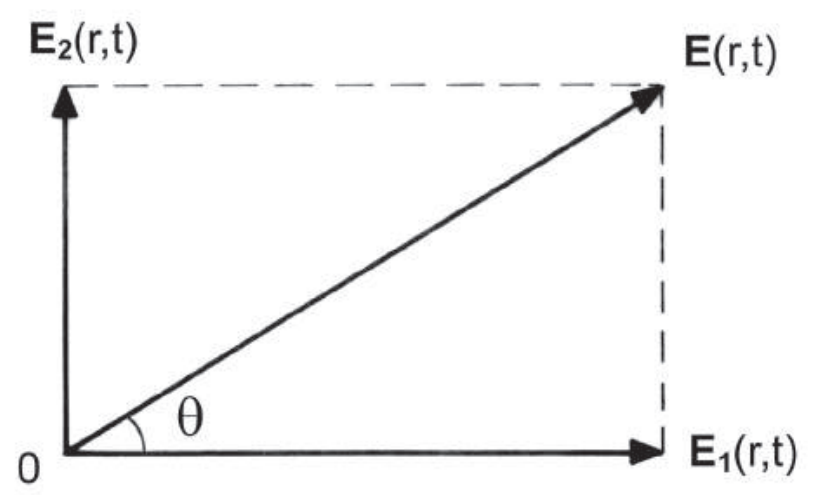

Fig. 6 Electric field vector of a linearly polarized wave, represented by the superposition of two independent, linearly polarized waves $\mathbf{E}_{1}$ and $\mathbf{E}_{2}$, having the same phase.

and its polarization vector makes an angle

$$
\theta=\tan ^{-1}\left(E_{2} / E_{1}\right)
$$

with the direction of $\widehat{\mathbf{e}}_{1}$. The electric field of this linearly polarized wave is represented in Fig. 6.

If $E_{1}$ and $E_{2}$ have different phases $\left(\alpha_{1} \neq \alpha_{2}\right)$, then (4.10) represents, in general, an elliptically polarized wave. The simplest case is the one in which the amplitudes of the components are equal, $E_{1}^{0}=E_{2}^{0}=E^{0}$, but the phases differ by $\pi / 2$, that is, $\alpha_{1}=0$ and $\alpha_{2}=\pi / 2$. Then, since $\exp ( \pm i \pi / 2)= \pm i$, the wave (4.10) becomes

$$
\mathbf{E}(\mathbf{r}, t)=E^{0}\left(\widehat{\mathbf{e}}_{1} \pm i \widehat{\mathbf{e}}_{2}\right) \exp [i(\mathbf{k} \cdot \mathbf{r}-\omega t)]
$$

This wave is said to be circularly polarized. In order to illustrate this point, consider a Cartesian coordinate system such that the wave is propagating in the $z$ direction, $\widehat{\mathbf{k}}=\widehat{\mathbf{z}}$, with $\widehat{\mathbf{e}}_{1}=\widehat{\mathbf{x}}$ and $\widehat{\mathbf{e}}_{2}=\widehat{\mathbf{y}}$. Thus, taking the real part of (4.13) we obtain for the $x$ and $y$ components of the actual electric field,

$$
\begin{gathered}
\mathbf{E}_{x}(z, t)=E^{0} \widehat{\mathbf{x}} \cos (k z-\omega t) \\
\mathbf{E}_{y}(z, t)=\mp E^{0} \widehat{\mathbf{y}} \sin (k z-\omega t)
\end{gathered}
$$

At a fixed plane $z=$ constant, the fields are such that the $\mathbf{E}$ vector has a constant magnitude $E^{0}$, but rotates around in a circle at the frequency $\omega$. 


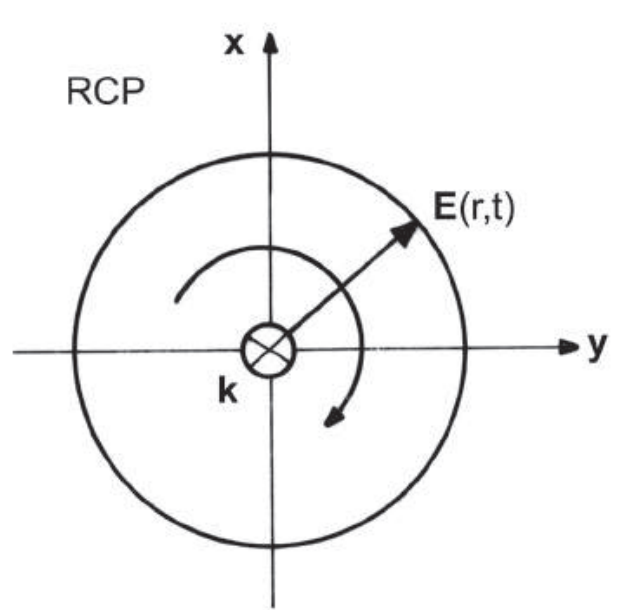

(a)

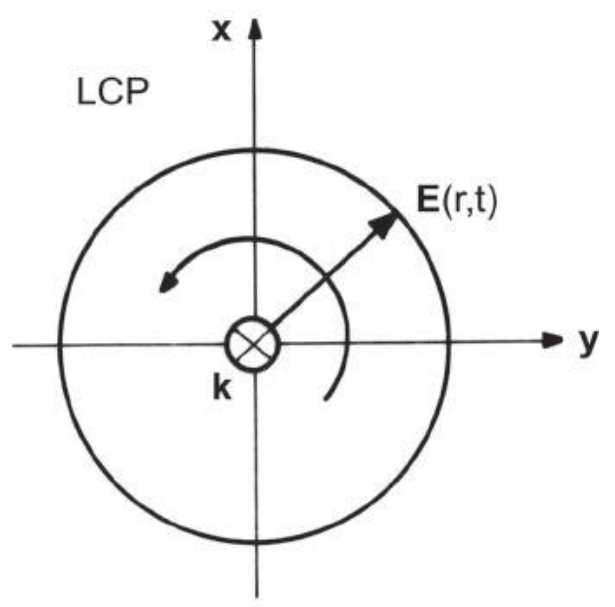

(b)

Fig. 7 Electric field vector of a right circularly polarized wave (a) and of a left circularly polarized wave (b). The propagation vector $\mathbf{k}$ points into the paper.

For the upper sign (that is, $\widehat{\mathbf{e}}_{1}+i \widehat{\mathbf{e}}_{2}$ ) the direction of rotation is clockwise if the wave is observed by looking at the outgoing wave front (i.e., looking along the positive $z$ direction), as shown in Fig. 7 (a). Such a wave is said to be right circularly polarized. For the lower sign $\left(\widehat{\mathbf{e}}_{1}-i \widehat{\mathbf{e}}_{2}\right)$ the rotation is in the counterclockwise direction as shown in Fig. 7 (b), and the wave is said to be left circularly polarized.

In the most general cases the amplitudes are different $\left(E_{1}^{0} \neq E_{2}^{0}\right)$ and the fields $\mathbf{E}_{1}$ and $\mathbf{E}_{2}$ have an arbitrary phase difference $\left(\alpha_{1} \neq \alpha_{2}\right)$. Considering $\left(\widehat{\mathbf{e}}_{1}, \widehat{\mathbf{e}}_{2}, \widehat{\mathbf{k}}\right)=(\widehat{\mathbf{x}}, \widehat{\mathbf{y}}, \widehat{\mathbf{z}})$ we have, from (4.10),

$$
\begin{aligned}
& \mathbf{E}_{x}(z, t)=\widehat{\mathbf{x}} E_{x}^{0} \exp \left(i \alpha_{1}\right) \exp [i(k z-\omega t)] \\
& \mathbf{E}_{y}(z, t)=\widehat{\mathbf{y}} E_{y}^{0} \exp \left(i \alpha_{2}\right) \exp [i(k z-\omega t)]
\end{aligned}
$$

Taking the real part of these expressions we obtain

$$
\begin{aligned}
& E_{x}(z, t)=E_{x}^{0} \cos \left(k z-\omega t+\alpha_{1}\right) \\
& E_{y}(z, t)=E_{y}^{0} \cos \left(k z-\omega t+\alpha_{2}\right)
\end{aligned}
$$

Squaring and adding (4.18) and (4.19), yields

$$
\left(\frac{E_{x}}{E_{x}^{0}}\right)^{2}+\left(\frac{E_{y}}{E_{y}^{0}}\right)^{2}=\cos ^{2}\left(k z-\omega t+\alpha_{1}\right)+\cos ^{2}\left(k z-\omega t+\alpha_{2}\right)
$$




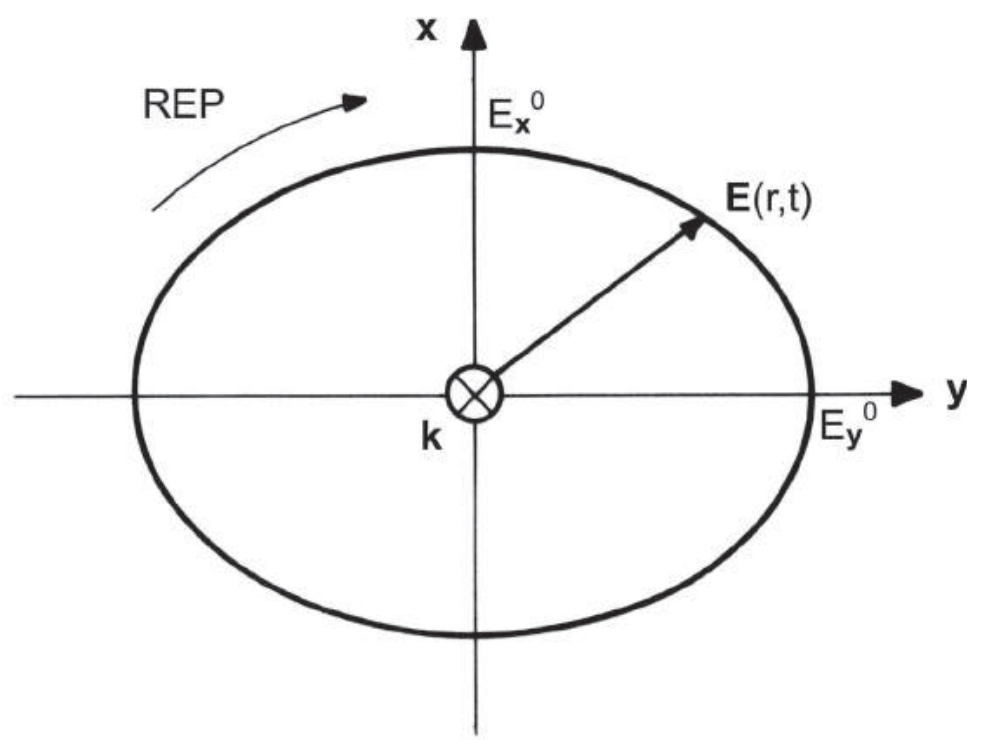

Fig. 8 Electric field vector of a right-hand elliptically polarized wave $\left(\alpha_{1}=0, \alpha_{2}=\pi / 2\right)$, rotating in the clockwise direction for an observer looking at the outgoing wave. The propagation vector $\mathbf{k}$ points into the paper.

This result shows that at a fixed plane $z=$ constant the electric field vector $\mathbf{E}$ performs, in general, an elliptical motion as a function of time.

For the case when $\alpha_{1}=0$ and $\alpha_{2}=\pi / 2,(4.20)$ becomes, at the plane $z=0$,

$$
\left(\frac{E_{x}}{E_{x}^{0}}\right)^{2}+\left(\frac{E_{y}}{E_{y}^{0}}\right)^{2}=\cos ^{2}(-\omega t)+\cos ^{2}(-\omega t+\pi / 2)=1
$$

since for any angle $\phi$ we have $\cos (\phi+\pi / 2)=-\sin (\phi)$. In this case, the $x$ and $y$ components of the electric field are given by

$$
\begin{aligned}
& E_{x}(0, t)=E_{x}^{0} \cos (\omega t) \\
& E_{y}(0, t)=E_{y}^{0} \sin (\omega t)
\end{aligned}
$$

Therefore, the tip of the electric field vector traces an ellipse in the $(x, y)$ plane rotating in the clockwise direction (right-hand polarization) when the wave is propagating away from the observer, as illustrated in Fig. 8.

For the case when $\alpha_{1}=0$ and $\alpha_{2}=-\pi / 2$, the electric field vector rotates in the counterclockwise direction when the wave is propagating 


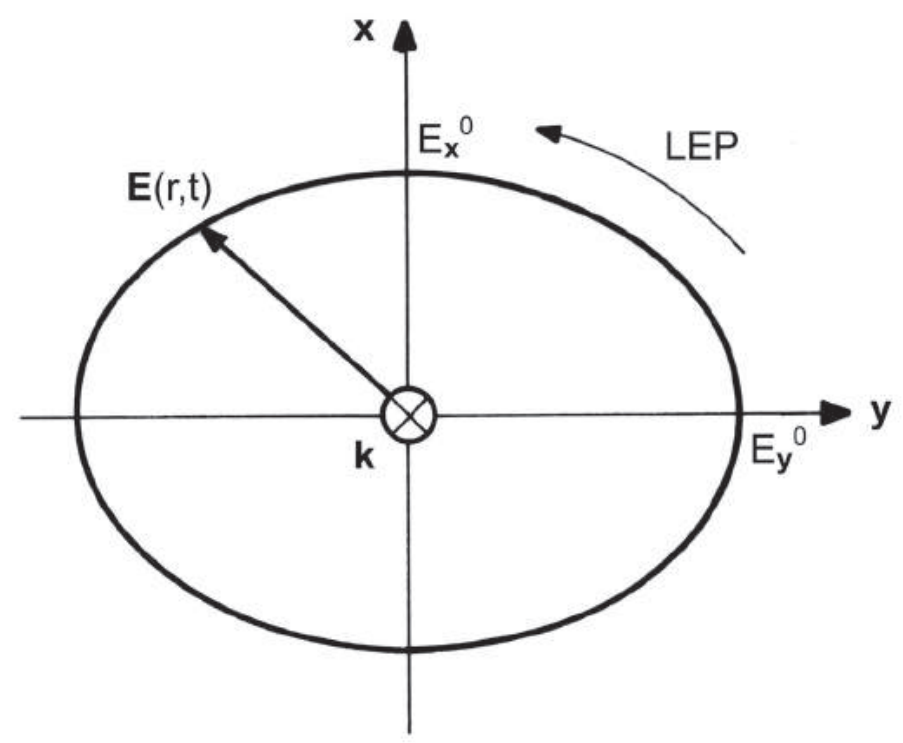

Fig. 9 Electric field vector of a left-hand elliptically polarized wave $\left(\alpha_{1}=0, \alpha_{2}=-\pi / 2\right)$, rotating in the counterclockwise direction for an observer looking at the outgoing wave (k points into the paper).

away from the observer, so that this wave is said to be left-hand elliptically polarized, as shown in Fig. 9.

The ellipticity of the ellipse traced and the orientation of the major axis, with respect to the $x$ axis, depend on the relative magnitude of the amplitudes $E_{x}^{0}$ and $E_{y}^{0}$, and on the phase difference $\left(\alpha_{2}-\alpha_{1}\right)$. Fig. 10 illustrates various wave polarizations, obtained from (4.18) and (4.19) at the plane $z=0$, for the case when $E_{x}^{0}=E_{y}^{0}$. The angle $\phi$ indicates the phase difference, $\phi=\left(\alpha_{2}-\alpha_{1}\right)$. In the figures shown, the sense of rotation of the $\mathbf{E}$ vector in the $(x, y)$ plane depends on whether $\phi$ lies between 0 and $\pi$, or between $\pi$ and $2 \pi$.

\section{ENERGY FLOW}

Associated with the electric and magnetic field vectors $\mathbf{E}$ and $\mathbf{H}$ of an electromagnetic wave, there is a flow of energy (energy per unit area and per unit time) in the direction perpendicular to both $\mathbf{E}$ and $\mathbf{H}$. This energy flow is given by the Poynting vector

$$
\mathbf{S}=\mathbf{E} \times \mathbf{H}
$$



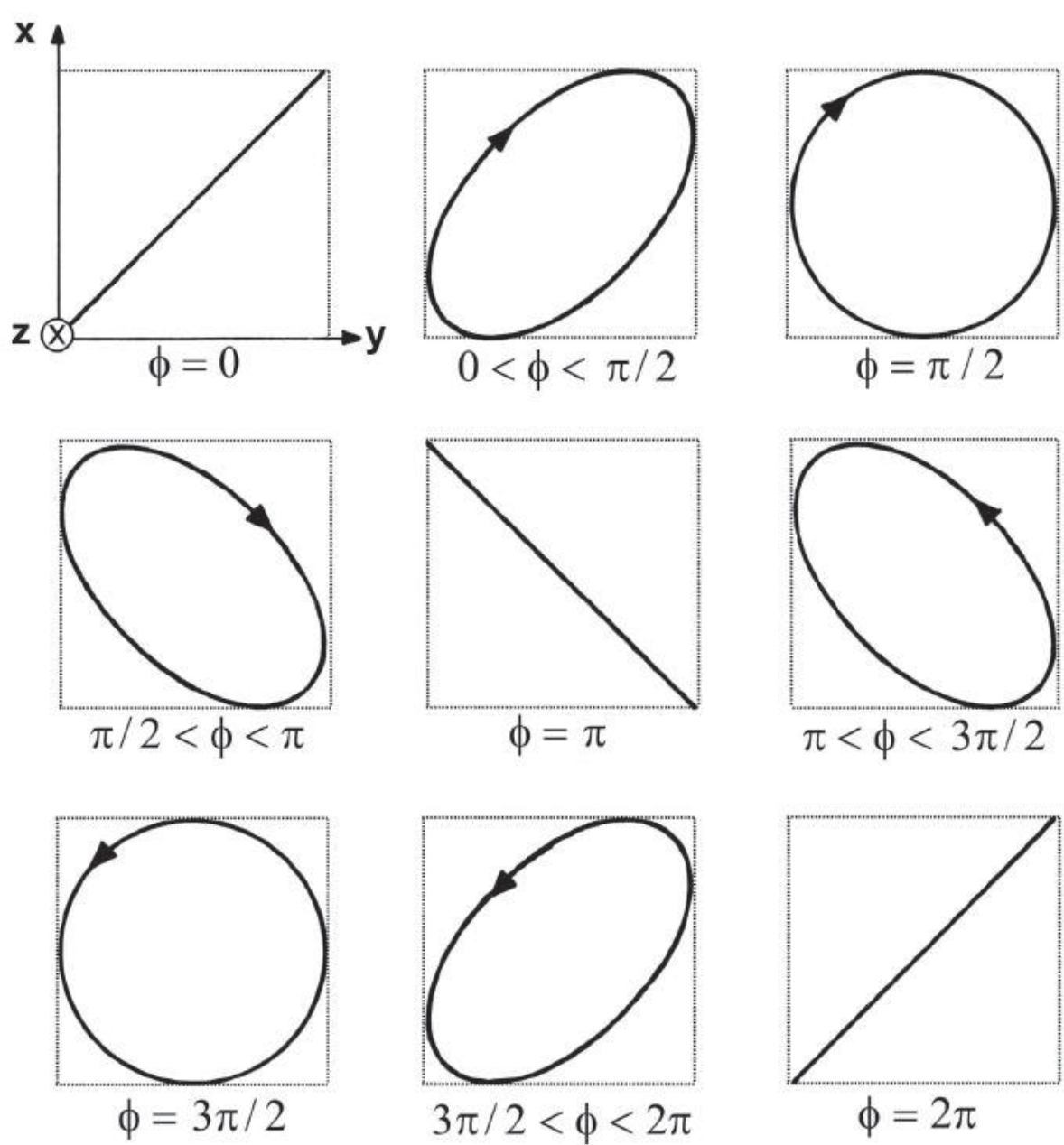

Fig. 10 Various wave polarizations for the case when $E_{x}^{0}=E_{y}^{0}$ obtained from (4.18) and from (4.19) at the plane $z=0$. The angle $\phi$ denotes the phase difference $\left(\phi=\alpha_{2}-\alpha_{1}\right)$.

In free space, $\mathbf{H}$ and $\mathbf{B}$ are related by $\mathbf{B}=\mu_{0} \mathbf{H}$. Also, in free space, the field vectors $\mathbf{E}$ and $\mathbf{H}$, and the propagation vector $\mathbf{k}$, form a mutually orthogonal set of vectors, although this may not be the case in a conducting medium as, for example, in a plasma. In such a medium, because of the presence of polarization charges, Maxwell equation $\nabla \cdot \mathbf{E}=\rho / \epsilon_{0}$ requires that $\mathbf{k} \cdot \mathbf{E} \neq 0$. Nevertheless, the direction of energy flow, given by the Poynting vector, is always perpendicular to $\mathbf{E}$ and $\mathbf{H}$.

When the field vectors $\mathbf{E}$ and $\mathbf{H}$ are expressed in terms of complex quantities, the energy flow vector given by (5.1) is called the complex Poynting vector. In this case, however, in order to obtain real physical 
quantities from the complex notation expressions, some caution must be taken since (5.1) involves the product of two complex quantities. The real energy flow in the wave is obtained by using the real part of both $\mathbf{E}$ and $\mathbf{H}$ in (5.1).

For the harmonic waves, the quantity of interest is the average energy flow (over one cycle) given by

$$
<\mathbf{S}>=<\mathcal{R}\{\mathbf{E}\} \times \mathcal{R}\{\mathbf{H}\}>
$$

where the time average is obtained by integrating the quantity over one period of the harmonic wave and dividing by the period, and where $\mathcal{R}$ means "the real part of". It must be stressed that the average energy flow $<\mathbf{S}>$ is not given by $<\mathcal{R}\{\mathbf{E} \times \mathbf{H}\}>$, nor by $\mathcal{R}\{<\mathbf{E} \times \mathbf{H}>\}$.

In what follows we show that the time-averaged energy flow $\langle\mathbf{S}>$, given by (5.2), can also be computed directly from the complex field vectors without performing an average over one cycle of the oscillation. For this purpose let us write

$$
\begin{gathered}
\mathbf{E}=\mathbf{E}_{0} \exp (-i \omega t)=\left(\mathbf{E}_{1}+i \mathbf{E}_{2}\right) \exp (-i \omega t) \\
\mathbf{H}=\mathbf{H}_{0} \exp (-i \omega t)=\left(\mathbf{H}_{1}+i \mathbf{H}_{2}\right) \exp (-i \omega t)
\end{gathered}
$$

where $\mathbf{E}_{1}, \mathbf{E}_{2}, \mathbf{H}_{1}$, and $\mathbf{H}_{2}$ are real amplitudes, and $\omega$ is the wave frequency. Taking the real part of (5.3) and (5.4), yields

$$
\begin{gathered}
\mathcal{R}\{\mathbf{E}\}=\mathbf{E}_{1} \cos (\omega t)+\mathbf{E}_{2} \sin (\omega t) \\
\mathcal{R}\{\mathbf{H}\}=\mathbf{H}_{1} \cos (\omega t)+\mathbf{H}_{2} \sin (\omega t)
\end{gathered}
$$

Consequently, (5.2) becomes

$$
\begin{gathered}
<\mathbf{S}>=\left(\mathbf{E}_{1} \times \mathbf{H}_{1}\right)<\cos ^{2}(\omega t)>+\left(\mathbf{E}_{2} \times \mathbf{H}_{2}\right)<\sin ^{2}(\omega t)>+ \\
\left(\mathbf{E}_{1} \times \mathbf{H}_{2}+\mathbf{E}_{2} \times \mathbf{H}_{1}\right)<\sin (\omega t) \cos (\omega t)>
\end{gathered}
$$

Using the following results for the integrals appearing in the average values contained in (5.7),

$$
\begin{aligned}
& <\cos ^{2}(\omega t)>=\frac{1}{T} \int_{0}^{T} \cos ^{2}(\omega t) d t=\frac{1}{2} \\
& <\sin ^{2}(\omega t)>=\frac{1}{T} \int_{0}^{T} \sin ^{2}(\omega t) d t=\frac{1}{2}
\end{aligned}
$$




$$
<\sin (\omega t) \cos (\omega t)>=\frac{1}{T} \int_{0}^{T} \sin (\omega t) \cos (\omega t) d t=0
$$

we obtain

$$
<\mathbf{S}>=\frac{1}{2}\left(\mathbf{E}_{1} \times \mathbf{H}_{1}+\mathbf{E}_{2} \times \mathbf{H}_{2}\right)
$$

Let us consider now the following quantity:

$$
\begin{gathered}
\mathcal{R}\left\{\mathbf{E} \times \mathbf{H}^{*}\right\}=\mathcal{R}\left\{\left(\mathbf{E}_{1}+i \mathbf{E}_{2}\right)[\cos (\omega t)-i \sin (\omega t)] \times\right. \\
\left.\times\left(\mathbf{H}_{1}-i \mathbf{H}_{2}\right)[\cos (\omega t)+i \sin (\omega t)]\right\} \\
=\mathbf{E}_{1} \times \mathbf{H}_{1}+\mathbf{E}_{2} \times \mathbf{H}_{2}
\end{gathered}
$$

Comparing this result with (5.11) we obtain the following alternative expression for the time-averaged energy flow (5.2):

$$
<\mathbf{S}>=\frac{1}{2} \mathcal{R}\left\{\mathbf{E} \times \mathbf{H}^{*}\right\}
$$

In a general medium (not free space) the time-averaged energy flow is given by

$$
<\mathbf{S}>=\frac{c}{4}\left(\mathbf{E} \cdot \mathbf{D}^{*}+\mathbf{B} \cdot \mathbf{H}^{*}\right) \widehat{\mathbf{e}}_{3}
$$

where $\widehat{\mathbf{e}}_{3}$ is a unit vector in the direction of $\mathbf{E} \times \mathbf{H}$.

\section{WAVE PACKETS AND GROUP VELOCITY}

So far we have considered simple waves having one specific value for $k$ and $\omega$. In practice, however, real disturbances consist of waves having some finite spread in the wave number $k$ and in the frequency $\omega$. A wave packet is a superposition of waves with different values of $k$ and $\omega$. This is equivalent to the statement that any wave-like disturbance can be decomposed by Fourier analysis into a superposition of simple harmonic oscillations with different frequencies $\omega$ and wave numbers $k$, with appropriate amplitudes.

A wave packet, consisting of a superposition of plane harmonic waves propagating in the $\zeta$ direction, can be represented by

$$
\psi(\zeta, t)=\int_{-\infty}^{+\infty} A(k) \exp [i(k \zeta-\omega t)] d k
$$

The wave packet concept is particularly useful when there is only a small spread in wave numbers about a central wave number $k_{0}$. This is equivalent to a small spread in frequencies about a central frequency $\omega_{0}$, since 
for any wave motion there is a functional relationship between $k$ and $\omega$, which depends on the medium, and which is called the dispersion relation. Thus, the amplitude function $A(k)$ is usually assumed to be peaked about some central wave number $k_{0}$. In (6.1) the wave number $k$ has been taken as the independent variable and $\omega$ is considered to be a function of $k$, determined by the dispersion relation $\omega=\omega(k)$.

The amplitude function $A(k)$ can be determined from the Fourier transform of $\psi(\zeta, 0)$. For this purpose, we multiply (6.1) (with $t=0$ ) by $\exp (-i k \zeta)$ and integrate the resultant equation over all possible values of $\zeta$. Thus,

$$
\int_{-\infty}^{+\infty} \psi(\zeta, 0) \exp (-i k \zeta) d \zeta=\int_{-\infty}^{+\infty} A\left(k^{\prime}\right) d k^{\prime} \int_{-\infty}^{+\infty} \exp \left[i\left(k^{\prime}-k\right) \zeta\right] d \zeta
$$

Using the following representation for the Dirac delta function

$$
\delta\left(k^{\prime}-k\right)=\frac{1}{2 \pi} \int_{-\infty}^{+\infty} \exp \left[i\left(k^{\prime}-k\right) \zeta\right] d \zeta
$$

and the property

$$
\int_{-\infty}^{+\infty} A\left(k^{\prime}\right) \delta\left(k^{\prime}-k\right) d k^{\prime}=A(k)
$$

we obtain, from (6.2),

$$
A(k)=\frac{1}{2 \pi} \int_{-\infty}^{+\infty} \psi(\zeta, 0) \exp (-i k \zeta) d \zeta
$$

The field quantity $\psi(\zeta, t)$ can also be synthesized in terms of time periodic functions of all possible frequencies, using the relation

$$
\psi(\zeta, t)=\int_{-\infty}^{+\infty} A(\omega) \exp [i(k \zeta-\omega t)] d \omega
$$

where the amplitude function $A(\omega)$ gives the frequency spectrum. In this case, $\omega$ is taken as the independent variable and the dispersion relation gives $k=k(\omega)$. The amplitude function $A(\omega)$ is usually peaked about some central frequency $\omega_{0}$ and it can be determined from the Fourier transform of $\psi(0, t)$,

$$
A(\omega)=\frac{1}{2 \pi} \int_{-\infty}^{+\infty} \psi(0, t) \exp (i \omega t) d t
$$


Consider now that we have a wave packet represented by (6.1), in which the range of values of $k$ is small and is centered about some specific wave number $k_{0}$,

$$
k_{0}-\delta k \leq k \leq k_{0}+\delta k
$$

If $\omega(k)$ is a slowly varying function of $k$, then $\omega(k)$ deviates only slightly from its value $\omega_{0}=\omega\left(k_{0}\right)$, and we can expand $\omega(k)$ in a Taylor series about $k_{0}$ retaining only the first two terms,

$$
\omega(k)=\omega_{0}+\left(k-k_{0}\right)\left(\frac{\partial \omega}{\partial k}\right)_{k_{0}}
$$

The phase factor in (6.1) can thus be written as

$$
[k \zeta-\omega(k) t]=k_{0} \zeta-\omega_{0} t+\left(k-k_{0}\right)\left[\zeta-\left(\frac{\partial \omega}{\partial k}\right)_{k_{0}} t\right]
$$

This allows the wave packet $\psi(\zeta, t)$ of $(6.1)$ to be recast in the form

$$
\psi(\zeta, t)=\psi_{m}(\zeta, t) \exp \left[i\left(k_{0} \zeta-\omega_{0} t\right)\right]
$$

where

$$
\psi_{m}(\zeta, t)=\int_{\left(k_{0}-\delta k\right)}^{\left(k_{0}+\delta k\right)} A(k) \exp \left\{i\left(k-k_{0}\right)\left[\zeta-\left(\frac{\partial \omega}{\partial k}\right)_{k_{0}} t\right]\right\} d k
$$

Therefore, the wave packet $\psi(\zeta, t)$ corresponds in this case to a carrier wave at the frequency $\omega_{0}$, modulated by the amplitude function $\psi_{m}(\zeta, t)$. A typical form for a wave packet is shown in Fig. 11, at a fixed instant of time.

The phases of constant packet amplitude are given by

$$
\zeta-\left(\frac{\partial \omega}{\partial k}\right)_{k_{0}} t=0
$$

and the velocity of propagation of these planes of constant phase, $d \zeta / d t$, called the group velocity, is obtained by differentiating (6.13) with respect to time,

$$
v_{g}=\left(\frac{\partial \omega}{\partial k}\right)_{k_{0}}
$$

The group velocity can be written in terms of the phase velocity $\left(v_{p h}=\right.$ $\omega / k)$ as

$$
v_{g}=\left(\frac{\partial \omega}{\partial k}\right)_{k_{0}}=\left[\frac{\partial\left(v_{p h} k\right)}{\partial k}\right]_{k_{0}}=v_{p h_{0}}+k_{0}\left(\frac{\partial v_{p h}}{\partial k}\right)_{k_{0}}
$$




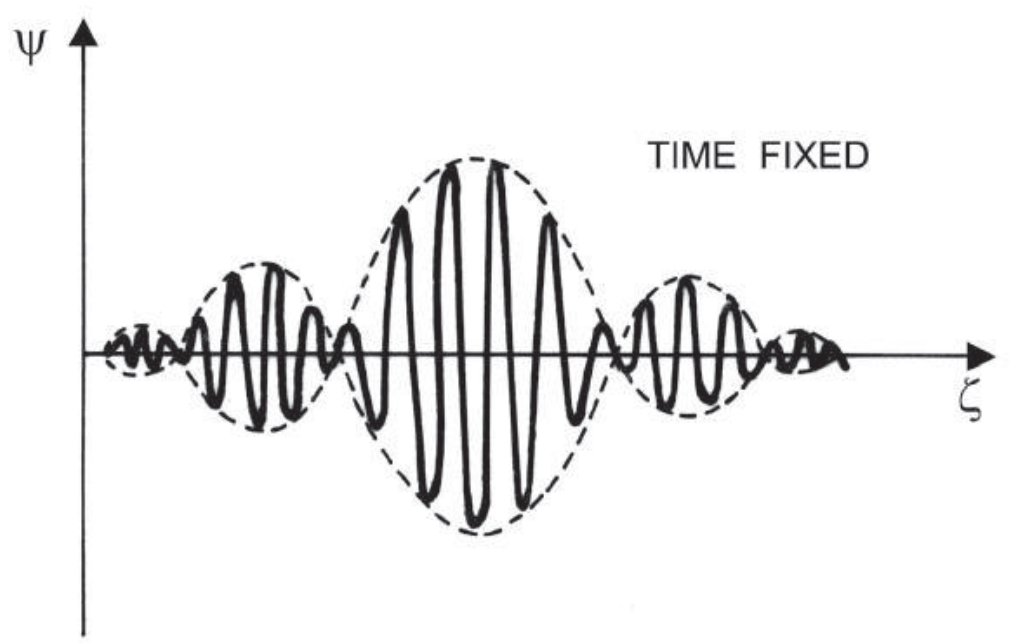

Fig. 11 Amplitude function $\psi(\zeta, t)$ of a typical wave packet. The dotted curve is the amplitude modulation function $\psi_{m}(\zeta, t)$.

If the medium is such that the phase velocity is not a function of $k$ [i.e., $\omega(k) \propto k$ ], then the group velocity is equal to the phase velocity and the medium is known as a nondispersive medium. This is the case for free space, for which $\omega=c k$. If the phase velocity decreases with increasing $k$ (i.e., $\partial v_{p h} / \partial k<0$ ), then $v_{g}$ is less than $v_{p h}$ and the medium is said to be normally dispersive. If the phase velocity increases with increasing $k$ (i.e., $\partial v_{p h} / \partial k>0$ ), then $v_{g}$ is larger than $v_{p h}$ and the medium is said to be anomalously dispersive.

It should be pointed out that, in many cases, the phase velocity of a wave in a plasma exceeds the velocity of light $c$. This fact is not in conflict with the relativity theory, because an infinitely long wave train of constant amplitude does not carry information. This information, however, is contained in the modulation of the carrier wave and this modulation travels at the group velocity, which is always less than the velocity of light.

In the derivation of (6.11) and (6.12) the Taylor expansion of $\omega(k)$ was carried out only up to a term linear in $\left(k-k_{0}\right)$. In this case the spatial variation of the amplitude function $\psi_{m}(\zeta, t)$, which gives the shape of the wave packet, remains the same at any time. If the higher order terms in $\left(k-k_{0}\right)$ are included in $(6.10)$, then the wave packet shape will change with time and the packet will spread out as it moves. Hence, the group velocity concept is useful only for wave packets with a very small spread in wave numbers and frequencies. 


\section{PROBLEMS}

14.1 Derive the vector wave equations, analogous to (1.8) and (1.9) for the electric and magnetic fields, considering a medium in which there is a space charge density distribution $\rho(\mathbf{r}, t)$ and a charge current density distribution $\mathbf{J}(\mathbf{r}, t)$.

14.2 Consider a plane electromagnetic harmonic wave traveling towards the positive $x$ direction in free space, having the frequency $\nu=5 \times 10^{15}$ hertz.

(a) What is the associated wavelength?

(b) Calculate the corresponding values of $\omega$ and $k$.

(c) Considering that

$$
\mathbf{E}=\widehat{\mathbf{y}} E_{0} \exp (i k x-i \omega t)
$$

calculate $(\nabla \times \mathbf{E})$ and $-(\partial \mathbf{B} / \partial t)$, and compare the results. Calculate also $(\nabla \times \mathbf{B})$ and $-(\partial \mathbf{B} / \partial t)$, and compare the results.

(d) Calculate the Poynting vector $\mathbf{S}$ and the average Poynting vector $<\mathbf{S}>$.

14.3 Consider a plane electromagnetic harmonic wave propagating along the positive $x$ direction in free space, which can be decomposed into the sum of two waves,

$$
\mathbf{E}=\widehat{\mathbf{y}} E_{1} \exp (i k x-i \omega t)+\widehat{\mathbf{z}} E_{2} \exp (i k x-i \omega t+i \alpha)
$$

Show that

$$
\begin{gathered}
\mathbf{S}=\left(\frac{\epsilon_{0}}{\mu_{0}}\right)^{1 / 2}\left[E_{1}^{2} \cos ^{2}(k x-\omega t)+E_{2}^{2} \cos ^{2}(k x-\omega t+\alpha)\right] \widehat{\mathbf{x}} \\
<\mathbf{S}>=\frac{1}{2}\left(\frac{\epsilon_{0}}{\mu_{0}}\right)^{1 / 2}\left(E_{1}^{2}+E_{2}^{2}\right) \widehat{\mathbf{x}}
\end{gathered}
$$

14.4 The electric field vector of an elliptically polarized plane wave, propagating in free space, can be expressed as

$$
\mathbf{E}=\left[\widehat{\mathbf{e}}_{1} E_{1}^{0} \exp \left(i \alpha_{1}\right)+\widehat{\mathbf{e}}_{2} E_{2}^{0} \exp \left(i \alpha_{2}\right)\right] \exp (i \mathbf{k} \cdot \mathbf{r}-i \omega t)
$$


(a) Show that the associated magnetic induction vector is

$$
\mathbf{B}=\frac{1}{c}\left[\widehat{\mathbf{e}}_{2} E_{1}^{0} \exp \left(i \alpha_{1}\right)-\widehat{\mathbf{e}}_{1} E_{2}^{0} \exp \left(i \alpha_{2}\right)\right] \exp (i \mathbf{k} \cdot \mathbf{r}-i \omega t)
$$

(b) Show that the time average of the energy density in this wave is

$$
<W>=\frac{1}{4}\left(\epsilon_{0} E^{2}+\frac{B^{2}}{\mu_{0}}\right)
$$

where

$$
E^{2}=\left(E_{1}^{0}\right)^{2}+\left(E_{2}^{0}\right)^{2}=c^{2} B^{2}
$$

(c) Show that the time-averaged energy flow $\langle\mathbf{S}>$ is equal to the phase velocity $(\omega / k=c)$ times the average energy density of the wave, that is,

$$
<\mathbf{S}>=c<W>\widehat{\mathbf{e}}_{3}=\frac{c}{4}\left(\epsilon_{0} E^{2}+\frac{B^{2}}{\mu_{0}}\right) \widehat{\mathbf{e}}_{3}
$$

where $\widehat{\mathbf{e}}_{3}$ denotes a unit vector in the direction of $\mathbf{E} \times \mathbf{H}$.

14.5 Show that in a general medium (not free space), the time average of the energy density (over one cycle) for harmonic plane waves is given by

$$
<W>=\frac{1}{4}\left(\mathbf{E} \cdot \mathbf{D}^{*}+\mathbf{B} \cdot \mathbf{H}^{*}\right)
$$

Consequently, show that the average Poynting vector (energy flux) is given by

$$
<\mathbf{S}>=\frac{c}{4}\left(\mathbf{E} \cdot \mathbf{D}^{*}+\mathbf{B} \cdot \mathbf{H}^{*}\right) \widehat{\mathbf{e}}_{3}
$$

14.6 Consider the superposition $\left(\mathbf{E}=\mathbf{E}_{1}+\mathbf{E}_{2}\right)$ of the following waves:

$$
\begin{aligned}
& \mathbf{E}_{1}=\widehat{\mathbf{e}}_{1} E_{1}^{0} \exp \left(i \alpha_{1}\right) \exp [i(\mathbf{k} \cdot \mathbf{r}-\omega t)] \\
& \mathbf{E}_{2}=\widehat{\mathbf{e}}_{2} E_{2}^{0} \exp \left(i \alpha_{2}\right) \exp [i(\mathbf{k} \cdot \mathbf{r}-\omega t)]
\end{aligned}
$$

Analyze the resultant polarization for the following cases:
(a) $\alpha_{2}=\alpha_{1}$ and $E_{2}^{0} \neq E_{1}^{0}$.
(b) $\alpha_{2}=\alpha_{1} \pm \pi / 2$ and $E_{2}^{0}=E_{1}^{0}=E^{0}$.
(c) $\alpha_{2}=\alpha_{1} \pm \pi / 2$ and $E_{2}^{0}>E_{1}^{0}$. 
(d) $\alpha_{2}=\alpha_{1}+\phi$ and $E_{2}^{0}=E_{1}^{0}$, with $\alpha_{1}=0$ and $\phi$ varying from 0 to $2 \pi$.

(e) Same as in (d), but with $E_{2}^{0}>E_{1}^{0}$ and $\alpha_{1}=\pi / 2$.

14.7 Generalize equations (6.1) through (6.5) for the three-dimensional case in Cartesian coordinates.

14.8 Show that the time evolution of a wave packet $\psi(\mathbf{r}, t)$ can be expressed in terms of the initial form of the wave packet $\psi\left(\mathbf{r}, t_{0}\right)$ as

$$
\psi(\mathbf{r}, t)=\int_{-\infty}^{+\infty} G\left(\mathbf{r}, t ; \mathbf{r}^{\prime}, t_{0}\right) \psi\left(\mathbf{r}^{\prime}, t_{0}\right) d^{3} r^{\prime}
$$

where $G\left(\mathbf{r}, t ; \mathbf{r}^{\prime}, t_{0}\right)$ denotes the Green function or kernel of the integral and which depends on the dispersion relation $\omega(k)$,

$$
G\left(\mathbf{r}, t ; \mathbf{r}^{\prime}, t_{0}\right)=\left(\frac{1}{2 \pi}\right)^{3} \int_{-\infty}^{+\infty} \exp \left[i \mathbf{k} \cdot\left(\mathbf{r}-\mathbf{r}^{\prime}\right)-i \omega\left(t-t_{0}\right)\right] d^{3} k
$$

14.9 Analyze the meaning of the Green function of problem 14.8 for the case when $\psi\left(\mathbf{r}, t_{0}\right)$ is given by the Dirac delta function

$$
\psi\left(\mathbf{r}, t_{0}\right)=\delta\left(\mathbf{r}-\mathbf{r}_{0}\right)
$$

14.10 Calculate the Green function of problem 14.8 for the case of free space, for which $\omega=c k$, where $c$ is the speed of light in free space. Show that, in this case, the initial wave packet $\psi\left(\mathbf{r}, t_{0}\right)$ maintains its original shape and there is no dispersion.

14.11 Consider a one-dimensional wave packet at the instant $t=0$, whose amplitude function $A(k)$ is given by

$$
\begin{array}{ll}
A(k)=1 & \text { for } \quad-a \leq k \leq a \\
A(k)=0 & \text { otherwise }
\end{array}
$$

Show that

$$
\psi(x, 0)=2 \frac{\sin (a x)}{x}
$$


Make a plot of both $A(k)$ and $\psi(x, 0)$ and verify that the uncertainties in $x$ and $k$ satisfy the following uncertainty relation:

$$
(\Delta x)(\Delta k) \geq 4 \pi
$$

14.12 Consider a one-dimensional wave packet at the instant $t=0$, whose amplitude function $A(k)$ is given by the Gaussian function

$$
A(k)=\exp \left[-a^{2}\left(k-k_{0}\right)^{2}\right]
$$

where $a$ and $k_{0}$ are constants.

(a) Show that $\psi(x, 0)$ is also a Gaussian function given by

$$
\psi(x, 0)=\frac{\sqrt{\pi}}{a} \exp \left(i k_{0} x\right) \exp \left(-\frac{x^{2}}{4 a^{2}}\right)
$$

Make a plot of both $A(k)$ and $\mathcal{R}\{\psi(x, 0)\}$, considering that $k_{0} \gg(1 / a)$. (b) The average extension of the wave packet $\overline{\Delta x}$ can be defined in terms of the root mean square deviation,

$$
\overline{\Delta x}=<(\Delta x)^{2}>^{1 / 2}
$$

where the dispersion is given by

$$
<(\Delta x)^{2}>=<(x-<x>)^{2}>=\frac{\int_{-\infty}^{+\infty}|\psi|^{2}(x-<x>)^{2} d x}{\int_{-\infty}^{+\infty}|\psi|^{2} d x}
$$

Similarly, we have

$$
\overline{\Delta k}=<(\Delta k)^{2}>^{1 / 2}
$$

where the dispersion in $k$ is

$$
<(\Delta k)^{2}>=<(k-<k>)^{2}>=\frac{\int_{-\infty}^{+\infty}|A|^{2}(k-<k>)^{2} d k}{\int_{-\infty}^{+\infty}|A|^{2} d k}
$$

Show that for this Gaussian wave packet we have

$$
\begin{aligned}
\overline{\Delta x} & =a \\
\overline{\Delta k} & =\frac{1}{2 a}
\end{aligned}
$$


Consequently, in this case,

$$
\overline{\Delta x} \overline{\Delta k}=\frac{1}{2}
$$

It can be shown that the Gaussian wave packet is the minimum uncertainty packet and that, in general, we have the uncertainty principle

$$
\overline{\Delta x} \overline{\Delta k} \geq \frac{1}{2}
$$

14.13 Calculate $\psi(x, 0)$ for a one-dimensional wave packet, when the amplitude function $A(k)$ is given by

$$
A(k)=\exp \left(-i k x_{0}\right)
$$

and when it is given by

$$
A(k)=\delta\left(k-k_{0}\right)
$$

For both cases, verify the validity of the uncertainty principle for wave packets, stated in the previous problem.

14.14 Consider an evanescent plane electromagnetic wave (for which $\mathbf{k}=i \alpha \widehat{\mathbf{x}}$, with $\alpha$ real), with the wave field vectors $\mathbf{E}$ and $\mathbf{H}$ proportional to $\exp [i(\mathbf{k} \cdot \mathbf{r}-\omega t)]$. Show that the average value of the Poynting vector $<\mathbf{S}>$ is zero for an evanescent wave. 


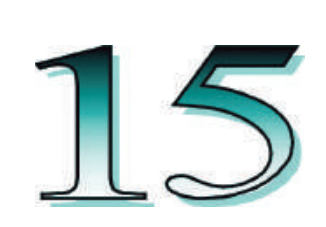

\section{MAGNETOHYDRODYNAMIC WAVES}

\section{INTRODUCTION}

The most fundamental type of wave motion that propagates in a compressible, nonconducting fluid is that of longitudinal sound waves. For these waves the variations in pressure $(p)$ and in density $\left(\rho_{m}\right)$, associated with the fluid compressions and rarefactions, obey the adiabatic energy equation commonly used in thermodynamics,

$$
p \rho_{m}^{-\gamma}=\text { constant }
$$

where $\gamma$ denotes the ratio of the specific heats at constant pressure and at constant volume. Differentiating (1.1) we obtain

$$
\nabla p=\frac{\gamma p}{\rho_{m}} \nabla \rho_{m}=V_{s}^{2} \nabla \rho_{m}
$$

where

$$
V_{s}=\left(\frac{\gamma p}{\rho_{m}}\right)^{1 / 2}=\left(\frac{\gamma k T}{m}\right)^{1 / 2}
$$

is the wave propagation speed, known as the adiabatic sound speed. Fig. 1 illustrates the regions of fluid compression and rarefaction associated with the longitudinal motion of sound waves. 


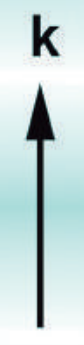

Fig. 1 Schematic representation of longitudinal sound waves that propagate in a compressible, nonconducting fluid, showing the regions of compression and rarefaction associated with the longitudinal wave motion.

\subsection{Alfvén Waves}

In the case of a compressible, conducting fluid immersed in a magnetic field, other types of wave motion are possible.

We have seen that, in a magnetic field of intensity $\mathbf{B}_{0}$, the magnetic stresses are equivalent to a tension $B_{0}^{2} / \mu_{0}$ along the field lines and an isotropic hydrostatic pressure $B_{0}^{2} / 2 \mu_{0}$ (see section 5 , Chapter 12). Since the latter can always be combined with the kinetic fluid pressure, the magnetic field lines behave effectively as elastic cords under a tension $B_{0}^{2} / \mu_{0}$. Further, in a perfectly conducting fluid the plasma particles behave as if they were tied to the magnetic field lines (see section 4, Chapter 12), so that the lines of force act as if they were mass-loaded strings under tension. Thus, by analogy with the transverse vibrations of elastic strings, we expect that, whenever the conducting fluid is slightly disturbed from the equilibrium conditions, the magnetic field lines will perform transverse vibrations. 


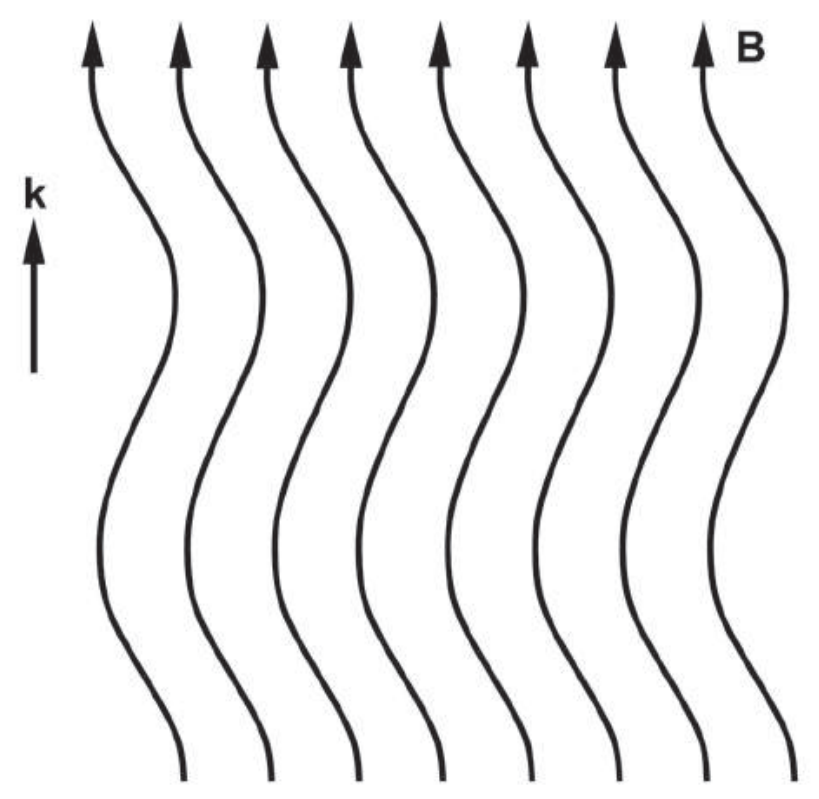

Fig. 2 Transverse Alfvén waves in a compressible, conducting magnetofluid. The velocity of propagation is along the magnetic field lines, whereas the fluid motion and magnetic field line perturbations are perpendicular to the field lines.

The speed of propagation of these transverse vibrations is expected to be given by

$$
V_{A}=\left(\frac{\text { tension }}{\text { density }}\right)^{1 / 2}=\left(\frac{B_{0}^{2}}{\mu_{0} \rho_{m}}\right)^{1 / 2}
$$

which is known as the Alfvén speed, since the existence of this type of lowfrequency wave motion in a conducting magnetized fluid was first pointed out by Alfvén, in 1942. An important property of these waves, as will be shown later, is the absence of any fluctuations in the fluid density $\left(\rho_{m}\right)$ or pressure $(p)$. Fig. 2 illustrates the transverse motions of the fluid (and of the frozen in field lines) for the Alfvén wave.

\subsection{Magnetosonic Waves}

Longitudinal oscillations are also expected to occur in a compressible, conducting fluid in a magnetic field. For motion of the particles (and propagation of the wave) along the magnetic field lines there will be no 


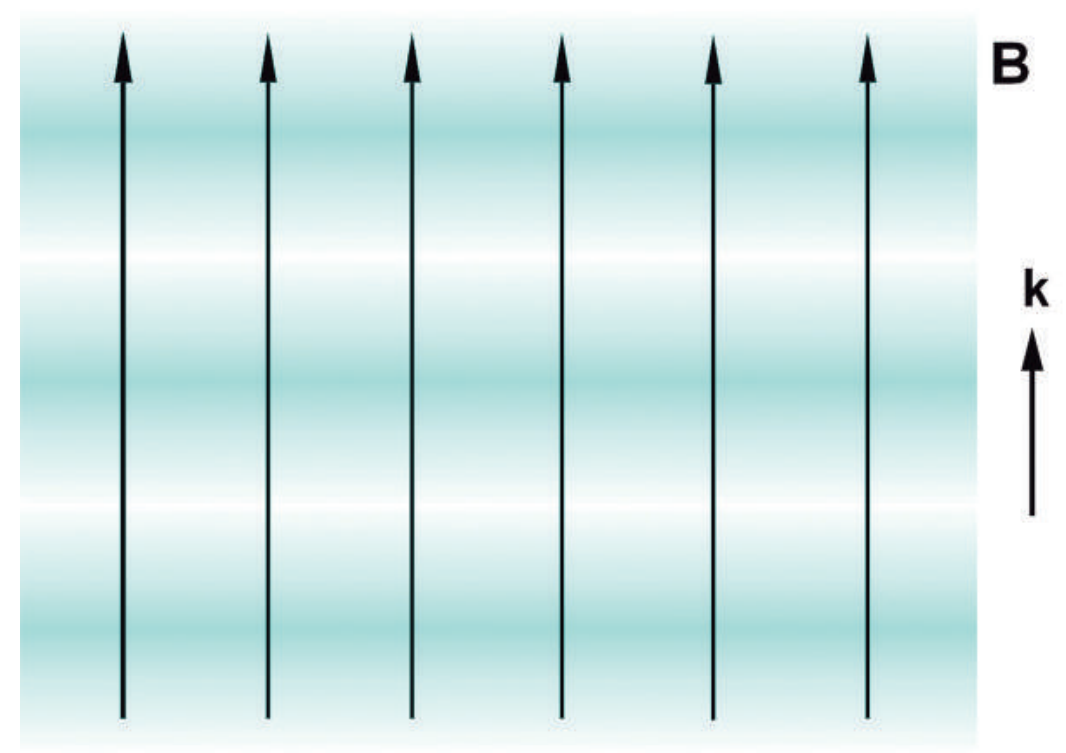

Fig. 3 Longitudinal sound waves propagating along the magnetic field lines in a compressible, conducting magnetofluid.

magnetic field perturbations, since the particles are free to move in this direction. Thus, in this case, the waves will be ordinary longitudinal sound waves propagating at the sound speed $V_{s}$ along the field lines (see Fig. 3).

On the other hand, for motion of the particles (and propagation of the wave) in a direction perpendicular to the magnetic field, a new type of longitudinal wave motion is possible since now, in addition to the kinetic fluid pressure $p$, there is also the magnetic pressure $B_{0}^{2} / 2 \mu_{0}$. Hence, the total pressure is $p+B_{0}^{2} / 2 \mu_{0}$ and, consequently, the speed $\left(V_{m}\right)$ of propagation of these so-called magnetosonic or magnetoacoustic waves (see Fig. 4) satisfies the following relation, analogous to (1.2),

$$
\nabla\left(p+\frac{B^{2}}{2 \mu_{0}}\right)=V_{m}^{2} \nabla \rho_{m}
$$

Therefore, we can write

$$
V_{m}^{2}=\frac{d}{d \rho_{m}}\left(p+\frac{B_{0}^{2}}{2 \mu_{0}}\right)_{\rho_{m 0}}=V_{s}^{2}+\frac{d}{d \rho_{m}}\left(\frac{B_{0}^{2}}{2 \mu_{0}}\right)_{\rho_{m 0}}
$$

where the suffix zero, in $\rho_{m}$, refers to the undisturbed state, and $V_{s}$ is the adiabatic sound speed. Since the lines of force are frozen in the conducting 


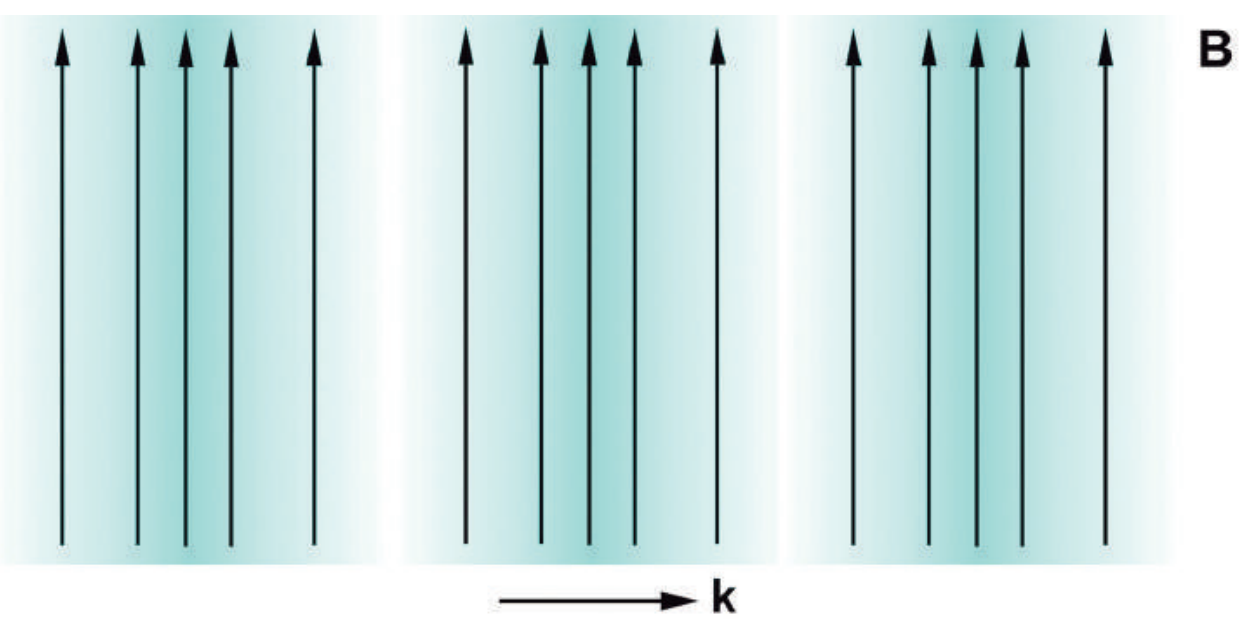

Fig. 4 The longitudinal magnetosonic wave propagates perpendicularly to the magnetic field lines, causing compressions and rarefactions of both the lines of force and the conducting fluid.

fluid, the magnetic flux $B d S$ across an element of surface $d \mathbf{S}$ (whose normal is oriented along the magnetic field) and the mass $\rho_{m} d S$ of a unit length of column having $d \mathbf{S}$ as base are both conserved during the wave motion, in such a way that $\left(B / \rho_{m}\right)=\left(B_{0} / \rho_{m 0}\right)$. Consequently, (1.6) becomes

$$
V_{m}^{2}=V_{s}^{2}+\frac{d}{d \rho_{m}}\left(\frac{B_{0}^{2} \rho_{m}^{2}}{2 \mu_{0} \rho_{m 0}^{2}}\right)_{\rho_{m 0}}=V_{s}^{2}+V_{A}^{2}
$$

where $V_{A}$ is the Alfvén speed defined in (1.4).

For propagation in a direction inclined with respect to the magnetic field the wave motion behavior is more complex. This subject will be considered in some detail in section 5 .

\section{MHD EQUATIONS FOR A COMPRESSIBLE NONVISCOUS CONDUCTING FLUID}

\subsection{Basic Equations}

To investigate the propagation of waves in a conducting magnetofluid, let us consider a compressible, nonviscous, perfectly conducting fluid immersed in a magnetic field. The appropriate system of equations, which 
governs the behavior of this type of fluid, with the assumptions involved, were summarized in section 1 of Chapter 12. These equations are

$$
\begin{gathered}
\frac{\partial \rho_{m}}{\partial t}+\nabla \cdot\left(\rho_{m} \mathbf{u}\right)=0 \\
\rho_{m} \frac{\partial \mathbf{u}}{\partial t}+\rho_{m}(\mathbf{u} \cdot \nabla) \mathbf{u}=-\nabla p+\mathbf{J} \times \mathbf{B} \\
\nabla p=V_{s}^{2} \nabla \rho_{m} \\
\nabla \times \mathbf{B}=\mu_{0} \mathbf{J} \\
\nabla \times \mathbf{E}=-\frac{\partial \mathbf{B}}{\partial t} \\
\mathbf{E}+\mathbf{u} \times \mathbf{B}=0
\end{gathered}
$$

To reduce this system of equations we combine equations (2.2) to (2.4) in the form

$$
\rho_{m} \frac{\partial \mathbf{u}}{\partial t}+\rho_{m}(\mathbf{u} \cdot \nabla) \mathbf{u}=-V_{s}^{2} \nabla \rho_{m}+\frac{1}{\mu_{0}}(\nabla \times \mathbf{B}) \times \mathbf{B}
$$

as well as (2.5) and (2.6) in the form

$$
\nabla \times(\mathbf{u} \times \mathbf{B})=\frac{\partial \mathbf{B}}{\partial t}
$$

Under equilibrium conditions, the fluid is assumed to be spatially uniform with constant density $\rho_{m 0}$, the equilibrium velocity is considered zero, and throughout the fluid the magnetic induction $\mathbf{B}_{0}$ is uniform and constant.

In order to deduce the dispersion relation for small-amplitude waves, consider small-amplitude departures from the equilibrium values, so that

$$
\begin{gathered}
\mathbf{B}(\mathbf{r}, t)=\mathbf{B}_{0}+\mathbf{B}_{1}(\mathbf{r}, t) \\
\rho_{m}(\mathbf{r}, t)=\rho_{m 0}+\rho_{m 1}(\mathbf{r}, t) \\
\mathbf{u}(\mathbf{r}, t)=\mathbf{u}_{1}(\mathbf{r}, t)
\end{gathered}
$$

Substituting (2.9) to (2.11) into (2.1), (2.7), and (2.8), and neglecting second-order terms, we obtain the following linearized equations in the small first-order quantities:

$$
\frac{\partial \rho_{m 1}}{\partial t}+\rho_{m 0}\left(\nabla \cdot \mathbf{u}_{1}\right)=0
$$




$$
\begin{gathered}
\rho_{m 0} \frac{\partial \mathbf{u}_{1}}{\partial t}+V_{s}^{2} \nabla \rho_{m 1}+\frac{1}{\mu_{0}} \mathbf{B}_{0} \times\left(\nabla \times \mathbf{B}_{1}\right)=0 \\
\frac{\partial \mathbf{B}_{1}}{\partial t}-\nabla \times\left(\mathbf{u}_{1} \times \mathbf{B}_{0}\right)=0
\end{gathered}
$$

\subsection{Development of an Equation for the Fluid Velocity}

Equations (2.12) to (2.14) can be combined to yield an equation for $\mathbf{u}_{1}$ alone. For this purpose, we first differentiate (2.13) with respect to time, obtaining

$$
\rho_{m 0} \frac{\partial^{2} \mathbf{u}_{1}}{\partial t^{2}}+V_{s}^{2} \nabla\left(\frac{\partial \rho_{m 1}}{\partial t}\right)+\frac{1}{\mu_{0}} \mathbf{B}_{0} \times\left[\nabla \times\left(\frac{\partial \mathbf{B}_{1}}{\partial t}\right)\right]=0
$$

Next, using (2.12) and (2.14), we can write (2.15) as

$$
\frac{\partial^{2} \mathbf{u}_{1}}{\partial t^{2}}-V_{s}^{2} \nabla\left(\nabla \cdot \mathbf{u}_{1}\right)+\mathbf{V}_{A} \times\left\{\nabla \times\left[\nabla \times\left(\mathbf{u}_{1} \times \mathbf{V}_{A}\right)\right]\right\}=0
$$

where we have introduced the vector Alfvén velocity

$$
\mathbf{V}_{A}=\frac{\mathbf{B}_{0}}{\left(\mu_{0} \rho_{m 0}\right)^{1 / 2}}
$$

Without loss of generality, we can consider plane wave solutions for (2.16) in the form

$$
\mathbf{u}_{1}(\mathbf{r}, t)=\mathbf{u}_{1} \exp (i \mathbf{k} \cdot \mathbf{r}-i \omega t)
$$

In what follows, $\mathbf{u}_{1}$ can stand for either the amplitude or the entire expression (2.18). Thus, in (2.16) we can replace the operator $\nabla$ by $i \mathbf{k}$ and the partial time derivative by $-i \omega$, so that

$$
-\omega^{2} \mathbf{u}_{1}+V_{s}^{2}\left(\mathbf{k} \cdot \mathbf{u}_{1}\right) \mathbf{k}-\mathbf{V}_{A} \times\left\{\mathbf{k} \times\left[\mathbf{k} \times\left(\mathbf{u}_{1} \times \mathbf{V}_{A}\right)\right]\right\}=0
$$

Since for any three vectors $\mathbf{A}, \mathbf{B}$, and $\mathbf{C}$ we have the vector identity

$$
\mathbf{A} \times(\mathbf{B} \times \mathbf{C})=(\mathbf{A} \cdot \mathbf{C}) \mathbf{B}-(\mathbf{A} \cdot \mathbf{B}) \mathbf{C}
$$

we can rearrange (2.19) to read

$$
-\omega^{2} \mathbf{u}_{1}+\left(V_{s}^{2}+V_{A}^{2}\right)\left(\mathbf{k} \cdot \mathbf{u}_{1}\right) \mathbf{k}+
$$




$$
\left(\mathbf{k} \cdot \mathbf{V}_{A}\right)\left[\left(\mathbf{k} \cdot \mathbf{V}_{A}\right) \mathbf{u}_{1}-\left(\mathbf{V}_{A} \cdot \mathbf{u}_{1}\right) \mathbf{k}-\left(\mathbf{k} \cdot \mathbf{u}_{1}\right) \mathbf{V}_{A}\right]=0
$$

Although this expression appears to be somewhat involved, it leads to remarkably simple solutions for waves propagating in the directions parallel or perpendicular to the magnetic field.

\section{PROPAGATION PERPENDICULAR TO THE MAGNETIC FIELD}

When the wave vector $\mathbf{k}$ is perpendicular to the magnetic induction $\mathbf{B}_{0}$, we have $\mathbf{k} \cdot \mathbf{V}_{A}=0$, and (2.21) simplifies to

$$
-\omega^{2} \mathbf{u}_{1}+\left(V_{s}^{2}+V_{A}^{2}\right)\left(\mathbf{k} \cdot \mathbf{u}_{1}\right) \mathbf{k}=0
$$

from which we obtain

$$
\mathbf{u}_{1}=\frac{1}{\omega^{2}}\left(V_{s}^{2}+V_{A}^{2}\right)\left(\mathbf{k} \cdot \mathbf{u}_{1}\right) \mathbf{k}
$$

Therefore, $\mathbf{u}_{1}$ is parallel to $\mathbf{k}$, so that $\mathbf{k} \cdot \mathbf{u}_{1}=k u_{1}$, and the solution for $\mathbf{u}_{1}$ is a longitudinal wave with the phase velocity

$$
\frac{\omega}{k}=\left(V_{s}^{2}+V_{A}^{2}\right)^{1 / 2}
$$

The magnetic field associated with this longitudinal wave can be obtained from (2.14). Taking

$$
\mathbf{B}_{1}(\mathbf{r}, t)=\mathbf{B}_{1} \exp (i \mathbf{k} \cdot \mathbf{r}-i \omega t)
$$

we obtain

$$
-\omega \mathbf{B}_{1}-\mathbf{k} \times\left(\mathbf{u}_{1} \times \mathbf{B}_{0}\right)=0
$$

Using the vector identity $(2.20)$ and noting that $\mathbf{k} \cdot \mathbf{B}_{0}=0$, we find

$$
\mathbf{B}_{1}=\frac{u_{1}}{(\omega / k)} \mathbf{B}_{0}
$$

The electric field associated with this wave is seen, from (2.6), to be given by

$$
\mathbf{E}=-\mathbf{u}_{1} \times \mathbf{B}_{0}
$$

This wave is somewhat similar to an electromagnetic wave, since the time-varying magnetic field is perpendicular to the direction of propagation, but parallel to the magnetostatic field, whereas the time-varying 
electric field is perpendicular to both the direction of propagation and the magnetostatic field. It is a longitudinal wave, however, since the velocity of mass flow and the fluctuating mass density associated with the wave motion are both in the wave propagation direction. For these reasons, this wave is called the magnetosonic wave. The phase velocity (3.3) is independent of frequency, so that it is a nondispersive wave. As illustrated in Fig. 4, the magnetosonic wave produces compressions and rarefactions in the magnetic field lines without changing their direction. Since the fluid is perfectly conducting, the lines of force and the fluid move together.

The restoring forces operating in the magnetosonic wave are the fluid pressure gradient and the gradient of the compressional stresses between the magnetic field lines. When the fluid pressure is much greater than the magnetic pressure, the effect of the magnetic field is negligible, so that $(\omega / k)=V_{s}$ and the wave becomes essentially an acoustic wave. On the other hand, when the magnetic field is very strong so that the magnetic pressure is much larger than the fluid pressure, then the phase velocity of the magnetosonic wave becomes equal to the Alfvén wave velocity $V_{A}$.

The magnetosonic wave mode is also known variously as the compressional Alfvén wave or the fast Alfvén wave.

\section{PROPAGATION PARALLEL TO THE MAGNETIC FIELD}

For waves propagating along the magnetic field $\left(\mathbf{k} \| \mathbf{B}_{0}\right)$, we have $\mathbf{k} \cdot \mathbf{V}_{A}=k V_{A}$, and (2.21) simplifies to

$$
\left(k^{2} V_{A}^{2}-\omega^{2}\right) \mathbf{u}_{1}+\left(V_{s}^{2} / V_{A}^{2}-1\right) k^{2}\left(\mathbf{u}_{1} \cdot \mathbf{V}_{A}\right) \mathbf{V}_{A}=0
$$

In this case, there are two types of wave motion possible.

For $\mathbf{u}_{1}$ parallel to $\mathbf{B}_{0}$ and $\mathbf{k}$, we find from (4.1) that a longitudinal mode is possible, with the phase velocity

$$
\frac{\omega}{k}=V_{s}
$$

This is an ordinary longitudinal sound wave, in which the velocity of mass flow is in the propagation direction (refer to Fig. 3). There is no electric field, electric current density, or magnetic field associated with this wave.

A transverse wave, with $\mathbf{u}_{1}$ perpendicular to $\mathbf{B}_{0}$ and $\mathbf{k}$, is the other possibility. In this case $\mathbf{u}_{1} \cdot \mathbf{V}_{A}=0$ and (4.1) gives for the phase velocity of this transverse wave, known as the Alfvén wave,

$$
\frac{\omega}{k}=V_{A}
$$


Since the phase velocity is independent of frequency, there is no dispersion.

The magnetic field associated with the Alfvén wave is found, from (2.14) and (3.5), to be given by

$$
\mathbf{B}_{1}=-\frac{B_{0}}{(\omega / k)} \mathbf{u}_{1}
$$

Hence, the magnetic field disturbance is normal to the original magnetostatic induction $\mathbf{B}_{0}$. The small component $\mathbf{B}_{1}$, when added to $\mathbf{B}_{0}$, gives the lines of force a sinusoidal ripple, shown in Fig. 5. The associated electric field is given by (3.7).

The Alfvén wave involves no fluctuations in the fluid density or pressure, although both the fluid and the magnetic field lines oscillate back and forth laterally, in the plane normal to $\mathbf{B}_{0}$. The magnetic energy density of this wave motion $\left(B_{1}^{2} / 2 \mu_{0}\right)$ is equal to the kinetic energy density of the fluid motion $\left(\rho_{m 0} u_{1}^{2} / 2\right)$. This equipartition of energy is easily verified from (4.4),

$$
\frac{B_{1}^{2}}{2 \mu_{0}}=\frac{B_{0}^{2} u_{1}^{2}}{2 \mu_{0}(\omega / k)^{2}}=\frac{B_{0}^{2} u_{1}^{2}}{2 \mu_{0} V_{A}^{2}}=\frac{1}{2} \rho_{m 0} u_{1}^{2}
$$

where we have used (4.3) and (2.17).

The Alfvén wave mode is also known variously as the shear Alfvén wave or the slow Alfvén wave.

\section{PROPAGATION AT ARBITRARY DIRECTIONS}

Let us now investigate the case of wave propagation in an arbitrary direction with respect to the magnetic induction $\mathbf{B}_{0}$. With no loss of generality, we introduce a Cartesian coordinate system such that the $y$ axis is normal to the plane defined by the direction of propagation $\mathbf{k}$ and the magnetic induction $\mathbf{B}_{0}$, and choose $\widehat{\mathbf{z}}$ to be along $\mathbf{B}_{0}$, as shown in Fig. 6 . Denoting the angle between $\mathbf{k}$ and $\mathbf{B}_{0}$ by $\theta$, we have

$$
\begin{gathered}
\mathbf{k}=k(\widehat{\mathbf{x}} \sin \theta+\widehat{\mathbf{z}} \cos \theta) \\
\mathbf{V}_{A}=V_{A} \widehat{\mathbf{z}} \\
\mathbf{u}_{1}=u_{1 x} \widehat{\mathbf{x}}+u_{1 y} \widehat{\mathbf{y}}+u_{1 z} \widehat{\mathbf{z}} \\
\mathbf{k} \cdot \mathbf{V}_{A}=k V_{A} \cos \theta \\
\mathbf{k} \cdot \mathbf{u}_{1}=k\left(u_{1 x} \sin \theta+u_{1 z} \cos \theta\right)
\end{gathered}
$$




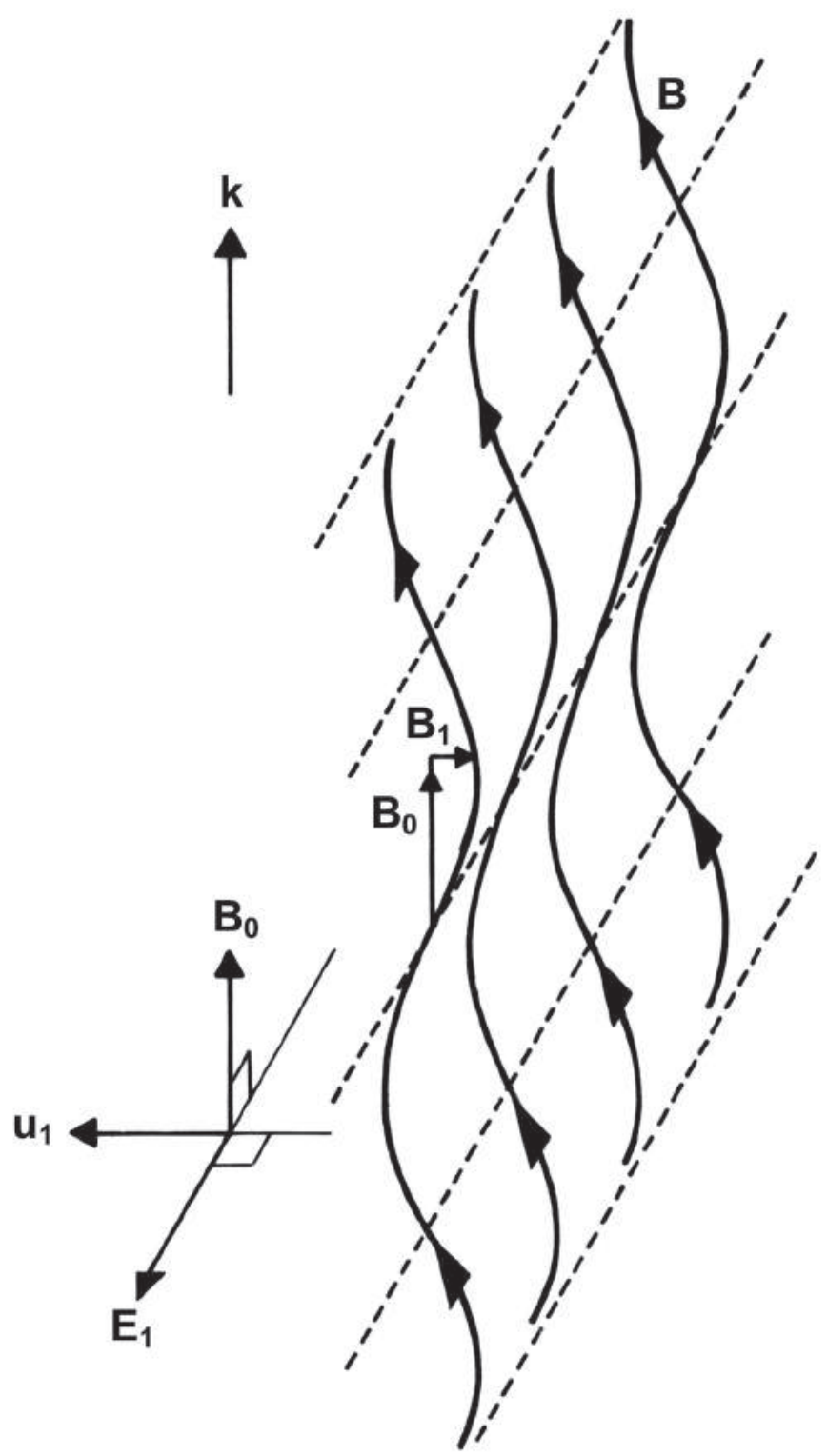

Fig. 5 Schematic illustration for Alfvén waves propagating along the magnetic field, showing the relations between the oscillating parameters.

$$
\mathbf{V}_{A} \cdot \mathbf{u}_{1}=V_{A} u_{1 z}
$$

Substituting these expressions into (2.21), performing the required alge- 


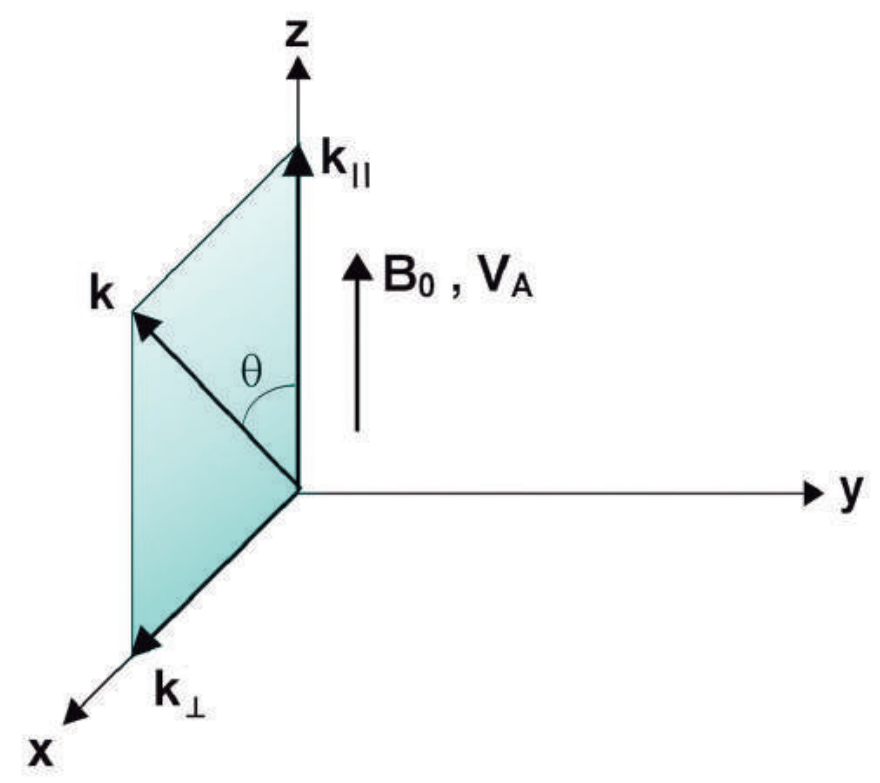

Fig. 6 Cartesian coordinate system showing the relative orientation of the vectors $\mathbf{k}$ and $\mathbf{B}_{0}$.

bra, and rearranging the terms, we obtain for the $x$ component equation,

$$
u_{1 x}\left(-\omega^{2}+k^{2} V_{A}^{2}+k^{2} V_{s}^{2} \sin ^{2} \theta\right)+u_{1 z}\left(k^{2} V_{s}^{2} \sin \theta \cos \theta\right)=0
$$

for the $y$ component equation,

$$
u_{1 y}\left(-\omega^{2}+k^{2} V_{A}^{2} \cos ^{2} \theta\right)=0
$$

and for the $z$ component equation,

$$
u_{1 x}\left(k^{2} V_{s}^{2} \sin \theta \cos \theta\right)+u_{1 z}\left(-\omega^{2}+k^{2} V_{s}^{2} \cos ^{2} \theta\right)=0
$$

\subsection{Pure Alfvén Wave}

From (5.8) we see that (for $u_{1 y} \neq 0$ ) there is a linearly polarized wave involving oscillations in the direction perpendicular to both $\mathbf{k}$ and $\mathbf{B}_{0}$, with a phase velocity given by

$$
\frac{\omega}{k}=V_{A} \cos \theta
$$


The field components associated with this wave can be seen to be $B_{1 y}$, $u_{1 y}, E_{1 x}$, and $J_{1 x}$, so that it is a transverse Alfvén wave. For this reason, this wave is generally referred to as the pure Alfvén wave. Note that for propagation along the magnetostatic field $(\theta=0)$ equation (5.10) gives $(\omega / k)=V_{A}$, while for propagation across the magnetostatic field $\left(\theta=90^{\circ}\right)$ this wave does not exist, since $(\omega / k)=0$. This wave mode is also known as the oblique Alfvén wave.

\subsection{Fast and Slow MHD Waves}

Equations (5.7) and (5.9) constitute a system of two coupled equations for the amplitudes of $u_{1 x}$ and $u_{1 z}$. To have a solution in which $u_{1 x}$ and $u_{1 z}$ are nonzero, the determinant of the matrix formed with the coefficients of this system must vanish. Therefore, setting to zero the determinant of the following matrix,

$$
\mathcal{M}=\left(\begin{array}{cc}
\left(-\omega^{2}+k^{2} V_{A}^{2}+k^{2} V_{s}^{2} \sin ^{2} \theta\right) & \left(k^{2} V_{s}^{2} \sin \theta \cos \theta\right) \\
\left(k^{2} V_{s}^{2} \sin \theta \cos \theta\right) & \left(-\omega^{2}+k^{2} V_{s}^{2} \cos ^{2} \theta\right)
\end{array}\right)
$$

we obtain the following dispersion relation, expressed in terms of the phase velocity $(\omega / k)$,

$$
\left(\frac{\omega}{k}\right)^{4}-\left(V_{s}^{2}+V_{A}^{2}\right)\left(\frac{\omega}{k}\right)^{2}+V_{s}^{2} V_{A}^{2} \cos ^{2} \theta=0
$$

Solving this equation for $(\omega / k)^{2}$, we obtain two real solutions:

$$
\left(\frac{\omega}{k}\right)^{2}=\frac{1}{2}\left(V_{s}^{2}+V_{A}^{2}\right) \pm \frac{1}{2}\left[\left(V_{s}^{2}+V_{A}^{2}\right)^{2}-4 V_{s}^{2} V_{A}^{2} \cos ^{2} \theta\right]^{1 / 2}
$$

The solutions with the plus and minus signs are called, respectively, the fast and the slow MHD wave modes. Note that taking the square root of $(\omega / k)^{2}$ does not give two different modes, but only waves with opposite directions of propagation.

\subsection{Phase Velocities}

All three MHD wave modes have constant phase velocities, given by (5.10) and (5.13), for all frequencies, and hence there is no wave dispersion. Fig. 7 displays the phase velocity, for each of these waves, as a 
function of the angle $\theta$ between $\mathbf{k}$ and $\mathbf{B}_{0}$, for both cases when $V_{A}>V_{s}$ and when $V_{A}<V_{s}$.

The phase velocity of the fast MHD wave increases from $V_{A}$ (or $V_{s}$ if $V_{s}>V_{A}$ ), when $\theta=0$, to $\left(V_{s}^{2}+V_{A}^{2}\right)^{1 / 2}$ when $\theta=90^{\circ}$, while that of the slow MHD wave mode decreases from $V_{s}$ (or $V_{A}$ if $V_{s}>V_{A}$ ), when $\theta=0$, to zero when $\theta=90^{\circ}$.

Therefore, if $V_{A}>V_{s}$, the fast MHD wave becomes the Alfvén wave for $\theta=0$, and the magnetosonic wave for $\theta=90^{\circ}$, while the slow MHD wave becomes the sound wave for $\theta=0$, and does not exist for $\theta=90^{\circ}$. On the other hand, if $V_{s}>V_{A}$, the fast MHD wave becomes the sound wave for $\theta=0$, and the magnetosonic wave for $\theta=90^{\circ}$, while the slow MHD wave becomes the Alfvén wave for $\theta=0$, and does not exist for $\theta=90^{\circ}$.

\subsection{Wave Normal Surfaces}

The dependence of the phase velocity on the angle between $\mathbf{k}$ and $\mathbf{B}_{0}$, for these waves, can be conveniently represented by means of diagrams called phase velocity surfaces or wave normal surfaces, which give the variations of the magnitude of the phase velocity with respect to the magnetic field direction. Fig. 8 shows the wave normal diagram for the pure Alfvén wave, constructed from (5.10). In this diagram the magnitude of a vector drawn from the origin to a point $P$ on the curve represents the phase velocity of a plane wave for a given direction $(\theta)$ of the wave normal with respect to $\mathbf{B}_{0}$. The actual state of affairs, in three dimensions, is obtained by rotating the curves shown in Fig. 8 about the axis oriented along $\mathbf{B}_{0}$. The three-dimensional surface, thus obtained, is called the wave normal surface.

Fig. 9 shows the wave normal diagrams for propagation of the pure Alfvén, the fast and the slow MHD waves, for the two cases $V_{A}>V_{s}$ and $V_{A}<V_{s}$. The three-dimensional wave normal surfaces are obtained by rotating the curves of shown in Fig. 9 about the axis oriented along $\mathbf{B}_{0}$. The wave normal surface corresponding to the fast MHD wave is a smooth, closed surface enclosing the two spheres passing through the origin 0 corresponding to the pure Alfvén wave. Within each of these spheres, there is another smooth, closed wave normal surface corresponding to the slow MHD wave. 


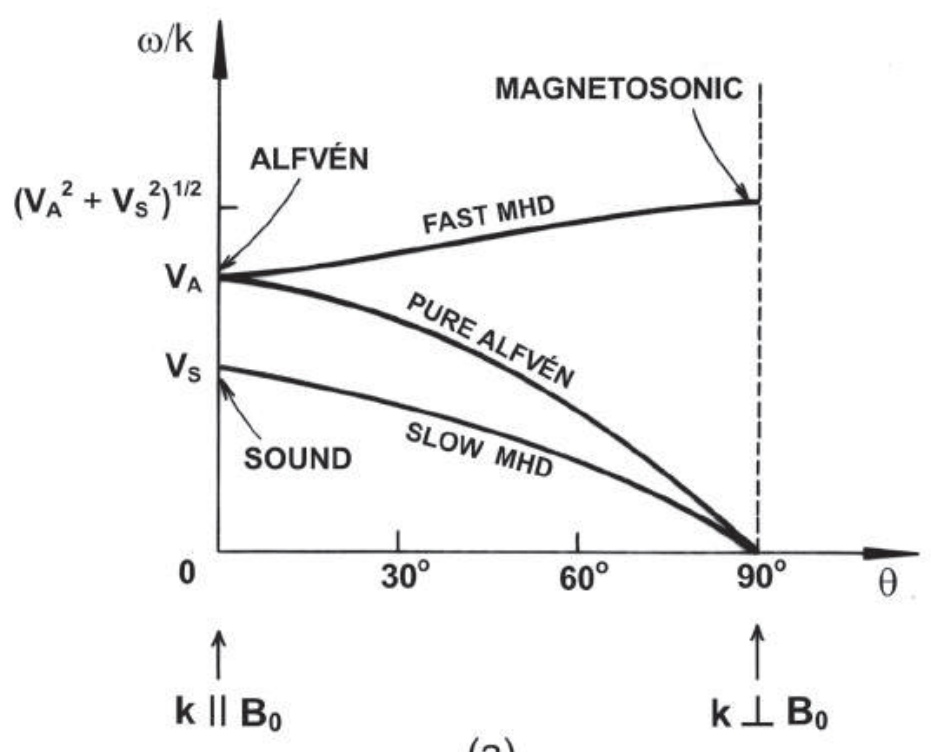

(a)

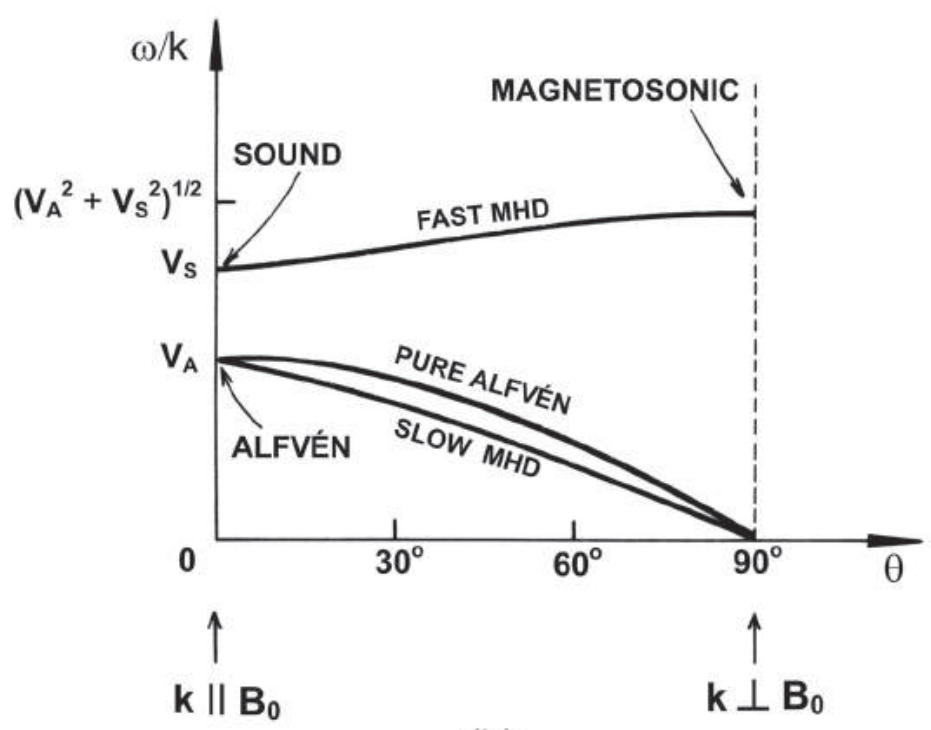

(b)

Fig. 7 Phase velocities (independent of frequency) as a function of the angle between $\mathbf{k}$ and $\mathbf{B}_{0}$ for the pure Alfvén, the fast and the slow MHD waves, for (a) $V_{A}>V_{s}$ and (b) $V_{A}<V_{s}$. 


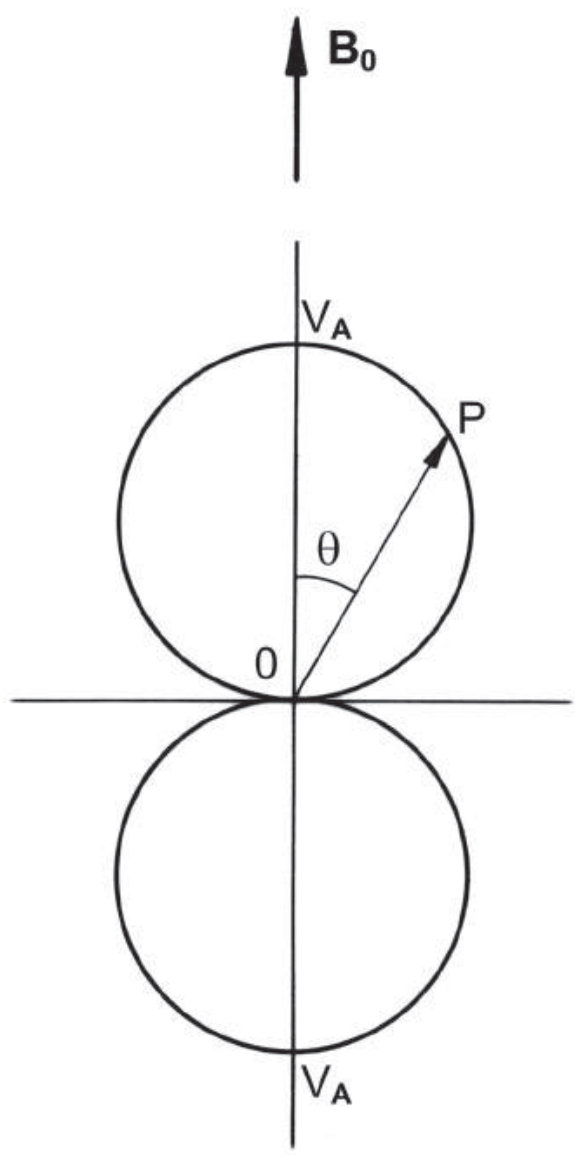

Fig. 8 Wave normal diagram for the pure Alfvén wave illustrating the variations of the phase velocity for a given direction $(\theta)$ of the wave normal with respect to the magnetic field direction.

\section{EFFECT OF DISPLACEMENT CURRENT}

In magnetohydrodynamics the displacement current term $\left(\epsilon_{0} \partial \mathbf{E} / \partial t\right)$, which appears in Maxwell $\nabla \times \mathbf{B}$ equation, is usually neglected. This approximation is valid only for highly conducting fluids at relatively low frequencies (well below the ion cyclotron frequency), as discussed in section 6 of Chapter 9. The inclusion of the displacement current in the basic equations modify the propagation characteristics of the Alfvén and magnetosonic waves. The results obtained, however, are valid only at frequencies where charge separation effects are unimportant (otherwise we must consider $\rho \neq 0$ ). 

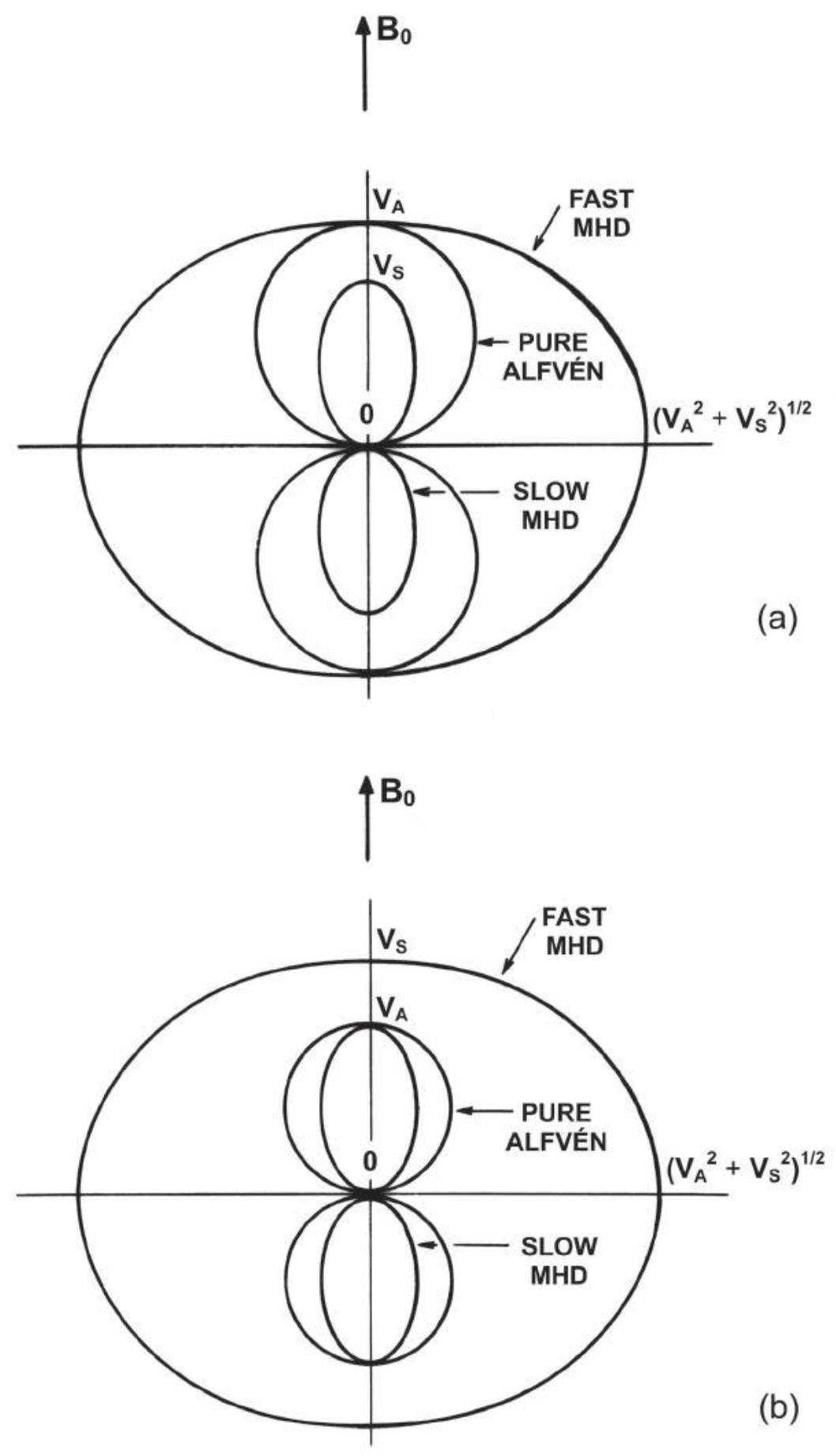

Fig. 9 Wave normal diagrams for the pure Alfvén, the fast and the slow MHD waves for the cases (a) $V_{A}>V_{s}$ and (b) $V_{A}<V_{s}$. 


\subsection{Basic Equations}

To investigate the effect of the displacement current on the propagation of MHD waves in a compressible, nonviscous, perfectly conducting fluid, (2.4) must be modified to read

$$
\nabla \times \mathbf{B}=\mu_{0} \mathbf{J}+\frac{1}{c^{2}} \frac{\partial \mathbf{E}}{\partial t}
$$

Consequently, the current density to be inserted into the $\mathbf{J} \times \mathbf{B}$ term, in the equation of motion (2.2), is now

$$
\mathbf{J}=\frac{1}{\mu_{0}}\left[\nabla \times \mathbf{B}+\frac{1}{c^{2}} \frac{\partial}{\partial t}(\mathbf{u} \times \mathbf{B})\right]
$$

where use was made of (2.6). Using expressions (2.9) to (2.11) for smallamplitude waves, the set of linearized equations (2.12) to (2.14) for the small quantities $\rho_{m 1}, \mathbf{u}_{1}$, and $\mathbf{B}_{1}$ become now

$$
\begin{gathered}
\frac{\partial \rho_{m 1}}{\partial t}+\rho_{m 0}\left(\nabla \cdot \mathbf{u}_{1}\right)=0 \\
\rho_{m 0} \frac{\partial \mathbf{u}_{1}}{\partial t}+V_{s}^{2} \nabla \rho_{m 1}+\frac{1}{\mu_{0}} \mathbf{B}_{0} \times\left(\nabla \times \mathbf{B}_{1}+\frac{1}{c^{2}} \frac{\partial \mathbf{u}_{1}}{\partial t} \times \mathbf{B}_{0}\right)=0 \\
\frac{\partial \mathbf{B}_{1}}{\partial t}-\nabla \times\left(\mathbf{u}_{1} \times \mathbf{B}_{0}\right)=0
\end{gathered}
$$

\subsection{Equation for the Fluid Velocity}

To obtain an equation involving only the variable $\mathbf{u}_{1}$, let us take the time derivative of (6.4), and use (6.3) and (6.5), which gives

$$
\begin{gathered}
\frac{\partial^{2} \mathbf{u}_{1}}{\partial t^{2}}-V_{s}^{2} \nabla\left(\nabla \cdot \mathbf{u}_{1}\right)+\mathbf{V}_{A} \times\left\{\nabla \times\left[\nabla \times\left(\mathbf{u}_{1} \times \mathbf{V}_{A}\right)\right]\right\}+ \\
\frac{1}{c^{2}} \mathbf{V}_{A} \times\left(\frac{\partial^{2} \mathbf{u}_{1}}{\partial t^{2}} \times \mathbf{V}_{A}\right)=0
\end{gathered}
$$

where $\mathbf{V}_{A}$ is the Alfvén velocity, defined in (2.17). From the vector indentity (2.20), we have

$$
\mathbf{V}_{A} \times\left(\frac{\partial^{2} \mathbf{u}_{1}}{\partial t^{2}} \times \mathbf{V}_{A}\right)=\frac{\partial^{2}}{\partial t^{2}}\left[V_{A}^{2} \mathbf{u}_{1}-\left(\mathbf{V}_{A} \cdot \mathbf{u}_{1}\right) \mathbf{V}_{A}\right]
$$


so that (6.6) can be rearranged in the form

$$
\begin{gathered}
\frac{\partial^{2}}{\partial t^{2}}\left[\left(1+\frac{V_{A}^{2}}{c^{2}}\right) \mathbf{u}_{1}-\left(\mathbf{V}_{A} \cdot \mathbf{u}_{1}\right) \frac{\mathbf{V}_{A}}{c^{2}}\right]-V_{s}^{2} \nabla\left(\nabla \cdot \mathbf{u}_{1}\right)+ \\
\mathbf{V}_{A} \times\left\{\nabla \times\left[\nabla \times\left(\mathbf{u}_{1} \times \mathbf{V}_{A}\right)\right]\right\}=0
\end{gathered}
$$

It is clear that this equation reduces to $(2.16)$ when $\left(V_{A} / c\right)^{2} \ll 1$.

Plane wave solutions of (6.8), in the form (2.18), give

$$
\begin{gathered}
-\omega^{2}\left[\left(1+\frac{V_{A}^{2}}{c^{2}}\right) \mathbf{u}_{1}-\left(\mathbf{V}_{A} \cdot \mathbf{u}_{1}\right) \frac{\mathbf{V}_{A}}{c^{2}}\right]+\left(V_{s}^{2}+V_{A}^{2}\right)\left(\mathbf{k} \cdot \mathbf{u}_{1}\right) \mathbf{k}+ \\
\left(\mathbf{k} \cdot \mathbf{V}_{A}\right)\left[\left(\mathbf{k} \cdot \mathbf{V}_{A}\right) \mathbf{u}_{1}-\left(\mathbf{V}_{A} \cdot \mathbf{u}_{1}\right) \mathbf{k}-\left(\mathbf{k} \cdot \mathbf{u}_{1}\right) \mathbf{V}_{A}\right]=0
\end{gathered}
$$

\subsection{Propagation Across the Magnetostatic Field}

For $\mathbf{k} \perp \mathbf{B}_{0}$ we have $\mathbf{k} \cdot \mathbf{V}_{A}=0$, so that (6.9) gives $\left(\mathbf{V}_{A} \cdot \mathbf{u}_{1}\right)=0$ and

$$
-\omega^{2}\left(1+\frac{V_{A}^{2}}{c^{2}}\right) \mathbf{u}_{1}+\left(V_{s}^{2}+V_{A}^{2}\right)\left(\mathbf{k} \cdot \mathbf{u}_{1}\right) \mathbf{k}=0
$$

This equation is similar to (3.1), except that the square of the frequency is multiplied by the factor $\left(1+V_{A}^{2} / c^{2}\right)$. Thus, the phase velocity of the longitudinal magnetosonic wave propagating across $\mathbf{B}_{0}$ now becomes

$$
\frac{\omega}{k}=\left(\frac{V_{s}^{2}+V_{A}^{2}}{1+V_{A}^{2} / c^{2}}\right)^{1 / 2}
$$

\subsection{Propagation Along the Magnetostatic Field}

For $\mathbf{k} \| \mathbf{B}_{0}$, inspection of (6.9) shows that for $\mathbf{u}_{1}$ parallel to $\mathbf{V}_{A}$ (i.e., $\mathbf{B}_{0}$ ) it becomes identical to (2.21). Thus, for the longitudinal sound wave propagating along the magnetic field there is no change from the results obtained before.

However, for the transverse Alfvén wave considering $\left(\mathbf{u}_{1} \perp \mathbf{k}\right)$ we have $\left(\mathbf{V}_{A} \cdot \mathbf{u}_{1}\right)=0$ and $(6.9)$ reduces to

$$
-\omega^{2}\left(1+\frac{V_{A}^{2}}{c^{2}}\right) \mathbf{u}_{1}+k^{2} V_{A}^{2} \mathbf{u}_{1}=0
$$


Consequently, the modification introduced in the Alfvén wave by the displacement current is that the square of the frequency must be multiplied by the factor $\left(1+V_{A}^{2} / c^{2}\right)$. Thus, the phase velocity of the Alfvén wave becomes

$$
\frac{\omega}{k}=\frac{V_{A}}{\left(1+V_{A}^{2} / c^{2}\right)^{1 / 2}}
$$

In the usual limit of $\left(V_{A} / c\right)^{2} \ll 1,(6.13)$ reduces to (4.3) and the effect of the displacement current is unimportant. When using these results, however, it must be kept in mind that they are valid only for frequencies such that charge separation effects are negligible, since the electric force term has been neglected in the equation of motion (2.13).

\section{DAMPING OF MHD WAVES}

In this section it is shown that when the fluid is not perfectly conducting, but has a finite conductivity, or if viscous effects are present, the MHD waves will be damped. Denoting the kinematic viscosity (fluid viscosity divided by mass density) by $\eta_{k}$ and the magnetic viscosity by $\eta_{m}$, as defined in (12.2.5) (Eq. 2.5 in Chapter 12), the linearized set of equations (2.12) to (2.14) are modified to include additional terms as follows:

$$
\begin{gathered}
\frac{\partial \rho_{m 1}}{\partial t}+\rho_{m 0}\left(\nabla \cdot \mathbf{u}_{1}\right)=0 \\
\rho_{m 0} \frac{\partial \mathbf{u}_{1}}{\partial t}+V_{s}^{2} \nabla \rho_{m 1}+\frac{1}{\mu_{0}} \mathbf{B}_{0} \times\left(\nabla \times \mathbf{B}_{1}\right)-\rho_{m 0} \eta_{k} \nabla^{2} \mathbf{u}_{1}=0 \\
\frac{\partial \mathbf{B}_{1}}{\partial t}-\nabla \times\left(\mathbf{u}_{1} \times \mathbf{B}_{0}\right)-\eta_{m} \nabla^{2} \mathbf{B}_{1}=0
\end{gathered}
$$

Although for a compressible fluid the use of the simple viscous force term $\rho_{m 0} \eta_{k} \nabla^{2} \mathbf{u}_{1}$ is not really adequate, it is, nevertheless, expected to give the correct order of magnitude behavior. The displacement current is not included in the treatment presented in this section in order to simplify matters.

For plane wave solutions, the differential operators $\frac{\partial}{\partial t}$ and $\nabla$ are replaced by $-i \omega$ and $i \mathbf{k}$, respectively, so that the set of differential equations (7.1) to (7.3) are replaced by a corresponding set of algebraic equations. Thus, we obtain

$$
\rho_{m 1}=\rho_{m 0} \frac{\left(\mathbf{k} \cdot \mathbf{u}_{1}\right)}{\omega}
$$




$$
\begin{gathered}
\omega \mathbf{u}_{1}=\frac{\rho_{m 1}}{\rho_{m 0}} V_{s}^{2} \mathbf{k}+\frac{1}{\mu_{0} \rho_{m 0}} \mathbf{B}_{0} \times\left(\mathbf{k} \times \mathbf{B}_{1}\right)-i \eta_{k} k^{2} \mathbf{u}_{1} \\
\mathbf{B}_{1}=-\frac{1}{\left(\omega+i \eta_{m} k^{2}\right)} \mathbf{k} \times\left(\mathbf{u}_{1} \times \mathbf{B}_{0}\right)
\end{gathered}
$$

Substituting (7.4) and (7.6) into (7.5), and rearranging, we get

$$
\begin{gathered}
-\omega^{2}\left(1+\frac{i \eta_{k} k^{2}}{\omega}\right)\left(1+\frac{i \eta_{m} k^{2}}{\omega}\right) \mathbf{u}_{1}+\left(1+\frac{i \eta_{m} k^{2}}{\omega}\right) V_{s}^{2}\left(\mathbf{k} \cdot \mathbf{u}_{1}\right) \mathbf{k}- \\
\mathbf{V}_{A} \times\left\{\mathbf{k} \times\left[\mathbf{k} \times\left(\mathbf{u}_{1} \times \mathbf{V}_{A}\right)\right]\right\}=0
\end{gathered}
$$

Comparing this equation for $\mathbf{u}_{1}$ with (2.19), we see that we obtain the same results as before, except that $\omega^{2}$ must be multiplied by the factor $\left(1+i \eta_{k} k^{2} / \omega\right)\left(1+i \eta_{m} k^{2} / \omega\right)$, and $V_{s}^{2}$ must be multiplied by the factor $\left(1+i \eta_{m} k^{2} / \omega\right)$.

\subsection{Alfvén Waves}

For the case of transverse Alfvén waves propagating along $\mathbf{B}_{0}$, the relation (4.3) between $\omega$ and $k$ becomes

$$
\begin{aligned}
& k^{2} V_{A}^{2}=\omega^{2}\left(1+\frac{i \eta_{k} k^{2}}{\omega}\right)\left(1+\frac{i \eta_{m} k^{2}}{\omega}\right) \\
& =\omega^{2}\left[1+\frac{i\left(\eta_{k}+\eta_{m}\right) k^{2}}{\omega}-\frac{\eta_{k} \eta_{m} k^{4}}{\omega^{2}}\right]
\end{aligned}
$$

In order to simplify this result we shall assume that the correction terms corresponding to the kinematic and magnetic viscosities are small, so that the term in the right-hand side of (7.8) can be neglected. Thus,

$$
\begin{gathered}
k^{2} V_{A}^{2} \simeq \omega^{2}\left[1+\frac{i\left(\eta_{k}+\eta_{m}\right) k^{2}}{\omega}\right] \\
\simeq \omega^{2}\left[1+\frac{i\left(\eta_{k}+\eta_{m}\right) \omega}{V_{A}^{2}}\right]
\end{gathered}
$$

where we have replaced $\omega / k$, in the right-hand side, by the first-order result $\left(V_{A}\right)$. Using the binomial expansion approximation $(1+x)^{1 / 2} \simeq 1+x / 2$, valid for $x \ll 1$, (7.9) can be further simplified to

$$
k \simeq \frac{\omega}{V_{A}}+\frac{i\left(\eta_{k}+\eta_{m}\right) \omega^{2}}{2 V_{A}^{3}}
$$


The positive imaginary part in this expression for $k(\omega)$ implies in wave damping. This is easily seen by writing $k=k_{r}+i k_{i}$, with $k_{r}$ and $k_{i}$ real numbers, and noting that

$$
\exp (i k z)=\exp \left(-k_{i} z\right) \exp \left(i k_{r} z\right)
$$

which represents a wave propagating along the $z$ axis with wave number $k_{r}$, but having an exponentially decreasing amplitude, which falls to $1 / e$ of its original intensity in a distance of $1 / k_{i}$.

Expression (7.10) shows that the attenuation of Alfvén waves increases rapidly with frequency (or wave number), but decreases rapidly with increasing magnetic field intensity. Also, the attenuation increases with the fluid viscosity and with the magnetic viscosity. The latter increases as the fluid conductivity decreases.

\subsection{Sound Waves}

For longitudinal sound waves propagating along $\mathbf{B}_{0},(4.2)$ is modified to read

$$
k^{2} V_{s}^{2}=\omega^{2}\left(1+\frac{i \eta_{k} k^{2}}{\omega}\right)
$$

Considering that the resistive and viscous correction terms are small, we find

$$
k \simeq \frac{\omega}{V_{s}}+\frac{i \eta_{k} \omega^{2}}{2 V_{s}^{3}}
$$

This result shows that the attenuation of sound waves also increases rapidly with frequency, but decreases with increasing sound velocity. It also increases with increasing fluid viscosity, as expected.

\subsection{Magnetosonic Waves}

For longitudinal magnetosonic waves propagating across $\mathbf{B}_{0}$, the dispersion relation becomes [see (3.3)]

$$
k^{2} V_{s}^{2}\left(1+\frac{i \eta_{m} k^{2}}{\omega}\right)+k^{2} V_{A}^{2}=\omega^{2}\left(1+\frac{i \eta_{k} k^{2}}{\omega}\right)\left(1+\frac{i \eta_{m} k^{2}}{\omega}\right)
$$

To simplify this expression we consider that the kinematic and magnetic viscosities are small and neglect the term involving the product $\eta_{m} \eta_{k} k^{4} / \omega^{2}$. Hence, (7.14) becomes, after some rearrangement,

$$
k^{2}\left(V_{s}^{2}+V_{A}^{2}\right) \simeq \omega^{2}\left\{1+i \frac{k^{2}}{\omega}\left[\eta_{k}+\eta_{m}\left(1-\frac{k^{2} V_{s}^{2}}{\omega^{2}}\right)\right]\right\}
$$


In the terms in the right-hand side of $(7.15)$ we can replace $\left(\omega^{2} / k^{2}\right)$ by the approximate result $\left(V_{s}^{2}+V_{A}^{2}\right)$, so that (7.15) can be further simplified to give the following dispersion relation:

$$
k=\frac{\omega}{\left(V_{s}^{2}+V_{A}^{2}\right)^{1 / 2}}+i \frac{\omega^{2}}{2\left(V_{s}^{2}+V_{A}^{2}\right)^{3 / 2}}\left[\eta_{k}+\frac{\eta_{m}}{\left(1+V_{s}^{2} / V_{A}^{2}\right)}\right]
$$

Thus, the attenuation of magnetosonic waves also increases with frequency and with kinematic and magnetic viscosities, but decreases with increasing magnetic field strength.

\section{PROBLEMS}

15.1 Calculate the speed of an Alfvén wave for the following cases:

(a) In the Earth's ionosphere, considering that $n_{e}=10^{5} \mathrm{~cm}^{-3}, B=0.5$ gauss, and that the positive charge carriers are atomic oxygen ions.

(b) In the solar corona, assuming $n_{e}=10^{6} \mathrm{~cm}^{-3}, B=10$ gauss, and that the positive charge carriers are protons.

(c) In the interstellar space, considering $n_{e}=10^{7} \mathrm{~m}^{-3}$ and $B=10^{-7}$ tesla, the positive charge carriers being protons.

15.2 Show that the Alfvén wave propagating along the magnetic field is circularly polarized.

15.3 For the pure Alfvén wave propagating at an angle $\theta$ with respect to the magnetostatic field $\mathbf{B}_{0}$, with phase velocity given by (5.10), determine relations between the associated field components $B_{1 y}, u_{1 y}, E_{1 x}$, and $J_{1 x}$.

15.4 Include the effect of finite conductivity in the derivation of the equations for the plane Alfvén wave propagating along the magnetic field. Show that the linearized equations are satisfied by solutions of the form $\exp (\alpha z-i \omega t)$ and determine the coefficient $\alpha$.

15.5 A plane electromagnetic wave is incident normally on the surface of a conducting fluid of large but finite conductivity $(\sigma)$, immersed in a uniform magnetic field $\mathbf{B}_{0}$ such that $\mathbf{k} \perp \mathbf{B}_{0}$. Assume that the magnetic field $(\mathbf{B})$ of the incoming wave is parallel to $\mathbf{B}_{0}$. Show that there are two wave modes that penetrate the fluid. One of them is an unattenuated magnetosonic wave and the other one is a mode that has an effective skin 
depth $\delta=\left(V_{s} / V_{m}\right) \delta_{r c}$, where $V_{s}$ and $V_{m}$ are the sound and magnetosonic velocities, respectively, and $\delta_{r c}$ is the skin depth in a rigid conductor.

15.6 For the fast and slow MHD waves, let $u_{\ell}$ and $u_{t}$ denote the components of the mass flow velocity that are longitudinal and transverse, respectively, to the direction of propagation. Show that $u_{\ell}$ and $u_{t}$ are in phase for the fast wave and $180^{\circ}$ out of phase for the slow wave. Also, show that the perturbations in the kinetic and magnetic pressures are in phase for the fast wave and $180^{\circ}$ out of phase for the slow wave.

15.7 Consider the following closed set of MHD equations in the so-called Chew, Goldberger, and Low approximation,

$$
\begin{gathered}
\frac{\partial \rho_{m}}{\partial t}+\nabla \cdot\left(\rho_{m} \mathbf{u}\right)=0 \\
\rho_{m} \frac{D \mathbf{u}}{D t}=\rho \mathbf{E}-\nabla\left(p_{\perp}+\frac{B^{2}}{2 \mu_{0}}\right)+(\mathbf{B} \cdot \nabla)\left[\left(\frac{1}{\mu_{0}}-\frac{p_{\|}-p_{\perp}}{B^{2}}\right) \mathbf{B}\right] \\
\frac{D}{D t}\left(\frac{p_{\|} B^{2}}{\rho_{m}^{3}}\right)=0 \\
\frac{D}{D t}\left(\frac{p_{\perp}}{\rho_{m} B}\right)=0 \\
\nabla \times \mathbf{B}=\mu_{0} \mathbf{J} \\
\nabla \times \mathbf{E}=-\frac{\partial \mathbf{B}}{\partial t} \\
\nabla \cdot \mathbf{E}=\frac{\rho}{\epsilon_{0}} \\
\mathbf{E}+\mathbf{u} \times \mathbf{B}=0
\end{gathered}
$$

In the equations of this set, involving the pressure tensor $\mathcal{P}$, it is considered that

$$
\mathcal{P}=\left(\begin{array}{ccc}
p_{\perp} & 0 & 0 \\
0 & p_{\perp} & 0 \\
0 & 0 & p_{\|}
\end{array}\right)
$$

(a) Taking the equilibrium mean velocity equal to zero, show that the dispersion relation for the magnetohydrodynamic waves can be written as 


$$
\begin{gathered}
\rho_{m 0} \omega^{2}+\cos \theta\left(p_{\|}-p_{\perp}-\frac{B_{0}^{2}}{\mu_{0}}\right)-k^{2} \sin ^{2} \theta\left(2 p_{\perp}+\frac{B_{0}^{2}}{\mu_{0}}\right)= \\
\frac{p_{\perp}^{2} k^{4} \sin ^{2} \theta \cos ^{2} \theta}{\rho_{m 0} \omega^{2}-3 p_{\|} k^{2} \cos ^{2} \theta}
\end{gathered}
$$

where $\theta$ is the angle between $\mathbf{k}$ and $\mathbf{B}_{0}$, and where $p_{\|}, p_{\perp}$, and $\mathbf{B}_{0}$ stand for the unperturbed quantities.

(b) Show that these waves are unstable for all values of $\theta$ less than a critical angle $\theta_{c}$, which satisfies the equation

$$
\frac{B_{0}^{2}}{\mu_{0}}+p_{\perp}\left(1+\sin ^{2} \theta_{c}\right)=\frac{p_{\perp}^{2}}{3 p_{\|}} \sin ^{2} \theta_{c}+2 p_{\|} \cos ^{2} \theta_{c} .
$$




\section{6}

\section{WAVES IN COLD PLASMAS}

\section{INTRODUCTION}

In this chapter we analyze the problem of wave propagation in cold plasmas. In the cold plasma model the thermal kinetic energy of the particles is ignored and the corresponding velocity distribution function is a Dirac delta function centered at the macroscopic fluid velocity. The study of waves in plasmas is very useful for plasma diagnostics, since it provides information on the plasma properties. The theory of wave propagation in a cold homogeneous plasma, immersed in a magnetic field, is commonly known as magnetoionic theory.

There are two main different methods of approach that are normally used in analyzing the problem of wave propagation in plasmas. In one of them, the plasma is characterized as a medium having either a conductivity or a dielectric constant and the wave equation for this medium is derived from Maxwell equations. In the presence of an externally applied magnetostatic field, the plasma is equivalent to an anisotropic dielectric characterized by a dielectric tensor or dyad. In another approach, Maxwell equations are solved simultaneously with the fluid equations describing the particle motions. In this case we do not explicity derive a wave equation, and expressions for the dielectric or conductivity dyad are not obtained directly. Instead, we obtain a dispersion relation, which relates the wave number $k$ to the wave frequency $\omega$. All the information about the propagation of a given wave mode is contained in the appropriate dispersion relation. This method of approach is often straightforward and simpler than the other one and is the method we shall adopt in this treatment. 
The pressure gradient term in the momentum equation can be neglected if the particle thermal velocity is small, when compared to the wave phase velocity. For this reason, the cold plasma model gives a satisfactory description except for waves with extremely small phase velocities. For waves with such small phase velocities, the pressure term becomes important and must be considered for a correct description. The propagation of waves in warm plasmas (which includes the pressure gradient term) is the subject of the next chapter.

The study presented here is restricted to small amplitude waves, so that the analysis will be based on a linear perturbation theory under the assumption that the variations in the plasma parameters (due to the presence of waves) are small (to the first order), as compared to the undisturbed parameters. The plasma is assumed to be homogeneous and infinite (no boundary effects), and the externally applied magnetic field is assumed to be constant and uniform. This medium is usually called a magnetoionic medium. For mathematical simplicity, the analysis will be made in terms of plane waves. This does not imply in loss of generality, since any physically realizable wave motion can, in principle, be synthesized in terms of plane waves.

In the usual magnetoionic theory only the electron motion is considered. This approximation is adequate for high-frequency waves, i.e., for frequencies that are large compared to the ion cyclotron frequency. The theory of high-frequency, small-amplitude plane waves propagating in an arbitrary direction with respect to the magnetostatic field, in a magnetoionic medium, is known as the Appleton-Hartree theory, in honor of E. V. Appleton and D. R. Hartree, who developed this theory when studying the problem of wave propagation in the Earth's ionosphere. At frequencies of the order of the ion cyclotron frequency and smaller, ion motion must be considered. The theory of wave propagation in a cold multicomponent plasma is commonly referred to as the hydromagnetic extension of magnetoionic theory.

\section{BASIC EQUATIONS OF MAGNETOIONIC THEORY}

In a cold electron gas the two hydrodynamic variables involved are the electron number density $n(\mathbf{r}, t)$ and the average electron velocity $\mathbf{u}(\mathbf{r}, t)$. They satisfy the continuity equation

$$
\frac{\partial n}{\partial t}+\nabla \cdot(n \mathbf{u})=0
$$


and the Langevin equation of motion for the electrons

$$
m \frac{D \mathbf{u}}{D t}=q(\mathbf{E}+\mathbf{u} \times \mathbf{B})-m \nu \mathbf{u}
$$

These two equations are complemented by Maxwell equations:

$$
\begin{gathered}
\nabla \cdot \mathbf{E}=\frac{\rho}{\epsilon_{0}} \\
\nabla \cdot \mathbf{B}=0 \\
\nabla \times \mathbf{E}=-\frac{\partial \mathbf{B}}{\partial t} \\
\nabla \times \mathbf{B}=\mu_{0}\left(\mathbf{J}+\epsilon_{0} \frac{\partial \mathbf{E}}{\partial t}\right)
\end{gathered}
$$

Considering one type of positive ions of charge $q_{i}$ and number density $n_{i}$, the total electric charge density is given by

$$
\rho=-e n+q_{i} n_{i}
$$

Since ion motion is not considered $\left(\mathbf{u}_{i}=0\right)$, the electric current density is

$$
\mathbf{J}=-e n \mathbf{u}
$$

As previously discussed, equation (2.4) is actually considered as an initial condition for (2.5). Furthermore, (2.3) and (2.6) can be combined to yield the electric charge conservation equation.

\section{PLANE WAVE SOLUTIONS AND LINEARIZATION}

Let us separate the total magnetic induction and the electron number density into two parts,

$$
\begin{aligned}
\mathbf{B}(\mathbf{r}, t) & =\mathbf{B}_{0}+\mathbf{B}_{1}(\mathbf{r}, t) \\
n(\mathbf{r}, t) & =n_{0}+n_{1}(\mathbf{r}, t)
\end{aligned}
$$

where $\mathbf{B}_{0}$ is a constant and uniform field, and $n_{0}$ is the undisturbed electron number density in the absence of waves. Denoting by $\psi_{j}$ any one of the components of the quantities $\mathbf{E}, \mathbf{B}_{1}, \mathbf{u}$, and $n_{1}$, we can write, for harmonic plane wave solutions,

$$
\psi_{j}(\mathbf{r}, t)=\psi_{j} \exp (i \mathbf{k} \cdot \mathbf{r}-i \omega t)
$$


where $\mathbf{k}$ is the wave propagation vector and $\omega$ is the wave frequency. The use of the same symbol to denote the complex amplitude as well as the entire expession in (3.3) should lead to no confusion, because in linear wave theory the same exponential factor will occur on both sides of any equation and can be canceled out.

Equation (2.2) is not yet quite tractable because of the nonlinear terms $(\mathbf{u} \cdot \nabla) \mathbf{u}$ and $\mathbf{u} \times \mathbf{B}$. This difficulty can be eliminated considering $\mathbf{u}$ and $\mathbf{B}_{1}$ as first-order quantities and neglecting second-order terms. As discussed in section 3 of Chapter 10, when dealing with wave phenomena in plasmas the second-order nonlinear term $\mathbf{u} \times \mathbf{B}_{1}$ can be neglected provided the average electron velocity is much less than the wave phase velocity $(u \ll \omega / k)$.

For harmonic plane wave solutions, the differential operators $\nabla$ and $\partial / \partial t$ are replaced, respectively, by $i \mathbf{k}$ and $-i \omega$, so that the differential equations become simple algebraic equations. Therefore, neglecting second-order terms, (2.2), (2.5), and (2.6) become, respectively,

$$
\begin{gathered}
-i \omega m \mathbf{u}=-e\left(\mathbf{E}+\mathbf{u} \times \mathbf{B}_{0}\right)-m \nu \mathbf{u} \\
\mathbf{k} \times \mathbf{E}=\omega \mathbf{B}_{1} \\
i \mathbf{k} \times \mathbf{B}_{1}=\mu_{0}\left(-e n_{0} \mathbf{u}-i \omega \epsilon_{0} \mathbf{E}\right)
\end{gathered}
$$

where use was made of (2.8) linearized. These three equations involving the dependent variables $\mathbf{u}, \mathbf{E}$, and $\mathbf{B}_{1}$ can be used to derive a dispersion relation for wave propagation in a cold electron gas. In order to keep matters as simple as possible, we investigate initially the characteristics of wave propagation in a cold isotropic plasma with $\mathbf{B}_{0}=0$.

\section{WAVE PROPAGATION IN ISOTROPIC ELECTRON PLASMAS}

\subsection{Derivation of the Dispersion Relation}

In the absence of an externally applied magnetic field $\left(\mathbf{B}_{0}=0\right)$ the Langevin equation (3.4) yields

$$
\mathbf{u}=-\frac{e}{m(\nu-i \omega)} \mathbf{E}
$$

Combining (3.5), (3.6), and (4.1), we obtain

$$
\mathbf{k} \times(\mathbf{k} \times \mathbf{E})=-\frac{i \omega \mu_{0} e^{2} n_{0}}{m(\nu-i \omega)} \mathbf{E}-\frac{\omega^{2}}{c^{2}} \mathbf{E}
$$




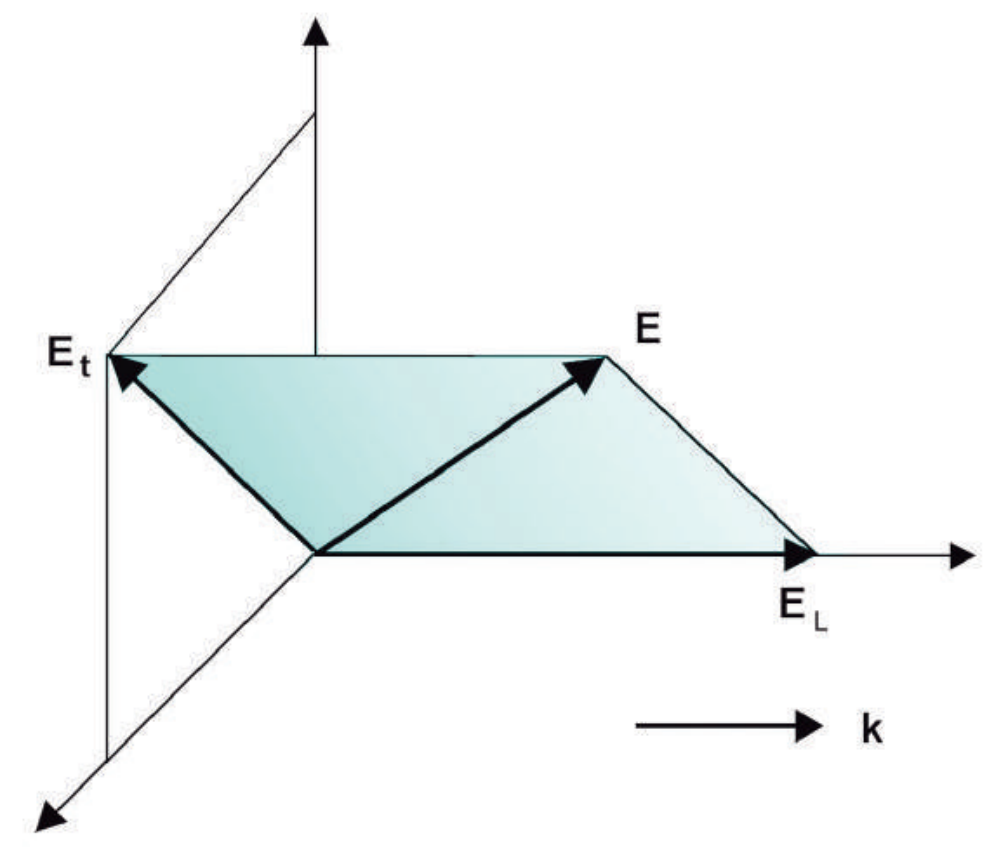

Fig. 1 Longitudinal and transverse components of the electric field vector with respect to the wave propagation vector $\mathbf{k}$.

It is convenient to separate the electric field vector in a longitudinal component $\mathbf{E}_{\ell}$ (parallel to $\mathbf{k}$ ) and a transverse component $\mathbf{E}_{t}$ (perpendicular to $\mathbf{k}$ ),

$$
\mathbf{E}=\mathbf{E}_{\ell}+\mathbf{E}_{t}
$$

as shown in Fig. 1. Note that the indices $\ell$ and $t$ refer to the direction of the wave vector $\mathbf{k}$, whereas the indices $\|$ and $\perp$, when used, refer to the direction of the externally applied magnetic induction vector $\mathbf{B}_{0}$. Therefore, we have

$$
\begin{aligned}
\mathbf{k} \times \mathbf{E}_{\ell} & =0 \\
\mathbf{k} \times\left(\mathbf{k} \times \mathbf{E}_{t}\right) & =-k^{2} \mathbf{E}_{t}
\end{aligned}
$$

and (4.2) becomes

$$
-k^{2} \mathbf{E}_{t}=-\left[\frac{i \omega \mu_{0} e^{2} n_{0}}{m(\nu-i \omega)}+\frac{\omega^{2}}{c^{2}}\right]\left(\mathbf{E}_{\ell}+\mathbf{E}_{t}\right)
$$

This equation can be separated into a longitudinal component,

$$
\left[\frac{\omega_{p e}^{2}}{c^{2}(1+i \nu / \omega)}-\frac{\omega^{2}}{c^{2}}\right] \mathbf{E}_{\ell}=0
$$


and a transverse component,

$$
-k^{2} \mathbf{E}_{t}=\left[\frac{\omega_{p e}^{2}}{c^{2}(1+i \nu / \omega)}-\frac{\omega^{2}}{c^{2}}\right] \mathbf{E}_{t}
$$

Equation (4.7) yields the following dispersion relation for a longitudinal $\operatorname{mode}\left(\mathbf{E}_{\ell} \neq 0\right)$ :

$$
\omega^{2}(1+i \nu / \omega)-\omega_{p e}^{2}=0
$$

For a transverse mode $\left(\mathbf{E}_{t} \neq 0\right)$ the dispersion relation is, from (4.8),

$$
\left(\omega^{2}-k^{2} c^{2}\right)(1+i \nu / \omega)-\omega_{p e}^{2}=0
$$

\subsection{Collisionless Plasma}

For simplicity, we consider first the case in which the collision frequency is much less than the wave frequency $(\nu \ll \omega)$, so that the effect of collisions can be ignored. In subsection 4.4 we shall take into consideration the effect of collisions. Thus, for $(\nu / \omega) \ll 1$ the dispersion relation (4.9) for the longitudinal mode becomes

$$
\omega^{2}=\omega_{p e}^{2}
$$

while, for the transverse mode, (4.10) becomes

$$
k^{2} c^{2}=\omega^{2}-\omega_{p e}^{2}
$$

Equation (4.11) shows that longitudinal oscillations $\left(\mathbf{E}_{\ell} \neq 0\right)$ can occur just at the plasma frequency $\omega_{p e}$. These longitudinal oscillations are the same plasma oscillations discussed in section 1 of Chapter 11. It is seen from (4.1) that the electrons oscillate with a velocity given by

$$
\mathbf{u}=-\frac{i e}{m \omega} \mathbf{E}_{\ell}
$$

From (4.4) and (3.5) it is clear that $\mathbf{B}_{1}=0$, so that there is no magnetic field associated with these longitudinal oscillations. Further, there is no wave propagation, since there is no relative phase variation from point to point. These oscillations are therefore longitudinal, electrostatic, and stationary. In the next chapter we shall consider wave propagation in a 
warm plasma, where it will be shown that these electron plasma oscillations correspond to the zero-temperature limit of the longitudinal mode of propagation called the electron plasma wave.

Considering now the dispersion relation (4.12) for transverse waves $\left(\mathbf{E}_{t} \neq 0\right)$, it is seen that $k^{2}$ is positive for $\omega>\omega_{p e}$ and negative for $\omega<\omega_{p e}$. Hence, for traveling waves (with $\omega$ real) $k$ becomes imaginary for $\omega<\omega_{p e}$. Writing $k=\beta+i \alpha$, where $\beta$ and $\alpha$ are real quantities, it is seen from (4.12) that for $\omega>\omega_{p e}(k=\beta ; \quad \alpha=0)$ the transverse wave propagates with a phase velocity ( $\omega$ divided by the real part of $k$ ) given by

$$
v_{p h}=\frac{\omega}{k}=\frac{c}{\left(1-\omega_{p e}^{2} / \omega^{2}\right)^{1 / 2}} \quad\left(\omega>\omega_{p e}\right)
$$

Also, for $\omega>\omega_{p e}$, the group velocity of the transverse wave can be obtained differentiating (4.12) with respect to $k$,

$$
v_{g}=\frac{\partial \omega}{\partial k}=\frac{c^{2}}{v_{p h}} \quad\left(\omega>\omega_{p e}\right)
$$

For $\omega<\omega_{p e}, k$ is imaginary $(k=i \alpha)$ and the transverse wave is exponentially damped, since

$$
E_{t} \propto \exp (i k \zeta-i \omega t)=\exp (-\alpha \zeta) \exp (-i \omega t)
$$

so that the wave dies out with increasing values of $\zeta$. Such exponentially damped fields are called evanescent waves and do not transport any timeaveraged power. Since $\beta=0$, it is easily seen that in this case

$$
\begin{array}{ll}
v_{p h}=\infty & \left(\omega<\omega_{p e}\right) \\
v_{g}=0 & \left(\omega<\omega_{p e}\right)
\end{array}
$$

Also, from (4.12) we find (for $\omega<\omega_{p e}$ )

$$
\alpha=\mathcal{I}\{k\}=\frac{\left(\omega_{p e}^{2}-\omega^{2}\right)^{1 / 2}}{c}
$$

where $\mathcal{I}$ denotes the imaginary part of.

A plot of phase velocity and group velocity as a function of the wave frequency is shown in Fig. 2(a) and the dependence of the attenuation factor on the wave frequency is shown in Fig. 2(b). Note that the phase velocity is always greater than the speed of light $c$, but the group velocity, 
which is the velocity at which a signal propagates, is always less than $c$, in agreement with the requirements of the relativity theory. For $\omega \gg \omega_{p e}$ we find, from (4.14) and (4.15),

$$
v_{p h}=v_{g}=c
$$

which shows that for very high frequencies the plane wave characteristics of a plasma degenerate to those of free space. This behavior is expected on a physical basis, since in the limiting case of infinite frequency even the electrons are unable to respond to the oscillating electric field.

The dispersion relation (4.12) is shown in Fig. 3, where it is plotted $\omega$ as a function of the real part of $k$. In this text we shall adopt the usual graphic representation of dispersion relations, plotting $\omega$ versus $k$, rather than $k$ versus $\omega$. Note that in the frequency region where $\omega<\omega_{p e}$ the transverse wave is evanescent.

From the definition $v_{g}=\partial \omega / \partial k$ it is clear that at a given point in the $\omega(k)$ curve the group velocity is given by the slope of the tangent to the curve at that point, whereas the phase velocity $\omega / k$ is equal to the slope of the line drawn from the origin to this point. This geometrical representation is illustrated in Fig. 3.

\subsection{Time-Averaged Poynting Vector}

We evaluate next the time-averaged Poynting vector $\langle\mathbf{S}\rangle$, which gives the time-averaged power flow for the transverse wave. From (3.5), taking $\mathbf{B}_{1}=\mu_{0} \mathbf{H}_{1}$, we have

$$
\mathbf{H}_{1}=\frac{1}{\mu_{0} \omega}(\mathbf{k} \times \mathbf{E})
$$

and the expression for $\langle\mathbf{S}>$, given in (14.5.13) (Eq. 5.13 in Chapter 14), becomes

$$
\begin{aligned}
<\mathbf{S}>= & \frac{1}{2} \mathcal{R}\left\{\mathbf{E} \times \mathbf{H}_{1}^{*}\right\}=\frac{1}{2 \mu_{0} \omega} \mathcal{R}\left\{\mathbf{E} \times\left(\mathbf{k}^{*} \times \mathbf{E}^{*}\right)\right\} \\
= & \frac{1}{2 \mu_{0} \omega} \mathcal{R}\left\{k^{*} E(\mathbf{r}, t) E^{*}(\mathbf{r}, t)\right\} \widehat{\mathbf{n}}
\end{aligned}
$$

where $\widehat{\mathbf{n}}$ is a unit vector in the direction of $\mathbf{E} \times \mathbf{H}_{1}$. Using (3.3) and considering $k$ to be a complex quantity, (4.22) becomes

$$
<\mathbf{S}>=\widehat{\mathbf{n}}\left(\frac{E^{2}}{2 \mu_{0} \omega}\right) \mathcal{R}\left\{k^{*} \exp \left[i\left(k-k^{*}\right) \zeta\right]\right\}
$$



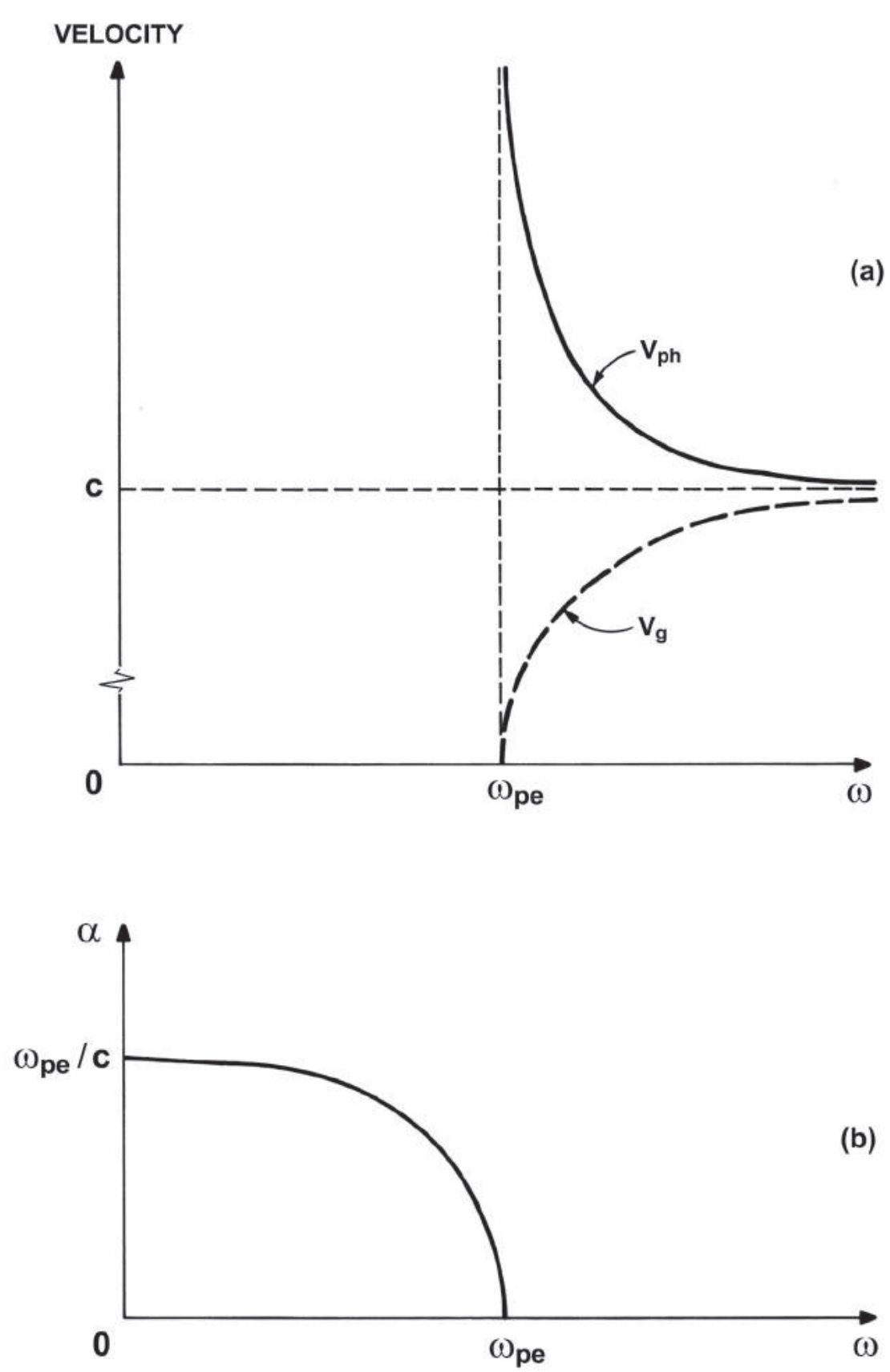

Fig. 2 Frequency dependence of the phase velocity, group velocity, and the attenuation factor $\alpha$, for transverse waves in a collisionless isotropic cold electron gas. 


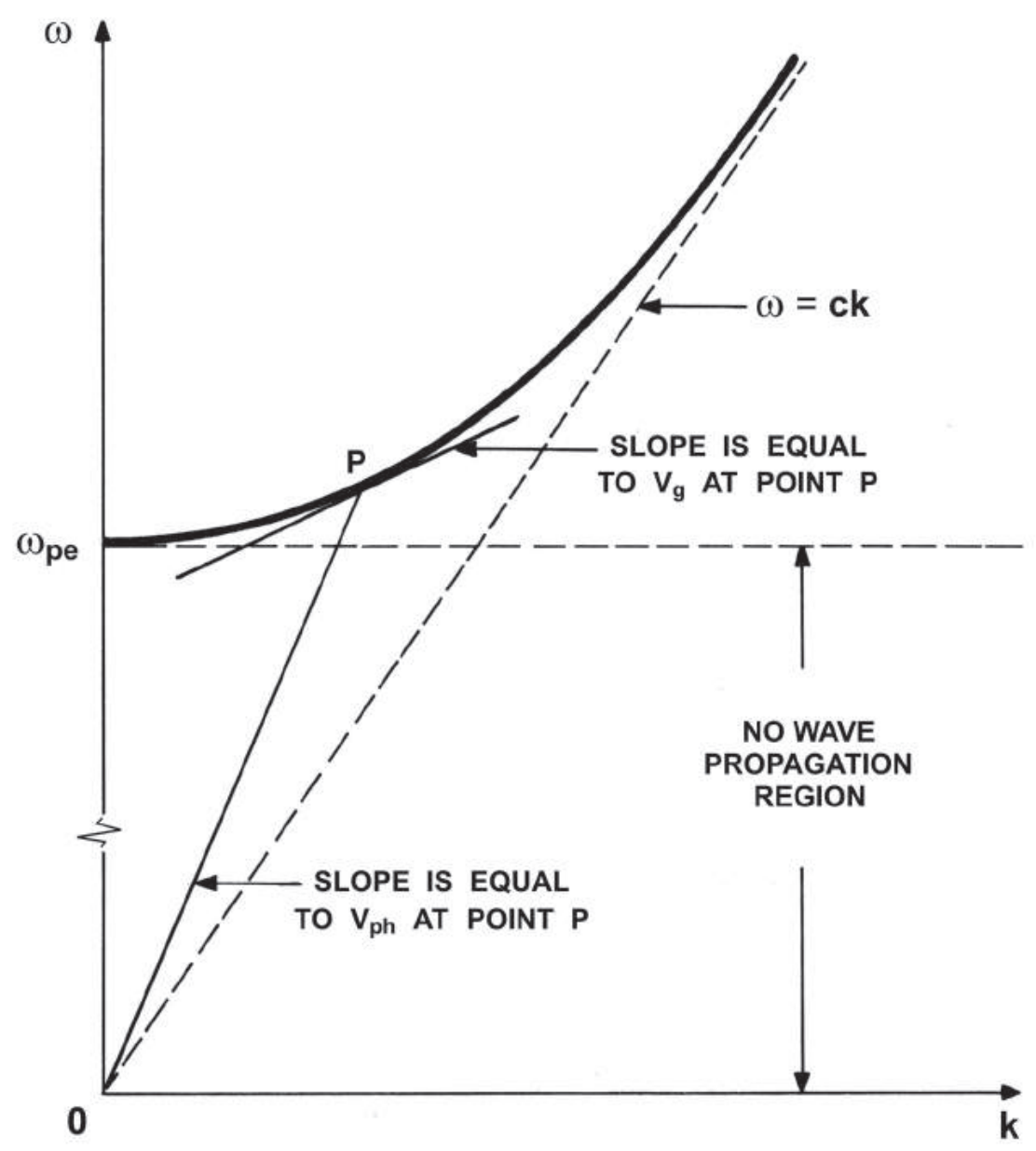

Fig. 3 Dispersion relation $\omega(k)$ for the transverse wave mode propagating in an isotropic cold electron plasma. Note the geometrical representations for the phase velocity and for the group velocity at the point $P$ on the curve.

Therefore, since $k$ is either real or imaginary according to whether $\omega>\omega_{p e}$ or $\omega<\omega_{p e}$, respectively, it follows from (4.23) that

$$
\begin{array}{ll}
<\mathbf{S}>=0 & \text { for }\left(\omega<\omega_{p e}\right) \\
<\mathbf{S}>=\widehat{\mathbf{n}}\left(\frac{1}{2} \epsilon_{0} E^{2}\right) v_{g} & \text { for }\left(\omega>\omega_{p e}\right)
\end{array}
$$

where, in (4.25), we have used the relation $c^{2} k / \omega=v_{g}$ given in (4.15). Thus, for $\omega>\omega_{p e}$ the fields transport power in the direction of $\mathbf{E} \times \mathbf{H}_{1}$, 
whereas for $\omega<\omega_{p e}$ there is no power flow and the wave is evanescent. For this reason, the region $\omega>\omega_{p e}$ is called the propagation region.

Since the wave is totally reflected for $\omega<\omega_{p e}$, the frequency $\omega=\omega_{p e}$ is often called a reflection point (where $k$ is zero and $v_{p h}$ is infinite). It can be shown that the power transmitted into a semi-infinite slab of plasma is zero if $\beta$ is zero, so that in a more general sense any frequency for which $\beta=0$ (i.e., $v_{p h}=\infty$ ) is referred to as a reflection point. However, if the plasma medium is finite, some energy can be transmitted through a finite plasma slab even if $\beta=0$. Details are provided in problem 16.2. This effect is known as the tunneling effect.

\subsection{The Effect of Collisions}

The principal effect of collisons is to produce damping of the waves. Before analyzing the dispersion relations (4.9) and (4.10), it is useful to discuss some general results concerning dispersion relations of the form

$$
k^{2}=A+i B
$$

where $A$ and $B$ are real quantities. If we separate $k$ into its real and imaginary parts,

$$
k=\beta+i \alpha
$$

where $\beta$ and $\alpha$ are both real, then it is a simple matter to verify that

$$
\begin{gathered}
A=\mathcal{R}\left\{k^{2}\right\}=\beta^{2}-\alpha^{2} \\
B=\mathcal{I}\left\{k^{2}\right\}=2 \beta \alpha
\end{gathered}
$$

On the other hand, since the waves are proportional to exp $(i k \zeta-i \omega t)$, we have

$$
\exp (i k \zeta-i \omega t)=\exp (-\alpha \zeta) \exp (i \beta \zeta-i \omega t)
$$

Thus, the sign of $\beta$ determines the direction of wave propagation, i.e., $\beta>0$ implies propagation in the positive direction, whereas $\beta<0$ implies propagation in the negative direction. The sign of $\alpha$ is related to growing or damping of the wave amplitude as the wave propagates. If both $\alpha$ and $\beta$ are positive, then the wave travels in the positive $\zeta$ direction and is exponentially damped. If both $\alpha$ and $\beta$ are negative, then the wave travels in the negative $\zeta$ direction and is also exponentially damped. On the other hand, if $\alpha$ and $\beta$ have opposite signs, then the wave is exponentially growing (see Fig. 4). In any case, the sign of $B$ determines whether the 


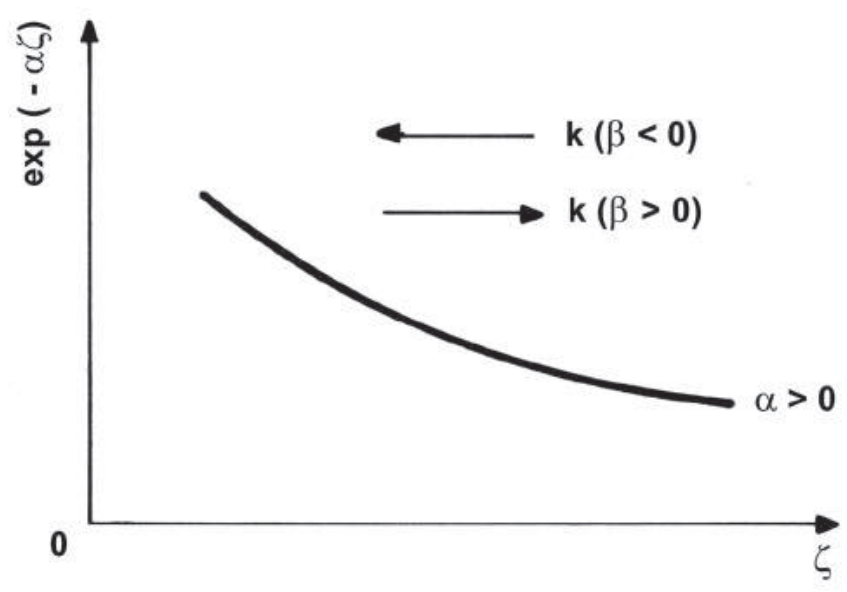

(a)

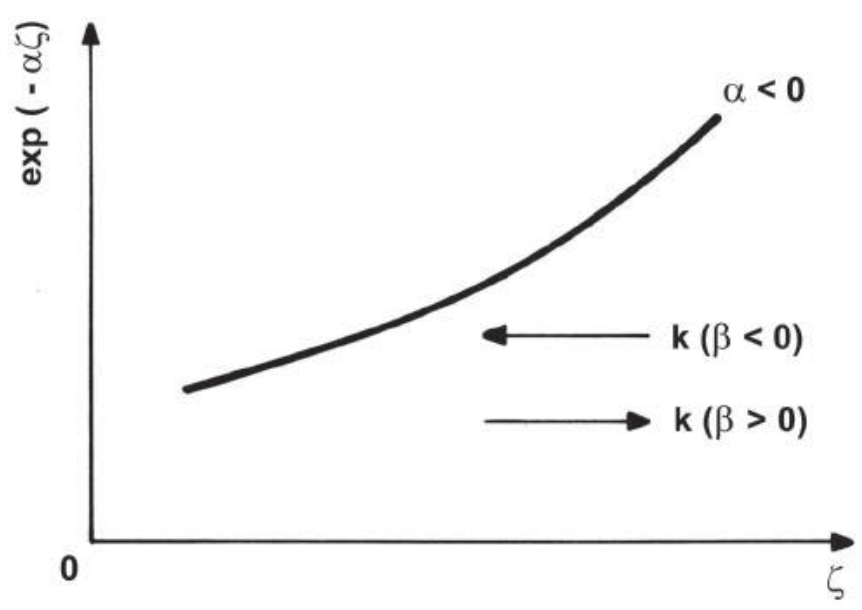

(b)

Fig. 4 (a) For $\alpha>0$ the wave amplitude is exponentially damped if it propagates in the positive $\zeta$ direction $(\beta>0)$, or exponentially growing if it propagates in the negative $\zeta$ direction $(\beta<0)$, whereas (b) for $\alpha<0$ the opposite situation holds.

traveling wave is growing or decaying. For $B>0$, the wave amplitude is damped with distance, whereas, for $B<0$, the wave amplitude grows. Similarly, for a dispersion relation having the form

$$
\omega^{2}=C+i D
$$

it can be easily verified that, for standing waves, if $D>0$, the wave amplitude grows in time, whereas if $D<0$, the wave amplitude is damped. 


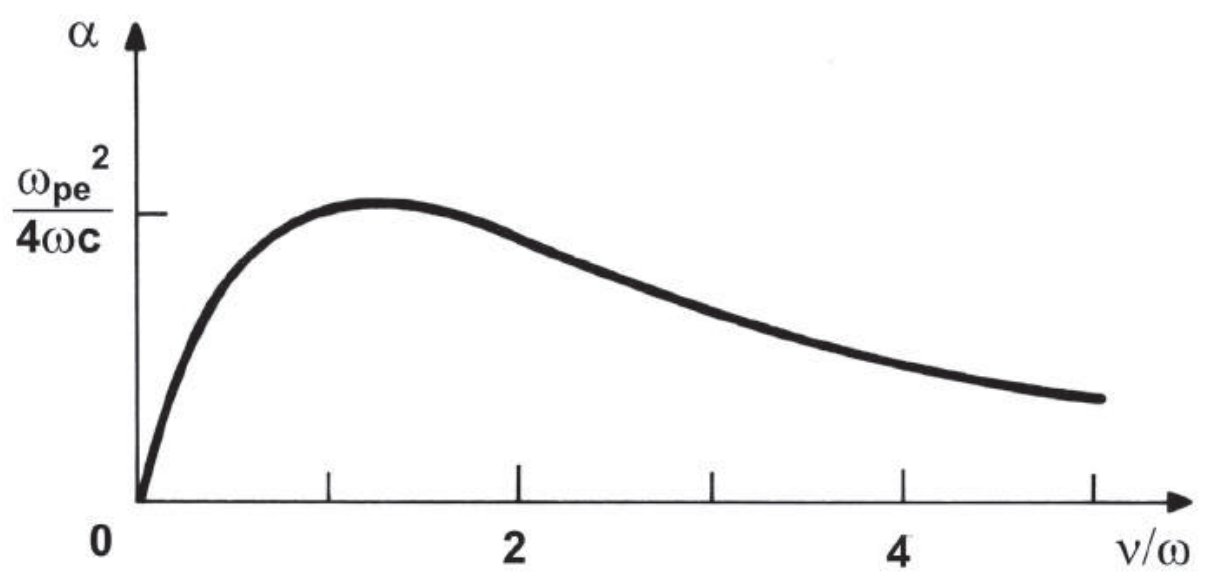

Fig. 5 Attenuation factor $\alpha$ as a function of collision frequency, considering a given frequency such that $\omega \gg \omega_{p e}$, for the transverse wave mode.

Let us consider now the dispersion relation (4.9) for longitudinal oscillations,

$$
\omega^{2}+i \nu \omega-\omega_{p e}^{2}=0
$$

or

$$
\omega=\frac{1}{2}\left[-i \nu \pm\left(4 \omega_{p e}^{2}-\nu^{2}\right)^{1 / 2}\right]
$$

This equation shows that for any value of $\nu$ the imaginary part of $\omega$ is negative, so that the oscillation is damped, since it is proportional to $\exp (-i \omega t)$.

For the transverse mode the dispersion relation (4.10) gives

$$
k^{2} c^{2}=\omega^{2}-\frac{\omega_{p e}^{2}}{1+(\nu / \omega)^{2}}+\frac{i \omega_{p e}^{2}(\nu / \omega)}{1+(\nu / \omega)^{2}}
$$

Consequently, in the propagation band $B=\mathcal{I}\left\{k^{2}\right\}$ is negative and the traveling waves are damped for all frequencies. A plot of the attenuation factor $\alpha=\mathcal{I}\{k\}$, as a function of the normalized collision frequency $(\nu / \omega)$, is shown in Fig. 5, calculated from (4.34) for a given frequency $\omega$ much larger than the plasma frequency $\omega_{p e}$. Fig. 6 shows the dispersion relation $\omega(k)$ for the transverse mode of propagation, considering several values of $\nu$, such that $\nu_{3}>\nu_{2}>\nu_{1}>0$. 


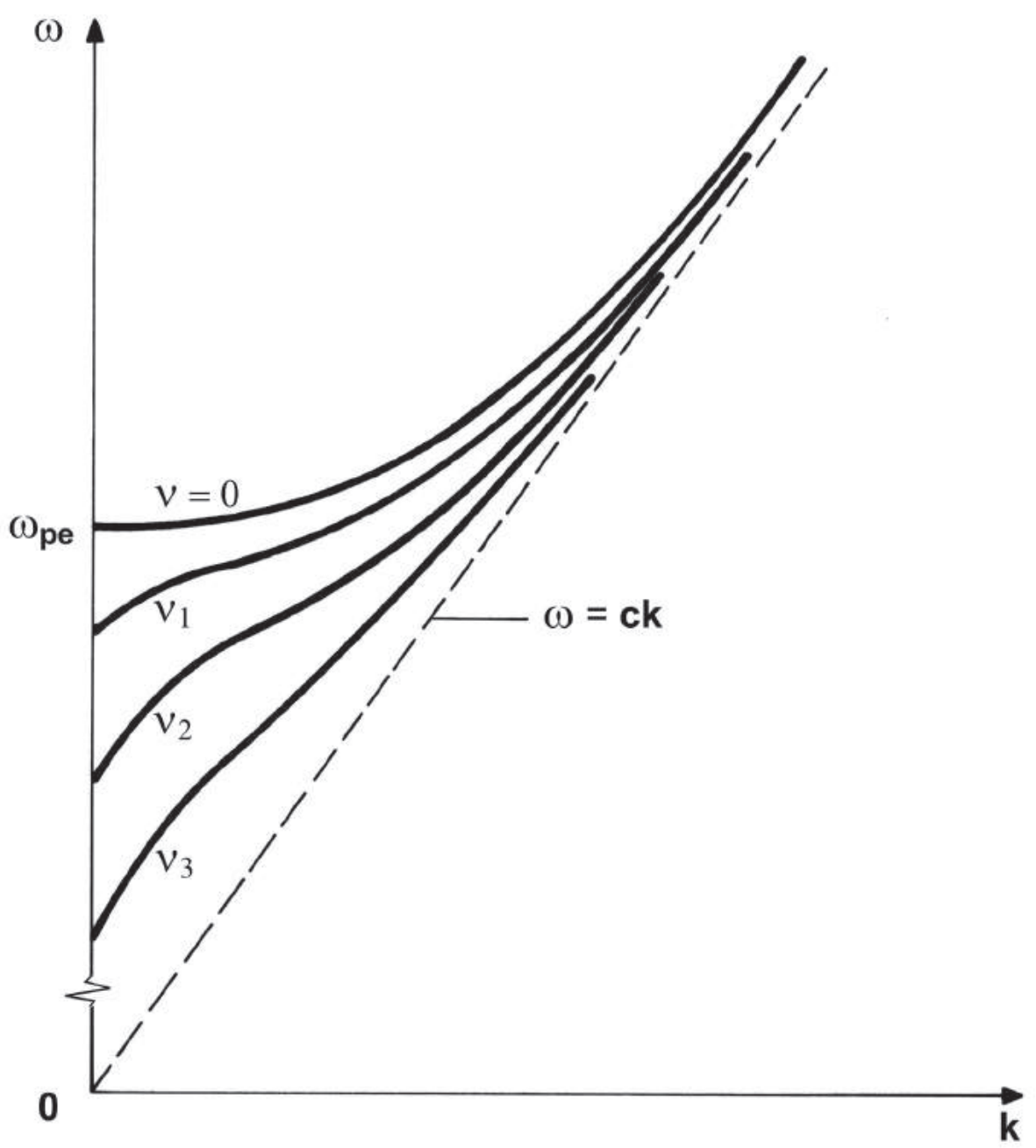

Fig. 6 Plot of the dispersion relation $\omega(k)$ for the transverse wave mode in an isotropic cold electron plasma, considering the effect of collisions $\left(\nu_{3}>\nu_{2}>\nu_{1}>0\right)$.

\section{WAVE PROPAGATION IN MAGNETIZED COLD PLASMAS}

Consider now the problem of wave propagation in a cold electron plasma, when there is a uniform magnetostatic field externally applied. The presence of the magnetostatic field $\mathbf{B}_{0}$ introduces a space anisotropy in the plasma. 


\subsection{Derivation of the Dispersion Relation}

To derive the dispersion relation for this case, we shall start from the coupled set of equations (3.4), (3.5), and (3.6). Combining (3.5) and (3.6), and rearranging, this set reduces to

$$
\begin{gathered}
\mathbf{k} \times(\mathbf{k} \times \mathbf{E})+\frac{\omega^{2}}{c^{2}} \mathbf{E}=\frac{i \omega e n_{0}}{\epsilon_{0} c^{2}} \mathbf{u} \\
\left(1+i \frac{\nu}{\omega}\right) \mathbf{u}+\frac{i e}{m \omega}\left(\mathbf{u} \times \mathbf{B}_{0}\right)=-\frac{i e}{m \omega} \mathbf{E}
\end{gathered}
$$

If we denote the angle between $\mathbf{B}_{0}$ and $\mathbf{k}$ by $\theta$ and choose a Cartesian coordinate system in which $\widehat{\mathbf{z}}$ is in the direction of $\mathbf{B}_{0}$, and $\widehat{\mathbf{y}}$ is perpendicular to the plane formed by $\mathbf{B}_{0}$ and $\mathbf{k}$ (see Fig. 7 ), then we have

$$
\begin{gathered}
\mathbf{B}_{0}=B_{0} \widehat{\mathbf{z}} \\
\mathbf{k}=k_{\perp} \widehat{\mathbf{x}}+k_{\|} \widehat{\mathbf{z}}=k \sin (\theta) \widehat{\mathbf{x}}+k \cos (\theta) \widehat{\mathbf{z}}
\end{gathered}
$$

Note that the index symbols $\|$ and $\perp$ are used to denote components parallel and perpendicular to the direction of the magnetostatic field $\mathbf{B}_{0}$, whereas the index symbols $\ell$ and $t$ (used in the previous section) refer to vector components longitudinal and transverse with respect to the wave vector $\mathbf{k}$, respectively.

With this choice of coordinate system, we obtain

$$
\begin{gathered}
\mathbf{k} \times(\mathbf{k} \times \mathbf{E})=k^{2} \cos \theta\left(\sin \theta E_{z}-\cos \theta E_{x}\right) \widehat{\mathbf{x}}- \\
k^{2} E_{y} \widehat{\mathbf{y}}+k^{2} \sin \theta\left(\cos \theta E_{x}-\sin \theta E_{z}\right) \widehat{\mathbf{z}}
\end{gathered}
$$

Using this result in (5.1), we find the following relations for the $x, y$, and $z$ components of this equation, respectively,

$$
\begin{gathered}
\left(1-\frac{k^{2} c^{2}}{\omega^{2}} \cos ^{2} \theta\right) E_{x}+\left(\frac{k^{2} c^{2}}{\omega^{2}} \sin \theta \cos \theta\right) E_{z}=\frac{i e n_{0}}{\epsilon_{0} \omega} u_{x} \\
\left(1-\frac{k^{2} c^{2}}{\omega^{2}}\right) E_{y}=\frac{i e n_{0}}{\epsilon_{0} \omega} u_{y} \\
\left(\frac{k^{2} c^{2}}{\omega^{2}} \sin \theta \cos \theta\right) E_{x}+\left(1-\frac{k^{2} c^{2}}{\omega^{2}} \sin ^{2} \theta\right) E_{z}=\frac{i e n_{0}}{\epsilon_{0} \omega} u_{z}
\end{gathered}
$$




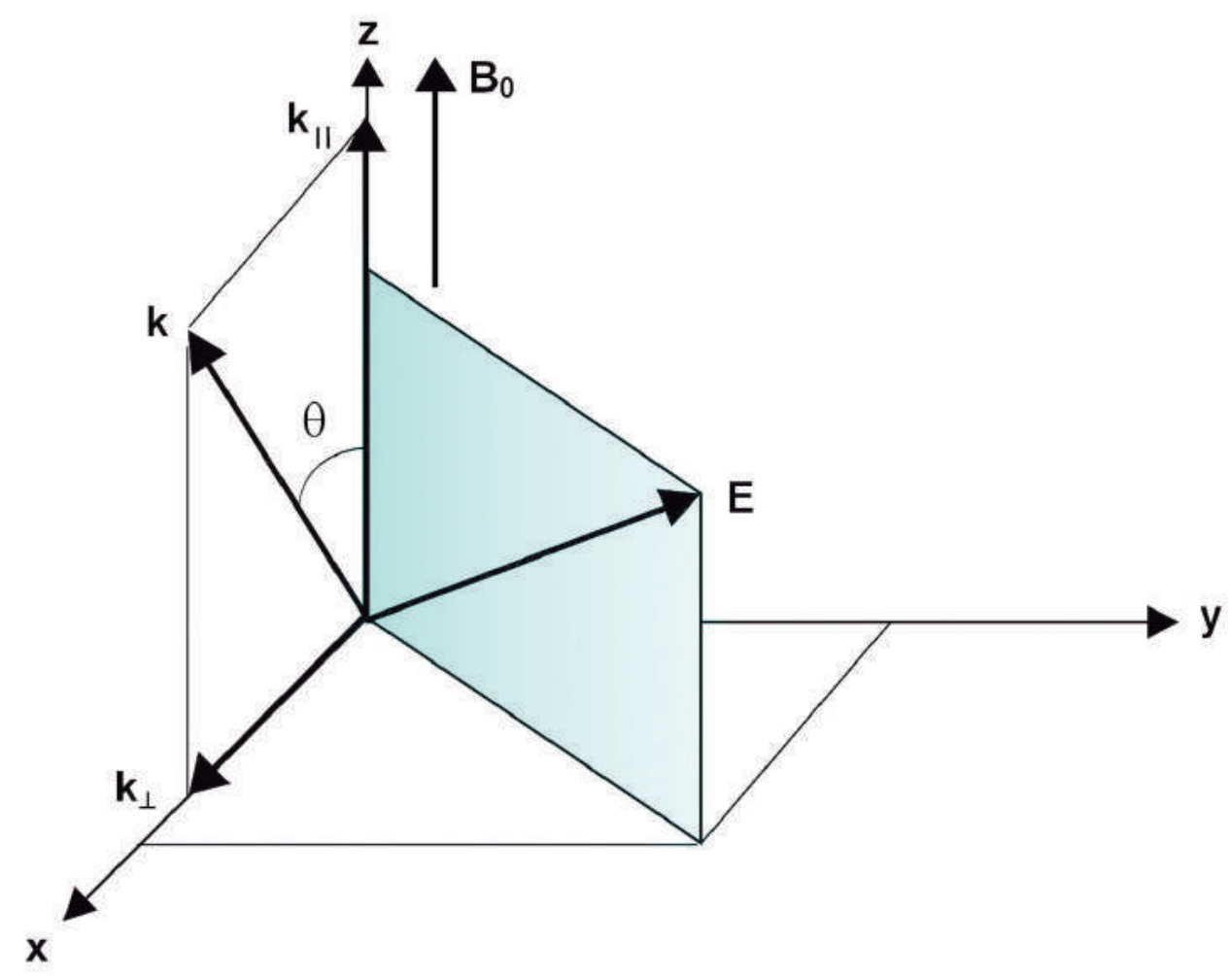

Fig. 7 Cartesian coordinate system $(x, y, z)$, chosen such that $\widehat{\mathbf{z}}$ is along $\mathbf{B}_{0}$ and $\widehat{\mathbf{y}}$ is perpendicular to the plane formed by $\mathbf{B}_{0}$ and $\mathbf{k}$.

which can be written, in matrix form, as

$$
\begin{gathered}
\left(\begin{array}{ccc}
\left(1-\eta^{2} \cos ^{2} \theta\right) & 0 & \eta^{2} \sin \theta \cos \theta \\
0 & \left(1-\eta^{2}\right) & 0 \\
\eta^{2} \sin \theta \cos \theta & 0 & \left(1-\eta^{2} \sin ^{2} \theta\right)
\end{array}\right)\left(\begin{array}{l}
E_{x} \\
E_{y} \\
E_{z}
\end{array}\right)= \\
=\frac{i e n_{0}}{\epsilon_{0} \omega}\left(\begin{array}{l}
u_{x} \\
u_{y} \\
u_{z}
\end{array}\right)
\end{gathered}
$$

The quantity $\eta=(k c / \omega)$ represents the index of refraction of the medium.

Next, in order to write (5.2) also in matrix form, we first note that (refer to Fig. 7)

$$
\mathbf{u} \times \mathbf{B}_{0}=B_{0}\left(u_{y} \widehat{\mathbf{x}}-u_{x} \widehat{\mathbf{y}}\right)
$$


Using this result in (5.2) and after some algebraic manipulations, we obtain for the $x, y$, and $z$ components of this equation, respectively,

$$
\begin{gathered}
\left(1+i \frac{\nu}{\omega}\right) u_{x}+i \frac{\Omega_{c e}}{\omega} u_{y}=-\frac{i e}{m \omega} E_{x} \\
-i \frac{\Omega_{c e}}{\omega} u_{x}+\left(1+i \frac{\nu}{\omega}\right) u_{y}=-\frac{i e}{m \omega} E_{y} \\
\left(1+i \frac{\nu}{\omega}\right) u_{z}=-\frac{i e}{m \omega} E_{z}
\end{gathered}
$$

where $\Omega_{c e}$ denotes the electron cyclotron frequency. Introducing now the notation

$$
\begin{gathered}
U=1+i \frac{\nu}{\omega} \\
Y=\frac{\Omega_{c e}}{\omega} \\
X=\frac{\omega_{p e}^{2}}{\omega^{2}}
\end{gathered}
$$

we can write $(5.11)$ to $(5.13)$ in matrix form as

$$
\left(\begin{array}{ccc}
U & i Y & 0 \\
-i Y & U & 0 \\
0 & 0 & U
\end{array}\right)\left(\begin{array}{l}
u_{x} \\
u_{y} \\
u_{z}
\end{array}\right)=-\frac{i e}{m \omega}\left(\begin{array}{l}
E_{x} \\
E_{y} \\
E_{z}
\end{array}\right)
$$

Inverting the $3 \times 3$ matrix of (5.17), and multiplying this equation by the inverted matrix, we find

$$
\begin{gathered}
\left(\begin{array}{l}
u_{x} \\
u_{y} \\
u_{z}
\end{array}\right)= \\
=-\frac{i e}{m \omega U\left(U^{2}-Y^{2}\right)}\left(\begin{array}{ccc}
U^{2} & -i U Y & 0 \\
i U Y & U^{2} & 0 \\
0 & 0 & \left(U^{2}-Y^{2}\right)
\end{array}\right)\left(\begin{array}{l}
E_{x} \\
E_{y} \\
E_{z}
\end{array}\right)
\end{gathered}
$$

Equations (5.9) and (5.18) can now be combined to eliminate the velocity components $u_{x}, u_{y}$, and $u_{z}$, yielding the following component equations involving only the electric field:

$$
\left(1-\frac{X U}{U^{2}-Y^{2}}-\eta^{2} \cos ^{2} \theta\right) E_{x}+\frac{i X Y}{U^{2}-Y^{2}} E_{y}+
$$




$$
\begin{gathered}
\left(\eta^{2} \sin \theta \cos \theta\right) E_{z}=0 \\
-\frac{i X Y}{U^{2}-Y^{2}} E_{x}+\left(1-\frac{X U}{U^{2}-Y^{2}}-\eta^{2}\right) E_{y}=0 \\
\left(\eta^{2} \sin \theta \cos \theta\right) E_{x}+\left(1-\frac{X}{U}-\eta^{2} \sin ^{2} \theta\right) E_{z}=0
\end{gathered}
$$

For reasons to become apparent later, it is appropriate to define the following quantities:

$$
\begin{gathered}
S=1-\frac{X U}{U^{2}-Y^{2}} \\
D=-\frac{X Y}{U^{2}-Y^{2}} \\
P=1-\frac{X}{U}
\end{gathered}
$$

With this notation, (5.19) to (5.21) can be written in matrix form as

$$
\left(\begin{array}{ccc}
\left(S-\eta^{2} \cos ^{2} \theta\right) & -i D & \eta^{2} \sin \theta \cos \theta \\
i D & \left(S-\eta^{2}\right) & 0 \\
\eta^{2} \sin \theta \cos \theta & 0 & \left(P-\eta^{2} \sin ^{2} \theta\right)
\end{array}\right)\left(\begin{array}{l}
E_{x} \\
E_{y} \\
E_{z}
\end{array}\right)=0
$$

In order to have a nontrivial solution $(\mathbf{E} \neq 0)$, the determinant of the $3 \times 3$ matrix in (5.25) must vanish. This condition gives the following dispersion relation, by direct calculation of the determinant,

$$
\begin{gathered}
\left(S \sin ^{2} \theta+P \cos ^{2} \theta\right) \eta^{4}-\left[R L \sin ^{2} \theta+S P\left(1+\cos ^{2} \theta\right)\right] \eta^{2}+ \\
+P R L=0
\end{gathered}
$$

where we have introduced the notation

$$
R=S+D \quad ; \quad L=S-D
$$

or

$$
S=\frac{1}{2}(R+L) \quad ; \quad D=\frac{1}{2}(R-L)
$$

As will become clear later, in this notation the symbols $R$ and $L$ stand for right and left, whereas $S$ and $D$ stand for sum and difference, respectively. Since (5.26) is a quadratic equation in $\eta^{2}$, there will be in general two solutions. Thus, at each frequency there can be two types of waves that propagate or two modes of propagation. Note, however, that if we take the square root of $\eta^{2}$ we have two values for $\eta$, which correspond to opposite directions of propagation and not to different wave modes. 


\subsection{The Appleton-Hartree Equation}

This well-known equation has been used with considerable success in the analysis of radio wave propagation in the ionospheric plasma, taking account of the Earth's magnetic field. It is just the dispersion relation given in (5.26), but written in a different form. In order to obtain the Appleton-Hartree equation, we first write (5.26) as

$$
A \eta^{4}-B \eta^{2}+C=0
$$

where

$$
\begin{gathered}
A=S \sin ^{2} \theta+P \cos ^{2} \theta \\
B=R L \sin ^{2} \theta+S P\left(1+\cos ^{2} \theta\right) \\
C=P R L
\end{gathered}
$$

Solving (5.29) for $\eta^{2}$, we find

$$
\eta^{2}=\frac{1}{2 A}\left(B \pm \sqrt{B^{2}-4 A C}\right)
$$

Now we add the quantity $A \eta^{2}$ to both sides of (5.29) and rearrange, to obtain

$$
\eta^{2}=\frac{A \eta^{2}-C}{A \eta^{2}+A-B}
$$

Next, we substitute $\eta^{2}$, from (5.33), into the right-hand side of (5.34) and manipulate, obtaining

$$
\eta^{2}=1-\frac{2(A-B+C)}{2 A-B \pm \sqrt{B^{2}-4 A C}}
$$

Finally, we substitute the appropriate expressions that define the quantities $A, B, C$ and $S, D, P, R, L$, to obtain

$$
\eta^{2}=1-\frac{X}{Q}
$$

where

$$
Q=U-\frac{Y^{2} \sin ^{2} \theta}{2(U-X)} \pm\left[\frac{Y^{4} \sin ^{4} \theta}{4(U-X)^{2}}+Y^{2} \cos ^{2} \theta\right]^{1 / 2}
$$


This expression is the well-known Appleton-Hartree equation. It is valid for high wave frequencies as compared to the ion cyclotron frequency, since ion motion was neglected in its derivation.

Because of the complexity of either (5.36) or (5.26), in order to simplify matters we shall first analyze the cold electron gas dispersion relation when $\mathbf{k}$ is either parallel or perpendicular to $\mathbf{B}_{0}$. Afterwards, we shall investigate some important aspects of wave propagation at an arbitrary angle $\theta$ with respect to $\mathbf{B}_{0}$ using the dispersion relation (5.36) or (5.26).

\section{PROPAGATION PARALLEL TO $B_{0}$}

For wave propagation along the magnetic field we have $\theta=0$ and (5.25) simplifies to

$$
\left(\begin{array}{ccc}
\left(S-\eta^{2}\right) & -i D & 0 \\
i D & \left(S-\eta^{2}\right) & 0 \\
0 & 0 & P
\end{array}\right)\left(\begin{array}{l}
E_{x} \\
E_{y} \\
E_{z}
\end{array}\right)=0
$$

In order to have a nontrivial solution $(\mathbf{E} \neq 0)$, the determinant of the $3 \times 3$ matrix in (6.1) must vanish. Thus, by direct calculation of this determinant we find three independent conditions:

$$
\begin{array}{lll}
P=0 \quad ; & & \left(\mathbf{E}_{\|} \equiv \mathbf{E}_{\ell} \neq 0\right) \\
\eta^{2}=S+D \equiv R & ; & \left(\mathbf{E}_{\perp} \equiv \mathbf{E}_{t} \neq 0\right) \\
\eta^{2}=S-D \equiv L & ; & \left(\mathbf{E}_{\perp} \equiv \mathbf{E}_{t} \neq 0\right)
\end{array}
$$

Using equations (5.22) to (5.24), and (5.14) to (5.16), which define $S$, $D, P$ and $U, Y, X$, respectively, we obtain from (6.2), neglecting collisions $(\nu=0)$,

$$
\omega^{2}=\omega_{p e}^{2}
$$

which corresponds to the longitudinal electron plasma oscillations discussed previously in section 4 . Thus, these oscillations along $\mathbf{B}_{0}$ are not affected by the presence of the magnetic field. Since there is no wave propagation in this case, these plasma oscillations do not constitute a propagation mode.

Equation (6.3) corresponds to transverse right-hand circularly polarized waves $(\mathrm{RCP})$, with the dispersion relation

$$
\eta_{R}^{2}=1-\frac{X}{(U-Y)}=R
$$


or, neglecting collisions $(\nu=0)$,

$$
\eta_{R}^{2}=1-\frac{\omega_{p e}^{2}}{\omega\left(\omega-\Omega_{c e}\right)}
$$

Equation (6.4) corresponds to transverse left-hand circularly polarized waves $(\mathrm{LCP})$, with the dispersion relation

$$
\eta_{L}^{2}=1-\frac{X}{(U+Y)}=L
$$

or, neglecting collisions $(\nu=0)$,

$$
\eta_{L}^{2}=1-\frac{\omega_{p e}^{2}}{\omega\left(\omega+\Omega_{c e}\right)}
$$

The polarization of these two transverse modes of propagation can be obtained from the $x$ component of (6.1), which gives

$$
\frac{E_{x}}{E_{y}}=\frac{i D}{S-\eta^{2}}
$$

Thus, for the $R C P$ wave mode, substituting $\eta^{2}=R$,

$$
\frac{E_{x}}{E_{y}}=-i
$$

whereas for the $L C P$ wave mode, substituting $\eta^{2}=L$,

$$
\frac{E_{x}}{E_{y}}=i
$$

Since the time dependence of $\mathbf{E}$ is of the form $\exp (-i \omega t)$, if we take $E_{x} \propto \cos (\omega t)$, then, for the RCP wave, we have $E_{y} \propto \sin (\omega t)$, whereas for the LCP wave, we have $E_{y} \propto-\sin (\omega t)$. Therefore, for an observer looking at the outgoing wave (along the positive $z$ direction), as time passes, the transverse electric field vector $\mathbf{E}_{t}$ rotates in the clockwise direction, for the RCP wave, and in the counterclockwise direction, for the LCP wave, as illustrated in Fig. 8. Note that the RCP wave rotates in the same direction as the electrons about the magnetic field. This means that, when $\omega=\Omega_{c e}$, the RCP wave is in resonance with the electron cyclotron motion 

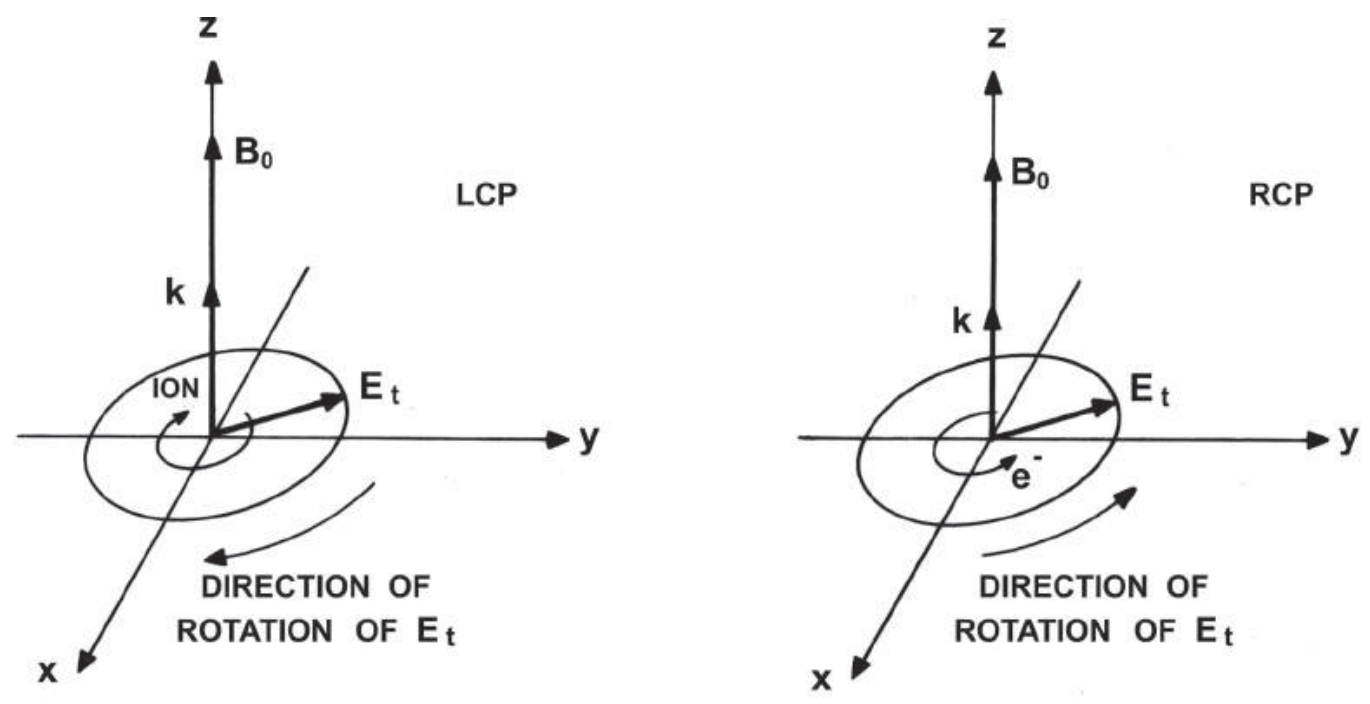

Fig. 8 For propagation along the magnetostatic field $(\theta=0)$ the $L C P$ wave rotates in the counterclockwise direction, whereas the $R C P$ wave rotates in the clockwise direction, for an observer looking at the outgoing wave.

and, therefore, energy is transferred from the wave field to the electrons. This absorption of energy by the electrons, from the RCP electromagnetic wave at the electron cyclotron frequency, is used as a means of heating the plasma electrons. When the motion of the ions is taken into account, these dispersion equations are slightly modified so that a resonance exists between the ion cyclotron motion and the LCP wave when $\omega=\Omega_{c i}$, since the ions gyrate in the same direction as the $\mathbf{E}_{t}$ vector of the LCP wave.

The resonance phenomenon occurs when the wave phase velocity goes to zero, $v_{p h}=0$ (or $\eta \rightarrow \infty$ ), whereas the phenomenon of wave reflection occurs when $v_{p h} \rightarrow \infty$ (or $\eta=0$ ). Thus, it is clear from (6.7) and from the physical argument just given that the RCP wave has a resonance when $\omega=\Omega_{c e}$, whereas (6.9) indicates no resonance for the LCP wave (when ion motion is included, the LCP wave shows a resonance when $\omega=\Omega_{c i}$ ). Also, from (6.9) it is easily verified that the LCP wave has a reflection point $(\mathrm{L}=0)$ at the frequency

$$
\omega_{01}=\frac{1}{2}\left[-\Omega_{c e}+\left(\Omega_{c e}^{2}+4 \omega_{p e}^{2}\right)^{1 / 2}\right]
$$


and, from (6.7), the $\mathrm{RCP}$ wave has a reflection point $(\mathrm{R}=0)$ at the frequency

$$
\omega_{02}=\frac{1}{2}\left[\Omega_{c e}+\left(\Omega_{c e}^{2}+4 \omega_{p e}^{2}\right)^{1 / 2}\right]=\omega_{01}+\Omega_{c e}
$$

The phase velocity of the LCP wave is obtained, from (6.9), as

$$
\left(v_{p h}\right)_{L}=\frac{c\left(1+\Omega_{c e} / \omega\right)^{1 / 2}}{\left(1+\Omega_{c e} / \omega-\omega_{p e}^{2} / \omega^{2}\right)^{1 / 2}}
$$

which holds for $\omega>\omega_{01}$. For $\omega<\omega_{01}$ the wave number $k$ is imaginary and the LCP wave is evanescent. Thus, the LCP wave propagates only for $\omega>\omega_{01}$.

Similarly, the phase velocity of the RCP wave is obtained, from (6.7), as

$$
\left(v_{p h}\right)_{R}=\frac{c\left(1-\Omega_{c e} / \omega\right)^{1 / 2}}{\left(1-\Omega_{c e} / \omega-\omega_{p e}^{2} / \omega^{2}\right)^{1 / 2}}
$$

which holds for $\omega<\Omega_{c e}$ and for $\omega>\omega_{02}$. Thus, the RCP wave propagates only in these two frequency ranges and is evanescent for $\Omega_{c e}<\omega<\omega_{02}$.

The group velocity for the LCP and RCP waves in their propagation bands are given, respectively, by

$$
\begin{aligned}
& \left(v_{g}\right)_{L}=\left(\frac{\partial \omega}{\partial k}\right)_{L}=\frac{2 c\left(\omega+\Omega_{c e}\right)^{3 / 2}\left[\omega\left(\omega^{2}+\omega \Omega_{c e}-\omega_{p e}^{2}\right)\right]^{1 / 2}}{2 \omega\left(\omega+\Omega_{c e}\right)^{2}-\Omega_{c e} \omega_{p e}^{2}} \\
& \left(v_{g}\right)_{R}=\left(\frac{\partial \omega}{\partial k}\right)_{R}=\frac{2 c\left(\omega-\Omega_{c e}\right)^{3 / 2}\left[\omega\left(\omega^{2}-\omega \Omega_{c e}-\omega_{p e}^{2}\right)\right]^{1 / 2}}{2 \omega\left(\omega-\Omega_{c e}\right)^{2}+\Omega_{c e} \omega_{p e}^{2}}
\end{aligned}
$$

A plot of phase velocity and group velocity as a function of frequency for these two transverse modes is shown in Fig. 9. The same dispersion relations (6.7) and (6.9) are plotted, in a different form, in Figs. 10 and 11 , respectively, where the frequency $\omega$ as a function of the real part of the wave number $k$ is shown. The frequency bands for which there is no wave propagation are indicated in the plots.

The RCP wave mode, in the lower branch that has $\omega<\Omega_{c e}$, is also known as the electron cyclotron wave. Similarly, when ion motion is taken into account, the LCP mode has also a lower branch of propagation for $\omega<\Omega_{c i}$, with a resonance at $\Omega_{c i}$. The LCP waves having $\omega<\Omega_{c i}$ are also known as ion cyclotron waves. 


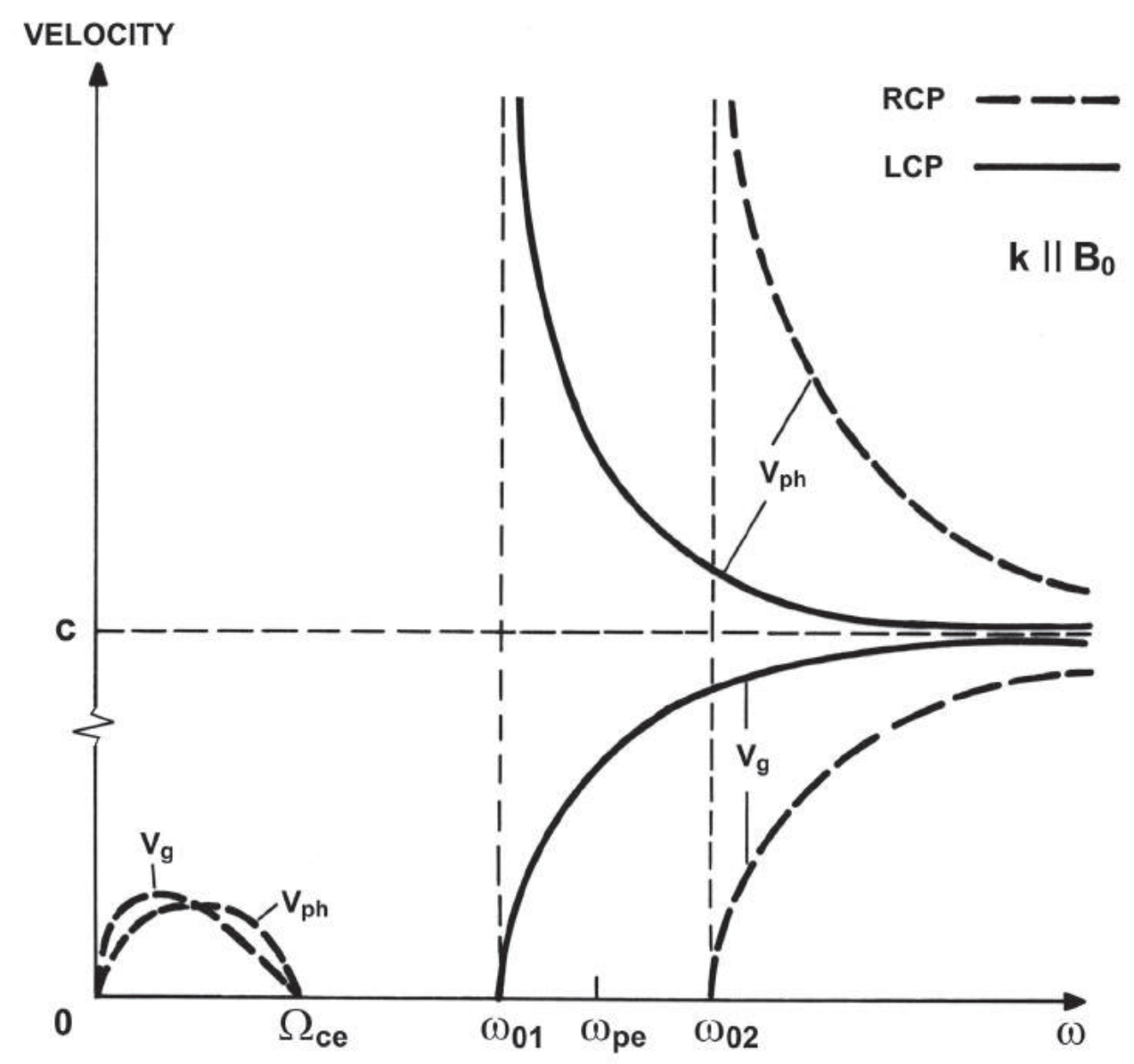

Fig. 9 Phase velocity and group velocity as a function of frequency for the transverse $R C P$ and $L C P$ waves propagating along the magnetostatic field $\left(\mathbf{k} \| \mathbf{B}_{0}\right)$.

\section{PROPAGATION PERPENDICULAR TO $\mathrm{B}_{0}$}

We consider now wave propagation in the direction perpendicular to the magnetostatic field $\left(\mathbf{k} \perp \mathbf{B}_{0}\right)$. For $\theta=90^{\circ}$, (5.25) simplifies to

$$
\left(\begin{array}{ccc}
S & -i D & 0 \\
i D & \left(S-\eta^{2}\right) & 0 \\
0 & 0 & \left(P-\eta^{2}\right)
\end{array}\right)\left(\begin{array}{c}
E_{x} \\
E_{y} \\
E_{z}
\end{array}\right)=0
$$

Again, for a nontrivial solution $(\mathbf{E} \neq 0)$ the determinant of the $3 \times 3$ matrix in (7.1) must vanish. Direct calculation of this determinant yields the following two independent modes of propagation: 


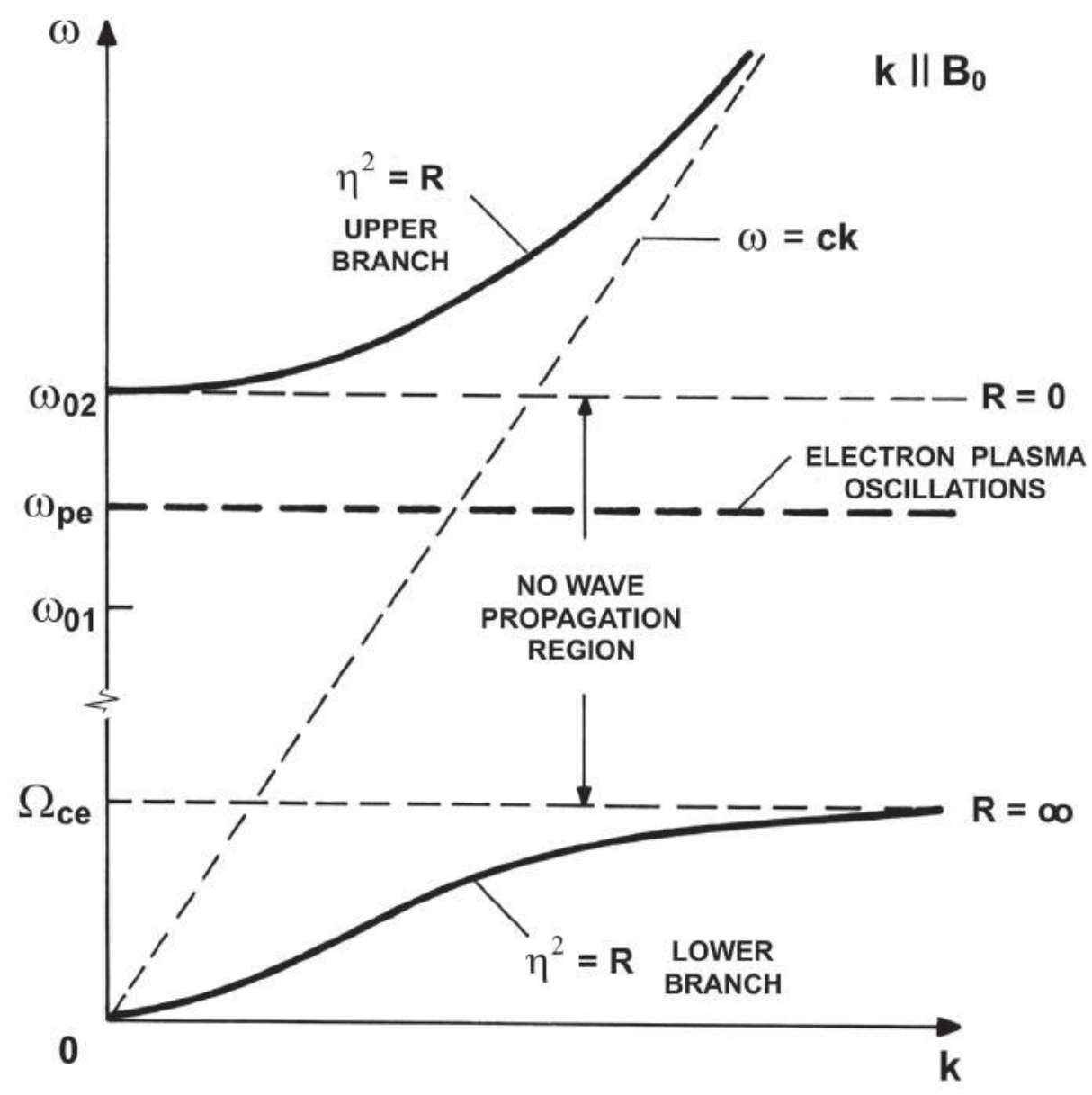

Fig. 10 Dispersion plot for the $R C P$ wave mode propagating along the magnetostatic field $\left(\mathbf{k} \| \mathbf{B}_{0}\right)$.

$$
\begin{array}{ccc}
\eta_{O}^{2}=P & ; \quad & \left(\mathbf{E}_{\|} \neq 0\right) \\
\eta_{X}^{2}=\frac{R L}{S} & ; \quad & \left(\mathbf{E}_{\perp} \neq 0\right)
\end{array}
$$

The indices $O$ and $X$ refer to ordinary and extraordinary modes, respectively, as will be explained shortly.

From (7.2) and using (5.24), we obtain the dispersion relation

$$
\eta_{O}^{2}=1-\frac{X}{U}
$$

or, using (5.14) and (5.16), neglecting collisions $(\nu=0)$,

$$
\eta_{O}^{2}=1-\frac{\omega_{p e}^{2}}{\omega^{2}}
$$




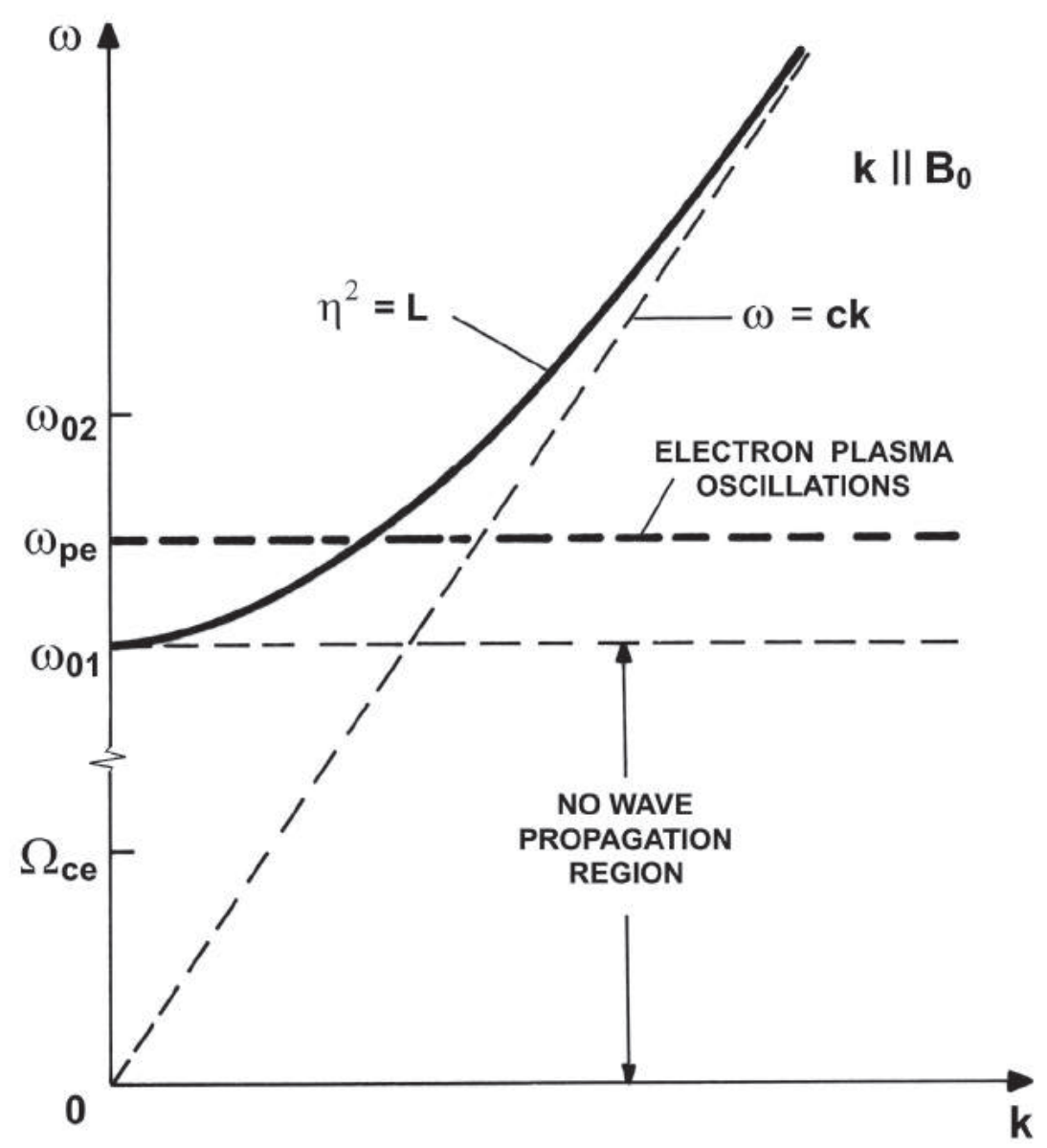

Fig. 11 Dispersion plot for the $L C P$ wave mode propagating along the magnetostatic field $\left(\mathbf{k} \| \mathbf{B}_{0}\right)$.

This relation is identical to (4.12) for transverse waves in an isotropic cold plasma. Hence, this mode of propagation is not affected by the presence of the magnetic field $\mathbf{B}_{0}$ and, for this reason, it is called an ordinary wave mode. For this mode propagating perpendicular to $\mathbf{B}_{0}$ the wave electric field $\left(\mathbf{E}_{\|} \neq 0\right)$ is parallel to $\mathbf{B}_{0}$, so that it involves electron velocities solely in the direction of $\mathbf{B}_{0}$. Consequently, there is no magnetic force acting on the electron motion $\left(\mathbf{u} \times \mathbf{B}_{0}=0\right)$ and the wave propagates as if $\mathbf{B}_{0}$ were zero. The ordinary mode is also commonly called a TEM (transverse electric magnetic) mode, since both the electric and magnetic fields are transverse to the direction of propagation $\left(\mathbf{E}_{\|} \perp \mathbf{k} ; \mathbf{B} \perp \mathbf{k}\right)$. The wave electric field is linearly polarized along $\mathbf{B}_{0}$. Fig. 12 illustrates the relative orientation of these vector fields. 


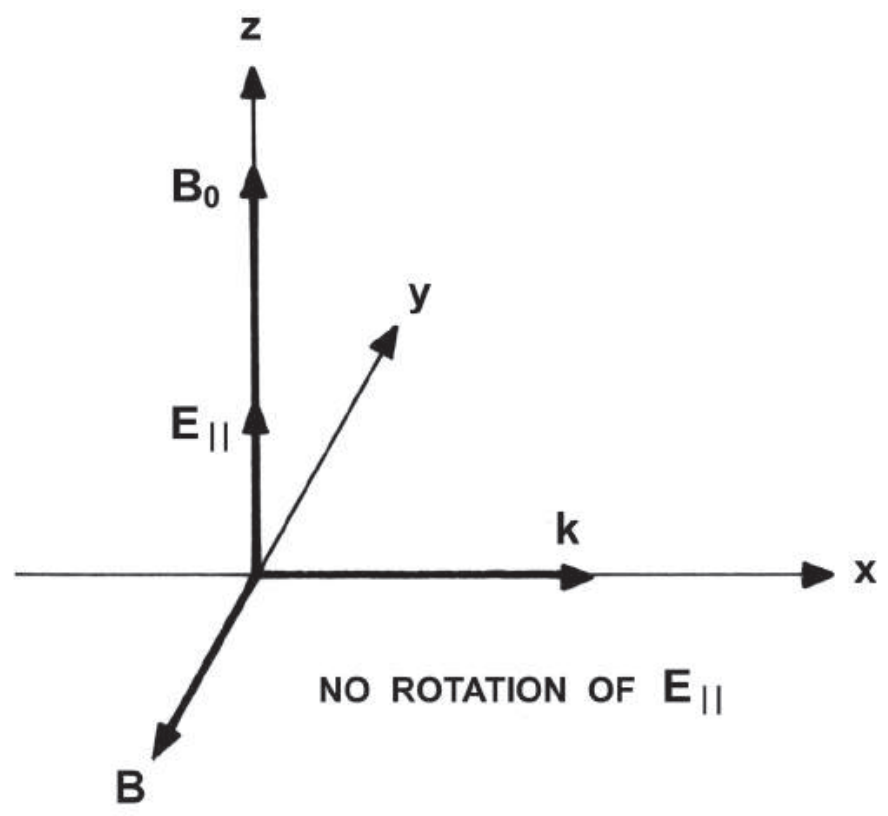

Fig. 12 Vector diagram for the ordinary wave propagating perpendicular to the magnetic field $(\theta=\pi / 2)$.

The other mode of propagation $\left(\mathbf{E}_{\perp} \neq 0\right)$ is called the extraordinary mode, since it depends on the $\mathbf{B}_{0}$ field, with the dispersion relation given by (7.3),

$$
\eta_{X}^{2}=\frac{R L}{S}=\left(1-\frac{X U}{U^{2}-Y^{2}}\right)^{-1}\left[\left(1-\frac{X U}{U^{2}-Y^{2}}\right)^{2}-\left(\frac{X Y}{U^{2}-Y^{2}}\right)^{2}\right]
$$

or, using (5.14), (5.15), and (5.16), after neglecting collisions $(\nu=0)$,

$$
\begin{gathered}
\eta_{X}^{2}=\frac{\left(\omega^{2}+\omega \Omega_{c e}-\omega_{p e}^{2}\right)\left(\omega^{2}-\omega \Omega_{c e}-\omega_{p e}^{2}\right)}{\omega^{2}\left(\omega^{2}-\Omega_{c e}^{2}-\omega_{p e}^{2}\right)}= \\
\frac{\left(\omega^{2}-\omega_{01}^{2}\right)\left(\omega^{2}-\omega_{02}^{2}\right)}{\omega^{2}\left(\omega^{2}-\omega_{u h}^{2}\right)}
\end{gathered}
$$

where $\omega_{01}$ and $\omega_{02}$ are given by (6.13) and (6.14), respectively, and where $\omega_{u h}$ denotes the upper hybrid frequency, defined by

$$
\omega_{u h}=\left(\omega_{p e}^{2}+\Omega_{c e}^{2}\right)^{1 / 2}
$$




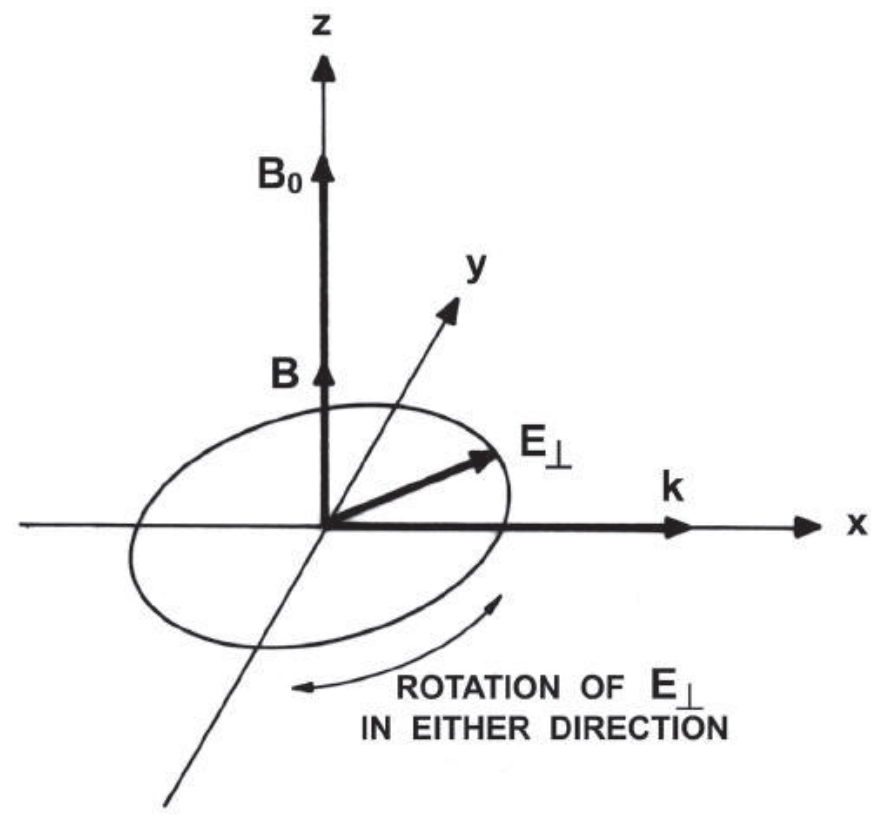

Fig. 13 Vector diagram for the extraordinary wave mode propagating perpendicular to the magnetic field $(\theta=\pi / 2)$.

For the extraordinary mode, the wave electric field $\mathbf{E}_{t}$ has in general a longitudinal component (along $\mathbf{k}$ ) and a transverse component (normal to $\mathbf{k}$ ), as shown in Fig. 13. Hence, these waves are partially longitudinal and partially transverse. From (7.1), the polarization of the extraordinary mode is determined by

$$
\frac{E_{x}}{E_{y}}=\frac{i D}{S}
$$

so that this mode is in general elliptically polarized. The extraordinary mode is also commonly called a TM (transverse magnetic) mode, since the wave magnetic field is transverse to the direction of propagation.

From (7.5) it is clear that the ordinary wave has a reflection point $\left(v_{p h} \rightarrow \infty\right.$ or $\left.\eta=0\right)$ at $\omega=\omega_{p e}$ and no resonances $\left(v_{p h}=0\right.$ or $\left.\eta \rightarrow \infty\right)$. For the extraordinary wave, (7.7) indicates a resonance at the upper hybrid frequency $\omega_{u h}$ and reflection points at $\omega_{01}$ and $\omega_{02}$ (when ion motion is included it turns out that the extraordinary wave has also a resonance approximately at the lower hybrid frequency, given by $\omega_{\ell h}^{2}=\Omega_{c e} \Omega_{c i}$ ).

The dispersion plot for the ordinary wave is the same as that presented in Fig. 3 for the transverse wave mode in an isotropic plasma. This mode propagates only for $\omega>\omega_{p e}$. For the extraordinary wave mode, the 


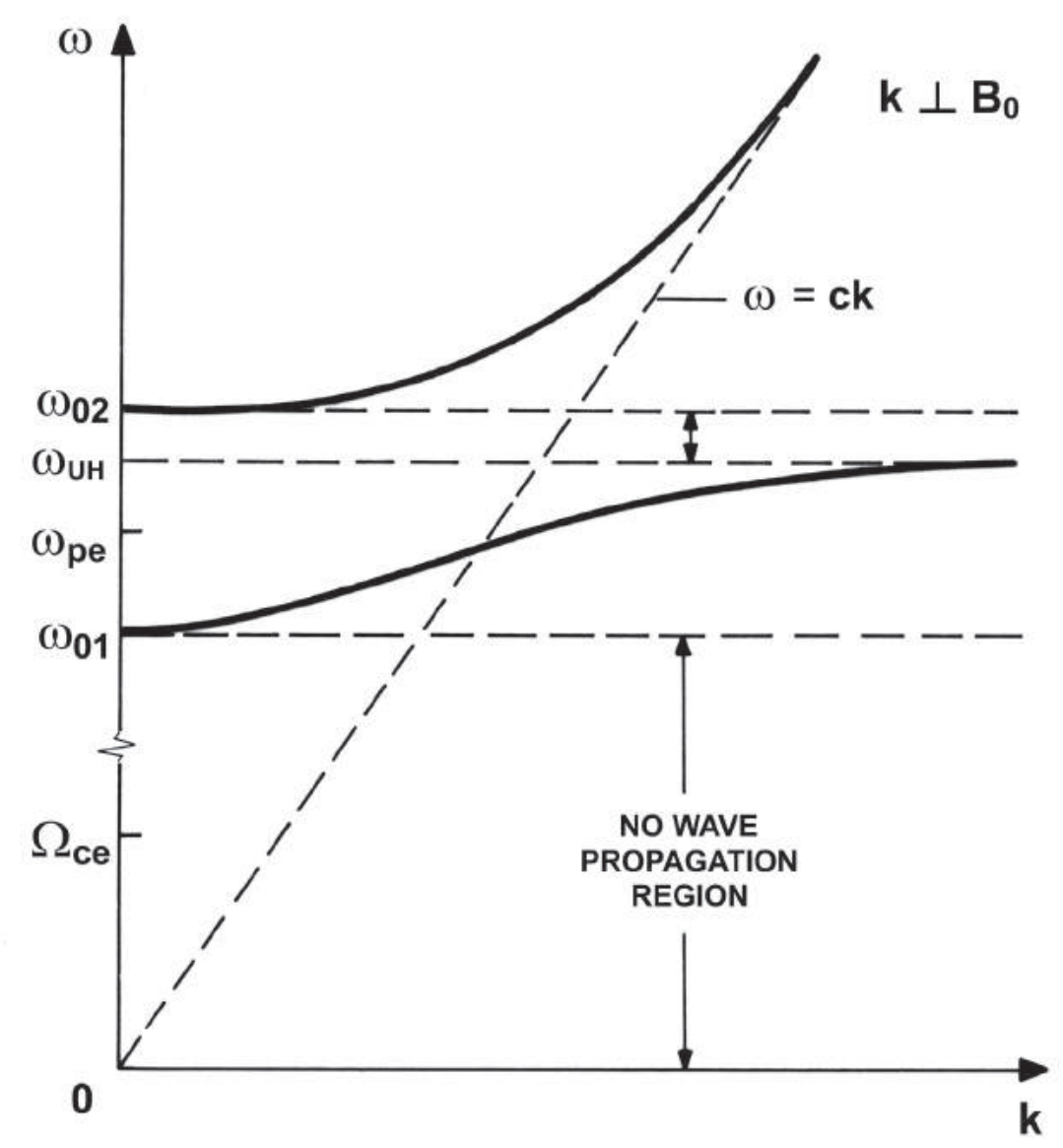

Fig. 14 Dispersion relation for the extraordinary wave mode $\left(\eta^{2}=R L / S\right)$ propagating perpendicular to the magnetic field $(\theta=\pi / 2)$.

dispersion plot shown in Fig. 14 indicates that this mode propagates only for $\omega>\omega_{02}$ and for $\omega$ in a band of frequencies between $\omega_{01}$ and $\omega_{u h}$. For other frequencies $k$ is imaginary and the phase velocity is infinite.

The phase velocities of the ordinary and extraordinary waves in their respective propagation bands are obtained from (7.5) and (7.7), respectively, as

$$
\begin{gathered}
\left(v_{p h}\right)_{O}=\frac{c \omega}{\left(\omega^{2}-\omega_{p e}^{2}\right)^{1 / 2}} \\
\left(v_{p h}\right)_{X}=\frac{c \omega\left(\omega^{2}-\omega_{u h}^{2}\right)^{1 / 2}}{\left(\omega^{2}-\omega_{01}^{2}\right)^{1 / 2}\left(\omega^{2}-\omega_{02}^{2}\right)^{1 / 2}}
\end{gathered}
$$

Expressions for the group velocities of these two modes can be derived 


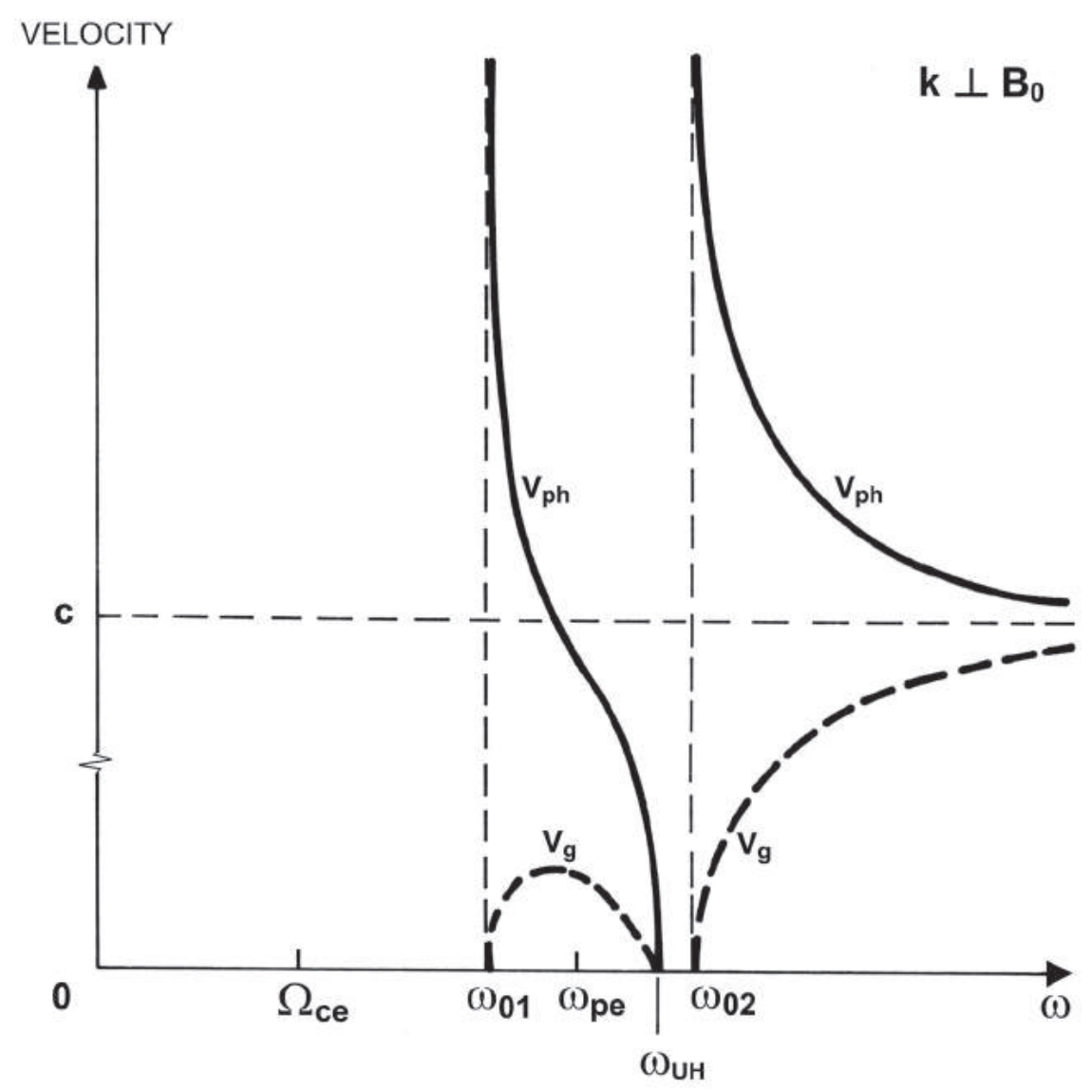

Fig. 15 Phase velocity and group velocity as a function of frequency for the extraordinary wave mode propagating perpendicular to the magnetic field $(\theta=\pi / 2)$.

with the help of (7.5) and (7.7), valid in their respective propagation bands,

$$
\begin{gathered}
\left(v_{g}\right)_{O}=c\left(1-\frac{\omega_{p e}^{2}}{\omega^{2}}\right)^{1 / 2} \\
\left(v_{g}\right)_{X}=\frac{c\left(\omega^{2}-\omega_{u h}^{2}\right)^{3 / 2}\left(\omega^{2}-\omega_{01}^{2}\right)^{1 / 2}\left(\omega^{2}-\omega_{02}^{2}\right)^{1 / 2}}{\omega\left[\omega^{4}-2 \omega^{2}\left(\Omega_{c e}^{2}+\omega_{p e}^{2}\right)+\Omega_{c e}^{4}+3 \Omega_{c e}^{2} \omega_{p e}^{2}+\omega_{p e}^{4}\right]}
\end{gathered}
$$

The dependence of the phase velocity and group velocity on the wave frequency, for the extraordinary (TM) mode, is shown in Fig. 15. A similar plot for the ordinary (TEM) mode has already been presented in Fig. 2 (the same one for the transverse wave in an isotropic plasma). 


\section{PROPAGATION AT ARBITRARY DIRECTIONS}

\subsection{Resonances and Reflection Points}

Going back now to (5.26), we shall first determine the resonances and the reflection points for arbitrary angles of propagation with respect to $\mathbf{B}_{0}$. From (5.33) and (5.30) it is seen that resonance occurs when

$$
S \sin ^{2} \theta+P \cos ^{2} \theta=0
$$

or

$$
\tan ^{2} \theta=-\frac{P}{S}
$$

Using (5.22) and (5.24), and neglecting collisions $(\nu=0),(8.2)$ yields

$$
1-X=Y^{2}\left(1-X \cos ^{2} \theta\right)
$$

or, using (5.15) and (5.16),

$$
\omega^{4}-\omega^{2}\left(\omega_{p e}^{2}+\Omega_{c e}^{2}\right)+\omega_{p e}^{2} \Omega_{c e}^{2} \cos ^{2} \theta=0
$$

Thus, the resonance frequencies are given in terms of the angle $\theta$ by

$$
\omega_{0 \pm}^{2}=\frac{1}{2}\left(\omega_{p e}^{2}+\Omega_{c e}^{2}\right) \pm\left[\frac{1}{4}\left(\omega_{p e}^{2}+\Omega_{c e}^{2}\right)^{2}-\omega_{p e}^{2} \Omega_{c e}^{2} \cos ^{2} \theta\right]^{1 / 2}
$$

These two resonance frequencies are plotted against $\theta$ in Fig. 16. From (8.5) it is clear that the sum of the square of these two frequencies $\left(\omega_{0+}^{2}+\right.$ $\left.\omega_{0-}^{2}\right)$ is always equal to $\left(\omega_{p e}^{2}+\Omega_{c e}^{2}\right)$ for any angle $\theta$. From Fig. 16 we see that the high-frequency resonance increases with increasing $\theta$, from the larger of $\omega_{p e}$ and $\Omega_{c e}$, at $\theta=0^{\circ}$, to the upper hybrid resonance frequency, $\left(\omega_{p e}^{2}+\Omega_{c e}^{2}\right)^{1 / 2}$, at $\theta=90^{\circ}$. The low-frequency resonance decreases correspondingly from the frequency that is the smaller of $\omega_{p e}$ and $\Omega_{c e}$, at $\theta=0^{\circ}$, to zero, at $\theta=90^{\circ}$. The resonances for $\theta=0^{\circ}$ and for $\theta=90^{\circ}$ are called the principal resonances. At $\theta=0^{\circ}$ the principal resonances are given by $S \rightarrow \infty$ and $P=0$, whereas at $\theta=90^{\circ}$ there is a principal resonance given by $S=0$.

The reflection points are seen, from (5.26), to be given by

$$
P R L=0
$$

This equation is satisfied whenever $P=0$, or $R=0$, or $L=0$. However, for $\theta=0^{\circ},(5.26)$ simplifies to

$$
\eta^{2}-2 S \eta+R L=0
$$



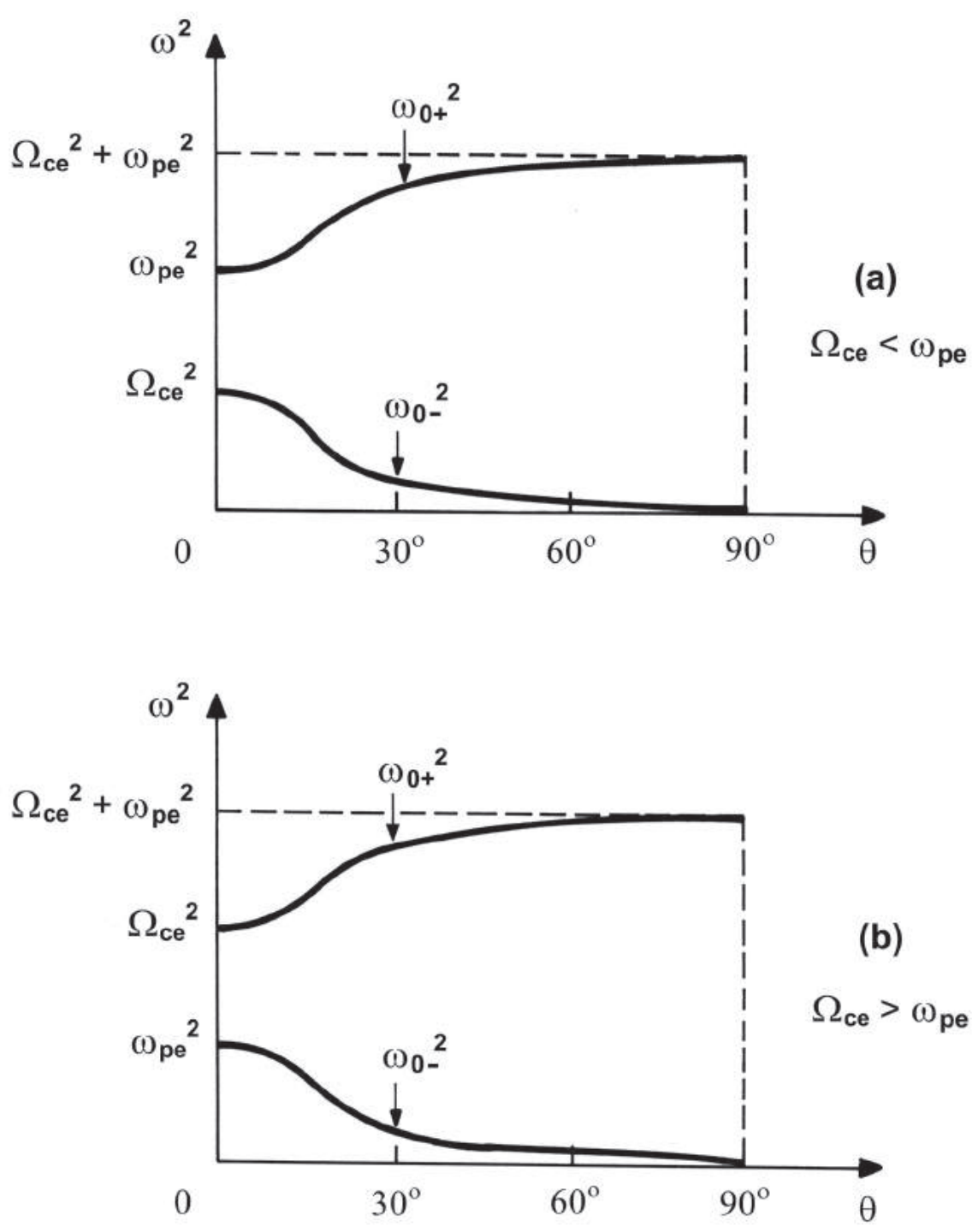

Fig. 16 Resonance frequencies as functions of the angle $\theta$ between $\mathbf{B}_{0}$ and the direction of wave propagation in a cold electron plasma, considering the cases (a) $\Omega_{c e}<\omega_{p e}$ and (b) $\Omega_{c e}>\omega_{p e}$.

so that $P=0$ is no longer a reflection point for $\theta=0^{\circ}$. Thus, for propagation exactly along the $\mathbf{B}_{0}$ field the reflection points are given by $R=0$ and $L=0$. But for $\theta \neq 0^{\circ}$, irrespective of how small $\theta$ is, $P=0$ also corresponds to a reflection point. Note that these cut-off frequencies are, otherwise, independent of $\theta$. The cut-off frequencies and the principal resonances are summarized in Table 1. 


\section{TABLE 1}

\section{CUT-OFFS AND PRINCIPAL RESONANCES FOR WAVES IN A COLD ELECTRON PLASMA}

\begin{tabular}{|c|cc|}
\hline Cut-offs & \multicolumn{2}{|c|}{ Principal Resonances } \\
& $\theta=0^{\circ}$ & $\theta=90^{\circ}$ \\
\hline $\mathrm{P}=0\left(\theta \neq 0^{\circ}\right)$ & $\mathrm{P}=0$ & $\mathrm{~S}=0$ \\
$\mathrm{R}=0$ & $R=\infty$ & \\
$\mathrm{L}=0$ & $L=\infty$ & \\
\hline
\end{tabular}

Expressions for the phase velocity and group velocity for arbitrary angles of propagation can be obtained from the dispersion relation (5.26) or (5.36). Since this involves considerable algebra, it will not be presented here. For this case the curves of $k, v_{p h}$, and $v_{g}$, as functions of $\omega$, must lie somewhere between the corresponding curves for $\theta=0^{\circ}$ (see Figs. 9, 10, and 11) and for $\theta=90^{\circ}$ (see Figs. 2, 3, 14, and 15). If the angle $\theta$ is continuously changed from $0^{\circ}$ to $90^{\circ}$, the corresponding curves for $0^{\circ}$ must change continuously into those for $90^{\circ}$.

Fig. 17 shows $\omega$ as a function of the real part of $k$, while Fig. 18 shows $v_{p h}$ and $v_{g}$ as functions of $\omega$ for the two modes of propagation at an angle $\theta=45^{\circ}$ with respect to $\mathbf{B}_{0}$. It is important to note that the branch of mode 2 (that propagates for $\omega_{01}<\omega<\omega_{0+}$ ) and the branch of mode 1 (that propagates for $\omega>\omega_{p e}$ ) are transformed, as $\theta$ goes to zero, into the LCP waves and the electron plasma oscillations at $\omega_{p e}$. This fact is indicated schematically in Fig. 19.

Fig. 20 is a plot showing the phase velocity versus frequency, illustrating how the two modes of wave propagation for $\theta=0^{\circ}$ (LCP and $\mathrm{RCP}$ waves) evolve into the two modes of wave propagation for $\theta=90^{\circ}$ (ordinary and extraordinary waves).

\subsection{Wave Normal Surfaces}

The wave normal surface, also known as the normalized phase velocity surface, is a polar plot of the normalized phase velocity $v_{p h} / c$ as a function of $\theta$. Because of the symmetry in the azimuthal angle $\theta$, it is a surface of revolution about $\mathbf{B}_{0}$. For any direction of propagation, the length (properly normalized) of the line drawn from the origin to intersect this 


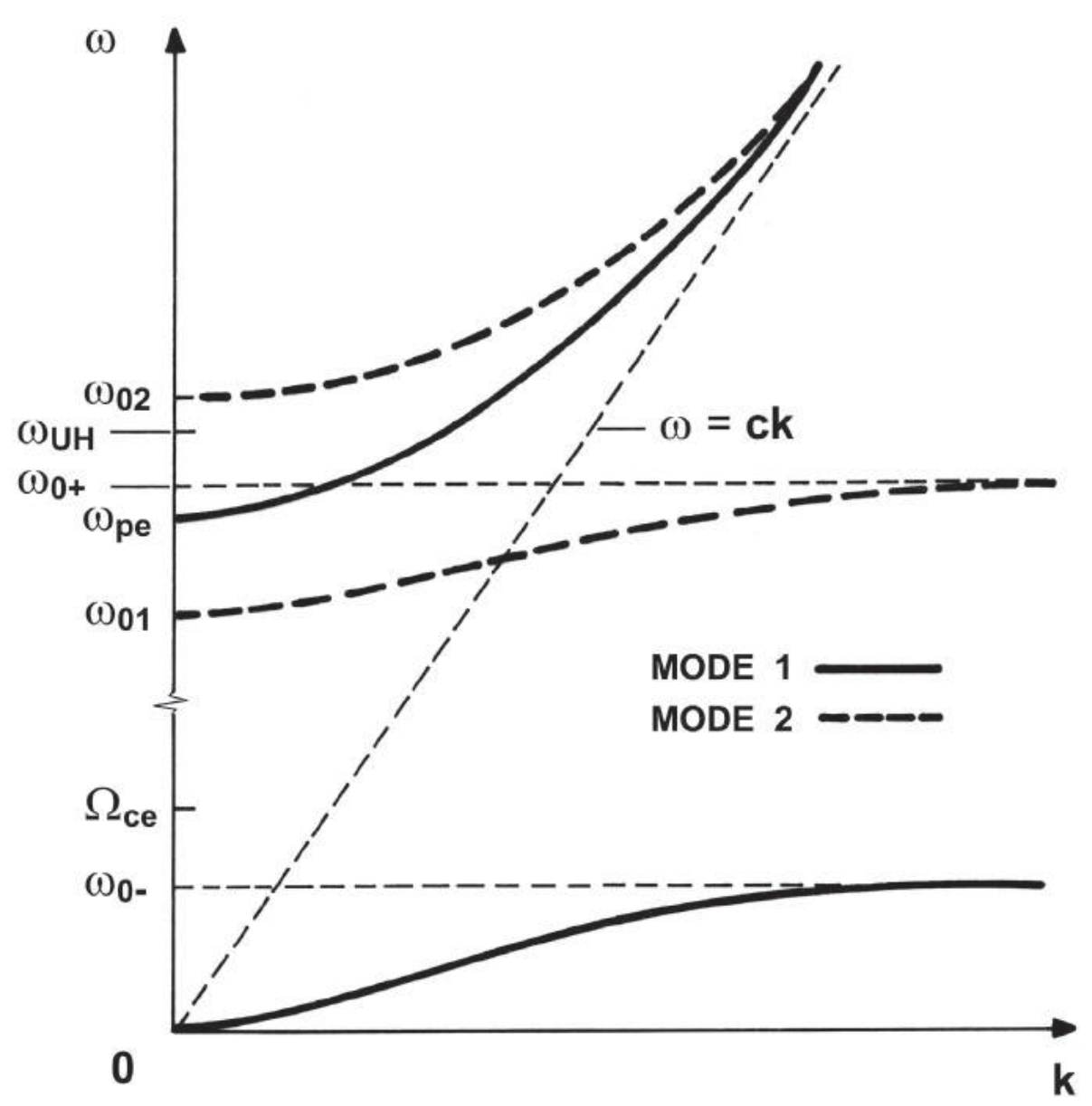

Fig. 17 Dispersion relation for the two modes of propagation at an angle of $45^{\circ}$ with respect to the magnetostatic field in a cold electron plasma.

surface corresponds to $v_{p h} / c$. The shape of the wave normal surface is generally not the same as the shape of a wave front. A typical wave normal surface is presented in Fig. 21, in which the velocity of light is shown as a dashed circle. The two solutions for $\eta^{2}$, from (5.26), are superimposed on the same coordinate axes as slow and fast waves. The denomination slow wave refers to the mode with the largest value of $\eta^{2}$, whereas the fast wave refers to the mode with the smallest value of $\eta^{2}$. With some exceptions, the fast wave has generally a phase velocity greater than $c$, while the phase velocity of the slow wave is generally less than $c$. 


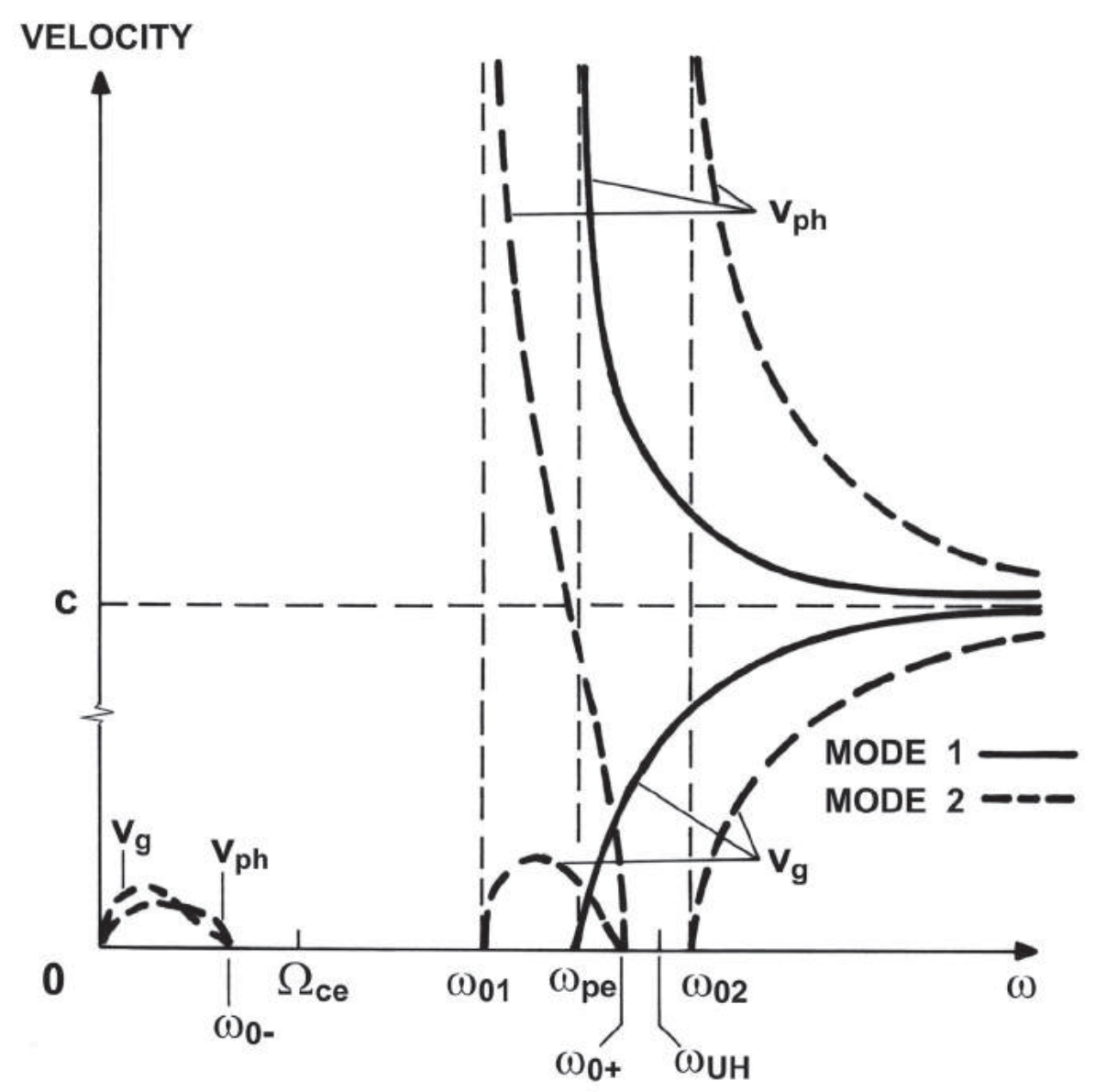

Fig. 18 Phase velocity and group velocity as a function of frequency for the two modes of propagation at an angle of $45^{\circ}$ with respect to the magnetostatic field in a cold electron plasma.

\subsection{The CMA Diagram}

The CMA (Clemmow-Mullaly-Allis) diagram is a very compact alternative way for presenting the solutions of the dispersion relation. This diagram is constructed in a two-dimensional parameter space having $X=$ $\omega_{p e}^{2} / \omega^{2}$ as the horizontal axis and $Y^{2}=\Omega_{c e}^{2} / \omega^{2}$ as the vertical axis, and displays all the resonances and reflection points as a function of both $X$ and $Y^{2}$. Thus, in this diagram, the magnetic field increases in the vertical direction, the plasma electron density increases in the horizontal direction, and the electromagnetic wave frequency decreases in the radial direction (in each case, considering all other parameters fixed). Furthermore, the 


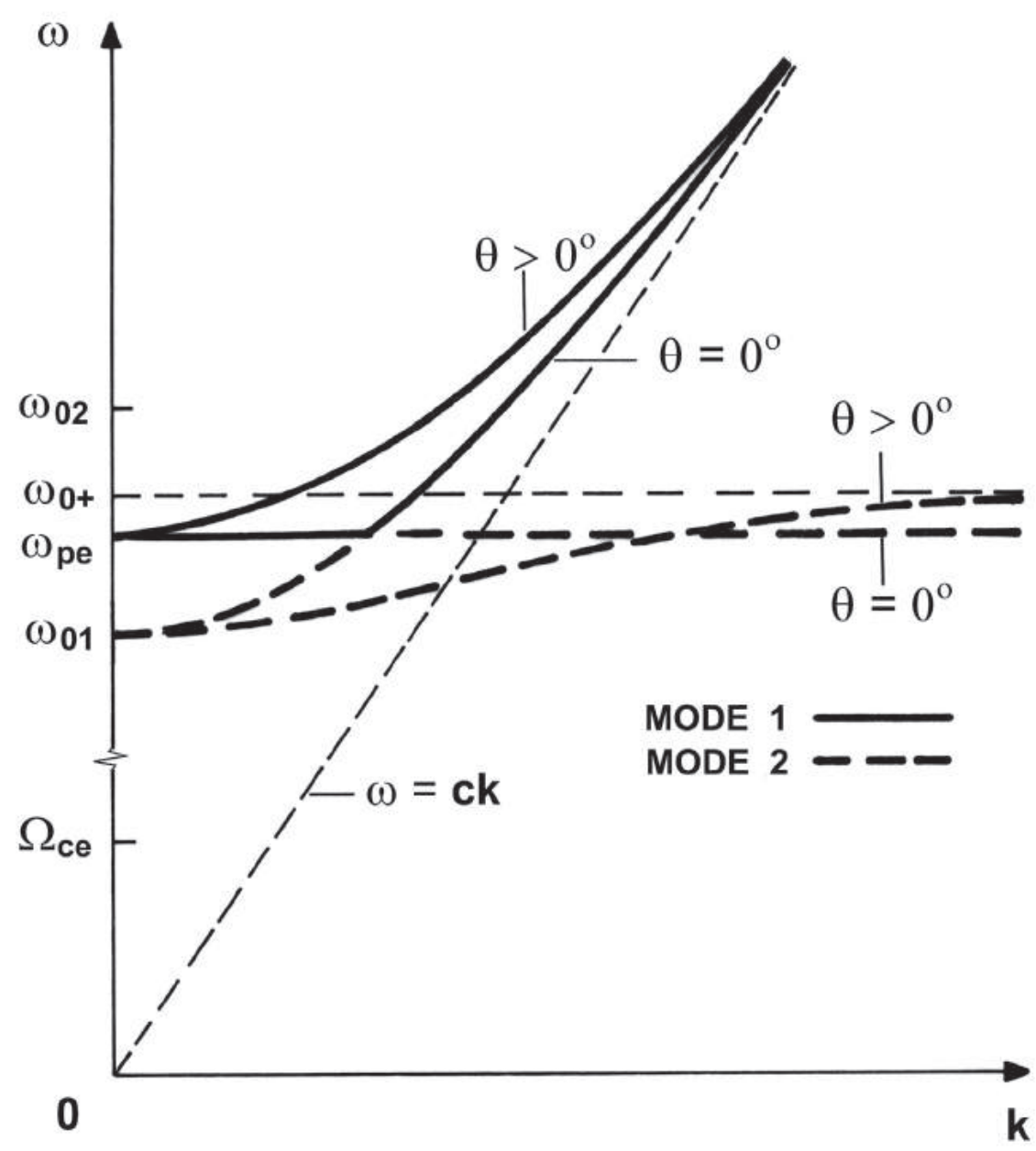

Fig. 19 Illustrating how the branch $\omega_{01}<\omega<\omega_{0+}$ of mode 2 and the branch $\omega>\omega_{p e}$ of mode 1 , for $\theta>0^{\circ}$, are related to the $L C P$ wave and the electron plasma oscillations when $\theta=0^{\circ}$.

CMA diagram divides the $\left(X, Y^{2}\right)$ plane into a number of regions such that, within each of these regions, the characteristic topological forms of the phase velocity surfaces remain unchanged.

From (8.3), which gives the resonance frequencies, we see that for $\theta=0^{\circ}$ the loci of the resonances are given in the CMA diagram by the straight line $Y^{2}=1$, and for $\theta=90^{\circ}$ by the straight line $Y^{2}=1-X$. The loci of the reflection points, as determined from (8.6), can be shown to be the curves $Y^{2}=(1-X)^{2}$ for any angle $\theta$, and $X=1$ for any angle except $\theta=0^{\circ}$. The two reflection point curves and the two principal resonance curves divide the $\left(X, Y^{2}\right)$ plane into eight regions. In each of these regions, a polar plot of the normalized phase velocity $\left(v_{p h} / c\right)$ as a function of $\theta$ 


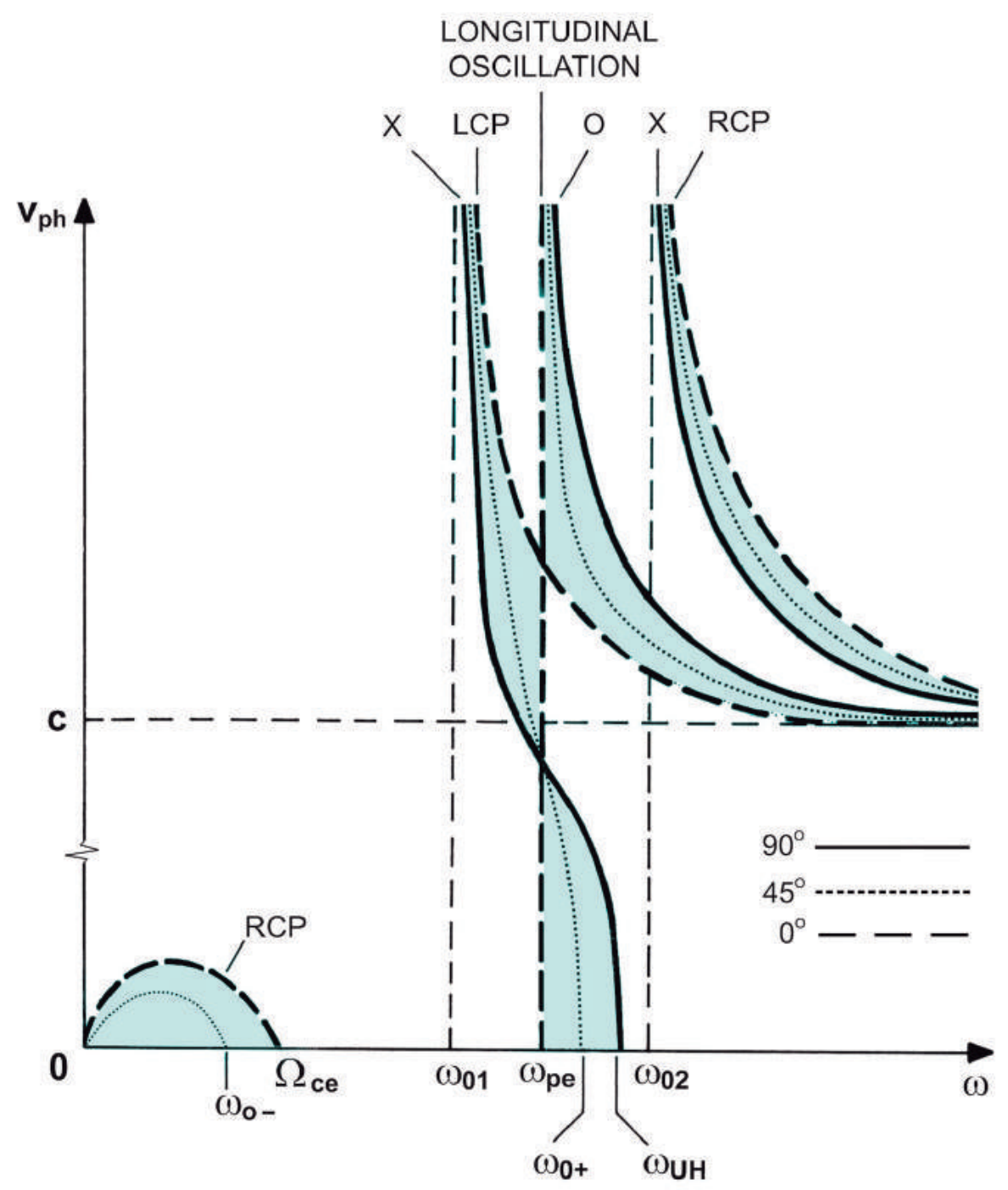

Fig. 20 Phase velocity versus frequency for waves in a cold electron plasma, illustrating how the two modes of propagation for $\theta=0^{\circ}$ ( $L C P$ and RCP) evolve into the two modes for $\theta=90^{\circ}$ $(O$ and $X)$.

(wave normal surface) is presented for each mode of propagation.

Fig. 22 shows the CMA diagram for wave propagation in a cold electron plasma. The dashed lines are the loci of the reflection points and the solid lines are the loci of the principal resonances (the dotted line indicates the loci of the resonances when $\theta=30^{\circ}$ ). The dashed circles 


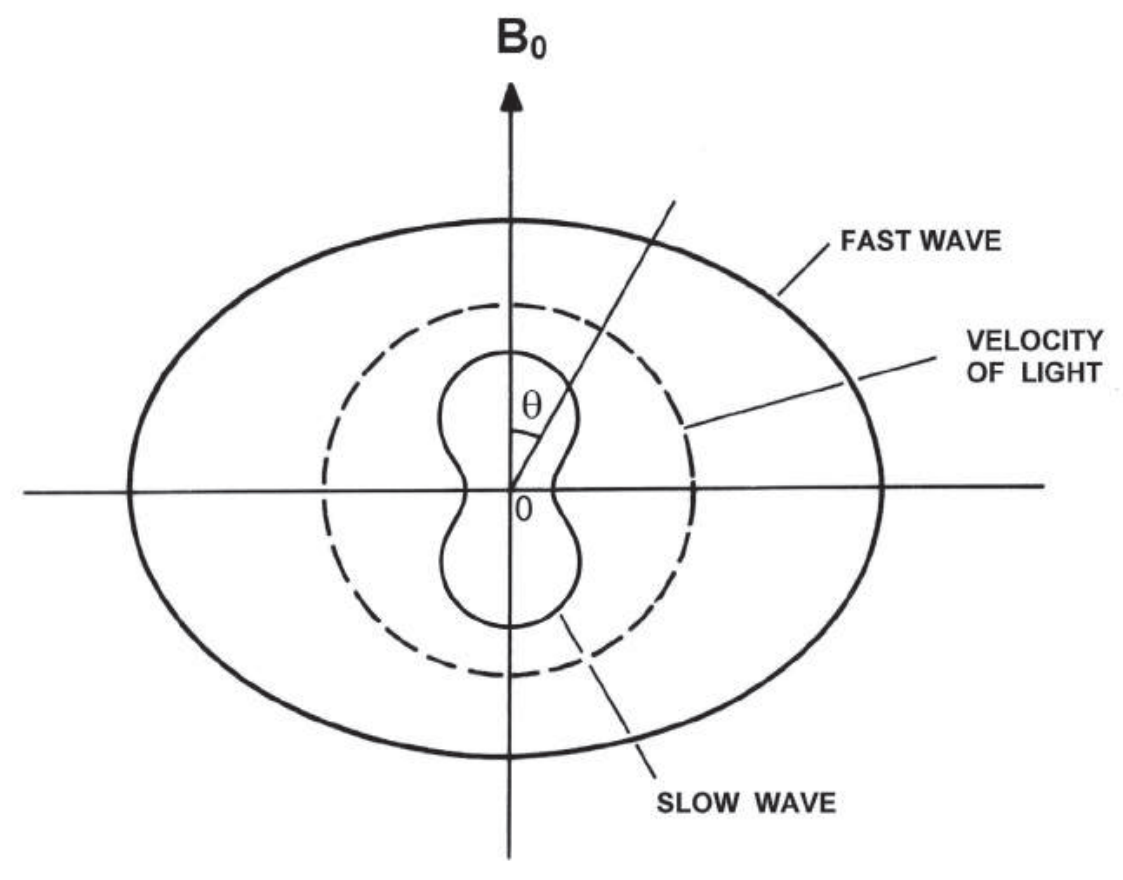

Fig. 21 Typical wave normal surface or phase velocity surface.

represent the wave normal surface corresponding to the speed of light. The slow and fast wave notation, used in Fig. 21, becomes now apparent. The labels $\mathrm{R}$ (right-hand polarization) and L (left-hand polarization) appear on the phase velocity surface only along the magnetic axis (up in the diagram). The labels 0 (ordinary) and X (extraordinary) appear only at $90^{\circ}$ with respect to the magnetic field axis.

In some regions of the CMA diagram certain modes are present and others are not. As the boundaries of these regions are crossed, the wave normal surfaces for the modes change their shape so that a given mode may appear or disappear. For instance, in region I both modes are present, but when we move to region II the fast wave disappears. Similarly, if the parameters are changed along a path that goes from region VIII to VII (decreasing electron density), the fast wave appears as the boundary $L=0$ is crossed and so on. Note that the same frequency may appear in the modes of different regions, depending upon the values of electron density and magnetic field. Note also that, although the characteristic shapes of the wave normal surfaces remain the same inside each bounded region, their magnitudes may change. A detailed examination of the CMA 


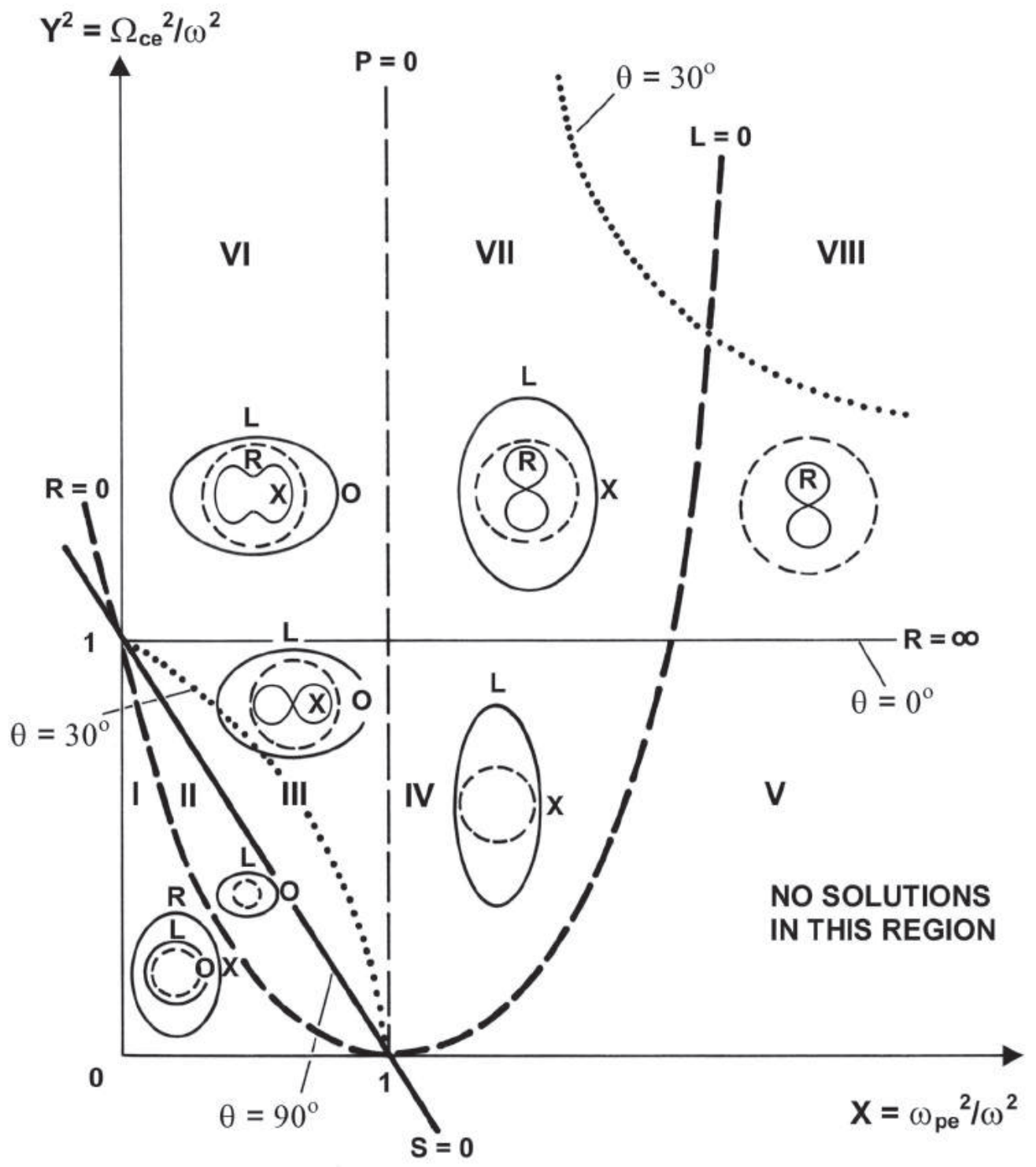

Fig. 22 The CMA diagram for waves in a cold electron gas. The solid lines represent the principal resonances and the dashed lines are the reflection points.

diagram shows that it provides a very broad picture of the nature of the waves that propagate in a cold electron plasma. 


\section{SOME SPECIAL WAVE PHENOMENA IN COLD PLASMAS}

\subsection{Atmospheric Whistlers}

The propagation of whistlers is a naturally occurring phenomenon, which can be originated by a lightning flash in the atmosphere. During thunderstorms and lightning, a pulse of electromagnetic radiation energy is produced that is rich in very low frequency components. This pulse, or wave packet, propagates through the ionosphere, being guided by ducts along the Earth's magnetic field to a distant point at the Earth's surface (the magnetic conjugate point). When the whistler is detected at this point (see Fig. 23) it is called a short whistler. However, this electromagnetic signal can be reflected at the Earth's surface and guided back along the Earth's magnetic field to a point close to where it originated. If the whistler is detected at this point it is called a long whistler.

As the wave packet, rich in low frequencies, propagates through the ionosphere along the Earth's magnetic field, it gets dispersed in such a way that the higher frequencies move faster than the lower ones. The frequencies in a whistler are in the audio range, usually between about $100 \mathrm{~Hz}$ and $10 \mathrm{kHz}$. Thus, at the point of detection, the higher frequencies arrive at the receiver sooner than the lower ones, and if the receiver is attached to a loudspeaker we hear a discending pitch whistle. These frequencies are usually much smaller than the electron cyclotron frequency in the Earth's ionosphere.

At various locations on the Earth there are stations that continuously record sonograms of whistler activity. A sonogram is a spectrun of the wave frequency versus time of arrival, as illustrated in Fig. 24. These sonograms are used as an effective diagnostic tool for studying the ionospheric conditions.

The phenomenon of atmospheric whistler propagation can be explained in terms of the very low frequency $\left(\omega<\Omega_{c e}\right)$ region of propagation of the right circularly polarized wave (refer to Fig. 20). For a simplified analysis of this phenomenon, consider the Appleton-Hartree equation (5.36), neglecting collisions $(\mathrm{U}=1)$. For propagation nearly along the magnetic field lines, and for $\omega \ll \Omega_{c e}$ and $\omega \ll \omega_{p e}$, we have $Y \cos \theta \gg Y^{2} \sin ^{2} \theta /[2(1-X)]$, so that (5.36), using the minus sign, simplifies to

$$
\eta^{2}=1-\frac{X}{1-Y \cos \theta}
$$




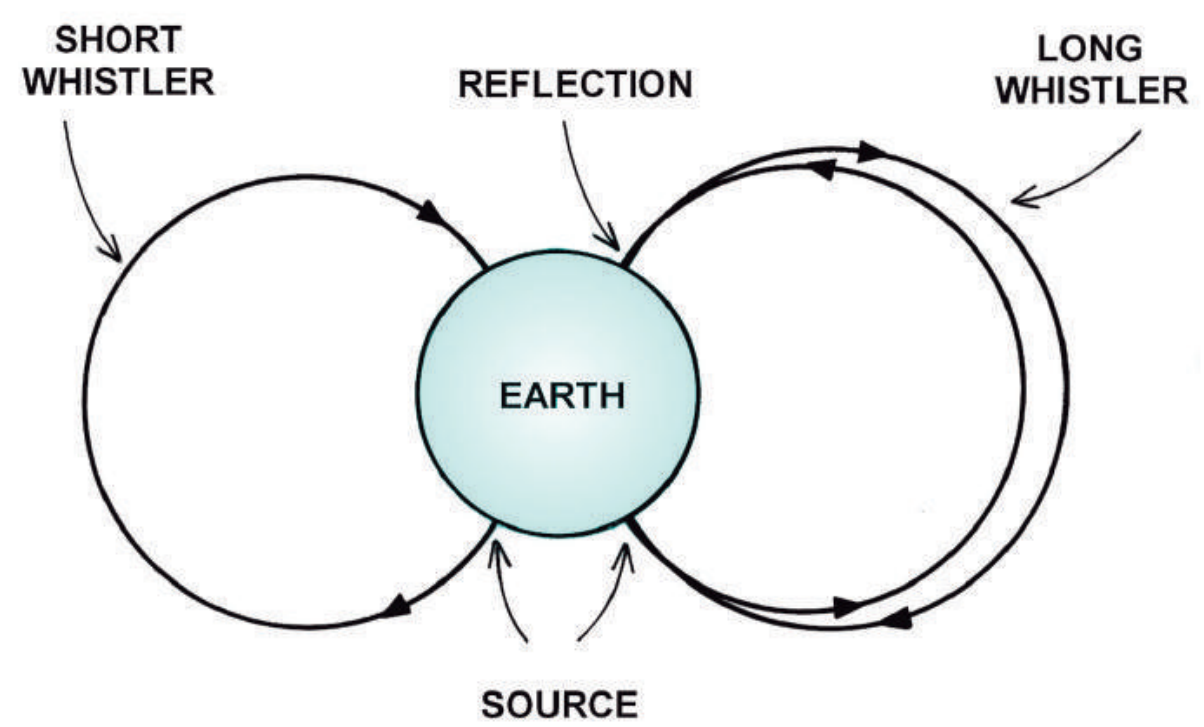

Fig. 23 Atmospheric whistler propagation, illustrating the detection of a short whistler and a long whistler.

This equation is often referred to as the dispersion relation for the quasilongitudinal mode. For $Y \cos \theta \gg 1$ (i.e., $\omega \ll \Omega_{c e} \cos \theta$ ), equation (9.1) becomes

$$
\eta^{2}=1+\frac{X}{Y \cos \theta}
$$

and considering further that $X \gg Y$ (i.e., $\omega_{p e}^{2} \gg \omega \Omega_{c e}$ ), we obtain

$$
\eta=\left(\frac{X}{Y \cos \theta}\right)^{1 / 2}
$$

The phase velocity is found directly from (9.3),

$$
v_{p h}=\frac{\omega}{k}=c\left(\frac{Y \cos \theta}{X}\right)^{1 / 2}
$$

or, substituting $Y=\Omega_{c e} / \omega$ and $X=\omega_{p e}^{2} / \omega^{2}$,

$$
v_{p h}=c \frac{\left(\omega \Omega_{c e} \cos \theta\right)^{1 / 2}}{\omega_{p e}}
$$




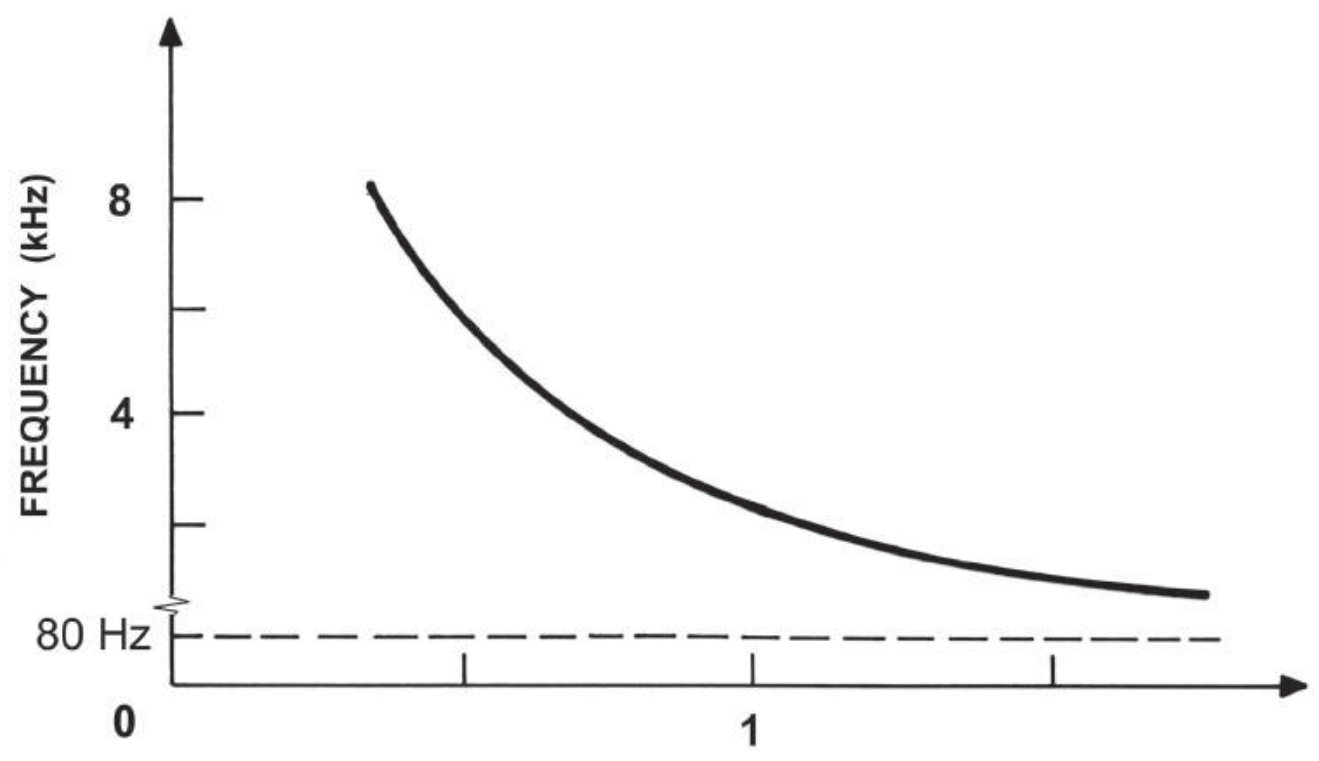

TIME (s)

Fig. 24 Typical sonogram of a whistler.

Also, from (9.3) we obtain the group velocity as

$$
v_{g}=\frac{\partial \omega}{\partial k}=2 c \frac{\left(\omega \Omega_{c e} \cos \theta\right)^{1 / 2}}{\omega_{p e}}
$$

Thus, both the phase velocity and the group velocity are proportional to the square root of the wave frequency and consequently the higher frequencies arrive at the receiver slightly ahead of the lower frequencies, producing a descending pitch whistle when received with a simple antenna and loudspeaker.

The characteristics of atmospheric whistler propagation are such that they are situated in region VIII of the CMA diagram. In this region, the wave normal diagram for the RCP wave is a lemniscate, as shown in Fig. 25. This wave normal surface has a resonant cone, which gives the maximum value that the angle $\theta$ may have. The angle between the direction of propagation of the wave packet and the magnetic field also has a maximum value, which specifies the maximum angular deviation (from the magnetic field) of the direction in which a wave packet can propagate. It can be shown that the maximum value of this angle is about $19.5^{\circ}$. Therefore, the wave packet is confined to a beam of less than $20^{\circ}$ about the magnetic field lines. 


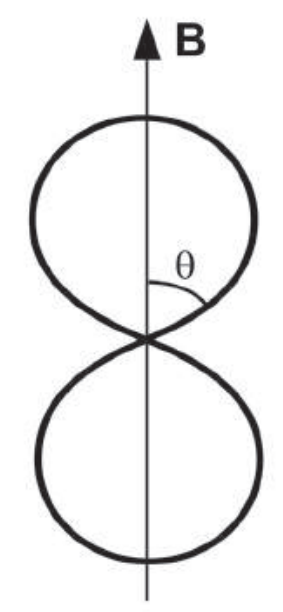

Fig. 25 Wave normal surface for whistlers and helicons.

Experiments carried out on whistlers have verified the results presented here. In addition, when the frequency is near (but smaller than) the electron cyclotron frequency, it is possible to have the frequency increasing with the time of arrival. These types of whistlers have been called ascending frequency whistlers. In the frequency regime where the whistlers change from the ascending to the descending tone they are known as nose whistlers. These types of whistlers have also been observed experimentally.

\subsection{Helicons}

The experimentally observed helicon waves, in a solid-state plasma, is also a phenomenon related with the right circularly polarized wave propagation in the very low frequency regime. The term helicons comes from the fact that the tip of the wave $\mathbf{B}$ vector traces a helix.

Consider a solid-state plasma slab of thickness $d$, the other two dimensions being very large, oriented perpendicularly to an externally applied B field, as indicated in Fig. 26. Suppose that a low-frequency $\left(\omega \ll \Omega_{c e}\right)$ $\mathrm{RCP}$ wave is launched in the direction of the $\mathbf{B}$ field. From the dispersion relation (6.7) for the RCP wave, in the low-frequency regime, we obtain

$$
k=\frac{\omega_{p e}}{c}\left(\frac{\omega}{\Omega_{c e}}\right)^{1 / 2} \quad ; \quad\left(\omega \ll \Omega_{c e}\right)
$$




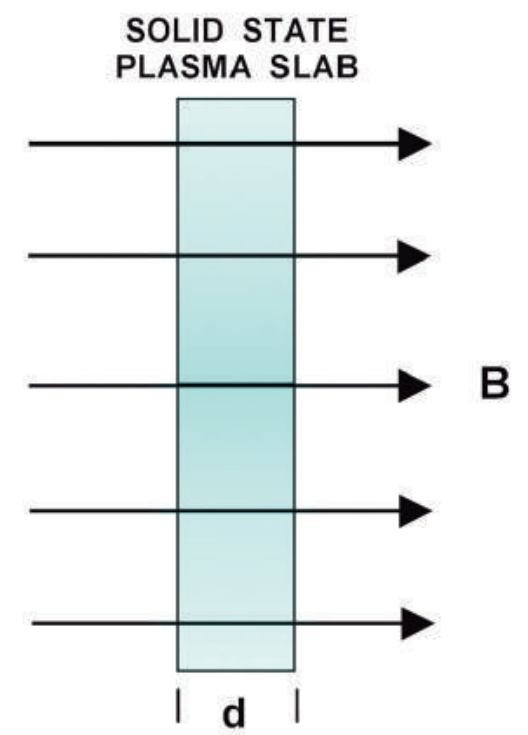

Fig. 26 Geometrical arrangement for the detection of helicon waves in a solid-state plasma slab.

Denoting the propagation coefficient of the electromagnetic wave in the medium external to the plasma slab by $k_{v}$, the magnitude of the reflection coefficient at the plasma boundary is given by $\left(k_{v}-k\right) /\left(k_{v}+k\right)=1$, since $\omega \ll \Omega_{c e}$. Consequently, the reflection of the waves at the plasma boundary is nearly complete. Therefore, the wave will be successively reflected at the boundaries of the plasma slab and will form a standing wave, whose resonances are given approximately by

$$
n \lambda=2 d
$$

where $\lambda$ is the wavelength inside the plasma slab of thickness $d$ and $n$ is an integer. Since $\lambda k=2 \pi$, we can combine (9.7) and (9.8) to obtain

$$
\frac{n \pi c}{\omega_{p e}}\left(\frac{\Omega_{c e}}{\omega}\right)^{1 / 2}=d
$$

This is the condition for standing wave resonance. It is appropriate to add the subscript $n$ to $\omega$, in order to identify the resonance frequency with the corresponding value of $n$, which gives the number of the standingwave pattern in the slab. Thus, (9.9) can be rearranged in the following convenient form:

$$
\omega_{n}=\left(\frac{n \pi c}{\omega_{p e} d}\right)^{2} \Omega_{c e}
$$


In some experiments carried out on helicons, the frequency of the wave excited along $\mathbf{B}$ is continuously varied, maintaining constant the values of $\omega_{p e}, \Omega_{c e}$, and $d$. At the frequencies where $\omega=\omega_{n}$, given by (9.10), there are standing-wave resonances inside the plasma slab, resulting in large wave amplitudes, which can be measured. A plot of wave amplitude inside the plasma slab, versus frequency, permits the identification of the resonant frequencies $\omega_{n}$.

In sodium, which contains about $10^{28}$ electrons $/ \mathrm{m}^{3}$, the first $(n=1)$ standing-wave resonant frequency is of the order of $10^{2} \mathrm{~Hz}$ in a typical magnetic field of about 1 tesla. Note that $\omega_{n}$ is proportional to $n^{2}$.

In some other experimental investigations, the parameters $d, \omega_{p e}$, and $\omega$ are kept fixed and the $\mathbf{B}$ field is varied. Then, the standing-wave resonant frequencies occur for those values of the $\mathbf{B}$ field for which

$$
\Omega_{c e}=\left(\Omega_{c e}\right)_{n}=\left(\frac{\omega_{p e} d}{n \pi c}\right)^{2} \omega
$$

\subsection{Faraday Rotation}

Let us consider now a phenomenon, known as Faraday rotation, which occurs in the range of frequencies where both the RCP and LCP waves propagate. When a plane polarized wave is sent along the magnetic field in a plasma, the plane of polarization of the wave gets rotated as it propagates in the plasma. Since a plane polarized wave can be considered as a superposition of RCP and LCP waves (as illustrated in Fig. 27) which propagate independently, this phenomenon can be understood in terms of the difference in phase velocity of the RCP and LCP waves.

If we take a look at Fig. 9 we see that the RCP wave (for frequencies greater than $\omega_{02}$ ) propagates faster than the LCP wave. After traveling a given distance, in which the RCP wave has undergone $N$ cycles, the LCP wave (which travels more slowly) will have undergone $N+\epsilon$ (with $\epsilon>0$ ) cycles. Obviously, both waves are considered to be at the same frequency. Therefore, the plane of polarization of the plane wave is rotated counterclockwise (looking along B), as indicated in Fig. 28.

In order to obtain an expression for the angle of rotation $\theta_{F}$, let us consider a Cartesian coordinate system with the propagation vector along the $z$ axis (also the direction of $\mathbf{B}_{0}$ ) and such that, at $z=0$, the electric field has only the $x$ component, as indicated in Fig. 27. Therefore, without loss of generality, we take

$$
\mathbf{E}(z=0, t)=\widehat{\mathbf{x}} E_{0} \exp (-i \omega t)
$$




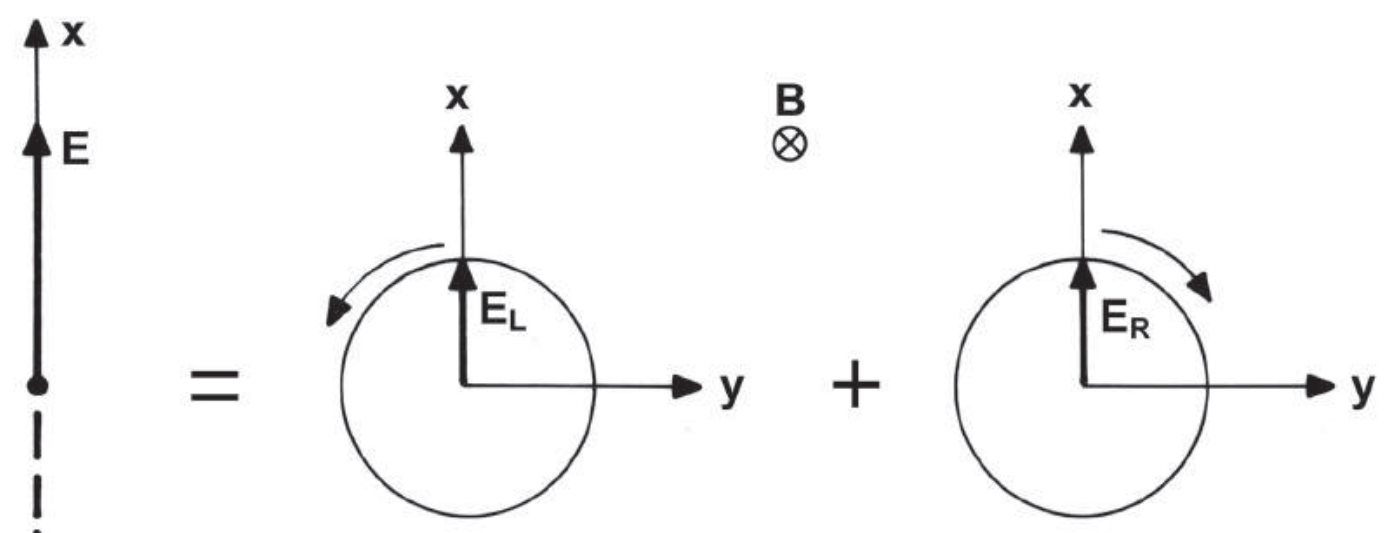

Fig. 27 A plane polarized wave as a superposition of left and right circularly polarized waves $\mathbf{E}=\mathbf{E}_{L}+\mathbf{E}_{R}$.

This equation can be rewritten as

$$
\mathbf{E}(0, t)=\frac{1}{2} E_{0}[(\widehat{\mathbf{x}}+i \widehat{\mathbf{y}})+(\widehat{\mathbf{x}}-i \widehat{\mathbf{y}})] \exp (-i \omega t)
$$

where the first and the second terms within brackets in the right-hand side correspond, respectively, to the RCP and the LCP components. These two components propagate independently, so that, for any $z>0$, the electric field vector is given by

$$
\begin{gathered}
\mathbf{E}(z, t)=\frac{1}{2} E_{0}(\widehat{\mathbf{x}}+i \widehat{\mathbf{y}}) \exp \left(i k_{R} z-i \omega t\right)+ \\
\frac{1}{2} E_{0}(\widehat{\mathbf{x}}-i \widehat{\mathbf{y}}) \exp \left(i k_{L} z-i \omega t\right)
\end{gathered}
$$

where $k_{R}$ and $k_{L}$ denote the wave number vectors for the RCP and LCP waves, respectively. Equation (9.14) can be rearranged as follows:

$$
\begin{gathered}
\mathbf{E}(z, t)=\frac{1}{2} E_{0} \exp \left[\frac{1}{2} i\left(k_{R}+k_{L}\right) z-i \omega t\right]\left\{(\widehat{\mathbf{x}}+i \widehat{\mathbf{y}}) \exp \left[\frac{1}{2} i\left(k_{R}-k_{L}\right) z\right]+\right. \\
\left.(\widehat{\mathbf{x}}-i \widehat{\mathbf{y}}) \exp \left[-\frac{1}{2} i\left(k_{R}-k_{L}\right) z\right]\right\}= \\
E_{0} \exp \left[\frac{1}{2} i\left(k_{R}+k_{L}\right) z-i \omega t\right]\left\{\widehat{\mathbf{x}} \cos \left[\frac{1}{2}\left(k_{R}-k_{L}\right) z\right]-\right. \\
\left.\widehat{\mathbf{y}} \sin \left[\frac{1}{2}\left(k_{R}-k_{L}\right) z\right]\right\}
\end{gathered}
$$




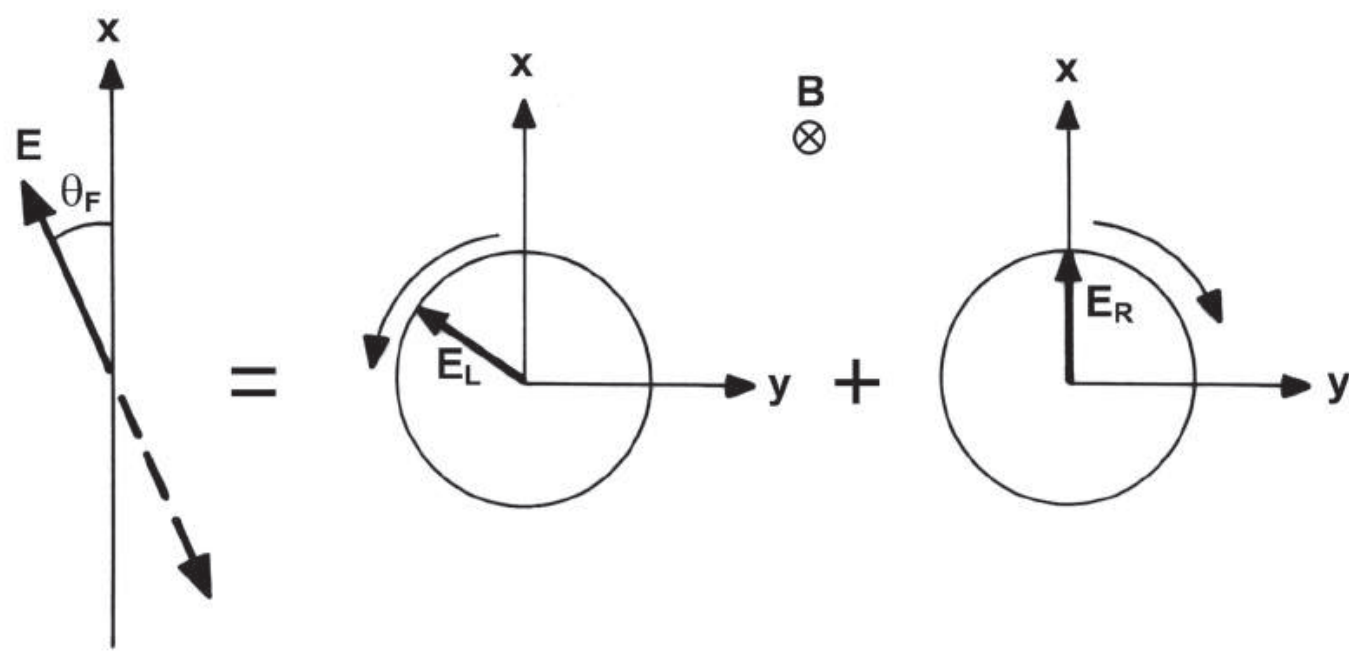

Fig. 28 After traveling a given distance in the plasma, the plane of polarization of the plane wave gets rotated since the $L C P$ wave moves slower than the $R C P$ wave.

Equation (9.12) represents a linearly polarized wave in the $x$ direction at $z=0$, while $(9.15)$ is also a linearly polarized wave, but with the polarization direction rotated in the counterclockwise direction (looking along $\mathbf{B}_{0}$ ) by the angle

$$
\theta_{F}=\frac{1}{2}\left(k_{R}-k_{L}\right) z
$$

Therefore, the angle of rotation per unit distance $\left(\theta_{F} / z\right)$ depends on the difference between the propagation coefficients of the RCP and LCP waves. Expressions for $k_{R}$ and $k_{L}$ are given in (6.6) and (6.8), respectively.

The measurement of Faraday rotation is a useful tool in plasma diagnostic and it has been widely used in the investigation of ionospheric properties. A linearly polarized wave, emitted by an orbiting satellite, has its plane of polarization rotated as it traverses the ionospheric plasma. A measurement of the rotation angle $\theta_{F}$, after the wave has traversed the plasma, provides information on the total electron content (i.e., the column integrated electron density) along the wave path. 


\section{PROBLEMS}

16.1 Consider a plane electromagnetic wave incident normally on a semiinfinite plasma occupying the semi-space $x>0$, with vacuum for $x<0$, as illustrated in Fig. 29. Denote the incident, reflected, and transmitted waves, respectively, by

$$
\begin{gathered}
\mathbf{E}_{i}=\widehat{\mathbf{y}} \exp \left(i k_{0} x-i \omega t\right) \\
\mathbf{E}_{r}=\widehat{\mathbf{y}} E_{r} \exp \left(-i k_{0} x-i \omega t\right) \\
\mathbf{E}_{t}=\widehat{\mathbf{y}} E_{t} \exp \left(i k_{1} x-i \omega t\right)
\end{gathered}
$$

(a) Show that the associated magnetic fields are given by

$$
\begin{gathered}
\mathbf{H}_{i}=\widehat{\mathbf{z}} \frac{k_{0}}{\omega \mu_{0}} \exp \left(i k_{0} x-i \omega t\right) \\
\mathbf{H}_{r}=-\widehat{\mathbf{z}} \frac{k_{0} E_{r}}{\omega \mu_{0}} \exp \left(-i k_{0} x-i \omega t\right) \\
\mathbf{H}_{t}=\widehat{\mathbf{z}} \frac{k_{1} E_{t}}{\omega \mu_{0}} \exp \left(i k_{1} x-i \omega t\right)
\end{gathered}
$$

(b) From the continuity of $E_{y}$ and $H_{z}$ at the boundary $x=0$, show that

$$
\begin{aligned}
& E_{r}=\frac{k_{0}-k_{1}}{k_{0}+k_{1}} \\
& E_{t}=\frac{2 k_{0}}{k_{0}+k_{1}}
\end{aligned}
$$

(c) Prove that the ratio of the transmitted average power to the incident average power, at the boundary $x=0$, is

$$
T=\frac{\mathcal{R}\left\{\mathbf{E}_{t} \times \mathbf{H}_{t}^{*}\right\}}{\mathcal{R}\left\{\mathbf{E}_{i} \times \mathbf{H}_{i}^{*}\right\}}=\frac{E_{t} E_{t}^{*}}{k_{0}} \beta
$$

where $k_{0}$ is real and $\beta=\mathcal{R}\left\{k_{1}\right\}$. Show that $T=0$ both at a reflection point and a resonance.

16.2 Consider a plane electromagnetic wave incident normally on an infinite plane plasma slab occupying the space $0 \leq x \leq L$, with vacuum for 


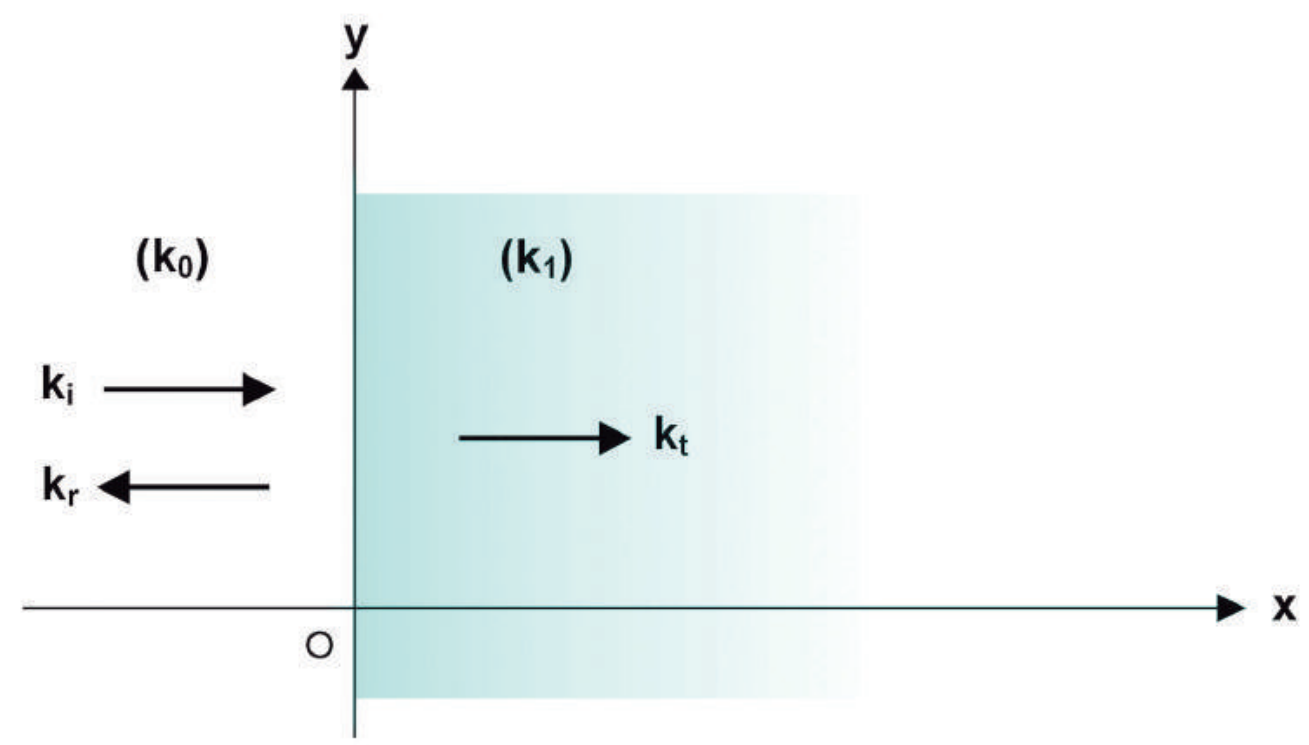

Fig. 29 Wave vectors of an electromagnetic wave incident on a semi-infinite plasma occupying the space $x>0$.

$x<0$ and $x>L$, as indicated in Fig. 30. Use the following representation for the wave electric field vector:

$$
\begin{aligned}
\mathbf{E}_{i}=\widehat{\mathbf{y}} \exp \left(i k_{0} x-i \omega t\right) & \text { (incident wave) } \\
\mathbf{E}_{r}=\widehat{\mathbf{y}} E_{r} \exp \left(-i k_{0} x-i \omega t\right) & \text { (reflected wave) } \\
\mathbf{E}_{f}=\widehat{\mathbf{y}} E_{f} \exp \left(i k_{1} x-i \omega t\right) & (\text { forward wave) } \\
\mathbf{E}_{b}=\widehat{\mathbf{y}} E_{b} \exp \left(-i k_{1} x-i \omega t\right) & \text { (backward wave) } \\
\left.\mathbf{E}_{t}=\widehat{\mathbf{y}} E_{t} \exp \left[i k_{0}(x-L)-i \omega t\right)\right] & \text { (transmitted wave) }
\end{aligned}
$$

(a) Calculate the corresponding expressions for the associated magnetic fields.

(b) Calculate the amplitudes $E_{r}, E_{f}, E_{b}$, and $E_{t}$ by applying the condition of continuity of $E_{y}$ and $H_{z}$ at the boundaries $x=0$ and $x=L$.

(c) Show that the ratio of the average power transmitted out of the plasma slab to the incident average power is given by

$$
T=\frac{\mathcal{R}\left\{\mathbf{E}_{t} \times \mathbf{H}_{t}^{*}\right\}_{x=L}}{\mathcal{R}\left\{\mathbf{E}_{i} \times \mathbf{H}_{i}^{*}\right\}_{x=0}}=E_{t} E_{t}^{*}
$$




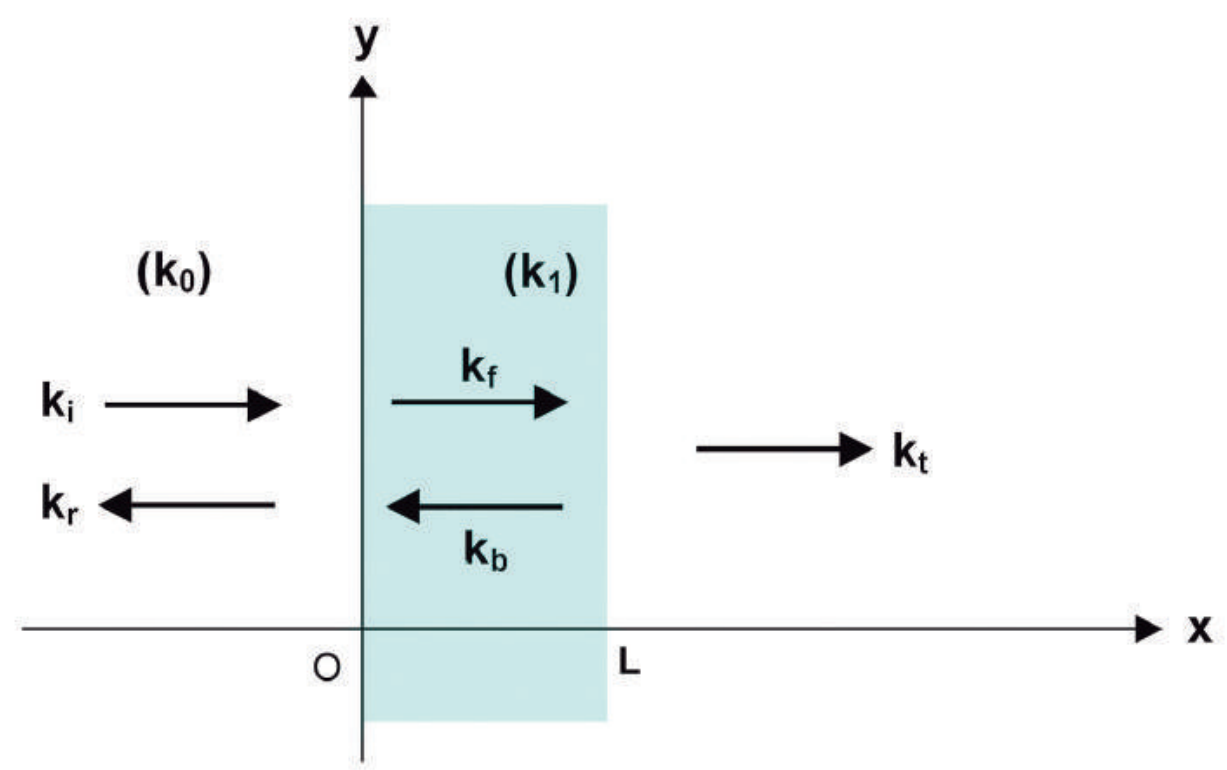

Fig. 30 Wave vectors associated with an electromagnetic wave incident normally on a plasma slab.

where

$$
E_{t}=4\left[\left(2+\frac{k_{0}}{k_{1}}+\frac{k_{1}}{k_{0}}\right) \exp \left(-i k_{1} L\right)+\left(2-\frac{k_{0}}{k_{1}}-\frac{k_{1}}{k_{0}}\right) \exp \left(i k_{1} L\right)\right]^{-1}
$$

(d) For $\omega<\omega_{p e}$, where $k_{1}=i \alpha$, with $\alpha$ real, show that

$$
\begin{aligned}
E_{t} & =4\left[4 \cosh (\alpha L)+2 i\left(\frac{\alpha}{k_{0}}-\frac{k_{0}}{\alpha}\right) \sinh (\alpha L)\right]^{-1} \\
T & =\left[\cosh ^{2}(\alpha L)+\frac{1}{4}\left(\frac{\alpha}{k_{0}}-\frac{k_{0}}{\alpha}\right)^{2} \sinh ^{2}(\alpha L)\right]^{-1}
\end{aligned}
$$

This result shows that some power is transmitted through the plasma slab, even with $\beta=\mathcal{R}\left\{k_{1}\right\}=0$. This effect is known as the tunneling effect.

16.3 Derive expressions for the phase velocity and group velocity from the dispersion relation (5.26), for wave propagation at arbitrary angles in a cold magnetoplasma.

16.4 Use the dispersion relation (4.10), for the transverse mode of propagation in a cold isotropic electron gas (with $\mathbf{B}_{0}=0$ ), to calculate the 
damping factor $\alpha=\mathcal{I}\{k\}$. Show that, when $\omega \gg \omega_{p e}$, the damping factor is given approximately by

$$
\alpha=\frac{2 \omega c \omega_{p e}^{2}(\nu / \omega)}{1+(\nu / \omega)^{2}}
$$

16.5 Consider the propagation of high-frequency waves in a solid-state plasma with equal number of electrons and holes (considering $m_{e}=m_{h}$ and $\nu_{e}=\nu_{h}$ ), immersed in a magnetostatic field $\mathbf{B}_{0}$. Let $\mathbf{k}=k \widehat{\mathbf{x}}$ and $\mathbf{B}_{0}=B_{0}(\cos \theta \widehat{\mathbf{x}}+\sin \theta \widehat{\mathbf{y}})$. Use the Langevin equation for the electrons and for the holes, together with Maxwell equations, to show that

$$
\begin{gathered}
\left(-2 U X-Y^{2} \sin ^{2} \theta+U^{2}\right) u_{x}+\left(Y^{2} \sin \theta \cos \theta\right) u_{y}=0 \\
\left(Y^{2} \sin \theta \cos \theta\right) u_{x}+\left(-2 U \phi-Y^{2} \cos ^{2} \theta+U^{2}\right) u_{y}=0 \\
\left(-2 U \phi-Y^{2}+U^{2}\right) u_{z}=0
\end{gathered}
$$

where

$$
\begin{gathered}
\mathbf{u}=\mathbf{u}_{e}-\mathbf{u}_{h} \\
U=1+i \frac{\nu}{\omega} \\
X=\frac{\omega_{p e}^{2}}{\omega^{2}} \\
Y=\frac{\Omega_{c e}}{\omega} \\
\phi=\frac{X}{1-\eta^{2}}
\end{gathered}
$$

From these component equations derive the following dispersion relations:

$$
\begin{gathered}
\phi=\frac{U}{2}-\frac{Y^{2} \cos ^{2} \theta}{2 U}-\frac{Y^{4} \sin ^{2} \theta \cos ^{2} \theta}{2 U\left(-2 U X-Y^{2} \sin ^{2} \theta+U^{2}\right)} \\
\phi=\frac{U}{2}-\frac{Y^{2}}{2 U}
\end{gathered}
$$

Obtain expressions for the reflection points and the resonances. In particular, for the collisionless case $(U=1)$ show that the conditions for resonance are

$$
\omega^{2}=\frac{1}{2}\left\{\Omega_{c e}^{2}+2 \omega_{p e}^{2} \pm\left[\left(\Omega_{c e}^{2}+2 \omega_{p e}^{2}\right)^{2}-8 \omega_{p e}^{2} \Omega_{c e}^{2} \cos ^{2} \theta\right]\right\}^{1 / 2}
$$




$$
\omega^{2}=\Omega_{c e}^{2}
$$

and the reflection points are given by

$$
\begin{gathered}
\omega^{2}=\frac{1}{2}\left(\Omega_{c e}^{2}+4 \omega_{p e}^{2} \pm \Omega_{c e}^{2}\right) \\
\omega^{2}=\Omega_{c e}^{2}+2 \omega_{p e}^{2}
\end{gathered}
$$

16.6 Use (6.17) and (6.18) for the group velocities of the left and right circularly polarized waves, respectively, to show that the group velocity vanishes at the resonances and reflection points.

16.7 Consider the problem of wave propagation at an arbitrary direction in a cold magnetoplasma, but including the motion of the ions (one type only).

(a) Show that the dispersion relation is obtained from an equation identical to (5.25), except that now we have (neglecting collisions)

$$
\begin{gathered}
S=1-\frac{X_{e}}{1-Y_{e}^{2}}-\frac{X_{i}}{1-Y_{i}^{2}} \\
D=-\frac{X_{e} Y_{e}}{1-Y_{e}^{2}}+\frac{X_{i} Y_{i}}{1-Y_{i}^{2}} \\
P=1-X_{e}-X_{i}
\end{gathered}
$$

where (with $\alpha=e, i$ )

$$
\begin{aligned}
& X_{\alpha}=\frac{\omega_{p \alpha}^{2}}{\omega^{2}} \\
& Y_{\alpha}=\frac{\Omega_{c \alpha}}{\omega}
\end{aligned}
$$

(b) Obtain the dispersion relation and show that it can be written in the form

$$
\tan ^{2} \theta=-\frac{P\left(\eta^{2}-R\right)\left(\eta^{2}-L\right)}{\left(S \eta^{2}-R L\right)\left(\eta^{2}-P\right)}
$$

where $\theta$ is the angle between $\mathbf{k}$ and $\mathbf{B}_{0}, R=S+D$, and $L=S-D$.

(c) Determine and plot the resonances and reflection points as a function of $\theta$.

16.8 Using the results of the previous problem, analyze the various modes of propagation for the particular cases when $\theta=0$ and $\theta=\pi / 2$. Compare 
the results with those for a cold electron gas. Make a plot analogous to the one presented in Fig. 20.

16.9 From the dispersion relations obtained in problem 16.8 show that, in the limit of $\omega \ll \Omega_{c i}$, we obtain the dispersion relation for the (shear) Alfvén wave (cold plasma limit of the magnetosonic wave) when $\mathbf{k}$ is parallel to $\mathbf{B}_{0}$. Furthermore, for $\omega \leq \Omega_{c i}$ and $\mathbf{k}$ parallel to $\mathbf{B}_{0}$, determine the following approximate dispersion relation for ion cyclotron waves (assume $\left.1+c^{2} / V_{A}^{2} \ll \eta^{2}\right)$,

$$
\eta^{2}=\frac{2 c^{2} \Omega_{c i}^{2}}{V_{A}^{2}\left(\Omega_{c i}^{2}-\omega^{2}\right)}=\frac{2 \omega_{p i}^{2}}{\Omega_{c i}^{2}-\omega^{2}}
$$

16.10 From (5.25) show that the polarization of the waves propagating at an angle $\theta$ with respect to $\mathbf{B}_{0}$ (considering the perpendicular electric field vector component) is determined by

$$
i \frac{E_{x}}{E_{y}}=\frac{\eta^{2}-S}{D}
$$

From this result verify that for $\theta=0$ the waves are left and right circularly polarized, whereas for $\theta=\pi / 2$ the polarization of the extraordinary mode is given by

$$
i \frac{E_{x}}{E_{y}}=-\frac{D}{S}
$$

so that this mode is in general elliptically polarized.

16.11 For a helicon wave, or a circularly polarized wave, show that the tip of the wave magnetic field vector traces out a helix.

16.12 Make a plot analogous to Fig. 20 for wave propagation in a cold magnetoplasma, but in terms of $\omega$ as a function of the real part of $k$.

16.13 Consider a plasma slab of thickness $L$ and number density specified by $n(x)$, where the $x$ axis is normal to the slab. A plane polarized monochromatic electromagnetic wave is normally incident on the slab (assume $\omega$ sufficiently large that $\eta^{2}>0$ ). Neglecting reflection from the slab surfaces, determine an expression for the Faraday rotation angle as the wave traverses the plasma slab. Then, simplify this expression considering the cases $n(x)=$ constant and $n(x)=x$, for $0<x<L$. 


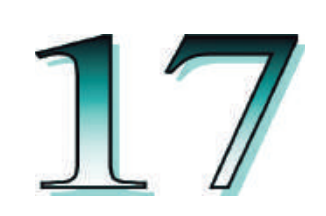

\section{WAVES IN WARM PLASMAS}

\section{INTRODUCTION}

In the previous chapter we analyzed the characteristics of wave propagation in a cold plasma. Now we will extend the theory already developed to include the pressure gradient term in the momentum equation. We shall consider wave propagation in a warm electron gas (neglecting ion motion) and, also, in a fully ionized warm plasma (considering electrons and only one type of ions), in the absence as well as in the presence of an externally applied magnetic field.

\section{WAVES IN A FULLY IONIZED ISOTROPIC WARM PLASMA}

\subsection{Derivation of the Equations for the Electron and Ion Velocities}

Consider now a fully ionized warm plasma, composed of electrons and only one ion species, with no externally applied magnetic field. The equations of conservation of mass and of momentum, for the electrons and for the ions, can be written as

$$
\begin{gathered}
\frac{\partial n_{\alpha}}{\partial t}+\nabla \cdot\left(n_{\alpha} \mathbf{u}_{\alpha}\right)=0 \\
m_{\alpha} \frac{D \mathbf{u}_{\alpha}}{D t}=q_{\alpha}\left(\mathbf{E}+\mathbf{u}_{\alpha} \times \mathbf{B}\right)-\frac{1}{n_{\alpha}} \nabla p_{\alpha}-m_{\alpha} \nu_{\alpha \beta}\left(\mathbf{u}_{\alpha}-\mathbf{u}_{\beta}\right)
\end{gathered}
$$


where for the electrons $\alpha=e$ and $\beta=i$ while for the ions $\alpha=i$ and $\beta=e$. These equations are complemented by the following adiabatic energy equation for each species,

$$
p_{\alpha} n_{\alpha}^{-\gamma}=\text { constant }
$$

where $\gamma=1+2 / N$ is the adiabatic constant and $N$ denotes the number of degrees of freedom. Applying the $\nabla$ operator to (2.3) and using the ideal gas law $p_{\alpha}=n_{\alpha} k T_{\alpha}$, we can rewrite (2.3) in the form

$$
\nabla p_{\alpha}=\gamma k_{B} T_{\alpha} \nabla n_{\alpha}
$$

We restrict our attention to small amplitude waves in order to linearize the equations and assume that

$$
\begin{gathered}
n_{\alpha}(\mathbf{r}, t)=n_{0}+n_{\alpha}^{\prime} \exp (i \mathbf{k} \cdot \mathbf{r}-i \omega t) ; \quad\left|n_{\alpha}^{\prime}\right| \ll n_{0} \\
\mathbf{u}_{\alpha}(\mathbf{r}, t)=\mathbf{u}_{\alpha} \exp (i \mathbf{k} \cdot \mathbf{r}-i \omega t) ; \quad u_{\alpha} \ll|\omega / k| \\
\mathbf{E}(\mathbf{r}, t)=\mathbf{E} \exp (i \mathbf{k} \cdot \mathbf{r}-i \omega t) \\
\mathbf{B}(\mathbf{r}, t)=\mathbf{B} \exp (i \mathbf{k} \cdot \mathbf{r}-i \omega t)
\end{gathered}
$$

Using these expressions in (2.1) and neglecting second-order terms, we find

$$
\frac{n_{\alpha}^{\prime}}{n_{0}}=\frac{1}{\omega}\left(\mathbf{k} \cdot \mathbf{u}_{\alpha}\right)
$$

Similarly, we obtain for (2.2) after the substitution of $\nabla p_{\alpha}$ from (2.4) and linearizing,

$$
-i \omega \mathbf{u}_{\alpha}=\frac{q_{\alpha}}{m_{\alpha}} \mathbf{E}-V_{s \alpha}^{2} i \mathbf{k} \frac{n_{\alpha}^{\prime}}{n_{0}}-\nu_{\alpha \beta}\left(\mathbf{u}_{\alpha}-\mathbf{u}_{\beta}\right)
$$

where $V_{s \alpha}=\left(\gamma k_{B} T_{\alpha} / m_{\alpha}\right)^{1 / 2}$ is the adiabatic sound speed for the type $\alpha$ particles. Substituting (2.9) into (2.10) and multiplying by $i \omega$, we obtain the following equation involving the variables $\mathbf{u}_{\alpha}, \mathbf{u}_{\beta}$, and $\mathbf{E}$,

$$
\omega^{2} \mathbf{u}_{\alpha}=i \omega \frac{q_{\alpha}}{m_{\alpha}} \mathbf{E}+V_{s \alpha}^{2} \mathbf{k}\left(\mathbf{k} \cdot \mathbf{u}_{\alpha}\right)-i \omega \nu_{\alpha \beta}\left(\mathbf{u}_{\alpha}-\mathbf{u}_{\beta}\right)
$$

A relationship between the electric field and the electron and ion velocities can be obtained from Maxwell curl equations with harmonic variations of $\mathbf{E}$ and $\mathbf{B}$, according to (2.7) and (2.8),

$$
\mathbf{k} \times \mathbf{E}=\omega \mathbf{B}
$$




$$
i \mathbf{k} \times \mathbf{B}=\mu_{0} \mathbf{J}-\frac{i \omega}{c^{2}} \mathbf{E}
$$

and from the linearized expression for the plasma current density,

$$
\mathbf{J}=n_{0} \sum q_{\alpha} \mathbf{u}_{\alpha}=n_{0} e\left(\mathbf{u}_{i}-\mathbf{u}_{e}\right)
$$

Combining (2.12), (2.13), and (2.14), we find

$$
\begin{aligned}
& \mathbf{E}_{\ell}=\frac{i e n_{0}}{\omega \epsilon_{0}}\left(\mathbf{u}_{e \ell}-\mathbf{u}_{i \ell}\right) \\
& \mathbf{E}_{t}=\frac{i e n_{0}}{\omega \epsilon_{0}} \frac{\left(\mathbf{u}_{e t}-\mathbf{u}_{i t}\right)}{\left(1-\eta^{2}\right)}
\end{aligned}
$$

where the subscripts $\ell$ and $t$ indicate components longitudinal and transverse, respectively, with respect to the wave propagation vector $\mathbf{k}$ (refer to Fig. 1, Chapter 16) and $\eta$ denotes the index of refraction $k c / \omega$.

Substituting (2.15) and (2.16) into (2.11), and writing this equation for each type of particles (electrons and ions), we obtain the following set of coupled equations for the longitudinal components of the electron and ion velocities,

$$
\begin{aligned}
& \mathbf{u}_{e \ell}\left(\omega^{2}-\omega_{p e}^{2}-k^{2} V_{s e}^{2}+i \omega \nu_{e i}\right)+\mathbf{u}_{i \ell}\left(\omega_{p e}^{2}-i \omega \nu_{e i}\right)=0 \\
& \mathbf{u}_{e \ell}\left(\omega_{p i}^{2}-i \omega \nu_{i e}\right)+\mathbf{u}_{i \ell}\left(\omega^{2}-\omega_{p i}^{2}-k^{2} V_{s i}^{2}+i \omega \nu_{i e}\right)=0
\end{aligned}
$$

and for the transverse components,

$$
\begin{aligned}
& \mathbf{u}_{e t}\left(\omega^{2}-\frac{\omega_{p e}^{2}}{1-\eta^{2}}+i \omega \nu_{e i}\right)+\mathbf{u}_{i t}\left(\frac{\omega_{p e}^{2}}{1-\eta^{2}}-i \omega \nu_{e i}\right)=0 \\
& \mathbf{u}_{e t}\left(\frac{\omega_{p i}^{2}}{1-\eta^{2}}-i \omega \nu_{i e}\right)+\mathbf{u}_{i t}\left(\omega^{2}-\frac{\omega_{p i}^{2}}{1-\eta^{2}}+i \omega \nu_{i e}\right)=0
\end{aligned}
$$

Note that the effect of the pressure gradient term appears only on the longitudinal component of the motion and consequently the transverse modes of propagation are the same ones as in the cold plasma model, but with the motion of the ions included. 


\subsection{Longitudinal Waves}

In what follows, in order to simplify the algebra, we shall neglect collisions $\left(\nu_{e i}=\nu_{i e}=0\right)$. For longitudinal waves the determinant of the coefficients in the system of equations (2.17) and (2.18) must vanish. This condition gives

$$
\left(\omega^{2}-\omega_{p e}^{2}-k^{2} V_{s e}^{2}\right)\left(\omega^{2}-\omega_{p i}^{2}-k^{2} V_{s i}^{2}\right)-\omega_{p e}^{2} \omega_{p i}^{2}=0
$$

Multiplying the terms within parenthesis, this equation can be recast into the form

$$
\begin{gathered}
\left(V_{s e}^{2} V_{s i}^{2}\right) k^{4}+\left[\omega_{p e}^{2} V_{s i}^{2}+\omega_{p i}^{2} V_{s e}^{2}-\omega^{2}\left(V_{s e}^{2}+V_{s i}^{2}\right)\right] k^{2}+ \\
+\omega^{2}\left(\omega^{2}-\omega_{p e}^{2}-\omega_{p i}^{2}\right)=0
\end{gathered}
$$

Note that in the case of the cold plasma model, in which the pressure gradient terms are ignored (i.e., $V_{s e}=V_{s i}=0$ ), equation (2.22) gives $\omega^{2}=\left(\omega_{p e}^{2}+\omega_{p i}^{2}\right)$, which corresponds to the longitudinal plasma oscillations when the motion of both electrons and ions are taken into account.

Equation (2.22) has two roots for $k^{2}$, so that there are two longitudinal modes of propagation. One of these is termed the longitudinal electron plasma wave and the other one is the longitudinal ion plasma wave. These plasma modes are electrostatic in character and contain all the charge accumulation and no magnetic field, whereas the transverse electromagnetic mode contains the entire magnetic field and has no charge accumulation (as we will see in section 2.3). Although it is not difficult to obtain the two exact solutions for $k^{2}$ from (2.22), it is more convenient to analyze it for some special cases that emphasize the role played by the inclusion of ion motion and the pressure gradient term.

For this purpose, let us first rewrite (2.22) for the case when ion motion is not taken into account, which becomes

$$
-V_{s e}^{2} \omega^{2} k^{2}+\omega^{2}\left(\omega^{2}-\omega_{p e}^{2}\right)=0
$$

or

$$
\omega^{2}=\omega_{p e}^{2}+V_{s e}^{2} k^{2}
$$

Now $V_{s e}^{2}=\gamma k_{B} T_{e} / m_{e}$ and since for plane waves the compression is onedimensional, we have $\gamma=3$, so that

$$
\omega^{2}=\omega_{p e}^{2}+\left(\frac{3 k_{B} T_{e}}{m_{e}}\right) k^{2}
$$


This equation is known as the Bohm-Gross dispersion relation for the longitudinal electron plasma wave. This relation shows a reflection point for $\omega=\omega_{p e}$. For very high frequencies $\left(\omega \gg \omega_{p e}\right)$ the phase velocity becomes $\omega / k=V_{\text {se }}$, which represents an electron acoustic wave.

Next, let us include the motion of the ions but under the assumption that their temperature is such that $V_{s i}=0$ (cold ions). Then, (2.22) simplifies to

$$
V_{s e}^{2}\left(\omega_{p i}^{2}-\omega^{2}\right) k^{2}+\omega^{2}\left(\omega^{2}-\omega_{p e}^{2}-\omega_{p i}^{2}\right)=0
$$

At very high frequencies $\left(\omega \gg \omega_{p e}\right)$ we still have $\omega / k=V_{\text {se }}$, but now (2.26) shows a reflection point at $\omega=\left(\omega_{p e}^{2}+\omega_{p i}^{2}\right)^{1 / 2}$.

Finally, let us analyze (2.22) in the limits of high and low frequencies. From the definitions of $\omega_{p e}$ and $V_{s i}$, we have

$$
\omega_{p e}^{2} V_{s i}^{2}=\frac{T_{i}}{T_{e}} \omega_{p i}^{2} V_{s e}^{2}
$$

Therefore, (2.22) can be rewritten as

$$
\begin{gathered}
V_{s e}^{2} V_{s i}^{2} k^{4}+\left[\omega_{p i}^{2} V_{s e}^{2}\left(1+T_{i} / T_{e}\right)-\omega^{2}\left(V_{s e}^{2}+V_{s i}^{2}\right)\right] k^{2}+ \\
\omega^{2}\left(\omega^{2}-\omega_{p e}^{2}-\omega_{p i}^{2}\right)=0
\end{gathered}
$$

For high frequencies, such that $\omega^{2} \gg \omega_{p i}^{2}\left(1+T_{i} / T_{e}\right),(2.28)$ becomes

$$
V_{s e}^{2} V_{s i}^{2} k^{4}-\omega^{2}\left(V_{s e}^{2}+V_{s i}^{2}\right) k^{2}+\omega^{2}\left(\omega^{2}-\omega_{p e}^{2}-\omega_{p i}^{2}\right)=0
$$

Further, considering $V_{s e}^{2} \omega^{2} \gg V_{s i}^{2}\left(\omega_{p e}^{2}+\omega_{p i}^{2}\right)$, a condition equivalent to $\omega^{2} \gg \omega_{p i}^{2}\left(T_{i} / T_{e}\right)\left(1+m_{e} / m_{i}\right)$ and that satisfies $\omega^{2} \gg \omega_{p i}^{2}\left(1+T_{i} / T_{e}\right)$, we can add the term $V_{s i}^{2}\left(\omega_{p e}^{2}+\omega_{p i}^{2}\right) k^{2}$ to the left-hand side of (2.29) and rearrange this equation in the following approximate form:

$$
\left(V_{s i}^{2} k^{2}-\omega^{2}\right)\left(V_{s e}^{2} k^{2}-\omega^{2}+\omega_{p e}^{2}+\omega_{p i}^{2}\right)=0
$$

From this equation we see that in the high-frequency limit the dispersion relation for the longitudinal ion plasma wave is

$$
\omega^{2}=V_{s i}^{2} k^{2}
$$

while for the electron plasma wave the dispersion relation is

$$
\omega^{2}=\omega_{p e}^{2}+\omega_{p i}^{2}+V_{s e}^{2} k^{2}
$$


Next, for low frequencies, such that $\omega^{2} \ll \omega_{p i}^{2}\left(1+T_{i} / T_{e}\right)$, (2.28) becomes

$$
V_{s e}^{2} V_{s i}^{2} k^{4}+V_{s e}^{2} \omega_{p i}^{2}\left(1+T_{i} / T_{e}\right) k^{2}-\omega^{2} \omega_{p e}^{2}=0
$$

Multiplying this equation by $-\omega^{2} /\left(\omega_{p e}^{2} k^{4}\right)$, assuming $k \neq 0$, it can be rewritten as

$$
\left(\frac{\omega}{k}\right)^{4}-V_{s e}^{2}\left(\frac{\omega_{p i}^{2}}{\omega_{p e}^{2}}\right)\left(1+\frac{T_{i}}{T_{e}}\right)\left(\frac{\omega}{k}\right)^{2}-V_{s e}^{2} V_{s i}^{2}\left(\frac{\omega^{2}}{\omega_{p e}^{2}}\right)=0
$$

Since we are considering low frequencies and as long as $(\omega / k)$ is not much larger than $V_{s i}$, the last term in the left-hand side of (2.34) can be neglected as compared to the second one. Therefore, (2.34) gives in the low-frequency limit

$$
\left(\frac{\omega}{k}\right)^{2}=V_{s e}^{2}\left(\frac{\omega_{p i}^{2}}{\omega_{p e}^{2}}\right)\left(1+\frac{T_{i}}{T_{e}}\right)
$$

Using the relation (2.27), this equation can be rewritten in the form

$$
\omega^{2}=V_{s p}^{2} k^{2}
$$

where

$$
V_{s p}^{2}=\frac{\gamma k_{B}\left(T_{e}+T_{i}\right)}{m_{i}}
$$

which is known as the plasma sound speed. It can be verified that the other root of (2.33) gives an evanescent wave at very low frequencies.

A plot of phase velocity versus frequency for the longitudinal waves is shown in Fig. 1. The longitudinal waves with phase velocities equal to $V_{s e}$ or $V_{s i}$ at high frequencies represent, respectively, acoustic oscillations due to the electrons and the ions. The low-frequency wave traveling at the plasma sound speed represents an acoustic oscillation of both the electrons and the ions, and is usually referred to as the ion acoustic wave.

\subsection{Transverse Wave}

For the transverse mode of propagation $\left(\mathbf{u}_{e t} \neq 0 ; \mathbf{u}_{i t} \neq 0\right)$ the determinant of the coefficients in the system of equations (2.19) and (2.20) must vanish. Neglecting collisions, we find

$$
\left(\omega^{2}-\frac{\omega_{p e}^{2}}{1-\eta^{2}}\right)\left(\omega^{2}-\frac{\omega_{p i}^{2}}{1-\eta^{2}}\right)-\frac{\omega_{p e}^{2} \omega_{p i}^{2}}{\left(1-\eta^{2}\right)^{2}}=0
$$




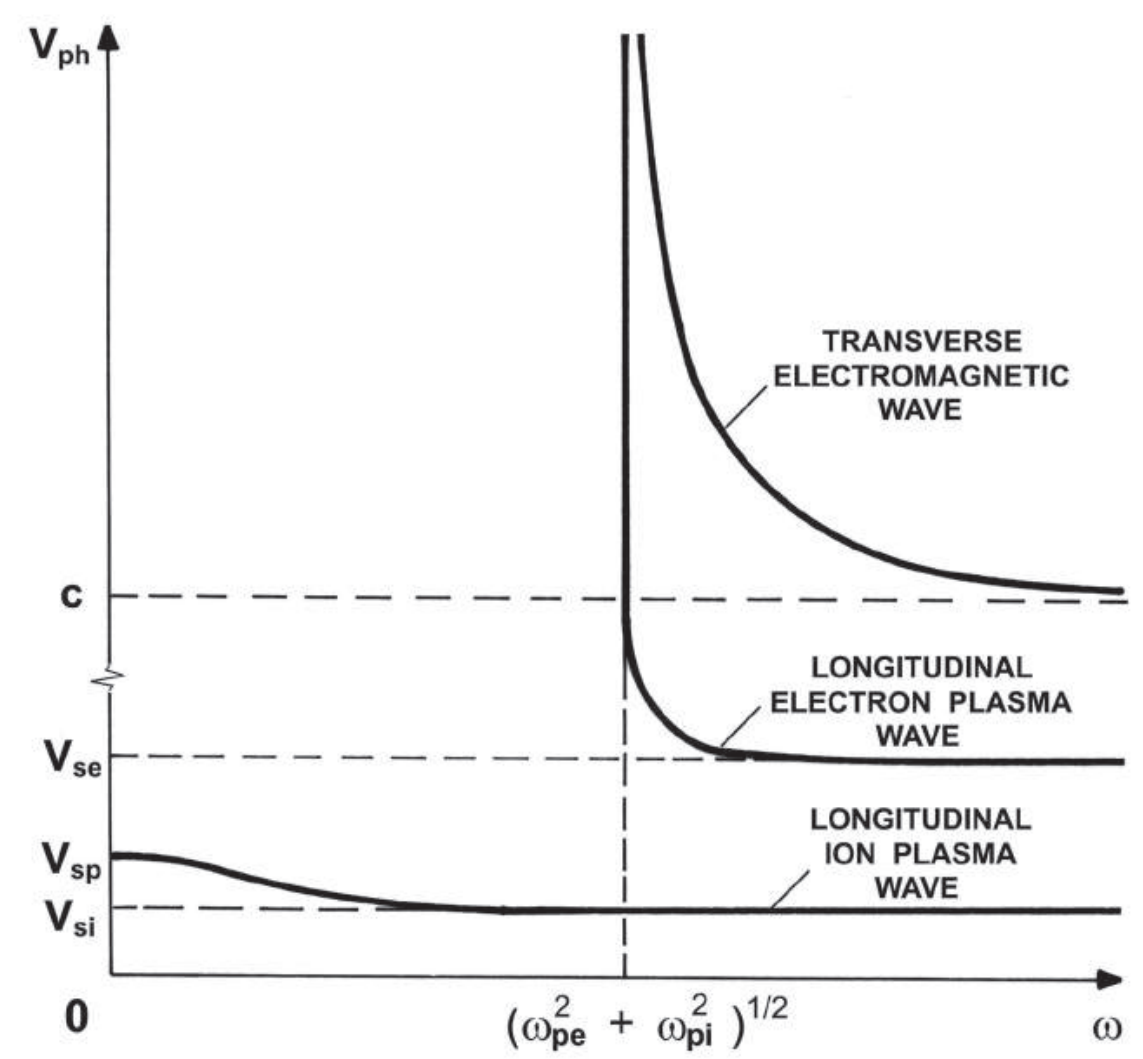

Fig. 1 Phase velocity as a function of frequency for waves in a fully ionized isotropic $\left(\mathbf{B}_{0}=0\right)$ warm plasma. The curves for the longitudinal waves also hold for propagation in the direction of $\mathbf{B}_{0}$, when $\mathbf{B}_{0} \neq 0$.

which simplifies to

$$
k^{2} c^{2}=\omega^{2}-\left(\omega_{p e}^{2}+\omega_{p i}^{2}\right)
$$

This equation is similar to the dispersion relation (16.4.12) (Eq. 4.12 in Chapter 16) for the propagation of transverse waves in a cold isotropic plasma, except that the reflection point is now $\left(\omega_{p e}^{2}+\omega_{p i}^{2}\right)^{1 / 2}$ as a consequence of the inclusion of ion motion. A plot of phase velocity as a function of frequency for the dispersion relation (2.39) is also shown in Fig. 1. A dispersion plot in terms of $\omega$ as a function of $k$ is displayed in Fig. 2 for the three modes of propagation.

In summary, there are three modes of wave propagation in a warm fully ionized isotropic plasma (as compared to only one mode in the case of cold isotropic plasma). They are the transverse electromagnetic mode 


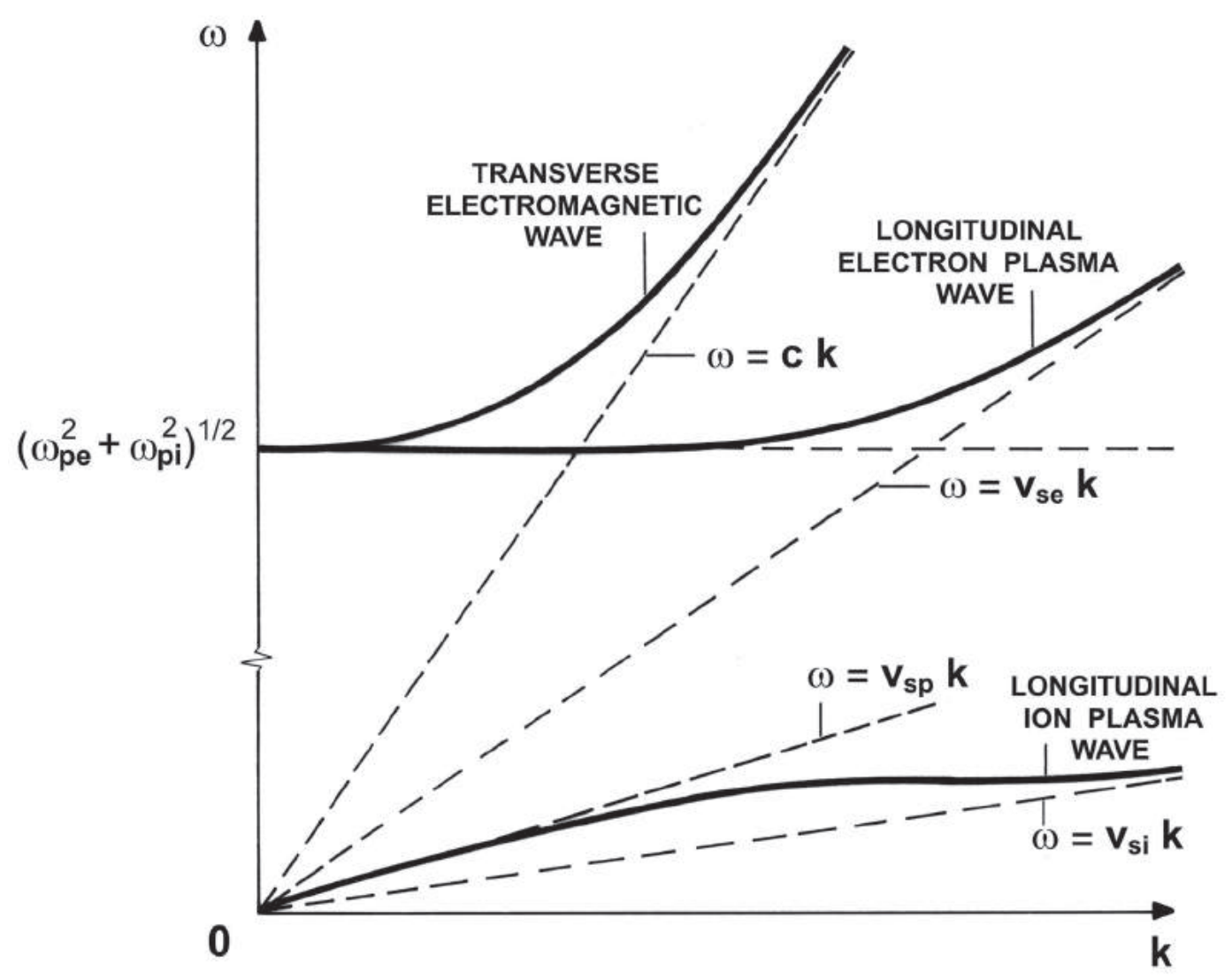

Fig. 2 Dispersion relation for the three modes of wave propagation in a warm isotropic fully ionized plasma.

(also present in the case of a cold plasma), the longitudinal electron plasma mode, and the longitudinal ion plasma mode.

\section{BASIC EQUATIONS FOR WAVES IN A WARM MAGNETOPLASMA}

The basic equations for the analysis of wave propagation in a warm fully ionized magnetoplasma are (2.1), (2.2), and (2.3). Proceeding in the same manner as in the previous section, but now considering an externally applied uniform magnetostatic field $\mathbf{B}_{0}$, we obtain, in place of (2.11),

$$
\omega^{2} \mathbf{u}_{\alpha}=i \omega \frac{q_{\alpha}}{m_{\alpha}}\left(\mathbf{E}+\mathbf{u}_{\alpha} \times \mathbf{B}_{0}\right)+V_{s \alpha}^{2} \mathbf{k}\left(\mathbf{k} \cdot \mathbf{u}_{\alpha}\right)-i \omega \nu_{\alpha \beta}\left(\mathbf{u}_{\alpha}-\mathbf{u}_{\beta}\right)
$$




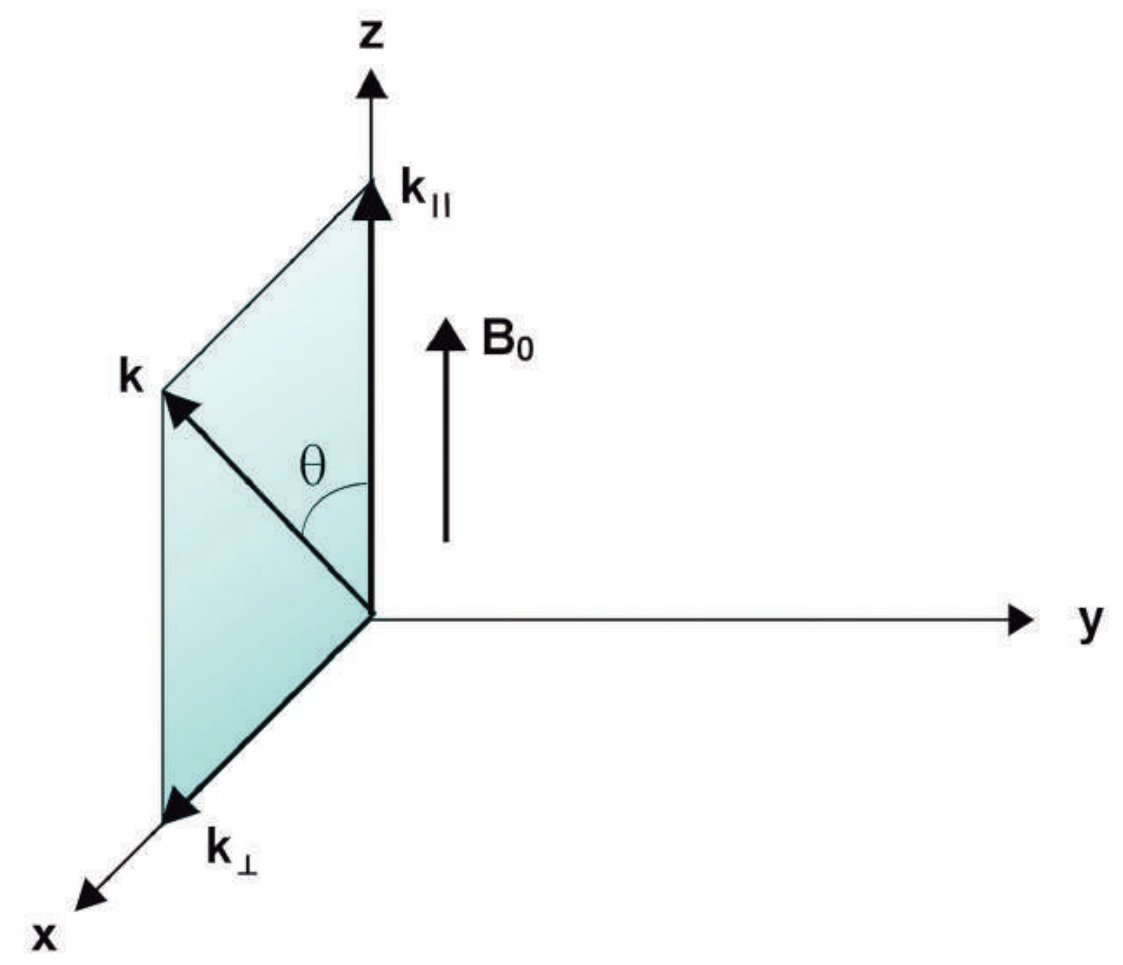

Fig. 3 Cartesian coordinate system chosen with $\mathbf{B}_{0}$ along the $\mathrm{z}$ axis and $\mathbf{k}$ in the $(\mathrm{x}, \mathrm{z})$ plane.

This equation is complemented by (2.15) and (2.16) or, equivalently, by

$$
\mathbf{k} \times(\mathbf{k} \times \mathbf{E})+\frac{\omega^{2}}{c^{2}} \mathbf{E}=-\frac{i \omega e n_{0}}{\epsilon_{0} c^{2}}\left(\mathbf{u}_{i}-\mathbf{u}_{e}\right)
$$

If we choose a Cartesian coordinate system such that the $z$ axis is along $\mathbf{B}_{0}$ and $\mathbf{k}$ is in the $(x, z)$ plane, as illustrated in Fig. 3, we have

$$
\begin{gathered}
\mathbf{B}_{0}=B_{0} \widehat{\mathbf{z}} \\
\mathbf{k}=\mathbf{k}_{\|}+\mathbf{k}_{\perp}=k \sin \theta \widehat{\mathbf{x}}+k \cos \theta \widehat{\mathbf{z}}
\end{gathered}
$$

and, consequently, (3.1) and (3.2) become, respectively [see (16.5.10) and $(16.5 .5)]$

$$
\omega^{2} \mathbf{u}_{\alpha}-i \omega \frac{q_{\alpha}}{m_{\alpha}} B_{0}\left(u_{\alpha y} \widehat{\mathbf{x}}-u_{\alpha x} \widehat{\mathbf{y}}\right)-V_{s \alpha}^{2} k^{2}\left(\sin \theta u_{\alpha x}+\right.
$$

$\left.\cos \theta u_{\alpha z}\right)(\sin \theta \widehat{\mathbf{x}}+\cos \theta \widehat{\mathbf{z}})+i \omega \nu_{\alpha \beta}\left(\mathbf{u}_{\alpha}-\mathbf{u}_{\beta}\right)=i \omega \frac{q_{\alpha}}{m_{\alpha}} \mathbf{E}$ 
and

$$
\mathcal{A} \cdot \mathbf{E}=-\frac{i e n_{0}}{\omega \epsilon_{0}}\left(\mathbf{u}_{i}-\mathbf{u}_{e}\right)
$$

where the components of the dyad $\mathcal{A}$, which represents the vector operator $\left[\left(c^{2} / \omega^{2}\right) \mathbf{k} \times(\mathbf{k} \times \ldots)+(\ldots)\right]$, can be arranged in matrix form as

$$
\mathcal{A}=\left(\begin{array}{ccc}
\left(1-\eta^{2} \cos ^{2} \theta\right) & 0 & \left(\eta^{2} \sin \theta \cos \theta\right) \\
0 & \left(1-\eta^{2}\right) & 0 \\
\eta^{2} \sin \theta \cos \theta & 0 & \left(1-\eta^{2} \sin ^{2} \theta\right)
\end{array}\right)
$$

With this matrix definition of $\mathcal{A}$, the dot product in (3.6) can be thought of as a matrix product between $\mathcal{A}$ and the vector column E. Taking the inverse of the matrix associated with $\mathcal{A}$ (assuming a nonvanishing determinant of its elements) and multiplying $(3.6)$ by $(\mathcal{A})^{-1}$, we obtain

$$
\mathbf{E}=-\frac{i e n_{0}}{\omega \epsilon_{0}}(\mathcal{A})^{-1} \cdot\left(\mathbf{u}_{i}-\mathbf{u}_{e}\right)
$$

since $(\mathcal{A})^{-1} \cdot(\mathcal{A})=\mathbf{1}$, where $\mathbf{1}$ is the unit dyad.

Equation (3.8) can be used to replace $\mathbf{E}$ in (3.5). For the electrons we take $\alpha=e$ and $\beta=i$ in (3.5), whereas for the ions $\alpha=i$ and $\beta=e$. We obtain, therefore, a system of six equations with the six unknowns $u_{\alpha j}$ (with $j=x, y, z$, and $\alpha=e, i$ ). The requirement that the determinant of its coefficients be equal to zero gives the dispersion relation.

\section{WAVES IN A WARM ELECTRON GAS IN A MAGNETIC FIELD}

In view of the complexity of the algebra involved, we shall initially consider the simple case of an electron gas immersed in an externally applied magnetic field, neglecting for the moment the macroscopic ion motion $\left(\mathbf{u}_{i}=0\right)$.

\subsection{Derivation of the Dispersion Relation}

From (3.5) we obtain for the electrons (taking $\mathbf{u}_{i}=0$ )

$$
\mathbf{u}_{e}+i\left(\frac{\Omega_{c e}}{\omega}\right)\left(u_{e y} \widehat{\mathbf{x}}-u_{e x} \widehat{\mathbf{y}}\right)-\left(\frac{V_{s e}^{2} k^{2}}{\omega^{2}}\right)\left(\sin \theta u_{e x}+\cos \theta u_{e z}\right)(\sin \theta \widehat{\mathbf{x}}+
$$




$$
\cos \theta \widehat{\mathbf{z}})+i\left(\frac{\nu_{e}}{\omega}\right) \mathbf{u}_{e}=\left(\frac{\omega_{p e}^{2}}{\omega^{2}}\right)(\mathcal{A})^{-1} \cdot \mathbf{u}_{e}
$$

Using the notation introduced in (16.5.14), (16.5.15), and (16.5.16), (4.1) can be rewritten in the form

$$
\begin{gathered}
U \mathbf{u}_{e}+\left(-\frac{V_{s e}^{2} k^{2}}{\omega^{2}} \sin ^{2} \theta u_{e x}+i Y u_{e y}-\frac{V_{s e}^{2} k^{2}}{\omega^{2}} \sin \theta \cos \theta u_{e z}\right) \widehat{\mathbf{x}}- \\
i Y u_{e x} \widehat{\mathbf{y}}+\left(-\frac{V_{s e}^{2} k^{2}}{\omega^{2}} \sin \theta \cos \theta u_{e x}-\frac{V_{s e}^{2} k^{2}}{\omega^{2}} \cos ^{2} \theta u_{e z}\right) \widehat{\mathbf{z}}= \\
X(\mathcal{A})^{-1} \cdot \mathbf{u}_{e}
\end{gathered}
$$

Defining a dyad $\mathcal{B}$ through the matrix

$$
\mathcal{B}=\left(\begin{array}{ccc}
\left(U-a^{2} \sin ^{2} \theta\right) & i Y & -\left(a^{2} \sin \theta \cos \theta\right) \\
-i Y & U & 0 \\
-\left(a^{2} \sin \theta \cos \theta\right) & 0 & \left(U-a^{2} \cos ^{2} \theta\right)
\end{array}\right)
$$

where $a^{2}=\left(V_{s e}^{2} k^{2} / \omega^{2}\right)$, equation (4.2) becomes

$$
\left(\mathcal{B}-X \mathcal{A}^{-1}\right) \cdot \mathbf{u}_{e}=0
$$

A nontrivial solution of this equation $\left(\mathbf{u}_{e} \neq 0\right)$ exists only if the determinant of the matrix multiplying $\mathbf{u}_{e}$ vanishes. Therefore, we must have

$$
\operatorname{det}\left(\mathcal{B}-X \mathcal{A}^{-1}\right)=0
$$

This condition gives the dispersion relation for wave propagation in a warm electron gas immersed in a magnetic field.

In order to simplify matters, in the two following subsections we shall examine the dispersion relation (4.5) for the special cases of propagation either parallel or perpendicular to the magnetic field.

\subsection{Wave Propagation Along the Magnetic Field}

For propagation along the magnetic field $\left(\mathbf{k} \| \mathbf{B}_{0}\right)$ we have $\mathbf{k}=k \widehat{\mathbf{z}}$ and $\theta=0$, so that (3.7) and (4.3) simplify to

$$
\mathcal{A}=\left(\begin{array}{ccc}
\left(1-\eta^{2}\right) & 0 & 0 \\
0 & \left(1-\eta^{2}\right) & 0 \\
0 & 0 & 1
\end{array}\right)
$$




$$
\mathcal{B}=\left(\begin{array}{ccc}
U & i Y & 0 \\
-i Y & U & 0 \\
0 & 0 & \left(U-a^{2}\right)
\end{array}\right)
$$

Therefore, the determinant (4.5) becomes

$$
\operatorname{det}\left(\begin{array}{ccc}
U-\frac{X}{\left(1-\eta^{2}\right)} & i Y & 0 \\
-i Y & U-\frac{X}{\left(1-\eta^{2}\right)} & 0 \\
0 & 0 & \left(U-a^{2}-X\right)
\end{array}\right)=0
$$

which gives the following dispersion relations for transverse waves $\left(u_{e x} \neq 0\right.$ and $u_{e y} \neq 0$ ),

$$
U-\frac{X}{\left(1-\eta^{2}\right)}= \pm Y
$$

and for a longitudinal wave $\left(u_{e z} \neq 0\right)$,

$$
U-a^{2}-X=0
$$

Note that in this case the $z$ component of (4.4) is uncoupled from the $x$ and $y$ components, so that the longitudinal mode is independent of the two transverse modes.

Equation (4.9) yields the following expressions corresponding, respectively, to the plus and minus signs,

$$
\begin{aligned}
& \eta^{2}=1-\frac{X}{U-Y} \\
& \eta^{2}=1-\frac{X}{U+Y}
\end{aligned}
$$

These dispersion relations correspond, respectively, to the right and left circularly polarized waves (RCP and LCP) obtained in section 6, Chapter 16, for transverse waves in a cold plasma [refer to (16.6.6) and (16.6.8)].

For the longitudinal wave, sustituting $U=1+i \nu_{e} / \omega$ and $X=\omega_{p e}^{2} / \omega^{2}$ in (4.10), the dispersion relation becomes

$$
\omega^{2}+i \nu_{e} \omega=\omega_{p e}^{2}+V_{s e}^{2} k^{2}
$$

Hence, as compared to the cold plasma model, instead of the longitudinal 


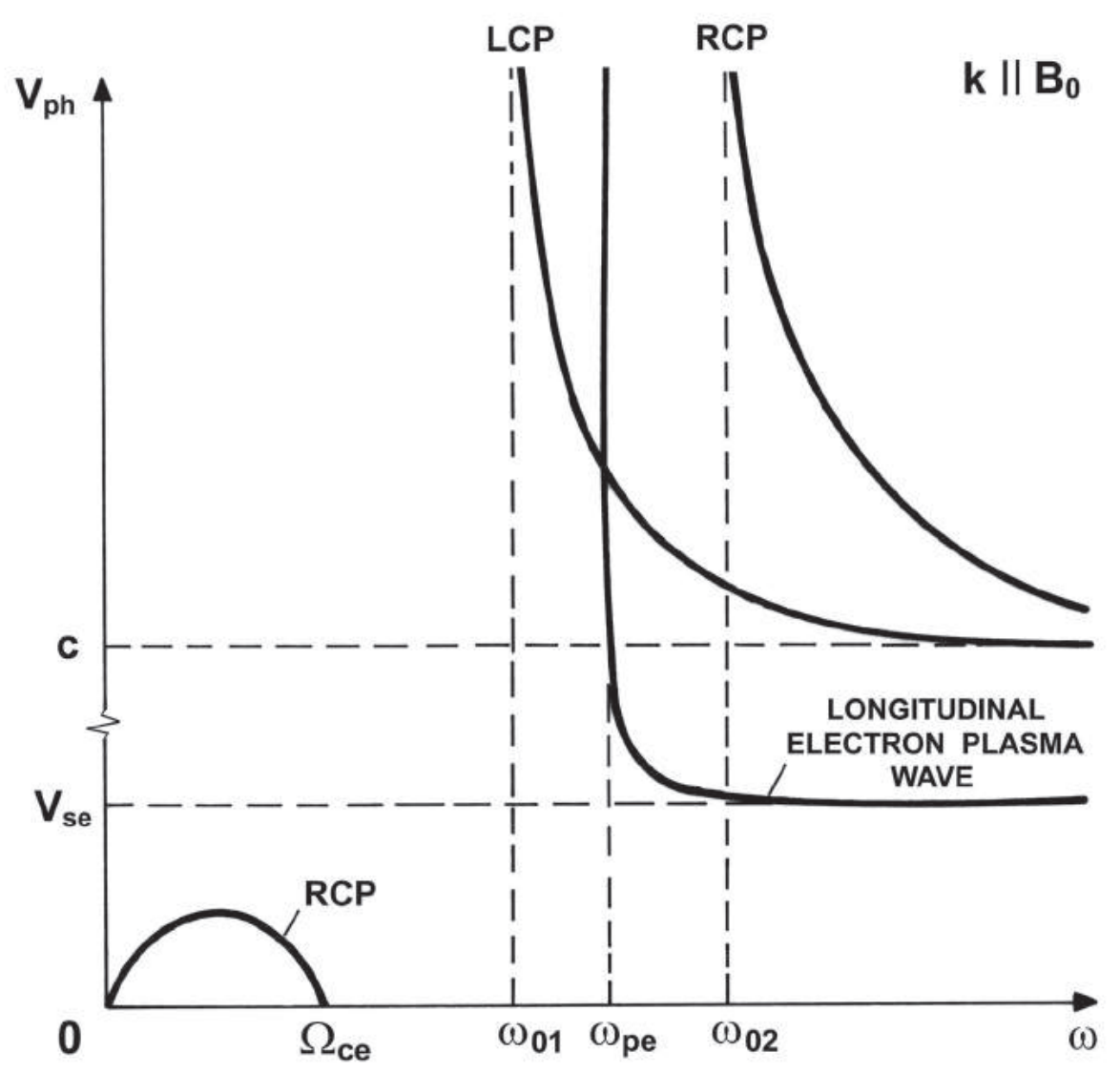

Fig. 4 Phase velocity as a function of frequency for waves propagating along the magnetic field in a warm electron gas.

oscillation at $\omega_{p e}$ (present in the cold plasma model) there is, in this case, an additional mode of propagation, known as the electron plasma wave. Neglecting collisions, (4.13) becomes the same dispersion relation as obtained in section 2, given in (2.24), for waves in an isotropic warm plasma. Hence, for propagation along the magnetic field, the longitudinal electron plasma wave is not affected by the presence of the magnetic field.

In summary, there are three modes of propagation in a warm electron gas for $\mathbf{k}$ parallel to the magnetic field. They are the transverse $R C P$ and LCP waves and the longitudinal electron plasma wave. The addition of the pressure gradient term in the equation of motion for the electrons has no effect on the transverse waves. A plot of phase velocity versus frequency for these three modes is displayed in Fig. 4. The corresponding $\omega(k)$ dispersion plot is shown in Fig. 5. 


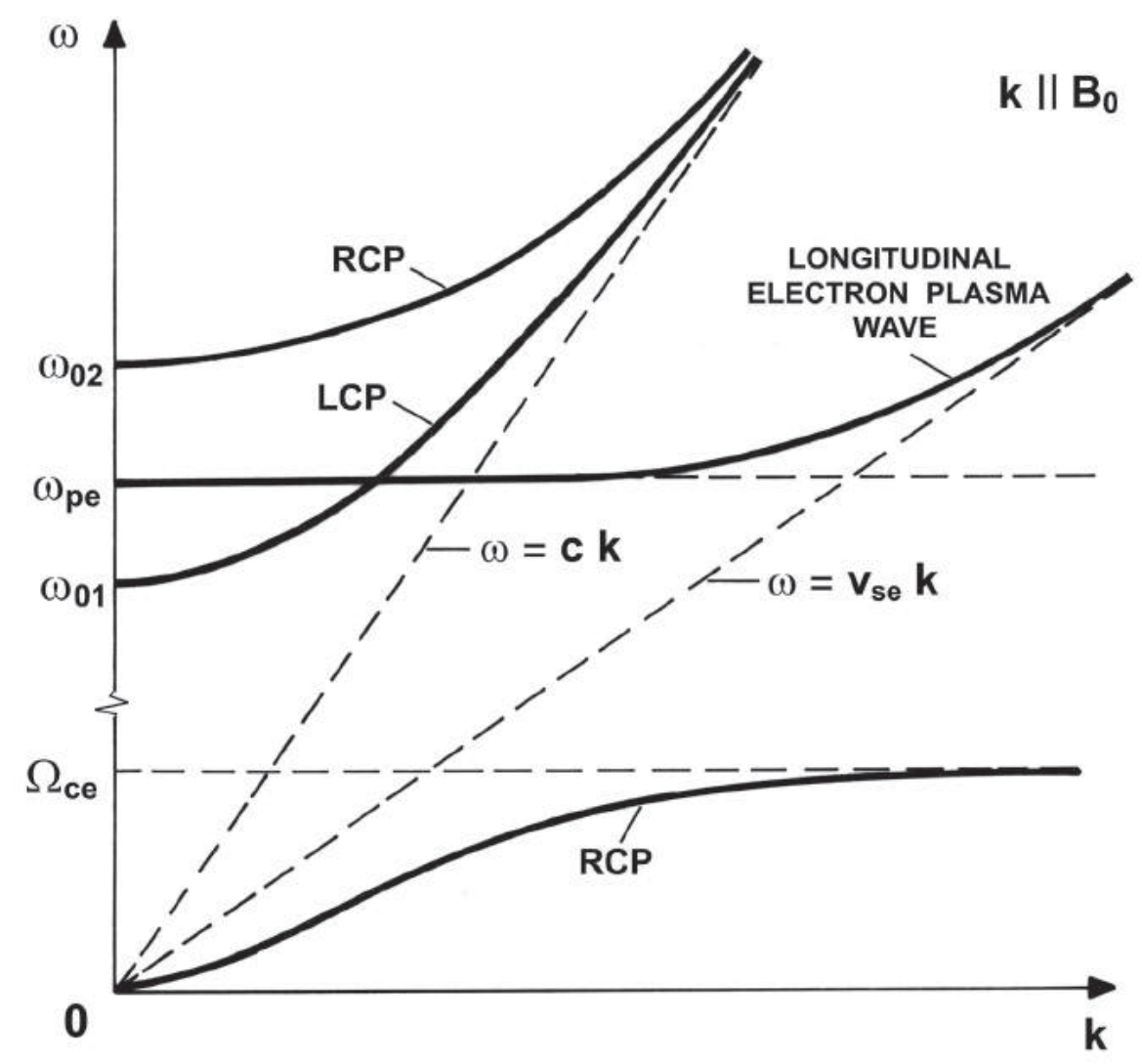

Fig. 5 Dispersion plot for waves propagating along the magnetic field in a warm electron gas.

\subsection{Wave Propagation Normal to the Magnetic Field}

For the case of propagation across the magnetic field $\left(\mathbf{k} \perp \mathbf{B}_{0}\right)$ we have $\mathbf{k}=k \widehat{\mathbf{x}}$ and $\theta=90^{\circ}$, so that (3.7) and (4.3) simplify to

$$
\begin{gathered}
\mathcal{A}=\left(\begin{array}{ccc}
1 & 0 & 0 \\
0 & \left(1-\eta^{2}\right) & 0 \\
0 & 0 & \left(1-\eta^{2}\right)
\end{array}\right) \\
\mathcal{B}=\left(\begin{array}{ccc}
\left(U-a^{2}\right) & i Y & 0 \\
-i Y & U & 0 \\
0 & 0 & U
\end{array}\right)
\end{gathered}
$$


From these expressions it is clear that the $z$ component of (4.4) is uncoupled from the $x$ and $y$ components. Thus, for transverse waves with electron motion along the $z$ axis $\left(u_{e z} \neq 0\right)$ we must have from the $z$ component of (4.4),

$$
U-\frac{X}{1-\eta^{2}}=0
$$

or

$$
\eta^{2}=1-\frac{X}{U}
$$

which is the familiar dispersion relation for the transverse ordinary wave (in which the electric field of the wave oscillates in the same direction as $\mathbf{B}_{0}$ ) found in section 7, Chapter 16 [see equation (16.7.4)].

From (4.4), (4.14), and (4.15) it is clear that the equations for $u_{e x}$ and $u_{e y}$ are coupled. Therefore, in order to have nontrivial solutions (longitudinal wave for $u_{e x} \neq 0$ and transverse wave for $u_{e y} \neq 0$ ) we must require the determinant formed with the coefficients of the $x$ and $y$ components of (4.4) to vanish,

$$
\operatorname{det}\left(\begin{array}{cc}
\left(U-a^{2}-X\right) & i Y \\
-i Y & \left(U-\frac{X}{1-\eta^{2}}\right)
\end{array}\right)=0
$$

This determinant gives, neglecting collisions,

$$
\left(\omega^{2}-V_{s e}^{2} k^{2}-\omega_{p e}^{2}\right)\left(\omega^{2}-\frac{\omega_{p e}^{2}}{1-\eta^{2}}\right)-\omega^{2} \Omega_{c e}^{2}=0
$$

Expanding this expression and rearranging, we get

$$
\begin{gathered}
c^{2} V_{s e}^{2} k^{4}-\left[V_{s e}^{2}\left(\omega^{2}-\omega_{p e}^{2}\right)+c^{2}\left(\omega^{2}-\omega_{p e}^{2}-\Omega_{c e}^{2}\right)\right] k^{2}+ \\
\left(\omega^{2}-\omega_{p e}^{2}\right)^{2}-\omega^{2} \Omega_{c e}^{2}=0
\end{gathered}
$$

This dispersion relation is quadratic in $k^{2}$, so that there will be in general two values of $k^{2}$ as a function of $\omega$, that is, two modes of propagation. Since generally we have $V_{s e} \ll c$, the first term within brackets in the left-hand side of (4.20) can be neglected as compared to the other one. With this approximation (4.20) becomes

$$
c^{2} V_{s e}^{2} k^{4}-c^{2}\left(\omega^{2}-\omega_{p e}^{2}-\Omega_{c e}^{2}\right) k^{2}+\left(\omega^{2}-\omega_{p e}^{2}\right)^{2}-\omega^{2} \Omega_{c e}^{2}=0
$$

Although it is not difficult to obtain the exact solution of this equation, it is more instructive to analyze it for some special limiting cases. 
First, let us obtain the approximate solution of (4.21) in the region where $\omega^{2} \gg k^{2} V_{s e}^{2}$, that is, when the term $c^{2} V_{s e}^{2} k^{4}$ is smaller than any of the others. For $k^{2}$ positive, this condition implies phase velocities much larger than $V_{s e}$ and, for this reason, it will be referred to as the high-phase velocity limit. With this condition, (4.21) reduces to

$$
-k^{2} c^{2}\left(\omega^{2}-\omega_{p e}^{2}-\Omega_{c e}^{2}\right)+\left(\omega^{2}-\omega_{p e}^{2}\right)^{2}-\omega^{2} \Omega_{c e}^{2}=0
$$

or

$$
k^{2} c^{2}=\frac{\left(\omega^{2}+\omega \Omega_{c e}-\omega_{p e}^{2}\right)\left(\omega^{2}-\omega \Omega_{c e}-\omega_{p e}^{2}\right)}{\left(\omega^{2}-\omega_{p e}^{2}-\Omega_{c e}^{2}\right)} ; \quad\left(\omega^{2} \gg k^{2} V_{s e}^{2}\right)
$$

This equation is similar to the dispersion relation found in section 7, Chapter 16 [see equation (16.7.7)], for the extraordinary wave in a cold plasma, except that now the condition $\omega^{2} \gg k^{2} V_{s e}^{2}$ must be satisfied for (4.23) to be applicable.

Next, let us obtain the approximate solution of (4.21) in the region where $\omega^{2} \ll k^{2} c^{2}$. For $k^{2}$ positive, this condition implies phase velocities much smaller than the velocity of light and, for this reason, it will be referred to as the low-phase velocity limit. Thus, in this limit (4.21) reduces to

$$
c^{2} V_{s e}^{2} k^{4}-c^{2}\left(\omega^{2}-\omega_{p e}^{2}-\Omega_{c e}^{2}\right) k^{2}=0
$$

or

$$
\omega^{2}=\omega_{p e}^{2}+\Omega_{c e}^{2}+V_{s e}^{2} k^{2} \quad ; \quad\left(\omega^{2} \ll k^{2} c^{2}\right)
$$

When $\mathbf{B}_{0}=0$ (i.e., $\Omega_{c e}=0$ ) this equation becomes identical to the dispersion relation for the longitudinal electron plasma wave [see equation (2.24)]. It is a valid solution for (4.21) only under the condition $\omega^{2} \ll k^{2} c^{2}$.

Fig. 6 displays the phase velocity as a function of frequency for the transverse ordinary mode (4.17) and for the two modes described by (4.20). Note that, of these last two modes, one is a purely transverse extraordinary wave, while the other one is partially transverse (i.e., electromagnetic extraordinary wave in the high-phase velocity limit) and partially longitudinal (i.e., electron plasma wave in the low-phase velocity limit). In this last mode the transition from a basically transverse electromagnetic wave to a basically longitudinal electron plasma wave occurs in the frequency range where the phase velocity lies between $c$ and $V_{s e}$. The corresponding $\omega(k)$ dispersion plot is shown in Fig. 7. 


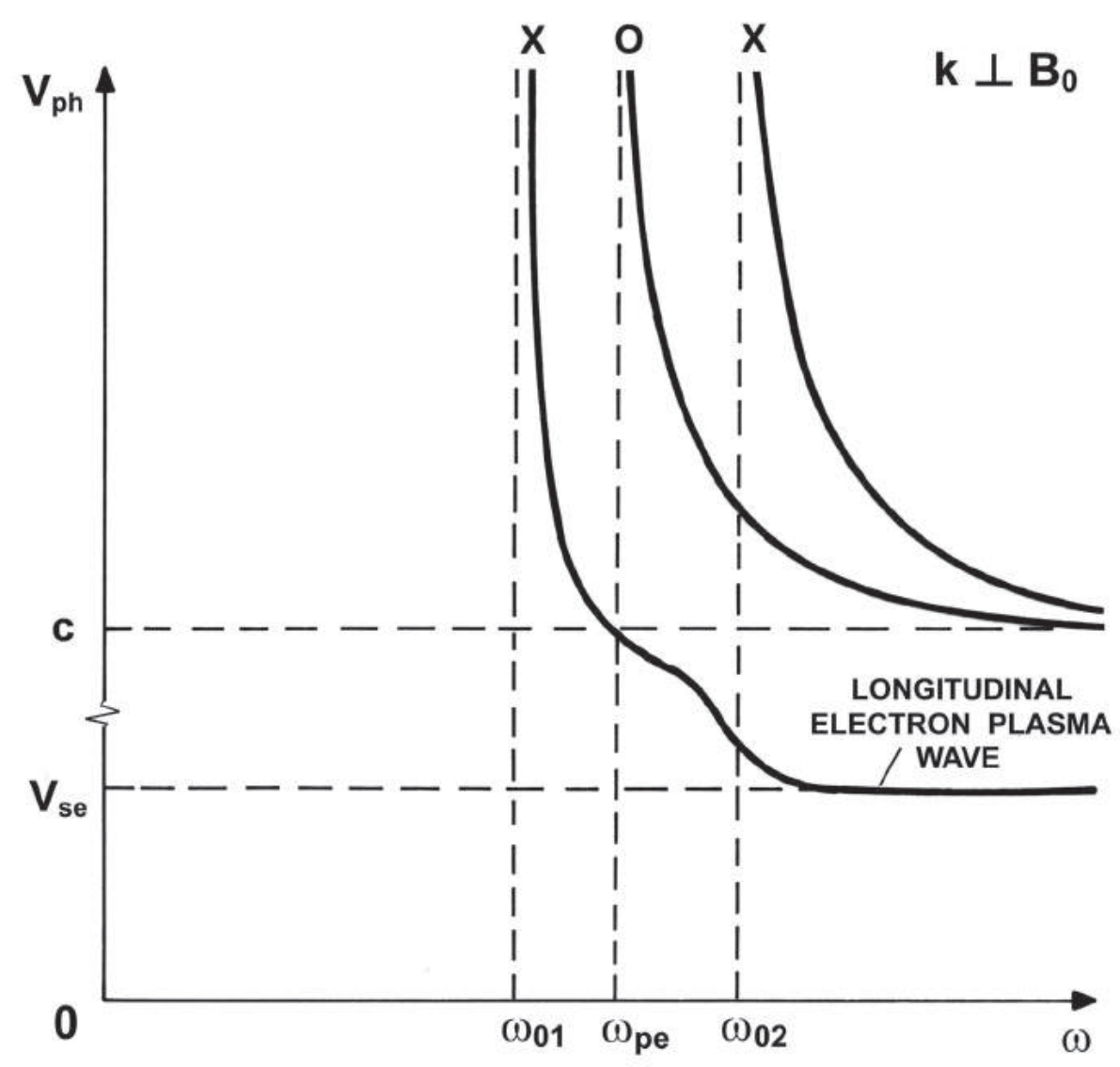

Fig. 6 Phase velocity as a function of frequency for waves propagating perpendicular to the magnetic field in a warm electron gas.

\subsection{Wave Propagation at Arbitrary Directions}

For propagation at an arbitrary direction with respect to the magnetic field, the dispersion relation can be obtained from (4.5), with the dyads $\mathcal{A}$ and $\mathcal{B}$ given by (3.7) and (4.3). For an arbitrary angle between $0^{\circ}$ and $90^{\circ}$, we expect the phase velocity versus frequency curves to lie between those of Figs. 4 and 6. Therefore, instead of getting involved in the cumbersome algebra behind (4.5), we shall present only the dispersion curves of Fig. 8, in which the shaded area illustrates how the transition occurs from $\theta=0^{\circ}$ to $\theta=90^{\circ}$. It can be easily verified that the only resonance that exists for an arbitrary angle occurs approximately at the frequency $\omega=\Omega_{c e} \cos \theta$. 


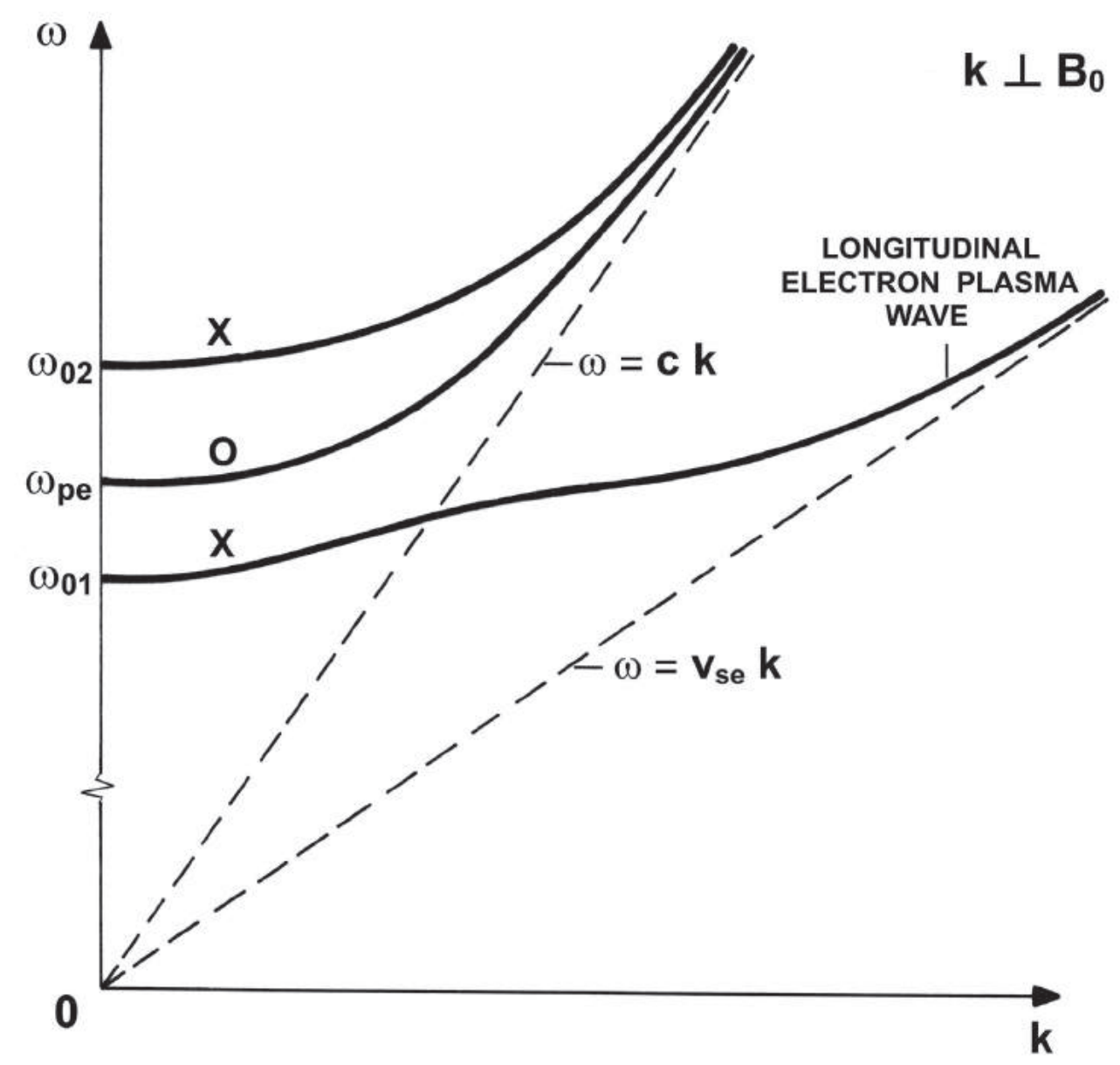

Fig. 7 Dispersion plot for waves propagating perpendicular to the magnetic field in a warm electron gas.

The reflection points, for any angle of propagation, occur at the frequencies $\omega_{01}, \omega_{p e}$, and $\omega_{02}$.

\section{WAVES IN A FULLY IONIZED WARM MAGNETOPLASMA}

We shall consider now the propagation of plane waves in a fully ionized warm plasma having only one ion species, immersed in an externally applied uniform magnetostatic field. 


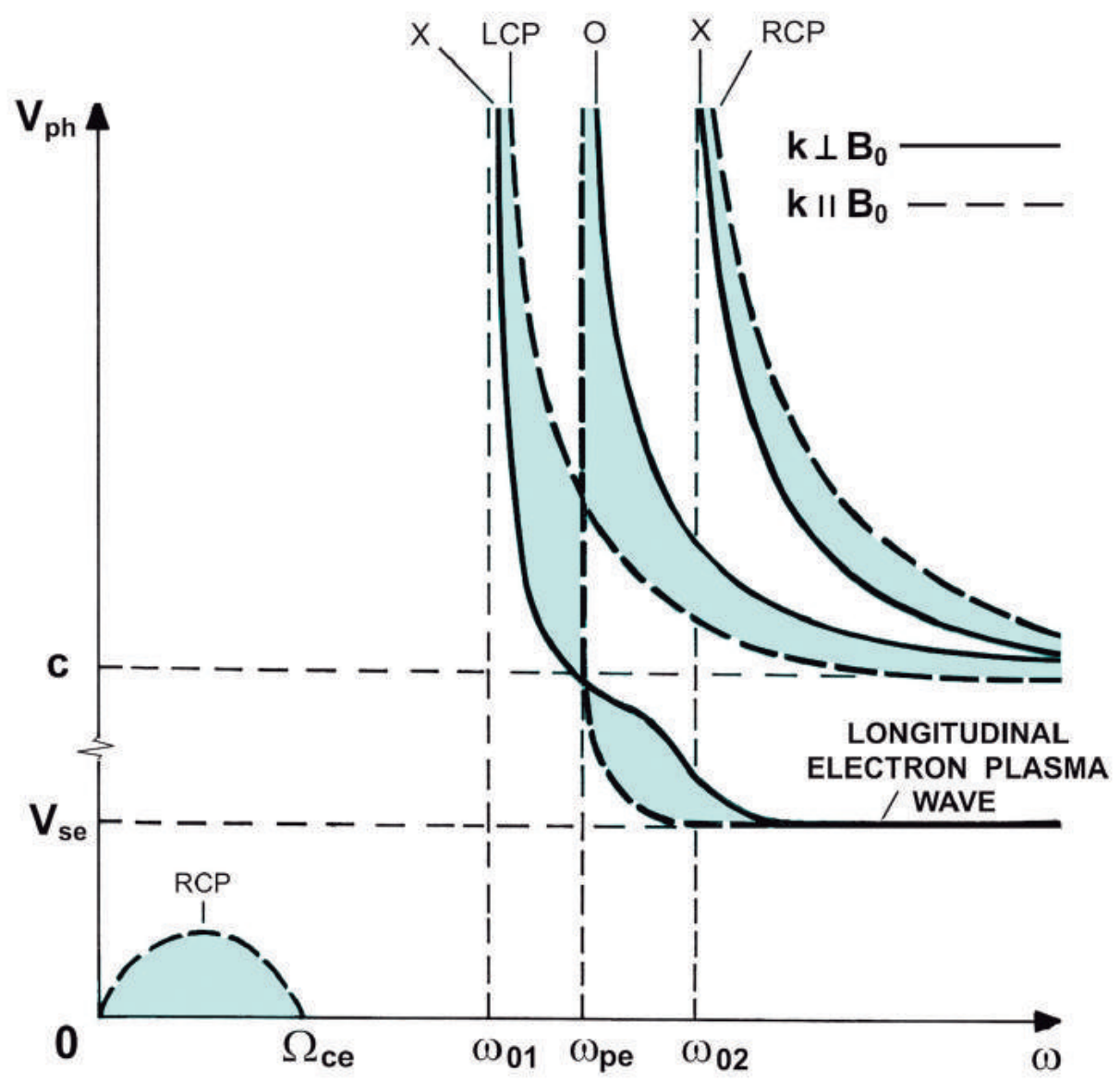

Fig. 8 Phase velocity versus frequency for wave propagation in a warm electron gas immersed in a magnetic field.

\subsection{Derivation of the Dispersion Relation}

The equation of motion for the electrons is, from (3.5),

$$
\begin{gathered}
\omega^{2} \mathbf{u}_{e}+i \omega \Omega_{c e}\left(u_{e y} \widehat{\mathbf{x}}-u_{e x} \widehat{\mathbf{y}}\right)- \\
V_{s e}^{2} k^{2}\left(\sin \theta u_{e x}+\cos \theta u_{e z}\right)(\sin \theta \widehat{\mathbf{x}}+\cos \theta \widehat{\mathbf{z}})+ \\
i \omega \nu_{e i}\left(\mathbf{u}_{e}-\mathbf{u}_{i}\right)=-\frac{i \omega e}{m_{e}} \mathbf{E}
\end{gathered}
$$

and for the ions,

$$
\begin{gathered}
\omega^{2} \mathbf{u}_{i}-i \omega \Omega_{c i}\left(u_{i y} \widehat{\mathbf{x}}-u_{i x} \widehat{\mathbf{y}}\right)- \\
V_{s i}^{2} k^{2}\left(\sin \theta u_{i x}+\cos \theta u_{i z}\right)(\sin \theta \widehat{\mathbf{x}}+\cos \theta \widehat{\mathbf{z}})+
\end{gathered}
$$




$$
i \omega \nu_{i e}\left(\mathbf{u}_{i}-\mathbf{u}_{e}\right)=\frac{i \omega e}{m_{i}} \mathbf{E}
$$

Equations (5.1) and (5.2), involving the variables $\mathbf{u}_{e}, \mathbf{u}_{i}$, and $\mathbf{E}$, are complemented by (3.6),

$$
\mathcal{A} \cdot \mathbf{E}=\frac{i e n_{0}}{\omega \epsilon_{0}}\left(\mathbf{u}_{e}-\mathbf{u}_{i}\right)
$$

where the dyad $\mathcal{A}$ is defined according to (3.7).

Equations (5.1) and (5.2) can be written, respectively, in compact form, as

$$
\mathcal{B}_{e} \cdot \mathbf{u}_{e}=-\frac{i e}{\omega m_{e}} \mathbf{E}-\frac{i \nu_{e i}}{\omega}\left(\mathbf{u}_{e}-\mathbf{u}_{i}\right)
$$

and

$$
\mathcal{B}_{i} \cdot \mathbf{u}_{i}=\frac{i e}{\omega m_{i}} \mathbf{E}-\frac{i \nu_{i e}}{\omega}\left(\mathbf{u}_{i}-\mathbf{u}_{e}\right)
$$

where the dyads $\mathcal{B}_{e}$ and $\mathcal{B}_{i}$ are appropriately defined by

$$
\begin{gathered}
\mathcal{B}_{e}=\left(\begin{array}{ccc}
\left(1-a^{2} \sin ^{2} \theta\right) & i Y_{e} & -a^{2} \sin \theta \cos \theta \\
-i Y_{e} & 1 & 0 \\
-a^{2} \sin \theta \cos \theta & 0 & \left(1-a^{2} \cos ^{2} \theta\right)
\end{array}\right) \\
\mathcal{B}_{i}=\left(\begin{array}{ccc}
\left(1-b^{2} \sin ^{2} \theta\right) & -i Y_{i} & -b^{2} \sin \theta \cos \theta \\
i Y_{i} & 1 & 0 \\
-b^{2} \sin \theta \cos \theta & 0 & \left(1-b^{2} \cos ^{2} \theta\right)
\end{array}\right)
\end{gathered}
$$

where $Y_{e}=\left(\Omega_{c e} / \omega\right), Y_{i}=\left(\Omega_{c i} / \omega\right)$, and $b^{2}=\left(V_{s i}^{2} k^{2} / \omega^{2}\right)$. Multiplying (5.4) and (5.5), respectively, by the inverse matrices corresponding to $\mathcal{B}_{e}$ and $\mathcal{B}_{i}$, we get

$$
\begin{gathered}
\mathbf{u}_{e}=-\frac{i e}{\omega m_{e}}\left(\mathcal{B}_{e}\right)^{-1} \cdot \mathbf{E}-\frac{i \nu_{e i}}{\omega}\left(\mathcal{B}_{e}\right)^{-1} \cdot\left(\mathbf{u}_{e}-\mathbf{u}_{i}\right) \\
\mathbf{u}_{i}=\frac{i e}{\omega m_{i}}\left(\mathcal{B}_{i}\right)^{-1} \cdot \mathbf{E}+\frac{i \nu_{i e}}{\omega}\left(\mathcal{B}_{i}\right)^{-1} \cdot\left(\mathbf{u}_{e}-\mathbf{u}_{i}\right)
\end{gathered}
$$

Subtracting (5.9) from (5.8) and rearranging, yields

$$
\begin{gathered}
{\left[\mathbf{1}+\frac{i \nu_{e i}}{\omega}\left(\mathcal{B}_{e}\right)^{-1}+\frac{i \nu_{i e}}{\omega}\left(\mathcal{B}_{i}\right)^{-1}\right] \cdot\left(\mathbf{u}_{e}-\mathbf{u}_{i}\right)+} \\
\frac{i e}{\omega}\left[\frac{1}{m_{e}}\left(\mathcal{B}_{e}\right)^{-1}+\frac{1}{m_{i}}\left(\mathcal{B}_{i}\right)^{-1}\right] \cdot \mathbf{E}=0
\end{gathered}
$$


Combining (5.10) and (5.3) to eliminate the variable $\left(\mathbf{u}_{e}-\mathbf{u}_{i}\right)$ results in the following equation involving only the electric field vector,

$$
\begin{gathered}
\left\{\left[\mathbf{1}+\frac{i \nu_{e i}}{\omega}\left(\mathcal{B}_{e}\right)^{-1}+\frac{i \nu_{i e}}{\omega}\left(\mathcal{B}_{i}\right)^{-1}\right] \cdot \mathcal{A}-\right. \\
\left.X_{e}\left(\mathcal{B}_{e}\right)^{-1}-X_{i}\left(\mathcal{B}_{i}\right)^{-1}\right\} \cdot \mathbf{E}=0
\end{gathered}
$$

where $X_{e}=\omega_{p e}^{2} / \omega^{2}$ and $X_{i}=\omega_{p i}^{2} / \omega^{2}$. This equation is of the form

$$
\mathcal{C} \cdot \mathbf{E}=0
$$

where $\mathcal{C}$ stands for the matrix within braces in (5.11). As before, the dispersion relation is obtained by setting the determinant corresponding to the matrix $\mathcal{C}$ equal to zero,

$$
\operatorname{det}(\mathcal{C})=0
$$

If collisions are neglected, (5.13) simplifies to

$$
\operatorname{det}\left[\mathcal{A}-X_{e}\left(\mathcal{B}_{e}\right)^{-1}-X_{i}\left(\mathcal{B}_{i}\right)^{-1}\right]=0
$$

In the following subsections, in order to simplify the algebra involved, we shall neglect collisions and analyze the problem using (5.14).

\subsection{Wave Propagation Along the Magnetic Field}

For $\theta=0^{\circ}$ we have from (3.7), (5.6), and (5.7), respectively,

$$
\begin{aligned}
\mathcal{A} & =\left(\begin{array}{ccc}
\left(1-\eta^{2}\right) & 0 & 0 \\
0 & \left(1-\eta^{2}\right) & 0 \\
0 & 0 & 1
\end{array}\right) \\
\mathcal{B}_{e} & =\left(\begin{array}{ccc}
1 & i Y_{e} & 0 \\
-i Y_{e} & 1 & 0 \\
0 & 0 & \left(1-a^{2}\right)
\end{array}\right) \\
\mathcal{B}_{i} & =\left(\begin{array}{ccc}
1 & -i Y_{i} & 0 \\
i Y_{i} & 1 & 0 \\
0 & 0 & \left(1-b^{2}\right)
\end{array}\right)
\end{aligned}
$$


The inverses of the matrices (5.16) and (5.17) are, respectively,

$$
\begin{aligned}
\left(\mathcal{B}_{e}\right)^{-1} & =\left(\begin{array}{ccc}
\frac{1}{\left(1-Y_{e}^{2}\right)} & -\frac{i Y_{e}}{\left(1-Y_{e}^{2}\right)} & 0 \\
\frac{i Y_{e}}{\left(1-Y_{e}^{2}\right)} & \frac{1}{\left(1-Y_{e}^{2}\right)} & 0 \\
0 & 0 & \frac{1}{\left(1-a^{2}\right)}
\end{array}\right) \\
\left(\mathcal{B}_{i}\right)^{-1} & =\left(\begin{array}{ccc}
\frac{1}{\left(1-Y_{i}^{2}\right)} & \frac{i Y_{i}}{\left(1-Y_{i}^{2}\right)} & 0 \\
-\frac{i Y_{i}^{2}}{\left(1-Y_{i}^{2}\right)} & \frac{1}{\left(1-Y_{i}^{2}\right)} & 0 \\
0 & 0 & \frac{1}{\left(1-b^{2}\right)}
\end{array}\right)
\end{aligned}
$$

Substituting the matrices (5.15), (5.18), and (5.19), into (5.12) and setting $\nu_{e i}=\nu_{i e}=0$, we obtain

$$
\left(\begin{array}{ccc}
\alpha_{1} & \alpha_{2} & 0 \\
-\alpha_{2} & \alpha_{1} & 0 \\
0 & 0 & \alpha_{3}
\end{array}\right)\left(\begin{array}{c}
E_{x} \\
E_{y} \\
E_{z}
\end{array}\right)=0
$$

where

$$
\begin{gathered}
\alpha_{1}=1-\eta^{2}-\frac{X_{i}}{1-Y_{i}^{2}}-\frac{X_{e}}{1-Y_{e}^{2}} \\
\alpha_{2}=-\frac{i X_{i} Y_{i}}{1-Y_{i}^{2}}+\frac{i X_{e} Y_{e}}{1-Y_{e}^{2}} \\
\alpha_{3}=1-\frac{X_{i}}{1-b^{2}}-\frac{X_{e}}{1-a^{2}}
\end{gathered}
$$

It is clear from this matrix equation that the longitudinal component of the electric field $\left(E_{z}\right)$ is uncoupled from the transverse component $\left(E_{x}\right.$ and $E_{y}$ ).

Therefore, for longitudinal waves $\left(E_{z} \neq 0\right)$, the coefficient of $E_{z}$ in (5.20) must be equal to zero, which gives the following dispersion relation:

$$
1-\frac{X_{i}}{1-b^{2}}-\frac{X_{e}}{1-a^{2}}=0
$$

This dispersion relation can be rearranged in the following form:

$$
V_{s e}^{2} V_{s i}^{2} k^{4}+\left[\omega_{p e}^{2} V_{s i}^{2}+\omega_{p i}^{2} V_{s e}^{2}-\omega^{2}\left(V_{s e}^{2}+V_{s i}^{2}\right)\right] k^{2}+
$$




$$
\omega^{2}\left(\omega^{2}-\omega_{p e}^{2}-\omega_{p i}^{2}\right)=0
$$

which is identical to (2.22). Therefore, since it is a quadratic equation in $k^{2}$, there are in general two longitudinal modes of propagation. Note that these two longitudinal modes propagating along $\mathbf{B}_{0}$ are not affected by the magnetic field strength. This dispersion relation has already been analyzed in section 2, where it was shown that these two longitudinal modes are the electron plasma wave and the ion plasma wave.

For transverse waves $\left(E_{x} \neq 0\right.$ and $\left.E_{y} \neq 0\right)$ the dispersion relation, from (5.20), is given by

$$
\left(1-\eta^{2}-\frac{X_{i}}{1-Y_{i}^{2}}-\frac{X_{e}}{1-Y_{e}^{2}}\right)^{2}-\left(\frac{X_{i} Y_{i}}{1-Y_{i}^{2}}-\frac{X_{e} Y_{e}}{1-Y_{e}^{2}}\right)^{2}=0
$$

Using the notation

$$
\begin{gathered}
S=1-\frac{X_{i}}{1-Y_{i}^{2}}-\frac{X_{e}}{1-Y_{e}^{2}} \\
D=\frac{X_{i} Y_{i}}{1-Y_{i}^{2}}-\frac{X_{e} Y_{e}}{1-Y_{e}^{2}}
\end{gathered}
$$

and letting

$$
\begin{aligned}
& R=S+D \\
& L=S-D
\end{aligned}
$$

then (5.23) becomes

$$
\left(\eta^{2}-R\right)\left(\eta^{2}-L\right)=0
$$

There are, therefore, two modes that propagate along the magnetic field with dispersion relations given by

$$
\left(\eta^{2}\right)_{R}=R
$$

and

$$
\left(\eta^{2}\right)_{L}=L
$$

From the $x$ component of (5.20) we have

$$
\frac{E_{y}}{E_{x}}=\frac{S-\eta^{2}}{i D}
$$

so that, using (5.29), we obtain

$$
\left(\frac{E_{y}}{E_{x}}\right)_{R}=i
$$

whereas, using (5.30),

$$
\left(\frac{E_{y}}{E_{x}}\right)_{L}=-i
$$

Therefore, the dispersion relation (5.29) corresponds to a right-hand circularly polarized wave and (5.30) to a left-hand circularly polarized wave. 


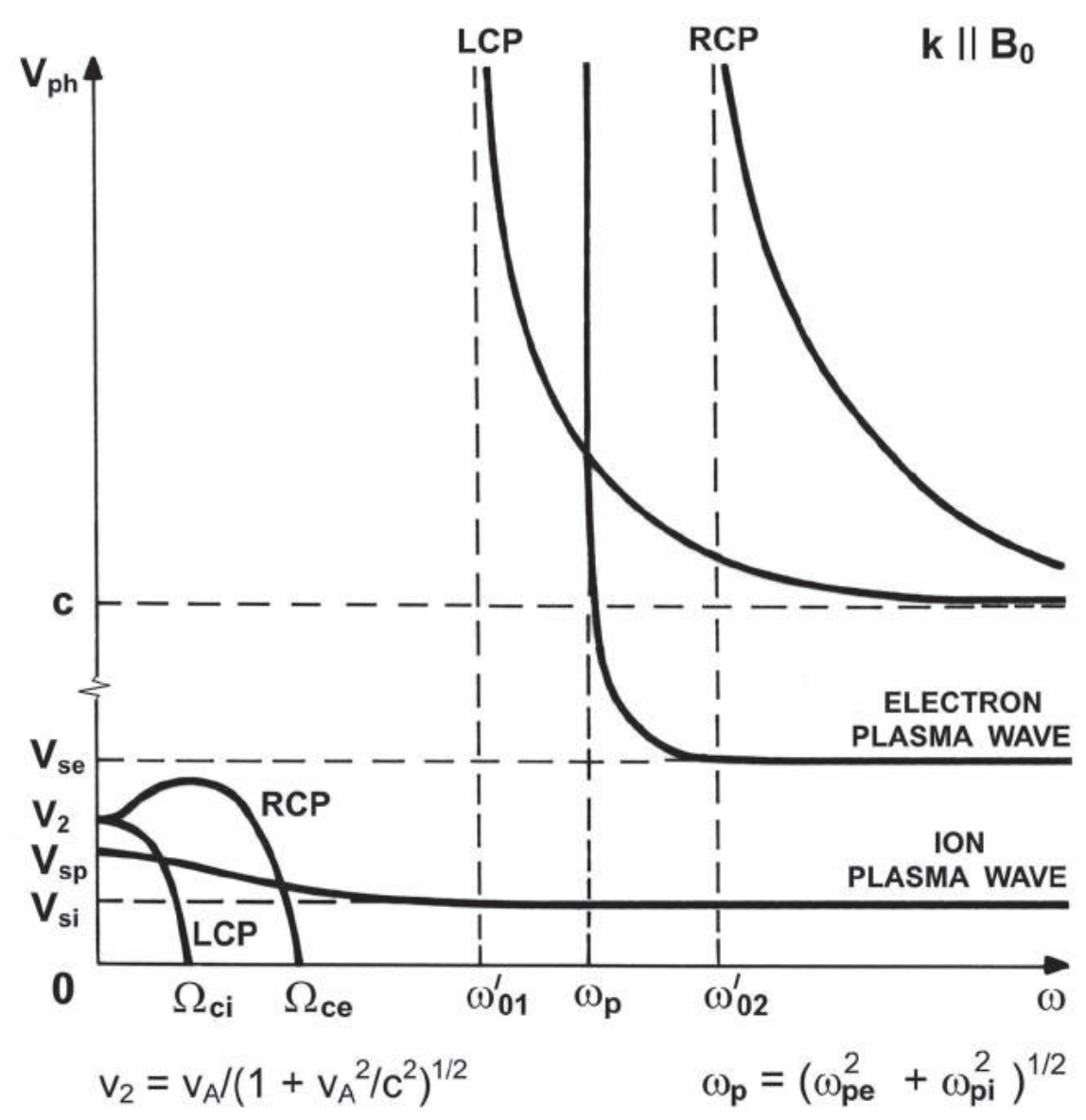

Fig. 9 Phase velocity as a function of frequency for plane waves traveling along the magnetic field in a warm fully ionized magnetoplasma.

The phase velocity as a function of frequency for propagation along $\mathbf{B}_{0}$ is shown in Fig. 9. The reflection points at $\omega_{01}^{\prime}$ and $\omega_{02}^{\prime}$ are not exactly the same ones given by equations (16.6.13) and (16.6.14), but are slightly different as a result of the inclusion of ion motion. Also, because ion motion has been taken into account, besides the resonance at $\Omega_{c e}$ for the RCP wave, there is also a resonance at $\Omega_{c i}$ for the LCP wave.

In the very low frequency limit, the phase velocities of the RCP and LCP waves tend to $V_{A} /\left(1+V_{A}^{2} / c^{2}\right)^{1 / 2}$, instead of going to zero as in the case of the cold electron plasma model. This result can be seen as follows. For very low frequency waves such that

$$
\omega \ll \Omega_{c i}
$$


we obtain, using (5.24) and (5.25),

$$
R=L=1+\frac{\omega_{p e}^{2}}{\Omega_{c e} \Omega_{c i}}
$$

Therefore, using the definitions of $\omega_{p e}, \Omega_{c e}$, and $\Omega_{c i}$, the dispersion relation for the RCP and LCP waves in the very low frequency limit becomes

$$
\eta^{2}=1+\frac{n_{0} m_{i}}{\epsilon_{0} B_{0}^{2}}
$$

The average mass density is $\rho_{m}=n_{0}\left(m_{e}+m_{i}\right) \simeq n_{0} m_{i}$, and since $\epsilon_{0}=$ $1 /\left(\mu_{0} c^{2}\right),(5.36)$ can be rewritten as

$$
\eta^{2}=1+\frac{c^{2} \mu_{0} \rho_{m}}{B_{0}^{2}}
$$

or

$$
\eta^{2}=1+\frac{c^{2}}{V_{A}^{2}}
$$

where $V_{A}=\left(B_{0}^{2} / \mu_{0} \rho_{m}\right)^{1 / 2}$ is the Alfvén velocity, defined in (15.1.4) (Eq. 1.4 in Chapter 15). Thus, from (5.38) in the very low frequency limit the phase velocity of both transverse waves is given by

$$
v_{p h}=\frac{\omega}{k}=\frac{V_{A}}{\left(1+V_{A}^{2} / c^{2}\right)^{1 / 2}}
$$

Note that, for plasmas in which $V_{A}^{2} \ll c^{2}$ (weak $\mathbf{B}_{0}$ field or high density), (5.39) reduces to $v_{p h}=V_{A}$. This very low frequency limit corresponds to the Alfvén wave discussed in Chapter 15.

\subsection{Wave Propagation Normal to the Magnetic Field}

Considering now $\theta=90^{\circ}$, we obtain from (3.7), (5.6), and (5.7),

$$
\begin{gathered}
\mathcal{A}=\left(\begin{array}{ccc}
1 & 0 & 0 \\
0 & \left(1-\eta^{2}\right) & 0 \\
0 & 0 & \left(1-\eta^{2}\right)
\end{array}\right) \\
\mathcal{B}_{e}=\left(\begin{array}{ccc}
\left(1-a^{2}\right) & i Y_{e} & 0 \\
-i Y_{e} & 1 & 0 \\
0 & 0 & 1
\end{array}\right)
\end{gathered}
$$




$$
\mathcal{B}_{i}=\left(\begin{array}{ccc}
\left(1-b^{2}\right) & -i Y_{i} & 0 \\
i Y_{i} & 1 & 0 \\
0 & 0 & 1
\end{array}\right)
$$

Taking the inverse of the matrices in (5.41) and (5.42), we obtain for (5.12), neglecting collisions,

$$
\left(\begin{array}{ccc}
S_{2} & -i D_{1} & 0 \\
i D_{1} & \left(S_{1}-\eta^{2}\right) & 0 \\
0 & 0 & \left(P-\eta^{2}\right)
\end{array}\right)\left(\begin{array}{c}
E_{x} \\
E_{y} \\
E_{z}
\end{array}\right)
$$

where

$$
\begin{gathered}
S_{1}=1-\frac{X_{i}\left(1-b^{2}\right)}{1-Y_{i}^{2}-b^{2}}-\frac{X_{e}\left(1-a^{2}\right)}{1-Y_{e}^{2}-a^{2}} \\
S_{2}=1-\frac{X_{i}}{1-Y_{i}^{2}-b^{2}}-\frac{X_{e}}{1-Y_{e}^{2}-a^{2}} \\
D_{1}=\frac{X_{i} Y_{i}}{1-Y_{i}^{2}-b^{2}}-\frac{X_{e} Y_{e}}{1-Y_{e}^{2}-a^{2}} \\
P=1-X_{i}-X_{e}
\end{gathered}
$$

From (5.43) it is clear that $E_{z}$ is uncoupled from the electric field components $E_{x}$ and $E_{y}$.

Therefore, the ordinary mode (the transverse mode, which has $E_{z} \neq 0$ and is not affected by the presence of the magnetostatic field) has the dispersion relation

$$
\eta^{2}=P
$$

or

$$
k^{2} c^{2}=\omega^{2}-\left(\omega_{p e}^{2}+\omega_{p i}^{2}\right)
$$

which is the same expression obtained in (2.39).

The modes involving the field components $E_{x}$ and $E_{y}$ (longitudinal for $E_{x} \neq 0$ and transverse for $E_{y} \neq 0$ ) are seen, from (5.43), to be coupled and have the following dispersion relation:

$$
S_{2}\left(S_{1}-\eta^{2}\right)-D_{1}^{2}=0
$$

Substituting the expressions for $S_{1}, S_{2}$, and $D_{1}$ into (5.50), results in a cubic equation in $k^{2}$, showing that in general there are three modes of propagation. A detailed analysis of this dispersion relation shows that 
these three modes of propagation are the partially transverse extraordinary wave, the longitudinal electron plasma wave, and the longitudinal ion plasma wave.

Fig. 10 shows the phase velocity as a function of frequency for the four modes of propagation in a direction normal to the magnetic field. The basic points to be noted in this plot are the following: (1) the presence of the reflection points at $\left(\omega_{p e}^{2}+\omega_{p i}^{2}\right)^{1 / 2}, \omega_{01}^{\prime}$ and $\omega_{02}^{\prime} ;(2)$ the transition from a basically longitudinal (electron plasma) wave to a basically transverse electromagnetic (extraordinary) wave, in the frequency region where the phase velocity lies between $V_{s e}$ and $c$; and (3) in the very low frequency limit the phase velocity of the ion plasma wave tends to

$$
v_{p h}=\left(\frac{V_{A}^{2}+V_{s p}^{2}}{1+V_{A}^{2} / c^{2}}\right)^{1 / 2}
$$

\subsection{Wave Propagation at Arbitrary Directions}

For arbitrary directions of propagation the dispersion relation is given by (5.14). Since a detailed analysis of this equation is a rather complex and tedious affair, we shall content ourselves by merely presenting the plot of phase velocity versus frequency in Fig. 11, in which the shaded areas give an indication of how the curves evolve from $\theta=0^{\circ}$ to $\theta=90^{\circ}$.

\section{SUMMARY}

The modes for wave propagation in a warm fully ionized plasma can be summarized as follows:

\section{(a) For $\mathbf{B}_{0}=0$ :}

Transverse electromagnetic wave.

Longitudinal electron plasma wave.

Longitudinal ion plasma wave.

\section{(b) For $\mathrm{k} \| \mathbf{B}_{0}$ :}

Transverse right-hand circularly polarized wave.

Transverse left-hand circularly polarized wave.

Longitudinal electron plasma wave.

Longitudinal ion plasma wave. 


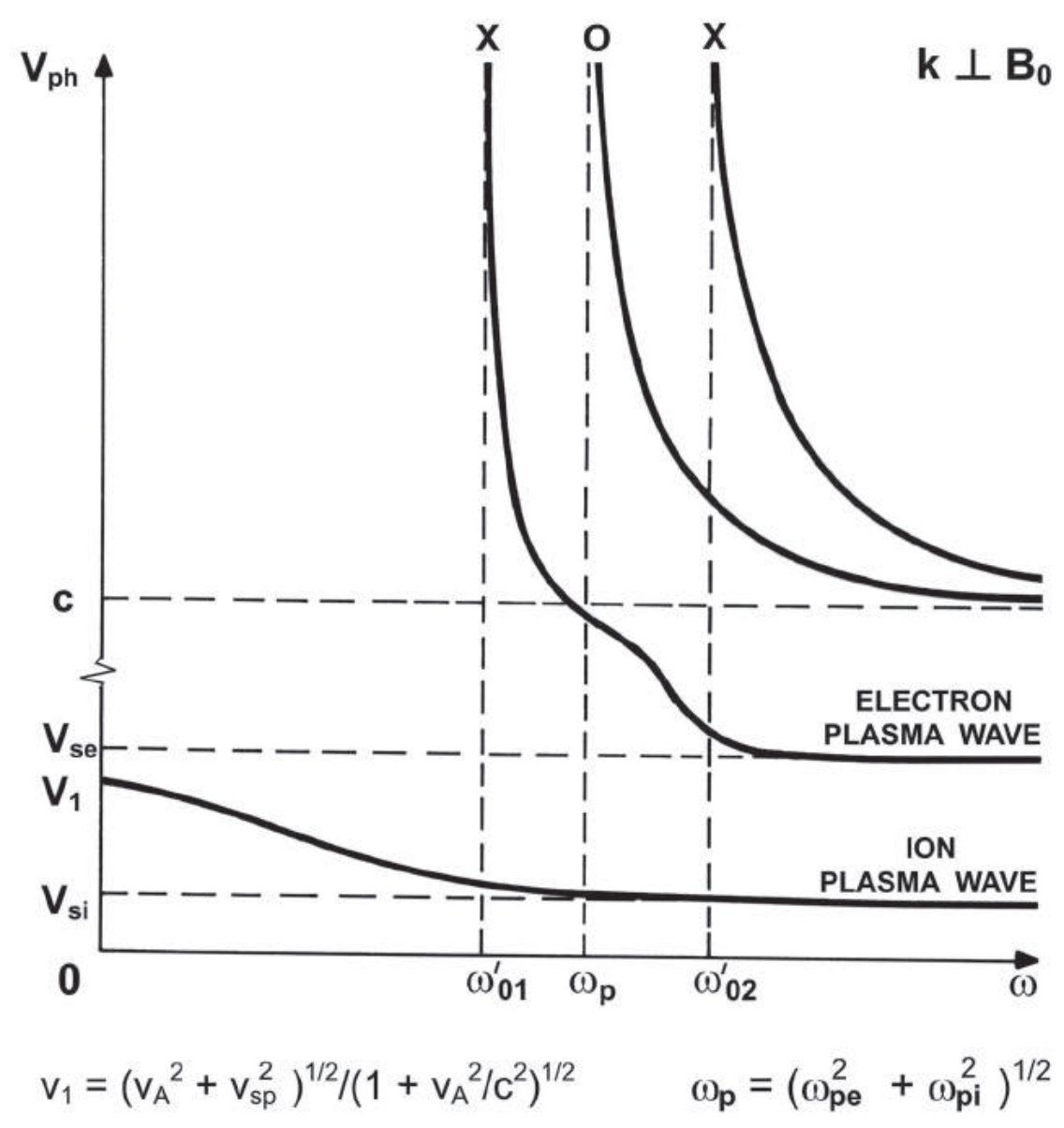

Fig. 10 Phase velocity as a function of frequency for waves propagating in a direction normal to the magnetic field in a warm fully ionized plasma.

\section{(c) For $\mathrm{k} \perp \mathbf{B}_{0}$ :}

Transverse ordinary wave.

Partially transverse extraordinary wave.

Longitudinal electron plasma wave.

Longitudinal ion plasma wave.

For a warm electron gas, in which the motion of the ions is ignored, the longitudinal ion plasma mode is absent. For a cold plasma, both the ion plasma and the electron plasma modes are absent. Note that for $\mathbf{k} \perp \mathbf{B}_{0}$ the electron mode and the extraordinary mode are coupled. 


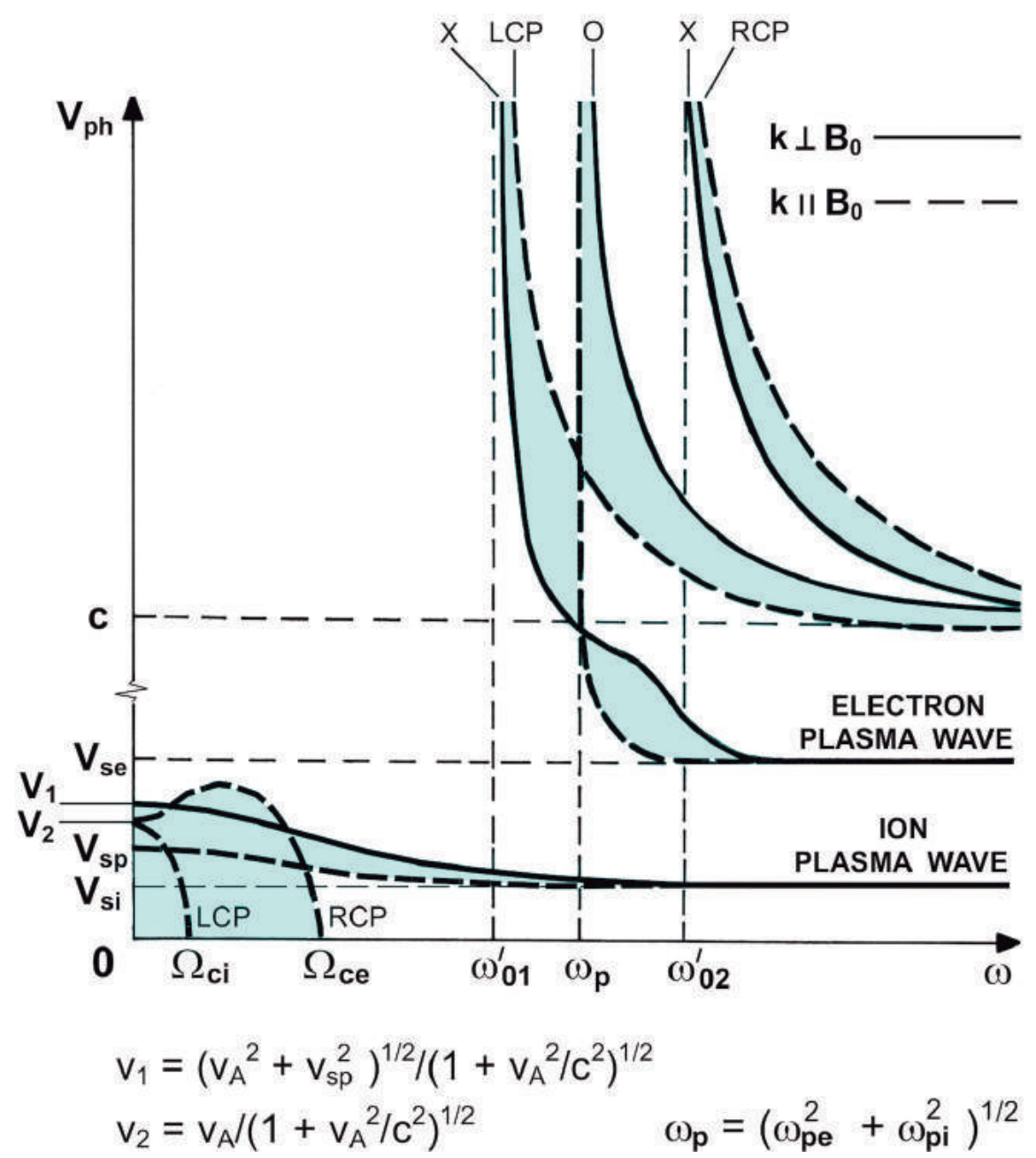

Fig. 11 Phase velocity as a function of frequency for wave propagation in a warm fully ionized plasma.

\section{PROBLEMS}

17.1 Show that one of the roots of the dispersion relation (2.33), at very low frequencies, corresponds to an evanescent wave.

17.2 Make a plot analogous to Fig. 8 for wave propagation in a warm electron gas immersed in a magnetic field, but in terms of $\omega$ as a function of the real part of $k$. 
17.3 Show that the reflection points $\omega_{01}^{\prime}$ and $\omega_{02}^{\prime}$, for the LCP and RCP waves propagating along the magnetic field in a fully ionized warm plasma (see Fig. 9) are given, respectively, by

$$
\begin{gathered}
\omega_{01}^{\prime}=-\frac{1}{2}\left(\Omega_{c e}-\Omega_{c i}\right)+\frac{1}{2}\left[\left(\Omega_{c e}+\Omega_{c i}\right)^{2}+4 \omega_{p e}^{2}\right]^{1 / 2} \\
\omega_{02}^{\prime}=\frac{1}{2}\left(\Omega_{c e}-\Omega_{c i}\right)+\frac{1}{2}\left[\left(\Omega_{c e}+\Omega_{c i}\right)^{2}+4 \omega_{p e}^{2}\right]^{1 / 2}
\end{gathered}
$$

Compare these expressions with (16.6.13) and (16.6.14).

17.4 Starting from (5.12), (5.40), (5.41), and (5.42), provide all the necessary steps to obtain (5.43).

17.5 Obtain a cubic equation in $k^{2}$, from (5.50), and analyze the dispersion relations for these three modes of wave propagation across the magnetic field in a fully ionized warm plasma.

17.6 Make plots analogous to Figs. 9, 10, and 11 for wave propagation in a fully ionized warm plasma, but in terms of $\omega$ as a function of the real part of $k$.

17.7 Show that the resonances in a warm fully ionized magnetoplasma, neglecting collisions, occur approximately at the frequencies $\omega=\Omega_{c e} \cos \theta$ and $\omega=\Omega_{c i} \cos \theta$. 


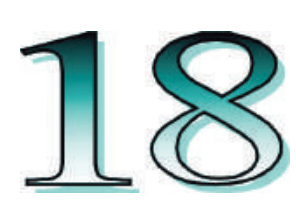

\section{WAVES IN HOT ISOTROPIC PLASMAS}

\section{INTRODUCTION}

We consider in this chapter the propagation of small-amplitude waves in unbounded hot plasmas that are close to equilibrium conditions, from the kinetic theory point of view. The problem will be examined using the Vlasov equation, and only electron motion will be considered. The ions, in view of their greater inertia, are assumed to stay immobile. A major point of this chapter will be to emphasize those effects that arise when the Vlasov equation is used and that were missing when the problem was treated using the cold and warm plasma models (Chapters 16 and 17).

The treatment present here is restricted to isotropic plasmas, in the absence of an externally applied magnetic field. It will be shown that the plasma waves can be separated into three groups, the first group being the longitudinal plasma wave (also known as space charge wave or Langmuir wave), and the second and third groups being the two different polarizations of the transverse electromagnetic wave. The chapter ends with a brief discussion of plasma instabilities that arise from the interaction of the plasma particles with the wave electric field. To illustrate the waveparticle interaction phenomenon we describe one important example, the so-called two-stream instability.

\section{BASIC EQUATIONS}

The relevant equations for the kinetic theory treatment of smallamplitude waves in a electron gas of infinite extent are the Vlasov and Maxwell equations. 
The Vlasov equation satisfied by the electron distribution function $f(\mathbf{r}, \mathbf{v}, t)$, can be written as

$$
\begin{gathered}
\frac{\partial f(\mathbf{r}, \mathbf{v}, t)}{\partial t}+\mathbf{v} \cdot \nabla f(\mathbf{r}, \mathbf{v}, t)+\left\{-\frac{e}{m_{e}}[\mathbf{E}(\mathbf{r}, t)+\mathbf{v} \times \mathbf{B}(\mathbf{r}, t)]+\right. \\
\left.\frac{\mathbf{F}_{e x t}}{m_{e}}\right\} \cdot \nabla_{v} f(\mathbf{r}, \mathbf{v}, t)=0
\end{gathered}
$$

where $\mathbf{F}_{\text {ext }}$ denotes any force externally applied to the plasma, and $\mathbf{E}(\mathbf{r}, t)$ and $\mathbf{B}(\mathbf{r}, t)$ are the internal smoothed, self-consistent, macroscopic electric and magnetic induction fields associated with the distributions of charge density and charge current density inside the plasma. The fields $\mathbf{E}(\mathbf{r}, t)$ and $\mathbf{B}(\mathbf{r}, t)$ satisfy Maxwell equations

$$
\begin{gathered}
\nabla \cdot \mathbf{E}(\mathbf{r}, t)=\frac{\rho(\mathbf{r}, t)}{\epsilon_{0}} \\
\nabla \cdot \mathbf{B}(\mathbf{r}, t)=0 \\
\nabla \times \mathbf{E}(\mathbf{r}, t)=-\frac{\partial \mathbf{B}(\mathbf{r}, t)}{\partial t} \\
\nabla \times \mathbf{B}(\mathbf{r}, t)=\mu_{0} \mathbf{J}(\mathbf{r}, t)+\frac{1}{c^{2}} \frac{\partial \mathbf{E}(\mathbf{r}, t)}{\partial t}
\end{gathered}
$$

where the charge and current densities are given, respectively, by

$$
\begin{gathered}
\rho(\mathbf{r}, t)=\sum_{\alpha} q_{\alpha} n_{\alpha}(\mathbf{r}, t)=\sum_{\alpha} q_{\alpha} \int_{v} f_{\alpha}(\mathbf{r}, \mathbf{v}, t) d^{3} v \\
\mathbf{J}(\mathbf{r}, t)=\sum_{\alpha} q_{\alpha} n_{\alpha}(\mathbf{r}, t) \mathbf{u}_{\alpha}(\mathbf{r}, t)=\sum_{\alpha} q_{\alpha} \int_{v} \mathbf{v} f_{\alpha}(\mathbf{r}, \mathbf{v}, t) d^{3} v
\end{gathered}
$$

Equations (2.1) to (2.7) form a complete self-consistent set of equations, which were first introduced in section 7 of Chapter 5 . It is worth noting that even though there is no explicit collision term in the Vlasov equation (2.1), an important contribution to the charged particle interactions is included through the internal self-consistent electromagnetic fields. 


\section{GENERAL RESULTS FOR A PLANE WAVE IN A HOT ISOTROPIC PLASMA}

Consider an unbounded uniform electron plasma with a fixed neutralizing ion background, without any externally applied field and under equilibrium conditions. Suppose that at a given instant some electrons are slightly displaced from their equilibrium positions. As a result of this small space-dependent perturbation in the electron gas, some sort of oscillatory or wave phenomenon can be expected to arise as a consequence of the internal electric fields produced by charge separation. The ions, because of their much larger mass, can be assumed to remain nearly stationary during the process, since the frequencies involved will be sufficiently high. Since we are dealing with small deviations from equilibrium, the equations can be linearized, that is, the products of two nonequilibrium quantities (which are considered to be of second order) can be neglected.

\subsection{Perturbation Charge Density and Current Density}

To describe small deviations from equilibrium we express the electron distribution function in the form

$$
f(\mathbf{r}, \mathbf{v}, t)=f_{0}(v)+f_{1}(\mathbf{r}, \mathbf{v}, t) \quad\left(\left|f_{1}\right| \ll f_{0}\right)
$$

where $f_{0}(v)$ is the equilibrium distribution function, considered to be homogeneous and isotropic, whereas $f_{1}(\mathbf{r}, \mathbf{v}, t)$ is a perturbation in the distribution function, always small compared to $f_{0}(v)$. Before the application of the perturbation, the plasma is in equilibrium so that the macroscopic self-consistent electric and magnetic fields, as well as the charge and current densities, vanish throughout the plasma. The equilibrium number density of the electrons is everywhere the same as that of the ions, and is given by

$$
n_{0}=\int_{v} f_{0}(v) d^{3} v
$$

Since $f_{1}(\mathbf{r}, \mathbf{v}, t)$ is a first-order quantity, the internal electric and magnetic fields that arise due to the perturbation are also small first-order quantities. From (2.6) the perturbation charge density is given by

$$
\rho(\mathbf{r}, t)=e n_{0}-e \int_{v} f(\mathbf{r}, \mathbf{v}, t) d^{3} v
$$


Using (3.1) and (3.2), we obtain

$$
\rho(\mathbf{r}, t)=-e \int_{v} f_{1}(\mathbf{r}, \mathbf{v}, t) d^{3} v
$$

The perturbation current density is obtained from (2.7), noting that the ions are assumed to stay immobile,

$$
\mathbf{J}(\mathbf{r}, t)=-e \int_{v} \mathbf{v} f(\mathbf{r}, \mathbf{v}, t) d^{3} v
$$

Substituting (3.1) into (3.5) and considering that the current density in the equilibrium state vanishes, that is,

$$
-e \int_{v} \mathbf{v} f_{0}(\mathbf{r}, \mathbf{v}, t) d^{3} v=0
$$

we find

$$
\mathbf{J}(\mathbf{r}, t)=-e \int_{v} \mathbf{v} f_{1}(\mathbf{r}, \mathbf{v}, t) d^{3} v
$$

\subsection{Solution of the Linearized Vlasov Equation}

Substituting (3.1) into the Vlasov equation (2.1), without any external fields present, we obtain

$$
\begin{gathered}
\frac{\partial f_{1}(\mathbf{r}, \mathbf{v}, t)}{\partial t}+\mathbf{v} \cdot \nabla f_{1}(\mathbf{r}, \mathbf{v}, t)-\frac{e}{m_{e}}[\mathbf{E}(\mathbf{r}, t)+\mathbf{v} \times \mathbf{B}(\mathbf{r}, t)] \cdot \nabla_{v} f_{0}(v)- \\
\frac{e}{m_{e}}[\mathbf{E}(\mathbf{r}, t)+\mathbf{v} \times \mathbf{B}(\mathbf{r}, t)] \cdot \nabla_{v} f_{1}(\mathbf{r}, \mathbf{v}, t)=0
\end{gathered}
$$

Since $\mathbf{E}(\mathbf{r}, t), \mathbf{B}(\mathbf{r}, t)$, and $f_{1}(\mathbf{r}, \mathbf{v}, t)$ are first-order quantities, the last term in the left-hand side of (3.8) involves the product of two first-order quantities and therefore it is of second order and can be neglected as compared to the remaining terms. Thus, the linearized Vlasov equation becomes

$$
\frac{\partial f_{1}(\mathbf{r}, \mathbf{v}, t)}{\partial t}+\mathbf{v} \cdot \nabla f_{1}(\mathbf{r}, \mathbf{v}, t)-\frac{e}{m_{e}}[\mathbf{E}(\mathbf{r}, t)+\mathbf{v} \times \mathbf{B}(\mathbf{r}, t)] \cdot \nabla_{v} f_{0}(v)=0
$$

A convenient way to solve this equation is to use the method of integral transforms. For an initial-value problem, the equation is simplified by 
taking its Laplace transform in the time domain and the Fourier transform with respect to the space variables. This method reduces the differential equation to an algebraic equation that can then be solved for the desired transform variables. Next, in order to regain the original variables, we have to invert the Laplace and Fourier transforms of the dependent variables. This mathematical treatment, however, involves the calculation of some complicated contour integrals in the complex plane, which is beyond the scope of this text. Therefore, in order to simplify the mathematical analysis of the problem, without losing the essentials of the plasma behavior under consideration, we shall look for periodic harmonic solutions for $f_{1}(\mathbf{r}, \mathbf{v}, t)$ in the space and time variables, according to

$$
f_{1}(\mathbf{r}, \mathbf{v}, t)=f_{1}(\mathbf{v}) \exp (i \mathbf{k} \cdot \mathbf{r}-i \omega t)
$$

where the vectors involved are referred to a Cartesian coordinate system. With this particular choice for $f_{1}(\mathbf{r}, \mathbf{v}, t)(3.4)$ and (3.7) become

$$
\begin{aligned}
& \rho(\mathbf{r}, t)=\rho \exp (i \mathbf{k} \cdot \mathbf{r}-i \omega t) \\
& \mathbf{J}(\mathbf{r}, t)=\mathbf{J} \exp (i \mathbf{k} \cdot \mathbf{r}-i \omega t)
\end{aligned}
$$

where

$$
\begin{gathered}
\rho=-e \int_{v} f_{1}(\mathbf{v}) d^{3} v \\
\mathbf{J}=-e \int_{v} \mathbf{v} f_{1}(\mathbf{v}) d^{3} v
\end{gathered}
$$

Consequently, the macroscopic self-consistent electric and magnetic fields have the same harmonic space and time dependence,

$$
\begin{aligned}
& \mathbf{E}(\mathbf{r}, t)=\mathbf{E} \exp (i \mathbf{k} \cdot \mathbf{r}-i \omega t) \\
& \mathbf{B}(\mathbf{r}, t)=\mathbf{B} \exp (i \mathbf{k} \cdot \mathbf{r}-i \omega t)
\end{aligned}
$$

Furthermore, since we are assuming that the equilibrium distribution function $f_{0}(v)$ is a function of only the magnitude of $\mathbf{v}$, we have the very useful identity

$$
\nabla_{v} f_{0}(v)=\frac{\mathbf{v}}{v} \frac{d f_{0}(v)}{d v}
$$

so that, for the term involving the magnetic force in (3.9), we have

$$
[\mathbf{v} \times \mathbf{B}(\mathbf{r}, t)] \cdot \nabla_{v} f_{0}(v)=[\mathbf{v} \times \mathbf{B}(\mathbf{r}, t)] \cdot \frac{\mathbf{v}}{v} \frac{d f_{0}(v)}{d v}=0
$$


Substituting (3.10), (3.15), (3.16), and (3.18) into the linearized Vlasov equation (3.9), we get

$$
-i \omega f_{1}(\mathbf{v})+i \mathbf{k} \cdot \mathbf{v} f_{1}(\mathbf{v})-\frac{e}{m_{e}} \mathbf{E} \cdot \nabla_{v} f_{0}(v)=0
$$

whose solution is

$$
f_{1}(\mathbf{v})=\frac{i e}{m_{e}} \frac{\mathbf{E} \cdot \nabla_{v} f_{0}(v)}{(\omega-\mathbf{k} \cdot \mathbf{v})}
$$

For definiteness we shall consider the direction of propagation of the plane waves as being the $x$ direction, that is, $\mathbf{k}=k \widehat{\mathbf{x}}$. Therefore, $\mathbf{k} \cdot \mathbf{v}=$ $k v_{x}$ and (3.20) becomes

$$
f_{1}(\mathbf{v})=\frac{i e}{m_{e}} \frac{\mathbf{E} \cdot \nabla_{v} f_{0}(v)}{\left(\omega-k v_{x}\right)}
$$

With this orientation chosen for the coordinate system, the longitudinal component of the wave electric field is $\mathbf{E}_{\ell}=E_{x} \widehat{\mathbf{x}}$, whereas the transverse component is $E_{t}=E_{y} \widehat{\mathbf{y}}+E_{z} \widehat{\mathbf{z}}$, as illustrated in Fig. 1 .

\subsection{Expression for the Current Density}

Next we derive expressions for the Cartesian components of the charge current density J. Substituting (3.21) into (3.14), we obtain

$$
\mathbf{J}=-\frac{i e^{2}}{m_{e}} \int_{v} \frac{\mathbf{v}\left[\mathbf{E} \cdot \nabla_{v} f_{0}(v)\right]}{\left(\omega-k v_{x}\right)} d^{3} v
$$

Note that the $x$ component of this equation in given by

$$
J_{x}=-\frac{i e^{2}}{m_{e}} \int_{v} \frac{v_{x}\left[\mathbf{E} \cdot \nabla_{v} f_{0}(v)\right]}{\left(\omega-k v_{x}\right)} d^{3} v
$$

where the triple integral with respect to the three variables $v_{x}, v_{y}$, and $v_{z}$ range from $-\infty$ to $+\infty$. Using the identity (3.17), we note that

$$
\int_{v} \frac{v_{x}}{\left(\omega-k v_{x}\right)} \frac{E_{j} v_{j}}{v} \frac{d f_{0}(v)}{d v} d^{3} v=0
$$

for $j=y, z$, since the integrand is an odd function of $v_{j}$. Consequently, the only contribution from the term $\mathbf{E} \cdot \nabla_{v} f_{0}(v)$ to the $x$ component of $\mathbf{J}$ comes from the term $E_{x} \partial f_{0}(v) / \partial v_{x}$, so that (3.23) can be written as

$$
J_{x}=-\frac{i e^{2}}{m_{e}} E_{x} \int_{v} \frac{v_{x}}{\left(\omega-k v_{x}\right)} \frac{\partial f_{0}(v)}{\partial v_{x}} d^{3} v
$$




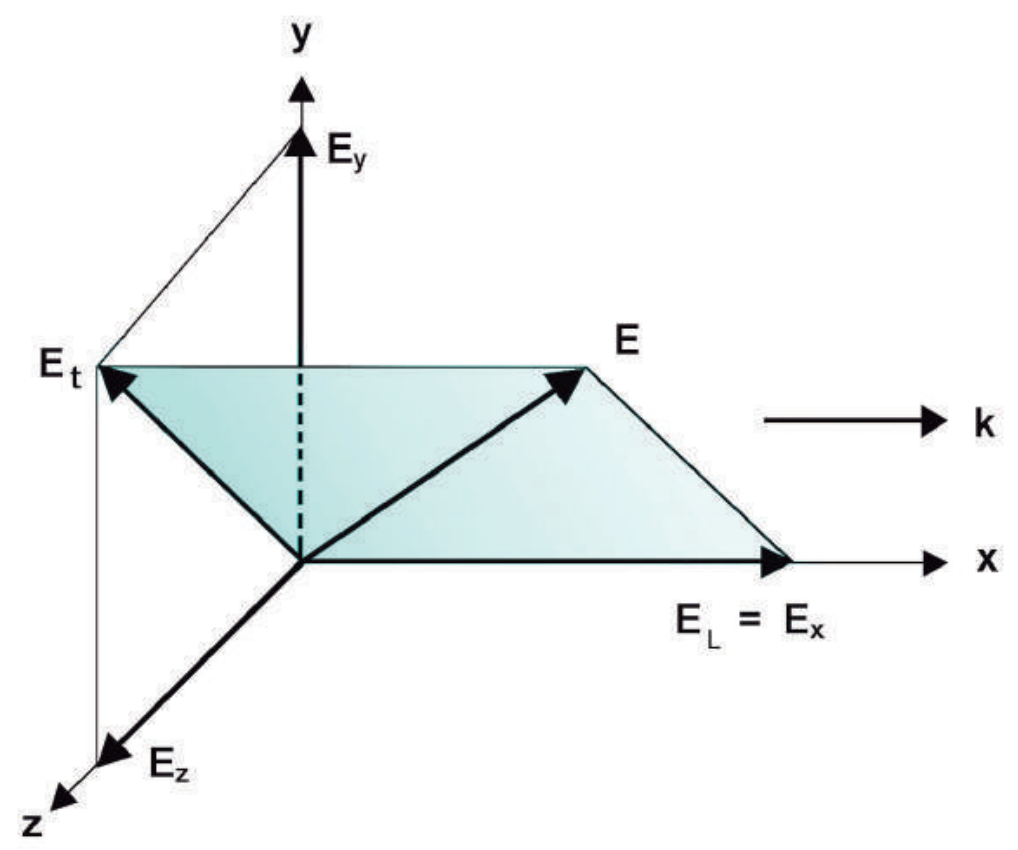

Fig. 1 Illustrating the relative orientations of the wave propagation vector $\mathbf{k}$ and the wave electric field $\mathbf{E}$ in a Cartesian coordinate system.

Similarly, the $y$ and $z$ components of (3.22) are found to be given by

$$
\begin{aligned}
& J_{y}=-\frac{i e^{2}}{m_{e}} E_{y} \int_{v} \frac{v_{y}}{\left(\omega-k v_{x}\right)} \frac{\partial f_{0}(v)}{\partial v_{y}} d^{3} v \\
& J_{z}=-\frac{i e^{2}}{m_{e}} E_{z} \int_{v} \frac{v_{z}}{\left(\omega-k v_{x}\right)} \frac{\partial f_{0}(v)}{\partial v_{z}} d^{3} v
\end{aligned}
$$

Note that $J_{x}, J_{y}$, and $J_{z}$ are linearly related to $E_{x}, E_{y}$, and $E_{z}$, respectively, a feature that is a consequence of the plasma isotropy, as expected in the absence of an external magnetic field.

\subsection{Separation into the Various Modes}

To complete the specification of the problem we use the two Maxwell curl equations (2.4) and (2.5), which for the fields given by (3.15) and (3.16) reduce to

$$
i k \widehat{\mathbf{x}} \times \mathbf{E}=i \omega \mathbf{B}
$$




$$
i k \widehat{\mathbf{x}} \times \mathbf{B}=\mu_{0} \mathbf{J}-\frac{i \omega}{c^{2}} \mathbf{E}
$$

In Cartesian coordinates, $\widehat{\mathbf{x}} \times \mathbf{E}=E_{y} \widehat{\mathbf{z}}-E_{z} \widehat{\mathbf{y}}$ so that the components of the vector equations (3.28) and (3.29) become, respectively,

$$
\begin{gathered}
\omega B_{x}=0 \\
\omega B_{y}=-k E_{z} \\
\omega B_{z}=k E_{y}
\end{gathered}
$$

and

$$
\begin{gathered}
\mu_{0} J_{x}-\frac{i \omega}{c^{2}} E_{x}=0 \\
-i k B_{z}=\mu_{0} J_{y}-\frac{i \omega}{c^{2}} E_{y} \\
i k B_{y}=\mu_{0} J_{z}-\frac{i \omega}{c^{2}} E_{z}
\end{gathered}
$$

where the components of $\mathbf{J}$ are given by (3.25) to (3.27).

An examination of these equations shows that the electromagnetic fields can be separated into four independent groups, each one of them involving the following variables:
(a) $J_{x}, E_{x}$ [Equation (3.33)].
(b) $B_{x}$ [Equation (3.30)].
(c) $J_{y}, E_{y}, B_{z}$ [Equations (3.32) and (3.34)].
(d) $J_{z}, E_{z}, B_{y}$ [Equations (3.31) and (3.35)].

The first group contains an electric field and a current density in the direction of the propagation coefficent $\mathbf{k}$, that is, parallel to the wave normal of the initial plane wave disturbance produced in the plasma, but contains no magnetic field. This group gives the longitudinal plasma wave mode, since the average particle velocity is also in the direction of $\mathbf{k}$. The second group does not constitute a natural wave mode, since it has no current associated with it and therefore is not influenced by the collective electron motion. It only indicates that there is no magnetic field associated with the longitudinal plasma wave so that these waves are electrostatic in character. The third and fourth groups involve electric and magnetic fields that are perpendicular to $\mathbf{k}$. The electric current density and therefore the average particle velocity are also perpendicular to the wave normal direction. Note that $\mathbf{E}, \mathbf{B}$, and $\mathbf{k}$ form a mutually perpendicular triad. These two groups constitute the two different polarizations 
of the transverse electromagnetic wave mode. In the next section we shall discuss the characteristics of the longitudinal plasma wave. The characteristics of the transverse electromagnetic wave will be discussed in section 5 .

\section{ELECTROSTATIC LONGITUDINAL WAVE IN A HOT ISOTROPIC PLASMA}

\subsection{Development of the Dispersion Relation}

The intrinsic behavior of the longitudinal plasma wave is contained in the dispersion relation. This equation, which relates the variables $\omega$ and $k$, determines the natural wave modes of the system. To obtain the dispersion relation for the longitudinal plasma wave we use (3.33) with $J_{x}$ as given by (3.25),

$$
E_{x}=\frac{\omega_{p e}^{2} E_{x}}{n_{0} \omega} \int_{v} \frac{v_{x}}{\left(k v_{x}-\omega\right)} \frac{\partial f_{0}(v)}{\partial v_{x}} d^{3} v
$$

Dividing this equation by $E_{x} \neq 0$, yields the dispersion relation for the longitudinal plasma wave:

$$
1=\frac{\omega_{p e}^{2}}{n_{0} \omega} \int_{v} \frac{v_{x}}{\left(k v_{x}-\omega\right)} \frac{\partial f_{0}(v)}{\partial v_{x}} d^{3} v
$$

It is convenient to simplify (4.2) by noting that

$$
\begin{gathered}
\int_{v} \frac{v_{x}}{\left(k v_{x}-\omega\right)} \frac{\partial f_{0}(v)}{\partial v_{x}} d^{3} v=\frac{1}{k} \int_{v} \frac{\partial f_{0}(v)}{\partial v_{x}}\left(\frac{\omega}{k v_{x}-\omega}+1\right) d^{3} v= \\
\frac{\omega}{k} \int_{v} \frac{\partial f_{0}(v)}{\partial v_{x}} \frac{1}{\left(k v_{x}-\omega\right)} d^{3} v
\end{gathered}
$$

since

$$
\int_{v} \frac{\partial f_{0}(v)}{\partial v_{x}} d^{3} v=\int_{-\infty}^{+\infty} d v_{y} \int_{-\infty}^{+\infty} d v_{z}\left[f_{0}(v)\right]_{-\infty}^{+\infty}=0
$$

because $f_{0}(v)$ vanishes at both limits. Therefore, the dispersion relation (4.2) becomes

$$
1=\frac{\omega_{p e}^{2}}{n_{0} k^{2}} \int_{v} \frac{\partial f_{0}(v)}{\partial v_{x}} \frac{1}{\left(v_{x}-\omega / k\right)} d^{3} v
$$


A useful alternative form of this dispersion relation can be obtained by an integration by parts in the $v_{x}$ variable. Thus, using the relation

$$
\int_{a}^{b} U d V=\left.U V\right|_{a} ^{b}-\int_{a}^{b} V d U
$$

for the integration with respect to $v_{x}$ in (4.5), where

$$
\begin{array}{cc}
U=\frac{1}{\left(v_{x}-\omega / k\right)} & d U=-\frac{d v_{x}}{\left(v_{x}-\omega / k\right)^{2}} \\
V=f_{0}(v) & d V=\frac{\partial f_{0}(v)}{\partial v_{x}} d v_{x}
\end{array}
$$

the triple integral in (4.5) becomes

$$
\begin{gathered}
\iiint_{-\infty}^{+\infty} \frac{\partial f_{0}(v)}{\partial v_{x}} \frac{1}{\left(v_{x}-\omega / k\right)} d v_{x} d v_{y} d v_{z}=\int_{-\infty}^{+\infty} d v_{y} \int_{-\infty}^{+\infty} d v_{z} \\
{\left[\left.\frac{f_{0}(v)}{\left(v_{x}-\omega / k\right)}\right|_{v_{x}=-\infty} ^{v_{x}=+\infty}+\int_{-\infty}^{+\infty} \frac{f_{0}(v)}{\left(v_{x}-\omega / k\right)^{2}} d v_{x}\right]=} \\
\int_{v} \frac{f_{0}(v)}{\left(v_{x}-\omega / k\right)^{2}} d^{3} v
\end{gathered}
$$

Therefore, the dispersion relation (4.5) can also be written as

$$
1=\frac{\omega_{p e}^{2}}{n_{0} k^{2}} \int_{v} \frac{f_{0}(v)}{\left(v_{x}-\omega / k\right)^{2}} d^{3} v
$$

\subsection{Limiting Case of a Cold Plasma}

Before proceeding further with the analysis of the dispersion relation (4.9), it is instructive to examine the results for the limiting case of a cold plasma, for which the electron velocity distribution, under equilibrium conditions and at rest, is given by

$$
f_{0}(v)=n_{0} \delta\left(v_{x}\right) \delta\left(v_{y}\right) \delta\left(v_{z}\right)
$$

where $\delta(x)$ is the Dirac delta function, defined by

$$
\delta(x)=\infty \quad \text { for } \quad x=0 ; \quad \int_{-\infty}^{+\infty} \delta(x) d x=1
$$


Substituting (4.10) into the dispersion relation (4.9) and using the following property of the Dirac delta function

$$
\int_{-\infty}^{+\infty} f(x) \delta\left(x-x_{0}\right) d x=f\left(x_{0}\right)
$$

we obtain

$$
1=\frac{\omega_{p e}^{2}}{k^{2}} \int_{v} \frac{\delta\left(v_{x}\right) \delta\left(v_{y}\right) \delta\left(v_{z}\right)}{\left(v_{x}-\omega / k\right)^{2}} d^{3} v
$$

or

$$
\omega^{2}=\omega_{p e}^{2}
$$

in agreement with the cold plasma result (section 4 of Chapter 16).

\subsection{High Phase Velocity Limit}

Another important result can be immediately obtained from the dispersion relation (4.9), for the limiting case in which the wave phase velocity $\omega / k$ is very large compared to the velocity of almost all of the electrons. In this high phase velocity limit it is reasonable to expand $\left(1-k v_{x} / \omega\right)^{-2}$ into a binomial series and retain only the first few terms, since $k v_{x} / \omega \ll 1$. Thus, recalling that for any $|\epsilon|<1$ we have

$$
(1-\epsilon)^{-2}=1+2 \epsilon+3 \epsilon^{2}+4 \epsilon^{3}+\cdots
$$

the dispersion relation (4.9) becomes (for $\left|v_{x}\right| \ll \omega / k$ ),

$$
\begin{gathered}
1=\frac{\omega_{p e}^{2}}{n_{0} \omega^{2}} \int_{v} \frac{f_{0}(v)}{\left(1-k v_{x} / \omega\right)^{2}} d^{3} v= \\
\frac{\omega_{p e}^{2}}{\omega^{2}}\left(1+2 \frac{k}{\omega}<v_{x}>_{0}+3 \frac{k^{2}}{\omega^{2}}<v_{x}^{2}>_{0}+\cdots\right)
\end{gathered}
$$

where the average value with the subscript 0 is calculated using the equilibrium distribution function $f_{0}(v)$. Since the plasma is considered to be stationary, we have $\left\langle v_{x}\right\rangle_{0}=u_{x}=0$, so that the second term in the right-hand side of (4.16) vanishes. To a first degree of approximation we obtain $\omega^{2}=\omega_{p e}^{2}$, which is again the cold plasma result given in (4.14). For a small correction to the cold plasma result, we consider the next nonzero term in the expansion (4.16). Assuming that the equilibrium distribution function is isotropic and using the definition of absolute temperature,

$$
<v_{x}^{2}>_{0}=<c_{x}^{2}>_{0}=\frac{1}{3}<c^{2}>_{0}=\frac{k_{B} T_{e}}{m_{e}}
$$


where $T_{e}$ is the temperature of the electron gas at equilibrium and $k_{B}$ is Boltzmann's constant, the dispersion relation (4.16) becomes

$$
\omega^{2}=\omega_{p e}^{2}\left(1+3 \frac{k^{2}}{\omega^{2}} \frac{k_{B} T_{e}}{m_{e}}+\cdots\right)
$$

Since the second term in the right-hand side of (4.18) is small in the high phase velocity limit, we can replace $\omega$, in just this small term, by $\omega_{p e}$ (which is the value of $\omega$ when this term is zero) and write (4.18) as

$$
\omega^{2}=\omega_{p e}^{2}+3 k^{2} \frac{k_{B} T_{e}}{m_{e}}
$$

This result is known as the Bohm-Gross dispersion relation. Note that it is identical to the result obtained using the warm plasma model when collisions are neglected and when the ratio of specific heats is taken equal to 3 . Since $\gamma$ is related to the number of degrees of freedom $N$ by the relation

$$
\gamma=\frac{(2+N)}{N}
$$

we see that $\gamma=3$ corresponds to the case when the electron gas has one degree of freedom $(N=1)$, so that the electrons move only in the direction of wave propagation.

If additional terms are retained in the binomial series expansion of (4.16), additional approximations can be obtained for the dispersion relation. In all these approximations we find that $\omega$ remains real, so that the longitudinal plasma wave has a constant amplitude in time. There is neither temporal growth nor decay. It is usual to terminate the approximations to this dispersion relation at the stage given by (4.19). Using the definition of the Debye length $\lambda_{D}$, the Bohm-Gross dispersion relation can be rewritten as

$$
\omega^{2}=\omega_{p e}^{2}\left(1+3 k^{2} \lambda_{D}^{2}\right)
$$

\subsection{Dispersion Relation for Maxwellian Distribution Function}

Next we evaluate the longitudinal wave dispersion relation (4.5) for the important case when $f_{0}(v)$ is the Maxwellian distribution function for a stationary equilibrium plasma,

$$
f_{0}(v)=n_{0}\left(\frac{m_{e}}{2 \pi k_{B} T_{e}}\right)^{3 / 2} \exp \left(-\frac{m_{e} v^{2}}{2 k_{B} T_{e}}\right)
$$


In this case, a careful analysis of (4.5) shows that $\omega$ has a negative imaginary part, causing a temporal damping of the electron plasma wave. This temporal damping, which arises in the absence of collisions, is known as Landau damping and will be discussed in the next subsection.

For the moment, we evaluate the dispersion relation for the longitudinal electron wave using the Maxwell-Boltzmann equilibrium distribution function. Substituting (4.22) into (4.5) yields

$$
\begin{gathered}
1=-\frac{\omega_{p e}^{2}}{n_{0} k^{2}} \frac{m_{e}}{k_{B} T_{e}} \int_{v} \frac{v_{x} f_{0}(v)}{\left(v_{x}-\omega / k\right)} d^{3} v= \\
-\frac{\omega_{p e}^{2}}{k^{2}} \frac{m_{e}}{k_{B} T_{e}}\left(\frac{m_{e}}{2 \pi k_{B} T_{e}}\right)^{3 / 2} \int_{-\infty}^{+\infty} \frac{v_{x}}{\left(v_{x}-\omega / k\right)} \exp \left(-\frac{m_{e} v_{x}^{2}}{2 k_{B} T_{e}}\right) d v_{x} \\
\int_{-\infty}^{+\infty} \exp \left(-\frac{m_{e} v_{y}^{2}}{2 k_{B} T_{e}}\right) d v_{y} \int_{-\infty}^{+\infty} \exp \left(-\frac{m_{e} v_{z}^{2}}{2 k_{B} T_{e}}\right) d v_{z}
\end{gathered}
$$

The second and third integrals are each equal to $\left(2 \pi k_{B} T_{e} / m_{e}\right)^{1 / 2}$. It is convenient to introduce the following dimensionless parameters,

$$
\begin{aligned}
& C=\frac{(\omega / k)}{\left(2 k_{B} T_{e} / m_{e}\right)^{1 / 2}} \\
& q=\frac{v_{x}}{\left(2 k_{B} T_{e} / m_{e}\right)^{1 / 2}}
\end{aligned}
$$

so that the dispersion relation $(4.23)$ reduces to

$$
k^{2}=-\omega_{p e}^{2} \frac{m_{e}}{k_{B} T_{e}} \frac{1}{\pi^{1 / 2}} \int_{-\infty}^{+\infty} \frac{q \exp \left(-q^{2}\right)}{(q-C)} d q
$$

Using the notation

$$
I(C)=\frac{1}{\pi^{1 / 2}} \int_{-\infty}^{+\infty} \frac{q \exp \left(-q^{2}\right)}{(q-C)} d q
$$

and substituting $\left(k_{B} T_{e} / m_{e}\right)$ by $\lambda_{D}^{2} \omega_{p e}^{2},(4.26)$ becomes

$$
k^{2} \lambda_{D}^{2}+I(C)=0
$$

The evaluation of the integral $I(C)$ is not straightforward because of the singularity at $q=C$, since for real $\omega(k)$ the denominator vanishes on the real $v_{x}$ axis. For complex $\omega(k)$, which corresponds to damped waves 
$(\mathcal{I}\{\omega\}<0)$, or unstable waves $(\mathcal{I}\{\omega\}>0)$, the singularity lies off the path of integration along the real $v_{x}$ axis. However, this simplified derivation of the dispersion relation gives no indication of the proper integration contour to be chosen in the complex $v_{x}$ plane. Possible contours of integration are shown in Fig. 2 for the cases: (a) unstable wave, with $\mathcal{I}\{\omega\}>0$; (b) real $\omega(k)$; and (c) damped wave, with $\mathcal{I}\{\omega\}<0$. Landau was the first to treat this problem properly as an initial-value problem. If we are interested in the evaluation of the plasma behavior after an initial perturbation, then the causality principle demands that there should be no fields before the starting of the source. According to the well-known theorem of residues in the theory of complex variables, the value of an integral in the complex domain with a closed contour of integration, such as in Fig. 2, is equal to $2 \pi i$ times the sum of the residues within the closed path. The integral vanishes if there are no singularities enclosed by the integration path. Thus, the nature of the singularities of the integrand determines the behavior of the fields after the initial perturbation. The correct contour prescribed by Landau is along the real $v_{x}$ axis, indented such as to pass below the singularities, and closed by an infinite semicircular path in the upper half of the complex $v_{x}$ plane, as shown in Fig. 2.

This technique of integration around a contour closed by an infinite semicircle in the upper half plane works if the contribution of the integral from the semicircular path vanishes as its radius goes to infinity. The integral $I(C)$ given in (4.27), the way it stands, cannot be handled by the usual method of residues, since the integrand diverges for $q= \pm i \infty$. To put this integral in a form suitable for evaluation by the method of residues, or by any other method, note first that we can write

$$
\frac{q}{(q-C)}=1+\frac{C}{(q-C)}
$$

so that we have

$$
I(C)=\frac{1}{\pi^{1 / 2}} \int_{-\infty}^{+\infty}\left(1+\frac{C}{q-C}\right) \exp \left(-q^{2}\right) d q
$$

The first integral in the right-hand side of this equation is equal to unity. Therefore,

$$
I(C)=1+\frac{C}{\pi^{1 / 2}} \int_{-\infty}^{+\infty} \frac{\exp \left(-q^{2}\right)}{(q-C)} d q
$$



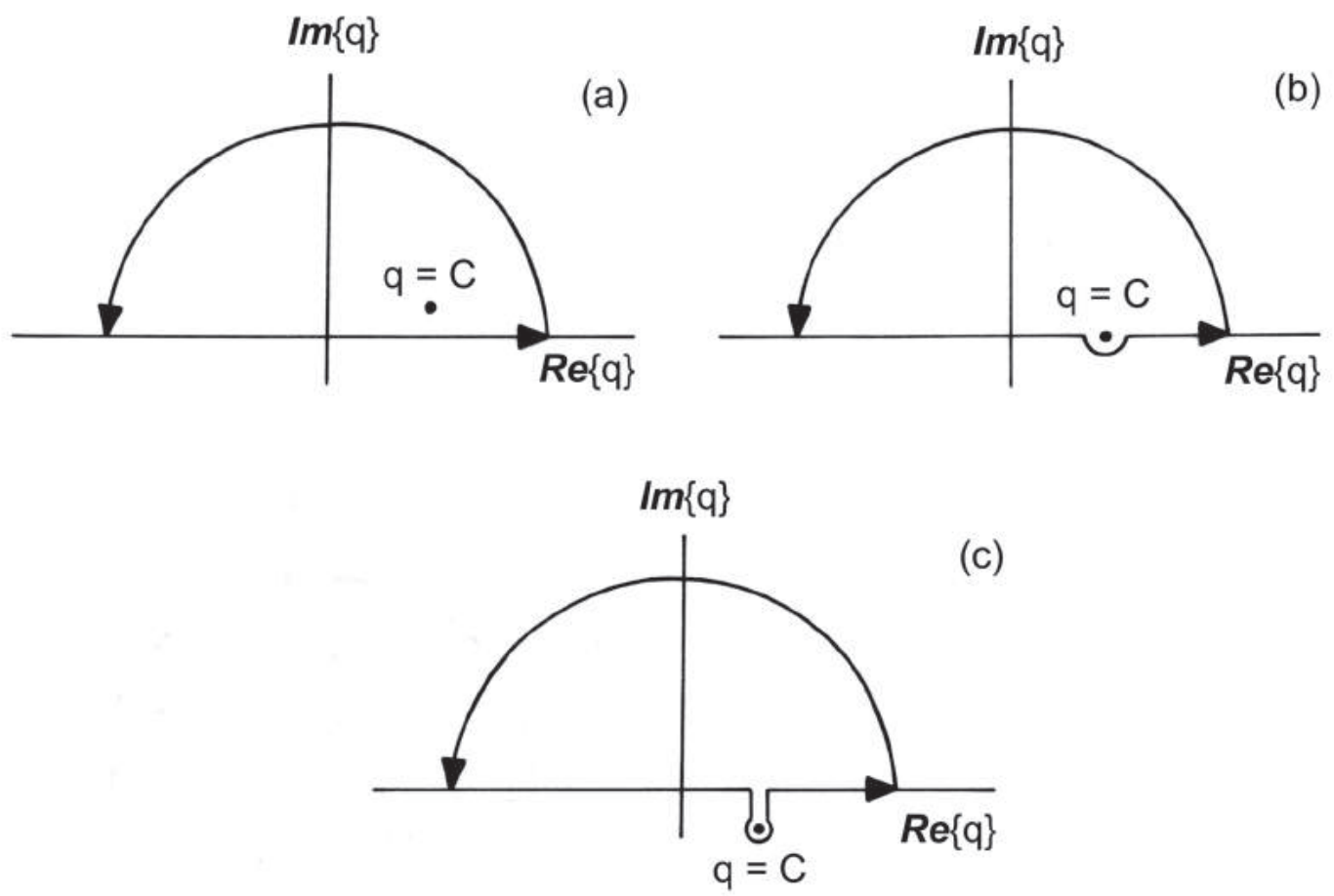

Fig. 2 Contours of integration in the complex $v_{x}$ plane for (a) $\mathcal{I}\{\omega\}>0$, (b) $\mathcal{I}\{\omega\}=0$, and (c) $\mathcal{I}\{\omega\}<0$.

For purposes of integration it is convenient to introduce a parameter $s$ in the integral of (4.31), by defining

$$
G(C, s)=\frac{1}{\pi^{1 / 2}} \int_{-\infty}^{+\infty} \frac{\exp \left(-s q^{2}\right)}{(q-C)} d q
$$

Hence, we identify the integral $\mathrm{I}(\mathrm{C})$ as

$$
I(C)=1+C G(C, 1)
$$

so that the dispersion relation (4.28) becomes

$$
k^{2} \lambda_{D}^{2}+1+C G(C, 1)=0
$$

The purpose of defining $G(C, s)$, as in (4.32), is that this relation allows us to evaluate $G(C, 1)$ through a transformation of the integral into a 
differential equation. Initially, note that the integral in (4.32) can also be written as

$$
G(C, s)=\frac{1}{\pi^{1 / 2}} \int_{-\infty}^{+\infty} \frac{(q+C)}{\left(q^{2}-C^{2}\right)} \exp \left(-s q^{2}\right) d q
$$

The first integral in the right-hand side of this equation vanishes, since the integrand is an odd function of $q$. Therefore, an alternative expression for $G(C, s)$ is

$$
G(C, s)=\frac{C}{\pi^{1 / 2}} \int_{-\infty}^{+\infty} \frac{\exp \left(-s q^{2}\right)}{\left(q^{2}-C^{2}\right)} d q
$$

Taking the derivative of (4.36) with respect to $s$, yields

$$
\begin{gathered}
\frac{d G(C, S)}{d s}=-\frac{C}{\pi^{1 / 2}} \int_{-\infty}^{+\infty} \frac{q^{2} \exp \left(-s q^{2}\right)}{\left(q^{2}-C^{2}\right)} d q= \\
-\frac{C}{\pi^{1 / 2}} \int_{-\infty}^{+\infty}\left(1+\frac{C^{2}}{q^{2}-C^{2}}\right) \exp \left(-s q^{2}\right) d q
\end{gathered}
$$

Evaluating the first integral we obtain $-C / s^{1 / 2}$, so that

$$
\frac{d G(C, s)}{d s}=-\frac{C}{s^{1 / 2}}-C^{2} G(C, s)
$$

Next, we multiply this differential equation by $\exp \left(s C^{2}\right)$ and note that

$$
\frac{d}{d s}\left[G(C, s) \exp \left(s C^{2}\right)\right]=\exp \left(s C^{2}\right)\left[\frac{d G(C, s)}{d s}+C^{2} G(C, s)\right]
$$

Thus, it is possible to write (4.38) in the form

$$
\frac{d}{d s}\left[G(C, s) \exp \left(s C^{2}\right)\right]=-\frac{C}{s^{1 / 2}} \exp \left(s C^{2}\right)
$$

Upon integrating both sides of this equation from $s=0$ to $s=1$, gives

$$
G(C, 1) \exp \left(C^{2}\right)-G(C, 0)=-C \int_{0}^{1} \frac{\exp \left(s C^{2}\right)}{s^{1 / 2}} d s
$$

or, rearranging,

$$
G(C, 1)=G(C, 0) \exp \left(-C^{2}\right)-C \exp \left(-C^{2}\right) \int_{0}^{1} \frac{\exp \left(s C^{2}\right)}{s^{1 / 2}} d s
$$


The integral $G(C, 0)$ is easily evaluated for the case of weak damping (large phase velocity). In this case, the pole at $v_{x}=\omega / k$ lies near the real $v_{x}$ axis and $G(C, 0)$ can be evaluated as an improper integral, as follows:

$$
\begin{gathered}
G(C, 0)=\frac{1}{\pi^{1 / 2}} \int_{-\infty}^{+\infty} \frac{d q}{(q-C)}=\lim _{X \rightarrow \infty}\left[\frac{1}{\pi^{1 / 2}} \int_{-X}^{X} \frac{d q}{(q-C)}\right]= \\
\lim _{X \rightarrow \infty}\left[\frac{1}{\pi^{1 / 2}} \ln \left(-\frac{X-C}{X+C}\right)\right]=\frac{1}{\pi^{1 / 2}} \ln (-1)= \\
\frac{1}{\pi^{1 / 2}} \ln \left(e^{i \pi}\right)=i \pi^{1 / 2}
\end{gathered}
$$

The integral $G(C, 0)$ can also be evaluated by the method of residues, using an appropriate contour of integration in the complex $q$-plane, as shown in Fig. 2(b), which gives the same result (4.43) for the Cauchy principal value of the integral. Therefore, (4.42) becomes

$$
G(C, 1)=i \pi^{1 / 2} \exp \left(-C^{2}\right)-C \exp \left(-C^{2}\right) \int_{0}^{1} \frac{\exp \left(s C^{2}\right)}{s^{1 / 2}} d s
$$

The remaining integral in the right-hand side of (4.44) can be rewritten in a different form by changing the variable $s$ to $W^{2} / C^{2}$. Consequently, $d s / s^{1 / 2}=2 d W / C$ and

$$
G(C, 1)=i \pi^{1 / 2} \exp \left(-C^{2}\right)-2 \int_{0}^{C} \exp \left(W^{2}-C^{2}\right) d W
$$

Although this integral cannot be evaluated explicitly, it is now in a more convenient form for numerical calculation.

Substituting (4.45) into (4.34) results in the following expression for the dispersion relation:

$$
-k^{2} \lambda_{D}^{2}=1+i \pi^{1 / 2} C \exp \left(-C^{2}\right)-2 C \int_{0}^{C} \exp \left(W^{2}-C^{2}\right) d W
$$

The integral remaining here can be evaluated numerically, while the imaginary term is known as the Landau damping term. The formal procedure to evaluate $k$ as a function of $\omega$ (or vice versa) from this dispersion relation consists in choosing a given value of $C$, as defined in (4.24), and finding the corresponding value of the dispersion function from numerical calculations. Equation (4.46) can then be used to evaluate the propagation coefficient $k$. 


\subsection{Landau Damping}

In order to show that (4.46) indicates temporal damping of the longitudinal plasma wave, it is convenient to perform an approximate evaluation of the dispersion relation. The special case of high phase velocity and weak damping can be analyzed in a straightforward way and, at the same time, provides a partial check on the accuracy of the Bohm-Gross dispersion relation obtained earlier. Furthermore, an explicit expression is obtained for the imaginary part of $\omega$. Thus, for the limiting case of $C \gg 1$, let us find an approximate expression for the dispersion function integal

$$
I_{1}=2 C \int_{0}^{C} \exp \left(W^{2}-C^{2}\right) d W
$$

As the first step, equation (4.47) can be rewritten by transforming the integration variable to $\xi=C^{2}-W^{2}$, which gives

$$
I_{1}=\int_{0}^{C^{2}}\left(1-\frac{\xi}{C^{2}}\right)^{-1 / 2} \exp (-\xi) d \xi
$$

Since $\xi$ is less than $C^{2}$ over the entire range of integration, we can expand $\left(1-\xi / C^{2}\right)^{-1 / 2}$ in a binomial series,

$$
\begin{gathered}
\left(1-\frac{\xi}{C^{2}}\right)^{-1 / 2}=1+\frac{\xi}{2 C^{2}}+\frac{3 \xi^{2}}{8 C^{4}}+\cdots+ \\
\frac{1 \times 3 \times \cdots \times(2 n-1)}{2^{n} n !}\left(\frac{\xi}{C^{2}}\right)^{n}+\cdots
\end{gathered}
$$

If this expansion is substituted into (4.48) and each term is integrated by noting that

$$
\begin{gathered}
\int_{0}^{C^{2}}\left(\frac{\xi}{C^{2}}\right)^{n} \exp (-\xi) d \xi=\frac{n !}{C^{2 n}}- \\
\exp \left(-C^{2}\right)\left[1+\frac{n}{C^{2}}+\frac{n(n-1)}{C^{4}}+\cdots+\frac{n !}{C^{2 n}}\right]
\end{gathered}
$$

we find

$$
\begin{gathered}
I_{1}=1+\frac{1}{2 C^{2}}+\frac{3}{4 C^{4}}+\cdots+\frac{1 \times 3 \times \cdots \times(2 n-1)}{2^{n} C^{2 n}}+\cdots \\
+\mathcal{O}\left\{\exp \left(-C^{2}\right)\right\}
\end{gathered}
$$


where $\mathcal{O}\left\{\exp \left(-C^{2}\right)\right\}$ denotes terms of order $\exp \left(-C^{2}\right)$. Although this is an asymptotic expansion and actually diverges when $n \rightarrow \infty$, a good estimate of $I_{1}$ can be obtained by retaining only the first few terms, provided $C$ is large. Therefore, on retaining only the first three terms of (4.51), the dispersion relation (4.46), in the high phase velocity limit, becomes

$$
k^{2} \lambda_{D}^{2}=\frac{1}{2 C^{2}}+\frac{3}{4 C^{4}}-i \pi^{1 / 2} C \exp \left(-C^{2}\right)
$$

With the help of (4.24), which defines $C$, and the definition of the Debye length $\lambda_{D}$, equation (4.52) can be written as

$$
\begin{gathered}
\frac{\omega^{2}}{\omega_{p e}^{2}}=1+3 k^{2} \lambda_{D}^{2}\left(\frac{\omega_{p e}}{\omega}\right)^{2}- \\
\frac{i(\pi / 2)^{1 / 2}}{k^{3} \lambda_{D}^{3}}\left(\frac{\omega}{\omega_{p e}}\right)^{3} \exp \left[-\frac{1}{2 k^{2} \lambda_{D}^{2}}\left(\frac{\omega}{\omega_{p e}}\right)^{2}\right]
\end{gathered}
$$

In the high phase velocity limit the second term in the right-hand side of (4.53) is small as compared to the first one, whereas the third term is exponentially small as compared to the first one, so that in this limit the plasma oscillates very close to the plasma frequency $\omega_{p e}$. Note that this limit corresponds also to a long-wavelength limit. Thus, (4.53) can be further approximated as

$$
\begin{gathered}
\omega^{2}=\omega_{p e}^{2}+\frac{3 k_{B} T_{e}}{m_{e}} k^{2}- \\
\frac{i(\pi / 2)^{1 / 2} \omega_{p e}^{5}}{k^{3}\left(k_{B} T_{e} / m_{e}\right)^{3 / 2}} \exp \left[-\frac{\omega_{p e}^{2}}{2 k^{2}\left(k_{B} T_{e} / m_{e}\right)}-\frac{3}{2}\right]
\end{gathered}
$$

where in the right-hand side of (4.53) we have replaced $\omega$ by $\omega_{p e}$, except in the exponential term where $\omega^{2}$ has been replaced by the Bohm-Gross results (4.19).

Note that the first two terms in (4.54) correspond to the Bohm-Gross result, whereas the imaginary term is new. Separating $\omega$ in its real and imaginary parts according to $\omega=\omega_{r}+i \omega_{i}$ and noting that

$$
\omega_{i}=\frac{\left(\omega^{2}\right)_{i}}{2 \omega_{r}}
$$

we obtain (taking $\omega_{r}=\omega_{p e}$ ) 


$$
\omega_{i}=-\frac{(\pi / 8)^{1 / 2} \omega_{p e}^{4}}{k^{3}\left(k_{B} T_{e} / m_{e}\right)^{3 / 2}} \exp \left[-\frac{\omega_{p e}^{2}}{2 k^{2}\left(k_{B} T_{e} / m_{e}\right)}-\frac{3}{2}\right]
$$

This negative imaginary term in $\omega$ leads to temporal decay of the wave amplitude, since for a standing wave (where $k$ is real) the waves are proportional to

$$
\exp (i k x-i \omega t)=\exp \left(i k x-i \omega_{r} t\right) \exp \left(\omega_{i} t\right)
$$

This temporal damping of the longitudinal plasma wave was first pointed out by L.D. Landau and, for this reason, the expression (4.56) is usually called the Landau damping factor.

The Landau damping of the longitudinal plasma wave amplitude arises in the absence of dissipative mechanisms, such as collisions of the electrons with heavy particles. The physical mechanism responsible for this collisionless Landau damping is the wave-particle interaction, i.e., the interaction of the electrons with the wave electric field $E_{x} \widehat{\mathbf{x}} \cos (k x-\omega t)$. The electrons that initially have velocities quite close to the phase velocity of the wave are trapped inside the moving potential wells of the wave, and this trapping results in a net interchange of energy between the electrons and the wave. For the Maxwell-Boltzmann velocity distribution function we find that, for small $k$, the phase velocity lies far out on the distribution tail and the damping is negligible, but for values of $k$ close to $1 / \lambda_{D}$ the phase velocity lies within the tail, as shown in Fig. 3, so that there is a velocity band $\Delta v$ around $v=\omega / k$ where there are more electrons in $\Delta v$ moving initially slower than $\omega / k$, than moving faster than $\omega / k$. Consequently, the trapping of the electrons in the wave potential troughs will cause a net increase in the electron energy at the expense of the wave energy. This happens in the region where $\partial f_{0} / \partial v_{x}$ is negative, like the one shown in Fig. 3. In some cases, the initial velocity distribution of the electrons may be appropriately chosen in such a way that $\omega_{i}$ becomes positive. This would indicate an unstable situation, with the wave amplitude growing in time. This happens when $\partial f_{0} / \partial v_{x}$ is positive at $v_{x}=\omega / k$.

It is important to note that the Landau damping factor is essentially due to the pole of the integrand in (4.31), which occurs at the value of the electron velocity component $v_{x}$ (parallel to $k$ ) equal to the phase velocity of the wave $(\omega / k)$. This property is a mathematical manifestation of the fact that the wave-particle interaction is effective only when the velocity of the electrons is very close to the phase velocity of the wave. 


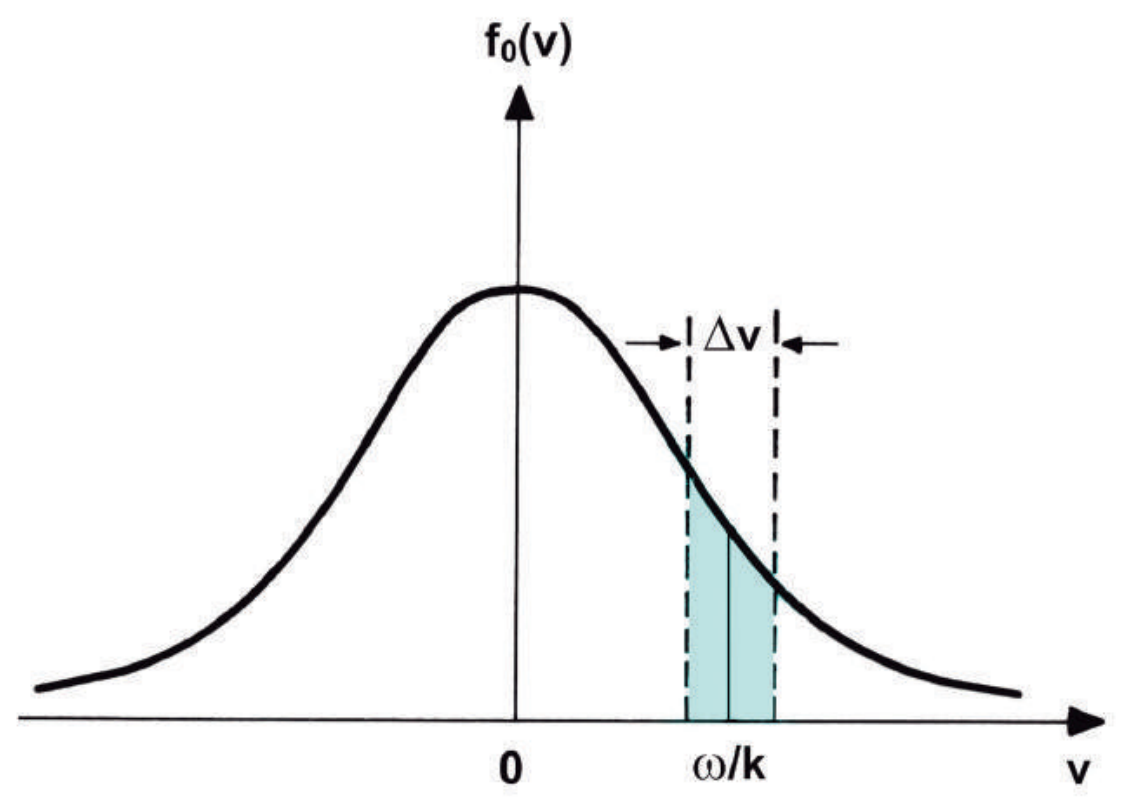

Fig. 3 Equilibrium distribution function of the electrons showing a velocity band $\Delta v$ around the phase velocity $(\omega / k)$, in which there are more electrons moving slower than $(\omega / k)$, than moving faster than $(\omega / k)$.

\section{TRANSVERSE WAVE IN A HOT ISOTROPIC PLASMA}

\subsection{Development of the Dispersion Relation}

The third and fourth independent groups of fields, consisting of $J_{y}$, $E_{y}, B_{z}$ and $J_{z}, E_{z}, B_{y}$, respectively, constitute the two different polarizations of the transverse wave mode. In order to deduce the dispersion relation for the transverse electromagnetic wave, let us consider initially equations (3.26), (3.32), and (3.34). Substituting $B_{z}$ from (3.32) into (3.34), yields

$$
E_{y}=\frac{i \omega}{\epsilon_{0}\left(k^{2} c^{2}-\omega^{2}\right)} J_{y}
$$

Combining this equation with (3.26), to eliminate $J_{y}$, we obtain

$$
E_{y}=\frac{\omega_{p e}^{2} \omega}{n_{0}\left(\omega^{2}-k^{2} c^{2}\right)} E_{y} \int_{v} \frac{v_{y}}{\left(k v_{x}-\omega\right)} \frac{\partial f_{0}(v)}{\partial v_{y}} d^{3} v
$$


In a similar way, combining (3.27), (3.31), and (3.35) we find that the equation for $E_{z}$ is identical to (5.2). The integral with respect to $v_{y}$ in (5.2) can be simplified by an integration by parts

$$
\int_{-\infty}^{+\infty} v_{y} \frac{\partial f_{0}(v)}{\partial v_{y}} d v_{y}=\left.v_{y} f_{0}(v)\right|_{v_{y}=-\infty} ^{v_{y}=+\infty}-\int_{-\infty}^{+\infty} f_{0}(v) d v_{y}
$$

The first term in the right-hand side of this equation vanishes, since $f_{0}(v)$ vanishes at $v_{y}= \pm \infty$. Thus, we obtain from (5.2) the following dispersion relation for the transverse electromagnetic wave:

$$
k^{2} c^{2}-\omega^{2}=\frac{\omega_{p e}^{2} \omega}{n_{0} k} \int_{v} \frac{f_{0}(v)}{\left(v_{x}-\omega / k\right)} d^{3} v
$$

\subsection{Cold Plasma Result}

Again, we shall examine first the limiting case of a cold plasma characterized by the distribution function (4.10). Substituting (4.10) into (5.4) and using the property (4.12) of the Dirac delta function, we find

$$
k^{2} c^{2}=\omega^{2}-\omega_{p e}^{2}
$$

This result is identical to the one obtained in Chapter 16 using the cold plasma model [see (16.4.12)].

\subsection{Dispersion Relation for Maxwellian Distribution Function}

Considering $f_{0}(v)$, in (5.4), as the Maxwell-Boltzmann distribution function, we find, after integrating over $v_{y}$ and $v_{z}$,

$$
k^{2} c^{2}-\omega^{2}=\omega_{p e}^{2} \frac{C}{\pi^{1 / 2}} \int_{-\infty}^{+\infty} \frac{\exp \left(-q^{2}\right)}{q-C} d q
$$

where, as before, we have introduced the dimensionless parameters $C$ and $q$, defined in (4.24) and (4.25), respectively. The integral appearing in (5.6) is the same as the integral $G(C, s)$ for $s=1$, defined in (4.32), so that we can write the dispersion relation (5.6) as

$$
k^{2} c^{2}-\omega^{2}=\omega_{p e}^{2} C G(C, 1)
$$


For weak damping we can use (4.45), obtaining

$$
k^{2} c^{2}-\omega^{2}=\omega_{p e}^{2}\left[i \pi^{1 / 2} C \exp \left(-C^{2}\right)-2 C \int_{0}^{C} \exp \left(W^{2}-C^{2}\right) d W\right]
$$

\subsection{Landau Damping of the Transverse Wave}

In contrast with the Landau damping of the longitudinal plasma wave, the Landau damping of the transverse electromagnetic wave, which is due to the small negative imaginary part of $\omega$ in (5.8), is negligibly small. For the purpose of establishing this result, it is convenient to evaluate approximately the dispersion relation (5.8) in the high phase velocity limit. In the limit when $C$ is very large we can use (4.51). To obtain a first approximation to the real part of $\omega$, it is sufficient to retain only the first term in (4.51), so that in the high phase velocity limit (5.8) reduces to

$$
k^{2} c^{2}=\omega^{2}-\omega_{p e}^{2}+i \pi^{1 / 2} \omega_{p e}^{2} C \exp \left(-C^{2}\right)
$$

This result is similar to the dispersion relation obtained using the cold plasma model without collisions, except for the Landau damping term.

In the high phase velocity limit $(C \gg 1)$ the Landau damping factor is very small and can be omitted in a first approximation, with the result that (5.9) reduces to the cold plasma result (5.5). From (5.5) we see that for $\omega>$ $\omega_{p e}$ the phase velocity $\omega / k$ is greater than $c$ (the speed of electromagnetic waves in free space). Thus, $C$ is of the order of $c /\left(2 k_{B} T_{e} / m_{e}\right)^{1 / 2}$ and is therefore a very large number. Since $C$ is very large, the Landau damping of the transverse electromagnetic wave is negligible.

As a matter of fact it can be argued that, for this case, the Landau damping term is really zero, since the integration over $v_{x}$ should really extend only from $-c$ to $+c$, while the phase velocity is always greater than c. This implies that the pole at $v_{x}=(\omega / k)$, or equivalently at $q=C$, lies outside the path of integration along the real axis. Therefore, the conditions for efficient wave-particle interaction are not met for the transverse electromagnetic wave throughout the frequency range of propagation (since $\omega / k$ is greater than $c$ ), resulting in no wave damping. On the other hand, for the longitudinal plasma wave there are frequencies for which the wave phase velocity is of the order of the electron thermal velocities, so that wave-particle interaction can take place efficiently, with the result that the Landau damping factor becomes important for the lower phase velocity longitudinal waves. 


\section{THE TWO-STREAM INSTABILITY}

As an example of a situation in which wave-particle interaction leads to a growing wave amplitude, at the expense of the kinetic energy of the plasma particles, we consider in this section the so-called two-stream instability. Although the instability arises under a wide range of beam conditions, we shall consider only the simple case of two counterstreaming uniform beams of electrons with the same number density $n_{0} / 2$. The first stream travels in the $x$ direction with drift velocity $\mathbf{v}_{D}=v_{D} \widehat{\mathbf{x}}$ and the second stream in the opposite direction with drift velocity $\mathbf{v}_{D}=-v_{D} \widehat{\mathbf{x}}$. We shall assume that each particle, in each stream, has exactly the stream velocity, i.e., the particles are assumed to be cold, so that the electron distribution function can be written in terms of the Dirac delta function as

$$
f_{0}(v)=\frac{1}{2} n_{0}\left[\delta\left(v_{x}-v_{D}\right)+\delta\left(v_{x}+v_{D}\right)\right] \delta\left(v_{y}\right) \delta\left(v_{z}\right)
$$

This distribution function is illustrated in Fig. 4 for the $v_{x}$ component only.

For longitudinal plasma waves propagating in the $x$ direction $(\mathbf{k}=k \widehat{\mathbf{x}})$ in an electron gas described by the Vlasov equation, the dispersion relation is, from (4.9),

$$
1=\frac{\omega_{p e}^{2}}{n_{0} k^{2}} \int_{v} \frac{f_{0}(v)}{\left(v_{x}-\omega / k\right)^{2}} d^{3} v
$$

Substituting (6.1) into (6.2), yields

$$
\begin{gathered}
1=\frac{1}{2} \omega_{p e}^{2} \int_{-\infty}^{+\infty} \frac{\delta\left(v_{x}-v_{D}\right)+\delta\left(v_{x}+v_{D}\right)}{\left(k v_{x}-\omega\right)^{2}} d v_{x} \\
\int_{-\infty}^{+\infty} \delta\left(v_{y}\right) d v_{y} \int_{-\infty}^{+\infty} \delta\left(v_{z}\right) d v_{z}
\end{gathered}
$$

and integrating over each of the $\delta$ functions, we obtain

$$
1=\frac{1}{2} \omega_{p e}^{2}\left[\frac{1}{\left(k v_{D}-\omega\right)^{2}}+\frac{1}{\left(k v_{D}+\omega\right)^{2}}\right]
$$

This is the dispersion relation for longitudinal waves (with the wave normal in the direction of the first electron stream) in a counterstreaming electron plasma characterized by the distribution function (6.1). We assume that the propagation coefficient $k$ of the longitudinal plasma wave is real (standing waves) and investigate the existence of temporal growth or decay of the wave amplitude. 


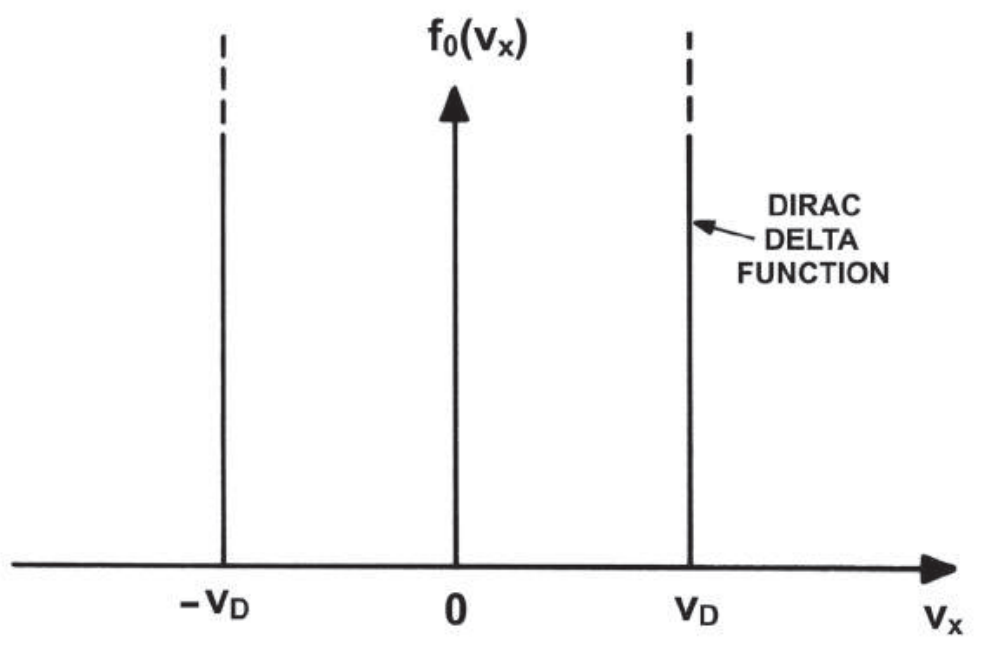

Fig. 4 Illustrating the $v_{x}$ component of the distribution function (6.1).

Equation (6.4) can be rearranged in the following polynomial form:

$$
\omega^{4}-B \omega^{2}+C=0
$$

where

$$
\begin{gathered}
B=\omega_{p e}^{2}+2 k^{2} v_{D}^{2} \\
C=k^{2} v_{D}^{2}\left(k^{2} v_{D}^{2}-\omega_{p e}^{2}\right)
\end{gathered}
$$

Note that B is always positive, whereas $\mathrm{C}$ can be either positive or negative, depending on whether $k^{2} v_{D}^{2}>\omega_{p e}^{2}$ or $k^{2} v_{D}^{2}<\omega_{p e}^{2}$, respectively. The polynomial equation (6.5) has two roots for $\omega^{2}$, which are

$$
\begin{aligned}
\omega_{1}^{2} & =\frac{1}{2} B+\left(\frac{1}{4} B^{2}-C\right)^{1 / 2} \\
\omega_{2}^{2} & =\frac{1}{2} B-\left(\frac{1}{4} B^{2}-C\right)^{1 / 2}
\end{aligned}
$$

In what follows we shall show that an instability can arise only when $k^{2} v_{D}^{2}<\omega_{p e}^{2}$.

First we note that for $k^{2} v_{D}^{2}>\omega_{p e}^{2}$ we have $C>0$, so that both $\omega_{1}^{2}$ and $\omega_{2}^{2}$ are positive real quantities and therefore there can be no temporal growth or decay of the wave amplitude. On the other hand, for $k^{2} v_{D}^{2}<\omega_{p e}^{2}$ we have $C<0$, so that $\omega_{1}^{2}$ is still a positive real quantity, whereas $\omega_{2}^{2}$ is a negative real quantity. Therefore, $\omega_{2}$ has two imaginary values (one positive and one negative). The positive imaginary value of $\omega_{2}$ corresponds 
to an unstable mode, since for $\omega_{2}=i \omega_{2 i}$, with $\omega_{2 i}$ real and positive, we have $\exp \left(-i \omega_{2} t\right)=\exp \left(\omega_{2 i} t\right)$. Hence the growth rate is given by

$$
\omega_{2 i}=\left[-\frac{1}{2} B+\left(\frac{1}{4} B^{2}-C\right)^{1 / 2}\right]^{1 / 2} ; C<0
$$

or, using (6.6) and (6.7),

$$
\begin{gathered}
\omega_{2 i}=\left\{-\left(\frac{1}{2} \omega_{p e}^{2}+k^{2} v_{D}^{2}\right)+\left[\left(\frac{1}{2} \omega_{p e}^{2}+k^{2} v_{D}^{2}\right)^{2}-\right.\right. \\
\left.\left.k^{2} v_{D}^{2}\left(k^{2} v_{D}^{2}-\omega_{p e}^{2}\right)\right]^{1 / 2}\right\}^{1 / 2}
\end{gathered}
$$

valid for $k^{2} v_{D}^{2}<\omega_{p e}^{2}$.

The maximum value of the growth rate (6.11) corresponds to the minimum value of $\omega_{2}^{2}$ in (6.9), since $\omega_{2 i}^{2}=-\omega_{2}^{2}$. Examining the derivative of $\omega_{2}^{2}$ with respect to $k$, we find that the minimum value of $\omega_{2}^{2}$ occurs when $k^{2} v_{D}^{2}=(3 / 8) \omega_{p e}^{2}$ and the corresponding value of $\omega_{2}^{2}$ is $-\omega_{p e}^{2} / 8$. Consequently, the maximum value of the growth rate is

$$
\omega_{2 i}^{(\max )}=\frac{1}{8^{1 / 2}} \omega_{p e}
$$

\section{SUMMARY}

\subsection{Longitudinal Mode}

The dispersion relation is (for $\mathbf{k}=k \widehat{\mathbf{x}}$ )

$$
1=\frac{\omega_{p e}^{2}}{n_{0} \omega} \int_{v} \frac{v_{x}}{\left(k v_{x}-\omega\right)} \frac{\partial f_{0}(v)}{\partial v_{x}} d^{3} v
$$

Alternative forms for this dispersion relation are

$$
\begin{gathered}
1=\frac{\omega_{p e}^{2}}{n_{0} k^{2}} \int_{v} \frac{\partial f_{0}(v)}{\partial v_{x}} \frac{1}{\left(v_{x}-\omega / k\right)} d^{3} v \\
1=\frac{\omega_{p e}^{2}}{n_{0} k^{2}} \int_{v} \frac{f_{0}(v)}{\left(v_{x}-\omega / k\right)^{2}} d^{3} v
\end{gathered}
$$

When $f_{0}(v)$ is the Maxwell-Boltzmann distribution function,

$$
-k^{2} \lambda_{D}^{2}=1+i \pi^{1 / 2} C \exp \left(-C^{2}\right)-2 C \int_{0}^{C} \exp \left(W^{2}-C^{2}\right) d W
$$


The cold plasma limit gives stationary electrostatic oscillations at the plasma frequency,

$$
\omega^{2}=\omega_{p e}^{2}
$$

The high phase velocity limit gives the warm plasma model result (BohmGross dispersion relation) for the electron plasma wave,

$$
\omega^{2}=\omega_{p e}^{2}+3 k^{2} \frac{k_{B} T_{e}}{m_{e}}
$$

The Landau (temporal) damping factor is (with $\omega=\omega_{r}+i \omega_{i}$ )

$$
\omega_{i}=-\frac{(\pi / 8)^{1 / 2} \omega_{p e}^{4}}{k^{3}\left(k_{B} T_{e} / m_{e}\right)^{3 / 2}} \exp \left[-\frac{\omega_{p e}^{2}}{2 k^{2}\left(k_{B} T_{e} / m_{e}\right)}-\frac{3}{2}\right]
$$

\subsection{Transverse Mode}

The dispersion relation is (for $\mathbf{k}=k \widehat{\mathbf{x}}$ )

$$
k^{2} c^{2}-\omega^{2}=\frac{\omega_{p e}^{2} \omega}{n_{0} k} \int_{v} \frac{f_{0}(v)}{\left(v_{x}-\omega / k\right)} d^{3} v
$$

For $f_{0}(v)$ as the Maxwell-Boltzmann distribution function,

$$
k^{2} c^{2}-\omega^{2}=\omega_{p e}^{2}\left[i \pi^{1 / 2} C \exp \left(-C^{2}\right)-2 C \int_{0}^{C} \exp \left(W^{2}-C^{2}\right) d W\right]
$$

The cold and the warm plasma limits give

$$
k^{2} c^{2}=\omega^{2}-\omega_{p e}^{2}
$$

The high phase velocity limit gives

$$
k^{2} c^{2}-\omega^{2}=\omega_{p e}^{2}+i \pi^{1 / 2} \omega_{p e}^{2} C \exp \left(-C^{2}\right)
$$

In this case, the Landau damping term is not relevant, since $v_{p h} \geq c$. 


\section{PROBLEMS}

18.1 Since the longitudinal plasma wave is an electrostatic oscillation, it is possible to derive its dispersion relation using Poisson equation, satisfied by the electrostatic potential $\phi(\mathbf{r}, t)$, instead of Maxwell equations. Consider the problem of small-amplitude longitudinal waves propagating in the $x$ direction in an electron gas (only electrons move in a background of stationary ions), in the absence of a magnetic field. Assume that

$$
\begin{gathered}
f(\mathbf{r}, \mathbf{v}, t)=f_{0}(\mathbf{v})+f_{1}(\mathbf{v}) \exp (i k x-i \omega t) \\
\mathbf{E}(\mathbf{r}, t)=\widehat{\mathbf{x}} E \exp (i k x-i \omega t)
\end{gathered}
$$

where $\left|f_{1}\right| \ll f_{0}$, with $f_{0}(\mathbf{v})$ the nonperturbed equilibrium distribution function and where $\mathbf{E}(\mathbf{r}, t)$ is the internal electric field due to the smallamplitude perturbation in the electron gas. Using the linearized Vlasov equation (neglecting second-order terms) determine the expression for $f_{1}(\mathbf{v})$ in terms of $\mathbf{E}=-\nabla \phi$ and $\nabla_{v} f_{0}$. Using this result in Poisson equation, obtain the following dispersion relation for longitudinal waves propagating in the $x$ direction

$$
1=\frac{\omega_{p e}^{2}}{n_{0} k^{2}} \int_{v} \frac{\partial f_{0}}{\partial v_{x}} \frac{1}{\left(v_{x}-\omega / k\right)} d^{3} v
$$

18.2 Show that

$$
\begin{gathered}
2 C \int_{0}^{C} \exp \left(W^{2}-C^{2}\right) d W= \\
2 C^{2} \sum_{n=0}^{\infty}(-1)^{n} \frac{2^{n} C^{2 n}}{1 \times 3 \times 5 \times \cdots(2 n-1)(2 n+1)}
\end{gathered}
$$

by making a series expansion of the integrand. For $C \ll 1$, that is, for $(\omega / k) \ll\left(2 k_{B} T_{e} / m_{e}\right)^{1 / 2}$, show that the dispersion relation for the longitudinal plasma wave reduces to

$$
k^{2} \lambda_{D}^{2}=-1
$$

or

$$
k^{2}\left(\frac{k_{B} T_{e}}{m_{e}}\right)^{1 / 2}=-\omega_{p e}^{2}
$$


This result is the low-frequency limit of the result obtained from the macroscopic warm plasma model, using the isothermal sound speed of the electron gas $V_{s e}=\left(k_{B} T_{e} / m_{e}\right)^{1 / 2}$.

18.3 (a) Show that the dispersion relation for the longitudinal plasma wave (with $\mathbf{k}=k \widehat{\mathbf{x}}$ ), for the case of an unbounded homogeneous plasma in which the motion of the electrons and the ions is taken into account, can be written as

$$
1=\sum_{\alpha} \frac{\omega_{p \alpha}^{2}}{n_{0} k^{2}} \int_{v} \frac{\partial f_{0 \alpha}(v)}{\partial v_{x}} \frac{1}{\left(v_{x}-\omega / k\right)} d^{3} v
$$

where $\alpha=e, i$. Show that this dispersion relation can be recast into the form

$$
1=\frac{1}{k^{2}}\left[\frac{\omega_{p e}^{2}}{<\left(v_{x}-\omega / k\right)^{2}>_{0 e}}+\frac{\omega_{p i}^{2}}{<\left(v_{x}-\omega / k\right)^{2}>_{0 i}}\right]
$$

where (with $\alpha=e, i$ )

$$
\frac{1}{<\left(v_{x}-\omega / k\right)^{2}>_{0 \alpha}}=\frac{1}{n_{0}} \int_{v} \frac{f_{0 \alpha}(v)}{\left(v_{x}-\omega / k\right)^{2}} d^{3} v
$$

(b) For the cold plasma model, for which

$$
f_{0 \alpha}(v)=n_{0} \delta\left(v_{x}\right) \delta\left(v_{y}\right) \delta\left(v_{z}\right)
$$

show that the dispersion relation reduces to

$$
\omega^{2}=\omega_{p e}^{2}+\omega_{p i}^{2}=\frac{n_{0} e^{2}}{\mu \epsilon_{0}}
$$

where $\mu=m_{e} m_{i} /\left(m_{e}+m_{i}\right)$ is the reduced mass of an electron and an ion.

(c) In the high phase velocity limit, show, by making a binomial expansion, that the dispersion relation becomes

$$
\begin{aligned}
& \frac{\omega_{p e}^{2}}{\omega^{2}}\left(1+3 \frac{k^{2}}{\omega^{2}} \frac{k_{B} T_{e}}{m_{e}}+\cdots\right)+ \\
& \frac{\omega_{p i}^{2}}{\omega^{2}}\left(1+3 \frac{k^{2}}{\omega^{2}} \frac{k_{B} T_{i}}{m_{i}}+\cdots\right)=1
\end{aligned}
$$


Show that this equation can be written as

$$
1=\frac{\omega_{p e}^{2}+\omega_{p i}^{2}}{\omega^{2}}\left(1+3 \frac{k^{2}}{\omega^{2}} \frac{k_{B} T_{h}}{\mu}+\cdots\right)
$$

where $T_{h}$ is a hybrid temperature given by

$$
T_{h}=\frac{\left(m_{i}^{2} T_{e}+m_{e}^{2} T_{i}\right)}{\left(m_{e}+m_{i}\right)^{2}}
$$

Under what conditions does this relation reduce to the Bohm-Gross dispersion relation for a warm electron plasma?

(d) Show that the dispersion relation of part (a) can be expressed as

$$
\begin{aligned}
1= & -\frac{1}{k^{2} \lambda_{D e}^{2}}\left[1+i \pi^{1 / 2} C_{e} \exp \left(-C_{e}^{2}\right)-2 C_{e} \int_{0}^{C_{e}} \exp \left(W^{2}-C_{e}^{2}\right) d W\right]- \\
& \frac{1}{k^{2} \lambda_{D i}^{2}}\left[1+i \pi^{1 / 2} C_{i} \exp \left(-C_{i}^{2}\right)-2 C_{i} \int_{0}^{C_{i}} \exp \left(W^{2}-C_{i}^{2}\right) d W\right]
\end{aligned}
$$

where (with $\alpha=e, i$ )

$$
\begin{gathered}
\lambda_{D \alpha}=\left(\frac{\epsilon_{0} k_{B} T_{\alpha}}{n_{0} e^{2}}\right)^{1 / 2} \\
C_{\alpha}=\frac{(\omega / k)}{\left(2 k_{B} T_{\alpha} / m_{\alpha}\right)^{1 / 2}}
\end{gathered}
$$

For weakly damped oscillations $\left(\omega_{i} \ll \omega_{r}\right)$ and in the low-frequency and low phase velocity range specified by the condition

$$
C_{i} \gg 1 \gg C_{e}
$$

show that the dispersion relation reduces to

$$
1=-\frac{1}{k^{2} \lambda_{D e}^{2}}\left(1+i \pi^{1 / 2} C_{e}-\frac{m_{e}}{2 m_{i}} C_{e}^{2}\right)
$$

Consequently, verify that the frequency of oscillation and the Landau damping constant are given by

$$
\omega_{r}=\left(\frac{k_{B} T_{e}}{m_{i}}\right)^{1 / 2} \frac{k}{\left(1+k^{2} \lambda_{D e}^{2}\right)^{1 / 2}}
$$




$$
\omega_{i}=-\left(\frac{\pi m_{e}}{8 m_{i}}\right)^{1 / 2}\left(\frac{k_{B} T_{e}}{m_{i}}\right)^{1 / 2} \frac{k}{\left(1+k^{2} \lambda_{D e}^{2}\right)^{2}}
$$

Note that the condition $C_{i} \gg 1 \gg C_{e}$ is fulfilled only if $T_{e} / T_{i} \gg(1+$ $k^{2} \lambda_{D e}^{2}$ ), which implies a strongly nonisothermal plasma, with hot electrons and cold ions. Show that in the long-wave range we find

$$
\omega_{r}=k^{2}\left(\frac{k_{B} T_{e}}{m_{i}}\right)^{1 / 2}
$$

which is essentially the same as the low-frequency ion acoustic waves that propagate at a sound speed determined by the ion mass and the electron temperature.

18.4 A longitudinal plasma wave is set up propagating in the $x$ direction $(\mathbf{k}=k \widehat{\mathbf{x}})$ in a plasma whose equilibrium state is characterized by the following so-called resonance distribution of velocities in the direction of the wave normal of the longitudinal plasma wave:

$$
f_{0}(\mathbf{v})=n_{0} \frac{A}{\pi} \frac{1}{\left(v_{x}^{2}+A^{2}\right)} \delta\left(v_{y}\right) \delta\left(v_{z}\right)
$$

where $A$ is a constant.

(a) Using this expression for $f_{0}(\mathbf{v})$ in the dispersion relation for the longitudinal plasma wave, given in (4.9), obtain the result

$$
1=\frac{\omega_{p e}^{2}}{k^{2}} \frac{A}{\pi} \int_{-\infty}^{+\infty} \frac{d v_{x}}{\left(v_{x}-\omega / k\right)^{2}\left(v_{x}^{2}+A^{2}\right)}
$$

(b) Evaluate the integral of part (a) by closing the integration contour in the upper half plane (note that there is a double pole at $v_{x}=\omega / k$ and a single pole at $v_{x}=i A$ ), to obtain the dispersion relation

$$
1=\frac{\omega_{p e}^{2}}{k^{2}} \frac{1}{(\omega / k+i A)^{2}}
$$

(c) Analyze this dispersion relation $\left(\omega=\omega_{r}+i \omega_{i}\right)$ to show that the longitudinal wave in this plasma is not unstable and determine the frequency of oscillation $\left(\omega_{r}\right)$ and the Landau damping constant $\left(\omega_{i}\right)$. Compare this Landau damping constant with the corresponding value for a Maxwellian distribution of velocities, for the cases when $k \lambda_{D} \ll 1$ and $k \lambda_{D} \geq 1$. 
18.5 Solve the linearized Vlasov equation (3.9) by the method of integral transforms, taking its Laplace transform in the time domain and the Fourier transform with respect to the space variables. Then, determine the dispersion relation for the modes of wave propagation in a hot isotropic plasma.

18.6 Evaluate the integral $G(C, 0)$, defined in (4.32) with $s=0$, by the method of residues using the contours of integration in the complex plane shown in Fig. 2.

18.7 Consider a longitudinal wave propagating along the $x$ direction in a plasma whose electric field is given by

$$
E_{x}(x, t)=E_{0} \sin (k x-\omega t)
$$

(a) Show that, for small displacements, the electrons that are moving with a velocity approximately equal to the phase velocity of the wave will oscillate with a frequency given by

$$
\omega^{\prime}=\left(\frac{e E_{0} k}{m_{e}}\right)^{1 / 2}
$$

(b) Establish the necessary conditions for trapping of the electrons by the wave.

18.8 Consider the two-stream instability using the macroscopic cold plasma equations for two beams of electrons having number densities given by

$$
n_{1,2}=\frac{1}{2} n_{0}+n_{1,2} \exp (i k x-i \omega t)
$$

and average velocities given by

$$
u_{1,2}= \pm u_{0}+u_{1,2} \exp (i k x-i \omega t)
$$

Consider that the electric field is given by

$$
E_{x}=E_{0} \exp (i k x-i \omega t)
$$

Determine the dispersion relation for this two-stream problem and verify if the oscillations with real $k$ are stable or unstable. 


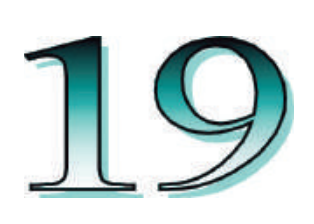

\section{WAVES IN HOT \\ MAGNETIZED PLASMAS}

\section{INTRODUCTION}

The analysis of small-amplitude waves propagating in a hot plasma, presented in the previous chapter, is now extended to anisotropic plasmas immersed in an externally applied magnetic field. Emphasis is given to the study of plasma waves having their propagation vector $\mathbf{k}$ either parallel or perpendicular to the externally applied magnetostatic field.

For propagation along the magnetostatic field the plasma waves separate again into three independent groups. The first group is the longitudinal plasma wave, whereas the second and third groups are the left and the right circularly polarized transverse electromagnetic waves.

For propagation across the magnetostatic field the plasma waves separate into two groups, which are designated as the TM (transverse magnetic) and the TEM (transverse electric magnetic) modes. The longitudinal plasma wave does not exist independently for any orientation of the magnetostatic field other than parallel to $\mathbf{k}$.

The mathematical analysis of the problem of wave propagation at an arbitrary direction relative to the magnetostatic field in a hot magnetized plasma is more complicated insofar as the details are concerned and will not be presented here. 


\section{WAVE PROPAGATION ALONG THE MAGNETOSTATIC FIELD IN A HOT PLASMA}

In this section we analyze the problem of wave propagation in an unbounded hot plasma consisting of mobile electrons in a neutralizing background of stationary ions, immersed in a uniform magnetostatic field $\mathbf{B}_{0}$.

In the equilibrium state, the electron number density (which is the same as that of the ions) is denoted by $n_{0}$. In the absence of perturbations, the homogeneous electron equilibrium distribution function has to satisfy the zero-order Vlasov equation

$$
\left(\mathbf{v} \times \mathbf{B}_{0}\right) \cdot \nabla_{v} f_{0}(\mathbf{v})=0
$$

The presence of the magnetostatic field introduces an anisotropy in the distribution function, so that the equilibrium distribution function will be denoted by $f_{0}\left(v_{\|}, v_{\perp}\right)$, where $v_{\|}$and $v_{\perp}$ represent the electron velocity in directions parallel and perpendicular to $\mathbf{B}_{0}$, respectively.

\subsection{Linearized Vlasov Equation}

As before, the perturbed distribution function is assumed to consist of a small perturbation, $f_{1}(\mathbf{r}, \mathbf{v}, t)$, superimposed on the equilibrium distribution function, $f_{0}\left(v_{\|}, v_{\perp}\right)$, that is,

$$
f(\mathbf{r}, \mathbf{v}, t)=f_{0}\left(v_{\|}, v_{\perp}\right)+f_{1}(\mathbf{r}, \mathbf{v}, t)
$$

where $\left|f_{1}\right| \ll f_{0}$. The electric field $\mathbf{E}(\mathbf{r}, t)$ and the magnetic induction $\mathbf{B}(\mathbf{r}, t)$ associated with the charge density and the current density inside the plasma, and which are related to the first-order pertubation $f_{1}(\mathbf{r}, \mathbf{v}, t)$, are also first-order quantities. Note, however, that $\mathbf{E}(\mathbf{r}, t)$ denotes the total electric field inside the plasma, whereas the total magnetic field $\mathbf{B}_{t}(\mathbf{r}, t)$ is given by

$$
\mathbf{B}_{t}(\mathbf{r}, t)=\mathbf{B}_{0}+\mathbf{B}(\mathbf{r}, t)
$$

Substituting (2.2) and (2.3) into the Vlasov equation (18.2.1) (Eq. 2.1 in Chapter 18), neglecting all second-order terms, and noting that the equilibrium distribution function is homogeneous, results in the following linearized Vlasov equation:

$$
\frac{\partial f_{1}(\mathbf{r}, \mathbf{v}, t)}{\partial t}+\mathbf{v} \cdot \nabla f_{1}(\mathbf{r}, \mathbf{v}, t)-\frac{e}{m_{e}}[\mathbf{E}(\mathbf{r}, t)+\mathbf{v} \times \mathbf{B}(\mathbf{r}, t)] .
$$




$$
\cdot \nabla_{v} f_{0}\left(v_{\|}, v_{\perp}\right)-\frac{e}{m_{e}}\left(\mathbf{v} \times \mathbf{B}_{0}\right) \cdot \nabla_{v} f_{1}(\mathbf{r}, \mathbf{v}, t)=0
$$

\subsection{Solution of the Linearized Vlasov Equation}

For the purpose of investigating the characteristic of plane waves propagating along the magnetostatic field, we shall assume that the spacetime dependence of all physical quantities is a periodic harmonic dependence according to

$$
\begin{aligned}
\mathbf{E}(\mathbf{r}, t) & =\mathbf{E} \exp (i \mathbf{k} \cdot \mathbf{r}-i \omega t) \\
\mathbf{B}(\mathbf{r}, t) & =\mathbf{B} \exp (i \mathbf{k} \cdot \mathbf{r}-i \omega t) \\
f_{1}(\mathbf{r}, \mathbf{v}, t) & =f_{1}(\mathbf{v}) \exp (i \mathbf{k} \cdot \mathbf{r}-i \omega t)
\end{aligned}
$$

where $\mathbf{E}, \mathbf{B}$, and $f_{1}(\mathbf{v})$ are phasor amplitudes (which in general may be complex quantities) independent of space and time. With this space-time dependence, the linearized Vlasov equation (2.4) reduces to

$$
\begin{gathered}
-i(\omega-\mathbf{k} \cdot \mathbf{v}) f_{1}(\mathbf{v})-\frac{e}{m_{e}}\left(\mathbf{v} \times \mathbf{B}_{0}\right) \cdot \nabla_{v} f_{1}(\mathbf{v})= \\
\frac{e}{m_{e}}(\mathbf{E}+\mathbf{v} \times \mathbf{B}) \cdot \nabla_{v} f_{0}\left(v_{\|}, v_{\perp}\right)
\end{gathered}
$$

In order to solve this differential equation for $f_{1}(\mathbf{v})$ in velocity space, we shall introduce cylindrical coordinates $\left(v_{\|}, v_{\perp}, \phi\right)$ with the vector component $v_{\|}$along the magnetostatic field, as shown in Fig. 1. Therefore, we have $\mathbf{B}_{0}=B_{0} \widehat{\mathbf{z}}$ and

$$
v_{x}=v_{\perp} \cos \phi \quad ; \quad v_{y}=v_{\perp} \sin \phi \quad ; \quad v_{z}=v_{\|}
$$

Using these relations, we can write

$$
\begin{gathered}
\frac{d}{d \phi} f_{1}(\mathbf{v})=\left(\frac{d v_{x}}{d \phi} \frac{\partial}{\partial v_{x}}+\frac{d v_{y}}{d \phi} \frac{\partial}{\partial v_{y}}+\frac{d v_{z}}{d \phi} \frac{\partial}{\partial v_{z}}\right) f_{1}(\mathbf{v})= \\
\left(-v_{y} \frac{\partial}{\partial v_{x}}+v_{x} \frac{\partial}{\partial v_{y}}\right) f_{1}(\mathbf{v})=-(\mathbf{v} \times \widehat{\mathbf{z}}) \cdot \nabla_{v} f_{1}(\mathbf{v})
\end{gathered}
$$

Substituting this expression into (2.8), we obtain

$$
-i(\omega-\mathbf{k} \cdot \mathbf{v}) f_{1}(\mathbf{v})+\frac{e B_{0}}{m_{e}} \frac{d f_{1}(\mathbf{v})}{d \phi}=\frac{e}{m_{e}}(\mathbf{E}+\mathbf{v} \times \mathbf{B}) \cdot \nabla_{v} f_{0}\left(v_{\|}, v_{\perp}\right)
$$




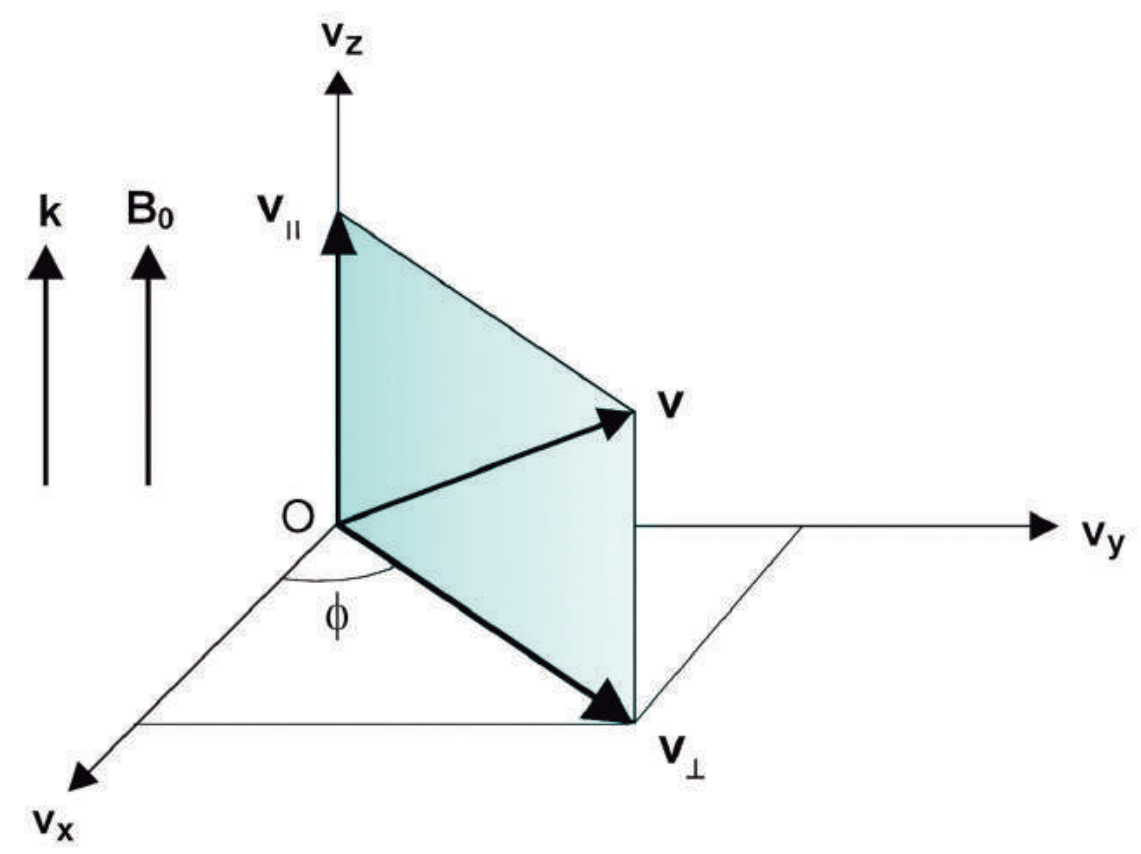

Fig. 1 Cylindrical coordinate system $\left(v_{\|}, v_{\perp}, \phi\right)$ in velocity space, with the $v_{\|}$axis along the magnetostatic field $\mathbf{B}_{0}$ and $v_{\perp}$ in the $\left(v_{x}, v_{y}\right)$ plane normal to $\mathbf{B}_{0}$.

Using the definition of the electron cyclotron frequency $\Omega_{c e}=\left(e B_{0} / m_{e}\right)$, (2.11) can be rewritten as

$$
\frac{d f_{1}(\mathbf{v})}{d \phi}-\frac{i(\omega-\mathbf{k} \cdot \mathbf{v})}{\Omega_{c e}} f_{1}(\mathbf{v})=\frac{e}{m_{e} \Omega_{c e}}(\mathbf{E}+\mathbf{v} \times \mathbf{B}) \cdot \nabla_{v} f_{0}\left(v_{\|}, v_{\perp}\right)
$$

From Maxwell $\nabla \times \mathbf{E}$ equation we can express the magnetic field as

$$
\mathbf{B}=\frac{1}{\omega}(\mathbf{k} \times \mathbf{E})
$$

Substituting (2.13) into (2.12), and making use of the vector identity $\mathbf{v} \times(\mathbf{k} \times \mathbf{E})=(\mathbf{v} \cdot \mathbf{E}) \mathbf{k}-(\mathbf{k} \cdot \mathbf{v}) \mathbf{E}$, we obtain for the right-hand side of $(2.12)$

$$
\begin{gathered}
\frac{e}{m_{e} \Omega_{c e}}(\mathbf{E}+\mathbf{v} \times \mathbf{B}) \cdot \nabla_{v} f_{0}= \\
\frac{e}{m_{e} \Omega_{c e}}\left[\left(1-\frac{k v_{\|}}{\omega}\right) \mathbf{E} \cdot \nabla_{v} f_{0}+\frac{k(\mathbf{v} \cdot \mathbf{E})}{\omega} \frac{\partial f_{0}}{\partial v_{\|}}\right]=
\end{gathered}
$$




$$
\begin{gathered}
\frac{e}{m_{e} \Omega_{c e}}\left\{\left(1-\frac{k v_{\|}}{\omega}\right)\left[\left(E_{x} \cos \phi+E_{y} \sin \phi\right) \frac{\partial f_{0}}{\partial v_{\perp}}+E_{\|} \frac{\partial f_{0}}{\partial v_{\|}}\right]+\right. \\
\left.\frac{k}{\omega}\left[\left(E_{x} \cos \phi+E_{y} \sin \phi\right) v_{\perp}+E_{\|} v_{\|}\right] \frac{\partial f_{0}}{\partial v_{\|}}\right\}= \\
\frac{e}{m_{e} \Omega_{c e}}\left\{\left[\left(1-\frac{k v_{\|}}{\omega}\right) \frac{\partial f_{0}}{\partial v_{\perp}}+\frac{k v_{\perp}}{\omega} \frac{\partial f_{0}}{\partial v_{\|}}\right]\left(E_{x} \cos \phi+E_{y} \sin \phi\right)+E_{\|} \frac{\partial f_{0}}{\partial v_{\|}}\right\}
\end{gathered}
$$

where we have taken $\mathbf{k} \cdot \mathbf{v}=k v_{\|}$and $\mathbf{k} \cdot \nabla_{v}=k\left(\partial / \partial v_{\|}\right)$, since we are considering wave propagation parallel to the magnetic field.

At this point it is convenient to express the component of the electric field vector in the plane perpendicular to $\mathbf{B}_{0}$ as a linear superposition of two, oppositely directed, circularly polarized components. Noting that $(\widehat{\mathbf{x}}+i \widehat{\mathbf{y}}) / \sqrt{2}$ and $(\widehat{\mathbf{x}}-i \widehat{\mathbf{y}}) / \sqrt{2}$ are unit complex vectors, the Cartesian components of the electric field vector

$$
\mathbf{E}=E_{x} \widehat{\mathbf{x}}+E_{y} \widehat{\mathbf{y}}+E_{\|} \widehat{\mathbf{z}}
$$

can be appropriately rewritten as

$$
\mathbf{E}=E_{+} \frac{(\widehat{\mathbf{x}}+i \widehat{\mathbf{y}})}{\sqrt{2}}+E_{-} \frac{(\widehat{\mathbf{x}}-i \widehat{\mathbf{y}})}{\sqrt{2}}+E_{\|} \widehat{\mathbf{z}}
$$

where the following notation is used:

$$
E_{ \pm}=\frac{\left(E_{x} \mp i E_{y}\right)}{\sqrt{2}}
$$

The first term in the right-hand side of (2.16) represents a circularly polarized field with the electric field vector rotating in the clockwise direction, whereas the second term represents a circularly polarized field with the electric field vector rotating in the counterclockwise direction, for an observer looking along $\mathbf{B}_{0}$. For the right (left) circularly polarized field, with the thumb of the right (left) hand pointing in the direction of propagation $(\widehat{\mathbf{z}})$, the fingers curl in the direction of rotation of the electric field vector. Thus, the two linearly polarized perpendicular components of the electric field in the plane $(x, y)$, normal to $\mathbf{B}_{0}$, can be recast as a superposition of two circularly polarized components with opposite directions of rotation. The advantage of using the two circularly polarized components is that it permits the final equations, involving the transverse modes of propagation, to be separated into two independent sets of transverse fields. 
It is a trivial matter to verify the relation

$$
E_{x} \cos \phi+E_{y} \sin \phi=\frac{1}{\sqrt{2}}\left(E_{+} e^{i \phi}+E_{-} e^{-i \phi}\right)
$$

so that (2.12) can be rewritten as

$$
\begin{gathered}
\frac{d f_{1}(\mathbf{v})}{d \phi}-\frac{i\left(\omega-k v_{\|}\right)}{\Omega_{c e}} f_{1}(\mathbf{v})=\frac{e}{m_{e} \Omega_{c e}}\left\{\left[\left(1-\frac{k v_{\|}}{\omega}\right) \frac{\partial f_{0}}{\partial v_{\perp}}+\right.\right. \\
\left.\left.\frac{k v_{\perp}}{\omega} \frac{\partial f_{0}}{\partial v_{\|}}\right] \frac{1}{\sqrt{2}}\left(E_{+} e^{i \phi}+E_{-} e^{-i \phi}\right)+E_{\|} \frac{\partial f_{0}}{\partial v_{\|}}\right\}
\end{gathered}
$$

Introducing the notation

$$
\begin{gathered}
F_{+}(\mathbf{v})=F_{+}\left(v_{\|}, v_{\perp}\right) e^{i \phi} \\
F_{-}(\mathbf{v})=F_{-}\left(v_{\|}, v_{\perp}\right) e^{-i \phi} \\
F_{\|}(\mathbf{v})=F_{\|}\left(v_{\|}, v_{\perp}\right)
\end{gathered}
$$

where

$$
\begin{gathered}
F_{+}\left(v_{\|}, v_{\perp}\right)=\frac{e}{m_{e} \Omega_{c e}}\left[\left(1-\frac{k v_{\|}}{\omega}\right) \frac{\partial f_{0}}{\partial v_{\perp}}+\frac{k v_{\perp}}{\omega} \frac{\partial f_{0}}{\partial v_{\|}}\right] \frac{E_{+}}{\sqrt{2}} \\
F_{-}\left(v_{\|}, v_{\perp}\right)=\frac{e}{m_{e} \Omega_{c e}}\left[\left(1-\frac{k v_{\|}}{\omega}\right) \frac{\partial f_{0}}{\partial v_{\perp}}+\frac{k v_{\perp}}{\omega} \frac{\partial f_{0}}{\partial v_{\|}}\right] \frac{E_{-}}{\sqrt{2}} \\
F_{\|}\left(v_{\|}, v_{\perp}\right)=\frac{e}{m_{e} \Omega_{c e}} \frac{\partial f_{0}}{\partial v_{\|}} E_{\|}
\end{gathered}
$$

equation (2.19) becomes

$$
\frac{d f_{1}(\mathbf{v})}{d \phi}-\frac{i\left(\omega-k v_{\|}\right)}{\Omega_{c e}} f_{1}(\mathbf{v})=F_{+}(\mathbf{v})+F_{-}(\mathbf{v})+F_{\|}(\mathbf{v})
$$

In order to solve this differential equation, let

$$
f_{1}(\mathbf{v})=f_{1+}(\mathbf{v})+f_{1-}(\mathbf{v})+f_{1 \|}(\mathbf{v})
$$

where $f_{1+}(\mathbf{v}), f_{1-}(\mathbf{v})$, and $f_{1 \|}(\mathbf{v})$ are the solutions of (2.26) corresponding, respectively, to $F_{+}(\mathbf{v}), F_{-}(\mathbf{v})$, and $F_{\|}(\mathbf{v})$, individually, in the right-hand 
side of (2.26). Thus, the differential equation for $f_{1+}(\mathbf{v})$, for example, can be written as

$$
\begin{aligned}
& \frac{d}{d \phi}\left\{f_{1+}(\mathbf{v}) \exp \left[-\frac{i\left(\omega-k v_{\|}\right)}{\Omega_{c e}} \phi\right]\right\}= \\
& F_{+}\left(v_{\|}, v_{\perp}\right) \exp \left[-\frac{i\left(\omega-k v_{\|}\right)}{\Omega_{c e}} \phi+i \phi\right]
\end{aligned}
$$

Integrating both sides of this equation with respect to $\phi$, from $\phi=-\infty$ to an arbitrary value of $\phi$, and noting that the exponential term vanishes at $\phi=-\infty$, since $\omega$ has a vanishingly small positive imaginary part, yields

$$
f_{1+}(\mathbf{v})=\frac{i \Omega_{c e}}{\left(\omega-k v_{\|}-\Omega_{c e}\right)} F_{+}\left(v_{\|}, v_{\perp}\right) e^{i \phi}+C_{+} \exp \left[\frac{i\left(\omega-k v_{\|}\right)}{\Omega_{c e}} \phi\right]
$$

The value of $f_{1+}(\mathbf{v})$ must not change if $\phi$ is increased or decreased by integral multiples of $2 \pi$, since by physical arguments $f_{1+}(\mathbf{v})$ must be a single valued function of $\mathbf{v}$. This requirement can be satisfied only if $C_{+}=0$. Therefore, we obtain

$$
f_{1+}(\mathbf{v})=f_{1+}\left(v_{\|}, v_{\perp}\right) e^{i \phi}
$$

where

$$
f_{1+}\left(v_{\|}, v_{\perp}\right)=\frac{i \Omega_{c e}}{\left(\omega-k v_{\|}-\Omega_{c e}\right)} F_{+}\left(v_{\|}, v_{\perp}\right)
$$

In a similar way, we find

$$
f_{1-}(\mathbf{v})=f_{1-}\left(v_{\|}, v_{\perp}\right) e^{-i \phi}
$$

where

$$
f_{1-}\left(v_{\|}, v_{\perp}\right)=\frac{i \Omega_{c e}}{\left(\omega-k v_{\|}+\Omega_{c e}\right)} F_{-}\left(v_{\|}, v_{\perp}\right)
$$

and

$$
f_{1 \|}(\mathbf{v})=f_{1 \|}\left(v_{\|}, v_{\perp}\right)=\frac{i \Omega_{c e}}{\left(\omega-k v_{\|}\right)} F_{\|}\left(v_{\|}, v_{\perp}\right)
$$

Substituting expressions (2.23), (2.24), and (2.25) into (2.31), (2.33), and (2.34), respectively, yields the following explicit expression for the phasor amplitude $f_{1}(\mathbf{v})$ of the perturbation of the velocity distribution function, in terms of the equilibrium distribution function of the electrons:

$$
f_{1}(\mathbf{v})=\frac{i e}{m_{e}\left(\omega-k v_{\|}-\Omega_{c e}\right)}\left[\left(1-\frac{k v_{\|}}{\omega}\right) \frac{\partial f_{0}}{\partial v_{\perp}}+\frac{k v_{\perp}}{\omega} \frac{\partial f_{0}}{\partial v_{\|}}\right] \frac{E_{+}}{\sqrt{2}} e^{i \phi}+
$$




$$
\begin{aligned}
\frac{i e}{m_{e}\left(\omega-k v_{\|}+\Omega_{c e}\right)} & {\left[\left(1-\frac{k v_{\|}}{\omega}\right) \frac{\partial f_{0}}{\partial v_{\perp}}+\frac{k v_{\perp}}{\omega} \frac{\partial f_{0}}{\partial v_{\|}}\right] \frac{E_{-}}{\sqrt{2}} e^{-i \phi}+} \\
& \frac{i e}{m_{e}\left(\omega-k v_{\|}\right)} \frac{\partial f_{0}}{\partial v_{\|}} E_{\|}
\end{aligned}
$$

\subsection{Perturbation Current Density}

Since the space-time dependence of the electromagnetic field is of the form $\exp (i \mathbf{k} \cdot \mathbf{r}-i \omega t)$, we expect the current density to behave also as

$$
\mathbf{J}(\mathbf{r}, t)=\mathbf{J} \exp (i \mathbf{k} \cdot \mathbf{r}-i \omega t)
$$

where the phasor amplitude of the current density is given by

$$
\mathbf{J}=-e \int_{v} \mathbf{v} f_{1}(\mathbf{v}) d^{3} v
$$

where the integration is to be performed over all of velocity space. It is also convenient to separate $\mathbf{J}$ into two, oppositely directed, circularly polarized components, as well as a longitudinal component along $\mathbf{B}_{0}$. For this purpose, we express the electron velocity in a form analogous to (2.16),

$$
\mathbf{v}=v_{+} \frac{(\widehat{\mathbf{x}}+i \widehat{\mathbf{y}})}{\sqrt{2}}+v_{-} \frac{(\widehat{\mathbf{x}}-i \widehat{\mathbf{y}})}{\sqrt{2}}+\widehat{\mathbf{z}} v_{\|}
$$

where

$$
v_{ \pm}=\frac{1}{\sqrt{2}}\left(v_{x} \mp i v_{y}\right)
$$

Thus, with this representation for $\mathbf{v}$, we obtain the following corresponding components for $\mathbf{J}$ :

$$
\begin{aligned}
& J_{+}=-e \int_{v} v_{+} f_{1}(\mathbf{v}) d^{3} v \\
& J_{-}=-e \int_{v} v_{-} f_{1}(\mathbf{v}) d^{3} v \\
& J_{\|}=-e \int_{v} v_{\|} f_{1}(\mathbf{v}) d^{3} v
\end{aligned}
$$

According to $(2.27),(2.30),(2.32)$, and $(2.34)$, we can replace $f_{1}(\mathbf{v})$ by

$$
f_{1}(\mathbf{v})=f_{1+}\left(v_{\|}, v_{\perp}\right) e^{i \phi}+f_{1-}\left(v_{\|}, v_{\perp}\right) e^{-i \phi}+f_{1 \|}\left(v_{\|}, v_{\perp}\right)
$$


Further, in view of (2.9), we also have

$$
v_{+}=\frac{1}{\sqrt{2}} v_{\perp} e^{-i \phi} \quad ; \quad v_{-}=\frac{1}{\sqrt{2}} v_{\perp} e^{i \phi}
$$

so that (2.40), (2.41), and (2.42) become

$$
\begin{gathered}
J_{+}=-\frac{e}{\sqrt{2}} \int_{v} v_{\perp} e^{-i \phi}\left[f_{1+}\left(v_{\|}, v_{\perp}\right) e^{i \phi}+f_{1-}\left(v_{\|}, v_{\perp}\right) e^{-i \phi}+\right. \\
\left.f_{1 \|}\left(v_{\|}, v_{\perp}\right)\right] d^{3} v \\
J_{-}=-\frac{e}{\sqrt{2}} \int_{v} v_{\perp} e^{i \phi}\left[f_{1+}\left(v_{\|}, v_{\perp}\right) e^{i \phi}+f_{1-}\left(v_{\|}, v_{\perp}\right) e^{-i \phi}+\right. \\
\left.f_{1 \|}\left(v_{\|}, v_{\perp}\right)\right] d^{3} v \\
J_{\|}=-e \int_{v} v_{\|}\left[f_{1+}\left(v_{\|}, v_{\perp}\right) e^{i \phi}+f_{1-}\left(v_{\|}, v_{\perp}\right) e^{-i \phi}+\right. \\
\left.f_{1 \|}\left(v_{\|}, v_{\perp}\right)\right] d^{3} v
\end{gathered}
$$

In cylindrical coordinates we have $d^{3} v=v_{\perp} d v_{\perp} d v_{\|} d \phi$. Evaluating the integrals with respect to $\phi$, from 0 to $2 \pi$, yields the following simple results:

$$
\begin{gathered}
J_{+}=-\sqrt{2} e \pi \int_{0}^{\infty} v_{\perp}^{2} d v_{\perp} \int_{-\infty}^{+\infty} f_{1+}\left(v_{\|}, v_{\perp}\right) d v_{\|} \\
J_{-}=-\sqrt{2} e \pi \int_{0}^{\infty} v_{\perp}^{2} d v_{\perp} \int_{-\infty}^{+\infty} f_{1-}\left(v_{\|}, v_{\perp}\right) d v_{\|} \\
J_{\|}=-2 e \pi \int_{0}^{\infty} v_{\perp} d v_{\perp} \int_{-\infty}^{+\infty} v_{\|} f_{1 \|}\left(v_{\|}, v_{\perp}\right) d v_{\|}
\end{gathered}
$$

since

$$
\begin{aligned}
\int_{0}^{2 \pi} e^{ \pm i n \phi} d \phi & =0 \quad ; \quad \text { for } \quad n=1,2,3, \cdots \\
& =2 \pi \quad ; \quad \text { for } \quad n=0
\end{aligned}
$$

From (2.31), (2.33), and (2.34), together with (2.23), (2.24), and (2.25), we see that $J_{+}, J_{-}$, and $J_{\|}$depend, respectively, only on $E_{+}, E_{-}$, and $E_{\|}$. This result justifies the use of the method of decomposition of the vectors into the sum of two, oppositely directed, circularly polarized components in the plane normal to $\mathbf{B}_{0}$, and a longitudinal component along $\mathbf{B}_{0}$. 


\subsection{Separation into the Various Modes}

From Maxwell equations, considering the special case in which all field vectors vary as $\exp (i \mathbf{k} \cdot \mathbf{r}-i \omega t)$, with $\mathbf{k}=k \widehat{\mathbf{z}}$, we have

$$
\begin{gathered}
k \widehat{\mathbf{z}} \times \mathbf{E}=\omega \mathbf{B} \\
i k \widehat{\mathbf{z}} \times \mathbf{B}=\mu_{0} \mathbf{J}-\frac{i \omega}{c^{2}} \mathbf{E}
\end{gathered}
$$

Noting that $\widehat{\mathbf{z}} \times \mathbf{E}=\widehat{\mathbf{y}} E_{x}-\widehat{\mathbf{x}} E_{y},(2.52)$ and (2.53) can be rewritten in component form as

$$
\begin{gathered}
\omega B_{x}=-k E_{y} \\
\omega B_{y}=k E_{x} \\
\omega B_{z}=0
\end{gathered}
$$

and

$$
\begin{gathered}
-i k B_{y}=\mu_{0} J_{x}-\frac{i \omega}{c^{2}} E_{x} \\
i k B_{x}=\mu_{0} J_{y}-\frac{i \omega}{c^{2}} E_{y} \\
0=\mu_{0} J_{\|}-\frac{i \omega}{c^{2}} E_{\|}
\end{gathered}
$$

Now we define, as in (2.17),

$$
B_{ \pm}=\frac{1}{\sqrt{2}}\left(B_{x} \mp i B_{y}\right)
$$

Multiplying (2.54) by $1 / \sqrt{2}$, and $(2.55)$ by $\mp i / \sqrt{2}$, and adding the resulting expressions, yields

$$
B_{ \pm}=\mp i \frac{k}{\omega} E_{ \pm}
$$

Note that the signs are coupled, that is, either upper signs or lower signs are to be used. Similarly, combining (2.57) and (2.58), and noting that

$$
J_{ \pm}=\frac{1}{\sqrt{2}}\left(J_{x} \mp i J_{y}\right)
$$

we obtain

$$
\mp k B_{ \pm}=-\mu_{0} J_{ \pm}+\frac{i \omega}{c^{2}} E_{ \pm}
$$


From these equations it is clear that the total electromagnetic field can be separated into four independent groups, involving the following quantities:

(a) $J_{\|}, E_{\|}$[equation (2.59)].

(b) $B_{z}$ [equation (2.56)].

(c) $J_{-}, E_{-}, B_{-}$[equations (2.61) and (2.63), lower signs].

(d) $J_{+}, E_{+}, B_{+}$[equations (2.61) and (2.63), upper signs].

Note that $J_{+}, J_{-}$, and $J_{\|}$depend, respectively, only on $E_{+}, E_{-}$, and $E_{\|}$. The first group contains an electric field and an electric current in the direction of $\mathbf{k}$, which, in this section, is also the direction of $\mathbf{B}_{0}$. Further, there is no associated magnetic field. Therefore, it represents the electrostatic longitudinal plasma wave. The second group does not constitute a propagation mode but only shows, through (2.56), that for a wave propagating parallel to $\mathbf{B}_{0}$ the time-varying magnetic field in the parallel direction is zero. The third and fourth groups represent, respectively, the left circularly polarized and the right circularly polarized transverse electromagnetic waves. Thus, we can separately analyze the characteristics of the longitudinal plasma wave and of the two polarized transverse electromagnetic waves.

\subsection{Longitudinal Plasma Wave}

To deduce the dispersion relation for the longitudinal plasma wave propagating along the magnetostatic field $\mathbf{B}_{0}$, we substitute $J_{\|}$from (2.50), into (2.59), obtaining

$$
E_{\|}=i \frac{2 \pi e}{\epsilon_{0} \omega} \int_{0}^{\infty} v_{\perp} d v_{\perp} \int_{-\infty}^{+\infty} v_{\|} f_{1 \|}\left(v_{\|}, v_{\perp}\right) d v_{\|}
$$

From (2.34) and (2.25) we can replace $f_{1 \|}\left(v_{\|}, v_{\perp}\right)$ in $(2.64)$, to obtain the following dispersion relation:

$$
1=-2 \pi \frac{\omega_{p e}^{2}}{n_{0} \omega} \int_{0}^{\infty} v_{\perp} d v_{\perp} \int_{-\infty}^{+\infty} \frac{v_{\|}}{\left(\omega-k v_{\|}\right)}\left(\frac{\partial f_{0}}{\partial v_{\|}}\right) d v_{\|}
$$

This dispersion relation can be conveniently recasted as

$$
1=-\frac{\omega_{p e}^{2}}{n_{0} \omega} \int_{v} \frac{v_{\|}}{\left(\omega-k v_{\|}\right)}\left(\frac{\partial f_{0}}{\partial v_{\|}}\right) d^{3} v
$$

since in cylindrical coordinates $d^{3} v=v_{\perp} d v_{\perp} d v_{\|} d \phi$ and $\int_{0}^{2 \pi} d \phi=2 \pi$. 
This equation is identical to the dispersion equation (18.4.2) (Eq. 4.2 in Chapter 18), deduced for longitudinal waves in an isotropic plasma, except for the fact the directions $x$ and $z$ are interchanged (here $\mathbf{k}$ is parallel to $\mathbf{B}_{0}$ and to $\widehat{\mathbf{z}}$ ). Thus, the characteristic behavior of the longitudinal plasma wave propagating along the magnetostatic field is identical to the case of an isotropic plasma with no external magnetostatic field. The magnetostatic field, therefore, has no influence on the longitudinal plasma wave. This result is due to the fact that the magnetostatic field exerts no force on the charged particles moving along it, and therefore it does not influence the distribution of the electrons in the longitudinal direction. It is the perturbation in the electron velocity distribution in the longitudinal direction that accounts for the characteristics of the longitudinal plasma wave. Recall that the longitudinal plasma wave separates out as an independent mode of propagation.

\subsection{Transverse Electromagnetic Waves}

Consider now the two circularly polarized transverse waves (E normal to the direction of propagation $\widehat{\mathbf{z}})$. To deduce the dispersion relation for both waves, we first eliminate $B_{ \pm}$from (2.61) and (2.63), and express $J_{ \pm}$ in terms of $E_{ \pm}$as

$$
J_{ \pm}=\frac{i \epsilon_{0}}{\omega}\left(\omega^{2}-k^{2} c^{2}\right) E_{ \pm}
$$

Substituting $J_{ \pm}$, from (2.48) and (2.49), with $f_{1+}\left(v_{\|}, v_{\perp}\right)$ and $f_{1-}\left(v_{\|}, v_{\perp}\right)$ given by (2.31) and (2.33), respectively, yields

$$
\begin{gathered}
-e \pi \Omega_{c e} \sqrt{2} \int_{0}^{\infty} v_{\perp}^{2} d v_{\perp} \int_{-\infty}^{+\infty} \frac{F_{ \pm}\left(v_{\|}, v_{\perp}\right)}{\left(\omega-k v_{\|} \mp \Omega_{c e}\right)} d v_{\|}= \\
\frac{\epsilon_{0}}{\omega}\left(\omega^{2}-k^{2} c^{2}\right) E_{ \pm}
\end{gathered}
$$

If $(2.23)$ and $(2.24)$ are used to replace $F_{ \pm}\left(v_{\|}, v_{\perp}\right)$, we find the following dispersion relation for the transverse electromagnetic waves $\left(E_{ \pm} \neq 0\right)$ :

$$
\begin{gathered}
k^{2} c^{2}=\omega^{2}+\frac{\omega_{p e}^{2} \pi}{n_{0}} \int_{0}^{\infty} v_{\perp}^{2} d v_{\perp} \\
\int_{-\infty}^{+\infty} \frac{\left(\omega-k v_{\|}\right)\left(\partial f_{0} / \partial v_{\perp}\right)+k v_{\perp}\left(\partial f_{0} / \partial v_{\|}\right)}{\left(\omega-k v_{\|} \mp \Omega_{c e}\right)} d v_{\|}
\end{gathered}
$$


where the upper sign refers to the right circularly polarized wave and the lower sign to the left circularly polarized wave.

An alternative form of this equation can be obtained integrating the right-hand side by parts. First, integrating over $v_{\|}$by parts, we have

$$
\begin{aligned}
& \int_{0}^{\infty} v_{\perp}^{2} d v_{\perp} \int_{-\infty}^{+\infty} \frac{k v_{\perp}\left(\partial f_{0} / \partial v_{\|}\right)}{\left(\omega-k v_{\|} \mp \Omega_{c e}\right)} d v_{\|}= \\
& -\int_{0}^{\infty} \int_{-\infty}^{+\infty} \frac{k^{2} v_{\perp}^{2} f_{0}}{\left(\omega-k v_{\|} \mp \Omega_{c e}\right)} v_{\perp} d v_{\perp} d v_{\|}
\end{aligned}
$$

and integrating over $v_{\perp}$ by parts, we have

$$
\begin{gathered}
\int_{-\infty}^{+\infty} \frac{\left(\omega-k v_{\|}\right)}{\left(\omega-k v_{\|} \mp \Omega_{c e}\right)} d v_{\|} \int_{0}^{\infty} v_{\perp}^{2}\left(\frac{\partial f_{0}}{\partial v_{\perp}}\right) d v_{\perp}= \\
-2 \int_{0}^{\infty} \int_{-\infty}^{+\infty} \frac{\left(\omega-k v_{\|}\right) f_{0}}{\left(\omega-k v_{\|} \mp \Omega_{c e}\right)} v_{\perp} d v_{\perp} d v_{\|}
\end{gathered}
$$

Since in cylindrical coordinates we have $d^{3} v=v_{\perp} d v_{\perp} d v_{\|} d \phi$ and $\int_{0}^{2 \pi} d \phi=$ $2 \pi$, we obtain the following alternative form of the dispersion relation (2.69):

$$
k^{2} c^{2}=\omega^{2}-\frac{\omega_{p e}^{2}}{n_{0}} \int_{v}\left[\frac{\left(\omega-k v_{\|}\right)}{\left(\omega-k v_{\|} \mp \Omega_{c e}\right)}+\frac{\frac{1}{2} k^{2} v_{\perp}^{2}}{\left(\omega-k v_{\|} \mp \Omega_{c e}\right)^{2}}\right] f_{0} d^{3} v
$$

Let us investigate first the plasma behavior for the case of an isotropic equilibrium distribution function. Thus, we choose $f_{0}$ to be the MaxwellBoltzmann distribution function (18.4.22). Note that, in this case, the vector $\nabla_{v} f_{0}(v)$ is parallel to $\mathbf{v}$, so that the magnetic force term $[\mathbf{v} \times \mathbf{B}(\mathbf{r}, t)] \cdot \nabla_{v} f_{0}(v)$, in the linearized Vlasov equation (2.4), vanishes. Consequently, for an isotropic equilibrium distribution function, the magnetic field $\mathbf{B}(\mathbf{r}, t)$ of the wave has no influence on the plasma behavior in the linear approximation. Also, it can be verified that, in the isotropic case, all factors in the numerator of the integrands in (2.69) and (2.72), which contain the propagation coefficient $k$, vanish. The dispersion equation (2.69) then reduces to

$$
k^{2} c^{2}=\omega^{2}+\frac{\omega_{p e}^{2} \pi}{n_{0}} \int_{0}^{\infty} v_{\perp}^{2} d v_{\perp} \int_{-\infty}^{+\infty} \frac{\omega\left(\partial f_{0} / \partial v_{\perp}\right)}{\left(\omega-k v_{\|} \mp \Omega_{c e}\right)} d v_{\|}
$$


or, equivalently,

$$
k^{2} c^{2}=\omega^{2}+\frac{\omega_{p e}^{2} \omega}{2 n_{0}} \int_{v} \frac{v_{\perp}\left(\partial f_{0} / \partial v_{\perp}\right)}{\left(\omega \mp \Omega_{c e}\right)-k v_{\|}} d^{3} v
$$

The alternative form of this equation, corresponding to (2.72) for the isotropic case, is

$$
k^{2} c^{2}=\omega^{2}-\frac{\omega_{p e}^{2} \omega}{n_{0}} \int_{v} \frac{f_{0}}{\left(\omega \mp \Omega_{c e}\right)-k v_{\|}} d^{3} v
$$

Substituting $f_{0}(v)$ from (18.4.22) and performing the integration over $v_{\perp}$ and $\phi$, the dispersion relation (2.75) becomes

$$
k^{2} c^{2}=\omega^{2}-\omega_{p e}^{2} \omega\left(\frac{m_{e}}{2 \pi k_{B} T_{e}}\right)^{1 / 2} \int_{-\infty}^{+\infty} \frac{\exp \left[-m_{e} v_{\|}^{2} /\left(2 k_{B} T_{e}\right)\right]}{\left(\omega \mp \Omega_{c e}\right)-k v_{\|}} d v_{\|}
$$

where the upper and the lower signs correspond to the right and the left circularly polarized waves, respectively.

At this point it is convenient to introduce the following dimensionless parameters

$$
\begin{aligned}
& \alpha_{ \pm}=\frac{\left(\omega \mp \Omega_{c e}\right) / k_{ \pm}}{\left(2 k_{B} T_{e} / m_{e}\right)^{1 / 2}} \\
& \beta_{ \pm}=\frac{\omega / k_{ \pm}}{\left(2 k_{B} T_{e} / m_{e}\right)^{1 / 2}}
\end{aligned}
$$

The subscripts $(+)$ and $(-)$ are used in $k$ to denote that it corresponds either to the right or to the left circularly polarized wave, respectively. Thus, $\beta_{ \pm}$represents the wave phase velocity normalized to the most probable speed of the electrons $\left(2 k_{B} T_{e} / m_{e}\right)^{1 / 2}$. Setting, as in (18.4.25),

$$
q=\frac{v_{\|}}{\left(2 k_{B} T_{e} / m_{e}\right)^{1 / 2}}
$$

the dispersion relation (2.76) can be rewritten in the following simplified form:

$$
k_{ \pm}^{2} c^{2}=\omega^{2}+\omega_{p e}^{2} \beta_{ \pm} I\left(\alpha_{ \pm}\right)
$$

where $I\left(\alpha_{ \pm}\right)$denotes the integral

$$
I\left(\alpha_{ \pm}\right)=\frac{1}{\sqrt{\pi}} \int_{-\infty}^{+\infty} \frac{\exp \left(-q^{2}\right)}{\left(q-\alpha_{ \pm}\right)} d q
$$


This integral is the same as that defined by (18.4.32) with $s=1$ and has been calculated in section 4 of Chapter 18. Hence, with the help of (18.4.32) and (18.4.45), equation (2.80) can be rewritten as

$k_{ \pm}^{2} c^{2}=\omega^{2}+i \sqrt{\pi} \omega_{p e}^{2} \beta_{ \pm} \exp \left(-\alpha_{ \pm}^{2}\right)-2 \omega_{p e}^{2} \beta_{ \pm} \int_{0}^{\alpha_{ \pm}} \exp \left(W^{2}-\alpha_{ \pm}^{2}\right) d W$

This is the dispersion relation for the right (upper sign) and for the left (lower sign) circularly polarized transverse electromagnetic waves propagating along the magnetostatic field in a hot plasma, whose equilibrium state is characterized by the isotropic Maxwell-Boltzmann distribution function.

\subsection{Temporal Damping of the Transverse Electromagnetic Waves}

A careful examination of (2.82) reveals that, for $k_{ \pm}$real, $\omega$ has a negative imaginary part, indicating that the wave amplitude decays in time.

To establish if this temporal damping is significant or not, let us evaluate the asymptotic series expansion of the integral in (2.82) for the case when $\left|\alpha_{ \pm}\right| \gg 1$. For this purpose, we expand the integral in (2.82) in inverse powers of $\alpha_{ \pm}$. According to (18.4.51), we find that, as the first approximation (retaining only the leading term),

$$
\int_{0}^{\alpha_{ \pm}} \exp \left(W^{2}-\alpha_{ \pm}^{2}\right) d W=\frac{1}{2 \alpha_{ \pm}}
$$

With this result, and making use of the definitions (2.77) and (2.78), the dispersion equation (2.82) simplifies to

$$
k_{ \pm}^{2} c^{2}=\omega^{2}-\omega_{p e}^{2} \frac{\omega}{\left(\omega \mp \Omega_{c e}\right)}+i \sqrt{\pi} \omega_{p e}^{2} \beta_{ \pm} \exp \left(-\alpha_{ \pm}^{2}\right)
$$

Furthermore, for $\left|\alpha_{ \pm}\right| \gg 1$ the exponential damping term may be omitted in a first approximation, so that (2.84) becomes

$$
k_{ \pm}^{2} c^{2}=\omega^{2}-\omega_{p e}^{2} \frac{\omega}{\left(\omega \mp \Omega_{c e}\right)}
$$

This dispersion equation corresponds to the results obtained using the cold plasma model, with the upper sign for the right circularly polarized wave 
and the lower sign for the left circularly polarized wave. Consequently, it follows that the results of the cold plasma model are valid only if $\left|\alpha_{ \pm}\right| \gg 1$. In the case of the left circularly polarized wave, for a given real propagation coefficient $k_{-}$, we find, from (2.85), that $\omega$ is real and satisfies the condition

$$
\omega>-\frac{1}{2} \Omega_{c e}+\left(\frac{1}{4} \Omega_{c e}^{2}+\omega_{p e}^{2}\right)^{1 / 2}
$$

The phase velocity $\left(\omega / k_{-}\right)$of the left circularly polarized wave is greater than the velocity of light $c$ for all $k_{-}$and therefore $\beta_{-}$is a large number of the order of the ratio of $c$ to the thermal velocity of the electrons. Since $\alpha_{-} / \beta_{-}=\left(\omega+\Omega_{c e}\right) / \omega$ is positive and greater than unity, it follows that $\alpha_{-} \gg 1$ for all $k_{-}$. Consequently, the Landau damping of the left circularly polarized wave propagating along the magnetostatic field in a hot plasma is always negligible. This result was also obtained for the case of transverse electromagnetic waves in a hot isotropic plasma. Further, as far as the characteristics of the left circularly polarized waves are concerned, the cold plasma model is a very good approximation for all real propagation coefficients.

In the case of the right circularly polarized wave, for a given real propagation coefficient $k_{+}$, it is seen, from equation (2.85), that $\omega$ is real and satisfies the conditions

$$
\begin{gathered}
0<\omega<\Omega_{c e} \\
\omega>\frac{1}{2} \Omega_{c e}+\left(\frac{1}{4} \Omega_{c e}^{2}+\omega_{p e}^{2}\right)^{1 / 2}
\end{gathered}
$$

An important feature associated with the right circularly polarized wave is the existence of two natural frequency ranges of propagation, whereas for the left circularly polarized wave there is only one natural frequency range of propagation. However, the results for $\omega$ in the range specified in (2.87) do not strictly hold for frequencies of the order of the ion plasma frequency and lower, since at these low frequencies the motion of the ions cannot be neglected. For this reason we omit, in the following discussion, the very low frequency region $\left(\omega<\Omega_{c i}\right)$ of $(2.87)$. In the frequency range (2.88), it is found that the phase velocity $\left(\omega / k_{+}\right)$of the right circularly polarized wave is always greater than the velocity of light $c$, whereas in the frequency range (2.87) the phase velocity is less than, but of the order of $c$, except in the close neighborhood of $\Omega_{c e}$. Therefore, we see that $\beta_{+}$ is a large number and, since $\left|\alpha_{+} / \beta_{+}\right|=\left|\left(\omega-\Omega_{c e}\right) / \omega\right|$ is of the order of unity, we conclude that $\left|\alpha_{+}\right| \gg 1$, except for $\omega$ close to $\Omega_{c e}$. Thus, the temporal damping of the right circularly polarized wave is also negligibly 
small and the cold plasma model is a very good approximation for $\omega$ not close to $\Omega_{c e}$.

\subsection{Cyclotron Damping of the RCP Transverse Wave}

For $\omega$ in the close neighborhood of $\Omega_{c e}$, the phase velocity $\left(\omega / k_{+}\right)$of the right circularly polarized wave is of the order of the thermal velocity of the electrons or lower, so that $\beta_{+} \leq 1$. Consequently, since $\left|\alpha_{+} / \beta_{+}\right|=$ $\left|\left(\omega-\Omega_{c e}\right) / \omega\right|$ is much less than unity, it follows that $\left|\alpha_{+}\right| \ll 1$. This implies that the asymptotic series expansion in inverse powers of $\alpha_{ \pm}$, as given in (2.83) and valid for $\left|\alpha_{ \pm}\right| \gg 1$, is not applicable for $\omega$ close to $\Omega_{c e}$.

As a first approximation to the dispersion relation (2.82) for the limiting case of $\left|\alpha_{+}\right| \ll 1$, we can set $\alpha_{+}$equal to zero in (2.82), to obtain

$$
\left(\frac{k_{+} c}{\omega}\right)^{3}-\left(\frac{k_{+} c}{\omega}\right)=\frac{i c \pi^{1 / 2}}{\left(2 k_{B} T_{e} / m_{e}\right)^{1 / 2}}\left(\frac{\omega_{p e}}{\Omega_{c e}}\right)^{2}
$$

The second term in the left-hand side of (2.89) can be omitted in a first approximation, as compared to the first term, since $(\omega / k) / c \ll 1$. Hence, (2.89) simplifies to

$$
\left(\frac{\omega}{k_{+} c}\right)^{3}=-i \frac{\left(2 k_{B} T_{e} / m_{e}\right)^{1 / 2}}{c \pi^{1 / 2}}\left(\frac{\Omega_{c e}}{\omega_{p e}}\right)^{2}
$$

Solving this equation explicity for $\omega$, gives

$$
\omega=\omega_{r}+i \omega_{i}
$$

where

$$
\begin{aligned}
& \omega_{r}=\frac{\sqrt{3}}{2} k_{+}\left[\frac{\left(2 k_{B} T_{e} / m_{e}\right)^{1 / 2}}{\pi^{1 / 2}} c^{2}\left(\frac{\Omega_{c e}}{\omega_{p e}}\right)^{2}\right]^{1 / 3} \\
& \omega_{i}=-\frac{1}{2} k_{+}\left[\frac{\left(2 k_{B} T_{e} / m_{e}\right)^{1 / 2}}{\pi^{1 / 2}} c^{2}\left(\frac{\Omega_{c e}}{\omega_{p e}}\right)^{2}\right]^{1 / 3}
\end{aligned}
$$

Since $\omega$ has a negative imaginary part, it follows that the right circularly polarized wave, which is initially set to propagate along the magnetostatic field, is damped in time for $\omega$ close to $\Omega_{c e}$. This temporal damping is usually called cyclotron damping and it is similar to the Landau damping of the longitudinal plasma wave. 
The cyclotron damping, however, differs from the Landau damping in some aspects. The most important one is the fact that the acceleration is perpendicular to the drift motion of the particles and, since the perpendicular electric acceleration does not, in the first approximation, modify the parallel drift velocity, there is no tendency toward trapping. Therefore, trapping is insignificant in cyclotron damping. The charged particles moving along lines of force will feel the oscillations of the perpendicular electric field at a frequency that differs from the plasma rest-frame frequency by the Doppler shift. Since the electrons rotate about $\mathbf{B}_{0}$ in the same direction as the electric field of the right circularly polarized wave (see Fig. 2), some of them will feel the oscillations at their own cyclotron frequency and they will absorb energy from the field. As a consequence of this wave-particle interaction at the resonance frequency $\omega=\Omega_{c e}$, the electrons absorb energy from the wave electric field, causing the plasma wave to damp out with time. In the absence of resonant particles, there is no energy exchange between the wave electric field and the particles, and hence $\omega$ is real.

As a final point, note that in the limiting case of $\Omega_{c e} \rightarrow 0$, that is, in the absence of the externally applied magnetostatic field, we have $\alpha_{ \pm}=\beta_{ \pm}=C$ and (2.82) becomes identical to the dispersion relation (18.5.8) for transverse waves in an isotropic plasma.

\subsection{Instabilities in the RCP Transverse Wave}

We have seen that for an isotropic equilibrium distribution function the resonance at $\omega=\Omega_{c e}$, between the electrons and the right circularly polarized wave, leads to a temporal damping of the wave amplitude. However, depending on the characteristics of the distribution function, resonance can also lead to instabilities (which are associated with a positive imaginary part of $\omega$ ).

Recall that for the case of an isotropic velocity distribution function the wave magnetic field has no effect on the plasma behavior in the linear approximation, since $\nabla_{v} f_{0}(v)$ is parallel to $\mathbf{v}$ and, consequently, the magnetic force term in the linearized Vlasov equation vanishes. However, when the condition of velocity isotropy is dropped, the effects that arise from the wave magnetic field become important and may lead to instabilities. Although the wave magnetic field itself does not exchange energy with the particles, it exerts a force in the parallel $(\widehat{\mathbf{z}})$ direction on the particles, which destroys the isotropy of the velocity distribution function in the plane perpendicular to $\mathbf{B}_{0}$. This effect can lead to instabilities 


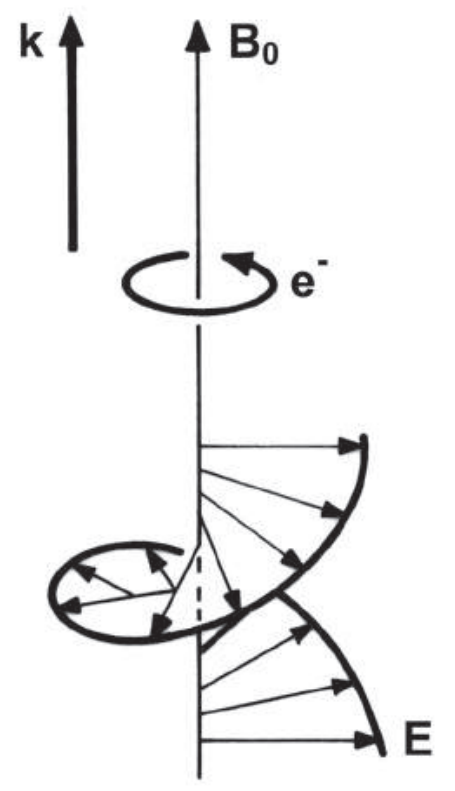

Fig. 2 Illustrating the resonance that occurs at $\omega=\Omega_{c e}$ between the electrons and the electric field of the right circularly polarized wave propagating along $\mathbf{B}_{0}$.

depending on the particle distribution function.

For the purpose of demonstrating such an instability, let us consider the following simple anisotropic equilibrium distribution function,

$$
f_{0}\left(v_{\|}, v_{\perp}\right)=\delta\left(v_{\|}\right) f_{0}\left(v_{\perp}\right)
$$

which represents cold electrons in the parallel $(\widehat{\mathbf{z}})$ direction, but with a Maxwellian velocity distribution function in the plane normal to $\mathbf{B}_{0}$. Inserting (2.94) into the dispersion relation (2.72) for the right circularly polarized wave (upper sign), gives

$$
\begin{gathered}
k^{2} c^{2}=\omega^{2}-\frac{\omega_{p e}^{2}}{n_{0}}\left[\int_{-\infty}^{+\infty} \frac{\left(\omega-k v_{\|}\right) \delta\left(v_{\|}\right)}{\left(\omega-k v_{\|}-\Omega_{c e}\right)} d v_{\|} \int_{0}^{\infty} f_{0}\left(v_{\perp}\right) v_{\perp} d v_{\perp} \int_{0}^{2 \pi} d \phi+\right. \\
\left.\int_{-\infty}^{+\infty} \frac{\delta\left(v_{\|}\right)}{\left(\omega-k v_{\|}-\Omega_{c e}\right)^{2}} d v_{\|} \int_{0}^{\infty} \frac{1}{2} k^{2} v_{\perp}^{2} f_{0}\left(v_{\perp}\right) v_{\perp} d v_{\perp} \int_{0}^{2 \pi} d \phi\right]
\end{gathered}
$$

Using the following property of the Dirac delta function,

$$
\int_{-\infty}^{+\infty} f(x) \delta\left(x-x_{0}\right) d x=f\left(x_{0}\right)
$$


substituting $f_{0}\left(v_{\perp}\right)$ by

$$
f_{0}\left(v_{\perp}\right)=n_{0}\left(\frac{m_{e}}{2 \pi k_{B} T_{e}}\right) \exp \left(-\frac{m_{e} v_{\perp}^{2}}{2 k_{B} T_{e}}\right)
$$

and performing the integrals, we obtain

$$
k^{2} c^{2}=\omega^{2}-\omega_{p e}^{2}\left[\frac{\omega}{\left(\omega-\Omega_{c e}\right)}+\frac{k^{2}\left(k_{B} T_{e} / m_{e}\right)}{\left(\omega-\Omega_{c e}\right)^{2}}\right]
$$

This equation can be rearranged in the form

$$
k^{2}=\frac{\omega^{2}\left(\omega-\Omega_{c e}\right)^{2}-\omega_{p e}^{2} \omega\left(\omega-\Omega_{c e}\right)}{c^{2}\left(\omega-\Omega_{c e}\right)^{2}+\omega_{p e}^{2}\left(k_{B} T_{e} / m_{e}\right)}
$$

It is a simple matter to verify that, for large values of $k^{2}$, the wave frequency $\omega$ becomes complex. Thus, in the limit of $k^{2} \rightarrow \infty$, the denominator of (2.99) vanishes and we obtain

$$
\omega^{2}-2 \omega \Omega_{c e}+\Omega_{c e}^{2}+\frac{\omega_{p e}^{2}\left(k_{B} T_{e} / m_{e}\right)}{c^{2}}=0
$$

The solution of this second-degree equation in $\omega$ is

$$
\omega=\Omega_{c e} \pm i \frac{\omega_{p e}\left(k_{B} T_{e} / m_{e}\right)^{1 / 2}}{c}
$$

which shows that growing modes (instabilities) can occur for $\omega_{r}=\Omega_{c e}$.

Choosing an anisotropic equilibrium distribution function with some velocity spread along the parallel $(\widehat{\mathbf{z}})$ direction, instead of $(2.94)$, we expect this instability to diminish, while turning into damping for an isotropic distribution function. The analysis of this statement is left as an exercise for the reader.

\section{WAVE PROPAGATION ACROSS THE MAGNETOSTATIC FIELD IN A HOT PLASMA}

We shall consider now the problem of wave propagation in a direction perpendicular to the externally applied uniform magnetostatic field $\mathbf{B}_{0}$. As before, we choose the $z$ axis along the magnetostatic field, that is, $\mathbf{B}_{0}=B_{0} \widehat{\mathbf{z}}$. The propagation coefficient $\mathbf{k}$ is normal to $\mathbf{B}_{0}$ and along the $x$ 


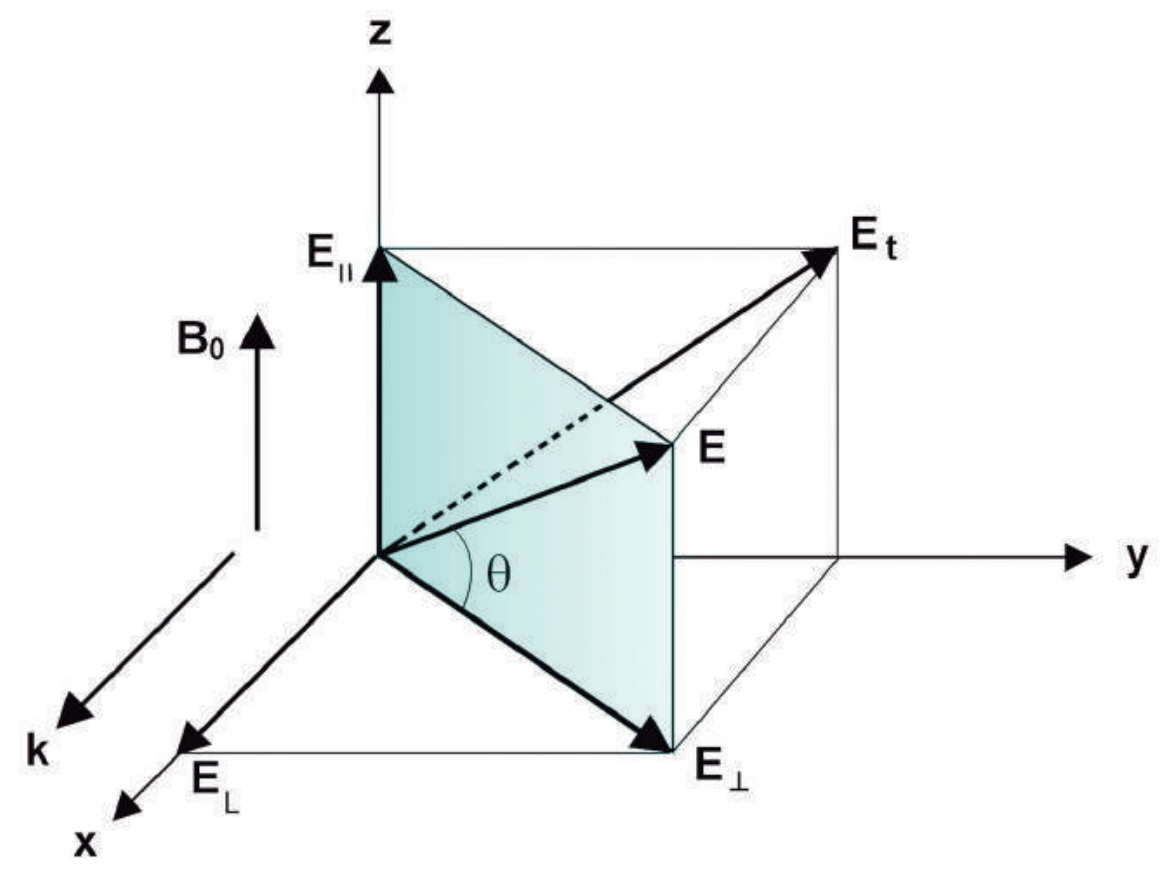

Fig. 3 Decomposition of the wave electric field vector into components parallel and perpendicular to $\mathbf{B}_{0}$, or in components longitudinal and transverse with respect to $\mathbf{k}$.

axis, that is, $\mathbf{k}=k \widehat{\mathbf{x}}$, as illustrated in Fig. 3, with $k$ considered to be real. All field quantities are assumed to vary harmonically in space and time, with the phase factor $\exp (i \mathbf{k} \cdot \mathbf{r}-i \omega t)$. As in the previous cases, we take

$$
f(\mathbf{r}, \mathbf{v}, t)=f_{0}\left(v_{\|}, v_{\perp}\right)+f_{1}(\mathbf{r}, \mathbf{v}, t) \quad ; \quad\left|f_{1}\right| \ll f_{0}
$$

where $f_{0}\left(v_{\|}, v_{\perp}\right)$ is the equilibrium distribution function of the electrons under the presence of the magnetostatic field, $v_{\|}=v_{z}$ is the velocity component of the electrons in the direction parallel to $\mathbf{B}_{0}$, and $v_{\perp} \mathrm{v}$ is the velocity component of the electrons in the plane $(x, y)$ normal to $\mathbf{B}_{0}$.

For the perturbation distribution function we take

$$
f_{1}(\mathbf{r}, \mathbf{v}, t)=f_{1}(\mathbf{v}) \exp (i k x-i \omega t)
$$

and for the wave electric and magnetic fields

$$
\begin{aligned}
& \mathbf{E}(\mathbf{r}, t)=\mathbf{E} \exp (i k x-i \omega t) \\
& \mathbf{B}(\mathbf{r}, t)=\mathbf{B} \exp (i k x-i \omega t)
\end{aligned}
$$


where $f_{1}(\mathbf{v}), \mathbf{E}$ and $\mathbf{B}$ are the phasor amplitudes, independent of space and time. As in the previous section, the purpose is to deduce the dispersion equation giving the functional relationship between $k$ and $\omega$. From an analysis of the dispersion relation we shall determine the intrinsic behavior of the plasma for the case under consideration.

\subsection{Solution of the Linearized Vlasov Equation}

From the linearized Vlasov equation (2.4), replacing the differential operators $\partial / \partial t$ and $\nabla$ by $-i \omega$ and $i k \widehat{\mathbf{x}}$, respectively, and making use of relation (2.10), we obtain

$$
\frac{d f_{1}(\mathbf{v})}{d \phi}-i \frac{(\omega-\mathbf{k} \cdot \mathbf{v})}{\Omega_{c e}} f_{1}(\mathbf{v})=\frac{e}{m_{e} \Omega_{c e}}(\mathbf{E}+\mathbf{v} \times \mathbf{B}) \cdot \nabla_{v} f_{0}\left(v_{\|}, v_{\perp}\right)
$$

where now $\mathbf{k} \cdot \mathbf{v}=k v_{x}=k v_{\perp} \cos \phi$. From Maxwell equation $\mathbf{k} \times \mathbf{E}=\omega \mathbf{B}$ we have

$$
\mathbf{B}=\frac{k}{\omega}\left(E_{y} \widehat{\mathbf{z}}-E_{z} \widehat{\mathbf{y}}\right)
$$

Using this expression for $\mathbf{B}$, we get

$$
\mathbf{v} \times \mathbf{B}=\frac{k}{\omega}\left[\left(v_{y} E_{y}+v_{z} E_{z}\right) \widehat{\mathbf{x}}-v_{x} E_{y} \widehat{\mathbf{y}}-v_{x} E_{z} \widehat{\mathbf{z}}\right]
$$

Noting that

$$
\begin{gathered}
\frac{\partial f_{0}}{\partial v_{x}}=\cos \phi \frac{\partial f_{0}}{\partial v_{\perp}} \\
\frac{\partial f_{0}}{\partial v_{y}}=\sin \phi \frac{\partial f_{0}}{\partial v_{\perp}} \\
\frac{\partial f_{0}}{\partial v_{z}}=\frac{\partial f_{0}}{\partial v_{\|}}
\end{gathered}
$$

we obtain

$$
\begin{gathered}
(\mathbf{E}+\mathbf{v} \times \mathbf{B}) \cdot \nabla_{v} f_{0}=\left[E_{x}+\frac{k}{\omega}\left(v_{y} E_{y}+v_{z} E_{z}\right)\right] \cos \phi \frac{\partial f_{0}}{\partial v_{\perp}}+ \\
\left(1-\frac{k v_{x}}{\omega}\right) E_{y} \sin \phi \frac{\partial f_{0}}{\partial v_{\perp}}+\left(1-\frac{k v_{x}}{\omega}\right) E_{z} \frac{\partial f_{0}}{\partial v_{z}}= \\
\frac{\partial f_{0}}{\partial v_{\perp}}\left(\cos \phi E_{x}+\sin \phi E_{y}\right)+\left[\frac{k}{\omega}\left(v_{z} \frac{\partial f_{0}}{\partial v_{\perp}}-v_{\perp} \frac{\partial f_{0}}{\partial v_{z}}\right) \cos \phi+\frac{\partial f_{0}}{\partial v_{z}}\right] E_{z}
\end{gathered}
$$


The linearized Vlasov equation (3.5) becomes, therefore,

$$
\begin{gathered}
\frac{d f_{1}(\mathbf{v})}{d \phi}-i \frac{\left(\omega-k v_{\perp} \cos \phi\right)}{\Omega_{c e}} f_{1}(\mathbf{v})=\frac{e}{m_{e} \Omega_{c e}}\left\{\frac{\partial f_{0}}{\partial v_{\perp}}\left(\cos \phi E_{x}+\sin \phi E_{y}\right)+\right. \\
\left.\left[\frac{k}{\omega}\left(v_{z} \frac{\partial f_{0}}{\partial v_{\perp}}-v_{\perp} \frac{\partial f_{0}}{\partial v_{z}}\right) \cos \phi+\frac{\partial f_{0}}{\partial v_{z}}\right] E_{z}\right\}
\end{gathered}
$$

The integrating factor for this first-order differential equation is found to be

$$
\begin{gathered}
h(\phi)=\exp \left[-\int_{0}^{\phi} i \frac{\left(\omega-k v_{\perp} \cos \phi\right)}{\Omega_{c e}} d \phi\right]= \\
\exp \left[-i\left(\frac{\omega}{\Omega_{c e}}\right) \phi+i\left(\frac{k v_{\perp}}{\Omega_{c e}}\right) \sin \phi\right]
\end{gathered}
$$

Multiplying both sides of (3.12) by the integrating factor (3.13), gives

$$
\begin{gathered}
\frac{d}{d \phi}\left\{f_{1}(\mathbf{v}) \exp \left[-i\left(\frac{\omega}{\Omega_{c e}}\right) \phi+i\left(\frac{k v_{\perp}}{\Omega_{c e}}\right) \sin \phi\right]\right\}= \\
\frac{e}{m_{e} \Omega_{c e}}\left\{\frac{\partial f_{0}}{\partial v_{\perp}}\left(\cos \phi E_{x}+\sin \phi E_{y}\right)+\left[\frac { k } { \omega } \left(v_{z} \frac{\partial f_{0}}{\partial v_{\perp}}-\right.\right.\right. \\
\left.\left.\left.v_{\perp} \frac{\partial f_{0}}{\partial v_{z}}\right) \cos \phi+\frac{\partial f_{0}}{\partial v_{z}}\right] E_{z}\right\} \exp \left[-i\left(\frac{\omega}{\Omega_{c e}}\right) \phi+i\left(\frac{k v_{\perp}}{\Omega_{c e}}\right) \sin \phi\right]
\end{gathered}
$$

The solution for $f_{1}(\mathbf{v})$ is obtained by integrating this equation over $\phi$,

$$
\begin{gathered}
f_{1}(\mathbf{v})=\frac{e}{m_{e} \Omega_{c e}} \exp \left[i\left(\frac{\omega}{\Omega_{c e}}\right) \phi-i\left(\frac{k v_{\perp}}{\Omega_{c e}}\right) \sin \phi\right] \int_{-\infty}^{\phi}\left\{\frac { \partial f _ { 0 } } { \partial v _ { \perp } } \left(\cos \phi^{\prime \prime} E_{x}+\right.\right. \\
\left.\left.\sin \phi^{\prime \prime} E_{y}\right)+\left[\frac{k}{\omega}\left(v_{z} \frac{\partial f_{0}}{\partial v_{\perp}}-v_{\perp} \frac{\partial f_{0}}{\partial v_{z}}\right) \cos \phi^{\prime \prime}+\frac{\partial f_{0}}{\partial v_{z}}\right] E_{z}\right\} \\
\exp \left[-i\left(\frac{\omega}{\Omega_{c e}}\right) \phi^{\prime \prime}+i\left(\frac{k v_{\perp}}{\Omega_{c e}}\right) \sin \phi^{\prime \prime}\right] d \phi^{\prime \prime}
\end{gathered}
$$

If the variable of integration is changed to $\phi^{\prime}=\phi-\phi^{\prime \prime}$, (3.15) becomes

$$
\begin{aligned}
& f_{1}(\mathbf{v})=\frac{e}{m_{e} \Omega_{c e}} \exp \left[-i\left(\frac{k v_{\perp}}{\Omega_{c e}}\right) \sin \phi\right] \int_{0}^{\infty}\left\{\frac { \partial f _ { 0 } } { \partial v _ { \perp } } \left[\cos \left(\phi-\phi^{\prime}\right) E_{x}+\right.\right. \\
& \left.\left.\sin \left(\phi-\phi^{\prime}\right) E_{y}\right]+\left[\frac{k}{\omega}\left(v_{z} \frac{\partial f_{0}}{\partial v_{\perp}}-v_{\perp} \frac{\partial f_{0}}{\partial v_{z}}\right) \cos \left(\phi-\phi^{\prime}\right)+\frac{\partial f_{0}}{\partial v_{z}}\right] E_{z}\right\}
\end{aligned}
$$




$$
\exp \left[i\left(\frac{\omega}{\Omega_{c e}}\right) \phi^{\prime}+i\left(\frac{k v_{\perp}}{\Omega_{c e}}\right) \sin \left(\phi-\phi^{\prime}\right)\right] d \phi^{\prime}
$$

Note that $\phi$ occurs only as the argument of periodic functions of period $2 \pi$, which is in agreement with the physical requirement that $f_{1}(\mathbf{v})$ be a single valued function of $\phi$.

\subsection{Current Density and the Conductivity Tensor}

The current density is given by

$$
\mathbf{J}(\mathbf{r}, t)=\mathbf{J} \exp (i k x-i \omega t)
$$

where the phasor amplitude $\mathbf{J}$ is

$$
\mathbf{J}=-e \int_{v} \mathbf{v} f_{1}(\mathbf{v}) d^{3} v
$$

or

$\mathbf{J}=-e \int_{0}^{\infty} v_{\perp} d v_{\perp} \int_{0}^{2 \pi} d \phi \int_{-\infty}^{+\infty} d v_{z} f_{1}(\mathbf{v})\left(v_{\perp} \cos \phi \widehat{\mathbf{x}}+v_{\perp} \sin \phi \widehat{\mathbf{y}}+v_{z} \widehat{\mathbf{z}}\right)$

For the purpose of calculating the components of $\mathbf{J}$, it is appropriate to express $\mathbf{J}$ as

$$
\mathbf{J}=\mathcal{S} \cdot \mathbf{E}
$$

or in explicit form, considering a Cartesian coordinate system,

$$
\begin{aligned}
& J_{x}=\sigma_{x x} E_{x}+\sigma_{x y} E_{y}+\sigma_{x z} E_{z} \\
& J_{y}=\sigma_{y x} E_{x}+\sigma_{y y} E_{y}+\sigma_{y z} E_{z} \\
& J_{z}=\sigma_{z x} E_{x}+\sigma_{z y} E_{y}+\sigma_{z z} E_{z}
\end{aligned}
$$

where $\mathcal{S}$ is the conductivity tensor, whose components can be arranged in matrix form as

$$
\mathcal{S}=\left(\begin{array}{lll}
\sigma_{x x} & \sigma_{x y} & \sigma_{x z} \\
\sigma_{y x} & \sigma_{y y} & \sigma_{y z} \\
\sigma_{z x} & \sigma_{z y} & \sigma_{z z}
\end{array}\right)
$$

If $f_{1}(\mathbf{v})$, from (3.16), is substituted into (3.19) and the resulting expression is compared with equations (3.21) to (3.23), we identify the components of the conductivity tensor as 


$$
\begin{aligned}
\sigma_{x x} & =\mathcal{A}_{1} B_{1}\left(v_{\|}, v_{\perp}, \phi\right) \\
\sigma_{x y} & =\mathcal{A}_{1} B_{2}\left(v_{\|}, v_{\perp}, \phi\right) \\
\sigma_{x z} & =\mathcal{A}_{1} B_{3}\left(v_{\|}, v_{\perp}, \phi\right) \\
\sigma_{y x} & =\mathcal{A}_{2} B_{1}\left(v_{\|}, v_{\perp}, \phi\right) \\
\sigma_{y y} & =\mathcal{A}_{2} B_{2}\left(v_{\|}, v_{\perp}, \phi\right) \\
\sigma_{y z} & =\mathcal{A}_{2} B_{3}\left(v_{\|}, v_{\perp}, \phi\right) \\
\sigma_{z x} & =\mathcal{A}_{3} B_{1}\left(v_{\|}, v_{\perp}, \phi\right) \\
\sigma_{z y} & =\mathcal{A}_{3} B_{2}\left(v_{\|}, v_{\perp}, \phi\right) \\
\sigma_{z z} & =\mathcal{A}_{3} B_{3}\left(v_{\|}, v_{\perp}, \phi\right)
\end{aligned}
$$

where we have used the notation

$$
\begin{gathered}
B_{1}\left(v_{\|}, v_{\perp}, \phi\right)=\int_{0}^{\infty} \frac{\partial f_{0}}{\partial v_{\perp}} \cos \left(\phi-\phi^{\prime}\right) \exp \left[g_{1}\left(\phi^{\prime}\right)\right] d \phi^{\prime} \\
B_{2}\left(v_{\|}, v_{\perp}, \phi\right)=\int_{0}^{\infty} \frac{\partial f_{0}}{\partial v_{\perp}} \sin \left(\phi-\phi^{\prime}\right) \exp \left[g_{1}\left(\phi^{\prime}\right)\right] d \phi^{\prime} \\
B_{3}\left(v_{\|}, v_{\perp}, \phi\right)=\int_{0}^{\infty}\left[\frac{k}{\omega}\left(v_{z} \frac{\partial f_{0}}{\partial v_{\perp}}-v_{\perp} \frac{\partial f_{0}}{\partial v_{z}}\right) \cos \left(\phi-\phi^{\prime}\right)+\frac{\partial f_{0}}{\partial v_{z}}\right] \\
\exp \left[g_{1}\left(\phi^{\prime}\right)\right] d \phi^{\prime} \\
g_{1}\left(\phi^{\prime}\right)=i \frac{\omega}{\Omega_{c e}} \phi^{\prime}+i \frac{k v_{\perp}}{\Omega_{c e}} \sin \left(\phi-\phi^{\prime}\right)
\end{gathered}
$$

which are to be considered as integrands of the integral operators defined by

$$
\begin{gathered}
\mathcal{A}_{1}=-\frac{e^{2}}{m_{e} \Omega_{c e}} \int_{0}^{\infty} v_{\perp}^{2} d v_{\perp} \int_{0}^{2 \pi} \cos \phi d \phi \int_{-\infty}^{+\infty} d v_{z} \exp \left(-i \frac{k v_{\perp}}{\Omega_{c e}} \sin \phi\right) \\
\mathcal{A}_{2}=-\frac{e^{2}}{m_{e} \Omega_{c e}} \int_{0}^{\infty} v_{\perp}^{2} d v_{\perp} \int_{0}^{2 \pi} \sin \phi d \phi \int_{-\infty}^{+\infty} d v_{z} \exp \left(-i \frac{k v_{\perp}}{\Omega_{c e}} \sin \phi\right) \\
\mathcal{A}_{3}=-\frac{e^{2}}{m_{e} \Omega_{c e}} \int_{0}^{\infty} v_{\perp} d v_{\perp} \int_{0}^{2 \pi} d \phi \int_{-\infty}^{+\infty} v_{z} d v_{z} \exp \left(-i \frac{k v_{\perp}}{\Omega_{c e}} \sin \phi\right)
\end{gathered}
$$




\subsection{Evaluation of the Integrals}

Let us consider initially the integrals in the equation (3.25) for $\sigma_{x x}$. In simplifying this expression for $\sigma_{x x}$ it is advantageous to calculate first the integral with respect to $\phi^{\prime}$. From (3.34a) consider, therefore, the integral

$$
I_{1}=\int_{0}^{\infty} \cos \left(\phi-\phi^{\prime}\right) \exp \left[g_{1}\left(\phi^{\prime}\right)\right] d \phi^{\prime}
$$

Differentiating $(3.34 d)$ with respect to $\phi^{\prime}$, we find

$$
\cos \left(\phi-\phi^{\prime}\right)=\frac{\omega}{k v_{\perp}}+i \frac{\Omega_{c e}}{k v_{\perp}} \frac{d g_{1}\left(\phi^{\prime}\right)}{d \phi^{\prime}}
$$

Thus, (3.35) becomes

$$
I_{1}=\frac{\omega}{k v_{\perp}} \int_{0}^{\infty} \exp \left[g_{1}\left(\phi^{\prime}\right)\right] d \phi^{\prime}+i \frac{\Omega_{c e}}{k v_{\perp}} \int_{0}^{\infty} d\left\{\exp \left[g_{1}\left(\phi^{\prime}\right)\right]\right\}
$$

since $d\left\{\exp \left[g_{1}\left(\phi^{\prime}\right)\right]\right\}=\exp \left[g_{1}\left(\phi^{\prime}\right)\right] d g_{1}\left(\phi^{\prime}\right)$. Therefore,

$$
I_{1}=\frac{\omega}{k v_{\perp}} \int_{0}^{\infty} \exp \left[g_{1}\left(\phi^{\prime}\right)\right] d \phi^{\prime}-i \frac{\Omega_{c e}}{k v_{\perp}} \exp \left(i \frac{k v_{\perp}}{\Omega_{c e}} \sin \phi\right)
$$

In order to evaluate the integral in (3.38), let us introduce the variable

$$
\xi=\frac{k v_{\perp}}{\Omega_{c e}}
$$

and express the term $\exp \left[g_{1}\left(\phi^{\prime}\right)\right]$ in a infinite series expansion in terms of the Bessel functions $J_{n}(\xi)$,

$$
\begin{gathered}
\exp \left(i \frac{\omega}{\Omega_{c e}} \phi^{\prime}\right) \exp \left[i \xi \sin \left(\phi-\phi^{\prime}\right)\right]= \\
\exp \left(i \frac{\omega}{\Omega_{c e}} \phi^{\prime}\right) \sum_{n=-\infty}^{+\infty} J_{n}(\xi) \exp \left[i n\left(\phi-\phi^{\prime}\right)\right]= \\
\sum_{n=-\infty}^{+\infty} J_{n}(\xi) \exp (i n \phi) \exp \left[i\left(\omega / \Omega_{c e}-n\right) \phi^{\prime}\right]
\end{gathered}
$$


where $J_{n}(\xi)$ is the Bessel function of the first kind and of order $n$. The factor $\exp \left[i \xi \sin \left(\phi-\phi^{\prime}\right)\right]$ is identified with the so-called generating function of the Bessel functions. Substituting (3.40) into (3.38), gives

$$
\begin{gathered}
I_{1}=-\frac{i}{\xi} \exp (i \xi \sin \phi)+\frac{\omega}{k v_{\perp}} \sum_{n=-\infty}^{+\infty} J_{n}(\xi) \exp (i n \phi) \\
\int_{0}^{\infty} \exp \left[i\left(\omega / \Omega_{c e}-n\right) \phi^{\prime}\right] d \phi^{\prime}
\end{gathered}
$$

or

$$
I_{1}=-\frac{i}{\xi} \exp (i \xi \sin \phi)+\frac{i \omega}{k v_{\perp}} \sum_{n=-\infty}^{+\infty} \frac{J_{n}(\xi) \exp (i n \phi)}{\left(\omega / \Omega_{c e}-n\right)}
$$

As the next step in evaluating $\sigma_{x x}$ we shall calculate now the integral with respect to $\phi$. Substituting (3.42) into the expression (3.25) for $\sigma_{x x}$, we find the integral with respect to $\phi$ to be

$$
I_{2}=\int_{0}^{2 \pi} \cos \phi\left[-\frac{i}{\xi}+\frac{i \omega}{k v_{\perp}} \sum_{n=-\infty}^{+\infty} \frac{J_{n}(\xi) \exp (i n \phi-i \xi \sin \phi)}{\left(\omega / \Omega_{c e}-n\right)}\right] d \phi
$$

The first term within square brackets in this equation integrates to zero. For the remaining terms note first that we can write

$$
\cos \phi=\frac{n}{\xi}+\frac{i}{\xi} \frac{d}{d \phi}(i n \phi-i \xi \sin \phi)
$$

so that the integral $I_{2}$ becomes

$$
\begin{gathered}
I_{2}=\frac{i \omega}{k v_{\perp}} \sum_{n=-\infty}^{+\infty} \frac{J_{n}(\xi)}{\left(\omega / \Omega_{c e}-n\right)}\left\{\frac{n}{\xi} \int_{0}^{2 \pi} \exp (i n \phi-i \xi \sin \phi) d \phi+\right. \\
\left.\frac{i}{\xi} \int_{0}^{2 \pi} d[\exp (i n \phi-i \xi \sin \phi)]\right\}
\end{gathered}
$$

The second integral within brackets in this equation vanishes, whereas the first integral can be expressed in terms of Bessel functions, according to the relation

$$
\int_{0}^{2 \pi} \exp (i n \phi-i \xi \sin \phi) d \phi=2 \pi J_{n}(\xi)
$$


which is known as the Bessel integral. Therefore, (3.45) becomes

$$
I_{2}=\frac{2 \pi i \omega}{\xi^{2} \Omega_{c e}} \sum_{n=-\infty}^{+\infty} \frac{n J_{n}^{2}(\xi)}{\left(\omega / \Omega_{c e}-n\right)}
$$

This result can be written in a slightly different form by noting that

$$
\begin{aligned}
I_{2}= & \frac{2 \pi i}{\xi^{2}} \sum_{n=-\infty}^{+\infty} \frac{n J_{n}^{2}(\xi)\left(\omega / \Omega_{c e}-n+n\right)}{\left(\omega / \Omega_{c e}-n\right)}= \\
& \frac{2 \pi i}{\xi^{2}} \sum_{n=-\infty}^{+\infty}\left[n J_{n}^{2}(\xi)+\frac{n^{2} J_{n}^{2}(\xi)}{\left(\omega / \Omega_{c e}-n\right)}\right]
\end{aligned}
$$

Now, since $J_{-n}(\xi)=(-1)^{n} J_{n}(\xi)$, we have

$$
\sum_{n=-\infty}^{+\infty} n J_{n}^{2}(\xi)=0
$$

and the integral (3.48) simplifies to

$$
I_{2}=\frac{2 \pi i}{\xi^{2}} \sum_{n=-\infty}^{+\infty} \frac{n^{2} J_{n}^{2}(\xi)}{\left(\omega / \Omega_{c e}-n\right)}
$$

From (3.25), (3.35), and (3.43), we see that the expression for $\sigma_{x x}$ can be written as

$$
\sigma_{x x}=-\frac{e^{2}}{m_{e} \Omega_{c e}} \int_{0}^{\infty} v_{\perp}^{2} d v_{\perp} \int_{-\infty}^{+\infty} \frac{\partial f_{0}}{\partial v_{\perp}} I_{2} d v_{z}
$$

Thus, the substitution of (3.50) into (3.51) yields

$$
\sigma_{x x}=-\frac{2 \pi i e^{2}}{m_{e} \Omega_{c e}} \int_{0}^{\infty} v_{\perp}^{2} d v_{\perp} \int_{-\infty}^{+\infty} d v_{z} \frac{\Omega_{c e}}{k v_{\perp}} \frac{\partial f_{0}}{\partial v_{\perp}} \sum_{n=-\infty}^{+\infty} \frac{n^{2} J_{n}^{2}\left(k v_{\perp} / \Omega_{c e}\right)}{\left(\omega / \Omega_{c e}-n\right)}
$$

This expression for $\sigma_{x x}$ is valid for any cylindrically symmetric equilibrium distribution function $f_{0}\left(v_{\|}, v_{\perp}\right)$.

However, for mathematical simplicity, in what follows, all the details will be restricted to the case in which the equilibrium state is characterized by the isotropic Maxwell-Boltzmann distribution function $f_{0}(v)$. Thus, we consider now

$$
f_{0}(v)=n_{0}\left(\frac{m_{e}}{2 \pi k_{B} T_{e}}\right)^{3 / 2} \exp \left[-\frac{m_{e}\left(v_{\perp}^{2}+v_{z}^{2}\right)}{2 k_{B} T_{e}}\right]
$$


for the evaluation of the integrals over $v_{\perp}$ and $v_{z}$ in (3.52). In order to perform the integral over $v_{\perp}$, it is convenient to introduce the following parameter:

$$
\bar{\nu}=\frac{k_{B} T_{e}}{m_{e}} \frac{k^{2}}{\Omega_{c e}^{2}}
$$

Performing the differentiation $\partial f_{0} / \partial v_{\perp}$ and using (3.54), the expression (3.52) simplifies to

$$
\sigma_{x x}=\frac{i n_{0} e^{2}}{m_{e} \Omega_{c e} \bar{\nu}^{2}} \sum_{n=-\infty}^{+\infty} \frac{n^{2}}{\left(\omega / \Omega_{c e}-n\right)} \int_{0}^{\infty} \xi J_{n}^{2}(\xi) \exp \left(-\frac{\xi^{2}}{2 \bar{\nu}}\right) d \xi
$$

From the theory of Bessel functions we have the following expression, known as Weber's second exponential integral,

$$
\int_{0}^{\infty} \exp \left(-p^{2} t^{2}\right) J_{n}(a t) J_{n}(b t) t d t=\frac{1}{2 p^{2}} \exp \left(-\frac{\left.a^{2}+b^{2}\right)}{4 p^{2}}\right) I_{n}\left(a b / 2 p^{2}\right)
$$

where $I_{n}(x)$ is the Bessel function of the second kind, which is related to the ordinary Bessel function with an imaginary argument, $J_{n}(i x)$, by

$$
I_{n}(x)=(-i)^{n} J_{n}(i x)
$$

Substituting (3.56) into (3.55), yields

$$
\sigma_{x x}=\frac{i n_{0} e^{2}}{m_{e} \Omega_{c e}} \frac{e^{-\bar{\nu}}}{\bar{\nu}} \sum_{n=-\infty}^{+\infty} \frac{n^{2} I_{n}(\bar{\nu})}{\left(\omega / \Omega_{c e}-n\right)}
$$

The components $\sigma_{x z}, \sigma_{y z}, \sigma_{z x}$, and $\sigma_{z y}$ of the conductivity tensor vanish, since the integrands in (3.27), (3.30), (3.31), and (3.32) are found to be odd functions of $v_{z}$. Thus, performing the integrations with respect to $v_{z}$ first, we find

$$
\sigma_{x z}=\sigma_{y z}=\sigma_{z x}=\sigma_{z y}=0
$$

The component $\sigma_{z z}$ of the conductivity tensor, for the case of the isotropic Maxwell-Boltzmann distribution function, simplifies to

$$
\begin{gathered}
\sigma_{z z}=-\frac{e^{2}}{m_{e} \Omega_{c e}} \int_{0}^{\infty} v_{\perp} d v_{\perp} \int_{0}^{2 \pi} d \phi \int_{-\infty}^{+\infty} v_{z} d v_{z} \exp (-i \xi \sin \phi) \\
\int_{0}^{\infty} \frac{\partial f_{0}}{\partial v_{z}} \exp \left[g_{1}\left(\phi^{\prime}\right)\right] d \phi^{\prime}
\end{gathered}
$$


The integrals appearing here can be evaluated as in the case of $\sigma_{x x}$, yielding the following result:

$$
\sigma_{z z}=\frac{i n_{0} e^{2}}{m_{e} \Omega_{c e}} e^{-\bar{\nu}} \sum_{n=-\infty}^{+\infty} \frac{I_{n}(\bar{\nu})}{\left(\omega / \Omega_{c e}-n\right)}
$$

The components $\sigma_{x y}, \sigma_{y x}$, and $\sigma_{y y}$ of the conductivity tensor will not be needed here, in order to investigate the characteristics of waves propagating across the magnetostatic field in a hot plasma. The derivation of explicit expressions for these components of the tensor $\mathcal{S}$ will be left as an exercise for the reader.

\subsection{Separation into the Various Modes}

Considering the space-time dependence of the fields, as given by (3.3) and (3.4), and expressing the electric current density as $\mathbf{J}=\mathcal{S} \cdot \mathbf{E}$, Maxwell curl equations reduce to

$$
\begin{gathered}
k \widehat{\mathbf{x}} \times \mathbf{E}=\omega \mathbf{B} \\
i k \widehat{\mathbf{x}} \times \mathbf{B}=\left(\mu_{0} \mathcal{S}-\mathbf{1} \frac{i \omega}{c^{2}}\right) \cdot \mathbf{E}=-\frac{i \omega}{c^{2}} \mathcal{E} \cdot \mathbf{E}
\end{gathered}
$$

where 1 denotes the unit dyad and

$$
\mathcal{E}=\mathbf{1}+\frac{i}{\omega \epsilon_{0}} \mathcal{S}
$$

is the relative permittivity dyad or tensor. In component form, (3.62) and (3.63) become, respectively,

$$
\begin{gathered}
B_{x}=0 \\
E_{z}=-\frac{\omega}{k} B_{y} \\
E_{y}=\frac{\omega}{k} B_{z}
\end{gathered}
$$

and

$$
\begin{gathered}
-\frac{\omega}{k c^{2}}\left(\epsilon_{x x} E_{x}+\epsilon_{x y} E_{y}\right)=0 \\
-\frac{\omega}{k c^{2}}\left(\epsilon_{y x} E_{x}+\epsilon_{y y} E_{y}\right)=-B_{z} \\
-\frac{\omega}{k c^{2}} \epsilon_{z z} E_{z}=B_{y}
\end{gathered}
$$


From (3.64), (3.58), and (3.61), it follows that

$$
\begin{aligned}
\epsilon_{x x} & =1-\frac{\omega_{p e}^{2}}{\omega \Omega_{c e}} \frac{e^{-\bar{\nu}}}{\bar{\nu}} \sum_{n=-\infty}^{+\infty} \frac{n^{2} I_{n}(\bar{\nu})}{\left(\omega / \Omega_{c e}-n\right)} \\
\epsilon_{z z} & =1-\frac{\omega_{p e}^{2}}{\omega \Omega_{c e}} e^{-\bar{\nu}} \sum_{n=-\infty}^{+\infty} \frac{I_{n}(\bar{\nu})}{\left(\omega / \Omega_{c e}-n\right)}
\end{aligned}
$$

and, from (3.59),

$$
\epsilon_{x z}=\epsilon_{y z}=\epsilon_{z x}=\epsilon_{z y}=0
$$

The expressions for the other components of $\mathcal{E}$ will not be needed in the mathematical treatment that follows.

An analysis of equations (3.65) to (3.70) shows that the waves are transverse magnetic $(\mathrm{TM})$ with respect to the direction $(\widehat{\mathbf{x}})$ of propagation, since $B_{x}=0$. Also, we see that the remaining field components can be separated into two independent groups, involving the following variables each:

(a) $E_{x}, E_{y}, B_{z}$ [equations (3.67), (3.68), (3.69)] (TM mode)

(b) $E_{z}, B_{y}$ [equations (3.66), (3.70)] (TEM mode).

The first group represents the TM mode, since there is no component of the wave magnetic field along the propagation direction $(\widehat{\mathbf{x}})$. The second group represents the TEM (transverse electric magnetic) mode, since it has no component of either the electric or the magnetic field along the propagation direction (refer to Fig. 3). It can be considered as a degenerate case of the TM mode. Since the electric field is in the direction of $\mathbf{B}_{0}$, the TEM mode is also called (in magnetoionic theory) the ordinary wave and it is not affected by $\mathbf{B}_{0}$.

\subsection{Dispersion Relations}

To deduce the dispersion equation for the TM mode, we shall first combine (3.68) and (3.69) to eliminate $E_{x}$, obtaining

$$
\frac{k c^{2}}{\omega} B_{z}=\left(\epsilon_{y y}-\frac{\epsilon_{x y} \epsilon_{y x}}{\epsilon_{x x}}\right) E_{y}
$$

Substituting $E_{y}$ from (3.67), into (3.74), yields

$$
\left(\frac{k^{2} c^{2}}{\omega^{2}}-\epsilon_{y y}+\frac{\epsilon_{x y} \epsilon_{y x}}{\epsilon_{x x}}\right) B_{z}=0
$$


For a nontrivial solution (i.e., for $B_{z} \neq 0$, as well as for $E_{x}$ and $E_{y}$ nonzero), the term within parenthesis in (3.75) must vanish, resulting in the following dispersion relation for the TM mode,

$$
\eta^{2}=\frac{1}{\epsilon_{x x}}\left(\epsilon_{x x} \epsilon_{y y}-\epsilon_{x y} \epsilon_{y x}\right)
$$

where $\eta=(k c / \omega)$ denotes the index of refraction.

To obtain the dispersion equation for the TEM mode, we substitute $E_{z}$ from (3.66), into (3.70), to find

$$
\left(\eta^{2}-\epsilon_{z z}\right) B_{y}=0
$$

For a nontrivial solution (i.e., for $B_{y} \neq 0$ and therefore for $E_{z} \neq 0$ ), we must require that

$$
\eta^{2}=\epsilon_{z z}
$$

which is the dispersion relation for the TEM mode.

\subsection{The Quasistatic Mode}

The TM mode corresponds to the extraordinary wave of magnetoionic theory in the zero-temperature limit. Since the dispersion relation (3.76), for the TM mode, is very complicated, in what follows we shall analyze this dispersion relation only for the limiting case of $k c / \omega$ tending to infinity. This limiting situation defines the resonance condition.

From (3.69) we see that, for finite values of $E_{x}$ and $E_{y}$, the wave magnetic field component $B_{z}$ must be equal to zero in the limiting case of $k c / \omega$ tending to infinity. From (3.67) it follows, therefore, that $E_{y}$ vanishes. Consequently, for a nontrivial solution $\left(E_{x} \neq 0\right)$, the dispersion relation becomes (for $k c / \omega \rightarrow \infty$ )

$$
\epsilon_{x x}=0
$$

This equation is known as the dispersion relation for the quasistatic wave propagating across the magnetostatic field, since the wave magnetic field is negligible and the wave electric field is essentially along the propagation direction. In this limit the longitudinal wave is already uncoupled from the transverse wave and the dispersion relation (3.79) refers to the longitudinal wave $\left(E_{x} \neq 0\right)$. As a matter of fact, the dispersion relation (3.79) can be derived directly from the laws of electrostatics, instead of 
using Maxwell equations. Thus, since the magnetic field can be omitted at the outset, (3.79) is also called the dispersion relation for the electrostatic wave. Although (3.79) is strictly correct only for $k c / \omega \rightarrow \infty$, it can be considered to be a reasonably good approximation for $k c / \omega \gg 1$.

From (3.71) the explicit expression for the dispersion relation (3.79), for the quasistatic wave, is found to be

$$
1=\frac{\omega_{p e}^{2}}{\omega \Omega_{c e}} \frac{e^{-\bar{\nu}}}{\bar{\nu}} \sum_{n=-\infty}^{+\infty} \frac{n^{2} I_{n}(\bar{\nu})}{\left(\omega / \Omega_{c e}-n\right)}
$$

Since $I_{-n}(\bar{\nu})=I_{n}(\bar{\nu})$, we have

$$
\sum_{n=-\infty}^{+\infty} n I_{n}(\bar{\nu})=0
$$

so that multiplying $(3.81)$ by $\left(\omega_{p e}^{2} / \omega \Omega_{c e}\right)\left(e^{-\bar{\nu}} / \bar{\nu}\right)$ and adding it to (3.80), we find

$$
\bar{\nu} \frac{\Omega_{c e}^{2}}{\omega_{p e}^{2}}=e^{-\bar{\nu}} \sum_{n=-\infty}^{+\infty} \frac{n I_{n}(\bar{\nu})}{\left(\omega / \Omega_{c e}-n\right)}
$$

This equation was extensively investigated by Bernstein, who showed that is has solutions for both $\omega$ and $k$ real. For this reason, these solutions are often called the Bernstein modes.

In order to show the absence of complex solutions for $\omega$, let us first write the dispersion equation (3.82) in a more convenient form. Making use of the expansion

$$
\exp (\bar{\nu} \cos y)=\sum_{n=-\infty}^{+\infty} I_{n}(\bar{\nu}) \exp (\text { iny })
$$

and setting $y=0$ in this expansion, we obtain

$$
1=e^{-\bar{\nu}} \sum_{n=-\infty}^{+\infty} I_{n}(\bar{\nu})
$$

Adding (3.84) and (3.82), gives

$$
1+\bar{\nu} \frac{\Omega_{c e}^{2}}{\omega_{p e}^{2}}=\omega e^{-\bar{\nu}} \sum_{n=-\infty}^{+\infty} \frac{I_{n}(\bar{\nu})}{\left(\omega-n \Omega_{c e}\right)}
$$


From (3.54) it is seen that $\bar{\nu}$ is real and positive, and therefore $I_{n}(\bar{\nu})$ is also real and positive. Hence, writing the angular frequency as

$$
\omega=\omega_{r}+i \omega_{i}
$$

where $\omega_{r}$ and $\omega_{i}$ are the real and imaginary parts of $\omega$, respectively, we can separate (3.85) into its real and imaginary parts. From the real part we have

$$
1+\bar{\nu} \frac{\Omega_{c e}^{2}}{\omega_{p e}^{2}}=e^{-\bar{\nu}} \sum_{n=-\infty}^{+\infty} I_{n}(\bar{\nu})\left[1+\frac{\Omega_{c e}+n\left(\omega_{r}-n \Omega_{c e}\right)}{\left(\omega_{r}-n \Omega_{c e}\right)^{2}+\omega_{i}^{2}}\right]
$$

and from the imaginary part,

$$
0=-\omega_{i} e^{-\bar{\nu}} \sum_{n=-\infty}^{+\infty} I_{n}(\bar{\nu}) \frac{n \Omega_{c e}}{\left(\omega_{r}-n \Omega_{c e}\right)^{2}+\omega_{i}^{2}}
$$

It can be shown that (3.88) can be satisfied only if $\omega_{i}=0$. This result means that the dispersion equation for the quasistatic wave has only real solutions for $\omega$, and therefore there is neither temporal damping nor instability for the quasistatic waves.

Next we shall obtain explicit real solutions for $\omega$ for two limiting cases. First, we consider the special case $\bar{\nu} \ll 1$, which, as seen from (3.54), corresponds to the zero-temperature limit, and afterwards we analyze the case $\bar{\nu} \gg 1$, which corresponds to the high-temperature limit.

For $\bar{\nu} \ll 1$ (zero-temperature limit), we have $I_{ \pm 1}(\bar{\nu})=\bar{\nu} / 2$, while $I_{ \pm n}(\bar{\nu})=\mathcal{O}\left(\bar{\nu}^{n}\right)$. If $\omega / \Omega_{c e}$ is not close to $n$, only the terms corresponding to $n= \pm 1$, in the infinite series on the right-hand side of (3.83), contribute significantly, whereas the other terms are small and can be neglected. Thus, (3.82) becomes, for $\bar{\nu} \ll 1$,

$$
\bar{\nu} \frac{\Omega_{c e}^{2}}{\omega_{p e}^{2}}=-\frac{I_{-1}(\bar{\nu})}{\left(\omega / \Omega_{c e}+1\right)}+\frac{I_{1}(\bar{\nu})}{\left(\omega / \Omega_{c e}-1\right)}
$$

which simplifies to

$$
\omega=\left(\omega_{p e}^{2}+\Omega_{c e}^{2}\right)^{1 / 2}
$$

This frequency is known as the upper hybrid resonant frequency. This resonant frequency is also predicted in the cold plasma model treatment of waves propagating across the magnetostatic field. Thus, we find that 
the hot plasma theory confirms the results predicted by the cold plasma model in the zero-temperature limit.

In addition, the hot plasma theory establishes the existence of other resonant frequencies not predicted by the cold plasma model. The dispersion equation (3.82) can also be satisfied by taking $\omega=n \Omega_{c e}$, for $n \geq 2$, and arranging such that only the $n^{\text {th }}$ term contributes, which it will if $\left(\omega / \Omega_{c e}-n\right)=\mathcal{O}\left(\bar{\nu}^{n-1}\right)$. Hence, in the zero-temperature limit, the hot plasma theory predicts resonant frequencies at each harmonic of the electron cyclotron frequency,

$$
\omega=n \Omega_{c e} \quad ; \quad n \geq 2 \quad(\text { for } \quad \bar{\nu} \ll 1)
$$

These resonant frequencies are not predicted by the cold plasma model.

In the high-temperature limit $(\bar{\nu} \gg 1)$, we have $e^{-\bar{\nu}} I_{n}(\bar{\nu})=\mathcal{O}\left(\bar{\nu}^{-1 / 2}\right)$ and it is found that the dispersion relation (3.82) is satisfied for

$$
\omega=n \Omega_{c e} \quad ; \quad n \geq 1 \quad(\text { for } \quad \bar{\nu} \gg 1)
$$

Therefore, in the limit $\bar{\nu} \gg 1$, the resonances occur at the fundamental, as well as at all the harmonics of the electron cyclotron frequency.

To obtain the resonant frequencies for intermediate values of $\bar{\nu}$, equation (3.82) needs to be solved numerically. It is convenient, for numerical purposes, to rewrite (3.82) in the form

$$
F\left(\omega / \Omega_{c e}, \bar{\nu}\right)=\bar{\nu} \frac{\Omega_{c e}^{2}}{\omega_{p e}^{2}}=2 e^{-\bar{\nu}} \sum_{n=1}^{+\infty} \frac{n^{2} I_{n}(\bar{\nu})}{\left(\omega / \Omega_{c e}\right)^{2}-n^{2}}
$$

The function $F\left(\omega / \Omega_{c e}, \bar{\nu}\right)$ is plotted in Fig. 4 in terms of $\omega / \Omega_{c e}$, for $\bar{\nu}=0.1$. The intersection points of this curve with the horizontal line corresponding to $\bar{\nu}\left(\Omega_{c e}^{2} / \omega_{p e}^{2}\right)$ give the resonant frequencies in the normalized form $\omega / \Omega_{c e}$.

In Fig. 5 we show the normalized resonant frequency, $\omega / \Omega_{c e}$, as a function of $\bar{\nu}^{1 / 2}$, for a specified value of $\Omega_{c e} / \omega_{p e}$. Note, from this figure, that below each resonant frequency curve, corresponding to frequencies greater than the upper hybrid resonant frequency, there is a frequency range in which resonance does not occur for any value of $\bar{\nu}$. Also, for $\bar{\nu} \ll 1$ it is verified, from Fig. 5, that the first harmonic of the electron cyclotron frequency is not a solution of the dispersion equation (3.82).

An important difference between the quasistatic waves considered here and the longitudinal plasma waves analyzed previously is the absence of Landau damping for the quasistatic waves. The analysis of quasistatic wave propagation at an arbitrary direction with respect to $\mathbf{B}_{0}$ is left as an exercise for the reader. 


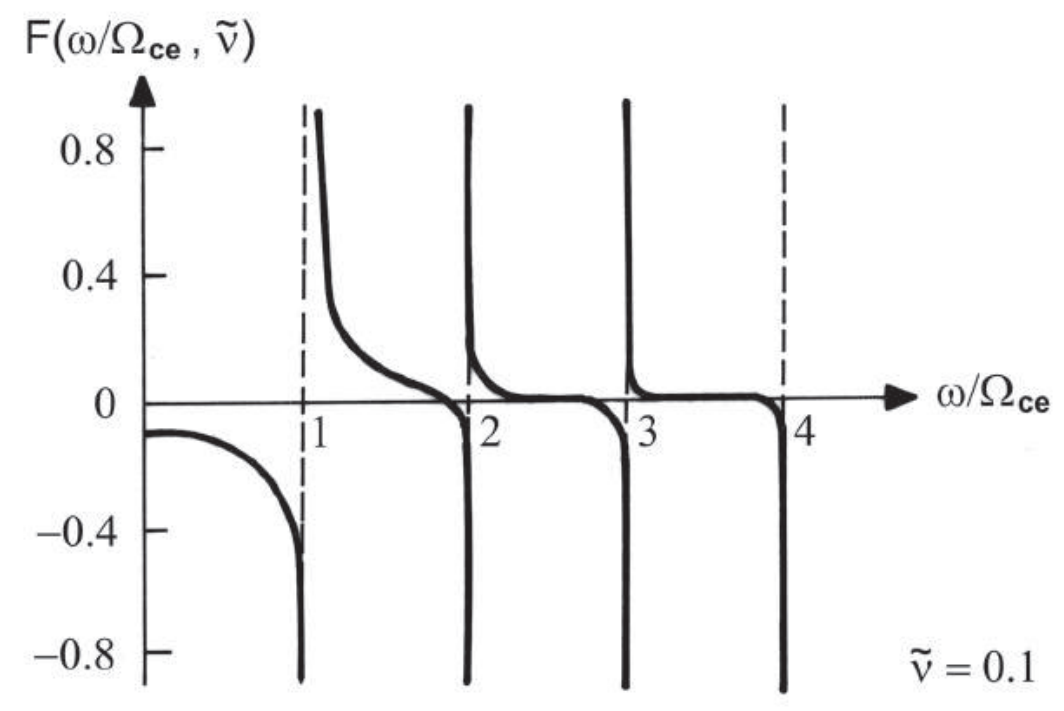

Fig. 4 Dependence of the function $F\left(\omega / \Omega_{c e}, \bar{\nu}\right)$, given by (3.93), in terms of $\omega / \Omega_{c e}$ for a fixed value of $\bar{\nu}$ (here $\bar{\nu}=0.1$ ), for the quasistatic wave.

\subsection{The TEM Mode}

From (3.78) and (3.72) the dispersion relation for the TEM mode propagating across the magnetostatic field in a hot plasma is given explicitly by

$$
\eta^{2}=1-\frac{\omega_{p e}^{2}}{\omega \Omega_{c e}} e^{-\bar{\nu}} \sum_{n=-\infty}^{+\infty} \frac{I_{n}(\bar{\nu})}{\left(\omega / \Omega_{c e}-n\right)}
$$

This equation has to be analyzed numerically. However, some useful results can be obtained directly, without resorting to numerical work, for some special limiting cases.

For the zero-temperature limiting case $\bar{\nu} \ll 1$, only the term corresponding to $n=0$ is significant, while all other terms are small and can be neglected. Therefore, for $\bar{\nu} \ll 1$, (3.94) simplifies to

$$
\eta^{2}=1-\omega_{p e}^{2} / \omega^{2}
$$

where we have used the relation $I_{0}(0)=1$. This result is just the dispersion relation for the ordinary (TEM) mode deduced from the cold plasma 


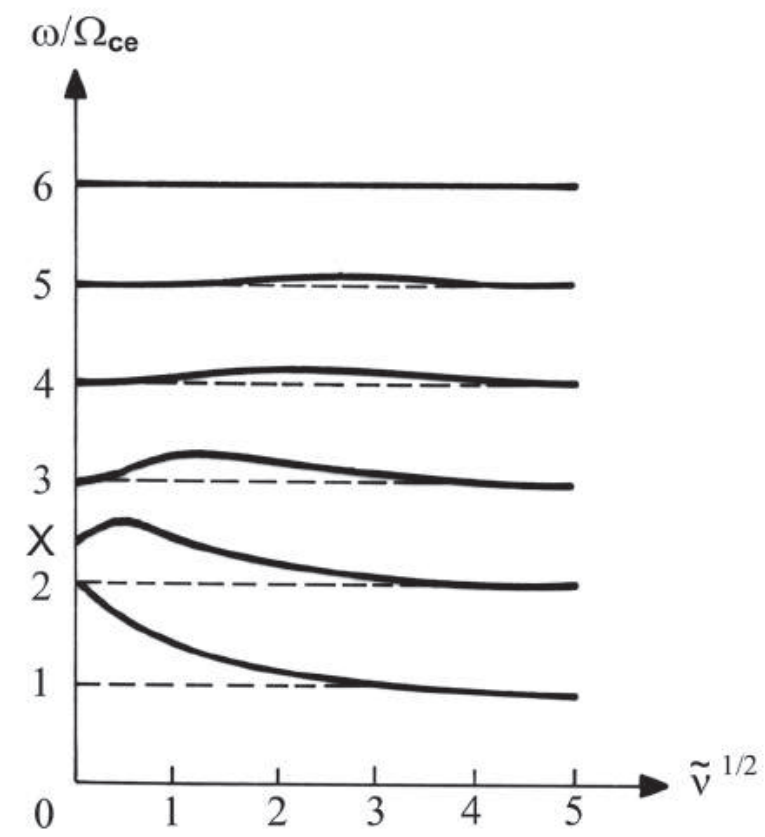

Fig. 5 Curves of resonant frequencies for the quasistatic wave propagating across the magnetostatic field, as a function of $(\bar{\nu})^{1 / 2}$, when $\left(\Omega_{c e} / \omega_{p e}\right)^{2}=0.2$. The resonant frequency, denoted by $X$, is the normalized upper hybrid frequency, given by $X=\left(\omega_{p e}^{2}+\Omega_{c e}^{2}\right)^{1 / 2} / \Omega_{c e}$.

model. Thus we find that, in the zero-temperature limit, the hot plasma theory agrees with the cold plasma model for the characteristics of the TEM mode propagating across the magnetostatic field.

For the high-temperature limiting case $\bar{\nu} \gg 1$, we have $e^{-\bar{\nu}} I_{n}(\bar{\nu})=$ $\mathcal{O}\left(\bar{\nu}^{-1 / 2}\right)$ and (3.94) reduces to

$$
\eta=1
$$

which is the dispersion relation for electromagnetic waves propagating in free space. Note that the condition $\bar{\nu} \gg 1$, together with (3.54) and (3.96), is equivalent to

$$
\omega \gg \Omega_{c e}\left(\frac{m_{e} c^{2}}{k_{B} T_{e}}\right)^{1 / 2}
$$

showing that the frequency must be very high. Hence, for $\bar{\nu} \gg 1$, or for very high frequencies, the results of the hot plasma theory are also in agreement with those predicted by the cold plasma model. 
Furthermore, according to the hot plasma theory, the TEM mode has resonances at the electron cyclotron frequency and at all its harmonics, since (3.94) shows that $k c / \omega \rightarrow \infty$ for

$$
\omega=n \Omega_{c e} \quad ; \quad n \geq 1
$$

The cold plasma model does not predict the existence of these harmonic resonances.

\section{SUMMARY}

\subsection{Propagation Along $\mathrm{B}_{0}$ in Hot Magnetoplasmas}

The dispersion relation for the longitudinal mode, considering $\mathbf{B}_{0}=$ $B_{0} \widehat{\mathbf{z}}$ and $\mathbf{k}=k \widehat{\mathbf{z}}$, is

$$
1=-\frac{\omega_{p e}^{2}}{n_{0} \omega} \int_{v} \frac{v_{\|}}{\left(\omega-k v_{\|}\right)}\left(\frac{\partial f_{0}}{\partial v_{\|}}\right) d^{3} v
$$

which is the same result obtained for the isotropic hot plasma.

The dispersion relation for the two transverse modes, is

$$
\begin{gathered}
k^{2} c^{2}=\omega^{2}+\frac{\omega_{p e}^{2} \pi}{n_{0}} \int_{0}^{\infty} v_{\perp}^{2} d v_{\perp} \\
\int_{-\infty}^{+\infty} \frac{\left(\omega-k v_{\|}\right)\left(\partial f_{0} / \partial v_{\perp}\right)+k v_{\perp}\left(\partial f_{0} / \partial v_{\|}\right)}{\left(\omega-k v_{\|} \mp \Omega_{c e}\right)} d v_{\|}
\end{gathered}
$$

The upper sign corresponds to the right circularly polarized wave, whereas the lower sign corresponds to the left circularly polarized wave. An alternative form for this dispersion relation is

$$
k^{2} c^{2}=\omega^{2}-\frac{\omega_{p e}^{2}}{n_{0}} \int_{v}\left[\frac{\left(\omega-k v_{\|}\right)}{\left(\omega-k v_{\|} \mp \Omega_{c e}\right)}+\frac{\frac{1}{2} k^{2} v_{\perp}^{2}}{\left(\omega-k v_{\|} \mp \Omega_{c e}\right)^{2}}\right] f_{0} d^{3} v
$$

When $f_{0}$ is the isotropic Maxwell-Boltzmann distribution function,

$k_{ \pm}^{2} c^{2}=\omega^{2}+i \sqrt{\pi} \omega_{p e}^{2} \beta_{ \pm} \exp \left(-\alpha_{ \pm}^{2}\right)-2 \omega_{p e}^{2} \beta_{ \pm} \int_{0}^{\alpha_{ \pm}} \exp \left(W^{2}-\alpha_{ \pm}^{2}\right) d W$ 
In the limits of the cold and warm plasma models,

$$
k_{ \pm}^{2} c^{2}=\omega^{2}-\omega_{p e}^{2} \frac{\omega}{\left(\omega \mp \Omega_{c e}\right)}
$$

The Landau (temporal) damping is negligible, since $v_{p h} \geq c$. The cyclotron temporal damping constant of the right circularly polarized wave, for $\omega_{r}=$ $\Omega_{c e}$, is given by

$$
\omega_{i}=-\frac{1}{2} k_{+}\left[\frac{\left(2 k_{B} T_{e} / m_{e}\right)^{1 / 2}}{\pi^{1 / 2}} c^{2}\left(\frac{\Omega_{c e}}{\omega_{p e}}\right)^{2}\right]^{1 / 3}
$$

\subsection{Propagation Across $\mathrm{B}_{0}$ in Hot Magnetoplasmas}

The dispersion relation for the TM mode, considering $\mathbf{B}_{0}=B_{0} \widehat{\mathbf{z}}$ and $\mathbf{k}=k \widehat{\mathbf{x}}$ and when $f_{0}$ is the isotropic Maxwell-Boltzmann distribution function, is

$$
\eta^{2}=\frac{1}{\epsilon_{x x}}\left(\epsilon_{x x} \epsilon_{y y}-\epsilon_{x y} \epsilon_{y x}\right)
$$

The TM mode corresponds to the extraordinary wave in magnetoionic theory (cold plasma). The longitudinal and transverse modes (with regard to $\mathbf{E})$ are coupled.

In the limit $k c / \omega \rightarrow \infty$ (resonance condition), $\epsilon_{x x}=0$, so that

$$
1=\frac{\omega_{p e}^{2}}{\omega \Omega_{c e}} \frac{e^{-\bar{\nu}}}{\bar{\nu}} \sum_{n=-\infty}^{+\infty} \frac{n^{2} I_{n}(\bar{\nu})}{\left(\omega / \Omega_{c e}-n\right)}
$$

which is called the dispersion relation for the quasistatic mode (longitudinal mode with $E_{x} \neq 0$ ). In the limit $k c / \omega \rightarrow \infty$ the two TM modes are uncoupled and the equation (3.79) applies to the longitudinal mode. The resonances are given by

$$
\omega=\left(\omega_{p e}^{2}+\Omega_{c e}^{2}\right)^{1 / 2}
$$

in the cold plasma limit, and by the so-called Bernstein modes

$$
\begin{aligned}
& \omega=n \Omega_{c e} \quad ; \quad n \geq 2 \quad(\text { for } \quad \bar{\nu} \ll 1) \\
& \omega=n \Omega_{c e} \quad ; \quad n \geq 1 \quad(\text { for } \quad \bar{\nu} \gg 1)
\end{aligned}
$$


The dispersion relation for the TEM mode, when $f_{0}$ is the MaxwellBoltzmann distribution function, is

$$
\eta^{2}=1-\frac{\omega_{p e}^{2}}{\omega \Omega_{c e}} e^{-\bar{\nu}} \sum_{n=-\infty}^{+\infty} \frac{I_{n}(\bar{\nu})}{\left(\omega / \Omega_{c e}-n\right)}
$$

The TEM mode corresponds to the ordinary wave in magnetoionic theory (cold plasma).

In the limit of the cold plasma model $(\bar{\nu} \ll 1)$,

$$
\eta^{2}=1-\omega_{p e}^{2} / \omega^{2}
$$

In hot plasma theory the resonances are given by

$$
\omega=n \Omega_{c e} \quad ; \quad n \geq 1
$$

\section{PROBLEMS}

19.1 Show that the first and second terms in the right-hand side of (2.16) represent, respectively, right and left circularly polarized wave fields.

19.2 Derive expression (3.61) for $\sigma_{z z}$ starting from (3.60).

19.3 Consider plane wave disturbances propagating along the magnetostatic field $\mathbf{B}_{0}$ in a hot electron gas, whose equilibrium distribution function is homogeneous and isotropic. In spherical coordinates in velocity space $(v, \theta, \phi)$ with $\mathbf{B}_{0}=B_{0} \widehat{\mathbf{z}}$ and $\mathbf{k}$ along $\mathbf{B}_{0}$, as illustrated in Fig. 6, show that the linearized Vlasov equation reduces to

$$
i \Omega_{c e} \frac{d f_{1}(\mathbf{v})}{d \phi}+(\omega-\mathbf{k} \cdot \mathbf{v}) f_{1}(\mathbf{v})=\frac{i e}{m_{e}} \mathbf{E} \cdot \nabla_{v} f_{0}(v)
$$

Verify that this differential equation has the formal solution

$$
f_{1}(\mathbf{v})=\frac{e}{m_{e} \Omega_{c e}} \int_{-\infty}^{\phi} \mathbf{E} \cdot \nabla_{v^{\prime}} f_{0}(v) \exp \left[\frac{i}{\Omega_{c e}}\left(\omega-\mathbf{k} \cdot \mathbf{v}^{\prime}\right)\left(\phi-\phi^{\prime}\right)\right] d \phi^{\prime}
$$




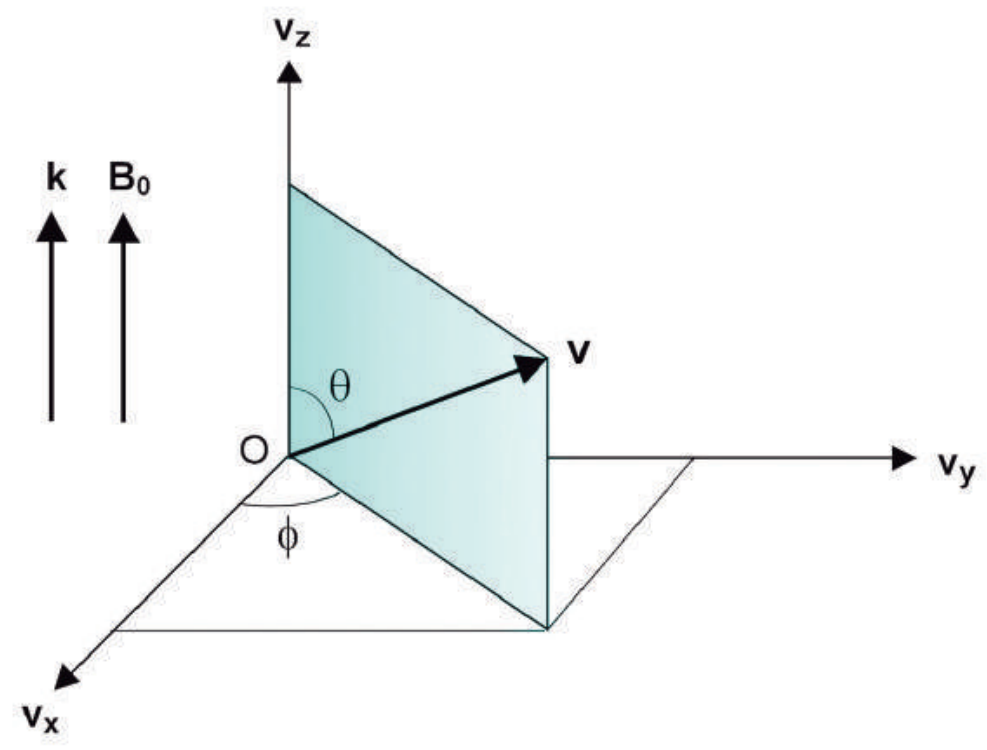

Fig. 6 Spherical coordinates in velocity space $(v, \theta, \phi)$, with $\mathbf{k}$ and $\mathbf{B}_{0}$ parallel to the third axis $(\widehat{\mathbf{z}})$.

where $\mathbf{v}^{\prime}$ is the velocity vector with components $\left(v, \theta, \phi^{\prime}\right)$. Note that

$$
\nabla_{v^{\prime}} f_{0}(v)=\frac{\mathbf{v}^{\prime}}{v} \frac{d f_{0}(v)}{d v}
$$

Perform the integral in this expression for $f_{1}(\mathbf{v})$ to obtain

$$
\begin{gathered}
f_{1}(\mathbf{v})=-\frac{i e}{m_{e} \Omega_{c e}}\left\{\frac { 1 } { ( A ^ { 2 } - 1 ) } \left[E_{x}\left(A \frac{\partial f_{0}}{\partial v_{x}}-i \frac{\partial f_{0}}{\partial v_{y}}\right)+\right.\right. \\
\left.\left.E_{y}\left(A \frac{\partial f_{0}}{\partial v_{y}}+i \frac{\partial f_{0}}{\partial v_{x}}\right)\right]+\frac{E_{z}}{A} \frac{\partial f_{0}}{\partial v_{z}}\right\}
\end{gathered}
$$

where $A=-(\omega-\mathbf{k} \cdot \mathbf{v}) / \Omega_{c e}$. From Maxwell equations obtain the relation

$$
\mathbf{E}-\eta^{2} \mathbf{E}_{t}=\frac{i e}{\omega \epsilon_{0}} \int_{v} \mathbf{v} f_{1}(\mathbf{v}) d^{3} v
$$

where $\mathbf{E}_{t}=\mathbf{E}-E_{z} \widehat{\mathbf{z}}$ is the transverse part of the electric field $\mathbf{E}$. Using the expression for $f_{1}(\mathbf{v})$ in this equation, show that we obtain a dispersion relation with three wave solutions, which are the usual Landau damped longitudinal waves and the left and the right circularly polarized waves (with $E_{x}= \pm i E_{y}$ ). 
19.4 Consider an electron gas immersed in a uniform magnetostatic field $\mathbf{B}_{0}$ and characterized by the following modified Maxwellian distribution function:

$$
f_{0}\left(v_{\|}, v_{\perp}\right)=n_{0}\left(\frac{m_{e}}{2 \pi k_{B} T_{\|}}\right)^{1 / 2}\left(\frac{m_{e}}{2 \pi k_{B} T_{\perp}}\right) \exp \left(-\frac{m_{e} v_{\|}^{2}}{2 k_{B} T_{\|}}-\frac{m_{e} v_{\perp}^{2}}{2 k_{B} T_{\perp}}\right)
$$

Use this distribution function in the dispersion relation for the right circularly polarized transverse wave propagating along $\mathbf{B}_{0}$, given in (2.69), and evaluate the integrals to obtain the following dispersion relation

$$
\begin{aligned}
k^{2} c^{2}= & \omega^{2}-\tau \omega_{p e}^{2}-i \pi^{1 / 2} \omega_{p e}^{2} \tau(\alpha-\beta) \exp \left(-\alpha^{2}\right)+ \\
& 2 \omega_{p e}^{2} \tau(\alpha-\beta) \int_{0}^{\alpha} \exp \left(W^{2}-\alpha^{2}\right) d W
\end{aligned}
$$

where

$$
\begin{gathered}
\tau=1-\frac{T_{\perp}}{T_{\|}} \\
\alpha=\frac{\left(\omega-\Omega_{c e}\right) / k}{\left(2 k_{B} T_{\|} / m_{e}\right)^{1 / 2}} \\
\beta=\frac{(\omega / k)}{\tau\left(2 k_{B} T_{\perp} / m_{e}\right)^{1 / 2}}
\end{gathered}
$$

Analyze this dispersion relation to verify the existence or not of instabilities (positive imaginary part of $\omega$ ) and/or damping (negative imaginary part of $\omega$ ) of the wave amplitude, considering the propagation coefficient $\mathbf{k}=k \widehat{\mathbf{z}}$ to be real. Determine the cyclotron damping coefficient. Analyze also the results considering the isotropic case for which $T_{\|}=T_{\perp}$.

19.5 In problem 19.4 suppose that in the equilibrium state the velocity distribution function of the electrons is given by

$$
f_{0}(\mathbf{v})=n_{0}\left(\frac{m_{e}}{2 \pi k_{B} T_{e}}\right)^{3 / 2} \exp \left\{-\frac{m_{e}}{2 k_{B} T_{e}}\left[v_{\perp}^{2}+\left(v_{\|}-u_{0}\right)^{2}\right]\right\}
$$

which corresponds to an isotropic distribution but with the electrons drifting with macroscopic speed $u_{0}$ along $\mathbf{B}_{0}$. Show that, with this choice of $f_{0}(\mathbf{v})$, the dispersion relation for the right circularly polarized wave reduces to

$$
k^{2} c^{2}=\omega^{2}-\frac{\omega_{p e}^{2}}{n_{0}} \int_{v} \frac{\left(\omega-k u_{0}\right)}{\left(\omega-k v_{\|}-\Omega_{c e}\right)} f_{0}(\mathbf{v}) d^{3} v
$$


For the limiting case of $T_{e}=0$, find the form of the distribution function $f_{0}(\mathbf{v})$ and show that the dispersion relation reduces to

$$
k^{2} c^{2}=\omega^{2}-\frac{\omega_{p e}^{2}\left(\omega-k u_{0}\right)}{\left(\omega-k u_{0}-\Omega_{c e}\right)}
$$

19.6 For an unbounded homogeneous electron gas, characterized by the following velocity distribution function,

$$
f_{0}(\mathbf{v})=n_{0} \frac{a_{0}}{\pi^{2}} \frac{1}{\left(v^{2}+a_{0}^{2}\right)^{2}}
$$

where $a_{0}$ is a constant, show that the dispersion relation for the right circularly polarized wave, propagating along the magnetostatic field $\mathbf{B}_{0}=$ $B_{0} \widehat{\mathbf{z}}$, is given by

$$
k^{2} c^{2}=\omega^{2}-\frac{\omega_{p e}^{2} \omega}{\left(\omega+i k a_{0}-\Omega_{c e}\right)}
$$

From this result show that the cyclotron damping coefficient is given approximately by

$$
\omega_{i}=-\frac{1}{2} k\left[a_{0} c^{2}\left(\frac{\Omega_{c e}}{\omega_{p e}}\right)^{2}\right]^{1 / 3}
$$

19.7 (a) Show that, starting from the Vlasov equation and the laws of electrostatics, we obtain the following dispersion relation for the quasistatic wave propagating at an arbitrary direction with respect to an externally applied magnetostatic field $\mathbf{B}_{0}$ in a hot plasma,

$$
\frac{\Omega_{c e}^{2} \bar{\nu}}{\omega_{p e}^{2} \sin ^{2} \theta}=\frac{k_{B} T_{e}}{m_{e}}\left(\frac{k}{\omega_{p e}}\right)^{2}=-\exp (-\bar{\nu}) \sum_{n=-\infty}^{+\infty}\left(1+\bar{\nu} H_{n}\right) I_{n}(\bar{\nu})
$$

where

$$
\begin{gathered}
\bar{\nu}=\frac{k_{B} T_{e}}{m_{e}} \frac{k^{2} \sin ^{2} \theta}{\Omega_{c e}^{2}} \\
H_{n}=\frac{1}{\pi^{1 / 2}} \int_{-\infty}^{+\infty} \frac{\exp \left(-\bar{v}_{z}^{2}\right)}{\left(\bar{v}_{z}+n \bar{\Omega}_{c e}-\bar{\omega}\right)} d \bar{v}_{z}
\end{gathered}
$$




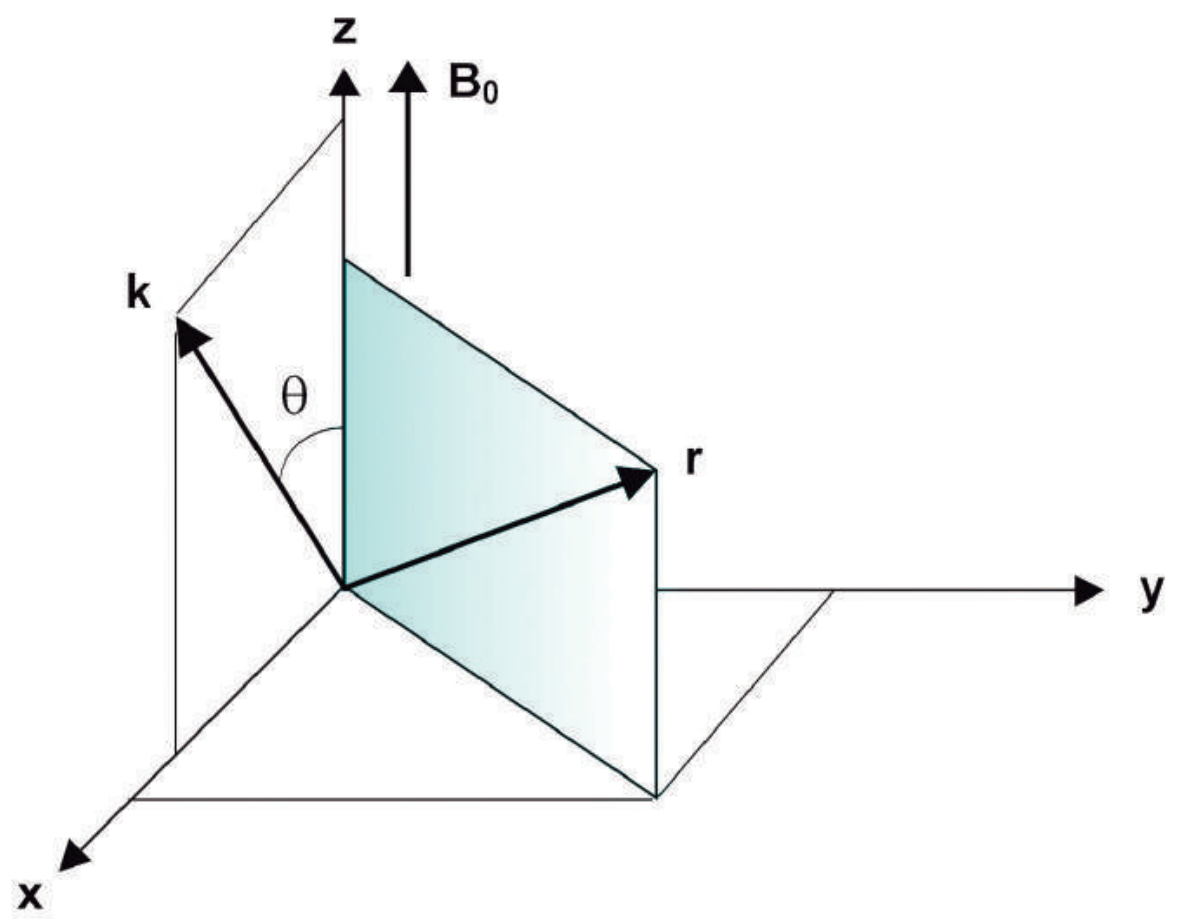

Fig. 7 Illustrating the relative orientation of the propagation vector $\mathbf{k}$ with respect to $\mathbf{B}_{0}$ in a Cartesian coordinate system, with $\mathbf{B}_{0}$ along the $z$ axis and $\mathbf{k}$ in the $(x, z)$ plane.

$$
\begin{gathered}
\bar{v}_{z}=\frac{v_{z}}{V_{e}} \\
V_{e}=\left(\frac{2 k_{B} T_{e}}{m_{e}}\right)^{1 / 2} \\
\bar{\omega}=\frac{\omega}{k \cos \theta V_{e}} \\
\bar{\Omega}_{c e}=\frac{\Omega_{c e}}{k \cos \theta V_{e}}
\end{gathered}
$$

The function $I_{n}(\bar{\nu})$ is the Bessel function of the second kind and $\theta$ is the angle between $\mathbf{k}$ and $\mathbf{B}_{0}$, as indicated in Fig. 7 .

(b) Rewrite this dispersion relation in the form

$$
1+\frac{k_{B} T_{e}}{m_{e}}\left(\frac{k}{\omega_{p e}}\right)^{2}=-i \omega \int_{0}^{\infty} d t \exp \{i \omega t-
$$




$$
\left.\frac{1}{2}\left[1-\cos \left(\Omega_{c e} t\right)\right]\left(k^{2} V_{e}^{2} / \Omega_{c e}^{2}\right) \sin ^{2} \theta-\frac{1}{4} k^{2} V_{e}^{2} t^{2} \cos ^{2} \theta\right\}
$$

(c) Simplify this expression for the case of a very weak magnetostatic field to obtain the following approximate expression for the frequency of oscillation

$$
\omega^{2}=\omega_{p e}^{2}+k^{2}\left(\frac{3 k_{B} T_{e}}{m_{e}}\right)+\Omega_{c e}^{2} \sin ^{2} \theta
$$

Compare this result with the cold and warm plasma model results for both the cases of $\mathbf{k}$ parallel to $\mathbf{B}_{0}$ and $\mathbf{k}$ perpendicular to $\mathbf{B}_{0}$.

19.8 Deduce the dispersion relation for small amplitude waves propagating at an arbitrary direction with respect to an externally applied magnetostatic field $\mathbf{B}_{0}=B_{0} \widehat{\mathbf{z}}$ in a hot plasma. Carry through the derivation as far as possible for an arbitrary value of the strength of the magnetostatic field. Then, particularize for the special case of a very weak magnetostatic field. For simplicity, assume the equilibrium distribution function to be the isotropic Maxwell-Boltzmann distribution. You may refer to the article Waves in a Plasma in a Magnetic Field, by Ira B. Bernstein, Physical Review, 109(1), 10-21, 1958. 


\section{0}

\section{Particle interactions \\ IN PLASMAS}

\section{INTRODUCTION}

The fundamental properties of a plasma depend upon the interactions between the plasma particles and the existing force fields. These fields may be externally applied or they can be internal fields associated with the nature and motion of the particles themselves. In this text the words collision and interaction are used synonymously. The notion of a collision as a physical contact between bodies loses its utility in the microscopic world. In the atomic level a collision between particles must be regarded as an interaction between the force fields associated with each of the interacting particles.

Collisional phenomena can be broadly divided into two categories: elastic and inelastic. In elastic collisions there is conservation of mass, of momentum, and of energy in such a way that there are no changes in the internal states of the particles involved and there is neither creation nor annihilation of particles. In inelastic collisions the internal states of some or all of the particles involved are changed and particles may be created as well as destroyed. In inelastic collisions a charged particle may recombine with another to form a neutral particle or it can attach itself with a neutral particle to form a heavier charged particle. Also, the energy state of an electron in an atom may be raised and electrons can be removed from their atoms, resulting in ionization.

In plasmas there is an important distinction to be made between charge-charge and charge-neutral interactions. Electrically charged particles interact with one another according to Coulomb's law. This coulomb 
interaction, in view of its $1 / r^{2}$ dependence, is a long-range interaction, so that the field of one particle interacts simultaneously with a large number of other particles. Therefore, it involves multiple interactions. In contrast, the fields associated with neutral particles are significantly strong only within the electronic shells of the particles. Thus, they are short-range fields, and a neutral particle only occasionally interacts with another particle and very rarely interacts simultaneously with more than one particle. Therefore, these short-range fields result primarily in binary interactions.

The multiple-particle coulomb interaction, however, can be thought of as a number of simultaneous binary interactions. In fact, one way of dealing with multiple interactions is to consider that a series of consecutive small-angle binary interactions describe the situation. The multiple interactions that result from the coulomb force are of essential importance in understanding the behavior of plasmas and underlines the validity of describing a plasma as the fourth state of matter. Nevertheless, binary collisions adequately describe plasma phenomena in the case of weakly ionized plasmas. In fact, we use the term weakly ionized plasma to mean a plasma in which multiple particle interactions can be ignored. In these plasmas the electrons tend to dominate the situation, since they respond quickly to the influence of electric and magnetic fields, in view of their low inertia.

In this chapter we deal with collisional processes that are of importance in plasmas, from the point of view of classical dynamics. The results are valid to a good approximation, even though the internal structure of the particles is ignored. More important, however, the procedures to be developed are useful whether the mechanics is classical or quantum.

\section{BINARY COLLISIONS}

Consider an elastic collision between two particles of mass $m$ and $m_{1}$, having velocities $\mathbf{v}$ and $\mathbf{v}_{1}$ before collision, and $\mathbf{v}^{\prime}$ and $\mathbf{v}_{1}^{\prime}$, after collision. This binary interaction is illustrated in Fig. 1, as seen from the laboratory system. In what follows, the variables indicated with a prime are aftercollision variables.

It is convenient to adopt a coordinate system in which the particle having mass $m$ is at rest and the particle having mass $m_{1}$ approaches with the relative velocity

$$
\mathbf{g}=\mathbf{v}_{1}-\mathbf{v}
$$




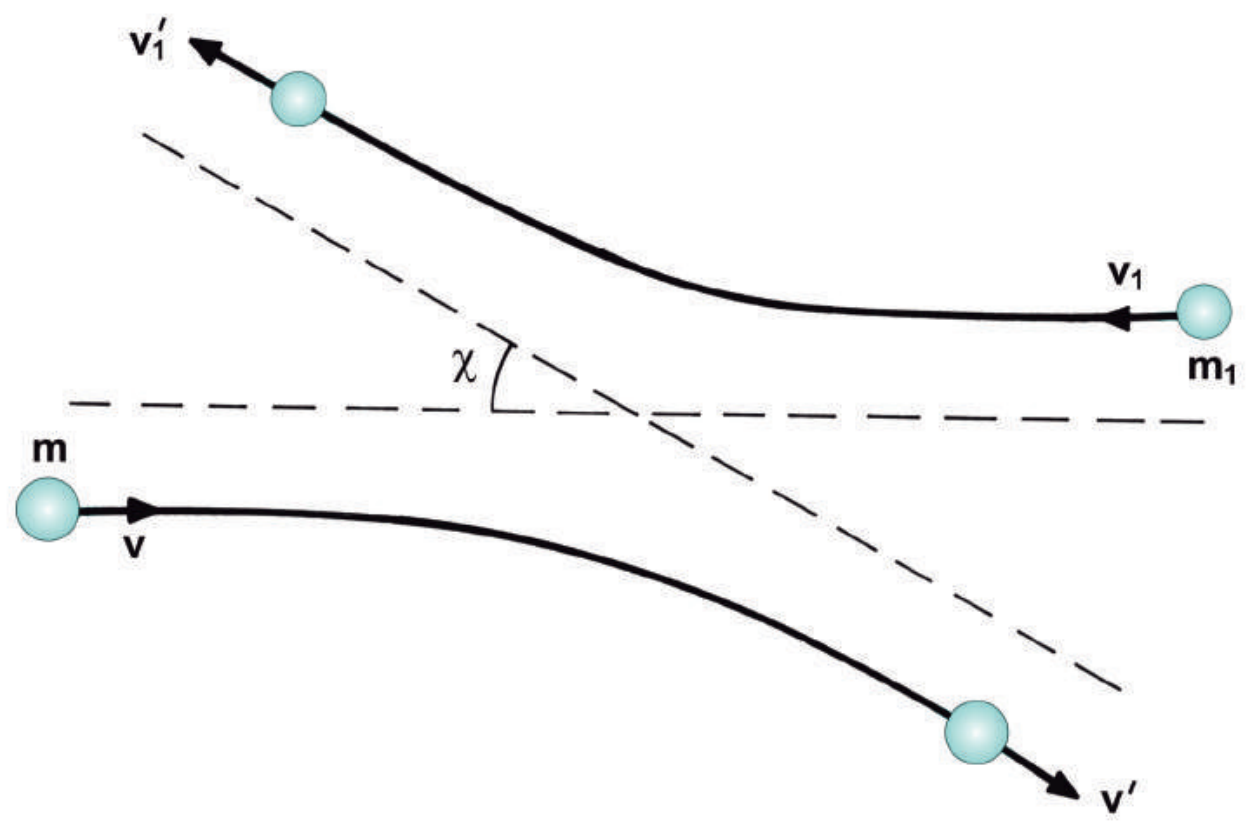

Fig. 1 Binary interaction between two particles having mass $m$ and $m_{1}$, with velocities $\mathbf{v}$ and $\mathbf{v}_{1}$, respectively, as seen from the laboratory system.

After collision, the relative velocity is

$$
\mathrm{g}^{\prime}=\mathbf{v}_{1}^{\prime}-\mathbf{v}^{\prime}
$$

The geometry of this interaction is shown in Fig. 2. The impact parameter, defined as the minimum distance of approach if there were no interaction, is denoted by $b$, the scattering angle by $\chi$, and the orientation of the orbital plane (or collision plane), with respect to some given direction in a plane normal to the orbital plane, is denoted by $\epsilon$.

The velocity of the center of mass of the colliding particles, before collision, is defined by

$$
\mathbf{c}_{0}=\frac{m \mathbf{v}+m_{1} \mathbf{v}_{1}}{m+m_{1}}
$$

and, after collision, by

$$
\mathbf{c}_{0}^{\prime}=\frac{m \mathbf{v}^{\prime}+m_{1} \mathbf{v}_{1}^{\prime}}{m+m_{1}}
$$

We can express the initial velocities in terms of $\mathbf{c}_{0}$ and $\mathbf{g}$. From (2.3) and (2.1), which define $\mathbf{c}_{0}$ and $\mathbf{g}$, respectively, we find

$$
\mathbf{v}=\mathbf{c}_{0}-\frac{\mu}{m} \mathbf{g}
$$




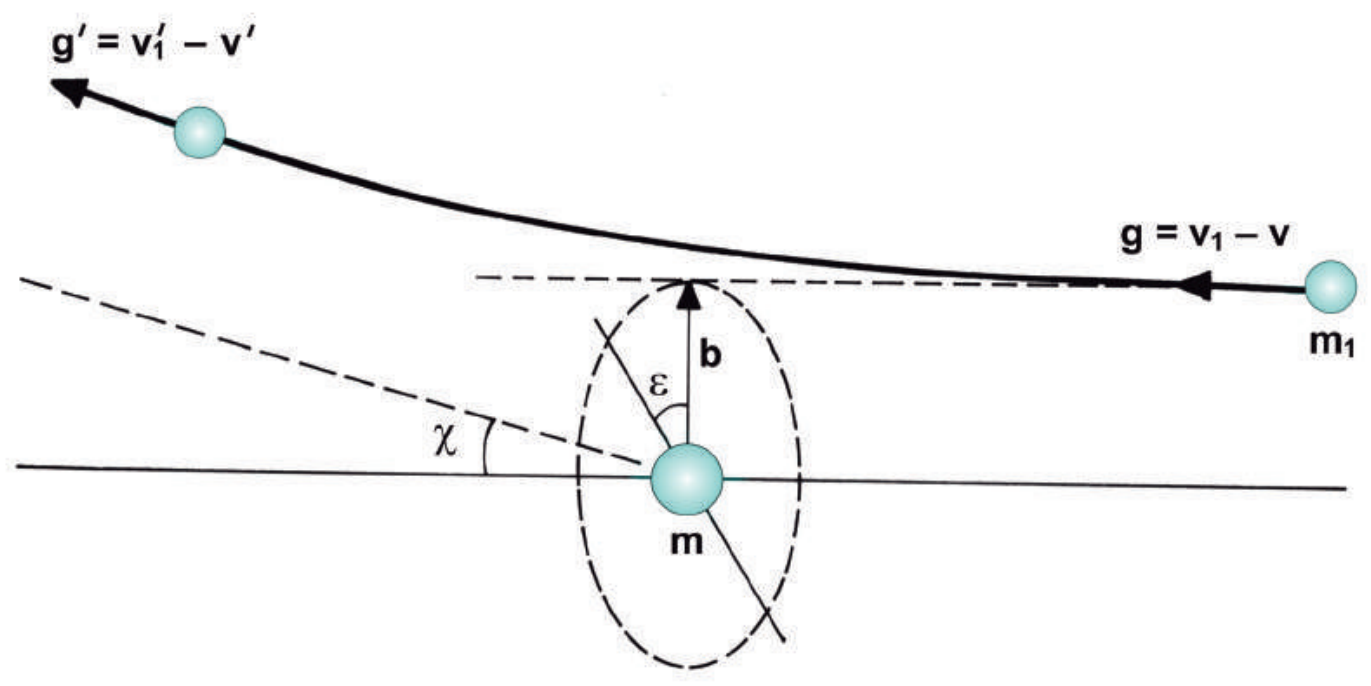

Fig. 2 Geometry of a collision between a particle of mass $m$ and velocity $\mathbf{v}$, and a particle of mass $m_{1}$ and velocity $\mathbf{v}_{1}$, viewed from a coordinate system in which the first particle is at rest.

$$
\mathbf{v}_{1}=\mathbf{c}_{0}+\frac{\mu}{m_{1}} \mathbf{g}
$$

where $\mu$ denotes the reduced mass, defined by

$$
\mu=\frac{m m_{1}}{m+m_{1}}
$$

Similarly, from (2.4) and (2.2) we obtain, for the after-collision velocities,

$$
\begin{aligned}
& \mathbf{v}^{\prime}=\mathbf{c}_{0}^{\prime}-\frac{\mu}{m} \mathbf{g}^{\prime} \\
& \mathbf{v}_{1}^{\prime}=\mathbf{c}_{0}^{\prime}+\frac{\mu}{m_{1}} \mathbf{g}^{\prime}
\end{aligned}
$$

From the law of conservation of momentum for the collision event, we have

$$
m \mathbf{v}+m_{1} \mathbf{v}_{1}=m \mathbf{v}^{\prime}+m_{1} \mathbf{v}_{1}^{\prime}
$$

or, using (2.3) and (2.4),

$$
\left(m+m_{1}\right) \mathbf{c}_{0}=\left(m+m_{1}\right) \mathbf{c}_{0}^{\prime}
$$


Thus, we conclude that

$$
\mathbf{c}_{0}=\mathbf{c}_{0}^{\prime}
$$

that is, the velocity of the center of mass is the same before and after the collision event.

From the law of conservation of energy for elastic collisions, we have

$$
\frac{1}{2}\left(m v^{2}+m_{1} v_{1}^{2}\right)=\frac{1}{2}\left[m\left(v^{\prime}\right)^{2}+m_{1}\left(v_{1}^{\prime}\right)^{2}\right]
$$

and using (2.5), (2.6), (2.8), and (2.9) we find, by direct calculation,

$$
\begin{gathered}
\frac{1}{2}\left(m v^{2}+m_{1} v_{1}^{2}\right)=\frac{1}{2}\left(m+m_{1}\right) c_{0}^{2}+\frac{1}{2} \mu g^{2} \\
\frac{1}{2}\left[m\left(v^{\prime}\right)^{2}+m_{1}\left(v_{1}^{\prime}\right)^{2}\right]=\frac{1}{2}\left(m+m_{1}\right)\left(c_{0}^{\prime}\right)^{2}+\frac{1}{2} \mu\left(g^{\prime}\right)^{2}
\end{gathered}
$$

Now, since $\mathbf{c}_{0}=\mathbf{c}_{0}^{\prime}$, we conclude that

$$
g=g^{\prime}
$$

Thus, the magnitude, but not the direction of the relative velocity is conserved in a binary elastic collision. Equations (2.14) and (2.15) show that the total instantaneous kinetic energy of the two-particle system is equivalent to that associated with the motion of the center of mass plus the motion of one particle relative to the other, but using the reduced mass.

The angle between $\mathbf{g}$ and $\mathbf{g}^{\prime}$ is the scattering angle, or deflection angle, which is denoted by $\chi$. To relate the relative velocity vectors $\mathbf{g}$ and $\mathbf{g}^{\prime}$, we can choose, for instance, a Cartesian coordinate system with the $z$ axis along $\mathbf{g}$, as shown in Fig. 3. Thus, we have

$$
\begin{gathered}
g_{x}=g_{y}=0 \\
g_{z}=g=g^{\prime} \\
g_{x}^{\prime}=g \sin \chi \cos \epsilon \\
g_{y}^{\prime}=g \sin \chi \sin \epsilon \\
g_{z}^{\prime}=g \cos \chi
\end{gathered}
$$

where $\epsilon$ defines the relative orientation of the collision plane. Therefore, knowing the initial velocities and the scattering angle $\chi$ we can determine the after-collision velocities. The opposite is also true, that is, if we know the final velocities and the scattering angle, we can find the initial velocities. 


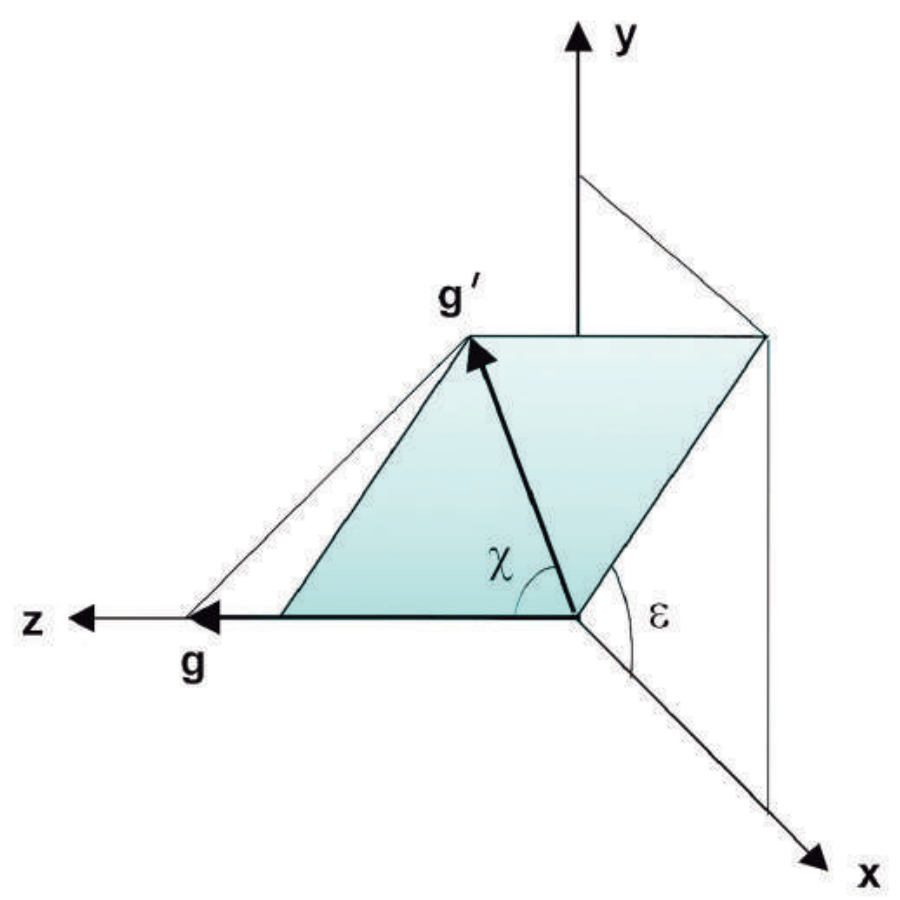

Fig. 3 Relative orientations of the velocities $\mathbf{g}$ and $\mathbf{g}^{\prime}$ in a Cartesian coordinate system chosen such that $\mathbf{g}=g \widehat{\mathbf{z}}$. The angle $\epsilon$ defines the relative orientation of the plane containing the trajectory of the particle.

It is of interest to consider the inverse collision (refer to Fig. 1 of Chapter 7 ), in which a particle with initial velocity $\mathbf{v}^{\prime}$ collides with another particle having initial velocity $\mathbf{v}_{1}^{\prime}$, the velocities after collision being $\mathbf{v}$ and $\mathbf{v}_{1}$, respectively. For the inverse collision, the scattering angle $\chi$ is the same as that for the direct collision, since the impact parameter $b$, the interparticle force law, and the relative speed $\mathbf{g}$ are all the same.

The scattering angle is the only quantity appearing in the analysis presented in this section that depends on the details of the collision process. For interparticle force laws that depend only on the distance between the interacting particles, $\chi$ depends on the following quantities:

(a) Interparticle force law;

(b) Magnitude of the relative velocity $g$;

(c) The value of the impact parameter $b$.

Therefore, in order to determine $\chi$ we must analyze the classical dynamics of binary collisions. 


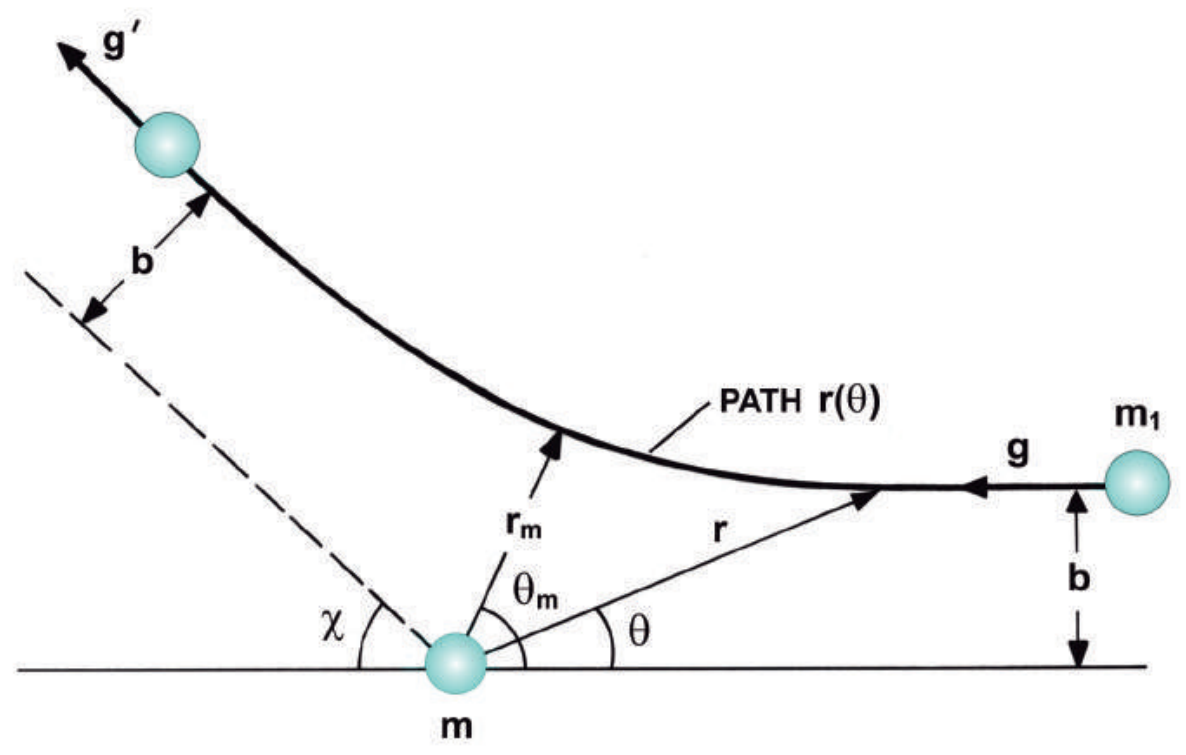

Fig. 4 Path $r(\theta)$ of the particle of mass $m_{1}$ relative to the particle of mass $m$.

\section{DYNAMICS OF BINARY COLLISIONS}

The dynamics of a binary collision is governed by the interparticle force law. For each impact parameter $b$ there will be associated a given scattering angle $\chi$, the relation being dependent on the interparticle force law. This information is contained in the differential cross section, which we shall define in section 5 .

Consider the collision between two particles having mass $m$ and $m_{1}$, viewed from a reference system in which the first particle is at rest. Let $\mathbf{r}$ be the position vector of the particle of mass $m_{1}$ with respect to that of mass $m$ (see Fig. 4). The force of interaction between the two particles is assumed to be a central force, which acts along the straight line joining the two particles, that is,

$$
\mathbf{F}(\mathbf{r})=F(r) \widehat{\mathbf{r}}
$$

This force is related to the potential energy $U(\mathbf{r})$ of the interaction by the condition

$$
\mathbf{F}(\mathbf{r})=-\nabla U(\mathbf{r})=-\frac{\partial U(r)}{\partial r} \widehat{\mathbf{r}}
$$




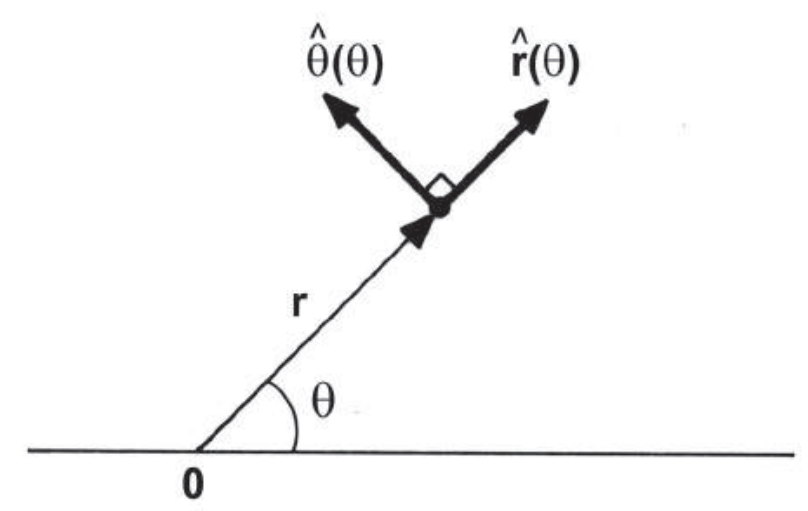

Fig. 5 Polar coordinates $(r, \theta)$, showing that the directions of the unit vectors $\widehat{\mathbf{r}}$ and $\widehat{\boldsymbol{\Theta}}$ depend on $\theta$.

For a central force the torque $\mathbf{N}=\mathbf{r} \times \mathbf{F}(\mathbf{r})$ vanishes, because $\mathbf{F}(\mathbf{r})$ is parallel to $\mathbf{r}$. Since the torque is the time rate of change of the angular momentum $\mathbf{L}$,

$$
\mathbf{N}=\frac{d \mathbf{L}}{d t}
$$

we conclude that the angular momentum is a constant of the motion. Furthermore, since $\mathbf{L}=\mathbf{r} \times \mathbf{p}$, we see that $\mathbf{r}$ is always normal to the constant direction of $\mathbf{L}$ in space so that the motion lies in a plane.

Using polar coordinates $(r, \theta)$ and noting that the unit vectors $\widehat{\mathbf{r}}$ and $\widehat{\Theta}$ depend on $\theta$, as illustrated in Fig. 5, we have, for the instantaneous relative velocity,

$$
\frac{d \mathbf{r}}{d t}=\frac{d r}{d t} \widehat{\mathbf{r}}+r \frac{d \widehat{\mathbf{r}}}{d t}=\frac{d r}{d t} \widehat{\mathbf{r}}+r \frac{d \widehat{\mathbf{r}}}{d \theta} \frac{d \theta}{d t}
$$

Since it can be shown that $d \widehat{\mathbf{r}} / d \theta=\widehat{\Theta}$, we obtain

$$
\frac{d \mathbf{r}}{d t}=\frac{d r}{d t} \widehat{\mathbf{r}}+r \frac{d \theta}{d t} \widehat{\boldsymbol{\Theta}}
$$

or, using a dot over the variable to denote the time derivative,

$$
\dot{\mathbf{r}}=\dot{r} \widehat{\mathbf{r}}+r \dot{\theta} \widehat{\Theta}
$$

The trajectory of the particle in the equivalent one-body problem can be easily found by using the laws of conservation of energy and of angular momentum. The kinetic energy of the relative motion is given by

$$
K=\frac{1}{2} \mu \dot{\mathbf{r}} \cdot \dot{\mathbf{r}}=\frac{1}{2} \mu\left(\dot{r}^{2}+r^{2} \dot{\theta}^{2}\right)
$$


From the law of conservation of energy we can equate the kinetic plus potential energy, at any point, to the initial kinetic energy $\frac{1}{2} \mu g^{2}$, since the initial potential energy is zero. Thus, we have

$$
\frac{1}{2} \mu\left(\dot{r}^{2}+r^{2} \dot{\theta}^{2}\right)+U(r)=\frac{1}{2} \mu g^{2}
$$

The angular momentum relative to the origin is given by

$$
\mathbf{L}=\mathbf{r} \times(\mu \dot{\mathbf{r}})=\mu r^{2} \dot{\theta}(\widehat{\mathbf{r}} \times \widehat{\boldsymbol{\Theta}})
$$

Setting the angular momentum, at any point, equal to its initial value, $b \mu g(\widehat{\mathbf{r}} \times \widehat{\mathbf{\Theta}})$, we get

$$
r^{2} \dot{\theta}=b g
$$

From (3.8) and (3.1) we can easily obtain a differential equation for the orbit $r(\theta)$. First, we write

$$
\frac{d r}{d t}=\frac{d r}{d \theta} \frac{d \theta}{d t}
$$

and use (3.10) and (3.8) to eliminate $d \theta / d t$ and $d r / d t$. The resulting differential equation for the trajectory $r(\theta)$ is found to be

$$
\left(\frac{d r}{d \theta}\right)^{2}=\frac{r^{4}}{b^{2}}\left[1-\frac{b^{2}}{r^{2}}-\frac{2 U(r)}{\mu g^{2}}\right]
$$

Rearranging (3.12) yields the following result:

$$
d \theta= \pm \frac{b}{r^{2}}\left[1-\frac{b^{2}}{r^{2}}-\frac{2 U(r)}{\mu g^{2}}\right]^{-1 / 2} d r
$$

The choice of sign must be made on physical grounds. The coordinates of the position of the particle, when it is at the distance of closest approach, are denoted by $r_{m}$ and $\theta_{m}$ (refer to Fig. 4). This position is called the vertex of the trajectory and the line connecting the origin to the vertex is called the apse line. Thus, $\theta_{m}$ specifies the orientation of the apse line. The plus sign in (3.13) must be used when $\theta$ is greater than $\theta_{m}$, since for $\theta>\theta_{m}$ we see that $r$ increases with $\theta$. On the other hand, for $\theta<\theta_{m}$ we see that $r$ decreases as $\theta$ increases, so that the minus sign in (3.13) must be used when $\theta$ is less than $\theta_{m}$. This result also shows that the particle trajectory is symmetrical about the apse line. 
The distance of closest approach $r_{m}$ can be obtained from (3.12) by noting that $d r / d \theta=0$ when $r=r_{m}$. Thus, we have

$$
1-\frac{b^{2}}{r_{m}^{2}}-\frac{2 U\left(r_{m}\right)}{\mu g^{2}}=0
$$

or

$$
r_{m}=b\left[1-\frac{2 U\left(r_{m}\right)}{\mu g^{2}}\right]^{-1 / 2}
$$

To determine the scattering angle $\chi$, we first note, from Fig. 5, that

$$
\chi=\pi-2 \theta_{m}
$$

In order to determine $\theta_{m}$ we integrate (3.13) from $\theta_{m}$ to some other angle $\theta$, obtaining

$$
\theta-\theta_{m}= \pm \int_{r_{m}}^{r} \frac{b}{\left(r^{\prime}\right)^{2}}\left[1-\frac{b^{2}}{\left(r^{\prime}\right)^{2}}-\frac{2 U\left(r^{\prime}\right)}{\mu g^{2}}\right]^{-1 / 2} d r^{\prime}
$$

where the plus sign is to be used when $\theta>\theta_{m}$ and the minus sign when $\theta<\theta_{m}$. When $r \rightarrow \infty$ we have $\theta_{(-)} \rightarrow 0$, while $\theta_{(+)} \rightarrow 2 \theta_{m}$, so that (3.17) gives for the orientation of the apse line,

$$
\theta_{m}=\int_{r_{m}}^{\infty} \frac{b}{r^{2}}\left[1-\frac{b^{2}}{r^{2}}-\frac{2 U(r)}{\mu g^{2}}\right]^{-1 / 2} d r
$$

The scattering angle is therefore given by

$$
\chi(b, g)=\pi-2 \int_{r_{m}}^{\infty} \frac{b}{r^{2}}\left[1-\frac{b^{2}}{r^{2}}-\frac{2 U(r)}{\mu g^{2}}\right]^{-1 / 2} d r
$$

To compute $\chi$ from this equation we must know the impact parameter $b$, the magnitude of the initial relative velocity $g$, and the interparticle potential energy function $U(r)$.

\section{EVALUATION OF THE SCATTERING ANGLE}

In this section we shall present two examples of the use of (3.19) to determine the scattering angle $\chi$ in terms of the impact parameter $b$ and of the initial relative speed $g$. First, we will consider the collision between 
two perfectly elastic hard spheres and afterwards the case of the coulomb potential interaction.

\subsection{Two Perfectly Elastic Hard Spheres}

Consider the collision between two perfectly elastic hard spheres of radii $R_{1}$ and $R_{2}$ (see Fig. 6). The potential energy of interaction is given by

$$
\begin{aligned}
U(r) & =0 & \text { for } & & r>R_{1}+R_{2} \\
& =\infty & \text { for } & & r<R_{1}+R_{2}
\end{aligned}
$$

For $b>R_{1}+R_{2}$ there is no interaction and we must have $r_{m}=b$, whereas for $b<R_{1}+R_{2}$ the particles collide and we have $r_{m}=R_{1}+R_{2}$. In either case, however, since the spheres are impenetrable, we have $r>R_{1}+R_{2}$, so that (3.19) becomes

$$
\chi=\pi-2 \int_{r_{m}}^{\infty} \frac{b}{r^{2}}\left(1-\frac{b^{2}}{r^{2}}\right)^{-1 / 2} d r
$$

To solve this integral it is convenient to define a new variable by $y=b / r$ and write (4.2) in the form

$$
\chi=\pi-2 \int_{0}^{b / r_{m}}\left(1-y^{2}\right)^{-1 / 2} d y
$$

which gives

$$
\chi=\pi-2 \sin ^{-1}\left(b / r_{m}\right)
$$

Therefore, we find that

$$
\begin{aligned}
& \chi=\pi-2 \sin ^{-1}\left(\frac{b}{R_{1}+R_{2}}\right) \quad \text { for } \quad b \leq R_{1}+R_{2} \\
& =0 \quad \text { for } \quad b \geq R_{1}+R_{2}
\end{aligned}
$$

\subsection{Coulomb Interaction Potential}

Let us consider now the important case of the coulomb potential field, whose interaction potential energy is given by

$$
U(r)=\frac{1}{4 \pi \epsilon_{0}} \frac{q q_{1}}{r}
$$




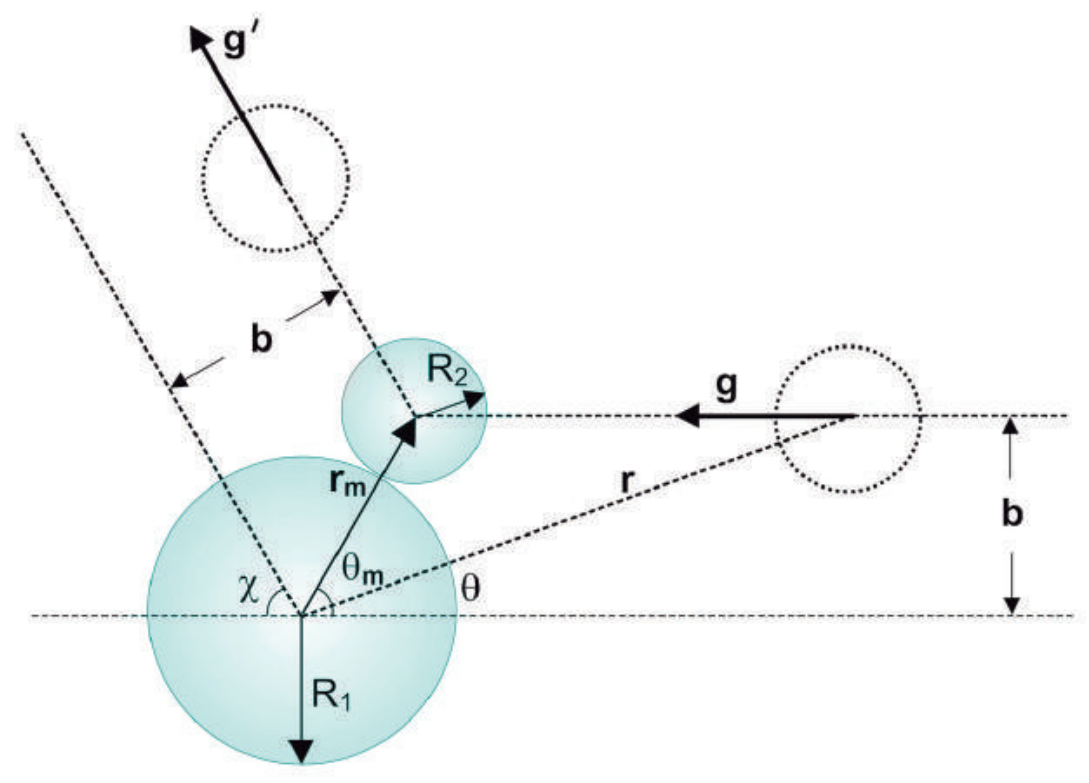

Fig. 6 Collision between two perfectly elastic, impenetrable spheres.

where $q$ and $q_{1}$ denote the electric charge of the particles of mass $m$ and $m_{1}$, respectively. Substituting (4.6) into (3.19), gives

$$
\chi(b, g)=\pi-2 \int_{r_{m}}^{\infty} \frac{b}{r^{2}}\left(1-\frac{b^{2}}{r^{2}}-\frac{q q_{1}}{2 \pi \epsilon_{0} \mu g^{2} r}\right)^{-1 / 2} d r
$$

The distance of closest approach $r_{m}$ in obtained from (3.15) and (4.6), and is found to be

$$
r_{m}=\frac{b^{2}}{-b_{0}+\left(b_{0}^{2}+b^{2}\right)^{1 / 2}}
$$

where, for convenience, we have introduced the notation

$$
b_{0}=\frac{q q_{1}}{4 \pi \epsilon_{0} \mu g^{2}}
$$

Thus, $b_{0}$ represents the distance at which the electrical potential energy of interaction is twice the relative kinetic energy at infinity. Making the change of variable $y=1 / r$ and inserting the value for $b_{0}$, given by (4.9), into (4.7), gives for the deflection angle

$$
\chi(b, g)=\pi-2 b \int_{0}^{1 / r_{m}}\left(1-b^{2} y^{2}-2 b_{0} y\right)^{-1 / 2} d y
$$


The integral appearing here is of the standard form

$$
\int\left(\alpha+\beta x+\gamma x^{2}\right)^{-1 / 2} d x=\frac{1}{\sqrt{-\gamma}} \sin ^{-1}\left[\frac{-2 \gamma x-\beta}{\left(\beta^{2}-4 \alpha \gamma\right)^{1 / 2}}\right]
$$

where, in our case, $\alpha=1, \beta=-2 b_{0}$, and $\gamma=-b^{2}$. Applying the limits of integration, with $r_{m}$ as given by (4.8), yields for the deflection angle

$$
\chi(b, g)=2 \sin ^{-1}\left[\frac{b_{0}}{\left(b_{0}^{2}+b^{2}\right)^{1 / 2}}\right]
$$

This equation for $\chi(b, g)$ can be written in the following alternative form:

$$
\tan \left(\frac{1}{2} \chi\right)=\frac{b_{0}}{b}
$$

Note that for $\chi=\pi / 2$ we have $b=b_{0}$, that is, $b_{0}$ is the value of the impact parameter for a $90^{\circ}$ deflection angle. If the signs of the two charged particles are the same, then $b_{0}$ and $\chi$ will both be positive. On the other hand, if the signs of the two charged particles are opposite, then $b_{0}$ and $\chi$ will be negative. These two situations are illustrated in Fig. 7 for a deflection angle of $90^{\circ}$. Further, we note, from (4.13), that $\chi=\pi$ for $b=0$. Also, $\chi$ decreases as $b$ increases and $\chi=0$ only in the limit of $b \rightarrow \infty$. Therefore, scattering occurs for all (finite) values of the impact parameter $b$, so that there is no cut-off value for $b$.

\section{CROSS SECTIONS}

So far we have considered specifically only the interaction between two particles. Cross sections are usually defined in terms of a beam of identical particles incident on a center of force (target particle). Therefore, let us imagine a steady beam of identical particles of mass $m_{1}$, uniformly spread out in space, incident with velocity $\mathbf{g}=\mathbf{v}_{1}-\mathbf{v}$ upon the center of force provided by the particle of mass $m$, in its rest frame of reference. For simplicity, the incident beam of particles is assumed to be monoenergetic, so that all particles in the beam have the same initial relative velocity, $\mathbf{g}$, with respect to the scattering particle. Since the particles in the incident beam are supposed to be identical, the interaction potential is the same for all particles in the beam.

The particles incident with an impact parameter $b$ will be scattered through some deflection angle $\chi$, whereas the particles incident with an 

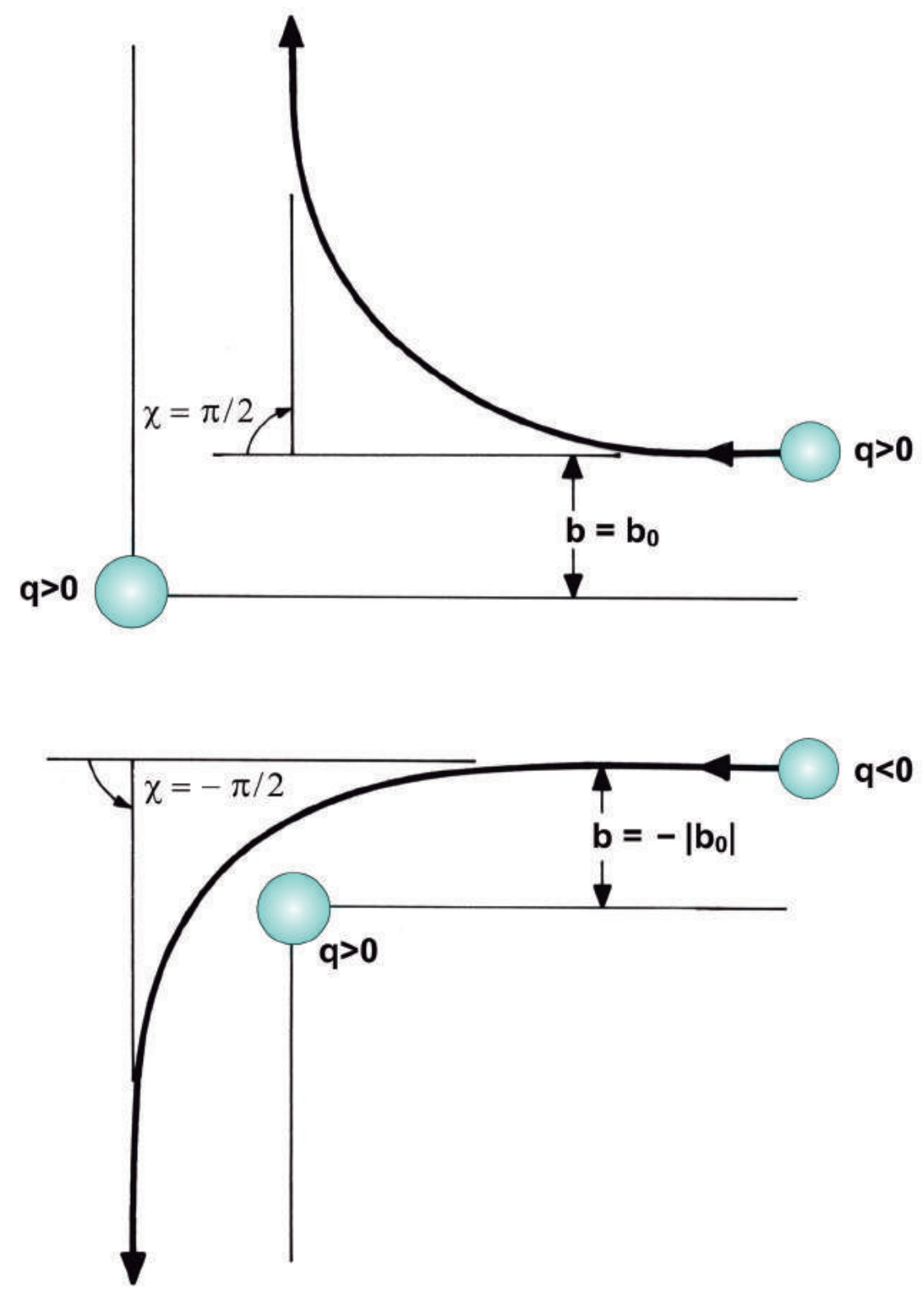

Fig. 7 Scattering in a coulomb potential field for a deflection angle $\chi$ of $90^{\circ}$.

impact parameter $b+d b$ will be scattered through the deflection angle $\chi+d \chi$, as illustrated in Fig. 8. The number of particles scattered per second, between the angles $\chi$ and $\chi+d \chi$, depends on the incident particle current density (particle flux) $\boldsymbol{\Gamma}$, that is, on the number of particles in the incident beam crossing a unit area normal to the beam per unit time. 


\subsection{Differential Scattering Cross Section}

Let $d N / d t$ denote the number of particles scattered per unit time into the differential element of solid angle $d \Omega$, oriented at $(\chi, \epsilon)$, as shown in Fig. 8. The differential scattering cross section $\sigma(\chi, \epsilon)$ (also referred to as the angular distribution function) is defined as the number of particles scattered per unit time, per unit incident flux, and per unit solid angle oriented at $(\chi, \epsilon)$. Thus, according to this definition we have

$$
\frac{d N}{d t}=\sigma(\chi, \epsilon) \Gamma d \Omega
$$

The number of particles incident per unit time, with impact parameter between $b$ and $b+d b$, and with the orbital plane oriented between $\epsilon$ and $\epsilon+d \epsilon$, is given by $\Gamma b d b d \epsilon$. These same particles are scattered per unit time into the differential element of solid angle $d \Omega$ contained between $\chi$ and $\chi+d \chi$, and between $\epsilon$ and $\epsilon+d \epsilon$. Thus, we have

$$
\frac{d N}{d t}=\Gamma b d b d \epsilon
$$

Comparing (5.1) and (5.2) we see that, according to the definition of the differential scattering cross section $\sigma(\chi, \epsilon)$, we have

$$
\sigma(\chi, \epsilon) d \Omega=b d b d \epsilon
$$

Since $d \Omega=\sin \chi d \chi d \epsilon$, this equation can also be written as

$$
\sigma(\chi, \epsilon) \sin \chi d \chi=b d b
$$

Solving for the differential scattering cross section, we obtain

$$
\sigma(\chi, \epsilon)=\frac{b}{\sin \chi}\left|\frac{d b}{d \chi}\right|
$$

The absolute value of $d b / d \chi$ is used here, because $\chi$ normally decreases when $b$ increases, whereas the differential scattering cross section $\sigma(\chi, \epsilon)$ is inherently a positive quantity, since it is associated with the number of particles being scattered. The quantity $d b / d \chi$ can be obtained from (3.19), which gives $\chi(b, g)$ once the potential energy function $U(r)$ is known.

The differential cross section has dimensions of area and can be interpreted, in a geometrical way, such that the number of particles scattered 

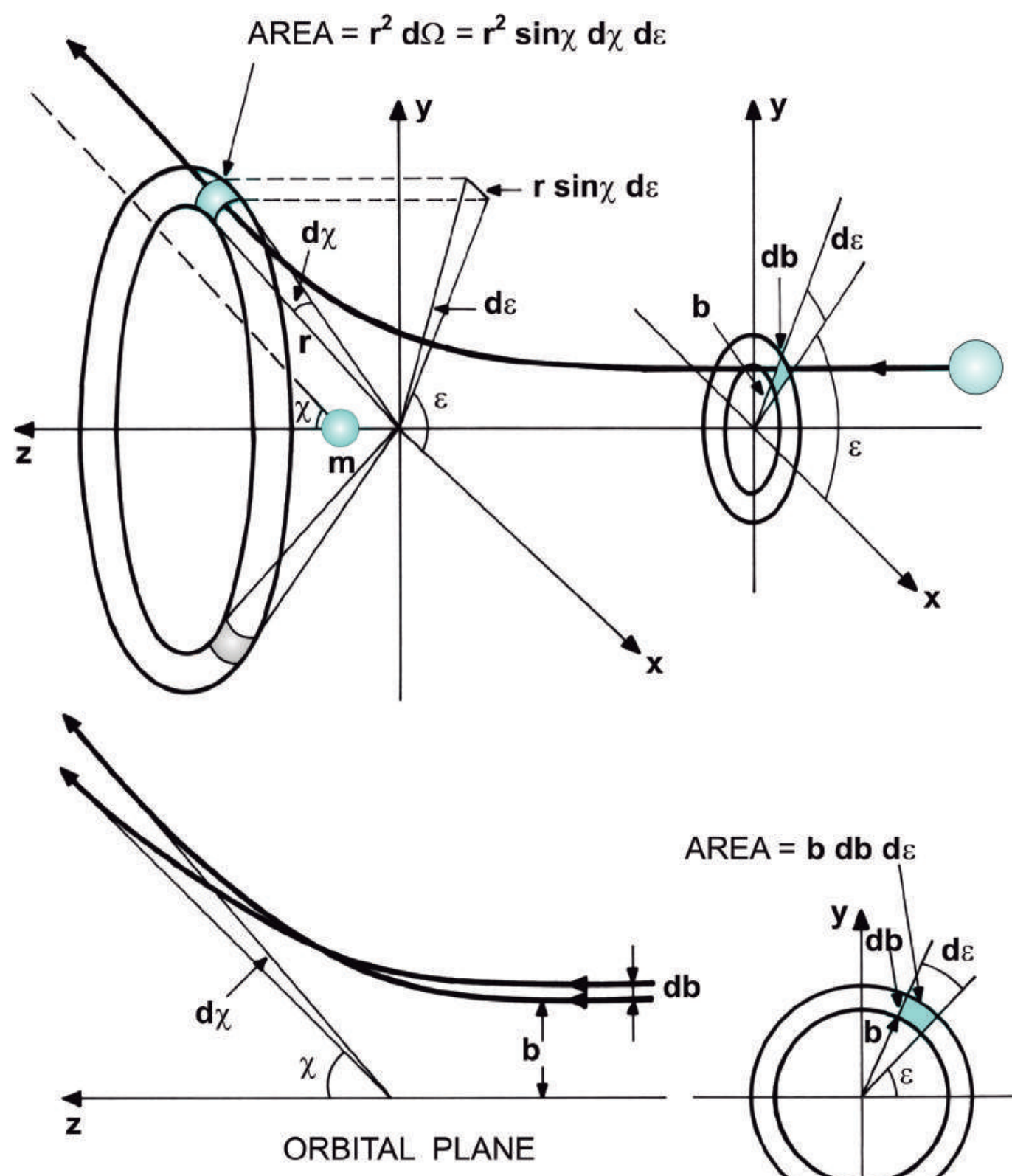

AREA $=\mathbf{b} \mathbf{d} \mathbf{b} \mathbf{d} \varepsilon$

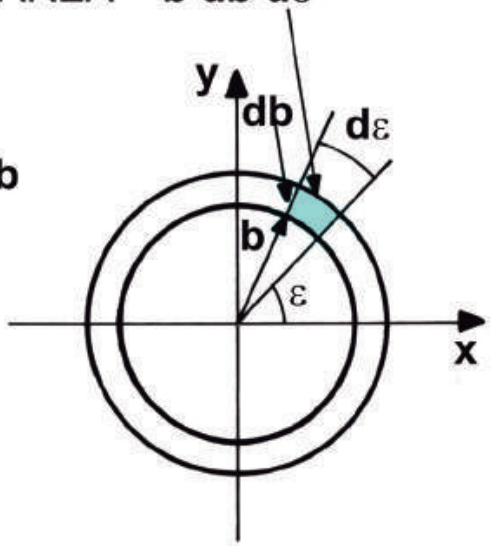

$(x, y)$ PLANE

Fig. 8 Particle scattering in a central field of force. 
into the solid angle element $d \Omega$ per unit time is equal to the number of particles crossing an area equal to $\sigma(\chi, \epsilon) d \Omega$ (or $b d b d \epsilon$ ) in the incident beam, per unit time.

The form of $\sigma(\chi, \epsilon)$ depends on the interparticle force law and, if this force law is known, $\sigma(\chi, \epsilon)$ can be calculated. However, a quantum mechanical calculation is necessary for this purpose, since the quantum wave packets of the colliding particles necessarily overlap and the problem is no longer a classical one. For a collection of atoms or molecules to be regarded classically, with each particle having a rather well-defined position and momentum, it is necessary that the particles be localized wave packets whose extensions are small compared to the average interparticle distance. For a classical treatment the average de Broglie wavelength of each particle therefore must be much smaller than the average interparticle separation.

The differential cross section is also a directly measurable quantity and can be obtained experimentally. For our purposes, we will consider the differential cross section $\sigma(\chi, \epsilon)$, which contains the nature of the collisional interaction, as a known quantity.

\subsection{Total Scattering Cross Section}

The total scattering cross section $\sigma_{t}$ is defined as the number of particles scattered per unit time and per unit incident flux, in all directions from the scattering center. It is obtained by integrating $\sigma(\chi, \epsilon) d \Omega$ over the entire solid angle,

$$
\sigma_{t}=\int_{\Omega} \sigma(\chi, \epsilon) d \Omega=\int_{0}^{2 \pi} d \epsilon \int_{0}^{\pi} \sigma(\chi, \epsilon) \sin \chi d \chi
$$

Both $\sigma(\chi, \epsilon)$ and $\sigma_{t}$ depend on the magnitude of the relative particle velocity $g$.

In the special case when the interaction potential is isotropic, that is, when the differential scattering cross section is independent of $\epsilon$, we can immediately perform the integral over $\epsilon$ in (5.6), to get

$$
\sigma_{t}=2 \pi \int_{0}^{\pi} \sigma(\chi) \sin \chi d \chi
$$

This is the case, for example, of the coulomb interaction potential. 


\subsection{Momentum Transfer Cross Section}

A cross section can be defined for various interaction processes. We shall see later that the transfer of momentum, during a collision, is the basic microscopic event in the transport phenomena of diffusion and mobility. Hence, it is appropriate to define a cross section for the rate of momentum transfer $\sigma_{m}$ as the total momentum transferred per unit time to the scattering center, per unit incident momentum flux (momentum per normal unit area per unit time),

$$
\sigma_{m}=\frac{(\text { momentum transferred per second })}{\text { (incident momentum flux })}
$$

The momentum of a particle in the beam, before interaction, is $\mu g$, where $\mu$ is the reduced mass and $g$ is the initial relative velocity. The incident momentum flux is therefore $\Gamma \mu \mathrm{g}$. After interaction, the momentum of a particle in the beam, in the direction of incidence, and which is scattered at an angle $\chi$, is $\mu g \cos \chi$. Therefore, the momentum transferred by this particle to the scattering center is $\mu g(1-\cos \chi)$. The total momentum transferred per second to the scattering center, by all the particles scattered in all directions in space, is given by the expression

$$
\Gamma \mu g \int_{\Omega}(1-\cos \chi) \sigma(\chi, \epsilon) d \Omega
$$

Recall that $\sigma(\chi, \epsilon)$ can be considered as an angular distribution function. Since the total incident momentum flux is $\Gamma \mu \mathrm{g}$, we obtain for the momentum transfer cross section,

$$
\sigma_{m}=\int_{\Omega}(1-\cos \chi) \sigma(\chi, \epsilon) d \Omega
$$

For the special case of an isotropic interaction potential, and noting that $d \Omega=\sin \chi d \chi d \epsilon$, we can perform the integral over $\epsilon$, in (5.10), obtaining

$$
\sigma_{m}=2 \pi \int_{0}^{\pi}(1-\cos \chi) \sigma(\chi) \sin \chi d \chi
$$

Since $\sigma(\chi)$ is an angular distribution function, it can be also be considered as a weight function to calculate the mean value of any function $F(\chi)$ of the scattering angle. The contribution to the total value of $F(\chi)$, resulting from the particles scattered into $d \Omega$, is $F(\chi) \sigma(\chi) d \Omega$. Since the 
total number of particles scattered is $\int \sigma(\chi) d \Omega$, it follows that the mean value of $F(\chi)$, averaged over all values of $\chi$, is given by

$$
<F(\chi)>=\frac{\int_{\Omega} F(\chi) \sigma(\chi) d \Omega}{\int_{\Omega} \sigma(\chi) d \Omega}
$$

which may be written as

$$
<F(\chi)>=\frac{2 \pi}{\sigma_{t}} \int_{0}^{\pi} F(\chi) \sigma(\chi) \sin \chi d \chi
$$

According to this definition of mean values we see that (5.11) can be written in the form

$$
\sigma_{m}=\sigma_{t}<1-\cos \chi>
$$

Thus, the cross section for momentum transfer is a weighted cross section in which scattering angles of zero degrees do not count at all, scattering of $90^{\circ}$ count as one, and scattering of $180^{\circ}$ count as two. This weighting is proportional to the amount of momentum transferred from the incident beam to the scattering center.

\section{CROSS SECTIONS FOR THE HARD SPHERE MODEL}

\subsection{Differential Scattering Cross Section}

To calculate $\sigma(\chi, \epsilon)$, as given in (5.5), we first obtain from (4.5), for $b \leq R_{1}+R_{2}$,

$$
b=\left(R_{1}+R_{2}\right) \cos \left(\frac{1}{2} \chi\right)
$$

and

$$
\left|\frac{d b}{d \chi}\right|=\frac{1}{2}\left(R_{1}+R_{2}\right) \sin \left(\frac{1}{2} \chi\right)
$$

Substituting these last two expressions into (5.5), yields for the differential scattering cross section

$$
\sigma=\frac{1}{4}\left(R_{1}+R_{2}\right)^{2}
$$




\subsection{Total Scattering Cross Section}

Integrating (6.3) over the whole solid angle, we obtain

$$
\sigma_{t}=2 \pi \int_{0}^{\pi} \frac{1}{4}\left(R_{1}+R_{2}\right)^{2} \sin \chi d \chi=\pi\left(R_{1}+R_{2}\right)^{2}
$$

Two special simple cases may be mentioned here. For the collision between an electron and a molecule of radius $R$, we have $\sigma=R^{2} / 4$ and $\sigma_{t}=\pi R^{2}$. For molecules colliding with themselves, their diameter being $D$, we have $\sigma=D^{2} / 4$ and $\sigma_{t}=\pi D^{2}$.

Note that in the case of the hard sphere model there is a cut-off value for the impact parameter $b$, beyond which collisions do not occur. It is the existence of this cut-off value for $b$ that leads to a finite value for the total scattering cross section $\sigma_{t}$. This conclusion will be made clear in the next section.

\subsection{Momentum Transfer Cross Section}

From (6.3) and (5.11) we obtain

$$
\begin{aligned}
& \sigma_{m}=2 \pi \int_{0}^{\pi} \frac{1}{4}\left(R_{1}+R_{2}\right)^{2}(1-\cos \chi) \sin \chi d \chi= \\
& \frac{1}{2} \pi\left(R_{1}+R_{2}\right)^{2}\left(\int_{0}^{\pi} \sin \chi d \chi-\int_{0}^{\pi} \cos \chi \sin \chi d \chi\right)
\end{aligned}
$$

Performing the integrals, yields

$$
\sigma_{m}=\pi\left(R_{1}+R_{2}\right)^{2}
$$

The average value of momentum loss per particle is found, from (5.13), to be given by

$$
<\mu g(1-\cos \chi)>=\frac{2 \pi}{\sigma_{t}} \int_{0}^{\pi} \mu g(1-\cos \chi) \sigma(\chi) \sin \chi d \chi
$$

Using (5.11), we get

$$
<\mu g(1-\cos \chi)>=\mu g \frac{\sigma_{m}}{\sigma_{t}}
$$


Thus, from (6.4), (6.6), and (6.8) we deduce that the average value of momentum loss, per particle, for the hard sphere model, is

$$
<\mu g(1-\cos \chi)>=\mu g
$$

For collisions between electrons and neutral particles, for example, in a weakly ionized plasma, the mass of the electron can be neglected as compared to the mass of the neutral particle, so that the reduced mass becomes equal to the electron mass. From (6.9) it is seen, in a first approximation, that the entire momentum of an electron is lost in a collision with a neutral particle. Assuming that the motion of the heavy particles can be ignored and denoting the collision frequency by $\nu$ (that is, the number of collisions between electrons and neutral particles per second), then the rate of momentum loss of an average electron is $\nu m_{e} \mathbf{u}$, where $\mathbf{u}$ denotes the electron velocity. However, in general an electron does not lose its entire momentum on a collision with a neutral particle. Furthermore, the perfectly elastic hard sphere model is not a very good representation for the interaction between an electron and a neutral particle. Consequently, the rate of loss of momentum is written as $\nu_{e n} m_{e} \mathbf{u}$, where $\nu_{e n}$ represents an effective collision frequency for momentum transfer between electrons and neutral particles. This is the term used in the Langevin equation, introduced in Chapter 10, to represent the time rate of momentum transfer due to collisions.

\section{CROSS SECTIONS FOR THE COULOMB POTENTIAL}

\subsection{Differential Scattering Cross Section}

Differentiating (4.13) we find

$$
\left|\frac{d b}{d \chi}\right|=\frac{b^{2}}{2 b_{0} \cos ^{2}(\chi / 2)}
$$

Thus, the differential scattering cross section, given in (5.5), becomes

$$
\sigma(\chi)=\frac{b^{3}}{2 b_{0} \sin \chi \cos ^{2}(\chi / 2)}
$$

Using (4.13) this equation can be rearranged as

$$
\sigma(\chi)=\frac{b_{0}^{2}}{4 \sin ^{4}(\chi / 2)}
$$


This equation is known as the Rutherford scattering formula. Since we have $2 \sin ^{2}(\chi / 2)=(1-\cos \chi)$, it can also be written as

$$
\sigma(\chi)=\frac{b_{0}^{2}}{(1-\cos \chi)^{2}}
$$

The Rutherford scattering formula shows that the differential scattering cross section is equal to $b_{0}^{2} / 4$ for the deflection angle $\chi=\pi$, increases monotonically as $\chi$ is decreased, and tends to infinity as $\chi$ tends to zero.

\subsection{Total Scattering Cross Section}

Since the differential scattering cross section increases rapidly to infinity as $\chi$ goes to zero, it turns out that the total scattering cross section $\sigma_{t}$ becomes infinite. From (5.7) and (7.4), we obtain

$$
\sigma_{t}=2 \pi \int_{\chi_{m i n}}^{\pi} \sigma(\chi) \sin \chi d \chi=2 \pi b_{0}^{2} \int_{\chi_{m i n}}^{\pi} \frac{\sin \chi}{(1-\cos \chi)^{2}} d \chi
$$

where $\chi_{\min }=0$. The lower limit has been written implicitly for reasons that will become apparent in what follows. Evaluating the integral in (7.5), yields

$$
\sigma_{t}=\pi b_{0}^{2}\left[\frac{1}{\sin ^{2}\left(\chi_{\min } / 2\right)}-1\right]
$$

which clearly gives $\sigma_{t}=\infty$ for $\chi_{\min }=0$. The particles with very small deflection angles contribute to make $\sigma_{t}$ infinite.

\subsection{Momentum Transfer Cross Section}

The substitution of (7.4) into (5.11) gives the following expression for the momentum transfer cross section

$$
\sigma_{m}=2 \pi \int_{\chi_{\min }}^{\pi}(1-\cos \chi) \sigma(\chi) \sin \chi d \chi=2 \pi b_{0}^{2} \int_{\chi_{\min }}^{\pi} \frac{\sin \chi}{(1-\cos \chi)} d \chi
$$

where, again, $\chi_{\min }=0$. Evaluating the integral we find

$$
\sigma_{m}=4 \pi b_{0}^{2} \ln \left[\frac{1}{\sin \left(\chi_{\min } / 2\right)}\right]
$$


Setting $\chi_{\min }=0$ we also find that $\sigma_{m}=\infty$. Thus, the coulomb potential gives infinite values for both $\sigma_{t}$ and $\sigma_{m}$, the particles with very small deflection angles being responsible for this infinite result.

\section{SCREENING OF THE COULOMB POTENTIAL}

The infinite results for $\sigma_{t}$ and $\sigma_{m}$, obtained in the previous section, may be interpreted as due to the absence of a cut-off value for the impact parameter $b$. Note that small values of $\chi$ correspond to large values of $b$, and that for $\chi_{\min }=0$ we must have, from $(4.13), b_{\max }=\infty$. In order to obtain finite and meaningful values for $\sigma_{t}$ and $\sigma_{m}$, it is necessary to modify the basis of the treatment of interactions between individual charged particles and introduce, on some plausible grounds, a cut-off value $b=b_{c}$ for the impact parameter.

From (5.3) and (5.6) we have for the total scattering cross section (considering $\sigma$ independent of $\epsilon$ )

$$
\sigma_{t}=2 \pi \int_{0}^{b_{c}} b d b
$$

where a cut-off value $b=b_{c}$ has been introduced for the impact parameter. With this cut-off, $\sigma_{t}$ for the coulomb potential is found to be

$$
\sigma_{t}=\pi b_{c}^{2}
$$

The introduction of a cut-off value for $b$ corresponds to the assumption that, for charged particles incident with $b$ greater than $b_{c}$ there will be no interaction, whereas for charged particles incident with $b$ less than $b_{c}$ there will be a coulomb-type interaction with the target particle.

The deflections that yield scattering angles between $\pi / 2$ and $\pi$, and which are associated with values of $b$ between 0 and $b_{0}$, are usually called large-angle deflections, or close encounters. If only the large-angle deflections are taken into account, we obtain

$$
\sigma_{t, \text { large }}=\pi b_{0}^{2} \quad ; \quad(\pi / 2<\chi<\pi)
$$

with $b_{0}$ as given by (4.9).

For a charged particle situated inside a plasma we know that it will be surrounded by a shielding cloud of particles of opposite sign. The scale 
length for an effective shielding of the charged particle under consideration is the Debye length defined by

$$
\lambda_{D}=\left(\frac{\epsilon_{0} k T}{n_{0} e^{2}}\right)^{1 / 2}
$$

The sphere of radius $\lambda_{D}$, surrounding the charged particle under consideration, is called the Debye sphere. We have seen in Chapter 11 that the charged particles lying within the Debye sphere shield the coulomb potential due to the charged particle under consideration, reducing significantly its effect on the particles lying outside its Debye sphere. Taking this screening effect into account, we find that the interaction potential energy is given by

$$
U(r)=\frac{1}{4 \pi \epsilon_{0}} \frac{q q_{1}}{r} \exp \left(-\frac{r}{\lambda_{D}}\right)
$$

Thus, when $r \ll \lambda_{D}$ the Debye potential, as given by (8.5), is very nearly equal to the coulomb potential, whereas when $r \gg \lambda_{D}$ the Debye potential is nearly equal to zero.

The analysis required for calculating $\sigma_{t}$, using the Debye potential, is excessively complicated and it must be done numerically. However, an alternative simple approach can be used that leads to results in very good agreement with those obtained numerically using the Debye potential. It consists in assuming that the interaction potential is exactly equal to the coulomb potential for $r<\lambda_{D}$ and is equal to zero for $r>\lambda_{D}$, as illustrated in Fig. 9. Therefore, it is convenient and more legitimate to introduce the cut-off in the impact parameter at $b_{c}=\lambda_{D}$ and not at $b_{c}=b_{0}$. In general, we have

$$
\lambda_{D} \gg b_{0}
$$

It is usual to denominate the deflections corresponding to $b_{0}<b<\lambda_{D}$, leading to $\chi<\pi / 2$, as small-angle deflections. The contribution to the total scattering cross section from the small-angle deflections is found to be given by

$$
\sigma_{t, \text { small }}=2 \pi \int_{b_{0}}^{\lambda_{D}} b d b=\pi\left(\lambda_{D}^{2}-b_{0}^{2}\right) \quad ; \quad(\chi<\pi / 2)
$$




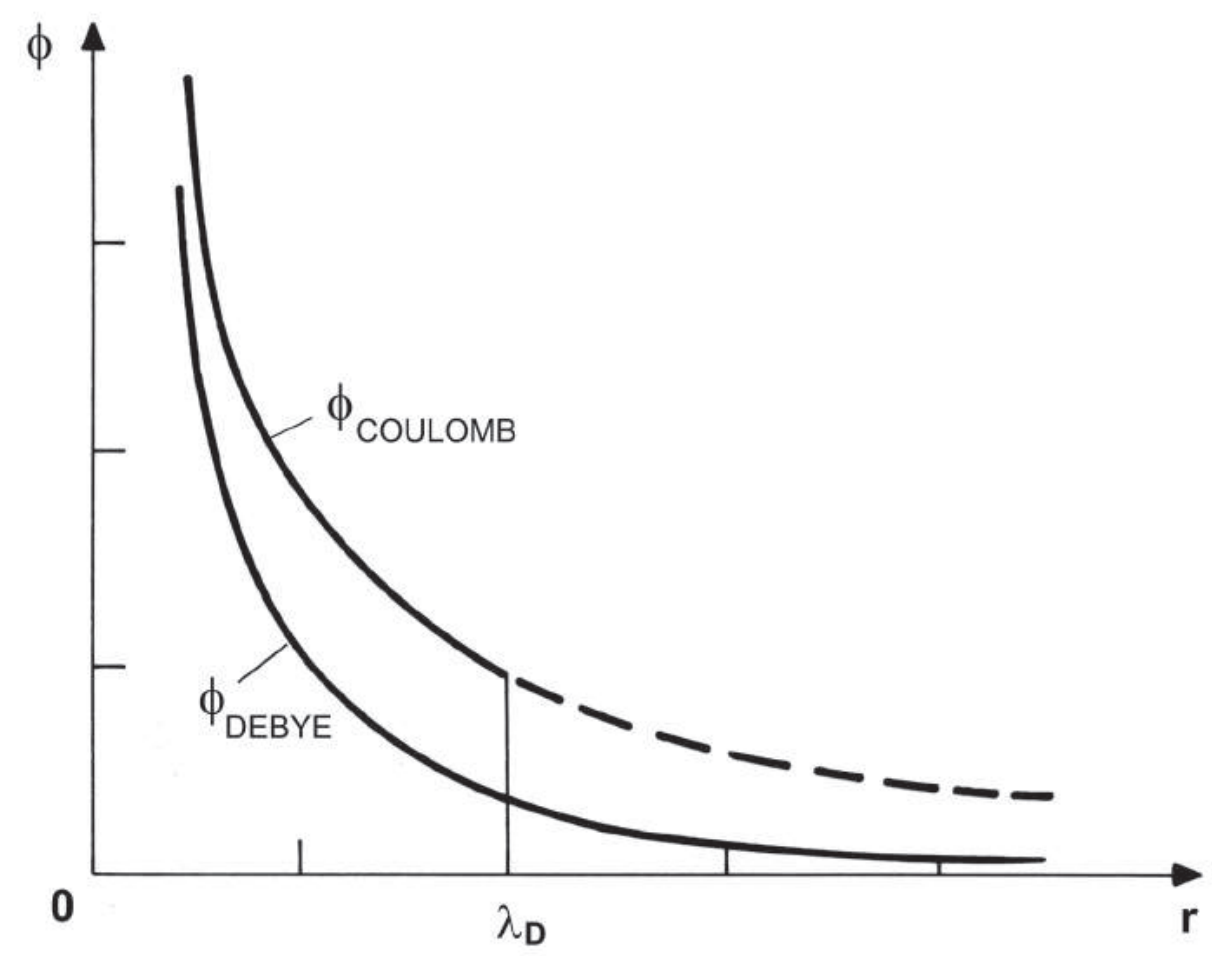

Fig. 9 The approximation used to obtain $\sigma_{t}=\pi \lambda_{D}^{2}$ consists in assuming that for $r<\lambda_{D}$ the shielding effect is completely omitted so that the particles interact according to the coulomb potential, whereas for $r>\lambda_{D}$ the shielding of the target particle is complete so that there is no interaction.

Therefore, it follows from (8.3) and (8.7) that

$$
\frac{\sigma_{t, \text { small }}}{\sigma_{t, \text { large }}}=\frac{\lambda_{D}^{2}}{b_{0}^{2}}-1 \simeq \frac{\lambda_{D}^{2}}{b_{0}^{2}}
$$

since $\lambda_{D} \gg b_{0}$. This result shows that the large number of particles interacting mildly with the target particle, and therefore producing only small-angle deflections, are much more important than the small number of particles interacting strongly with the target particle and producing large-angle deflections. Therefore, if the impact parameter is cutoff at $b_{c}=\lambda_{D}$ we obtain, from (8.1), the following result for the total scattering cross section:

$$
\sigma_{t}=\pi \lambda_{D}^{2}
$$


For the momentum transfer cross section, introducing the cut-off at $b_{c}=\lambda_{D}$, we obtain from $(7.8)$,

$$
\sigma_{m}=2 \pi b_{0}^{2} \ln \left(1+\frac{\lambda_{D}^{2}}{b_{0}^{2}}\right)
$$

since from (4.12), letting $\chi=\chi_{c}$ for $b=b_{c}$, we have

$$
\sin \left(\frac{1}{2} \chi_{c}\right)=\left(1+\frac{b_{c}^{2}}{b_{0}^{2}}\right)^{-1 / 2}
$$

Using the notation

$$
\Lambda=\frac{\lambda_{D}}{b_{0}}
$$

and noting that in general $\Lambda \gg 1,(8.10)$ becomes

$$
\sigma_{m}=4 \pi b_{0}^{2} \ln \Lambda
$$

It can be shown that for $\sigma_{m}$, as well as for $\sigma_{t}$, the large number of particle producing small-angle deflections are much more important than the small number of particles producing large-angle deflections.

The function ln $\Lambda$ varies very slowly over a large range of variation of the parameters on which $\Lambda$ depends. For most laboratory plasmas $\ell n \Lambda$ lies between 10 and 20. In order to calculate $\Lambda$ it is usual to make some approximations. For this purpose consider the interaction between an electron gas (charge $q=-e)$ and a singly charged ion gas $\left(q_{1}=e, Z=1\right)$. Let $n_{0}$ denote the number density of both the electrons and the ions in the gas, which we assume to constitute a plasma, and let the temperature of both species be equal to $T$. If we further assume that the electron and ion velocities have a Maxwellian equilibrium distribution function with no drift velocity, then we find, by direct calculation,

$$
\begin{gathered}
<g^{2}>=\frac{1}{n_{0}^{2}} \int_{v} \int_{v_{1}} f_{e} f_{i 1}\left(\mathbf{v}_{1}-\mathbf{v}\right)^{2} d^{3} v d^{3} v_{1}= \\
\frac{1}{n_{0}} \int_{v} f_{e}\left(\frac{3 k T}{m_{i}}+v^{2}\right) d^{3} v=\frac{3 k T}{\mu}
\end{gathered}
$$

where $k$ is Boltzmann's constant and $\mu$ is the reduced mass. Replacing $g^{2}$, in (4.9), by its average value, we find (for $q_{1}=-q=e$ )

$$
b_{0}=\frac{e^{2}}{4 \pi \epsilon_{0} \mu<g^{2}>}=\frac{e^{2}}{12 \pi \epsilon_{0} k T}
$$




\section{TABLE 1}

VALUES OF $\ln \Lambda$ FOR $Z=1$

AS A FUNCTION OF $T(K)$ AND $n_{e}\left(\mathrm{~cm}^{-3}\right)$

\begin{tabular}{|r|c|c|c|c|c|c|c|}
\hline $\mathbf{T} / \mathbf{n}_{e}$ & $10^{3}$ & $10^{6}$ & $10^{9}$ & $10^{12}$ & $10^{15}$ & $10^{18}$ & $10^{21}$ \\
\hline $10^{2}$ & 12.8 & 9.43 & 5.97 & & & & \\
$10^{3}$ & 16.3 & 12.8 & 9.43 & 5.97 & & & \\
$10^{4}$ & 19.7 & 16.3 & 12.8 & 9.43 & 5.97 & & \\
$10^{5}$ & 23.2 & 19.7 & 16.3 & 12.8 & 9.43 & 5.97 & \\
$10^{6}$ & 26.3 & 22.8 & 19.3 & 15.9 & 12.4 & 8.96 & 5.54 \\
$10^{7}$ & 28.5 & 25.1 & 21.6 & 18.1 & 14.7 & 11.2 & 7.85 \\
$10^{8}$ & 30.9 & 27.4 & 24.0 & 20.5 & 17.0 & 13.6 & 10.1 \\
\hline
\end{tabular}

Substituting this result into the expression (8.12), with $\lambda_{D}$ as given by (8.4), gives

$$
\Lambda=\frac{12 \pi \epsilon_{0} k T}{e^{2}} \lambda_{D}=12 \pi n_{0} \lambda_{D}^{3}=9 N_{D}
$$

where $N_{D}$ denotes the number of electrons in a Debye sphere. Table 1 presents values for $\ln \Lambda$, for various values of the electron number density $n_{e}$ and the electron temperature $T$.

\section{PROBLEMS}

20.1 For a differential scattering cross section with an angular dependence given by

$$
\sigma(\chi)=\frac{1}{2} \sigma_{0}\left(3 \cos ^{2} \chi-1\right)
$$

where $\sigma_{0}$ is a constant, calculate the total cross section and the momentum transfer cross section.

20.2 Consider a collision between two particles of mass $m$ and $m_{1}$, in which the particle of mass $m_{1}$ is initially at rest. Denote the scattering angle in the center of mass coordinate system by $\chi$ and in the laboratory coordinate system (as seen by an observer at rest) by $\chi_{L}$.

(a) Show that

$$
\tan \chi_{L}=\frac{\sin \chi}{\cos \chi+\left(m / m_{1}\right)}
$$


(b) Show that the relationship between the differential scattering cross section in the laboratory system $\sigma_{L}\left(\chi_{L}\right)$ and in the center of mass coordinate system $\sigma(\chi)$ is given by

$$
\sigma_{L}\left(\chi_{L}\right)=\sigma(\chi) \frac{\left[1+2\left(m / m_{1}\right) \cos \chi+\left(m / m_{1}\right)^{2}\right]^{3 / 2}}{1+\left(m / m_{1}\right) \cos \chi}
$$

Note that when $m_{1}=\infty$ we have $\chi_{L}=\chi$ and $\sigma_{L}\left(\chi_{L}\right)=\sigma(\chi)$.

(c) Verify that when $m=m_{1}$ we obtain $\chi_{L}=\chi / 2$ and $\sigma_{L}\left(\chi_{L}\right)=$ $4 \cos (\chi / 2) \sigma(\chi)$.

20.3 Consider two particles whose interaction is governed by the following rectangular-well potential:

$$
\begin{array}{lll}
U(r)=0 & \text { for } & r>a \\
U(r)=-U_{0} & \text { for } & r \leq a
\end{array}
$$

(a) Calculate the differential scattering cross section $\sigma(\chi)$ and show that it is given by (considering $b<a$ )

$$
\sigma(\chi)=\frac{p^{2} a^{2}[p \cos (\chi / 2)-1][p-\cos (\chi / 2)]}{4 \cos (\chi / 2)\left[1-2 p \cos (\chi / 2)+p^{2}\right]^{2}}
$$

where

$$
p=\left(1+\frac{2 U_{0}}{\mu g^{2}}\right)^{1 / 2}
$$

(b) Show that the total scattering cross section is given by

$$
\sigma_{t}=2 \pi \int_{0}^{a} b d b=\pi a^{2}
$$

20.4 Consider a general inverse-power interparticle force of the form

$$
F(r)=\frac{K}{r^{p}}
$$

where $K$ is a constant and $p$ is a positive integer number.

(a) Determine expressions for the scattering angle $\chi$, for the differential scattering cross section $\sigma(\chi, \epsilon)$, for the total scattering cross section $\sigma_{t}$, and for the momentum transfer cross section $\sigma_{m}$. 
(b) Calculate $\chi, \sigma(\chi, \epsilon), \sigma_{t}$, and $\sigma_{m}$ for the case of Maxwell molecules, for which $p=5$.

20.5 From the expression for $\sigma_{m}$, obtained in part (a) of problem 20.4, verify that for $p=2$ the momentum transfer cross section is given by

$$
\sigma_{m}=2 \pi A_{1}(2)\left(\frac{K}{\mu}\right)^{2} \frac{1}{g^{4}}
$$

where $A_{1}(2)$ is given by (with $\ell=1$ and $p=2$ )

$$
\begin{gathered}
A_{\ell}(p)=\int_{0}^{\infty}\left(1-\cos ^{\ell} \chi\right) v_{0} d v_{0} \\
v_{0}=b\left(\frac{\mu g^{2}}{K}\right)^{1 /(p-1)}
\end{gathered}
$$

Consequently, the velocity-dependent collision frequency, defined by

$$
\nu_{r}(g)=n \sigma_{m} g
$$

varies as $g^{-3}$. This inverse dependence on $g$ accounts for the so-called electron runaway effect. This implies that in the presence of a sufficiently large electric field $\mathbf{E}$, some electrons will gain enough kinetic energy between collisions so as to decrease their cross section and the collision frequency, which in turn allows them to pick up more energy from the field and decrease even further their cross section and collision frequency. If $\mathbf{E}$ is large enough, the collision frequency may fall so fast that these electrons will form an accelerated beam of runaway electrons.

20.6 Show that for the case of coulomb interactions $(p=2)$ we have

$$
\begin{gathered}
A_{1}(2)=\left(\frac{\mu g^{2}}{K}\right)^{2} b_{0}^{2} \ln \left(1+\Lambda^{2}\right) \\
A_{2}(2)=\left(\frac{\mu g^{2}}{k}\right)^{2} 2 b_{0}^{2}\left[\ln \left(1+\Lambda^{2}\right)-\frac{\Lambda^{2}}{\left(1+\Lambda^{2}\right)}\right]
\end{gathered}
$$

where $b_{0}=q q_{1} /\left(4 \pi \epsilon_{0} \mu g^{2}\right), A_{\ell}(p)$ is as defined in problem 20.5 , and $\Lambda=$ $\lambda_{D} / b_{0}$. For $\Lambda \gg 1$ verify that

$$
\begin{gathered}
A_{1}(2)=\left(\frac{\mu g^{2}}{K}\right)^{2} 2 b_{0}^{2} \ln \Lambda=2 \ln \Lambda \\
A_{2}(2)=\left(\frac{\mu g^{2}}{K}\right)^{2} 2 b_{0}^{2}(2 \ln \Lambda-1)=2(2 \ln \Lambda-1)
\end{gathered}
$$

Note that, since $K=q q_{1} /\left(4 \pi \epsilon_{0}\right)$ for the coulomb interaction potential, we have $\left(\mu g^{2} / K\right)^{2} b_{0}^{2}=1$. 


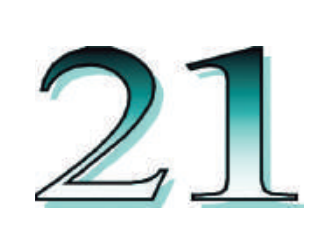

\section{THE BOLTZMANN AND THE FOKKER-PLANCK EQUATIONS}

\section{INTRODUCTION}

When the Boltzmann equation was first introduced in Chapter 5, the collisional effects were incorporated in its right-hand side [see equation (5.5.27)] through a general collision term $\left(\delta f_{\alpha} / \delta t\right)_{\text {coll }}$ still to be specified. We present now a derivation of the Boltzmann collision term, which takes into account only binary collisions. This collision term involves integrals over the particle velocities, so that the Boltzmann equation turns out to be an integro-differential equation. The fact that the Boltzmann collision integral takes into account only binary collisions limits considerably its applicability for a plasma, where each charged particle interacts simultaneously with a large number of neighboring charged particles. Although these multiple coulomb collisions are very important for a plasma, there are some cases (as in weakly ionized plasmas) where the binary chargeneutral collisions play a dominant role.

The collision term originally proposed by Boltzmann applies to a low density gas, in which only binary elastic collisions are important. These binary collisions may involve neutral atoms or molecules in a dilute gas, or charged and neutral particles in a plasma. We have seen that in a plasma these are not the only particle interactions of importance. The multiple coulomb interactions need to be taken into account and in most cases are much more important than the binary collisions. Nevertheless, the Boltzmann collision term can in some cases be used for a plasma, but the results obtained have to be interpreted cautiously. 
Furthermore, the Fokker-Planck collision term, which applies to charged particle interactions, can be derived from the Boltzmann collision term by considering the charged particle encounters as a series of consecutive weak (small deflection angle) binary collisions.

\section{THE BOLTZMANN EQUATION}

\subsection{Derivation of the Boltzmann Collision Integral}

The collision term $\left(\delta f_{\alpha} / \delta t\right)_{\text {coll }}$ represents the rate of change of the distribution function $f_{\alpha}(\mathbf{r}, \mathbf{v}, t)$ as a result of collisions. Some of the particles of type $\alpha$ originally situated inside the volume element $d^{3} r d^{3} v$ at $(\mathbf{r}, \mathbf{v})$ in phase space may leave this volume element, whereas some particles of type $\alpha$ originally situated outside this volume element may end inside it, as a result of collisions during the time interval $d t$. Let $\Delta N_{\alpha}$ denote this net gain or loss of particles of type $\alpha$ in $d^{3} r d^{3} v$ at $(\mathbf{r}, \mathbf{v})$ during $d t$, that is,

$$
\Delta N_{\alpha}=\left(\frac{\delta f_{\alpha}}{\delta t}\right)_{\text {coll }} d^{3} r d^{3} v d t
$$

It is convenient to separate $\Delta N_{\alpha}$ into two parts

$$
\Delta N_{\alpha}=\Delta N_{\alpha}^{+}-\Delta N_{\alpha}^{-}
$$

where $\Delta N_{\alpha}^{+}$represents the gain term due to collisions in which a particle of type $\alpha$ situated inside $d^{3} r$ about $\mathbf{r}$ has, after collision, a velocity lying within $d^{3} v$ about $\mathbf{v}$, and $\Delta N_{\alpha}^{-}$represents the loss term due to collisions in which a particle of type $\alpha$ situated inside $d^{3} r$ about $\mathbf{r}$ has, before collision, a velocity lying within $d^{3} v$ about $\mathbf{v}$. We proceed now to determinte $\Delta N_{\alpha}$, defined in (2.1), by calculating initially $\Delta N_{\alpha}^{-}$and afterwards $\Delta N_{\alpha}^{+}$.

To calculate $\Delta N_{\alpha}^{-}$we consider the particles of type $\alpha$ situated within the volume element $d^{3} r$ at $\mathbf{r}$, whose velocities lie within $d^{3} v$ about $\mathbf{v}$, and which are scattered out of this velocity range, as a result of collisions with particles of some type (which may or may not be type $\alpha$ particles) lying in the same volume element $d^{3} r$ at $\mathbf{r}$ and having some velocity within $d^{3} v_{1}$ about $\mathbf{v}_{1}$. Let us focus attention on a single particle of type $\alpha$ situated within the phase space volume element $d^{3} r d^{3} v$ at the coordinates $(\mathbf{r}, \mathbf{v})$. The particles of type $\beta$ inside $d^{3} r d^{3} v_{1}$ at $\left(\mathbf{r}, \mathbf{v}_{1}\right)$ may be viewed as constituting a particle flux incident on this type $\alpha$ particle. Noting that $f_{\beta}\left(\mathbf{r}, \mathbf{v}_{1}, t\right) d^{3} v_{1}$ is the number of type $\beta$ particles per unit volume, 
with velocities within $d^{3} v_{1}$ about $\mathbf{v}_{1}$, the flux of this incident beam can be expressed as

$$
\Gamma_{\beta}=f_{\beta}\left(\mathbf{r}, \mathbf{v}_{1}, t\right) d^{3} v_{1}\left|\mathbf{v}_{1}-\mathbf{v}\right|=f_{\beta}\left(\mathbf{r}, \mathbf{v}_{1}, t\right) d^{3} v_{1} g
$$

Consider the type $\beta$ particles that approach with an impact parameter between $b$ and $b+d b$, in a collision plane lying between the angles $\epsilon$ and $\epsilon+d \epsilon$. The average number of interactions of these type $\beta$ particles with the type $\alpha$ particle, occurring in the time interval $d t$, is equal to the number of particles crossing the element of area $b d b d \epsilon$ during $d t$. This number can be obtained by multiplying the flux of type $\beta$ particles, given in (2.3), by the element of area $b d b d \epsilon$ and by the time interval $d t$,

$$
\Gamma_{\beta} b d b d \epsilon d t=f_{\beta}\left(\mathbf{r}, \mathbf{v}_{1}, t\right) d^{3} v_{1} g b d b d \epsilon d t
$$

This expression is just the number of particles of type $\beta$ with velocities lying within $d^{3} v_{1}$ about $\mathbf{v}_{1}$ and situated inside the elementary cylindrical volume of length $g d t$ and cross-sectional area $b d b d \epsilon$, as shown in Fig. 1 , and whose volume is $g b d b d \epsilon d t$. It is assumed here that $d t$ is large compared to the time of interaction of the colliding particles. To determine the number of collisions between the type $\beta$ particles indicated in (2.4), with all the type $\alpha$ particles lying within $d^{3} r d^{3} v$ at $(\mathbf{r}, \mathbf{v})$, during $d t$, we multiply (2.4) by $f_{\alpha}(\mathbf{r}, \mathbf{v}, t) d^{3} r d^{3} v$, which is the number of type $\alpha$ particles lying within this phase space volume element, obtaining

$$
f_{\alpha}(\mathbf{r}, \mathbf{v}, t) d^{3} r d^{3} v f_{\beta}\left(\mathbf{r}, \mathbf{v}_{1}, t\right) d^{3} v_{1} g b d b d \epsilon d t
$$

In deducing this expression it has been assumed that the number of collisions between the particles of the types $\alpha$ and $\beta$, with velocities in $d^{3} v$ about $\mathbf{v}$, and in $d^{3} v_{1}$ about $\mathbf{v}_{1}$, respectively, lying in the same volume element $d^{3} r$ about $\mathbf{r}$, is proportional to the product $f_{\alpha}(\mathbf{r}, \mathbf{v}, t) f_{\beta}\left(\mathbf{r}, \mathbf{v}_{1}, t\right)$. However, in a system of interacting particles the existence of a particle within a given volume element $d^{3} r$ at $\mathbf{r}$, with a given velocity $\mathbf{v}$, affects the probability that another particle be found with a specified velocity $\mathbf{v}_{1}$ in the same volume element, at the same time. Thus, in expression (2.5) we are neglecting any possible correlation that may exist between the velocity of a particle and its position. This approximation, known as the molecular chaos assumption, is introduced as a mathematical convenience, but although it may represent a possible condition for a system of particles, it is not a general condition.

The total number of particles of type $\alpha$ in $d^{3} r$ about $\mathbf{r}$ that are scattered out of the velocity space element $d^{3} v$ about $\mathbf{v}$, during $d t$, is obtained 
integrating expression (2.5) over all possible values of $b, \epsilon$, and $\mathbf{v}_{1}$, and summing over all species $\beta$,

$$
\Delta N_{\alpha}^{-}=f_{\alpha}(\mathbf{r}, \mathbf{v}, t) d^{3} r d^{3} v d t \sum_{\beta} \int_{v_{1}} \int_{b} \int_{\epsilon} f_{\beta}\left(\mathbf{r}, \mathbf{v}_{1}, t\right) d^{3} v_{1} g b d b d \epsilon
$$

where the triple integral over $\mathbf{v}_{1}$ is represented again by a single integral sign.

To determine the gain term $\Delta N_{\alpha}^{+}$, we proceed in a way similar to the determination of $\Delta N_{\alpha}^{-}$, by considering the inverse collision, in which a particle of type $\alpha$ with initial velocity in $d^{3} v^{\prime}$ about $\mathbf{v}^{\prime}$, collides with a particle of type $\beta$ having initial velocity in $d^{3} v_{1}^{\prime}$ about $\mathbf{v}_{1}^{\prime}$, resulting in the particle of type $\alpha$ scattered into the velocity element $d^{3} v$ about $\mathbf{v}$, the event occurring inside the volume element $d^{3} r$ about $\mathbf{r}$. The average number of interactions between a single particle of type $\alpha$, inside the volume element of phase space $d^{3} r d^{3} v^{\prime}$ at $\left(\mathbf{r}, \mathbf{v}^{\prime}\right)$, with the particles of type $\beta$ inside $d^{3} r d^{3} v_{1}^{\prime}$ at $\left(\mathbf{r}, \mathbf{v}_{1}^{\prime}\right)$, which approach with an impact parameter between $b$ and $b+d b$, and with the collision plane oriented between the angles $\epsilon$ and $\epsilon+d \epsilon$, is given by

$$
f_{\beta}\left(\mathbf{r}, \mathbf{v}_{1}^{\prime}, t\right) d^{3} v_{1}^{\prime} g^{\prime} b d b d \epsilon d t
$$

To take into account all collisions occurring within $d^{3} r$, at $\mathbf{r}$, during the time interval $d t$, between the particles of type $\alpha$ and $\beta$, which scatter the particles of type $\alpha$ into the volume element $d^{3} v$, about $\mathbf{v}$, we must multiply (2.7) by the number of particles of type $\alpha$ that lie initially inside $d^{3} r d^{3} v^{\prime}$, that is, by $f_{\alpha}\left(\mathbf{r}, \mathbf{v}^{\prime}, t\right) d^{3} r d^{3} v^{\prime}$, integrate the result over all possible values of $b, \epsilon$, and $\mathbf{v}_{1}^{\prime}$, and sum over all species $\beta$,

$$
\Delta N_{\alpha}^{+}=f_{\alpha}\left(\mathbf{r}, \mathbf{v}^{\prime}, t\right) d^{3} r d^{3} v^{\prime} d t \sum_{\beta} \int_{v_{1}^{\prime}} \int_{b} \int_{\epsilon} f_{\beta}\left(\mathbf{r}, \mathbf{v}_{1}^{\prime}, t\right) d^{3} v_{1}^{\prime} g^{\prime} b d b d \epsilon
$$

We have seen that $g^{\prime}=g=\left|\mathbf{v}_{1}-\mathbf{v}\right|$ and from the theory of Jacobians

$$
d^{3} v^{\prime} d^{3} v_{1}^{\prime}=|J| d^{3} v d^{3} v_{1}
$$

We will show in the following subsection that for this transformation of velocities we have $|J|=1$, so that

$$
d^{3} v^{\prime} d^{3} v_{1}^{\prime}=d^{3} v d^{3} v_{1}
$$




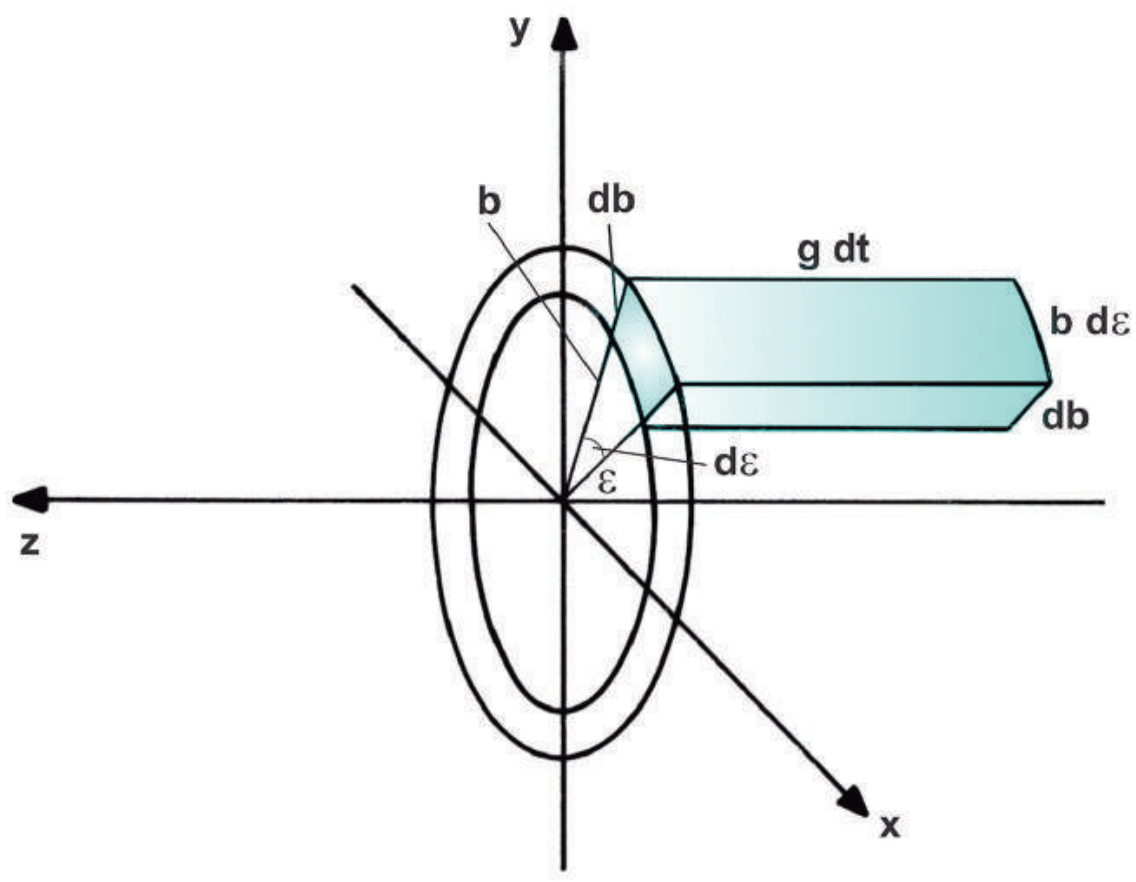

Fig. 1 The volume element of height $g d t$ and cross-sectional area $b d b d \epsilon$, with sides lying between $b$ and $b+d b$, and between $\epsilon$ and $\epsilon+d \epsilon$.

Consequently, (2.8) becomes

$$
\Delta N_{\alpha}^{+}=f_{\alpha}\left(\mathbf{r}, \mathbf{v}^{\prime}, t\right) d^{3} r d^{3} v d t \sum_{\beta} \int_{v_{1}} \int_{b} \int_{\epsilon} f_{\beta}\left(\mathbf{r}, \mathbf{v}_{1}^{\prime}, t\right) d^{3} v_{1} g b d b d \epsilon
$$

If we now combine the expressions for $\Delta N_{\alpha}^{-}$and $\Delta N_{\alpha}^{+}$, and substitute $b d b d \epsilon$ by $\sigma(\Omega) d \Omega$, we obtain the following expression for the Boltzmann collision integral,

$$
\begin{gathered}
\left(\frac{\delta f_{\alpha}}{\delta t}\right)_{\text {coll }}=\left(\frac{\Delta N_{\alpha}^{+}-\Delta N_{\alpha}^{-}}{d^{3} r d^{3} v d t}\right)= \\
\sum_{\beta} \int_{v_{1}} \int_{\Omega}\left(f_{\alpha}^{\prime} f_{\beta 1}^{\prime}-f_{\alpha} f_{\beta 1}\right) d^{3} v_{1} g \sigma(\Omega) d \Omega
\end{gathered}
$$

where we have introduced the notation 


$$
\begin{aligned}
f_{\alpha}^{\prime} & =f_{\alpha}\left(\mathbf{r}, \mathbf{v}^{\prime}, t\right) \\
f_{\beta 1}^{\prime} & =f_{\beta}\left(\mathbf{r}, \mathbf{v}_{1}^{\prime}, t\right) \\
f_{\alpha} & =f_{\alpha}(\mathbf{r}, \mathbf{v}, t) \\
f_{\beta 1} & =f_{\beta}\left(\mathbf{r}, \mathbf{v}_{1}, t\right)
\end{aligned}
$$

In explicit form, the Boltzmann equation can finally be written as

$$
\frac{\partial f_{\alpha}}{\partial t}+\mathbf{v} \cdot \nabla f_{\alpha}+\mathbf{a} \cdot \nabla_{v} f_{\alpha}=\sum_{\beta} \int_{v_{1}} \int_{\Omega}\left(f_{\alpha}^{\prime} f_{\beta 1}^{\prime}-f_{\alpha} f_{\beta 1}\right) d^{3} v_{1} g \sigma(\Omega) d \Omega
$$

The Boltzmann equation is therefore an integro-differential equation, involving integrals and partial derivatives of the distribution function. The external force $\mathbf{F}=m_{\alpha} \mathbf{a}$, in the case of a plasma, includes also the electromagnetic Lorentz force $\mathbf{F}=q_{\alpha}(\mathbf{E}+\mathbf{v} \times \mathbf{B})$ due to externally applied fields.

For a system consisting of various different species of particles, there is one equation for each species. For an ionized gas composed of electrons, one type of positive ions, and one type of neutral particles, for example, we have a system of three Boltzmann equations coupled through the collision term. In the Boltzmann equation for the electrons, for example, the collision term contains the distribution function for the electrons $f_{e}$, the distribution function for the ions $f_{i}$, and the distribution function for the neutral particles $f_{n}$. Since the collision term involves products of the distribution functions, the Boltzmann equation is also nonlinear. For a system consisting of only one type of particles, the summation over the type $\beta$ particles disappears and the collision term involves only the product of distribution functions of the same particle species.

\subsection{Jacobian of the Transformation}

The relation between the velocity elements $d^{3} v d^{3} v_{1}$ and $d^{3} v^{\prime} d^{3} v_{1}^{\prime}$ is given by

$$
d^{3} v^{\prime} d^{3} v_{1}^{\prime}=|J| d^{3} v d^{3} v_{1}
$$

where $J$ is the Jacobian of the transformation from the velocity variables $\left(\mathbf{v}, \mathbf{v}_{1}\right)$ to $\left(\mathbf{v}^{\prime}, \mathbf{v}_{1}^{\prime}\right)$ and can be expressed as

$$
J=\frac{\partial\left(\mathbf{v}^{\prime}, \mathbf{v}_{1}^{\prime}\right)}{\partial\left(\mathbf{v}, \mathbf{v}_{1}\right)}=\frac{\partial\left(v_{x}^{\prime}, v_{y}^{\prime}, v_{z}^{\prime}, v_{1 x}^{\prime}, v_{1 y}^{\prime}, v_{1 z}^{\prime}\right)}{\partial\left(v_{x}, v_{y}, v_{z}, v_{1 x}, v_{1 y}, v_{1 z}\right)}
$$


which corresponds to the determinant of the matrix

$$
(J)=\left(\begin{array}{cccc}
\frac{\partial v_{x}^{\prime}}{\partial v_{x}} & \frac{\partial v_{y}^{\prime}}{\partial v_{x}} & \ldots & \frac{\partial v_{1 z}^{\prime}}{\partial v_{x}} \\
\frac{\partial v_{x}^{\prime}}{\partial v_{y}} & \frac{\partial v_{y}^{\prime}}{\partial v_{y}} & \ldots & \frac{\partial v_{1 z}^{\prime}}{\partial v_{y}} \\
\cdots & \ldots & \ldots & \ldots \\
\frac{\partial v_{x}^{\prime}}{\partial v_{1 z}} & \frac{\partial v_{y}^{\prime}}{\partial v_{1 z}} & \ldots & \frac{\partial v_{1 z}^{\prime}}{\partial v_{1 z}}
\end{array}\right)
$$

Using (20.2.5) and (20.2.6) (Eqs. 2.5 and 2.6 in Chapter 20) we can express $d^{3} v$ and $d^{3} v_{1}$ in terms of $d^{3} c_{0}$ and $d^{3} g$,

$$
d^{3} v d^{3} v_{1}=\left|J_{c}\right| d^{3} c_{0} d^{3} g
$$

where $J_{c}$ denotes the Jacobian of the transformation indicated in equations (20.2.5) and (20.2.6). Let us consider initially only the $x$ component of (2.18),

$$
d v_{x} d v_{1 x}=\left|\frac{\partial\left(v_{x}, v_{1 x}\right)}{\partial\left(c_{0 x}, g_{x}\right)}\right| d c_{0 x} d g_{x}
$$

From (20.2.5) and (20.2.6) we can calculate the determinant of the $2 \times 2$ matrix indicated in (2.19), obtaining

$$
d v_{x} d v_{1 x}=\left(\frac{\mu}{m_{1}}+\frac{\mu}{m}\right) d c_{0 x} d g_{x}=d c_{0 x} d g_{x}
$$

Taking the product of three such terms, corresponding to the components $x, y$, and $z$, gives

$$
d^{3} v d^{3} v_{1}=d^{3} c_{0} d^{3} g
$$

In a similar way, using (20.2.8) and (20.2.9) we find

$$
d^{3} v^{\prime} d^{3} v_{1}^{\prime}=d^{3} c_{0}^{\prime} d^{3} g^{\prime}
$$

We have seen that $\mathbf{c}_{0}=\mathbf{c}_{0}^{\prime}$. Furthermore, $g$ and $g^{\prime}$ differ only in direction, having the same magnitude, and since volume elements are not changed by a simple rotation of coordinates, we must have $d^{3} g=d^{3} g^{\prime}$. Consequently, (2.21) and (2.22) yields

$$
d^{3} v d^{3} v_{1}=d^{3} v^{\prime} d^{3} v_{1}^{\prime}
$$




\subsection{Assumptions in the Derivation of the Boltzmann Collision Integral}

The derivation of the Boltzmann collision integral presented here involves four basic assumptions:

(a) The distribution function does not vary appreciably over a distance of the order of the range of the interparticle force law, as well as over time scales of the order of the interaction time.

(b) Effects of the external force, on the magnitude of the collision cross section, are ignored.

(c) Only binary collisions are taken into account.

(d) The velocities of the interacting particles, before collision, are assumed to be uncorrelated.

The first assumption is quite reasonable and is incorporated in the calculation of $\left(\delta f_{\alpha} / \delta t\right)_{\text {coll }}$ when we evaluate all the distribution functions at the position $\mathbf{r}$ and at the instant $t$. The element of volume $d^{3} r$ is considered to be large compared to the range of the interparticle forces and the time interval $d t$ is taken to be large compared to the time of interaction. On the other hand, as far as variations in the distribution functions are concerned, the elements $d^{3} r$ and $d t$ must be infinitesimally small quantities.

Next, the external force was assumed to have a negligible effect on the two-body collision problem. This is valid if the external force is negligibly small compared to the interaction force between the particles. When external forces of magnitude comparable to the short-range interparticle forces are present, the collision process is modified. The constancy of the relative speed $g=\left|\mathbf{v}_{1}-\mathbf{v}\right|$ is strictly valid only in the absence of external forces.

The assumption of binary collisions is justified for a dilute gas, whose molecules interact through short-range forces. However, it is not valid for the coulomb interactions in a plasma. Because of the long-range characteristic of the coulomb force, a charged particle in a plasma interacts simultaneously with all the charged particles inside its Debye sphere. Since there is a large number of charged particles inside a Debye sphere, each charged particle in the plasma does not move freely, as does a neutral particle between collisions, but interacts continuously with a large number of charged particles. However, the Boltzmann collision integral may also be used as a starting point in the derivation of the Fokker-Plamck collision term for a plasma, under the assumption that each long-range individual interaction results only in a small deflection in the particle trajectory and 
since each individual interaction is relatively weak, the collective effect of many simultaneous interactions can be considered as a cumulative succession of weak binary collisions. Thus, in general, the Boltzmann collision term is not strictly valid for a plasma and the results obtained for the case of charge-neutral particle interactions in weakly ionized plasmas must be interpreted cautiously.

Assumption (d) is known as the molecular chaos assumption. It is justified for a gas in which the density is sufficiently small, so that the mean free path is much larger than the characteristic range of the interparticle forces. This is certainly not a general situation for a plasma, in view of the long-range characteristic of the coulomb force. Generally, the joint probability of having, at the position $\mathbf{r}$ and at the instant $t$, a particle of type $\alpha$ with velocity $\mathbf{v}$ and a particle of type $\beta$ with velocity $\mathbf{v}_{1}$ is proportional to

$$
f_{\alpha}(\mathbf{r}, \mathbf{v}, t) f_{\beta}\left(\mathbf{r}, \mathbf{v}_{1}, t\right)\left[1+\psi_{\alpha \beta}\left(\mathbf{v}, \mathbf{v}_{1}, \mathbf{r}, t\right)\right]
$$

where $\psi_{\alpha \beta}\left(\mathbf{v}, \mathbf{v}_{1}, \mathbf{r}, t\right)$ is known as the correlation function. In the derivation of the Boltzmann collision integral we have neglected the correlation effects and we have taken this joint probability as being proportional to the product $f_{\alpha}(\mathbf{r}, \mathbf{v}, t) f_{\beta}\left(\mathbf{r}, \mathbf{v}_{1}, t\right)$. The irreversible character of the Boltzmann equation, to be discussed in the next section, is a consequence of the molecular chaos assumption. In order to avoid this approximation the only alternative is to work with the reversible equations of the $B B G K Y$ (Bogoliubov, Born, Green, Kirkwood, and Yvon) hierarchy. This kinetic treatment, however, is beyond the scope of this text.

For gaseous systems in which the characteristic interaction length is much less than the average interparticle distance and where temporal and spatial gradients are not very large, the Boltzmann equation is nevertheless very well verified experimentally and, in this respect, constitutes one of the basic relations of the kinetic theory of gases.

\subsection{Rate of Change of a Physical Quantity as a Result of Collisions}

In section 2 of Chapter 8 we have denoted the time rate of change of a physical quantity $\chi(\mathbf{v})$ per unit volume, for the particles of type $\alpha$, due to collisions with the other particles in the plasma, by

$$
\left[\frac{\delta\left(n_{\alpha}<\chi>_{\alpha}\right)}{\delta t}\right]_{\text {coll }}=\int_{v} \chi\left(\frac{\delta f_{\alpha}}{\delta t}\right)_{\text {coll }} d^{3} v
$$


Using the Boltzmann collision integral,

$$
\left(\frac{\delta f_{\alpha}}{\delta t}\right)_{\text {coll }}=\sum_{\beta} \int_{\Omega} \int_{v_{1}}\left(f_{\alpha}^{\prime} f_{\beta 1}^{\prime}-f_{\alpha} f_{\beta 1}\right) g \sigma(\Omega) d \Omega d^{3} v_{1}
$$

we obtain the following expression for (2.25):

$$
\left[\frac{\delta\left(n_{\alpha}<\chi>_{\alpha}\right)}{\delta t}\right]_{\text {coll }}=\sum_{\beta} \int_{\Omega} \int_{v_{1}} \int_{v}\left(f_{\alpha}^{\prime} f_{\beta 1}^{\prime}-f_{\alpha} f_{\beta 1}\right) \chi g \sigma(\Omega) d \Omega d^{3} v d^{3} v_{1}
$$

Recall that for each direct collision there is a corresponding inverse collision with the same cross section. Hence, the integrals over $\mathbf{v}$ and $\mathbf{v}_{1}$ can be replaced by integrals over $\mathbf{v}^{\prime}$ and $\mathbf{v}_{1}^{\prime}$, respectively, without altering the result. Therefore, the first group of integrals in (2.27) may be written as

$$
\begin{gathered}
\sum_{\beta} \int_{\Omega} \int_{v_{1}} \int_{v} f_{\alpha}^{\prime} f_{\beta 1}^{\prime} \chi g \sigma(\Omega) d \Omega d^{3} v d^{3} v_{1}= \\
\sum_{\beta} \int_{\Omega} \int_{v_{1}} \int_{v} f_{\alpha} f_{\beta 1} \chi^{\prime} g \sigma(\Omega) d \Omega d^{3} v d^{3} v_{1}
\end{gathered}
$$

where we have replaced $d^{3} v^{\prime} d^{3} v_{1}^{\prime}$ by $d^{3} v d^{3} v_{1}$. Using this expression, we obtain the following alternative form for the collision term in (2.27),

$$
\left[\frac{\delta\left(n_{\alpha}<\chi>_{\alpha}\right)}{\delta t}\right]_{\text {coll }}=\sum_{\alpha} \int_{\Omega} \int_{v_{1}} \int_{v} f_{\alpha} f_{\beta 1}\left(\chi^{\prime}-\chi\right) g \sigma(\Omega) d \Omega d^{3} v d^{3} v_{1}
$$

Note that the property $\chi(\mathbf{v})$ is associated with the particles of type $\alpha$ and that $\chi^{\prime}$ denotes $\chi\left(\mathbf{v}^{\prime}\right)$. Note also that only the quantity $\chi^{\prime}$ on the right-hand side of (2.29) is a function of the after-collision velocity $\mathbf{v}^{\prime}$. Recall that the result just derived applies to the special case of binary elastic collisions in a dilute gas, when processes of particle creation and disappearance, as well as radiation losses are unimportant.

\section{THE BOLTZMANN'S H FUNCTION}

An important characteristic of the Boltzmann collision term is that it drives the distribution function towards the equilibrium state in an $\mathrm{ir}$ reversible way. This irreversible characteristic of the Boltzmann collision 
term, as mentioned before, is a consequence of the molecular chaos assumption, which neglects the correlation effects between the particles.

In order to investigate this aspect of the Boltzmann collision term, we introduce now the Boltzmann's function $H(t)$. For simplicity we will consider a gas consisting of only one particle species, with the particles uniformly distributed in space (having no density gradients) and isolated from the action of external forces. The distribution function, therefore, is independent of $\mathbf{r}$ and can be denoted by $f(\mathbf{v}, t)$. We define, according to Boltzmann, the function $H(t)$ by

$$
H(t)=\int_{v} f(\mathbf{v}, t) \ln [f(\mathbf{v}, t)] d^{3} v
$$

For problems involving spatial gradients, the function $H(t)$, defined in (3.1), corresponds to $H_{\text {total }}(t)$ per unit volume, where

$$
H_{\text {total }}(t)=\int_{r} \int_{v} f(\mathbf{r}, \mathbf{v}, t) \ln [f(\mathbf{r}, \mathbf{v}, t)] d^{3} r d^{3} v
$$

The function $H(t)$ is proportional to the entropy per unit volume of the system according to

$$
\frac{S}{V}=-k H
$$

where $S$ denotes the total entropy, $V$ is the volume of the system, and $k$ is Boltzmann's constant. More generally, for systems in which spatial gradients are present we have

$$
S=-k H_{\text {total }}
$$

\subsection{Boltzmann's H Theorem}

The Boltzmann's $\mathrm{H}$ theorem states that if $f(\mathbf{v}, t)$ is a solution of the Boltzmann equation, that is, if

$$
\frac{\partial f(\mathbf{v}, t)}{\partial t}=\int_{\Omega} \int_{v_{1}}\left[f\left(\mathbf{v}^{\prime}, t\right) f\left(\mathbf{v}_{1}^{\prime}, t\right)-f(\mathbf{v}, t) f\left(\mathbf{v}_{1}, t\right)\right] g \sigma(\Omega) d \Omega d^{3} v_{1}
$$

then

$$
\frac{\partial H(t)}{\partial t} \leq 0
$$


To prove this theorem let us take the derivative of (3.1) with respect to time, which gives

$$
\frac{\partial H}{\partial t}=\int_{v}(1+\ln f) \frac{\partial f}{\partial t} d^{3} v
$$

Substituting (3.5) into (3.7) gives

$$
\frac{\partial H}{\partial t}=\int_{\Omega} \int_{v} \int_{v_{1}}(1+\ln f)\left(f^{\prime} f_{1}^{\prime}-f f_{1}\right) g \sigma(\Omega) d \Omega d^{3} v_{1} d^{3} v
$$

where the notation $f_{1}^{\prime}=f\left(\mathbf{v}_{1}^{\prime}, t\right)$, and so on, has been used. The variables of integration $\mathbf{v}$ and $\mathbf{v}_{1}$ are dummy variables and can be interchanged in the integrand of (3.8) without changing the value of the integral, since $\sigma(\Omega)$ and $g=\left|\mathbf{v}_{1}-\mathbf{v}\right|$ are also invariants. Thus, (3.8) can be written as

$$
\frac{\partial H}{\partial t}=\int_{\Omega} \int_{v_{1}} \int_{v}\left(1+\ln f_{1}\right)\left(f_{1}^{\prime} f^{\prime}-f_{1} f\right) g \sigma(\Omega) d \Omega d^{3} v d^{3} v_{1}
$$

Adding equations (3.8) and (3.9), and dividing by 2, gives

$$
\frac{\partial H}{\partial t}=\frac{1}{2} \int_{\Omega} \int_{v} \int_{v_{1}}\left[2+\ln \left(f f_{1}\right)\right]\left(f^{\prime} f_{1}^{\prime}-f f_{1}\right) g \sigma(\Omega) d \Omega d^{3} v_{1} d^{3} v
$$

In this equation we can replace the velocities before collision, $\mathbf{v}$ and $\mathbf{v}_{1}$, by the velocities after collision, $\mathbf{v}^{\prime}$ and $\mathbf{v}_{1}^{\prime}$, respectively, without altering the value of the integral, since for each direct collision there exists an inverse collision with the same differential cross section $\sigma(\Omega)$. We have already seen that $d^{3} v^{\prime} d^{3} v_{1}^{\prime}=d^{3} v d^{3} v_{1}$ and $g=g^{\prime}$. Consequently, (3.10) may be written as

$$
\frac{\partial H}{\partial t}=\frac{1}{2} \int_{\Omega} \int_{v} \int_{v_{1}}\left[2+\ln \left(f^{\prime} f_{1}^{\prime}\right)\right]\left(f f_{1}-f^{\prime} f_{1}^{\prime}\right) g \sigma(\Omega) d \Omega d^{3} v_{1} d^{3} v
$$

We now combine (3.10) and (3.11) to obtain

$$
\frac{\partial H}{\partial t}=\frac{1}{4} \int_{\Omega} \int_{v} \int_{v_{1}}\left[\ln \left(\frac{f f_{1}}{f^{\prime} f_{1}^{\prime}}\right)\right]\left(f^{\prime} f_{1}^{\prime}-f f_{1}\right) g \sigma(\Omega) d \Omega d^{3} v_{1} d^{3} v
$$

In this expression it is clear that if $f^{\prime} f_{1}^{\prime}>f f_{1}$ then $\ln \left(f f_{1} / f^{\prime} f_{1}^{\prime}\right)<0$ and, consequently, $(\partial H / \partial t)<0$, since all other factors appearing in the right-hand side of (3.12) are positive. On the other hand, if $f^{\prime} f_{1}^{\prime}<f f_{1}$ 
then $\ln \left(f f_{1} / f^{\prime} f_{1}^{\prime}\right)>0$ and, again, $(\partial H / \partial t)<0$. When $f^{\prime} f_{1}^{\prime}=f f_{1}$, both factors are zero and $(\partial H / \partial t)=0$, which corresponds to the equilibrium state.

This result proves the $H$ theorem, showing that when $f$ satisfies the Boltzmann equation, the functional $H(t)$ always decreases monotonically in time until it reaches a limiting value, which occurs when there is no further change with time in the system. This limiting value is reached only when

$$
f^{\prime} f_{1}^{\prime}=f f_{1}
$$

so that this condition is necessary for $(\partial H / \partial t)=0$ and, consequently, it is also a necessary condition for the equilibrium state. According to the Boltzmann equation (3.5), the equilibrium distribution function satisfies the following integral equation,

$$
\int_{\Omega} \int_{v_{1}}\left[f\left(\mathbf{v}^{\prime}\right) f\left(\mathbf{v}_{1}^{\prime}\right)-f(\mathbf{v}) f\left(\mathbf{v}_{1}\right)\right] g \sigma(\Omega) d \Omega d^{3} v_{1}=0
$$

so that the condition (3.13) is also a sufficient condition for the equilibrium state.

It is instructive to note that (3.13) can be considered as an example of the general principle of detailed balance of statistical mechanics, as discussed in section 1 of Chapter 7, where it was used to derive the MaxwellBoltzmann equilibrium distribution function. An important conclusion that can be drawn from (3.13) is that the equilibrium distribution function is independent of the differential collision cross section $\sigma(\Omega)$, considered to be nonzero. The Maxwell-Boltzmann distribution function, therefore, is the only distribution for the equilibrium state that can exist in a uniform gas in the absence of external forces.

\subsection{Analysis of Boltzmann's H Theorem}

According to (3.3), the $\mathrm{H}$ theorem states that the entropy of a given isolated system always increases with time until it reaches the equilibrium state. Although this irreversible behavior is compatible with the laws of thermodynamics, it is nevertheless in disagreement with the laws of mechanics, which are reversible. If, at a given instant of time, the velocities of all the particles in a system were reversed, the laws of mechanics predict that each particle would describe, in the opposite sense, its previous trajectory. However, we have seen that the Boltzmann collision term leads to a irreversible temporal evolution of the distribution function and of the 
function $H(t)$. The existence of this paradox has its origin in the molecular chaos assumption that was used in the derivation of the Boltzmann collision term.

Recall that the molecular chaos assumption considers that, if $f(\mathbf{r}, \mathbf{v}, t)$ is proportional to the probability of finding in a given volume element $d^{3} r$, about $\mathbf{r}$, a particle with velocity $\mathbf{v}$, at the instant $t$, then the joint probability of simultaneously finding in the same volume element $d^{3} r$, about $\mathbf{r}$, a particle with velocity $\mathbf{v}$ and another particle with velocity $\mathbf{v}_{1}$, at the instant $\mathrm{t}$, is proportional to the product $f(\mathbf{r}, \mathbf{v}, t) f\left(\mathbf{r}, \mathbf{v}_{1}, t\right)$. Thus, it neglects any possible correlation that may exist between the particle velocities. Generally, the state of the gas may or may not satisfy the molecular chaos assumption and consequently the distribution function describing the gas may or may not satisfy the Boltzmann equation. The distribution function, which characterizes the state of the gas, will obey the Boltzmann equation only at the instants of time when the molecular chaos assumption holds true for the gas. The $\mathrm{H}$ theorem, therefore, is also valid only when this condition is satisfied.

We shall show now that at the instants of time when the state of the gas satisfies the molecular chaos assumption, the function $H(t)$ is at a local maximum. For this purpose consider a gas not in equilibrium, which is in the state of molecular chaos at the instant $t=t_{0}$. The $\mathrm{H}$ theorem implies that at the instant $t_{0}+d t$ we have $(\partial H / \partial t) \leq 0$. Consider a second gas that at the instant $t=t_{0}$ is exactly identical to the first one, except that the velocities of all the particles have directions opposite to the velocities of the first one, has the same function $H(t)$ as the first one, and is in a state of molecular chaos at $t=t_{0}$. Consequently, at the instant $t_{0}+d t$ we must have $(\partial H / \partial t) \leq 0$, according to the $\mathrm{H}$ theorem. On the other hand, due to the invariance of the equations of motion under time reversal, the time evolution of the second gas corresponds to the past of the first. This means that for the first gas we must have

$$
\begin{array}{llll}
\frac{\partial H}{\partial t} \leq 0 & \text { at } & t & =t_{0}+d t \\
\frac{\partial H}{\partial t} \geq 0 & \text { at } & t & =t_{0}-d t
\end{array}
$$

which shows that, at the instant when the condition of molecular chaos is satisfied, the function $H(t)$ is at a local maximum. This situation is illustrated in Fig. 2 at the instant $t=t_{0}$ indicated by the number (2). At the instants when $H(t)$ does not present a local maximum, as for example at the instants indicated by the numbers (1) and (3) in Fig. 2, the gas is 


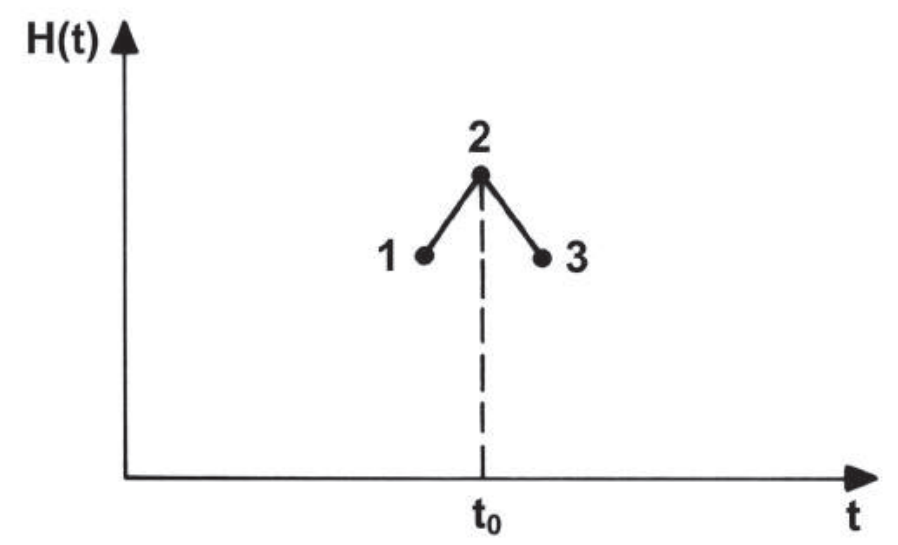

Fig. 2 When the gas satisfies the molecular chaos condition, the function $H(t)$ is at a local maximum, indicated here by the point denoted (2).

not in a state of molecular chaos. Note that $(\partial H / \partial t)$ need not necessarily be a continuous function of time and may change abruptly as a result of collisions.

The time evolution of $H(t)$ is governed by the collisional interactions between the particles, which occur at random and which can establish as well as destroy the state of molecular chaos as time passes. Fig. 3 illustrates how $H(t)$ may vary in time. Some of the instants when the condition of molecular chaos is satisfied are indicated by dots in the curve of $H(t)$. If the condition of molecular chaos prevails during most of the time, as in a dilute gas for example, $H(t)$ will be at a local maximum most of the time. Due to the random characteristic of the sequence of collisions, these instants of molecular chaos will probably be distributed in time in an almost uniform way. On the other hand, the time variation of $H(t)$, obtained using the distribution function that satisfies the Boltzmann equation, is represented by a smooth curve of negative slope which tries to fit, with a minimum deviation, all the points (instants) of the real curve of $H(t)$ in which the condition of molecular chaos is satisfied, as shown by the dashed line of Fig. 3. The state of molecular chaos, therefore, can be considered as a convenient mathematical model to describe a state not in equilibrium.

The Boltzmann equation, although strictly valid only at the instants 


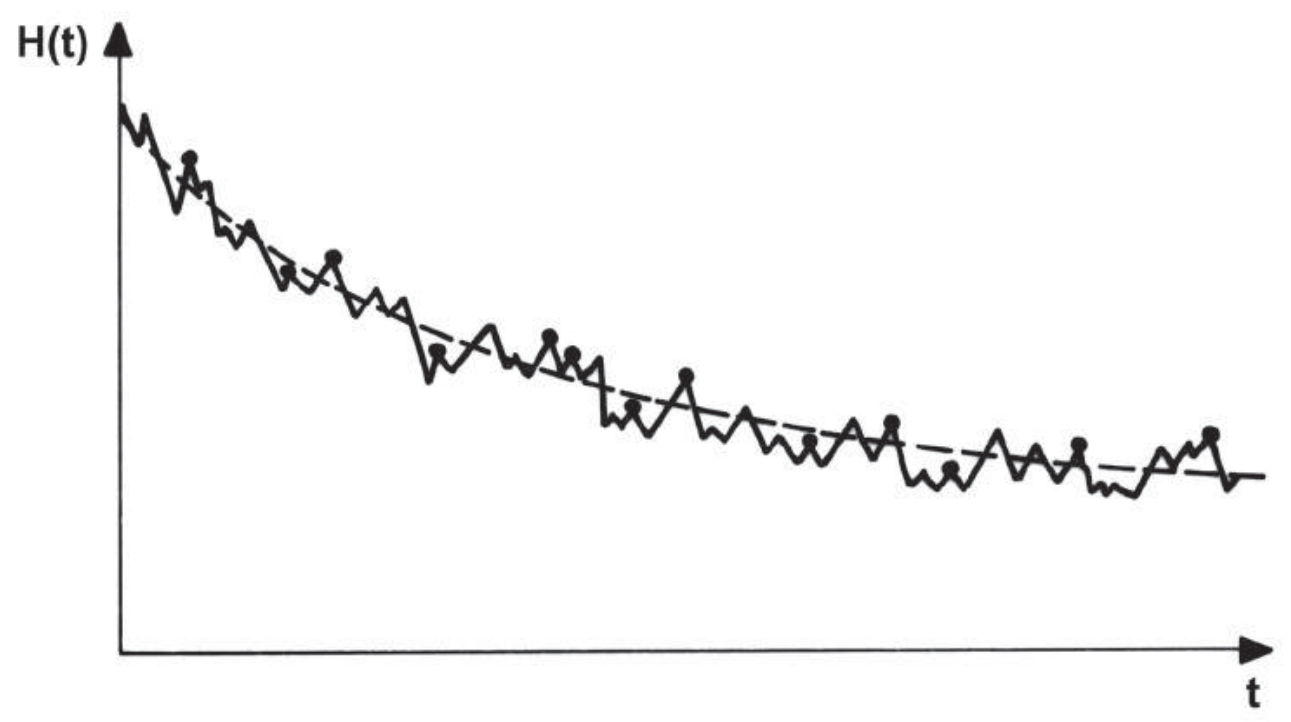

Fig. 3 The time evolution of $H(t)$ for a gas, initially not in an equilibrium state, is indicated by the solid curve. The dashed curve represents the time variation of $H(t)$ predicted by the Boltzmann equation. The dots indicate some of the instants when the condition of molecular chaos is satisfied.

when the gas is in the state of molecular chaos, nevertheless can be considered generally valid in a statistical sense at any instant, and the same applies for the $\mathrm{H}$ theorem.

\subsection{Maximum Entropy or Minimum H Approach for Deriving the Equilibrium Distribution Function}

The Maxwell-Boltzmann equilibrium distribution function can also be derived by performing a variational calculation on the function $H(t)$. We have seen that, under equilibrium conditions, $H(t)$ is a minimum, so that for a one-component uniform gas we must have, at equilibrium,

$$
\delta H=\delta\left[\int_{v} f \ln (f) d^{3} v\right]=0
$$

where the symbol $\delta$, before a given quantity, indicates a variation in this quantity as a result of a small change in the distribution function. Carrying 
out the variation indicated in (3.16) in a formal way, we have

$$
\delta H=\int_{v}(1+\ln f) \delta f d^{3} v=0
$$

There are, however, certain macroscopic constraints imposed on the system. When we vary $f$ slightly, we cannot violate the basic laws of conservation of mass, of momentum, and of energy for the system as a whole. Therefore, the variational integral (3.17) is subjected to the constraints that the total mass, momentum, and energy densities of the uniform gas remain constant under the variation in $f$. The constancy of the mass density, under a small change $\delta f$ in $f$, requires that

$$
\delta\left(\rho_{m}\right)=m \int_{v} \delta f d^{3} v=0
$$

Similarly, for the constancy of the momentum density,

$$
\delta\left(\rho_{m}<\mathbf{v}>\right)=m \int_{v} \mathbf{v} \delta f d^{3} v=0
$$

and for the energy density,

$$
\delta\left(\frac{1}{2} \rho_{m}<v^{2}>\right)=\frac{1}{2} m \int_{v} v^{2} \delta f d^{3} v=0
$$

We can now solve the variational integral in (3.17), subjected to the constraints expressed by (3.18), (3.19), and (3.20), using the method of the Langrange multipliers. Multiplying (3.18) by the Lagrange multiplier $a_{1}$, the $i^{\text {th }}$ component of (3.19) by the Lagrange multiplier $a_{2 i}$ (for $\left.i=x, y, z\right)$, (3.20) by the Lagrange multiplier $a_{3}$, and adding the resulting equations together with (3.17), yields

$$
m \int_{v}\left(1+\ln f+a_{1}+\mathbf{a}_{2} \cdot \mathbf{v}+\frac{1}{2} a_{3} v^{2}\right) \delta f d^{3} v=0
$$

where we have used the notation $\mathbf{a}_{2} \cdot \mathbf{v}=a_{2 x} v_{x}+a_{2 y} v_{y}+a_{2 z} v_{z}$. The variation in $f$ is now completely arbitrary, since all the constraints imposed on the system have been taken into account in (3.21). Thus, this integral can be equal to zero if and only if

$$
\ln f=-\left(1+a_{1}+\mathbf{a}_{2} \cdot \mathbf{v}+\frac{1}{2} a_{3} v^{2}\right)
$$


This equation is identical to (7.1.9), which we solved in Chapter 7 to obtain the Maxwell-Boltzmann distribution function. Hence, it leads in identical fashion to the equilibrium distribution function

$$
f=n\left(\frac{m}{2 \pi k T}\right)^{3 / 2} \exp \left(-\frac{m \mathbf{c}^{2}}{2 k T}\right)
$$

with $\mathbf{c}=\mathbf{v}-\mathbf{u}$.

The Maxwellian distribution function, besides being the equilibrium solution of the Boltzmann equation, is therefore also the most probable distribution consistent with the specified macroscopic parameters $n, \mathbf{u}$, and $T$ of the system.

\subsection{Mixture of Various Particle Species}

For the case of a mixture containing different species of particles, each species having a given number density $n_{\alpha}$, average velocity $\mathbf{u}_{\alpha}$, and temperature $T_{\alpha}$, we can still perform a variational calculation to determine the most probable distribution subjected to the constraints provided by the set of macroscopic parameters $n_{\alpha}, \mathbf{u}_{\alpha}$, and $T_{\alpha}$, for each species. Note that this is not an equilibrium situation unless the temperatures and mean velocities of all species are equal.

In order to determine the most probable distribution function for this nonequilibrium gas mixture (each species having its own number density, mean velocity, and temperature), we independently minimize each $H_{\alpha}$,

$$
H_{\alpha}=\int_{v} f_{\alpha} \ln f_{\alpha} d^{3} v
$$

This also minimizes $H$ for the mixture, since

$$
H=\sum_{\alpha} H_{\alpha}
$$

For the species of type $\alpha$, when $H_{\alpha}$ is at its minimum, we must have $\delta H_{\alpha}=0$ for a small variation $\delta f_{\alpha}$ in $f_{\alpha}$. The macroscopic parameters $n_{\alpha}, \mathbf{u}_{\alpha}$, and $T_{\alpha}$ must all remain fixed when $f_{\alpha}$ is varied. The problem is completely analogous to the one we solved in the previous subsection for a one-component gas and leads, in identical fashion, to equation (3.23) for each species. Therefore, each particle species has a Maxwellian distribution function, but with its own number density, mean velocity, and 
temperature. Although this it not an equilibrium situation for the whole gas (unless the mean velocities and temperatures of all species are the same), it is nevertheless the most probable distribution function for this system under the specified constraints.

\section{BOLTZMANN COLLISION TERM FOR A WEAKLY IONIZED PLASMA}

In this section we shall derive, from the Boltzmann equation, an approximate expression for the collision term for a weakly ionized plasma, in which the collisions between electrons and neutral particles play a dominant role. The distribution function for the neutral particles is assumed to be homogeneous and isotropic. The external force acting on the electrons is assumed to be small, so that the electrons are not very far from the equilibrium state. Consequently, the spatial inhomogeneity and the anisotropy of the nonequilibrium distribution function for the electrons are very small, since the nonequilibrium state is only slightly perturbed from the equilibrium state. Under equilibrium conditions the electrons are assumed to have no drift velocity and their distribution function is isotropic and homogeneous.

\subsection{Spherical Harmonic Expansion of the Distribution Function}

Let $(v, \theta, \phi)$ denote spherical coordinates in velocity space, as shown in Fig. 4. Since the anisotropy of the nonequilibrium distribution function is very small, the dependence of $f(\mathbf{r}, \mathbf{v}, t)$ on $\theta$ and $\phi$ is very small. Hence, it is appropriate to expand $f(\mathbf{r}, \mathbf{v}, t)$ in terms of the velocity space angular variables $\theta$ and $\phi$, and retain only the first few terms of this expansion. Since $\phi$ varies between 0 and $2 \pi$, we can expand $f(\mathbf{r}, \mathbf{v}, t)$ in a Fourier series in $\phi$. Furthermore, $\theta$ varies between 0 and $\pi$, and consequently $\cos \theta$ varies between +1 and -1 , which means that we can expand $f(\mathbf{r}, \mathbf{v}, t)$ in a series of Legendre polynomials in $\cos \theta$. Therefore, we can make a spherical harmonic expansion of the distribution function, as follows,

$$
\begin{gathered}
f(\mathbf{r . v}, t)=\sum_{m=0}^{\infty} \sum_{n=0}^{\infty} P_{n}^{m}(\cos \theta) \\
{\left[f_{m n}(\mathbf{r}, v, t) \cos (m \phi)+g_{m n}(\mathbf{r}, v, t) \sin (m \phi)\right]}
\end{gathered}
$$




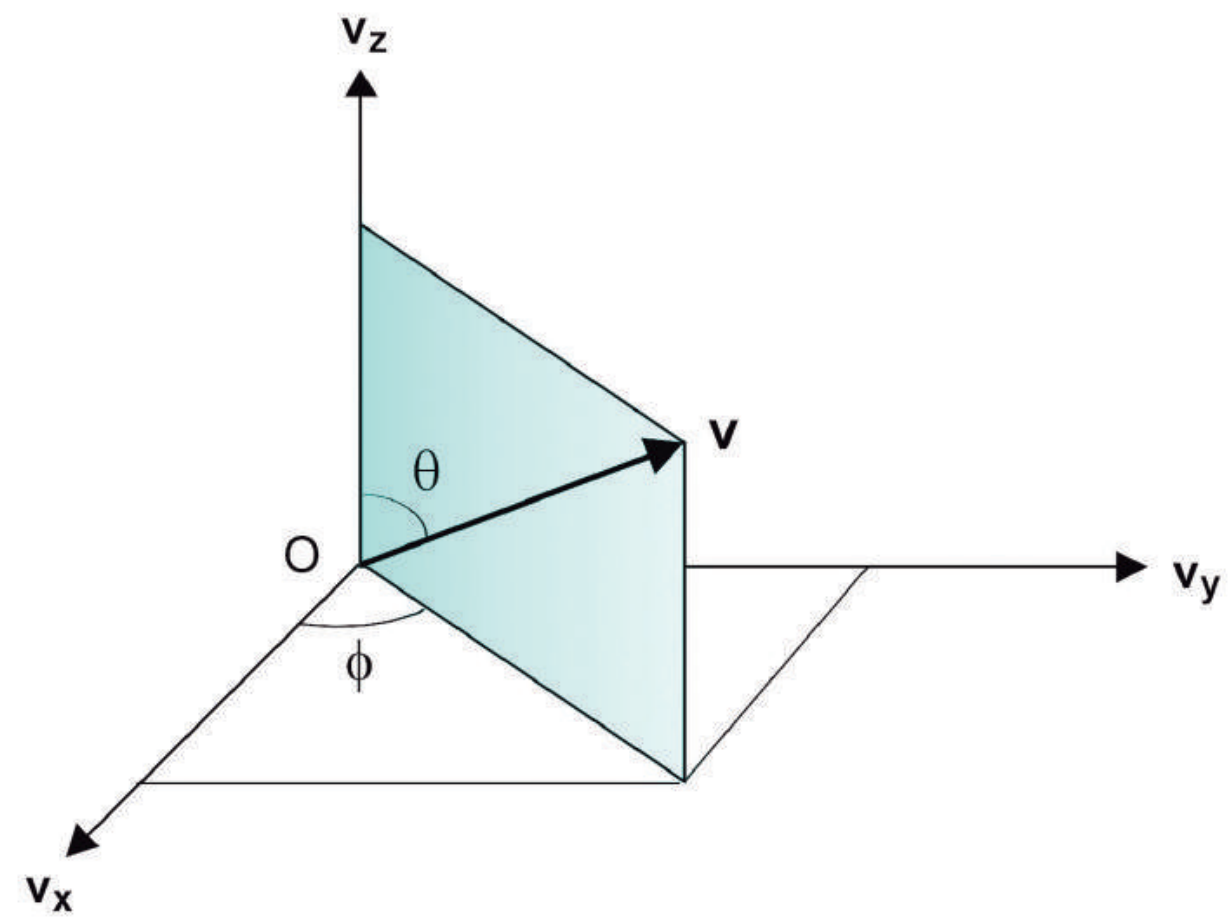

Fig. 4 Spherical coordinates $(v, \theta, \phi)$ in velocity space.

where the functions $P_{n}^{m}(\cos \theta)$ represent the associated Legendre polynomials and the functions $f_{m n}$ and $g_{m n}$ can be considered as coefficients of the expansion.

The first term in the expansion (4.1) corresponds to $m=0$ and $n=0$, and since $P_{0}^{0}(\cos \theta)=1$, it follows that it is given by $f_{00}(\mathbf{r}, v, t)$. This leading term is the isotropic distribution function corresponding to the equilibrium state of the electrons. The term corresponding to $m=1$ and $n=0$ vanishes, since $P_{0}^{1}(\cos \theta)=0$. The next highest order term in (4.1) corresponds to $m=0$ and $n=1$, and since $P_{1}^{0}(\cos \theta)=\cos \theta$, it is given by $f_{01}(\mathbf{r}, v, t) \cos \theta$. Therefore, retaining only the first two nonzero terms of the spherical harmonic expansion (4.1), in view of the fact that the anisotropy is assumed to be small, we obtain

$$
f(\mathbf{r}, \mathbf{v}, t)=f_{00}(\mathbf{r}, v, t)+\frac{\mathbf{v} \cdot \widehat{\mathbf{v}}_{z}}{v} f_{01}(\mathbf{r}, v, t)
$$

where we have replaced $\cos \theta$ by $\left(\mathbf{v} \cdot \widehat{\mathbf{v}}_{z}\right) / v$ (see Fig. 4). The second term in the right-hand side of (4.2) corresponds to the small anisotropy due to the spatial inhomogeneity and the external forces on the electrons. 


\subsection{Approximate Expression for the Boltzmann Collision Term}

The Boltzmann collision integral, given in (2.12), can be written for the case of binary electron-neutral collisions as

$$
\left(\frac{\delta f_{e}}{\delta t}\right)_{\text {coll }}=\int_{b} \int_{\epsilon} \int_{v_{1}}\left(f_{e}^{\prime} f_{n 1}^{\prime}-f_{e} f_{n 1}\right) g b d b d \epsilon d^{3} v_{1}
$$

where we have replaced $\sigma(\Omega) d \Omega$ by $b d b d \epsilon$. Here $f_{e}$ represents the nonequilibrium distribution function for the electrons and $f_{n}$ is the isotropic equilibrium function for the neutral particles.

In a first approximation we may assume the neutral particles to be stationary and not affected by collisions with the electrons, since the mass of a neutral particle is much larger than that of an electron. Hence, we assume that

$$
\begin{gathered}
\mathbf{v}_{1}=\mathbf{v}_{1}^{\prime}=0 \\
f_{n 1}=f_{n 1}^{\prime}
\end{gathered}
$$

Therefore, (4.3) becomes

$$
\left(\frac{\delta f_{e}}{\delta t}\right)_{\text {coll }}=\int_{v_{1}} f_{n 1} d^{3} v_{1} \int_{0}^{2 \pi} d \epsilon \int_{0}^{\infty}\left(f_{e}^{\prime}-f_{e}\right) g b d b
$$

Since the number density of the neutral particles is given by

$$
n_{n}=\int_{v_{1}} f_{n 1} d^{3} v_{1}
$$

we can write (4.6) as

$$
\left(\frac{\delta f_{e}}{\delta t}\right)_{\text {coll }}=n_{n} \int_{0}^{2 \pi} d \epsilon \int_{0}^{\infty}\left(f_{e}^{\prime}-f_{e}\right) g b d b
$$

Further, from (4.2) the distribution function for the electrons, before collision, is given by

$$
f_{e}=f_{e}(\mathbf{r}, \mathbf{v}, t)=f_{00}(\mathbf{r}, v, t)+\frac{\mathbf{v} \cdot \widehat{\mathbf{v}}_{z}}{v} f_{01}(\mathbf{r}, v, t)
$$

and, after collision, by

$$
f_{e}^{\prime}=f_{e}\left(\mathbf{r}, \mathbf{v}^{\prime}, t\right)=f_{00}\left(\mathbf{r}, v^{\prime}, t\right)+\frac{\mathbf{v}^{\prime} \cdot \widehat{\mathbf{v}}_{z}}{v^{\prime}} f_{01}\left(\mathbf{r}, v^{\prime}, t\right)=
$$




$$
f_{00}(\mathbf{r}, v, t)+\frac{\mathbf{v}^{\prime} \cdot \widehat{\mathbf{v}}_{z}}{v} f_{01}(\mathbf{r}, v, t)
$$

In this last equation we have considered $v^{\prime}=v$, in view of the fact that the electrons do not lose energy on collisions, since the neutrals are much more massive and considered at rest in a first approximation. This means that $\mathbf{v}=\mathbf{g}$ and $\mathbf{v}^{\prime}=\mathbf{g}^{\prime}$ [see equation (4.4)], and since $g=g^{\prime}$ [see equation (20.2.16) (Eq. 2.16 in Chapter 20)] we have $v=v^{\prime}$. Note, however, that $\mathbf{v} \neq \mathbf{v}^{\prime}$. Therefore, from (4.9) and (4.10), we have

$$
f_{e}^{\prime}-f_{e}=\frac{\left(\mathbf{v}^{\prime}-\mathbf{v}\right) \cdot \widehat{\mathbf{v}}_{z}}{v} f_{01}(\mathbf{r}, v, t)
$$

Without any loss of generality we can choose the $v_{z}$ axis as being parallel to the initial relative velocity $\mathbf{g}$ of the electron. Therefore,

$$
\left(\mathbf{v}^{\prime}-\mathbf{v}\right) \cdot \widehat{\mathbf{v}}_{z}=\left(\mathbf{g}^{\prime}-\mathbf{g}\right) \cdot \widehat{\mathbf{v}}_{z}=g(\cos \chi-1)=v(\cos \chi-1)
$$

where $\chi$ denotes the scattering angle (the angle between $\mathbf{g}$ and $\mathbf{g}^{\prime}$, as indicated in Fig. 3 of Chapter 20). Substituting (4.12) into (4.11), we obtain

$$
f_{e}^{\prime}-f_{e}=-(1-\cos \chi) f_{01}(\mathbf{r}, v, t)
$$

Plugging this result into (4.8), yields

$$
\left(\frac{\delta f_{e}}{\delta t}\right)_{\text {coll }}=-n_{n} g f_{01}(\mathbf{r}, v, t) \int_{0}^{2 \pi} d \epsilon \int_{0}^{\infty}(1-\cos \chi) b d b
$$

Since the momentum transfer cross section $\sigma_{m}$ for collisions between electrons and neutral particles is defined by [see (20.5.10)]

$$
\sigma_{m}=\int_{\Omega}(1-\cos \chi) \sigma(\Omega) d \Omega=\int_{0}^{2 \pi} d \epsilon \int_{0}^{\infty}(1-\cos \chi) b d b
$$

we can write (4.14) as

$$
\left(\frac{\delta f_{e}}{\delta t}\right)_{\text {coll }}=-n_{n} g \sigma_{m} f_{01}(\mathbf{r}, v, t)
$$

If we substitute $f_{01}(\mathbf{r}, v, t)$ in (4.16), using (4.9), and noting that for this case $\left(\mathbf{v} \cdot \widehat{\mathbf{v}}_{z}\right) / v=\left(\mathbf{g} \cdot \widehat{\mathbf{v}}_{z}\right) / g=1$, we obtain

$$
\left(\frac{\delta f_{e}}{\delta t}\right)_{\text {coll }}=-n_{n} v \sigma_{m}\left(f_{e}-f_{e 0}\right)=-\nu_{r}(v)\left(f_{e}-f_{e 0}\right)
$$


where we have introduced the velocity-dependent relaxation collision frequency $\nu_{r}(v)=n_{n} v \sigma_{m}$ and where $f_{00}$ (which characterizes the isotropic equilibrium state of the electrons) has been replaced by $f_{e 0}$, in accordance with the notation used previously. Expression (4.17) is similar to the relaxation model (or Krook model) for the collision term introduced in section 6 of Chapter 5, except for the velocity-dependent collision frequency. Once the interaction force between the electrons and the neutral particles has been specified, the momentum transfer cross section $\sigma_{m}$ and, consequently, the relaxation collision frequency $\nu_{r}(v)$ can be determined as functions of velocity.

\subsection{Rate of Change of Momentum Due to Collisions}

The time rate of change of momentum per unit volume of the electron gas, due to collisions with neutral particles, is given, from (8.4.3), by

$$
\mathbf{A}_{e}=\left[\frac{\delta\left(\rho_{m e} \mathbf{u}_{e}\right)}{\delta t}\right]_{\text {coll }}=m_{e} \int_{v} \mathbf{v}\left(\frac{\delta f_{e}}{\delta t}\right)_{\text {coll }} d^{3} v
$$

Substituting (4.17) into (4.18), we obtain

$$
\mathbf{A}_{e}=-m_{e} \int_{v} \nu_{r}(v) \mathbf{v} f_{e} d^{3} v+m_{e} \int_{v} \nu_{r}(v) \mathbf{v} f_{e 0} d^{3} v
$$

If we assume that the relaxation collision frequency $\nu_{r}$ does not depend on velocity and if we consider that the electron gas has no drift velocity in the equilibrium state, that is,

$$
\mathbf{u}_{e 0}=\frac{1}{n_{e}} \int_{v} \mathbf{v} f_{e 0} d^{3} v=0
$$

then (4.19) becomes

$$
\mathbf{A}_{e}=-n_{e} m_{e} \nu_{r} \mathbf{u}_{e}=-\rho_{m e} \nu_{r} \mathbf{u}_{e}
$$

where $\mathbf{u}_{e}$ is the average velocity of the electrons in the nonequilibrium state. Equation (4.21) corresponds to the expression used in the Langevin equation for the time rate of change of momentum per unit volume, as a result of collisions, in which a constant collisions frequency $\nu_{c}$ was introduced phenomenologically. 


\section{THE FOKKER-PLANCK EQUATION}

In this section we shall present a derivation of the Fokker-Planck equation, in which the collision term takes into account the simultaneous Coulomb interactions between the charged particles. For this purpose we assume that the large-angle deflection of a charged particle, in a multiple Coulomb interaction, can be considered as a series of consecutive weak binary collisions (or grazing collisions), that is, as a succession of small-angle scatterings. Therefore, the Fokker-Planck collision term can be derived directly from the Boltzmann collision integral, which is valid for binary collisions, under the assumption that a series of consecutive weak (small-angle deflection) binary collisions is a valid representation for the multiple Coulomb interaction. In the derivation that follows, we will consider collisions between species of particles represented by the indices $\alpha$ and $\beta$.

\subsection{Derivation of the Fokker-Planck Collision Term}

If $\chi(\mathbf{v})$ is some arbitrary function of velocity associated with the particles of type $\alpha$, then, according to (2.27) and (2.29), the time rate of change of the quantity $\chi(\mathbf{v})$ per unit volume, as a result of collision between particles of type $\alpha$ and those of type $\beta$, can be expressed as

$$
\begin{gathered}
\int_{v} \chi(\mathbf{v})\left(\frac{\delta f_{\alpha}}{\delta t}\right)_{c o l l} d^{3} v=\int_{\Omega} \int_{v_{1}} \int_{v}\left(f_{\alpha}^{\prime} f_{\beta 1}^{\prime}-f_{\alpha} f_{\beta 1}\right) \chi g \sigma(\Omega) d \Omega d^{3} v_{1} d^{3} v= \\
\int_{\Omega} \int_{v_{1}} \int_{v} f_{\alpha} f_{\beta 1}\left(\chi^{\prime}-\chi\right) g \sigma(\Omega) d \Omega d^{3} v_{1} d^{3} v
\end{gathered}
$$

where $\chi^{\prime}$ denotes $\chi\left(\mathbf{v}^{\prime}\right)$. In this last expression only the quantity $\chi^{\prime}$ is a function of the after-collision velocity $\mathbf{v}^{\prime}$.

For weak binary collisions (or grazing collisions), we can write

$$
\mathbf{v}^{\prime}=\mathbf{v}+\Delta \mathbf{v}
$$

where the change $\Delta \mathbf{v}$, due to collision, is assumed to be small. Since

$$
\chi^{\prime}=\chi\left(\mathbf{v}^{\prime}\right)=\chi(\mathbf{v}+\Delta \mathbf{v})
$$

we can expand $\chi^{\prime}$ in a Taylor series about the velocity $\mathbf{v}$, as

$$
\chi(\mathbf{v}+\Delta \mathbf{v})=\chi(\mathbf{v})+\sum_{i} \frac{\partial \chi}{\partial v_{i}} \Delta v_{i}+\frac{1}{2} \sum_{i j} \frac{\partial^{2} \chi}{\partial v_{i} \partial v_{j}} \Delta v_{i} \Delta v_{j}+\ldots
$$


Substituting (5.4) into (5.1) we obtain

$$
\begin{gathered}
\int_{v} \chi\left(\frac{\delta f_{\alpha}}{\delta t}\right)_{\text {coll }} d^{3} v=\int_{\Omega} \int_{v_{1}} \int_{v} f_{\alpha} f_{\beta 1}\left(\sum_{i} \frac{\partial \chi}{\partial v_{i}} \Delta v_{i}+\right. \\
\left.\frac{1}{2} \sum_{i j} \frac{\partial^{2} \chi}{\partial v_{i} \partial v_{j}} \Delta v_{i} \Delta v_{j}\right) g \sigma(\Omega) d \Omega d^{3} v_{1} d^{3} v
\end{gathered}
$$

where the higher order terms have been neglected.

The next step is to factor out the arbitrary function $\chi(\mathbf{v})$ from (5.5). This can be accomplished by integrating the first group of integrals involving $\partial \chi / \partial v_{i}$ by parts once and the second group of integrals involving $\partial^{2} \chi /\left(\partial v_{i} \partial v_{j}\right)$ by parts twice. For example, for the $x$ component of the first group of integrals involving $\partial \chi / \partial v_{i}$, we have

$$
\begin{gathered}
\int_{\Omega} \int_{v_{1}} \int_{v} \frac{\partial \chi(\mathbf{v})}{\partial v_{x}} \Delta v_{x} f_{\alpha}(\mathbf{v}) f_{\beta}\left(\mathbf{v}_{1}\right) g \sigma(\Omega) d \Omega d^{3} v_{1} d^{3} v= \\
\int_{\Omega} \int_{v_{1}}\left[\int_{v} d v_{y} d v_{z} \frac{\partial \chi(\mathbf{v})}{\partial v_{x}} d v_{x}\left(v_{x}^{\prime}-v_{x}\right)\right. \\
\left.f_{\alpha}(\mathbf{v}) g \sigma(\Omega) d \Omega\right] f_{\beta}\left(\mathbf{v}_{1}\right) d^{3} v_{1}
\end{gathered}
$$

For the term within brackets we can take

$$
d V=\frac{\partial \chi(\mathbf{v})}{\partial v_{x}} d v_{x}
$$

and

$$
U=\left(v_{x}^{\prime}-v_{x}\right) f_{\alpha}(\mathbf{v}) g \sigma(\Omega) d \Omega
$$

and perform the integral over $v_{x}$ by parts to obtain

$$
\begin{gathered}
\int_{v_{x}} \frac{\partial \chi(\mathbf{v})}{\partial v_{x}} d v_{x}\left(v_{x}^{\prime}-v_{x}\right) f_{\alpha}(\mathbf{v}) g \sigma(\Omega) d \Omega= \\
-\int_{v_{x}} \chi(\mathbf{v}) \frac{\partial}{\partial v_{x}}\left[\left(v_{x}^{\prime}-v_{x}\right) f_{\alpha}(\mathbf{v}) g \sigma(\Omega) d \Omega\right] d v_{x}
\end{gathered}
$$

where the integrated term is equal to zero, since $f$ must vanish at $\pm \infty$. Therefore, we find for the integral in (5.6), 


$$
\begin{array}{r}
\int_{\Omega} \int_{v_{1}} \int_{v} \frac{\partial \chi(\mathbf{v})}{\partial v_{x}} \Delta v_{x} f_{\alpha}(\mathbf{v}) f_{\beta}\left(\mathbf{v}_{1}\right) g \sigma(\Omega) d \Omega d^{3} v_{1} d^{3} v= \\
-\int_{\Omega} \int_{v_{1}} \int_{v} \chi(\mathbf{v}) \frac{\partial}{\partial v_{x}}\left[\Delta v_{x} f_{\alpha}(\mathbf{v}) g \sigma(\Omega) d \Omega\right] f_{\beta}\left(\mathbf{v}_{1}\right) d^{3} v_{1} d^{3} v
\end{array}
$$

Performing the other integrals in (5.5) by parts, in a similar way, we obtain the following expression for the collision term:

$$
\begin{gathered}
\int_{v} \chi\left(\frac{\delta f_{\alpha}}{\delta t}\right)_{\text {coll }} d^{3} v= \\
-\int_{\Omega} \int_{v_{1}} \int_{v} \chi \sum_{i} \frac{\partial}{\partial v_{i}}\left[\Delta v_{i} f_{\alpha} g \sigma(\Omega) d \Omega\right] f_{\beta 1} d^{3} v_{1} d^{3} v+ \\
\int_{\Omega} \int_{v_{1}} \int_{v} \frac{1}{2} \chi \sum_{i j} \frac{\partial^{2}}{\partial v_{i} \partial v_{j}}\left[\Delta v_{i} \Delta v_{j} f_{\alpha} g \sigma(\Omega) d \Omega\right] f_{\beta 1} d^{3} v_{1} d^{3} v= \\
-\int_{v} \chi\left[\sum_{i} \frac{\partial}{\partial v_{i}}\left(f_{\alpha} \int_{\Omega} \int_{v_{1}} \Delta v_{i} g \sigma(\Omega) d \Omega f_{\beta 1} d^{3} v_{1}\right)\right] d^{3} v+ \\
\int_{v} \chi\left[\frac{1}{2} \sum_{i j} \frac{\partial^{2}}{\partial v_{i} \partial v_{j}}\left(f_{\alpha} \int_{\Omega} \int_{v_{1}} \Delta v_{i} \Delta v_{j} g \sigma(\Omega) d \Omega f_{\beta 1} d^{3} v_{1}\right)\right] d^{3} v
\end{gathered}
$$

We now define the quantities

$$
<\Delta v_{i}>_{a v}=\int_{\Omega} \int_{v_{1}} \Delta v_{i} g \sigma(\Omega) d \Omega f_{\beta 1} d^{3} v_{1}
$$

and

$$
<\Delta v_{i} \Delta v_{j}>_{a v}=\int_{\Omega} \int_{v_{1}} \Delta v_{i} \Delta v_{j} g \sigma(\Omega) d \Omega f_{\beta 1} d^{3} v_{1}
$$

which are modified averages over the scattering angle and the velocity distribution function of the scatterers. Using this notation, (5.11) becomes

$$
\int_{v} \chi\left(\frac{\delta f_{\alpha}}{\delta t}\right)_{c o l l} d^{3} v=-\int_{v} \chi\left[\sum_{i} \frac{\partial}{\partial v_{i}}\left(f_{\alpha}<\Delta v_{i}>_{a v}\right)+\right.
$$




$$
\left.\frac{1}{2} \sum_{i j} \frac{\partial^{2}}{\partial v_{i} \partial v_{j}}\left(f_{\alpha}<\Delta v_{i} \Delta v_{j}>_{a v}\right)\right] d^{3} v
$$

Since this equation holds for any arbitrary function of velocity $\chi(\mathbf{v})$, it follows that for $\chi=1$ we must have

$$
\begin{gathered}
\left(\frac{\delta f_{\alpha}}{\delta t}\right)_{\text {coll }}=-\sum_{i} \frac{\partial}{\partial v_{i}}\left(f_{\alpha}<\Delta v_{i}>_{a v}\right)+ \\
\frac{1}{2} \sum_{i j} \frac{\partial^{2}}{\partial v_{i} \partial v_{j}}\left(f_{\alpha}<\Delta v_{i} \Delta v_{j}>_{a v}\right)
\end{gathered}
$$

This is the collision term of the Fokker-Planck equation. The average quantitites $\left\langle\Delta v_{i}>_{a v}\right.$ and $\left\langle\Delta v_{i} \Delta v_{j}>_{a v}\right.$ are known as the FokkerPlanck coefficients of dynamic friction and of diffusion in velocity space, respectively. They give the mean rate at which $\Delta v_{i}$ and $\Delta v_{i} \Delta v_{j}$ are changed due to many consecutive weak coulomb collisions.

Note that the Fokker-Planck collision term (5.15) includes two terms having opposite sign, which may result in no net change in $f_{\alpha}$ as a result of collisions. A dimensional analysis of (5.12) shows that the FokkerPlanck coefficient $\left\langle\Delta v_{i}\right\rangle_{a v}$ has dimensions of force per unit mass and tends to accelerate or decelerate the particles until they reach the average equilibrium velocity. This process is usually called dynamic friction. On the other hand, the Fokker-Planck coefficient $\left\langle\Delta v_{i} \Delta v_{j}\right\rangle_{a v}$ represents diffusion in velocity space, until equilibrium is reached. Under equilibrium conditions, diffusion in velocity space is balanced by dynamic friction and there is no net change in $f_{\alpha}$ as a result of collisions, so that the collision term in (5.15) vanishes. Fig. 5 illustrates schematically the effect of these processes on the distribution function.

In principle, the expansion procedure used to obtain the FokkerPlanck collision term can be extended to any number of terms. However, in practice only the first two terms of the expansion shown in (5.15) are ever used, so that (5.15) can be considered as a reasonable approximation to the collision term when $\Delta \mathbf{v}=\left(\mathbf{v}^{\prime}-\mathbf{v}\right)$ is small for most collisions. This is generally considered to be the case for the long-range coulomb force. Recall that, in section 8 of Chapter 20, we have shown that the large number of particles interacting mildly with the target particle (resulting in small-angle deflections) are much more important than the small number of particles interacting strongly with the target particle (resulting in large-angle deflections) and have a dominant contribution to the total scattering cross section. 


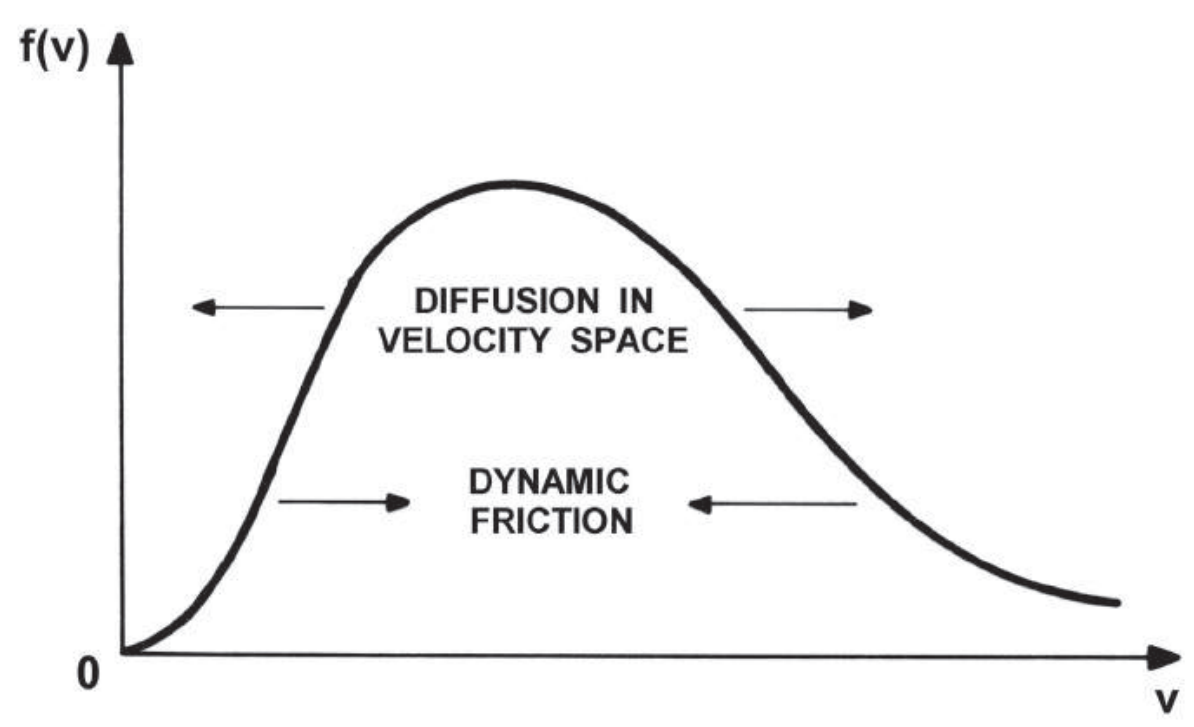

Fig. 5 Schematic illustration of the processes of dynamic friction and of diffusion in velocity space, associated with the Fokker-Planck coefficients.

\subsection{The Fokker-Planck Coefficients for Coulomb Interactions}

We shall evaluate now the coefficients of dynamic friction $\left\langle\Delta v_{i}\right\rangle_{a v}$ and of diffusion in velocity space $\left\langle\Delta v_{i} \Delta v_{j}>_{a v}\right.$, which appear in the Fokker-Planck collision term (5.15). It is convenient to perform first the integral over the solid angle $\Omega$, since it does not require knowledge of the distribution function $f_{\beta}\left(\mathbf{v}_{1}\right)$. For this purpose let us write

$$
<\Delta v_{i}>_{a v}=\int_{v_{1}}\left\{\Delta v_{i}\right\} f_{\beta 1} d^{3} v_{1}
$$

and

$$
<\Delta v_{i} \Delta v_{j}>_{a v}=\int_{v_{1}}\left\{\Delta v_{i} \Delta v_{j}\right\} f_{\beta 1} d^{3} v_{1}
$$

where the curly bracket notation has been introduced to represent the following integrals over solid angle:

$$
\left\{\Delta v_{i}\right\}=\int_{\Omega} \Delta v_{i} g \sigma(\Omega) d \Omega
$$


and

$$
\left\{\Delta v_{i} \Delta v_{j}\right\}=\int_{\Omega} \Delta v_{i} \Delta v_{j} g \sigma(\Omega) d \Omega
$$

In order to calculate these integrals over the solid angle, recall first that in the center of mass coordinate system we have, from (20.2.5) and (20.2.8),

$$
\begin{aligned}
\mathbf{v} & =\mathbf{c}_{0}-\frac{m_{\beta}}{\left(m_{\alpha}+m_{\beta}\right)} \mathbf{g} \\
\mathbf{v}^{\prime} & =\mathbf{c}_{0}-\frac{m_{\beta}}{\left(m_{\alpha}+m_{\beta}\right)} \mathbf{g}^{\prime}
\end{aligned}
$$

so that

$$
\Delta \mathbf{v}=\mathbf{v}^{\prime}-\mathbf{v}=\frac{m_{\beta}}{\left(m_{\alpha}+m_{\beta}\right)}\left(\mathbf{g}-\mathbf{g}^{\prime}\right)
$$

In a Cartesian coordinate system in which the vector $\mathbf{g}$ is along the $z$ axis (as shown in Fig. 3 of Chapter 20), we have

$$
g_{z}=g \quad ; \quad g_{x}=g_{y}=0
$$

and

$$
\begin{gathered}
g_{x}^{\prime}=g \sin \chi \cos \epsilon \\
g_{y}^{\prime}=g \sin \chi \sin \epsilon \\
g_{z}^{\prime}=g \cos \chi
\end{gathered}
$$

Plugging equations (5.23) to (5.26) into (5.22) gives

$$
\Delta \mathbf{v}=\frac{m_{\beta}}{\left(m_{\alpha}+m_{\beta}\right)} g[(1-\cos \chi) \widehat{\mathbf{z}}-\sin \chi(\cos \epsilon \widehat{\mathbf{x}}+\sin \epsilon \widehat{\mathbf{y}})]
$$

The differential scattering cross section for the coulomb potential was calculated in section 7 of Chapter 20 and was found to be given by

$$
\sigma(\chi)=\frac{b_{0}^{2}}{4 \sin ^{4}(\chi / 2)}=\frac{b_{0}^{2}}{(1-\cos \chi)^{2}}
$$

where $b_{0}$ is defined in (20.4.9).

Proceeding in the evaluation of $\left\{\Delta v_{i}\right\}$ for $i=x, y, z$, let us first calculate $\left\{\Delta v_{z}\right\}$. From (5.18), (5.27), and (5.28), we have

$$
\left\{\Delta v_{z}\right\}=\frac{m_{\beta}}{\left(m_{\alpha}+m_{\beta}\right)} g^{2} b_{0}^{2} \int_{0}^{2 \pi} d \epsilon \int_{\chi_{m i n}}^{\pi} \frac{\sin \chi}{(1-\cos \chi)} d \chi
$$


where the lower limit of the integral in $\chi$ was taken to be $\chi_{\min }$, in order to avoid the divergence of the integral that would result if we take zero as the lower limit. As we have seen, the charged particles in the plasma that are separated by distances greater than the Debye length $\lambda_{D}$ are effectively shielded from one another. Therefore, in order to avoid an infinite result for the integral in (5.29), we take the lower limit $\chi_{\min }$ to be the value of the scattering angle corresponding to an impact parameter $b$ equal to $\lambda_{D}$.

With reference to (20.4.13) let us introduce the new variable

$$
u=\frac{b}{b_{0}}=\cot \left(\frac{1}{2} \chi\right)
$$

from which we obtain

$$
d u=-\frac{d \chi}{(1-\cos \chi)}
$$

and

$$
\sin \chi=\frac{2 u}{\left(1+u^{2}\right)}
$$

With this change of variable and introducing the cut-off value for the impact paramter at $\lambda_{D}$, that is, at

$$
u_{c}=\frac{\lambda_{D}}{b_{0}}=\Lambda
$$

we obtain for (5.29),

$$
\begin{gathered}
\left\{\Delta v_{z}\right\}=2 \pi \frac{m_{\beta}}{\left(m_{\alpha}+m_{\beta}\right)} g^{2} b_{0}^{2} \int_{\Lambda}^{0} \frac{2 u}{\left(1+u^{2}\right)}(-d u)= \\
2 \pi \frac{m_{\beta}}{\left(m_{\alpha}+m_{\beta}\right)} g^{2} b_{0}^{2} \ln \left(1+\Lambda^{2}\right)
\end{gathered}
$$

In general $\Lambda \gg 1$, so that $\ln \left(1+\Lambda^{2}\right) \simeq 2 \ln \Lambda$ and (5.34) simplifies to

$$
\left\{\Delta v_{z}\right\}=\frac{m_{\beta}}{\left(m_{\alpha}+m_{\beta}\right)} \frac{Z^{2} e^{4} \ln \Lambda}{4 \pi \epsilon_{0}^{2} \mu^{2} g^{2}}
$$

where we have substituted $b_{0}$ by the expression given in (20.4.9). Introducing the notation

$$
\Theta=\frac{Z^{2} e^{4} \ln \Lambda}{4 \pi \epsilon_{0}^{2} \mu^{2}}
$$


we can write $(5.35)$ as

$$
\left\{\Delta v_{z}\right\}=\frac{m_{\beta}}{\left(m_{\alpha}+m_{\beta}\right)} \frac{\Theta}{g^{2}}
$$

Next let us consider the quantities $\left\{\Delta v_{x}\right\}$ and $\left\{\Delta v_{y}\right\}$. From (5.18) and (5.27) we see that these quantities involve integrals of either $\cos \epsilon$ or $\sin \epsilon$ from 0 to $2 \pi$, which are clearly equal to zero. Therefore,

$$
\left\{\Delta v_{x}\right\}=\left\{\Delta v_{y}\right\}=0
$$

In a similar way it can be shown, from (5.19) and (5.27), that

$$
\left\{\Delta v_{i} \Delta v_{j}\right\}=0 \quad \text { for } \quad i \neq j
$$

since the integrals over $\epsilon$ from 0 to $2 \pi$ vanish.

To evaluate $\left\{\Delta v_{z} \Delta v_{z}\right\}=\left\{\Delta v_{z}^{2}\right\}$, we use (5.19), (5.27), and (5.28), which yield

$$
\left\{\Delta v_{z}^{2}\right\}=2 \pi \frac{m_{\beta}^{2}}{\left(m_{\alpha}+m_{\beta}\right)^{2}} g^{3} b_{0}^{2} \int_{\chi_{m i n}}^{\pi} \sin \chi d \chi
$$

Changing variables according to (5.30), we obtain

$$
\begin{gathered}
\left\{\Delta v_{z}^{2}\right\}=2 \pi \frac{m_{\beta}^{2}}{\left(m_{\alpha}+m_{\beta}\right)^{2}} g^{3} b_{0}^{2} \int_{0}^{\Lambda} \frac{4 u}{\left(1+u^{2}\right)^{2}} d u= \\
4 \pi \frac{m_{\beta}^{2}}{\left(m_{\alpha}+m_{\beta}\right)^{2}} g^{3} b_{0}^{2} \frac{\Lambda^{2}}{\left(1+\Lambda^{2}\right)}
\end{gathered}
$$

Since $\Lambda \gg 1,(5.41)$ simplifies to

$$
\left\{\Delta v_{z}^{2}\right\}=4 \pi \frac{m_{\beta}^{2}}{\left(m_{\alpha}+m_{\beta}\right)^{2}} g^{3} b_{0}^{2}=\frac{m_{\beta}^{2}}{\left(m_{\alpha}+m_{\beta}\right)^{2}} \frac{Z^{2} e^{4}}{4 \pi \epsilon_{0}^{2} \mu^{2} g}
$$

or, using the notation introduced in (5.36),

$$
\left\{\Delta v_{z}^{2}\right\}=\frac{m_{\beta}^{2}}{\left(m_{\alpha}+m_{\beta}\right)^{2}} \frac{\Theta}{g \ln \Lambda}
$$


In a similar way, we can calculate $\left\{\Delta v_{x}^{2}\right\}$ and $\left\{\Delta v_{y}^{2}\right\}$ from (5.19), (5.27), and (5.28), which give

$$
\begin{aligned}
& \left\{\Delta v_{x}^{2}\right\}=\frac{m_{\beta}^{2}}{\left(m_{\alpha}+m_{\beta}\right)^{2}} g^{3} b_{0}^{2} \int_{0}^{2 \pi} \cos ^{2} \epsilon d \epsilon \int_{\chi_{m i n}}^{\pi} \frac{\sin ^{3} \chi}{(1-\cos \chi)^{2}} d \chi \\
& \left\{\Delta v_{y}^{2}\right\}=\frac{m_{\beta}^{2}}{\left(m_{\alpha}+m_{\beta}\right)^{2}} g^{3} b_{0}^{2} \int_{0}^{2 \pi} \sin ^{2} \epsilon d \epsilon \int_{\chi_{m i n}}^{\pi} \frac{\sin ^{3} \chi}{(1-\cos \chi)^{2}} d \chi
\end{aligned}
$$

Therefore, evaluating the integral over $\epsilon$, we find

$$
\left\{\Delta v_{x}^{2}\right\}=\left\{\Delta v_{y}^{2}\right\}=\pi \frac{m_{\beta}^{2}}{\left(m_{\alpha}+m_{\beta}\right)^{2}} g^{3} b_{0}^{2} \int_{\chi_{\min }}^{\pi} \frac{\sin ^{3} \chi}{(1-\cos \chi)^{2}} d \chi
$$

If we change variables according to (5.30), we readily find

$$
\begin{gathered}
\left\{\Delta v_{x}^{2}\right\}=\left\{\Delta v_{y}^{2}\right\}=4 \pi \frac{m_{\beta}^{2}}{\left(m_{\alpha}+m_{\beta}\right)^{2}} g^{3} b_{0}^{2} \int_{0}^{\Lambda} \frac{u^{3}}{\left(1+u^{2}\right)^{2}} d u= \\
2 \pi \frac{m_{\beta}^{2}}{\left(m_{\alpha}+m_{\beta}\right)^{2}} g^{3} b_{0}^{2}\left[\ln \left(1+\Lambda^{2}\right)-\frac{\Lambda^{2}}{\left(1+\Lambda^{2}\right)}\right]
\end{gathered}
$$

Since $\Lambda \gg 1$ and replacing $b_{0}$ using (20.4.9), we obtain finally

$$
\left\{\Delta v_{x}^{2}\right\}=\left\{\Delta v_{y}^{2}\right\}=\frac{m_{\beta}^{2}}{\left(m_{\alpha}+m_{\beta}\right)^{2}} \frac{Z^{2} e^{4} \ln \Lambda}{4 \pi \epsilon_{0}^{2} \mu^{2} g}=\frac{m_{\beta}^{2}}{\left(m_{\alpha}+m_{\beta}\right)^{2}} \frac{\Theta}{g}
$$

The next step in the evaluation of the Fokker-Planck coefficients consists in integrating the expressions for $\left\{\Delta v_{i}\right\}$ and $\left\{\Delta v_{i} \Delta v_{j}\right\}$, for $i, j=x, y, z$, over the distribution function of the particles that constitute the scattering centers. Thus, using the results we have just calculated, we find

$$
\begin{gathered}
<\Delta v_{z}>_{a v}=\frac{m_{\beta}}{\left(m_{\alpha}+m_{\beta}\right)} \int_{v_{1}} \frac{\Theta}{g^{2}} f_{\beta 1} d^{3} v_{1} \\
<\Delta v_{z}^{2}>_{a v}=\frac{m_{\beta}^{2}}{\left(m_{\alpha}+m_{\beta}\right)^{2}} \int_{v_{1}} \frac{\Theta}{g \ln \Lambda} f_{\beta 1} d^{3} v_{1} \\
<\Delta v_{x}^{2}>_{a v}=<\Delta v_{y}^{2}>_{a v}=\frac{m_{\beta}^{2}}{\left(m_{\alpha}+m_{\beta}\right)^{2}} \int_{v_{1}} \frac{\Theta}{g} f_{\beta 1} d^{3} v_{1}
\end{gathered}
$$

All other coefficients vanish. 


\subsection{Application to Electron-Ion Collisions}

Let us calculate the Fokker-Planck coefficients for the case of electronion collisions. For simplicity we shall assume that the electron is colliding with a field of heavy stationary positive ions. This assumption is reasonable, since on the average the electron velocities are much larger than the ion velocities $\left(<v_{i}^{2}>=3 k T_{i} / m_{i}\right.$ while $<v_{e}^{2}>=3 k T_{e} / m_{e}$ and generally $\left.T_{e} / m_{e} \gg T_{i} / m_{i}\right)$. Thus, assuming that the positive ions are motionless, we can represent their velocity distribution function by the Dirac delta function

$$
f_{\beta 1}=n_{0} \delta\left(v_{1 x}\right) \delta\left(v_{1 y}\right) \delta\left(v_{1 z}\right)
$$

In addition, because $m_{i} \gg m_{e}$, we can take $\left(m_{e}+m_{i}\right) \simeq m_{i}$ and $\mu \simeq m_{e}$. Substituting (5.52) into equations (5.49) through (5.51), we obtain at once

$$
\begin{gathered}
<\Delta v_{z}>_{a v}=\frac{n_{0} \Theta}{g^{2}} \\
<\Delta v_{z}^{2}>_{a v}=\frac{n_{0} \Theta}{g \ln \Lambda} \\
<\Delta v_{x}^{2}>_{a v}=<\Delta v_{y}^{2}>_{a v}=\frac{n_{0} \Theta}{g}
\end{gathered}
$$

where $\Theta$ is defined according to (5.36). Note that $\mathbf{v}_{z}$ is along the direction of the initial relative velocities, whereas $\mathbf{v}_{x}$ and $\mathbf{v}_{y}$ are in the plane perpendicular to the initial relative velocities.

\section{PROBLEMS}

21.1 Consider a system consisting of a mixture of two types of particles having masses $m$ and $M$, and subjected to an external force $\mathbf{F}$. Denote the corresponding distribution functions by $f$ and $g$, respectively. Write down the set of coupled Boltzmann transport equations for the system.

21.2 Consider a plasma in which the electrons and the ions are characterized, respectively, by the following distribution functions:

$$
\begin{aligned}
& f_{e}=n_{0}\left(\frac{m_{e}}{2 \pi k T_{e}}\right)^{3 / 2} \exp \left[-\frac{m_{e}\left(\mathbf{v}-\mathbf{u}_{e}\right)^{2}}{2 k T_{e}}\right] \\
& f_{i}=n_{0}\left(\frac{m_{i}}{2 \pi k T_{i}}\right)^{3 / 2} \exp \left[-\frac{m_{i}\left(\mathbf{v}-\mathbf{u}_{i}\right)^{2}}{2 k T_{i}}\right]
\end{aligned}
$$

(a) Calculate the difference $\left(f_{e}^{\prime} f_{i 1}^{\prime}-f_{e} f_{i 1}\right)$. 
(b) Show that this plasma of electrons and ions will be in the equilibrium state, that is, the difference $\left(f_{e}^{\prime} f_{i 1}^{\prime}-f_{e} f_{i 1}\right)$ will vanish if and only if $\mathbf{u}_{e}=\mathbf{u}_{i}$ and $T_{e}=T_{i}$.

21.3 Use a Lagrange multiplier technique to show that for a system characterized by the following modified Maxwell-Boltzmann distribution function

$$
f(\mathbf{r}, v)=n(\mathbf{r})\left(\frac{m}{2 \pi k T}\right)^{3 / 2} \exp \left(-\frac{m v^{2}}{2 k T}\right)
$$

where $T$ is constant, the entropy $S$, defined by

$$
S=-k \int_{r} \int_{v} f \ln (f) d^{3} v d^{3} r
$$

is a maximum when the density $n$ is constant, independent of $\mathbf{r}$. Consider that the system has a total of $N$ particles in a fixed volume $V$ at a temperature $T$.

21.4 Consider the case of Maxwell molecules, for which the interparticle force is of the form

$$
\mathbf{F}(\mathbf{r})=\frac{K}{r^{5}} \widehat{\mathbf{r}}
$$

where $K$ is a constant.

(a) Without specifying the form of the distribution functions $f_{\alpha}(\mathbf{v})$ and $f_{\beta}\left(\mathbf{v}_{1}\right)$ for the particles of type $\alpha$ and $\beta$, show that the time rate of change of momentum for the particles of type $\alpha$ per unit volume, due to collisions, is given by

$$
\left(\frac{\delta \mathcal{P}_{\alpha}}{\delta t}\right)_{\text {coll }}=\int_{v} m_{\alpha} \mathbf{c}_{\alpha}\left(\frac{\delta f_{\alpha}}{\delta t}\right)_{\text {coll }} d^{3} v=\sum_{\beta} n_{\alpha} m_{\alpha} \nu_{\alpha \beta}\left(\mathbf{u}_{\beta}-\mathbf{u}_{\alpha}\right)
$$

where $\nu_{\alpha \beta}$ is the collision frequency for momentum transfer, given explicity by

$$
\nu_{\alpha \beta}=2 \pi \frac{(K \mu)^{1 / 2}}{m_{\alpha}} n_{\beta} A_{1}(5)
$$

where $A_{1}(5)$ is a dimensionless number (of order unity) defined by (with $p=5$ and $\ell=1$ )

$$
A_{\ell}(p)=\int_{0}^{\infty}\left(1-\cos ^{\ell} \chi\right) v_{0} d v_{0}
$$


with

$$
v_{0}=b\left(\frac{\mu g^{2}}{K}\right)^{1 /(p-1)}
$$

Also,

$$
\begin{aligned}
n_{\alpha}=\int_{v} f_{\alpha} d^{3} v & ; & \mathbf{u}_{\alpha} & =\frac{1}{n_{\alpha}} \int_{v} \mathbf{v} f_{\alpha} d^{3} v \\
n_{\beta}=\int_{v_{1}} f_{\beta 1} d^{3} v_{1} & ; & \mathbf{u}_{\beta} & =\frac{1}{n_{\beta}} \int_{v_{1}} \mathbf{v}_{1} f_{\beta 1} d^{3} v_{1}
\end{aligned}
$$

(b) For the same case, show that the time rate of change of energy for the particles of type $\alpha$ per unit volume, due to collisions, is given by

$$
\begin{gathered}
\left(\frac{\delta \mathcal{E}_{\alpha}}{\delta t}\right)_{\text {coll }}=\frac{1}{2} \int_{v} m_{\alpha} c_{\alpha}^{2}\left(\frac{\delta f_{\alpha}}{\delta t}\right)_{\text {coll }} d^{3} v= \\
\frac{n_{\alpha} m_{\alpha} \nu_{\alpha \beta}}{\left(m_{\alpha}+m_{\beta}\right)}\left[3 k\left(T_{\beta}-T_{\alpha}\right)+m_{\beta}\left(\mathbf{u}_{\beta}-\mathbf{u}_{\alpha}\right)^{2}\right]
\end{gathered}
$$

where

$$
\begin{gathered}
T_{\alpha}=\frac{m_{\alpha}}{3 k}<c_{\alpha}^{2}>=\frac{m_{\alpha}}{3 k n_{\alpha}} \int_{v} c_{\alpha}^{2} f_{\alpha} d^{3} v \\
T_{\beta}=\frac{m_{\beta}}{3 k}<c_{\beta}^{2}>=\frac{m_{\beta}}{3 k n_{\beta}} \int_{v_{1}} c_{\beta 1}^{2} f_{\beta 1} d^{3} v_{1}
\end{gathered}
$$

21.5 Consider a gas mixture of two types of particles $(\alpha=1,2)$, each one characterized by a Maxwellian distribution function

$$
f_{\alpha}(\mathbf{v})=n_{\alpha}\left(\frac{m_{\alpha}}{2 \pi k T_{\alpha}}\right)^{3 / 2} \exp \left(-\frac{m_{\alpha} v^{2}}{2 k T_{\alpha}}\right)
$$

with its own mass, density, and temperature.

(a) Make the following transformation of velocity variables,

$$
\begin{aligned}
& \mathbf{v}_{1}=\mathbf{v}_{c}^{\prime}+\bar{M}_{2} \mathbf{g} \\
& \mathbf{v}_{2}=\mathbf{v}_{c}^{\prime}-\bar{M}_{1} \mathbf{g}
\end{aligned}
$$

where $\mathbf{v}_{c}^{\prime}$ is a velocity similar to the center of mass velocity, $\mathbf{g}$ is the relative velocity between the two species $\left(\mathbf{g}=\mathbf{v}_{1}-\mathbf{v}_{2}\right)$, and

$$
\bar{M}_{1}=\frac{m_{1} / T_{1}}{m_{1} / T_{1}+m_{2} / T_{2}}
$$




$$
\bar{M}_{2}=\frac{m_{2} / T_{2}}{m_{1} / T_{1}+m_{2} / T_{2}}
$$

Show that the Jacobian of this transformation,

$$
J=\frac{\partial\left(\mathbf{v}_{c}^{\prime}, \mathbf{g}\right)}{\partial\left(\mathbf{v}_{1}, \mathbf{v}_{2}\right)}
$$

satisfies $|J|=1$, so that $d^{3} v_{c}^{\prime} d^{3} g=d^{3} v_{1} d^{3} v_{2}$.

(b) The relative speed between the two species, $g=\left|\mathbf{v}_{1}-\mathbf{v}_{2}\right|$, when averaged over both their velocity distribution functions, is given by

$$
<g>=\frac{1}{n_{1} n_{2}} \int_{v_{1}} \int_{v_{2}} g f_{1}\left(\mathbf{v}_{1}\right) f_{2}\left(\mathbf{v}_{2}\right) d^{3} v_{1} d^{3} v_{2}
$$

Transform the variables of integration $\mathbf{v}_{1}$ and $\mathbf{v}_{2}$ to $\mathbf{v}_{c}^{\prime}$ and $\mathbf{g}$, and perform the integrals over $\mathbf{v}_{c}^{\prime}$ and $\mathbf{g}$ to show that

$$
<g>=\left(\frac{8 k}{\pi}\right)^{1 / 2}\left(\frac{T_{1}}{m_{1}}+\frac{T_{2}}{m_{2}}\right)^{1 / 2}
$$

(c) If only one kind of particle is present, so that $m_{1}=m_{2}=m, T_{1}=$ $T_{2}=T$, and $n_{1}=n_{2}=n$, show that

$$
<g>=\sqrt{2}<v>=\left(\frac{8 k T}{\pi \mu}\right)^{1 / 2}
$$

where $\langle v\rangle=(8 k T / \pi m)^{1 / 2}$ is the average speed and $\mu=m / 2$ is the reduced mass. If the mutual scattering cross section is $\sigma$, show that the collision frequency in a homogeneous Maxwellian gas is given by

$$
\nu=n \sigma<g>=4 n \sigma\left(\frac{k T}{\pi m}\right)^{1 / 2}
$$

21.6 Consider the following expressions that define the Fokker-Planck coefficients of dynamic friction and of diffusion in velocity space:

$$
\begin{gathered}
<\Delta v_{i}>_{a v}=\int_{\Omega} \int_{v_{1}} \Delta v_{i} g \sigma(\Omega) d \Omega f_{\beta 1} d^{3} v_{1} \\
<\Delta v_{i} \Delta v_{j}>_{a v}=\int_{\Omega} \int_{v_{1}} \Delta v_{i} \Delta v_{j} g \sigma(\Omega) d \Omega f_{\beta 1} d^{3} v_{1}
\end{gathered}
$$


(a) With reference to Fig. 6, verify that

$$
\begin{aligned}
& \Delta v_{x}=-\frac{m_{\beta}}{\left(m_{\alpha}+m_{\beta}\right)} g \sin \chi \cos \epsilon \\
& \Delta v_{y}=-\frac{m_{\beta}}{\left(m_{\alpha}+m_{\beta}\right)} g \sin \chi \sin \epsilon \\
& \Delta v_{z}=\frac{m_{\beta}}{\left(m_{\alpha}+m_{\beta}\right)} g(1-\cos \chi)
\end{aligned}
$$

For a general inverse-power interparticle force of the form

$$
\mathbf{F}(\mathbf{r})=\frac{K}{r^{p}} \widehat{\mathbf{r}}
$$

where $K$ is a constant and $p$ is a positive integer number, show that [see (5.18)]

$$
\begin{gathered}
\left\{\Delta v_{x}\right\}=\left\{\Delta v_{y}\right\}=0 \\
\left\{\Delta v_{z}\right\}=\frac{\mu g^{2} \sigma_{m}}{m_{\alpha}}
\end{gathered}
$$

where $\mu$ is the reduced mass and $\sigma_{m}$ is the momentum transfer cross section given by

$$
\sigma_{m}=2 \pi\left(\frac{K}{\mu g^{2}}\right)^{2 /(p-1)} A_{1}(p)
$$

where

$$
\begin{gathered}
A_{\ell}(p)=\int_{0}^{\infty}\left(1-\cos ^{\ell} \chi\right) v_{0} d v_{0} \\
v_{0}=b\left(\frac{\mu g^{2}}{K}\right)^{1 /(p-1)}
\end{gathered}
$$

Verify also that [see (5.19)]

$$
\begin{gathered}
\left\{\Delta v_{i} \Delta v_{j}\right\}=0 \quad \text { for } \quad i \neq j \\
\left\{\Delta v_{x}^{2}\right\}=\left\{\Delta v_{y}^{2}\right\}=\pi \frac{\mu^{2} g^{3}}{m_{\alpha}^{2}}\left(\frac{K}{\mu g^{2}}\right)^{2 /(p-1)} A_{2}(p) \\
\left\{\Delta v_{z}^{2}\right\}=2 \pi \frac{\mu^{2} g^{3}}{m_{\alpha}^{2}}\left(\frac{K}{\mu g^{2}}\right)^{2 /(p-1)}\left[2 A_{1}(p)-A_{2}(p)\right]
\end{gathered}
$$




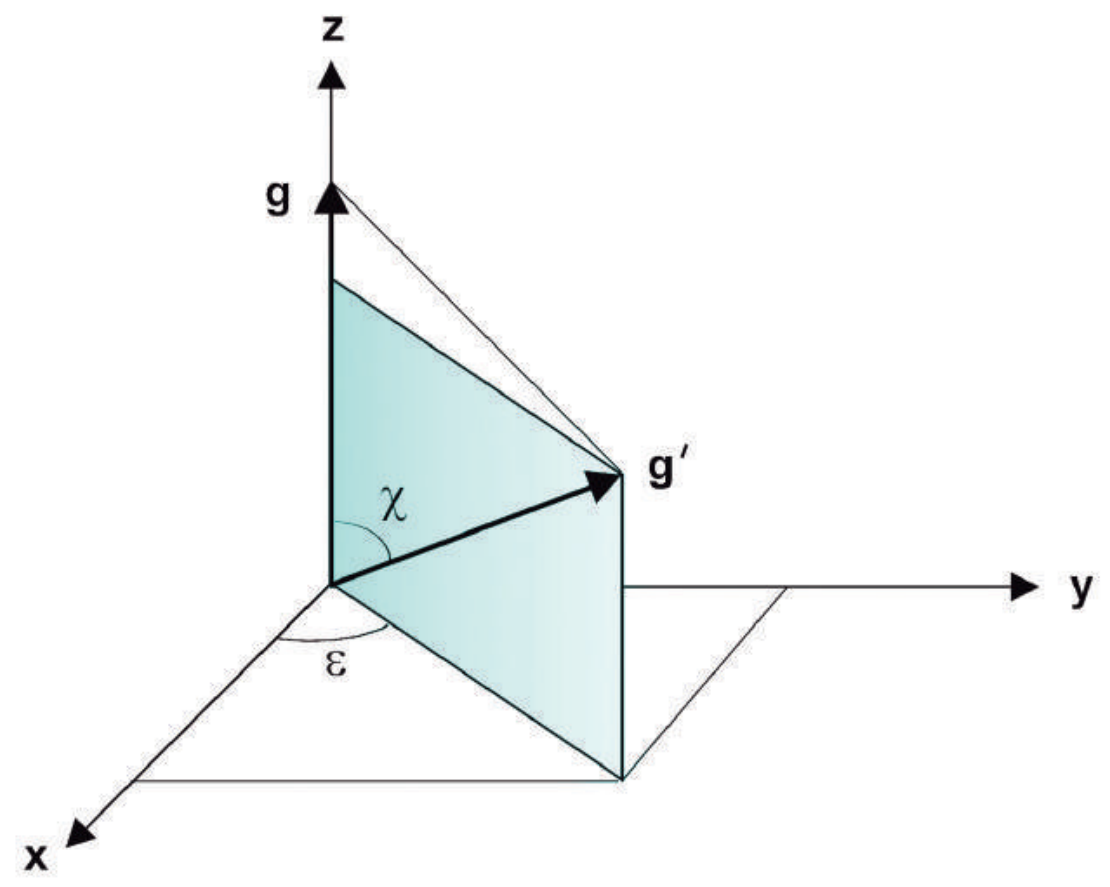

Fig. 6 Coordinate system showing the relative orientations of the velocity vectors $\mathbf{g}$ and $\mathbf{g}^{\prime}$.

(b) For the case of Maxwell molecules $(p=5)$, where the results are independent of $f_{\beta 1}$, show that the Fokker-Planck coefficients are given by

$$
\begin{gathered}
<\Delta v_{x}>_{a v}=<\Delta v_{y}>_{a v}=0 \\
<\Delta v_{z}>_{a v}=\nu_{\alpha \beta}<g>_{\beta} \\
<\Delta v_{i} \Delta v_{j}>_{a v}=0 \quad \text { for } \quad i \neq j \\
<\Delta v_{x}^{2}>_{a v}=<\Delta v_{y}^{2}>_{a v}=\frac{\mu}{2 m_{\alpha}} \frac{A_{2}(5)}{A_{1}(5)} \nu_{\alpha \beta}<g^{2}>_{\beta} \\
<\Delta v_{z}^{2}>_{a v}=\frac{\mu}{m_{\alpha}}\left[2-\frac{A_{2}(5)}{A_{1}(5)}\right] \nu_{\alpha \beta}<g^{2}>_{\beta}
\end{gathered}
$$

where

$$
\begin{gathered}
\nu_{\alpha \beta}=2 \pi \frac{(K \mu)^{1 / 2}}{m_{\alpha}} n_{\beta} A_{1}(5) \\
<g^{i}>_{\beta}=\frac{1}{n_{\beta}} \int_{v_{1}} g^{i} f_{\beta 1} d^{3} v_{1} \quad ; \quad(i=1,2)
\end{gathered}
$$


(c) Calculate the Fokker-Planck coefficients for the case of the coulomb interaction $(p=2)$ using the results of part (a) and of problem 20.6, in terms of integrals over $f_{\beta 1}$, and compare with the results derived in subsection 5.2.

(d) Calculate the Fokker-Planck coefficients for electron-electron interactions, when $f_{\beta 1}$ is the Maxwellian distribution function. Refer to (5.49), (5.50), and (5.51). 


\section{2}

\section{TRANSPORT PROCESSES}

IN PLASMAS

\section{INTRODUCTION}

Transport phenomena in plasmas can be promoted by external and by internal forces. In a spatially homogeneous plasma under the influence of external forces a drifting of the electrons can occur. This motion induced by external forces is referred to as mobility. Since the electrons have mass and electric charge, their motion implies transport of mass and conduction of electricity when acted upon by an external electric field. On the other hand, in a spatially inhomogeneous plasma, collisions cause the electrons to drift from the high-pressure to the low-pressure regions. The existence of pressure gradients is associated with the existence of either density gradients or temperature gradients, or both. This motion of the electrons, induced by internal pressure gradients, is called diffusion. Since the electrons also have kinetic energy associated with their random thermal motion, their drift implies the transport of thermal energy and therefore heat conduction. When the plasma is spatially inhomogeneous and is also acted upon by external forces, then the particle flux is due to both diffusion and mobility.

In this chapter we shall analyze the basic transport phenomena of electric conduction, particle diffusion, and thermal energy flux in a weakly ionized plasma, using the Boltzmann equation with the relaxation model for the collision term and considering a velocity-dependent collision frequency. 


\section{ELECTRIC CONDUCTIVITY IN A NONMAGNETIZED PLASMA}

Initially we shall derive an expression for the AC conductivity of a weakly ionized plasma taking into account only the electron-neutral collisions. We will consider that the spatial inhomogeneity and the anisotropy of the electron nonequilibrium distribution function are both very small, so that we can apply the results derived in section 4 of Chapter 21. Thus, according to (21.4.17) (Eq. 4.17 in Chapter 21) we have

$$
\left[\frac{\delta f(\mathbf{r}, \mathbf{v}, t)}{\delta t}\right]_{\text {coll }}=-\nu_{r}(v)\left[f(\mathbf{r}, \mathbf{v}, t)-f_{0}(v)\right]
$$

where $f_{0}(v)$ denotes the homogeneous isotropic equilibrium distribution function of the electrons and $\nu_{r}(v)$ is the velocity-dependent relaxation collision frequency. Expression (2.1) assumes that the neutral particles are stationary and do not recoil as they collide with the electrons, in view of their much larger mass.

\subsection{Solution of Boltzmann Equation}

Assuming that the electron distribution function $f(\mathbf{r}, \mathbf{v}, t)$ deviates only slightly from the equilibrium distribution function $f_{0}(v)$, we can take

$$
f(\mathbf{r}, \mathbf{v}, t)=f_{0}(v)+f_{1}(\mathbf{r}, \mathbf{v}, t) \quad ; \quad\left|f_{1}\right| \ll f_{0}
$$

where $f_{1}(\mathbf{r}, \mathbf{v}, t)$ corresponds to the small anisotropy and spatial inhomogeneity of the electrons in the nonequilibrium state. Using (2.2) and the relaxation model (2.1), the collision term in the Boltzmann equation becomes

$$
\left[\frac{\delta f(\mathbf{r}, \mathbf{v}, t)}{\delta t}\right]_{\text {coll }}=-\nu_{r}(v) f_{1}(\mathbf{r}, \mathbf{v}, t)
$$

Substituting (2.2) and (2.3) into the Boltzmann equation and neglecting second-order quantities, we obtain

$$
\begin{gathered}
\frac{\partial f_{1}(\mathbf{r}, \mathbf{v}, t)}{\partial t}+(\mathbf{v} \cdot \nabla) f_{1}(\mathbf{r}, \mathbf{v}, t)-\frac{e}{m_{e}} \mathbf{E}(\mathbf{r}, t) \cdot \nabla_{v} f_{0}(v)= \\
-\nu_{r}(v) f_{1}(\mathbf{r}, \mathbf{v}, t)
\end{gathered}
$$

where we have considered the electric field $\mathbf{E}(\mathbf{r}, t)$ as the only field externally applied to the plasma. 
For the purpose of evaluating the electric conductivity, the perturbation $f_{1}(\mathbf{r}, \mathbf{v}, t)$ in the velocity distribution function can be assumed to be essentially independent of the position coordinate $\mathbf{r}$ and therefore can be denoted by $f_{1}(\mathbf{v}, t)$, since the main effect associated with a spatial gradient is the diffusion of particles and, for the moment, we are primarily interested in the particle current density induced by an externally applied electric field. The electric field is considered to vary harmonically in time at a frequency $\omega$, according to

$$
\mathbf{E}(\mathbf{r}, t)=\mathbf{E}(\mathbf{r}) \exp (-i \omega t)
$$

Consequently, we assume that $f_{1}(\mathbf{v}, t)$ has also the same time variation,

$$
f_{1}(\mathbf{v}, t)=f_{1}(\mathbf{v}) \exp (-i \omega t)
$$

Therefore, for the phasor amplitudes, the Boltzmann equation (2.4) simplifies to

$$
-i \omega f_{1}(\mathbf{v})-\frac{e}{m_{e}} \mathbf{E}(\mathbf{r}) \cdot \nabla_{v} f_{0}(v)=-\nu_{r}(v) f_{1}(\mathbf{v})
$$

Using the following identity, given in (18.3.17),

$$
\nabla_{v} f_{0}(v)=\frac{\mathbf{v}}{v} \frac{d f_{0}(v)}{d v}
$$

we obtain, from (2.7),

$$
f_{1}(\mathbf{v})=\frac{i e}{m_{e}} \frac{\mathbf{E}(\mathbf{r}) \cdot \mathbf{v}}{v\left[\omega+i \nu_{r}(v)\right]} \frac{d f_{0}(v)}{d v}
$$

\subsection{Electric Current Density and Conductivity}

The electric current density is given by

$$
\mathbf{J}(\mathbf{r}, t)=-e n_{e}<\mathbf{v}>_{e}=-e \int_{v} \mathbf{v} f(\mathbf{r}, \mathbf{v}, t) d^{3} v
$$

Using (2.2), (2.6), and (2.9), we find that

$$
\mathbf{J}(\mathbf{r}, t)=\mathbf{J}(\mathbf{r}) \exp (-i \omega t)
$$




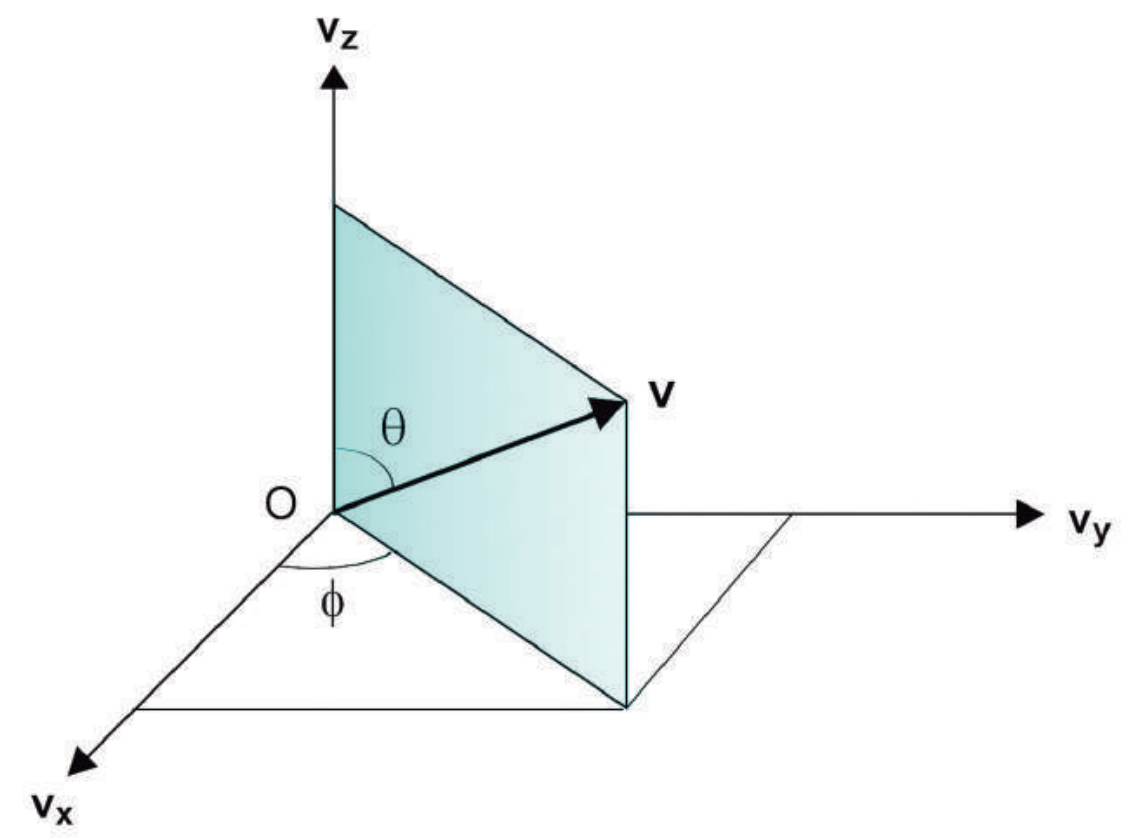

Fig. 1 Spherical coordinates $(v, \theta, \phi)$ in velocity space.

where

$$
\mathbf{J}(\mathbf{r})=-e \int_{v} \mathbf{v} f_{1}(\mathbf{v}) d^{3} v=-\frac{i e^{2}}{m_{e}} \int_{v} \frac{\mathbf{v}[\mathbf{E}(\mathbf{r}) \cdot \mathbf{v}]}{v\left[\omega+i \nu_{r}(v)\right]} \frac{d f_{0}(v)}{d v} d^{3} v
$$

In this result we have assumed that the electrons have no average flow velocity in the equilibrium state, that is,

$$
\mathbf{u}_{0}=\frac{1}{n_{0}} \int_{v} \mathbf{v} f_{0}(v) d^{3} v=0
$$

In spherical coordinates $(v, \theta, \phi)$ in velocity space (refer to Fig. 1), we have $d^{3} v=v^{2} d v \sin \theta d \theta d \phi$, so that (2.12) can be rewritten as

$$
\mathbf{J}(\mathbf{r})=-\frac{i e^{2}}{m_{e}} \int_{0}^{\infty} \frac{v d v}{\left[\omega+i \nu_{r}(v)\right]} \frac{d f_{0}(v)}{d v} \int_{0}^{\pi} \sin \theta d \theta \int_{0}^{2 \pi} \mathbf{v}[\mathbf{E}(\mathbf{r}) \cdot \mathbf{v}] d \phi
$$

Using the following orthogonality relation,

$$
\int_{0}^{\pi} \int_{0}^{2 \pi} v_{i} v_{j} \sin \theta d \theta d \phi=\frac{4 \pi}{3} v^{2} \delta_{i j}
$$


with $i, j=x, y, z$, we obtain

$$
\int_{0}^{\pi} \sin \theta d \theta \int_{0}^{2 \pi} \mathbf{v}[\mathbf{E}(\mathbf{r}) \cdot \mathbf{v}] d \phi=\frac{4 \pi}{3} v^{2} \mathbf{E}(\mathbf{r})
$$

Consequently, (2.14) becomes

$$
\mathbf{J}(\mathbf{r})=-\frac{4 \pi i e^{2}}{3 m_{e}} \mathbf{E}(\mathbf{r}) \int_{0}^{\infty} \frac{v^{3}}{\left[\omega+i \nu_{r}(v)\right]} \frac{d f_{0}(v)}{d v} d v
$$

From the relation $\mathbf{J}=\sigma \mathbf{E}$ for isotropic plasmas, we identify the following expression for the electric conductivity:

$$
\sigma=-\frac{4 \pi i e^{2}}{3 m_{e}} \int_{0}^{\infty} \frac{v^{3}}{\left[\omega+i \nu_{r}(v)\right]} \frac{d f_{0}(v)}{d v} d v
$$

An alternative expression for the electric conductivity can be obtained by integrating (2.18) by parts:

$$
\begin{gathered}
\sigma=-\left.\frac{4 \pi i e^{2}}{3 m_{e}}\left\{\frac{v^{3} f_{0}(v)}{\left[\omega+i \nu_{r}(v)\right]}\right\}\right|_{0} ^{\infty}+ \\
\frac{4 \pi i e^{2}}{3 m_{e}} \int_{0}^{\infty} f_{0}(v) \frac{d}{d v}\left\{\frac{v^{3}}{\left[\omega+i \nu_{r}(v)\right]}\right\} d v
\end{gathered}
$$

The integrated-out term on the right-hand side of this expression vanishes, since $f_{0}(v)$ goes to zero faster than $v^{3}$ goes to infinity, as $v$ approaches infinity [the isotropic equilibrium distribution function $f_{0}(v)$ decreases exponentially as $v$ goes to infinity].

The integrals that appear in expressions (2.18) and (2.19) can be calculated explicitly only after specifying $f_{0}(v)$ and $\nu_{r}(v)$. The functional dependence of the collision frequency on $v$ is generally determined experimentally from cross-section measurements.

If we assume that the collision frequency is independent of velocity, then we obtain from $(2.19)$, for any $f_{0}(v)$,

$$
\begin{gathered}
\sigma=\frac{4 \pi i e^{2}}{3 m_{e}\left(\omega+i \nu_{r}\right)} \int_{0}^{\infty} f_{0}(v) 3 v^{2} d v=\frac{i n_{0} e^{2}}{m_{e}\left(\omega+i \nu_{r}\right)}= \\
\frac{n_{0} e^{2} \nu_{r}}{m_{e}\left(\omega^{2}+\nu_{r}^{2}\right)}+i \frac{n_{0} e^{2} \omega}{m_{e}\left(\omega^{2}+\nu_{r}^{2}\right)}
\end{gathered}
$$


where $n_{0}$ denotes the electron number density at equilibrium,

$$
n_{0}=4 \pi \int_{0}^{\infty} f_{0}(v) v^{2} d v
$$

The result (2.20) is identical to the one obtained in section 5 of Chapter 10 [see equation (10.5.5)], for the longitudinal conductivity.

\subsection{Conductivity for Maxwellian Distribution Function}

Let us consider now that the equilibrium state distribution function $f_{0}(v)$ is given by the Maxwellian distribution,

$$
f_{0}(v)=n_{0}\left(\frac{m_{e}}{2 \pi k T}\right)^{3 / 2} \exp \left(-\frac{m_{e} v^{2}}{2 k T}\right)
$$

Defining a dimensionless variable by

$$
\xi=\left(\frac{m_{e}}{2 k T}\right)^{1 / 2} v
$$

we have

$$
v^{3} \frac{d f_{0}(v)}{d v} d v=-\frac{2}{\pi^{3 / 2}} n_{0} \xi^{4} \exp \left(-\xi^{2}\right) d \xi
$$

Substituting this expression into (2.18) and rationalizing, we find

$$
\begin{gathered}
\sigma=\frac{8 n_{0} e^{2}}{3 \sqrt{\pi} m_{e}}\left[\int_{0}^{\infty} \frac{\nu_{r}(\xi) \xi^{4} \exp \left(-\xi^{2}\right)}{\nu_{r}^{2}(\xi)+\omega^{2}} d \xi+\right. \\
\left.i \omega \int_{0}^{\infty} \frac{\xi^{4} \exp \left(-\xi^{2}\right)}{\nu_{r}^{2}(\xi)+\omega^{2}} d \xi\right]
\end{gathered}
$$

This equation can be used to calculate the electric conductivity of a weakly ionized plasma when the equilibrium distribution function of the electrons is the Maxwell-Boltzmann distribution, for any velocity dependence of the collision frequency $\nu_{r}(v)$. In particular, if the collision frequency is independent of velocity, it can be easily verified that (2.25) reduces directly to the result (2.20). 


\section{ELECTRIC CONDUCTIVITY IN A MAGNETIZED PLASMA}

We consider now a weakly ionized plasma immersed in an externally applied magnetostatic field $\mathbf{B}_{0}$. As in the previous section, we assume that the distribution function of the electrons in the nonequilibrium state is only slightly perturbed from the equilibrium function. For purposes of calculating the conductivity, it can also be assumed that the plasma is spatially homogeneous. Therefore, we can write

$$
f(\mathbf{v}, t)=f_{0}(v)+f_{1}(\mathbf{v}, t) \quad ; \quad\left|f_{1}\right| \ll f_{0}
$$

Suppose that an AC electric field is applied to the plasma, having a harmonic time dependence according to

$$
\mathbf{E}(\mathbf{r}, t)=\mathbf{E}(\mathbf{r}) \exp (-i \omega t)
$$

Consequently, we also have

$$
f_{1}(\mathbf{v}, t)=f_{1}(\mathbf{v}) \exp (-i \omega t)
$$

The total magnetic field will be denoted by

$$
\mathbf{B}_{t}(\mathbf{r}, t)=\mathbf{B}_{0}+\mathbf{B}(\mathbf{r}) \exp (-i \omega t)
$$

where $\mathbf{B}_{0}$ is the externally field and $\mathbf{B}(\mathbf{r}, t)$ is a first-order quantity that has the same harmonic time dependence as the electric field.

\subsection{Solution of Boltzmann Equation}

The Boltzmann equation satisfied by the homogeneous distribution function of the electrons can be written as

$$
\begin{aligned}
\frac{\partial f_{1}(\mathbf{v}, t)}{\partial t}-\frac{e}{m_{e}}[\mathbf{E}(\mathbf{r}, t) & \left.+\mathbf{v} \times \mathbf{B}_{t}(\mathbf{r}, t)\right] \cdot \nabla_{v}\left[f_{0}(v)+f_{1}(\mathbf{v}, t)\right]= \\
& -\nu_{r}(v) f_{1}(\mathbf{v}, t)
\end{aligned}
$$

where we have used the relaxation model (2.3) for the collision term. From the identity (2.8) we see that the term $\left(\mathbf{v} \times \mathbf{B}_{t}\right) \cdot \nabla_{v} f_{0}(v)$ vanishes, since it involves the dot product of two mutually orthogonal vector functions. 


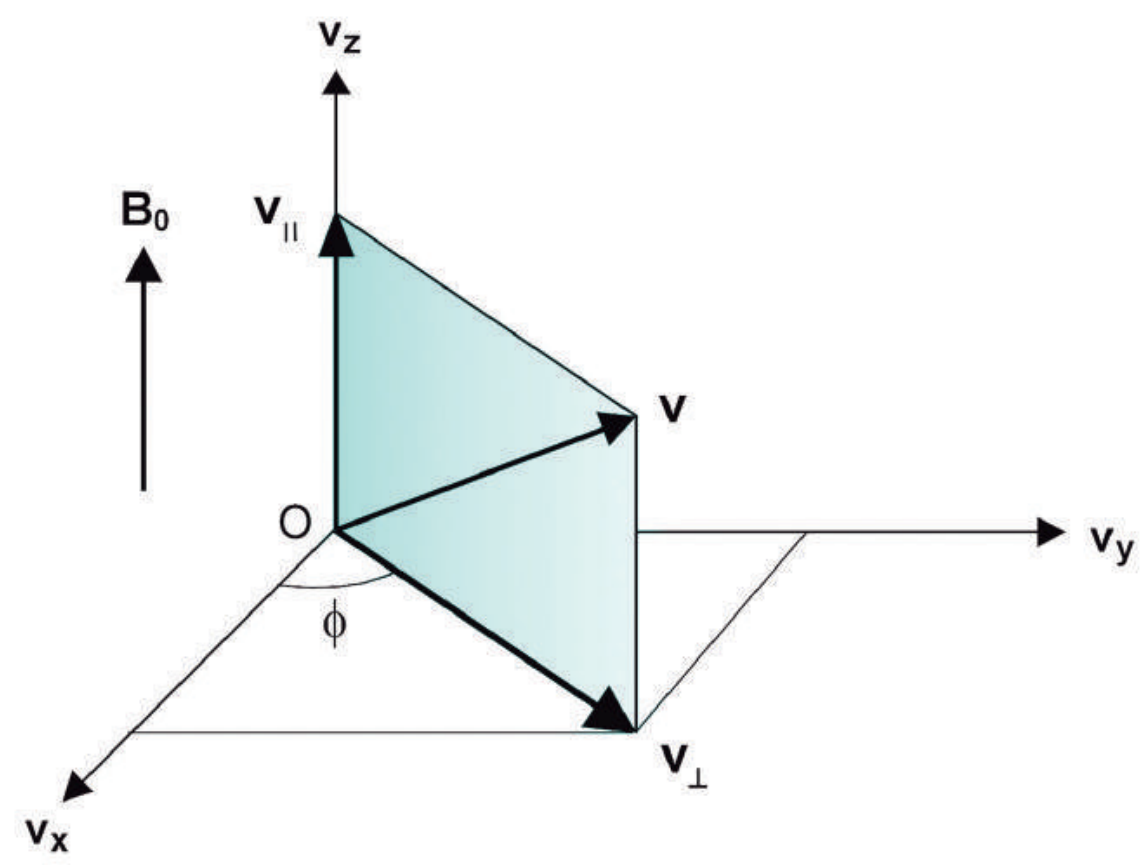

Fig. 2 Cylindrical coordinates $\left(v_{\perp}, v_{\|}, \phi\right)$ in velocity space.

Neglecting second-order terms, the linearized Boltzmann equation for the phasor amplitudes becomes

$$
\left[\nu_{r}(v)-i \omega\right] f_{1}(\mathbf{v})-\frac{e}{m_{e}}\left(\mathbf{v} \times \mathbf{B}_{0}\right) \cdot \nabla_{v} f_{1}(\mathbf{v})=\frac{e}{m_{e}} \mathbf{E} \cdot \nabla_{v} f_{0}(v)
$$

In cylindrical coordinates $\left(v_{\perp}, v_{\|}, \phi\right)$ in velocity space (refer to Fig. 2), with the $\mathbf{v}_{\|}$vector along the magnetostatic field $\mathbf{B}_{0}$, we have, from equation (19.2.10) (Eq. 2.10 in Chapter 19),

$$
\left(\mathbf{v} \times \mathbf{B}_{0}\right) \cdot \nabla_{v} f_{1}(\mathbf{v})=-\frac{d f_{1}(\mathbf{v})}{d \phi}
$$

Substituting (3.7) into (3.6) and using the identity (2.8), we obtain

$$
\frac{d f_{1}(\mathbf{v})}{d \phi}+\frac{\nu_{r}(v)-i \omega}{\Omega_{c e}} f_{1}(\mathbf{v})=\frac{e}{m_{e} \Omega_{c e}} \mathbf{E} \cdot \frac{\mathbf{v}}{v} \frac{d f_{0}(v)}{d v}
$$

where we have used the notation $\Omega_{c e}=e B_{0} / m_{e}$, which represents the electron cyclotron frequency. Notice that the speed $v$ does not depend on $\phi$, since $v^{2}=v_{\perp}^{2}+v_{\|}^{2}$. 
It is convenient to decompose the electric field vector into right circularly polarized $\left(E_{+}\right)$, left circularly polarized $\left(E_{-}\right)$, and longitudinal $\left(E_{\|}\right)$ components, that is,

$$
\mathbf{E}=\frac{(\widehat{\mathbf{x}}+i \widehat{\mathbf{y}})}{\sqrt{2}} E_{+}+\frac{(\widehat{\mathbf{x}}-i \widehat{\mathbf{y}})}{\sqrt{2}} E_{-}+E_{\|} \widehat{\mathbf{z}}
$$

where

$$
E_{ \pm}=\frac{1}{\sqrt{2}}\left(E_{x} \mp i E_{y}\right)
$$

Similarly, we can also decompose the electron velocity as

$$
\mathbf{v}=\frac{(\widehat{\mathbf{x}}+i \widehat{\mathbf{y}})}{\sqrt{2}} v_{+}+\frac{(\widehat{\mathbf{x}}-i \widehat{\mathbf{y}})}{\sqrt{2}} v_{-}+v_{\|} \widehat{\mathbf{z}}
$$

where

$$
v_{ \pm}=\frac{1}{\sqrt{2}}\left(v_{x} \mp i v_{y}\right)=\frac{1}{\sqrt{2}} v_{\perp} \exp (\mp i \phi)
$$

since $v_{x}=v_{\perp} \cos \phi, \quad v_{y}=v_{\perp} \sin \phi$, and $\exp ( \pm i \phi)=\cos \phi \pm i \sin \phi$. Thus, using (3.9) and (3.11), we have

$$
\mathbf{E} \cdot \mathbf{v}=E_{+} v_{-}+E_{-} v_{+}+E_{\|} v_{\|}=\frac{v_{\perp}}{\sqrt{2}}\left(E_{+} e^{i \phi}+E_{-} e^{-i \phi}\right)+E_{\|} v_{\|}
$$

Substituting this expression into the Boltzmann equation (3.8), we obtain

$$
\begin{gathered}
\frac{d f_{1}(\mathbf{v})}{d \phi}+\frac{\nu_{r}(v)-i \omega}{\Omega_{c e}} f_{1}(\mathbf{v})= \\
\frac{e}{m_{e} \Omega_{c e}}\left[\frac{v_{\perp}}{\sqrt{2}}\left(E_{+} e^{i \phi}+E_{-} e^{-i \phi}\right)+E_{\|} v_{\|}\right] \frac{1}{v} \frac{d f_{0}(v)}{d v}
\end{gathered}
$$

As in subsection 2.2 of Chapter 19, we now introduce the notation

$$
\begin{gathered}
F_{+}(\mathbf{v})=\frac{e}{m_{e} \Omega_{c e}} \frac{v_{\perp} e^{i \phi}}{\sqrt{2} v} \frac{d f_{0}(v)}{d v} E_{+} \\
F_{-}(\mathbf{v})=\frac{e}{m_{e} \Omega_{c e}} \frac{v_{\perp} e^{-i \phi}}{\sqrt{2} v} \frac{d f_{0}(v)}{d v} E_{-} \\
F_{\|}(\mathbf{v})=\frac{e}{m_{e} \Omega_{c e}} \frac{v_{\|}}{v} \frac{d f_{0}(v)}{d v} E_{\|}
\end{gathered}
$$


which allows (3.14) to be written as

$$
\frac{d f_{1}(\mathbf{v})}{d \phi}+\frac{\nu_{r}(v)-i \omega}{\Omega_{c e}} f_{1}(\mathbf{v})=F_{+}(\mathbf{v})+F_{-}(\mathbf{v})+F_{\|}(\mathbf{v})
$$

This differential equation is similar to (19.2.26), replacing the term $-k v_{\|}$ by $i \nu_{r}(v)$. Therefore, its solution can be obtained by inspection of the corresponding results contained in subsection 2.2 of Chapter 19. Hence, using equations (19.2.27) to (19.2.34), we obtain

$$
\begin{gathered}
f_{1}(\mathbf{v})=\frac{i \Omega_{c e}}{\omega+i \nu_{r}(v)-\Omega_{c e}} F_{+}(\mathbf{v})+\frac{i \Omega_{c e}}{\omega+i \nu_{r}(v)+\Omega_{c e}} F_{-}(\mathbf{v})+ \\
\frac{i \Omega_{c e}}{\omega+i \nu_{r}(v)} F_{\|}(\mathbf{v})
\end{gathered}
$$

or substituting (3.15), (3.16), and (3.17) into (3.19),

$$
\begin{gathered}
f_{1}(\mathbf{v})=\frac{i e}{m_{e}} \frac{1}{v} \frac{d f_{0}(v)}{d v}\left\{\frac{v_{\perp}}{\sqrt{2}}\left[\frac{E_{+} e^{i \phi}}{\omega+i \nu_{r}(v)-\Omega_{c e}}+\frac{E_{-} e^{-i \phi}}{\omega+i \nu_{r}(v)+\Omega_{c e}}\right]+\right. \\
\left.\frac{v_{\|} E_{\|}}{\omega+i \nu_{r}(v)}\right\}
\end{gathered}
$$

\subsection{Electric Current Density and Conductivity}

Assuming that the electron gas has no average flow velocity in the equilibrium state $\left(\mathbf{u}_{0}=0\right)$, we can write for the electric current density,

$$
\mathbf{J}=-e \int_{v} \mathbf{v} f_{1}(\mathbf{v}) d^{3} v
$$

As in equations (3.9) to (3.12), we can also decompose the current density vector into three components, according to

$$
\begin{aligned}
& J_{+}=-e \int_{v} v_{+} f_{1}(\mathbf{v}) d^{3} v \\
& J_{-}=-e \int_{v} v_{-} f_{1}(\mathbf{v}) d^{3} v
\end{aligned}
$$




$$
J_{\|}=-e \int_{v} v_{\|} f_{1}(\mathbf{v}) d^{3} v
$$

For purposes of calculating the conductivity, it is convenient to use spherical coordinates $(v, \theta, \phi)$ in velocity space (refer to Fig. 1 ), so that $v_{\perp}=$ $v \sin \theta, \quad v_{\|}=v \cos \theta$, and $d^{3} v=v^{2} d v \sin \theta d \theta d \phi$. Plugging $f_{1}(\mathbf{v})$, from (3.20), into the expressions for $J_{+}, J_{-}$, and $J_{\|}$, given in (3.22), (3.23), and (3.24), respectively, transforming to spherical coordinates and performing the integrals over $\phi$, making use of (19.5.21), we find

$$
\begin{aligned}
& J_{ \pm}=-\frac{\pi i e^{2}}{m_{e}} E_{ \pm} \int_{0}^{\pi} \sin ^{3} \theta d \theta \int_{0}^{\infty} \frac{v^{3}}{\omega+i \nu_{r}(v) \mp \Omega_{c e}} \frac{d f_{0}(v)}{d v} d v \\
& J_{\|}=-\frac{2 \pi i e^{2}}{m_{e}} E_{\|} \int_{0}^{\pi} \cos ^{2} \theta \sin \theta d \theta \int_{0}^{\infty} \frac{v^{3}}{\omega+i \nu_{r}(v)} \frac{d f_{0}(v)}{d v} d v
\end{aligned}
$$

Note that in (3.25) either upper signs or lower signs are to be used. Carrying out the integrations over $\theta$ in these last two equations, yields

$$
\begin{gathered}
J_{ \pm}=-\frac{4 \pi i e^{2}}{3 m_{e}} E_{ \pm} \int_{0}^{\infty} \frac{v^{3}}{\omega+i \nu_{r}(v) \mp \Omega_{c e}} \frac{d f_{0}(v)}{d v} d v \\
J_{\|}=-\frac{4 \pi i e^{2}}{3 m_{e}} E_{\|} \int_{0}^{\infty} \frac{v^{3}}{\omega+i \nu_{r}(v)} \frac{d f_{0}(v)}{d v} d v
\end{gathered}
$$

The advantage of using the right and left circularly polarized components in the plane normal to $\mathbf{B}_{0}$ is that the corresponding equations for $J_{+}$and $J_{-}$are uncoupled such that $J_{+}$depends only on $E_{+}$, whereas $J_{-}$ depends only on $E_{-}$. Therefore, writing $\mathbf{J}=\mathcal{S} \cdot \mathbf{E}$, where $\mathcal{S}$ denotes the conductivity tensor of an anisotropic plasma, we obtain, from (3.27) and (3.28),

$$
\left(\begin{array}{c}
J_{+} \\
J_{-} \\
J_{\|}
\end{array}\right)=\left(\begin{array}{ccc}
\sigma_{+} & 0 & 0 \\
0 & \sigma_{-} & 0 \\
0 & 0 & \sigma_{\|}
\end{array}\right)\left(\begin{array}{c}
E_{+} \\
E_{-} \\
E_{\|}
\end{array}\right)
$$

with the following expressions for the elements of the conductivity tensor:

$$
\begin{gathered}
\sigma_{ \pm}=-\frac{4 \pi i e^{2}}{3 m_{e}} \int_{0}^{\infty} \frac{v^{3}}{\omega+i \nu_{r}(v) \mp \Omega_{c e}} \frac{d f_{0}(v)}{d v} d v \\
\sigma_{\|}=-\frac{4 \pi i e^{2}}{3 m_{e}} \int_{0}^{\infty} \frac{v^{3}}{\omega+i \nu_{r}(v)} \frac{d f_{0}(v)}{d v} d v
\end{gathered}
$$


Note that the longitudinal conductivity $\sigma_{\|}$is the same as that for the case of a nonmagnetized plasma, deduced in the previous section.

The elements of the conductivity tensor, expressed in Cartesian coordinates with the $z$ axis along $\mathbf{B}_{0}$, can be obtained as follows. From (3.9) and (3.10) we can write in matrix form

$$
\left(\begin{array}{c}
E_{+} \\
E_{-} \\
E_{\|}
\end{array}\right)=\frac{1}{\sqrt{2}}\left(\begin{array}{ccc}
1 & -i & 0 \\
1 & i & 0 \\
0 & 0 & \sqrt{2}
\end{array}\right)\left(\begin{array}{c}
E_{x} \\
E_{y} \\
E_{z}
\end{array}\right)
$$

Using a matrix relation analogous to (3.32) for the current density $\mathbf{J}$ and inverting it, we obtain

$$
\left(\begin{array}{l}
J_{x} \\
J_{y} \\
J_{z}
\end{array}\right)=\frac{1}{\sqrt{2}}\left(\begin{array}{ccc}
1 & 1 & 0 \\
i & -i & 0 \\
0 & 0 & \sqrt{2}
\end{array}\right)\left(\begin{array}{c}
J_{+} \\
J_{-} \\
J_{\|}
\end{array}\right)
$$

Substituting (3.29) into (3.33) and combining the resulting expression with (3.32), we find that

$$
\left(\begin{array}{l}
J_{x} \\
J_{y} \\
J_{z}
\end{array}\right)=\left(\begin{array}{ccc}
\sigma_{\perp} & -\sigma_{H} & 0 \\
\sigma_{H} & \sigma_{\perp} & 0 \\
0 & 0 & \sigma_{\|}
\end{array}\right)\left(\begin{array}{l}
E_{x} \\
E_{y} \\
E_{z}
\end{array}\right)
$$

where

$$
\begin{gathered}
\sigma_{\perp}=\frac{1}{2}\left(\sigma_{+}+\sigma_{-}\right) \\
\sigma_{H}=\frac{1}{2} i\left(\sigma_{+}-\sigma_{-}\right)
\end{gathered}
$$

with $\sigma_{+}, \sigma_{-}$, and $\sigma_{\|}$as given in (3.30) and (3.31).

The integrals over $v$ can only be evaluated after specifying the dependence of $\nu_{r}$ on $v$. In general, when the collision frequency is an arbitrary function of velocity, the elements of the conductivity tensor have to be determined by a numerical procedure. In cases when the collision frequency can be expressed as a polynominal in $v$, it is possible to obtain simple expressions for the conductivities in the limiting cases of very high and very low collision frequencies. In particular, for the special case when $\nu_{r}$ is independent of $v$, the integrals over $v$ in (3.30) and (3.31) can be explicitly evaluated, yielding

$$
\sigma_{ \pm}=\frac{i n_{0} e^{2}}{m_{e}\left(\omega+i \nu_{r} \mp \Omega_{c e}\right)}
$$




$$
\sigma_{\|}=\frac{i n_{0} e^{2}}{m_{e}\left(\omega+i \nu_{r}\right)}
$$

If these expressions are substituted into (3.35) and (3.36), we obtain the following results for the Cartesian components $\sigma_{\perp}$ and $\sigma_{H}$ of the conductivity tensor:

$$
\begin{aligned}
\sigma_{\perp} & =\frac{i n_{0} e^{2}\left(\omega+i \nu_{r}\right)}{m_{e}\left[\left(\omega+i \nu_{r}\right)^{2}-\Omega_{c e}^{2}\right]} \\
\sigma_{H} & =\frac{n_{0} e^{2} \Omega_{c e}}{m_{e}\left[\left(\omega+i \nu_{r}\right)^{2}-\Omega_{c e}^{2}\right]}
\end{aligned}
$$

These are the same results deduced in section 5 of Chapter 10, which were calculated using the macroscopic transport equations with a constant collision frequency.

\section{FREE DIFFUSION}

In this section we derive an expression for the free diffusion coefficient of a weakly ionized plasma, considering a velocity-dependent relaxation collision frequency $\nu_{r}(v)$. For the analysis of diffusion phenomena let us consider specifically a spatial inhomogeneity in the electron density. Hence, we assume that the equilibrium velocity distribution function of the electrons has a spatial inhomogeneity, but is isotropic in velocity space, so that it will be denoted by $f_{0}(\mathbf{r}, v)$. Since we are interested in calculating the electron flux due to diffusion only, we also assume that there are no external electromagnetic fields applied to the plasma. Furthermore, we shall analyze the free diffusion problem only under steady-state conditions, in which all physical parameters are time-independent.

\subsection{Perturbation Distribution Function}

We assume that, under diffusion, the actual distribution function of the electrons $f(\mathbf{r}, \mathbf{v})$ deviates only slightly from the equilibrium function $f_{0}(\mathbf{r}, v)$, so that we can write

$$
f(\mathbf{r}, \mathbf{v})=f_{0}(\mathbf{r}, v)+f_{1}(\mathbf{r}, \mathbf{v})
$$

where $f_{1}(\mathbf{r}, \mathbf{v})$ is a first-order quantity such that $\left|f_{1}\right| \ll f_{0}$. Under steadystate conditions in the absence of external forces and using the relaxation model for the collision term, the Boltzmann equation simplifies to

$$
\mathbf{v} \cdot \nabla f_{0}(\mathbf{r}, v)=-\nu_{r}(v) f_{1}(\mathbf{r}, \mathbf{v})
$$


where only the first-order terms have been retained. Thus, we obtain directly for the perturbation distribution function

$$
f_{1}(\mathbf{r}, \mathbf{v})=-\frac{1}{\nu_{r}(v)} \mathbf{v} \cdot \nabla f_{0}(\mathbf{r}, v)
$$

\subsection{Particle Flux}

The expression for the particle current density (or flux) for the electrons, considering $\mathbf{u}_{0}=0$, is

$$
\boldsymbol{\Gamma}_{e}=n_{e}<\mathbf{v}>_{e}=\int_{v} \mathbf{v} f_{1}(\mathbf{r}, \mathbf{v}) d^{3} v
$$

Substituting (4.3) into (4.4), gives

$$
\boldsymbol{\Gamma}_{e}=-\int_{v} \frac{1}{\nu_{r}(v)} \mathbf{v}\left[\mathbf{v} \cdot \nabla f_{0}(\mathbf{r}, v)\right] d^{3} v
$$

In spherical coordinates $(v, \theta, \phi)$ in velocity space (refer to Fig. 1 ) we have $d^{3} v=v^{2} \sin \theta d v d \theta d \phi$ and using the result contained in (2.16) we obtain

$$
\int_{0}^{\pi} \sin \theta d \theta \int_{0}^{2 \pi} \mathbf{v}\left[\mathbf{v} \cdot \nabla f_{0}(\mathbf{r}, v)\right] d \phi=\frac{4 \pi}{3} v^{2} \nabla f_{0}(\mathbf{r}, v)
$$

Therefore, the electron flux vector (4.5) can be written as

$$
\boldsymbol{\Gamma}_{e}=-\frac{4 \pi}{3} \int_{0}^{\infty} \frac{v^{4}}{\nu_{r}(v)} \nabla f_{0}(\mathbf{r}, v) d v
$$

\subsection{Free Diffusion Coefficient}

The distribution function $f_{0}(\mathbf{r}, v)$ is in general a function of the electron number density $n_{e}$, the electron speed $v$, and the electron temperature $T_{e}$, so that it can generally be written in the form

$$
f_{0}(\mathbf{r}, v)=n_{e} F\left(v, T_{e}\right)
$$

since the number density appears only as a result of normalization of the distribution function. Usually the function $f_{0}(\mathbf{r}, v)$ is a local Maxwellian distribution. 
For the purpose of calculating the electron free diffusion coefficient, we will assume that the electron temperature has no spatial variation, so that

$$
\nabla f_{0}(\mathbf{r}, v)=\nabla n_{e}(\mathbf{r}) F\left(v, T_{e}\right)
$$

or, using (4.8),

$$
\nabla f_{0}(\mathbf{r}, v)=\nabla n_{e}(\mathbf{r}) \frac{f_{0}(\mathbf{r}, v)}{n_{e}(\mathbf{r})}
$$

Substituting (4.10) into (4.7), we obtain

$$
\boldsymbol{\Gamma}_{e}=-\frac{4 \pi}{3} \frac{\nabla n_{e}(\mathbf{r})}{n_{e}(\mathbf{r})} \int_{0}^{\infty} \frac{v^{4}}{\nu_{r}(v)} f_{0}(\mathbf{r}, v) d v
$$

Defining the electron free diffusion coefficient $D_{e}$ by the relation

$$
\boldsymbol{\Gamma}_{e}=-D_{e} \nabla n_{e}(\mathbf{r})
$$

we deduce the following expression for $D_{e}$, by inspection of (4.11),

$$
D_{e}=\frac{4 \pi}{3} \frac{1}{n_{e}(\mathbf{r})} \int_{0}^{\infty} \frac{v^{4}}{\nu_{r}(v)} f_{0}(\mathbf{r}, v) d v
$$

Note that this expression for $D_{e}$ is constant, independent of $\mathbf{r}$ and $v$, in view of (4.8) and (4.9).

If we consider $f_{0}(\mathbf{r}, v)$ as being a local Maxwellian distribution function given by

$$
f_{0}(\mathbf{r}, v)=n_{e}(\mathbf{r})\left(\frac{m_{e}}{2 \pi k T_{e}}\right)^{3 / 2} \exp \left(-\frac{m_{e} v^{2}}{2 k T_{e}}\right)
$$

then (4.13) becomes

$$
D_{e}=\frac{4 \pi}{3}\left(\frac{m_{e}}{2 \pi k T_{e}}\right)^{3 / 2} \int_{0}^{\infty} \frac{v^{4}}{\nu_{r}(v)} \exp \left(-\frac{m_{e} v^{2}}{2 k T_{e}}\right) d v
$$

Furthermore, if the relaxation collision frequency is taken to be constant, independent of $v$, then the integral in (4.15) can be explicitly evaluated [see (7.4.22) (Eq. 4.22 in Chapter 7)], yielding

$$
D_{e}=\frac{k T_{e}}{m_{e} \nu_{r}}
$$


This is the same result obtained in section 8 of Chapter 10 [see (10.8.9)], which was deduced using the macroscopic transport equations for a warm plasma, with a constant collision frequency.

\section{DIFFUSION IN A MAGNETIC FIELD}

In this section we will analyze the problem of electron diffusion in a weakly ionized plasma, including the presence of an externally applied magnetostatic field $\mathbf{B}_{0}$. We shall consider the same assumptions made in the previous section, except for the inclusion of the external magnetic field.

\subsection{Solution of Boltzmann Equation}

Retaining only the first-order terms, the linearized Boltzmann equation is now

$$
\mathbf{v} \cdot \nabla f_{0}(\mathbf{r}, v)-\frac{e}{m_{e}}\left(\mathbf{v} \times \mathbf{B}_{0}\right) \cdot \nabla_{v} f_{1}(\mathbf{r}, \mathbf{v})=-\nu_{r}(v) f_{1}(\mathbf{r}, \mathbf{v})
$$

Note that in view of the velocity isotropy of $f_{0}(\mathbf{r}, v)$ we can use the identity (2.8), so that

$$
\left(\mathbf{v} \times \mathbf{B}_{0}\right) \cdot \nabla_{v} f_{0}(\mathbf{r}, v)=0
$$

In cylindrical coordinates $\left(v_{\perp}, v_{\|}, \phi\right)$ in velocity space (refer to Fig. 2) we have, from (3.7),

$$
\left(\mathbf{v} \times \mathbf{B}_{0}\right) \cdot \nabla_{v} f_{1}(\mathbf{r}, \mathbf{v})=-\frac{d f_{1}(\mathbf{r}, \mathbf{v})}{d \phi}
$$

Choosing the unit vector $\widehat{\mathbf{z}}$ along the magnetic field $\mathbf{B}_{0}$, we can write

$$
\mathbf{v} \cdot \nabla f_{0}(\mathbf{r}, v)=\left(v_{\perp} \cos \phi \frac{\partial}{\partial x}+v_{\perp} \sin \phi \frac{\partial}{\partial y}+v_{\|} \frac{\partial}{\partial z}\right) f_{0}(\mathbf{r}, v)
$$

Substituting (5.4) and (5.3) into (5.1), and rearranging, yields

$$
\begin{gathered}
{\left[\frac{d}{d \phi}+\frac{\nu_{r}(v)}{\Omega_{c e}}\right] f_{1}(\mathbf{r}, \mathbf{v})=} \\
-\frac{1}{\Omega_{c e}}\left(v_{\perp} \cos \phi \frac{\partial}{\partial x}+v_{\perp} \sin \phi \frac{\partial}{\partial y}+v_{\|} \frac{\partial}{\partial z}\right) f_{0}(\mathbf{r}, v)
\end{gathered}
$$


In order to solve this linear differential equation let

$$
f_{1}(\mathbf{r}, \mathbf{v})=F_{1}(\mathbf{r}, \mathbf{v})+F_{2}(\mathbf{r}, \mathbf{v})+F_{3}(\mathbf{r}, \mathbf{v})
$$

where $F_{1}, F_{2}$, and $F_{3}$ are the solutions of (5.5) corresponding, respectively, to only the first, the second, and the third terms within parentheses in the right-hand side of (5.5), that is,

$$
\begin{gathered}
{\left[\frac{d}{d \phi}+\frac{\nu_{r}(v)}{\Omega_{c e}}\right] F_{1}(\mathbf{r}, \mathbf{v})=-\frac{1}{\Omega_{c e}} v_{\perp} \cos \phi \frac{\partial f_{0}(\mathbf{r}, v)}{\partial x}} \\
{\left[\frac{d}{d \phi}+\frac{\nu_{r}(v)}{\Omega_{c e}}\right] F_{2}(\mathbf{r}, \mathbf{v})=-\frac{1}{\Omega_{c e}} v_{\perp} \sin \phi \frac{\partial f_{0}(\mathbf{r}, v)}{\partial y}} \\
{\left[\frac{d}{d \phi}+\frac{\nu_{r}(v)}{\Omega_{c e}}\right] F_{3}(\mathbf{r}, \mathbf{v})=-\frac{1}{\Omega_{c e}} v_{\|} \frac{\partial f_{0}(\mathbf{r}, v)}{\partial z}}
\end{gathered}
$$

To solve (5.7) let us first rewrite it in the form

$$
\begin{gathered}
{\left[\frac{d}{d \phi}+\frac{\nu_{r}(v)}{\Omega_{c e}}\right] F_{1}(\mathbf{r}, \mathbf{v}) \equiv \exp \left[-\frac{\nu_{r}(v)}{\Omega_{c e}} \phi\right]} \\
\frac{d}{d \phi}\left\{F_{1}(\mathbf{r}, \mathbf{v}) \exp \left[\frac{\nu_{r}(v)}{\Omega_{c e}} \phi\right]\right\}=-\frac{1}{\Omega_{c e}} v_{\perp} \cos \phi \frac{\partial f_{0}(\mathbf{r}, v)}{\partial x}
\end{gathered}
$$

The solution of this differential equation is given by

$$
\begin{gathered}
F_{1}(\mathbf{r}, \mathbf{v})=-\frac{v_{\perp}}{\Omega_{c e}} \\
\frac{\partial f_{0}(\mathbf{r}, v)}{\partial x} \exp \left[-\frac{\nu_{r}(v)}{\Omega_{c e}} \phi\right] \int_{-\infty}^{\phi} \cos \phi^{\prime} \exp \left[\frac{\nu_{r}(v)}{\Omega_{c e}} \phi^{\prime}\right] d \phi^{\prime} \\
=-v_{\perp} \frac{\partial f_{0}(\mathbf{r}, v)}{\partial x} \frac{\nu_{r}(v) \cos \phi+\Omega_{c e} \sin \phi}{\nu_{r}^{2}(v)+\Omega_{c e}^{2}}
\end{gathered}
$$

Note that $F_{1}(\mathbf{r}, \mathbf{v})$ is a periodic function of $\phi$, with period $2 \pi$.

In a similar way, the solutions of (5.8) and (5.9) are given, respectively, by

$$
\begin{gathered}
F_{2}(\mathbf{r}, \mathbf{v})=-v_{\perp} \frac{\partial f_{0}(\mathbf{r}, v)}{\partial y} \frac{\nu_{r}(v) \sin \phi-\Omega_{c e} \cos \phi}{\nu_{r}^{2}(v)+\Omega_{c e}^{2}} \\
F_{3}(\mathbf{r}, \mathbf{v})=-v_{\|} \frac{\partial f_{0}(\mathbf{r}, \mathbf{v})}{\partial z} \frac{1}{\nu_{r}(v)}
\end{gathered}
$$

Adding expressions (5.11), (5.12), and (5.13), gives the solution for $f_{1}(\mathbf{r}, \mathbf{v})$ in terms of $f_{0}(\mathbf{r}, v)$ and $\nu_{r}(v)$. 


\subsection{Particle Flux and Diffusion Coefficients}

From (4.4) the expression for the $x$ component of the electron flux vector is found to be

$$
\Gamma_{e x}=\int_{v} v_{x} f_{1}(\mathbf{r}, \mathbf{v}) d^{3} v
$$

In cylindrical coordinates (refer to Fig. 2) in velocity space we have $d^{3} v=$ $v_{\perp} d v_{\perp} d v_{\|} d \phi$ and $v_{x}=v_{\perp} \cos \phi$. Therefore,

$$
\Gamma_{e x}=\int_{0}^{\infty} d v_{\perp} \int_{0}^{2 \pi} d \phi \int_{-\infty}^{+\infty} d v_{\|} v_{\perp}^{2} \cos \phi f_{1}(\mathbf{r}, \mathbf{v})
$$

Using (5.6), (5.11), (5.12), and (5.13), and performing the integration over $\phi$, we obtain

$\Gamma_{e x}=-\pi \int_{0}^{\infty} d v_{\perp} \int_{-\infty}^{+\infty} d v_{\|} \frac{v_{\perp}^{3}}{\nu_{r}^{2}(v)+\Omega_{c e}^{2}}\left[\nu_{r}(v) \frac{\partial f_{0}(\mathbf{r}, v)}{\partial x}-\Omega_{c e} \frac{\partial f_{0}(\mathbf{r}, v)}{\partial y}\right]$

To perform the integrals in (5.16) it is convenient to use spherical coordinates $(v, \theta, \phi)$ in velocity space (Fig. 1$)$. Transforming to spherical coordinates, (5.16) becomes

$$
\Gamma_{e x}=-\pi \int_{0}^{\infty} d v \int_{0}^{\pi} d \theta \frac{v^{4} \sin ^{3} \theta}{\nu_{r}^{2}(v)+\Omega_{c e}^{2}}\left[\nu_{r}(v) \frac{\partial f_{0}(\mathbf{r}, v)}{\partial x}-\Omega_{c e} \frac{\partial f_{0}(\mathbf{r}, v)}{\partial y}\right]
$$

Carrying out the integration over $\theta$, we obtain

$$
\begin{gathered}
\Gamma_{e x}=-\frac{4 \pi}{3} \int_{0}^{\infty} \frac{v^{4} \nu_{r}(v)}{\nu_{r}^{2}(v)+\Omega_{c e}^{2}} \frac{\partial f_{0}(\mathbf{r}, v)}{\partial x} d v- \\
\frac{4 \pi}{3} \int_{0}^{\infty} \frac{v^{4} \Omega_{c e}}{\nu_{r}^{2}(v)+\Omega_{c e}^{2}} \frac{\partial f_{0}(\mathbf{r}, v)}{\partial y} d v
\end{gathered}
$$

This equation can be written in the form

$$
\Gamma_{e x}=-\frac{\partial}{\partial x}\left[D_{\perp} n_{e}(\mathbf{r})\right]-\frac{\partial}{\partial y}\left[-D_{H} n_{e}(\mathbf{r})\right]
$$

where the electron diffusion coefficients $D_{\perp}$ and $D_{H}$ are given by

$$
D_{\perp}=\frac{4 \pi}{3} \frac{1}{n_{e}(\mathbf{r})} \int_{0}^{\infty} \frac{v^{4} \nu_{r}(v)}{\nu_{r}^{2}(v)+\Omega_{c e}^{2}} f_{0}(\mathbf{r}, v) d v
$$




$$
D_{H}=\frac{4 \pi}{3} \frac{1}{n_{e}(\mathbf{r})} \int_{0}^{\infty} \frac{v^{4} \Omega_{c e}}{\nu_{r}^{2}(v)+\Omega_{c e}^{2}} f_{0}(\mathbf{r}, v) d v
$$

Along similar lines, we obtain for the $y$ component of the electron flux vector,

$$
\Gamma_{e y}=-\frac{\partial}{\partial x}\left[D_{H} n_{e}(\mathbf{r})\right]-\frac{\partial}{\partial y}\left[D_{\perp} n_{e}(\mathbf{r})\right]
$$

and for the $z$ component

$$
\Gamma_{e z}=-\frac{\partial}{\partial z}\left[D_{\|} n_{e}(\mathbf{r})\right]
$$

where

$$
D_{\|}=\frac{4 \pi}{3} \frac{1}{n_{e}(\mathbf{r})} \int_{0}^{\infty} \frac{v^{4}}{\nu_{r}(v)} f_{0}(\mathbf{r}, v) d v
$$

Equations (5.19), (5.22), and (5.23) can be written in a succinct vector form as

$$
\boldsymbol{\Gamma}_{e}=-\nabla \cdot\left[\mathcal{D} n_{e}(\mathbf{r})\right]
$$

where $\mathcal{D}$ denotes the dyadic coefficient for electron diffusion in a magnetic field. In matrix form, considering a Cartesian coordinate system with the $z$ axis along $\mathbf{B}_{0}$, it can be written as

$$
\mathcal{D}=\left(\begin{array}{ccc}
D_{\perp} & D_{H} & 0 \\
-D_{H} & D_{\perp} & 0 \\
0 & 0 & D_{\|}
\end{array}\right)
$$

The diffusion coefficient $D_{\|}$is the same as that obtained in the absence of a magnetostatic field $\left(D_{\|}=D_{e}\right)$. Therefore, the diffusion of particles along the magnetic field is the same as when there is no field present, whereas the diffusion in the plane perpendicular to $\mathbf{B}_{0}$ is inhibited by the magnetic field, since $D_{\perp}<D_{\|}$, as can be verified from (5.20) and (5.24).

For the special case in which $f_{0}(\mathbf{r}, v)$ is given by a local Maxwellian distribution function, as in (4.14), and when the collision frequency is independent of velocity, the integrals in (5.20), (5.21), and (5.24) can be evaluated directly, yielding

$$
\begin{aligned}
& D_{\perp}=\frac{\nu_{r}^{2}}{\nu_{r}^{2}+\Omega_{c e}^{2}} D_{e} \\
& D_{H}=\frac{\nu_{r} \Omega_{c e}}{\nu_{r}^{2}+\Omega_{c e}^{2}} D_{e}
\end{aligned}
$$




$$
D_{\|}=D_{e}=\frac{k T_{e}}{m_{e} \nu_{r}}
$$

which are the same results obtained in section 9 of Chapter 10, deduced from the macroscopic transport equations for a warm plasma [see equations (10.9.4) to (10.9.7)].

\section{HEAT FLOW}

We shall now derive expressions for the heat flow vector $\mathbf{q}_{e}$ and for the thermal conductivity $K_{e}$, due to the random motion of the electrons in a weakly ionized plasma. As in the previous sections, we will calculate the nonequilibrium distribution function $f(\mathbf{r}, \mathbf{v})$, under steady-state conditions, by applying a perturbation technique to the Boltzmann equation, using the relaxation model for the collision term. To simplify matters we assume that there are no externally applied electromagnetic fields. The analysis of heat flow in weakly ionized plasmas under the presence of externally applied electromagnetic fields will be left as an exercise for the reader and is included in the problems at the end of this chapter.

Using (4.1) we find that the Boltzmann equation for this case is the same as that given by (4.2). Therefore, as in subsection 4.1, we have

$$
f_{1}(\mathbf{r}, \mathbf{v})=-\frac{1}{\nu_{r}(v)} \mathbf{v} \cdot \nabla f_{0}(\mathbf{r}, v)
$$

\subsection{General Expression for the Heat Flow Vector}

The expression for the heat flow vector due to the thermal motion of the electrons, considering $\mathbf{u}_{0}=0$, is

$$
\mathbf{q}_{e}=\frac{1}{2} m_{e} \int_{v} v^{2} \mathbf{v} f_{1}(\mathbf{r}, \mathbf{v}) d^{3} v
$$

Substituting (6.1) into (6.2), yields

$$
\mathbf{q}_{e}=-\frac{1}{2} m_{e} \int_{v} \frac{v^{2}}{\nu_{r}(v)} \mathbf{v}\left[\mathbf{v} \cdot \nabla f_{0}(\mathbf{r}, v)\right] d^{3} v
$$

In spherical coordinates in velocity space and using (4.6), we obtain

$$
\mathbf{q}_{e}=-\frac{2 \pi}{3} m_{e} \int_{0}^{\infty} \frac{v^{6}}{\nu_{r}(v)} \nabla f_{0}(\mathbf{r}, v) d v
$$


This expression gives the electron heat flow vector in terms of the distribution function $f_{0}(\mathbf{r}, v)$ and the relaxation collision frequency $\nu_{r}(v)$.

\subsection{Thermal Conductivity for a Constant Kinetic Pressure}

We shall next evaluate (6.4) for the case when $f_{0}(\mathbf{r}, v)$ is given by a local Maxwellian distribution function,

$$
f_{0}(\mathbf{r}, v)=n_{e}(\mathbf{r})\left[\frac{m_{e}}{2 \pi k T_{e}(\mathbf{r})}\right]^{3 / 2} \exp \left[-\frac{m_{e} v^{2}}{2 k T_{e}(\mathbf{r})}\right]
$$

in which both $n_{e}$ and $T_{e}$ may have a spatial variation, but such that the electron kinetic pressure stays constant, that is,

$$
p_{e}=n_{e}(\mathbf{r}) k T_{e}(\mathbf{r})=\text { constant }
$$

From (6.6) we have

$$
T_{e}(\mathbf{r}) \nabla n_{e}(\mathbf{r})=-n_{e}(\mathbf{r}) \nabla T_{e}(\mathbf{r})
$$

and calculating the gradient of (6.5) we find

$$
\nabla f_{0}(\mathbf{r}, v)=\left[-\frac{5}{2}+\frac{m_{e} v^{2}}{2 k T_{e}(\mathbf{r})}\right] \frac{\nabla T_{e}(\mathbf{r})}{T_{e}(\mathbf{r})} f_{0}(\mathbf{r}, v)
$$

Substituting (6.8) into (6.4), gives

$$
\mathbf{q}_{e}=-\frac{2 \pi}{3} m_{e} \frac{\nabla T_{e}(\mathbf{r})}{T_{e}(\mathbf{r})} \int_{0}^{\infty} \frac{v^{6}}{\nu_{r}(v)}\left[-\frac{5}{2}+\frac{m_{e} v^{2}}{2 k T_{e}(\mathbf{r})}\right] f_{0}(\mathbf{r}, v) d v
$$

This equation can be written in the form

$$
\mathbf{q}_{e}=-K_{e} \nabla T_{e}(\mathbf{r})
$$

where $K_{e}$ is the thermal conductivity coefficient, given by

$$
K_{e}=\frac{2 \pi}{3} \frac{m_{e}}{T_{e}(\mathbf{r})} \int_{0}^{\infty} \frac{v^{6}}{\nu_{r}(v)}\left[-\frac{5}{2}+\frac{m_{e} v^{2}}{2 k T_{e}(\mathbf{r})}\right] f_{0}(\mathbf{r}, v) d v
$$

In the special case when the collision frequency is independent of velocity, we can write (6.11) as

$$
K_{e}=\frac{2 \pi}{3} \frac{m_{e}}{\nu_{r} T_{e}(\mathbf{r})}\left[-\frac{5}{2} \int_{0}^{\infty} v^{6} f_{0}(\mathbf{r}, v) d v+\frac{m_{e}}{2 k T_{e}(\mathbf{r})} \int_{0}^{\infty} v^{8} f_{0}(\mathbf{r}, v) d v\right]
$$


It can be easily verified that

$$
\begin{gathered}
\int_{0}^{\infty} v^{6} f_{0}(\mathbf{r}, v) d v=\frac{15 k T_{e}(\mathbf{r}) p_{e}}{4 \pi m_{e}^{2}} \\
\int_{0}^{\infty} v^{8} f_{0}(\mathbf{r}, v) d v=\frac{105 k^{2} T_{e}^{2}(\mathbf{r}) p_{e}}{4 \pi m_{e}^{3}}
\end{gathered}
$$

Consequently, substituting (6.13) and (6.14) into (6.12) and simplifying, we obtain the following expression for the thermal conductivity, when $\nu_{r}$ is constant:

$$
K_{e}=\frac{5 k p_{e}}{2 m_{e} \nu_{r}}
$$

\subsection{Thermal Conductivity for the Adiabatic Case}

We consider now the case when the electron kinetic pressure is not constant, but obeys the adiabatic law

$$
p_{e}(\mathbf{r})\left[n_{e}(\mathbf{r})\right]^{-\gamma}=\mathrm{constant}
$$

where $\gamma$ is the adiabatic constant, defined as the ratio of the specific heats at constant pressure and at constant volume, which may be expressed as

$$
\gamma=\frac{2+N}{N}
$$

where $N$ denotes the number of degrees of freedom. Equation (6.16) can also be written as

$$
n_{e}(\mathbf{r})\left[T_{e}(\mathbf{r})\right]^{1 /(1-\gamma)}=\mathrm{constant}
$$

Taking the gradient of the local Maxwellian distribution function (6.5) and making use of (6.18), we obtain

$$
\nabla f_{0}(\mathbf{r}, v)=\left[\frac{1}{\gamma-1}-\frac{3}{2}+\frac{m_{e} v^{2}}{2 k T_{e}(\mathbf{r})}\right] \frac{\nabla T_{e}(\mathbf{r})}{T_{e}(\mathbf{r})} f_{0}(\mathbf{r}, v)
$$

Now we substitute (6.19) into (6.4), which yields the following expression for the heat flow vector:

$$
\mathbf{q}_{e}=-\frac{2 \pi}{3} m_{e} \frac{\nabla T_{e}(\mathbf{r})}{T_{e}(\mathbf{r})} \int_{0}^{\infty} \frac{v^{6}}{\nu_{r}(v)}\left[\frac{1}{\gamma-1}-\frac{3}{2}+\frac{m_{e} v^{2}}{2 k T_{e}(\mathbf{r})}\right] f_{0}(\mathbf{r}, v) d v
$$


With reference to (6.10) we identify the following expression for the thermal conductivity:

$$
K_{e}=\frac{2 \pi}{3} \frac{m_{e}}{T_{e}(\mathbf{r})} \int_{0}^{\infty} \frac{v^{6}}{\nu_{r}(v)}\left[\frac{1}{\gamma-1}-\frac{3}{2}+\frac{m_{e} v^{2}}{2 k T_{e}(\mathbf{r})}\right] f_{0}(\mathbf{r}, v) d v
$$

For the special case in which the collision frequency does not depend on velocity, we can use the results given in (6.13) and (6.14), so that (6.21) simplifies to

$$
K_{e}=\left(2+\frac{1}{\gamma-1}\right) \frac{5 k p_{e}}{2 m_{e} \nu_{r}}
$$

If three degrees of freedom corresponding to the three-dimensional translational motion are considered, we have $\gamma=5 / 3$, so that

$$
K_{e}=\frac{35 k p_{e}}{4 m_{e} \nu_{r}}
$$

When the plasma is immersed in an externally applied magnetostatic field $\mathbf{B}_{0}$, an anisotropy is introduced in the thermal energy flux, so that the thermal conductivity coefficient is replaced by a thermal conductivity dyad $\mathcal{K}$, according to

$$
\mathbf{q}_{e}=-\mathcal{K} \cdot \nabla T_{e}(\mathbf{r})
$$

Expressions for the components of the thermal conductivity dyad can be deduced along lines similar to the calculations presented for the diffusion coefficient dyad in section 5 of this chapter. The derivation of explicit expressions for the components of $\mathcal{K}$ in a magnetized plasma will be left as an exercise for the reader.

\section{PROBLEMS}

22.1 In Cartesian coordinates in velocity space (refer to Fig. 1), with the components expressed in spherical coordinates $(v, \theta, \phi)$, we have

$$
\mathbf{v}=v \widehat{\mathbf{v}}=v\left(\sin \theta \cos \phi \widehat{\mathbf{v}}_{x}+\sin \theta \sin \phi \widehat{\mathbf{v}}_{y}+\cos \theta \widehat{\mathbf{v}}_{z}\right)
$$

(a) Show that the dyad $(\mathbf{v v}) / v^{2}$ can be written in matrix form as

$$
\left(\begin{array}{ccc}
\left(\sin ^{2} \theta \cos ^{2} \phi\right) & \left(\sin ^{2} \theta \sin \phi \cos \phi\right) & (\sin \theta \cos \theta \cos \phi) \\
\left(\sin ^{2} \theta \sin \phi \cos \phi\right) & \left(\sin ^{2} \theta \sin ^{2} \phi\right) & (\sin \theta \cos \theta \sin \phi) \\
(\sin \theta \cos \theta \cos \phi) & (\sin \theta \cos \theta \sin \phi) & \left(\cos ^{2} \theta\right)
\end{array}\right)
$$


(b) Prove the following orthogonality relations

$$
\begin{gathered}
\int_{0}^{\pi} \int_{0}^{2 \pi} \sin \theta d \theta d \phi=4 \pi \\
\int_{0}^{\pi} \int_{0}^{2 \pi} \sin \theta d \theta d \phi v_{i}=0 \\
\int_{0}^{\pi} \int_{0}^{2 \pi} \sin \theta d \theta d \phi v_{i} v_{j}=\frac{4 \pi}{3} v^{2} \delta_{i j} \\
\int_{0}^{\pi} \int_{0}^{2 \pi} \sin \theta d \theta d \phi v_{i} v_{j} v_{k}=0
\end{gathered}
$$

with $i, j, k=x, y, z$ and where $\delta_{i j}$ is the Kronecker delta.

22.2 Using the Maxwell-Boltzmann distribution function (2.22) and the definition (2.23), verify equation (2.24).

22.3 Show that, when the collision frequency is independent of velocity, equation (2.25) reduces to (2.20).

22.4 Consider equation (2.25), which gives the AC electric conductivity of a weakly ionized plasma for a velocity-dependent collision frequency $\nu_{r}(v)$.

(a) Show that in the high-frequency limit $\left(\omega^{2} \gg \nu_{r}^{2}\right)$, we have

$$
\sigma=\frac{\left(\nu_{c}+i \omega\right) n_{0} e^{2}}{m_{e} \omega^{2}}
$$

where

$$
\nu_{c}=\frac{8}{3 \sqrt{\pi}} \int_{0}^{\infty} \nu_{r}(\xi) \xi^{4} \exp \left(-\xi^{2}\right) d \xi
$$

(b) Show that in the low-frequency limit $\left(\omega^{2} \ll \nu_{r}^{2}\right)$, we have

$$
\sigma=\frac{n_{0} e^{2}}{m_{e}}\left[\frac{1}{\nu_{c}^{\prime}}+\frac{i \omega}{\left(\nu_{c}^{\prime \prime}\right)^{2}}\right]
$$

where

$$
\frac{1}{\nu_{c}^{\prime}}=\frac{8}{3 \sqrt{\pi}} \int_{0}^{\infty} \frac{1}{\nu_{r}(\xi)} \xi^{4} \exp \left(-\xi^{2}\right) d \xi
$$




$$
\frac{1}{\left(\nu_{c}\right)^{2}}=\frac{8}{3 \sqrt{\pi}} \int_{0}^{\infty} \frac{1}{\nu_{r}(\xi)^{2}} \xi^{4} \exp \left(-\xi^{2}\right) d \xi
$$

(c) For intermediate frequencies, show that

$$
\sigma=\frac{n_{0} e^{2}}{m_{e}}\left(\nu_{c} K_{1}+i \omega K_{2}\right)
$$

where

$$
\begin{gathered}
K_{1}=\frac{8}{3 \sqrt{\pi}} \frac{1}{\nu_{c} \omega^{2}} \int_{0}^{\infty}\left[1-\frac{\nu_{r}^{2}(\xi)}{\omega^{2}}+\frac{\nu_{r}^{4}(\xi)}{\omega^{4}}+\cdots\right] \nu_{r}(\xi) \xi^{4} \exp \left(-\xi^{2}\right) d \xi \\
K_{2}=\frac{8}{3 \sqrt{\pi}} \frac{1}{\omega^{2}} \int_{0}^{\infty}\left[1-\frac{\nu_{r}^{2}(\xi)}{\omega^{2}}+\frac{\nu_{r}^{4}(\xi)}{\omega^{4}}+\cdots\right] \xi^{4} \exp \left(-\xi^{2}\right) d \xi
\end{gathered}
$$

and where $\nu_{c}$ is the same quantity defined in part (a) for the high-frequency limit.

22.5 If we define an effective collision frequency $\nu_{e f}(\omega)$ such that the longitudinal electric conductivity is given by

$$
\sigma=\frac{i n_{0} e^{2}}{m_{e}\left[\omega+i \nu_{e f}(\omega)\right]}
$$

then, by comparison with (2.18), we find that

$$
\frac{1}{\omega+i \nu_{e f}(\omega)}=-\frac{4 \pi}{3} \frac{1}{n_{0}} \int_{0}^{\infty} \frac{v^{3}}{\omega+i \nu_{r}(v)} \frac{d f_{0}(v)}{d v} d v
$$

(a) Show that in the low-frequency limit $\left(\omega \ll \nu_{e f}\right)$ we have

$$
\frac{1}{\nu_{e f}}=-\frac{4 \pi}{3} \frac{1}{n_{0}} \int_{0}^{\infty} \frac{v^{3}}{\nu_{r}(v)} \frac{d f_{0}(v)}{d v} d v
$$

(b) Show that in the high-frequency limit $\left(\omega \gg \nu_{e f}\right)$ we have

$$
\nu_{e f}=-\frac{4 \pi}{3} \frac{1}{n_{0}} \int_{0}^{\infty} v^{3} \nu_{r}(v) \frac{d f_{0}(v)}{d v} d v
$$

Thus, in both limits $\nu_{e f}$ is independent of $\omega$. 
22.6 In the expression deduced for $\nu_{e f}$ in part (b) of the previous problem (high-frequency limit), consider that $f_{0}$ is the Maxwell-Boltzmann distribution function and that $\nu_{r}(v)=\nu_{0} v^{n}$, where $\nu_{0}$ is a constant and $n$ is an integer.

(a) Show that in this case we have

$$
\nu_{e f}=\frac{4 \nu_{0}}{3 \sqrt{\pi}}\left(\frac{2 k T}{m}\right)^{n / 2} \Gamma\left(\frac{n+5}{2}\right)
$$

where $\Gamma(z)$ is the gamma function defined by

$$
\Gamma(z)=\int_{0}^{\infty} t^{(z-1)} e^{-t} d t
$$

(b) Calculate the average value of the collision frequency $<\nu_{r}(v)>_{0}$, using the Maxwell-Boltzmann distribution function and show that

$$
<\nu_{r}(v)>_{0}=\frac{\nu_{e f}}{1+n / 3}
$$

22.7 Derive (3.34), from equations (3.29) to (3.33).

22.8 Show that (3.30) and (3.31) yield, respectively, (3.37) and (3.38), when $\nu_{r}$ is independent of $v$ for any $f_{0}(v)$.

22.9 Deduce (5.22) and (5.23) starting from the definition of the electron flux vector and the expression for $f_{1}(\mathbf{r}, \mathbf{v})$ given by (5.6), (5.11), (5.12), and (5.13).

22.10 Analyze the heat flow problem in a weakly ionized plasma immersed in an externally applied magnetostatic field $\mathbf{B}_{0}$ and derive expressions for the heat flow vector $\mathbf{q}_{e}$ and for the components of the thermal conductivity dyad $\mathcal{K}$, considering a velocity-dependent collision frequency $\nu_{r}(v)$. Analyze the problem for the adiabatic case and for the case of a constant kinetic pressure. 



\section{APPENDIX}

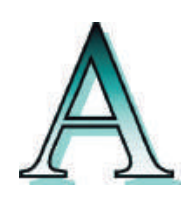

\section{USEFUL VECTOR RELATIONS}

In the following relations, $\mathbf{A}, \mathbf{B}, \mathbf{C}$, and $\mathbf{D}$ represent vector functions, whereas $\phi$ and $\psi$ represent scalar functions:

$$
\begin{gathered}
\mathbf{A} \cdot \mathbf{B}=\mathbf{B} \cdot \mathbf{A}=A_{x} B_{x}+A_{y} B_{y}+A_{z} B_{z} \\
\mathbf{A} \times \mathbf{B}=-\mathbf{B} \times \mathbf{A}=\left|\begin{array}{ccc}
\widehat{\mathbf{x}} & \widehat{\mathbf{y}} & \widehat{\mathbf{z}} \\
A_{x} & A_{y} & A_{z} \\
B_{x} & B_{y} & B_{z}
\end{array}\right|= \\
\left(A_{y} B_{z}-A_{z} B_{y}\right) \widehat{\mathbf{x}}+\left(A_{x} B_{z}-A_{z} B_{x}\right) \widehat{\mathbf{y}}+\left(A_{y} B_{x}-A_{x} B_{y}\right) \widehat{\mathbf{z}} \\
\mathbf{A} \cdot(\mathbf{B} \times \mathbf{C})=(\mathbf{A} \times \mathbf{B}) \cdot \mathbf{C}=(\mathbf{C} \times \mathbf{A}) \cdot \mathbf{B} \\
\mathbf{A} \times(\mathbf{B} \times \mathbf{C})=(\mathbf{A} \cdot \mathbf{C}) \mathbf{B}-(\mathbf{A} \cdot \mathbf{B}) \mathbf{C} \\
(\mathbf{A} \times \mathbf{B}) \times \mathbf{C}=(\mathbf{A} \cdot \mathbf{C}) \mathbf{B}-(\mathbf{B} \cdot \mathbf{C}) \mathbf{A} \\
(\mathbf{A} \times \mathbf{B}) \cdot(\mathbf{C} \times \mathbf{D})=(\mathbf{A} \cdot \mathbf{C})(\mathbf{B} \cdot \mathbf{D})-(\mathbf{A} \cdot \mathbf{D})(\mathbf{B} \cdot \mathbf{C}) \\
(\mathbf{A} \times \mathbf{B}) \times(\mathbf{C} \times \mathbf{D})=[\mathbf{A} \cdot(\mathbf{B} \times \mathbf{D})] \mathbf{C}-[\mathbf{A} \cdot(\mathbf{B} \times \mathbf{C})] \mathbf{D} \\
\nabla(\phi \psi)=\phi \nabla \psi+\psi \nabla \phi \\
\nabla \cdot(\phi \mathbf{A})=\phi \nabla \cdot \mathbf{A}+\mathbf{A} \cdot \nabla \phi \\
\nabla \times(\phi \mathbf{A})=\phi(\nabla \times \mathbf{A})+(\nabla \phi) \times \mathbf{A}
\end{gathered}
$$




$$
\begin{gathered}
\nabla \cdot(\mathbf{A} \times \mathbf{B})=\mathbf{B} \cdot(\nabla \times \mathbf{A})-\mathbf{A} \cdot(\nabla \times \mathbf{B}) \\
\nabla(\mathbf{A} \cdot \mathbf{B})=(\mathbf{A} \cdot \nabla) \mathbf{B}+(\mathbf{B} \cdot \nabla) \mathbf{A}+\mathbf{A} \times(\nabla \times \mathbf{B})+\mathbf{B} \times(\nabla \times \mathbf{A}) \\
\nabla \times(\mathbf{A} \times \mathbf{B})=\mathbf{A}(\nabla \cdot \mathbf{B})+(\mathbf{B} \cdot \nabla) \mathbf{A}-\mathbf{B}(\nabla \cdot \mathbf{A})-(\mathbf{A} \cdot \nabla) \mathbf{B} \\
\nabla \times(\nabla \times \mathbf{A})=\nabla(\nabla \cdot \mathbf{A})-(\nabla \cdot \nabla) \mathbf{A} \\
\nabla \cdot(\nabla \times \mathbf{A})=0 \\
\nabla \times(\nabla \phi)=0 \\
(\nabla \cdot \nabla) \phi=\nabla^{2} \phi
\end{gathered}
$$

If $\mathbf{r}$ is the radius vector, of magnitude $r$, drawn from the origin of a coordinate system to a general point $(x, y, z)$, then

$$
\begin{gathered}
\nabla \cdot \mathbf{r}=3 \\
\nabla \times \mathbf{r}=0 \\
\nabla r=\frac{\mathbf{r}}{r} \\
\nabla\left(\frac{1}{r}\right)=-\frac{\mathbf{r}}{r^{3}} \\
\nabla \cdot\left(\frac{\mathbf{r}}{r^{3}}\right)=-\nabla^{2}\left(\frac{1}{r}\right)=4 \pi \delta(\mathbf{r})
\end{gathered}
$$

In the following integral relations, $V$ is the volume bounded by the closed surface $S$ and $\widehat{\mathbf{n}}$ is a unit vector drawn outwardly to the closed surface $S$ :

$$
\oint_{S} \phi \widehat{\mathbf{n}} d S=\int_{V}(\nabla \phi) d V
$$

Gauss's divergence theorem:

$$
\begin{gathered}
\oint_{S} \mathbf{A} \cdot \widehat{\mathbf{n}} d S=\int_{V}(\nabla \cdot \mathbf{A}) d V \\
\oint_{S}(\widehat{\mathbf{n}} \times \mathbf{A}) d S=\int_{V}(\nabla \times \mathbf{A}) d V
\end{gathered}
$$


Green's first identity:

$$
\oint_{S} \phi(\nabla \psi) \cdot \widehat{\mathbf{n}} d S=\int_{V}\left[\phi\left(\nabla^{2} \psi\right)+(\nabla \phi) \cdot(\nabla \psi)\right] d V
$$

Green's second identity or Green's theorem:

$$
\oint_{S}(\phi \nabla \psi-\psi \nabla \phi) \cdot \widehat{\mathbf{n}} d S=\int_{V}\left(\phi \nabla^{2} \psi-\psi \nabla^{2} \phi\right) d V
$$

Vector version of Green's theorem:

$$
\begin{gathered}
\oint_{S}[\mathbf{B} \times(\nabla \times \mathbf{A})-\mathbf{A} \times(\nabla \times \mathbf{B}) \cdot \widehat{\mathbf{n}} d S= \\
\int_{V}\{\mathbf{A} \cdot[\nabla \times(\nabla \times \mathbf{B})]-\mathbf{B} \cdot[\nabla \times(\nabla \times \mathbf{A})]\} d V
\end{gathered}
$$

If $S$ is an open surface bounded by the closed contour $C$, of which the vector line element is $d \mathbf{l}$, then

$$
\oint_{C} \phi d \mathbf{l}=\int_{S} \widehat{\mathbf{n}} \times(\nabla \phi) d S
$$

Stokes's theorem:

$$
\oint_{C} \mathbf{A} \cdot d \mathbf{l}=\int_{S}(\nabla \times \mathbf{A}) \cdot \widehat{\mathbf{n}} d S
$$

If $\mathcal{D}$ denotes a dyad or a second-order tensor, then

$$
\begin{gathered}
\nabla \cdot(\phi \mathcal{D})=\phi(\nabla \cdot \mathcal{D})+(\nabla \phi) \cdot \mathcal{D} \\
\oint_{S} \mathcal{D} \cdot \widehat{\mathbf{n}} d S=\int_{V}(\nabla \cdot \mathcal{D}) d V
\end{gathered}
$$




\section{APPENDIX}

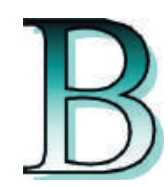

\section{USEFUL RELATIONS IN CARTESIAN AND IN CURVILINEAR COORDINATES}

\section{Cartesian Coordinates}

Orthogonal unit vectors:

$$
\widehat{\mathbf{x}}, \quad \widehat{\mathbf{y}}, \quad \widehat{\mathbf{z}}
$$

Orthogonal line elements:

$$
d x, \quad d y, \quad d z
$$

Components of gradient of a scalar function $\psi$ :

$$
\begin{aligned}
(\nabla \psi)_{x} & =\frac{\partial \psi}{\partial x} \\
(\nabla \psi)_{y} & =\frac{\partial \psi}{\partial y} \\
(\nabla \psi)_{z} & =\frac{\partial \psi}{\partial z}
\end{aligned}
$$

Divergence of a vector function $\mathbf{A}$ :

$$
\nabla \cdot \mathbf{A}=\frac{\partial A_{x}}{\partial x}+\frac{\partial A_{y}}{\partial y}+\frac{\partial A_{z}}{\partial z}
$$

Components of curl of a vector function $\mathbf{A}$ :

$$
\begin{aligned}
& (\nabla \times \mathbf{A})_{x}=\frac{\partial A_{z}}{\partial y}-\frac{\partial A_{y}}{\partial z} \\
& (\nabla \times \mathbf{A})_{y}=\frac{\partial A_{x}}{\partial z}-\frac{\partial A_{z}}{\partial x}
\end{aligned}
$$




$$
(\nabla \times \mathbf{A})_{z}=\frac{\partial A_{y}}{\partial x}-\frac{\partial A_{x}}{\partial y}
$$

Laplacian of a scalar function $\psi$ :

$$
\nabla^{2} \psi=\frac{\partial^{2} \psi}{\partial x^{2}}+\frac{\partial^{2} \psi}{\partial y^{2}}+\frac{\partial^{2} \psi}{\partial z^{2}}
$$

Components of divergence of a dyad or tensor $\mathcal{D}$ :

$$
\begin{aligned}
& (\nabla \cdot \mathcal{D})_{x}=\frac{\partial D_{x x}}{\partial x}+\frac{\partial D_{y x}}{\partial y}+\frac{\partial D_{z x}}{\partial z} \\
& (\nabla \cdot \mathcal{D})_{y}=\frac{\partial D_{x y}}{\partial x}+\frac{\partial D_{y y}}{\partial y}+\frac{\partial D_{z y}}{\partial z} \\
& (\nabla \cdot \mathcal{D})_{z}=\frac{\partial D_{x z}}{\partial x}+\frac{\partial D_{y z}}{\partial y}+\frac{\partial D_{z z}}{\partial z}
\end{aligned}
$$

\section{Cylindrical Coordinates}

Orthogonal unit vectors:

$$
\widehat{\boldsymbol{\rho}}, \widehat{\boldsymbol{\phi}}, \widehat{\mathbf{z}}
$$

Orthogonal line elements:

$$
d \rho, \quad \rho d \phi, \quad d z
$$

Components of gradient of a scalar function $\psi$ :

$$
\begin{gathered}
(\nabla \psi)_{\rho}=\frac{\partial \psi}{\partial \rho} \\
(\nabla \psi)_{\phi}=\frac{1}{\rho} \frac{\partial \psi}{\partial \phi} \\
(\nabla \psi)_{z}=\frac{\partial \psi}{\partial z}
\end{gathered}
$$

Divergence of a vector function $\mathbf{A}$ :

$$
\nabla \cdot \mathbf{A}=\frac{1}{\rho} \frac{\partial\left(\rho A_{\rho}\right)}{\partial \rho}+\frac{1}{\rho} \frac{\partial A_{\phi}}{\partial \phi}+\frac{\partial A_{z}}{\partial z}
$$


Components of curl of a vector function $\mathbf{A}$ :

$$
\begin{gathered}
(\nabla \times \mathbf{A})_{\rho}=\frac{1}{\rho} \frac{\partial A_{z}}{\partial \phi}-\frac{\partial A_{\phi}}{\partial z} \\
(\nabla \times \mathbf{A})_{\phi}=\frac{\partial A_{\rho}}{\partial z}-\frac{\partial A_{z}}{\partial \rho} \\
(\nabla \times \mathbf{A})_{z}=\frac{1}{\rho} \frac{\partial\left(\rho A_{\phi}\right)}{\partial \rho}-\frac{1}{\rho} \frac{\partial A_{\rho}}{\partial \phi}
\end{gathered}
$$

Laplacian of a scalar function $\psi$ :

$$
\nabla^{2} \psi=\frac{1}{\rho} \frac{\partial}{\partial \rho}\left(\rho \frac{\partial \psi}{\partial \rho}\right)+\frac{1}{\rho^{2}} \frac{\partial^{2} \psi}{\partial \phi^{2}}+\frac{\partial^{2} \psi}{\partial z^{2}}
$$

Components of divergence of a dyad or tensor $\mathcal{D}$ :

$$
\begin{gathered}
(\nabla \cdot \mathcal{D})_{\rho}=\frac{1}{\rho} \frac{\partial\left(\rho D_{\rho \rho}\right)}{\partial \rho}+\frac{1}{\rho} \frac{\partial D_{\phi \rho}}{\partial \phi}+\frac{\partial D_{z \rho}}{\partial z}-\frac{1}{\rho} D_{\phi \phi} \\
(\nabla \cdot \mathcal{D})_{\phi}=\frac{1}{\rho} \frac{\partial\left(\rho D_{\rho \phi}\right)}{\partial \rho}+\frac{1}{\rho} \frac{\partial D_{\phi \phi}}{\partial \phi}+\frac{\partial D_{z \phi}}{\partial z}+\frac{1}{\rho} D_{\phi \rho} \\
(\nabla \cdot \mathcal{D})_{z}=\frac{1}{\rho} \frac{\partial\left(\rho D_{\rho z}\right)}{\partial \rho}+\frac{1}{\rho} \frac{\partial D_{\phi z}}{\partial \phi}+\frac{\partial D_{z z}}{\partial z}
\end{gathered}
$$

\section{Spherical Coordinates}

Orthogonal unit vectors:

$$
\widehat{\mathbf{r}}, \widehat{\Theta}, \widehat{\phi}
$$

Orthogonal line elements:

$$
d r, \quad r d \theta, \quad r \sin \theta d \phi
$$

Components of gradient of a scalar function $\psi$ :

$$
\begin{gathered}
(\nabla \psi)_{r}=\frac{\partial \psi}{\partial r} \\
(\nabla \psi)_{\theta}=\frac{1}{r} \frac{\partial \psi}{\partial \theta}
\end{gathered}
$$




$$
(\nabla \psi)_{\phi}=\frac{1}{r \sin \theta} \frac{\partial \psi}{\partial \phi}
$$

Divergence of a vector function A:

$$
\nabla \cdot \mathbf{A}=\frac{1}{r^{2}} \frac{\partial\left(r^{2} A_{r}\right)}{\partial r}+\frac{1}{r \sin \theta} \frac{\partial\left(\sin \theta A_{\theta}\right)}{\partial \theta}+\frac{1}{r \sin \theta} \frac{\partial A_{\phi}}{\partial \phi}
$$

Components of curl of a vector function $\mathbf{A}$ :

$$
\begin{gathered}
(\nabla \times \mathbf{A})_{r}=\frac{1}{r \sin \theta} \frac{\partial\left(\sin \theta A_{\phi}\right)}{\partial \theta}-\frac{1}{r \sin \theta} \frac{\partial A_{\theta}}{\partial \phi} \\
(\nabla \times \mathbf{A})_{\theta}=\frac{1}{r \sin \theta} \frac{\partial A_{r}}{\partial \phi}-\frac{1}{r} \frac{\partial\left(r A_{\phi}\right)}{\partial r} \\
(\nabla \times \mathbf{A})_{\phi}=\frac{1}{r} \frac{\partial\left(r A_{\theta}\right)}{\partial r}-\frac{1}{r} \frac{\partial A_{r}}{\partial \theta}
\end{gathered}
$$

Laplacian of a scalar function $\psi$ :

$$
\nabla^{2} \psi=\frac{1}{r^{2}} \frac{\partial}{\partial r}\left(r^{2} \frac{\partial \psi}{\partial r}\right)+\frac{1}{r^{2} \sin \theta} \frac{\partial}{\partial \theta}\left(\sin \theta \frac{\partial \psi}{\partial \theta}\right)+\frac{1}{r^{2} \sin ^{2} \theta} \frac{\partial^{2} \psi}{\partial \phi^{2}}
$$

Components of divergence of a dyad or tensor $\mathcal{D}$ :

$$
\begin{aligned}
&(\nabla \cdot \mathcal{D})_{r}= \frac{1}{r^{2}} \frac{\partial\left(r^{2} D_{r r}\right)}{\partial r}+\frac{1}{r \sin \theta} \frac{\partial\left(\sin \theta D_{\theta r}\right)}{\partial \theta}+ \\
& \frac{1}{r \sin \theta} \frac{\partial D_{\phi r}}{\partial \phi}-\frac{1}{r}\left(D_{\theta \theta}+D_{\phi \phi}\right) \\
&(\nabla \cdot \mathcal{D})_{\theta}= \frac{1}{r^{2}} \frac{\partial\left(r^{2} D_{r \theta}\right)}{\partial r}+\frac{1}{r \sin \theta} \frac{\partial\left(\sin \theta D_{\theta \theta}\right)}{\partial \theta}+ \\
& \frac{1}{r \sin \theta} \frac{\partial D_{\phi \theta}}{\partial \phi}+\frac{1}{r}\left(D_{\theta r}-\cot \theta D_{\phi \phi}\right) \\
&(\nabla \cdot \mathcal{D})_{\phi}=\frac{1}{r^{2}} \frac{\partial\left(r^{2} D_{r \phi}\right)}{\partial r}+\frac{1}{r \sin \theta} \frac{\partial\left(\sin \theta D_{\theta \phi}\right)}{\partial \theta}+ \\
& \frac{1}{r \sin \theta} \frac{\partial D_{\phi \phi}}{\partial \phi}+\frac{1}{r}\left(D_{\phi r}+\cot \theta D_{\phi \theta}\right)
\end{aligned}
$$




\section{APPENDIX}

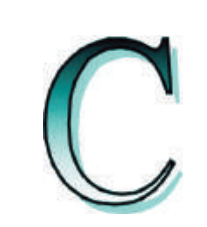

\section{Physical constants \\ (MKSA)}

$\begin{array}{lll}a_{0} & \text { Bohr radius } & 5.292 \times 10^{-11} \mathrm{~m} \\ a m u & \text { Unified atomic mass unit } & 1.661 \times 10^{-27} \mathrm{~kg} \\ c & \text { Speed of light in vacuum } & 2.998 \times 10^{8} \mathrm{~m} / \mathrm{s} \\ e & \text { Electron charge (absolute value) } & 1.602 \times 10^{-19} \text { coulomb } \\ g & \text { Standard acceleration of gravity } & 9.807 \mathrm{~m} / \mathrm{s}^{2} \\ G & \text { Gravitational constant } & 6.671 \times 10^{-11} \text { newton } \mathrm{m}^{2} / \mathrm{kg}^{2} \\ h & \text { Planck's constant } & 6.626 \times 10^{-34} \mathrm{joule} \cdot \mathrm{s} \\ k & \text { Boltzmann's constant } & 1.381 \times 10^{-23} \mathrm{joule} / \mathrm{K} \\ m_{e} & \text { Rest mass of electron } & 9.109 \times 10^{-31} \mathrm{~kg} \\ m_{n} & \text { Rest mass of neutron } & 1.675 \times 10^{-27} \mathrm{~kg} \\ m_{p} & \text { Rest mass of proton } & 1.673 \times 10^{-27} \mathrm{~kg} \\ m_{p} / m_{e} & \text { Proton/electron mass ratio } & 1.836 \times 10^{3} \\ N_{A} & \text { Avogadro's number } & 6.022 \times 10^{23} \mathrm{~mol}-1 \\ N_{L} & \text { Loschmidt's number } & 2.687 \times 10^{25} \mathrm{~m}-3 \\ R & \text { Gas constant }\left(N_{A} k\right) & 8.314 \mathrm{joule} /(\mathrm{K} \mathrm{mol}) \\ r_{e} & \text { Classical electron radius } & 2.818 \times 10^{-15} \mathrm{~m} \\ V_{0} & \text { Molar volume at STP } & 22.4 \times 10^{-3} \mathrm{~m}^{3} / \mathrm{mol} \\ \epsilon_{0} & \text { Permittivity of vacuum } & 8.854 \times 10^{-12} \mathrm{farad} / \mathrm{m} \\ \mu_{0} & \text { Permeability of vacuum } & 4 \pi \times 10^{-7} \mathrm{henry}^{-3} \mathrm{~m} \\ & & \end{array}$

The MKSA system of units is based on four primary quantities: length, mass, time, and current. Its name derives from the units meter $(\mathrm{m})$, kilogram (kg), second (s), and ampere (A). 


\section{APPENDIX}

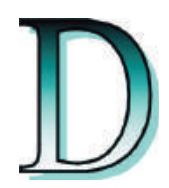

\section{Conversion factors FOR PHYSICAL UNITS}

Capacitance:

Charge:

Conductivity:

Current:

Electric field:

Energy:

Force:

Magnetic field:

Magnetic flux:

Magnetic induction:

Potential:

Power:

Pressure:

Resistance:
1 farad $=(2.998)^{2} \times 10^{11} \mathrm{~cm}$

1 coulomb $=2.998 \times 10^{9}$ statcoulomb

$1 \mathrm{mho} / \mathrm{m}=(2.998)^{2} \times 10^{9} \mathrm{~s}^{-1}$

1 ampere $\equiv 1$ coulomb $/ \mathrm{s}=2.998 \times 10^{9}$ statampere

$1 \mathrm{volt} / \mathrm{m}=\left(2.998 \times 10^{4}\right)^{-1}$ statvolt $/ \mathrm{cm}$

1 joule $=10^{7} \mathrm{erg}$

1 electron volt $(\mathrm{eV})=1.602 \times 10^{-19}$ joule

$1 \mathrm{eV}=k T\left(\right.$ for $\left.T=1.160 \times 10^{4} \mathrm{~K}\right)$

(where $k$ is Boltzmann's constant)

1 rydberg $=13.61 \mathrm{eV}$

1 newton $=10^{5}$ dyne

1 ampere.turn $/ \mathrm{m}=4 \pi \times 10^{-3}$ oersted

1 weber $=10^{8}$ gauss $\cdot \mathrm{cm}^{2}$ (or maxwells)

1 weber $/ \mathrm{m}^{2} \equiv 1$ tesla $=10^{4}$ gauss

1 volt $=\left(2.998 \times 10^{2}\right)^{-1}$ statvolt

1 watt $\equiv 1$ joule $/ \mathrm{s}=10^{2} \mathrm{erg} / \mathrm{s}$

1 newton $/ \mathrm{m}^{2}=10$ dyne $/ \mathrm{cm}^{2}$

$1 \mathrm{~atm}=760 \mathrm{~mm} \mathrm{Hg}=1.013 \times 10^{5}$ newton $/ \mathrm{m}^{2}$

1 torr $=1 \mathrm{~mm} \mathrm{Hg}$

$1 \mathrm{ohm}=(2.998)^{-2} \times 10^{-11} \mathrm{~s} / \mathrm{cm}$ 


\section{APPENDIX}

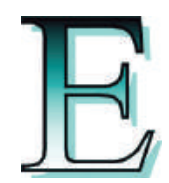

\section{SOME IMPORTANT PLASMA PARAMETERS}

1. Electron plasma frequency

$$
\begin{aligned}
\omega_{p e}= & \left(\frac{n_{e} e^{2}}{m_{e} \epsilon_{0}}\right)^{1 / 2}=56.5 n_{e}^{1 / 2} \quad(\text { in } \mathrm{rad} / \mathrm{s}) \\
& \left(\text { with } n_{e} \text { expressed in } \mathrm{m}^{-3}\right)
\end{aligned}
$$

2. Ion plasma frequency

$$
\omega_{p i}=\left(\frac{n_{i} Z^{2} e^{2}}{m_{i} \epsilon_{0}}\right)^{1 / 2}
$$

3. Debye length

$$
\lambda_{D}=\left(\frac{\epsilon_{0} k T}{n_{e} e^{2}}\right)^{1 / 2}=69.0\left(\frac{T}{n_{e}}\right)^{1 / 2} \quad \text { (in m) }
$$

(with $n_{e}$ in $\mathrm{m}^{-3}$ and $T$ in degrees $\mathrm{K}$ )

4. Electron cyclotron frequency

$$
\begin{aligned}
\Omega_{c e}= & \frac{e B}{m_{e}}=1.76 \times 10^{11} B \quad(\text { in } \mathrm{rad} / \mathrm{s}) \\
& (\text { with } B \text { expressed in tesla) }
\end{aligned}
$$


5. Ion cyclotron frequency

$$
\Omega_{c i}=\frac{Z e B}{m_{i}}
$$

6. Particle magnetic moment

$$
\mathbf{m}=-\frac{W_{\perp}}{B^{2}} \mathbf{B}=-\frac{\left(m v_{\perp}^{2}\right) / 2}{B^{2}} \mathbf{B}
$$

7. Electron cyclotron radius

$$
r_{c e}=\frac{v_{e \perp}}{\Omega_{c e}}=\frac{m_{e} v_{e \perp}}{e B}
$$

8. Ion cyclotron radius

$$
r_{c i}=\frac{v_{i \perp}}{\Omega_{c i}}=\frac{m_{i} v_{i \perp}}{Z e B}
$$

9. Number of electrons in a Debye sphere

$$
\begin{gathered}
N_{D}=\frac{4 \pi}{3} n_{e} \lambda_{D}^{3}=1.37 \times 10^{6} \frac{T^{3 / 2}}{n_{e}^{1 / 2}} \\
\left(\text { with } T \text { in degrees } \mathrm{K} \text { and } n_{e} \text { in } \mathrm{m}^{-3}\right. \text { ) }
\end{gathered}
$$

10. Alfvén velocity

$$
\mathbf{V}_{\mathbf{A}}=\frac{\mathbf{B}}{\left(\mu_{0} \rho_{m}\right)^{1 / 2}}
$$

11. DC conductivity

$$
\sigma_{0}=\frac{n_{e} e^{2}}{m_{e} \nu_{e}}
$$

12. Electron free diffusion coefficient

$$
D_{e}=\frac{k T_{e}}{m_{e} \nu_{e}}
$$


13. Ambipolar diffusion coefficient

$$
D_{a}=\frac{k\left(T_{e}+T_{i}\right)}{\left(m_{e} \nu_{e n}+m_{i} \nu_{i n}\right)}
$$

14. Magnetic pressure

$$
p_{m}=\frac{B_{0}^{2}}{2 \mu_{0}}
$$

15. Magnetic viscosity

$$
\eta_{m}=\frac{1}{\mu_{0} \sigma_{0}}
$$

16. Magnetic Reynolds number

$$
R_{m}=\frac{u L}{\eta_{m}}
$$

17. Coulomb cut-off parameter

$$
\Lambda=12 \pi n_{e} \lambda_{D}^{3}=9 N_{D}=1.23 \times 10^{7} \frac{T^{3 / 2}}{n_{e}^{1 / 2}}
$$

(with $T$ in degrees $\mathrm{K}$ and $n_{e}$ in $\mathrm{m}^{-3}$ )

18. Electron collision frequencies for momentum transfer

$$
\begin{gathered}
\nu_{e i}=3.62 \times 10^{-6} n_{i} T_{e}^{-3 / 2} \ln \Lambda \quad\left(\text { in s }^{-1}\right) \\
\nu_{e n}=2.60 \times 10^{4} \sigma^{2} n_{n} T_{e}^{1 / 2} \quad\left(\text { in s s }^{-1}\right)
\end{gathered}
$$

(with $T$ in degrees $\mathrm{K}$ and $n_{i, n}$ expressed in $\mathrm{m}^{-3}$. The parameter $\sigma$ denotes the sum of the radii of the colliding particles and is of the order of $10^{-10} \mathrm{~m}$, whereas $\ln \Lambda$ is typically about 10 .) 


\section{APPENDIX}

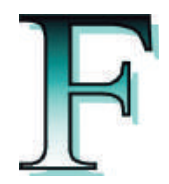

\section{ApProximate MAGNITUDES IN SOME TYPICAL PLASMAS}

\begin{tabular}{|r|c|c|c|c|c|}
\hline PLASMA TYPE & $n_{0}$ & $\mathrm{~T}$ & $\omega_{p e}$ & $\lambda_{D}$ & $n_{0} \lambda_{D}^{3}$ \\
\hline Interstellar gas & $10^{6}$ & $10^{-1}$ & $6 \times 10^{4}$ & 1 & $10^{6}$ \\
Interplanetary gas & $10^{8}$ & 1 & $6 \times 10^{5}$ & 1 & $10^{8}$ \\
Solar corona & $10^{12}$ & $10^{2}$ & $6 \times 10^{7}$ & $10^{-1}$ & $10^{9}$ \\
Solar atmosphere & $10^{20}$ & 1 & $6 \times 10^{11}$ & $10^{-6}$ & $10^{2}$ \\
Ionosphere & $10^{12}$ & $10^{-1}$ & $6 \times 10^{7}$ & $10^{-3}$ & $10^{4}$ \\
Gas discharge & $10^{20}$ & 1 & $6 \times 10^{11}$ & $10^{-6}$ & $10^{2}$ \\
Hot plasma & $10^{20}$ & $10^{2}$ & $6 \times 10^{11}$ & $10^{-5}$ & $10^{5}$ \\
Diffuse hot plasma & $10^{18}$ & $10^{2}$ & $6 \times 10^{10}$ & $10^{-4}$ & $10^{6}$ \\
Dense hot plasma & $10^{22}$ & $10^{2}$ & $6 \times 10^{12}$ & $10^{-6}$ & $10^{4}$ \\
Thermonuclear & $10^{22}$ & $10^{4}$ & $6 \times 10^{12}$ & $10^{-5}$ & $10^{7}$ \\
\hline
\end{tabular}

(Values of $n_{0}$ expressed in $\mathrm{m}^{-3}, T$ in $\mathrm{eV}, \omega_{p e}$ in $\mathrm{s}^{-1}$, and $\lambda_{D}$ in $\mathrm{m}$ ). 



\section{INDEX}

Adiabatic gas law, 208, 212, 299 cylindrical compression, 305 linear compression, 305 spherical compression, 306

Adiabatic invariants, 75, 81, 108 longitudinal, 81-84 magnetic flux, 75, 88, 111 magnetic moment, 75-77, 111 Adiabatic sound speed, 209, 376 Alfvén approximation, 60 Alfvén velocity, 377, 381 Alfvén waves, 376, 383, 391 compressional, 383 oblique, 387 pure, 386 shear, 384

Ambipolar diffusion, 5, 256-260 Angular momentum, 39, 568

Anomalous dispersion, 369

Appleton-Hartree equation, 418

Apse line, 568

Archimede's spiral, 13, 14

Atmospheric whistlers, 439-442

Attachment, 200

Average values, 141, 142
B BGKY hierarchy, 597

Bennett pinch, 332-335

Bernstein modes, 546-550

Bessel function, 540-543

of first kind, 541

of second kind, 543

Beta parameter, 321

Binary collisions, 162, 561-569

Blackbody radiation, 6

Bohm criterion, 288

Bohm diffusion, 5, 262

Bohm-Gross dispersion relation, 456

Boltzmann collision term, 590-598 assumptions involved, 596, 597 weakly ionized plasma, 607-611

Boltzmann equation,

129-136, 590-598

conditions for validity, 596, 597

derivation of, 128-134, 590-594

integro-differential

equation, 594

Boltzmann factor, 181-183

Boltzmann's constant, 662

Boltzmann's H theorem, 599-601

Bouncing phenomenon, 340 
Bound-bound transitions, 6

Boundary layer, 279-288

between wall and plasma, 279

Bremsstrahlung radiation, 6

Carrier frequency, 368

Causality principle, 496

Center of mass, 562

Central force, 566

Centrifugal force, 85-87

Characteristic plasma parameters, 12

Charge density, 137, 198, 220

Charged particle orbits, 33-121

Chew-Goldberger-Low equations, 302-304

Circular polarization, 102,360

Circularly polarized wave, 102, 360, 419

Coefficient of viscosity, 297, 310

Cold plasma model, 210

Collision, 162, 561-569

binary, 162, 561

direct, 162

elastic, 560

inelastic, 560

inverse, 162

Collision frequency, 135, 203, 588

Collisionless Boltzmann

equation, 129

Collisionless damping, 5, 500-503

Conductivity, 242-250, 629-640

for constant collision

frequency, 245

electric, 243, 245

thermal, 268, 648-650

for velocity-dependent

collision frequency, 638

Configuration space, 123
Confinement of plasmas, 18-22, 319

Confinement schemes, 18

Confinement time, 21

Constitutive relations, 32, 250

Continuity equation, 197, 222

Contour of integration, 496

Controlled thermonuclear fusion, 18

Conversion factors, 663

Correlation, 597

Coulomb collisions, 570

Coulomb cross section, 580

Coulomb force, 561, 570

Counterstreaming plasmas, 506, 514

Cross section, 572-578

differential, 574

momentum transfer, 577

total, 576

Current density, 137, 146, 198, 220

electric, 137, 198, 220

mass, 147, 220

Curvature drift, 84, 87

Cusp field, 347

Cut-off frequency, 406

Cycloidal trajectory, 52

Cyclotron damping, 531

Cyclotron frequency, 37, 39

Cyclotron heating, 108

Cyclotron motion, 37-44

Cyclotron radiation, 6

Cyclotron resonance, 106

Cyclotron waves, 422, 452

Damping, 394, 406, 500

collisional, 406

collisionless, 5, 500-503

of MHD waves, 394

Debye length, 7

Debye potential, 276, 292

Debye shielding, 7, 273, 278 
Debye sphere, 8

Deflection angle, 562, 564

Degeneracy factor, 184

Delta function, 367, 506

Detailed balance principle, 162, 601

Dielectric constant, 251

Differential cross section, 574

Diffusion coefficient, 251-255, 640-647

ambipolar diffusion, 256

for constant collision

frequency, 254

free diffusion, 251

for velocity-dependent

collision frequency, 645, 646

Diffusion current density, 254

Diffusion equation, 253, 255

Diffusion time constant, 253

Diffusion in velocity space, 615,616

Dipole moment, magnetic, 44

Dirac's delta function, 367, 506

Dispersion relations, 414, 457

$471,494,525,545$

definition of, 367

for electrostatic plasma

waves, 494

for propagation across

B field, 545

for propagation along

B field, 525

Dispersive medium, 369

Distribution function, 126, 127

definition of, 126

local Maxwellian, 169

loss-cone, 138

Maxwell-Boltzmann, 165

modified Maxwellian, 1169

properties of, 126, 127

Doppler shift, 190, 532

Double adiabatic equations, 302
Drift velocity, 52, 115

curvature of B, 84

electromagnetic, 52

external force, 55

gradient of $\mathrm{B}, 74$

polarization, 97

summary, 115

Dynamic pinch, 335

Earth's magnetic field, 14, 80

Einstein relation, 255

Electric charge density, 137, 198, 219

Electric conductivity, 242-250

Electric current density,

137, 198, 220

Electric permittivity of vacuum, 662

Electrokinetic pressure dyad, 230

Electromagnetic stress dyad, 213

Electromagnetic waves,

$351,515,552$

circularly polarized, 360

in free space, 351-374

group velocity of, 368

in hot isotropic plasma, 503

phase velocity of, 354

propagation across $\mathrm{B}$ in a

hot plasma, 534

propagation along $\mathrm{B}$ in a

hot plasma, 516

Electron gas, 240, 242

Electron plasma frequency, 9, 10

Electron plasma oscillations, 269

Electron plasma waves, 456, 490

Electron runaway effect, 588

Electrostatic mode, 491

Electrostatic potential, 7

Electrostatic shielding, 7, 273 
Energy conservation equation, 204, 226

Energy density, 204, 226

Energy distribution function, 178

Energy flow in electromagnetic waves, 363

Energy integral, 307

Entropy, 189, 599

Entropy-maximum principle, 170,604

Equation of charge conservation, 31,228

Equation of continuity, 197, 222

Equation of motion, 33, 200, 223

for conducting fluids, 223

for Lorentz force, 33

for multispecies plasma, 202

Equation of state, 153, 209

Equilibrium, 161-192

distribution function, 165

pinch, 326

solution of Boltzmann equation, 161

Error function, 294

Evanescent wave, 406

Extraordinary mode, 424, 546, 553

$\mathbf{F}$ araday rotation, 444

Fermi's acceleration, 84

First adiabatic invariant, 75-77

First-order orbit theory, 60

Fluid models, 193-237

cold plasma, 210

warm plasma, 211

Flux, 143-146

definition of, 143

energy, 155

heat, 154

momentum, 147 particle, 146

Fokker-Planck coefficients, 614, 615

Fokker-Planck equation, 612

Fourier integral, 366

Fourth state of matter, 1

Free diffusion, 251, 640

Free diffusion coefficient, 253, 641

Free-bound transition, 6

Free-free transition, 6

Frozen field lines, 312

Fusion, thermonuclear, 18

Fusion cross sections, 19

Fusion reactions, 18

Gas discharges, 3

Gauss's theorem, 151, 656

Generalized Ohm's law, 229

Gradient drift, 74

Gradient of magnetic field, 64

Gravitational constant, 662

Gravitational force, 55

Group velocity, 368

Guiding center, 38, 39

Gyrofrequency, 37-40

Gyromagnetic resonance, 104

Gyroperiod, 45

Gyroradius, 40

H-theorem, 599

Hall conductivity, 246

Hall current, 52, 246

Hall effect term, 235

Hard sphere collisions, 578

Heat conduction coefficient, $208,268,650$

Heat conduction equation, 208, 268, 650

Heat flux triad, 154

Heat flux vector, 154 
Helical orbit in B field, 39

Helicon, 442

Hierarchy of equation, 193

High-frequency plasma oscillations, 9, 269

High-frequency waves, 400-452 propagating across $\mathrm{B}$ in cold plasmas, 423 propagating along $\mathrm{B}$ in cold plasmas, 419

Hydrodynamic derivative, 202 Hydrodynamic viscosity, 310 Hydromagnetic equations, 235, 299 Hydromagnetic waves, 375-399

Alfvén, 376, 383, 386

fast, 387,391

magnetosonic, 377, 383

slow, 387, 391

Hydrostatic pressure, 148, 152

Ideal gas law, 153

Impact parameter, 562 cut-off value, 583 definition of, 562

Index of refraction, 415

Inelastic collisions, 560

Inertial confinement, 21

Infinite conductivity limit, 236

Inner radiation belt, 14, 15

Instabilities, 341, 506, 532 configuration space, 341 growth rate, 534

kink, 345

sausage, 342

in two plasma streams, 506 velocity space, 532

Interaction potential, 566

Interplanetary magnetic field, 13, 14

Interplanetary plasma, 13

Inverse collisions, 162

Ion acoustic wave, 458

Ion cyclotron damping, 531

Ion cyclotron resonance, 420

Ion plasma frequency, 664

Ion plasma mode, 456

Ionization, 2, 200

Ionization energy, 184

Ionization by photons, 2

Ionosphere, 16

Irreversibility, 601

Isobaric surfaces, 318

Isothermal atmosphere, 191

Isothermal compression, 305

Isothermal sound speed, 209

Isotropic pressure tensor, 153

J acobian of coordinate transformation, 132, 594

Joint probability function, 597

Joule heating, 227

Kinematic viscosity, 310

Kinetic energy density, 156

Kinetic pressure, 152, 221

Kinetic temperature, 152

Kronecker delta, 152

Krook collision model, 134

Lagrange multipliers, 605

Lagrangian of charged particle, 35,36

Landau damping, 500-505 descriptive account, 502 electromagnetic wave, 505 longitudinal wave, 500-503

Landau damping constant, 502 
Langevin equation, 238-242

Langmuir oscillation, 269-273

Laplace equation, 273

Laplacian operator, 658-661

Larmor period, 45

Larmor radius, 40

Lawson criterion, 22

Left circular polarization, 361

Left circularly polarized wave, 361

Legendre polynomial, 607

Linearly polarized wave, 358

Liouville's theorem, 131

Longitudinal adiabatic invariant, 81-84

Longitudinal pinch, 325

Longitudinal plasma wave, 456, 491

Long-range force, 561

Lorentz factor, 34

Lorentz force, 33

Lorentz gas, 240

Loss cone, 79, 80

Loss-cone distribution, 138

Lower hybrid resonance, 427

Lumped macroscopic variables, 219

Macroinstabilities, 341

Macroscopic equations, 193-237

for cold plasma, 210

for conducting fluid, 219

for warm plasma, 211

Magnetic axis, 319

Magnetic bottle, 77

Magnetic compression, 112

Magnetic cusp field, 347

Magnetic energy density, 32, 214

Magnetic field geometry, 61-66

curvature terms, 64

divergence terms, 62 gradient terms, 64

shear terms, 65

Magnetic flux tube, 312

Magnetic force term, 34

Magnetic heating, 113

Magnetic mirror effect, 77

Magnetic moment, 44

Magnetic permeability of vacuum, 662

Magnetic pressure, 316

Magnetic pumping, 113

Magnetic Reynolds number, 309

Magnetic rigidity, 40

Magnetic scalar potential, 273

Magnetic stress dyad, 316

Magnetic surfaces, 318

Magnetic susceptibility, 49

Magnetic tension, 317

Magnetic viscosity, 309

Magnetically trapped particles, 14, 77

Magnetization current density, 48

Magnetization vector, 47

Magnetohydrodynamic equations, 234

Magnetohydrodynamic waves, 375-399

fast wave, 383,387

for propagation across B field, 382

for propagation along B field, 383

for propagation at arbitrary directions, 384

slow wave, 384,387

Magnetohydrostatics, 316

Magnetoionic medium, 401

Magnetoionic theory, 400

Magnetopause, 14, 15 
Magnetosheath, 15

Magnetosonic wave, 377, 383

Magnetosphere, 14, 15

Mass conservation

equation, 197, 222

Mass density, 219

Mass of electron, 662

Mass flux, 147

Mass of proton, 662

Maxwell-Boltzmann distribution function, 165

Maxwell equations, 26, 32

Maxwell molecules, 622, 626

Maxwell stress tensor, 213

Maxwellian distribution (same as Maxwell-Boltzmann distribution)

Mean free path, 624

Mean velocity, 128

MHD approximations, 234

MHD generator, 22

Mirror loss cone, 79, 80

Mirror ratio, 77

Mobility, 105, 242, 247

Molecular chaos, 591

Moments of Boltzmann equation, 193

Moments of distribution function, 157

Momentum conservation equation, 200, 223

Momentum flux, 147

Most probable energy, 189

Most probable speed, 177

Nabla $(\nabla)$ operator, 131, 658-661 in configuration space, 131 in velocity space, 131

Natural coordinates,
102-104, 519, 638

Navier-Stokes equation, 310

Newton's law of motion, 33

Nuclear fusion devices, 18

Number density, 128, 142

Ohm's law, 229 generalyzed, 229 simplified, 233

One-fluid theory, 219-236

Orbital magnetic moment, 44

Ordinary mode, 424, 545, 550

Outer radiation belt, 14, 15

Parallel conductivity, 246

Parker momentum equation, 300

Particle current density, 146, 220

Particle drifts, 115

Particle interactions, 560-588

Peculiar velocity, 142

Pedersen conductivity, 246

Perfect ambipolar diffusion, 259

Perfect gas, 153

Permeability of free space, 662

Permittivity of free space, 662

Perpendicular conductivity, 246

Phase space, 123

Phase velocity, 354

Phase velocity diagrams, 388, 432

Physical constants, 662

Photoionization, 2

Pinch effect, 325-350

Bennett pinch, 332

dynamic pinch, 335

equilibrium pinch, 326

sheath current model, 330

snowplow model, 336

Pitch angle, 39

Planck's constant, 662 
Plane waves, 352

Plasma, 1-32

applications, 17

definition, 1, 6

general properties, 1

macroscopic phenomena, 4

naturally occurring, 11

physical parameters, 12, 664

quantitative criteria for definition, 6

Plasma angular frequency, 9, 271

for electrons, 10, 664

for ions, 664

Plasma behavior, 4

Plasma confinement, 18, 319

Plasma dynamics, 25

self-consistent formulation, 25

Plasma heating, 108, 112

by electromagnetic wave, 108

by magnetic compression, 112

Plasma oscillations, 9, 269

Plasma parameter g, 9

Plasma probe, 288

Plasma production, 2

Plasma propulsion, 23

Plasma sheath, 279

Plasma sound speed, 458

Plasma wave, 457

Poisson equation, 292, 510

Polarization current density, 97

Polarization drift, 95, 97

Polarization of waves, 358

Poles in contour integration, 496

Potential, electric, 35

Debye, 276

magnetic, 35

Potential at a wall, 281

Poynting vector, 363
Poynting's theorem, 32

Pressure, definition, 148

dyad, 149

magnetic, 316

scalar, 152

Pressure force, 149

Propagation bands, 410, 422, 427

Propagation vector, 354

Quantum effects, 24, 184

Quasistatic mode, 546, 553

Radiation, 6

blackbody, 6

bremsstrahlung, 6

cyclotron, 6

Radio communication, 24

Radius of gyration, 40

Random velocity, 142

Ratio of specific heats, 208

Recoil angle, 564

Recombination, 3, 200

Reduced mass, 563

Reentry plasma sheath, 24

Reflection by magnetic mirror, 77

Reflection points, 430, 476

cold plasma, 430, 431

warm plasma, 476

Relative permittivity, 98, 251

Relative velocity, 561

Relaxation collision

frequency, 135

Relaxation model, 135

Relaxation time, 135

Residue of integrand, 496

Resonance, 430, 476

cold plasma, 430, 431

cyclotron, 106 
upper hybrid, 426, 427

warm plasma, 476

Resonance distribution function, 513

Reversibility paradox, 598

Reynolds number, 309

kinematic, 311

magnetic, 310

Right circularly polarized waves, 361

Root mean square velocity, 173

Runaway effect, 588

Rutherford scattering, 581

Saha equation, 184

Scalar potential, 35, 273

electrostatic, 35 magnetic, 273

Scalar pressure, 152, 221

Scattering, 569

by coulomb force, 580

large angle, 582

small angle, 583

Scattering angle, 564

Scattering cross section, 572-582

differential, 574

momentum transfer, 577

total, 576

Semiconductor, 25

Shear force, 150

Shear stress, 150

Shielding, 7, 273, 278

Short-range force, 4, 622, 626

Simple pole singularity, 496, 513

Single-particle distribution function, 126

Skin depth, 398

Snow-plow model, 336

Solar wind, 13
Solid-state plasma, 24

Sound speed, 375, 454

electron gas, 456

fluid, 375

plasma, 458

Sound wave, 375

Space charge wave, 273, 456

Space physics, 11-17

Speed distribution

function, 174

Speed of light, 662

Spherical harmonic expansion, 607

Stable equilibrium, 347

Standing waves, 411

Statistical mechanics, 122, 184

Stellarator, 19

Stokes's theorem, 657

Summation invariants, 164

TEM wave mode (same as ordinary mode), 425, 554 cold plasma, 425 hot plasma, 545, 550, 554 warm plasma, 478

Temperature, 152

Test particle, 273

Thermal conductivity, 268, 647-650

scalar, 648, 650

tensor, 268, 650

Thermal energy, 154, 222

density, 222

flux, 154

Thermal velocity, 142

Thermionic energy converter, 24

Thermodynamic

equilibrium, 161-192

Thermonuclear process, 18

Theta pinch, 21, 321 
TM wave mode (extraordinary mode), 424, 545

cold plasma, 424, 554

hot plasma, 545,553

warm plasma, 468

Tokamak, 19

Toroidal magnetic field, 19, 91

Total time derivative, 202

in configuration space, 202

in phase space, 131

Transmission through plasma slab, 447

Transmission at vacuum-plasma interface, 447

Transport coefficients, 238, 628

diffusion, 254, 646

electric conductivity, 245, 638

thermal conductivity, 268, 648

viscosity, 297

Trapping process, 502

Traveling waves, 410

Triad, heat flux, 154

total energy flux, 155

Truncation of moment

equations, 194

Tunneling effect, 410, 449

Two-body collisions, 561

Two-stream instability, 506, 514
Ultraviolet radiation, 16

Uncertainty principle, 372, 373

Unit dyad, 121

Upper hybrid resonance, 427

V an Allen radiation belts, 14, 15

Variational technique, 604

Vector potential, 35

Velocity distribution function, 126, 127

Velocity space, 123

Velocity of whole fluid, 220

Viscosity, 310

kinematic, 310

magnetic, 310

Viscous-stress equation, 217

Vlasov equation, 136

W arm plasma model, 211

Wave energy, 363

Wave-normal plane, 352

Wave number, 354

Wave packet, 366

Wave-particle interaction, 502, 532

Wavelength, 354

Weakly ionized plasma, 4

Whistler waves, 439 


\section{About the author}

Jose Augusto BitTencourt (born June 08, 1947, in Araguari, Minas Gerais, Brazil) is a physicist who for more than 40 years has been actively researching Plasma Physics, Space Science, and Aeronomy. More recently, in the past 15 years, he has also done extensive research on Nutritional Biochemistry.

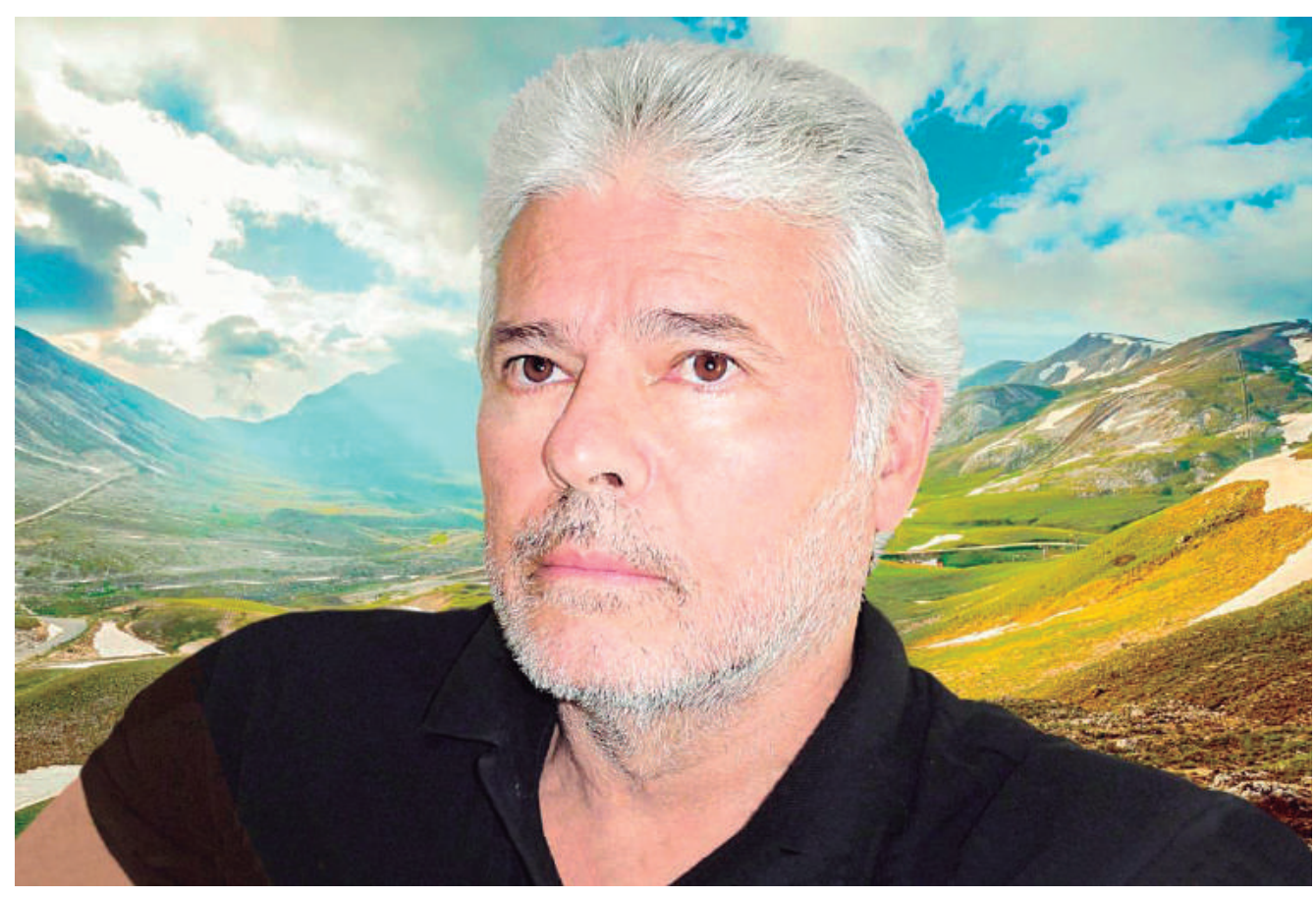

He received his Ph.D. degree in Physics from the University of Texas at Dallas UTD (USA) in 1975. Since then he has been a Research Scientist and Graduate-level Professor at the National Institute for Space Research - INPE (Sao Jose dos Campos, Sao Paulo, Brazil) and has served many scientific organizations. He is also graduated (B.Sc.) in Chemical Engineering (1970) from the Federal University of Minas Gerais - UFMG (Belo Horizonte, Brazil) and received a Master of Sciences (M.Sc.) degree in Space Science from the National Institute for Space Research INPE in 1972. 
His research interests include basic and applied plasma physics, ionospheric and magnetospheric plasmas, upper atmosphere physics, aeronomy, plasma dynamical processes, and computer simulation of plasma phenomena both in the laboratory and in space.

He has published more than 200 papers and scientific articles in well-known international research journals such as Journal of Geophysical Research (Space Science), Geophysical Research Letters, Journal of Atmospheric and SolarTerrestrial Physics, Planetary and Space Sciences, Advances in Space Research, Plasma Physics and Controlled Fusion, Applied Physics Letters, and Geophysical Journal International Annales Geophysicae.

$\mathrm{He}$ is also author of the book Fundamentals of Plasma Physics (704p., Third Edition, 2004 -Hardcover; 2010 - Paperback) published by Springer (USA).

Since 1975 he has taught graduate-level courses at INPE on plasma physics, electrodynamics, propagation of electromagnetic waves, thermodynamics, kinetic theory, quantum mechanics, ionospheric physics, and dynamics of the upper atmosphere. He has been also a graduate-level Conference Professor on plasma physics at the Aeronautics Institute of Technology - ITA (Sao Jose dos Campos) and, since 2004, a research collaborator at the University of Paraiba Valley - UNIVAP (Sao Jose dos Campos).

At INPE he was Head of the graduate courses on Space Science and on Plasma Physics, and also Head of the Aeronomy Department. He has been also an official member of the Commission $\mathrm{H}$ (Waves in Plasmas) of the International Union of Radio Science - URSI and Associate Editor of the Brazilian Journal of Geophysics, in the field of Aeronomy. Since 1989 he is a Research Fellowship member of the National Council for Scientific and Technological Development - CNPq (Brazil).

An especial interest has been dedicated to the study of Nutritional Science and the biochemical effects of the many nutrients in the human body. This research resulted in the books Nutricao e Saude: Como Fazer Escolhas Sensatas em Dieta e Nutricao (Portuguese, Sixth Edition, 2018) and The Power of Carbohydrates, Proteins, and Lipids (Fourth Edition, 2018), both printed by CreateSpace, an Amazon.com Company (USA). 



\section{J.A. BITTENCOURT}

\section{PUNDAIVIENTALS OP PLASIVIA PHYSICS}

\section{FOURTH EDITION}

Fundamentals of Plasma Physics is a comprehensive textbook designed to present a logical and unified treatment of the fundamentals of plasma physics based on statistical kinetic theory, with applications to a variety of important plasma phenomena. The clarity and completeness of the text makes it suitable for self-learning.

Throughout the text the emphasis is on clarity, rather than formality. The various derivations are explained in detail and, wherever possible, the physical interpretations are emphasized. The mathematical treatment is set out in great detail, carrying out steps that are usually left to the reader. The problems form an integral part of the text and most of them were designed in such a way as to provide a guideline for the student, stating intermediate steps with answers.

The book is intended primarily for advanced undergraduate and first year graduate students meeting the subject of plasma physics for the first time and is suitable for those who have taken classical mechanics, electrodynamics and mathematics beyond sophomore level.

It is a valuable compendium for any serious student of plasma physics at the level of research student or research worker and it is also of interest to researchers in other related fields, such as space physics and applied electromagnetism. 


\section{J.A. BitTENCOURT}

\section{FUNDAIVIENTALS OF PLASIVIA PHYSICS}

\section{FOURTH EDITION}

Fundamentals of Plasma Physics is a comprehensive textbook designed to present a logical and unified treatment of the fundamentals of plasma physics based on statistical kinetic theory, with applications to a variety of important plasma phenomena. The clarity and completeness of the text makes it suitable for self-learning.

Throughout the text the emphasis is on clarity, rather than formality. The various derivations are explained in detail and, wherever possible, the physical interpretations are emphasized. The mathematical treatment is set out in great detail, carrying out steps that are usually left to the reader. The problems form an integral part of the text and most of them were designed in such a way as to provide a guideline for the student, stating intermediate steps with answers.

The book is intended primarily for advanced undergraduate and first year graduate students meeting the subject of plasma physics for the first time and is suitable for those who have taken classical mechanics, electrodynamics and mathematics beyond sophomore level.

It is a valuable compendium for any serious student of plasma physics at the level of research student or research worker and it is also of interest to researchers in other related fields, such as space physics and applied electromagnetism.

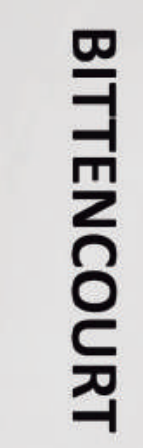

J.A.BITTENCOURT

FUNDAMENTALS OF
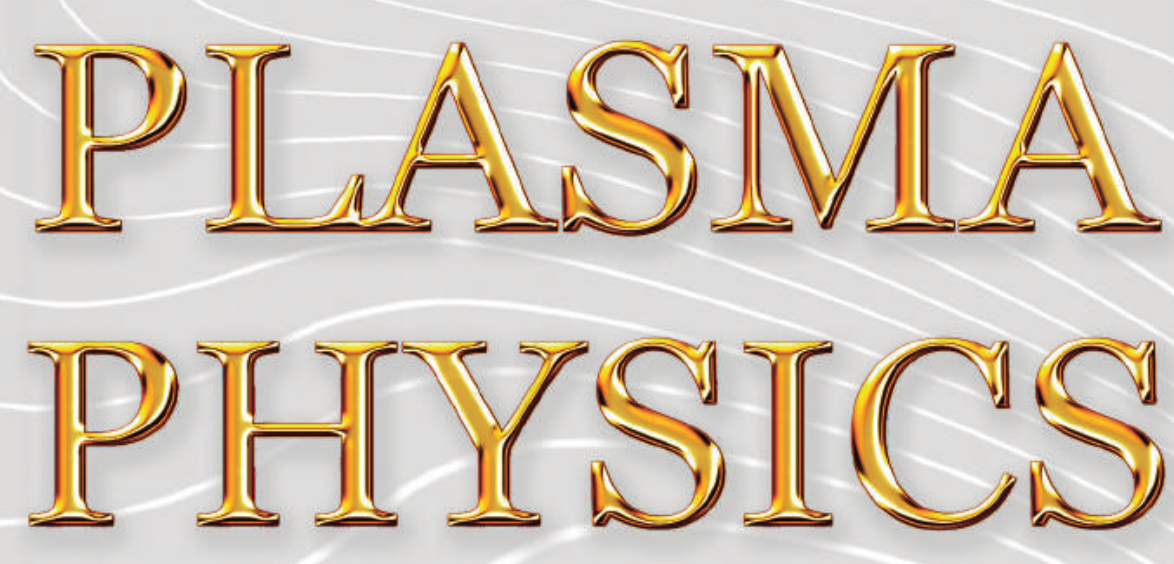

FOURTH

EDITION 